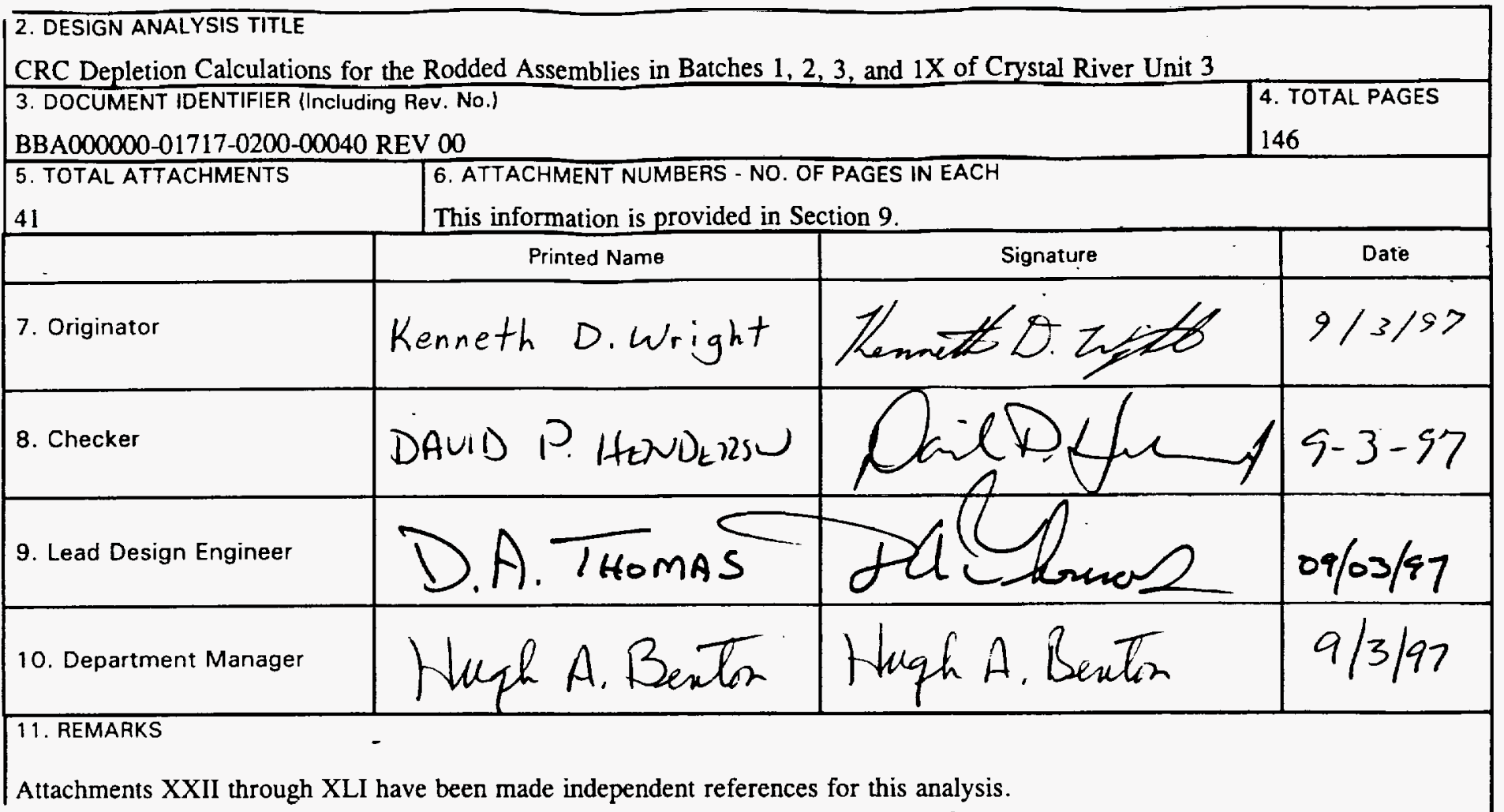

This analysis was started under procedure QAP-3-9, REV 06. During the development of this analysis QAP-3-9 was modified to REV 07. Impact review HAB-IR-002 was performed, and it was determined that this analysis should be completed under procedure QAP-3-9, REV 06, with some procedure modifications as documented in the impact review. 
2. DESIGN ANALYSIS TITLE

CRC Depletion Calculations for the Rodded Assemblies in Batches 1, 2, 3, and 1X of Crystal River Unit 3

3. DOCUMENT IDENTIFIER (Including Rev. No.)

BBA000000-01717-0200-00040 REV 00

4. Revision No.

00

Initial Issuance Copy
5. Description of Revision

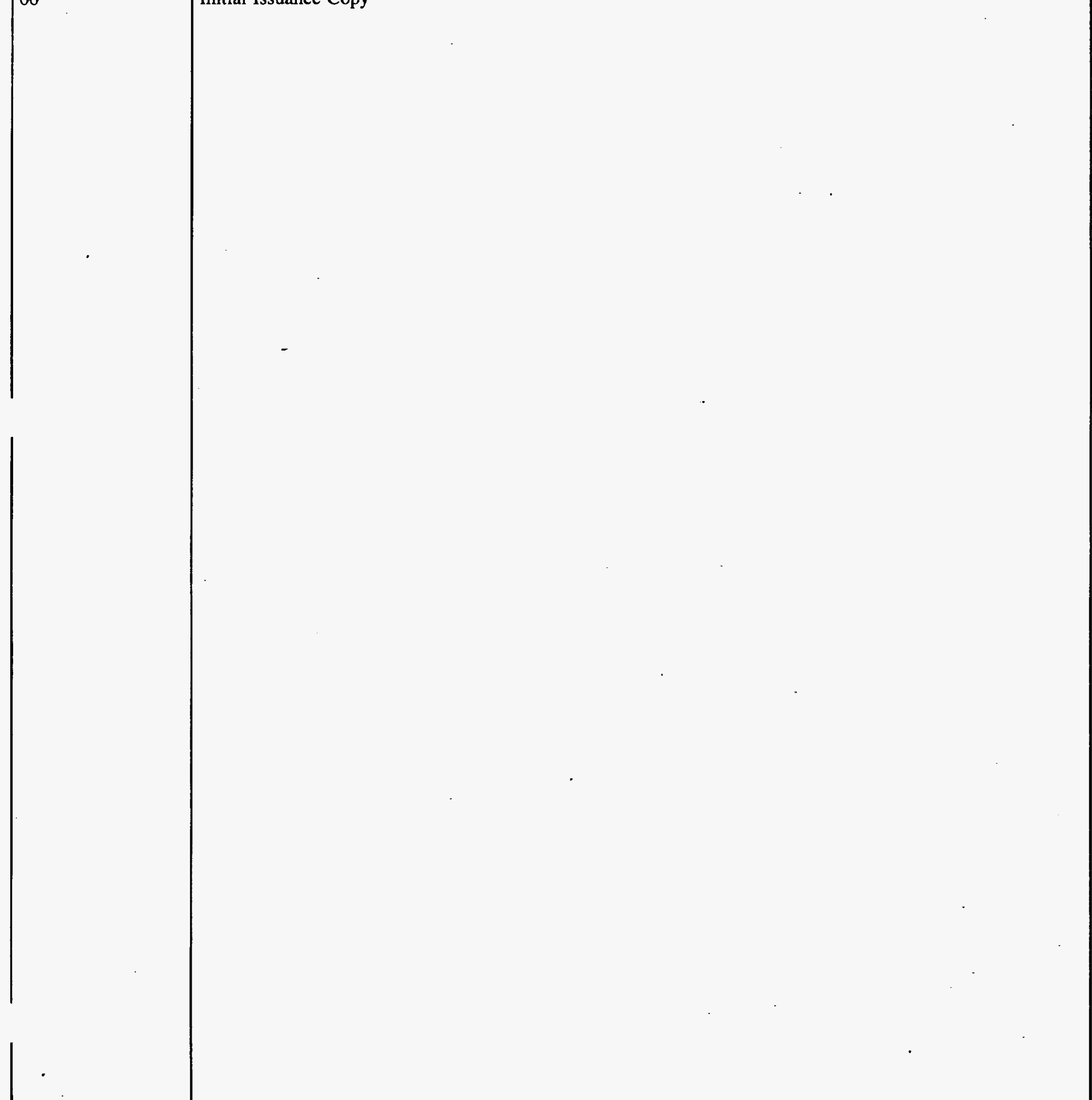




\section{Table of Contents}

Item

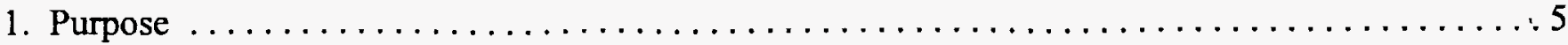

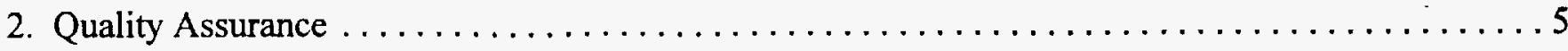

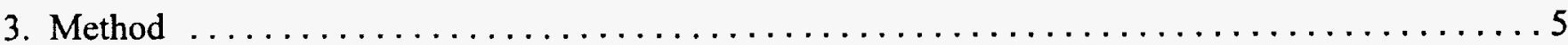

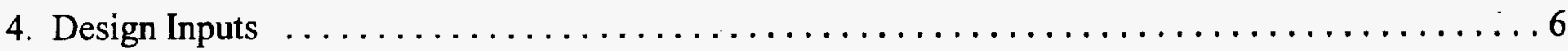

4.1 Design Parameters ........................................ 6

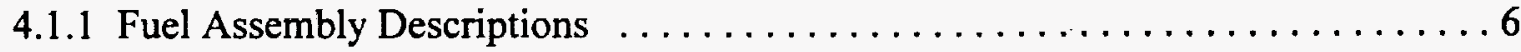

4.1.2 Burnable Poison Rod Assembly (BPRA) Description $\ldots \ldots \ldots \ldots \ldots \ldots \ldots$

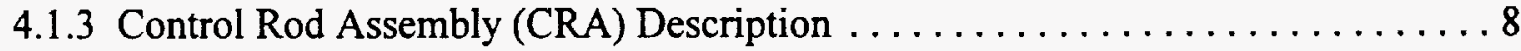

4.1.4 Axial Power Shaping Rod Assembly (APSRA) Description . . . . . . . . . . 8

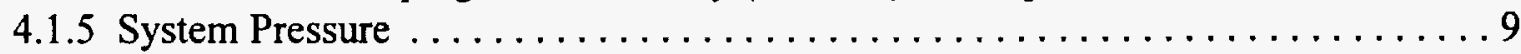

4.1.6 Fuel Assembly Insertion, Burnable Poison Loading, and Control Bank Insertion

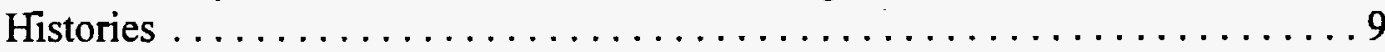

4.1.7 Fuel Assembly Insertion Position Histories . . . . . . . . . . . . . . . 10

4.1 .8 Reactor Cycle History Data $\ldots \ldots \ldots \ldots \ldots \ldots \ldots \ldots \ldots \ldots \ldots \ldots \ldots \ldots \ldots \ldots \ldots$

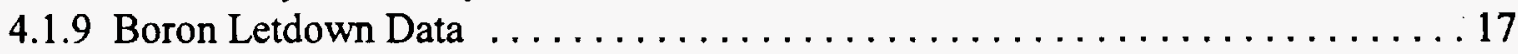

4.1.10 Burnup, Fuel Temperature, and Moderator Specific Volume Data ......... 23

4.1.11 Insertion History Data for CRA's and APSRA's $\ldots \ldots \ldots \ldots \ldots \ldots \ldots$

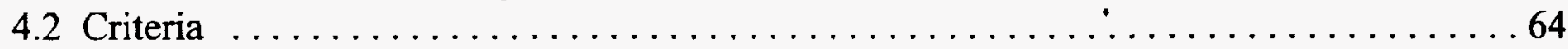

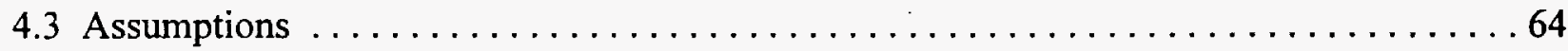

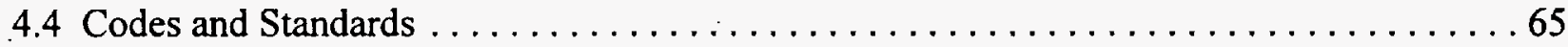

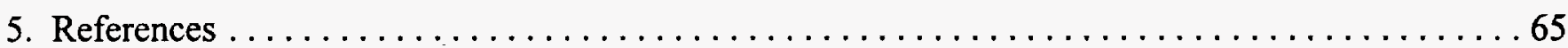

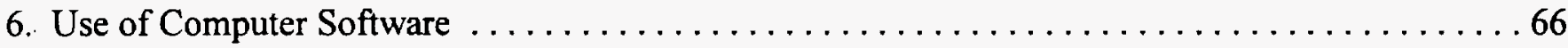

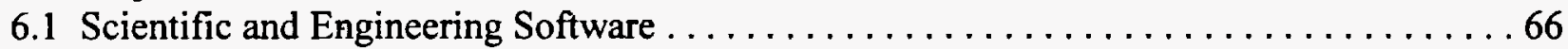

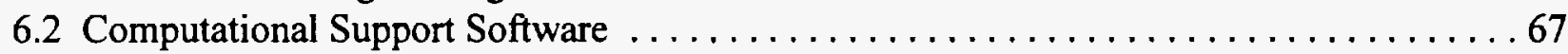

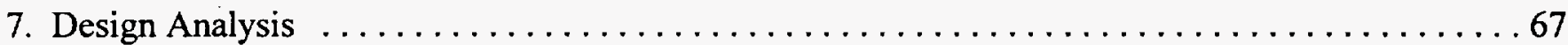

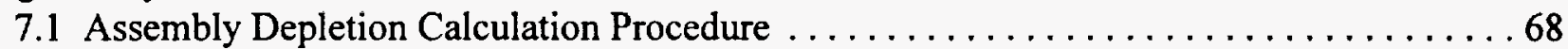

7.2 Path B Unit Cell Model Development . . . . . . . . . . . . . . . . . . . . 68

7.3 Cycle Irradiation History Layouts for the Depletion of the Rodded Assemblies in Batches 1,

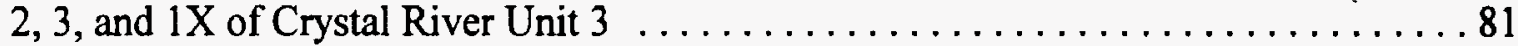

7.4 The Commercial Reactor Assembly Follow Taskmaster (CRAFT) Code \& Usage . . . . . 114

7.5 Input \& Output Filename Descriptions for CRAFT and SAS2H . . . . . . . . . . 117

7.6 Rodded Assembly Depletion Calculations for Fuel Batches 1, 2, 3, and 1X of Crystal River

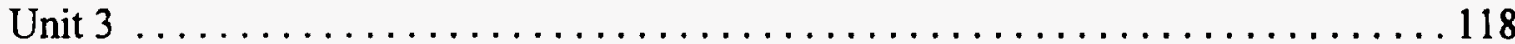

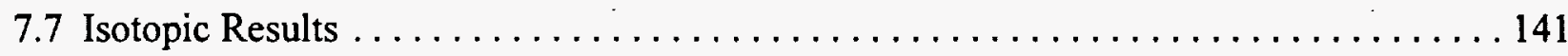




\section{Waste Package Development}

Design Analysis

Title: CRC Depletion Calculations for the Rodded Assemblies in Batches 1, 2, 3, and $1 \mathrm{X}$ of Crystal River Unit 3

Document Identifier: BBA000000-01717-0200-00040 REV 00

Page 4 of 146

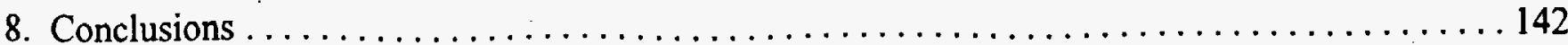

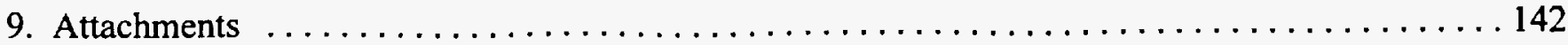


Title: CRC Depletion Calculations for the Rodded Assemblies in Batches 1, 2, 3, and $1 X$ of Crystal River Unit 3 Document Identifier: BBA000000-01717-0200-00040 REV 00

Page 5 of 146

\section{Purpose}

The purpose of this design analysis is to document the SAS2H depletion calculations of certain rodded fuel assemblies from batches 1,2,3, and $1 \mathrm{X}$ of the Crystal River Unit 3 pressurized water reactor (PWR) that are required for Commercial Reactor Critical (CRC) evaluations to support development of the disposal criticality methodology. A rodded assembly is one that contains a control rod assembly (CRA) or an axial power shaping rod assembly (APSRA) for some period of time during its irradiation history. The objective of this analysis is to provide SAS2H calculated isotopic compositions of depleted fuel and depleted burnable poison for each fuel assembly to be used in subsequent CRC reactivity calculations containing the fuel assemblies.

\section{Quality Assurance}

The Quality Assurance (QA) program applies to this analysis. The work reported in this document is part of the criticality disposal methodology development that will eventually support the License Application Design phase. This activity, when appropriately confirmed, can impact the proper functioning of the Mined Geologic Disposal System (MGDS) waste package; the waste package has been identified as an MǴDS Q-List item important to safety and waste isolation (pp. 4, 15, Ref. 5.6). The waste package is on the Q-List by direct inclusion by the Department of Energy (DOE), without conducting a QAP-2-3 evaluation. As determined by an evaluation performed in accordance with QAP2-0, Conduct of Activities, the work performed for this analysis is subject to Quality Assurance Requirements and Description (QARD; Ref. 5.2) requirements. As specified in NLP-3-18, "Documentation of QA Controls on Drawings, Specifications, Design Analyses, and Technical Documents", the development of this analysis is subject to QA controls. The Waste Package Development Department (WPDD) responsible manager has selected the applicable procedural controls for this activity commensurate with the work control activity evaluation entitled "Perform Criticality, Thermal, Structural, and Shielding Analyses" (Ref. 5.1).

The work reported in this document is part of the CRC neutronic analyses to support the development of the disposal criticality methodology. All design parameters utilized in this analysis are from a qualified source (Ref. 5.3) which was developed under a U. S. Nuclear Regulatory Commission approved QA program. Therefore, all design parameters utilized in this analysis are qualified.

\section{Method}

The method for obtaining fuel and burnable poison isotopic compositions at specific points during each assembly's irradiation history is based upon the use of the SAS2H control module of the SCALE 4.3 modular code system (Ref. 5.4). The effective full-power day (EFPD) times during reactor operation that correspond to a CRC evaluation are called "statepoints". An assembly depletion calculation between two CRC statepoints is called a "statepoint calculation". The depleted fuel and depleted burnable poison compositions may be used in subsequent $\mathrm{CRC}$ reactivity calculations. The SAS2H input decks are automatically developed by the CRAFT program which is a software routine documented in Sections 7.4 and 7.5 and Attachment I of this analysis. The SAS2H input decks and depletion models are developed using actual assembly specifications, actual assembly irradiation 
Title: CRC Depletion Calculations for the Rodded Assemblies in Batches 1, 2, 3, and $1 \mathrm{X}$ of Crystal River Unit 3 Document Identifier: BBA000000-01717-0200-00040 REV 00

Page 6 of 146

histories, and actual CRA and APSRA insertion histories. The isotopic results obtained from the SAS2H depletion calculations are reviewed and analyzed to identify any anomalous results which may propagate to subsequent $\mathrm{CRC}$ reactivity calculations and ultimately impact the development of the disposal criticality methodology.

\section{Design Inputs}

The design inputs documented in this analysis describe the design specifications and irradiation histories for certain rodded fuel assemblies in fuel batches 1, 2, 3, and $1 \mathrm{X}$ of the Crystal River Unit 3 PWR. All of the design inputs listed in this analysis are obtained from reference 5.3 , which is a reference summarizing the necessary input parameters.

\subsection{Design Parameters}

\subsubsection{Fuel Assembly Descriptions}

Table 4.1.1-1 contains a description of the rodded fuel assemblies corresponding to fuel batches 1, 2, 3, and $1 \mathrm{X}$ of Crystal River Unit 3. All fuel assemblies within a given fuel batch have the same characteristics as identified in Table 4.1.1-1.

Table 4.1.1-1 Fuel Assembly Descriptions for Batches 1, 2, 3, and $1 \mathrm{X}$ of Crystal River Unit 3

\begin{tabular}{||c|c|c|c|c||}
\cline { 2 - 5 } \multicolumn{1}{c|}{} & \multicolumn{4}{c|}{ Fuel Batch Identifier } \\
\hline \hline Parameter & $\mathbf{1}$ & $\mathbf{2}$ & $\mathbf{3}$ & $\mathbf{1 X}$ \\
\hline Assembly Type & Mark-B3 & Mark-B3 & Mark-B3 & Mark-B2 \\
\hline Weight Percent U-235 & 1.93 & 2.54 & 2.83 & 2.00 \\
\hline kg of U per Assembly & 463.63 & 463.63 & 463.63 & 468.62 \\
\hline Fuel Height $(\mathrm{cm})$ & 360.172 & 360.172 & 360.172 & 360.172 \\
\hline Fuel Pellet OD $(\mathrm{cm})$ & 0.9398 & 0.9398 & 0.9398 & 0.9398 \\
\hline Fuel Rod Clad OD $(\mathrm{cm})$ & 1.0922 & 1.0922 & 1.0922 & 1.0922 \\
\hline Fuel Rod Clad ID ${ }^{2}(\mathrm{~cm})$ & 0.95758 & 0.95758 & 0.95758 & 0.95758 \\
\hline Spacer Grid Material & Inconel & Inconel & Inconel & Inconel \\
\hline $\begin{array}{c}\text { Volume Fraction of Spacer } \\
\text { Grid in Moderator }\end{array}$ & 0.005757609 & 0.005757609 & 0.005757609 & 0.005757609 \\
\hline Guide Tube Material & Zircaloy & Zircaloy & Zircaloy & Zircaloy \\
\hline Guide Tube OD $(\mathrm{cm})$ & 1.3462 & 1.3462 & 1.3462 & 1.3462 \\
\hline
\end{tabular}


Title: CRC Depletion Calculations for the Rodded Assemblies in Batches 1, 2, 3, and $1 \mathrm{X}$ of Crystal River Unit 3

\begin{tabular}{||c|c|c|c|c||}
\cline { 2 - 5 } \multicolumn{1}{c|}{} & \multicolumn{4}{c|}{ Fuel Batch Identifier } \\
\hline Parameter & 1 & $\mathbf{2}$ & $\mathbf{3}$ & $\mathbf{1 X}$ \\
\hline Guide Tube ID (cm) & 1.26492 & 1.26492 & 1.26492 & 1.26492 \\
\hline Instrument Tube Material & Zircaloy & Zircaloy & Zircaloy & Zircaloy \\
\hline Instrument Tube OD (cm) & 1.38193 & 1.38193 & 1.38193 & 1.38193 \\
\hline Instrument Tube ID (cm) & 1.12014 & 1.12014 & 1.12014 & 1.12014 \\
\hline Array Size & $15 \times 15$ & $15 \times 15$ & $15 \times 15$ & $15 \times 15$ \\
\hline Number of Fuel Rods & 208 & 208 & 208 & 208 \\
\hline Number of Guide Tubes & 16 & 16 & 16 & 16 \\
\hline Number of Instr. Tubes & 1 & 1 & 1 & 1 \\
\hline Pin Pitch (cm) & 1.44272 & 1.44272 & 1.44272 & 1.44272 \\
\hline Assembly Pitch (cm) & 21.81098 & 21.81098 & 21.81098 & 21.81098 \\
\hline
\end{tabular}

${ }^{1} \mathrm{OD}=$ Outer Diameter

${ }^{2} \mathrm{ID}=$ Inner Diameter

\subsubsection{Burnable Poison Rod Assembly (BPRA) Description}

Table 4.1.2-1 contains a description of the burnable poison rod assembly utilized in the various fuel assemblies from fuel batches 1,2,3, and $1 \mathrm{X}$ of Crystal River Unit 3. The rods of the BPRA are inserted into the guide tubes of the fuel assembly during irradiation to produce a lower thermal flux which ultimately allows for longer fuel assembly burnup and better core power distributions.

Table 4.1.2-1 BPRA Descriptions for Use in Batches 1, 2, 3, and $1 \mathrm{X}$ of Crystal River Unit 3

\begin{tabular}{|c|c|}
\hline Parameter & Value \\
\hline \hline Burnable Poison (BP) Material & $\mathrm{Al}_{2} \mathrm{O}_{3}-\mathrm{B}_{4} \mathrm{C}$ \\
\hline BP Density (g/cc) & 3.7 \\
\hline BP Pellet OD (cm) & 0.8636 \\
\hline Burnable Poison Rod (BPR) Cladding Material & Zircaloy \\
\hline BPR Cladding OD (cm) & 1.0922 \\
\hline BPR Cladding ID (cm) & 0.9144 \\
\hline Number of BPR's in a BPRA & 16 \\
\hline
\end{tabular}


Title: CRC Depletion Calculations for the Rodded Assemblies in Batches 1, 2, 3, and $1 \mathrm{X}$ of Crystal River Unit 3 Document Identifier: BBA000000-01717-0200-00040 REV 00

Page 8 of 146

\subsubsection{Control Rod Assembly (CRA) Description}

Table 4.1.3-1 contains a description of the control rod assembly utilized in the various fuel assemblies from fuel batches 1,2,3, and $1 \mathrm{X}$ of Crystal River Unit 3. The rods of the CRA are inserted into the guide tubes of the fuel assembly during irradiation to produce a local thermal flux depression which provides a mechanism for controlling the core power distribution (both radially and axially). Operating with CRAs inserted may also allow for extended fuel assembly burnup.

Table 4.1.3-1 CRA Descriptions for Use in Batches 1, 2,3, and 1X of Crystal River Unit 3

\begin{tabular}{|c|c|}
\hline Parameter & Value \\
\hline Control Rod Neutron Absorbing Material & $\begin{array}{c}\text { Ag-In-Cd with a 79.8, 15.0, and 5.0 weight } \\
\text { percent by mass composition, respectively }\end{array}$ \\
\hline Ag-In-Cd Density (g/cc) & 10.17 \\
\hline Absorber Pellet OD (cm) & 0.99568 \\
\hline Control Rod (CR) Cladding Material & Stainless Steel 304 (SS304) \\
\hline CR Cladding OD (cm) & 1.11760 \\
\hline CR Cladding ID (cm) & 1.01092 \\
\hline Number of CR's in a CRA & 16 \\
\hline
\end{tabular}

\subsubsection{Axial Power Shaping Rod Assembly (APSRA) Description}

Table 4.1.4-1 contains a description of the axial power shaping rod assembly utilized in the various fuel assemblies from fuel batches 1,2,3, and 1X of Crystal River Unit 3. The rods of the APSRA are inserted into the guide tubes of the fuel assembly during irradiation to produce a local thermal flux depression which provides a mechanism for controlling the core power distribution (both radially and axially). Operating with APSRAs inserted allows for a more uniform axial burnup which results in longer average fuel assembly burnups. There are two types of APSRAs (black and grey) utilized in Crystal River Unit 3. The black APSRAs utilize Ag-In-Cd as the neutron absorbing material. The grey APSRAs utilize Inconel as the neutron absorbing material. As the names indicate, the black APSRAs have a larger macroscopic neutron absorption cross-section than the grey APSRAs. The grey APSRAs were not inserted in any of the assemblies documented in this analysis.

Table 4.1.4-1 Black APSRA Descriptions for Use in Batches 1, 2, 3, and 1X of Crystal River Unit 3

\begin{tabular}{|c|c||}
\hline Parameter & Value \\
\hline APSRA Neutron Absorbing Material & $\begin{array}{l}\text { Ag-In-Cd with a 79.8, 15.0, and 5.0 weight } \\
\text { percent by mass composition, respectively }\end{array}$ \\
\hline
\end{tabular}


Title: CRC Depletion Calculations for the Rodded Assemblies in Batches 1, 2, 3, and $1 \mathrm{X}$ of Crystal River Unit 3 Document Identifier: BBA000000-01717-0200-00040 REV 00

Page 9 of 146

\begin{tabular}{|c|c|}
\hline Parameter & Value \\
\hline \hline Ag-In-Cd Density $(\mathrm{g} / \mathrm{cc})$ & 10.17 \\
\hline Absorber Pellet OD (cm) & 0.99568 \\
\hline $\begin{array}{c}\text { Mxial Power Shaping Rod (APSR) Cladding } \\
\text { Material }\end{array}$ & Stainless Steel 304 (SS304) \\
\hline APSR Cladding OD (cm) & 1.11760 \\
\hline APSR Cladding ID (cm) & 1.01092 \\
\hline Number of APSR's in an APSRA & 16 \\
\hline
\end{tabular}

\subsubsection{System Pressure}

Crystal River Unit 3 is a pressurized water reactor that operates at a constant pressure of $2200 \mathrm{psi}$ (pounds per square inch).

\subsubsection{Fuel Assembly Insertion, Burnable Poison Loading, and Control Bank Insertion Histories}

The actual irradiation histories of the fuel assemblies in batches 1,2,3, and $1 \mathrm{X}$ must be used to perform the various assembly depletion calculations relevant to the CRC analyses. Table 4.1.6-1 contains the assembly insertion, burnable poison (BP) loading, and control bank insertion histories for the rodded assemblies in fuel batches 1, 2, 3, and IX which are required for the CRC analyses of Crystal River Unit 3. For fuel management purposes, some fuel assemblies were removed from the reactor and re-inserted in a later cycle as shown in Table 4.1.6-1.

Table 4.1.6-1 Crystal River Unit 3, Batches 1, 2, 3, and 1X, Rodded Fuel Assembly Insertion, BP Loading, and Control Bank Insertion Histories

\begin{tabular}{|c|c|c|c|c|c|c|c|c|}
\hline \multirow{2}{*}{$\begin{array}{c}\text { Assembly } \\
\text { Number/Batch }\end{array}$} & \multicolumn{7}{|c|}{ Assembly Location in Cycle } & \multirow[b]{2}{*}{ Comments } \\
\hline & $1 \mathrm{~A}$ & 1B & 2 & 3 & 7 & 8 & 9 & \\
\hline $\mathrm{Al} / 2$ & CR7 & CR7 & $\mathrm{X}$ & $\mathrm{X}$ & & & & The "A" designation \\
\hline $\mathrm{A} 4 / 2$ & 1.18 & $\mathrm{X}$ & CR6 & & & & & in the assembly \\
\hline $\mathrm{A} 5 / 1$ & CR6 & CR6 & & & & & & number indicates \\
\hline A7/3 & CR4 & CR7 & CR6 & $\mathrm{X}$ & & & & that Cycle $1 \dot{A}$ is the \\
\hline $\mathrm{A} 14 / 2$ & 1.34 & $\mathrm{X}$ & $\mathrm{X}$ & & $\mathrm{CR} 7$ & & & assembly's initial \\
\hline $\mathrm{A} 18 / 1$ & CR8 & & & & & $\mathrm{X}$ & & insertion cycle. \\
\hline $\mathrm{A} 18 \mathrm{a} / 1$ & CR8 & & & & & & $\mathrm{X}$ & For BP rods, the wt $\%$ \\
\hline $\mathrm{A} 18 \mathrm{~b} / 1$ & CR8 & CR8 & & & & & & of $\mathrm{BAC}$ in the $\mathrm{BP}$ \\
\hline $\mathrm{A} 20 / 3$ & CR7 & $\mathrm{X}$ & $\mathrm{X}$ & $\mathrm{x}$ & & & & material is given. \\
\hline
\end{tabular}


Title: CRC Depletion Calculations for the Rodded Assemblies in Batches 1, 2, 3, and $1 \mathrm{X}$ of Crystal River Unit 3 Document Identifier: BBA000000-01717-0200-00040 REV 00

Page 10 of 146

\begin{tabular}{|c|c|c|c|c|c|c|c|c|}
\hline \multirow{2}{*}{$\begin{array}{c}\text { Assembly } \\
\text { Number/Batch }\end{array}$} & \multicolumn{7}{|c|}{ Assembly Location in Cycle } & \multirow[b]{2}{*}{ Comments } \\
\hline & $1 \mathrm{~A}$ & $1 \mathrm{~B}$ & 2 & 3 & 7 & 8 & 9 & \\
\hline $\mathrm{A} 22 / 1$ & $\mathrm{CR} 6$ & CR6 & & & & & & CR4=Control Bank 4 \\
\hline $\mathrm{A} 23 / 2$ & 1.01 & $\mathrm{X}$ & $\mathrm{CR} 7$ & $\mathrm{X}$ & & & & CR6 $=$ Control Bank 6 \\
\hline $\mathrm{A} 23 \mathrm{a} / 2$ & 1.01 & $\mathrm{X}$ & CR7 & $\mathrm{X}$ & & & & CR7=Control Bank 7 \\
\hline $\mathrm{A} 25 / 3$ & $\mathrm{X}$ & $\mathrm{X}$ & $\mathrm{X}$ & CR7 & & & & CR8=Control Bank 8 \\
\hline $\mathrm{A} 25 \mathrm{a} / 3$ & $\mathrm{X}$ & $\mathrm{X}$ & $\bar{X}$ & CR6 & & & & CR8=Black APSR \\
\hline $\mathrm{A} 26 / 1$ & $\mathrm{CR} 4$ & CR8 & & & & & & The "X" indicates \\
\hline A28 / 3 & $\bar{x}$ & $\mathrm{X}$ & CR8 & $\mathrm{X}$ & & & & that the assembly is \\
\hline $\mathrm{A} 29 / 3$ & $X$ & $\mathrm{X}$ & $X$ & CR7 & & & & present in the cycle \\
\hline $01 / 1 \mathrm{x}$ & $X^{1}$ & $\mathrm{CR7}$ & & & & & & indicated. \\
\hline
\end{tabular}

'The "O1" assembly has a control rod insertion history from when it was inserted in Cycle 1 of Oconee.

\subsubsection{Fuel Assembly Insertion Position Histories}

The positions of the various assemblies in the core must be known to correlate the burnup, fuel temperature, and moderator specific volume data with the appropriate assembly. The assembly position data is also used to document the depletion cases so that the isotopic results may be identified at a later time for a specific assembly in a particular position of the core. Table 4.1.7-1 contains the assembly position histories for the rodded assemblies in batches 1,2,3, and $1 \mathrm{X}$ of Crystal River Unit 3 which are relevant to the $\mathrm{CRC}$ analyses. The assembly position identifiers refer to locations in a one-eighth core symmetrical arrangement for Crystal River Unit 3 as shown in Figure 4.1.7-1. The integer values (1-29) shown in Figure 4.1.7-1 are used in the SAS2H depletion calculations to identify the various assembly locations.

Table 4.1.7-1 Assembly Position Histories for the Rodded Assemblies from Batches 1, 2, 3, and $1 \mathrm{X}$ of Crystal River Unit 3

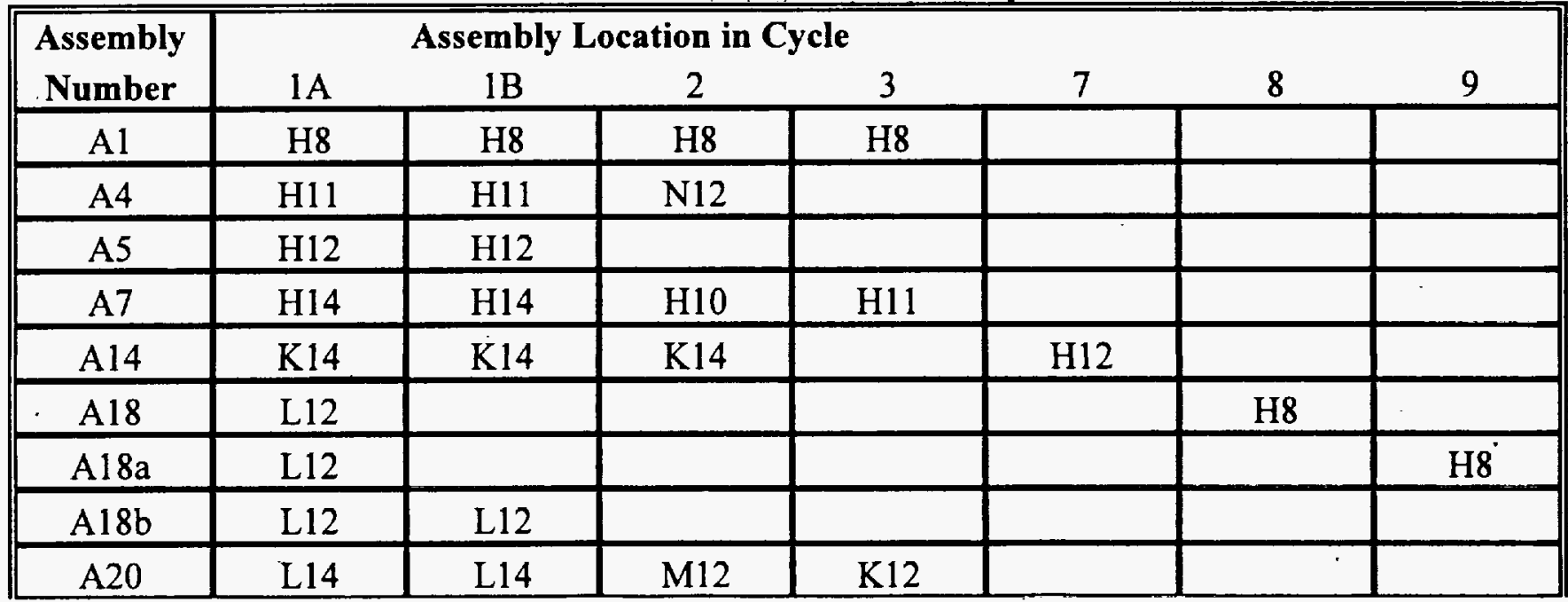


Title: CRC Depletion Calculations for the Rodded Assemblies in Batches 1, 2, 3, and $1 \mathrm{X}$ of Crystal River Unit 3

\begin{tabular}{|c|c|c|c|c|c|c|c||}
\hline $\begin{array}{c}\text { Assembly } \\
\text { Number }\end{array}$ & 1A & 1B & 2 & 3 & 7 & 9 \\
\hline A22 & M11 & M11 & & & & & \\
\hline A23 & M12 & M12 & H14 & H13 & & & \\
\hline A23a & M12 & M12 & L10 & M11 & & & \\
\hline A25 & M14 & M14 & K13 & H14 & & & \\
\hline A25a & M14 & M14 & K13 & N12 & & & \\
\hline A26 & N12 & L12 & & & & & \\
\hline A28 & N14 & N14 & L12 & K10 & & & \\
\hline A29 & O13 & O13 & M11 & L10 & & & \\
\hline O1 & K9 & N12 & & & & & \\
\hline
\end{tabular}

'Assembly "O1" in was in location K9 in Cycle 1 of Oconee. There are no CRC statepoints for Oconee. Therefore, an arbitrary assembly position for assembly "Ol" in Cycle 1 of Oconee may be documented in the calculations as long as the correct (from "K9") data (i.e., burnups, fuel temperatures, moderator specific volumes, etc.) are utilized in the calculations.

\begin{tabular}{|c|c|c|c|c|c|c|c|c|}
\hline & 8 & 9 & 10 & 11 & 12 & 13 & 14 & 15 \\
\hline $\mathbf{H}$ & 1 & 2 & 3 & 4 & 5 & 6 & 7 & 8 \\
\hline $\mathbf{K}$ & & 9 & 10 & 11 & 12 & 13 & 14 & 15 \\
\hline $\mathbf{L}$ & & & 16 & 17 & 18 & 19 & 20 & 21 \\
\hline $\mathbf{M}$ & & & & 22 & 23 & 24 & 25 & \\
\hline $\mathbf{N}$ & & & & & 26 & 27 & 28 & \\
\hline $\mathbf{O}$ & & & & & & 29 & & \\
\hline
\end{tabular}

Figure 4.1.7-1 One-Eighth Symmetry Core Layout for Crystal River Unit 3

\subsubsection{Reactor Cycle History Data}

Table 4.1.8-1 contains a listing of the Crystal River Unit 3 reactor cycle history data that is relevant to the SAS2H depletion calculations documented in this analysis. The time durations other than the days of downtime and the total cycle effective full power days presented in Table 4.1.8-1 are calculated using the appropriate dates from Table 4.1.8-1 and the Lotus 1-2-3 "DATEDIF" function. 
Table 4.1.8-1 Crystal River Unit 3 Reactor Cycle History Data Relevant to the Depletion Calculations for the Rodded Assemblies in Batches 1, 2, 3, and $1 \mathrm{X}$

\section{Crystal River, Unit-3, Cycle-1A Summary}

01/14/77 : Cycle Start Date

03/03/78 : Cycle End Date

413 : Cycle Length (Calendar Days)

268.8 : Cycle Effective Full Power Day (EFPD) Value

195.292 : Calendar Days of Downtime Between Cycle 1A and 1B

\section{Crystal River. Unit-3. Cycle-1B Summary}

09/18/78 : Cycle Start Date (09/15/78: Ref. 5.3)

03/03/79 : 411.0 EFPD Shutdown Date

03/18/79 : Restart Date After the 411.0 EFPD Shutdown

04/23/79 : Cycle End Date

166 : Cycle Length to 411.0 EFPD Date

36 : Cycle Length from 411.0 EFPD Restart to End of Cycle (EOC)

217 : Total Cycle Length (Calendar Days )

14.792 : Days of Downtime During Shutdown at 411.0 EFPD

171.3 : Total Cycle Effective Full Power Days

97 : Calendar Days of Downtime Between Cycle 1B and 2

\section{Crystal River, Unit-3. Cycle-2 Summary}

07/29/79 : Cycle Start Date

02/26/80 : Cycle End Date

212 : Cycle Length (Calendar Days )

166.5 : Cycle Effective Full Power Days

164 : Calendar Days of Downtime Between Cycle 2 and 3 
Title: CRC Depletion Calculations for the Rodded Assemblies in Batches 1, 2, 3, and $1 \mathrm{X}$ of Crystal River Unit 3 Document Identifier: BBA000000-01717-0200-00040 REV 00

Page 13 of 146

Table 4.1.8-1 Crystal River Unit 3 Reactor Cycle History Data Relevant to the Depletion Calculations for the Rodded Assemblies in Batches 1, 2, 3, and 1X

\section{Crystal River, Unit-3, Cycle-3 Summary}

08/08/80 : Cycle Start Date

02/17/81 : 168.5 EFPD Shutdown Date

03/06/81 : Restart Date After the 168.5 EFPD Shutdown

06/30/81 : 250.0 EFPD Shutdown Date

07/13/81 : Restart Date After the 250.0 EFPD Shutdown

09/28/81 : Cycle End Date

193 : Cycle Length to 168.5 EFPD Date

116 : Cycle Length from 168.5 EFPD Restart to 250.0 EFPD Date

77 : Cycle Length from 250.0 EFPD Restart to EOC Date

416 : Total Cycle Length (Calendar Days)

16.792 : Days of Downtime During Shutdown at 168.5 EFPD

12.333 : Days of Downtime During Shutdown at 250.0 EFPD

323 : Total Cycle Effective Full Power Days

73 : Calendar Days of Downtime Between Cycle 3 and 4

\section{Crystal River, Unit-3, Cycle-4 Summary}

12/10/81 : Cycle Start Date

10/14/82 : 228.1 EFPD Shutdown Date

10/31/82 : Restart Date After the 228.1 EFPD Shutdown (10/29/82: Ref. 5.3)

11/25/82 : 253.0 EFPD Shutdown Date (11/26/82: Ref. 5.3)

12/20/82 : Restart Date After the 253.0 EFPD Shutdown

03/19/83 : Cycle End Date

308 : Cycle Length to 228.1 EFPD Date

25 : Cycle Length from 228.1 EFPD Restart to 253.0 EFPD Date

89 : Cycle Length from 253.0 EFPD Restart to EOC Date

464 : Total Cycle Length (Calendar Days )

15.167 : Days of Downtime During Shutdown at 228.1 EFPD

24 : Days of Downtime During Shutdown at 253.0 EFPD

336.6 : Total Cycle Effective Full Power Days

127 : Calendar Days of Downtime Between Cycle 4 and 5 
Table 4.1.8-1 Crystal River Unit 3 Reactor Cycle History Data Relevant to the Depletion Calculations for the Rodded Assemblies in Batches 1, 2, 3, and $1 \mathrm{X}$

\section{Crystal River, Unit-3, Cycle-5 Summary}

07/24/83 : Cycle Start Date

11/05/84 : 388.5 EFPD Shutdown Date

$11 / 10 / 84$ : Restart Date After the 388.5 EFPD Shutdown

03/08/85 : Cycle End Date

470 : Cycle Length to 388.5 EFPD Date

118 : Cycle Length from 388.5 EFPD Restart to EOC Date

593 : Total Cycle Length (Calendar Days )

4.958 : Days of Downtime During Shutdown at 388.5 EFPD

484.4 : Total Cycle Effective Full Power Days

163 : Calendar Days of Downtime Between Cycle-5 and 6

\section{Crystal River, Unit-3, Cycle-6 Summary}

08/18/85 : Cycle Start Date

01/01/86 : 96.0 EFPD Shutdown Date

06/19/86 : Restart Date After the 96.0 EFPD Shutdown

08/21/87 : 400.0 EFPD Shutdown Date

08/31/87 : Restart Date After the 400.0 EFPD Shutdown (09/01/87: Ref. 5.3)

09/18/87 : Cycle End Date

136 : Cycle Length to 96.0 EFPD Date

428 : Cycle Length from 96.0 EFPD Restart to 400.0 EFPD Date

18 : Cycle Length from 400.0 EFPD Restart to EOC Date

761 : Total Cycle Length (Calendar Days )

168.917 : Days of Downtime During Shutdown at 96.0 EFPD

10.417 : Days of Downtime During Shutdown at 400.0 EFPD

412.07 : Total Cycle Effective Full Power Days

113 : Calendar Days of Downtime Between Cycle 6 and 7 
Title: CRC Depletion Calculations for the Rodded Assemblies in Batches 1, 2, 3, and $1 \mathrm{X}$ of Crystal River Unit 3 Document Identifier: BBA000000-01717-0200-00040 REV 00

Page 15 of 146

Table 4.1.8-1 Crystal River Unit 3 Reactor Cycle History Data Relevant to the Depletion Calculations for the Rodded Assemblies in Batches 1, 2, 3, and 1X

\section{Crystal River, Unit-3, Cycle-7 Summary}

01/08/88 : Cycle Start Date

10/09/88 : 260.3 EFPD Shutdown Date

10/28/88 : Restart Date After the 260.3 EFPD Shutdown (10/27/88: Ref. 5.3)

12/07/88 : 291.0 EFPD Shutdown Date

01/16/89 : Restart Date After the 291.0 EFPD Shutdown (01/15/89: Ref. 5.3)

02/26/89 : 319.0 EFPD Shutdown Date

06/16/89 : Restart Date After the 319.0 EFPD Shutdown

01/22/90 : 462.3 EFPD Shutdown Date

01/24/90 : Restart Date After the 462.3 EFPD Shutdown

02/12/90 : 479.0 EFPD Shutdown Date (02/13/90: Ref. 5.3)

02/19/90 : Restart Date After the 479.0 EFPD Shutdown (02/20/90: Ref. 5.3)

03/14/90 : Cycle End Date

275 : Cycle Length to 260.3 EFPD Date

40 : Cycle Length from 260.3 EFPD Restart to 291.0 EFPD Date

41 : Cycle Length from 291.0 EFPD Restart to 319.0 EFPD Date

220 : Cycle Length from 319.0 EFPD Restart to 462.3 EFPD Date

19 : Cycle Length from 462.3 EFPD Restart to 479.0 EFPD Date

23 : Cycle Length from 479.0 EFPD Restart to EOC

796 : Total Cycle Length (Calendar Days )

18.875 : Days of Downtime During Shutdown at 260.3 EFPD

39.5 : Days of Downtime During Shutdown at 291.0 EFPD

109.5 : Days of Downtime During Shutdown at 319.0 EFPD

2.229 : Days of Downtime During Shutdown at 462.3 EFPD

7.208 : Days of Downtime During Shutdown at 479.0 EFPD

497.9 : Total Cycle Effective Full Power Days

99 : Calendar Days of Downtime Between Cycle 7 and 8

\section{Crystal River. Unit-3, Cycle-8 Summary}

06/21/90 : Cycle Start Date

10/09/90 : 97.6 EFPD Shutdown Date

10/25/90 : Restart Date After the 97.6 EFPD Shutdown 
Title: CRC Depletion Calculations for the Rodded Assemblies in Batches 1, 2, 3, and $1 \mathrm{X}$ of Crystal River Unit 3 Document Identifier: BBA000000-01717-0200-00040 REV 00

Page 16 of 146

Table 4.1.8-1 Crystal River Unit 3 Reactor Cycle History Data Relevant to the Depletion Calculations for the Rodded Assemblies in Batches 1, 2, 3, and $1 X$

12/12/90: 139.8 EFPD Shutdown Date

$12 / 18 / 90$ : Restart Date After the 139.8 EFPD Shutdown

10/14/91 : 404.0 EFPD Shutdown Date

11/27/91 : Restart Date After the 404.0 EFPD Shutdown

12/02/91 : 409.6 EFPD Shutdown Date

12/07/91 : Restart Date After the 409.6 EFPD Shutdown

03/27/92 : 515.5 EFPD Shutdown Date

04/04/92 : Restart Date After the 515.5 EFPD Shutdown

04/30/92 : Cycle End Date

110 : Cycle Length to 97.6 EFPD Date

48 : Cycle Length from 97.6 EFPD Restart to 139.8 EFPD Date

300 : Cycle Length from 139.8 EFPD Restart to 404.0 EFPD Date

5 : Cycle Length from 404.0 EFPD Restart to 409.6 EFPD Date

111 : Cycle Length from 409.6 EFPD Restart to 515.5 EFPD Date

26 : Cycle Length from 515.5 EFPD Restart to EOC

679 : Total Cycle Length (Calendar Days)

15.5 : Days of Downtime During Shutdown at 97.6 EFPD

6.2 : Days of Downtime During Shutdown at 139.8 EFPD

44.4 : Days of Downtime During Shutdown at 404.0 EFPD

4.9 : Days of Downtime During Shutdown at 409.6 EFPD

7.6 : Days of Downtime During Shutdown at 515.5 EFPD

535.9 : Total Cycle Effective Full Power Days

75 : Calendar Days of Downtime Between Cycle 8 and 9

\section{Crystal River. Unit-3, Cycle-9 Summary}

07/14/92 : Cycle Start Date

12/29/92: 158.8 EFPD Shutdown Date

12/31/92 : Restart Date After the 158.8 EFPD Shutdown

03/04/93 : 219.0 EFPD Shutdown Date

04/26/93 : Restart Date After the 219.0 EFPD Shutdown

09/18/93 : 363.1 EFPD Shutdown Date

09/20/93 : Restart Date After the 363.1 EFPD Shutdown

04/07/94 : Cycle End Date 
Title: CRC Depletion Calculations for the Rodded Assemblies in Batches 1, 2, 3, and IX of Crystal River Unit 3

Document Identifler: BBA000000-01717-0200-00040 REV 00

Page 17 of 146

Table 4.1.8-1 Crystal River Unit 3 Reactor Cycle History Data Relevant to the Depletion Calculations for the Rodded Assemblies in Batches 1, 2, 3, and 1X

168 : Cycle Length to 158.8 EFPD Date

63 : Cycle Length from 158.8 EFPD Restart to 219.0 EFPD Date

145 : Cycle Length from 219.0 EFPD Restart to 363.1 EFPD Date

199 : Cycle Length from 363.1 EFPD Restart to EOC

632 : Total Cycle Length (Calendar Days )

2.146 : Days of Downtime During Shutdown at 158.8 EFPD

53.125 : Days of Downtime During Shutdown at 219.0 EFPD

1.625 : Days of Downtime During Shutdown at 363.1 EFPD

557.23 : Total Cycle Effective Full Power Days

55 : Calendar Days of Downtime Between Cycle 9 and 10

A number of the dates presented in Table 4.1.8-1 do not correspond directly with the dates presented in reference 5.3. The date contained in reference 5.3, is presented in parentheses next to each inconsistency. Inconsistencies in the restart and shutdown date values do not affect the calculations due to the fact that the depletions are based upon EFPD durations rather than calendar day durations. The various calendar day time periods between statepoints as presented in Table 4.1.8-1 are used for documentation purposes only. The cycle starting and ending dates are the only dates presented in Table 4.1.8-1 which are involved in calculations that are documented in this analysis. A cycle's starting and ending dates are used to calculate calendar day decay durations for fuel assemblies which skip that particular cycle. Cycle-1B is the only cycle relevant to this analysis which used a starting date that does not directly correspond to the dates provided in reference 5.3. There are no assembly depletion and decay calculations documented in this analysis which were in Cycle-1A and skipped Cycle-1B to be inserted at a later time. The days of downtime between cycles are not calculated from the dates presented in Table 4.1.8-1. The days of downtime between cycles are obtained directly from reference 5.3 in units of hours that are converted to days for presentation in Table 4.1.8-1 and use in this analysis. Therefore, no calculations documented in this analysis are affected by the date inconsistencies between Table 4.1.8-1 and reference 5.3.

\subsubsection{Boron Letdown Data}

The boron letdown data provided in the Core Operations Reports for Cycles 1, 2, 3, 7, 8, and 9 of Crystal River Unit 3 is used to determine the soluble boron concentration in the moderator at the mid-point of each irradiation step in the various SAS2H depletion calculations performed to deplete the rodded fuel assemblies of batches 1,2,3, and 1X. The boron concentrations at the irradiation step mid-point effective full-power day (EFPD) times are determined by linear interpolation between the measured values listed in Tables 4.1.9-1 through 4.1.9-7. The boron letdown data tables presented in this section are obtained from reference 5.3 , which is a summary compilation of data pertinent to CRC analyses for Crystal River Unit 3. 
Title: CRC Depletion Calculations for the Rodded Assemblies in Batches 1, 2, 3, and $1 \mathrm{X}$ of Crystal River Unit 3 Document Identifier: BBA000000-01717-0200-00040 REV 00

Table 4.1.9-1 Boron Letdown Data for Cycle 1 A of Crystal River Unit 3

\begin{tabular}{|c|c||}
\hline Exposure (EFPD) & Boron Concentration (ppm) \\
\hline 0.0 & 1147 \\
\hline 7.2 & 968 \\
\hline 18.6 & 912 \\
\hline 55.2 & 934 \\
\hline 63.8 & 909 \\
\hline 69.9 & 909 \\
\hline 94.9 & 884 \\
\hline 184.7 & 705 \\
\hline 192.3 & 683 \\
\hline 216.0 & 627 \\
\hline 224.8 & 610 \\
\hline 228.5 & 666 \\
\hline 238.0 & 584 \\
\hline 244.0 & 575 \\
\hline 250.8 & 614 \\
\hline 254.7 & 588 \\
\hline
\end{tabular}

- The acronym "ppm" means parts per million by mass of moderator.

Table 4.1.9-2 Boron Letdown Data for Cycle 1B of Crystal River Unit 3

\begin{tabular}{|c|c|}
\hline $\begin{array}{c}\text { EFPD with Cycles 1A \& 1B Continuous } \\
\text { [EFPD with BOC*1B set to 0.0 EFPD] }\end{array}$ & Boron Concentration (ppm) \\
\hline \hline $269.4[0.6]$ & 843 \\
\hline $269.8[1.0]$ & 783 \\
\hline $272.0[3.2]$ & 748 \\
\hline $280.2[11.4]$ & 558 \\
\hline $287.2[18.4]$ & 571 \\
\hline
\end{tabular}


Title: CRC Depletion Calculations for the Rodded Assemblies in Batches 1, 2, 3, and $1 \mathrm{X}$ of Crystal River Unit 3 Document Identifier: BBA000000-01717-0200-00040 REV 00

Table 4.1.9-2 Boron Letdown Data for Cycle $1 B$ of Crystal River Unit 3

\begin{tabular}{|c|c|}
\hline $\begin{array}{c}\text { EFPD with Cycles 1A \& 1B Continuous } \\
\text { [EFPD with BOC }\end{array}$ & -1B set to 0.0 EFPD] \\
\hline $306.2[37.4]$ & 513 \\
\hline $313.2[44.4]$ & 441 \\
\hline $337.2[68.4]$ & 419 \\
\hline $345.7[76.9]$ & 346 \\
\hline $364.2[95.4]$ & 309 \\
\hline $377.6[108.8]$ & 246 \\
\hline $389.5[120.7]$ & 279 \\
\hline $401.7[132.9]$ & 290 \\
\hline $419.3[150.5]$ & 272 \\
\hline $427.1[158.3]$ & 229 \\
\hline $431.8[163.0]$ & 231 \\
\hline $437.1[168.3]$ & 229 \\
\hline $440.1[171.3]$ & 242 \\
\hline
\end{tabular}

- The acronym "BOC" means beginning of cycle.

Table 4.1.9-3 Boron Letdown Data for Cycle 2 of Crystal River Unit 3

\begin{tabular}{|c|c|}
\hline Exposure (EFPD) & Boron Concentration (ppm) \\
\hline 0.6 & 930 \\
\hline 0.8 & 930 \\
\hline 0.9 & 930 \\
\hline 2.1 & 826 \\
\hline 3.0 & 809 \\
\hline 4.4 & 778 \\
\hline 11.4 & 809 \\
\hline 15.8 & 735 \\
\hline
\end{tabular}


Title: CRC Depletion Calculations for the Rodded Assemblies in Batches 1, 2, 3, and $1 \mathrm{X}$ of Crystal River Unit 3 Document Identifier: BBA000000-01717-0200-00040 REV 00

Table 4.1.9-3 Boron Letdown Data for Cycle 2 of Crystal River Unit 3

\begin{tabular}{|c|c|}
\hline Exposure (EFPD) & Boron Concentration (ppm) \\
\hline 22.5 & 709 \\
\hline 29.3 & 683 \\
\hline 35.3 & 666 \\
\hline 42.3 & 644 \\
\hline 50.0 & 623 \\
\hline 55.8 & $614^{\circ}$ \\
\hline 60.8 & 592 \\
\hline 69.1 & 571 \\
\hline 75.2 & 558 \\
\hline 83.1 & 528 \\
\hline 89.8 & 506 \\
\hline 97.8 & 480 \\
\hline 104.7 & 463 \\
\hline 116.4 & 441 \\
\hline 122.5 & 406 \\
\hline 129.1 & 385 \\
\hline 135.9 & 372 \\
\hline 139.9 & 346 \\
\hline 148.6 & 333 \\
\hline 156.4 & 320 \\
\hline 161.4 & 316 \\
\hline
\end{tabular}

Table 4.1.9-4 Boron Letdown Data for Cycle 3 of Crystal River Unit 3

\begin{tabular}{|c|c|}
\hline Exposure (EFPD) & Boron Concentration (ppm) \\
\hline 0.7 & 1090 \\
\hline 2.0 & 1020 \\
\hline
\end{tabular}


Title: CRC Depletion Calculations for the Rodded Assemblies in Batches 1, 2, 3, and 1X of Crystal River Unit 3 Document Identifier: BBA000000-01717-0200-00040 REV 00

Table 4.1.9-4 Boron Letdown Data for Cycle 3 of Crystal River Unit 3

\begin{tabular}{|c|c|}
\hline Exposure (EFPD) & Boron Concentration (ppm) \\
\hline 4.0 & 947 \\
\hline 6.7 & 951 \\
\hline 12.6 & 908 \\
\hline 26.8 & 891 \\
\hline 32.6 & 843 \\
\hline 50.7 & 822 \\
\hline 66.0 & 757 \\
\hline 69.9 & 746 \\
\hline 85.0 & 692 \\
\hline 100.2 & 666 \\
\hline 111.2 & 636 \\
\hline 130.5 & 562 \\
\hline 143.8 & 528 \\
\hline 163.9 & 467 \\
\hline 174.0 & 432 \\
\hline 184.2 & 394 \\
\hline 212.9 & 324 \\
\hline 227.5 & 272 \\
\hline 246.4 & 229 \\
\hline 262.9 & 250 \\
\hline 283.8 & 190 \\
\hline 304.0 & 130 \\
\hline 322.0 & 86 \\
\hline
\end{tabular}




\section{Waste Package Development}

Title: CRC Depletion Calculations for the Rodded Assemblies in Batches 1, 2, 3, and $1 \mathrm{X}$ of Crystal River Unit 3 Document Identifier: BBA000000-01717-0200-00040 REV 00

Table 4.1.9-5 Boron Letdown Data for Cycle 7 of Crystal River Unit 3

\begin{tabular}{|c|c|}
\hline Exposure (EFPD) & Boron Concentration (ppm) \\
\hline 7.5 & 1478 \\
\hline 41.4 & 1405 \\
\hline 60.3 & 1367 \\
\hline 81.7 & 1333 \\
\hline 102.9 & 1290 \\
\hline 122.3 & 1245 \\
\hline 139.8 & 1204 \\
\hline 160.5 & 1167 \\
\hline 180.8 & 1102 \\
\hline 202.8 & 1040 \\
\hline 230.9 & 963 \\
\hline 251.2 & 898 \\
\hline 306.7 & 803 \\
\hline 317.9 & 775 \\
\hline 345.3 & 593 \\
\hline 412.3 & 398 \\
\hline 459.8 & 260 \\
\hline 485.0 & 193 \\
\hline
\end{tabular}

Table 4.1.9-6 Boron Letdown Data for Cycle 8 of Crystal River Unit 3

\begin{tabular}{|c|c|}
\hline Exposure (EFPD) & Boron Concentration (ppm) \\
\hline 11.2 & 1537 \\
\hline 52.4 & 1455 \\
\hline 78 & 1411 \\
\hline 111.4 & 1332 \\
\hline 154.4 & 1176 \\
\hline
\end{tabular}


Title: CRC Depletion Calculations for the Rodded Assemblies in Batches 1, 2, 3, and $1 \mathrm{X}$ of Crystal River Unit 3

Table 4.1.9-6 Boron Letdown Data for Cycle 8 of Crystal River Unit 3

\begin{tabular}{|c|c|}
\hline Exposure (EFPD) & Boron Concentration (ppm) \\
\hline 194.8 & 1103 \\
\hline 234.6 & 999 \\
\hline 271.5 & 887 \\
\hline 338 & 701 \\
\hline 390.7 & 522 \\
\hline 445.7 & 394 \\
\hline 474 & 311 \\
\hline 513.1 & 216 \\
\hline
\end{tabular}

Table 4.1.9-7 Boron Letdown Data for Cycle 9 of Crystal River Unit 3

\begin{tabular}{|c|c|}
\hline Exposure (EFPD) & Boron Concentration (ppm) \\
\hline 22.1 & 1608 \\
\hline 61.5 & 1535 \\
\hline 145.7 & 1329 \\
\hline 192.8 & 1201 \\
\hline 211.3 & 1157 \\
\hline 262 & 994 \\
\hline 303.7 & 869 \\
\hline 345.7 & 750 \\
\hline 397.9 & 577 \\
\hline 432.5 & 473 \\
\hline 452.4 & 412 \\
\hline 495.4 & 283 \\
\hline 543.4 & 136 \\
\hline
\end{tabular}


Title: CRC Depletion Calculations for the Rodded Assemblies in Batches 1, 2, 3, and $1 \mathrm{X}$ of Crystal River Unit 3 Document Identifler: BBA000000-01717-0200-00040 REV 00

Page 24 of 146

Burnup, fuel temperature, and moderator specific volume data are required for each node of each assembly in each SAS2H depletion calculation. A set of nodal burnup data at the beginning and end of each SAS2H depletion calculation is required. A set of nodal fuel temperature and moderator specific volume data representative of full-power operation during each depletion calculation of interest is required. Tables 4.1.10-1 through 4.1.10-18 contain the burnup, fuel temperature, and moderator specific volume data necessary to perform all depletion calculations for each of the rodded fuel assemblies from batches 1,2,3, and $1 \mathrm{X}$ of Crystal River Unit 3. The assembly heights corresponding to the axial nodes presented in Tables 4.1.10-1 through 4.1.10-18 are as follow: the top node (node 1) is $17.78 \mathrm{~cm}$, the bottom node (node 18) is $22.352 \mathrm{~cm}$, all other nodes are $20.0025 \mathrm{~cm}$. The top of node 1 begins at the top of the active fuel region. The burnup data is presented in units of gigawatt-days per metric ton of uranium (GWd/MTU). The fuel temperature data is presented in units of degrees Fahrenheit. The moderator specific volume data is presented in units of cubic feet per pound. The statepoint numbers shown in the tables identify the relative reactivity statepoint calculations that fuel and burnable poison isotopic data will be generated to support for the evaluation of that particular assembly. The EFPD statepoint and cycle number corresponding to each set of fuel temperature and moderator specific volume data are presented above their respective columns in the tables. Each set of fuel temperature and moderator specific volume data listed in the tables is applicable to the depletion calculation performed between the statepoint number identified above the particular data and the previous statepoint number.

Table 4.1.10-1 Burnup, Fuel Temperature, and Moderator Specific Volume Data for Assembly A1 of Crystal River Unit 3

\begin{tabular}{|c|c|c|c|c|c|c|c|c|c|}
\hline & \multicolumn{9}{|c|}{ Assembly Number A1 } \\
\hline & \multicolumn{3}{|c|}{ Statepoint 1 (BOC Cycle 1A) } & \multicolumn{3}{|c|}{ Statepoint 2 (0.0 Cycle 1B) } & \multicolumn{3}{|c|}{ Statepoint 3 (142.2 Cycle 1B) } \\
\hline & $\begin{array}{c}\text { Burnup } \\
\text { (GWd/MTU) }\end{array}$ & $\begin{array}{l}\text { Fuel } \\
\text { Temp. }\end{array}$ & $\begin{array}{l}\text { Moderator } \\
\text { Spec. Vol. }\end{array}$ & $\begin{array}{c}\text { Burnup } \\
\text { (GWd/MTU) }\end{array}$ & $\begin{array}{l}\text { Fuel } \\
\text { Temp. }\end{array}$ & $\begin{array}{l}\text { Moderator } \\
\text { Spec. Vol. }\end{array}$ & $\begin{array}{l}\text { Burnup } \\
\text { (GWd/MTU) }\end{array}$ & $\begin{array}{l}\text { Fuel } \\
\text { Temp. }\end{array}$ & $\begin{array}{l}\text { Moderator } \\
\text { Spec. Vol. }\end{array}$ \\
\hline & & & & 0.0 Cy1B & 184.7 Cy1A & 184.7 Cy1A & $142.2 C_{y 1 B}$ & 89.5 Cy1B & 89.5 Cy1B \\
\hline 1 & 0.0 & & & 2.816 & 881.9 & 0.0231 & 4.819 & 974.7 & 0.0230 \\
\hline 2 & 0.0 & & & 4.715 & 1055.5 & 0.0231 & 7.860 & 1135.9 & 0.0229 \\
\hline 3 & 0.0 & & & 6.179 & 1169.8 & 0.0230 & 9.908 & 1186.3 & 0.0228 \\
\hline 4 & 0.0 & \multicolumn{2}{|c|}{ Data not required. } & 7.134 & 1222.7 & 0.0229 & 11.075 & 1178.1 & 0.0227 \\
\hline 5 & 0.0 & & & 7.720 & 1247.6 & 0.0228 & 11.694 & 1153.1 & 0.0226 \\
\hline 6 & 0.0 & & & 8.056 & 1257.8 & 0.0228 & 11.953 & 1128.4 & 0.0225 \\
\hline 7 & 0.0 & & & 8.232 & 1262.6 & 0.0227 & 12.045 & 1108.3 & 0.0225 \\
\hline 8 & 0.0 & & & 8.306 & 1267.6 & 0.0226 & 12.060 & 1094.5 & 0.0224 \\
\hline 9 & 0.0 & & & 8.314 & 1275.2 & 0.0225 & 12.042 & 1086.6 & 0.0223 \\
\hline 10 & 0.0 & & & 8.275 & 1285.4 & 0.0224 & 12.010 & 1083.8 & 0.0222 \\
\hline 11 & 0.0 & & & 8.206 & 1295.9 & 0.0223 & 11.979 & 1085.1 & 0.0222 \\
\hline 12 & 0.0 & & & 8.129 & 1303.4 & 0.0222 & 11.963 & 1089.7 & 0.0221 \\
\hline 13 & 0.0 & & & 8.077 & 1305.7 & 0.0221 & 11.987 & 1096.8 & 0.0220 \\
\hline 14 & 0.0 & . & & 8.211 & 1302.6 & 0.0220 & 12.208 & 1104.0 & 0.0219 \\
\hline 15 & 0.0 & & & 9.357 & 1318.7 & 0.0219 & 13.574 & 1114.5 & 0.0219 \\
\hline .16 & 0.0 & & & 11.135 & 1561.3 & 0.0218 & 16.249 & 1257.0 & 0.0218 \\
\hline
\end{tabular}




\section{Waste Package Development}

Design Analysis

Title: CRC Depletion Calculations for the Rodded Assemblies in Batches 1, 2, 3, and $1 \mathrm{X}$ of Crystal River Unit 3

Document Identifier: BBA000000-01717-0200-00040 REV 00

Page 25 of 146

Table 4.1.10-1 Burnup, Fuel Temperature, and Moderator

Specific Volume Data for Assembly A1 of Crystal River Unit 3

\begin{tabular}{|l|l|l|l|r|r|r|r|r|r|}
\hline 17 & 0.0 & & & 10.619 & 1475.9 & 0.0217 & 15.740 & 1271.5 & 0.0217 \\
\hline 18 & 0.0 & & & 6.746 & 1191.0 & 0.0216 & 10.259 & 1100.7 & 0.0216 \\
\hline
\end{tabular}

\begin{tabular}{|c|c|c|c|c|c|c|c|c|c|}
\hline & \multicolumn{3}{|c|}{ Statepoint 4 (BOC Cycle 2) } & \multicolumn{3}{|c|}{ Statepoint 5 (BOC Cycle 3) } & \multicolumn{3}{|c|}{ Statepoint 6 (168.5 Cycle 3) } \\
\hline $\begin{array}{l}\text { Node } \\
\text { No. }\end{array}$ & $\begin{array}{c}\text { Burnup } \\
\text { (GWd/MTU) }\end{array}$ & $\begin{array}{l}\text { Fuel } \\
\text { Temp. }\end{array}$ & $\begin{array}{l}\text { Moderator } \\
\text { Spec. Vol. }\end{array}$ & $\begin{array}{c}\text { Burnup } \\
\text { (GWd/MTU) }\end{array}$ & $\begin{array}{l}\text { Fuel } \\
\text { Temp. }\end{array}$ & $\begin{array}{l}\text { Moderator } \\
\text { Spec. Vol. }\end{array}$ & \begin{tabular}{|c|} 
Burmup \\
(GWd/MTU)
\end{tabular} & $\begin{array}{l}\text { Fuel } \\
\text { Temp. }\end{array}$ & $\begin{array}{l}\text { Moderator } \\
\text { Spec. Vol. }\end{array}$ \\
\hline & BOC Cy2 & 139.6 Cy1B & 139.6 Cy1B & $\mathrm{BOC} \mathrm{Cy3}$ & 89.8 Cy2 & $89.8 \mathrm{Cy2}$ & $168.5 \mathrm{Cy}_{3}$ & $85.0 \mathrm{Cy} 3$ & $85.0 \mathrm{Cy3}$ \\
\hline 1 & 5.191 & 912.6 & 0.0237 & 7.992 & 974.1 & 0.0231 & 10.720 & 968.2 & 0.0232 \\
\hline 2 & 8.429 & 1033.5 & 0.0236 & 12.840 & 1120.5 & 0.0231 & 17.114 & 1107.5 & 0.0231 \\
\hline 3 & 10.599 & 1084.4 & 0.0235 & 15.827 & 1189.6 & 0.0230 & 20.924 & 1151.9 & 0.0230 \\
\hline 4 & 11.995 & 1168.3 & 0.0234 & 17.481 & 1209.5 & 0.0229 & 22.904 & 1149.8 & 0.0229 \\
\hline 5 & 12.770 & 1428.3 & 0.0233 & 18.282 & 1211.3 & 0.0227 & 23.812 & 1138.4 & 0.0228 \\
\hline 6 & 13.190 & 1490.9 & 0.0232 & 18.646 & 1205.6 & 0.0226 & 24.195 & 1124.8 & 0.0227 \\
\hline 7 & 13.318 & 1501.4 & 0.0230 & 18.721 & 1201.9 & 0.0225 & 24.276 & 1116.0 & 0.0226 \\
\hline 8 & 13.341 & 1504.9 & 0.0229 & 18.702 & 1199.5 & 0.0224 & 24.275 & 1112.9 & 0.0225 \\
\hline 9 & 13.329 & 1507.4 & 0.0227 & 18.662 & 1196.7 & 0.0223 & 24.283 & 1116.6 & 0.0224 \\
\hline 10 & 13.308 & 1510.2 & 0.0225 & 18.630 & 1191.9 & 0.0222 & 24.335 & 1126.7 & 0.0223 \\
\hline 11 & 13.294 & 1512.9 & 0.0224 & 18.616 & 1183.6 & 0.0221 & 24.424 & 1140.4 & 0.0222 \\
\hline 12 & 13.297 & 1513.2 & 0.0223 & 18.619 & 1171.5 & 0.0220 & 24.518 & 1153.2 & 0.0221 \\
\hline 13 & 13.332 & 1505.7 & 0.0221 & 18.633 & 1155.5 & 0.0219 & 24.577 & 1161.0 & 0.0220 \\
\hline 14 & 13.535 & 1478.7 & 0.0220 & 18.754 & 1132.8 & 0.0219 & 24.659 & 1158.9 & 0.0219 \\
\hline 15 & 14.814 & 1390.7 & 0.0219 & 19.753 & 1084.0 & 0.0218 & 25.403 & 1128.4 & 0.0218 \\
\hline 16 & 17.321 & 1233.5 & 0.0217 & 21.717 & 1005.7 & 0.0217 & 26.806 & 1061.8 & 0.0217 \\
\hline 17 & 16.644 & 1128.2 & 0.0217 & 20.470 & 957.9 & 0.0216 & 24.902 & 1009.0 & 0.0216 \\
\hline 18 & 10.859 & 974.7 & 0.0216 & 13.470 & 866.9 & 0.0216 & 16.497 & 915.6 & 0.0216 \\
\hline
\end{tabular}

\begin{tabular}{|c|c|c|c|c|c|c|c|c|c|}
\hline & \multicolumn{3}{|c|}{ Statepoint $7(250.0$ Cycle 3$)$} & \multicolumn{3}{|c|}{ Statepoint 8} & \multicolumn{3}{|c|}{ Statepoint 9} \\
\hline $\begin{array}{l}\text { Node } \\
\text { No. }\end{array}$ & $\begin{array}{c}\text { Burnup } \\
\text { (GWdMTU) }\end{array}$ & $\begin{array}{l}\text { Fuel } \\
\text { Temp. }\end{array}$ & $\begin{array}{l}\text { Moderator } \\
\text { Spec. Vol. }\end{array}$ & $\begin{array}{c}\text { Burnup } \\
\text { (GWdMTU) }\end{array}$ & $\begin{array}{l}\text { Fuel } \\
\text { Temp. }\end{array}$ & $\begin{array}{l}\text { Moderator } \\
\text { Spec. Vol. }\end{array}$ & $\begin{array}{c}\text { Burnup } \\
\text { (GWdMTU) }\end{array}$ & $\begin{array}{l}\text { Fuel } \\
\text { Temp. }\end{array}$ & $\begin{array}{l}\text { Moderator } \\
\text { Spec. Vol. }\end{array}$ \\
\hline & $250.0 \mathrm{cy} 3$ & 246.4 Cy3 & $246.4 \mathrm{Cy}^{3}$ & & & & & & \\
\hline 1 & 12.280 & 1006.3 & 0.0232 & No additio & I states: & & No additio & states & \\
\hline 2 & 19.411 & 1108.0 & 0.0231 & & & & & & \\
\hline 3 & 23.509 & 1126.5 & 0.0230 & & & & & & \\
\hline 4 & 25.524 & 1111.8 & 0.0229 & & & & & & \\
\hline 5 & 26.395 & 1093.3 & 0.0228 & & & & & & \\
\hline 6 & 26.737 & 1079.3 & 0.0227 & & & & & & \\
\hline 7 & 26.797 & 1071.7 & 0.0226 & & & & & & \\
\hline 8 & 26.793 & 1068.2 & 0.0225 & & & & & & \\
\hline 9 & 26.815 & 1067.3 & 0.0224 & & & & & & \\
\hline 10 & 26.893 & 1068.0 & 0.0223 & & & & & & \\
\hline 11 & 27.016 & 1069.3 & 0.0222 & & & & & & \\
\hline
\end{tabular}


Title: CRC Depletion Calculations for the Rodded Assemblies in Batches 1, 2, 3, and $1 \mathrm{X}$ of Crystal River Unit 3 Document Identifier: BBA000000-01717-0200-00040 REV 00

Page 26 of 146

Table 4.1.10-1 Burnup, Fuel Temperature, and Moderator

Specific Volume Data for Assembly A1 of Crystal River Unit 3

\begin{tabular}{|l|r|r|r|l|l|l|l|l|l|}
\hline 12 & 27.148 & 1070.6 & 0.0221 & & & & & & \\
\hline 13 & 27.247 & 1072.0 & 0.0220 & & & & & & \\
\hline 14 & 27.358 & 1071.6 & 0.0219 & & & & & & \\
\hline 15 & 28.056 & 1054.6 & 0.0218 & & & & & & \\
\hline 16 & 29.285 & 1012.1 & 0.0217 & & & & & & \\
\hline 17 & 27.150 & 985.7 & 0.0217 & & & & & & \\
\hline 18 & 18.111 & 923.6 & 0.0216 & & & & & & \\
\hline
\end{tabular}

Table 4.1.10-2 Burnup, Fuel Temperature, and Moderator Specific Volume Data for Assembly A4 of Crystal River Unit 3

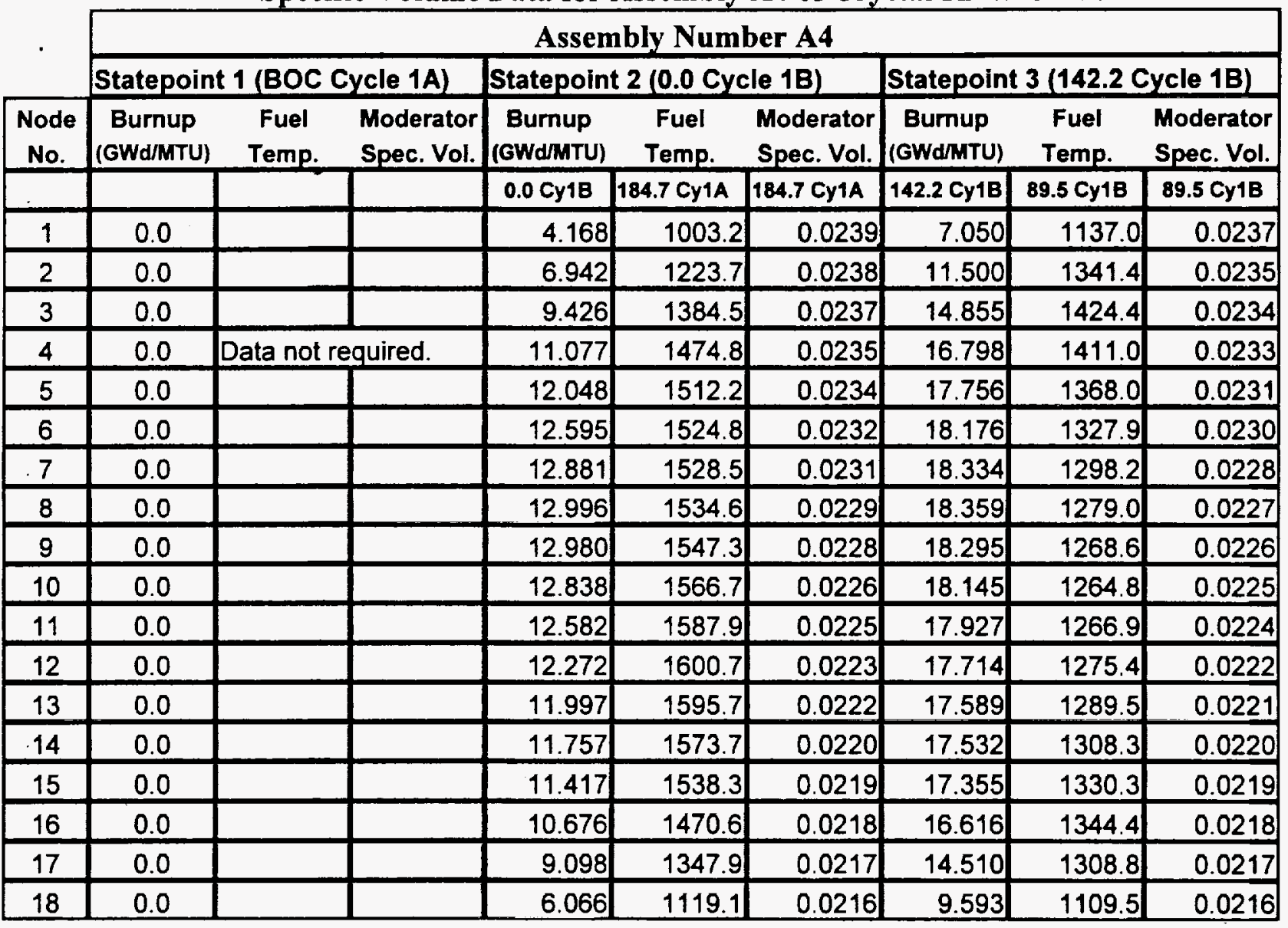

\begin{tabular}{|c|c|c|c|c|c|c|c|c|c|}
\hline & \multicolumn{3}{|c|}{ Statepoint 4 (BOC Cycle 2) } & \multicolumn{3}{|c|}{ Statepoint 5 (BOC Cycle 3) } & \multicolumn{3}{|c|}{ Statepoint 6 (168.5 Cycle 3) } \\
\hline $\begin{array}{l}\text { Node } \\
\text { No. }\end{array}$ & $\begin{array}{c}\text { Burnup } \\
\text { (GWd/MTU) }\end{array}$ & $\begin{array}{c}\text { Fuel } \\
\text { Temp. }\end{array}$ & $\begin{array}{l}\text { Moderator } \\
\text { Spec. Vol. }\end{array}$ & \begin{tabular}{|c|} 
Burnup \\
(GWd/MTU)
\end{tabular} & $\begin{array}{c}\text { Fuel } \\
\text { Temp. }\end{array}$ & $\begin{array}{l}\text { Moderator } \\
\text { Spec. Vol. }\end{array}$ & \begin{tabular}{|c|} 
Burnup \\
(GWdMTU)
\end{tabular} & $\begin{array}{c}\text { Fuel } \\
\text { Temp. }\end{array}$ & $\begin{array}{l}\text { Moderator } \\
\text { Spec. Vol. }\end{array}$ \\
\hline & BOC Cy2 & 139.6 Cy1B & 139.6 Cy1B & $\mathrm{BOC}$ Cy3 & 89.8 Cy2 & 89.8 Cy2 & $168.5 \mathrm{Cy} 3$ & $85.0 \mathrm{Cy3}$ & $85.0 \mathrm{Cy} 3$ \\
\hline 1 & 7.708 & 1111.1 & 0.0234 & & & & & & \\
\hline 2 & 12.474 & 1267.6 & 0.0233 & & & & & & \\
\hline
\end{tabular}


Title: CRC Depletion Calculations for the Rodded Assemblies in Batches 1, 2, 3, and $1 \mathrm{X}$ of Crystal River Unit 3

Document Identifier: BBA000000-01717-0200-00040 REV 00

Page 27 of 146

Table 4.1.10-2 Burnup, Fuel Temperature, and Moderator Specific Volume Data for Assembly A4 of Crystal River Unit 3

\begin{tabular}{|c|r|r|r|l|l|l|l|l|l|}
\hline 3 & 15.921 & 1288.1 & 0.0231 & No additional statepoints. & \multicolumn{3}{|l|}{ No additional statepoints. } \\
\hline 4 & 17.847 & 1255.8 & 0.0230 & & & & & & \\
\hline 5 & 18.769 & 1226.7 & 0.0229 & & & & & & \\
\hline 6 & 19.161 & 1209.2 & 0.0228 & & & & & & \\
\hline 7 & 19.298 & 1199.5 & 0.0227 & & & & & & \\
\hline 8 & 19.306 & 1193.5 & 0.0226 & & & & & & \\
\hline 9 & 19.230 & 1188.7 & 0.0224 & & & & & & \\
\hline 10 & 19.077 & 1185.4 & 0.0223 & & & & & & \\
\hline 11 & 18.874 & 1186.3 & 0.0222 & & & & & & \\
\hline 12 & 18.692 & 1191.8 & 0.0221 & & & & & & \\
\hline 13 & 18.604 & 1197.4 & 0.0220 & & & & & & \\
\hline 14 & 18.575 & 1199.5 & 0.0219 & & & & & & \\
\hline 15 & 18.406 & 1196.3 & 0.0218 & & & & & & \\
\hline 16 & 17.637 & 1183.5 & 0.0217 & & & & & & \\
\hline 17 & 15.425 & 1141.3 & 0.0217 & & & & & & \\
\hline 18 & 10.199 & 983.4 & 0.0216 & & & & & & \\
\hline
\end{tabular}

Table 4.1.10-3 Burnup, Fuel Temperature, and Moderator

Specific Volume Data for Assembly A5 of Crystal River Unit 3

\begin{tabular}{|c|c|c|c|c|c|c|c|c|c|}
\hline & \multicolumn{9}{|c|}{ Assembly Number A5 } \\
\hline & \multicolumn{3}{|c|}{ Statepoint 1 (BOC Cycle 1A) } & \multicolumn{3}{|c|}{ Statepoint 2 (0.0 Cycle 1B) } & \multicolumn{3}{|c|}{ Statepoint 3 (142.2 Cycle 1B) } \\
\hline & $\begin{array}{c}\text { Bumup } \\
\text { (GWd/MTU) }\end{array}$ & $\begin{array}{l}\text { Fuel } \\
\text { Temp. }\end{array}$ & $\begin{array}{l}\text { Moderator } \\
\text { Spec. Vol. }\end{array}$ & $\begin{array}{c}\text { Burnup } \\
\text { (GWd/MTU) }\end{array}$ & $\begin{array}{c}\text { Fuel } \\
\text { Temp. }\end{array}$ & $\begin{array}{l}\text { Moderator } \\
\text { Spec. Vol. }\end{array}$ & $\begin{array}{c}\text { Burnup } \\
\text { (GWdMTU) }\end{array}$ & $\begin{array}{c}\text { Fuel } \\
\text { Temp. }\end{array}$ & $\begin{array}{l}\text { Moderator } \\
\text { Spec. Vol. }\end{array}$ \\
\hline & & & & 0.0 Cy1B & 184.7 Cy1A & 184.7 Cy $1 \mathrm{~A}$ & 142.2 Cy1B & 89.5 Cy1B & 89.5 Cy1B \\
\hline 1 & 0.0 & & & 2.445 & 825.3 & 0.0236 & 4.124 & 925.7 & 0.0233 \\
\hline 2 & 0.0 & & & 5.137 & 993.2 & 0.0235 & 7.935 & 1131.7 & 0.0233 \\
\hline 3 & 0.0 & & & 8.442 & 1279.6 & 0.0234 & 12.475 & 1344.4 & 0.0232 \\
\hline 4 & 0.0 & \multicolumn{2}{|c|}{ Data not required. } & 10.198 & 1403.0 & 0.0233 & 15.090 & 1340.7 & 0.0230 \\
\hline 5 & 0.0 & & & 11.117 & 1443.2 & 0.0232 & 16.172 & 1300.8 & 0.0229 \\
\hline 6 & 0.0 & & & 11.623 & 1456.5 & 0.0230 & 16.599 & 1265.2 & 0.0228 \\
\hline 7 & 0.0 & & & 11.884 & 1462.9 & 0.0229 & 16.758 & 1238.8 & 0.0227 \\
\hline 8 & 0.0 & & & 11.985 & 1472.1 & 0.0228 & 16.782 & 1221.7 & 0.0226 \\
\hline 9 & 0.0 & & & 11.956 & 1488.3 & 0.0226 & 16.708 & 1212.1 & 0.0225 \\
\hline 10 & 0.0 & & & 11.795 & 1511.8 & 0.0225 & 16.527 & 1207.7 & 0.0224 \\
\hline 11 & 0.0 & & & 11.501 & 1534.7 & 0.0224 & 16.247 & 1208.0 & 0.0223 \\
\hline 12 & 0.0 & & & 11.149 & 1545.9 & 0.0222 & 15.966 & 1214.4 & 0.0222 \\
\hline 13 & 0.0 & & & 10.864 & 1535.9 & 0.0221 & 15.807 & 1226.6 & 0.0221 \\
\hline 14 & 0.0 & & & 10.663 & 1503.9 & 0.0220 & 15.768 & 1243.8 & 0.0220 \\
\hline 15 & 0.0 & & & 10.418 & 1458.5 & 0.0219 & 15.684 & 1265.3 & 0.0219 \\
\hline 16 & 0.0 & & & 9.841 & 1393.7 & 0.0217 & 15.130 & 1279.9 & 0.0218 \\
\hline
\end{tabular}


Title: CRC Depletion Calculations for the Rodded Assemblies in Batches 1, 2, 3, and 1X of Crystal River Unit 3

Document Identifier: BBA000000-01717-0200-00040 REV 00

Page 28 of 146

Table 4.1.10-3 Burnup, Fuel Temperature, and Moderator

Specific Volume Data for Assembly A5 of Crystal River Unit 3

\begin{tabular}{|l|l|l|l|r|r|r|r|r|r|}
\hline 17 & 0.0 & & & 8.445 & 1283.3 & 0.0217 & 13.267 & 1245.7 & 0.0217 \\
\hline 18 & 0.0 & & & 5.210 & 1040.6 & 0.0216 & 8.365 & 1073.7 & 0.0216 \\
\hline
\end{tabular}

Table 4.1.10-4 Burnup, Fuel Temperature, and Moderator

Specific Volume Data for Assembly A7 of Crystal River Unit 3

\begin{tabular}{|c|c|c|c|c|c|c|c|c|c|}
\hline \multirow{3}{*}{$\begin{array}{c}\text { Node } \\
\text { No. }\end{array}$} & \multicolumn{9}{|c|}{ Assembly Number A7 } \\
\hline & \multicolumn{3}{|c|}{ Statepoint 1 (BOC Cycle 1A) } & \multicolumn{3}{|c|}{ Statepoint 2 (0.0 Cycle 1B) } & \multicolumn{3}{|c|}{ Statepoint 3 (142.2 Cycle 1B) } \\
\hline & $\begin{array}{c}\text { Burnup } \\
\text { (GWd/MTU) }\end{array}$ & $\begin{array}{l}\text { Fuel } \\
\text { Temp. }\end{array}$ & $\begin{array}{l}\text { Moderator } \\
\text { Spec. Vol. }\end{array}$ & $\begin{array}{c}\text { Bumup } \\
\text { (GWd/MTU) }\end{array}$ & $\begin{array}{l}\text { Fuel } \\
\text { Temp. }\end{array}$ & $\begin{array}{l}\text { Moderator } \\
\text { Spec. Vol. }\end{array}$ & $\begin{array}{c}\text { Bumup } \\
\text { (GWd/MTU) }\end{array}$ & $\begin{array}{l}\text { Fuel } \\
\text { Temp. }\end{array}$ & $\begin{array}{l}\text { Moderator } \\
\text { Spec. Vol. }\end{array}$ \\
\hline & & & & 0.0 Cy $1 \mathrm{~B}$ & 184.7 Сy1А & 184.7 Cy1A & 142.2 Cy1B & 89.5 Cy18 & 89.5 Cy1B \\
\hline 1 & 0.0 & & & 3.645 & 975.5 & 0.0236 & 5.260 & 897.9 & 0.0228 \\
\hline 2 & 0.0 & & & 6.341 & 1202.1 & 0.0236 & 8.891 & 1024.9 & 0.0227 \\
\hline 3 & 0.0 & & & 8.389 & 1328.9 & 0.0235 & 11.451 & 1066.6 & 0.0227 \\
\hline 4 & 0.0 & \multicolumn{2}{|c|}{ Data not required. } & 9.750 & 1397.0 & 0.0233 & 13.043 & 1066.3 & 0.0226 \\
\hline 5 & 0.0 & & & 10.606 & 1429.2 & 0.0232 & 13.979 & 1048.5 & 0.0225 \\
\hline 6 & 0.0 & & & 11.121 & 1443.3 & 0.0231 & 14.462 & 1028.6 & 0.0224 \\
\hline 7 & 0.0 & & & 11.414 & 1451.7 & 0.0229 & 14.706 & 1012.6 & 0.0224 \\
\hline 8 & 0.0 & & & 11.561 & 1461.8 & 0.0228 & 14.817 & 1002.0 & 0.0223 \\
\hline 9 & 0.0 & & & 11.597 & 1476.9 & 0.0227 & 14.839 & 996.2 & 0.0222 \\
\hline 10 & 0.0 & & & 11.542 & 1495.3 & 0.0225 & 14.791 & 994.3 & 0.0222 \\
\hline 11 & 0.0 & & & 11.417 & 1513.4 & 0.0224 & 14.695 & 995.7 & 0.0221 \\
\hline 12 & 0.0 & & & 11.273 & 1525.1 & 0.0223 & 14.602 & 1000.0 & 0.0221 \\
\hline 13 & 0.0 & & & 11.175 & 1525.5 & 0.0221 & 14.569 & 1006.5 & 0.0220 \\
\hline 14 & 0.0 & & & 11.168 & 1515.8 & 0.0220 & 14.652 & 1016.2 & 0.0219 \\
\hline 15 & 0.0 & & & 11.291 & 1498.4 & 0.0219 & 15.074 & 1047.3 & 0.0219 \\
\hline 16 & 0.0 & & & 10.892 & 1458.3 & 0.0218 & 15.739 & 1232.9 & 0.0218 \\
\hline 17 & 0.0 & & & 9.417 & 1357.3 & 0.0217 & 14.395 & 1279.6 & 0.0217 \\
\hline 18 & 0.0 & & & 5.813 & 1103.7 & 0.0216 & 9.214 & 1109.5 & 0.0216 \\
\hline
\end{tabular}

\begin{tabular}{|c|c|c|c|c|c|c|c|c|c|}
\hline & \multicolumn{3}{|c|}{ Statepoint 4 (BOC Cycle 2) } & \multicolumn{3}{|c|}{ Statepoint 5 (BOC Cycle 3) } & \multicolumn{3}{|c|}{ Statepoint 6 (168.5 Cycle 3) } \\
\hline $\begin{array}{c}\text { Node } \\
\text { No. }\end{array}$ & $\begin{array}{c}\text { Burnup } \\
\text { (GWd/MTU) } \\
\end{array}$ & $\begin{array}{l}\text { Fuel } \\
\text { Temp. }\end{array}$ & $\begin{array}{l}\text { Moderator } \\
\text { Spec. Vol. }\end{array}$ & $\begin{array}{c}\text { Burnup } \\
\text { (GWd/MTU) }\end{array}$ & $\begin{array}{l}\text { Fuel } \\
\text { Temp. }\end{array}$ & $\begin{array}{l}\text { Moderator } \\
\text { Spec. Vol. }\end{array}$ & $\begin{array}{c}\text { Burnup } \\
\text { (GWd/MTU) }\end{array}$ & $\begin{array}{l}\text { Fuel } \\
\text { Temp. }\end{array}$ & $\begin{array}{l}\text { Moderator } \\
\text { Spec. Vol. }\end{array}$ \\
\hline & BOC Cy2 & 139.6 Cy1B & 139.6 Cy1B & BOC Cy3 & 89.8 Cy2 & $89.8 \mathrm{Cy} 2$ & $168.5 \mathrm{Cy} 3$ & 85.0 Суз & $85.0 \mathrm{Cy} 3$ \\
\hline 1 & 5.593 & 874.2 & 0.0236 & 7.656 & 871.5 & 0.0233 & 10.803 & 1020.4 & 0.0234 \\
\hline 2 & 9.400 & 973.4 & 0.0236 & 12.915 & 977.1 & 0.0232 & 17.876 & 1176.9 & 0.0233 \\
\hline 3 & 12.075 & 1022.8 & 0.0235 & 17.273 & 1121.4 & 0.0232 & 23.097 & 1200.7 & 0.0232 \\
\hline 4 & 13.887 & 1101.7 & 0.0234 & 19.795 & 1241.2 & 0.0230 & 25.889 & 1188.3 & 0.0231 \\
\hline 5 & 14.978 & 1337.1 & 0.0233 & 21.042 & 1250.0 & 0.0229 & 27.189 & 1167.2 & 0.0230 \\
\hline 6 & 15.622 & 1403.6 & 0.0232 & 21.673 & 1239.1 & 0.0228 & 27.785 & 1145.2 & 0.0228 \\
\hline 7 & 15.911 & 1423.0 & 0.0230 & 21.905 & 1232.8 & 0.0227 & 27.950 & 1126.3 & 0.0227 \\
\hline
\end{tabular}


Title: CRC Depletion Calculations for the Rodded Assemblies in Batches 1, 2, 3, and $1 \mathrm{X}$ of Crystal River Unit 3

Document Identifier: BBA000000-01717-0200-00040 REV 00

Page 29 of 146

Table 4.1.10-4 Burnup, Fuel Temperature, and Moderator Specific Volume Data for Assembly A7 of Crystal River Unit 3

\begin{tabular}{|c|r|r|r|r|r|r|r|r|r|}
\hline 8 & 16.036 & 1432.8 & 0.0229 & 21.966 & 1229.6 & 0.0226 & 27.922 & 1111.6 & 0.0226 \\
\hline 9 & 16.067 & 1439.7 & 0.0227 & 21.942 & 1226.0 & 0.0224 & 27.817 & 1106.1 & 0.0225 \\
\hline 10 & 16.034 & 1446.6 & 0.0226 & 21.868 & 1218.8 & 0.0223 & 27.726 & 1113.7 & 0.0224 \\
\hline 11 & 15.964 & 1454.5 & 0.0224 & 21.778 & 1206.9 & 0.0222 & 27.706 & 1133.0 & 0.0223 \\
\hline 12 & 15.902 & 1460.8 & 0.0223 & 21.716 & 1190.9 & 0.0221 & 27.779 & 1158.1 & 0.0222 \\
\hline 13 & 15.891 & 1458.6 & 0.0221 & 21.714 & 1172.9 & 0.0220 & 27.934 & 1180.9 & 0.0221 \\
\hline 14 & 15.970 & 1439.1 & 0.0220 & 21.789 & 1154.5 & 0.0219 & 28.121 & 1191.9 & 0.0220 \\
\hline 15 & 16.335 & 1385.4 & 0.0219 & 22.075 & 1132.7 & 0.0218 & 28.346 & 1181.1 & 0.0219 \\
\hline 16 & 16.879 & 1288.2 & 0.0218 & 22.359 & 1101.9 & 0.0217 & 28.278 & 1143.5 & 0.0218 \\
\hline 17 & 15.372 & 1195.1 & 0.0217 & 20.344 & 1073.3 & 0.0217 & 25.590 & 1094.2 & 0.0217 \\
\hline 18 & 9.856 & 1021.7 & 0.0216 & 13.277 & 963.8 & 0.0216 & 16.831 & 980.9 & 0.0216 \\
\hline
\end{tabular}

\begin{tabular}{|c|c|c|c|c|c|c|c|c|c|}
\hline & \multicolumn{3}{|c|}{ Statepoint 7 (250.0 Cycle 3 ) } & \multicolumn{3}{|c|}{ Statepoint 8} & \multicolumn{3}{|c|}{ Statepoint 9} \\
\hline $\begin{array}{l}\text { Node } \\
\text { No. }\end{array}$ & $\begin{array}{l}\text { Burnup } \\
\text { (GWdMTU) }\end{array}$ & $\begin{array}{l}\text { Fuel } \\
\text { Temp: }\end{array}$ & $\begin{array}{l}\text { Moderator } \\
\text { Spec. Vol. }\end{array}$ & $\begin{array}{l}\text { Burnup } \\
\text { (GWd/MTU) }\end{array}$ & $\begin{array}{l}\text { Fuel } \\
\text { Temp. }\end{array}$ & $\begin{array}{l}\text { Moderator } \\
\text { Spec. Vol. }\end{array}$ & \begin{tabular}{|c|} 
Bumup \\
(GWdMTU)
\end{tabular} & $\begin{array}{l}\text { Fuel } \\
\text { Temp. }\end{array}$ & $\begin{array}{l}\text { Moderator } \\
\text { Spec. Vol. }\end{array}$ \\
\hline & 250.0 cy3 & 246.4 Cy3 & $246.4 \mathrm{Cy} 3$ & & & & & & \\
\hline 1 & 12.512 & 1041.4 & 0.0233 & No additio & I state & & No addition & a statep & nts. \\
\hline 2 & 20.413 & 1147.0 & 0.0232 & & & & & & \\
\hline 3 & 25.906 & 1150.4 & 0.0231 & & & & & & \\
\hline 4 & 28.701 & 1121.7 & 0.0230 & & & & & & \\
\hline 5 & 29.947 & 1098.0 & 0.0229 & & & & & & \\
\hline 6 & 30.492 & 1081.9 & 0.0228 & & & & & & \\
\hline 7 & 30.624 & 1071.6 & 0.0227 & & & & & & \\
\hline 8 & 30.571 & 1063.9 & 0.0225 & & & & & & \\
\hline 9 & 30.449 & 1058.6 & 0.0224 & & & & & & \\
\hline 10 & 30.361 & 1057.7 & 0.0223 & & & & & & \\
\hline 11 & 30.371 & 1061.0 & 0.0222 & & & & & & \\
\hline 12 & 30.497 & 1066.9 & 0.0222 & & & & & & \\
\hline 13 & 30.727 & 1074.6 & 0.0221 & & & & & & \\
\hline 14 & 30.994 & 1082.8 & 0.0220 & & & & & & \\
\hline 15 & 31.250 & 1082.9 & 0.0219 & & & & & & \\
\hline 16 & 31.101 & 1066.6 & 0.0218 & & & & & & \\
\hline 17 & 28.192 & 1044.3 & 0.0217 & & & & & & \\
\hline 18 & 18.697 & 973.0 & 0.0216 & & & & & & \\
\hline
\end{tabular}


Title: CRC Depletion Calculations for the Rodded Assemblies in Batches 1, 2, 3, and $1 \mathrm{X}$ of Crystal River Unit 3

Document Identifier: BBA000000-01717-0200-00040 REV 00

Page 30 of 146

Table 4.1.10-5 Burnup, Fuel Temperature, and Moderator

Specific Volume Data for Assembly A14 of Crystal River Unit 3

\begin{tabular}{|c|c|c|c|c|c|c|c|c|c|}
\hline & \multicolumn{9}{|c|}{ Assembly Number A14 } \\
\hline & \multicolumn{3}{|c|}{ Statepoint 1 (BOC Cycle 1A) } & \multicolumn{3}{|c|}{ Statepoint $2(0.0$ Cycle 1B) } & \multicolumn{3}{|c|}{ Statepoint 3 (142.2 Cycle 1B) } \\
\hline & $\begin{array}{l}\text { Bumup } \\
\text { (GWd/MTU) }\end{array}$ & $\begin{array}{l}\text { Fuel } \\
\text { Temp. }\end{array}$ & $\begin{array}{l}\text { Moderator } \\
\text { Spec. Vol. }\end{array}$ & $\begin{array}{l}\text { Burnup } \\
\text { (GWd/MTU) }\end{array}$ & $\begin{array}{l}\text { Fuel } \\
\text { Temp. }\end{array}$ & $\begin{array}{l}\text { Moderator } \\
\text { Spec. Vol. }\end{array}$ & $\begin{array}{c}\text { Bumup } \\
\text { (GWd/MTU) }\end{array}$ & $\begin{array}{l}\text { Fuel } \\
\text { Temp. }\end{array}$ & $\begin{array}{l}\text { Moderator } \\
\text { Spec. Vol. }\end{array}$ \\
\hline & & & & 0.0 Cy1B & 184.7 Cy1A & 184.7 Cy1A & 142.2 Cy1B & 89.5 Cy1B & 89.5 Cy1B \\
\hline 1 & 0.0 & & & 3.042 & 898.5 & 0.0232 & 5.483 & 1066.3 & 0.0234 \\
\hline 2 & 0.0 & \multicolumn{2}{|c|}{ Data not required. } & 4.939 & 1070.7 & 0.0231 & 8.892 & 1268.6 & 0.0233 \\
\hline 3 & 0.0 & & & 6.561 & 1193.8 & 0.0230 & 11.299 & 1338.5 & 0.0232 \\
\hline 4 & 0.0 & & & 7.668 & 1260.2 & 0.0230 & 12.673 & 1340.7 & 0.0231 \\
\hline 5 & 0.0 & & & 8.361 & 1294.1 & 0.0229 & 13.401 & 1318.5 & 0.0229 \\
\hline 6 & 0.0 & & & 8.770 & 1310.7 & 0.0228 & 13.759 & 1292.8 & 0.0228 \\
\hline 7 & 0.0 & & & 8.995 & 1320.6 & 0.0226 & 13.920 & 1272.1 & 0.0227 \\
\hline 8 & 0.0 & & & 9.097 & 1330.3 & 0.0225 & 13.980 & 1258.7 & 0.0226 \\
\hline 9 & 0.0 & & & 9.105 & 1342.9 & 0.0224 & 13.977 & 1252.1 & 0.0225 \\
\hline 10 & 0.0 & & & 9.030 & 1358.2 & 0.0223 & 13.922 & 1250.9 & 0.0224 \\
\hline 11 & 0.0 & & & 8.888 & 1372.4 & 0.0222 & 13.831 & 1254.2 & 0.0223 \\
\hline 12 & 0.0 & & & 8.731 & 1378.6 & 0.0221 & 13.759 & 1261.6 & 0.0222 \\
\hline 13 & 0.0 & & & 8.635 & 1372.7 & 0.0220 & 13.768 & 1271.6 & 0.0221 \\
\hline 14 & 0.0 & & & 8.663 & 1358.8 & 0.0219 & 13.903 & 1282.7 & 0.0220 \\
\hline 15 & 0.0 & & & 8.790 & 1348.7 & 0.0218 & 14.142 & 1299.2 & 0.0219 \\
\hline 16 & 0.0 & & & 8.660 & 1334.6 & 0.0217 & 14.055 & 1319.4 & 0.0218 \\
\hline 17 & 0.0 & & & 7.628 & 1251.7 & 0.0217 & 12.572 & 1284.6 & 0.0217 \\
\hline 18 & 0.0 & & & 5.173 & 1049.6 & 0.0216 & 8.373 & 1088.6 & 0.0216 \\
\hline
\end{tabular}

\begin{tabular}{|c|c|c|c|c|c|c|c|c|c|}
\hline & \multicolumn{3}{|c|}{ Statepoint 4 (BOC Cycle 2) } & \multicolumn{3}{|c|}{ Statepoint 16 (BOC Cycle 7) } & \multicolumn{3}{|c|}{ Statepoint 17 (260.3 Cycle 7) } \\
\hline $\begin{array}{l}\text { Node } \\
\text { No. }\end{array}$ & $\begin{array}{c}\text { Burnup } \\
\text { (GWd/MTU) }\end{array}$ & $\begin{array}{l}\text { Fuel } \\
\text { Temp. }\end{array}$ & $\begin{array}{l}\text { Moderator } \\
\text { Spec. Vol. }\end{array}$ & $\begin{array}{l}\text { Burnup } \\
\text { (GWd/MTU) }\end{array}$ & $\begin{array}{l}\text { Fuel } \\
\text { Temp. }\end{array}$ & $\begin{array}{l}\text { Moderator } \\
\text { Spec. Vol. }\end{array}$ & $\begin{array}{c}\text { Bumup } \\
\text { (GWd/MTU) }\end{array}$ & $\begin{array}{l}\text { Fuel } \\
\text { Temp. }\end{array}$ & $\begin{array}{l}\text { Moderator } \\
\text { Spec. Vol. }\end{array}$ \\
\hline & BOC Cy2 & 139.6 Cy1B & 139.6 Cy1B & BOC Cy7 & $89.8 C y 2+d t$ & 89.8 Cy $2+d t$ & $260.3 C_{y 7}$ & $160.5 \mathrm{Cy} 7$ & 160.5 Cy7 \\
\hline 1 & 5.980 & 1002.2 & 0.0236 & 8.374 & 927.3 & 0.0230 & 11.725 & 981.7 & 0.0232 \\
\hline 2 & 666 & 1160.9 & 35 & 13.458 & 061.1 & 0229 & 19.446 & 100.8 & 0231 \\
\hline 3 & 12.216 & 1226.2 & 0.0234 & 16.706 & 1115.0 & 0.0228 & 24.253 & 1123.5 & 0.0230 \\
\hline 4 & 13.671 & 1270.2 & 0.0233 & 18.408 & 1126.9 & 0.0227 & 26.522 & 1126.8 & 0.0229 \\
\hline 5 & 14.457 & 1323.1 & 0.0232 & 19.258 & 1127.2 & 0.0226 & 588 & 21.4 & 0228 \\
\hline 6 & 14.858 & 1357.9 & 230 & 659 & 6.4 & 0.0225 & 28.078 & 1114.2 & 0.0227 \\
\hline 7 & 044 & 1374.9 & 0.0229 & 19.822 & 1126.8 & 0.0225 & 28.285 & 1108.0 & 0.0226 \\
\hline 8 & 113 & 1384.7 & 0.0228 & 19.857 & 1127.4 & 0.0224 & 28.349 & 1103.6 & 0.0225 \\
\hline 9 & 15.116 & 1391.5 & 0.0226 & 19.812 & 1125.5 & 0.0223 & 28.328 & 100.7 & 0.0224 \\
\hline 10 & 15.072 & .1397 .8 & 0.0225 & 19.713 & 1118.0 & 0.0222 & 28.244 & 1098.8 & 0.0223 \\
\hline 11 & 15.005 & 1405.1 & 0.0224 & 19.598 & 1104.3 & 0.0221 & 28.131 & 1097.4 & 0.0222 \\
\hline 12 & 14.962 & 1410.8 & 0.0222 & 19.528 & 1087.2 & 0.0220 & 28.041 & 1095.5 & 0.0221 \\
\hline
\end{tabular}


Title: CRC Depletion Calculations for the Rodded Assemblies in Batches 1, 2, 3, and $1 \mathrm{X}$ of Crystal River Unit 3

Table 4.1.10-5 Burnup, Fuel Temperature, and Moderator Specific Volume Data for Assembly A14 of Crystal River Unit 3

\begin{tabular}{|c|c|c|c|c|c|c|c|c|c|}
\hline 13 & 14.994 & 1407.9 & 0.0221 & 19.554 & 1069.7 & 0.0219 & 28.005 & 1091.8 & 0.0220 \\
\hline 14 & 15.127 & 1386.9 & 0.0220 & 19.696 & 1053.7 & 0.0218 & 28.008 & 1083.9 & 0.0219 \\
\hline 15 & 15.321 & 1340.3 & 0.0219 & 19.899 & 1041.6 & 0.0218 & 27.941 & 1068.7 & 0.0218 \\
\hline 16 & 15.142 & 1273.1 & 0.0218 & 19.649 & 1035.0 & 0.0217 & 27.219 & 1044.7 & 217 \\
\hline 17 & 13.506 & 1186.3 & 0.0217 & 17.597 & 1013.6 & 0.0216 & 24.303 & 1008.4 & 0.0216 \\
\hline 18 & 8.974 & 1002.4 & 0.0216 & 11.653 & 889.8 & 0.0216 & 16.167 & 907.6 & 0.0216 \\
\hline
\end{tabular}

\begin{tabular}{|c|c|c|c|c|c|c|c|c|c|}
\hline \multirow[b]{2}{*}{$\begin{array}{l}\text { Node } \\
\text { No. }\end{array}$} & \multicolumn{3}{|c|}{ Statepoint 18 (291.0 Cycle 7$)$} & \multicolumn{3}{|c|}{ Statepoint 19 (319.0 Cycle 7) } & \multicolumn{3}{|c|}{ Statepoint 20 (462.3 Cycle 7) } \\
\hline & $\begin{array}{l}\text { Burnup } \\
\text { (GWd/MTU) }\end{array}$ & $\begin{array}{l}\text { Fuel } \\
\text { Temp. }\end{array}$ & $\begin{array}{l}\text { Moderator } \\
\text { Spec. Vol. }\end{array}$ & $\begin{array}{c}\text { Burmup } \\
\text { (GWd/MTU) }\end{array}$ & $\begin{array}{l}\text { Fuel } \\
\text { Temp. }\end{array}$ & $\begin{array}{l}\text { Moderator } \\
\text { Spec. Vol. }\end{array}$ & $\begin{array}{l}\text { Bumup } \\
\text { (GWd/MTU) }\end{array}$ & $\begin{array}{l}\text { Fuel } \\
\text { Temp. }\end{array}$ & $\begin{array}{l}\text { Moderator } \\
\text { Spec. Vol. }\end{array}$ \\
\hline & $291.0 \mathrm{Cy} 7$ & $345.3 \mathrm{Cy} 7$ & 345.3 Cy7 & 319.0 Cy7 & $345.3 C_{y 7}$ & $345.3 C_{y 7}$ & $462.3 \mathrm{Cy} 7$ & $402.3 \mathrm{Cy} 7$ & $402.3 \mathrm{Cy}$ \\
\hline 1 & 12.243 & 1001.0 & 0.0232 & 12.775 & 1001.0 & 0.0232 & 15.529 & 1014.2 & 0.0232 \\
\hline 2 & 20.297 & 1074.7 & 0.0231 & 21.119 & 1074.7 & 0.0231 & 25.257 & 1079.0 & 0.0231 \\
\hline 3 & 25.222 & 1077.8 & 0.0230 & 26.141 & 1077.8 & 0.0230 & 30.701 & 1075.1 & 0.0230 \\
\hline 4 & 27.518 & 1063.7 & 0.0229 & 28.452 & 1063.7 & 0.0229 & 33.047 & 1062.0 & 0.0229 \\
\hline 5 & 581 & 1051.3 & 0.0228 & 29.505 & 1051.3 & 0.0228 & 34.037 & 1049.6 & 0.0228 \\
\hline 6 & 29.061 & 1041.6 & 0.0227 & 29.969 & 1041.6 & 0.0227 & 34.436 & 1039.4 & 0.0227 \\
\hline 7 & 29.260 & 1036.2 & 0.0226 & 30.154 & 1036.2 & 0.0226 & 34.579 & 33.0 & 0.0226 \\
\hline 8 & 29.319 & 4.4 & & & 1034.4 & & 611 & 29.6 & 2225 \\
\hline 9 & 297 & 1035.2 & 0.0224 & 30.175 & 1035.2 & 0.0224 & 34.584 & 1028.4 & 0.0224 \\
\hline 10 & 29.214 & 1037.9 & 0.0223 & 30.090 & 1037.9 & 0.0223 & 34.512 & 1028.8 & 0.0223 \\
\hline 11 & 29.105 & 1042.1 & 0.0222 & 29.979 & 1042.1 & 0.0222 & 34.426 & 1030.4 & 0.0222 \\
\hline 12 & 29.019 & 1047.5 & 0.0221 & 29.895 & 1047.5 & 0.0221 & 34.378 & 1033.0 & 0.0221 \\
\hline 13 & 28.989 & 1053.7 & 0.0220 & 29.867 & 1053.7 & 0.0220 & 34.397 & 1036.7 & 0.0220 \\
\hline 14 & 28.996 & 1059.5 & 0.0219 & 29.876 & 1059.5 & 0.0219 & 34.457 & 1040.9 & 0.0219 \\
\hline 15 & 28.925 & 1062.7 & 0.0218 & 29.799 & 1062.7 & 0.0218 & 34.416 & 1044.2 & 0.0218 \\
\hline 16 & 28.177 & 1059.9 & 0.0218 & 29.030 & 1059.9 & 0.0218 & 33.614 & 1042.5 & 0.0218 \\
\hline 17 & 25.179 & 1045.6 & 0.0217 & 25.962 & 1045.6 & 0.0217 & 30.269 & 1028.2 & 0.0217 \\
\hline 18 & 16.777 & 963.2 & 0.0216 & 17.325 & 963.2 & 0.0216 & 20.436 & 957.4 & 0.0216 \\
\hline
\end{tabular}

\begin{tabular}{|c|c|c|c|c|c|c|c|c|c|}
\hline \multirow[b]{2}{*}{$\begin{array}{l}\text { Node } \\
\text { No. }\end{array}$} & \multicolumn{3}{|c|}{ Statepoint 21 (479.0 Cycle 7) } & \multicolumn{3}{|c|}{ Statepoint 22} & \multicolumn{3}{|c|}{ Statepoint 23} \\
\hline & $\begin{array}{c}\text { Burnup } \\
\text { (GWd/MTU) }\end{array}$ & $\begin{array}{l}\text { Fuel } \\
\text { Temp. }\end{array}$ & $\begin{array}{l}\text { Moderator } \\
\text { Spec. Vol. }\end{array}$ & $\begin{array}{c}\text { Burnup } \\
\text { (GWdMTU) }\end{array}$ & $\begin{array}{l}\text { Fuel } \\
\text { Temp. }\end{array}$ & $\begin{array}{l}\text { Moderator } \\
\text { Spec. Vol. }\end{array}$ & $\begin{array}{c}\text { Burnup } \\
\text { (GWdMTU) }\end{array}$ & $\begin{array}{l}\text { Fuel } \\
\text { Temp. }\end{array}$ & $\begin{array}{l}\text { Moderator } \\
\text { Spec. Vol. }\end{array}$ \\
\hline & 479.0 Cy 7 & 402.3 Cy7 & 402.3 Cy7 & & & & & & \\
\hline 1 & 15.897 & 1014.2 & 0.0232 & No addition & a statep & & No additior & al statep & ints. \\
\hline 2 & 25.756 & 1079.0 & 0.0231 & & & & & & \\
\hline 3 & 31.236 & 1075.1 & 0.0230 & & & & & & \\
\hline 4 & 33.579 & 1062.0 & 0.0229 & & & & & & \\
\hline 5 & 34.559 & 1049.6 & 0.0228 & & & & & & \\
\hline 6 & 34.949 & 1039.4 & 0.0227 & & & & & & \\
\hline 7 & 35.087 & 1033.0 & 0.0226 & & & & & & \\
\hline
\end{tabular}


Title: CRC Depletion Calculations for the Rodded Assemblies in Batches 1, 2, 3, and 1X of Crystal River Unit 3

Document Identifier: BBA000000-01717-0200-00040 REV 00

Page 32 of 146

Table 4.1.10-5 Burnup, Fuel Temperature, and Moderator

Specific Volume Data for Assembly A14 of Crystal River Unit 3

\begin{tabular}{|c|r|r|r|l|l|l|l|l|l|}
\hline 8 & 35.118 & 1029.6 & 0.0225 & & & & & & \\
\hline 9 & 35.091 & 1028.4 & 0.0224 & & & & & & \\
\hline 10 & 35.021 & 1028.8 & 0.0223 & & & & & & \\
\hline 11 & 34.938 & 1030.4 & 0.0222 & & & & & & \\
\hline 12 & 34.893 & 1033.0 & 0.0221 & & & & & & \\
\hline 13 & 34.917 & 1036.7 & 0.0220 & & & & & & \\
\hline 14 & 34.984 & 1040.9 & 0.0219 & & & & & & \\
\hline 15 & 34.950 & 1044.2 & 0.0218 & & & & & & \\
\hline 16 & 34.150 & 1042.5 & 0.0218 & & & & & & \\
\hline 17 & 30.781 & 1028.2 & 0.0217 & & & & & & \\
\hline 18 & 20.815 & 957.4 & 0.0216 & & & & & & \\
\hline
\end{tabular}

Table 4.1.10-6 Burnup, Fuel Temperature, and Moderator

Specific Volume Data for Assembly A18 of Crystal River Unit 3

\begin{tabular}{|c|c|c|c|c|c|c|c|c|c|}
\hline & \multicolumn{9}{|c|}{ Assembly Number A18 } \\
\hline & \multicolumn{3}{|c|}{ Statepoint 1 (BOC Cycle 1A) } & \multicolumn{3}{|c|}{ Statepoint 22 (BOC Cycle 8) } & \multicolumn{3}{|c|}{ Statepoint 23 (97.6 Cycle 8) } \\
\hline & $\begin{array}{c}\text { Burnup } \\
\text { (GWd/MTU) }\end{array}$ & $\begin{array}{l}\text { Fuel } \\
\text { Temp. }\end{array}$ & $\begin{array}{l}\text { Moderator } \\
\text { Spec. Vol. }\end{array}$ & $\begin{array}{l}\text { Burnup } \\
\text { (GWd/MTU) }\end{array}$ & $\begin{array}{l}\text { Fuel } \\
\text { Temp. }\end{array}$ & $\begin{array}{l}\text { Moderator } \\
\text { Spec. Vol. }\end{array}$ & $\begin{array}{c}\text { Burnup } \\
\text { (GWd/MTU) }\end{array}$ & $\begin{array}{l}\text { Fuel } \\
\text { Temp. }\end{array}$ & $\begin{array}{l}\text { Moderator } \\
\text { Spec. Vol. }\end{array}$ \\
\hline & & & & BOC Cy8 & $184.7 C_{y 1} A+$ & 184.7 Cy1A+ & 97.6 Cy8 & 111.4 Cy8 & 111.4 Cy8 \\
\hline 1 & 0.0 & & & 3.416 & 929.4 & 0.0232 & 4.858 & 975.0 & 0.0231 \\
\hline 2 & 0.0 & & & 6.039 & 1153.1 & 0.0232 & 8.345 & 1124.6 & 0.0230 \\
\hline 3 & 0.0 & & & 8.119 & 1287.8 & 0.0231 & 10.833 & 1168.5 & 0.0229 \\
\hline 4 & 0.0 & \multicolumn{2}{|c|}{ Data not required. } & 9.507 & 1364.2 & 0.0230 & 12.370 & 1173.9 & 0.0228 \\
\hline 5 & 0.0 & & & 10.341 & 1399.1 & 0.0229 & 13.263 & 1168.5 & 0.0227 \\
\hline 6 & 0.0 & & & 10.809 & 1413.1 & 0.0227 & 13.759 & 1162.1 & 0.0226 \\
\hline 7 & 0.0 & & & 11.047 & 1421.0 & 0.0226 & 14.013 & 1156.8 & 0.0225 \\
\hline 8 & 0.0 & & & 11.116 & 1431.8 & 0.0225 & 14.095 & 1153.4 & 0.0224 \\
\hline 9 & 0.0 & & & 10.998 & 1451.4 & 0.0224 & 13.994 & 1153.4 & 0.0223 \\
\hline 10 & 0.0 & & & 10.622 & 1482.1 & 0.0222 & 13.650 & 1158.8 & 0.0223 \\
\hline 11 & 0.0 & & & 9.391 & 1531.2 & 0.0221 & 12.517 & 1184.1 & 0.0222 \\
\hline 12 & 0.0 & & & 6.666 & 1591.7 & 0.0220 & 10.008 & 1246.7 & 0.0221 \\
\hline 13 & 0.0 & & & 5.922 & 1390.6 & 0.0219 & 9.284 & 1259.9 & 0.0220 \\
\hline 14 & 0.0 & & & 5.786 & 1185.1 & 0.0218 & 9.078 & 1252.9 & 0.0219 \\
\hline 15 & 0.0 & & & 5.910 & 1122.0 & 0.0217 & 9.058 & 1230.6 & 0.0218 \\
\hline 16 & 0.0 & & & 6.969 & 1052.3 & 0.0217 & 9.803 & 1165.4 & 0.0217 \\
\hline 17 & 0.0 & & & 7.477 & 969.8 & 0.0216 & 9.821 & 1073.4 & 0.0216 \\
\hline 18 & 0.0 & & & 4.839 & 927.0 & 0.0216 & 6.305 & 926.3 & 0.0216 \\
\hline
\end{tabular}


Title: CRC Depletion Calculations for the Rodded Assemblies in Batches 1, 2, 3, and 1X of Crystal River Unit 3

Table 4.1.10-6 Burnup, Fuel Temperature, and Moderator Specific Volume Data for Assembly A18 of Crystal River Unit 3

Statepoint 24 (139.8 Cycle 8) Statepoint 25 (404.0 Cycle 8) Statepoint 26 (409.6 Cycle 8)

\begin{tabular}{|c|c|c|c|c|c|c|c|c|c|}
\hline $\begin{array}{l}\text { Node } \\
\text { No. }\end{array}$ & $\begin{array}{c}\text { Bumup } \\
\text { (GWd/MTU) }\end{array}$ & $\begin{array}{c}\text { Fuel } \\
\text { Temp. }\end{array}$ & $\begin{array}{l}\text { Moderator } \\
\text { Spec. Vol. }\end{array}$ & $\begin{array}{l}\text { Burnup } \\
\text { (GWd/MTU) }\end{array}$ & $\begin{array}{l}\text { Fuel } \\
\text { Temp. }\end{array}$ & $\begin{array}{l}\text { Moderator } \\
\text { Spec. Vol. }\end{array}$ & $\begin{array}{l}\text { Bumup } \\
\text { (GWdMTU) }\end{array}$ & $\begin{array}{l}\text { Fuel } \\
\text { Temp. }\end{array}$ & $\begin{array}{l}\text { Moderator } \\
\text { Spec. Vol. }\end{array}$ \\
\hline & 139.8 Сy8 & 111.4 Cy8 & 111.4 Cy8 & $404.0 C_{y} 8$ & 234.6 Cy8 & 234.6 Cy8 & 409.6 Cy8 & 234.6 Cy8 & 234.6 Cy8 \\
\hline 1 & 5.507 & 975.0 & 0.0231 & 9.904 & 977.2 & 0.0230 & 10.003 & 977.2 & 0.0230 \\
\hline 2 & 9.362 & 1124.6 & 0.0230 & 15.935 & 1101.7 & 0.0230 & 16.078 & 1101.7 & 0.0230 \\
\hline 3 & 12.012 & 1168.5 & 0.0229 & 19.409 & 1134.2 & 0.0229 & 19.566 & 1134.2 & 0.0229 \\
\hline 4 & 13.600 & 1173.9 & 0.0228 & 21.159 & 1128.2 & 0.0228 & 21.317 & 1128.2 & 0.0228 \\
\hline 5 & 14.507 & 1168.5 & 0.0227 & 22.042 & 1116.0 & 0.0227 & 22.198 & 1116.0 & .0227 \\
\hline 6 & 15.006 & 1162.1 & 0.0226 & 22.481 & 1105.3 & 0.0226 & 22.634 & 1105.3 & 0.0226 \\
\hline 7 & .259 & 1156.8 & 0.0225 & 22.681 & 1097.4 & 0.0225 & 22.833 & 1097.4 & 0.0225 \\
\hline 8 & 15.341 & 1153.4 & 0.0224 & 22.729 & 1092.3 & 0.0224 & 22.880 & 1092.3 & .0224 \\
\hline 9 & 15.243 & 1153.4 & 0.0223 & 22.627 & 1090.9 & 0.0223 & 22.778 & 1090.9 & 0223 \\
\hline 10 & 908 & 58.8 & 223 & 331 & 94.6 & 0.0222 & 22.483 & 1094.6 & 0.0222 \\
\hline 11 & 13.810 & 1184.1 & 0.0222 & 21.408 & 1115.0 & 0.0221 & 21.563 & 1115.0 & 0.0221 \\
\hline 12 & 11.380 & 1246.7 & 0.0221 & 19.386 & 1166.1 & 0.0221 & 19.548 & 1166.1 & 0.0221 \\
\hline 13 & 10.668 & 1259.9 & 0.0220 & 18.788 & 1178.9 & 0.0220 & 18.953 & 1178.9 & .0220 \\
\hline 14 & 10.445 & 1252.9 & 0.0219 & 18.573 & 1178.4 & 0.0219 & 18.739 & 1178.4 & 0.0219 \\
\hline 15 & 10.384 & 1230.6 & 0.0218 & 18.427 & 1169.2 & 0.0218 & 18.595 & 1169.2 & 0.0218 \\
\hline 16 & 11.023 & 1165.4 & 0.0217 & 18.657 & 1129.3 & 0.0217 & 18.820 & 1129.3 & 0.0217 \\
\hline 17 & 10.854 & 1073.4 & 0.0216 & 17.588 & 1063.3 & 0.0216 & 17.737 & 1063.3 & 0.0216 \\
\hline 18 & 6.967 & 926.3 & 0.0216 & 11.499 & 931.6 & 0.0216 & 11.603 & 931.6 & 0.0216 \\
\hline
\end{tabular}

\begin{tabular}{|c|c|c|c|c|c|c|}
\hline & \multicolumn{3}{|c|}{ Statepoint 27 (515.5 Cycle 8) } & \multicolumn{3}{|c|}{ Statepoint 28 (BOC Cycle 9) } \\
\hline $\begin{array}{l}\text { Node } \\
\text { No. }\end{array}$ & $\begin{array}{c}\text { Burnup } \\
\text { (GWdMTU) } \\
\end{array}$ & $\begin{array}{l}\text { Fuel } \\
\text { Temp. }\end{array}$ & $\begin{array}{l}\text { Moderator } \\
\text { Spec. Vol. }\end{array}$ & $\begin{array}{c}\text { Burnup } \\
\text { (GWd/MTU) }\end{array}$ & $\begin{array}{l}\text { Fuel } \\
\text { Temp. }\end{array}$ & $\begin{array}{l}\text { Moderatór } \\
\text { Spec. Vol. }\end{array}$ \\
\hline & 515.5 Cy8 & 470.7 Cy8 & 470.7 Cy8 & 800 Cy9 & 470.7 Cy8 & 470.7 Cy8 \\
\hline 1 & 11.964 & 983.9 & 0.0230 & & & \\
\hline 2 & 18.845 & 1074.1 & 0.0229 & No additiol & al statepo & \\
\hline 3 & 22.566 & 1076.1 & 0.0228 & & & \\
\hline 4 & 24.308 & 1058.8 & 0.0227 & & & \\
\hline 5 & 25.131 & 1041.2 & 0.0226 & & & \\
\hline 6 & 25.516 & 1027.9 & 0.0225 & & & \\
\hline 7 & 25.678 & 1018.7 & 0.0225 & & & \\
\hline 8 & 25.702 & 1012.7 & 0.0224 & & & \\
\hline 9 & 25.591 & 1009.9 & 0.0223 & & & \\
\hline 10 & 25.302 & 1010.8 & 0.0222 & & & \\
\hline 11 & 24.433 & 1022.9 & 0.0221 & & & \\
\hline 12 & 22.549 & 1055.0 & 0.0221 & & & \\
\hline 13 & 22.002 & 1064.7 & 0.0220 & & & \\
\hline
\end{tabular}




\section{Waste Package Development}

Design Analysis

Title: CRC Depletion Calculations for the Rodded Assemblies in Batches 1, 2, 3, and $1 \mathrm{X}$ of Crystal River Unit 3

Document Identifier: BBA000000-01717-0200-00040 REV 00

Page 34 of 146

Table 4.1.10-6 Burnup, Fuel Temperature, and Moderator

Specific Volume Data for Assembly A18 of Crystal River Unit 3

\begin{tabular}{|r|r|r|r|l|l|l|}
\hline 14 & 21.820 & 1068.9 & 0.0219 & & & \\
\hline 15 & 21.698 & 1071.7 & 0.0218 & & & \\
\hline 16 & 21.858 & 1058.6 & 0.0217 & & & \\
\hline 17 & 20.539 & 1030.9 & 0.0216 & & & \\
\hline 18 & 13.607 & 944.7 & 0.0216 & & & \\
\hline
\end{tabular}

Table 4.1.10-7 Burnup, Fuel Temperature, and Moderator

Specific Volume Data for Assembly A18a of Crystal River Unit 3

\begin{tabular}{|c|c|c|c|c|c|c|c|c|c|}
\hline \multirow{3}{*}{$\begin{array}{c}\text { Node } \\
\text { No. }\end{array}$} & \multicolumn{9}{|c|}{ Assembly Number A18a } \\
\hline & \multicolumn{3}{|c|}{ Statepoint 1 (BOC Cycle 1A) } & \multicolumn{3}{|c|}{ Statepoint 28 (BOC Cycle 9) } & \multicolumn{3}{|c|}{ Statepoint 29 (158.8 Cycle 9) } \\
\hline & $\begin{array}{c}\text { Burnup } \\
\text { (GWd/MTU) }\end{array}$ & $\begin{array}{l}\text { Fuel } \\
\text { Temp. }\end{array}$ & $\begin{array}{l}\text { Moderator } \\
\text { Spec. Vol. }\end{array}$ & $\begin{array}{c}\text { Burnup } \\
\text { (GWd/MTU) }\end{array}$ & $\begin{array}{l}\text { Fuel } \\
\text { Temp. }\end{array}$ & $\begin{array}{l}\text { Moderator } \\
\text { Spec. Vol. }\end{array}$ & $\begin{array}{c}\text { Burnup } \\
\text { (GWd/MTU) }\end{array}$ & $\begin{array}{l}\text { Fuel } \\
\text { Temp. }\end{array}$ & $\begin{array}{l}\text { Moderator } \\
\text { Spec. Vol. }\end{array}$ \\
\hline & & & & BOC Cy9 & 184.7 Cy1A+ & 184.7 Cy1A+ & 158.8 Cy9 & 110.5 Cy9 & 110.5 Cy9 \\
\hline 1 & 0.0 & & & 3.416 & 929.4 & 0.0232 & 5.836 & 983.5 & 0.0231 \\
\hline 2 & 0.0 & & & 6.039 & 1153.1 & 0.0232 & 9.936 & 1142.6 & 0.0231 \\
\hline 3 & 0.0 & & & 8.119 & 1287.8 & 0.0231 & 12.740 & 1195.4 & 0.0230 \\
\hline 4 & 0.0 & \multicolumn{2}{|c|}{ Data not required. } & 9.507 & 1364.2 & 0.0230 & 14.408 & 1206.0 & 0.0229 \\
\hline 5 & 0.0 & & & 10.341 & 1399.1 & 0.0229 & 15.354 & 1203.0 & 0.0228 \\
\hline 6 & 0.0 & & & 10.809 & 1413.1 & 0.0227 & 15.868 & 1197.2 & 0.0227 \\
\hline 7 & 0.0 & & & 11.047 & 1421.0 & 0.0226 & 16.124 & 1191.6 & 0.0226 \\
\hline 8 & 0.0 & & & 11.116 & 1431.8 & 0.0225 & 16.202 & 1187.6 & 0.0225 \\
\hline 9 & 0.0 & & & 10.998 & 1451.4 & 0.0224 & 16.102 & 1187.1 & 0.0224 \\
\hline 10 & 0.0 & & & 10.622 & 1482.1 & 0.0222 & 15.770 & 1192.5 & 0.0223 \\
\hline 11 & 0.0 & & & 9.391 & 1531.2 & 0.0221 & 14.700 & 1219.6 & 0.0222 \\
\hline 12 & 0.0 & & & 6.666 & 1591.7 & 0.0220 & 12.336 & 1286.7 & 0.0221 \\
\hline 13 & 0.0 & & & 5.922 & 1390.6 & 0.0219 & 11.651 & 1300.8 & 0.0220 \\
\hline 14 & 0.0 & & & 5.786 & 1185.1 & 0.0218 & 11.434 & 1295.2 & 0.0219 \\
\hline 15 & 0.0 & & & 5.910 & 1122.0 & 0.0217 & 11.352 & 1273.0 & 0.0218 \\
\hline 16 & 0.0 & & & 6.969 & 1052.3 & 0.0217 & 11.911 & 1205.9 & 0.0218 \\
\hline 17 & 0.0 & & & 7.477 & 969.8 & 0.0216 & 11.595 & 1107.5 & 0.0217 \\
\hline 18 & 0.0 & & & 4.839 & 927.0 & 0.0216 & 7.424 & 949.6 & 0.0216 \\
\hline
\end{tabular}

\begin{tabular}{|c|c|c|c|c|c|c|c|c|c|}
\hline & \multicolumn{3}{|c|}{ Statepoint 30 (219.0 Cycle 9) } & \multicolumn{3}{|c|}{ Statepoint 31 (363.1 Cycle 9) } & \multicolumn{3}{|c|}{ Statepoint 32 (BOC Cycle 10) } \\
\hline $\begin{array}{l}\text { Node } \\
\text { No. }\end{array}$ & $\begin{array}{c}\text { Burnup } \\
\text { (GWd/MTU) }\end{array}$ & $\begin{array}{l}\text { Fuel } \\
\text { Temp. }\end{array}$ & $\begin{array}{l}\text { Moderator } \\
\text { Spec. Vol. }\end{array}$ & $\begin{array}{c}\text { Burnup } \\
\text { (GWd/MTU) }\end{array}$ & $\begin{array}{l}\text { Fuel } \\
\text { Temp. }\end{array}$ & $\begin{array}{l}\text { Moderator } \\
\text { Spec. Vol. }\end{array}$ & $\begin{array}{c}\text { Burnup } \\
\text { (GWd/MTU) }\end{array}$ & $\begin{array}{l}\text { Fuel } \\
\text { Temp. }\end{array}$ & $\begin{array}{l}\text { Moderator } \\
\text { Spec. Vol. }\end{array}$ \\
\hline & 219.0 Cy9 & 192.8 Сy9 & 192.8 Cy9 & 363.1 Cy9 & 303.7 Cy9 & 303.7 Cy9 & BOC Cy10 & 452.4 Cy9 & 452.4 Cy9 \\
\hline 1 & 6.823 & 988.8 & 0.0231 & 9.342 & 995.4 & 0.0231 & & & \\
\hline 2 & 11.468 & 1127.7 & 0.0231 & 15.254 & 1119.6 & 0.0230 & & & \\
\hline 3 & 14.512 & 1173.1 & 0.0230 & 18.790 & 1145.0 & 0.0229 & \multicolumn{3}{|c|}{ No additional statepoints. } \\
\hline 4 & 16.251 & 1173.5 & 0.0229 & 20.633 & 1134.0 & 0.0228 & & & \\
\hline
\end{tabular}


Title: CRC Depletion Calculations for the Rodded Assemblies in Batches 1, 2, 3, and $1 \mathrm{X}$ of Crystal River Unit 3

Document Identifier: BBA000000-01717-0200-00040 REV 00

Page 35 of 146

Table 4.1.10-7 Burnup, Fuel Temperature, and Moderator

Specific Volume Data for Assembly A18a of Crystal River Unit 3

\begin{tabular}{|l|r|r|r|r|r|r|l|l|l|}
\hline 5 & 17.210 & 1164.3 & 0.0228 & 21.580 & 1118.7 & 0.0227 & & & \\
\hline 6 & 17.721 & 1154.7 & 0.0227 & 22.052 & 1106.7 & 0.0226 & & & \\
\hline 7 & 17.969 & 1147.0 & 0.0226 & 22.265 & 1098.1 & 0.0226 & & & \\
\hline 8 & 18.040 & 1141.7 & 0.0225 & 22.313 & 1092.4 & 0.0225 & & & \\
\hline 9 & 17.939 & 1140.2 & 0.0224 & 22.207 & 1090.2 & 0.0224 & & & \\
\hline 10 & 17.617 & 1144.4 & 0.0223 & 21.908 & 1092.6 & 0.0223 & & & \\
\hline 11 & 16.594 & 1167.4 & 0.0222 & 20.985 & 1111.4 & 0.0222 & & & \\
\hline 12 & 14.339 & 1225.0 & 0.0221 & 18.963 & 1160.3 & 0.0221 & & & \\
\hline 13 & 13.681 & 1239.4 & 0.0220 & 18.383 & 1174.5 & 0.0220 & & & \\
\hline 14 & 13.458 & 1238.3 & 0.0219 & 18.182 & 1177.8 & 0.0219 & & & \\
\hline 15 & 13.339 & 1225.9 & 0.0218 & 18.034 & 1174.5 & 0.0218 & & & \\
\hline 16 & 13.768 & 1177.6 & 0.0218 & 18.247 & 1143.5 & 0.0218 & & & \\
\hline 17 & 13.197 & 1099.4 & 0.0217 & 17.168 & 1088.5 & 0.0217 & & & \\
\hline 18 & 8.469 & 953.8 & 0.0216 & 11.149 & 957.9 & 0.0216 & & & \\
\hline
\end{tabular}

Table 4.1.10-8 Burnup, Fuel Temperature, and Moderator

Specific Volume Data for Assembly A18b of Crystal River Unit 3

\begin{tabular}{|c|c|c|c|c|c|c|c|c|c|}
\hline & \multicolumn{9}{|c|}{ Assembly Number A18b } \\
\hline & \multicolumn{3}{|c|}{ Statepoint 1 (BOC Cycle 1A) } & \multicolumn{3}{|c|}{ Statepoint 2 (0.0 Cycle 1B) } & \multicolumn{3}{|c|}{ Statepoint 3 (142.2 Cycle 1B) } \\
\hline & $\begin{array}{c}\text { Burnup } \\
\text { (GWd/MTU) }\end{array}$ & $\begin{array}{l}\text { Fuel } \\
\text { Temp. }\end{array}$ & $\begin{array}{l}\text { Moderator } \\
\text { Spec. Vol. }\end{array}$ & $\begin{array}{c}\text { Burnup } \\
\text { (GWd/MTU) }\end{array}$ & $\begin{array}{l}\text { Fuel } \\
\text { Temp. }\end{array}$ & $\begin{array}{l}\text { Moderator } \\
\text { Spec. Vol. }\end{array}$ & $\begin{array}{c}\text { Burnup } \\
\text { (GWd/MTU) }\end{array}$ & $\begin{array}{l}\text { Fuel } \\
\text { Temp. }\end{array}$ & $\begin{array}{l}\text { Moderator } \\
\text { Spec. Vol. }\end{array}$ \\
\hline & & & & 0.0 Cy1B & $184.7 \mathrm{Cy} 1 \mathrm{~A}$ & 184.7 Cy1A & 142.2 Cy1B & 89.5 Cy1B & 89.5 Cy1B \\
\hline 1 & 0.0 & & & 3.416 & 929.4 & 0.0232 & 5.861 & 1064.3 & 0.0231 \\
\hline 2 & 0.0 & & & 6.039 & 1153.1 & 0.0232 & 9.956 & 1253.8 & 0.0231 \\
\hline 3 & 0.0 & & & 8.119 & 1287.8 & 0.0231 & 12.792 & 1321.8 & 0.0229 \\
\hline 4 & 0.0 & \multicolumn{2}{|c|}{ Data not required. } & 9.507 & 1364.2 & 0.0230 & 14.424 & 1317.4 & 0.0228 \\
\hline 5 & 0.0 & & & 10.341 & 1399.1 & 0.0229 & 15.255 & 1285.0 & 0.0227 \\
\hline 6 & 0.0 & & & 10.809 & 1413.1 & 0.0227 & 15.627 & 1251.8 & 0.0226 \\
\hline 7 & 0.0 & & & 11.047 & 1421.0 & 0.0226 & 15.760 & 1226.7 & 0.0225 \\
\hline 8 & 0.0 & & & 11.116 & 1431.8 & 0.0225 & 15.750 & 1210.8 & 0.0224 \\
\hline 9 & 0.0 & & & 10.998 & 1451.4 & 0.0224 & 15.564 & 1202.2 & 0.0223 \\
\hline 10 & 0.0 & & & 10.622 & 1482.1 & 0.0222 & 14.951 & 1185.3 & 0.0222 \\
\hline 11 & 0.0 & & & 9.391 & 1531.2 & 0.0221 & 12.957 & 1094.9 & 0.0221 \\
\hline 12 & 0.0 & & & 6.666 & 1591.7 & 0.0220 & 9.795 & 1048.4 & 0.0220 \\
\hline 13 & 0.0 & & & 5.922 & 1390.6 & 0.0219 & 9.090 & 1056.4 & 0.0220 \\
\hline 14 & 0.0 & & & 5.786 & 1185.1 & 0.0218 & 9.113 & 1070.0 & 0.0219 \\
\hline 15 & 0.0 & & & 5.910 & 1122.0 & 0.0217 & 9.779 & 1102.8 & 0.0218 \\
\hline 16 & 0.0 & & & 6.969 & 1052.3 & 0.0217 & 11.959 & 1269.7 & 0.0218 \\
\hline 17 & 0.0 & & & 7.477 & 969.8 & 0.0216 & 12.257 & 1252.8 & 0.0217 \\
\hline 18 & 0.0 & & & 4.839 & 927.0 & 0.0216 & 7.957 & 1075.8 & 0.0216 \\
\hline
\end{tabular}


Title: CRC Depletion Calculations for the Rodded Assemblies in Batches 1, 2, 3, and $1 \mathrm{X}$ of Crystal River Unit 3

Document Identifier: BBA000000-01717-0200-00040 REV 00

Page 36 of 146

Table 4.1.10-9 Burnup, Fuel Temperature, and Moderator

Specific Volume Data for Assembly A20 of Crystal River Unit 3 Assembly Number A20

Statepoint 1 (BOC Cycle 1A) Statepoint 2 (0.0 Cycle 1B) Statepoint 3 (142.2 Cycle 1B)

\begin{tabular}{|c|c|c|c|c|c|c|c|c|c|}
\hline $\begin{array}{l}\text { Node } \\
\text { No. }\end{array}$ & $\begin{array}{c}\text { Bumup } \\
\text { (GWd/MTU) }\end{array}$ & $\begin{array}{l}\text { Fuel } \\
\text { Temp. }\end{array}$ & $\begin{array}{l}\text { Moderator } \\
\text { Spec. Vol. }\end{array}$ & $\begin{array}{c}\text { Burnup } \\
\text { (GWd/MTU) }\end{array}$ & $\begin{array}{l}\text { Fuel } \\
\text { Temp. }\end{array}$ & $\begin{array}{l}\text { Moderator } \\
\text { Spec. Vol. }\end{array}$ & $\begin{array}{l}\text { Burnup } \\
\text { (GWdMTU) }\end{array}$ & $\begin{array}{l}\text { Fuel } \\
\text { Temp. }\end{array}$ & $\begin{array}{l}\text { Moderator } \\
\text { Spec. Vol. }\end{array}$ \\
\hline & & & & 0.0 Cy1B & 184.7 Cy1A & 184.7 Cy1A & 142.2 Cy1B & 89.5 Cy1B & 89.5 Cy1B \\
\hline 1 & 0.0 & & & 1.929 & 766.4 & 0.0226 & 4.564 & 1116.6 & 0.0236 \\
\hline 2 & 0.0 & & & 3.349 & 890.3 & 0.0226 & 7.656 & 1349.1 & 0.0235 \\
\hline 3 & 0.0 & & & 4.454 & 980.9 & 0.0225 & 9.656 & 1443.2 & 0.0234 \\
\hline 4 & 0.0 & \multicolumn{2}{|c|}{ Data not required. } & 5.176 & 1034.5 & 0.0225 & 10.692 & 1455.2 & 0.0232 \\
\hline 5 & 0.0 & & & 5.618 & 1063.3 & 0.0224 & 11.186 & 1437.0 & 0.0231 \\
\hline 6 & 0.0 & & & 5.871 & 1077.4 & 0.0224 & 11.397 & 1412.9 & 0.0229 \\
\hline 7 & 0.0 & & & 6.003 & 1085.8 & 0.0223 & 11.473 & 1393.2 & 0.0228 \\
\hline 8 & 0.0 & & & 6.054 & 1093.3 & 0.0222 & 11.490 & 1380.6 & 0.0227 \\
\hline 9 & 0.0 & - & & 6.041 & 1102.2 & 0.0222 & 11.473 & 1374.8 & 0.0226 \\
\hline 10 & 0.0 & & & 5.965 & 1112.6 & 0.0221 & 11.419 & 1374.1 & 0.0224 \\
\hline 11 & 0.0 & & & 5.831 & 1121.4 & 0.0220 & 11.340 & 1377.9 & 0.0223 \\
\hline 12 & 0.0 & & & 5.685 & 1122.4 & 0.0220 & 11.287 & 1386.3 & 0.0222 \\
\hline 13 & 0.0 & & & 5.608 & 1112.5 & 0.0219 & 11.324 & 1397.1 & 0.0221 \\
\hline 14 & 0.0 & & & 5.721 & 1098.0 & 0.0219 & 11.522 & 1403.6 & 0.0220 \\
\hline 15 & 0.0 & & & 6.476 & 1109.7 & 0.0218 & 12.214 & 1385.3 & 0.0219 \\
\hline 16 & 0.0 & & & 7.826 & 1298.5 & 0.0217 & 13.223 & 1329.1 & 0.0218 \\
\hline 17 & 0.0 & & & 7.524 & 1237.3 & 0.0216 & 12.242 & 1253.5 & 0.0217 \\
\hline 18 & 0.0 & & & 4.698 & 1003.7 & 0.0216 & 7.730 & 1068.4 & 0.0216 \\
\hline
\end{tabular}

\begin{tabular}{|c|c|c|c|c|c|c|c|c|c|}
\hline & \multicolumn{3}{|c|}{ Statepoint 4 (BOC Cycle 2) } & \multicolumn{3}{|c|}{ Statepoint 5 (BOC Cycle 3) } & \multicolumn{3}{|c|}{ Statepoint 6 ( 168.5 Cycle 3) } \\
\hline $\begin{array}{l}\text { Node } \\
\text { No. }\end{array}$ & $\begin{array}{l}\text { Burnup } \\
\text { (GWd/MTU) }\end{array}$ & $\begin{array}{l}\text { Fuel } \\
\text { Temp. }\end{array}$ & $\begin{array}{l}\text { Moderator } \\
\text { Spec. Vol. }\end{array}$ & $\begin{array}{c}\text { Burnup } \\
\text { (GWd/MTU) }\end{array}$ & $\begin{array}{l}\text { Fuel } \\
\text { Temp. }\end{array}$ & $\begin{array}{l}\text { Moderator } \\
\text { Spec. Vol. }\end{array}$ & $\begin{array}{c}\text { Burnup } \\
\text { (GWd/MTU) }\end{array}$ & $\begin{array}{l}\text { Fuel } \\
\text { Temp. }\end{array}$ & $\begin{array}{l}\text { Moderator } \\
\text { Spec. Vol. }\end{array}$ \\
\hline & BOC Cy2 & 139.6 Cy1B & 139.6 Cy1B & BOC Cy3 & 89.8 Cy2 & $89.8 \mathrm{Cy} 2$ & $168.5 \mathrm{Cy}^{3}$ & $85.0 C_{y 3}$ & $85.0 \mathrm{Cy3}$ \\
\hline 1 & 5.094 & 1038.6 & 0.0235 & 8.349 & 1050.3 & 0.0236 & 11.641 & 1022.2 & 0.0234 \\
\hline 2 & 8.487 & 1220.9 & 0.0235 & 13.645 & 1217.7 & 0.0235 & 18.735 & 1167.1 & 0.0233 \\
\hline 3 & 10.635 & 1294.5 & 0.0233 & 16.910 & 1311.6 & 0.0234 & 22.886 & 1207.6 & 0.0232 \\
\hline 4 & 11.730 & 1327.4 & 0.0232 & 18.514 & 1360.1 & 0.0232 & 24.819 & 1211.1 & 0.0231 \\
\hline 5 & 12.251 & 1351.3 & 0.0231 & 19.233 & 1380.6 & 0.0231 & 25.637 & 1201.2 & 0.0230 \\
\hline 6 & 12.480 & 1370.9 & 0.0230 & 19.520 & 1389.8 & 0.0229 & 25.916 & 1184.3 & 0.0228 \\
\hline 7 & 12.568 & 1385.1 & 0.0228 & 19.589 & 1397.4 & 0.0228 & 25.895 & 1159.4 & 0.0227 \\
\hline 8 & 12.589 & 1394.4 & 0.0227 & 19.522 & 1404.3 & 0.0227 & 25.615 & 1126.3 & 0.0226 \\
\hline 9 & 12.573 & 1400.1 & 0.0226 & 19.319 & 1404.1 & 0.0225 & 25.089 & 1103.4 & 0.0225 \\
\hline 10 & 12.528 & 1404.1 & 0.0224 & 18.973 & 1379.8 & 0.0224 & 24.534 & 1107.0 & 0.0224 \\
\hline 11 & 12.470 & 1409.1 & 0.0223 & 18.605 & 1318.8 & 0.0223 & 24.168 & 1130.3 & 0.0223 \\
\hline
\end{tabular}


Title: CRC Depletion Calculations for the Rodded Assemblies in Batches 1, 2, 3, and $1 \mathrm{X}$ of Crystal River Unit 3

Document Identifier: BBA000000-01717-0200-00040 REV 00

Page 37 of 146

Table 4.1.10-9 Burnup, Fuel Temperature, and Moderator

Specific Volume Data for Assembly A20 of Crystal River Unit 3

\begin{tabular}{|r|r|r|r|r|r|r|r|r|r|}
\hline 12 & 12.449 & 1414.7 & 0.0222 & 18.406 & 1260.1 & 0.0221 & 24.130 & 1169.9 & 0.0222 \\
\hline 13 & 12.515 & 1412.9 & 0.0221 & 18.467 & 1220.0 & 0.0220 & 24.503 & 1221.0 & 0.0221 \\
\hline 14 & 12.718 & 1393.5 & 0.0220 & 18.792 & 1193.8 & 0.0219 & 25.199 & 1248.4 & 0.0220 \\
\hline 15 & 13.362 & 1337.7 & 0.0218 & 19.541 & 1179.2 & 0.0218 & 26.016 & 1234.1 & 0.0219 \\
\hline 16 & 14.259 & 1244.4 & 0.0217 & 20.283 & 1168.9 & 0.0217 & 26.402 & 1185.8 & 0.0218 \\
\hline 17 & 13.123 & 1150.2 & 0.0217 & 18.570 & 1141.1 & 0.0217 & 23.960 & 1126.2 & 0.0217 \\
\hline 18 & 8.299 & 985.1 & 0.0216 & 11.970 & 1005.6 & 0.0216 & 15.579 & 999.8 & 0.0216 \\
\hline
\end{tabular}

\begin{tabular}{|c|c|c|c|c|c|c|c|c|c|}
\hline & \multicolumn{3}{|c|}{ Statepoint 7 (250.0 Cycle 3) } & \multicolumn{3}{|c|}{ Statepoint 8} & \multicolumn{3}{|c|}{ Statepoint 9} \\
\hline $\begin{array}{l}\text { Node } \\
\text { No. }\end{array}$ & $\begin{array}{c}\text { Burnup } \\
\text { (GWdMTU) }\end{array}$ & $\begin{array}{l}\text { Fuel } \\
\text { Temp. }\end{array}$ & $\begin{array}{l}\text { Moderator } \\
\text { Spec. Vol. }\end{array}$ & $\begin{array}{c}\text { Burnup } \\
\text { (GWd/MTU) }\end{array}$ & $\begin{array}{l}\text { Fuel } \\
\text { Temp. }\end{array}$ & $\begin{array}{l}\text { Moderator } \\
\text { Spec. Vol. }\end{array}$ & \begin{tabular}{|c|} 
Bumup \\
(GWdMMTU)
\end{tabular} & $\begin{array}{l}\text { Fuel } \\
\text { Temp }\end{array}$ & $\begin{array}{l}\text { Moderator } \\
\text { Spec. Vol. }\end{array}$ \\
\hline & 250.0 cy3 & $246.4 C_{y 3}$ & 246.4 Cy3 & & & & & & \\
\hline 1 & 13.385 & 1044.7 & 0.0233 & \multicolumn{3}{|c|}{ No additional statepoints } & \multicolumn{3}{|c|}{ No additional statepoints. } \\
\hline 2 & 21.262 & 1137.9 & 0.0233 & & & & & & \\
\hline 3 & 25.705 & 1153.8 & 0.0231 & & & & & & \\
\hline 4 & 27.687 & 1140.6 & 0.0230 & & & & & & \\
\hline 5 & 28.483 & 1125.2 & 0.0229 & & & & & & \\
\hline 6 & 28.733 & 1113.4 & 0.0228 & & & & & & \\
\hline 7 & 28.682 & 1103.9 & 0.0227 & & & & & & \\
\hline 8 & 28.343 & 1090.3 & 0.0226 & & & & & & \\
\hline 9 & 27.715 & 1071.2 & 0.0225 & & & & & & \\
\hline 10 & 27.095 & 1065.2 & 0.0224 & & & & & & \\
\hline 11 & 26.738 & 1070.7 & 0.0223 & & & & & & \\
\hline 12 & 26.761 & 1081.5 & 0.0222 & & & & & & \\
\hline 13 & 27.260 & 1099.8 & 0.0221 & & & & & & \\
\hline 14 & 28.139 & 1121.4 & 0.0220 & & & & & & \\
\hline 15 & 29.033 & 1120.3 & 0.0219 & & & & & & \\
\hline 16 & 29.332 & 1098.5 & 0.0218 & & & & & & \\
\hline 17 & 26.649 & 1075.1 & 0.0217 & & & & & & \\
\hline 18 & 17.494 & 995.2 & 0.0216 & & & & & & \\
\hline
\end{tabular}

Table 4.1.10-10 Burnup, Fuel Temperature, and Moderator Specific Volume Data for Assembly A22 of Crystal River Unit 3 Assembly Number A22

Statepoint 1 (BOC Cycle 1A) Statepoint 2 (0.0 Cycle 1B) Statepoint 3 (142.2 Cycle 1B)

\begin{tabular}{|c|c|c|c|c|c|c|c|c|c|}
\hline $\begin{array}{l}\text { Node } \\
\text { No. }\end{array}$ & $\begin{array}{l}\text { Bumup } \\
\text { (GWd/MTU) }\end{array}$ & $\begin{array}{l}\text { Fuel } \\
\text { Temp. }\end{array}$ & $\begin{array}{l}\text { Moderator } \\
\text { Spec. Vol. }\end{array}$ & $\begin{array}{l}\text { Burnup } \\
\text { (GWd/MTU) }\end{array}$ & $\begin{array}{c}\text { Fuel } \\
\text { Temp. }\end{array}$ & $\begin{array}{l}\text { Moderator } \\
\text { Spec. Vol. }\end{array}$ & $\begin{array}{c}\text { Burnup } \\
\text { (GWd/MTU) }\end{array}$ & $\begin{array}{l}\text { Fuel } \\
\text { Temp. }\end{array}$ & $\begin{array}{l}\text { Moderator } \\
\text { Spec. Vol. }\end{array}$ \\
\hline & & & & 0.0 Cy1B & 184.7 Cy1A & 184.7 Cy1A & 142.2 Cy1B & 89.5 Cy1B & 89.5 Cy1B \\
\hline 1 & 0.0 & & & 2.356 & 817.6 & 0.0235 & 3.912 & 901.0 & 0.0232 \\
\hline 2 & 0.0 & & & 4.987 & 984.0 & 0.0234 & 7.595 & 1099.8 & 0.02 \\
\hline
\end{tabular}


Title: CRC Depletion Calculations for the Rodded Assemblies in Batches 1, 2, 3, and $1 \mathrm{X}$ of Crystal River Unit 3

Table 4.1.10-10 Burnup, Fuel Temperature, and Moderator Specific Volume Data for Assembly A22 of Crystal River Unit 3

\begin{tabular}{|c|c|c|c|c|c|c|c|c|}
\hline 3 & 0.0 & & 8.232 & 1269.2 & 0.0233 & 12.009 & 1302.3 & 0.0230 \\
\hline 4 & 0.0 & Data not required. & 9.957 & 1391.9 & 0.0232 & 14.545 & 1300.9 & 0.0229 \\
\hline 5 & 0.0 & & 10.858 & 1432.1 & 0.0231 & 15.588 & 1261.8 & 0.0228 \\
\hline 6 & 0.0 & & 11.352 & 1445.9 & 0.0230 & 15.992 & 1226.2 & 0.0227 \\
\hline 7 & 0.0 & & 11.601 & 1453.4 & 0.0228 & 16.133 & 1200.1 & 0.0226 \\
\hline 8 & 0.0 & & 11.681 & 1464.5 & 0.0227 & 16.129 & 1183.4 & 0.0225 \\
\hline 9 & 0.0 & & 11.603 & 1484.2 & 0.0226 & 15.985 & 1173.3 & 0.0224 \\
\hline 10 & 0.0 & & 11.320 & 1514.1 & 0.0224 & 15.619 & 1164.2 & 0.0223 \\
\hline 11 & 0.0 & & 10.761 & 1546.4 & 0.0223 & 14.973 & 1154.9 & 0.0222 \\
\hline 12 & 0.0 & & 10.054 & 1559.1 & 0.0222 & 14.263 & 1154.6 & 0.0221 \\
\hline 13 & 0.0 & & 9.553 & 1523.6 & 0.0220 & 13.875 & 1166.3 & 0.0220 \\
\hline 14 & 0.0 & & 9.311 & 1454.6 & 0.0219 & 13.841 & 1187.8 & 0.0219 \\
\hline 15 & 0.0 & & 9.181 & 1384.7 & 0.0218 & 14.005 & 1223.7 & 0.0219 \\
\hline 16 & 0.0 & & 8.884 & 1306.8 & 0.0217 & 13.923 & 1262.0 & 0.0218 \\
\hline 17 & 0.0 & & 7.816 & 1200.9 & 0.0216 & 12.514 & 1240.6 & 0.0217 \\
\hline 18 & 0.0 & & 4.869 & 981.4 & 0.0216 & 7.959 & 1072.3 & 0.0216 \\
\hline
\end{tabular}

Table 4.1.10-11 Burnup, Fuel Temperature, and Moderator Specific Volume Data for Assembly A23 of Crystal River Unit 3

Assembly Number A23

Statepoint 1 (BOC Cycle 1A) Statepoint 2 (0.0 Cycle 1B)

Statepoint 3 (142.2 Cycle 1B)

\begin{tabular}{|c|c|c|c|c|c|c|c|c|c|}
\hline $\begin{array}{l}\text { Node } \\
\text { No. }\end{array}$ & $\begin{array}{c}\text { Bumup } \\
\text { (GWd/MTU) }\end{array}$ & $\begin{array}{l}\text { Fuel } \\
\text { Temp. }\end{array}$ & $\begin{array}{l}\text { Moderator } \\
\text { Spec. Vol. }\end{array}$ & $\begin{array}{l}\text { Bumup } \\
\text { (GWd/MTU) }\end{array}$ & $\begin{array}{l}\text { Fuel } \\
\text { Temp. }\end{array}$ & $\begin{array}{l}\text { Moderator } \\
\text { Spec. Vol. }\end{array}$ & $\begin{array}{c}\text { Burnup } \\
\text { (GWd/MTU) }\end{array}$ & $\begin{array}{l}\text { Fuel } \\
\text { Temp. }\end{array}$ & $\begin{array}{l}\text { Moderator } \\
\text { Spec. Vol. }\end{array}$ \\
\hline & & & & 0.0 Cy1B & 184.7 Cy1A & 184.7 Cy1A & 142.2 Cy1B & 89.5 Cy1B & 89.5 Cy1B \\
\hline 1 & 0.0 & & & 3.449 & 938.7 & 0.0235 & 5.805 & 1052.6 & 0.0233 \\
\hline 2 & 0.0 & & & 5.866 & 1149.6 & 0.0234 & 9.673 & 1250.7 & 0.0232 \\
\hline 3 & 0.0 & & & 8.075 & 1304.2 & 0.0233 & 12.706 & 1331.4 & 0.0231 \\
\hline 4 & 0.0 & \multicolumn{2}{|c|}{ Data not required. } & 9.565 & 1395.0 & 0.0232 & 14.516 & 1329.1 & 0.0230 \\
\hline 5 & 0.0 & & & 10.449 & 1436.6 & 0.0231 & 15.410 & 1292.3 & 0.0229 \\
\hline 6 & 0.0 & & & 10.949 & 1454.3 & 0.0230 & 15.800 & 1255.8 & 0.0228 \\
\hline 7 & 0.0 & & & 11.206 & 1464.9 & 0.0228 & 15.948 & 1229.3 & 0.0226 \\
\hline 8 & 0.0 & & & 11.291 & 1478.0 & 0.0227 & 15.955 & 1213.1 & 0.0225 \\
\hline 9 & 0.0 & & & 11.214 & 1498.6 & 0.0226 & 15.819 & 1204.0 & 0.0224 \\
\hline 10 & 0.0 & & & 10.922 & 1526.7 & 0.0224 & 15.443 & 1195.3 & 0.0223 \\
\hline 11 & 0.0 & & & 10.308 & 1557.5 & 0.0223 & 14.716 & 1183.6 & 0.0222 \\
\hline 12 & 0.0 & & & 9.490 & 1567.9 & 0.0222 & 13.885 & 1182.9 & 0.0222 \\
\hline 13 & 0.0 & & & 8.973 & 1520.3 & 0.0220 & 13.484 & 1195.7 & 0.0221 \\
\hline 14 & 0.0 & & & 8.769 & 1441.6 & 0.0219 & 13.499 & 1217.9 & 0.0220 \\
\hline .15 & 0.0 & & & 8.724 & 1372.0 & 0.0218 & 13.812 & 1260.9 & 0.0219 \\
\hline 16 & 0.0 & & & 8.565 & 1295.7 & 0.0217 & 14.009 & 1322.0 & 0.0218 \\
\hline
\end{tabular}


Title: CRC Depletion Calculations for the Rodded Assemblies in Batches 1, 2, 3, and $1 \mathrm{X}$ of Crystal River Unit 3

Document Identifier: BBA000000-01717-0200-00040 REV 00

Page 39 of 146

Table 4.1.10-11 Burnup, Fuel Temperature, and Moderator

Specific Volume Data for Assembly A23 of Crystal River Unit 3

\begin{tabular}{|l|l|l|l|r|r|r|r|r|r|}
\hline 17 & 0.0 & & & 7.653 & 1192.2 & 0.0216 & 12.790 & 1307.0 & 0.0217 \\
\hline 18 & 0.0 & & & 5.186 & 1005.8 & 0.0216 & 8.552 & 1110.7 & 0.0216 \\
\hline
\end{tabular}

\begin{tabular}{|c|c|c|c|c|c|c|c|c|c|}
\hline \multirow[b]{2}{*}{$\begin{array}{l}\text { Node } \\
\text { No. }\end{array}$} & \multicolumn{3}{|c|}{ Statepoint 4 (BOC Cycle 2) } & \multicolumn{3}{|c|}{ Statepoint 5 (BOC Cycle 3) } & \multicolumn{3}{|c|}{ Statepolnt 6 ( 168.5 Cycle 3 ) } \\
\hline & $\begin{array}{c}\text { Burnup } \\
\text { (GWd/MTU) }\end{array}$ & $\begin{array}{l}\text { Fuel } \\
\text { Temp. }\end{array}$ & $\begin{array}{c}\text { Moderator } \\
\text { Spec. Vol. }\end{array}$ & $\begin{array}{c}\text { Burnup } \\
\text { (GWd/MTU) }\end{array}$ & $\begin{array}{l}\text { Fuel } \\
\text { Temp. }\end{array}$ & $\begin{array}{l}\text { Moderator } \\
\text { Spec. Vol. }\end{array}$ & $\begin{array}{l}\text { Burnup } \\
\text { (GWd/MTU) }\end{array}$ & $\begin{array}{l}\text { Fuel } \\
\text { Temp. }\end{array}$ & $\begin{array}{l}\text { Moderator } \\
\text { Spec. Vol. }\end{array}$ \\
\hline & BOC Cy2 & 139.6 Cy1B & 139.6 Cy1B & BOC Cy3 & $89.8 \mathrm{Cy} 2$ & $89.8 \mathrm{Cy2}$ & $168.5 \mathrm{Cy} 3$ & $85.0 \mathrm{Cy3}$ & $85.0 \mathrm{Cy3}$ \\
\hline 1 & 6.376 & 1048.6 & 0.0234 & 7.865 & 788.9 & 0.0224 & 10.524 & 939.6 & 0.0231 \\
\hline 2 & 10.542 & 1200.9 & 0.0233 & 12.836 & 864.6 & 0.0224 & 16.971 & 1065.8 & 0.0230 \\
\hline 3 & 13.698 & 1242.4 & 0.0232 & 16.381 & 892.7 & 0.0223 & 21.207 & 1094.9 & 0.0229 \\
\hline 4 & 15.549 & 1249.2 & 0.0231 & 18.343 & 894.4 & 0.0223 & 23.400 & 1090.8 & 0.0228 \\
\hline 5 & 16.458 & 1266.8 & 0.0230 & 19.264 & 891.3 & 0.0222 & 24.380 & 1080.2 & 0.0227 \\
\hline 6 & 16.855 & 1274.6 & 0.0229 & 19.645 & 889.0 & 0.0222 & 24.747 & 1066.7 & 0.0226 \\
\hline 7 & 16.997 & 1274.3 & 0.0227 & 19.764 & 888.1 & 0.0221 & 24.805 & 1051.9 & 0.0225 \\
\hline 8 & 16.982 & 1271.8 & 0.0226 & 19.724 & 887.9 & 0.0221 & 24.668 & 1038.6 & 0.0224 \\
\hline 9 & 16.807 & 1264.4 & 0.0225 & 19.524 & 887.4 & 0.0220 & 24.366 & 1033.2 & 0.0224 \\
\hline 10 & 16.392 & 1249.6 & 0.0224 & 19.089 & 886.2 & 0.0220 & 23.889 & 1041.7 & 0.0223 \\
\hline 11 & 15.663 & 1242.7 & 0.0223 & 18.356 & 884.8 & 0.0219 & 23.219 & 1065.6 & 0.0222 \\
\hline 12 & 14.867 & 1261.5 & 0.0222 & 17.572 & 882.2 & 0.0219 & 22.586 & 1098.9 & 0.0221 \\
\hline 13 & 14.529 & 1281.9 & 0.0221 & 17.243 & 875.2 & 0.0218 & 22.437 & 1127.2 & 0.0220 \\
\hline 14 & 14.621 & 1296.4 & 0.0220 & 17.361 & 865.7 & 0.0218 & 22.707 & 1140.4 & 0.0219 \\
\hline 15 & 14.972 & 1303.3 & 0.0219 & 17.917 & 861.2 & 0.0217 & 23.302 & 1134.9 & 0.0218 \\
\hline 16 & 15.122 & 1274.3 & 0.0218 & 18.555 & 908.1 & 0.0217 & 23.728 & 1109.1 & 0.0217 \\
\hline 17 & 13.767 & 1198.0 & 0.0217 & 17.404 & 973.2 & 0.0216 & 21.941 & 1054.6 & 0.0217 \\
\hline 18 & 9.189 & 1015.0 & 0.0216 & 11.711 & 872.0 & 0.0216 & 14.702 & 930.3 & 0.0216 \\
\hline
\end{tabular}

\begin{tabular}{|c|c|c|c|c|c|c|c|c|c|}
\hline & \multicolumn{3}{|c|}{ Statepoint 7 (250.0 Cycle 3) } & \multicolumn{3}{|c|}{ Statepoint 8} & \multicolumn{3}{|c|}{ Statepoint 9} \\
\hline $\begin{array}{l}\text { Node } \\
\text { No. }\end{array}$ & $\begin{array}{l}\text { Burnup } \\
\text { (GWdMTU) }\end{array}$ & $\begin{array}{l}\text { Fuel } \\
\text { Temp. }\end{array}$ & $\begin{array}{l}\text { Moderator } \\
\text { Spec. Vol. }\end{array}$ & \begin{tabular}{|c} 
Burnup \\
(GWd/MTU)
\end{tabular} & $\begin{array}{c}\text { Fuel } \\
\text { Temp. }\end{array}$ & $\begin{array}{l}\text { Moderator } \\
\text { Spec. Vol. }\end{array}$ & \begin{tabular}{|c|} 
Bumup \\
(GWdMTU)
\end{tabular} & $\begin{array}{l}\text { Fuel } \\
\text { Temp. }\end{array}$ & $\begin{array}{l}\text { Moderator } \\
\text { Spec. Vol. }\end{array}$ \\
\hline & $250.0 \mathrm{cys}$ & 246.4 Cy3 & $246.4 C y^{3}$ & & & & & & \\
\hline 1 & 11.962 & 974.7 & 0.0231 & No additio & al statep & & No addition & statep & \\
\hline 2 & 19.074 & 1066.7 & 0.0230 & & & & & & \\
\hline 3 & 23.549 & 1074.2 & 0.0229 & & & & & & \\
\hline 4 & 25.776 & 1058.6 & 0.0228 & & & & & & \\
\hline 5 & 26.735 & 1043.2 & 0.0227 & & & & & & \\
\hline 6 & 27.077 & 1032.4 & 0.0226 & & & & & & \\
\hline 7 & 27.114 & 1024.9 & 0.0225 & & & & & & \\
\hline 8 & 26.957 & 1019.4 & 0.0224 & & & & & & \\
\hline 9 & 26.639 & 1016.6 & 0.0224 & & & & & & \\
\hline 10 & 26.167 & 1020.0 & 0.0223 & & & & & & \\
\hline 11 & 25.535 & 1031.2 & 0.0222 & & & & & & \\
\hline
\end{tabular}


Title: CRC Depletion Calculations for the Rodded Assemblies in Batches 1, 2, 3, and 1X of Crystal River Unit 3

Document Identifier: BBA000000-01717-0200-00040 REV 00

Page 40 of 146

Table 4.1.10-11 Burnup, Fuel Temperature, and Moderator

Specific Volume Data for Assembly A23 of Crystal River Unit 3

\begin{tabular}{|l|r|r|r|l|l|l|l|l|l|}
\hline 12 & 24.973 & 1046.7 & 0.0221 & & & & & & \\
\hline 13 & 24.914 & 1060.4 & 0.0220 & & & & & & \\
\hline 14 & 25.305 & 1074.7 & 0.0219 & & & & & & \\
\hline 15 & 26.011 & 1086.1 & 0.0218 & & & & & & \\
\hline 16 & 26.384 & 1072.9 & 0.0217 & & & & & & \\
\hline 17 & 24.346 & 1041.8 & 0.0217 & & & & & & \\
\hline 18 & 16.375 & 953.9 & 0.0216 & & & & & & \\
\hline
\end{tabular}

Table 4.1.10-12 Burnup, Fuel Temperature, and Moderator Specific Volume Data for Assembly A23a of Crystal River Unit 3

\begin{tabular}{|c|c|c|c|c|c|c|c|c|c|}
\hline & \multicolumn{9}{|c|}{ Assembly Number A23a } \\
\hline & \multicolumn{3}{|c|}{ Statepoint 1 (BOC Cycle 1A) } & \multicolumn{3}{|c|}{ Statepoint 2 ( 0.0 Cycle 1B) } & \multicolumn{3}{|c|}{ Statepoint 3 (142.2 Cycle 1B) } \\
\hline & $\begin{array}{c}\text { Burnup } \\
\text { (GWd/MTU) }\end{array}$ & $\begin{array}{l}\text { Fuel } \\
\text { Temp. }\end{array}$ & $\begin{array}{l}\text { Moderator } \\
\text { Spec. Vol. }\end{array}$ & \begin{tabular}{|c|} 
Burnup \\
(GWdMTU)
\end{tabular} & $\begin{array}{l}\text { Fuel } \\
\text { Temp. }\end{array}$ & $\begin{array}{l}\text { Moderator } \\
\text { Spec. Vol. }\end{array}$ & \begin{tabular}{|c|} 
Bumup \\
(GWdMTU)
\end{tabular} & $\begin{array}{l}\text { Fuel } \\
\text { Temp. }\end{array}$ & $\begin{array}{l}\text { Moderator } \\
\text { Spec. Vol. }\end{array}$ \\
\hline & & & & $0.0 C_{y 1 B}$ & 184.7 Cy1A & 184.7 Cy1A & 142.2 Cy1B & $89.5 \mathrm{Cy} 1 \mathrm{~B}$ & $89.5 \mathrm{Cy} 1 \mathrm{~B}$ \\
\hline 1 & 0.0 & & & 3.449 & 938.7 & 0.0235 & 5.805 & 1052.6 & 0.0233 \\
\hline 2 & 0.0 & & & 5.866 & 1149.6 & 0.0234 & 9.673 & 1250.7 & 0.0232 \\
\hline 3 & 0.0 & & & 8.075 & 1304.2 & 0.0233 & 12.706 & 1331.4 & 0.0231 \\
\hline 4 & 0.0 & \multicolumn{2}{|c|}{ Data not required. } & 9.565 & 1395.0 & 0.0232 & 14.516 & 1329.1 & 0.0230 \\
\hline 5 & 0.0 & & & 10.449 & 1436.6 & 0.0231 & 15.410 & 1292.3 & 0.0229 \\
\hline 6 & 0.0 & & & 10.949 & 1454.3 & 0.0230 & 15.800 & 1255.8 & 0.0228 \\
\hline 7 & 0.0 & & & 11.206 & 1464.9 & 0.0228 & 15.948 & 1229.3 & 0.0226 \\
\hline 8 & 0.0 & & & 11.291 & 1478.0 & 0.0227 & 15.955 & 1213.1 & 0.0225 \\
\hline 9 & 0.0 & & & 11.214 & 1498.6 & 0.0226 & 15.819 & 1204.0 & 0.0224 \\
\hline 10 & 0.0 & & & 10.922 & 1526.7 & 0.0224 & 15.443 & 1195.3 & 0.0223 \\
\hline 11 & 0.0 & & & 10.308 & 1557.5 & 0.0223 & 14.716 & 1183.6 & 0.0222 \\
\hline 12 & 0.0 & & & 9.490 & 1567.9 & 0.0222 & 13.885 & 1182.9 & 0.0222 \\
\hline 13 & 0.0 & & & 8.973 & 1520.3 & 0.0220 & 13.484 & 1195.7 & 0.0221 \\
\hline 14 & 0.0 & & & 8.769 & 1441.6 & 0.0219 & 13.499 & 1217.9 & 0.0220 \\
\hline 15 & 0.0 & & & 8.724 & 1372.0 & 0.0218 & 13.812 & 1260.9 & 0.0219 \\
\hline 16 & 0.0 & & & 8.565 & 1295.7 & 0.0217 & 14.009 & 1322.0 & 0.0218 \\
\hline 17 & 0.0 & & & 7.653 & 1192.2 & 0.0216 & 12.790 & 1307.0 & 0.0217 \\
\hline 18 & 0.0 & & & 5.186 & 1005.8 & 0.0216 & 8.552 & 1110.7 & 0.0216 \\
\hline
\end{tabular}

\begin{tabular}{|c|c|c|c|c|c|c|c|c|c|}
\hline & \multicolumn{3}{|c|}{ Statepoint 4 (BOC Cycle 2) } & \multicolumn{3}{|c|}{ Statepoint 5 (BOC Cycle 3) } & \multicolumn{3}{|c|}{ Statepoint 6 (168.5 Cycle 3$)$} \\
\hline $\begin{array}{l}\text { Node } \\
\text { No. }\end{array}$ & \begin{tabular}{|c|} 
Bumup \\
(GWd/MTU)
\end{tabular} & $\begin{array}{l}\text { Fuel } \\
\text { Temp. }\end{array}$ & $\begin{array}{l}\text { Moderator } \\
\text { Spec. Vol. }\end{array}$ & \begin{tabular}{|c|} 
Burnup \\
(GWdMTU)
\end{tabular} & $\begin{array}{c}\text { Fuel } \\
\text { Temp. }\end{array}$ & $\begin{array}{l}\text { Moderator } \\
\text { Spec. Vol. }\end{array}$ & \begin{tabular}{|c|} 
Burnup \\
(GWdMTMU)
\end{tabular} & $\begin{array}{c}\text { Fuel } \\
\text { Temp. }\end{array}$ & $\begin{array}{l}\text { Moderator } \\
\text { Spec. Vol. }\end{array}$ \\
\hline & BOC Cy2 & $39.6 \mathrm{Cy} 1 \mathrm{~B}$ & 139 & $\mathrm{BOCCy3}$ & $=y 2$ & $-y 2$ & 168.5 Cy3 & $5.0 \mathrm{Cy} 3$ & $y^{3}$ \\
\hline & & & & & 50.2 & & & 964.8 & 0232 \\
\hline 2 & .522 & 00.9 & 0233 & 3.52 & 942.5 & 0.0226 & 17.963 & $\overline{1104.5}$ & 0.023 \\
\hline
\end{tabular}


Title: CRC Depletion Calculations for the Rodded Assemblies in Batches 1, 2, 3, and $1 \mathrm{X}$ of Crystal River Unit 3

Document Identifier: BBA000000-01717-0200-00040 REV 00

Page 41 of 146

Table 4.1.10-12 Burnup, Fuel Temperature, and Moderator

Specific Volume Data for Assembly A23a of Crystal River Unit 3

\begin{tabular}{|r|r|r|r|r|r|r|r|r|r|}
\hline 3 & 13.671 & 1242.4 & 0.0232 & 17.174 & 977.7 & 0.0225 & 22.439 & 1139.5 & 0.0230 \\
\hline 4 & 15.519 & 1249.2 & 0.0231 & 19.167 & 982.5 & 0.0225 & 24.749 & 1139.8 & 0.0229 \\
\hline 5 & 16.427 & 1266.8 & 0.0230 & 20.086 & 980.3 & 0.0224 & 25.766 & 1127.7 & 0.0228 \\
\hline 6 & 16.824 & 1274.6 & 0.0229 & 20.451 & 977.5 & 0.0223 & 26.121 & 1109.7 & 0.0227 \\
\hline 7 & 16.966 & 1274.3 & 0.0227 & 20.549 & 976.0 & 0.0223 & 26.117 & 1083.8 & 0.0226 \\
\hline 8 & 16.953 & 1271.8 & 0.0226 & 20.485 & 975.6 & 0.0222 & 25.827 & 1051.6 & 0.0225 \\
\hline 9 & 16.782 & 1264.4 & 0.0225 & 20.252 & 974.3 & 0.0221 & 25.283 & 1029.5 & 0.0224 \\
\hline 10 & 16.376 & 1249.6 & 0.0224 & 19.783 & 969.5 & 0.0221 & 24.606 & 1032.5 & 0.0223 \\
\hline 11 & 15.664 & 1242.7 & 0.0223 & 19.033 & 961.4 & 0.0220 & 23.864 & 1060.1 & 0.0222 \\
\hline 12 & 14.889 & 1261.5 & 0.0222 & 18.259 & 952.1 & 0.0220 & 23.291 & 1108.6 & 0.0222 \\
\hline 13 & 14.563 & 1281.9 & 0.0221 & 17.958 & 940.4 & 0.0219 & 23.337 & 1163.0 & 0.0221 \\
\hline 14 & 14.661 & 1296.4 & 0.0220 & 18.129 & 929.4 & 0.0218 & 23.895 & 1194.5 & 0.0220 \\
\hline 15 & 15.010 & 1303.3 & 0.0219 & 18.816 & 928.8 & 0.0218 & 24.731 & 1188.6 & 0.0219 \\
\hline 16 & 15.145 & 1274.3 & 0.0218 & 19.682 & 994.1 & 0.0217 & 25.340 & 1148.7 & 0.0218 \\
\hline 17 & 13.769 & 1198.0 & 0.0217 & 18.652 & 1087.9 & 0.0217 & 23.595 & 1083.3 & 0.0217 \\
\hline 18 & 9.184 & 1015.0 & 0.0216 & 12.650 & 976.3 & 0.0216 & 15.922 & 956.3 & 0.0216 \\
\hline
\end{tabular}

\begin{tabular}{|c|c|c|c|c|c|c|c|c|c|}
\hline & \multicolumn{3}{|c|}{ Statepoint $7(250.0$ Cycle 3$)$} & \multicolumn{3}{|c|}{ Statepoint 8} & \multicolumn{3}{|c|}{ Statepoint 9} \\
\hline $\begin{array}{l}\text { Node } \\
\text { No. }\end{array}$ & $\begin{array}{c}\text { Burnup } \\
\text { (GWd/MTU) }\end{array}$ & $\begin{array}{l}\text { Fuel } \\
\text { Temp. }\end{array}$ & $\begin{array}{l}\text { Moderator } \\
\text { Spec. Vol. }\end{array}$ & \begin{tabular}{|c|} 
Burnup \\
(GWdMTU)
\end{tabular} & $\begin{array}{c}\text { Fuel } \\
\text { Temp. }\end{array}$ & $\begin{array}{l}\text { Moderator } \\
\text { Spec. Vol. }\end{array}$ & \begin{tabular}{|c|} 
Burnup \\
(GWdMTMU)
\end{tabular} & $\begin{array}{l}\text { Fuel } \\
\text { Temp. }\end{array}$ & $\begin{array}{l}\text { Moderator } \\
\text { Spec. Vol. }\end{array}$ \\
\hline & 250.0 Cy3 & 246.4 Cy3 & 246.4 Cy3 & & & & & & \\
\hline 1 & 12.699 & 996.5 & 0.0232 & No additior & a statep & & No additior & statep & nts. \\
\hline 2 & 20.238 & 1092.0 & 0.0231 & & & & & & \\
\hline 3 & 24.987 & 1103.4 & 0.0230 & & & & & & \\
\hline 4 & 27.335 & 1086.1 & 0.0229 & & & & & & \\
\hline 5 & 28.325 & 1068.4 & 0.0228 & & & & & & \\
\hline 6 & 28.647 & 1055.5 & 0.0227 & & & & & & \\
\hline 7 & 28.606 & 1044.8 & 0.0226 & & & & & & \\
\hline 8 & 28.252 & 1030.4 & 0.0225 & & & & & & \\
\hline 9 & 27.612 & 1013.5 & 0.0224 & & & & & & \\
\hline 10 & 26.872 & 1008.6 & 0.0223 & & & & & & \\
\hline 11 & 26.143 & 1018.5 & 0.0222 & & & & & & \\
\hline 12 & 25.648 & 1037.7 & 0.0221 & & & & & & \\
\hline 13 & 25.835 & 1061.7 & 0.0220 & & & & & & \\
\hline 14 & 26.583 & 1086.2 & 0.0219 & & & & & & \\
\hline 15 & 27.535 & 1092.6 & 0.0218 & & & & & & \\
\hline 16 & 28.085 & 1073.5 & 0.0217 & & & & & & \\
\hline 17 & 26.085 & 1041.6 & 0.0217 & & & & & & \\
\hline 18 & 17.667 & 957.0 & 0.0216 & & & & & & \\
\hline
\end{tabular}


Title: CRC Depletion Calculations for the Rodded Assemblies in Batches 1, 2, 3, and IX of Crystal River Unit 3

Document Identifier: BBA000000-01717-0200-00040 REV 00

Page 42 of 146

Table 4.1.10-13 Burnup, Fuel Temperature, and Moderator

Specific Volume Data for Assembly A25 of Crystal River Unit 3

\begin{tabular}{|c|c|c|c|c|c|c|c|c|c|}
\hline & \multicolumn{9}{|c|}{ Assembly Number A25 } \\
\hline & \multicolumn{3}{|c|}{ Statepoint 1 (BOC Cycle 1A) } & \multicolumn{3}{|c|}{ Statepoint 2 (0.0 Cycle 1B) } & \multicolumn{3}{|c|}{ Statepoint 3 (142.2 Cycle 1B) } \\
\hline & $\begin{array}{l}\text { Bumup } \\
\text { (GWd/MTU) }\end{array}$ & $\begin{array}{l}\text { Fuel } \\
\text { Temp. }\end{array}$ & $\begin{array}{l}\text { Moderator } \\
\text { Spec. Vol. }\end{array}$ & $\begin{array}{l}\text { Burnup } \\
\text { (GWd/MTU) }\end{array}$ & $\begin{array}{l}\text { Fuel } \\
\text { Temp. }\end{array}$ & $\begin{array}{c}\text { Moderator } \\
\text { Spec. Vol. }\end{array}$ & $\begin{array}{c}\text { Burnup } \\
\text { (GWd/MTU) }\end{array}$ & $\begin{array}{l}\text { Fuel } \\
\text { Temp. }\end{array}$ & $\begin{array}{l}\text { Moderator } \\
\text { Spec. Vol. }\end{array}$ \\
\hline & & & & 0.0 Cy1B & 184.7 Cy1A & 184.7 Cy1A & 142.2 Cy1B & 89.5 Cy1B & 89.5 Cy1B \\
\hline 1 & 0.0 & & & 2.109 & 798.7 & 0.0227 & 4.098 & 983.8 & 0.0230 \\
\hline 2 & 0.0 & & & 3.769 & 950.4 & 0.0227 & 7.024 & 1172.9 & 0.0230 \\
\hline 3 & 0.0 & & & 5.015 & 1053.3 & 0.0226 & 8.938 & 1242.8 & 0.0229 \\
\hline 4 & 0.0 & \multicolumn{2}{|c|}{ Data not required. } & 5.823 & 1106.3 & 0.0226 & 9.967 & 1248.0 & 0.0228 \\
\hline 5 & 0.0 & & & 6.323 & 1134.1 & 0.0225 & 10.484 & 1231.1 & 0.0227 \\
\hline 6 & 0.0 & & & 6.613 & 1148.4 & 0.0224 & 10.722 & 1210.0 & 0.0226 \\
\hline 7 & 0.0 & & & 6.765 & 1157.4 & 0.0223 & 10.818 & 1192.8 & 0.0225 \\
\hline 8 & 0.0 & & & 6.822 & 1166.3 & 0.0223 & 10.840 & 1181.8 & 0.0224 \\
\hline 9 & 0.0 & & & 6.801 & 1177.7 & 0.0222 & 10.809 & 1176.7 & 0.0223 \\
\hline 10 & 0.0 & - & & 6.704 & 1191.4 & 0.0221 & 10.726 & 1176.3 & 0.0222 \\
\hline 11 & 0.0 & & & 6.540 & 1203.4 & 0.0220 & 10.605 & 1180.2 & 0.0221 \\
\hline 12 & 0.0 & & & 6.358 & 1206.4 & 0.0220 & 10.497 & 1188.0 & 0.0221 \\
\hline 13 & 0.0 & & & 6.239 & 1195.7 & 0.0219 & 10.470 & 1197.9 & 0.0220 \\
\hline 14 & 0.0 & & & 6.245 & 1176.9 & 0.0218 & 10.555 & 1206.2 & 0.0219 \\
\hline 15 & 0.0 & & & 6.383 & 1166.0 & 0.0217 & 10.701 & 1207.2 & 0.0218 \\
\hline 16 & 0.0 & & & 6.393 & 1160.2 & 0.0217 & 10.561 & 1192.4 & 0.0217 \\
\hline 17 & 0.0 & & & 5.646 & 1090.4 & 0.0216 & 9.314 & 1140.9 & 0.0216 \\
\hline 18 & 0.0 & & & 3.409 & 888.4 & 0.0216 & 5.732 & 971.1 & 0.0216 \\
\hline
\end{tabular}

Statepoint 4 (BOC Cycle 2) $\quad$ Statepoint 5 (BOC Cycle 3)

Statepoint 6 (168.5 Cycle 3)

\begin{tabular}{|c|c|c|c|c|c|c|c|c|c|}
\hline $\begin{array}{l}\text { Node } \\
\text { No. }\end{array}$ & $\begin{array}{c}\text { Burnup } \\
\text { (GWd/MTU) }\end{array}$ & $\begin{array}{l}\text { Fuel } \\
\text { Temp. }\end{array}$ & $\begin{array}{l}\text { Moderator } \\
\text { Spec. Vol. }\end{array}$ & $\begin{array}{c}\text { Burnup } \\
\text { (GWd/MTU) }\end{array}$ & $\begin{array}{l}\text { Fuel } \\
\text { Temp. }\end{array}$ & $\begin{array}{l}\text { Moderator } \\
\text { Spec. Vol. }\end{array}$ & $\begin{array}{c}\text { Burnup } \\
\text { (GWd/MTU) }\end{array}$ & $\begin{array}{l}\text { Fuel } \\
\text { Temp. }\end{array}$ & $\begin{array}{l}\text { Moderator } \\
\text { Spec. Vol. }\end{array}$ \\
\hline & BOC Cy2 & 139.6 Cy1B & 139.6 Cy1B & BOC Cy3 & $89.8 \mathrm{Cy} 2$ & $89.8 \mathrm{Cy} 2$ & 168.5 Cy3 & $85.0 \mathrm{Cy} 3$ & 85.0 Cyз \\
\hline 1 & 4.503 & 928.5 & 0.0230 & 7.848 & 1077.0 & 0.0234 & 9.228 & 762.6 & 0.0224 \\
\hline 2 & 7.663 & 1079.4 & 0.0229 & 12.816 & 1241.9 & 0.0233 & 14.942 & 828.4 & 0.0224 \\
\hline 3 & 9.691 & 1137.8 & 0.0229 & 15.675 & 1306.2 & 0.0232 & 18.205 & 856.1 & 0.0223 \\
\hline 4 & 10.762 & 1160.7 & 0.0228 & 16.993 & 1319.7 & 0.0231 & 19.683 & 861.8 & 0.0223 \\
\hline 5 & 11.295 & 1174.0 & 0.0227 & 17.562 & 1320.1 & 0.0230 & 20.304 & 859.6 & 0.0222 \\
\hline 6 & 11.542 & 1184.1 & 0.0226 & 17.780 & 1320.1 & 0.0228 & 20.526 & 854.3 & 0.0222 \\
\hline 7 & 11.642 & 1191.3 & 0.0225 & 17.830 & 1322.6 & 0.0227 & 20.553 & 847.9 & 0.0221 \\
\hline 8 & 11.664 & 1196.0 & 0.0224 & 17.779 & 1326.0 & 0.0226 & 20.463 & 842.4 & 0.0221 \\
\hline 9 & 11.633 & 1199.1 & 0.0223 & 17.636 & 1324.7 & 0.0225 & 20.281 & 840.5 & 0.0221 \\
\hline 10 & 11.557 & .1202 .1 & 0.0222 & 17.409 & 1309.0 & 0.0223 & 20.036 & 844.3 & 0.0220 \\
\hline 11 & 11.454 & 1207.3 & 0.0221 & 17.162 & 1275.9 & 0.0222 & 19.807 & 853.5 & 0.0220 \\
\hline 12 & 11.373 & 1213.6 & 0.0220 & 17.007 & 1240.4 & 0.0221 & 19.702 & 865.4 & 0.0219 \\
\hline
\end{tabular}


Title: CRC Depletion Calculations for the Rodded Assemblies in Batches 1, 2, 3, and $1 \mathrm{X}$ of Crystal River Unit 3 Document Identifier: BBA000000-01717-0200-00040 REV 00

Page 43 of 146

Table 4.1.10-13 Burnup, Fuel Temperature, and Moderator

Specific Volume Data for Assembly A25 of Crystal River Unit 3

\begin{tabular}{|r|r|r|r|r|r|r|r|r|r|}
\hline 13 & 11.371 & 1215.0 & 0.0220 & 17.013 & 1211.8 & 0.0220 & 19.779 & 876.1 & 0.0219 \\
\hline 14 & 11.463 & 1204.0 & 0.0219 & 17.177 & 1191.5 & 0.0219 & 20.070 & 884.4 & 0.0218 \\
\hline 15 & 11.586 & 1175.3 & 0.0218 & 17.383 & 1184.6 & 0.0218 & 20.741 & 913.8 & 0.0218 \\
\hline 16 & 11.382 & 1128.6 & 0.0217 & 17.138 & 1187.8 & 0.0217 & 21.239 & 1031.6 & 0.0217 \\
\hline 17 & 10.017 & 1059.8 & 0.0216 & 15.289 & 1163.2 & 0.0217 & 19.155 & 1017.0 & 0.0216 \\
\hline 18 & 6.179 & 910.1 & 0.0216 & 9.716 & 1020.3 & 0.0216 & 12.322 & 898.4 & 0.0216 \\
\hline
\end{tabular}

\begin{tabular}{|c|c|c|c|c|c|c|c|c|c|}
\hline & \multicolumn{3}{|c|}{ Statepoint 7 (250.0 Cycle 3) } & \multicolumn{3}{|c|}{ Statepoint 8} & \multicolumn{3}{|c|}{ Statepolnt 9} \\
\hline $\begin{array}{l}\text { Node } \\
\text { No. }\end{array}$ & $\begin{array}{c}\text { Burnup } \\
\text { (GWd/MTU) }\end{array}$ & $\begin{array}{l}\text { Fuel } \\
\text { Temp. }\end{array}$ & $\begin{array}{l}\text { Moderator } \\
\text { Spec. Vol. }\end{array}$ & $\begin{array}{c}\text { Burmup } \\
\text { (GWd/MTU) }\end{array}$ & $\begin{array}{l}\text { Fuel } \\
\text { Temp. }\end{array}$ & $\begin{array}{l}\text { Moderator } \\
\text { Spec. Vol. }\end{array}$ & $\begin{array}{c}\text { Bumup } \\
\text { (GWd/MTU) }\end{array}$ & $\begin{array}{l}\text { Fuel } \\
\text { Temp. }\end{array}$ & $\begin{array}{l}\text { Moderator } \\
\text { Spec. Vol. }\end{array}$ \\
\hline & 250.0 Су3 & 246.4 Cy 3 & $246.4 C_{y 3}$ & & & & & & \\
\hline 1 & 10.021 & 805.0 & 0.0225 & \multicolumn{3}{|c|}{ No additional statepoints. } & \multicolumn{3}{|c|}{ No additional statepoints. } \\
\hline 2 & 16.110 & 869.5 & 0.0225 & & & & & & \\
\hline 3 & 19.542 & 885.3 & 0.0225 & & & & & & \\
\hline 4 & 21.063 & 882.6 & 0.0224 & & & & & & \\
\hline 5 & 21.685 & 876.8 & 0.0224 & & & & & & \\
\hline 6 & 21.898 & 871.7 & 0.0223 & & & & & & \\
\hline 7 & 21.916 & 868.0 & 0.0222 & & & & & & \\
\hline 8 & 21.819 & 865.5 & 0.0222 & & & & & & \\
\hline 9 & 21.635 & 864.8 & 0.0221 & & & & & & \\
\hline 10 & 21.396 & 866.4 & 0.0221 & & & & & & \\
\hline 11 & 21.186 & 870.3 & 0.0220 & & & & & & \\
\hline 12 & 21.112 & 875.6 & 0.0220 & & & & & & \\
\hline 13 & 21.244 & 882.5 & 0.0219 & & & & & & \\
\hline 14 & 21.712 & 906.4 & 0.0219 & & & & & & \\
\hline 15 & 23.126 & 1040.8 & 0.0218 & & $\therefore$ & & & & \\
\hline 16 & 23.701 & 1060.1 & 0.0217 & & & & & & \\
\hline 17 & 21.407 & 1038.8 & 0.0217 & & & & & & \\
\hline 18 & 13.883 & 950.7 & 0.0216 & & & & & & \\
\hline
\end{tabular}

Table 4.1.10-14 Burnup, Fuel Temperature, and Moderator

Specific Volume Data for Assembly A25a of Crystal River Unit 3

\begin{tabular}{|c|c|c|c|c|c|c|c|c|c|}
\hline & \multicolumn{9}{|c|}{ Assembly Number A25a } \\
\hline & \multicolumn{3}{|c|}{ Statepoint 1 (BOC Cycle 1A) } & \multicolumn{3}{|c|}{ Statepolnt 2 (0.0 Cycle 1B) } & \multicolumn{3}{|c|}{ Statepoint 3 (142.2 Cycle 1B) } \\
\hline & $\begin{array}{c}\text { Burnup } \\
\text { (GWd/MTU) }\end{array}$ & $\begin{array}{l}\text { Fuel } \\
\text { Temp. }\end{array}$ & $\begin{array}{l}\text { Moderator } \\
\text { Spec. Vol. }\end{array}$ & $\begin{array}{c}\text { Burnup } \\
\text { (GWd/MTU) }\end{array}$ & $\begin{array}{l}\text { Fuel } \\
\text { Temp. }\end{array}$ & $\begin{array}{l}\text { Moderator } \\
\text { Spec. Vol. }\end{array}$ & $\begin{array}{c}\text { Bumup } \\
\text { (GWd/MTU) }\end{array}$ & $\begin{array}{l}\text { Fuel } \\
\text { Temp. }\end{array}$ & $\begin{array}{l}\text { Moderator } \\
\text { Spec. Vol. }\end{array}$ \\
\hline & & & & 0.0 Cy1B & 184.7 Cy1A & 184.7 Cy1A & 142.2 Cy1B & 89.5 Cy1B & 89.5 Cy1B \\
\hline 1 & 0.0 & & & 2.109 & 798.7 & 0.0227 & 4.098 & 983.8 & 0.0230 \\
\hline 2 & 0.0 & & & 3.769 & 950.4 & 0.0227 & 7.024 & 1172.9 & 0.0230 \\
\hline 3 & 0.0 & & & 5.015 & 1053.3 & 0.0226 & 8.938 & 1242.8 & 0.0229 \\
\hline
\end{tabular}


Title: CRC Depletion Calculations for the Rodded Assemblies in Batches 1, 2, 3, and $1 \mathrm{X}$ of Crystal River Unit 3

Table 4.1.10-14 Burnup, Fuel Temperature, and Moderator

Specific Volume Data for Assembly A25a of Crystal River Unit 3

\begin{tabular}{|c|c|c|c|c|c|c|c|c|}
\hline 4 & 0.0 & Data not required. & 5.823 & 1106.3 & 0.0226 & 9.967 & 1248.0 & 0.0228 \\
\hline 5 & 0.0 & & 6.323 & 1134.1 & 0.0225 & 10.484 & 1231.1 & 0.0227 \\
\hline 6 & 0.0 & & 6.613 & 1148.4 & 0.0224 & 10.722 & 1210.0 & 0.0226 \\
\hline 7 & 0.0 & & 6.765 & 1157.4 & 0.0223 & 10.818 & 1192.8 & 0.0225 \\
\hline 8 & 0.0 & & 6.822 & 1166.3 & 0.0223 & 10.840 & 1181.8 & 0.0224 \\
\hline 9 & 0.0 & & 6.801 & 1177.7 & 0.0222 & 10.809 & 1176.7 & 0.0223 \\
\hline 10 & 0.0 & & 6.704 & 1191.4 & 0.0221 & 10.726 & 1176.3 & 0.0222 \\
\hline 11 & 0.0 & & 6.540 & 1203.4 & 0.0220 & 10.605 & 1180.2 & 0.0221 \\
\hline 12 & 0.0 & & 6.358 & 1206.4 & 0.0220 & 10.497 & 1188.0 & 0.0221 \\
\hline 13 & 0.0 & & 6.239 & 1195.7 & 0.0219 & 10.470 & 1197.9 & 0.0220 \\
\hline 14 & 0.0 & & 6.245 & 1176.9 & 0.0218 & 10.555 & 1206.2 & 0.0219 \\
\hline 15 & 0.0 & & 6.383 & 1166.0 & 0.0217 & 10.701 & 1207.2 & 0.0218 \\
\hline 16 & 0.0 & & 6.393 & 1160.2 & 0.0217 & 10.561 & 1192.4 & 0.0217 \\
\hline 17 & 0.0 & & 5.646 & 1090.4 & 0.0216 & 9.314 & 1140.9 & 0.0216 \\
\hline 18 & 0.0 & - & 3.409 & 888.4 & 0.0216 & 5.732 & 971.1 & 0.0216 \\
\hline
\end{tabular}

Statepoint 4 (BOC Cycle 2) Statepoint 5 (BOC Cycle 3)

Statepoint 6 (168.5 Cycle 3)

\begin{tabular}{|c|c|c|c|c|c|c|c|c|c|}
\hline $\begin{array}{l}\text { Node } \\
\text { No. }\end{array}$ & $\begin{array}{l}\text { Bumup } \\
\text { (GWd/MTU) }\end{array}$ & $\begin{array}{l}\text { Fuel } \\
\text { Temp. }\end{array}$ & $\begin{array}{l}\text { Moderator } \\
\text { Spec. Vol. }\end{array}$ & $\begin{array}{c}\text { Bumup } \\
\text { (GWd/MTU) }\end{array}$ & $\begin{array}{l}\text { Fuel } \\
\text { Temp. }\end{array}$ & $\begin{array}{l}\text { Moderator } \\
\text { Spec. Vol. }\end{array}$ & $\begin{array}{c}\text { Burnup } \\
\text { (GWd/MTU) }\end{array}$ & $\begin{array}{l}\text { Fuel } \\
\text { Temp. }\end{array}$ & $\begin{array}{l}\text { Moderator } \\
\text { Spec. Vol. }\end{array}$ \\
\hline & BOC Cy2 & 139.6 Cy1B & 139.6 Cy1B & BOC Cy3 & 89.8 Cy2 & $89.8 \mathrm{Cy} 2$ & $168.5 \mathrm{Cy}^{3}$ & $85.0 \mathrm{Cy} 3$ & 85.0 Cy3 \\
\hline 1 & 4.503 & 928.5 & 0.0230 & 7.856 & 1077.0 & 0.0234 & 9.710 & 855.6 & 0.0234 \\
\hline 2 & 7.663 & 1079.4 & 0.0229 & 12.829 & 1241.9 & 0.0233 & 16.384 & 1077.9 & 0.0233 \\
\hline 3 & 9.691 & 1137.8 & 0.0229 & 15.690 & 1306.2 & 0.0232 & 20.857 & 1183.4 & 0.0232 \\
\hline 4 & 10.762 & 1160.7 & 0.0228 & 17.010 & 1319.7 & 0.0231 & 22.822 & 1206.9 & 0.0231 \\
\hline 5 & 11.295 & 1174.0 & 0.0227 & 17.580 & 1320.1 & 0.0230 & 23.707 & 1202.7 & 0.0230 \\
\hline 6 & 11.542 & 1184.1 & 0.0226 & 17.798 & 20.1 & 228 & 4.005 & 90.9 & 2229 \\
\hline 7 & 11.642 & 1191.3 & 0.0225 & 17.847 & 1322.6 & 0.0227 & 24.015 & 1174.0 & 0.0227 \\
\hline 8 & 11.664 & 1196.0 & 0.0224 & 17.794 & 1326.0 & 0.0226 & 23.842 & 1156.6 & 0.0226 \\
\hline 9 & 11.633 & 1199.1 & 0.0223 & 17.648 & 1324.7 & 0.0225 & 23.542 & 1147.8 & 0.0225 \\
\hline 10 & 557 & 1202.1 & 0.0222 & 17.414 & 1309.0 & 0.0223 & 23.213 & 55.1 & 0.0224 \\
\hline 11 & 454 & 7.3 & 221 & 158 & 5.9 & 0.0222 & 22.980 & 1178.1 & 0.0223 \\
\hline 12 & 11.373 & 1213.6 & 0.0220 & 16.992 & 1240.4 & 0.0221 & 22.947 & 1210.9 & 0.0222 \\
\hline 13 & 11.371 & 1215.0 & 0.0220 & 16.991 & 1211.8 & 0.0220 & 23.144 & 1242.2 & .0221 \\
\hline 14 & 11.463 & 1204.0 & 0.0219 & 17.152 & 1191.5 & 0.0219 & 23.472 & 1256.6 & 0.0220 \\
\hline 15 & 11.586 & 1175.3 & 0.0218 & 17.361 & 1184.6 & 0.0218 & 23.667 & 1245.6 & 0.0219 \\
\hline 16 & 11.382 & 1128.6 & 0.0217 & 17.123 & 1187.8 & 0.0217 & 23.121 & 1211.0 & 0.0218 \\
\hline 17 & 10.017 & 1059.8 & 0.0216 & 15.283 & 1163.2 & 0.0217 & 20.510 & 1148.8 & 0.0217 \\
\hline 18 & 6.179 & 910.1 & 0.0216 & 9.715 & 1020.3 & 0.0216 & 13.098 & 993.2 & 0.0216 \\
\hline
\end{tabular}


Title: CRC Depletion Calculations for the Rodded Assemblies in Batches 1, 2, 3, and $1 \mathrm{X}$ of Crystal River Unit 3 Document Identifier: BBA000000-01717-0200-00040 REV 00

Page 45 of 146

Table 4.1.10-14 Burnup, Fuel Temperature, and Moderator Specific Volume Data for Assembly A25a of Crystal River Unit 3

\begin{tabular}{|c|c|c|c|c|c|c|c|c|c|}
\hline & \multicolumn{3}{|c|}{ Statepoint 7 (250.0 Cycle 3$)$} & \multicolumn{3}{|c|}{ Statepoint 8} & \multicolumn{3}{|c|}{ Statepoint 9} \\
\hline $\begin{array}{l}\text { Node } \\
\text { No. }\end{array}$ & $\begin{array}{l}\text { Burnup } \\
\text { (GWd/MTU) }\end{array}$ & $\begin{array}{l}\text { Fuel } \\
\text { Temp. }\end{array}$ & $\begin{array}{l}\text { Moderator } \\
\text { Spec. Vol. }\end{array}$ & $\begin{array}{c}\text { Burnup } \\
\text { (GWd/MTU) }\end{array}$ & $\begin{array}{l}\text { Fuel } \\
\text { Temp. }\end{array}$ & $\begin{array}{l}\text { Moderator } \\
\text { Spec. Vol. }\end{array}$ & $\begin{array}{c}\text { Bumup } \\
\text { (GWd/MTU) }\end{array}$ & $\begin{array}{l}\text { Fuel } \\
\text { Temp. }\end{array}$ & $\begin{array}{l}\text { Moderator } \\
\text { Spec. Vol. }\end{array}$ \\
\hline & $250.0 \mathrm{Cy}^{3}$ & 246.4 Cy3 & $246.4 \mathrm{Cy}^{3}$ & & & & & & \\
\hline 1 & 10.832 & 894.2 & 0.0233 & \multicolumn{3}{|c|}{ No additional statepoints. } & \multicolumn{3}{|c|}{ No additional statepoints. } \\
\hline 2 & 18.586 & 1110.1 & 0.0233 & & & & & & \\
\hline 3 & 23.555 & 1151.9 & 0.0232 & & & & & & \\
\hline 4 & 25.624 & 1146.4 & 0.0230 & & & & & & \\
\hline 5 & 26.507 & 1133.1 & 0.0229 & & & & & & \\
\hline 6 & 26.787 & 1123.1 & 0.0228 & & & & & & \\
\hline 7 & 26.779 & 1115.7 & 0.0227 & & & & & & \\
\hline 8 & 26.583 & 1109.0 & 0.0226 & & & & & & \\
\hline 9 & 26.256 & 1104.0 & 0.0225 & & & & & & \\
\hline 10 & 25.918 & 1104.4 & 0.0224 & & & & & & \\
\hline 11 & 25.708 & $111 \theta .2$ & 0.0223 & & & & & & \\
\hline 12 & 25.727 & 1118.6 & 0.0222 & & & & & & \\
\hline 13 & 25.998 & 1127.3 & 0.0221 & & & & & & \\
\hline 14 & 26.400 & 1133.2 & 0.0220 & & & & & & \\
\hline 15 & 26.626 & 1132.0 & 0.0219 & & & & & & \\
\hline 16 & 26.020 & 1123.4 & 0.0218 & & & & & & \\
\hline 17 & 23.164 & 1099.7 & 0.0217 & & & & & & \\
\hline 18 & 14.939 & 1003.9 & 0.0216 & & & & & & \\
\hline
\end{tabular}

Table 4.1.10-15 Burnup, Fuel Temperature, and Moderator Specific Volume Data for Assembly A26 of Crystal River Unit 3

\begin{tabular}{|c|c|c|c|c|c|c|c|c|}
\hline \multicolumn{9}{|c|}{ Assembly Number A26 } \\
\hline \multicolumn{3}{|c|}{ Statepoint 1 (BOC Cycle 1A) } & \multicolumn{3}{|c|}{ Statepoint $2(0.0$ Cycle $1 \mathrm{~B})$} & \multicolumn{3}{|c|}{ Statepoint 3 (142.2 Cycle 1B) } \\
\hline $\begin{array}{l}\text { Burnup } \\
\text { (GWd/MTU) }\end{array}$ & $\begin{array}{l}\text { Fuel } \\
\text { Temp. }\end{array}$ & $\begin{array}{l}\text { Moderator } \\
\text { Spec. Vol. }\end{array}$ & $\begin{array}{c}\text { Burnup } \\
\text { (GWd/MTU) }\end{array}$ & $\begin{array}{l}\text { Fuel } \\
\text { Temp. }\end{array}$ & $\begin{array}{l}\text { Moderator } \\
\text { Spec. Vol. }\end{array}$ & \begin{tabular}{|c|} 
Burnup \\
(GWd/MTU)
\end{tabular} & $\begin{array}{l}\text { Fuel } \\
\text { Temp. }\end{array}$ & $\begin{array}{l}\text { Moderator } \\
\text { Spec. Vol. }\end{array}$ \\
\hline & & & $0.0 \mathrm{Cy} 1 \mathrm{~B}$ & 184.7 Cy1A & 184.7 Cy1A & $142.2 \mathrm{Cy} 1 \mathrm{~B}$ & $89.5 \mathrm{Cy} 1 \mathrm{~B}$ & $89.5 \mathrm{Cy} 1 \mathrm{~B}$ \\
\hline 0.0 & & & 2.845 & 894.7 & 0.0232 & 5.336 & 1080.1 & 0.0231 \\
\hline 0.0 & & & 5.061 & 1100.1 & 0.0232 & 9.077 & 1285.2 & 0.0231 \\
\hline 0.0 & & & 6.838 & 1233.0 & 0.0231 & 11.651 & 1358.8 & 0.0229 \\
\hline 0.0 & Data not & quired. & 8.044 & 1305.8 & 0.0230 & 13.123 & 1357.7 & 0.0228 \\
\hline 0.0 & & & 8.786 & 1341.5 & 0.0229 & 13.869 & 1325.9 & 0.0227 \\
\hline 0.0 & & & 9.214 & 1357.9 & 0.0228 & 14.202 & 1292.0 & 0.0226 \\
\hline 0.0 & & & 9.439 & 1368.1 & 0.0227 & 14.320 & 1266.0 & 0.0225 \\
\hline 0.0 & & & 9.518 & 1380.0 & 0.0226 & 14.314 & 1248.9 & 0.0224 \\
\hline 0.0 & & & 9.470 & 1397.3 & 0.0224 & 14.182 & 1237.5 & 0.0223 \\
\hline 0.0 & & & 9.282 & 1420.2 & 0.0223 & 13.718 & 1213.7 & 0.0222 \\
\hline
\end{tabular}


Title: CRC Depletion Calculations for the Rodded Assemblies in Batches 1, 2, 3, and IX of Crystal River Unit 3

Document Identifier: BBA000000-01717-0200-00040 REV 00

Page 46 of 146

Table 4.1.10-15 Burnup, Fuel Temperature, and Moderator

Specific Volume Data for Assembly A26 of Crystal River Unit 3

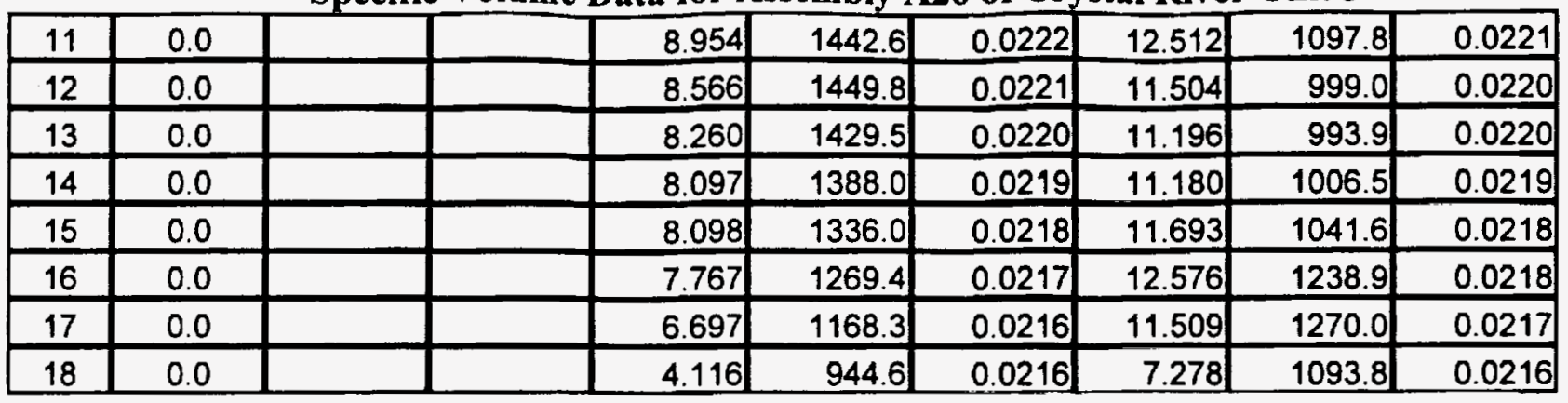

Table 4.1.10-16 Burnup, Fuel Temperature, and Moderator

Specific Volume Data for Assembly A28 of Crystal River Unit 3

\begin{tabular}{|c|c|c|c|c|c|c|c|c|c|}
\hline \multirow[b]{3}{*}{$\begin{array}{l}\text { Node } \\
\text { No. }\end{array}$} & \multicolumn{9}{|c|}{ Assembly Number A28 } \\
\hline & \multicolumn{3}{|c|}{ Statepoint 1 (BOC Cycle 1) } & \multicolumn{3}{|c|}{ Statepoint 2 (0.0 Cycle 1B) } & \multicolumn{3}{|c|}{ Statepoint 3 (142.2 Cycle 1B) } \\
\hline & $\begin{array}{c}\text { Burnup } \\
\text { (GWd/MTU) }\end{array}$ & $\begin{array}{l}\text { Fuel } \\
\text { Temp. }\end{array}$ & $\begin{array}{l}\text { Moderator } \\
\text { Spec. Vol. }\end{array}$ & $\begin{array}{l}\text { Burnup } \\
\text { (GWd/MTU) }\end{array}$ & $\begin{array}{l}\text { Fuel } \\
\text { Temp. }\end{array}$ & $\begin{array}{l}\text { Moderator } \\
\text { Spec. Vol. }\end{array}$ & $\begin{array}{c}\text { Burnup } \\
\text { (GWd/MTU) }\end{array}$ & $\begin{array}{l}\text { Fuel } \\
\text { Temp. }\end{array}$ & $\begin{array}{l}\text { Moderator } \\
\text { Spec. Vol. }\end{array}$ \\
\hline & & & & 0.0 Cy1B & 184.7 Cy1A & 184.7 Cy1A & 142.2 Cy1B & 89.5 Cy1B & 89.5 Cy1B \\
\hline 1 & 0.0 & & & 1.510 & 736.2 & 0.0224 & 2.757 & 833.2 & 0.0225 \\
\hline 2 & 0.0 & & & 2.701 & 851.2 & 0.0223 & 4.779 & 976.4 & 0.0224 \\
\hline 3 & 0.0 & & & 3.602 & 931.2 & 0.0223 & 6.129 & 1035.4 & 0.0224 \\
\hline 4 & 0.0 & \multicolumn{2}{|c|}{ Data not required. } & 4.196 & 978.1 & 0.0223 & 6.873 & 1043.6 & 0.0223 \\
\hline 5 & 0.0 & & & 4.565 & 1002.3 & 0.0222 & 7.250 & 1031.3 & 0.0223 \\
\hline 6 & 0.0 & & & 4.781 & 1015.2 & 0.0222 & 7.425 & 1014.9 & 0.0222 \\
\hline 7 & 0.0 & & & 4.893 & 1023.4 & 0.0221 & 7.494 & 1001.4 & 0.0221 \\
\hline 8 & 0.0 & & & 4.934 & 1030.9 & 0.0221 & 7.506 & 992.7 & 0.0221 \\
\hline 9 & 0.0 & & & 4.917 & 1039.7 & 0.0220 & 7.480 & 988.6 & 0.0220 \\
\hline 10 & 0.0 & & & 4.849 & 1049.5 & 0.0219 & 7.421 & 988.4 & 0.0220 \\
\hline 11 & 0.0 & & & 4.740 & 1057.3 & 0.0219 & 7.341 & 991.5 & 0.0219 \\
\hline 12 & 0.0 & & & 4.620 & 1058.6 & 0.0218 & 7.271 & 997.7 & 0.0219 \\
\hline 13 & 0.0 & & & 4.526 & 1050.4 & 0.0218 & 7.242 & 1006.3 & 0.0218 \\
\hline 14 & 0.0 & & & 4.474 & 1034.4 & 0.0217 & 7.260 & 1016.4 & 0.0218 \\
\hline 15 & 0.0 & & & 4.414 & 1013.1 & 0.0217 & 7.248 & 1026.4 & 0.0217 \\
\hline 16 & 0.0 & & & 4.192 & 979.7 & 0.0216 & 6.980 & 1026.7 & 0.0217 \\
\hline 17 & 0.0 & & & 3.561 & 912.5 & 0.0216 & 6.030 & 988.9 & 0.0216 \\
\hline 18 & 0.0 & & & 2.121 & 770.4 & 0.0216 & 3.657 & 845.1 & 0.0216 \\
\hline
\end{tabular}

\begin{tabular}{|c|c|c|c|c|c|c|c|c|c|}
\hline \multirow[b]{2}{*}{$\begin{array}{l}\text { Node } \\
\text { No. }\end{array}$} & \multicolumn{3}{|c|}{ Statepoint 4 (BOC Cycle 2) } & \multicolumn{3}{|c|}{ Statepoint 5 (BOC Cycle 3) } & \multicolumn{3}{|c|}{ Statepoint 6 (168.5 Cycle 3) } \\
\hline & $\begin{array}{l}\text { Burnup } \\
\text { (GWdMTU) }\end{array}$ & $\begin{array}{l}\text { Fuel } \\
\text { Temp. }\end{array}$ & $\begin{array}{l}\text { Moderator } \\
\text { Spec. Vol. }\end{array}$ & \begin{tabular}{|c|} 
Burnup \\
(GWd/MTU)
\end{tabular} & $\begin{array}{l}\text { Fuel } \\
\text { Temp. }\end{array}$ & $\begin{array}{l}\text { Moderator } \\
\text { Spec. Vol. }\end{array}$ & $\begin{array}{l}\text { Bumup } \\
\text { (GWd/MTU) }\end{array}$ & $\begin{array}{l}\text { Fuel } \\
\text { Temp. }\end{array}$ & $\begin{array}{l}\text { Moderator } \\
\text { Spec. Vol. }\end{array}$ \\
\hline & BOC Cy2 & 139.6 Cy1B & 139.6 Cy1B & $\mathrm{BOCC} \mathrm{Cy}^{3}$ & 89.8 Cy2 & 89.8 Cy2 & 168.5 Cy3 & $85.0 \mathrm{Cy3}$ & $85.0 \mathrm{Cy3}$ \\
\hline$\overline{1}$ & 3.017 & 803.4 & 0.0225 & 6.700 & 1138.8 & 0.0235 & 9.416 & 982.8 & 0.0234 \\
\hline
\end{tabular}




\section{Waste Package Development}

\section{Design Analysis}

Title: CRC Depletion Calculations for the Rodded Assemblies in Batches I, 2, 3, and $1 \mathrm{X}$ of Crystal River Unit 3

Document Identifier: BBA000000-01717-0200-00040 REV 00

Page 47 of 146

Table 4.1.10-16 Burnup, Fuel Temperature, and Moderator

Specific Volume Data for Assembly A28 of Crystal River Unit 3

\begin{tabular}{|c|c|c|c|c|c|c|c|c|c|}
\hline 2 & 5.200 & 921.9 & 0.0225 & 10.966 & 1342.7 & 0.0234 & 15.299 & 1136.1 & 0.0233 \\
\hline 3 & 6.634 & 976.2 & 0.0224 & 13.433 & 1432.2 & 0.0233 & 18.714 & 1199.8 & 0.0232 \\
\hline 4 & 7.414 & 999.7 & 0.0224 & 14.577 & 1462.7 & 0.0231 & 20.263 & 1209.1 & 0.0231 \\
\hline 5 & 7.809 & 1015.7 & 0.0223 & 15.070 & 1472.3 & 0.0230 & 20.898 & 1199.9 & 0.0230 \\
\hline 6 & 7.992 & 1026.8 & 0.0223 & 15.248 & 1477.4 & 0.0228 & 21.104 & 1186.1 & 0.0229 \\
\hline 7 & 8.065 & 1032.9 & 0.0222 & 15.230 & 1485.2 & 0.0227 & 21.072 & 1174.0 & 0.0228 \\
\hline 8 & 8.078 & 1036.3 & 0.0221 & 15.044 & 1495.6 & 0.0225 & 20.869 & 1168.1 & 0.0227 \\
\hline 9 & 8.053 & 1038.7 & 0.0221 & 14.610 & 1502.7 & 0.0224 & 20.464 & 1175.9 & 0.0226 \\
\hline 10 & 8.000 & 1041.6 & 0.0220 & 13.631 & 1472.4 & 0.0223 & 19.642 & 1208.6 & 0.0225 \\
\hline 11 & 7.933 & 1045.8 & 0.0219 & 12.560 & 1229.1 & 0.0221 & 18.816 & 1256.3 & 0.0224 \\
\hline 12 & 7.881 & 1050.5 & 0.0219 & 12.045 & 1101.8 & 0.0221 & 18.517 & 1295.9 & 0.0222 \\
\hline 13 & 7.870 & 1051.9 & 0.0218 & 12.149 & 1062.8 & 0.0220 & 18.757 & 1316.0 & 0.0221 \\
\hline 14 & 7.892 & 1045.4 & 0.0218 & 12.672 & 1042.4 & 0.0219 & 19.343 & 1316.2 & 0.0220 \\
\hline 15 & 7.867 & 1027.7 & 0.0217 & 13.627 & 1072.3 & 0.0219 & 20.264 & 1299.1 & 0.0219 \\
\hline 16 & 7.558 & 996.2 & 0.0217 & 14.062 & 1291.0 & 0.0218 & 20.525 & 1282.4 & 0.0218 \\
\hline 17 & 6.524 & 941.4 & 0.0216 & 12.694 & 1297.0 & 0.0217 & 18.523 & 1236.5 & 0.0217 \\
\hline 18 & 3.963 & 810.7 & 0.0216 & 8.065 & 1114.9 & 0.0216 & 11.954 & 1066.8 & 0.0216 \\
\hline
\end{tabular}

\begin{tabular}{|c|c|c|c|c|c|c|c|c|c|}
\hline & Statepoin & $7(250.0 \mathrm{C}$ & Eycle 3) & Statepoin & & & Statepoin & & \\
\hline $\begin{array}{l}\text { Node } \\
\text { No. }\end{array}$ & $\begin{array}{l}\text { Bumup } \\
\text { (GWd/MTU) }\end{array}$ & $\begin{array}{l}\text { Fuel } \\
\text { Temp. }\end{array}$ & $\begin{array}{l}\text { Moderator } \\
\text { Spec. Vol. }\end{array}$ & $\begin{array}{c}\text { Burnup } \\
\text { (GWd/MTU) }\end{array}$ & $\begin{array}{l}\text { Fuel } \\
\text { Temp. }\end{array}$ & $\begin{array}{l}\text { Moderator } \\
\text { Spec. Vol. }\end{array}$ & $\begin{array}{c}\text { Burnup } \\
\text { (GWd/MTU) }\end{array}$ & $\begin{array}{l}\text { Fuel } \\
\text { Temp. }\end{array}$ & $\begin{array}{l}\text { Moderator } \\
\text { Spec. Vol. }\end{array}$ \\
\hline & 250.0 Cy 3 & 246.4 Cy3 & 246.4 Cy3 & & & & & & \\
\hline 1 & 10.947 & 1010.3 & 0.0234 & No additio & al statep & ints. & No additio & al statep & nts. \\
\hline 2 & 17.603 & 1130.6 & 0.0233 & & & & & & \\
\hline 3 & 21.346 & 1156.0 & 0.0232 & & & & & & \\
\hline 4 & 22.952 & 1146.9 & 0.0230 & & & & & & \\
\hline 5 & 23.563 & 1132.6 & 0.0229 & & & & & & \\
\hline 6 & 23.738 & 1121.3 & 0.0228 & & & & & & \\
\hline 7 & 23.686 & 1114.5 & 0.0227 & & & & & & \\
\hline 8 & 23.477 & 1111.8 & 0.0226 & & & & & & \\
\hline 9 & 23.089 & 1115.2 & 0.0225 & & & & & & \\
\hline 10 & 22.330 & 1131.3 & 0.0224 & & & & & & \\
\hline 11 & 21.595 & 1152.6 & 0.0223 & & & & & & \\
\hline 12 & 21.379 & 1166.6 & 0.0222 & & & & & & \\
\hline 13 & 21.699 & 1173.1 & 0.0221 & & & & & & \\
\hline 14 & 22.398 & 1180.6 & 0.0220 & & & & & & \\
\hline 15 & 23.432 & 1187.8 & 0.0219 & & & & & . & \\
\hline 16 & 23.658 & 1178.8 & 0.0218 & & & & & & \\
\hline 17 & 21.413 & 1153.8 & 0.0217 & & & & & & \\
\hline 18 & 13.987 & 1049.9 & 0.0216 & & & & & & \\
\hline
\end{tabular}


Title: CRC Depletion Calculations for the Rodded Assemblies in Batches 1, 2, 3, and $1 \mathrm{X}$ of Crystal River Unit 3

Document Identifier: BBA000000-01717-0200-00040 REV 00

Page 48 of 146

Table 4.1.10-17 Burnup, Fuel Temperature, and Moderator

Specific Volume Data for Assembly A29 of Crystal River Unit 3

\begin{tabular}{|c|c|c|c|c|c|c|c|c|c|}
\hline & \multicolumn{9}{|c|}{ Assembly Number A29 } \\
\hline & \multicolumn{3}{|c|}{ Statepoint 1 (BOC Cycle 1A) } & \multicolumn{3}{|c|}{ Statepoint 2 (0.0 Cycle 1B) } & \multicolumn{3}{|c|}{ Statepoint 3 (142.2 Cycle 1B) } \\
\hline & $\begin{array}{c}\text { Bumup } \\
\text { (GWd/MTU) }\end{array}$ & $\begin{array}{l}\text { Fuel } \\
\text { Temp. }\end{array}$ & $\begin{array}{l}\text { Moderator } \\
\text { Spec. Vol. }\end{array}$ & $\begin{array}{c}\text { Bumup } \\
\text { (GWd/MTU) }\end{array}$ & $\begin{array}{c}\text { Fuel } \\
\text { Temp. }\end{array}$ & $\begin{array}{l}\text { Moderator } \\
\text { Spec. Vol. }\end{array}$ & $\begin{array}{l}\text { Burnup } \\
\text { (GWd/MTU) }\end{array}$ & $\begin{array}{l}\text { Fuel } \\
\text { Temp. }\end{array}$ & $\begin{array}{l}\text { Moderator } \\
\text { Spec. Vol. }\end{array}$ \\
\hline & & & & $0.0 \mathrm{Cy} 1 \mathrm{~B}$ & 184.7 Cy1A & 184.7 Cy1A & 142.2 Cy1B & 89.5 Cy1B & 89.5 Cy1B \\
\hline 1 & 0.0 & & & 1.731 & 765.0 & 0.0225 & 2.984 & 834.9 & 0.0225 \\
\hline 2 & 0.0 & & & 3.056 & 894.2 & 0.0225 & 5.144 & 977.7 & 0.0224 \\
\hline 3 & 0.0 & & & 4.068 & 984.8 & 0.0224 & 6.608 & 1035.9 & 0.0224 \\
\hline 4 & 0.0 & \multicolumn{2}{|c|}{ Data not required. } & 4.750 & 1036.6 & 0.0224 & 7.439 & 1041.2 & 0.0223 \\
\hline 5 & 0.0 & & & 5.179 & 1064.9 & 0.0223 & 7.864 & 1025.0 & 0.0223 \\
\hline 6 & 0.0 & & & 5.430 & 1080.0 & 0.0223 & 8.060 & 1005.4 & 0.0222 \\
\hline 7 & 0.0 & & & 5.563 & 1089.6 & 0.0222 & 8.140 & 990.2 & 0.0222 \\
\hline 8 & 0.0 & & & 5.611 & 1098.5 & 0.0221 & 8.155 & 980.7 & 0.0221 \\
\hline 9 & 0.0 & - & & 5.593 & 1108.9 & 0.0221 & 8.123 & 976.3 & 0.0220 \\
\hline 10 & 0.0 & & & 5.514 & 1120.1 & 0.0220 & 8.051 & 975.9 & 0.0220 \\
\hline 11 & 0.0 & & & 5.389 & 1128.8 & 0.0219 & 7.954 & 979.1 & 0.0219 \\
\hline 12 & 0.0 & & & 5.249 & 1130.5 & 0.0219 & 7.863 & 985.7 & 0.0219 \\
\hline 13 & 0.0 & & & 5.130 & 1121.6 & 0.0218 & 7.817 & 995.7 & 0.0218 \\
\hline 14 & 0.0 & & & 5.042 & 1101.7 & 0.0218 & 7.825 & 1010.4 & 0.0218 \\
\hline 15 & 0.0 & & & 4.920 & 1071.3 & 0.0217 & 7.822 & 1032.5 & 0.0217 \\
\hline 16 & 0.0 & & & 4.607 & 1025.1 & 0.0216 & 7.567 & 1052.2 & 0.0217 \\
\hline 17 & 0.0 & & & 3.890 & 947.7 & 0.0216 & 6.588 & 1026.3 & 0.0216 \\
\hline 18 & 0.0 & & & 2.347 & 793.9 & 0.0216 & 4.052 & 876.4 & 0.0216 \\
\hline
\end{tabular}

\begin{tabular}{|c|c|c|c|c|c|c|c|c|c|}
\hline & \multicolumn{3}{|c|}{ Statepoint 4 (BOC Cycle 2) } & \multicolumn{3}{|c|}{ Statepoint 5 (BOC Cycle 3) } & \multicolumn{3}{|c|}{ Statepoint 6 (168.5 Cycle 3) } \\
\hline $\begin{array}{l}\text { Node } \\
\text { No. }\end{array}$ & $\begin{array}{l}\text { Burnup } \\
\text { (GWd/MTU) }\end{array}$ & $\begin{array}{l}\text { Fuel } \\
\text { Temp. }\end{array}$ & $\begin{array}{l}\text { Moderator } \\
\text { Spec. Vol. }\end{array}$ & $\begin{array}{c}\text { Burnup } \\
\text { (GWd/MTU) }\end{array}$ & $\begin{array}{l}\text { Fuel } \\
\text { Temp. }\end{array}$ & $\begin{array}{l}\text { Moderator } \\
\text { Spec. Vol. }\end{array}$ & $\begin{array}{c}\text { Burnup } \\
\text { (GWd/MTU) }\end{array}$ & $\begin{array}{l}\text { Fuel . } \\
\text { Temp. }\end{array}$ & $\begin{array}{l}\text { Moderator } \\
\text { Spec. Vol. }\end{array}$ \\
\hline & BOC Cy2 & 139.6 Cy1B & 139.6 Cy1B & BOC Cy3 & $89.8 \mathrm{Cy}_{2}$ & $89.8 \mathrm{Cy2}$ & $168.5 \mathrm{Cy3}$ & B5.0 Cy3 & $85.0 \mathrm{Cy3}$ \\
\hline 1 & 3.249 & 809.5 & 0.0226 & 6.778 & 1114.3 & 0.0237 & 8.657 & 850.5 & 0.0228 \\
\hline 2 & $5: 575$ & 929.2 & 0.0226 & 11.124 & 1309.4 & 0.0236 & 14.019 & 943.4 & 0.0227 \\
\hline 3 & 7.134 & 987.1 & 0.0225 & 13.736 & 1401.2 & 0.0235 & 17.187 & 982.9 & 0.0227 \\
\hline 4 & 8.016 & 1020.3 & 0.0225 & 15.025 & 1438.2 & 0.0233 & 18.694 & 987.5 & 0.0226 \\
\hline 5 & 8.471 & 1051.2 & 0.0224 & 15.609 & 1452.7 & 0.0232 & 19.345 & 980.3 & 0.0225 \\
\hline 6 & 8.685 & 1069.8 & 0.0223 & 15.841 & 1459.7 & 0.0230 & 19.575 & 969.2 & 0.0225 \\
\hline 7 & 8.773 & 1077.4 & 0.0223 & 15.887 & 1466.4 & 0.0229 & 19.583 & 956.5 & 0.0224 \\
\hline 8 & 8.790 & 1080.9 & 0.0222 & 15.804 & 1473.4 & 0.0227 & 19.434 & 945.3 & 0.0223 \\
\hline 9 & 8.760 & .1083 .5 & 0.0221 & 15.584 & 1473.3 & 0.0226 & 19.153 & 941.9 & 0.0223 \\
\hline 10 & 8.695 & 1086.9 & 0.0221 & 15.235 & 1449.3 & 0.0224 & 18.801 & 951.4 & 0.0222 \\
\hline 11 & 8.612 & 1092.1 & 0.0220 & 14.872 & 1389.0 & 0.0223 & 18.508 & $971: 3$ & 0.0221 \\
\hline
\end{tabular}




\section{Waste Package Development}

Design Analysis

Title: CRC Depletion Calculations for the Rodded Assemblies in Batches 1, 2, 3, and $1 \mathrm{X}$ of Crystal River Unit 3

Document Identifier: BBA000000-01717-0200-00040 REV 00

Page 49 of 146

Table 4.1.10-17 Burnup, Fuel Temperature, and Moderator

Specific Volume Data for Assembly A29 of Crystal River Unit 3

\begin{tabular}{|r|r|r|r|r|r|r|r|r|r|}
\hline 12 & 8.543 & 1098.0 & 0.0219 & 14.651 & 1328.2 & 0.0222 & 18.403 & 995.1 & 0.0221 \\
\hline 13 & 8.516 & 1100.6 & 0.0219 & 14.649 & 1285.8 & 0.0221 & 18.538 & 1015.8 & 0.0220 \\
\hline 14 & 8.532 & 1094.9 & 0.0218 & 14.836 & 1262.9 & 0.0220 & 18.925 & 1029.2 & 0.0219 \\
\hline 15 & 8.515 & 1076.5 & 0.0217 & 15.050 & 1267.8 & 0.0219 & 19.776 & 1068.7 & 0.0219 \\
\hline 16 & 8.216 & 1042.7 & 0.0217 & 14.823 & 1294.0 & 0.0218 & 20.613 & 1233.3 & 0.0218 \\
\hline 17 & 7.143 & 982.2 & 0.0216 & 13.246 & 1277.2 & 0.0217 & 18.771 & 1214.6 & 0.0217 \\
\hline 18 & 4.398 & 840.4 & 0.0216 & 8.474 & 1104.7 & 0.0216 & 12.244 & 1051.5 & 0.0216 \\
\hline
\end{tabular}

\begin{tabular}{|c|c|c|c|c|c|c|c|c|c|}
\hline & \multicolumn{3}{|c|}{ Statepoint $7(250.0$ Cycle 3$)$} & \multicolumn{3}{|c|}{ Statepoint 8} & \multicolumn{3}{|c|}{ Statepoint 9} \\
\hline $\begin{array}{l}\text { Node } \\
\text { No. }\end{array}$ & $\begin{array}{l}\text { Burnup } \\
\text { (GWd/MTU) }\end{array}$ & $\begin{array}{l}\text { Fuel } \\
\text { Temp. }\end{array}$ & $\begin{array}{l}\text { Moderator } \\
\text { Spec. Vol. }\end{array}$ & $\begin{array}{c}\text { Burnup } \\
\text { (GWd/MTU) }\end{array}$ & $\begin{array}{l}\text { Fuel } \\
\text { Temp. }\end{array}$ & $\begin{array}{l}\text { Moderator } \\
\text { Spec. Vol. }\end{array}$ & $\begin{array}{c}\text { Bumup } \\
\text { (GWdMTU) }\end{array}$ & $\begin{array}{l}\text { Fuel } \\
\text { Temp. }\end{array}$ & $\begin{array}{l}\text { Moderator } \\
\text { Spec. Vol. }\end{array}$ \\
\hline & $250.0 \mathrm{Cy3}$ & 246.4 Cy3 & 246.4 Cy3 & & & & & & \\
\hline$\overline{1}$ & 9.702 & 878.7 & 0.0228 & No addition & I stater & & No additio & statep & \\
\hline 2 & 15.545 & 959.9 & 0.0228 & & & & & & \\
\hline 3 & 18.921 & 978.2 & 0.0227 & & & & & & \\
\hline 4 & 20.464 & 970.4 & 0.0226 & & & & & & \\
\hline 5 & 21.099 & 959.3 & 0.0225 & & & & & & \\
\hline 6 & 21.305 & 950.4 & 0.0225 & & & & & & \\
\hline 7 & 21.292 & 943.7 & 0.0224 & & & & & & \\
\hline 8 & 21.122 & 938.0 & 0.0223 & & & & & & \\
\hline 9 & 20.823 & 934.5 & 0.0223 & & & & & & \\
\hline 10 & 20.475 & 936.6 & 0.0222 & & & & & & \\
\hline 11 & 20.212 & 943.6 & 0.0222 & & & & & & \\
\hline 12 & 20.157 & 952.5 & 0.0221 & & & & & & \\
\hline 13 & 20.371 & 962.9 & 0.0220 & & & & & & \\
\hline 14 & 20.990 & 991.6 & 0.0220 & & & & & & \\
\hline 15 & 22.760 & 1148.8 & 0.0219 & & & & & & \\
\hline 16 & 23.682 & 1166.1 & 0.0218 & & & & & & - \\
\hline 17 & 21.602 & 1141.4 & 0.0217 & & & & & & \\
\hline 18 & 14.238 & 1039.3 & 0.0216 & & & & & & \\
\hline
\end{tabular}

Table 4.1.10-18 Burnup, Fuel Temperature, and Moderator Specific Volume Data for Assembly 01 of Crystal River Unit 3

\begin{tabular}{|c|c|c|c|c|c|c|c|c|}
\hline \multicolumn{9}{|c|}{ Assembly Number 01} \\
\hline \multicolumn{3}{|c|}{ Statepoint 1 (BOC Cycle 1A) } & \multicolumn{3}{|c|}{ Statepoint $2(0.0$ Cycle 1B) } & \multicolumn{3}{|c|}{ Statepoint 3 (142.2 Cycle 1B) } \\
\hline $\begin{array}{l}\text { Bumup } \\
\text { (GWd/MTU }\end{array}$ & $\begin{array}{l}\text { Fuel } \\
\text { Temp. }\end{array}$ & $\begin{array}{l}\text { Moderator } \\
\text { Spec. Vol. }\end{array}$ & $\begin{array}{c}\text { Burnup } \\
\text { (GWd/MTU) }\end{array}$ & $\begin{array}{l}\text { Fuel } \\
\text { Temp. }\end{array}$ & $\begin{array}{l}\text { Moderator } \\
\text { Spec. Vol. }\end{array}$ & $\begin{array}{c}\text { Burnup } \\
\text { (GWd/MTU) }\end{array}$ & $\begin{array}{l}\text { Fuel } \\
\text { Temp. }\end{array}$ & $\begin{array}{l}\text { Moderator } \\
\text { Spec. Vol. }\end{array}$ \\
\hline & & & 0.0 Cy4B & $146.00 c 01$ & 146.0 Oco1 & $142.2 \mathrm{Cy} 1 \mathrm{~B}$ & 89.5 Cy1B & 89.5 Cy1B \\
\hline 0.0 & & & 2.270 & 825.0 & 0.0236 & 3.557 & 841.6 & 0.0225 \\
\hline 0.0 & & & 3.977 & 982.4 & 0.0236 & 6.090 & 981.2 & 0.0225 \\
\hline
\end{tabular}


Title: CRC Depletion Calculations for the Rodded Assemblies in Batches 1, 2, 3, and 1X of Crystal River Unit 3 Document Identifier: BBA000000-01717-0200-00040 REV 00

Page 50 of 146

Table 4.1.10-18 Burnup, Fuel Temperature, and Moderator Specific Volume Data for Assembly 01 of Crystal River Unit 3

\begin{tabular}{|r|r|l|r|r|r|r|r|r|r|}
\hline 3 & 0.0 & & & 5.373 & 1099.5 & 0.0235 & 7.986 & 1043.8 & 0.0224 \\
\hline 4 & 0.0 & \multicolumn{1}{|c|}{ Data not required. } & 6.781 & 1195.7 & 0.0234 & 9.617 & 1037.1 & 0.0223 \\
\hline 5 & 0.0 & & & 10.599 & 1418.1 & 0.0233 & 13.312 & 961.7 & 0.0223 \\
\hline 6 & 0.0 & & & 12.825 & 1512.9 & 0.0232 & 15.391 & 918.2 & 0.0222 \\
\hline 7 & 0.0 & & & 13.478 & 1519.9 & 0.0230 & 15.966 & 898.9 & 0.0222 \\
\hline 8 & 0.0 & & & 13.638 & 1509.5 & 0.0228 & 16.080 & 888.8 & 0.0221 \\
\hline 9 & 0.0 & & & 13.636 & 1498.8 & 0.0227 & 16.048 & 882.9 & 0.0221 \\
\hline 10 & 0.0 & & & 13.609 & 1492.4 & 0.0226 & 15.996 & 878.2 & 0.0220 \\
\hline 11 & 0.0 & & & 13.621 & 1491.5 & 0.0224 & 15.991 & 873.7 & 0.0220 \\
\hline 12 & 0.0 & & & 13.679 & 1495.8 & 0.0223 & 16.060 & 871.9 & 0.0219 \\
\hline 13 & 0.0 & & & 13.741 & 1503.7 & 0.0221 & 16.174 & 875.2 & 0.0219 \\
\hline 14 & 0.0 & & & 13.709 & 1512.2 & 0.0220 & 16.247 & 885.8 & 0.0218 \\
\hline 15 & 0.0 & & & 13.411 & 1513.7 & 0.0219 & 16.258 & 920.9 & 0.0218 \\
\hline 16 & 0.0 & & & 12.554 & 1488.1 & 0.0218 & 16.316 & 1081.4 & 0.0217 \\
\hline 17 & 0.0 & & & 10.596 & 1388.5 & 0.0216 & 14.517 & 1130.2 & 0.0217 \\
\hline 18 & 0.0 & & & 6.368 & 1109.9 & 0.0216 & 9.061 & 1000.2 & 0.0216 \\
\hline
\end{tabular}

\subsubsection{Insertion History Data for CRA's and APSRA's}

The CRA and APSRA time of insertion in a particular axial position in a fuel assembly is required data for performing appropriate depletion calculations for a rodded assembly. Hardening (locally increasing the average energy of the neutron population due to less local thermalization and increased local capture of neutrons at thermal energies) the neutron spectrum in a particular axial region of an assembly at a time during its irradiation history effects the isotopic composition of the depleted fuel. The CRC depletion calculations of rodded assemblies as performed in this analysis requires rod insertion time input in terms of EFPD's inserted for either a CRA or APSRA in each axial node of each fuel assembly for each statepoint depletion calculation of interest. Tables 4.1.11-1 through 4.1.11-17 present the CRA and APSRA insertion time data relevant to each assembly depletion calculation documented in this analysis. Assembly A04 was located in a control bank 6 location during Cycle-2. Since Cycle- 2 was the last cycle of insertion for assembly A04 and there are no CRC statepoints other than the beginning-ofcycle statepoint in Cycle-2, the depletion of assembly A04 through Cycle-2 was not needed. Therefore, no control rod insertion data is presented for assembly A04 in this analysis. The assembly heights corresponding to the axial nodes presented in Tables 4.1.11-1 through 4.1.11-17 are as follow: the top node (node 1) is $17.78 \mathrm{~cm}$, the bottom node (node 18) is $22.352 \mathrm{~cm}$, all other nodes are $20.0025 \mathrm{~cm}$. The top of node 1 begins at the top of the active fuel region.

Table 4.1.11-1 CRA Insertion Time Data (EFPDs Inserted) for Assembly Number A1

\begin{tabular}{|c|c|c|c|}
\hline $\begin{array}{c}\text { Axial Node } \\
(1=\text { Top })\end{array}$ & $\begin{array}{c}\text { Cycle-1A 0.0 EFPD to } \\
\text { Cycle-1A 268.8 EFPD }\end{array}$ & $\begin{array}{c}\text { Cycle-1B 0.0 EFPD to } \\
\text { Cycle-1B 142.2 EFPD }\end{array}$ & $\begin{array}{c}\text { Cycle-1B 142.2 EFPD to } \\
\text { Cycle-1B.171.3 EFPD }\end{array}$ \\
\hline 1 & 268.80 & 142.20 & 29.10 \\
\hline
\end{tabular}


Title: CRC Depletion Calculations for the Rodded Assemblies in Batches 1, 2, 3, and IX of Crystal River Unit 3

\begin{tabular}{|l|l|l|l|}
\hline 2 & 268.80 & 142.20 & 29.10 \\
\hline 3 & 268.80 & 142.20 & 29.10 \\
\hline 4 & 268.80 & 134.91 & 12.02 \\
\hline 5 & 268.80 & 127.78 & 6.57 \\
\hline 6 & 268.80 & 126.76 & 0.00 \\
\hline 7 & 268.80 & 126.28 & 0.00 \\
\hline 8 & 268.80 & 125.99 & 0.00 \\
\hline 9 & 268.80 & 125.84 & 0.00 \\
\hline 10 & 268.80 & 125.83 & 0.00 \\
\hline 11 & 268.80 & 125.96 & 0.00 \\
\hline 12 & 268.80 & 126.21 & 0.00 \\
\hline 13 & 268.77 & 126.67 & 0.00 \\
\hline 14 & 265.13 & 127.51 & 0.00 \\
\hline 15 & 179.03 & 116.74 & 0.00 \\
\hline 16 & 41.67 & 30.17 & 0.00 \\
\hline 17 & 0.00 & 4.67 & 0.00 \\
\hline 18 & 0.00 & 0.00 & 0.00 \\
\hline
\end{tabular}

Table 4.1.11-2 CRA Insertion Time Data (EFPDs Inserted) for Assembly Number A5

\begin{tabular}{|c|c|c|}
\hline $\begin{array}{c}\text { Axial Node } \\
(1=\text { Top })\end{array}$ & $\begin{array}{c}\text { Cycle-1A 0.0 EFPD to } \\
\text { Cycle-1 A 268.8 EFPD }\end{array}$ & $\begin{array}{c}\text { Cycle-1B 0.0 EFPD to } \\
\text { Cycle-1B 142.2 EFPD }\end{array}$ \\
\hline 1 & 239.78 & 125.52 \\
\hline 2 & 124.80 & 117.60 \\
\hline 3 & 5.03 & 46.54 \\
\hline 4 & 0.00 & 4.51 \\
\hline 5 & 0.00 & 0.00 \\
\hline 6 & 0.00 & 0.00 \\
\hline 7 & 0.00 & 0.00 \\
\hline
\end{tabular}


Title: CRC Depletion Calculations for the Rodded Assemblies in Batches 1, 2, 3, and $1 \mathrm{X}$ of Crystal River Unit 3 Document Identifier: BBA000000-01717-0200-00040 REV 00

Page 52 of 146

\begin{tabular}{|l|l|l|}
\hline 8 & 0.00 & 0.00 \\
\hline 9 & 0.00 & 0.00 \\
\hline 10 & 0.00 & 0.00 \\
\hline 11 & 0.00 & 0.00 \\
\hline 12 & 0.00 & 0.00 \\
\hline 13 & 0.00 & 0.00 \\
\hline 14 & 0.00 & 0.00 \\
\hline 15 & 0.00 & 0.00 \\
\hline 16 & 0.00 & 0.00 \\
\hline 17 & 0.00 & 0.00 \\
\hline 18 & 0.00 & 0.00 \\
\hline
\end{tabular}

Table 4.1.11-3 CRA Insertion Time Data (EFPDs Inserted) for Assembly Number A7

\begin{tabular}{|c|c|c|c|c|}
\hline $\begin{array}{c}\text { Axial Node } \\
(1=\text { Top })\end{array}$ & $\begin{array}{c}\text { Cycle-1A 0.0 } \\
\text { EFP to Cycle-1A } \\
268.8 \text { EFPD }\end{array}$ & $\begin{array}{c}\text { Cycle-1B 0.0 EFPD } \\
\text { to Cycle-1B 142.2 } \\
\text { EFPD }\end{array}$ & $\begin{array}{c}\text { Cycle-1B 142.2 } \\
\text { EFPD to Cycle-1B } \\
171.3 \text { EFPD }\end{array}$ & $\begin{array}{c}\text { Cycle-2 0.0 EFPD } \\
\text { to Cycle-2 166.5 } \\
\text { EFPD }\end{array}$ \\
\hline 1 & 14.01 & 142.29 & 29.10 & 161.25 \\
\hline 2 & 12.97 & 142.20 & 29.10 & 125.38 \\
\hline 3 & 12.08 & 142.25 & 29.10 & 30.17 \\
\hline 4 & 11.22 & 134.21 & 11.83 & 8.45 \\
\hline 5 & 10.52 & 126.31 & 6.47 & 3.21 \\
\hline 6 & 9.96 & 125.18 & 0.00 & 0.41 \\
\hline 7 & 9.58 & 124.53 & 0.00 & 0.00 \\
\hline 8 & 9.37 & 124.16 & 0.00 & 0.00 \\
\hline 9 & 9.34 & 123.87 & 0.00 & 0.00 \\
\hline 10 & 9.43 & 123.82 & 0.00 & 0.00 \\
\hline 11 & 9.70 & 123.81 & 0.00 & 0.00 \\
\hline 12 & 10.09 & 124.00 & 0.00 & 0.00 \\
\hline 13 & 10.68 & 124.48 & 0.00 & 0.00 \\
\hline
\end{tabular}


Title: CRC Depletion Calculations for the Rodded Assemblies in Batches 1, 2, 3, and $1 \mathrm{X}$ of Crystal River Unit 3 Document Identifier: BBA000000-01717-0200-00040 REV 00

Page 53 of 146

\begin{tabular}{|l|l|l|l|l|}
\hline 14 & 11.72 & 125.38 & 0.00 & 0.00 \\
\hline 15 & 2.50 & 114.68 & 0.00 & 0.00 \\
\hline 16 & 0.00 & 28.90 & 0.00 & 0.00 \\
\hline 17 & 0.00 & 4.46 & 0.00 & 0.00 \\
\hline 18 & 0.00 & 0.00 & 0.00 & 0.00 \\
\hline
\end{tabular}

Table 4.1.11-4 CRA Insertion Time Data (EFPDs Inserted) for Assembly Number A14

\begin{tabular}{|c|c|c|c|c|c|}
\hline $\begin{array}{c}\text { Axial Node } \\
(1=\text { Top) }\end{array}$ & $\begin{array}{c}\text { Cycle-7 0.0 } \\
\text { EFPD to } \\
\text { Cycle-7 260.3 } \\
\text { EFPD }\end{array}$ & $\begin{array}{c}\text { Cycle-7 260.3 } \\
\text { EFPD to } \\
\text { Cycle-7 291.0 } \\
\text { EFPD }\end{array}$ & $\begin{array}{c}\text { Cycle-7 291.0 } \\
\text { EFPD to } \\
\text { Cycle-7 319.0 } \\
\text { EFPD }\end{array}$ & $\begin{array}{c}\text { Cycle-7 319.0 } \\
\text { EFPD to } \\
\text { Cycle-7 462.3 } \\
\text { EFPD }\end{array}$ & $\begin{array}{c}\text { Cycle-7 462.3 } \\
\text { EFPD to } \\
\text { Cycle-7 479.0 } \\
\text { EFPD }\end{array}$ \\
\hline 1 & 146.27 & 10.13 & 5.32 & 27.11 & 0.23 \\
\hline 2 & 35.38 & 0.00 & 0.00 & 0.00 & 0.00 \\
\hline 3 & 0.00 & 0.00 & 0.00 & 0.00 & 0.00 \\
\hline 4 & 0.00 & 0.00 & 0.00 & 0.00 & 0.00 \\
\hline 5 & 0.00 & 0.00 & 0.00 & 0.00 & 0.00 \\
\hline 6 & 0.00 & 0.00 & 0.00 & 0.00 & 0.00 \\
\hline 7 & 0.00 & 0.00 & 0.00 & 0.00 & 0.00 \\
\hline 8 & 0.00 & 0.00 & 0.00 & 0.00 & 0.00 \\
\hline 9 & 0.00 & 0.00 & 0.00 & 0.00 & 0.00 \\
\hline 10 & 0.00 & 0.00 & 0.00 & 0.00 & 0.00 \\
\hline 11 & 0.00 & 0.00 & 0.00 & 0.00 & 0.00 \\
\hline 12 & 0.00 & 0.00 & 0.00 & 0.00 & 0.00 \\
\hline 13 & 0.00 & 0.00 & 0.00 & 0.00 & 0.00 \\
\hline 14 & 0.00 & 0.00 & 0.00 & 0.00 & 0.00 \\
\hline 15 & 0.00 & 0.00 & 0.00 & 0.00 & 0.00 \\
\hline 16 & 0.00 & 0.00 & 0.00 & 0.00 & 0.00 \\
\hline 17 & 0.00 & 0.00 & 0.00 & 0.00 & 0.00 \\
\hline 18 & 0.00 & 0.00 & 0.00 & 0.00 & 0.00 \\
\hline
\end{tabular}


Title: CRC Depletion Calculations for the Rodded Assemblies in Batches 1, 2, 3, and 1X of Crystal River Unit 3

Table 4.1.11-5 APSRA Insertion Time Data (EFPDs Inserted) for Assembly Number A18

\begin{tabular}{|c|c|}
\hline Axial Node (1=Top) & Cycle-1A 0.0 EFPD to Cycle-1A 268.8 EFPD \\
\hline 1 & 0.00 \\
\hline 2 & 0.00 \\
\hline 3 & 0.00 \\
\hline 4 & 0.00 \\
\hline 5 & 0.00 \\
\hline 6 & 0.00 \\
\hline 7 & 0.00 \\
\hline 8 & 0.00 \\
\hline $9 \quad-$ & 2.20 \\
\hline 10 & 4.48 \\
\hline 11 & 31.29 \\
\hline 12 & 238.15 \\
\hline 13 & 261.36 \\
\hline 14 & 257.56 \\
\hline 15 & 250.06 \\
\hline 16 & 122.12 \\
\hline 17 & 4.49 \\
\hline 18 & 0.78 \\
\hline
\end{tabular}

Table 4.1.11-6 APSRA Insertion Time Data (EFPDs Inserted) for Assembly Number A18a Axial Node (1=Top) Cycle-1A 0.0 EFPD to Cycle-1A 268.8 EFPD

\begin{tabular}{l|l}
1 & 0.00 \\
\hline 2 & 0.00 \\
\hline 3 & 0.00 \\
\hline 4 & 0.00 \\
\hline 5 & 0.00 \\
\hline
\end{tabular}


Title: CRC Depletion Calculations for the Rodded Assemblies in Batches 1, 2, 3, and IX of Crystal River Unit 3 Document Identifier: BBA000000-01717-0200-00040 REV 00

\begin{tabular}{|c|c|}
\hline 6 & 0.00 \\
\hline 7 & 0.00 \\
\hline 8 & 0.00 \\
\hline 9 & 2.20 \\
\hline 10 & 4.48 \\
\hline 11 & 31.29 \\
\hline 12 & 238.15 \\
\hline 13 & 261.36 \\
\hline 14 & 257.56 \\
\hline 15 & 250.06 \\
\hline 16 & 122.12 \\
\hline 17 & 4.49 \\
\hline 18 & 0.78 \\
\hline
\end{tabular}

Table 4.1.11-7 APSRA Insertion Time Data (EFPDs Inserted) for Assembly Number A18b

\begin{tabular}{|c|c|c|}
\hline Axial Node (1=Top) & $\begin{array}{c}\text { Cycle-1A 0.0 EFPD to Cycle-1A } \\
268.8 \text { EFPD }\end{array}$ & $\begin{array}{c}\text { Cycle-1B 0.0 EFPD to Cycle-1A } \\
\text { 142.2 EFPD }\end{array}$ \\
\hline 1 & 0.00 & 0.00 \\
\hline 2 & 0.00 & 0.00 \\
\hline 3 & 0.00 & 0.00 \\
\hline 4 & 0.00 & 0.00 \\
\hline 5 & 0.00 & 0.00 \\
\hline 6 & 0.00 & 0.00 \\
\hline 7 & 0.00 & 0.21 \\
\hline 8 & 0.00 & 0.46 \\
\hline 9 & 2.20 & 0.44 \\
\hline 10 & 4.48 & 6.34 \\
\hline 11 & 31.29 & 71.70 \\
\hline
\end{tabular}




\begin{tabular}{|c|c|c|}
\hline 12 & 238.15 & 140.47 \\
\hline 13 & 261.36 & 140.05 \\
\hline 14 & 257.56 & 139.51 \\
\hline 15 & 250.06 & 109.34 \\
\hline 16 & 122.12 & 16.84 \\
\hline 17 & 4.49 & 0.00 \\
\hline 18 & 0.78 & 0.00 \\
\hline
\end{tabular}

Table 4.1.11-8 CRA Insertion Time Data (EFPDs Inserted) for Assembly Number A20

\begin{tabular}{|c|c|}
\hline Axial Node (1=Top) & Cycle-1A 0.0 EFPD to Cycle-1A 268.8 EFPD \\
\hline 1 & 224.35 \\
\hline 2 & 225.46 \\
\hline 3 & $227: 76$ \\
\hline 4 & 230.06 \\
\hline 5 & 232.01 \\
\hline 6 & 233.45 \\
\hline 7 & 234.37 \\
\hline 8 & 234.74 \\
\hline 9 & 234.54 \\
\hline 10 . & 233.79 \\
\hline 11 & 232.47 \\
\hline 12 & 230.64 \\
\hline 13 & 228.83 \\
\hline 14 & 225.25 \\
\hline 15 & 164.24 \\
\hline 16 & 37.78 \\
\hline 17 & 0.00 \\
\hline 18 & 0.00 \\
\hline
\end{tabular}


Title: CRC Depletion Calculations for the Rodded Assemblies in Batches 1, 2, 3, and $1 \mathrm{X}$ of Crystal River Unit 3 Document Identifier: BBA000000-01717-0200-00040 REV 00

Page 57 of 146

Table 4.1.11-9 CRA Insertion Time Data (EFPDs Inserted) for Assembly Number A22

\begin{tabular}{|c|c|c|}
\hline Axial Node $(1=$ Top $)$ & $\begin{array}{c}\text { Cycle-1A } 0.0 \text { EFPD to Cycle-1A } \\
268.8 \text { EFPD }\end{array}$ & $\begin{array}{c}\text { Cycle-1B 0.0 EFPD to Cycle-1 A } \\
142.2 \text { EFPD }\end{array}$ \\
\hline 1 & 240.16 & 125.38 \\
\hline 2 & 125.32 & 117.34 \\
\hline 3 & 5.03 & 46.31 \\
\hline 4 & 0.00 & 4.49 \\
\hline 5 & 0.00 & 0.00 \\
\hline 6 & 0.00 & 0.00 \\
\hline 7 & 0.00 & 0.00 \\
\hline - & 0.00 & 0.00 \\
\hline 9 & 0.00 & 0.00 \\
\hline 10 & 0.00 & 0.00 \\
\hline 11 & 0.00 & 0.00 \\
\hline 12 & 0.00 & 0.00 \\
\hline 13 & 0.00 & 0.00 \\
\hline 14 & 0.00 & 0.00 \\
\hline 15 & 0.00 & 0.00 \\
\hline 16 & 0.00 & 0.00 \\
\hline 17 & 0.00 & 0.00 \\
\hline 18 & 0.00 & 0.00 \\
\hline
\end{tabular}

Table 4.1.11-10 CRA Insertion Time Data (EFPDs Inserted) for Assembly Number A23

\begin{tabular}{|c|c|}
\hline Axial Node (1=Top) & Cycle-2 0.0 EFPD to Cycle-2 166.5 EFPD \\
\hline 1 & 165.61 \\
\hline 2 & 166.50 \\
\hline 3 & 166.50 \\
\hline
\end{tabular}


Title: CRC Depletion Calculations for the Rodded Assemblies in Batches 1, 2, 3, and $1 \mathrm{X}$ of Crystal River Unit 3

\begin{tabular}{|c|c|}
\hline 4 & 166.50 \\
\hline 5 & 166.56 \\
\hline 6 & 166.56 \\
\hline 7 & 166.50 \\
\hline 8 & 166.50 \\
\hline 9 & 166.50 \\
\hline 10 & 166.50 \\
\hline 11 & 166.56 \\
\hline 12 & 166.50 \\
\hline 13 & 166.50 \\
\hline 14 & 166.20 \\
\hline 15 & 137.89 \\
\hline 16 & 64.84 \\
\hline 17 & 10.12 \\
\hline 18 & 0.99 \\
\hline
\end{tabular}

Table 4.1.11-11 CRA Insertion Time Data (EFPDs Inserted) for Assembly Number A23a

\begin{tabular}{|c|c|}
\hline Axial Node (1=Top) & Cycle-2 0.0 EFPD to Cycle-2 166.5 EFPD \\
\hline 1 & 165.65 \\
\hline 2 & 166.50 \\
\hline 3 & 166.50 \\
\hline 4 & 166.45 \\
\hline 5 & 166.50 \\
\hline 6 & 166.55 \\
\hline 7 & 166.50 \\
\hline 8 & 166.45 \\
\hline 9 & 166.50 \\
\hline 10 & 166.50 \\
\hline
\end{tabular}


Title: CRC Depletion Calculations for the Rodded Assemblies in Batches 1, 2, 3, and $1 \mathrm{X}$ of Crystal River Unit 3 Document Identifier: BBA000000-01717-0200-00040 REV 00

\begin{tabular}{|c|c|}
\hline 11 & 166.50 \\
\hline 12 & 166.45 \\
\hline 13 & 166.50 \\
\hline 14 & 166.26 \\
\hline 15 & 136.66 \\
\hline 16 & 61.87 \\
\hline 17 & 10.02 \\
\hline 18 & 1.06 \\
\hline
\end{tabular}

Table 4.1.11-12 CRA Insertion Time Data (EFPDs Inserted) for Assembly Number A25

\begin{tabular}{|c|c|c|}
\hline Axial Node (1=Top) & $\begin{array}{c}\text { Cycle-3 0.0 EFPD to Cycle-3 } \\
168.5 \text { EFPD }\end{array}$ & $\begin{array}{c}\text { Cycle-3 168.5 EFPD to Cycle-3 } \\
250.0 \text { EFPD }\end{array}$ \\
\hline 1 & 168.38 & 81.60 \\
\hline 2 & 168.50 & 81.50 \\
\hline 3 & 168.50 & 81.50 \\
\hline 4 & 168.44 & 81.50 \\
\hline 5 & 168.50 & 81.50 \\
\hline 6 & 168.50 & 81.50 \\
\hline 7 & 168.50 & 81.56 \\
\hline 8 & 168.50 & 81.56 \\
\hline 9 & 168.50 & 81.50 \\
\hline 10 & 168.50 & 81.50 \\
\hline 11 & 168.50 & 81.44 \\
\hline 12 & 168.50 & 81.50 \\
\hline 13 & 168.50 & 81.50 \\
\hline 14 & 165.24 & 76.88 \\
\hline 15 & 111.40 & 3.04 \\
\hline 16 & 15.24 & 0.00 \\
\hline
\end{tabular}




\begin{tabular}{|l|l|l|}
\hline 17 & 5.49 & 0.00 \\
\hline 18 & 0.26 & 0.00 \\
\hline
\end{tabular}

Table 4.1.11-13 CRA Insertion Time Data (EFPDs Inserted) for Assembly Number A25a

\begin{tabular}{|c|c|c|}
\hline Axial Node (1=Top) & $\begin{array}{c}\text { Cycle-3 } 0.0 \text { EFPD to Cycle-3 } \\
168.5 \text { EFPD }\end{array}$ & $\begin{array}{c}\text { Cycle-3 168.5 EFPD to Cycle-3 } \\
\text { 250.0 EFPD }\end{array}$ \\
\hline 1 & 156.32 & 71.98 \\
\hline 2 & 76.64 & 8.99 \\
\hline 3 & 10.17 & 0.00 \\
\hline 4 & 6.84 & 0.00 \\
\hline 5 & 0.17 & 0.00 \\
\hline 6 & 0.00 & 0.00 \\
\hline 7 & 0.00 & 0.00 \\
\hline 8 & 0.00 & 0.00 \\
\hline 9 & 0.00 & 0.00 \\
\hline 10 & 0.00 & 0.00 \\
\hline 11 & 0.00 & 0.00 \\
\hline 12 & 0.00 & 0.00 \\
\hline 13 & 0.00 & 0.00 \\
\hline 14 & 0.00 & 0.00 \\
\hline 15 & 0.00 & 0.00 \\
\hline 16 & 0.00 & 0.00 \\
\hline 17 & 0.00 & 0.00 \\
\hline 18 & 0.00 & 0.00 \\
\hline
\end{tabular}

Table 4.1.11-14 CRA \& APSRA Insertion Time Data (EFPDs Inserted) for Assembly Num. A26 Axial Node (1=Top)

Cycle-1A 0.0 EFPD to Cycle-1A 268.8 EFPD (CRA Inserted)
Cycle-1B 0.0 EFPD to Cycle-1B 142.2 EFPD (APSRA Inserted) 
Title: CRC Depletion Calculations for the Rodded Assemblies in Batches 1, 2, 3, and IX of Crystal River Unit 3

\begin{tabular}{|c|c|c|}
\hline 2 & 12.27 & 0.00 \\
\hline 3 & 11.36 & 0.00 \\
\hline 4 & 10.46 & 0.00 \\
\hline 5 & 9.73 & 0.00 \\
\hline 6 & 9.19 & 0.00 \\
\hline 7 & 8.86 & 0.20 \\
\hline 8 & 8.67 & 0.44 \\
\hline 9 & 8.66 & 0.42 \\
\hline 10 & 8.80 & 6.32 \\
\hline 11 & 9.16 & 71.66 \\
\hline 12 & 9.73 & 140.55 \\
\hline 13 & 10.58 & 140.21 \\
\hline 14 & 11.95 & 139.62 \\
\hline 15 & 2.69 & 109.45 \\
\hline 16 & 0.00 & 16.83 \\
\hline 17 & 0.00 & 0.00 \\
\hline 18 & 0.00 & 0.00 \\
\hline
\end{tabular}

Table 4.1.11-15 APSRA Insertion Time Data (EFPDs Inserted) for Assembly Number A28

\begin{tabular}{|c|c|}
\hline Axial Node (1=Top) & Cycle-2 0.0 EFPD to Cycle-2 166.5 EFPD \\
\hline 1 & 0.00 \\
\hline 2 & 0.00 \\
\hline 3 & 0.00 \\
\hline 4 & 0.00 \\
\hline 5 & 0.00 \\
\hline 6 & 0.00 \\
\hline 7 & 1.93 \\
\hline 8 & 6.55 \\
\hline
\end{tabular}


Title: CRC Depletion Calculations for the Rodded Assemblies in Batches 1, 2, 3, and $1 \mathrm{X}$ of Crystal River Unit 3

\begin{tabular}{|c|c|}
\hline 9 & 15.29 \\
\hline 10 & 57.78 \\
\hline 11 & 122.85 \\
\hline 12 & 156.14 \\
\hline 13 & 142.18 \\
\hline 14 & 109.13 \\
\hline 15 & 47.29 \\
\hline 16 & 7.78 \\
\hline 17 & 0.00 \\
\hline 18 & 0.00 \\
\hline
\end{tabular}

Table 4.1.11-16 CंRA Insertion Time Data (EFPDs Inserted) for Assembly Number A29

\begin{tabular}{|c|c|c|}
\hline Axial Node (1=Top) & $\begin{array}{c}\text { Cycle-3 0.0 EFPD to Cycle-3 } \\
168.5 \text { EFPD }\end{array}$ & $\begin{array}{c}\text { Cycle-3 168.5 EFPD to Cycle-3 } \\
250.0 \text { EFPD }\end{array}$ \\
\hline 1 & 168.59 & 81.50 \\
\hline 2 & 168.50 & 81.55 \\
\hline 3 & 168.50 & 81.50 \\
\hline 4 & 168.50 & 81.50 \\
\hline 5 & 168.50 & 81.50 \\
\hline 6 & 168.55 & 81.50 \\
\hline 7 & 168.50 & 81.50 \\
\hline 8 & 168.50 & 81.50 \\
\hline 9 & 168.50 & 81.50 \\
\hline 10 & 168.50 & 81.50 \\
\hline 11 & 168.55 & 81.45 \\
\hline 12 & 168.50 & 81.50 \\
\hline 13 & 168.50 & 81.50 \\
\hline 14 & 165.37 & 76.92 \\
\hline & & \\
\hline
\end{tabular}


Title: CRC Depletion Calculations for the Rodded Assemblies in Batches 1, 2, 3, and $1 \mathrm{X}$ of Crystal River Unit 3

\begin{tabular}{|c|c|c|}
\hline 15 & 114.16 & 3.00 \\
\hline 16 & 16.44 & 0.00 \\
\hline 17 & 6.04 & 0.00 \\
\hline 18 & 0.27 & 0.00 \\
\hline
\end{tabular}

Table 4.1.11-17 CRA Insertion Time Data (EFPDs Inserted) for Assembly Number 01

\begin{tabular}{|c|c|c|}
\hline Axial Node (1=Top) & $\begin{array}{l}\text { Cycle-1 } 0.0 \text { EFPD to Cycle-1 } \\
\text { 309.3 EFPD of Oconee }\end{array}$ & $\begin{array}{c}\text { Cycle-1B 0.0 EFPD to Cycle-1B } \\
142.2 \text { EFPD }\end{array}$ \\
\hline 1 & 309.60 & 142.20 \\
\hline 2 & 309.60 & 142.20 \\
\hline 3 & 309.60 & 142.20 \\
\hline 4 & 309.60 & 133.93 \\
\hline 5 & 54.07 & 126.00 \\
\hline 6 & 0.00 & 125.02 \\
\hline 7 & 0.00 & 124.60 \\
\hline 8 & 0.00 & 124.32 \\
\hline 9 & 0.00 & 124.16 \\
\hline 10 & 0.00 & 124.09 \\
\hline 11 & 0.00 & 124.14 \\
\hline 12 & 0.00 & 124.22 \\
\hline 13 & 0.00 & 124.55 \\
\hline 14 & 0.00 & 125.39 \\
\hline 15 & 0.00 & 114.38 \\
\hline 16 & 0.00 & 28.95 \\
\hline 17 & 0.00 & 4.64 \\
\hline 18 & 0.00 & 0.00 \\
\hline
\end{tabular}


Title: CRC Depletion Calculations for the Rodded Assemblies in Batches 1, 2, 3, and $1 \mathrm{X}$ of Crystal River Unit 3

\subsection{Criteria}

The design of the waste package will depend on waste package configuration criticality analyses performed using an acceptable disposal criticality analysis methodology. Criteria that relate to the development and design of repository and engineered barrier components are derived from the applicable requirements and planning documents. The Engineered Barrier Design Requirements Document (EBDRD, Ref. 5.8) provides requirements for engineered barrier segment design. The Repository Design Requirements Document (RDRD, Ref. 5.9) provides requirements for repository design. The Controlled Design Assumptions Document (Ref. 5.10) provides guidance for requirements listed in the EBDRD and RDRD which have unqualified or unconfirmed data associated with the requirement.

This analysis supports the disposal criticality analysis methodology by providing input, in the form of fuel and burnable poison depletion results, to benchmark calculations which address the prediction of both spent fuel isotopic compositions and their associated reactivity. These benchmark calculations will contribute to the determination of bias values in the method of critical multiplication factor calculation that is implemented by the analytic tools to be used in the disposal criticality methodology. The requirements for utilizing the bias in the method of calculation of the critical multiplication factor for disposal configurations containing spent nuclear fuel are located in Section 3.2.2.5 of the RDRD and Section 3.2.2.6 of the EBDRD. This analysis does not satisfy these requirements, but the results from this analysis will be used as input to subsequent analyses which will satisfy these requirements.

\subsection{Assumptions}

4.3.1 The inherent approximation of uniformly distributed non-fuel lattice cells in the Path B unit cell models of the SAS2H calculations as described in Section 7.2 is considered acceptable within the fidelity of these calculations as documented in Section S2.2.3.1 of Volume 1, Rev. 5 in reference 5.4. The basis for this assumption is provided in the previously identified section of reference 5.4. This assumption is used throughout all depletion calculations documented in Section 7.

4.3.2 With the utilization of one cross-section update per irradiation time step, the maximum duration of any time step in any reactor cycle irradiation layout of this analysis should not exceed 80 days. The basis for this assumption is that the $\mathbf{8 0}$ day irradiation time step limit assures that the isotopic concentrations of the system (primarily fuel and borated moderator) will not alter the neutron spectrum radically enough to cause a time step of the depletion calculation to be performed without the availability of cross-sections which have been properly weighted with an updated neutron spectrum and spatial flux. This assumption is used throughout all depletion calculations documented in Section 7.

4.3.3 Distributing the spacer grid material uniformly in the moderator composition of the Path A and B models is acceptable. The basis for this assumption is that the limited reactivity worth of the spacer grid materials will have negligible impact on the neutron spectrum when placed homogeneously in axial regions of the assembly. This assumption is used throughout all depletion calculations documented in Section 7. 
Title: CRC Depletion Calculations for the Rodded Assemblies in Batches 1, 2, 3, and 1X of Crystal River Unit 3

\subsection{Codes and Standards}

Not applicable.

\section{References}

5.1 Activity Evaluation: Perform Criticality, Thermal, Structural, and Shielding Analyses. Document Identifier Number (DI\#): BB0000000-01717-2200-00025 REV 02, Civilian Radioactive Waste Management System (CRWMS) Management and Operating Contractor (M\&O).

5.2 Quality Assurance Requirements and Description. DOE/RW-0333P REV 07, DOE (U.S. Department of Energy).

5.3 Summary Report of Commercial Reactor Criticality Data for Crystal River Unit 3. DI\#: B00000000-01717-5705-00060 REV 00, CRWMS M\&O.

5.4 SCALE 4.3: Modular Code System for Performing Standardized Computer Analyses for Licensing Evaluation. User's Manual Volumes 0 through 3, Oak Ridge National Laboratory, Document Number: CCC-545.

5.5 Software Qualification Report for the SCALE Modular Code System Version 4.3. SCALE Version 4.3 Configuration Software Configuration Identifier (CSCI): 30011 V4.3, DI\#: 30011 2002 REV 00, CRWMS M\&O.

5.6 Q-List. YMP/90-55Q, REV 04, YMP (Yucca Mountain Site Characterization Project).

5.7 This reference is intentionally left blank.

5.8 Engineered Barrier Design Requirements Document. YMP/CM-0024, REV 0, ICN 1, DOE OCRWM.

5.9 Repository Design Requirements Document. YMP/CM-0023, REV 0, ICN 1, DOE OCRWM.

5.10 Controlled Design Assumptions Document. DI\#: B00000000-01717-4600-00032 REV 04, ICN 01, CRWMS M\&O.

5.11 CRC Depletion Calculations for the Non-Rodded Assemblies in Batches 1, 2, and 3 of Crystal River Unit 3. DI\#: BBA000000-01717-0200-00032 REV 00, CRWMS M\&O.

5.12 Attachments for BBA000000-01717-0200-00040 REV 00 - CRC Depletion Calculations for the Rodded Assemblies in Batches 1, 2, 3, and $1 \mathrm{X}$ of Crystal River Unit 3. Batch Number: MOY970820-04. 
Title: CRC Depletion Calculations for the Rodded Assemblies in Batches 1, 2, 3, and IX of Crystal River Unit 3

Document Identifier: BBA000000-01717-0200-00040 REV 00

Page 66 of 146

5.13 CRC Depletion Calculations for the Rodded Assemblies in Batches 1, 2, 3, and 1X of Crystal River Unit 3 (DI\#: BBA000000-01717-0200-00040 REV 00) - Attachments XL and XII - 2 Data Cartridges. Batch Number: MOY-970820-03.

\section{Use of Computer Software}

\subsection{Scientific and Engineering Software}

The SAS2H control module of the SCALE 4.3 modular code system (Ref. 5.4) was used in this analysis to perform fuel assembly depletion calculations required for CRC evaluations. The SCALE 4.3 code system is subject to the requirements of the QARD (Ref. 5.2). The SCALE 4.3 code system was obtained from the Software Configuration Management in accordance with appropriate procedures. The CSCI number for SCALE 4.3 is 30011 V4.3. The SAS2H calculations documented in this analysis were performed on Hewlett Packard (HP) 9000 series workstations. The SAS2H control module utilizes the BONAMI, NITAWL, XSDRNPM, COUPLE, and ORIGEN-S calculational modules to perform isotopic depletion calculations. A detailed description of the SAS2H control module is provided in Volume 1, Section S2 of reference 5.4. The SAS2H control module of the SCALE 4.3 code system is applicable to the engineering application within this analysis and is used within the range of verification and validation as documented in reference 5.5 .

A software routine entitled "Commercial Reactor Assembly Follow Taskmaster" (CRAFT) was written to automate the production of SAS2H input decks as required to support fuel assembly depletion calculations relevant to CRC evaluations. The CRAFT code does not generate data. All calculations performed by the CRAFT code are verified by visual inspection and/or hand calculations. The CRAFT code, Version 3.0, compiled on February 25, 1997, was utilized in this analysis to orchestrate the depletion calculations for the fuel assemblies. The CRAFT 3.0 source code ("CRAFT.f.V3.compiled_on_02_25_97") and executable file ("CRAFT3.0") exist in the directory "/users/wright/CRAFT_V3" on the Waste Package Development Department (WPDD) HP 9000 series workstation designated "Opus". The CRAFT code is subject to the requirements of the QARD as defined by Section I.2.1 Part C of Supplement I Rev. 1 of the QARD. Complete documentation of the CRAFT code, Version 3.0, including code description, user information, and documentation that the software provides correct results for a specified range of input parameters is included in Attachment I of this analysis.

A software routine entitled "CRC_DATA_TABULIZER" was written to automate the production of tables containing the isotopic results and other pertinent data for a set of 29 principal isotopes at each CRC statepoint for each assembly. The CRC_DATA_TABULIZER code does not generate data. All calculations performed by the CRC_DATA_TABULIZER code are verified by visual inspection and/or hand calculations. The CRC_DATA_TABULIZER code, Version 2.0, compiled on March 20, 1997, was utilized to tabulate the principal isotope results for each fuel assembly at each CRC statepoint. The CRC_DATA_TABULIZER, Version 2.0, source code (CRC_DATA_TABULIZER_V2.f) and executable file (CRC_DATA_TABULIZER_V2.exe) exist in the directory "/users/wright/CRC_DATA_TABULIZER" on the WPDD HP 9000 series workstation designated "Opus". The CRC_DATA_TABULIZER code is subject to the requirements of the QARD as defined 
by Section I.2.1 Part C of Supplement I Rev. I of the QARD. Complete documentation of the CRC_DATA_TABULIZER code including code description, user information, and documentation that the software provides correct results for a specified range of input parameters is presented in Attachment $\mathrm{V}$ of reference 5.11 .

A software routine entitled "RLAYOUT" was written to automate the development of appropriate irradiation time step layouts for depletion calculations involving rod insertion histories in which rod movements must be followed. The RLAYOUT code does not generate data. All calculations performed by the RLAYOUT code are verified by visual inspection and/or hand calculations. The RLAYOUT code, compiled on February 4, 1997, was utilized to develop appropriate irradiation time step layouts for the statepoint depletion calculations having associated rod insertion histories. The RLAYOUT source code (RLAYOUT.f) and executable file (RLAYOUT.exe) exist in the directory "/users/wright/RLAYOUT" on the WPDD HP 9000 series workstation designated "Opus". The RLAYOUT code is subject to the requirements of the QARD as defined by Section I.2.1 Part C of Supplement I Rev. 1 of the QARD. Complete documentation of the RLAYOUT code including code description, user information, and documentation that the software provides correct results for a specified range of input parameters is presented in Attachment III of this analysis.

\subsection{Computational Support Software}

The Excel, Version 5.0, and Lotus 1-2-3, Version 4.0, spreadsheet packages are two of the computational support software packages utilized in this analysis. The user-defined formulas, inputs, and results for all calculations performed with these spreadsheet packages are documented, where applicable, throughout this analysis. The "sed" line editor (Revision: 70.12) available in the "/bin" directory on the HP 9000 series workstations is utilized in the "sedexecute" script file which is called and executed by the CRAFT code. The usage of the "sed" line editor is described in Section 6 of Attachment I.

\section{Design Analysis}

This design analysis documents the fuel assembly SAS2H depletion calculations for the rodded assemblies of fuel batches 1,2,3, and $1 \mathrm{X}$ which are required for the CRC evaluations of Crystal River Unit 3. Sections 7.1 through 7.5 describe how the parameters listed in Section 4.1 are utilized to perform the appropriate SAS2H depletion calculations relevant to CRC evaluations. The CRAFT description and user information provided in Attachment I is essential for understanding the SAS2H modeling techniques employed in this analysis. The information in Attachment I, the input parameters in Section 4.1, and the CRAFT input decks in Attachments IV through XXI work together to provide a complete description of how all of the SAS2H depletion calculations in this analysis were performed. 
Title: CRC Depletion Calculations for the Rodded Assemblies in Batches 1, 2, 3, and $1 \mathrm{X}$ of Crystal River Unit 3 Document Identifier: BBA000000-01717-0200-00040 REV 00

\subsection{Assembly Depletion Calculation Procedure}

The calculational procedure for the fuel assembly SAS2H depletion calculations performed in this analysis is based on the utilization of the CRAFT Version 3.0 code. The CRAFT code is described generally in Sections 7.4 and 7.5. The complete detailed description of the CRAFT Version 3.0 code is provided in Attachment I of this analysis. The procedure for performing a fuel assembly depletion calculation with CRAFT Version 3.0 consists of the following four steps:

1) Create a CRAFT input deck for the assembly depletion calculation.

2) Assure that the CRAFT executable file and the CRAFT input deck entitled "datain" and the "sedexecute" executable file are in the same directory. The "sedexecute" executable file is a script file which is used in conjunction with the CRAFT code to create the consolidated output files described in Section 7.5.

3) Execute CRAFT.

4) Check and analyze the CRAFT generated SAS2H input decks and the SAS2H isotopic results.

The various CRAFT generated and consolidated SAS2H output files contain unique filenames which specify the following information:

1) reactor identifier,

2) one-eighth core symmetry assembly number in current reactor cycle,

3) axial node number,

4) reactor cycle number in which the SAS2H calculation begins,

5) EFPD statepoint at which the SAS2H calculation begins,

6) reactor cycle number in which the SAS2H calculation ends,

7) EFPD statepoint at which the SAS2H calculation ends.

A complete detailed description of the filename content and format is provided in Attachment I. Specific isotopic results contained in the various consolidated output files generated by CRAFT may be retrieved using the output filename information.

\subsection{Path B Unit Cell Model Development}

The SAS2H control module uses ORIGEN-S to perform a point depletion calculation for the fuel assembly or section of the fuel assembly described in the SAS2H input deck. The ORIGEN-S calculational module uses cell-weighted cross-sections based on one-dimensional (1-D) transport calculations performed by XSDRNPM. One-dimensional transport calculations are performed on two unit cell models, Path A and Path B, to calculate energy dependent spatial neutron flux distributions necessary to perform cross-section cell-weighting calculations.

The Path A unit cell model is simply a unit cell of the fuel assembly lattice containing a fuel rod. In the Path A model, the fuel pellet, gap, and clad are modeled explicitly. The only modification required to develop the Path A model is the conversion of the fuel assembly's square lattice unit cell perimeter to a 
Title: CRC Depletion Calculations for the Rodded Assemblies in Batches 1, 2, 3, and $1 \mathrm{X}$ of Crystal River Unit 3 Document Identifier: BBA000000-01717-0200-00040 REV 00

Page 69 of 146

radial perimeter conserving moderator volume within the unit cell, exterior to the fuel rod cladding. This modification is performed automatically by the SAS2H control module. A 1-D transport calculation is performed on the Path A unit cell model for each energy group, and the unit cell spatial flux distributions for each energy group are used to calculate cell-weighted cross-sections for the fuel.

The Path B unit cell model is a larger unit cell representation than the Path A model; hence, it is sometimes referred to as the larger unit cell model. The Path B unit cell model represents all or part of the fuel assembly. The Path B unit cell model attempts to account for spectral effects due to heterogeneities within the fuel assembly such as water gaps, burnable poison rods, control rods, or axial power shaping rods. Typically, fuel assemblies contain a number of similar non-fuel lattice cells dispersed somewhat uniformly throughout the assembly lattice. The structure of the Path B unit cell model is based on a uniform distribution of these non-fuel lattice cells. In reality, most fuel assemblies do not have uniformly distributed non-fuel lattice cells, but the approximation of uniformly distributed non-fuel lattice cells is considered acceptable within the fidelity of these calculations as documented in Section S2.2.3.1 of Volume 1, Rev. 5 in reference 5.4.

The basic structure of the Path B unit cell model for the fuel assembly depletion calculations performed in this analysis includes an inner region composed of an explicit representation of the non-fuel lattice cell. This inner region has essentially the same format as the Path A model with the fuel rod replaced by the non-fuel rod. A region representing the homogenization of the remainder of the fuel assembly surrounds the inner region in the Path B unit cell model. A final region representing the moderator in the assembly-to-assembly spacing surrounds the homogenized region in the Path $\mathrm{B}$ unit cell model. The size of each radial region surrounding the inner region in the Path $\mathrm{B}$ unit cell model is determined by conserving the fuel-to-moderator volume ratio in the system. The cell-weighted cross-sections from the Path A model are used with the fuel of the homogenized region during the Path B model transport calculations. New cell-weighted cross-sections for each energy group are then developed using the unit cell spatial flux distribution results from the Path B model transport calculations. These cell-weighted cross-sections are used in point depletion calculations performed by ORIGEN-S to calculate depleted fuel and depleted burnable poison, if present, isotopics in the fuel assembly. A detailed description of the calculations used to produce time-dependent cross-sections by SAS2H is documented in Section S2.2.4 of Volume 1, Rev. 5 in reference 5.4.

The Path B unit cell models for the various fuel assembly configurations must be developed manually and input to the SAS2H control module. The primary concern in the development of the Path B model for PWR assemblies is the conservation of the fuel-to-moderator volume ratio in the system. For the fuel assemblies in batches 1, 2, 3, and 1X of Crystal River Unit 3 a combination of the following sets of Path $B$ models must be utilized:

Set 1) This set is composed of one Path B model representing the base fuel assembly configuration with sixteen water-filled guide tubes and one water-filled instrument tube. This Path B model may only be employed in a statepoint depletion calculation which does not have any BPRA, CRA, or APSRA insertion history. 
Title: CRC Depletion Calculations for the Rodded Assemblies in Batches 1, 2, 3, and $1 \mathrm{X}$ of Crystal River Unit 3 Document Identifier: BBA000000-01717-0200-00040 REV 00

Page 70 of 146

Set 2) This set is composed of three Path B models that are utilized in statepoint depletion calculations that have a BPRA insertion history. One of the Path B models in this set represents a fuel assembly axial region containing sixteen BPRs inserted into the guide tubes with one water-filled instrument tube. Another Path B model in this set represents a fuel assembly axial region containing sixteen non-absorbing BPRs inserted into the guide tubes with one water-filled instrument tube. The last Path B model in this set represents a fuel assembly axial region with the BPRA removed. Since a constant number of Path B model radial zones must be maintained during a given SAS2H calculation (i.e., a statepoint depletion calculation), it is necessary to define a Path B model equivalent to that previously described in Set 1 , but with the same number of radial zones as those previously described in this set.

Set 3) This set is composed of two Path B models that are utilized in statepoint depletion calculations that have a CRA insertion history. One of the Path B models in this set represents a fuel assembly axial region containing sixteen CRs inserted into the guide tubes with one water-filled instrument tube. The other Path B model in this set represents a fuel assembly axial region with the CRA removed. Since a constant number of Path B model radial zones must be maintained during a given SAS2H calculation (i.e., a statepoint depletion calculation), it is necessary to define a Path B model equivalent to that previously described in Set 1 , but with the same number of radial zones as the first Path B model described in this set.

Set 4) This set is composed of three Path B models that are utilized in statepoint depletion calculations that have a APSRA insertion history. One of the Path B models in this set represents a fuel assembly axial region containing sixteen APSRs (absorbing region present in the guide tubes) with one water-filled instrument tube. Another Path B model in this set represents a fuel assembly axial region containing sixteen APSRs (only the follow rod region present in the guide tubes) with one water-filled instrument tube. The last Path $B$ model in this set represents a fuel assembly axial region with the APSRA removed. Since a constant number of Path $B$ model radial zones must be maintained during a given SAS2H calculation (i.e., a statepoint depletion calculation), it is necessary to define a Path B model equivalent to that previously described in Set 1 , but with the same number of radial zones as those previously described in this set.

The Path B model development spreadsheets in Tables 7.2-1 through 7.2-4, present the input parameters required, the parameters calculated, references to equations used to calculate the parameters, and the final Path B unit cell model dimensions available for direct implementation into SAS2H input decks for the rodded assembly depletion analyses of batches $1,2,3$, and 1X. The assembly specifications required to develop the Path $\mathrm{B}$ models are the same for batches $1,2,3$, and $1 \mathrm{X}$. The spreadsheet presented in Table 7.2-1, calculates the dimensions of the Path B unit cell model for Set 1 , as previously described. The spreadsheet presented in Table 7.2-2, calculates the dimensions of the Path B unit cell models for Set 2, as previously described. The spreadsheet presented in Table 7.2-3, calculates the dimensions of the Path B unit cell models for Set 3, as previously described. The spreadsheet presented in Table 7.2-4, calculates the dimensions of the Path B unit cell models for Set 4, as previously described. Table 7.2-5, 
Title: CRC Depletion Calculations for the Rodded Assemblies in Batches 1, 2, 3, and IX of Crystal River Unit 3 Document Identifler: BBA000000-01717-0200-00040 REV 00

Page 71 of 146

contains a listing of the equations referenced and utilized in each of the spreadsheets presented in Tables 7.2-1 and 7.2-4.

Table 7.2-1 Set 1 Path B Unit Cell Model Dimension Calculation Spreadsheet for the Rodded Assembly Axial Regions from Fuel Batches 1, 2, 3, and $1 X$ of Crystal River Unit 3

\author{
SAS2H Path B Unit Cell Model Dimension Calculations for the \\ Rodded Assembly Axial Regions in Fuel Batches 1,2,3, and $1 X$ of \\ Crystal River Unit 3 that Contain 16 Water-Filled Guide Tubes \\ and 1 Water-Filled Instrument Tube
}

Input Parameters

Number of unit cells in assembly: $\quad 225$

Number of fuel rods in assembly: 208

Number of guide tubes in assembly: 16

- Rod pitch in assembly $(\mathrm{cm}): \quad 1.44272$

Fuel pellet diameter $(\mathrm{cm}): \quad 0.9398$

Fuel cladding outer diameter $(\mathrm{cm}): \quad 1.0922$

Guide tube outer diameter $(\mathrm{cm}): \quad 1.3462$

Guide tube inner diameter $(\mathrm{cm}): \quad 1.26492$

Instrument tube outer diameter $(\mathrm{cm}): \quad 1.38193$

Instrument tube inner diameter $(\mathrm{cm}): \quad 1.12014$

Assembly pitch (cm): 21.81098

Fuel-to-Moderator Volume Ratio Calculation:

Identifier of Equation(s) Utilized: $\quad 1$ (Table 7.2-5)

Fuel-to-Moderator Volume Ratio $=\mathbf{0 . 5 3 3 8 6 4}$

Moderator Unit Volume in Central Unit Cell of Path B Model:

Identifier of Equation(s) Utilized: $\quad 2$ (Table 7.2-5)

Moderator Unit Volume in Central Unit Cell of Path B Model $=1.914755$

Fuel Unit Volume in Fuel Rod Unit Cell:

Identifier of Equation(s) Utilized: $\quad 3$ (Table 7.2-5)

Fuel Unit Volume in Fuel Rod Unit Cell $=0.693683$ 
Title: CRC Depletion Calculations for the Rodded Assemblies in Batches 1, 2, 3, and $1 \mathrm{X}$ of Crystal River Unit 3

Table 7.2-1 Set 1 Path B Unit Cell Model Dimension Calculation Spreadsheet for the Rodded Assembly Axial Regions from Fuel Batches 1, 2, 3, and 1X of Crystal River Unit 3

Moderator Unit Volume in Fuel Rod Unit Cell:

Identifier of Equation(s) Utilized: $\quad 4$ (Table 7.2-5)

Moderator Unit Volume in Fuel Rod Unit Cell $=1.144539$

Number of Fuel Rod Unit Cells that must be Represented

in the Homogenized Zone of the Path B Model:

Identifier of Equation(s) Utilized: $\quad 5$ (Table 7.2-5)

Number of Fuel Rod Unit Cells that must be Represented

in the Homogenized Zone of the Path B Model $=12.36742$

Path B Unit Cell Model Dimensions:

\begin{tabular}{|c|c|c|c|}
\hline & Zone \# & $\begin{array}{c}\text { Outer } \\
\text { Radius } \\
\text { (cm) }\end{array}$ & Zone Description \\
\hline \multirow[t]{4}{*}{ Inner } & 1 & 0.63246 & Water filled gap \\
\hline & 2 & 0.67310 & Guide tube \\
\hline & 3 & 0.81397 & Guide tube unit cell moderator \\
\hline & 4 & 2.97599 & Homogenized region \\
\hline Outer & 5 & 2.99939 & Moderator in the assembly-to-assembly gap \\
\hline
\end{tabular}

Notes: The Zone 4 outer radius is calculated using Equation 6 .

The Zone 5 outer radius is calculated using Equation 7. 
Title: CRC Depletion Calculations for the Rodded Assemblies in Batches 1, 2, 3, and $1 \mathrm{X}$ of Crystal River Unit 3 Document Identifier: BBA000000-01717-0200-00040 REV 00

Page 73 of 146

Table 7.2-2 Set 2 Path B Unit Cell Model's Dimension

Calculation Spreadsheet for the Rodded Assembly Axial

Regions from Fuel Batches 1, 2, 3, and 1X of Crystal River Unit 3

\section{SAS2H Path B Unit Cell Model Dimension Calculations for the Rodded}

Path B Models for Use in Assembly Axial Regions of Fuel Batches 1.2.3, and $1 X$ of Crystal

Biver Unit 3 that have a BPRA Insertion History

Input Parameters

Number of unit cells in assembly: $\quad 225$

Number of fuel rods in assembly: 208

Number of guide tubes in assembly: $\quad 16$

Number of BPR's in assembly: $\quad 16$

Rod pitch in assembly $(\mathrm{cm}): \quad 1.44272$

Fuel pellet diameter $(\mathrm{cm}): 0.9398$

Fuel cladding outer diameter $(\mathrm{cm}): \quad 1.0922$

Guide tube outer diameter $(\mathrm{cm}): \quad 1.3462$

Guide tube inner diameter $(\mathrm{cm}): \quad 1.26492$

BPR cladding outer diameter $(\mathrm{cm}): \quad 1.0922$

BPR cladding inner diameter $(\mathrm{cm}): \quad 0.9144$

BP pellet diameter $(\mathrm{cm}): \quad 0.8636$

Instrument tube outer diameter $(\mathrm{cm}): \quad 1.38193$

Instrument tube inner diameter $(\mathrm{cm}): \quad 1.12014$

Assembly pitch (cm): 21.81098

Fuel-to-Moderator Volume Ratio Calculation:

Identifier of Equation(s) Utilized: $\quad 1$ (Table 7.2-5)

Fuel-to-Moderator Volume Ratio $=0.565214$

Moderator Unit Volume in Central Unit Cell of Path B Model:

Identifier of Equation(s) Utilized: $\quad 2$ (Table 7.2-5)

Moderator Unit Volume in Central Unit Cell of Path B Model $=0.977852$

Fuel Unit Volume in Fuel Rod Unit Cell:

Identifier of Equation(s) Utilized: $\quad 3$ (Table 7.2-5)

Fuel Unit Volume in Fuel Rod Unit Cell $=0.693683$ 
Title: CRC Depletion Calculations for the Rodded Assemblies in Batches 1, 2, 3, and $1 \mathrm{X}$ of Crystal River Unit 3

Document Identifier: BBA000000-01717-0200-00040 REV 00

Page 74 of 146

Table 7.2-2 Set 2 Path B Unit Cell Model's Dimension

Calculation Spreadsheet for the Rodded Assembly Axial

Regions from Fuel Batches 1, 2, 3, and 1X of Crystal River Unit 3

Moderator Unit Volume in Fuel Rod Unit Cell:

Identifier of Equation(s) Utilized: $\quad 4$ (Table 7.2-5)

Moderator Unit Volume in Fuel Rod Unit Cell $=1.144539$

Number of Fuel Rod Unit Cells that must be Represented

in the Homogenized Zone of the Path B Model:

Identifier of Equation(s) Utilized: $\quad 5$ (Table 7.2-5)

Number of Fuel Rod Unit Cells that must be Represented in the Homogenized Zone of the Path B Model =

11.81651

Path B Unit Cell Model Dimensions:

\begin{tabular}{ccccc} 
Inner & $\begin{array}{c}\text { Outer } \\
\text { Radius } \\
(\mathbf{c m})\end{array}$ & $\begin{array}{c}\text { With Absorbing } \\
\text { BPRA Inserted }\end{array}$ & $\begin{array}{c}\text { Zone Descriptions } \\
\text { With Non-Absorbing } \\
\text { BPRA Inserted }\end{array}$ \\
\cline { 2 - 5 } & 1 & 0.43180 & Absorbing BP Material & Non-Absorbing BP Material \\
& 2 & 0.45720 & Helium Gap & Helium Gap \\
& 3 & 0.54610 & BPR cladding & BPR cladding \\
& 4 & 0.63246 & Water Filled Gap & Water Filled Gap \\
& 5 & 0.67310 & Guide tube & Guide tube \\
& 6 & 0.81397 & Unit cell moderator & Unit cell moderator \\
Outer & 7 & 2.91402 & Homogenized region & Homogenized region \\
& 8 & 2.93693 & Moderator Outside & Moderator Outside \\
& & & Assembly & Assembly
\end{tabular}

Notes: The Zone 7 outer radius is calculated using Equation 6.

The Zone 8 outer radius is calculated using Equation 7.

The Path B model that is used after the removal of the BPRA during a statepoint depletion calculation must use the same number of radial zones as the Path $B$ model with the BPRA inserted. One difference between the Path B model with the BPRA removed and the Path B model with the BPRA inserted is that the materials in zones 1 through 3 are changed to water. Another difference is that the outer radius of zones 7 and 8 are adjusted to match the homogenized region and outer water region dimensions of the - base Path B model (the Path B model with all empty guide tubes). Typically, a BPRA is not moved or removed during a reactor cycle. In this analysis there is no instance when a BPRA would need to be 
removed from an assembly axial node during a statepoint calculation. For this reason, the Path B model for the assembly node after removal of a BPRA during a statepoint calculation is not used in any of the assembly depletion calculations documented in this analysis.

Table 7.2-3 Set 3 Path B Unit Cell Model's Dimension Calculation Spreadsheet for the Rodded Assembly Axial Regions from Fuel Batches 1, 2, 3, and 1X of Crystal River Unit 3

Path B Models for Use in Assembly Axial Regions of Fuel Batches 1, 2,3, and 1X of Crystal River Unit 3 that have a CRA Insertion History

Input Parameters

Number of unit cells in assembly: $\quad 225$

Number of fuel rods in assembly: 208

Number of guide tubes in assembly: 16

Number of CR's in assembly: $\quad 16$

Rod pitch in assembly (cm): $\quad 1.44272$

Fuel pellet diameter $(\mathrm{cm}): \quad 0.9398$

Fuel cladding outer diameter $(\mathrm{cm}): \quad 1.0922$

Guide tube outer diameter $(\mathrm{cm}): \quad 1.3462$

Guide tube inner diameter $(\mathrm{cm}): \quad 1.26492$

CR cladding outer diameter $(\mathrm{cm}): \quad 1.1176$

CR cladding inner diameter $(\mathrm{cm}): \quad 1.01092$

CR absorber material diameter $(\mathrm{cm}): 0.99568$

Instrument tube outer diameter $(\mathrm{cm}): \quad 1.38193$

Instrument tube inner diameter $(\mathrm{cm}): \quad 1.12014$

Assembly pitch $(\mathrm{cm}): \quad 21.81098$

Fuel-to-Moderator Volume Ratio Calculation:

Identifier of Equation(s) Utilized: $\quad 1$ (Table 7.2-5)

Fuel-to-Moderator Volume Ratio $=0.56678$

Moderator Unit Volume in Central Unit Cell of Path B Model:

Identifier of Equation(s) Utilized: $\quad 2$ (Table 7.2-5)

Moderator Unit Volume in Central Unit Cell of Path B Model $=0.933769$

Fuel Unit Volume in Fuel Rod Unit Cell:

Identifier of Equation(s) Utilized: $\quad 3$ (Table 7.2-5) 
Title: CRC Depletion Calculations for the Rodded Assemblies in Batches 1, 2, 3, and $1 \mathrm{X}$ of Crystal River Unit 3 Document Identifier: BBA000000-01717-0200-00040 REV 00

Page 76 of 146

Table 7.2-3 Set 3 Path B Unit Cell Model's Dimension Calculation Spreadsheet for the Rodded Assembly Axial Regions from Fuel Batches 1, 2, 3, and 1X of Crystal River Unit 3

Fuel Unit Volume in Fuel Rod Unit Cell $=0.693683$

Moderator Unit Volume in Fuel Rod Unit Cell:

Identifier of Equation(s) Utilized: $\quad 4$ (Table 7.2-5)

Moderator Unit Volume in Fuel Rod Unit Cell $=1.144539$

Number of Fuel Rod Unit Cells that must be Represented

in the Homogenized Zone of the Path B Model:

Identifier of Equation(s) Utilized: $\quad 5$ (Table 7.2-5)

Number of Fuel Rod Unit Cells that must be Represented

in the Homogenized Zone of the Path B Model $=11.76595$

Path B Unit Cell Model Dimensions:

\begin{tabular}{|c|c|c|c|c|}
\hline & Zone \# & $\begin{array}{c}\text { Outer } \\
\text { Radius } \\
\text { (cm) }\end{array}$ & $\begin{array}{c}\text { With CRA Inserted } \\
\text { in Axial Region } \\
\end{array}$ & $\begin{array}{l}\text { With CRA Removed } \\
\text { from Axial Region }\end{array}$ \\
\hline \multirow[t]{7}{*}{ Inner } & 1 & 0.49784 & CR Absorber Material & Water \\
\hline & 2 & 0.50546 & Helium Gap & Water \\
\hline & 3 & 0.55880 & CR cladding & Water \\
\hline & 4 & 0.63246 & Water & Water \\
\hline & 5 & 0.67310 & Guide tube & Guide tube \\
\hline & 6 & 0.81397 & Unit cell moderator & Unit cell moderator \\
\hline & 7 & 2.90826 & Homogenized region & -- \\
\hline \multirow[t]{2}{*}{ Outer } & 8 & 2.93113 & $\begin{array}{l}\text { Moderator Outside } \\
\text { Assembly }\end{array}$ & - \\
\hline & 7 & 2.97599 & $\ldots$ & Homogenized region \\
\hline Outer & 8 & 2.99939 & - & $\begin{array}{l}\text { Moderator Outside } \\
\text { Assembly }\end{array}$ \\
\hline
\end{tabular}

Notes: The Zone 7 outer radius is calculated using Equation 6.

The Zone 8 outer radius is calculated using Equation 7.

The outer radius values for zones 7 and 8 with the control rod removed are calculated as shown in Table 7.2-1. 
Title: CRC Depletion Calculations for the Rodded Assemblies in Batches 1, 2, 3, and $1 \mathrm{X}$ of Crystal River Unit 3 Document Identifier: BBA000000-01717-0200-00040 REV 00

Table 7.2-4 Set 4 Path B Unit Cell Model's Dimension Calculation Spreadsheet for the Rodded Assembly Axial Regions from Fuel Batches 1, 2, 3, and 1X of Crystal River Unit 3

\section{Path B Models for Use in Assembly Axial Regions of Fuel Batches 1, 2,3, and $1 \mathrm{X}$ of Crystal} River Unit 3 that have a Black APSRA Insertion History

Input Parameters

Number of unit cells in assembly: $\quad 225$

Number of fuel rods in assembly: 208

Number of guide tubes in assembly: 16

Number of APSR's in assembly: $\quad 16$

Rod pitch in assembly $(\mathrm{cm}): \quad 1.44272$

Fuel pellet diameter $(\mathrm{cm}): \quad 0.9398$

Fuel cladding outer diameter $(\mathrm{cm}): \quad 1.0922$

Guide tube outer diameter $(\mathrm{cm}): \quad 1.3462$

Guide tube inner diameter $(\mathrm{cm}): \quad 1.26492$

APSR cladding outer diameter $(\mathrm{cm}): \quad 1.1176$

APSR cladding inner diameter $(\mathrm{cm}): \quad 1.01092$

APSR absorber material diameter $(\mathrm{cm}): 0.99568$

Instrument tube outer diameter $(\mathrm{cm}): \quad 1.38193$

Instrument tube inner diameter $(\mathrm{cm}): \quad 1.12014$

Assembly pitch $(\mathrm{cm}): \quad 21.81098$

The APSR follow rod has the same dimensions as the APSR cladding and is filled with water.

Fuel-to-Moderator Volume Ratio Calculation:

Identifier of Equation(s) Utilized: $\quad$ I (Table 7.2-5)

Fuel-to-Moderator Volume Ratio for the cross-section of the assembly containing the absorbing region of the APSRA $=0.56678$

Fuel-to-Moderator Volume Ratio for the cross-section of the assembly containing the follow rod region of the APSRA $=0.53956$

Moderator Unit Volume in Central Unit Cell of Path B Model:

Identifier of Equation(s) Utilized: $\quad 2$ (Table 7.2-5)

Moderator Unit Volume in the Central Unit Cell of the

Path B Model for the Inserted APSR Absorber Region $=0.933769$

Moderator Unit Volume in the Central Unit Cell of the

Path B Model for the Inserted APSR Follow Rod $=1.736414$ 
Table 7.2-4 Set 4 Path B Unit Cell Model's Dimension

Calculation Spreadsheet for the Rodded Assembly Axial Regions from Fuel Batches 1, 2, 3, and 1X of Crystal River Unit 3

Fuel Unit Volume in Fuel Rod Unit Cell:

$$
\text { Identifier of Equation(s) Utilized: } \quad 3 \text { (Table 7.2-5) }
$$

Fuel Unit Volume in Fuel Rod Unit Cell $=0.693683$

Moderator Unit Volume in Fuel Rod Unit Cell:

$$
\text { Identifier of Equation(s) Utilized: } \quad 4 \text { (Table 7.2-5) }
$$

Moderator Unit Volume in Fuel Rod Unit Cell $=1.144539$

Number of Fuel Rod Unit Cells that must be Represented

in the Homogenized Zone of the Path B Model:

$$
\text { Identifier of Equation(s) Utilized: } \quad 5 \text { (Table 7.2-5) }
$$

Number of Fuel Rod Unit Cells that must be Represented in the Homogenized Zone of the Path B Model with the APSR Absorber Region Inserted $=11.76595$

Number of Fuel Rod Unit Cells that must be Represented in the Homogenized

\begin{tabular}{|c|c|c|c|c|c|}
\hline & Zone \# & $\begin{array}{c}\text { Outer } \\
\text { Radius } \\
\text { (cm) }\end{array}$ & $\begin{array}{c}\text { With APSRA Inserted } \\
\text { in Axial Region } \\
\end{array}$ & $\begin{array}{l}\text { With APSRA Removed } \\
\text { from Axial Region }\end{array}$ & $\begin{array}{l}\text { With APSRA Follow Rod } \\
\text { Axial Region Inserted }\end{array}$ \\
\hline \multirow[t]{7}{*}{ Inner } & 1 & 0.49784 & APSR Absorber Material & Water & Water \\
\hline & 2 & 0.50546 & Helium Gap & Water & Water \\
\hline & 3 & 0.55880 & APSR cladding & Water & APSR cladding \\
\hline & 4 & 0.63246 & Water & Water & Water \\
\hline & 5 & 0.67310 & Guide tube & Guide tube & Guide tube \\
\hline & 6 & 0.81397 & Unit cell moderator & Unit cell moderator & Unit cell moderator \\
\hline & 7 & 2.90826 & Homogenized region & - & - \\
\hline \multirow[t]{2}{*}{ Outer } & 8 & 2.93113 & $\begin{array}{l}\text { Moderator Outside } \\
\text { Assembly }\end{array}$ & - & - \\
\hline & 7 & 2.97599 & - & Homogenized region & - \\
\hline Outer & 8 & 2.99939 & - & $\begin{array}{l}\text { Moderator Outside } \\
\text { Assembly }\end{array}$ & - \\
\hline
\end{tabular}

Zone of the Path B Model with the APSR Follow Rod Region Inserted $=12.30592$

Path B Unit Cell Model Dimensions: 
Title: CRC Depletion Calculations for the Rodded Assemblies in Batches 1, 2, 3, and 1X of Crystal River Unit 3 Document Identifier: BBA000000-01717-0200-00040 REV 00

Page 79 of 146

Table 7.2-4 Set 4 Path B Unit Cell Model's Dimension

Calculation Spreadsheet for the Rodded Assembly Axial

Regions from Fuel Batches 1, 2, 3, and 1X of Crystal River Unit 3

\begin{tabular}{|c|c|c|c|c|c|}
\hline & 7 & 2.96913 & -- & --- & Homogenized region \\
\hline Outer & 8 & 2.99248 & -- & $\ldots$ & $\begin{array}{l}\text { Moderator Outside } \\
\text { Assembly }\end{array}$ \\
\hline
\end{tabular}

Notes: The Zone 7 outer radius is calculated using Equation 6.

The Zone 8 outer radius is calculated using Equation 7.

The outer radius values for zones 7 and 8 with the APSR removed are calculated as shown in Table 7.2-1.

Table 7.2-5 Equations Utilized in the Path B Model Dimension Calculation Spreadsheets Presented in Tables 7.2-1 and 7.2-4

Equation 1 (Fuel-to-Moderator Volume Ratio in Actual Assembly):

$$
\begin{aligned}
& \frac{F}{M} \text { Ratio }=\frac{(\text { Number of Fuel Rods })\left(\frac{\pi}{4}\right)(\text { Fuel Pellet Diameter })^{2}}{\left\{(\text { Number of Fuel Rods })\left[\text { Rod Pitch }{ }^{2}-(\text { Clad OD })^{2}\left(\frac{\pi}{4}\right)\right]+\right.} \\
& (\text { Number of Empty GT's })\left[\text { Rod Pitch }-(G T O D)^{2}\left(\frac{\pi}{4}\right)+(G T I D)^{2}\left(\frac{\pi}{4}\right)\right]+
\end{aligned}
$$

(Number of Rodded $\left.G T^{\prime} s\right)\left[\right.$ Rod Pitch ${ }^{2}-(G T O D)^{2}\left(\frac{\pi}{4}\right)+(G T I D)^{2}\left(\frac{\pi}{4}\right)-(\text { Inserted Rod } O D)^{2}\left(\frac{\pi}{4}\right)+$

$$
\left.\left.(\text { APSR Follow Rod ID })^{2}\left(\frac{\pi}{4}\right)\right]+\left[\operatorname{Rod} \text { Pitch }^{2}-(I T O D)^{2}\left(\frac{\pi}{4}\right)+(I T I D)^{2}\left(\frac{\pi}{4}\right)\right]\right\}
$$

where GT means guide tube, IT means instrument tube, and ID means inner diameter, OD means outer diameter. This equation assumes that there is no instrument inserted in the instrument tube, and the instrument tube is filled with moderator. The APSR Follow Rod ID is only specified if the follow rod region of an APSRA is being represented in the Path B model.

Equation 2 (Central Unit Cell Moderator Volume):

$$
C U C M V=\text { Rod Pitch }{ }^{2}-(G T O D)^{2}\left(\frac{\pi}{4}\right)+(G T I D)^{2}\left(\frac{\pi}{4}\right)-(\text { Inserted Rod } O D)^{2}\left(\frac{\pi}{4}\right)
$$

Equation 3 (Fuel Volume in an Assembly Lattice Cell Containing a Fuel Rod):

$$
F V=(\text { Fuel Pellet } O D)^{2}\left(\frac{\pi}{4}\right)
$$


Title: CRC Depletion Calculations for the Rodded Assemblies in Batches 1, 2, 3, and $1 \mathrm{X}$ of Crystal River Unit 3 Document Identifier: BBA000000-01717-0200-00040 REV 00

Equation 4 (Moderator Volume in an Assembly Lattice Cell Containing a Fuel Rod):

$$
M V=\text { Rod Pitch }{ }^{2}-(\text { Fuel Clad } O D)^{2}\left(\frac{\pi}{4}\right)
$$

Base equation from which Equation 5 is derived:

$$
\frac{F}{M} \text { Ratio }=\frac{x(F V)}{C U C M V+x(M V)}
$$

where $\mathrm{x}$ is the number of assembly lattice cells containing fuel rods that must be represented in the Path $B$ homogenized region.

Equation 5:

$$
x=\frac{\left(\frac{F}{M} \text { Ratio }\right)(C U C M V)}{F V-\left(\frac{F}{M} \text { Ratio }\right)(M V)}
$$

Base equations from which Equations 6 and 7 are derived:

Area of Any Annular Region in the Path B Model = $\pi$ (Outer Radius of Annular Region ${ }^{2}$-Inner Radius of Annular Region ${ }^{2}$ )

$$
\text { Outer Radius of Annular Region }=\sqrt{\frac{\text { Area of Annular Region }}{\pi}+I R^{2}}
$$

where IR means the inner radius of the annular region.

Equation 6:

$$
\text { Path B Model Homogenized Region Outer Radius }=\sqrt{\frac{x(\text { Rod Pitch })^{2}}{\pi}+I R^{2}}
$$

Equation 7: 
Title: CRC Depletion Calculations for the Rodded Assemblies in Batches 1, 2, 3, and IX of Crystal River Unit 3 Document Identifier: BBA000000-01717-0200-00040 REV 00

Page 81 of 146

\author{
Assembly-to-Assembly Spacing Moderator Zone Outer Radius $=$ \\ $\left\{\frac{(x+1)}{\text { Number of Lattice Cells in Assembly }} *\right.$ \\ $\left[\right.$ Assembly Pitch ${ }^{2}-(\text { Rod Pitch })^{2}($ Number of Lattice Cells in Assembly $\left.\left.)\right]\left(\frac{1}{\pi}\right)+I R^{2}\right\}^{0.5}$.
}

\title{
7.3 Cycle Irradiation History Layouts for the Depletion of the Rodded Assemblies in Batches 1, 2, 3, and 1X of Crystal River Unit 3
}

The irradiation time step layouts for the statepoint depletion calculations performed with the SAS2H control module, as documented in this analysis, will vary depending on whether or not the analyzed assembly has a CRA or APSRA insertion history in the statepoint calculation of interest. This variation in irradiation time step layouts between statepoint calculations containing rod insertion histories occurs because the rod insertion histories vary between statepoint calculations for different assemblies. The rod insertion histories for an assembly must be modeled such that the appropriate axial nodes of the fuel assembly are depleted using the appropriate neutron flux and spectrum over the correct exposure duration. The presence of a CRA or APSRA will effect the isotopic inventory in a fuel assembly local axial region as a result of the hardened neutron spectrum. In general, a hardened neutron spectrum (a higher average energy for the neutron population) will be produced as a result of decreased neutron moderation and increased parasitic capture of thermal neutrons. A locally hardened neutron spectrum in a thermal reactor for a short period of time will result in a local decrease in reactivity due to the following:

1) a decrease in the thermal utilization factor (the ratio of thermal neutron absorptions in the fuel to total thermal neutron absorptions);

2) a decrease in the resonance escape probability (the fraction of fission neutrons that manage to slow down from fission to thermal energies without being absorbed).

The presence of a locally hardened neutron spectrum for longer periods of time will result in the buildup of Pu-239 through the parasitic capture of fast neutrons by U-238 with subsequent beta decay through $\mathrm{Np}-239$. Due to the lower depletion of U-235 through fission and the increase production of Pu-239 through parasitic capture by U-238, the fissile content and hence reactivity of the fuel will be greater upon transition back to a thermal neutron spectrum rather than if the fuel had experienced a continuous thermal neutron spectrum. Therefore, the use of BPRAs, CRAs and APSRAs in reactor operation is not only for power regulation, but also for fuel assembly burnup extension. The isotopic inventory may be quite different between fuel with and without an absorbing rod assembly insertion history. These isotopic inventory differences must be accounted for in the CRC depletion calculations to allow for correct prediction of core $\mathrm{k}_{\text {eff }}$ values in subsequent $\mathrm{CRC}$ reactivity evaluations.

In SAS2H, the duration of an irradiation interval may be separated into a number of time steps of variable length. Typically, an irradiation interval is a CRC statepoint depletion calculation interval, or the continuous irradiation time required to go from one $\mathrm{CRC}$ statepoint to another. To follow the CRA 
Title: CRC Depletion Calculations for the Rodded Assemblies in Batches 1, 2, 3, and $1 \mathrm{X}$ of Crystal River Unit 3 Document Identifier: BBA000000-01717-0200-00040 REV 00

Page 82 of 146

or APSRA insertion histories, detailed intra-cycle variable irradiation time steps are required. This is due to the fact that the CRs and APSRs are only present in a given axial node of an assembly for a given period of exposure during a statepoint depletion calculation. A user specified number of cross-section library updates are performed during each time step of an irradiation interval. The boron letdown curve of the reactor cycle may also be followed by specifying, at each irradiation step, a fraction of the soluble boron concentration defined in the base moderator material specification. This boron concentration is applied uniformly over the irradiation time step. The boron concentration fraction at the mid-point of each irradiation time step is specified in the SAS2H depletion calculations of this analysis to appropriately follow boron letdown curves. Considering the cross-section update frequency, the boron letdown data, and the absorber rod assembly insertion histories, the following three primary requirements apply to determining an appropriate reactor cycle irradiation layout for a rodded assembly.

1) The duration of each time step should be specified such that a maximum of 80 days of irradiation is not exceeded between cross-section updates. The SAS2H calculations in this analysis utilize one cross-section update per irradiation step. Therefore, the maximum duration of any time step in any reactor cycle irradiation layout of this analysis should not exceed 80 days. The 80 day limit is an arbitrary limit based on engineering judgement. The 80 day irradiation time step limit should assure that the isotopic concentrations of the system (primarily fuel and borated moderator) will not alter the neutron spectrum radically enough to cause a time step of the depletion calculation to be performed without the availability of cross-sections which have been properly weighted with an appropriate neutron spectrum and spatial flux.

2) Any radical perturbations in the boron letdown curve should be followed by defining irradiation time step durations such that the average boron concentration over each time step is representative of the actual boron letdown. Usually, the 80 day time step limit imposed for cross-section update frequency is adequate to properly follow a reactor cycle's boron letdown curve.

3) The duration of each time step should be specified such that the insertion of a CRA or APSRA in a given assembly axial node may be modeled for the correct exposure time in terms of EFPD. A more detailed description of the meaning of this statement is warranted. In SAS2H, there is an option to vary the Path B unit cell model between irradiation steps as long as the number of radial zones in the Path $B$ models of a given SAS2H calculation (i.e., statepoint depletion calculation) remain the same. Therefore, an assembly axial node represented in a given SAS2H statepoint depletion calculation that has a CRA or APSRA insertion history for a specified period of exposure (that is a fraction of the exposure covered by the statepoint depletion calculation) may be modeled appropriately by changing the Path $B$ model from one representing the insertion of a CRA or APSRA to one representing the removal of a CRA or APSRA at the appropriate time step (corresponding to the CRA or APSRA removal time).

All three of the requirements previously described must be correctly addressed in the SAS2H input decks developed for each axial node of an assembly in each statepoint depletion calculation. Assuring 
Title: CRC Depletion Calculations for the Rodded Assemblies in Batches 1, 2, 3, and IX of Crystal River Unit 3

that the cross-section update frequency and the boron letdown curves are properly modeled is usually a by-product of developing the irradiation layouts for the statepoint depletion calculations containing either CRA or APSRA insertion history. The irradiation time step layout for a given statepoint depletion calculation must be developed such that breakpoints exist between irradiation time steps that allow for the appropriate removal or insertion of a CRA or APSRA to obtain the correct integrated neutron spectrum exposure for each axial node of the assembly. It becomes obvious then that the complexity of the irradiation time step layout for a given statepoint calculation is proportional to the number of CRC axial nodes being modeled and the frequency of CRA or APSRA movement during the assembly depletion. The time steps developed to model CRA or APSRA insertion histories are also designed to encompass the cross-section update and boron letdown requirements. A program entitled "RLAYOUT" was written to automate the development of appropriate irradiation time step layouts for the statepoint depletion calculations of an assembly containing either a CRA or APSRA insertion history. The RLAYOUT program is described in Attachment III of this analysis.

The RLAYOUT program is only utilized to determine the irradiation time step layouts for the CRC statepoint depletion calculations that contain either a CRA or APSRA insertion history. A single assembly may have a combination of CRC statepoint calculations that either require or do not require the RLAYOUT developed ifradiation time step layouts. For the CRC statepoint depletion calculations that do not require the consideration of CRA or APSRA insertion histories, the irradiation time step layouts are developed by considering the cross-section update frequency and the boron letdown data. Tables 7.3-1 through 7.3-7 contain the CRC statepoint depletion calculation time step layouts for each reactor cycle that is relevant to statepoint calculations documented in this analysis which do not have either a CRA or APSRA insertion history. The mid-step boron concentrations presented in Tables 7.3-1 through 7.3-7 are obtained by using linear interpolation within the data presented in Tables 4.1.9-1 through 4.1.97. A description of the linear interpolation procedures employed are presented in the "UNITS_CONVERSION" subroutine description section of the CRAFT code description in Attachment I of this analysis.

The irradiation time step layouts developed with the RLAYOUT program for the assemblies documented in this analysis are presented in Tables 7.3-8 through 7.3-24. Tables 7.3-8 through 7.3-24 contain information required to prepare the irradiation layout portion and the CRA or APSRA insertion history portion of the CRAFT input decks for assemblies containing either a CRA or APSRA insertion history. The boron letdown data utilized by RLAYOUT in developing the irradiation layouts that are presented in Tables 7.3-8 through 7.3-24 is not exactly the same as that utilized in developing the irradiation history layouts for the non-rodded statepoint depletion calculations as presented in Tables 7.3-1 through 7.3-7. The boron letdown data provided to the RLAYOUT program is taken from the data presented in 4.1.9-1 through 4.1.9-7. However, some of the measured boron concentrations shown in Tables 4.1.9-1 through 4.1.9-7 were eliminated due to the fact that the particular boron concentration measurements in question were not made at nominal full-power core operation conditions. The use of the entire set of boron letdown data from Tables 4.1.9-1 through 4.1.9-7 in the non-rodded statepoint depletion calculation layouts has no adverse effect on the depletion calculation results. The modified boron letdown data from Tables 4.1.9-1 through 4.1.9-7 is presented in Tables 7.3-25 through 7.3-31. The acronym "ppmb" in the following tables means part per million of natural boron by mass of moderator. 
Title: CRC Depletion Calculations for the Rodded Assemblies in Batches 1, 2, 3, and $1 \mathrm{X}$ of Crystal River Unit 3 Document Identifier: BBA000000-01717-0200-00040 REV 00

Table 7.3-1 Crystal River Unit 3 Cycle-1A Irradiation History Layout for Non-Rodded Assemblies

\begin{tabular}{|c|c|c|}
\hline \multicolumn{3}{|c|}{$\begin{array}{l}\text { Cycle-1A } \\
\text { BOC to EOC (268.8 EFPD of Cycle-1) }\end{array}$} \\
\hline 67. & $\begin{array}{l}\text { Number of Irrad } \\
\text { : Length of Each }\end{array}$ & $\begin{array}{l}\text { ion Steps } \\
\text { adiation Step (EFPD) }\end{array}$ \\
\hline Step Number & Mid-Step ppmb & Mid-Step EFPD \\
\hline 1 & 921.02 & 33.60 \\
\hline 2 & 872.24 & 100.80 \\
\hline 3 & 738.29 & 168.00 \\
\hline 4 & 608.17 & 235.20 \\
\hline
\end{tabular}

Table 7.3-2 Crystal River Unit 3 Cycle-1B Irradiation History Layout for Non-Rodded Assemblies

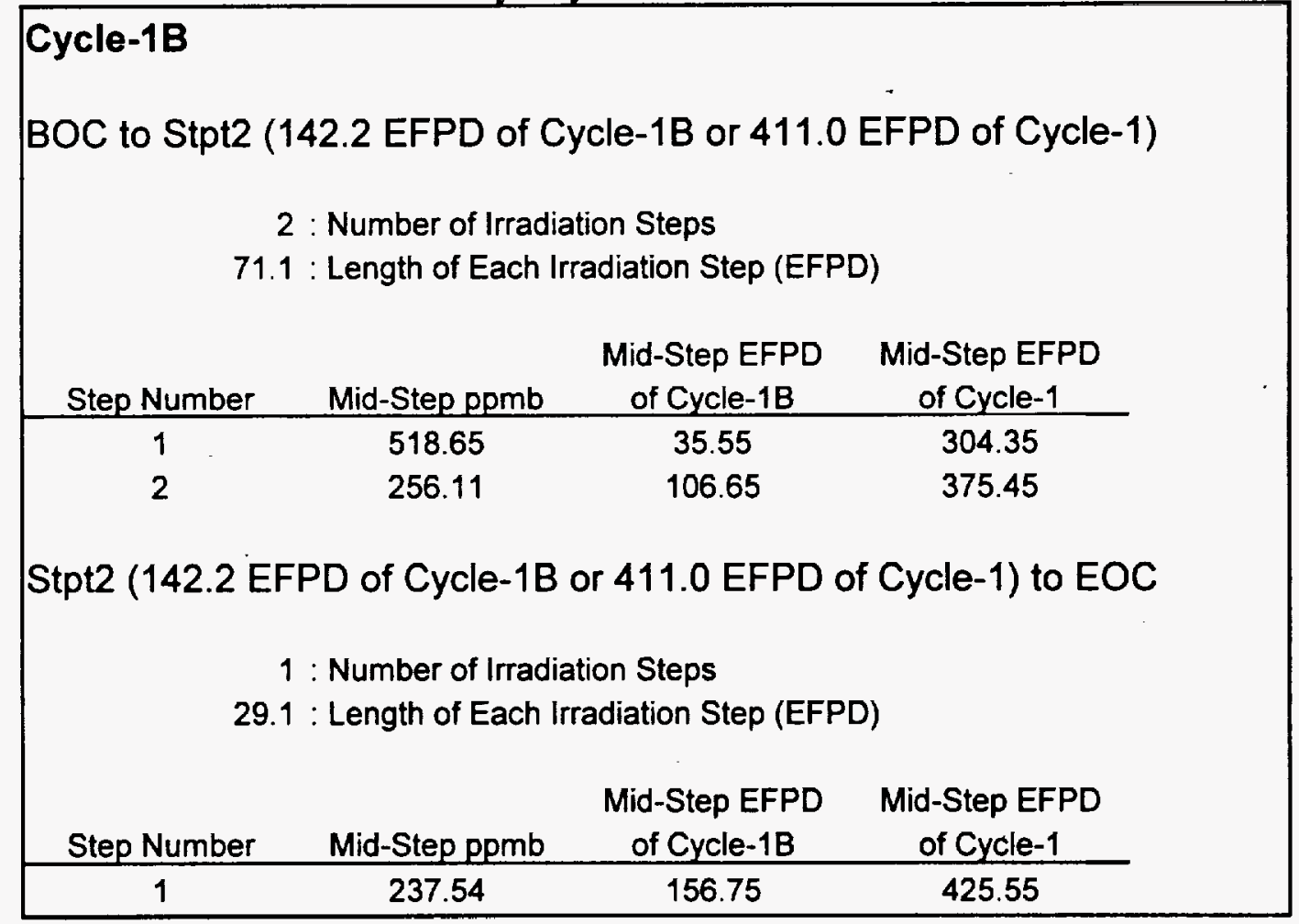


Title: CRC Depletion Calculations for the Rodded Assemblies in Batches 1, 2, 3, and $1 \mathrm{X}$ of Crystal River Unit 3

Table 7.3-3 Crystal River Unit 3 Cycle-2 Irradiation History Layout for Non-Rodded Assemblies Cycle-2
BOC to EOC

3 : Number of Irradiation Steps

55.5 : Length of Each Irradiation Step (EFPD)

Step Number Mid-Step ppmb Mid-Step EFPD

$\begin{array}{lll}1 & 688.93 & 27.75\end{array}$

$2 \quad 527.51 \quad 83.25$

$3 \quad 353.48 \quad 138.75$

Table 7.3-4 Crystal River Unit 3 Cycle-3

Irradiation History Layout for Non-Rodded Assemblies

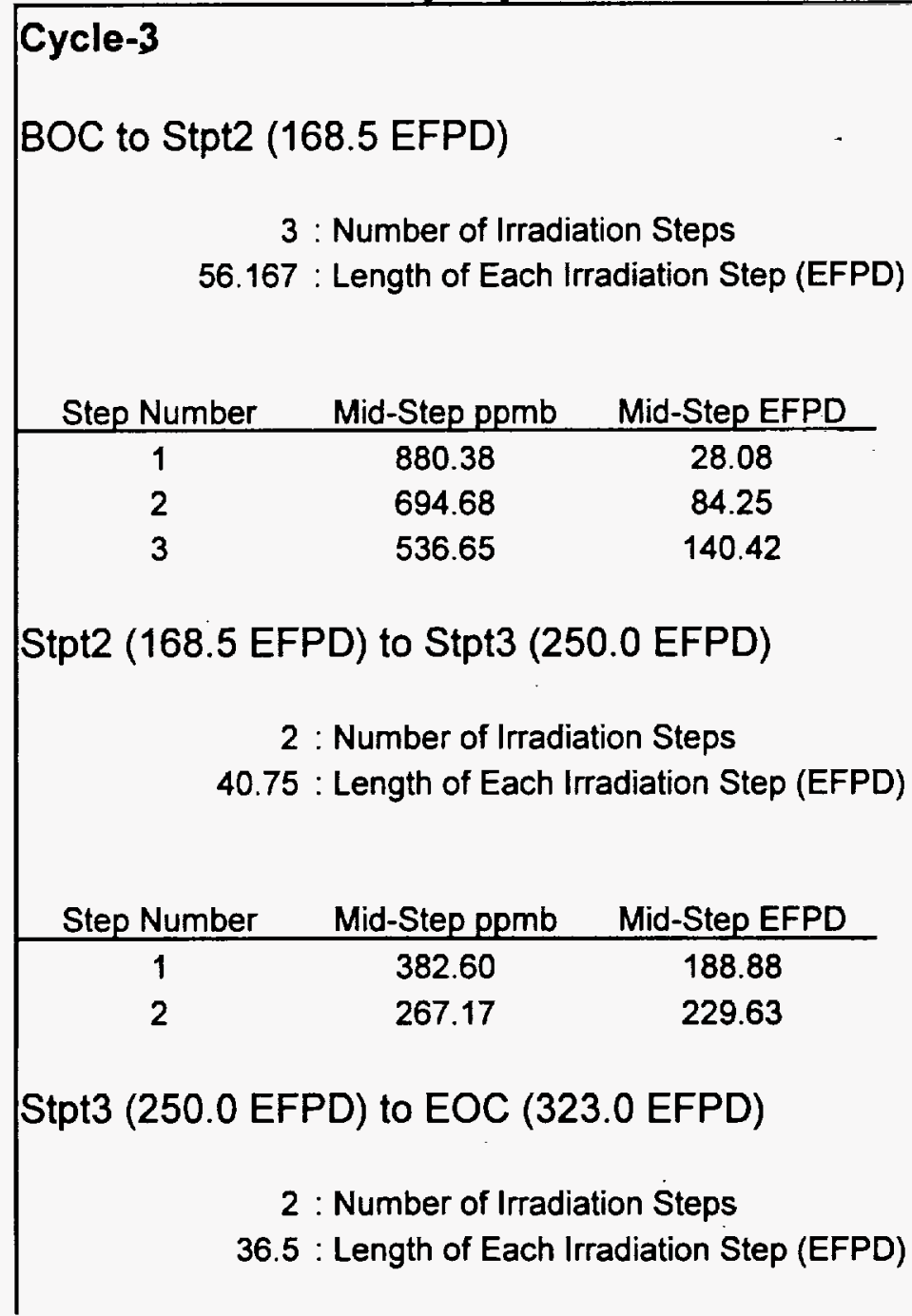




\begin{tabular}{|ccc|} 
& & \\
Step Number & Mid-Step ppmb & Mid-Step EFPD \\
\hline 1 & 234.64 & 268.25 \\
2 & 128.17 & 304.75 \\
\hline
\end{tabular}

Table 7.3-5 Crystal River Unit 3 Cycle-7 Irradiation History Layout for Non-Rodded Assemblies

\section{Cycle-7}

BOC to Stpt2 (260.3 EFPD)

4 : Number of Irradiation Steps

65.075 : Length of Each Irradiation Step (EFPD)

Step Number Mid-Step ppmb Mid-Step EFPD

$-1$ 1424.17 32.54 1300.75

97.61

3

1159.96

162.69

4

971.50

227.76

Stpt2 (260.3 EFPD) to Stpt3 (291.0 EFPD)

1 : Number of Irradiation Steps

30.7 : Length of Each Irradiation Step (EFPD)

Step Number Mid-Step ppmb Mid-Step EFPD

1 856.06 275.65

Stpt3 (291.0 EFPD) to Stpt4 (319.0 EFPD)

1 : Number of Irradiation Steps

28 : Length of Each Irradiation Step (EFPD)

Step Number Mid-Step ppmb Mid-Step EFPD

1 805.91 305.00

Stpt4 (319.0 EFPD) to Stpt5 (462.3 EFPD)

2 : Number of Irradiation Steps

71.65 : Length of Each Irradiation Step (EFPD) 


\begin{tabular}{|c|c|c|}
\hline Step Number & Mid-Step ppmb & Mid-Step EFPD \\
\hline 1 & 565.35 & 354.83 \\
\hline 2 & 356.75 & 426.48 \\
\hline \multicolumn{3}{|c|}{ Stpt5 (462.3 EFPD) to Stpt6 (479.0 EFPD) } \\
\hline \multicolumn{3}{|c|}{$1:$ Number of Irradiation Steps } \\
\hline Step Number & Mid-Step ppmb & Mid-Step EFPD \\
\hline 1 & 231.02 & 470.65 \\
\hline \multicolumn{3}{|c|}{ Stpt6 (479.0 EFPD) to EOC (497.9 EFPD) } \\
\hline- & $\begin{array}{l}\text { Number of Irradi } \\
\text { Length of Each }\end{array}$ & $\begin{array}{l}\text { ion Steps } \\
\text { adiation Step (EFPD) }\end{array}$ \\
\hline & & - \\
\hline Step Number & Mid-Step ppmb & Mid-Step EFPD \\
\hline 1 & 183.83 & 488.45 \\
\hline
\end{tabular}

Table 7.3-6 Crystal River Unit 3 Cycle-8 Irradiation History Layout for Non-Rodded Assemblies

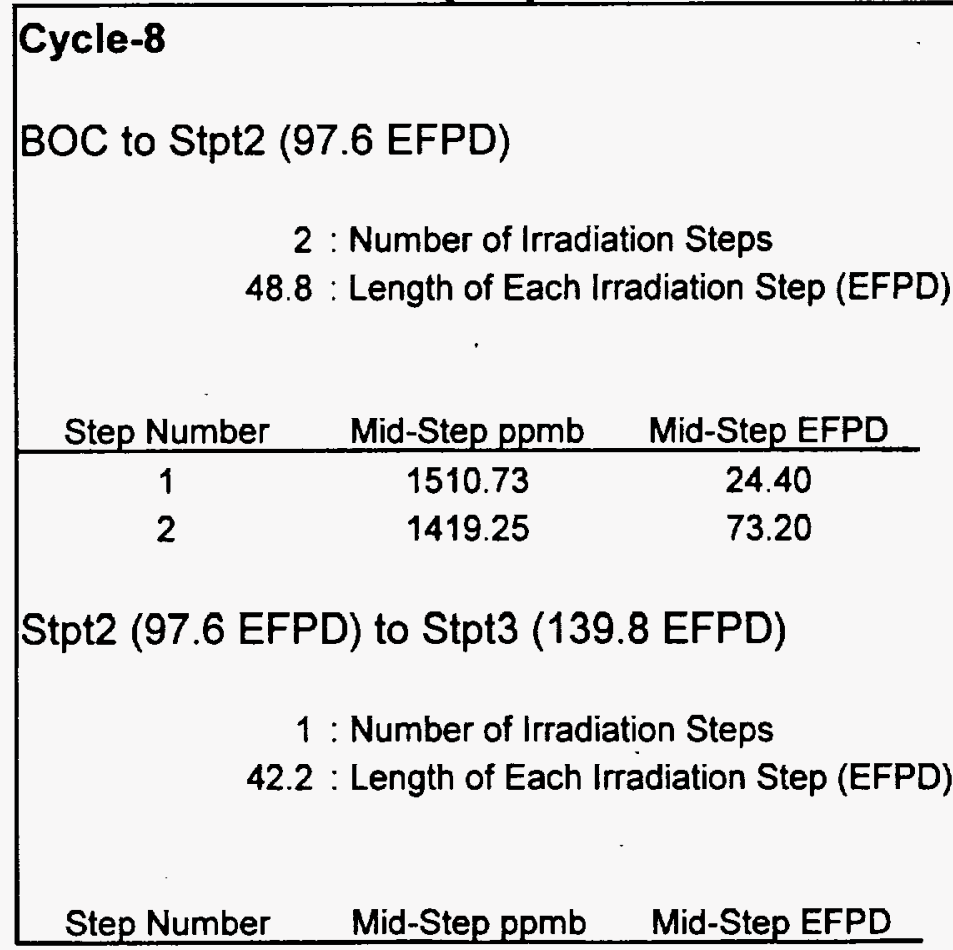




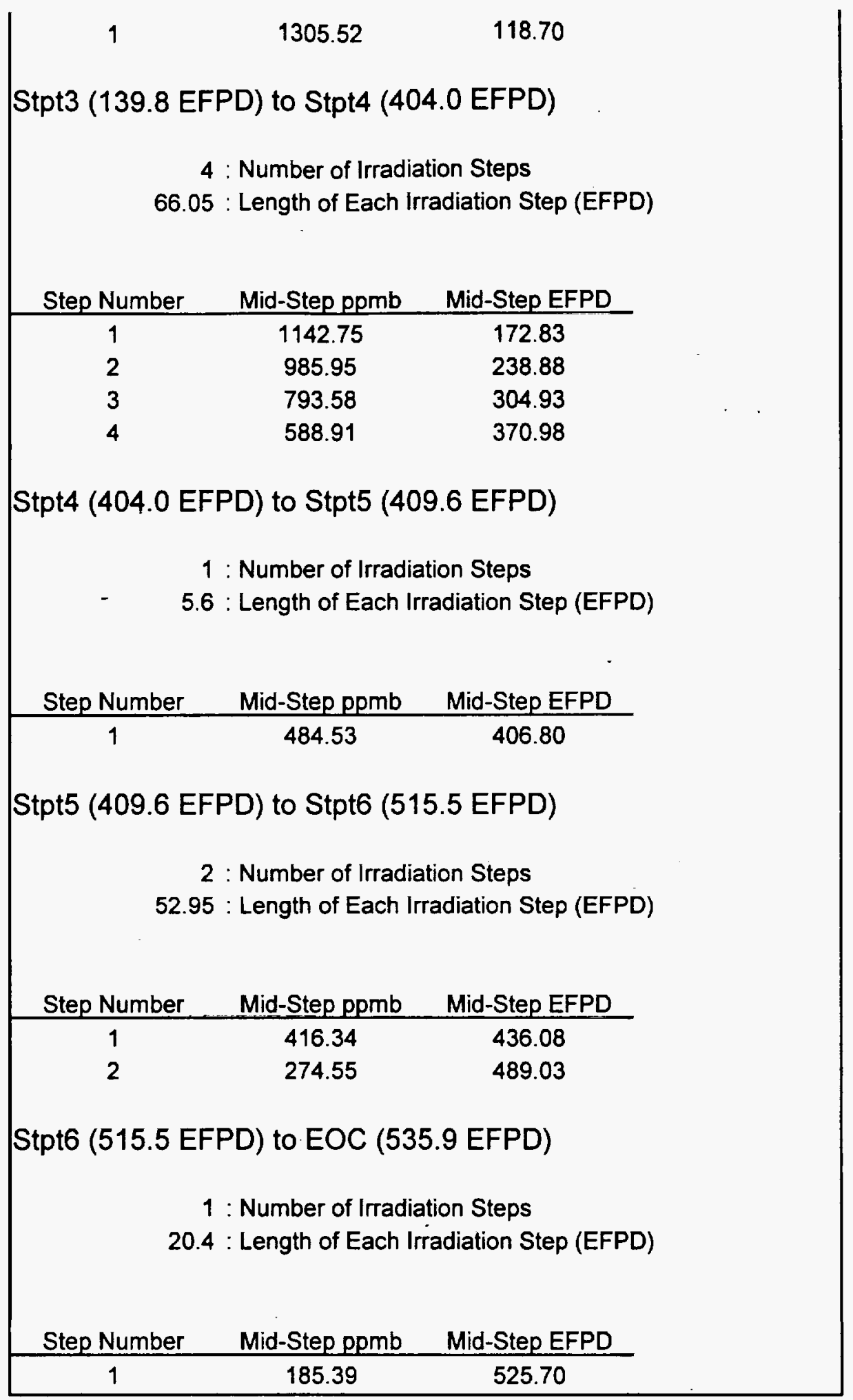


Title: CRC Depletion Calculations for the Rodded Assemblies in Batches 1, 2, 3, and IX of Crystal River Unit 3

Table 7.3-7 Crystal River Unit 3 Cycle-9

Irradiation History Layout for Non-Rodded Assemblies

Cycle-9

BOC to Stpt2 (158.8 EFPD)

3 : Number of Irradiation Steps

52.93 : Length of Each Irradiation Step (EFPD)

Step Number Mid-Step ppmb Mid-Step EFPD

$1 \quad 26.47$

$\begin{array}{lll}1 & 1491.21 & 79.40\end{array}$

$\begin{array}{lll}3 & 1361.78 & 132.33\end{array}$

Stpt2 (158.8 EFPD) to Stpt3 (219.0 EFPD)

1 : Number of Irradiation Steps

60.2 : Length of Each Irradiation Step (EFPD)

Step Number Mid-Step ppmb Mid-Step EFPD

$\begin{array}{lll}1 & 1211.60 & 188.90\end{array}$

Stpt3 (219.0 EFPD) to Stpt4 (363.1 EFPD)

2 : Number of Irradiation Steps

72.05 : Length of Each Irradiation Step (EFPD)

Step Number Mid-Step ppmb Mid-Step EFPD

$1 \quad 1016.51 \quad 255.03$

$\begin{array}{lll}2 & 802.70 & 327.08\end{array}$

Stpt4 (363.1 EFPD) to EOC (557.23 EFPD)

3 : Number of Irradiation Steps

64.71 : Length of Each Irradiation Step (EFPD)

Step Number Mid-Step ppmb Mid-Step EFPD

$1 \quad 584.95 \quad 395.46$

$2 \quad 388.60 \quad 460.17$

\begin{tabular}{l}
382.60 \\
\hline
\end{tabular} 
Title: CRC Depletion Calculations for the Rodded Assemblies in Batches 1, 2, 3, and $1 \mathrm{X}$ of Crystal River Unit 3 Document Identifler: BBA000000-01717-0200-00040 REV 00

Page 90 of 146

Table 7.3-8 Rodded Irradiation Time Step Layout for Assembly A1

IRRADIATION LAYOUT FOR ASSEMBLY: A01

Cycle-1A, .0 EFPD to Cycle-1A, 268.8 EFPD Statepoint Calculation

\begin{tabular}{cccc}
$\begin{array}{c}\text { Irradiation } \\
\text { Step Number }\end{array}$ & $\begin{array}{c}\text { Step } \\
\text { Duation } \\
\text { (EFPD) }\end{array}$ & $\begin{array}{c}\text { Exposure at } \\
\text { End of Step } \\
\text { (EFPD) }\end{array}$ & $\begin{array}{l}\text { Mid-Step-Boron } \\
\text { Concentration } \\
\text { (ppm) }\end{array}$ \\
\hline 1 & 41.67 & 41.67 & 913.3 \\
2 & 68.68 & 110.35 & 902.9 \\
3 & 68.68 & 179.03 & 784.8 \\
4 & 43.05 & 222.08 & 663.5 \\
5 & 43.05 & 265.13 & 575.6 \\
6 & 3.87 & 269.00 & 505.6
\end{tabular}

Cycle-1B, $\quad .0$ EFPD to Cycle-1B, 142.2 EFPD Statepoint Calculation

\begin{tabular}{cccc}
$\begin{array}{c}\text { Irradiation } \\
\text { Step Number }\end{array}$ & $\begin{array}{c}\text { Exposure at } \\
\text { Nuration } \\
\text { (EFPD) }\end{array}$ & $\begin{array}{c}\text { End of Step } \\
\text { (EFPD) }\end{array}$ & $\begin{array}{c}\text { Mid-Step Boron } \\
\text { Concentration } \\
\text { (ppm) }\end{array}$ \\
\hline 1 & 4.67 & 4.67 & 541.2 \\
2 & 25.50 & 30.17 & 569.2 \\
3 & 43.28 & 73.46 & 434.2 \\
4 & 43.28 & 116.74 & 309.6 \\
5 & 9.26 & 126.00 & 279.6 \\
6 & 1.00 & 127.00 & 284.2 \\
7 & 1.00 & 128.00 & 285.1 \\
8 & 6.91 & 134.91 & 288.7 \\
9 & 7.09 & 142.00 & 284.3 \\
10 & .20 & 142.20 & 280.6
\end{tabular}

Cycle-1B, 142.2 EFPD to Cycle-1B, 171.3 EFPD Statepoint Calculation

\begin{tabular}{cccc}
$\begin{array}{c}\text { Irradiation } \\
\text { Step Number }\end{array}$ & $\begin{array}{c}\text { Suration } \\
\text { (EFPD) }\end{array}$ & $\begin{array}{c}\text { Exposure at } \\
\text { End of Step }\end{array}$ & $\begin{array}{l}\text { Mid-Step Boron } \\
\text { Concentration } \\
\text { (EFPD) }\end{array}$ \\
\hline 1 & 6.57 & 6.57 & 277.1 \\
2 & 5.45 & 12.02 & 266.5 \\
3 & 16.98 & 29.00 & 204.7 \\
4 & .10 & 29.10 & 157.6
\end{tabular}

NODAL ROD ASSEMBLY INSERTION LAYOUT FOR FUEL ASSEMBLY: AOI

COLUMN A: Cycle-IA, .0 EFPD to Cycle-1A, 268.8 EFPD statepoint Calculation COLUMN B: Cycle-1B, .0 EFPD to Cycle-1B, 142.2 EFPD Statepoint Calculation COLUMN C: Cycle-1B, 142.2 EFPD to Cycle-1B, 171.3 EFPD Statepoint Calculation

$\mathrm{X}=$ Rod assembly inserted in corresponding node during the irradiation step

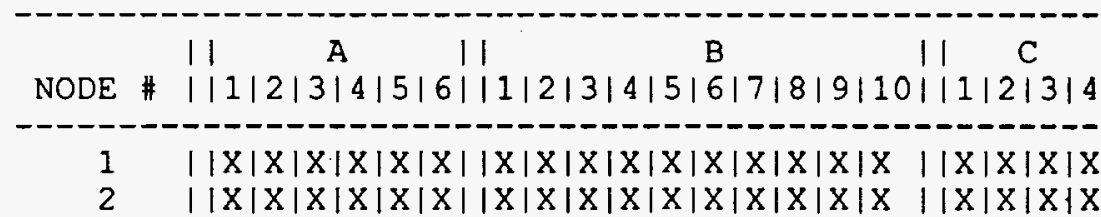


Title: CRC Depletion Calculations for the Rodded Assemblies in Batches 1, 2, 3, and 1X of Crystal River Unit 3 Document Identifier: BBA000000-01717-0200-00040 REV 00

Page 91 of 146

\begin{tabular}{|c|c|c|}
\hline 3 & ||$X|X| X|X| X|X||X| X|X| X|X| X|X| X|X| X$ & ||$X|X| X \mid$ \\
\hline 4 & ||$X|X| X|X| X|X||X| X|X| X|X| X|X| X||$ & ||$x|x| \mid$ \\
\hline 5 & ||$x|x| x|x| x|x||x| x|x| x|x| x|x| i \mid$ & ||$x||$ \\
\hline 6 & ||$X|X| X|X| X|X||X| X|X| X|X| X|| \mid 1$ & 1111 \\
\hline 7 & ||$X|X| X|X| X|X||X| X|X| X|X| \quad|\quad| 1$ & 1111 \\
\hline 8 & ||$X|X| X|X| X|X||X| X|X| X|X| 1111$ & 1111 \\
\hline$y$ & 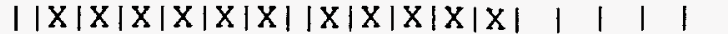 & 1111 \\
\hline & ||$X|X| X|X| X|X||X| X|X| X|X| 1|1|$ & 1111 \\
\hline & ||$X|X| X|X| X|X||X| X|X| X|X| 1|1|$ & 1111 \\
\hline & ||$X|X| X|X| X|X||X| X|X| X|X| 1$ & 1111 \\
\hline & ||$X|X| X|X| X|X||X| X|X| X|X| X|| 1 \mid$ & 1111 \\
\hline & ||$X|X| X|X| X|||X| X|X| X|X| X|X| 11$ & II I I \\
\hline & ||$X|X| X|\quad| \quad|||X| X|X| X|\quad| \quad \mid$ & 1111 \\
\hline & $\mid \begin{array}{llllllllllll}|X| & \mid & \mid & \mid & \mid & |X| X \mid & |X| & 1 & 1 & 1 & 1\end{array}$ & 1111 \\
\hline & 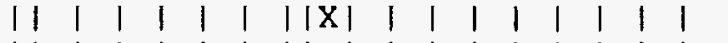 & 1111 \\
\hline & 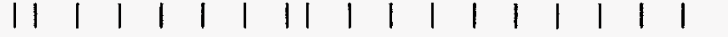 & 1111 \\
\hline
\end{tabular}

\section{Table 7.3-9 Rodded Irradiation Time Step Layout for Assembly A5}

IRRADIATION LAYOUT FOR ASSEMBLY: A05

Cycle-1A, .0 EEPD to Cycle-1A, 268.8 EFPD Statepoint Calculation

\begin{tabular}{cccc} 
Irradiation & $\begin{array}{c}\text { Step } \\
\text { Duration } \\
\text { (EFPD) }\end{array}$ & $\begin{array}{c}\text { Exposure at } \\
\text { End of Step }\end{array}$ & $\begin{array}{l}\text { Mid-Step Boron } \\
\text { Concentration } \\
\text { (ppm) }\end{array}$ \\
\hline 1 & 5.03 & (EFPD) & 902.3 \\
2 & 59.89 & 5.03 & 921.8 \\
3 & 59.89 & 124.80 & 884.0 \\
4 & 57.49 & 182.29 & 767.1 \\
5 & 57.49 & 239.78 & 638.7 \\
6 & 29.02 & 268.80 & 590.7
\end{tabular}

Cycle-1B, $\quad .0$ EFPD to Cycle-1B, 142.2 EFPD Statepoint Calculation

\begin{tabular}{cccc}
$\begin{array}{c}\text { Irradiation } \\
\text { Step Number }\end{array}$ & $\begin{array}{c}\text { Exposure at } \\
\text { Dution } \\
\text { (EFPD) }\end{array}$ & $\begin{array}{c}\text { End of Step } \\
\text { (EFPD) }\end{array}$ & $\begin{array}{l}\text { Mid-Step Boron } \\
\text { Concentration } \\
\text { (ppm) }\end{array}$ \\
\hline 1 & 4.51 & 4.51 & 541.0 \\
2 & 42.03 & 46.54 & 549.3 \\
3 & 35.53 & 82.07 & 422.8 \\
4 & 35.53 & 117.60 & 288.1 \\
5 & 7.92 & 125.52 & 279.8 \\
6 & 16.68 & 142.20 & 289.0
\end{tabular}

NODAL ROD ASSEMBLY INSERTION LAYOUT FOR FUEL ASSEMBLY: A05

COLUMN A: Cycle-1A, .0 EFPD to Cycle-1A, 268.8 EFPD Statepoint Calculation COLUMN B: Cycle-1B, .0 EFPD to Cycle-1B, 142.2 EFPD Statepoint Calculation

$\mathrm{X}=$ Rod assembly inserted in corresponding node during the irradiation step

\begin{tabular}{c|l|l|l|l|}
$|1|$ & A
\end{tabular}

NODE \# ||1|2|3|4|5|6||1|2|3|4|5|6

$1 \quad|X| X|X| X|X||X| X|X| X|X|$ 
Table 7.3-10 Rodded Irradiation Time Step Layout for Assembly A7

IRRADIATION LAYOUT FOR ASSEMBLY: A07

Cycle-1A, .0 EFPD to Cycle-1A, 268.8 EFPD Statepoint Calculation

\begin{tabular}{cccc}
$\begin{array}{c}\text { Irradiation } \\
\text { Step Number }\end{array}$ & $\begin{array}{c}\text { Step } \\
\text { Duration } \\
\text { (EFPD) }\end{array}$ & $\begin{array}{c}\text { Exposure at } \\
\text { End of Step } \\
\text { (EFPD) }\end{array}$ & $\begin{array}{c}\text { Mid-Step Boron } \\
\text { Concentration } \\
\text { (ppm) }\end{array}$ \\
\hline 1 & 2.50 & 2.50 & 901.6 \\
2 & 6.50 & 9.00 & 904.3 \\
3 & 1.00 & 10.00 & 906.5 \\
4 & 1.00 & 11.00 & 907.1 \\
5 & 1.00 & 12.00 & 907.7 \\
6 & .97 & 12.97 & 908.3 \\
7 & 1.04 & 14.01 & 908.9 \\
8 & 63.70 & 77.71 & 928.4 \\
9 & 63.70 & 141.40 & 854.8 \\
10 & 63.70 & 205.10 & 727.8 \\
11 & 63.70 & 268.80 & 593.1
\end{tabular}

Cycle-1B, .0 EFPD to Cycle-1B, 142.2 EFPD Statepoint Calculation

\begin{tabular}{cccc}
$\begin{array}{c}\text { Irradiation } \\
\text { Step Number }\end{array}$ & $\begin{array}{c}\text { Step } \\
\text { Duration } \\
\text { (EFPD) }\end{array}$ & $\begin{array}{c}\text { Exposure at } \\
\text { End of Step } \\
\text { (EFPD) }\end{array}$ & $\begin{array}{l}\text { Mid-Step Boron } \\
\text { Concentration } \\
\text { (ppm) }\end{array}$ \\
\hline 1 & 4.46 & 4.46 & 541.0 \\
2 & 24.44 & 28.90 & 567.8 \\
3 & 42.89 & 71.79 & 435.6 \\
4 & 42.89 & 114.68 & 313.3 \\
5 & 9.32 & 124.00 & 275.2 \\
6 & 1.00 & 125.00 & 282.4 \\
7 & 1.31 & 126.31 & 283.5 \\
8 & 7.90 & 134.21 & 287.6 \\
9 & 7.79 & 142.00 & 284.7 \\
10 & .20 & 142.20 & 280.6
\end{tabular}

Cycle-1B, 142.2 EFPD to Cycle-1B, 171.3 EFPD Statepoint Calculation 
Title: CRC Depletion Calculations for the Rodded Assemblies in Batches 1, 2, 3, and 1X of Crystal River Unit 3 Document Identifier: BBA000000-01717-0200-00040 REV 00

Page 93 of 146

\begin{tabular}{cccc}
$\begin{array}{c}\text { Irradiation } \\
\text { Step Number }\end{array}$ & $\begin{array}{c}\text { Step } \\
\text { Duration } \\
\text { (EFPD) }\end{array}$ & $\begin{array}{c}\text { Exposure at } \\
\text { End of Step } \\
\text { (EFPD) }\end{array}$ & $\begin{array}{c}\text { Mid-Step Boron } \\
\text { Concentration } \\
\text { (ppm) }\end{array}$ \\
\hline 1 & 6.47 & 6.47 & 277.2 \\
2 & 5.36 & 11.83 & 267.3 \\
3 & 17.17 & 29.00 & 205.2 \\
4 & .10 & 29.10 & 157.6
\end{tabular}

Cycle-02, .0 EFPD to Cycle-02, 166.5 EFPD Statepoint Calculation

\begin{tabular}{cccc}
$\begin{array}{c}\text { Step } \\
\text { Step Number }\end{array}$ & $\begin{array}{c}\text { Exposure at } \\
\text { Duration } \\
\text { (EFPD) }\end{array}$ & $\begin{array}{c}\text { End of Step } \\
\text { (EFPD) }\end{array}$ & $\begin{array}{l}\text { Mid-Step Boron } \\
\text { Concentration } \\
\text { (ppm) }\end{array}$ \\
\hline 1 & 3.21 & 3.21 & 839.9 \\
2 & 5.24 & 8.45 & 784.3 \\
3 & 21.72 & 30.17 & 721.4 \\
4 & 47.60 & 77.78 & 618.1 \\
5 & 47.60 & 125.38 & 470.7 \\
6 & 35.87 & 161.25 & 340.9 \\
7 & 5.25 & 166.50 & 314.0
\end{tabular}

NODAL ROD ASSEMBLY INSERTION LAYOUT FOR FUEL ASSEMBLY: A07

COLUMN A: Cycle-1A, .0 EFPD to Cycle-1A, 268.8 EFPD Statepoint Calculation COLUMN B: Cycle-1B, .0 EFPD to Cycle-1B, 142.2 EFPD Statepoint Calculation COLUMN C: Cycle-1B, 142.2 EFPD to Cycle-1B, 171.3 EFPD Statepoint Calculation COLUMN D: Cycle-02, .0 EFPD to Cycle-02, 166.5 EFPD Statepoint Calculation

$\mathrm{x}=$ Rod assembly inserted in corresponding node during the irradiation step

11

A

11

B

$11 \quad \mathrm{C} \quad \mid 1$

D

NODE \# ||1|2|3|4|5|6|7|8|9|10|11||1|2|3|4|5|6|7|8|9|10||1|2|3|4||1|2|3|4|5|6|7

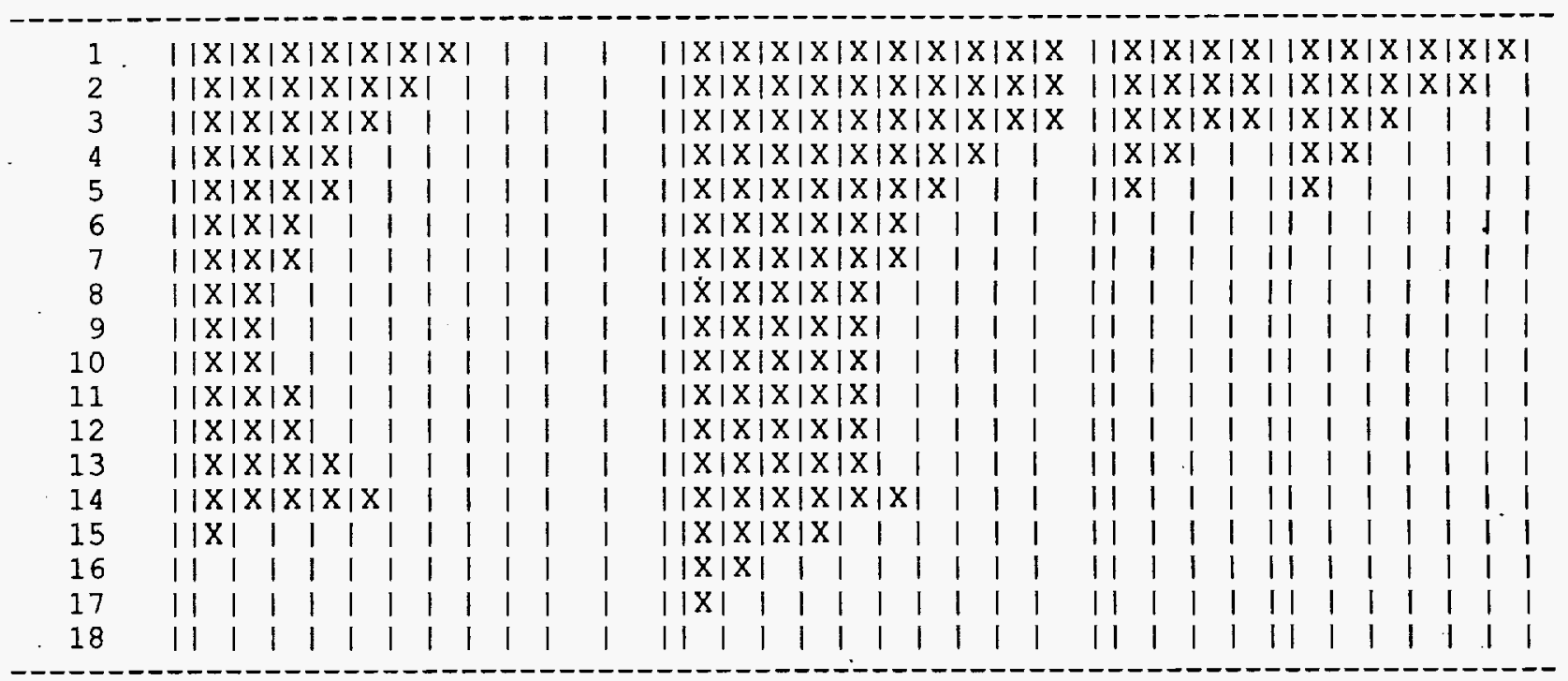


Title: CRC Depletion Calculations for the Rodded Assemblies in Batches 1, 2, 3, and $1 \mathrm{X}$ of Crystal River Unit 3 Document Identifier: BBA000000-01717-0200-00040 REV 00

Page 94 of 146

\section{Table 7.3-11 Rodded Irradiation Time Step Layout for Assembly A14}

IRRADIATION LAYOUT FOR ASSEMBLY: A14

Cycle-07, .0 EFPD to Cycle-07, 260.3 EFPD Statepoint Calculation

\begin{tabular}{cccc}
$\begin{array}{c}\text { Step } \\
\text { Step Number }\end{array}$ & $\begin{array}{c}\text { Duration } \\
\text { (EFPD) }\end{array}$ & $\begin{array}{c}\text { Exposure at } \\
\text { End of Step } \\
\text { (EFPD) }\end{array}$ & $\begin{array}{l}\text { Mid-Step Boron } \\
\text { Concentration } \\
\text { (ppm) }\end{array}$ \\
\hline 1 & 35.38 & 35.38 & 1456.1 \\
2 & 55.44 & 90.82 & 1362.5 \\
3 & 55.44 & 146.27 & 1253.7 \\
4 & 57.01 & 203.28 & 1121.3 \\
5 & 57.01 & 260.30 & 960.1
\end{tabular}

Cycle-07, 260.3 EFPD to Cycle-07, 291.0 EFPD Statepoint Calculation

\begin{tabular}{cccc}
$\begin{array}{c}\text { Irradiation } \\
\text { Step Number }\end{array}$ & $\begin{array}{c}\text { Step } \\
\text { Duration } \\
\text { (EFPD) }\end{array}$ & $\begin{array}{c}\text { Exposure at } \\
\text { End of Step } \\
\text { (EFPD) }\end{array}$ & $\begin{array}{c}\text { Mid-Step Boron } \\
\text { Concentration } \\
\text { (ppm) }\end{array}$ \\
\hline 1 & 10.13 & 10.13 & 852.1 \\
12 & 20.57 & 30.70 & 802.3
\end{tabular}

Cycle-07, 291.0 EFPD to Cycle-07, 319.0 EFPD Statepoint Calculation

\begin{tabular}{cccc}
$\begin{array}{c}\text { Irradiation } \\
\text { Step Number }\end{array}$ & $\begin{array}{c}\text { Step } \\
\text { Duration } \\
\text { (EFPD) }\end{array}$ & $\begin{array}{c}\text { Exposure at } \\
\text { End of Step } \\
\text { (EFPD) }\end{array}$ & $\begin{array}{l}\text { Mid-Step Boron } \\
\text { Concentration } \\
\text { (ppm) }\end{array}$ \\
\hline 1 & 5.32 & 5.32 & 760.4 \\
\hline 2 & 22.68 & 28.00 & 715.0
\end{tabular}

Cycle-07, 319.0 EFPD to Cycle-07, 462.3 EFPD Statepoint Calculation

\begin{tabular}{cccc}
$\begin{array}{c}\text { Irradiation } \\
\text { Step Number }\end{array}$ & $\begin{array}{c}\text { Step } \\
\text { Duration } \\
\text { (EFPD) }\end{array}$ & $\begin{array}{c}\text { Exposure at } \\
\text { End of Step } \\
\text { (EFPD) }\end{array}$ & $\begin{array}{l}\text { Mid-Step Boron } \\
\text { Concentration } \\
\text { (ppm) }\end{array}$ \\
\hline 1 & 27.11 & 27.11 & 634.3 \\
2 & 58.09 & 85.20 & 506.1 \\
3 & 58.09 & 143.30 & 337.1
\end{tabular}

Cycle-07, 462.3 EFPD to Cycle-07, 479.0 EFPD Statepoint Calculation

\begin{tabular}{lccc} 
Irradiation & $\begin{array}{c}\text { Step } \\
\text { Duration } \\
\text { (EFPD) }\end{array}$ & $\begin{array}{c}\text { Exposure at } \\
\text { End of Step } \\
\text { (EFPD) }\end{array}$ & $\begin{array}{l}\text { Mid-Step Boron } \\
\text { Concentration } \\
\text { (ppm) }\end{array}$ \\
\hline 1 & 16.70 & 16.70 & 231.2
\end{tabular}

NODAL ROD ASSEMBLY INSERTION LAYOUT FOR FUEL ASSEMBLY: AI4

COLUMN A: Cycle-07, .0 EFPD to Cycle-07, 260.3 EFPD Statepoint Calculation COLUMN B: Cycle-07, 260.3 EFPD to Cycle-07, 291.0 EFPD Statepoint Calculation COLUMN C: Cycle-07, 291.0 EFPD to Cycle-07, 319.0 EFPD Statepoint Calculation COLUMN D: Cycle-07, 319.0 EFPD to Cycle-07, 462.3 EFPD Statepoint Calculation COLUMN E: Cycle-07, 462.3 EFPD to Cycle-07, 479.0 EFPD Statepoint Calculation 
Title: CRC Depletion Calculations for the Rodded Assemblies in Batches 1, 2, 3, and IX of Crystal River Unit 3 Document Identifier: BBA000000-01717-0200-00040 REV 00

Page 95 of 146

$\mathrm{X}=$ Rod assembly inserted in corresponding node during the irradiation step

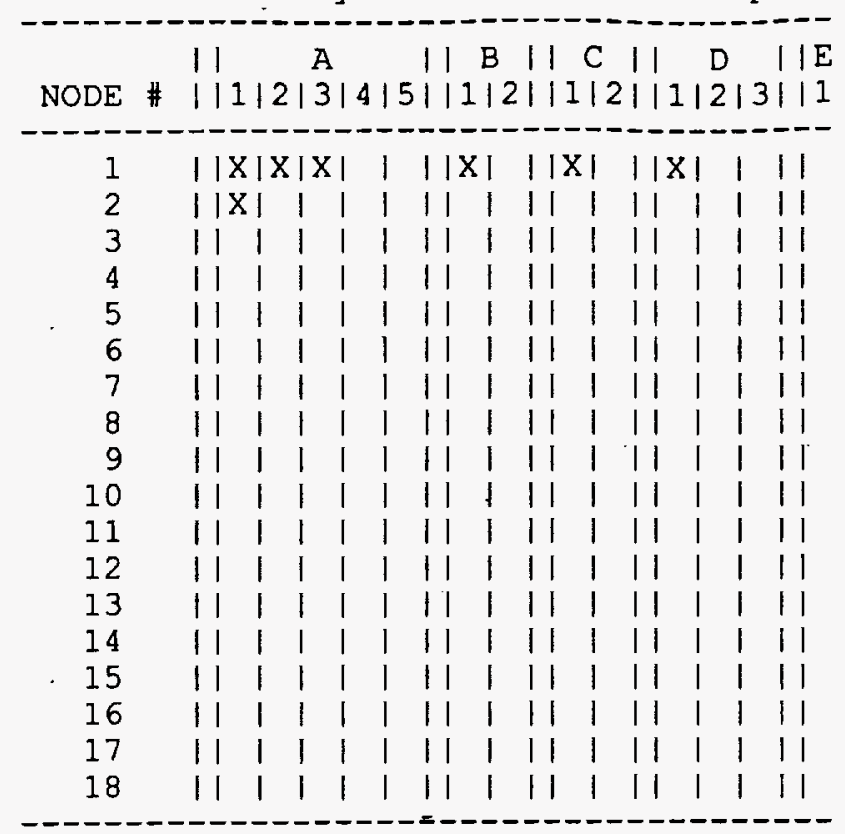

Table 7.3-12 Rodded Irradiation Time Step Layout for Assembly A18

IRRADIATION LAYOUT FOR ASSEMBLY: A18

Cycle-1A, .0 EFPD to Cycle-1A, 268.8 EFPD Statepoint Calculation

\begin{tabular}{cccc}
$\begin{array}{c}\text { Irradiation } \\
\text { Step Number }\end{array}$ & $\begin{array}{c}\text { Step } \\
\text { Duration } \\
\text { (EFPD) }\end{array}$ & $\begin{array}{c}\text { Exposure at } \\
\text { End of Step } \\
\text { (EFPD) }\end{array}$ & $\begin{array}{c}\text { Mid-Step Boron } \\
\text { Concentration } \\
\text { (Ppm) }\end{array}$ \\
\hline 1 & .78 & .78 & 901.1 \\
2 & 1.42 & 2.20 & 901.7 \\
3 & 1.80 & 4.00 & 902.7 \\
4 & 27.29 & 31.29 & 911.4 \\
5 & 45.42 & 76.71 & 933.3 \\
6 & 45.42 & 122.12 & 875.0 \\
7 & 58.01 & 180.13 & 771.9 \\
8 & 58.01 & 238.15 & 643.2 \\
9 & 11.91 & 250.06 & 575.6 \\
10 & 7.50 & 257.56 & 593.9 \\
11 & 3.80 & 261.36 & 556.3 \\
12 & 7.44 & 268.80 & 518.8
\end{tabular}

NODAL ROD ASSEMBLY INSERTION LAYOUT FOR FUEL ASSEMBLY: A18

COLUMN A: Cycle-1A, .0 EFPD to Cycle-1A, 268.8 EFPD Statepoint Calculation

$\mathrm{X}=$ Rod assembly inserted in corresponding node during the irradiation step

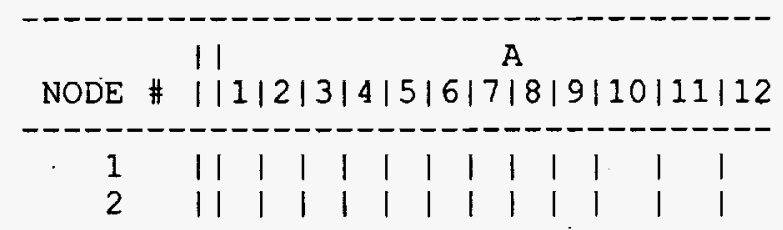


Title: CRC Depletion Calculations for the Rodded Assemblies in Batches 1, 2, 3, and $1 \mathrm{X}$ of Crystal River Unit 3

\begin{tabular}{|c|c|c|c|c|c|}
\hline 3 & 111111 & 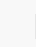 & 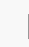 & & \\
\hline 4 & $\begin{array}{llll}11 & 1 & 1 & 1\end{array}$ & & & & \\
\hline 5 & $\begin{array}{llll}1 & 1 & 1 & 1\end{array}$ & 1 & & & \\
\hline 6 & 11111 & 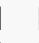 & & & \\
\hline 7 & 1111 & & & & \\
\hline 8 & 1111 & & . & & \\
\hline 9 & ||$X|X|$ & & . & & \\
\hline 10 & ||$x|x| x$ & & & & \\
\hline 11 & ||$X|X| X$ & & & & \\
\hline 12 & ||$x|X| X$ & & & & \\
\hline 13 & ||$x|X| x$ & & & & \\
\hline 14 & ||$x|x| x$ & & & & \\
\hline 15 & ||$x|x| x$ & & & & \\
\hline 16 & ||$x|x| x$ & & & & \\
\hline 17 & ||$x|x| x$ & & & & \\
\hline 18 & ||$x||$ & & & & \\
\hline
\end{tabular}

Table 7.3-13 Rodded Irradiation Time Step Layout for Assembly A18a

IRRADIATION LAYOUT FOR ASSEMBLY: A18a

Cycle-1A, .0 EFPD to Cycle-1A, 268.8 EFPD Statepoint Calculation

\begin{tabular}{cccc}
$\begin{array}{c}\text { Irradiation } \\
\text { Step Number }\end{array}$ & $\begin{array}{c}\text { Step } \\
\text { Duration } \\
\text { (EFPD) }\end{array}$ & $\begin{array}{c}\text { Exposure at } \\
\text { End of Step } \\
\text { (EFPD) }\end{array}$ & $\begin{array}{c}\text { Mid-Step Boron } \\
\text { Concentration } \\
\text { (ppm) }\end{array}$ \\
\hline 1 & .78 & .78 & 901.1 \\
2 & 1.42 & 2.20 & 901.7 \\
3 & 1.80 & 4.00 & 902.7 \\
4 & 27.29 & 31.29 & 911.4 \\
5 & 45.42 & 76.71 & 933.3 \\
6 & 45.42 & 122.12 & 875.0 \\
7 & 58.01 & 180.13 & 771.9 \\
8 & 58.01 & 238.15 & 643.2 \\
9 & 11.91 & 250.06 & 575.6 \\
10 & 7.50 & 257.56 & 593.9 \\
11 & 3.80 & 261.36 & 556.3 \\
12 & 7.44 & 268.80 & 518.8
\end{tabular}

NODAL ROD ASSEMBLY INSERTION LAYOUT FOR FUEL ASSEMBLY: A18a

COLUMN A: Cycle-1A, .0 EFPD to Cycle-1A, 268.8 EFPD Statepoint Calculation $\mathrm{X}=$ Rod assembly inserted in corresponding node during the irradiation step

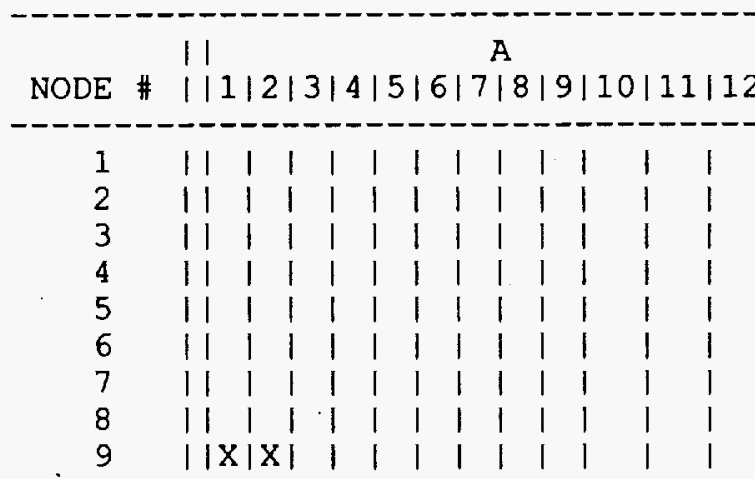


Title: CRC Depletion Calculations for the Rodded Assemblies in Batches 1, 2, 3, and $1 \mathrm{X}$ of Crystal River Unit 3 Document Identifier: BBA000000-01717-0200-00040 REV 00

Page 97 of 146

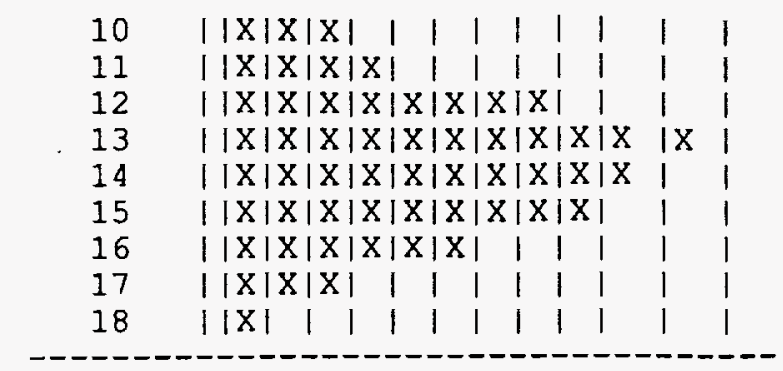

\section{Table 7.3-14 Rodded Irradiation Time Step Layout for Assembly A18b}

IRRADIATION LAYOUT FOR ASSEMBLY: A18b

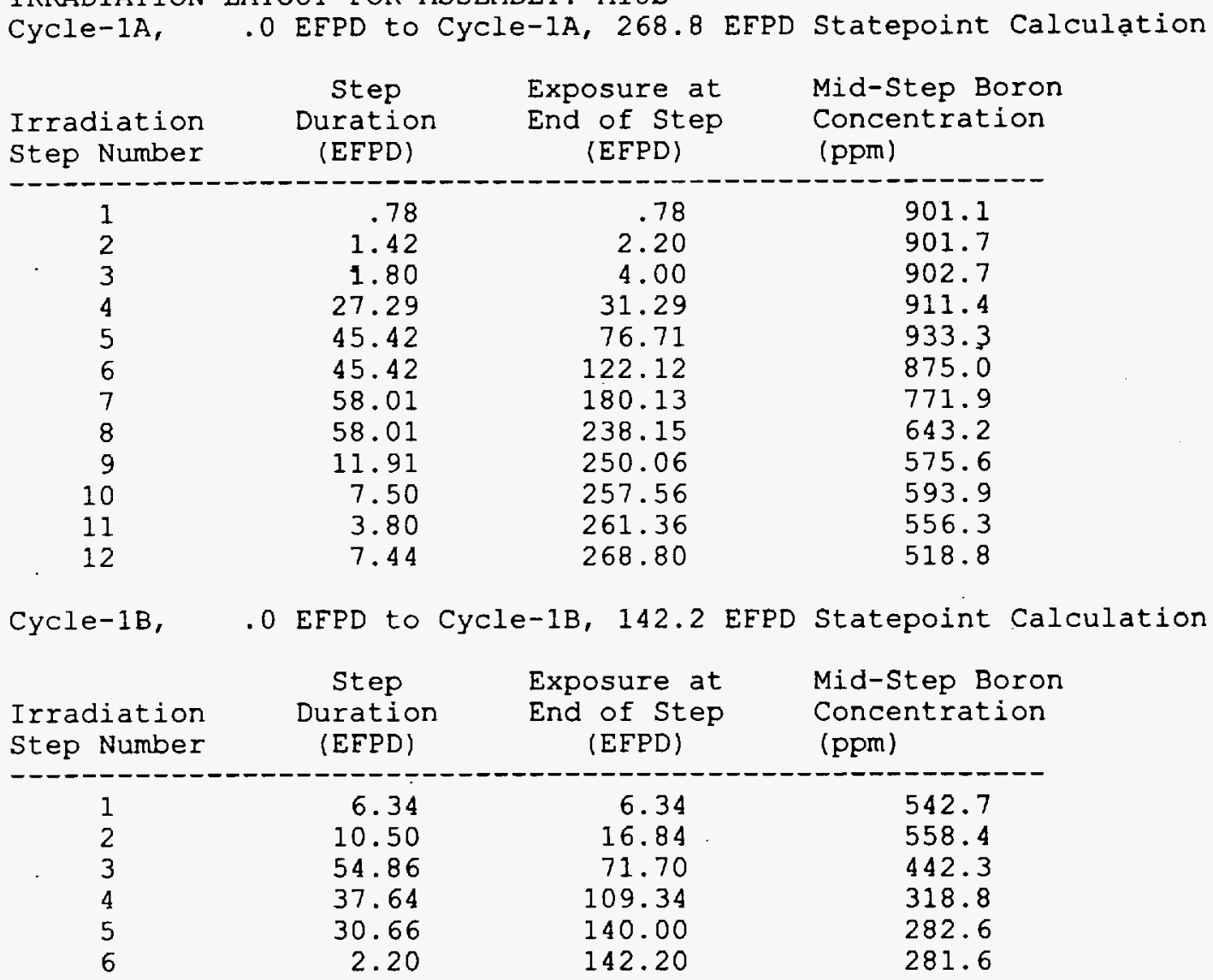

NODAL ROD ASSEMBLY INSERTION LAYOUT FOR FUEL ASSEMBLY: A18b

COLUMN A: Cycle-1A, .0 EFPD to Cycle-1A, 268.8 EFPD Statepoint Calculation COLUMN B: Cycle-1B, .0 EFPD to Cycle-1B, 142.2 EFPD Statepoint Calculation

$\mathrm{X}=$ Rod assembly inserted in corresponding node during the irradiation step

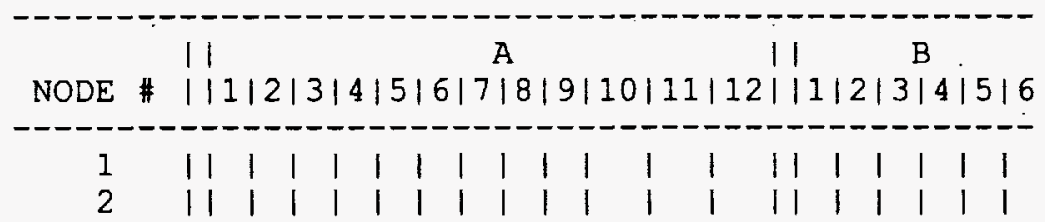




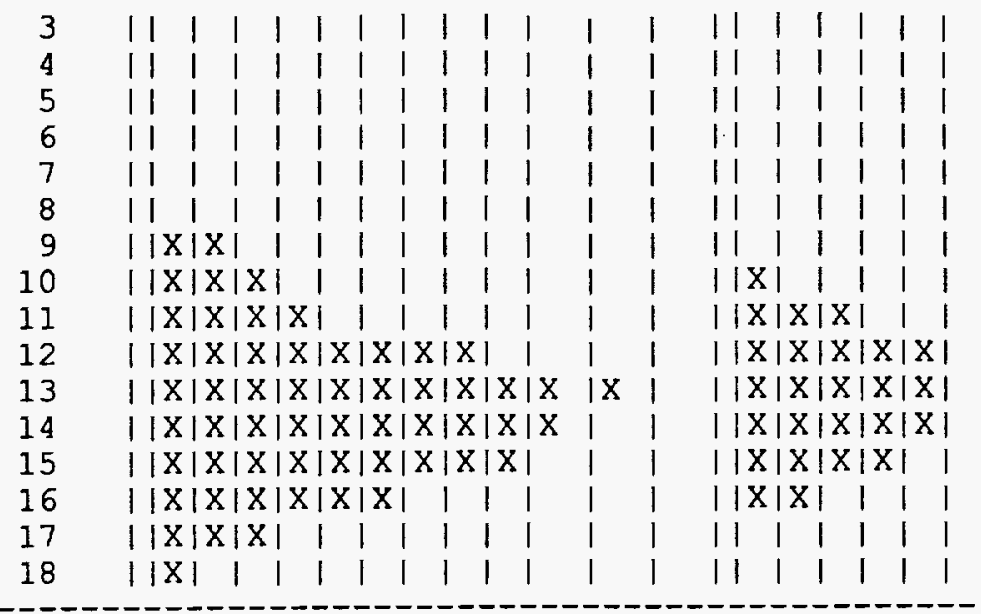

Table 7.3-15 Rodded Irradiation Time Step Layout for Assembly A20

IRRADIATION LAYOUT FOR ASSEMBLY: A20

Cycle-1A, .0 EFPD to Cycle-1A, 268.8 EFPD Statepoint Calculation

\begin{tabular}{cccc}
$\begin{array}{c}\text { Irradiation } \\
\text { Step Number }\end{array}$ & $\begin{array}{c}\text { Step } \\
\text { Duration } \\
\text { (EFPD) }\end{array}$ & $\begin{array}{c}\text { Exposure at } \\
\text { End of Step } \\
\text { (EFPD) }\end{array}$ & $\begin{array}{l}\text { Mid-Step Boron } \\
\text { Concentration } \\
\text { (ppm) }\end{array}$ \\
\hline 1 & 37.78 & 37.78 & 912.2 \\
2 & 63.23 & 101.01 & 909.0 \\
3 & 63.23 & 164.24 & 808.8 \\
4 & 60.11 & 224.35 & 678.3 \\
5 & .65 & 225.00 & 610.2 \\
6 & 2.76 & 227.76 & 633.9 \\
7 & 1.07 & 228.83 & 662.9 \\
8 & 1.23 & 230.06 & 657.8 \\
9 & 1.58 & 230.64 & 650.0 \\
10 & 1.36 & 232.00 & 641.7 \\
11 & 1.45 & 233.45 & 629.5 \\
12 & 1.55 & 234.00 & 620.9 \\
13 & 1.00 & 235.00 & 614.2 \\
14 & 33.80 & 268.80 & 606.7
\end{tabular}

NODAL ROD ASSEMBLY INSERTION LAYOUT FOR FUEL ASSEMBLY: A20

COLUMN A: Cycle-1A, .0 EFPD to Cycle-1A, 268.8 EFPD Statepoint Calculation $\mathrm{X}=$ Rod assembly inserted in corresponding node during the irradiation step II

NODE \# ||1|2|3|4|5|6|7|8|9|10|11|12|13|14

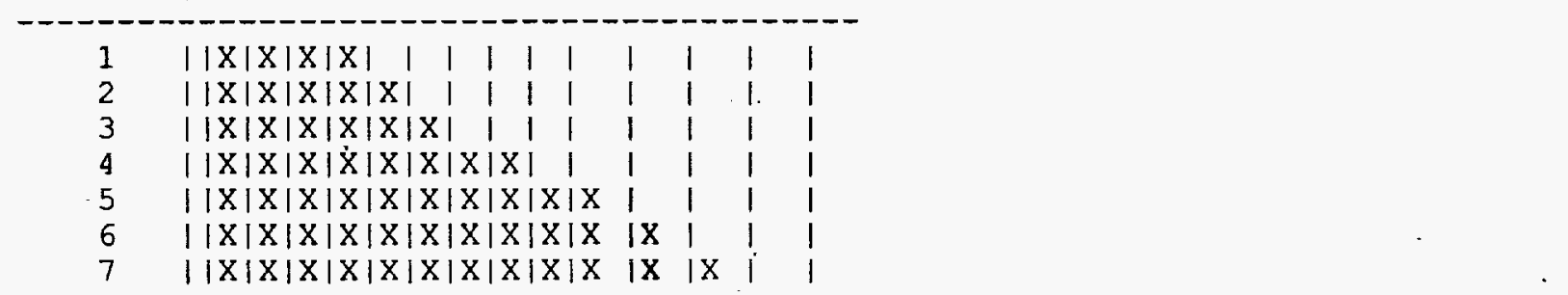


Title: CRC Depletion Calculations for the Rodded Assemblies in Batches 1, 2, 3, and $1 \mathrm{X}$ of Crystal River Unit 3

Document Identifier: BBA000000-01717-0200-00040 REV 00

Page 99 of 146

\begin{tabular}{|c|c|c|c|c|}
\hline 8 & ||$X|X| X|X| X|X| X|X| X \mid X$ & $\mathrm{IX}$ & |X & $\mid x$ \\
\hline 9 & ||$X|X| X|X| X|X| X|X| X \mid X$ & $\mathrm{I} X$ & | $\mathrm{X}$ & IX \\
\hline 0 & ||$X|X| X|X| X|X| X|X| X \mid X$ & $\mathrm{X}$ & $\mathrm{IX}$ & 1 \\
\hline & ||$x|x| X|X| X|X| X|x| x \mid X$ & I & l & i \\
\hline & ||$X|X| X|X| X|X| X|X| X \mid$ & I & 1 & i \\
\hline & ||$X|X| X|X| X|X| X|1|$ & 1 & | & I \\
\hline & ||$x|x| x|x| x|i| i \mid$ & 1 & i & i \\
\hline & ||$x|x| x|\quad| \quad \mid 1111$ & 1 & 1 & 1 \\
\hline & $\mid \begin{array}{lllllllll}|x| & 1 & 1 & 1 & 1 & 1 & 1 & 1 & 1\end{array}$ & 1 & 1 & 1 \\
\hline & 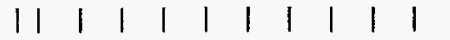 & 1 & 1 & 1 \\
\hline & $\begin{array}{llllllllll}1 & 1 & 1 & 1 & 1 & 1 & 1 & 1 & 1 & 1\end{array}$ & 1 & I & 1 \\
\hline
\end{tabular}

Table 7.3-16 Rodded Irradiation Time Step Layout for Assembly A22

IRRADIATION LAYOUT FOR ASSEMBLY: A22

Cycle-IA, $\quad$. EFPD to Cycle-IA, 268.8 EFPD Statepoint Calculation

\begin{tabular}{|c|c|c|c|}
\hline $\begin{array}{l}\text { Irradiation } \\
\text { Step Number }\end{array}$ & $\begin{array}{l}\text { Step } \\
\text { Duration } \\
\text { (EFPD) }\end{array}$ & $\begin{array}{c}\text { Exposure at } \\
\text { End of Step } \\
\text { (EFPD) }\end{array}$ & $\begin{array}{l}\text { Mid-Step Boron } \\
\text { Concentration } \\
\text { (ppm) }\end{array}$ \\
\hline $\begin{array}{l}1 \\
2 \\
3 \\
4 \\
5 \\
6\end{array}$ & $\begin{array}{r}5.03 \\
60.15 \\
60.15 \\
57.42 \\
57.42 \\
28.64\end{array}$ & $\begin{array}{r}5.03 \\
65.18 \\
125.32 \\
182.74 \\
240.16 \\
268.80\end{array}$ & $\begin{array}{l}902.3 \\
921.9 \\
883.3 \\
766.1 \\
637.8 \\
589.5\end{array}$ \\
\hline
\end{tabular}

Cycle-1B, .0 EFPD to Cycle-1B, 142.2 EFPD. Statepoint Calculation

\begin{tabular}{cccc}
$\begin{array}{c}\text { Step } \\
\text { Irradiation }\end{array}$ & $\begin{array}{c}\text { Exposure at } \\
\text { Duration } \\
\text { (EFPD) }\end{array}$ & $\begin{array}{c}\text { End of Step } \\
\text { (EFPD) }\end{array}$ & $\begin{array}{l}\text { Mid-Step Boron } \\
\text { Concentration } \\
\text { (ppm) }\end{array}$ \\
\hline $\begin{array}{c}\text { Step Number } \\
1\end{array}$ & 4.49 & 4.49 & 541.0 \\
2 & 41.82 & 46.31 & 549.6 \\
3 & 35.51 & 81.82 & 423.0 \\
4 & 35.51 & 117.34 & 289.3 \\
5 & 8.04 & 125.38 & 279.6 \\
6 & 16.82 & 142.20 & 289.1
\end{tabular}

NODAL ROD ASSEMBLY INSERTION LAYOUT FOR FUEL ASSEMBLY: A22

COLUMN A: Cycle-1A, .0 EFPD to Cycle-1A, 268.8 EFPD Statepoint Calculation COLUMN B: Cycle-1B, .0 EFPD to Cycle-1B, 142.2 EFPD Statepoint Calculation

$\mathrm{x}=$ Rod assembly inserted in corresponding node during the irradiation step

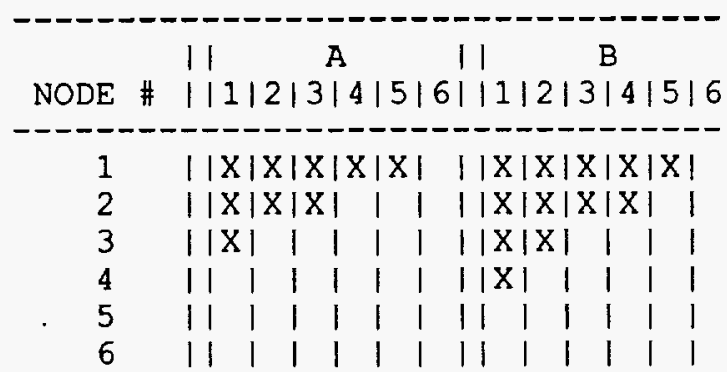




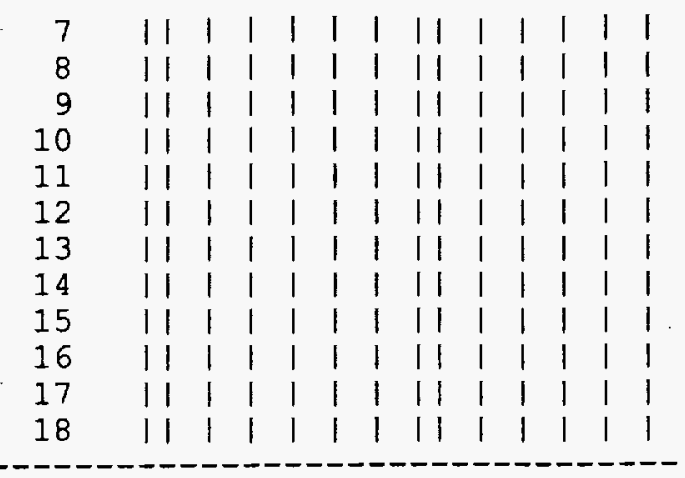

Table 7.3-17 Rodded Irradiation Time Step Layout for Assembly A23

IRRADIATION LAYOUT FOR ASSEMBLY: A23

Cycle-02, .0 EFPD to Cycle-02, 166.5 EFPD Statepoint Calculation

\begin{tabular}{cccc}
$\begin{array}{c}\text { Irradiation } \\
\text { Step Number }\end{array}$ & $\begin{array}{c}\text { Step } \\
\text { Duration } \\
\text { (EFPD) }\end{array}$ & $\begin{array}{c}\text { Exposure at } \\
\text { End of Step } \\
\text { (EFPD) }\end{array}$ & $\begin{array}{l}\text { Mid-Step Boron } \\
\text { Concentration } \\
\text { (Ppm) }\end{array}$ \\
\hline 1 & .99 & .99 & 864.5 \\
2 & 9.13 & 10.12 & 783.1 \\
3 & 54.72 & 64.84 & 659.1 \\
4 & 36.53 & 101.36 & 528.0 \\
5 & 36.53 & 137.89 & 422.5 \\
6 & 28.11 & 166.00 & 327.4 \\
7 & 1.00 & 167.00 & 311.9
\end{tabular}

NODAL ROD ASSEMBLY INSERTION LAYOUT FOR FUEL ASSEMBLY: A23

COLUMN A: Cycle-02, .0 EFPD to Cycle-02, 166.5 EFPD Statepoint Calculation $\mathrm{X}=$ Rod assembly inserted in corresponding node during the irradiation step

\begin{tabular}{|c|c|}
\hline & $\begin{array}{l}\mid 1 \\
|| 1|2| 3|4| 5|6| 7\end{array}$ \\
\hline 1 & ||$X|X| X|X| X|X|$ \\
\hline 2 & ||$x|x| x|x| x|x| x$ \\
\hline 3 & ||$x|x| x|x| x|x| x$ \\
\hline 4 & ||$x|x| x|x| x|x| x$ \\
\hline 5 & ||$x|x| x|x| x|x| x$ \\
\hline 6 & ||$x|x| x|x| x|x| x$ \\
\hline 7 & ||$x|x| x|x| x|x| x$ \\
\hline 8 & |ixixixixixixix \\
\hline 9 & ||$x|x| x|x| x|x| x$ \\
\hline 10 & $|i x| x|x| x|x| x \mid x$ \\
\hline 11 & ||$x|x| x|x| x|x| x$ \\
\hline 12 & ||$x|x| x|x| x|x| x$ \\
\hline 13 & ||$x|x| x|x| x|x| x$ \\
\hline 14 & ||$x|x| x|x| x|x|$ \\
\hline 15 & ||$x|x| x|x| x||$ \\
\hline 16 & ||$x|x| x|| 1 \mid$ \\
\hline 17 & ||$x|x||| 1 \mid$ \\
\hline 18 & ||$x|1| 1 \mid 1$ \\
\hline
\end{tabular}


Table 7.3-18 Rodded Irradiation Time Step Layout for Assembly A23a

IRRADIATION LAYOUT FOR ASSEMBLY: A23a

Cycle-02, .0 EFPD to Cycle-02, 166.5 EFPD Statepoint Calculation

\begin{tabular}{cccc}
$\begin{array}{c}\text { Irradiation } \\
\text { Step Number }\end{array}$ & $\begin{array}{c}\text { Step } \\
\text { Duration } \\
\text { (EFPD) }\end{array}$ & $\begin{array}{c}\text { Exposure at } \\
\text { End of Step } \\
\text { (EFPD) }\end{array}$ & $\begin{array}{l}\text { Mid-Step Boron } \\
\text { Concentration } \\
\text { (ppm) }\end{array}$ \\
\hline 1 & 1.06 & 1.06 & 863.7 \\
2 & 8.96 & 10.02 & 783.0 \\
3 & 51.85 & 61.87 & 664.0 \\
4 & 37.40 & 99.26 & 537.6 \\
5 & 37.40 & 136.66 & 432.0 \\
6 & 29.34 & 166.00 & 328.5 \\
7 & 1.00 & 167.00 & 311.9
\end{tabular}

NODAL ROD ASSEMBLY INSERTION LAYOUT FOR FUEL ASSEMBLY: A23a

COLUMN A: Cycle-02,- .0 EFPD to Cycle-02, 166.5 EFPD Statepoint Calculation $\mathrm{X}=$ Rod assembly inserted in corresponding node during.the irradiation step

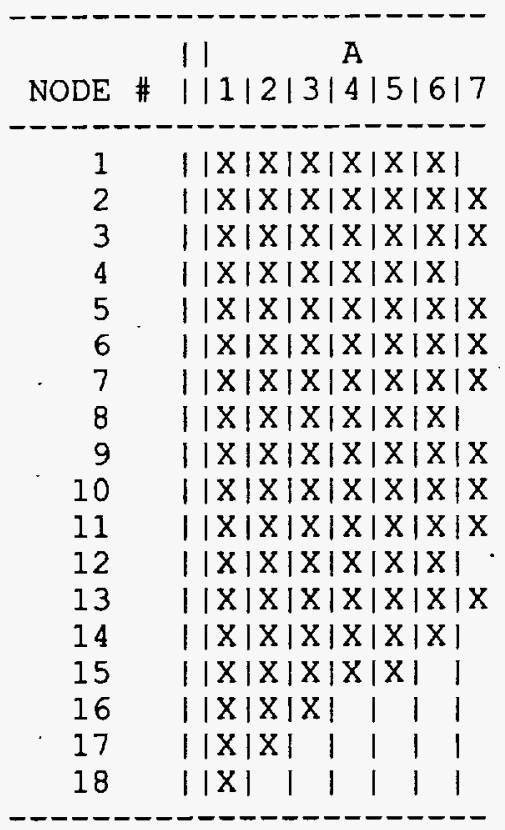




\section{Table 7.3-19 Rodded Irradiation Time Step Layout for Assembly A25}

IRRADIATION LAYOUT FOR ASSEMBLY: A25

Cycle-03, .0 EFPD to Cycle-03, 168.5 EFPD Statepoint Calculation

\begin{tabular}{cccc}
$\begin{array}{c}\text { Irradiation } \\
\text { Step Number }\end{array}$ & $\begin{array}{c}\text { Step } \\
\text { (EFation }\end{array}$ & $\begin{array}{c}\text { Exposure at } \\
\text { End of Step } \\
\text { (EFPD) }\end{array}$ & $\begin{array}{c}\text { Mid-Step Boron } \\
\text { Concentration } \\
\text { (ppm) }\end{array}$ \\
\hline 1 & .26 & .26 & 941.3 \\
2 & 5.23 & 5.49 & 945.3 \\
3 & 9.75 & 15.24 & 940.1 \\
4 & 48.08 & 63.32 & 835.2 \\
5 & 48.08 & 111.40 & 688.0 \\
6 & 53.84 & 165.24 & 542.0 \\
7 & 2.76 & 168.00 & 457.6 \\
8 & 1.00 & 169.00 & 451.1
\end{tabular}

Cycle-03, 168.5 EFPD to Cycle-03, 250.0 EFPD Statepoint Calculation

\begin{tabular}{cccc} 
Irradiation & $\begin{array}{c}\text { Step } \\
\text { Duration } \\
\text { (EEPD) }\end{array}$ & $\begin{array}{c}\text { Exposure at } \\
\text { End of Step } \\
\text { (EFPD) }\end{array}$ & $\begin{array}{l}\text { Mid-Step Boron } \\
\text { Concentration } \\
\text { (ppm) }\end{array}$ \\
\hline 1 & 3.04 & 3.04 & 444.1 \\
2 & 36.92 & 39.96 & 378.6 \\
3 & 36.92 & 76.88 & 272.3 \\
4 & 4.56 & 81.44 & 231.2 \\
5 & .56 & 82.00 & 234.5
\end{tabular}

NODAL ROD ASSEMBLY INSERTION LAYOUT FOR FUEL ASSEMBLY: A25

COLUMN A: Cycle-03, .0 EFPD to Cycle-03, 168.5 EFPD Statepoint Calculation COLUMN B: Cycle-03, 168.5 EFPD to Cycle-03, 250.0 EEPD Statepoint Calculation

$X=$ Rod assembly inserted in corresponding node during the irradiation step

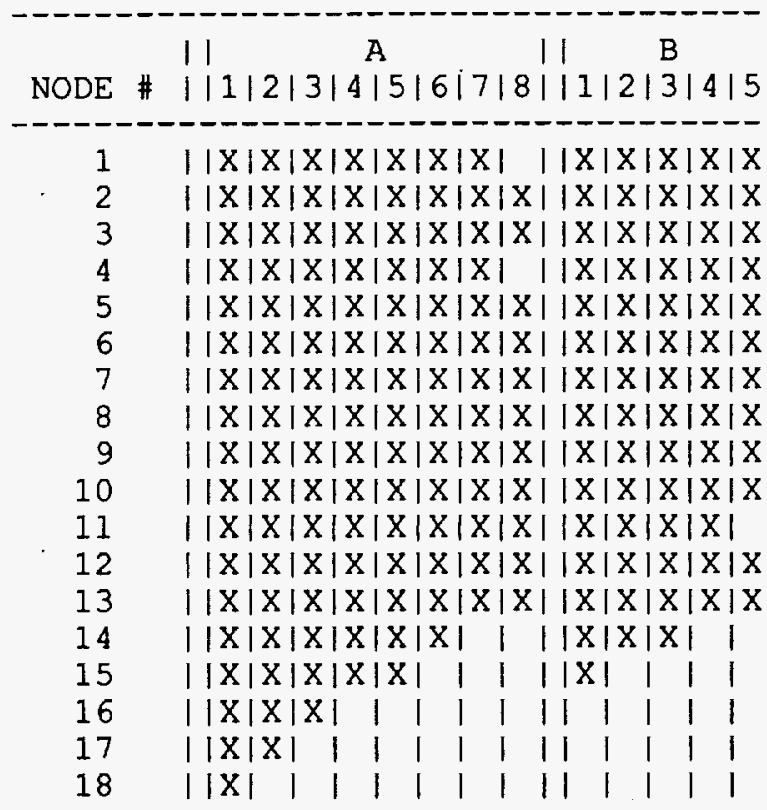




\section{Table 7.3-20 Rodded Irradiation Time Step Layout for Assembly A25a}

IRRADIATION LAYOUT FOR ASSEMBLY: A25a

Cycle-03, .0 EFPD to Cycle-03, 168.5 EFPD Statepoint Calculation

\begin{tabular}{cccc}
$\begin{array}{c}\text { Irradiation } \\
\text { Step Number }\end{array}$ & $\begin{array}{c}\text { Step } \\
\text { Duration } \\
\text { (EFPD) }\end{array}$ & $\begin{array}{c}\text { Exposure at } \\
\text { End of Step } \\
\text { (EFPD) }\end{array}$ & $\begin{array}{c}\text { Mid-Step Boron } \\
\text { Concentration } \\
\text { (ppm) }\end{array}$ \\
\hline 1 & 6.84 & 6.84 & 946.1 \\
2 & 3.33 & 10.17 & 945.6 \\
3 & 66.47 & 76.64 & 830.5 \\
4 & 39.84 & 116.48 & 672.2 \\
5 & 39.84 & 156.32 & 546.9 \\
6 & 12.18 & 168.50 & 471.5
\end{tabular}

Cycle-03, 168.5 EFPD to Cycle-03, 250.0 EFPD Statepoint Calculation

\begin{tabular}{|c|c|c|c|}
\hline $\begin{array}{l}\text { Irradiation } \\
\text { Step Number }\end{array}$ & $\begin{array}{c}\text { Step } \\
\text { Duration } \\
\text { (EFPD) }\end{array}$ & $\begin{array}{c}\text { Exposure at } \\
\text { End of Step } \\
\text { (EFPD) }\end{array}$ & $\begin{array}{l}\text { Mid-Step Boron } \\
\text { Concentration } \\
\text { (ppm) }\end{array}$ \\
\hline $\begin{array}{r}1 \\
2 \\
3\end{array}$ & $\begin{array}{r}8.99 \\
62.99 \\
9.52\end{array}$ & $\begin{array}{r}8.99 \\
71.98 \\
81.50\end{array}$ & $\begin{array}{l}435.5 \\
333.5 \\
231.6\end{array}$ \\
\hline
\end{tabular}

NODAL ROD ÁSSEMBLY INSERTION LAYOUT FOR FUEL ASSEMBLY: A25a

COLUMN A: Cycle-03, $\quad .0$ EFPD to Cycle-03, 168.5 EFPD Statepoint Calculation COLUMN B: Cycle-03, 168.5 EFPD to Cycle-03, 250.0 EFPD Statepoint Calculation $\mathrm{X}=$ Rod assembly inserted in corresponding node during the irradiation step

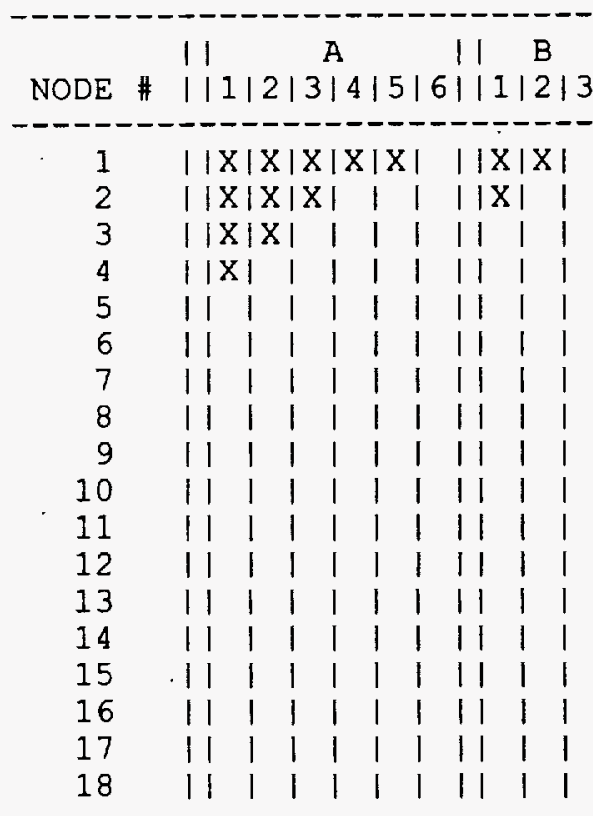


Title: CRC Depletion Calculations for the Rodded Assemblies in Batches 1, 2, 3, and $1 \mathrm{X}$ of Crystal River Unit 3

Table 7.3-21 Rodded Irradiation Time Step Layout for Assembly A26

IRRADIATION LAYOUT FOR ASSEMBLY: A26

Cycle-1A, .0 EFPD to Cycle-1A, 268.8 EFPD Statepoint Calculation

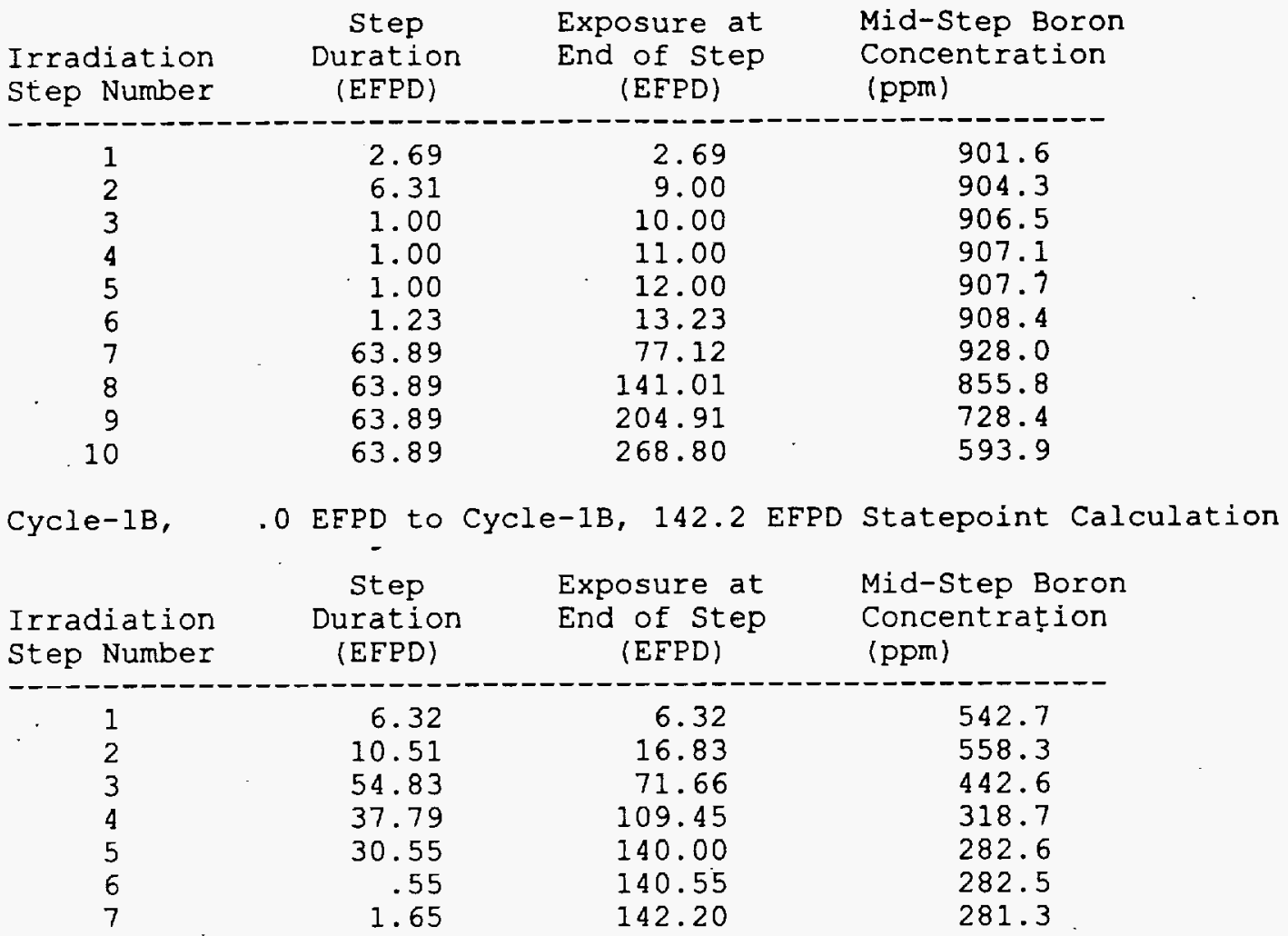

NODAL ROD ASSEMBLY INSERTION LAYOUT FOR FUEL ASSEMBLY: A26

COLUMN A: Cycle-1A, $\quad .0$ EFPD to Cycle-1A; 268.8 EFPD Statepoint Calculation COLUMN B: Cycle-1B, .0 EFPD to Cycle-1B, 142.2 EFPD Statepoint Calculation

$\mathrm{X}=$ Rod assembly inserted in corresponding node during the irradiation step

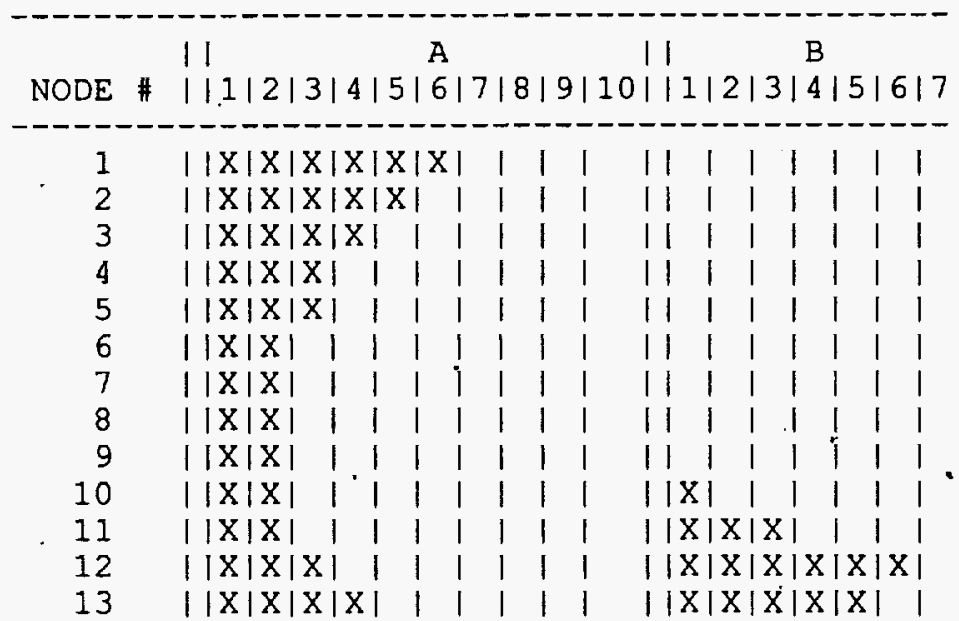




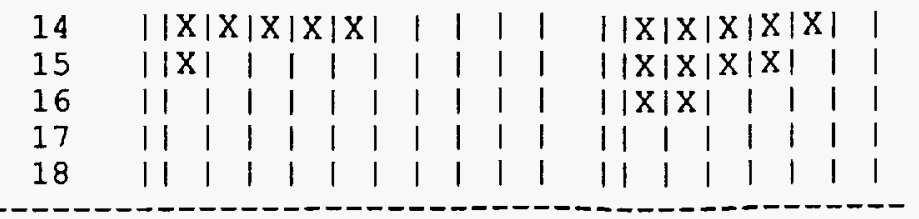

Table 7.3-22 Rodded Irradiation Time Step Layout for Assembly A28

IRRADIATION LAYOUT FOR ASSEMBLY: A28

Cycle-02, .0 EFPD to Cycle-02, 166.5 EFPD Statepoint Calculation

\begin{tabular}{cccc}
$\begin{array}{c}\text { Irradiation } \\
\text { Step Number }\end{array}$ & $\begin{array}{c}\text { Step } \\
\text { (EFation }\end{array}$ & $\begin{array}{c}\text { Exposure at } \\
\text { End of Step } \\
\text { (EFPD) }\end{array}$ & $\begin{array}{l}\text { Mid-Step Boron } \\
\text { Concentration } \\
\text { (ppm) }\end{array}$ \\
\hline 1 & 1.93 & 1.93 & 854.1 \\
2 & 4.62 & 6.55 & 781.5 \\
3 & 1.23 & 7.78 & 790.2 \\
4 & 7.51 & 15.29 & 806.7 \\
5 & 32.00 & 47.29 & 677.4 \\
6 & 10.49 & 57.78 & 621.3 \\
7 & 51.35 & 109.13 & 526.8 \\
8 & 13.72 & 122.85 & 441.8 \\
9 & 19.33 & 142.18 & 378.5 \\
10 & 13.96 & 156.14 & 332.1 \\
11 & 10.36 & 166.50 & 316.1
\end{tabular}

NODAL ROD ASSEMBLY INSERTION LAYOUT FOR FUEL ASSEMBLY: A28

COLUMN A: Cycle-02, .0 EFPD to Cycle-02, 166.5 EFPD Statepoint Calculation

$\mathrm{X}=$ Rod assembly inserted in corresponding node during the irradiation step

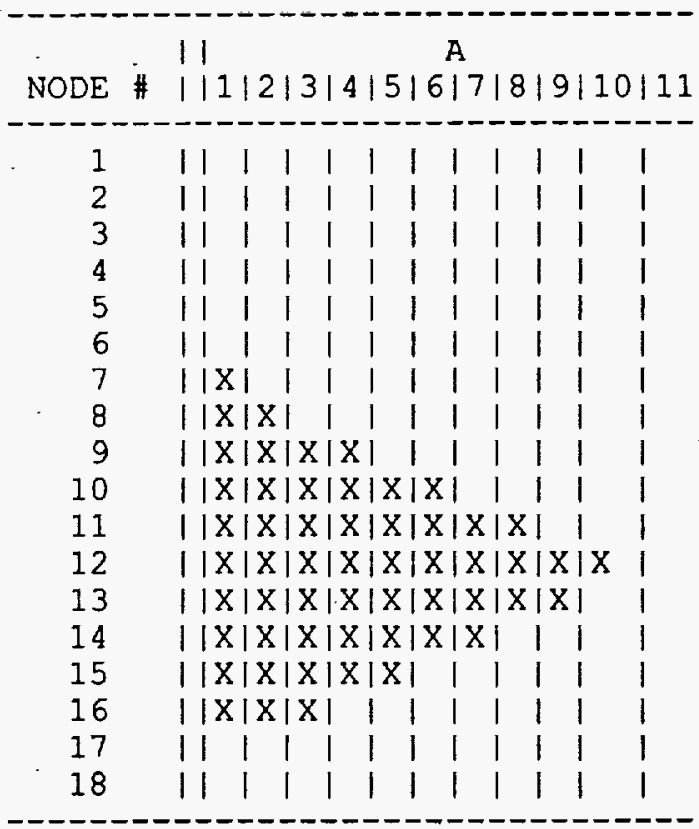


Title: CRC Depletion Calculations for the Rodded Assemblies in Batches 1, 2, 3, and $1 \mathrm{X}$ of Crystal River Unit 3

Table 7.3-23 Rodded Irradiation Time Step Layout for Assembly A29

IRRADIATION LAYOUT FOR ASSEMBLY: A29

Cycle-03, .0 EFPD to Cycle-03, 168.5 EFPD Statepoint Calculation

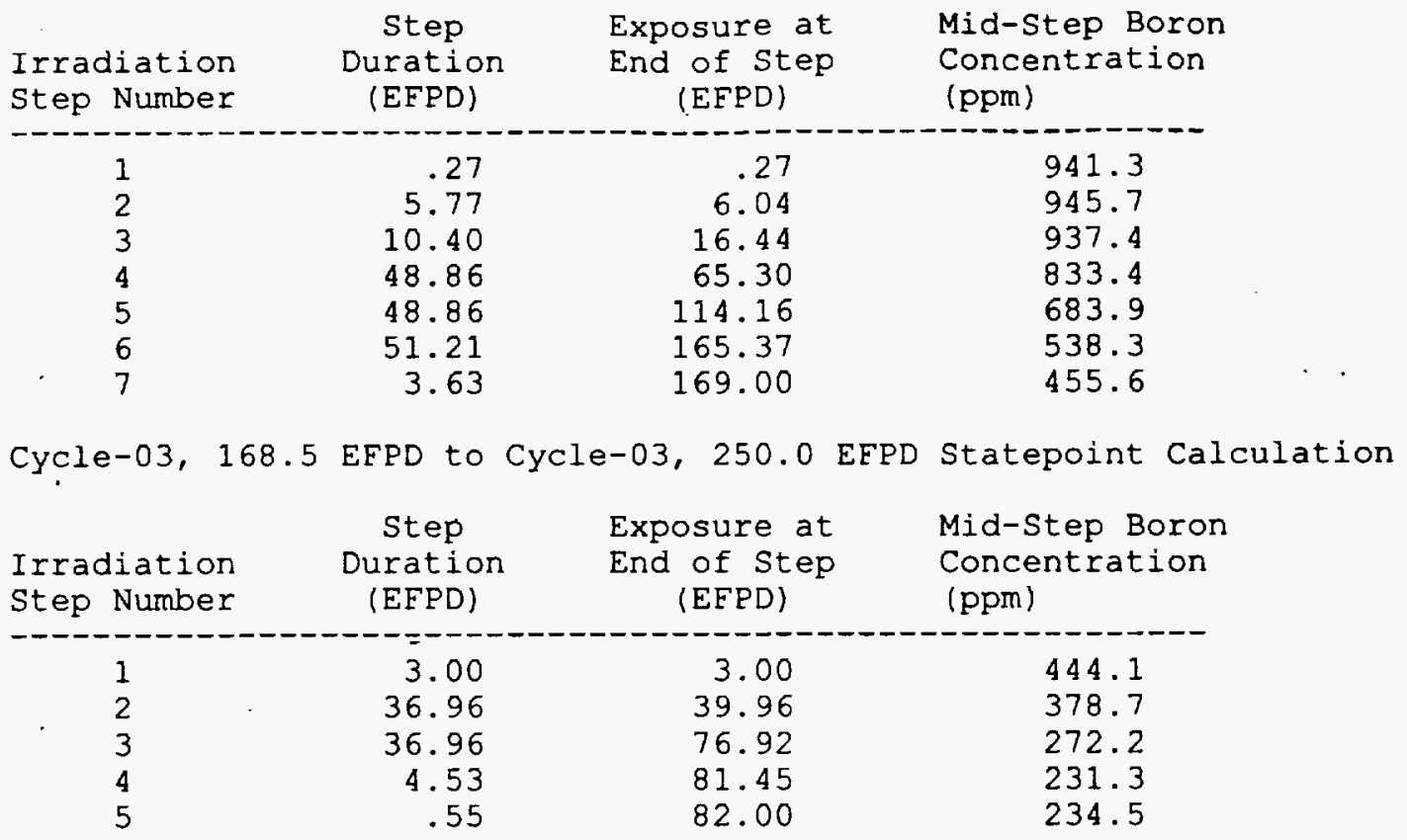

NODAL ROD ASSEMBLY INSERTION LAYOUT FOR FUEL ASSEMBLY: A29

COLUMN A: Cycle-03, .0 EFPD to Cycle-03, 168.5 EFPD Statepoint Calculation COLUMN B: Cycle-03, 168.5 EFPD to Cycle-03, 250.0 EFPD Statepoint Calculation

$\mathrm{X}=$ Rod assembly inserted in corresponding node during the irradiation step

\begin{tabular}{|c|c|}
\hline & 11 \\
\hline NODE \# & || $1|2| 3|4| 5|6| 7|| 1 \mid 2$ \\
\hline & \\
\hline 1 & ||$X|X| X|X| X|X| X|| X|X| X|X| X$ \\
\hline 2 & ||$X|X| X|X| X|X| X|| X|X| X|X| X$ \\
\hline 3 & ||$x|X| X|X| X|X| X|| X|X| X|X| X$ \\
\hline 4 & ||$X|X| X|X| X|X| X|| X|X| X|X| X$ \\
\hline 5 & ||$X|X| X|X| X|X| X|| X|X| X|X| X$ \\
\hline 6 & ||$X|X| X|X| X|X| X|| X|X| X|X| X$ \\
\hline 7 & ||$X|X| X|X| X|X| X|| X|X| X|X| X$ \\
\hline 8 & ||$X|X| X|X| X|X| X|| X|X| X|X| X$ \\
\hline 9 & ||$X|X| X|X| X|X| X|| X|X| X|X| X$ \\
\hline 10 & ||$X|X| X|X| X|X| X|| X|X| X|X| X$ \\
\hline 11 & ||$X|X| X|X| X|X| X|| X|X| X|X|$ \\
\hline 12 & ||$X|X| X|X| X|X| X|| X|X| X|X| X$ \\
\hline 13 & ||$X|X| X|X| X|X| X|| X|X| X|X| X$ \\
\hline 14 & ||$X|X| X|X| X|X||| X|X| X||$ \\
\hline 15 & 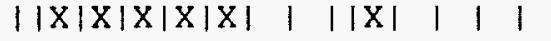 \\
\hline 16 & 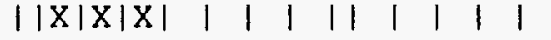 \\
\hline 17 & 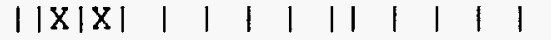 \\
\hline 18 & 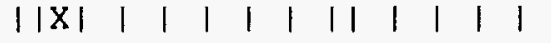 \\
\hline
\end{tabular}




\section{Table 7.3-24 Rodded Irradiation Time Step Layout for Assembly 01}

IRRADIATION LAYOUT FOR ASSEMBLY: 01

Cycle-01, .0 EFPD to Cycle-01, 309.3 EFPD Statepoint Calculation

\begin{tabular}{|c|c|c|c|}
\hline $\begin{array}{l}\text { Irradiation } \\
\text { Step Number }\end{array}$ & $\begin{array}{l}\text { Step } \\
\text { Duration } \\
\text { (EFPD) }\end{array}$ & $\begin{array}{c}\text { Exposure at } \\
\text { End of Step } \\
\text { (EFPD) }\end{array}$ & $\begin{array}{l}\text { Mid-Step Boron } \\
\text { Concentration } \\
\text { (ppm) }\end{array}$ \\
\hline $\begin{array}{l}1 \\
2 \\
3 \\
4 \\
5\end{array}$ & $\begin{array}{l}54.07 \\
63.98 \\
63.98 \\
63.98 \\
63.98\end{array}$ & $\begin{array}{r}54.07 \\
118.05 \\
182.04 \\
246.02 \\
310.00\end{array}$ & $\begin{array}{r}1005.9 \\
867.4 \\
654.9 \\
450.1 \\
245.4\end{array}$ \\
\hline
\end{tabular}

Cycle-1B, .0 EFPD to Cycle-1B, 142.2 EFPD Statepoint Calculation

\begin{tabular}{cccc}
$\begin{array}{c}\text { Irradiation } \\
\text { Step Number }\end{array}$ & $\begin{array}{c}\text { Suration } \\
\text { (EFPD) }\end{array}$ & $\begin{array}{c}\text { Exposure at } \\
\text { End of Step } \\
\text { (EFPD) }\end{array}$ & $\begin{array}{c}\text { Mid-Step Boron } \\
\text { Concentration } \\
\text { (ppm) }\end{array}$ \\
\hline 1 & 4.64 & 4.64 & 541.1 \\
2 & 24.31 & 28.95 & 568.0 \\
3 & 42.71 & 71.66 & 435.6 \\
4 & 42.71 & 114.38 & 313.8 \\
5 & 9.62 & 124.00 & 274.8 \\
6 & 1.00 & 125.00 & 282.4 \\
7 & 1.00 & 126.00 & 283.3 \\
8 & 7.93 & 133.93 & 287.4 \\
9 & 8.07 & 142.00 & 284.8 \\
10 & .20 & 142.20 & 280.6
\end{tabular}

NODAL ROD ASSEMBLY INSERTION LAYOUT FOR FUEL ASSEMBLỴ: OI

COLUMN A: Cycle-01, .o EFPD to Cycle-01, 309.3 EFPD Statepoint Calculation COLUMN B: Cycle-1B, .0 EFPD to Cycle-1B, 142.2 EFPD Statepoint Calculation

$\mathrm{X}=$ Rod assembly inserted in corresponding node during the irradiation step

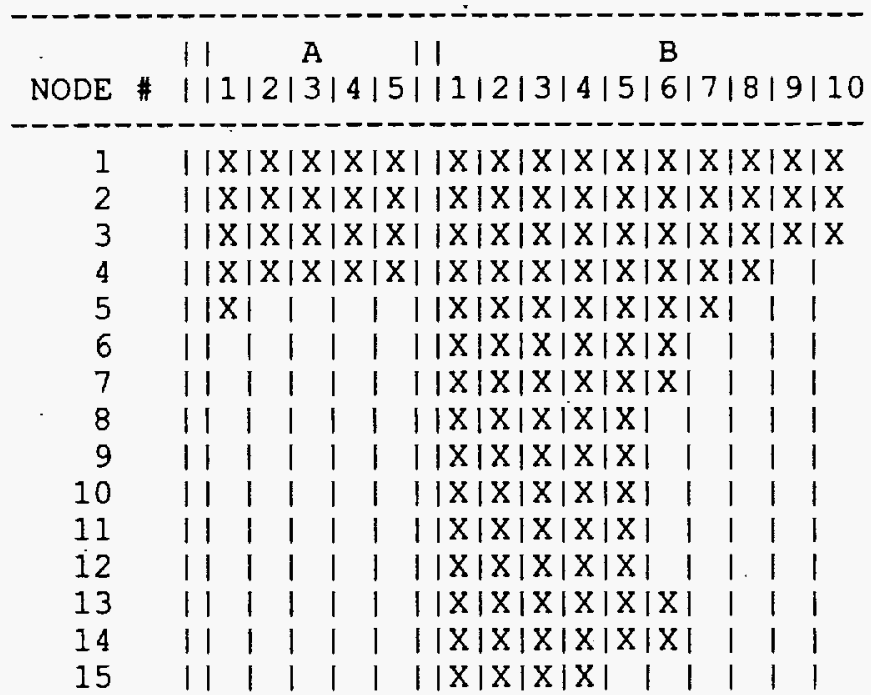


Title: CRC Depletion Calculations for the Rodded Assemblies in Batches 1, 2, 3, and 1X of Crystal River Unit 3 Document Identifier: BBA000000-01717-0200-00040 REV 00

Page 108 of 146

Table 7.3-25 Boron Letdown Data Provided to RLAYOUT for Cycle 1A of Crystal River Unit 3

\begin{tabular}{|c|c|}
\hline Exposure (EFPD) & Boron Concentration (ppm) \\
\hline 18.6 & 912 \\
\hline 55.2 & 934 \\
\hline 63.8 & 909 \\
\hline 69.9 & 909 \\
\hline 94.9 & 884 \\
\hline 184.7 & 705 \\
\hline 192.3 & 683 \\
\hline 216.0 & 627 \\
\hline 224.8 & 610 \\
\hline 228.5 & 666 \\
\hline 238.0 & 584 \\
\hline 244.0 & 575 \\
\hline 250.8 & 614 \\
\hline 254.7 & 588 \\
\hline
\end{tabular}

- The acronym "ppm" means parts per million by mass.

Table 7.3-26 Boron Letdown Data Provided to RLAYOUT for Cycle 1B of Crystal River Unit 3

\begin{tabular}{|c|c|}
\hline $\begin{array}{c}\text { EFPD with Cycles 1A \& 1B Continuous } \\
\text { [EFPD with BOC*1B set to 0.0 EFPD] }\end{array}$ & Boron Concentration (ppm) \\
\hline $280.2[11.4]$ & 558 \\
\hline $287.2[18.4]$ & 571 \\
\hline $306.2[37.4]$ & 513 \\
\hline $313.2[44.4]$ & 441 \\
\hline
\end{tabular}


Title: CRC Depletion Calculations for the Rodded Assemblies in Batches 1, 2, 3, and $1 \mathrm{X}$ of Crystal River Unit 3

Table 7.3-26 Boron Letdown Data Provided to RLAYOUT for Cycle 1B of Crystal River Unit 3

\begin{tabular}{|c|c|}
\hline $\begin{array}{c}\text { EFPD with Cycles 1A \& 1B Continuous } \\
\text { [EFPD with BOC*'1B set to 0.0 EFPD] }\end{array}$ & Boron Concentration (ppm) \\
\hline $337.2[68.4]$ & 419 \\
\hline $345.7[76.9]$ & 346 \\
\hline $364.2[95.4]$ & 309 \\
\hline $377.6[108.8]$ & 246 \\
\hline $389.5[120.7]$ & 279 \\
\hline $401.7[132.9]$ & 290 \\
\hline $419.3[150.5]$ & 272 \\
\hline $427.1[158.3]$ & 229 \\
\hline
\end{tabular}

- The acronym "BOC" means beginning of cycle.

Table 7.3-27 Boron Letdown Data Provided to RLAYOUT for Cycle 2 of Crystal River Unit 3

\begin{tabular}{|c|c|}
\hline Exposure (EFP) & Boron Concentration (ppm) \\
\hline 3.0 & 809 \\
\hline 4.4 & 778 \\
\hline 11.4 & 809 \\
\hline 15.8 & 735 \\
\hline 22.5 & 709 \\
\hline 29.3 & 683 \\
\hline 35.3 & 666 \\
\hline 42.3 & 644 \\
\hline 55.8 & 614 \\
\hline 60.8 & 592 \\
\hline 75.2 & 558 \\
\hline 83.1 & 528 \\
\hline & \\
\hline & \\
\hline
\end{tabular}


Table 7.3-27 Boron Letdown Data Provided to RLAYOUT for Cycle 2 of Crystal River Unit 3

\begin{tabular}{|c|c|}
\hline Exposure (EFPD) & Boron Concentration (ppm) \\
\hline 89.8 & 506 \\
\hline 97.8 & 480 \\
\hline 104.7 & 463 \\
\hline 116.4 & 441 \\
\hline 122.5 & 406 \\
\hline 129.1 & 385 \\
\hline 135.9 & 372 \\
\hline 139.9 & 346 \\
\hline 148.6 & 333 \\
\hline 156.4 & 320 \\
\hline 161.4 & 316 \\
\hline
\end{tabular}

Table 7.3-28 Boron Letdown Data Provided to RLAYOUT for Cycle 3 of Crystal River Unit 3

\begin{tabular}{|c|c|}
\hline Exposure (EFPD) & Boron Concentration (ppm) \\
\hline \hline 4.0 & 947 \\
\hline 6.7 & 951 \\
\hline 26.8 & 891 \\
\hline 32.6 & 843 \\
\hline 50.7 & 822 \\
\hline 66.0 & 757 \\
\hline 69.9 & 746 \\
\hline 85.0 & 692 \\
\hline 100.2 & 666 \\
\hline 111.2 & 636 \\
\hline 130.5 & 562 \\
\hline 143.8 & 528 \\
\hline
\end{tabular}




\section{Waste Package Development}

Title: CRC Depletion Calculations for the Rodded Assemblies in Batches 1, 2, 3, and $1 \mathrm{X}$ of Crystal River Unit 3

Document Identifier: BBA000000-01717-0200-00040 REV 00

Page 111 of 146

Table 7.3-28 Boron Letdown Data Provided to RLAYOUT for Cycle 3 of Crystal River Unit 3

\begin{tabular}{|c|c|}
\hline Exposure (EFPD) & Boron Concentration (ppm) \\
\hline 163.9 & 467 \\
\hline 174.0 & 432 \\
\hline 184.2 & 394 \\
\hline 212.9 & 324 \\
\hline 227.5 & 272 \\
\hline 246.4 & 229 \\
\hline 262.9 & 250 \\
\hline 283.8 & 190 \\
\hline 304.0 & 130 \\
\hline 322.0 & 86 \\
\hline
\end{tabular}

Table 7.3-29 Boron Letdown Data Provided to RLAYOUT for Cycle 7 of Crystal River Unit 3

\begin{tabular}{|c|c||}
\hline Exposure (EFPD) & Boron Concentration (ppm) \\
\hline 7.5 & 1478 \\
\hline 41.4 & 1405 \\
\hline 60.3 & 1367 \\
\hline 81.7 & 1333 \\
\hline 102.9 & 1290 \\
\hline 122.3 & 1245 \\
\hline 139.8 & 1204 \\
\hline 160.5 & 1167 \\
\hline 180.8 & 1102 \\
\hline 202.8 & 1040 \\
\hline 230.9 & 963 \\
\hline 251.2 & 898 \\
\hline 345.3 & 593 \\
\hline & \\
\hline
\end{tabular}


Table 7.3-29 Boron Letdown Data Provided to RLAYOUT for Cycle 7 of Crystal River Unit 3

\begin{tabular}{|c|c|}
\hline Exposure (EFPD) & Boron Concentration (ppm) \\
\hline 412.3 & 398 \\
\hline 459.8 & 260 \\
\hline 485.0 & 193 \\
\hline
\end{tabular}

Table 7.3-30 Boron Letdown Data Provided to RLAYOUT for Cycle 8 of Crystal River Unit 3

\begin{tabular}{|c|c|}
\hline Exposure (EFPD) & Boron Concentration (ppm) \\
\hline 11.2 & 1537 \\
\hline 52.4 & 1455 \\
\hline 78.0 & 1411 \\
\hline 111.4 & 1332 \\
\hline 154.4 & 1176 \\
\hline 194.8 & 1103 \\
\hline 234.6 & 999 \\
\hline 271.5 & 887 \\
\hline 338.0 & 701 \\
\hline 390.7 & 522 \\
\hline 445.7 & 394 \\
\hline 474.0 & 311 \\
\hline 513.1 & 216 \\
\hline & \\
\hline
\end{tabular}

Table 7.3-31 Boron Letdown Data for Cycle 9 of Crystal River Unit 3

\begin{tabular}{|c|c|}
\hline Exposure (EFPD) & Boron Concentration (ppm) \\
\hline 22.1 & 1608 \\
\hline 61.5 & 1535 \\
\hline 145.7 & 1329 \\
\hline 192.8 & 1201 \\
\hline
\end{tabular}


Title: CRC Depletion Calculations for the Rodded Assemblies in Batches 1, 2, 3, and $1 \mathrm{X}$ of Crystal River Unit 3 Document Identifier: BBA000000-01717-0200-00040 REV 00

Page 113 of 146

Table 7.3-31 Boron Letdown Data for Cycle 9 of Crystal River Unit 3

\begin{tabular}{|c|c|}
\hline Exposure (EFPD) & Boron Concentration (ppm) \\
\hline \hline 211.3 & 1157 \\
\hline 262.0 & 994 \\
\hline 303.7 & 869 \\
\hline 345.7 & 750 \\
\hline 397.9 & 577 \\
\hline 432.5 & 473 \\
\hline 452.4 & 412 \\
\hline 495.4 & 283 \\
\hline 543.4 & 136 \\
\hline
\end{tabular}

Some interesting behavior appears in the CRA nodal insertion history layouts presented in Tables 7.3-8 through 7.3-24. This interesting behavior refers to the axially staggered nodal CRA insertion locations in some of the irradiation steps that are defined. At first glance, the axial staggering of CRA nodal insertion locations in a given irradiation time step does not make any sense. This is true from a physical perspective based on knowledge of actual CRA design and reactor operation. However, certain approximations are utilized in performing the CRC depletion calculations as documented in this analysis which result in this strange presentation of CRA insertion histories. The following discussion describes the modeling techniques utilized in the CRC depletion calculations of this analysis which contribute to this staggered CRA nodal insertion phenomena.

In the SAS2H CRC depletion calculations, the time dependency of the CRA and APSRA insertion histories are treated on an integral exposure basis rather than on an actual physical irradiation history basis. This does not mean that the CRA and APSRA insertion history data utilized in the CRC depletion calculations of this analysis is not the actual insertion history data from the reactor. In fact, the CRA and APSRA insertion history data for each axial node in each statepoint depletion calculation is based on the true CRA and APSRA movement data obtained during the actual reactor operation. The implementation of this true measured data in the SAS2H CRC depletion calculations is based on modeling CRA and APSRA insertion durations (measured in EFPD) in each axial node of the assembly at the beginning of each CRC statepoint calculation. An average nodal power is utilized in each SAS2H CRC statepoint depletion calculation for a given axial node. This average nodal power is calculated based on the average nodal burnup at the beginning and end of the statepoint depletion calculation. Therefore, EFPD durations in a SAS2H CRC statepoint depletion calculation are calculated based on this average nodal power that is being utilized in the statepoint depletion calculation. The CRA nodal insertion durations (measured in EFPDs of exposure) will need to be modeled in SAS2H based on the assembly average nodal power. Due to the fact that the assembly average nodal power may be less than the actual assembly nodal power during a given period of core operation, the SAS2H insertion time of a CRA in a 
Title: CRC Depletion Calculations for the Rodded Assemblies in Batches 1, 2, 3, and $1 \mathrm{X}$ of Crystal River Unit 3

given axial node may vary, relative to the other assembly axial nodes, depending on the average power that is being utilized in the SAS2H calculation and the nodal exposure (EFPD) required with CRA inserted. This results in the staggered CRA insertion phenomena that is present in some of the data presented in Tables 7.3-8 through 7.3-24.

\subsection{The Commercial Reactor Assembly Follow Taskmaster (CRAFT) Code \& Usage}

The Commercial Reactor Assembly Follow Taskmaster (CRAFT) code directs the performance of assembly depletion and decay calculations relevant to CRC evaluations. The CRAFT code generates input decks for the SAS2H control module of the SCALE 4.3 modular code system based on userdefined input which describes the fuel assembly's specifications and irradiation history. Appropriate isotopic concentrations relevant to both the CRC evaluations containing the fuel assembly and subsequent depletion and decay calculations of the fuel assembly are extracted and stored by CRAFT as it generates and executes SAS2H cases required to simulate the complete fuel assembly irradiation history.

The CRAFT code is developed with a high degree of flexibility that provides for the depletion and decay of fuel assemblies with widely varying features under either standard or non-standard core operating procedures. The following listing describes some of the capabilities and usage of the CRAFT code.

- $\quad$ The CRAFT code generates and executes appropriate SAS2H cases required to perform a prescribed depletion and decay sequence for a fuel assembly. The depletion and decay sequence is orchestrated from the BOC statepoint calculation of the initial prescribed insertion cycle through the final statepoint calculation of the last prescribed insertion cycle. The CRAFT code extracts and saves fuel and burnable poison isotopics at each statepoint, including BOC statepoints, during the fuel assembly's depletion and decay sequence. A certain number of the generated isotopics in the depleted fuel composition obtained from a SAS2H calculation are not used in the initial charge composition to the next SAS2H calculation due to a lack of crosssection data in the specified cross-section library. The CRAFT code provides a listing of the fuel isotopics from the output of a SAS2H calculation which are not used in the initial charge to the next SAS2H calculation. The isotopics left out of the initial charge are fission products whose reactivity worth is small relative to the isotopics retained in the initial charge composition. The listing of excluded initial charge isotopics allows for a determination of the impact upon the reactivity worth of the initial fuel composition in the subsequent depletion calculation.

- Any assembly design may be analyzed within the bounds of the SAS2H control module through the use of the CRAFT code. This includes both PWR and BWR fuel assemblies.

- An axial blanket fuel modeling option is available in the CRAFT code. Any $\mathrm{UO}_{2}$ enrichment may be specified for the axial blanket fuel. The axial blanket fuel may be defined to exist in any of the CRC axial nodes which are defined for the CRAFT calculation.

- A spacer grid modeling technique is available with the CRAFT code. The modeling technique homogenizes the spacer grid material throughout the moderator of the fuel assembly by utilizing 
a user-defined spacer material and spacer material volume fraction in the moderator. The available spacer grid materials include the following-- ZIRC-4, INCONEL, SS316, SS316S, SS304, SS304S. Any volume fraction of spacer material in the moderator may be specified (including zero).

The fuel cladding, BPR cladding, axial power shaping rod (APSR) cladding, or control rod (CR) cladding in the CRAFT calculation may be designated as any of the following materials-- ZIRC4, INCONEL, SS316, SS316S, SS304, SS304S.

The insertion of a BPR assembly during the irradiation of the fuel assembly may be modeled in the CRAFT calculation. Up to 10 unique BPR assembly designs may be specified for use during the depletion of a fuel assembly. Any type of BPR assembly design may be specified. The default $\mathrm{BP}$ material for use in CRAFT calculation is $\mathrm{Al}_{2} \mathrm{O}_{3}-\mathrm{B}_{4} \mathrm{C}$. Any arbitrary BP material may be specified for use in a BPR assembly design. A maximum of 10 unique BP materials may be specified. A maximum of 20 unique elements or isotopes may be specified in any given BP material. A BPR assembly may be inserted in any reactor cycle specified in the CRAFT calculation. Only one BPR assembly design may be specified per cycle. The position of the BPR assembly in the fuel assembly is specified by identifying the top and bottom axial nodes of the BP material. The BPR assembly remains fixed during a given reactor cycle. The depletion of the BP material is tracked during the CRAFT calculation. The appropriate depleted BP material is utilized in statepoint calculations following the BOC to statepoint 2 calculation for a given reactor cycle. Depleted BP material isotopic concentrations are also retained for use in subsequent mid-cycle statepoint reactivity calculations which may be performed as part of the CRC evaluation process.

The insertion of a CR assembly during the irradiation of the fuel assembly may be modeled in the CRAFT calculation. Up to 10 unique CR assembly designs may be specified for use during the depletion of a fuel assembly. Any type of $\mathrm{CR}$ assembly design may be specified. Any arbitrary $\mathrm{CR}$ absorber material may be specified for use in a $\mathrm{CR}$ assembly design. A maximum of 10 unique $\mathrm{CR}$ absorber materials may be specified. A maximum of 10 unique elements or isotopes may be specified in any given CR absorber material. A CR assembly may be inserted in any reactor cycle specified in the CRAFT calculation. Multiple $C R$ assembly designs may be specified per cycle. The position of the CR assembly in the fuel assembly is specified by identifying the top and bottom axial nodes of sections of the fuel assembly which contain the $\mathrm{CR}$ absorber material. The $\mathrm{CR}$ assembly position may be changed between each irradiation step of a SAS2H calculation generated by CRAFT. The CR assembly design may also be changed between any two CRC statepoint depletion calculations in a given reactor cycle.

- The insertion of an APSR assembly during the irradiation of the fuel assembly may be modeled in the CRAFT calculation. Up to 10 unique APSR assembly designs may be specified for use during the depletion of a fuel assembly. Any type of APSR assembly design may be specified. Any arbitrary APSR absorber material may be specified for use in an APSR assembly design. A maximum of 10 unique APSR absorber materials may be specified. A maximum of 10 unique elements or isotopes may be specified in any given APSR absorber material. An APSR assembly 
Title: CRC Depletion Calculations for the Rodded Assemblies in Batches 1, 2, 3, and IX of Crystal River Unit 3

may be inserted in any reactor cycle specified in the CRAFT calculation. Multiple APSR assembly designs may be specified per cycle. The position of the APSR assembly in the fuel assembly is specified by identifying the top and bottom axial nodes of the APSR absorber material. The APSR assembly position may be changed between each irradiation step of a SAS2H calculation generated by CRAFT. The APSR assembly design may also be changed between any statepoint calculations in a given reactor cycle. For any APSRA modeled, the APSR follow rods are modeled in the axial region above the absorbing region of the APSR. The APSR follow rod material may be specified as a cladding material in the CRAFT input deck.

- A fuel assembly may be inserted in a maximum of 10 reactor cycles during a CRAFT calculation.

- A maximum of 20 statepoints (BOC is always considered a statepoint) may be specified in any given reactor cycle in a CRAFT calculation.

- A maximum of 23 irradiation steps of variable duration may be specified in any given SAS2H statepoint calculation to be generated during a CRAFT calculation.

- A maximum of 50 axial nodes may be specified in the CRC nodal format for use in a CRAFT calculation. Each axial node may have a unique height.

- The CRAFT code utilizes a user-defined input format for fuel temperature, moderator specific volume, and burnup data. The input data must be specified for each axial node in a user-defined nodal format of up to 50 nodes of arbitrary height. The total assembly active fuel height for the input data descriptions may be different than that specified in the CRC nodal format. Depending on the users needs, the fuel temperature, moderator specific volume and burnup input data may be specified in a different nodal format each time an assembly set of this input data is provided. Nominal fuel temperature input data representing full-power reactor operation must be provided in units of degrees Fahrenheit for each node in each statepoint calculation to be generated by the CRAFT calculation. Nominal moderator specific volume input data representing full-power reactor operation must be provided in units of cubic feet per pound for each node in each statepoint calculation to be generated by the CRAFT calculation. The nodal average burnup input data must be provided in units of GWd/MTU for each node at each statepoint including the BOC statepoint. All burnup input data that is specified must be cumulative from the initial insertion of the fuel assembly in the reactor.

- A continuation CRAFT calculation for an assembly may be initiated from any statepoint in any reactor cycle if all of the nodal consolidated output files ("*.cut" files) from the statepoint calculation immediately preceding the continuation calculation exist in the CRAFT execution directory.

Additional information on the CRAFT code is provided in the CRAFT user information in Attachment I of this analysis. Instructions on how to develop CRAFT input decks and execute CRAFT calculations are also provided in Attachment I. This attachment also discusses specific modeling procedures and details relevant to the SAS2H fuel assembly depletion calculations which are generated by CRAFT. 


\subsection{Input \& Output Filename Descriptions for CRAFT and SAS2H}

The CRAFT code generates five types of files identified as either "*.input", "*.output", "*.cut", "*.msgs", or "*.notes", where the "*" is the base file set identifier for the statepoint calculation of interest. The "*.cut" and "*.notes" files are the only files that must be retained for CRC evaluation and documentation purposes. All files are generated in the working directory in which the CRAFT calculation is performed.

All CRAFT generated filenames utilize the following format-- " $\{$ Base File Set Identifier $\}$. suffix $\}$ ". Where the suffix corresponds to one of the five file types previously mentioned, and the base file set identifier is a 25 character name containing essential information necessary to delineate one CRAFT generated SAS2H calculation from another.

The base file set identifier for a statepoint calculation contains the following information:

1) reactor identifier (three character);

2) one-eighth core symmetry assembly number in current reactor cycle (two digit);

3) axial node number (node 1 is always the top node) (two digit);

4) reactor cycle number in which the SAS2H calculation starts (two character);

5) EFPD statepoint at which the SAS2H calculation starts (three digit);

6) reactor cycle number in which the SAS2H calculation ends (two character);

7) EFPD statepoint at which the SAS2H calculation ends (three digit).

The format of the base file set identifier is as follows where the numbers identified as \#\{number\} correspond to one of the seven items previously listed-- \#1 A \#2 N\#3 DC \#4 T\#5 AC \#6 T \#7. The letters contained in the base file set identifier are presented explicitly as shown in the previous format. The base file set identifier does not contain any spaces.

The "*.input" files contain a CRAFT generated SAS2H input deck. The "*.output" files contain a complete SAS2H calculation output file. The "*.cut" files contain the corresponding SAS2H input deck followed by an output extraction, from the final ORIGEN-S pass of the SAS2H calculation, which contains data relevant to CRC evaluations. The "*.msgs" files contain the standard run-time messages associated with the SAS2H calculation. The "*.notes" files contain a listing of the isotopes and their concentration which were left behind in generating the initial charge fuel composition for a continuation SAS2H calculation. The "*.notes" files are only created for CRAFT generated SAS2H calculations which represent continuation depletion and decay calculations. The "*.cut" and "*.notes" files contain all of the information which is required to perform CRC evaluations or repeat calculations as necessary for quality assurance purposes. The remainder of the CRAFT generated files may be discarded once the "*.cut" and "*.notes" files have been produced correctly. 
Title: CRC Depletion Calculations for the Rodded Assemblies in Batches 1, 2, 3, and 1 X of Crystal River Unit 3 Document Identifier: BBA000000-01717-0200-00040 REV 00

Page 118 of 146

\subsection{Rodded Assembly Depletion Calculations for Fuel Batches 1, 2, 3, and 1X of Crystal River Unit 3}

Depletion calculations for 18 rodded fuel assemblies from fuel batches 1,2,3, and $1 \mathrm{X}$ of Crystal River Unit 3 are documented in this analysis. The depleted fuel and depleted burnable poison isotopics for these fuel assemblies must be calculated at a number of statepoints during several reactor cycles of Crystal River Unit 3 for use in subsequent $\mathrm{CRC}$ reactivity calculations. The assembly depletion calculations documented in this analysis are applicable to CRC statepoints in Cycles-1A, $-1 \mathrm{~B},-2,-3,-7$, -8 , and -9 of Crystal River Unit 3. Table 7.6-1 identifies the CRC statepoint EFPD values in each of these cycles for which isotopic compositions are required.

Table 7.6-1 CRC Statepoint EFPD Values Relevant to Rodded Assembly Depletion Calculations for Fuel Batches 1, 2, 3, and 1X of Crystal River Unit 3

\begin{tabular}{|c|c|}
\hline Crystal River Unit 3 Reactor Cycle & CRC Statepoint EFPD Values \\
\hline $1 \mathrm{~A}$ & 0.0 (BOC) \\
\hline 1B & $0.0(\mathrm{BOC})$ \\
\hline $1 \mathrm{~B}$ & 142.2 \\
\hline 2 & $0.0(\mathrm{BOC})$ \\
\hline 3 & 0.0 (BOC) \\
\hline 3 & 168.5 \\
\hline 3 & 250.0 \\
\hline 7 & $0.0(\mathrm{BOC})$ \\
\hline 7 & 260.3 \\
\hline 7 & 291.0 \\
\hline 7 & 319.0 \\
\hline 7 & 462.3 \\
\hline 7 & 479.0 \\
\hline 8 & $0.0(\mathrm{BOC})$ \\
\hline 8 & 97.6 \\
\hline 8 & 139.8 \\
\hline 8 & 404.0 \\
\hline
\end{tabular}


Title: CRC Depletion Calculations for the Rodded Assemblies in Batches 1, 2, 3, and $1 \mathrm{X}$ of Crystal River Unit 3

\begin{tabular}{|c|c|}
\hline Crystal River Unit 3 Reactor Cycle & CRC Statepoint EFPD Values \\
\hline 8 & 409.6 \\
\hline 8 & 515.5 \\
\hline 9 & $0.0(\mathrm{BOC})$ \\
\hline 9 & 158.8 \\
\hline 9 & 219.0 \\
\hline 9 & 363.1 \\
\hline
\end{tabular}

CRAFT input decks for each of the fuel assemblies identified in Tables 4.1.6-1 and 4.1.7-1 were developed and executed such that their depleted fuel and depleted burnable poison (if applicable) isotopic concentrations were retained at each of the CRC statepoints identified in Table 7.6-1 for which a particular assembly is inserted. The CRAFT input decks were developed in accordance with the instructions presented in Sections 5 and 7 of Attachment I of this analysis. SAS2H modeling features incorporated in the depletion calculations of this analysis are described in Attachment I. The CRAFT input decks for the assembly depletions documented in this analysis are provided in Attachments IV through XXI, as documented in Section 9.

The SAS2H input decks generated for the various depletion calculations have similar structures depending on the characteristics of the fuel assembly axial node that is being depleted. The following listing presents the base SAS2H input deck descriptions.

- Fuel assembly axial node containing empty guide tubes

- Fuel assembly axial node containing an absorbing BPRA inserted in the guide tubes

- Fuel assembly axial node (top node) containing a non-absorbing BPRA region inserted in the guide tubes

- Fuel assembly axial node containing a CRA insertion in the guide tubes (with or without CRA removal during the depletion calculation)

- Fuel assembly axial node containing an APSRA insertion in the guide tubes (with or without APSRA removal and/or APSRA follow rod region insertion during the depletion calculation)

All of the SAS2H input decks generated by CRAFT in this analysis will correspond to one of the aforementioned base SAS2H input decks depending on the assembly characteristics being modeled in the specific depletion calculation. The material compositions of the fuel, burnable absorber, and moderator are modified for each SAS2H case depending on the depleted material compositions at the beginning of the SAS2H case and the irradiation parameters for the SAS2H case as defined in the CRAFT input deck. The material specifications for the fuel and burnable absorber have different formats in the SAS2H input 
decks depending on whether the depletion case represents the initial depletion calculation for the assembly axial node or a continuation depletion calculation for the axial node utilizing previously calculated fuel and burnable poison (if applicable) isotopics for the initial charge compositions.

The following ten example SAS2H input decks are presented to demonstrate the modeling techniques employed by CRAFT in generating appropriate SAS2H depletion cases for the fuel assembly depletion calculations relevant to this analysis. These example input decks are actual SAS2H input decks which were generated and executed during the depletion of fuel assemblies relevant to CRC evaluations (the assemblies from which these examples are obtained may not be documented in this analysis). Each section of the SAS2H input decks are modified as necessary to perform each depletion calculation according to the pertinent information provided in the CRAFT input deck.

\section{SAS2H Depletion Input Deck Example 1: BOL Depletion Calculation for a B\&W Fuel Assembly Axial Node Containing 208 Fuel Rods, 16 Empty Guide Tubes, and 1 Empty Instrument Tube}

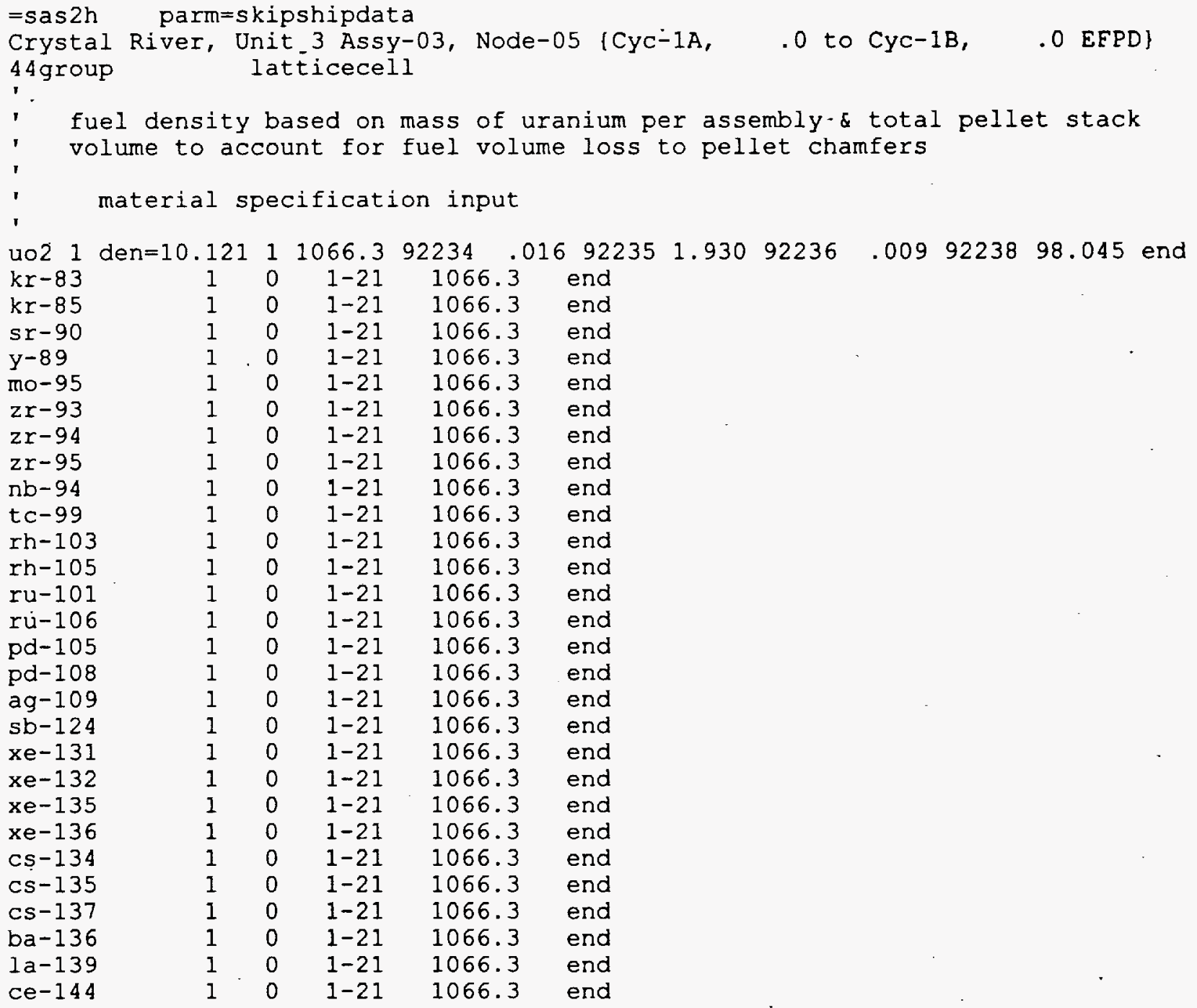


Title: CRC Depletion Calculations for the Rodded Assemblies in Batches 1, 2, 3, and 1X of Crystal River Unit 3 Document Identifier: BBA000000-01717-0200-00040 REV 00

Page 121 of 146

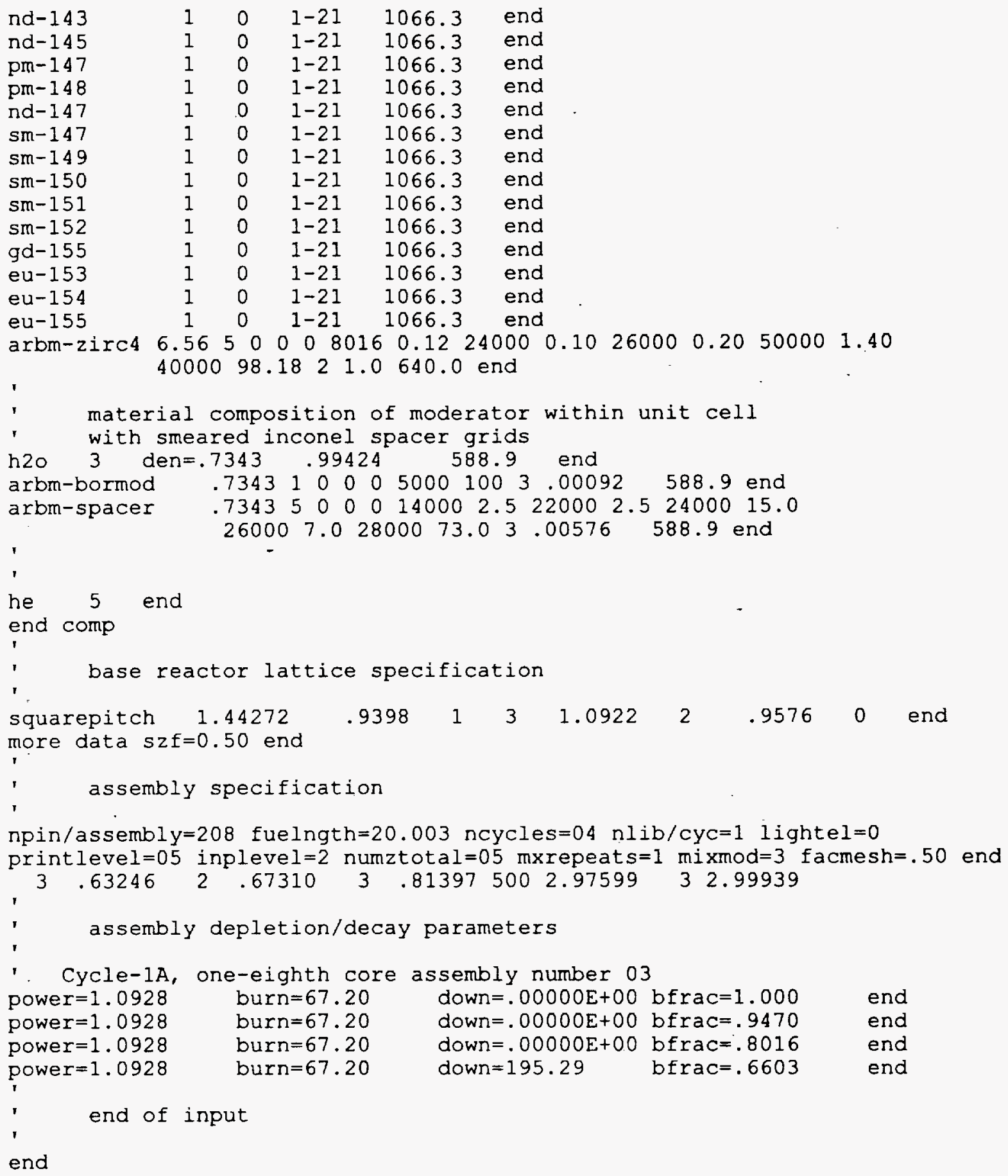

SAS2H Depletion Input Deck Example 2: Continuation Depletion Calculation for a B\&W Fuel Assembly Axial Node Containing 208 Fuel Rods, 16 Empty Guide Tubes, and 1 Empty Instrument Tube 


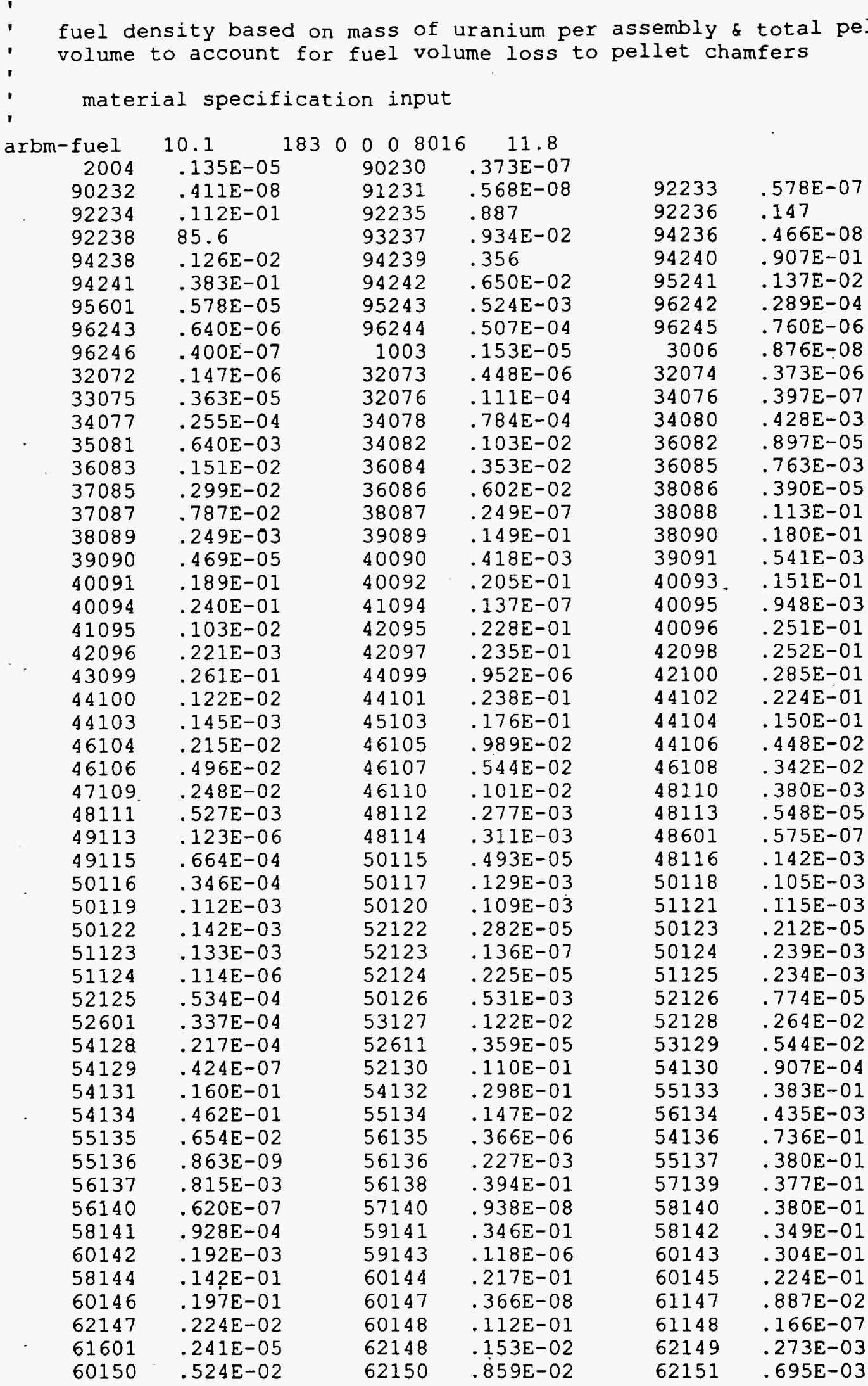


Title: CRC Depletion Calculations for the Rodded Assemblies in Batches 1, 2, 3, and $1 \mathrm{X}$ of Crystal River Unit 3 Document Identifier: BBA000000-01717-0200-00040 REV 00

Page 123 of 146

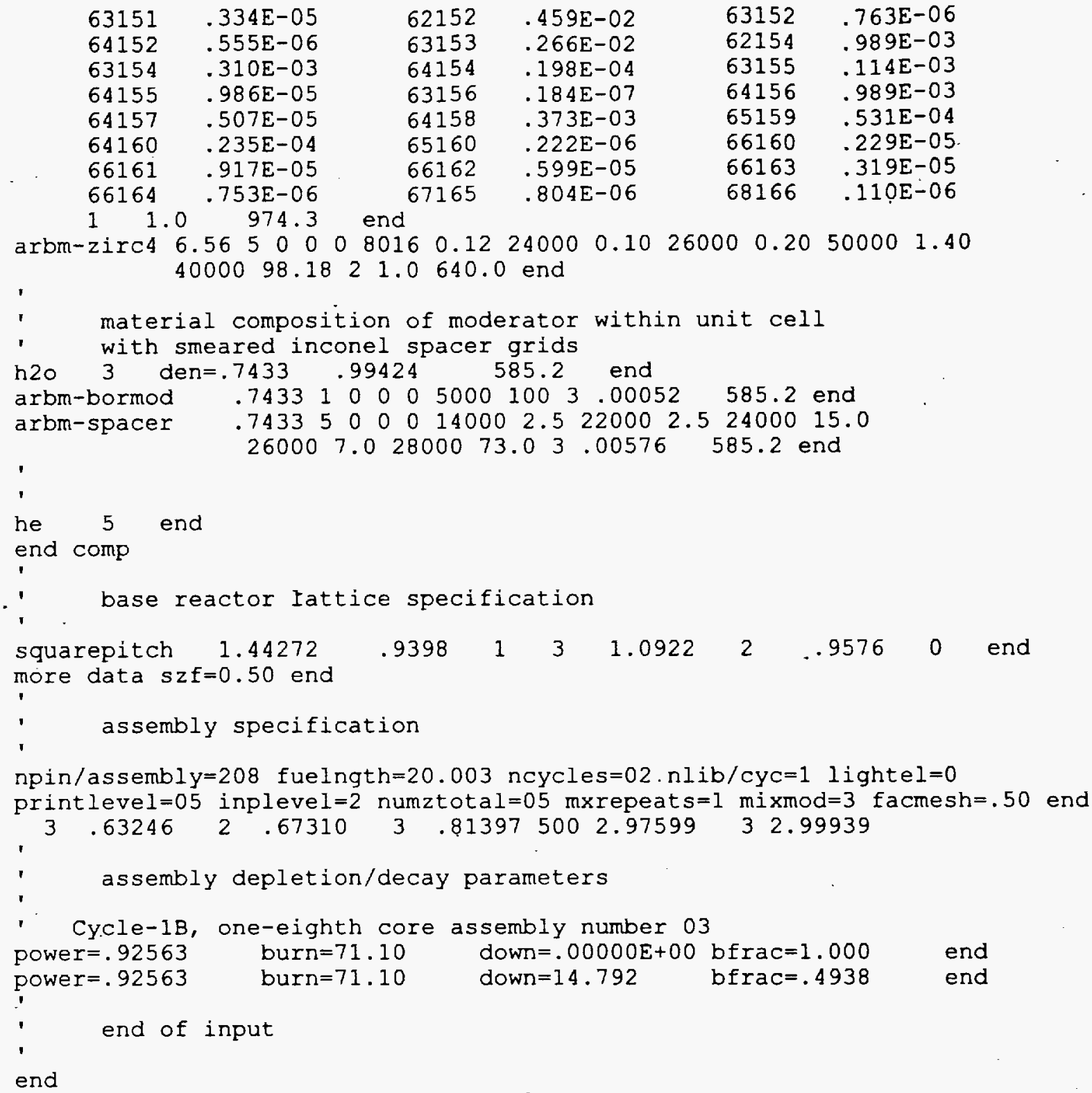

SAS2H Depletion Input Deck Example 3: BOL Depletion Calculation for a B\&W Fuel Assembly Top Axial Node Containing 208 Fuel Rods, 16 Guide Tubes with BPR's Inserted, and 1 Empty Instrument Tube

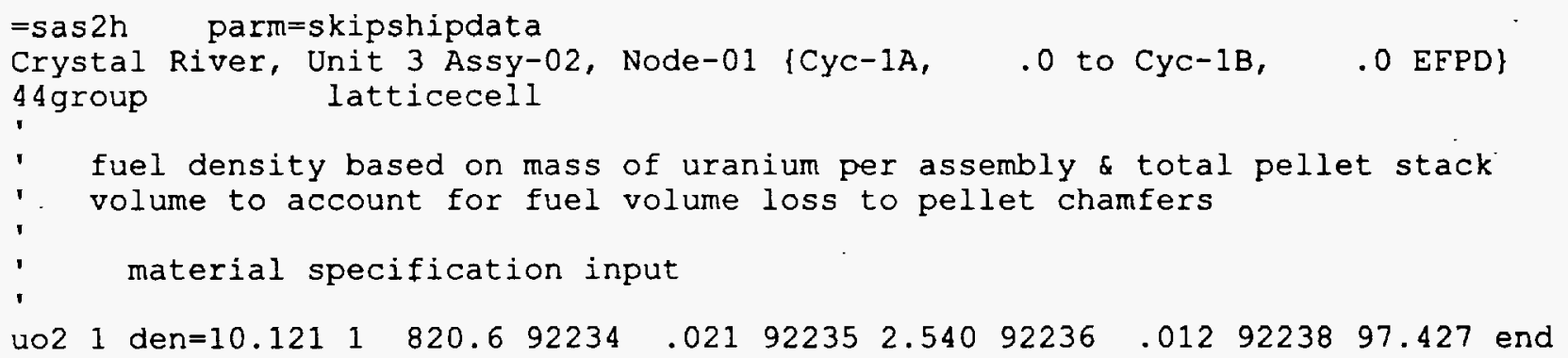


Title: CRC Depletion Calculations for the Rodded Assemblies in Batches 1, 2, 3, and $1 \mathrm{X}$ of Crystal River Unit 3 Document Identifier: BBA000000-01717-0200-00040 REV 00

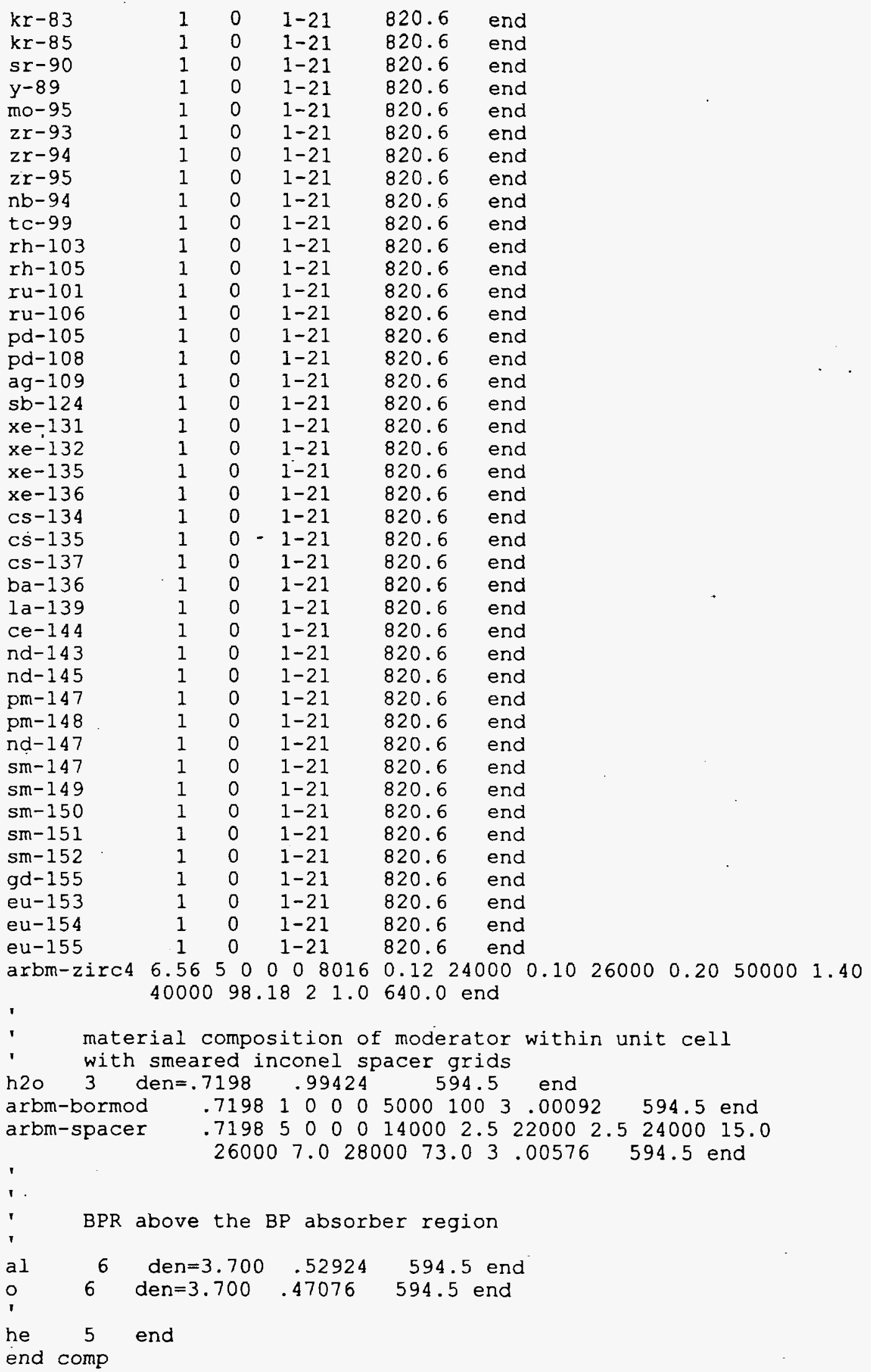


Title: CRC Depletion Calculations for the Rodded Assemblies in Batches 1, 2, 3, and $1 \mathrm{X}$ of Crystal River Unit 3 Document Identifier: BBA000000-01717-0200-00040 REV 00

Page 125 of 146

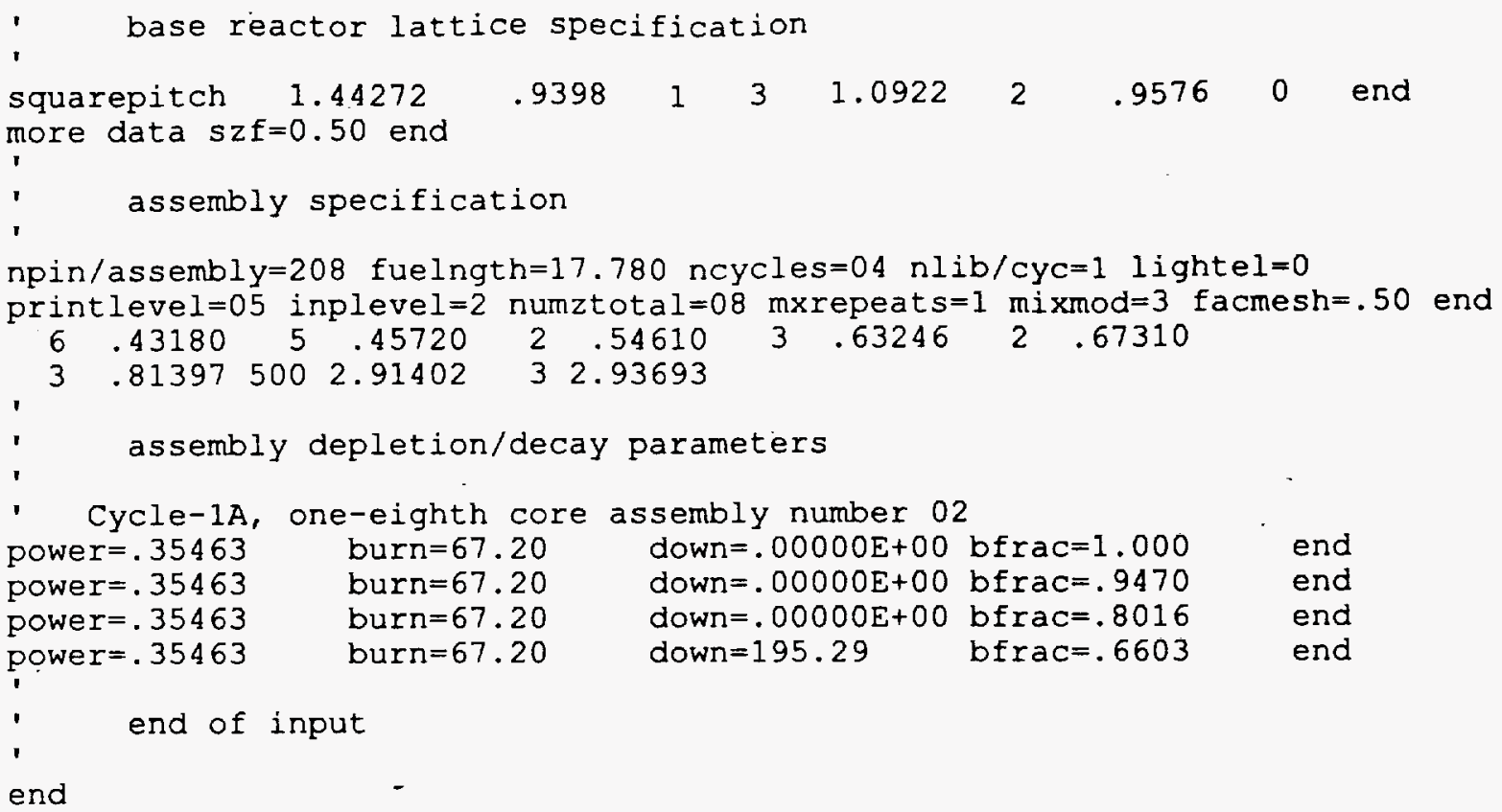

\section{SAS2H Depletion Input Deck Example 4: Continuation Depletion Calculation for a B\&W Fuel Assembly Top Axial Node Containing 208 Fuel Rods, 16 Guide Tubes with BPR's Inserted, and 1 Empty Instrument Tube}

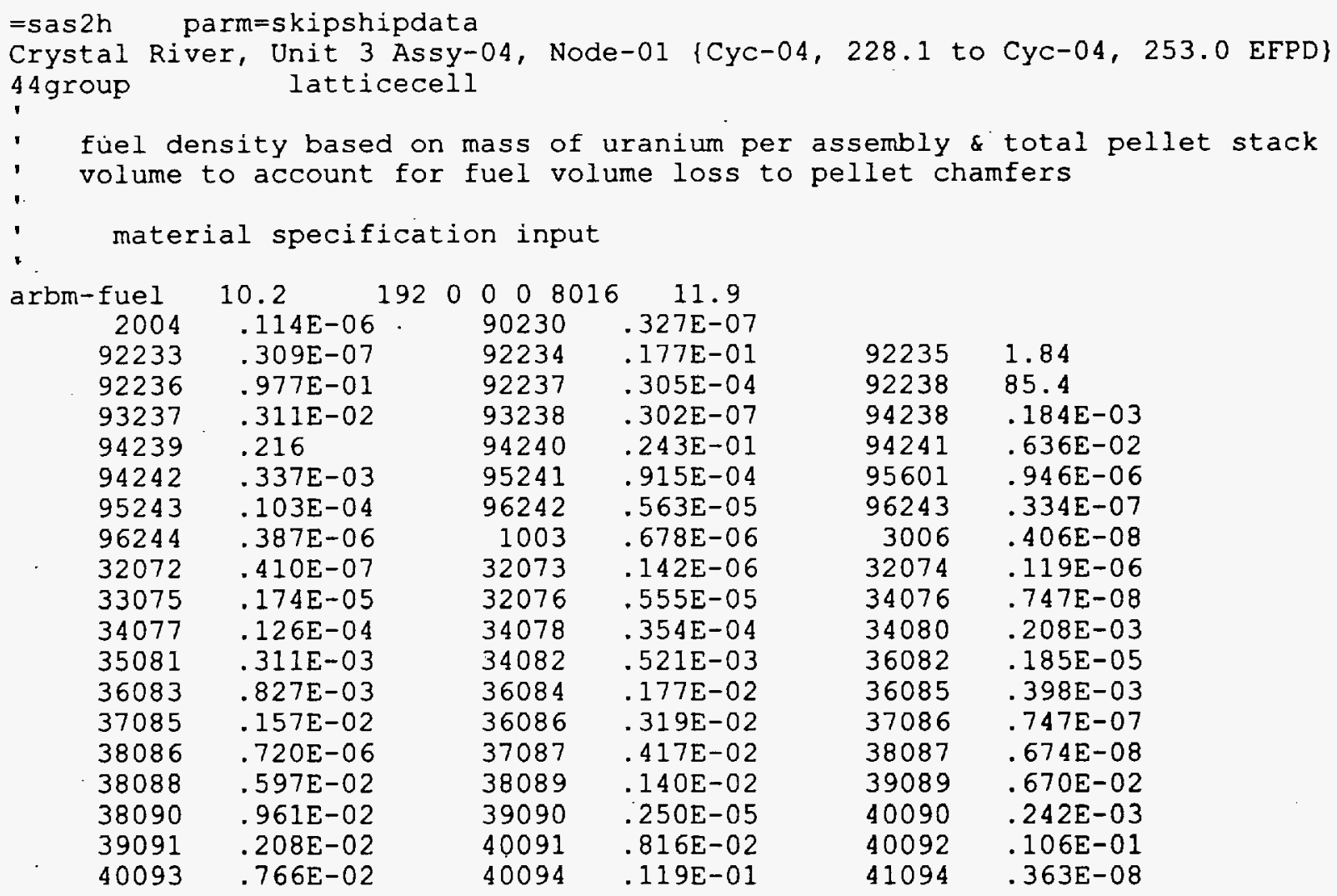




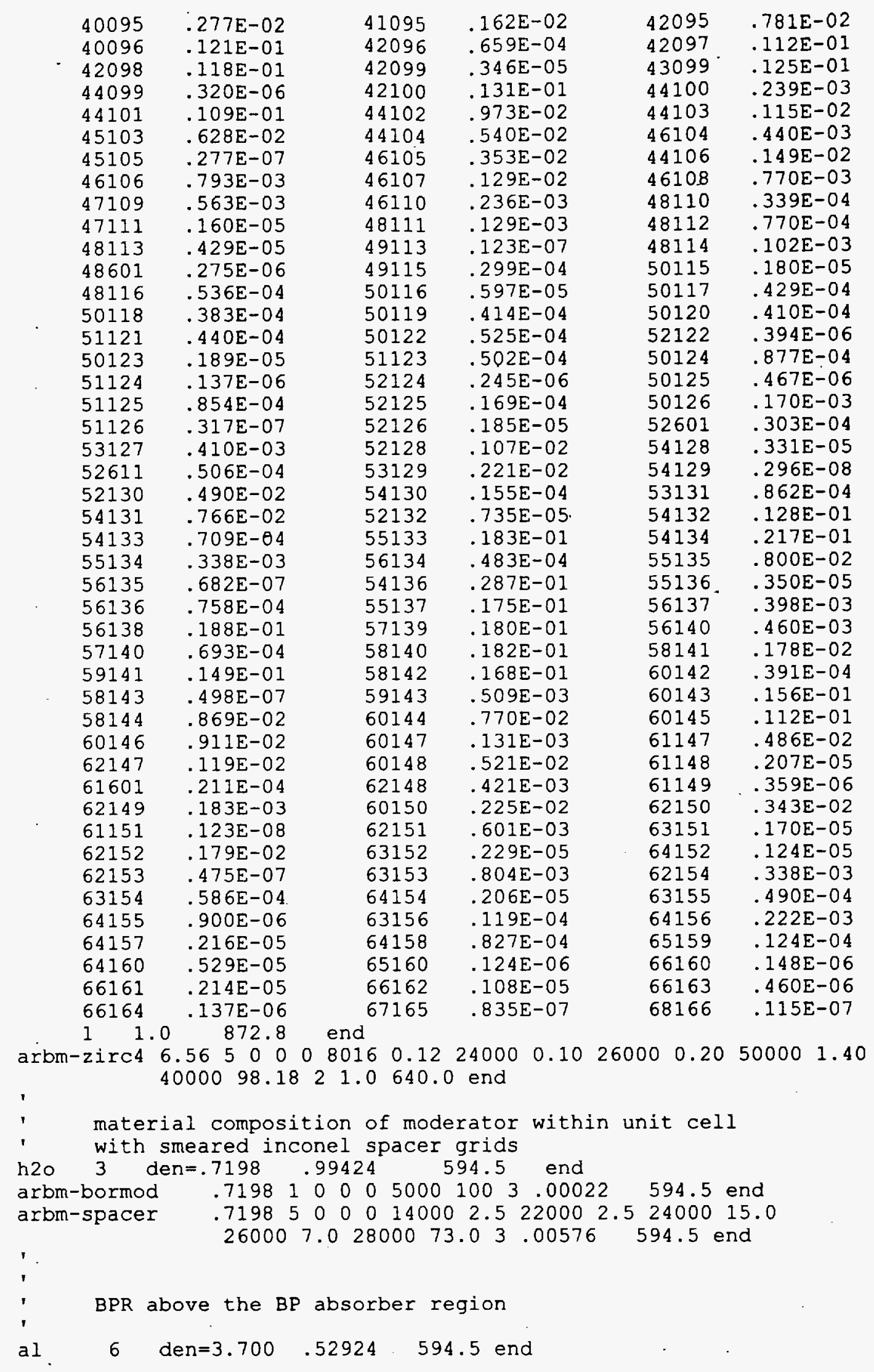


Title: CRC Depletion Calculations for the Rodded Assemblies in Batches 1, 2, 3, and $1 \mathrm{X}$ of Crystal River Unit 3 Document Identifier: BBA000000-01717-0200-00040 REV 00

Page 127 of 146

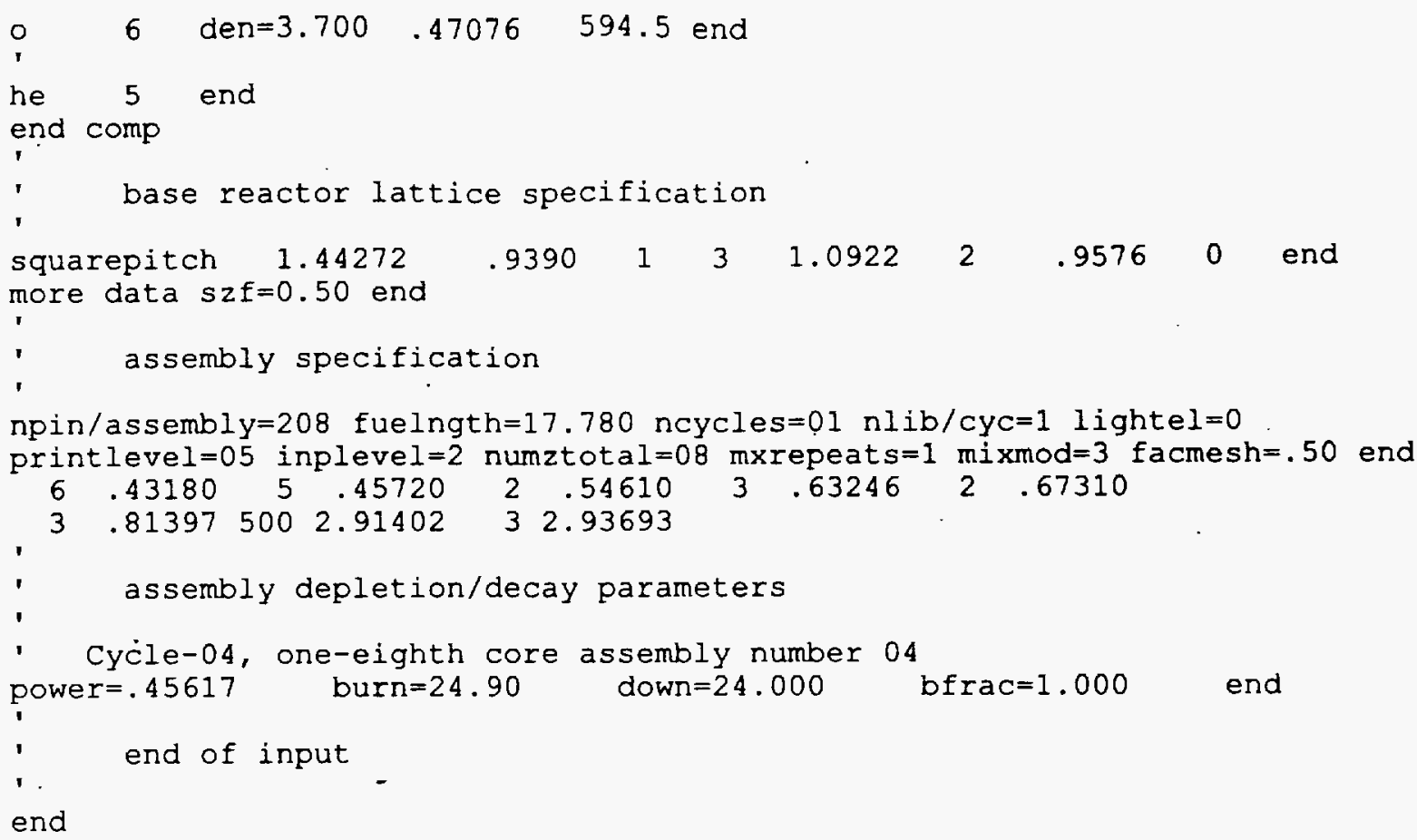

SAS2H Depletion Input Deck Example 5: BOL Depletion Calculation for a B\&W Fuel Assembly Axial Node (Other than Top Node) Containing 208 Fuel Rods, 16 Guide Tubes with BPR's Inserted, and 1 Empty Instrument Tube

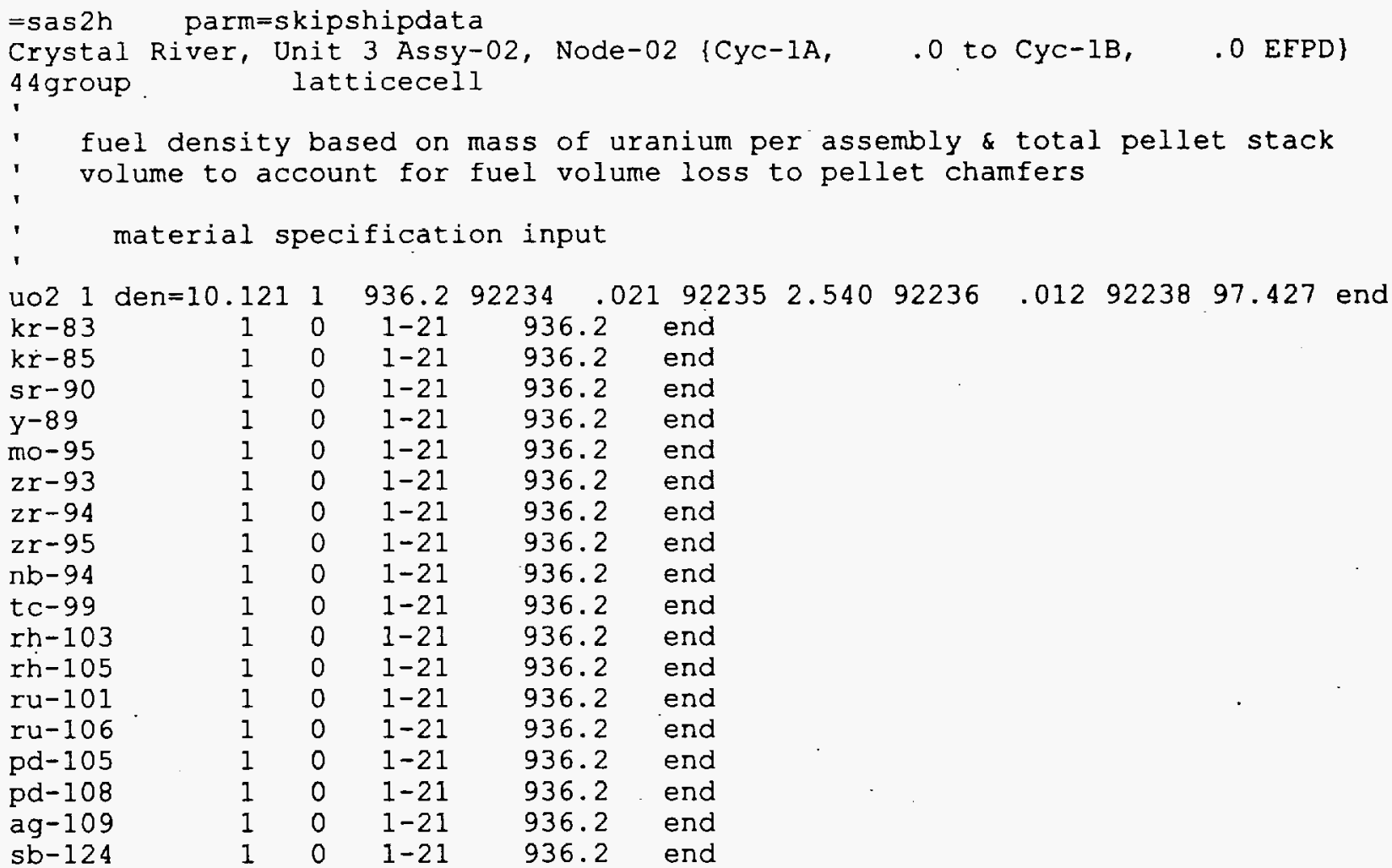


Title: CRC Depletion Calculations for the Rodded Assemblies in Batches 1, 2, 3, and $1 \mathrm{X}$ of Crystal River Unit 3

$\begin{array}{llllll}\text { xe-131 } & 1 & 0 & 1-21 & 936.2 & \text { end } \\ \text { xe-132 } & 1 & 0 & 1-21 & 936.2 & \text { end } \\ \text { xe-135 } & 1 & 0 & 1-21 & 936.2 & \text { end } \\ \text { xe-136 } & 1 & 0 & 1-21 & 936.2 & \text { end } \\ \text { cs-134 } & 1 & 0 & 1-21 & 936.2 & \text { end } \\ \text { cs-135 } & 1 & 0 & 1-21 & 936.2 & \text { end } \\ \text { cs-137 } & 1 & 0 & 1-21 & 936.2 & \text { end } \\ \text { ba-136 } & 1 & 0 & 1-21 & 936.2 & \text { end } \\ 1 \mathrm{a}-139 & 1 & 0 & 1-21 & 936.2 & \text { end } \\ \text { ce-144 } & 1 & 0 & 1-21 & 936.2 & \text { end } \\ \text { nd-143 } & 1 & 0 & 1-21 & 936.2 & \text { end } \\ \text { nd-145 } & 1 & 0 & 1-21 & 936.2 & \text { end } \\ \text { pm-147 } & 1 & 0 & 1-21 & 936.2 & \text { end } \\ \text { pm-148 } & 1 & 0 & 1-21 & 936.2 & \text { end } \\ \text { nd-147 } & 1 & 0 & 1-21 & 936.2 & \text { end } \\ \text { sm-147 } & 1 & 0 & 1-21 & 936.2 & \text { end } \\ \text { sm-149 } & 1 & 0 & 1-21 & 936.2 & \text { end } \\ \text { sm-150 } & 1 & 0 & 1-21 & 936.2 & \text { end } \\ \text { sm-151 } & 1 & 0 & 1-21 & 936.2 & \text { end } \\ \text { sm-152 } & 1 & 0 & 1-21 & 936.2 & \text { end } \\ \text { gd-155 } & 1 & 0 & 1-21 & 936.2 & \text { end } \\ \text { eu-153 } & 1 & 0 & 1-21 & 936.2 & \text { end } \\ \text { eu-154 } & 1 & 0 & 1-21 & 936.2 & \text { end } \\ \text { eu-155 } & 1 & 0 & 1-21 & 936.2 & \text { end }\end{array}$

arbm-zirc4 $6.56 \quad 5 \quad 0 \quad 0 \quad 0 \quad 8016 \quad 0.12 \quad 24000 \quad 0.10 \quad 26000 \quad 0.20 \quad 50000 \quad 1.40$

4000098.1821 .0640 .0 end

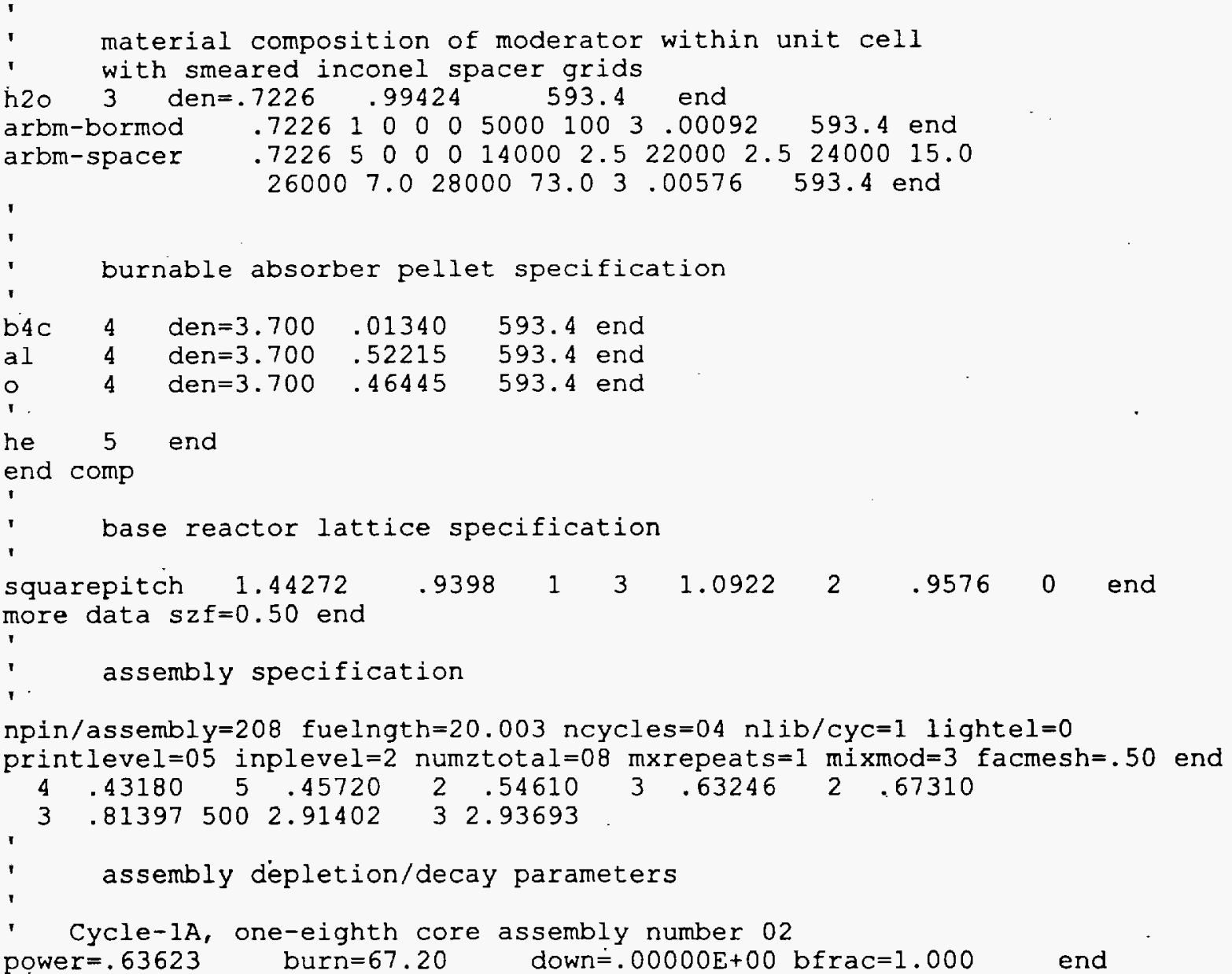


Title: CRC Depletion Calculations for the Rodded Assemblies in Batches 1, 2, 3, and $1 \mathrm{X}$ of Crystal River Unit 3 Document Identifier: BBA000000-01717-0200-00040 REV 00

Page 129 of 146

$\begin{array}{llllll}\text { power }=.63623 & \text { burn=67.20 } & \text { down=.00000E+00 bfrac }=.9470 & \text { end } \\ \text { power }=.63623 & \text { burn=67.20 } & \text { down=.00000E+00 bfrac }=.8016 & \text { end } \\ \text { power }=.63623 & \text { burn=67.20 } & \text { down=195.29 } & \text { bfrac }=.6603 & \text { end } \\ \text { ind of input } & & & & \end{array}$

\section{SAS2H Depletion Input Deck Example 6: Continuation Depletion Calculation for a B\&W Fuel Assembly Axial Node (Other than Top Node) Containing 208 Fuel Rods, 16 Guide Tubes with BPR's Inserted, and 1 Empty Instrument Tube}

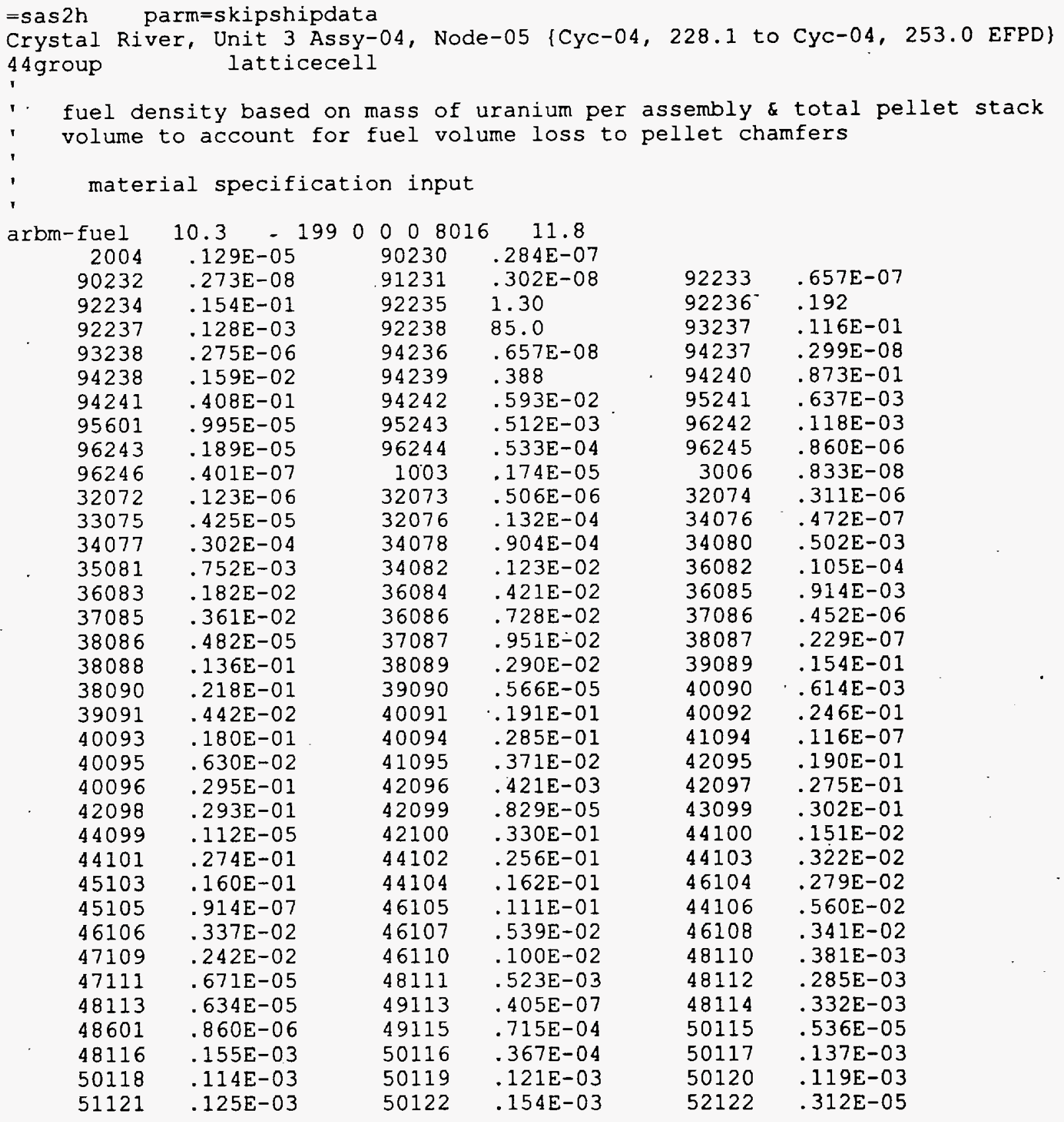




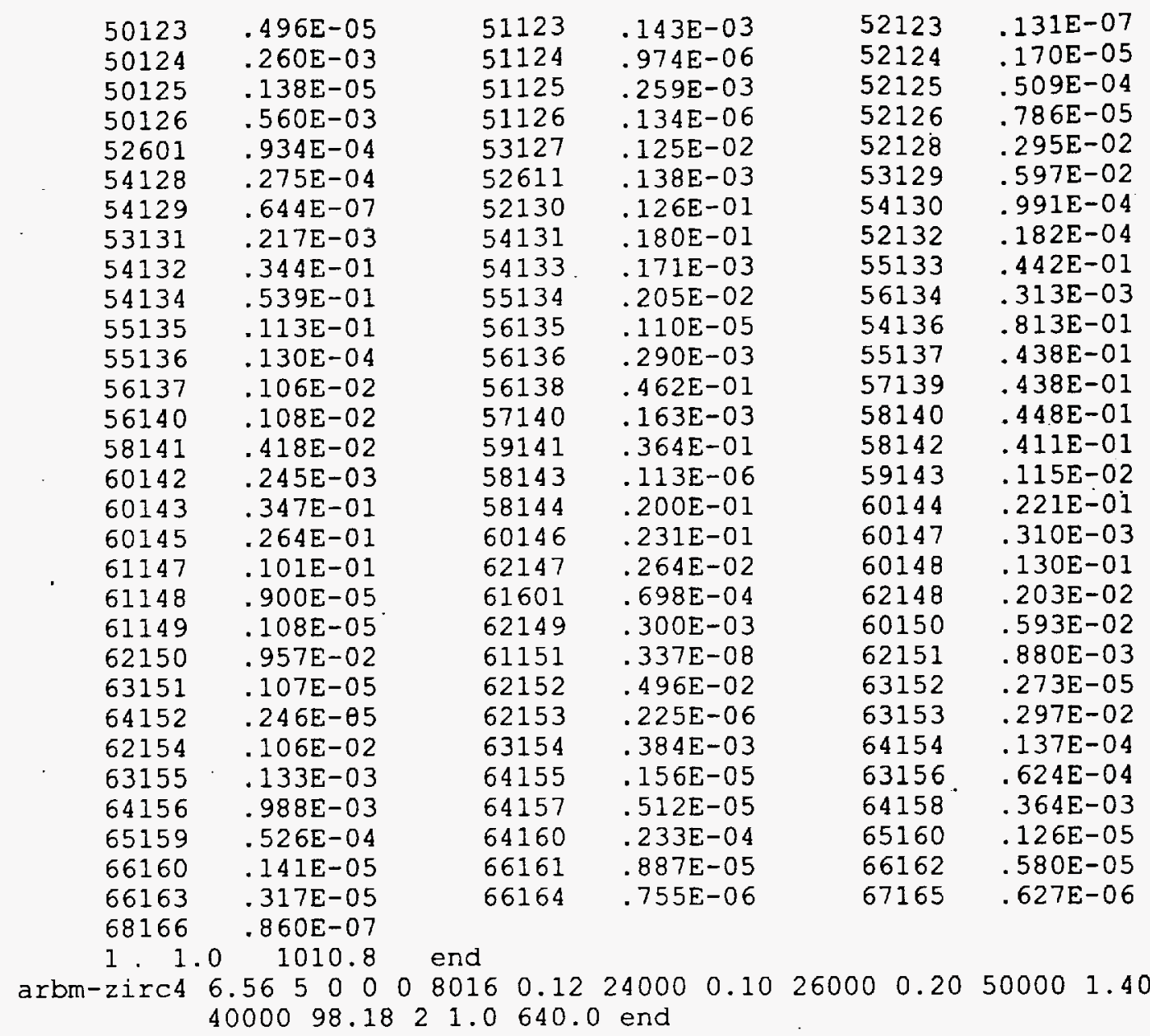

burnable absorber pellet specification

$$
\begin{array}{cccc}
\text { arbm-bp } & 3.6995000 & \\
5010 & .225 \mathrm{E}-02 & \\
5011 & .140 & \\
6012 & .435 \mathrm{E}-01 & \\
13027 & 52.827 \quad 8016 & 46.987 \\
41.0 & 588.9 \text { end } &
\end{array}
$$

he 5 end

end comp

base reactor lattice specification

$\begin{array}{llllllllll}\text { squarepitch } & 1.44272 & .9390 & 1 & 3 & 1.0922 & 2 & .9576 & 0 & \text { end }\end{array}$ more data $s z f=0.50$ end 
Title: CRC Depletion Calculations for the Rodded Assemblies in Batches 1, 2, 3, and $1 \mathrm{X}$ of Crystal River Unit 3

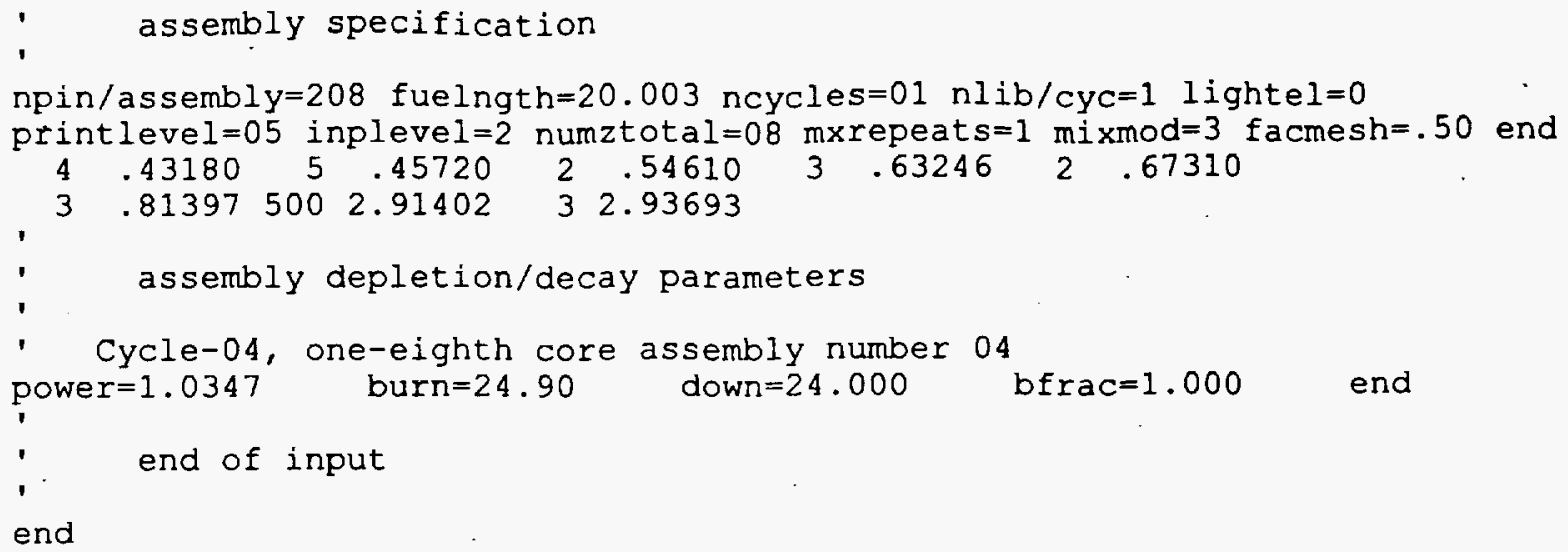

SAS2H Depletion Input Deck Example 7: BOL Depletion Calculation for a B\&W Fuel Assembly Axial Node Containing 208 Fuel Rods, 16 Guide Tubes with a 16 Rod CRA Inserted for a Portion of the Depletion, and 1 Empty (Water-filled) Instrument Tube

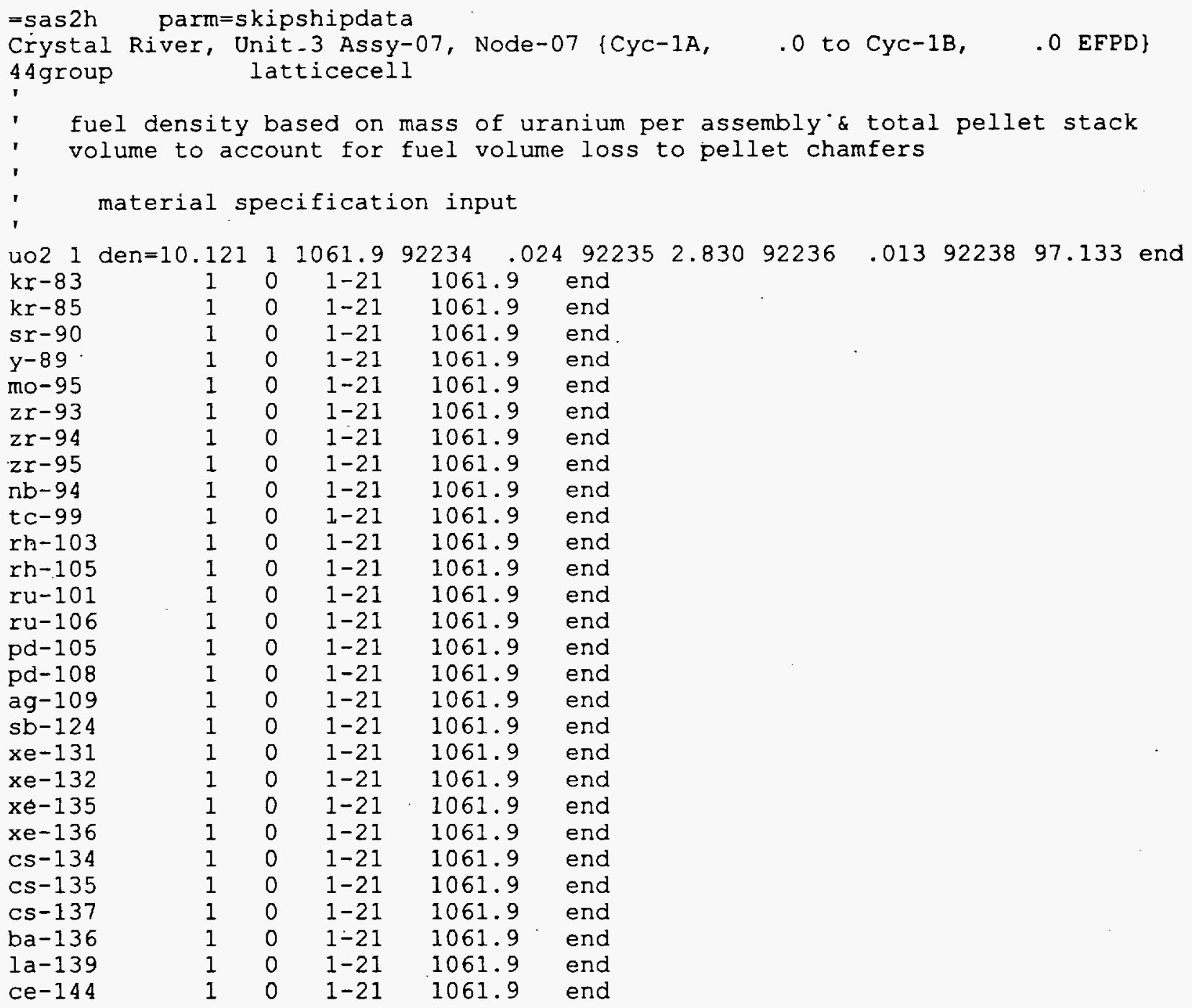


Title: CRC Depletion Calculations for the Rodded Assemblies in Batches 1, 2, 3, and $1 \mathrm{X}$ of Crystal River Unit 3 Document Identifler: BBA000000-01717-0200-00040 REV 00

Page 132 of 146

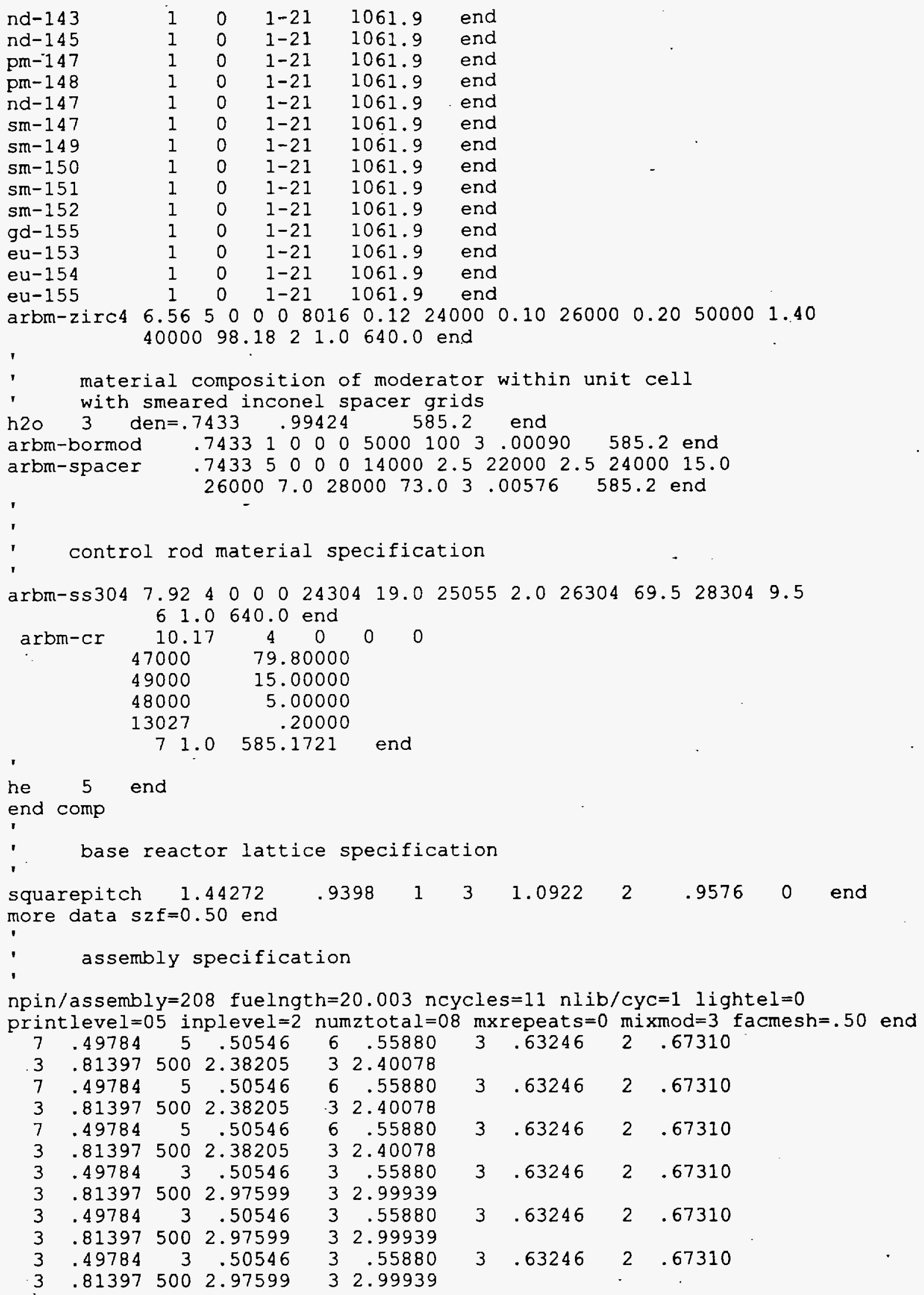


Title: CRC Depletion Calculations for the Rodded Assemblies in Batches 1, 2, 3, and $1 \mathrm{X}$ of Crystal River Unit 3 Document Identifier: BBA000000-01717-0200-00040 REV 00

Page 133 of 146

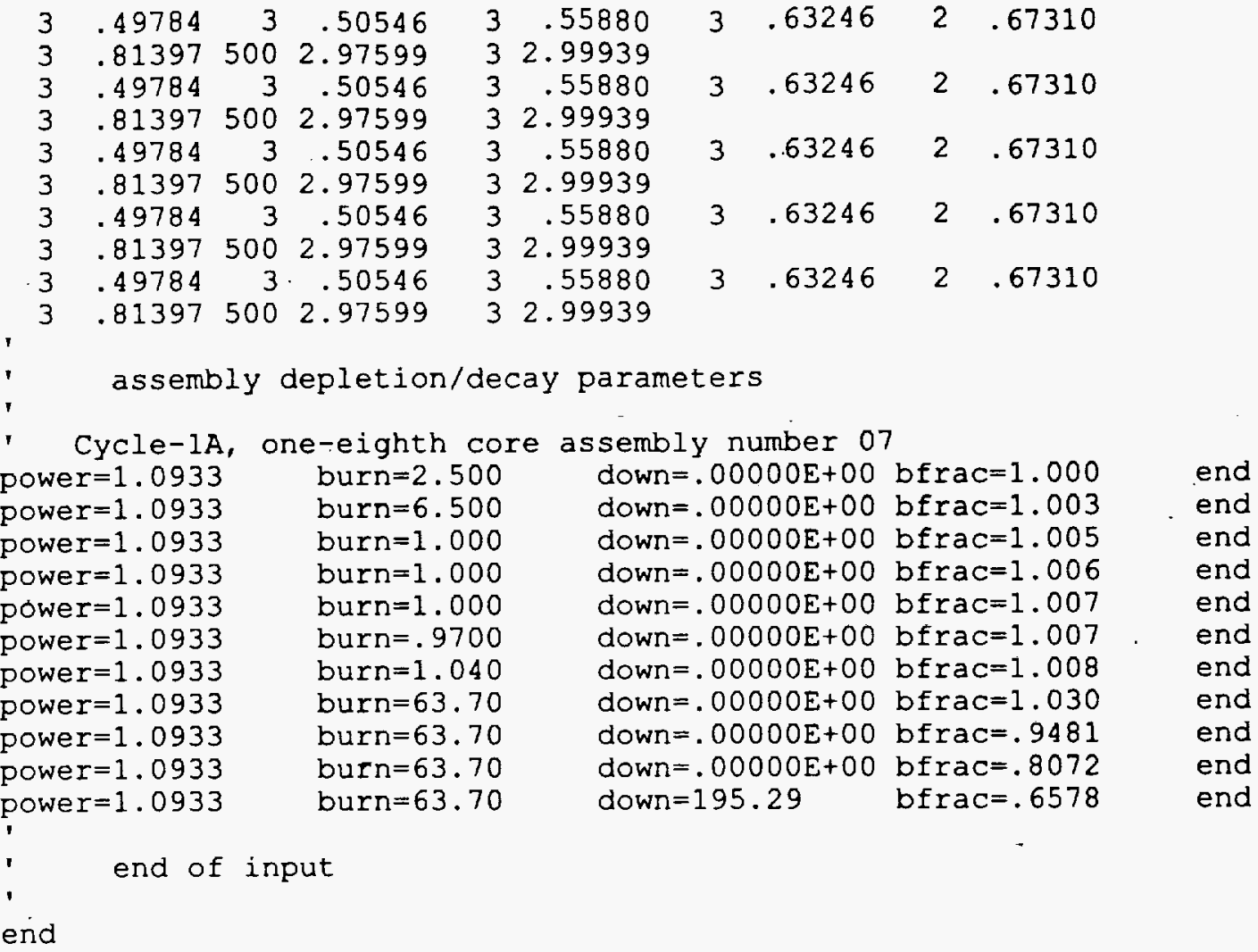

SAS2H Depletion Input Deck Example 8: Continuation Depletion Calculation for a B\&W Fuel Assembly Axial Node Containing 208 Fuel Rods, 16 Guide Tubes with a 16 Rod CRA Inserted for a Portion of the Depletion, and 1 Empty (Water-filled) Instrument Tube

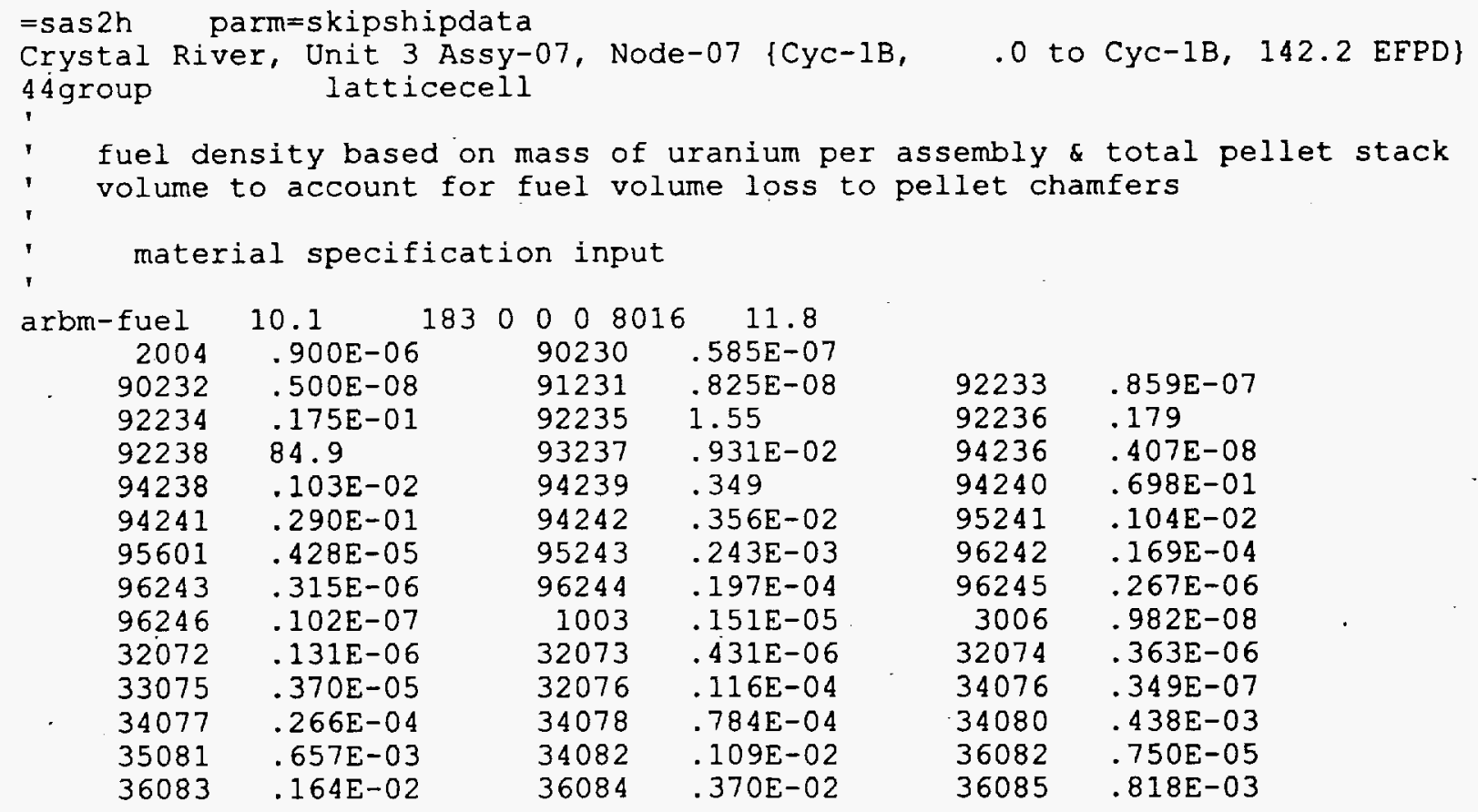


Title: CRC Depletion Calculations for the Rodded Assemblies in Batches 1, 2, 3, and IX of Crystal River Unit 3

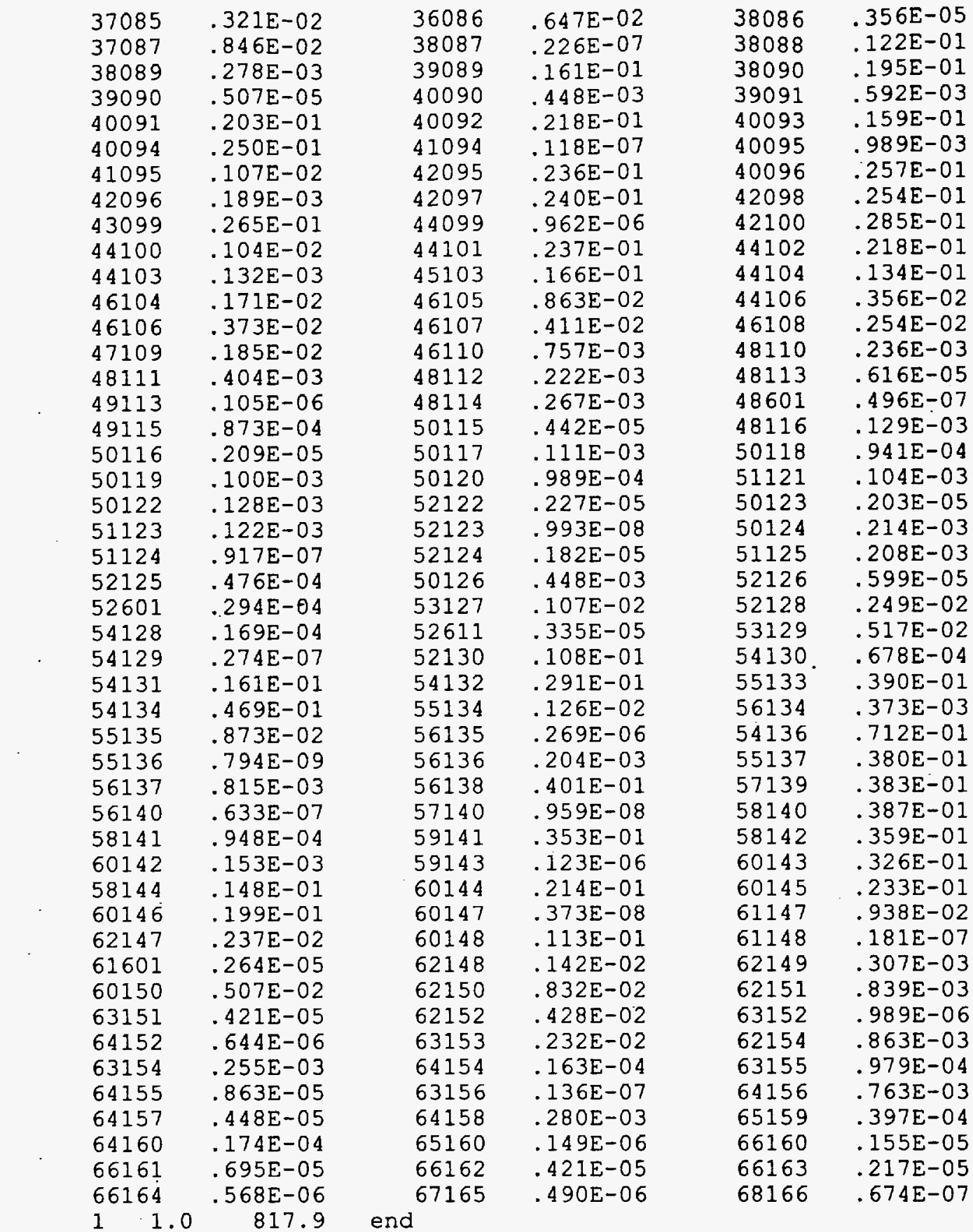

arbm-zirc4 $6.56 \quad 5 \quad 0 \quad 0 \quad 0 \quad 8016 \quad 0.12 \quad 24000 \quad 0.10 \quad 26000 \quad 0.20 \quad 50000 \quad 1.40$ 4000098.1821 .0640 .0 end

- material composition of moderator within unit cell

' with smeared inconel spacer grids

h20 3 den $=.7588 \quad .99424 \quad 578.4$ end

arbm-bormod $\quad .7588 \quad 100005000100 \quad 3 \quad .00054 \quad 578.4$ end

arbm-spacer $\quad .7588 \quad 5 \quad 0 \quad 0 \quad 0 \quad 14000 \quad 2.5 \quad 220002.52400015 .0$

$260007.02800073 .0 \quad 3.00576 \quad 578.4$ end 
Title: CRC Depletion Calculations for the Rodded Assemblies in Batches 1, 2, 3, and $1 \mathrm{X}$ of Crystal River Unit 3

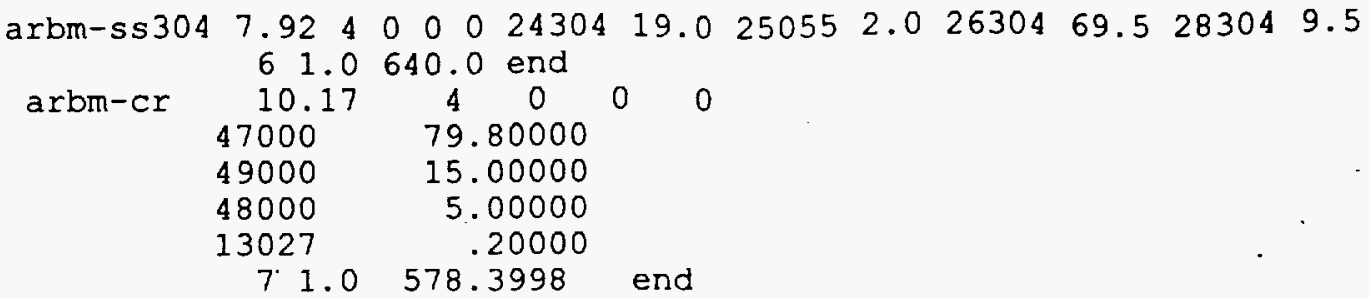


Title: CRC Depletion Calculations for the Rodded Assemblies in Batches 1, 2, 3, and $1 X$ of Crystal River Unit 3 Document Identifier: BBA000000-01717-0200-00040 REV 00

Page 136 of 146

\section{SAS2H Depletion Input Deck Example 9: BOL Depletion Calculation for a B\&W Fuel Assembly Axial Node Containing 208 Fuel Rods, 16 Guide Tubes with a 16 Rod APSRA Inserted for a Portion of the Depletion with a Subsequent APSRA Follow Rod Region Insertion with a Subsequent APSRA Removal, and 1 Empty (Water-filled) Instrument Tube}

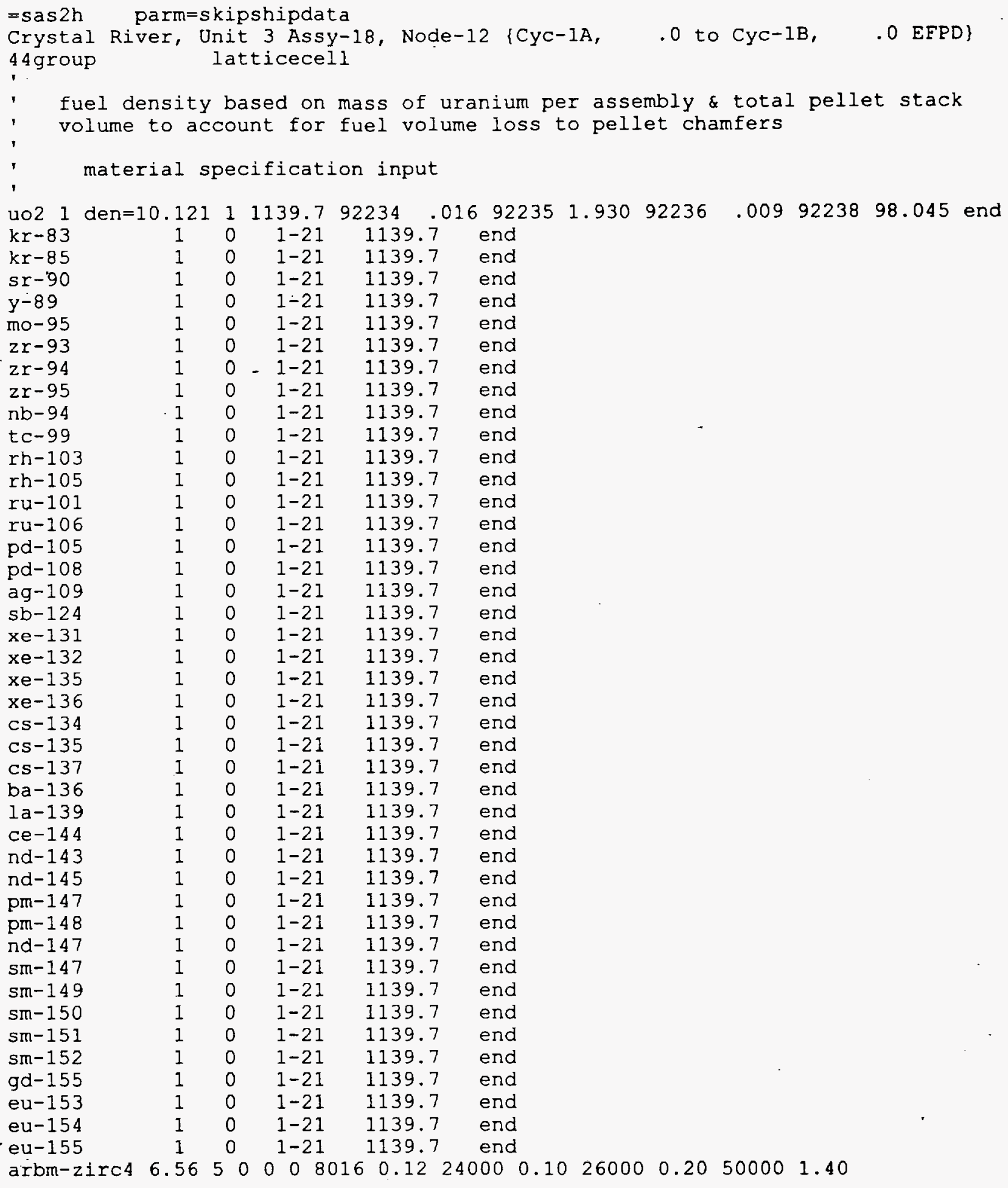




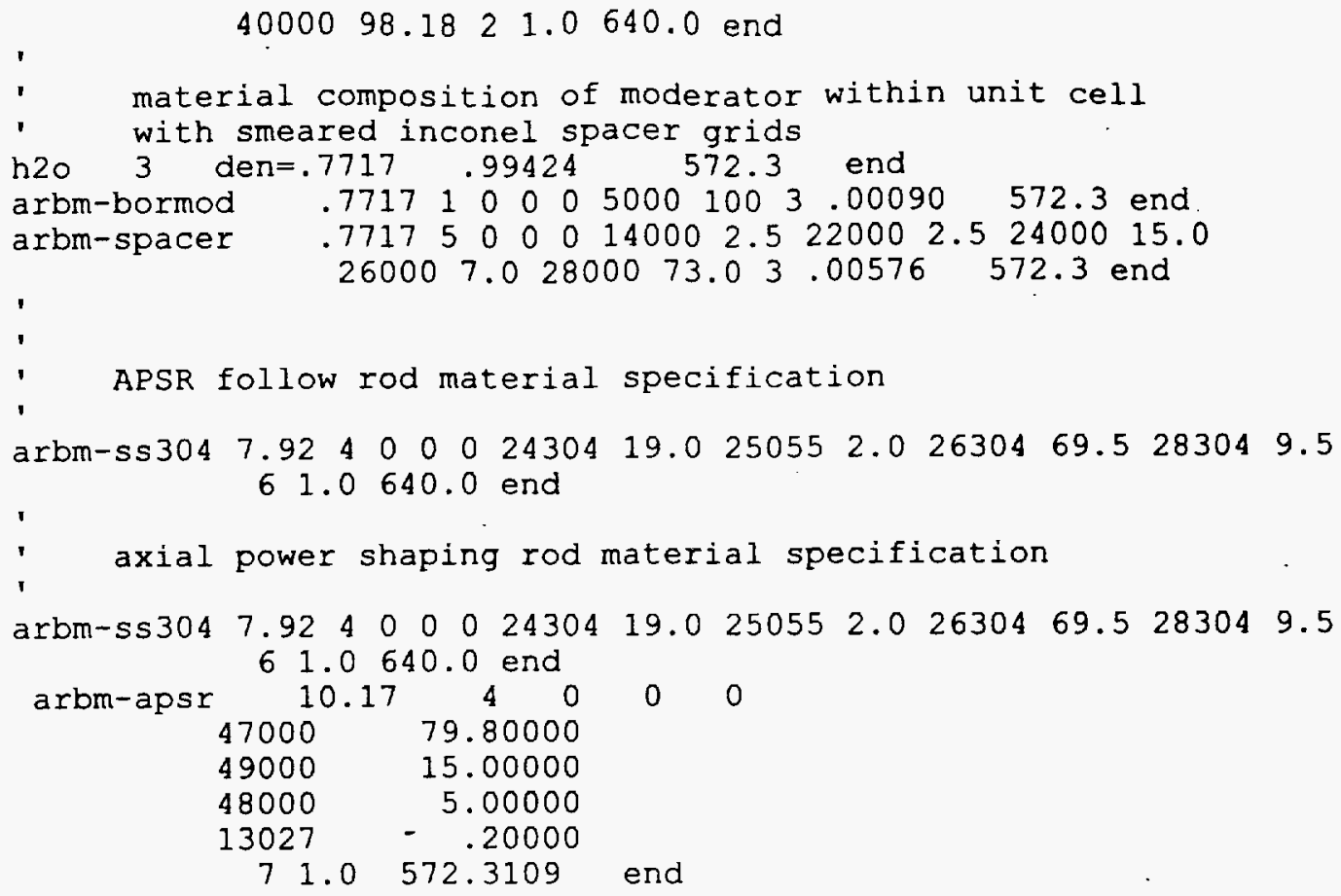

he 5 end end comp

base reactor lattice specification

$\begin{array}{llllllllll}\text { squarepitch } & 1.44272 & .9398 & 1 & 3 & 1.0922 & 2 & .9576 & 0 & \text { end }\end{array}$
more data szf=0.50 end

assembly specification

npin/assembly $=208$ fuelngth $=20.003$ ncycles $=12 \mathrm{nlib} / \mathrm{cyc}=1$ lightel=0

printlevel $=05$ inplevel $=2$ numztotal $=08 \mathrm{mxrepeats}=0 \mathrm{mixmod}=3$ facmesh $=.50$ end

$\begin{array}{rrrrrrrrrr}7 & .49784 & 5 & .50546 & 6 & .55880 & 3 & .63246 & 2 & .67310 \\ 3 & .81397 & 500 & 2.38205 & 3 & 2.40078 & & & & \\ 7 & .49784 & 5 & .50546 & 6 & .55880 & 3 & .63246 & 2 & .67310 \\ 3 & .81397 & 500 & 2.38205 & 3 & 2.40078 & & & & \\ 7 & .49784 & 5 & .50546 & 6 & .55880 & 3 & .63246 & 2 & .67310 \\ 3 & .81397 & 500 & 2.38205 & 3 & 2.40078 & & & & \\ 7 & .89784 & 5 & .50546 & 6 & .55880 & 3 & .63246 & 2 & .67310 \\ 3 & .81397 & 500 & 2.38205 & 3 & 2.40078 & & & & \\ 7 & .89784 & 5 & .50546 & 6 & .55880 & 3 & .63246 & 2 & .67310 \\ 3 & .81397 & 500 & 2.38205 & 3 & 2.40078 & & & & \\ 7 & .89784 & 5 & .50546 & 6 & .55880 & 3 & .63246 & 2 & .67310 \\ 3 & .81397 & 500 & 2.38205 & 3 & 2.40078 & & & & \\ 7 & .49784 & 5 & .50546 & 6 & .55880 & 3 & .63246 & 2 & .67310 \\ 3 & .81397 & 500 & 2.38205 & 3 & 2.40078 & & & & \\ 7 & .49784 & 5 & .50546 & 6 & .55880 & 3 & .63246 & 2 & .67310 \\ 3 & .81397 & 500 & 2.38205 & 3 & 2.40078 & & & & \\ 3 & .89784 & 3 & .50546 & 6 & .55880 & 3 & .63246 & 2 & .67310 \\ 3 & .81397 & 500 & 2.38205 & 3 & 2.40078 & & & & \\ 3 & .89784 & 3 & .50546 & 6 & .55880 & 3 & .63246 & 2 & .67310 \\ 3 & .81397 & 500 & 2.38205 & 3 & 2.40078 & & & & \\ 3 & .49784 & 3 & .59546 & 6 & .55880 & 3 & .63246 & 2 & .67310 \\ 3 & .81397 & 500 & 2.38205 & 3 & 2.40078 & & & & \end{array}$


Title: CRC Depletion Calculations for the Rodded Assemblies in Batches 1, 2, 3, and $1 \mathrm{X}$ of Crystal River Unit 3

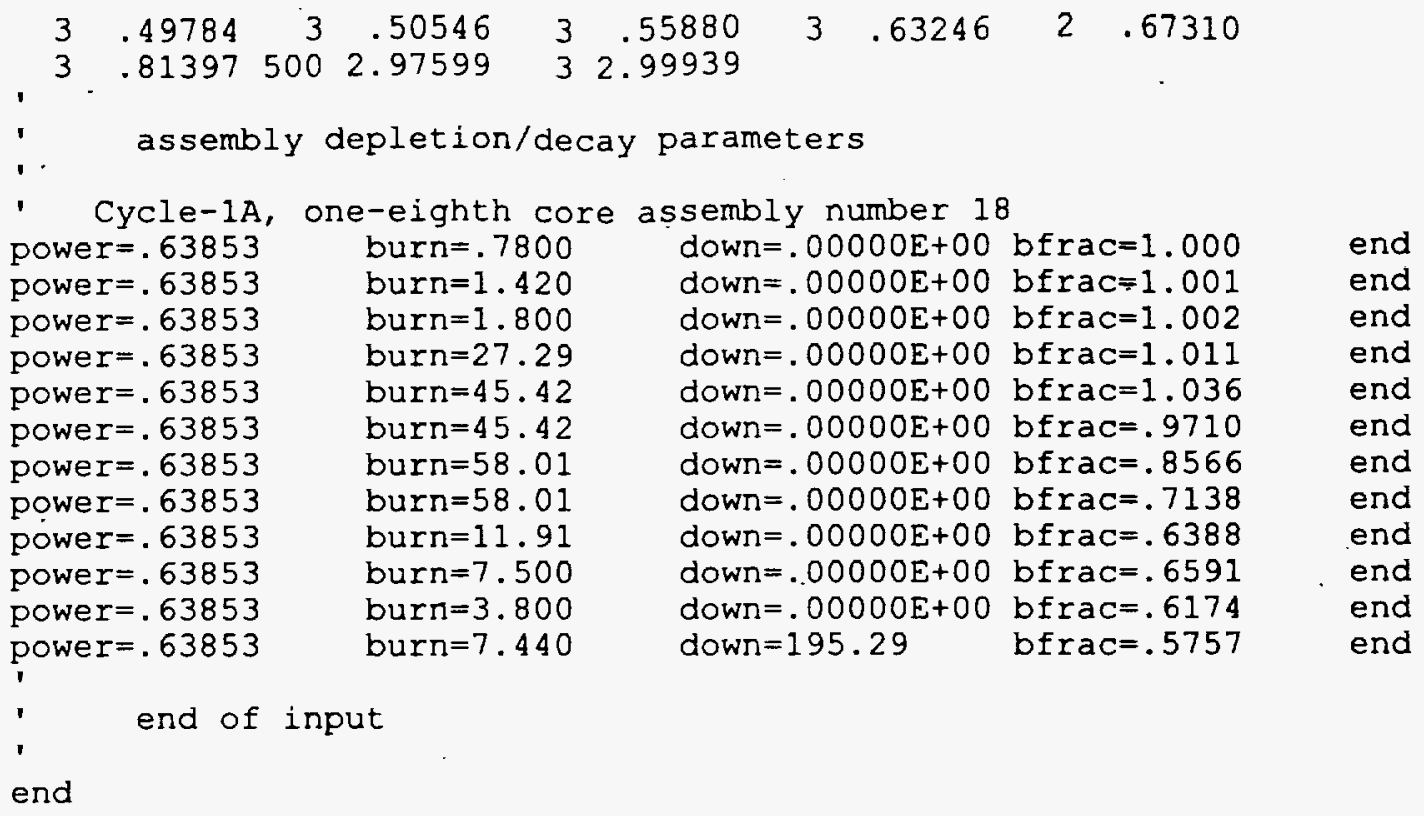

SAS2H Depletion Input Deck Example 10: Continuation Depletion Calculation for a B\&W Fuel Assembly Axial Node Containing 208 Fuel Rods, 16 Guide Tubes with a 16 Rod APSRA Inserted for a Portion of the Depletion with a Subsequent APSRA Follow Rod Region Insertion with a Subsequent APSRA Removal, and 1 Empty (Water-filled) Instrument Tube

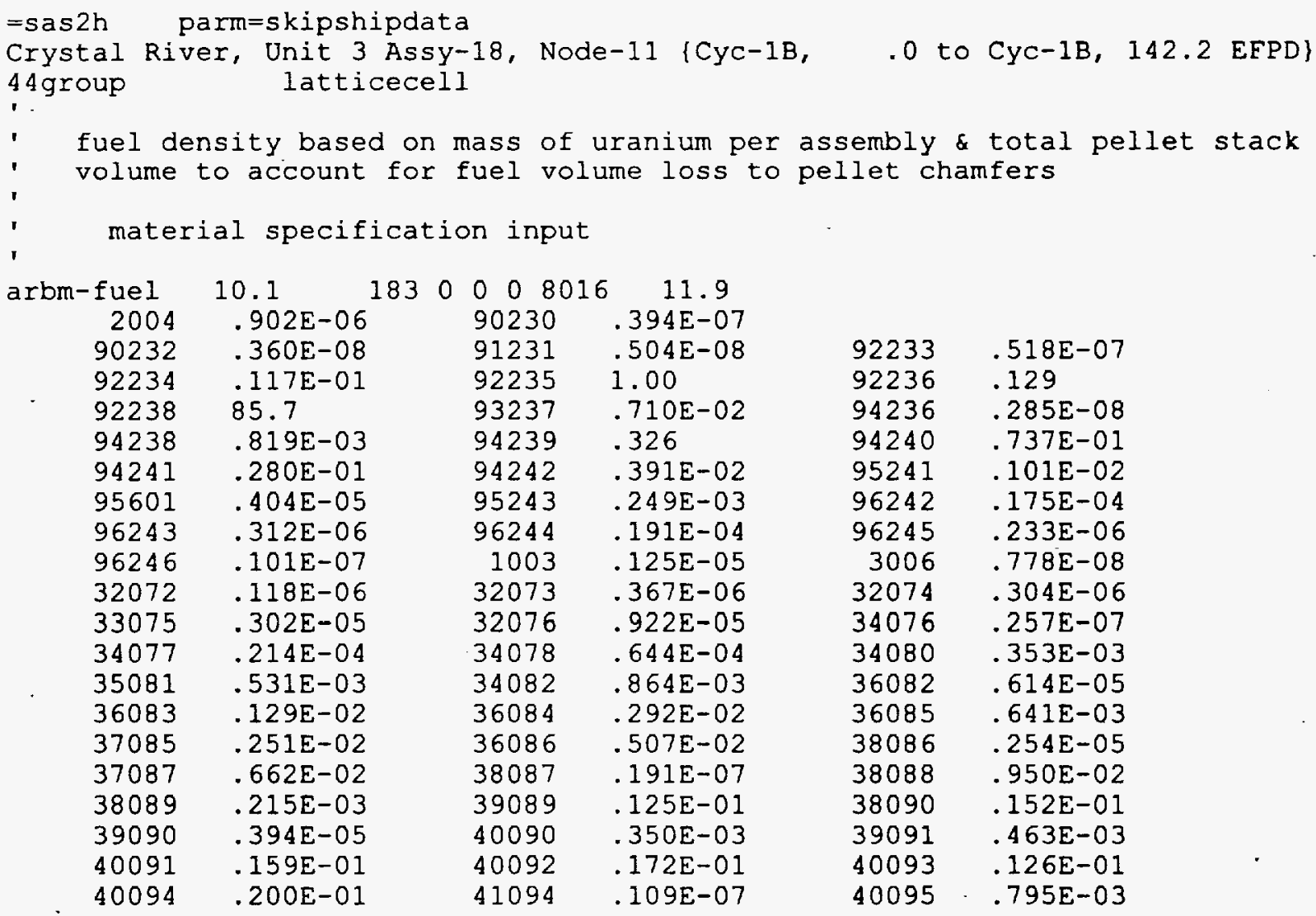


Title: CRC Depletion Calculations for the Rodded Assemblies in Batches 1, 2, 3, and IX of Crystal River Unit 3

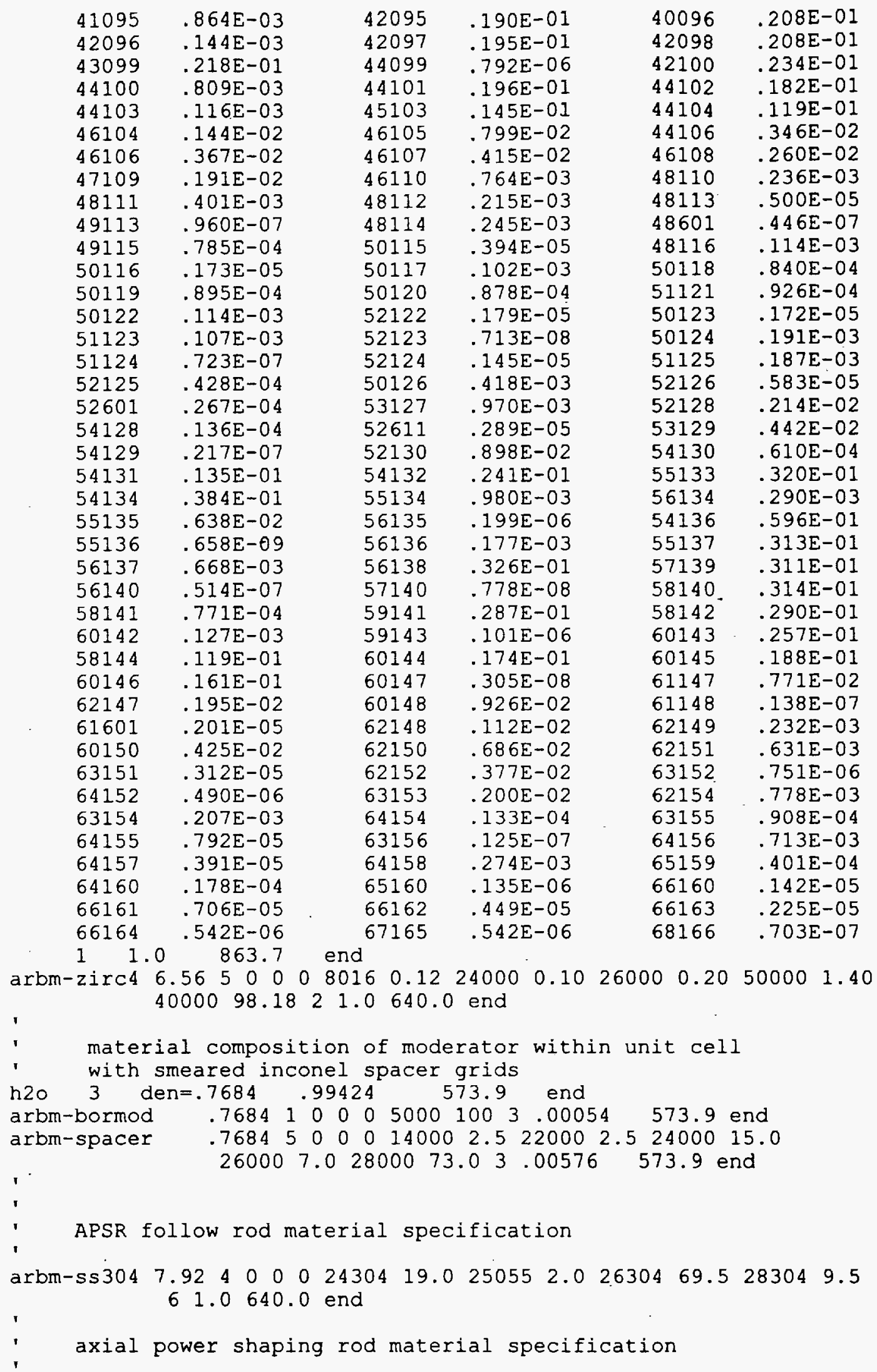




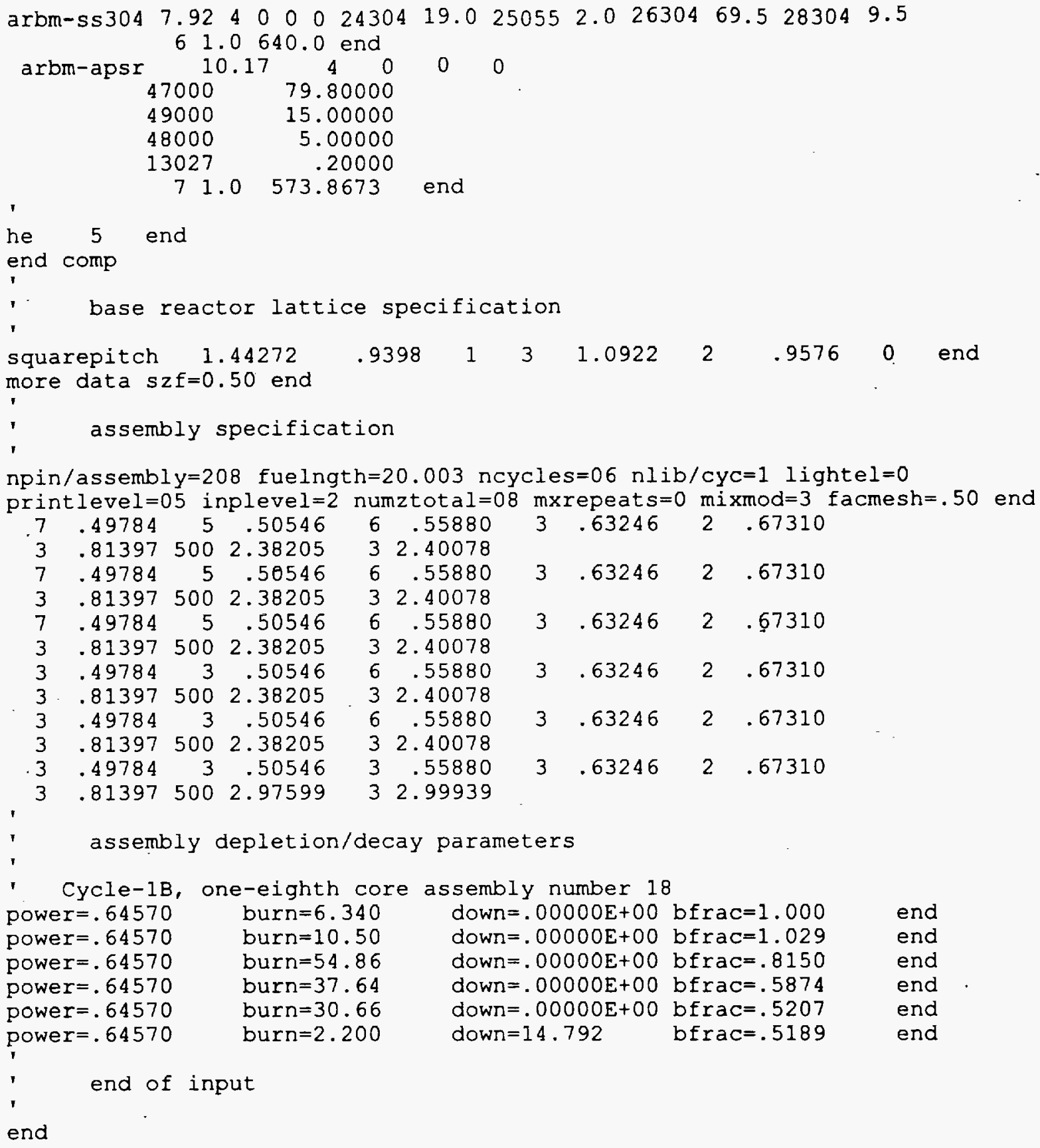

Attachment XL (moved to reference 5.13) contains the CRAFT generated consolidated SAS2H output files for the depletion calculations documented in this analysis as identified in the attachment listing of Section 9. The consolidated output files contain the following information:

time/date stamp for when the SAS2H depletion calculation was performed,

- echo of the SAS2H input deck generated by CRAFT, 
Title: CRC Depletion Calculations for the Rodded Assemblies in Batches 1, 2, 3, and IX of Crystal River Unit 3 Document Identifier: BBA000000-01717-0200-00040 REV 00

Page 141 of 146

- the output extraction of information pertinent to CRC evaluations from the final ORIGEN-S calculation of the SAS2H depletion calculation.

\subsection{Isotopic Results}

Isotopic results for the set of 29 principal isotopes identified in Table 7.7-1 are tabulated for each axial node of each fuel assembly at each CRC statepoint other than beginning of life (BOC of first reactor cycle of in which the assembly is inserted) statepoints. The program entitled "CRC_DATA_TABULIZER.exe" as described in Section 6.1, and Attachment V of reference 5.11, was used to create the principal isotope result tables included in this analysis. Attachments XXII through XXXIX (these attachments were moved to reference 5.12) contain the principal isotope tabulations for the assemblies documented in this analysis. The consolidated output files for the SAS2H depletion calculations contain isotopic concentrations for all isotopes included in the ORIGEN-S cross-section library. The ORIGEN-S cross-section library contains a considerably larger number of isotopes than the 29 isotopes included in the principal isotope set. Isotopic concentrations may be extracted from the consolidated SAS2H output files for subsequent evaluation and/or use in CRC reactivity analyses.

Table 7.7-1 Principal Isotopes

\begin{tabular}{|c|c|c|c|c|}
\hline Mo-95 & Tc-99 & Ru-101 & Rh-103 & Ag-109 \\
\hline Nd-143 & Nd-145 & Sm-147 & Sm-149 & Sm-150 \\
\hline Sm-151 & Sm-152 & Eu-151 & Eu-153 & Gd-155 \\
\hline U-233 & U-234 & U-235 & U-236 & U-238 \\
\hline Np-237 & Pu-238 & Pu-239 & Pu-240 & Pu-241 \\
\hline Pu-242 & Am-241 & Am-242m & Am-243 & --- \\
\hline
\end{tabular}

Between CRC statepoints in the depletion sequence for a fuel assembly axial region, a new SAS2H input deck must be created using the fuel isotopic results from the previous calculation as the initial charge. Since the 44-group cross-section library utilized in the SAS2H depletion calculations of this analysis has a reduced isotopic inventory relative to the ORIGEN-S cross-section library, a number of isotopes present in the ORIGEN-S output cannot be transferred to the initial fuel charge of the subsequent SAS2H depletion calculation. The isotopic inventory in the ORIGEN-S output which cannot be propagated to the following SAS2H depletion calculation does not significantly affect integral reactivity or the energy dependent neutron spectrum as documented in Section 4.9.1 of Attachment I. The nonpropagated isotopic inventory is written to a file entitled "\{depletion case identifier\}.notes" to allow for subsequent analysis of the impact of excluding these isotopics in the initial charge to the subsequent SAS2H depletion calculation. The "* notes" files are contained in Attachment XLI (moved to reference 5.13) as documented in Section 9. 
Title: CRC Depletion Calculations for the Rodded Assemblies in Batches 1, 2, 3, and $1 \mathrm{X}$ of Crystal River Unit 3

\section{Conclusions}

The SAS2H depletion calculations of the rodded fuel assemblies from batches $1,2,3$, and $1 \mathrm{X}$ of the Crystal River Unit 3 PWR that are required for CRC evaluations to support development of the disposal criticality methodology are fully documented in this analysis. The isotopic compositions of depleted fuel and depleted burnable poison for the various assemblies documented in this analysis are available in the consolidated SAS2H output files of Attachment XL (moved to reference 5.13) for subsequent evaluation and/or use in CRC reactivity evaluations. The inputs for the depletion calculations are obtained from a qualified source (Ref. 5.3). The SAS2H modeling techniques employed in the depletion calculations within this analysis are dictated by the CRAFT Version 3.0 code which is fully documented in Attachment I of this analysis.

\section{Attachments}

The attachments referenced throughout this design analysis are listed in Table 9-1. Attachment XL (moved to reference 5.13) contains the consolidated SAS2H output files for the assembly depletion calculations documented in this analysis. Attachment XLI (moved to reference 5.13) contains the "* .notes" files which are generated during the CRAFT calculations for each assembly documented in this analysis. Attachments XL and XLI are written in an ASCII format to an attachment tape (Ref. 5.13). Detailed listings of the content of Attachments XL and XLI on the attachment tape are provided in a hard-copy format in their corresponding attachment locations of this analysis. The listing of the tape content (Ref. 5.13) for Attachments XL and XLI contain the following information for each of the files that are written to the tape:

- the directory and filename as taken from the HP workstation,

- the corresponding filename on the tape attachment,

- the number of text pages in the file on tape after the addition of page headers,

- the date that the file was created on the HP workstation,

- the size of the file on the HP workstation in bytes,

- the file type (ASCII or BINARY).

The tape (Ref. 5.13) for Attachments XL and XLI contain text files only. This tape is written using the HP Colorado Trakker Model T1000e External Parallel Port Backup System for personal computers. The following assembly identifier combinations refer to the same assembly, respectively, in the attachments: $\mathrm{A} 18 \mathrm{z}=\mathrm{A} 18, \mathrm{~A} 18 \mathrm{az}=\mathrm{A} 18 \mathrm{a}, \mathrm{A} 18 \mathrm{bz}=\mathrm{A} 18 \mathrm{~b}, \mathrm{~A} 26 \mathrm{z}=\mathrm{A} 26$, and $\mathrm{A} 28 \mathrm{z}=\mathrm{A} 28$.

Table 9-1 Attachment Listing

\begin{tabular}{||c|c|c|c||}
\hline Attachment \# & $\begin{array}{c}\text { Number of } \\
\text { Pages }\end{array}$ & $\begin{array}{c}\text { Generation } \\
\text { Date }\end{array}$ & Description \\
\hline \hline $\mathrm{I}$ & 84 & $07 / 31 / 97$ & CRAFT Version 3.0 User Information \\
\hline
\end{tabular}


Title: CRC Depletion Calculations for the Rodded Assemblies in Batches 1, 2, 3, and $1 \mathrm{X}$ of Crystal River Unit 3

\begin{tabular}{|c|c|c|c|}
\hline Attachment \# & $\begin{array}{c}\text { Number of } \\
\text { Pages }\end{array}$ & $\begin{array}{c}\text { Generation } \\
\text { Date }\end{array}$ & Description \\
\hline II & 82 & $04 / 03 / 97$ & $\begin{array}{c}\text { CRAFT Version } 3.0 \\
\text { Fortran Source Code Listing } \\
\text { (This is Appendix A: CRAFT Version 3.0 } \\
\text { Fortran Source Code Listing of the CRAFT } \\
\text { User Information Documented in Attachment I) }\end{array}$ \\
\hline III & 24 & $04 / 04 / 97$ & RLAYOUT Version 1.0 Program Description \\
\hline IV & 14 & $04 / 03 / 97$ & CRAFT Input Deck for Fuel Assembly A1 \\
\hline $\mathrm{V}$ & 8 & $04 / 03 / 97$ & CRAFT Input Deck for Fuel Assembly A4 \\
\hline VI & 6 & 04/03/97 & CRAFT Input Deck for Fuel Assembly A5 \\
\hline VII & 14 & 04/03/97 & CRAFT Input Deck for Fuel Assembly A7 \\
\hline VIII & 19 & $04 / 03 / 97$ & CRAFT Input Deck for Fuel Assembly A14 \\
\hline IX & 13 & $07 / 30 / 97$ & CRAFT Input Deck for Fuel Assembly A18 \\
\hline $\mathrm{X}$ & 10 & $07 / 30 / 97$ & CRAFT Input Deck for Fuel Assembly A18a \\
\hline $\mathrm{XI}$ & 6 & $07 / 30 / 97$ & CRAFT Input Deck for Fuel Assembly A18b \\
\hline XII & 13 & 04/03/97 & CRAFT Input Deck for Fuel Assembly A20 \\
\hline XIII & 6 & 04/03/97. & CRAFT Input Deck for Fuel Assembly A22 \\
\hline XIV & 14 & $04 / 03 / 97$ & CRAFT Input Deck for Fuel Assembly A23 \\
\hline $\mathrm{XV}$ & 14 & $04 / 03 / 97$ & CRAFT Input Deck for Fuel Assembly A23a \\
\hline XVI & 13 & $04 / 03 / 97$ & CRAFT Input Deck for Fuel Assembly A25 \\
\hline XVII & 13 & $04 / 03 / 97$ & CRAFT Input Deck for Fuel Assembly A25a \\
\hline XVIII & 7 & 07/30/97 & CRAFT Input Deck for Fuel Assembly A26 \\
\hline $\mathrm{XIX}$ & 13 & 07/30/97 & CRAFT Input Deck for Fuel Assembly A28 \\
\hline $\mathrm{XX}$ & 13 & $04 / 03 / 97$ & CRAFT Input Deck for Fuel Assembly A29 \\
\hline $\mathrm{XXI}$ & 6 & $04 / 03 / 97$ & CRAFT Input Deck for Fuel Assembly O1 \\
\hline XXII & 80 & $04 / 03 / 97$ & $\begin{array}{l}\text { Principal Isotope Results for Assembly A1 } \\
\text { This attachment was moved to reference } 5.12 \\
\text { after the initial check and backcheck of this } \\
\text { analysis was completed. }\end{array}$ \\
\hline
\end{tabular}


Title: CRC Depletion Calculations for the Rodded Assemblies in Batches 1, 2, 3, and $1 \mathrm{X}$ of Crystal River Unit 3

\begin{tabular}{|c|c|c|c|}
\hline Attachment \# & $\begin{array}{c}\text { Number of } \\
\text { Pages } \\
\end{array}$ & $\begin{array}{c}\text { Generation } \\
\text { Date } \\
\end{array}$ & Description \\
\hline XXIII & 40 & $04 / 03 / 97$ & $\begin{array}{l}\text { Principal Isotope Results for Assembly A4 } \\
\text { This attachment was moved to reference } 5.12 \\
\text { after the initial check and backcheck of this } \\
\text { analysis was completed. }\end{array}$ \\
\hline XXIV & 27 & $04 / 03 / 97$ & $\begin{array}{l}\text { Principal Isotope Results for Assembly A5 } \\
\text { This attachment was moved to reference } 5.12 \\
\text { after the initial check and backcheck of this } \\
\text { analysis was completed. }\end{array}$ \\
\hline $\mathrm{XXV}$ & 80 & $04 / 03 / 97$ & $\begin{array}{l}\text { Principal Isotope Results for Assembly A7 } \\
\text { This attachment was moved to reference } 5.12 \\
\text { after the initial check and backcheck of this } \\
\text { analysis was completed. }\end{array}$ \\
\hline XXVI & -120 & $04 / 03 / 97$ & $\begin{array}{l}\text { Principal Isotope Results for Assembly A14 } \\
\text { This attachment was moved to reference } 5.12 \\
\text { after the initial check and backcheck of this } \\
\text { analysis was completed. }\end{array}$ \\
\hline XXVII & 80 & $07 / 30 / 97$ & $\begin{array}{l}\text { Principal Isotope Results for Assembly A18 } \\
\text { This attachment was moved to reference } 5.12 \\
\text { after the initial check and backcheck of this } \\
\text { analysis was completed. }\end{array}$ \\
\hline XXVIII & 53 & $07 / 30 / 97$ & $\begin{array}{l}\text { Principal Isotope Results for Assembly A18a } \\
\text { This attachment was moved to reference } 5.12 \\
\text { after the initial check and backcheck of this } \\
\text { analysis was completed. }\end{array}$ \\
\hline XXIX & 27 & $07 / 30 / 97$ & $\begin{array}{l}\text { Principal Isotope Results for Assembly A18b } \\
\text { This attachment was moved to reference } 5.12 \\
\text { after the initial check and backcheck of this } \\
\text { analysis was completed. }\end{array}$ \\
\hline $\mathrm{XXX}$ & 80 & $04 / 03 / 97$ & $\begin{array}{l}\text { Principal Isotope Results for Assembly A20 } \\
\text { This attachment was moved to reference } 5.12 \\
\text { after the initial check and backcheck of this } \\
\text { analysis was completed. }\end{array}$ \\
\hline XXXI & 27 & $04 / 03 / 97$ & $\begin{array}{l}\text { Principal Isotope Results for Assembly A22 } \\
\text { This attachment was moved to reference } 5.12 \\
\text { after the initial check and backcheck of this } \\
\text { analysis was completed. }\end{array}$ \\
\hline
\end{tabular}


Title: CRC Depletion Calculations for the Rodded Assemblies in Batches 1, 2, 3, and $1 \mathrm{X}$ of Crystal River Unit 3

\begin{tabular}{|c|c|c|c|}
\hline Attachment \# & $\begin{array}{c}\text { Number of } \\
\text { Pages }\end{array}$ & $\begin{array}{c}\text { Generation } \\
\text { Date } \\
\end{array}$ & Description \\
\hline XXXII & 80 & $04 / 03 / 97$ & $\begin{array}{l}\text { Principal Isotope Results for Assembly A23 } \\
\text { This attachment was moved to reference } 5.12 \\
\text { after the initial check and backcheck of this } \\
\text { analysis was completed. }\end{array}$ \\
\hline XXXIII & 53 & $04 / 03 / 97$ & $\begin{array}{l}\text { Principal Isotope Results for Assembly A23a } \\
\text { This attachment was moved to reference } 5.12 \\
\text { after the initial check and backcheck of this } \\
\text { analysis was completed. }\end{array}$ \\
\hline XXXIV & 80 & $04 / 03 / 97$ & $\begin{array}{l}\text { Principal Isotope Results for Assembly A25 } \\
\text { This attachment was moved to reference } 5.12 \\
\text { after the initial check and backoheck of this } \\
\text { analysis was completed. }\end{array}$ \\
\hline $\mathrm{XXXV}$ & -40 & $04 / 03 / 97$ & $\begin{array}{l}\text { Principal Isotope Results for Assembly A25a } \\
\text { This attachment was moved to reference } 5.12 \\
\text { after the initial check and backcheck of this } \\
\text { analysis was completed. }\end{array}$ \\
\hline XXXVI & 27 & $07 / 30 / 97$ & $\begin{array}{l}\text { Principal Isotope Results for Assembly A26 } \\
\text { This attachment was moved to reference } 5.12 \\
\text { after the initial check and backcheck of this } \\
\text { analysis was completed. }\end{array}$ \\
\hline XXXVII & 80 & $07 / 30 / 97$ & $\begin{array}{l}\text { Principal Isotope Results for Assembly A28 } \\
\text { This attachment was moved to reference } 5.12 \\
\text { after the initial check and backcheck of this } \\
\text { analysis was completed. }\end{array}$ \\
\hline XXXVIII & 80 & $04 / 03 / 97$ & $\begin{array}{l}\text { Principal Isotope Results for Assembly A29 } \\
\text { This attachment was moved to reference } 5.12 \\
\text { after the initial check and backcheck of this } \\
\text { analysis was completed. }\end{array}$ \\
\hline XXXIX & 27 & $04 / 03 / 97$ & $\begin{array}{l}\text { Principal Isotope Results for Assembly O1 } \\
\text { This attachment was moved to reference } 5.12 \\
\text { after the initial check and backcheck of this } \\
\text { analysis was completed. }\end{array}$ \\
\hline
\end{tabular}


Title: CRC Depletion Calculations for the Rodded Assemblies in Batches 1, 2, 3, and $1 \mathrm{X}$ of Crystal River Unit 3

\begin{tabular}{|c|c|c|c|}
\hline Attachment \# & $\begin{array}{c}\text { Number of } \\
\text { Pages }\end{array}$ & $\begin{array}{c}\text { Generation } \\
\text { Date } \\
\end{array}$ & Description \\
\hline XL & $\begin{array}{c}\text { Total Page } \\
\text { Count for } \\
\text { Hard-Copy } \\
\text { Listing of Tape } \\
\text { Content }=29\end{array}$ & 07/30/97 & $\begin{array}{c}\text { Tape Containing CRAFT Generated } \\
\text { Consolidated SAS2H Output Decks for } \\
\text { Assemblies A1, A4, A5, A7, A14, A18, A18a, } \\
\text { A18b, A20, A22, A23, A23a, A25, A25a, A26, } \\
\text { A28, A29, O1 }\end{array}$ \\
\hline XLI & $\begin{array}{l}\text { Total Page } \\
\text { Count for } \\
\text { Hard-Copy } \\
\text { Listing of Tape } \\
\text { Content }=23\end{array}$ & 07/30/97 & $\begin{array}{c}\text { CRAFT Generated “* .notes" files for } \\
\text { Assemblies A1, A4, A5, A7, A14, A18, A18a, } \\
\text { A18b, A20, A22, A23, A23a, A25, A25a, A26, } \\
\text { A28, A29, O1 } \\
\text { The files listed in these two attachments are } \\
\text { contained on magnetic media identified by } \\
\text { reference } 5.13 \text {. }\end{array}$ \\
\hline
\end{tabular}




\section{Attachment I}




\section{CRAFT, Version 3.0}

Commercial Reactor Assembly Follow Taskmaster

Developed by Kenneth D. Wright

Framatome Cogema Fuels

High-Level Waste Division

under contract with the

Management and Operating Contractor for the

Yucca Mountain High-Level Radioactive Waste Repository Project 


\section{Table of Contents}

\section{Item}

Page

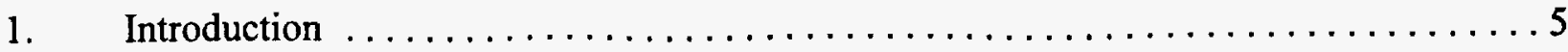

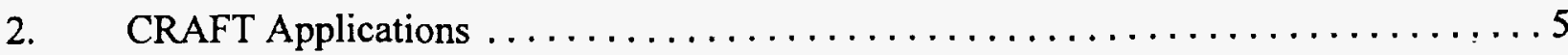

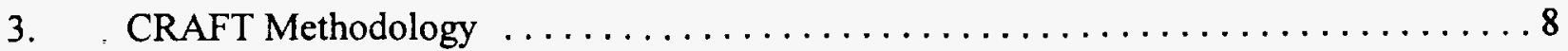

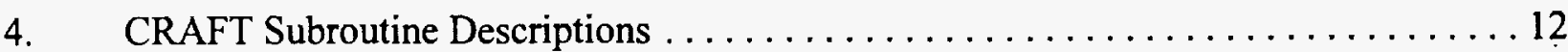

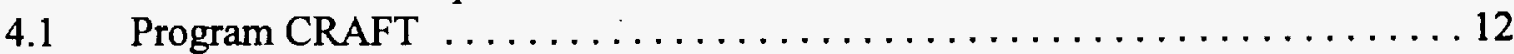

4.2 DATA_AQUISITION Subroutine $\ldots \ldots \ldots \ldots \ldots \ldots \ldots \ldots \ldots \ldots \ldots \ldots \ldots \ldots \ldots \ldots$

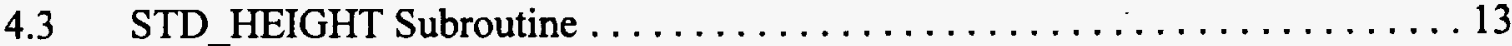

4.4 FUELTEMP_FORMAT, MODSPECVOL_FORMAT, and BURNUP_FORMAT

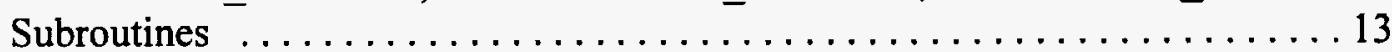

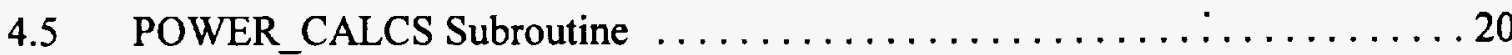

4.6 UNITS CONVERSION Subroutine $\ldots \ldots \ldots \ldots \ldots \ldots \ldots \ldots \ldots \ldots \ldots \ldots \ldots \ldots$

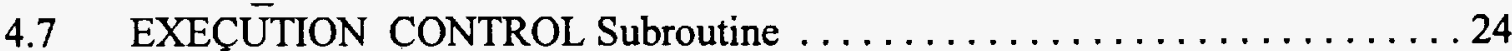

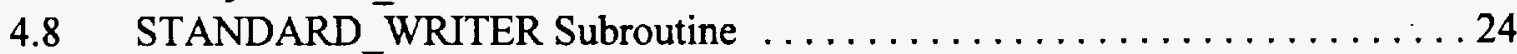

4.8.1 Identification and Global Comment Section ................ 24

4.8.2 Material Specification Section ...................... 25

4.8.3 Base Fuel Assembly Lattice Specification Section ........... 27

4.8.4 SAS2H Control Specifications and Unit Cell Models Section . . . . . . 28

4.8.5 Irradiation History Specification Section ................. 28

4.8.6 Calculations Performed within the STANDARD_WRITER Subroutine

4.9 CONTINUATION_WRITER Subroutine $\ldots \ldots \ldots \ldots \ldots \ldots \ldots \ldots \ldots \ldots \ldots \ldots$

4.9.1 Initial Charge Fuel and Burnable Poison Material Composition Specifications .............................. 31

4.9.2 Calculations Performed within the CONTINUATION_WRITER

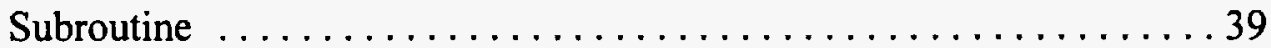

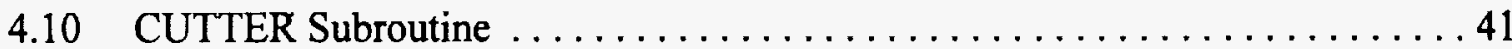

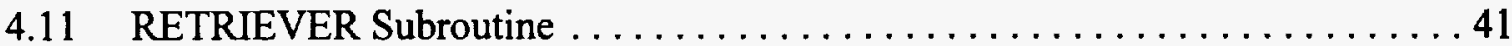

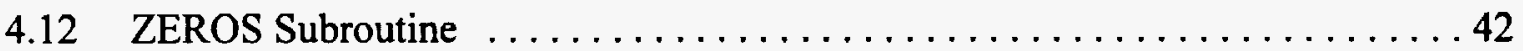

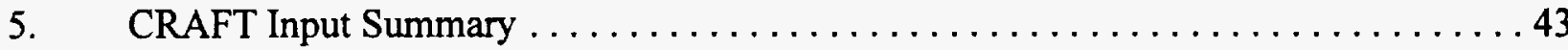

6. CRAFT Code Limits and Execution Instructions $\ldots \ldots \ldots \ldots \ldots \ldots \ldots \ldots \ldots \ldots \ldots \ldots \ldots$

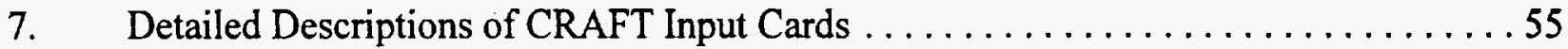

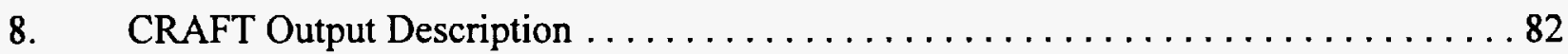

9. Modifications Made Between CRAFT Version 1.0 and Version $2.0 \ldots \ldots \ldots \ldots 83$ 


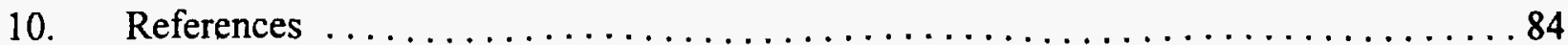

Appendix A: CRAFT Version 3.0

Fortran Source Code Listing 


\section{Introduction}

The Commercial Reactor Assembly Follow Taskmaster (CRAFT) code directs the performance of assembly depletion and decay calculations relevant to Commercial Reactor Critical (CRC) evaluations. The CRAFT code generates input decks for the SAS2H control module of the SCALE modular code system (Ref. 1) based on user defined input which describes the fuel assembly's irradiation history. Appropriate isotopic concentrations relevant to both the CRC evaluations containing the fuel assembly and the subsequent depletion and decay calculations for the fuel assembly are extracted and stored by CRAFT as it generates and executes SAS2H cases.

\section{CRAFT Applications}

The CRAFT code directs the performance of depletion and decay calculations required to simulate the complete irradiation history of a fuel assembly. During the CRAFT orchestration of the fuel assembly depletion and decay calculations, fuel and burnable poison isotopic concentrations are retained at user-defined statepoints. The fuel and burnable poison isotopic concentrations may be used for input to subsequent $\mathrm{CRC}$ statepoint reactivity calculations or in other analyses concerning spent nuclear fuel from commercial power reactors.

The CRAFT code is developed with a high degree of flexibility that provides for the depletion and decay of fuel assemblies with widely varying features under either standard or non-standard core operating procedures. The following list describes some of the capabilities of the CRAFT code.

1) The CRAFT code generates and executes appropriate SAS2H cases required to perform a prescribed depletion and decay sequence for a fuel assembly. The depletion and decay sequence is orchestrated from the beginning of cycle (BOC) statepoint calculation of the initial prescribed insertion cycle through the final statepoint calculation of the last prescribed insertion cycle. The CRAFT code extracts and saves fuel and burnable poison isotopics at each statepoint, including BOC statepoints, during the fuel assembly's depletion and decay sequence. A certain portion of generated isotopics in the depleted fuel composition obtained from a SAS2H calculation are not used in the charge composition to the next SAS2H calculation due to a lack of cross-section data in the specified crosssection library. The CRAFT code provides a listing of the fuel isotopics from the output of a SAS2H calculation which are not used in the initial charge to the next $\mathrm{SAS} 2 \mathrm{H}$ calculation. The isotopics left out of the initial charge are fission products whose reactivity worth is small relative to the isotopics retained in the charge composition. The listing of excluded charge isotopics allows for a determination of the impact upon the reactivity of the initial fuel composition in the subsequent calculation.

2) Any assembly design may be analyzed within the bounds of the SAS2H control module through the use of the CRAFT code. This includes both PWR and BWR fuel assemblies. 
3) An axial blanket fuel modeling option is available in the CRAFT code. Any $\mathrm{UO}_{2}$ enrichment may be specified for the axial blanket fuel. The axial blanket fuel may be defined to exist in any of the CRC axial nodes which are defined for the CRAFT calculation.

4) A spacer grid modeling technique is available with the CRAFT code. The modeling technique homogenizes the spacer grid material throughout the moderator of the fuel assembly by utilizing a user defined spacer material and spacer material volume fraction in the moderator. The available spacer grid materials include the following-- ZIRC-4, INCONEL, SS316, SS316S, SS304, SS304S. Any volume fraction of spacer material in the moderator may be specified (including zero).

5) The fuel cladding, burnable poison rod (BPR) cladding, axial power shaping rod (APSR) cladding, or control rod (CR) cladding in the CRAFT calculation may be designated as any of the following materials-- ZIRC-4, SS316, SS316S, SS304, SS304S, or INCONEL.

6) The insertion of a BPR assembly during the irradiation of the fuel assembly may be modeled in the CRAFT calculation. Up to 10 unique BPR assembly designs may be specified for use during the depletion of a fuel assembly. Any type of BPR assembly design may be specified. The default $\mathrm{BP}$ material for use in CRAFT calculation is $\mathrm{Al}_{2} \mathrm{O}_{3}-\mathrm{B}_{4} \mathrm{C}$. Any arbitrary $\mathrm{BP}$ material may be specified for use in a BPR assembly design. A maximum of 10 unique $\mathrm{BP}$ materials may be specified. A maximum of 20 unique elements or isotopes may be specified in any given BP material. A BPR assembly may be inserted in any reactor cycle specified in the CRAFT calculation. Only one BPR assembly design may be specified per cycle. The position of the BPR assembly in the fuel assembly is specified by identifying the top and bottom axial nodes of the BP material. The BPR assembly remains fixed during a given reactor cycle. The depletion of the BP material is tracked during the CRAFT calculation. The appropriate depleted $\mathrm{BP}$ material is utilized in statepoint calculations following the BOC to statepoint 1 calculation for a given reactor cycle. Depleted BP material isotopic concentrations are also retained for use in subsequent mid-cycle statepoint reactivity calculations which may be performed as part of the CRC evaluation process.

7) The insertion of a CR assembly during the irradiation of the fuel assembly may be modeled in the CRAFT calculation. Up to 10 unique CR assembly designs may be specified for use during the depletion of a fuel assembly. Any type of $\mathrm{CR}$ assembly design may be specified. Any arbitrary CR absorber material may be specified for use in a CR assembly design. A maximum of 10 unique $C R$ absorber materials may be specified. A maximum of 10 unique elements or isotopes may be specified in any given CR absorber material. A CR assembly may be inserted in any reactor cycle specified in the CRAFT calculation. Multiple 
$\mathrm{CR}$ assembly designs may be specified per cycle. The position of the $\mathrm{CR}$ assembly in the fuel assembly is specified by identifying number of $C R$ absorber regions and the top and bottom axial nodes of each region. The CR assembly position may be changed between each irradiation step of a SAS2H calculation generated by CRAFT. The CR assembly design may also be changed between any two statepoint calculations in a given reactor cycle.

8) The insertion of an APSR assembly during the irradiation of the fuel assembly may be modeled in the CRAFT calculation. Up to 10 unique APSR assembly designs may be specified for use during the depletion of a fuel assembly. Any type of APSR assembly design may be specified. Any arbitrary APSR absorber material may be specified for use in an APSR assembly design. A maximum of 10 unique APSR absorber materials may be specified. A maximum of 10 unique elements or isotopes may be specified in any given APSR absorber material. An APSR assembly may be inserted in any reactor cycle specified in the CRAFT calculation. Multiple APSR assembly designs may be specified per cycle. The position of the APSR assembly in the fuel assembly is specified by identifying the top and bottom axial nodes of the APSR absorber material. The APSR assembly position may be changed between each irradiation step of a SAS2H calculation generated by CRAFT. The APSR assembly design may also be changed between any statepoint calculations in a given reactor cycle. For any APSRA modeled, the APSR follow rods are modeled in the axial region above the poison region of the APSR's. The APSR follow rod material may be specified as a cladding material as previously described in item number five of this listing.

9) A fuel assembly may be inserted in a maximum of 10 reactor cycles during a CRAFT calculation.

10) A maximum of 20 statepoints ( $\mathrm{BOC}$ is always considered a statepoint) may be specified in any given reactor cycle in a CRAFT calculation.

11) A maximum of 23 irradiation steps of variable duration may be specified in any given SAS2H statepoint calculation to be generated during a CRAFT calculation.

12) A maximum of 50 axial nodes may be specified in the $\mathrm{CRC}$ nodal format for use in a CRAFT calculation. Each axial node may have a unique height.

13) The CRAFT code utilizes a user-defined input format for fuel temperature, moderator specific volume, and burnup data. The input data must be specified for each axial node in a user-defined nodal format of up to 50 nodes of arbitrary height. The total assembly active fuel height for the input data descriptions may be different than that specified in the CRC nodal format. Depending on the users needs, the fuel temperature, moderator specific volume, and burnup input data may be specified in a different nodal format each time an assembly set of this input data is provided. Nominal full-power operation nodal average fuel 
temperature input data must be provided in units of degrees Fahrenheit for each node in each statepoint calculation to be generated by the CRAFT calculation. Nominal full-power operation nodal average specific moderator input data must be provided in units of cubic feet per pound for each node in each statepoint calculation to be generated by the CRAFT calculation. The nodal average burnup input data must be provided in units of gigawatt-days per metric ton of uranium (GWd/MTU) for each node at each statepoint including the BOC statepoint. All burnup input data that is specified must be cumulative from the initial insertion of the fuel assembly in the reactor.

14) Up to 50 axial nodes of arbitrary height may be specified in a CRC nodal format.

15) A continuation CRAFT calculation for an assembly may be initiated from any statepoint in any reactor cycle if all of the nodal consolidated output files ("*.cut" files, see Section 8 ) from the statepoint calculation immediately preceding the continuation calculation exist in the CRAFT execution directory.

\section{CRAFT Methodology}

The objective of the CRAFT methodology was to develop a mechanism by which fuel assembly depletion and decay calculations required to support CRC evaluations could be performed most efficiently with minimal required user interface. The result was the CRAFT code which automates the process of performing numerous complex SAS2H depletion and decay calculations while extracting and archiving results pertinent to CRC analyses. The information provided in this section describes the general flow of a CRAFT calculation. Figure 3-1, presents a general calculational flow diagram for the CRAFT code. The identifiers for the CRAFT subroutines where the various processes and calculations take place are identified in this section. Detailed information on the calculations performed by the craft code may be found in Section 4, "CRAFT Subroutine Descriptions".

The CRAFT calculation begins by reading a well-defined yet flexible user input which describes the fuel assembly depletion and decay calculation to be performed. The input contains all data necessary to describe the fuel assembly and any insertion assemblies such as burnable poison rod assemblies (BPRA's), axial power shaping rod assemblies (APSRA's), or control rod assemblies (CRA's). Fuel temperature and moderator specific volume data (which may be obtained from reactor design core-follow codes) is also utilized to provide input to the depletion calculations which are to be generated by the CRAFT code. The use of nominal full-power fuel temperatures and moderator specific volumes from core-follow codes provide an additional level of detail in the calculation due to the fact that feedback and flux redistribution effects are incorporated into the development of this input parameter data. The "DATA_AQUISITION" subroutine performs the input data acquisition functions in the CRAFT code. A detailed description of the CRAFT input deck format is provided in the CRAFT input description in Sections 5 and 7.

After data acquisition, the next procedure is to standardize all fuel assembly heights corresponding to the input data specification to a prescribed CRC fuel assembly height. The fuel 
assembly depletion and decay calculations must be performed on an assembly which has the same total active fuel height as that prescribed for the CRC calculation. The fuel assembly nodal formats used for providing various input to the CRAFT code are allowed to have an arbitrary active fuel height which may differ from that required for the CRC calculation. The assembly height standardization procedure performed by the "STD_HEIGHT" subroutine puts all of the input data specification active fuel heights on a common basis with the CRC active fuel height.

After active fuel height standardization, the next procedure is to convert all of the axial node formats for the fuel temperature, moderator specific volume, and burnup input data to the prescribed CRC axial node format. There must be a one-to-one correspondence between the various axial node input data and the CRC axial nodes. The input data nodal format conversions are performed by the "FUELTEMP_FORMAT", "MODSPECVOL_FORMAT", and "BURNUP_FORMAT" subroutines for the fuel temperature data, moderator specific volume data, and burnup data, respectively.

After the input data nodal formats are converted, the next procedure is to calculate the power to be specified in each SAS2H statepoint calculation that will be generated by the CRAFT code. The power is calculated in units of megawatts for each axial node of the fuel assembly based upon the nodal burnup during the statepoint calculation, the initial mass of uranium in the node (fresh fuel), and the duration of the statepoint calculation irradiation period in days. The nodal power calculations are performed by the "POWER_CALCS" subroutine.

After the nodal powers are calculated, the next procedure is to convert units and calculate moderator densities and temperatures. At this point in the CRAFT calculation, there is a nominal full-power fuel temperature and moderator specific volume value for each axial node of the assembly in each statepoint calculation. The fuel temperatures, initially input in units of degrees Fahrenheit, are converted to units of degrees Kelvin. The moderator specific volume, initially input in units of cubic feet per pound, are converted to densities in units of grams per cubic centimeter. The system pressure and moderator density are used to determine the moderator temperature in units of degrees Kelvin. The units conversions and moderator density and temperature calculations are performed by the "UNITS_CONVERSION" subroutine.

After the "UNITS_CONVERSION" subroutine is finished, the next procedure is to initiate the "EXECUTION_CONTROL" subroutine. The "EXECUTION_CONTROL" subroutine directs the development and execution of SAS2H cases required to appropriately deplete and decay the fuel assembly. The subroutine also directs the extraction of results pertinent to $\mathrm{CRC}$ evaluations. The development of a unique SAS2H case is required for each CRC axial node in each statepoint calculation. The CRAFT code directs the development and execution of SAS2H cases beginning with the top assembly node (always identified as node number one) working sequentially through the assembly to the bottom node. The complete irradiation history of the assembly as defined in the CRAFT input deck is performed for each axial node before initiating the development and execution of SAS2H cases for the next axial node. Three subroutines are called by the "EXECUTION_CONTROL" subroutine--

1) the "STANDARD_WRITER" subroutine 
2) the "CONTINUATION_WRITER" subroutine

3) the "CUTTER" subroutine.

Two of these called subroutines create SAS2H input decks, and one extracts isotopic results for use in subsequent CRC analyses. The "EXECUTION_CONTROL" subroutine then calls either "STANDARD_WRITER" or "CONTINUATION_WRITER" to create the next SAS2H input deck. "EXECUTION_CONTROL" then executes the generated SAS2H calculation. Upon completion of the SAS2H calculation, "EXECUTION_CONTROL" calls the "CUTTER" subroutine to extract and archive the fuel and burnable poison isotopic compositions calculated by SAS2H. The next SAS2H input deck is then generated as appropriate. This cycle continues until the prescribed fuel assembly depletion and decay history is completed.

The subroutine called "STANDARD_WRITER", as previously mentioned in relation to the "EXECUTION_CONTROL" subroutine, creates an appropriate SAS2H input deck for the initial statepoint calculation in the initial insertion reactor cycle for a fuel assembly axial node. The fuel and burnable poison compositions in the SAS2H cases generated by the "STANDARD_WRITER" subroutine are always fresh. The sole source of input data for the SAS2H cases generated by the "STANDARD_WRITER" subroutine is the CRAFT input deck.

The subroutine called "CONTINUATION_WRITER", as previously mentioned in relation to the "EXECUTION_CONTROL" subroutine, writes SAS2H input decks for all statepoint calculations other than the initial statepoint calculation in the initial insertion reactor cycle. The "CONTINUATION_WRITER" subroutine calls a subroutine "RETRIEVER" to access and retrieve the fuel and burnable poison, if applicable, initial charge compositions for the statepoint calculation. The "CONTINUATION_WRITER" subroutine generates SAS2H input decks utilizing the appropriate depleted compositions such that the fuel assembly depletion and decay history continues uninterrupted.

The subroutine "CUTTER", as previously mentioned in relation to the "EXECUTION_CONTROL" subroutine, creates a CRC depletion output file for each statepoint. The file created by "CUTTER" contains the time/date stamp printed in the SAS2H output, the echoed SAS2H input deck for the statepoint calculation printed in the SAS2H output, and the pertinent section of the final ORIGEN output from the SAS2H output containing the desired depleted and decayed fuel and burnable poison isotopic concentrations. The CRC depletion output files created by "CUTTER" are identified by the same base filename identifier as the SAS2H statepoint calculation to which they apply followed by a ".cut" suffix. The CRAFT generated filenames are described in detail in Section 8.

The subroutine "RETRIEVER" reads through the appropriate "*.cut" file to obtain the fuel and burnable poison initial charge compositions for the next SAS2H calculation as previously mentioned in relation to the "CONTINUATION_WRITER" subroutine. Additionally, the "RETRIEVER" subroutine writes a file which contains a listing of all isotopes and their concentrations which were present in the ORIGEN output of the SAS2H calculation, but not utilized in the initial charge composition of the next SAS2H calculation. This file is identified by the base filename identifier corresponding to the SAS2H case which is being generated followed 
by a "*.notes" suffix. The CRAFT generated filenames are described in detail in Section 8.

Figure 3-1

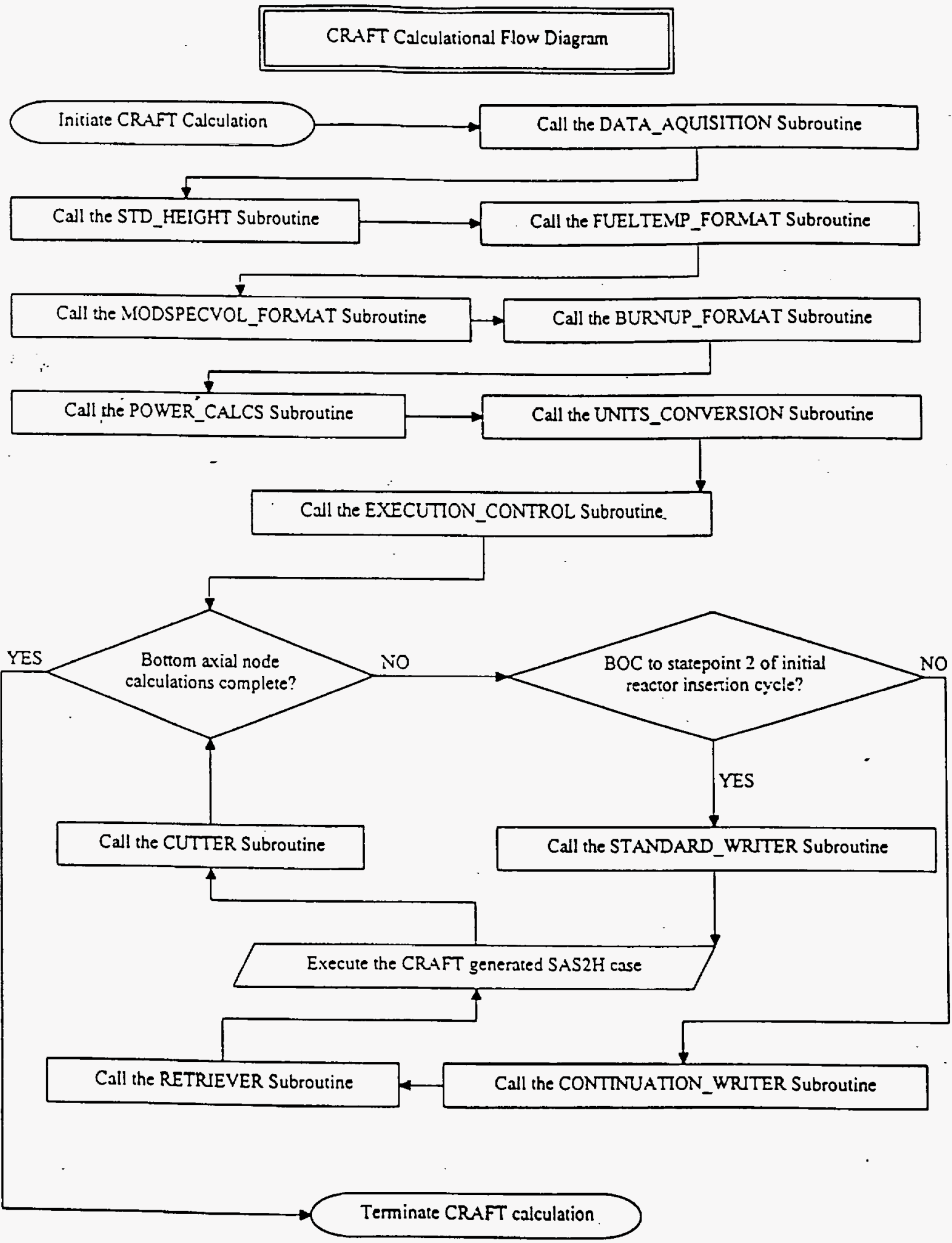




\section{CRAFT Subroutine Descriptions}

The CRAFT code is organized into 14 subroutines. Each of the subroutines has a specific responsibility in performing a CRAFT calculation. The following sections provide descriptions of the structure and task of each subroutine. The subroutines comprising the CRAFT code include the following:

1) Main program block-"PROGRAM CRAFT"

2) Reactor and problem data acquisition subroutine-"DATA_AQUISITION"

3) Assembly height standardization subroutine-"STD_HEIGHT"

4) Fuel temperature input nodal format conversion subroutine-"FUELTEMP_FORMAT"

5) Moderator specific volume input nodal format conversion subroutine-"MODSPECVOL_FORMAT"

6) Burnup input nodal format conversion subroutine-"BURNUP_FORMAT"

7) Nodal power calculation subroutine-"POWER_CALCS"

8) Units conversion subroutine-"UNITS_CONVERSION"

9) SAS2H input deck creation and execution control subroutine-"EXECUTION_CONTROL"

10) Standard beginning of assembly life SAS2H input deck writing subroutine-"STANDARD_WRITER"

11) Continuation SAS2H input deck writing subroutine-"CONTINUATION_WRITER"

12) CRC statepoint depletion/decay output file generator subroutine-"CUTTER"

13) Fuel and burnable poison composition retrieval subroutine-"RETRIEVER"

14) Two digit integer conversion utility subroutine.-"ZEROS"

\subsection{Program CRAFT}

The main program block is the orchestrator of the CRAFT calculation. The purpose of the main program block is to define fixed data sets and initiate the sequential execution of appropriate subroutines to perform the CRAFT calculation. The subroutines initiated by the main program block of the CRAFT code include the following, in order of initiation-- DATA_AQUISITION, STD_HEIGHT, FUELTEMP_FORMAT, MODSPECVOL_FORMAT, BURNUP_FORMAT, POWER_CALCS, UNITS_CONVERSION, and EXECUTION_CONTROL. 


\subsection{DATA_AQUISITION Subroutine}

A sufficient description of the DATA_AQUISITION subroutine is provided in Section 3. A detailed description of the CRAFT input deck format is provided in Sections 5 and 7.

\subsection{STD_HEIGHT Subroutine}

This subroutine standardizes all assembly total active fuel heights as specified in the user-defined input to the standard assembly active fuel height being utilized in the CRC evaluation. The active fuel height standardization calculation performed on the various input data requires the adjustment of input data nodal heights. The input data nodal height adjustment is performed by multiplying each input data node height by a factor equal to the ratio of the CRC assembly total active fuel height to the input data assembly total active fuel height. This calculation is summarized in the following equation--

Original Input Node Height *

$$
\text { Standardized Input Node Height }=\left(\frac{C R C \text { Assembly Total Active Fuel Height }}{\text { Input Data Assembly Total Active Fuel Height }}\right)
$$

All nodal input data which is a constituent of a complete set of assembly input data is adjusted using the equation above such that all sets of assembly input data have the same total active fuel height corresponding to the prescribed CRC total active fuel height.

\subsection{FUELTEMP_FORMAT, MODSPECVOL_FORMAT, and BURNUP_FORMAT Subroutines}

This subroutine standardizes all nodal input data such that there exists a one-to-one correspondence between input data values and CRC axial nodes. This basically means that the assembly axial node formats in which the input data is provided are adjusted such that they identically match the prescribed CRC axial node format. Appropriate averaging of the nodal input data values must be performed to adjust the input parameter nodal formats to the CRC nodal format. A nodal shadowing technique is used to calculate appropriate nodal average input values corresponding to the specified CRC nodal format using the data as provided in the arbitrary input nodal formats. The shadowing technique consists of determining which input data axial nodes shadow a particular CRC axial node. The relative shadowing contributions from the input data nodes upon the CRC axial node are used to determine the appropriate average input value for the $\mathrm{CRC}$ axial node. Average input data values for fuel temperature, moderator specific volume, and burnup are determined for each $\mathrm{CRC}$ axial node using each set of assembly input data provided in the CRAFT input deck.

The method for implementing the nodal shadowing technique consists of determining all of the possible combinations of input axial node to CRC axial node shadows that may exists. Three classes of input axial node to CRC axial node shadows are defined: 
1) shadows created by input axial nodes which are the same height as the CRC axial nodes;

2) . shadows created by input axial nodes which are smaller than the CRC axial nodes;

3) shadows created by input axial nodes which are larger than the CRC axial nodes.

Determining the average input parameter for a given CRC axial node requires that the input data values in the nodes which contribute to the average input data value for the CRC axial node be averaged appropriately. This averaging requires the determination of the relative weight which should be attributed to each of the contributing input data values. The shadowing technique determines the relative contribution of each input data axial node to the average input data value for the CRC axial node by weighting the input data values by their relative shadow contributions. The nodal shadowing descriptions below demonstrate how the contribution from each input data node to a CRC axial node is calculated. The CRAFT code calculates an average input data value for each CRC axial node by summing the contributions from all input data nodes which shadow the CRC axial node. This averaging process is performed for all fuel temperature input data, moderator specific volume input data, and burnup input data.

Shadows created by input axial nodes which are the same height as the CRC axial nodes--

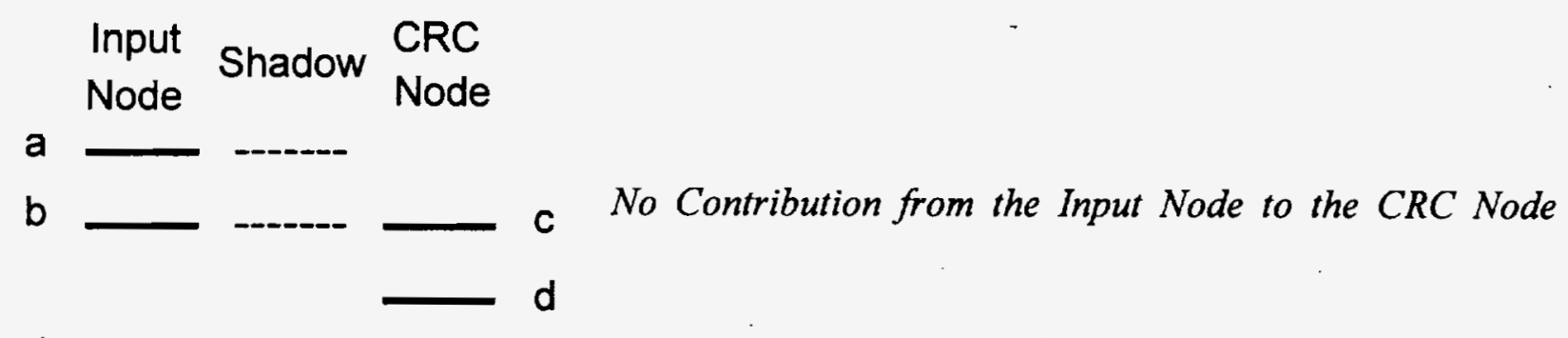

Input Shadow $\begin{gathered}\text { CRC } \\ \text { Node }\end{gathered}$
a - C Input Node Contribution to CRC Node $=\left(\frac{b-c}{d-c}\right) *($ Input Data Value $)$ 


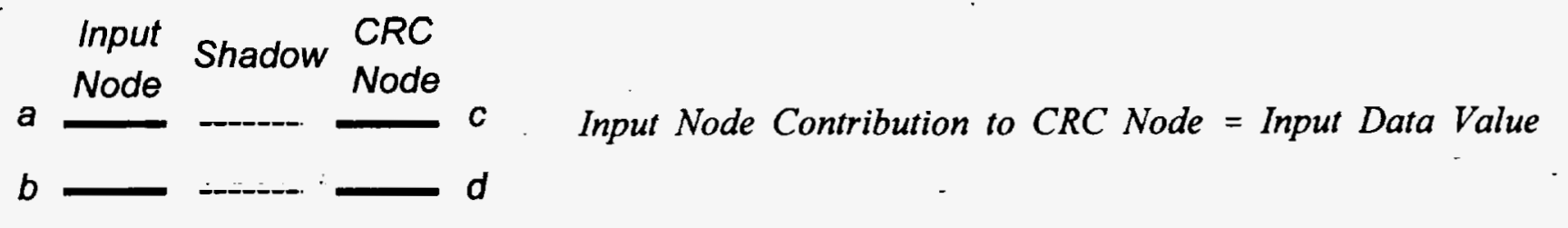

Input Shadow $\begin{aligned} & \text { CRC } \\ & \text { Node }\end{aligned}$
Node
$b-$

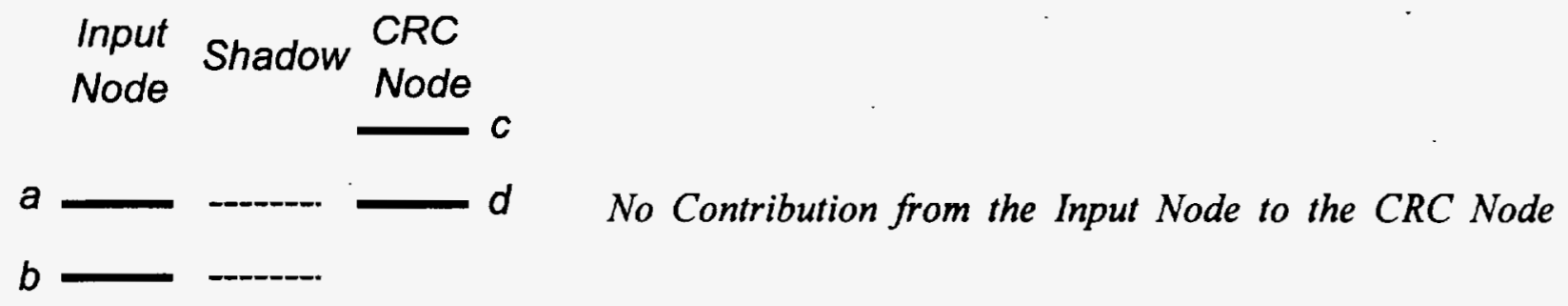


Shadows created by input axial nodes which are smaller than the CRC axial nodes--

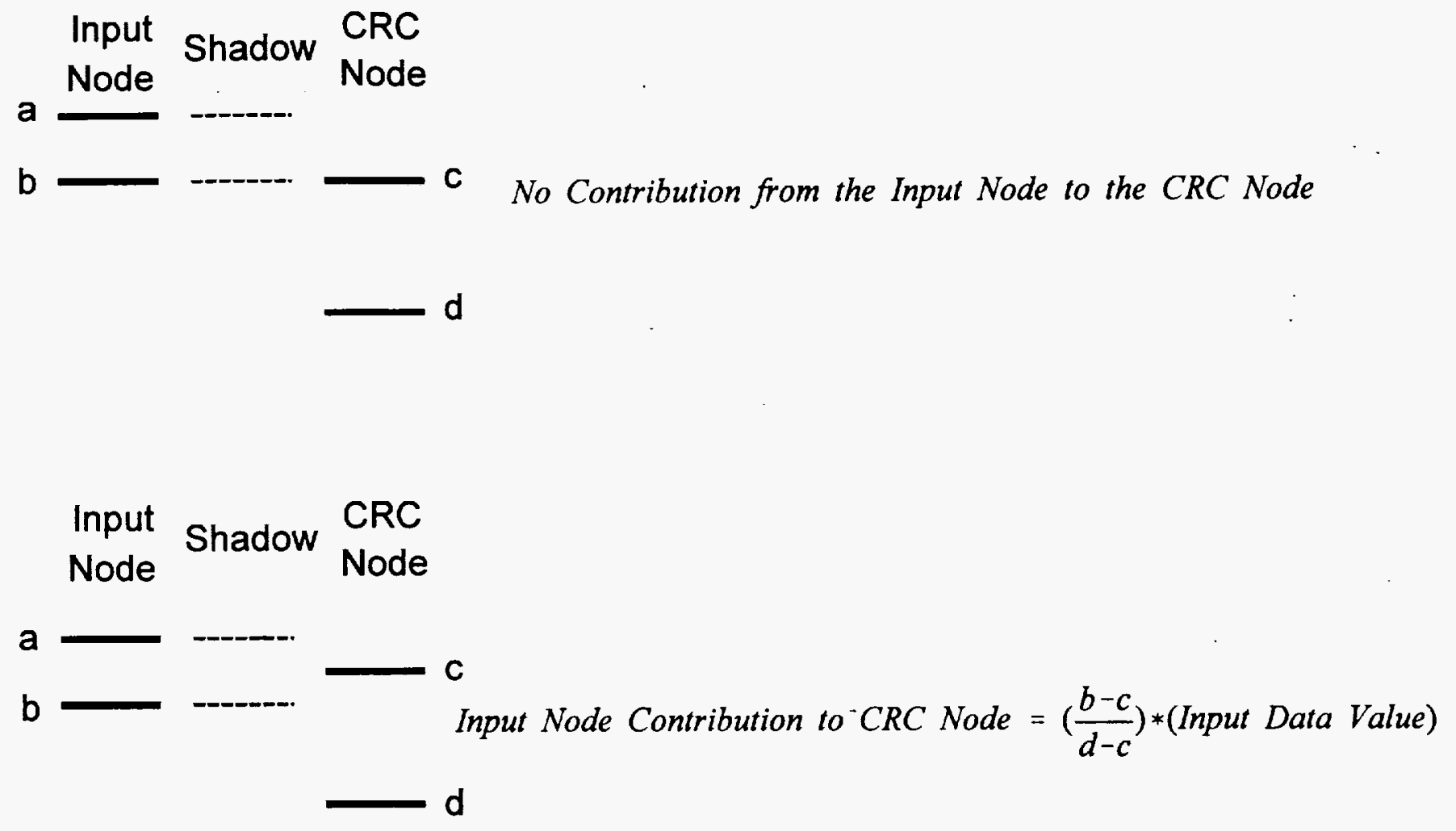
Input
Node
Shadow $\begin{aligned} & \text { CRC } \\ & \text { Node }\end{aligned}$

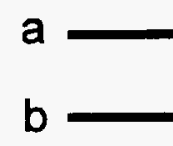

C Input Node Contribution to CRC Node $=\left(\frac{b-a}{d-c}\right) *($ Input Data Value $)$ d 


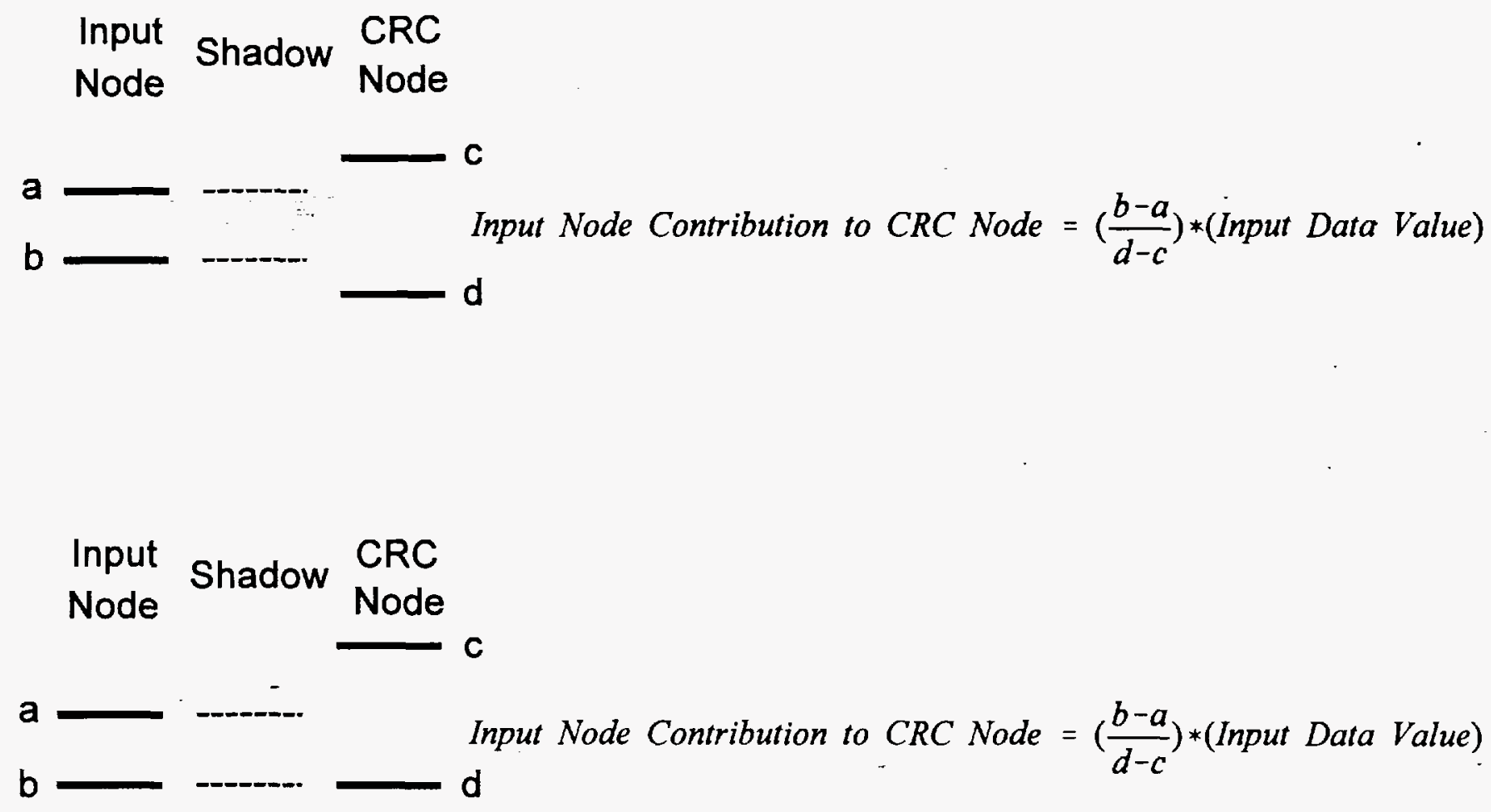

Input Shadow CRC

Node Node $\quad$ c

$a-\cdots$

Input Node Contribution to CRC Node $=\left(\frac{d-a}{d-c}\right) *($ Input Data Value $)$

\begin{abstract}
b
\end{abstract}


$\begin{array}{ll}\text { Input Shadow } & \begin{array}{l}\text { CRC } \\ \text { Node }\end{array} \\ & \end{array}$

No Contribution from the Input Node to the CRC Node

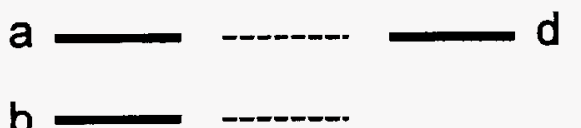

Shadows created by input axial nodes which are larger than the CRC axial nodes-

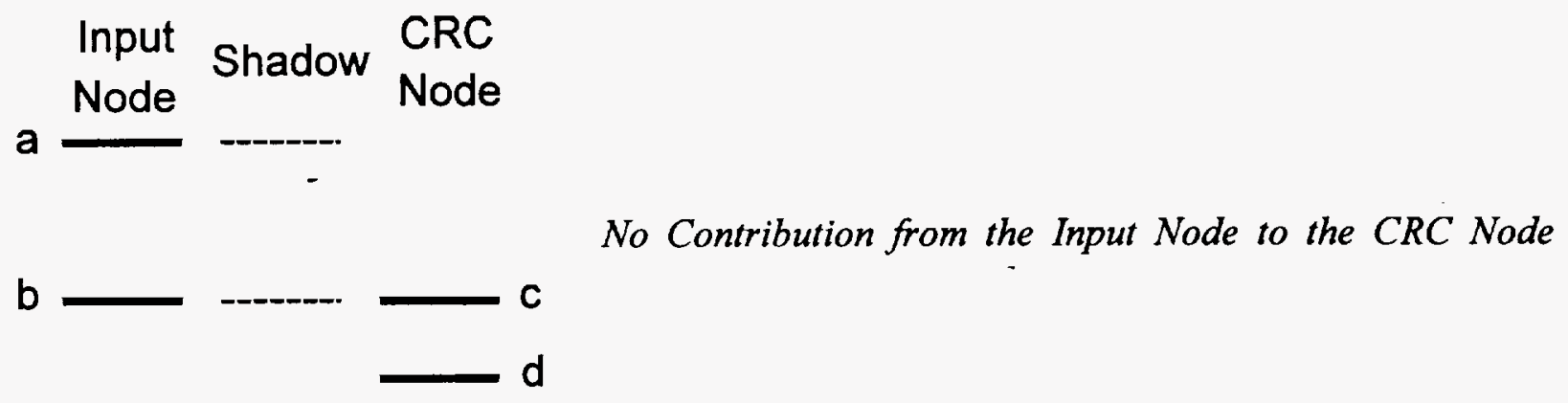

$\begin{array}{ll}\text { Input Shadow } & \text { CRC } \\ \text { Node } & \text { Node }\end{array}$

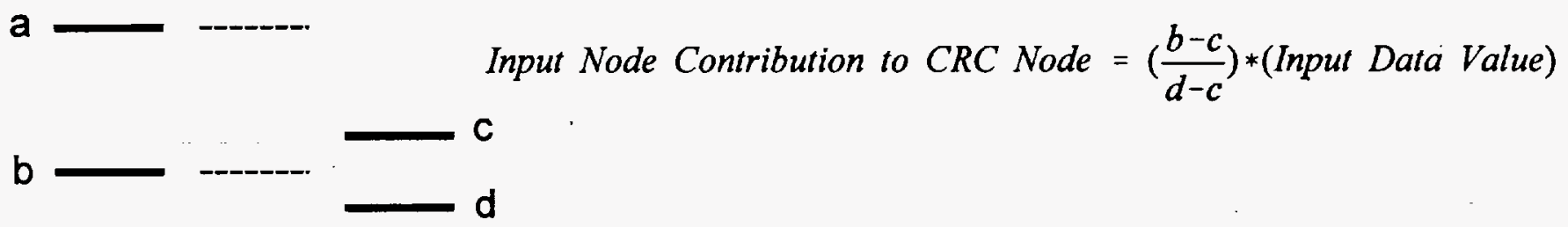



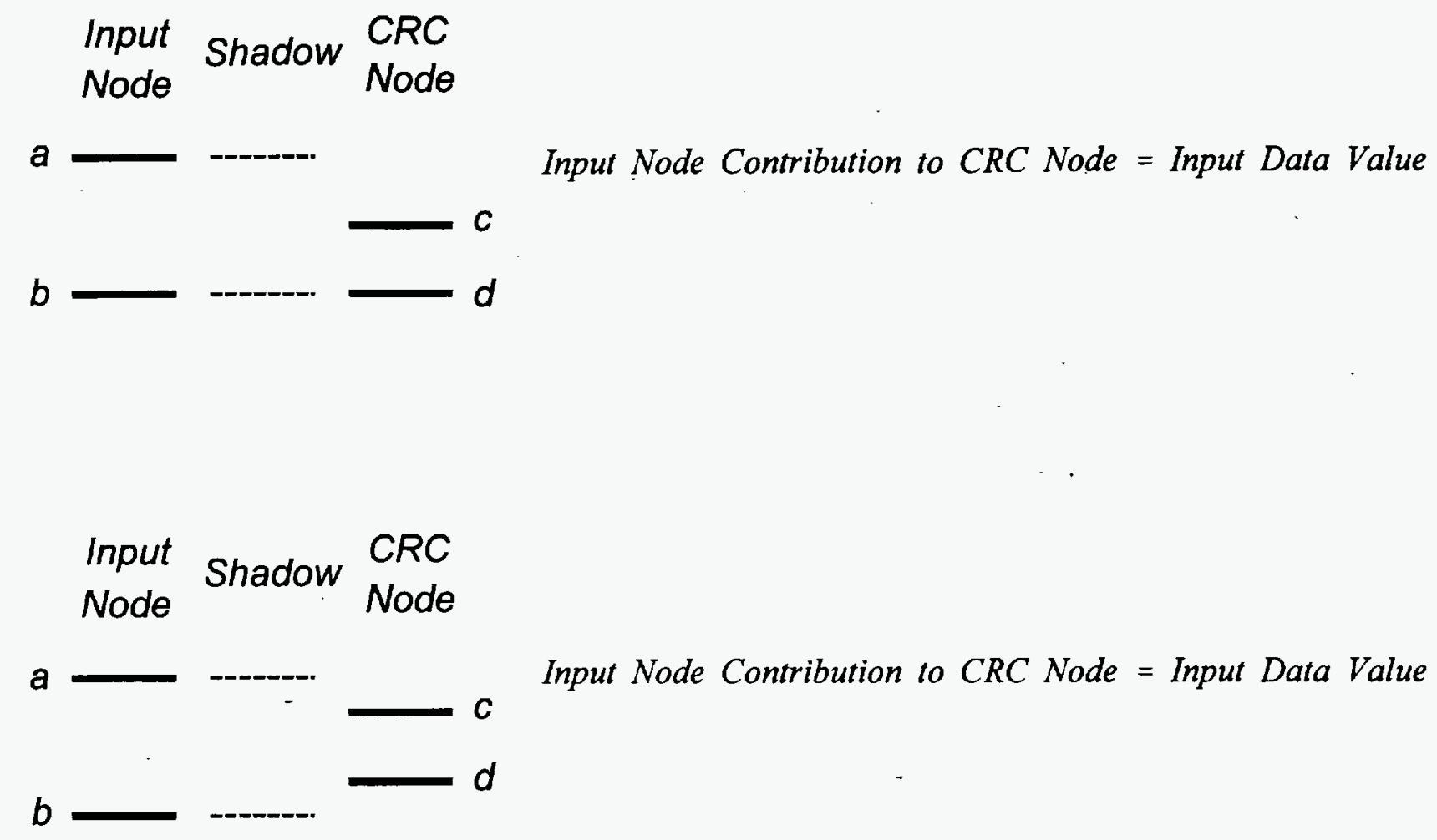

Input Node Contribution to CRC Node = Input Data Value

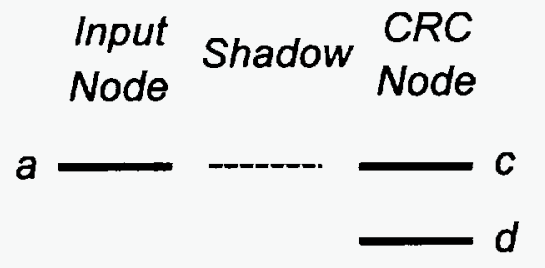

Input Node Contribution to CRC Node = Input Data Value

$b$ 

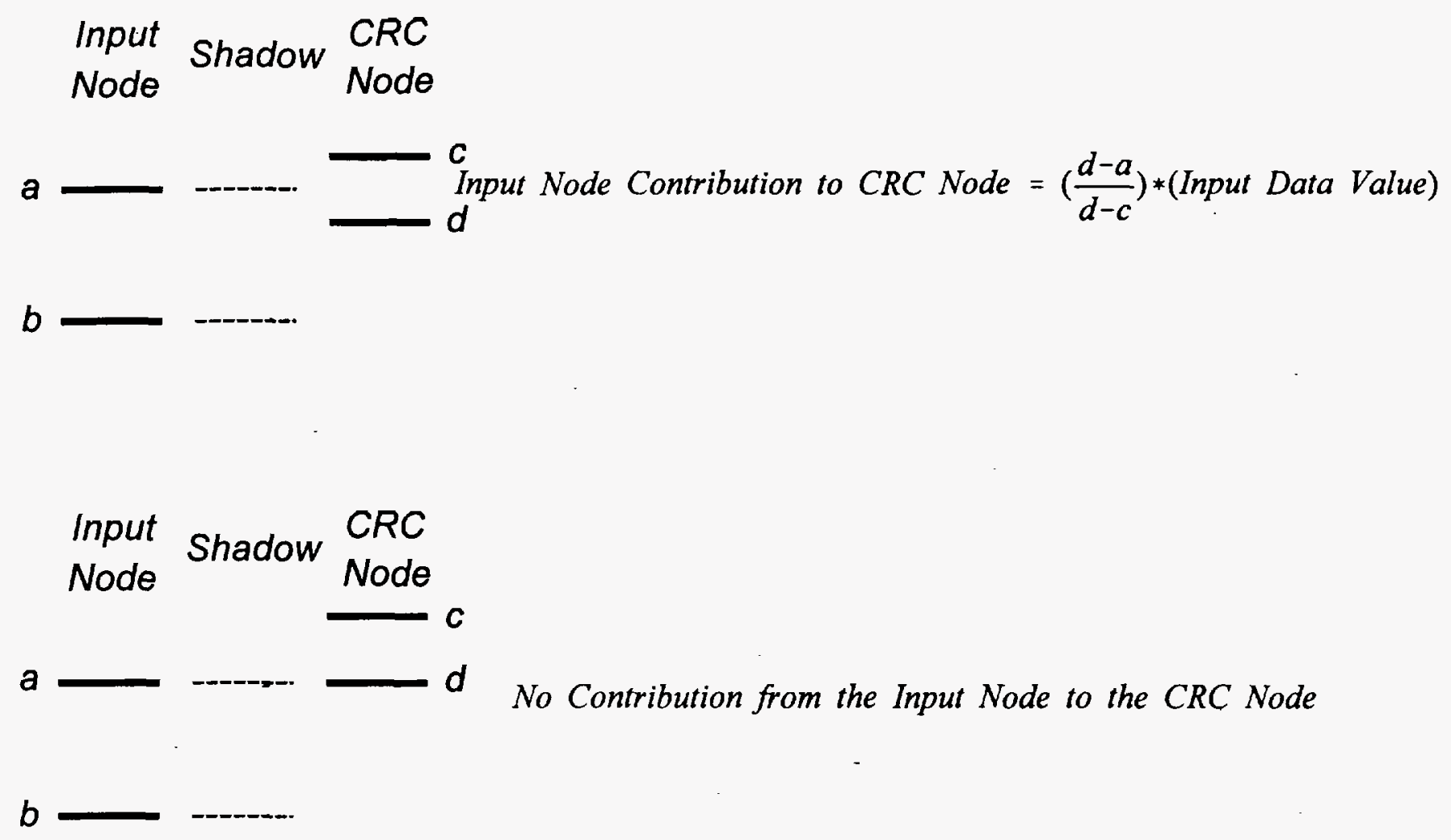

No Contribution from the Input Node to the CRC Node

\subsection{POWER_CALCS Subroutine}

This subroutine calculates the average nodal power to be applied to each CRC axial node in the CRAFT generated statepoint calculations. The average nodal powers are calculated in megawatts using the average nodal burnup during the entire statepoint calculation, the initial uranium mass in the node, and the duration which the statepoint calculation covers in EFPD. The following equation shows how an average nodal power is calculated for a given statepoint calculation.

$$
\text { Average Nodal Power }(M W)=\left(\begin{array}{c}
\text { (Average Nodal Burnup } \\
\text { During Statepoint Calculation in GWd/MTU) } \\
\text { (Initial Uranium Mass in Node in Grams)* }
\end{array}\right.
$$

where, 


$$
\text { Initial Uranium Mass in Node }=\frac{(\text { Initial Uranium Mass in Assembly }) *}{\left(\frac{\text { CRC Node Height }}{\text { CRC Total Active Fuel Height }}\right)}
$$

An average nodal power in units of megawatts is calculated for each node of the assembly for each statepoint calculation. The average nodal power is constant for a given node during a given statepoint calculation. The average nodal powers are not adjusted between the irradiation steps of a given SAS2H calculation. The use of the average nodal burnup in the determination of the average nodal power results in a final total burnup for the node which is equivalent to the node's total average burnup.

\subsection{UNITS_CONVERSION Subroutine}

This subroutine converts all of the $\mathrm{CRC}$ formatted fuel temperature input data from units of degrees Fahrenheit to units of degrees Kelvin. The following equation is used to make this units conversion.

$$
\text { Temperature }(K)=\left[(\text { Temperature }(F)-32.0) *\left(\frac{5}{9}\right)\right]+273.15
$$

This subroutine also converts the $\mathrm{CRC}$ formatted moderator specific volume input data from units of cubic feet per pound to density input data in units of grams per cubic centimeter. The following equation is used to make this conversion. The (1/62.42691) conversion factor appearing in the following equation is obtained from conversion data in reference 3.

$$
\text { Density }\left(\mathrm{g} / \mathrm{cm}^{3}\right)=\frac{1}{\left(\text { Specific Volume }\left(\mathrm{ft}^{3} / l b\right)\right) *(62.42691)}
$$

This subroutine also calculates the $\mathrm{CRC}$ formatted moderator temperature input data in units of degrees Fahrenheit using linear interpolation in the following density versus temperature versus pressure table for subcooled water shown in Table 4.6-1. Table 4.6-1 is obtained from the SCALE-4.3 user documentation (Ref. 1, p. S2.5.12). 
Table 4.6-1

Density $\left(\mathrm{g} / \mathrm{cm}^{3}\right)$ of Subcooled Water at Various Temperatures and Pressures

\begin{tabular}{|c|c|c|c|c|c|c|c|c|c|}
\hline & \multicolumn{9}{|c|}{ Pressure, psia } \\
\hline $\begin{array}{c}\text { Temp. } \\
\left({ }^{\circ} \mathrm{F}\right)\end{array}$ & 3000 & 2500 & 2000 & 1500 & 1000 & 800 & 600 & 400 & 200 \\
\hline 50 & 1.0084 & 1.0069 & 1.0055 & 1.0040 & 1.0025 & 1.0019 & 1.0013 & 1.0007 & 1.0000 \\
\hline 100 & 1.0018 & 1.0004 & 0.9989 & 0.9975 & 0.9960 & 0.9954 & 0.9948 & 0.9942 & 0.9936 \\
\hline 150 & 0.9893 & 0.9878 & 0.9864 & 0.9849 & 0.9834 & 0.9828 & 0.9822 & 0.9815 & 0.9809 \\
\hline 200 & 0.9725 & 0.9709 & 0.9694 & 0.9679 & 0.9663 & 0.9656 & 0.9650 & 0.9644 & 0.9637 \\
\hline 250 & 0.9522 & 0.9505 & 0.9489 & 0.9472 & 0.9455 & 0.9449 & 0.9442 & 0.9435 & 0.9428 \\
\hline 300 & 0.9289 & 0.9271 & 0.9252 & 0.9234 & 0.9215 & 0.9208 & 0.9200 & 0.9192 & 0.9185 \\
\hline 350 & 0.9026 & 0.9006 & 0.8985 & 0.8964 & 0.8943 & 0.8934 & 0.8925 & 0.8916 & \\
\hline 400 & 0.8733 & 0.8709 & 0.8685 & 0.8660 & 0.8634 & 0.8624 & 0.8613 & 0.8603 & \\
\hline 450 & 0.8405 & 0.8375 & 0.8345 & 0.8314 & 0.8281 & 0.8268 & 0.8255 & & \\
\hline 500 & 0.8029 & 0.7992 & 0.7952 & 0.7911 & 0.7869 & 0.7851 & & & \\
\hline 510 & 0.7947 & 0.7907 & 0.7866 & 0.7822 & 0.7776 & & & & \\
\hline 520 & 0.7862 & 0.7820 & 0.7776 & 0.7729 & 0.7680 & & & & \\
\hline 530 & 0.7775 & 0.7729 & 0.7682 & 0.7632 & 0.7579 & & & & \\
\hline 540 & 0.7683 & 0.7635 & 0.7584 & 0.7530 & 0.7472 & & & & \\
\hline 550 & 0.7589 & 0.7537 & 0.7482 & 0.7423 & & & & & \\
\hline 560 & 0.7490 & 0.7434 & 0.7374 & 0.7310 & & & & & \\
\hline 570 & 0.7386 & 0.7326 & 0.7261 & 0.7190 & & & & & \\
\hline 580 & 0.7278 & 0.7212 & 0.7141 & 0.7062 & & & & & \\
\hline 590 & 0.7164 & 0.7092 & 0.7012 & 0.6923 & & & & & \\
\hline 600 & 0.7043 & 0.6963 & 0.6874 & & & & & & \\
\hline 610 & 0.6915 & 0.6825 & 0.6724 & & & & & & \\
\hline 620 & 0.6777 & 0.6676 & 0.6558 & & & & & & \\
\hline 630 & 0.6629 & 0.6512 & 0.6370 & & & & & & \\
\hline 640 & 0.6467 & 0.6329 & & & & & & & \\
\hline
\end{tabular}




\begin{tabular}{|c|c|c|c|c|c|c|c|c|c||}
\cline { 2 - 10 } \multicolumn{1}{c|}{} & \multicolumn{9}{c|}{ Pressure, psia } \\
\hline $\begin{array}{c}\text { Temp. } \\
\left({ }^{\circ} \mathbf{F}\right)\end{array}$ & $\mathbf{3 0 0 0}$ & $\mathbf{2 5 0 0}$ & $\mathbf{2 0 0 0}$ & $\mathbf{1 5 0 0}$ & $\mathbf{1 0 0 0}$ & $\mathbf{8 0 0}$ & $\mathbf{6 0 0}$ & $\mathbf{4 0 0}$ & $\mathbf{2 0 0}$ \\
\hline \hline 650 & 0.6288 & 0.6119 & & & & & & & \\
\hline 660 & 0.6086 & 0.5866 & & & & & & & \\
\hline 670 & 0.5850 & & & & & & & & \\
\hline 680 & 0.5559 & & & & & & & & \\
\hline
\end{tabular}

Once the moderator temperature is determined in degrees Fahrenheit, the same units conversion equation previously described for use with the fuel temperature data is used to convert the moderator temperature to degrees Kelvin.

The CRAFT code utilizes a standard linear interpolation scheme to determine the moderator temperature values once the pressure and density are known. Linear interpolation is performed using the following equation:

$$
\frac{\text { Target Value }-x_{1}}{\text { Reference Value }-y_{1}}=\frac{x_{2}-x_{1}}{y_{2}-y_{1}}
$$

where,

Target Value $=$ the value for which the interpolation is being performed to obtain;

Reference Value = the known value which has a one-to-one correspondence to the Target Value;

$x_{1}=$ the target parameter value displayed in the table which corresponds to $y_{1}$;

$x_{2}=$ the target parameter value displayed in the table which corresponds to $y_{2}$;

$y_{1}=$ the reference parameter value displayed in the table which is

the largest value less than the Reference Value;

$y_{2}=$ the reference parameter value displayed in the table which is the smallest value greater than the Reference Value.

The UNITS_CONVERSION subroutine utilizes the following procedure to perform the linear interpolation.

1) Determine which two adjacent columns of densities in the table correspond to pressures which bound the user-defined system pressure.

2) Linear interpolate between each of the columns defined in step 1 for each row of the table to create a new density column which corresponds to the system pressure.

3) Determine which two adjacent rows in the new density column created in step 2 

4) Linear interpolate between the two bounding density rows to determine the moderator temperature which corresponds to the system pressure and moderator density.

Once the moderator temperatures are calculated in degrees Kelvin for each of the CRC nodes in each statepoint calculation, the UNITS_CONVERSION subroutine's duties are complete.

\subsection{EXECUTION_CONTROL Subroutine}

A description of the EXECUTION_CONTROL subroutine is provided in Section 3.

\subsection{STANDARD_WRITER Subroutine}

This subroutine generates all SAS2H input decks which correspond to BOC to statepoint 2 depletion cases for the initial insertion cycle of the fuel assembly in the reactor. The SAS2H input decks created by the STANDARD_WRITER subroutine contain all fresh fuel. A detailed explanation of how to develop a SAS2H input deck to perform a fuel assembly depletion and decay calculation is provided in reference 1 . The purpose of this discussion is not to explain how to develop a SAS2H input deck, but to explain the general format of the CRAFT generated SAS2H input decks.

The SAS2H input decks generated by the CRAFT code incorporate a general format consisting of the following five sections:

1) Identification and Global Comment Section;

2) Material Specification Section;

3) Base Fuel Assembly Lattice Specification Section;

4) SAS2H Control Specifications and Unit Cell Models Section;

5) Irradiation History Specification Section.

\subsubsection{Identification and Global Comment Section}

The first line of every SAS2H input deck relevant to CRC evaluations contains the SAS2H control module identifier and the "skipshipdata" parameter which tells the SAS2H control module not to perform an optional shielding analysis for a shipping container. The second line of every SAS2H input deck relevant to CRC evaluations is a case identification card. This card identifies the reactor in which the assembly is inserted, the relative one-eighth core symmetry assembly number, the CRC axial node to which the case pertains, the reactor cycle and statepoint at which the case begins, and the reactor cycle and statepoint at which the case ends. The third line identifies the cross-section library which is utilized in the SAS2H calculation. The ENDF/B-V based 44-group cross-section library is currently the suggested library for use in all CRAFT calculations relevant to $\mathrm{CRC}$ analyses. The remainder of the Identification and Global Comment Section contains general comments related to the SAS2H calculation. 


\subsubsection{Material Specification Section}

The material specification section defines the fuel composition, the burnable poison composition, the control rod absorber material composition, the axial power shaping rod absorber material composition, the moderator composition, the fill gas composition, the fuel cladding composition, and other cladding compositions for use in either BPRA's, CRA's, or APSRA's. Only the material compositions necessary for use in a given CRAFT generated SAS2H calculation are specified in the SAS2H input deck. Each material composition specification has a unique material mixture identifier. The fuel composition's material mixture number is always 1 . The fuel cladding's material mixture number is always 2 . The moderator's material mixture is always 3. The $\mathrm{Al}_{2} \mathrm{O}_{3}-\mathrm{B}_{4} \mathrm{C}$ burnable poison's material mixture number is always 4 . The helium fill gas' material mixture number is always 5 . Other compositions such as control rod or axial power shaping rod absorber materials, cladding materials other than the fuel cladding material, or burnable absorber materials other than $\mathrm{Al}_{2} \mathrm{O}_{3}-\mathrm{B}_{4} \mathrm{C}$ must be given unique material mixture identifier numbers greater than 5 . These additional material mixture number specifications are provided by the user in the CRAFT input deck.

The material specification section defines the $\mathrm{UO}_{2}$ fresh fuel composition for the axial node to which the CRAFT generated SAS2H calculation pertains. The $\mathrm{UO}_{2}$ fresh fuel composition is characterized by the fuel density, fuel temperature, and weight percentages of U-234, U-235, U236, and U-238. For fresh fuel SAS2H cases, a number of additional isotopes are specified in trace amounts in the fuel composition to assure that their buildup and decay is tracked during the depletion calculation. Table 4.8.2-1 contains a listing of the trace isotopes which are always specified as each having a concentration of $1 \mathrm{E}-21$ atoms $/ \mathrm{b}-\mathrm{cm}$ in the fresh fuel composition.

Table 4.8.2-1

Trace Isotopes Specified in Fresh Fuel Compositions

\begin{tabular}{|c|c|c|c|c|c|c|}
\hline $\mathrm{kr}-83$ & $\mathrm{kr}-85$ & sr-90 & $\mathrm{y}-89$ & mo-95 & zr-93 & zr-94 \\
\hline $\mathrm{zr}-95$ & nb-94 & tc-99 & rh-103 & rh-105 & ru-101 & ru-106 \\
\hline pd-105 & pd-108 & ag-109 & sb-124 & xe-131 & xe-132 & xe-135 \\
\hline xe-136 & cs-134 & cs-135 & cs-137 & ba-136 & la-139 & ce-144 \\
\hline nd-143 & nd-145 & pm-147 & pm-148 & nd-147 & sm-147 & sm-149 \\
\hline sm-150 & sm-151 & sm-152 & gd-155 & eu-153 & eu-154 & eu-155 \\
\hline
\end{tabular}

Several of the additional material composition specifications that must be provided in the SAS2H input decks include cladding materials for either fuel rods, control rod assemblies, axial power shaping rod assemblies, or burnable poison rod assemblies. The cladding materials available for specification include ZIRC-4, INCONEL, SS316, SS316S, SS304, and SS304S. The SS316/SS316S and SS304/SS304S materials are delineated by the use of two special weighting functions. The special weighting functions affect the generation of multigroup cross-sections for iron, nickel, and chromium. One of the special weighting functions corresponds to $1 / \mathrm{E} \sigma_{t}(E)$, 
where $\sigma_{t}(E)$ is the total cross-section of the stainless steel material. In the other special weighting function, $\sigma_{t}(E)$ is the total cross-section for the referenced nuclide. The stainless steel material identifiers ending in " $\mathrm{S}$ " use the weighting function where $\sigma_{t}(\mathrm{E})$ is the total cross-section for the referenced nuclide. The compositions and SCALE nuclide identifiers for the various cladding material compositions are shown in Table 4.8.2-2.

Table 4.8.2-2

Cladding Material Compositions Available in the CRAFT Code

\begin{tabular}{|c|c|c|c|c|c|c|c|}
\hline \multirow{2}{*}{$\begin{array}{l}\text { Element/ } \\
\text { Isotope }\end{array}$} & \multirow{2}{*}{$\begin{array}{l}\text { SCALE } \\
\text { Identifier }\end{array}$} & \multicolumn{6}{|c|}{ Constituent wt $\%$ in Each Cladding Material Composition } \\
\hline & & ZIRC-4 & INCONEL & SS316 & SS316S & SS304 & SS304S \\
\hline C & 6012 & -- & - & 0.08 & 0.08 & -.. & -- \\
\hline $\mathrm{O}$ & 8016 & 0.12 & --- & --- & -- & -- & --- \\
\hline $\mathrm{Si}$ & 14000 & -- & 2.5 & 1.0 & 1.0 & -- & --- \\
\hline $\mathrm{Ti}$ & 22000 & --- & 2.5 & --- & - & -- & -- \\
\hline $\mathrm{Cr}$ & 24000 & 0.10 & -- & - & 17.0 &.- & 19.0 \\
\hline $\mathrm{Cr}^{*}$ & 24304 & -- & --- & 17.0 & - & 19.0 & -- \\
\hline $\mathrm{Cr}^{*}$ & 24404 & --- & 15.0 & --- & - & --- & -- \\
\hline $\mathrm{Mn}$ & 25055 & --- & -- & 2.0 & 2.0 & 2.0 & 2.0 \\
\hline $\mathrm{Fe}$ & 26000 & 0.20 & -- & -- & 65.42 & --- & 69.5 \\
\hline $\mathrm{Fe}^{*}$ & 26304 & -- & -- & 65.42 & --- & 69.5 & -- \\
\hline $\mathrm{Fe}^{*}$ & 26404 & - & 7.0 & - & -- & --- & -- \\
\hline $\mathrm{Ni}$ & 28000 & --- & -- & -- & 12.0 & --- & 9.5 \\
\hline $\mathrm{Ni}^{*}$ & 28304 & -- & --- & 12.0 & -- & 9.5 & --- \\
\hline $\mathrm{Ni}^{*}$ & 28404 & - & 73.0 & --- & --- & --- & -- \\
\hline $\mathrm{Zr}$ & 40000 & 98.18 & -- & - & --- &.- & --- \\
\hline Mo & 42000 & --- & --- & 2.5 & 2.5 & -- & --- \\
\hline $\mathrm{Sn}$ & 50000 & 1.40 & -- & -- & -- & -- & $\ldots$ \\
\hline
\end{tabular}

These SCALE nuclide identifiers refer to the special $1 / E \sigma_{t}(E)$ weighted multigroup cross-sections.

Once the fuel material specification is complete, the fuel cladding material specification is defined. The fuel cladding material may be either ZIRC-4, INCONEL, SS316, SS316S, SS304, 
or SS404S. The compositions of these materials are hard-wired in the CRAFT code. The user is required to define an average fuel cladding temperature that will be applied to all fuel cladding material specifications.

The moderator material specification may contain homogenized spacer grid materials and/or soluble boron. The appropriate CRAFT calculated moderator density and temperature values are utilized in the moderator material composition description. The soluble boron concentration corresponding to the first irradiation step of the SAS2H case is used to define the base soluble boron content in the moderator composition. The soluble boron concentrations in each of the irradiation steps of the SAS2H calculation are defined by specifying a fraction of the initial boron concentration specified in the base moderator material composition description. The material and volume fraction of spacer grids displacing moderator in the fuel assembly are specified by the user in the CRAFT input deck. The spacer grid materials available for specification include ZIRC-4, INCONEL, SS316, SS316S, SS404, and SS404S. The spacer grids are homogenized in the moderator composition based on the volume fraction of spacer grids in the moderator that is specified in the CRAFT input deck. The sum of the volume fractions of spacer grid material and moderator material (light-water) should equal unity.

If the fuel assembly contains a BPRA during the CRAFT generated SAS2H calculation, the material specifications for the BPR cladding and burnable poison material are specified. The BPR cladding may be designated as either ZIRC-4, INCONEL, SS316, SS316S, SS304, and SS304S. The default burnable poison material is $\mathrm{Al}_{2} \mathrm{O}_{3}-\mathrm{B}_{4} \mathrm{C}$, but any arbitrary burnable poison material may be specified. The BPR cladding and burnable poison material compositions are given the same temperature as the moderator.

If the fuel assembly contains a CRA or APSRA during the CRAFT generated SAS2H calculation, the material specifications for the CR or APSR cladding and absorber material are specified. The CR or APSR cladding may be designated as either ZIRC-4, INCONEL, SS316, SS316S, SS304, and SS304S. The CR or APSR cladding and absorber material compositions are given the same temperature as the moderator.

The fuel rod fill gas material is always specified as helium. The helium material temperature is allowed to default to 293 degrees Kelvin.

\subsubsection{Base Fuel Assembly Lattice Specification Section}

The base fuel assembly lattice specification section describes the fuel assembly configuration and specifies special control parameters that are to be utilized in performing the XSDRNPM calculations associated with the CRAFT generated SAS2H calculation. The fuel assembly lattice specification includes a "squarepitch" designator which tells SAS2H that the fuel assembly is a square array of unit cells with a constant pitch. The fuel rod pitch, fuel pellet outer diameter, fuel rod cladding inner diameter, and fuel rod cladding outer diameter are specified. The number of fuel rods per fuel assembly and active fuel length are also specified. The active fuel length represents the fuel stack height for the CRC node that the CRAFT generated SAS2H calculation represents. The special parameters that allow more control over the XSDRNPM calculation are 
Title: CRC Depletion Calculations for the Rodded Assemblies in Batches 1, 2, 3, and $1 \mathrm{X}$ of Crystal River Unit 3 Document Identifier: BBA000000-01717-0200-00040 REV 00 Date Printed: 7/31/97 Attachment I, Page 28 of 84

described in Section 7. One special control parameter is always specified in the CRAFT generated SAS2H calculations. This parameter is designated "szf", and represents the spatial mesh factor for use in defining the XSDRNPM one-dimensional transport calculations.

\subsubsection{SAS2H Control Specifications and Unit Cell Models Section}

The SAS2H control specifications and unit cell models are provided in this section of the SAS2H input deck. The control specifications for SAS2H include the number of irradiation steps in the calculation, the number of cross-section libraries to be specified per irradiation step, and the SAS2H output print level. The unit cell model specification includes the following:

1) the unit cell model input level;

2) the number of radial zones to be specified in all unit cells of the SAS2H calculation;

3) the moderator material mixture number in the unit cell models;

4) the XSDRNPM spatial mesh factor;

5) the signal to specify if a single unit cell model description will be provided for all irradiation steps or if multiple unit cell model descriptions will be provided to accommodate each irradiation step.

\subsubsection{Irradiation History Specification Section}

The irradiation history specification section includes the following data for each irradiation step:

1) the assembly (node) power in megawatts;

2) the irradiation step burn duration in calendar days;

3) the down time following the irradiation step in calendar days;

4) the fraction of the soluble boron concentration specified in the base moderator material composition that corresponds to the average soluble boron concentration in the moderator over the duration of the irradiation step.

The irradiation history specification section is always the final section in CRAFT generated SAS2H input decks.

\subsubsection{Calculations Performed within the STANDARD_WRITER Subroutine}

- The density of $\mathrm{UO}_{2}$ in the fresh fuel composition is calculated by the STANDARD_WRITER subroutine based on the initial mass loading of uranium in the assembly. The initial mass loading of uranium in an axial node is calculated using the following equation. 


$$
\text { Initial Uranium Mass in Node }=\left(\begin{array}{c}
(\text { Initial Uranium Mass in Assembly }) * \\
\left(\frac{C R C \text { Node Height }}{C R C \text { Total Active Fuel Height }}\right)
\end{array}\right.
$$

The mass of oxygen in the $\mathrm{UO}_{2}$ of the node must be calculated after the initial uranium mass in the node is determined. The following equation is used to calculate the mass of oxygen in the fuel. The weight percentages of the uranium isotopes (U-234, U-235, U236 , and U-238) are calculated using the equations presented in the next bulleted calculation.

Oxygen Mass in $\mathrm{UO}_{2}=\frac{\left[\left(\text { Mass of Uranium in } U O_{2}\right) *(2) *(15.994915) *(100)\right]}{\left[\begin{array}{c}\left(w t \% U^{235}\right) *(235.043915)+\left(w t \% U^{234}\right) *(234.040904)+ \\ \left(w t \% U^{236}\right) *(236.045637)+\left(w t \% U^{238}\right) *(238.05077)\end{array}\right]}$

The mass of $\mathrm{UO}_{2}$ in the axial node is then calculated by summing the mass of the uranium in the axial node and the mass of oxygen in the axial node.

The fuel volume in the axial node must be calculated prior to calculating the fuel density. The fuel volume is calculated using the following equation.

$$
\begin{gathered}
\text { Fuel Volume } \\
\text { in Axial Node }
\end{gathered}=\begin{gathered}
\left(\frac{\pi}{4}\right) * \begin{array}{l}
(\text { Fuel Outer Diameter })^{2} *(\text { Node Height }) * \\
(\text { Number of Fuel Rods in Assembly })
\end{array}
\end{gathered}
$$

The fuel density in the axial node is then calculated by dividing the $\mathrm{UO}_{2}$ mass in the node by the fuel volume in the node.

- The weight percentages of the various isotopes in the uranium of the fresh $\mathrm{UO}_{2}$ fuel composition are calculated using the following equations (Ref. 2).

$$
\begin{gathered}
U^{234} w t \%=(0.007731) *\left(U^{235} w t \%\right)^{1.0837} \\
U^{236} w t \%=(0.0046) *\left(U^{235} w t \%\right) \\
U^{238} w t \%=100-U^{234} w t \%-U^{235} w t \%-U^{236} w t \%
\end{gathered}
$$

- The volume fraction of $\mathrm{H}_{2} \mathrm{O}$ in the homogenized moderator composition must be calculated by the STANDARD_WRITER subroutine to define the moderator material composition. The following equation is used to calculate the appropriate volume fraction of $\mathrm{H}_{2} \mathrm{O}$. 

$n$ Homogenized Moderator Composition $=1.0-$ in Homogenized Moderator Compositio

- The volume fraction of soluble boron in the $\mathrm{H}_{2} \mathrm{O}$ of the homogenized moderator composition must be calculated by the STANDARD_WRITER subroutine to define the moderator material composition. The following equation is used to calculate the appropriate volume fraction of soluble boron.

\author{
Boron \\ Volume Fraction of Soluble Boron \\ (Concentration) * $(1.0 E-6) *$ \\ ppm \\ in Homogenized Moderator Composition \\ ( $\left.\begin{array}{c}\text { Volume Fraction of } \mathrm{H}_{2} \mathrm{O} \text { in } \\ \text { Homogenized Moderator Composition }\end{array}\right)$
}

- The density of the homogenized moderator composition must be calculated by the STANDARD_WRITER subroutine to define the moderator material composition. The following equation is used to calculate the appropriately averaged homogenized moderator density in grams per cubic centimeter.

$\left.\left.\begin{array}{l}\text { Density of Homogenized } \\ \text { Moderator Composition }\end{array}=\begin{array}{c}\left.\begin{array}{c}\text { Actual } \\ {[(\text { Moderator })} \\ \text { Density }\end{array} *\left(\begin{array}{c}\text { Volume Fraction } \\ \text { of } \mathrm{H}_{2} \mathrm{O} \text { in the } \\ \text { Moderator Composition }\end{array}\right)\right]+ \\ \text { Actual Density } \\ {\left[\begin{array}{c}\text { Actume Fraction } \\ \text { Spacer Material }\end{array}\right)}\end{array}\right) \begin{array}{c}\text { Volume } \\ \text { (of Spacer Material in the }) \\ \text { Moderator Composition }\end{array}\right]$

- If the fuel assembly contains a BPRA with $\mathrm{Al}_{2} \mathrm{O}_{3}-\mathrm{B}_{4} \mathrm{C}$ burnable absorber material during the irradiation history covered in a SAS2H calculation, the aluminum and oxygen weight fractions must be calculated to define the fresh burnable absorber material composition. The following equation are used to calculate the aluminum and oxygen weight fractions in $\mathrm{Al}_{2} \mathrm{O}_{3}-\mathrm{B}_{4} \mathrm{C}$.

$$
\underset{\text { Fraction in } \mathrm{Al}_{2} \mathrm{O}_{3}-B_{4} C}{\text { Aluminum Weight }}=\begin{aligned}
& \left(\frac{100-B_{4} C \text { wt } \% \text { in } \mathrm{Al}_{2} \mathrm{O}_{3}-B_{4} C}{100}\right) *\left(\text { Density of } \mathrm{Al}_{2} \mathrm{O}_{3}-B_{4} C\right)^{2} * \\
& (2) *(26.981539) *\left(\frac{1}{101.9631}\right)
\end{aligned}
$$




$$
\begin{aligned}
& \text { Oxygen Weight } \\
& \text { Faction in } \mathrm{Al}_{2} \mathrm{O}_{3}-B_{4} \mathrm{C}
\end{aligned}=1-\left(\frac{\mathrm{B}_{4} \mathrm{C} \text { wt\% in } \mathrm{Al}_{2} \mathrm{O}_{3}-\mathrm{B}_{4} \mathrm{C}}{100}\right)-\left(\begin{array}{c}
\text { Aluminum Weight } \\
\text { Fraction in } \mathrm{Al}_{2} \mathrm{O}_{3}-B_{4} C
\end{array}\right)
$$

- The soluble boron fraction must be calculated by the STANDARD_WRITER subroutine for all irradiation steps. The soluble boron fraction for a given irradiation step is calculated using the following equation.

$$
\begin{aligned}
& \text { Soluble Boron Fraction }=\text { Soluble Boron ppm in Irradiation Step } \\
& \text { in Irradiation Step }=\frac{\text { Soluble Boron ppm in Base Moderator }}{\text { Compostion }} \\
& \text { Composition of the SAS2H Input Deck }
\end{aligned}
$$

\subsection{CONTINUATION_WRITER Subroutine}

- This subroutine generates all SAS2H input decks which correspond to continuation cases in which the fuel and burnable poison isotopic initial charge compositions are obtained from the output of a previous CRAFT generated SAS2H calculation. A detailed explanation of how to develop a SAS2H input deck to perform a fuel assembly depletion and decay calculation is provided in reference 1. The purpose of this discussion is not to explain how to develop a SAS2H input deck, but to explain the general format and calculations utilized by CRAFT in generating SAS2H input decks for calculations which initially contain spent fuel and burnable poison material compositions.

The format of the CRAFT generated SAS2H input decks for the continuation of a fuel assembly depletion and decay calculation relevant to CRC analyses is the same as that previously described for the standard beginning-of-life SAS2H input decks. The material specification section of the SAS2H input deck is the only input section where the continuation case differs from the standard case.

The CRAFT code tracks the depletion and decay of the fuel and burnable absorber materials during the fuel assembly depletion and decay calculation. The CONTINUATION_WRITER subroutine is designed to locate the appropriate fuel and burnable poison isotopic concentrations, and utilize them in developing the correct fuel and burnable poison initial charge compositions to allow for continuation of the fuel assembly depletion calculation. All calculations performed by the STANDARD_WRITER subroutine other than those related to the fuel and burnable poison material composition specifications are performed identically by the CONTINUATION_WRITER subroutine.

\subsubsection{Initial Charge Fuel and Burnable Poison Material Composition Specifications}

The initial charge fuel material composition specification for a continuation SAS2H calculation utilizes all available isotopic concentrations from the appropriate previous SAS2H depletion and 
decay calculation's output for which cross-section data is available in the SCALE 44-group library (Vol. 3, p. M4.2.19, Ref. 1) (recommended CRC cross-section library). Table 4.9.1-1 contains a listing of all the isotopes for which data is available in the 44-group cross-section library.

Table 4.9.1-1

Isotopic Inventory of the 44-group Cross-Section Library

\begin{tabular}{|c|c|c|c|c|c|c|c|c|}
\hline $\mathrm{H}-1$ & $\mathrm{H}-2$ & $\mathrm{H}-3$ & $\mathrm{He}-3$ & $\mathrm{He}-4$ & $\mathrm{Li}-6$ & $\mathrm{Li}-7$ & $\mathrm{Be}-9$ & B-10 \\
\hline B-11 & $\mathrm{C}-12$ & $N-14$ & $\mathrm{~N}-15$ & $0-16$ & $0-17$ & F-19 & $\mathrm{Na}-23$ & $\mathrm{Mg}$ \\
\hline Al-27 & $\mathrm{Si}$ & P-31 & S & $S-32$ & $\mathrm{Cl}$ & $\mathrm{K}$ & $\mathrm{Ca}$ & $\mathrm{Ti}$ \\
\hline V & $\mathrm{Cr}$ & $\mathrm{Mn}-55$ & $\mathrm{Fe}$ & Co-59 & $\mathrm{Ni}$ & $\mathrm{Cu}$ & $\mathrm{Ga}$ & $\mathrm{Ge}-72$ \\
\hline $\mathrm{Ge}-73$ & $\mathrm{Ge}-74$ & Ge-76 & As-75 & $\mathrm{Se}-74$ & $\mathrm{Se}-76$ & Se-77 & $\mathrm{Se}-78$ & Se-80 \\
\hline $\mathrm{Se}-82$ & $\mathrm{Br}-79$ & $\mathrm{Br}-81$ & $\mathrm{Kr}-78$ & $\mathrm{Kr}-80$ & $\mathrm{Kr}-82$ & $\mathrm{Kr}-83$ & $\mathrm{Kr}-84$ & $\mathrm{Kr}-85$ \\
\hline $\mathrm{Kr}-86$ & $\mathrm{Rb}-85$ & $\mathrm{Rb}-86$ & $\mathrm{Rb}-87$ & $\mathrm{Sr}-84$ & Sr-86 & Sr-87 & Sr-88 & Sr-89 \\
\hline Sr-90 & $Y-89$ & $Y-90$ & $Y-91$ & $\mathrm{Zr}$ & $\mathrm{Zr}-90$ & $\mathrm{Zr}-91$ & $\mathrm{Zr}-92$ & $\mathrm{Zr}-93$ \\
\hline $\mathrm{Zr}-94$ & $\mathrm{Zr}-95$ & $\mathrm{Zr}-96$ & $\mathrm{Nb}-93$ & $\mathrm{Nb}-94$ & $\mathrm{Nb}-95$ & Mo & Mo-92 & Mo-94 \\
\hline Mo-95 & Mo-96 & Mo-97 & Mo-98 & Mo-99 & Mo-100 & Tc-99 & Ru-96 & Ru-98 \\
\hline Ru-99 & $\mathrm{Ru}-100$ & $\mathrm{Ru}-101$ & Ru-102 & Ru-103 & $\mathrm{Ru}-104$ & $\mathrm{Ru}-105$ & $\mathrm{Ru}-106$ & $\mathrm{Rh}-103$ \\
\hline Rh-105 & Pd-102 & Pd-104 & Pd-105 & Pd-106 & Pd-107 & Pd-108 & Pd-110 & Ag-107 \\
\hline Ag-109 & Ag-111 & $\mathrm{Cd}$ & Cd-106 & $\mathrm{Cd}-108$ & $\mathrm{Cd}-110$ & Cd-111 & $\mathrm{Cd}-112$ & Cd-113 \\
\hline Cd-114 & Cd-116 & $\mathrm{Cd}-115 \mathrm{~m}$ & In-113 & In-115 & $\mathrm{Sn}-112$ & Sn-114 & Sn-115 & Sn-116 \\
\hline Sn-117 & Sn-118 & Sn-119 & $\mathrm{Sn}-120$ & Sn-122 & $\mathrm{Sn}-123$ & Sn-124 & Sn-125 & $\mathrm{Sn}-126$ \\
\hline $\mathrm{Sb}-121$ & $\mathrm{Sb}-123$ & Sb-124 & Sb-125 & $\mathrm{Sb}-126$ & $\mathrm{Te}-120$ & Te-122 & $\mathrm{Te}-123$ & $\mathrm{Te}-124$ \\
\hline $\mathrm{Te}-125$ & Te-126 & $\mathrm{Te}-128$ & Te-130 & $\mathrm{Te}-132$ & $\mathrm{Te}-127 \mathrm{~m}$ & $\mathrm{Te}-129 \mathrm{~m}$ & $\mathrm{I}-127$ & I-129 \\
\hline $\mathrm{I}-130$ & I-131 & I- 135 & $\mathrm{Xe}-124$ & $\mathrm{Xe}-126$ & $\mathrm{Xe}-128$ & $\mathrm{Xe}-129$ & $\mathrm{Xe}-130$ & $\mathrm{Xe}-131$ \\
\hline $\mathrm{Xe}-132$ & $\mathrm{Xe}-133$ & $\mathrm{Xe}-134$ & $\mathrm{Xe}-135$ & $\mathrm{Xe}-136$ & Cs-133 & Cs-134 & $\mathrm{Cs}-135$ & $\mathrm{Cs}-136$ \\
\hline Cs-137 & $\mathrm{Ba}-134$ & Ba-135 & $\mathrm{Ba}-136$ & $\mathrm{Ba}-137$ & $\mathrm{Ba}-138$ & $\mathrm{Ba}-140$ & La-139 & $\mathrm{La}-140$ \\
\hline Ce- 140 & $\mathrm{Ce}-141$ & $\mathrm{Ce}-142$ & $\mathrm{Ce}-143$ & $\mathrm{Ce}-144$ & Pr-141 & Pr-142 & Pr-143 & $\mathrm{Nd}-142$ \\
\hline $\mathrm{Nd}-143$ & Nd-144 & $\mathrm{Nd}-145$ & $\mathrm{Nd}-146$ & $\mathrm{Nd}-147$ & $\mathrm{Nd}-148$ & $\mathrm{Nd}-150$ & Pm-147 & Pm-148 \\
\hline$P m-149$ & Pm-151 & Pm-148m & $\mathrm{Sm}-144$ & $\mathrm{Sm}-147$ & Sm-148 & Sm-149 & $\mathrm{Sm}-150$ & Sm-151 \\
\hline
\end{tabular}


Table 4.9.1-1

Isotopic Inventory of the 44-group Cross-Section Library

\begin{tabular}{|c|c|c|c|c|c|c|c|c|}
\hline Sm-152 & Sm-153 & Sm-154 & Eu & Eu-151 & Eu-152 & Eu-153 & Eu-154 & Eu-155 \\
\hline Eu-156 & Eu-157 & Gd-152 & Gd-154 & Gd-155 & Gd-156 & Gd-157 & Gd-158 & Gd-160 \\
\hline Tb-159 & Tb-160 & Dy-160 & Dy-161 & Dy-162 & Dy-163 & Dy-164 & Ho-165 & Er-166 \\
\hline Er-167 & Lu-175 & Lu-176 & Hf & Hf-174 & Hf-176 & Hf-177 & Hf-178 & Hf-179 \\
\hline Hf-180 & Ta-181 & Ta-182 & W & W-182 & W-183 & W-184 & W-186 & Re-185 \\
\hline Re-187 & Au-197 & Pb & Bi-209 & Th-230 & Th-232 & Pa-231 & Pa-233 & U-232 \\
\hline U-233 & U-234 & U-235 & U-236 & U-237 & U-238 & Np-237 & Np-238 & Pu-236 \\
\hline Pu-237 & Pu-238 & Pu-239 & Pu-240 & Pu-241 & Pu-242 & Pu-243 & Pu-244 & Am-241 \\
\hline Am-242 & Am-243 & Am-242m & Cm-241 & Cm-242 & Cm-243 & Cm-244 & Cm-245 & Cm-246 \\
\hline Cm-247 & Cm-248 & Bk-249 & Cf-249 & Cf-250 & Cf-251 & Cf-252 & Cf-253 & Es-253 \\
\hline
\end{tabular}

The fuel composition is composed of the initial oxygen mass in the fresh $\mathrm{UO}_{2}$ and the mass of each of the actinides and fission products of the depleted fuel composition which are available in the 44-group library. There are some isotopes listed in the ORIGEN output of the spent fuel composition which are not available in the 44-group library. These isotopes are excluded from the initial charge composition for the continuation of the fuel assembly depletion. A listing of all excluded isotopes and their abundance in grams per node is retained in the CRAFT generated "*.notes" file corresponding to the SAS2H calculation for which the initial charge composition is obtained. The total mass of all isotopes (including oxygen) in the fuel composition is calculated to assist in determining the weight percentages of each isotope in the composition and the density of the composition. The fuel composition is then defined as an arbitrary material specification in the SAS2H input deck with the appropriate nodal fuel temperature applied.

Excluding the isotopic concentrations from the ORIGEN-S output, that are not available in the 44-group library, from the fuel charge composition of a subsequent depletion calculation has a negligible effect on the neutron spectrum. The neutron spectrum must be predicted correctly during the SAS2H depletion calculations to obtain the proper cell-weighting of the crosssections. For an absorber isotope to have a significant effect on the neutron spectrum, the absorber isotope must be present in a significant quantity and have a significant absorption crosssection. Three simple calculations were performed to demonstrate that the isotopes excluded from the continuation SAS2H depletion calculations (as identified in the "* notes" files) do not effect the neutron spectrum significantly enough to result in a change in the final depleted composition. The first two of the three calculations represent a simple fuel depletion calculation that was split into parts and continued via CRAFT. The third of these calculations is a continuous calculation equivalent to the simple depletion represented by the first two calculations. The final depleted isotopic results at the end of the second calculation (the second 
part of the total depletion composed of the first two calculations) are identical to the final depleted isotopic results obtained from the third calculation. The SAS2H input decks for these three calculations are presented in Figure 4.9.1-1 through Figure 4.9.1-3.

\section{Figure 4.9.1-1 Calculation 1 of the Isotopic Exclusion Test Depletion Calculation}

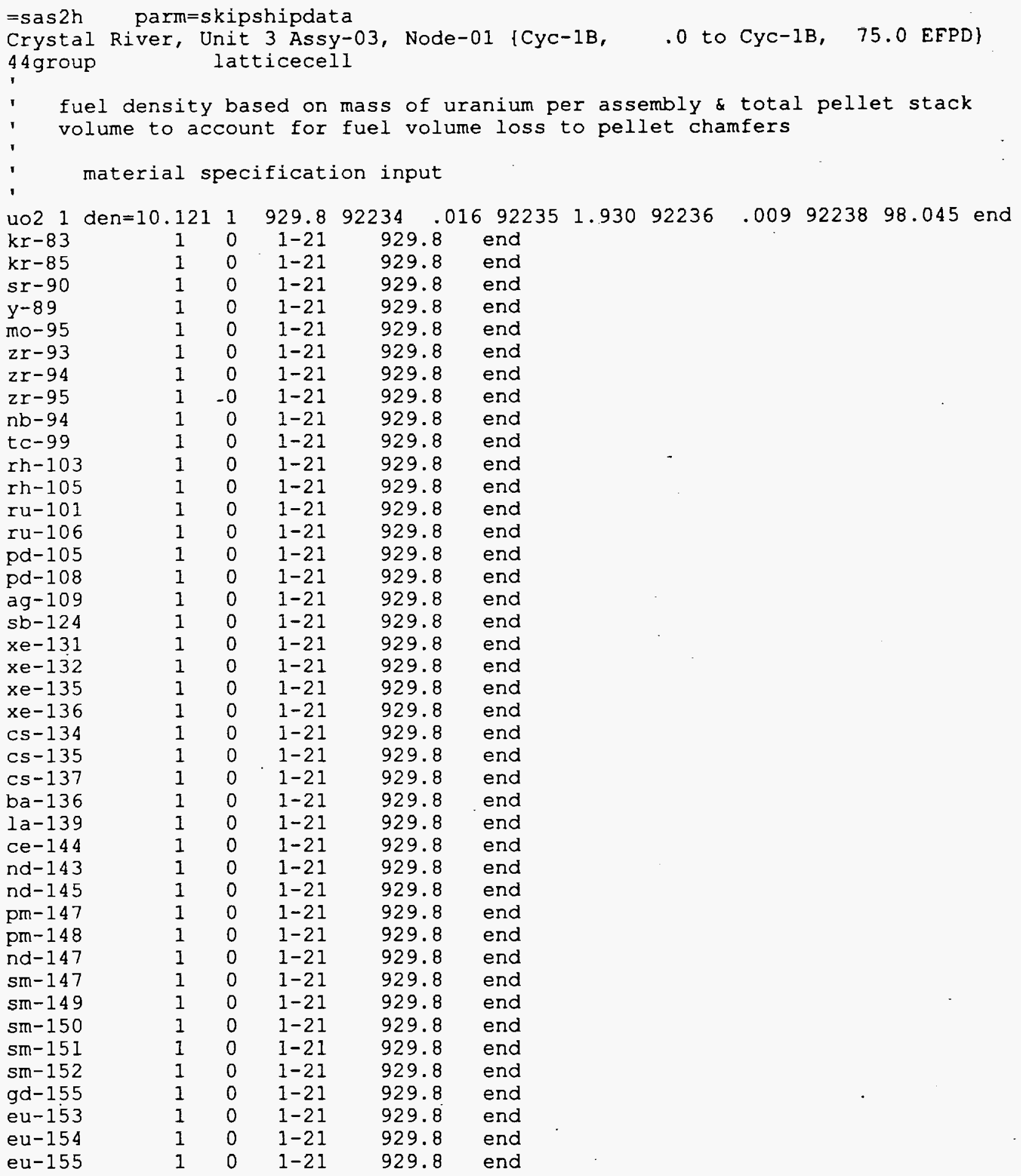




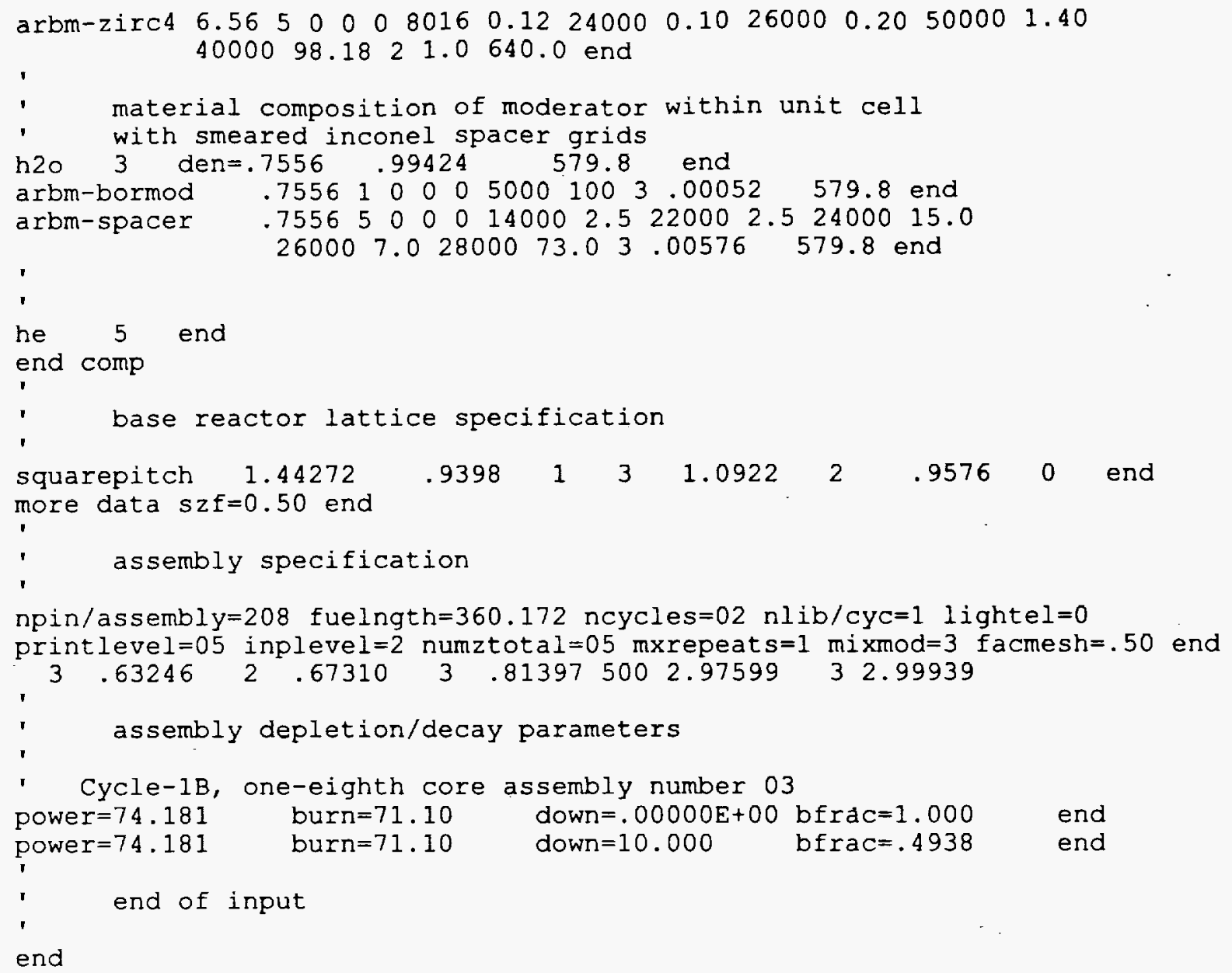

Figure 4.9.1-2 Calculation 2 of the Isotopic Exclusion Test Depletion Calculation

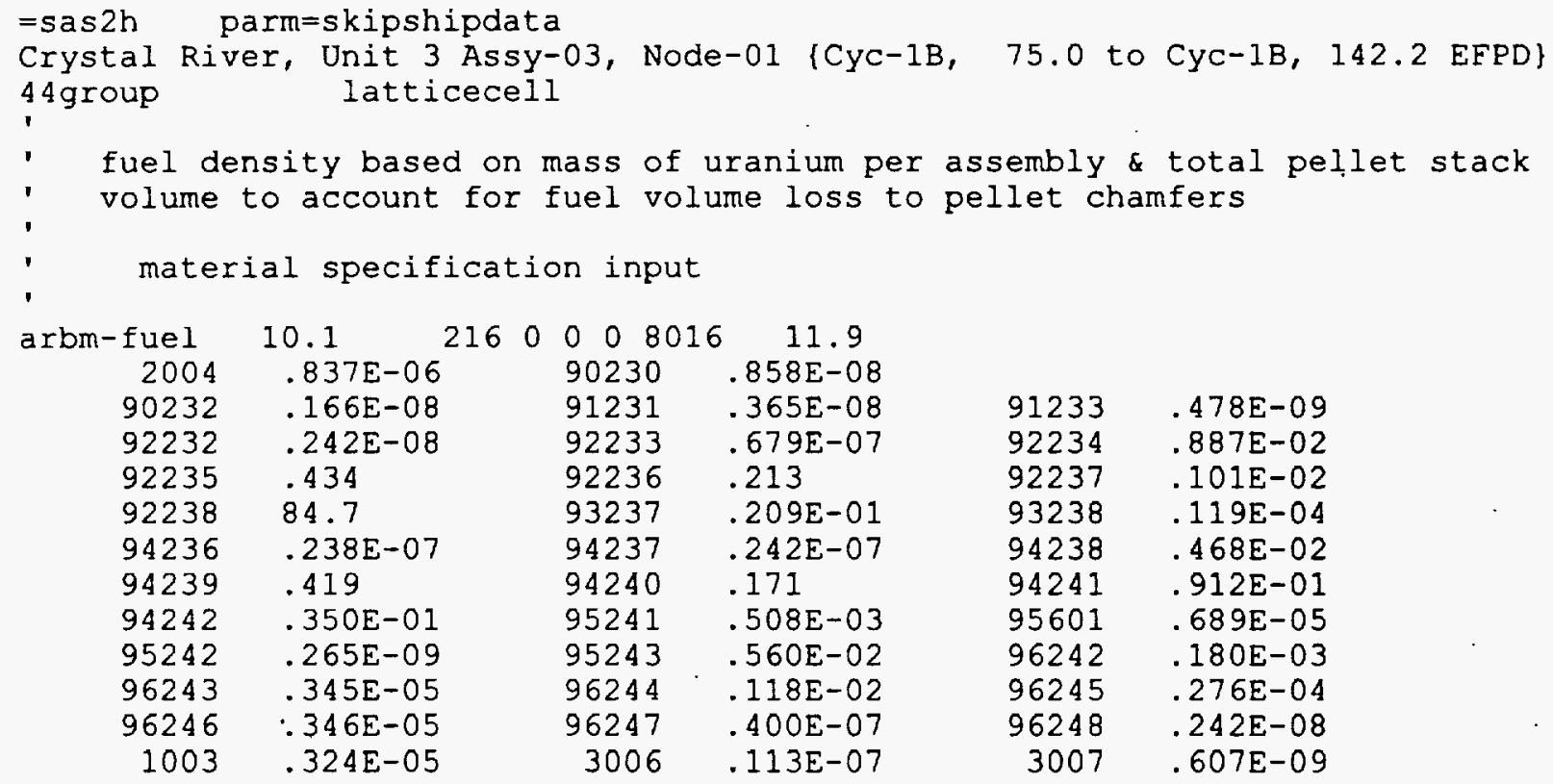




\begin{tabular}{|c|c|c|c|c|c|}
\hline & & & & & \\
\hline $\begin{array}{r}4009 \\
32074\end{array}$ & $\begin{array}{r}117 \mathrm{E}-08 \\
.767 \mathrm{E}-06\end{array}$ & $\begin{array}{l}32072 \\
33075\end{array}$ & $\begin{array}{l}.327 \mathrm{E}-06 \\
.685 \mathrm{E}-05\end{array}$ & $\begin{array}{l}32073 \\
32076\end{array}$ & $\begin{array}{l}.914 E-06 \\
.202 E-04\end{array}$ \\
\hline 34076 & $148 E-06$ & 34077 & $.468 \mathrm{E}-04$ & 34078 & $156 \mathrm{E}-03$ \\
\hline 35079 & $.246 E-09$ & 34080 & $.796 \mathrm{E}-03$ & 36080 & $.377 E-08$ \\
\hline 35081 & $.119 E-02$ & 34082 & $.189 \mathrm{E}-02$ & 36082 & $.299 E-04$ \\
\hline 36083 & $.240 E-02$ & 36084 & $.660 \mathrm{E}-02$ & 36085 & $.142 E-02$ \\
\hline 37085 & $.522 E-02$ & 36086 & $.106 \mathrm{E}-01$ & 37086 & $.299 E-05$ \\
\hline 38086 & $.112 \mathrm{E}-04$ & 37087 & $.138 \mathrm{E}-01$ & 38087 & $.674 E-07$ \\
\hline 38088 & $.196 \mathrm{E}-01$ & 38089 & $.923 \mathrm{E}-02$ & 39089 & $.170 E^{\circ}-01$ \\
\hline 38090 & $.318 \mathrm{E}-01$ & 39090 & $.837 \mathrm{E}-05$ & 40090 & $.200 \mathrm{E}-03$ \\
\hline 39091 & $.140 E-01$ & 40091 & $.206 \mathrm{E}-01$ & 40092 & $.369 \mathrm{E}-01$ \\
\hline 40093 & $.276 E-01$ & 41093 & $.695 E-09$ & 40094 & $.447 \mathrm{E}-01$ \\
\hline 41094 & $.310 E-07$ & 40095 & $.209 \mathrm{E}-01$ & 41095 & $.104 \mathrm{E}-01$ \\
\hline 42095 & $.150 \mathrm{E}-01$ & 40096 & $.476 E-01$ & 42096 & $.468 \mathrm{E}-03$ \\
\hline 42097 & $.455 \mathrm{E}-01$ & 42098 & $.493 \mathrm{E}-01$ & 42099 & $.118 \mathrm{E}-03$ \\
\hline 43099 & $.489 E-01$ & 44099 & $.171 \mathrm{E}-05$ & 42100 & $.561 \mathrm{E}-01$ \\
\hline 44100 & $.449 \mathrm{E}-02$ & 44101 & $.468 \mathrm{E}-01$ & 44102 & $.466 \mathrm{E}-01$ \\
\hline 44103 & $.142 \mathrm{E}-01$ & 45103 & $.223 E-01$ & 44104 & $.346 \mathrm{E}-01$ \\
\hline 46104 & $.617 \mathrm{E}-02$ & 45105 & $.402 E-05$ & 46105 & $.177 \mathrm{E}-01$ \\
\hline 44106 & $.183 E-01$ & 46106 & $.130 \mathrm{E}-01$ & 46107 & $.149 \mathrm{E}-01$ \\
\hline 47107 & $.280 E-09$ & 46108 & $.971 \mathrm{E}-02$ & 48108 & $.942 \mathrm{E}-08$ \\
\hline 47109 & $.636 \mathrm{E}-02$ & 46110 & $.287 \mathrm{E}-02$ & 48110 & $.198 \mathrm{E}-02$ \\
\hline 47111 & $.672 E-04$ & 48111 & $.141 \mathrm{E}-02$ & 48112 & $.759 \mathrm{E}-03$ \\
\hline 48113 & $.746 \mathrm{E}-05$ & 49113 & $.699 \mathrm{E}-07$ & 48114 & $.773 E-03$ \\
\hline 50114 & $.142 \mathrm{E}-08$ & 48601 & $.392 E-05$ & 49115 & $.109 \mathrm{E}-03$ \\
\hline 50115 & $.111 E-04$ & 48116 & $.312 E-03$ & 50116 & $.121 \mathrm{E}-03$ \\
\hline 50117 & $.299 E-03$ & 50118 & $.236 E-03$ & 50119 & $.247 E-03$ \\
\hline 50120 & $.242 \mathrm{E}-03$ & 51121 & $.249 E-03$ & 50122 & $.316 E-03$ \\
\hline 52122 & $.121 \mathrm{E}-04$ & 50123 & $.153 E-04$ & $51 I 23$ & $.274 E-03$ \\
\hline 52123 & $.617 \mathrm{E}-07$ & 50124 & $.527 \mathrm{E}-03$ & 51124 & $.556 \mathrm{E}-05$ \\
\hline 52124 & $.423 E-05$ & 50125 & $.925 E-05$ & 51125 & $.613 E-03$ \\
\hline 52125 & $.263 E-04$ & 50126 & $.124 \mathrm{E}-02$ & 51126 & $.105 E-05$ \\
\hline 52126 & $.198 \mathrm{E}-04$ & 52601 & $.327 \mathrm{E}-03$ & 53127 & $.246 E-02$ \\
\hline 52128 & $.556 \mathrm{E}-02$ & 54128 & $.908 E-04$ & 52611 & $.615 E-03$ \\
\hline 53129 & $.106 \mathrm{E}-01$ & 54129 & $.333 E-06$ & 52130 & $.219 \mathrm{E}-01$ \\
\hline 54130 & $.369 E-03$ & 53131 & $.137 E-02$ & 54131 & $.274 \mathrm{E}-01$ \\
\hline 52132 & $.217 E-03$ & 54132 & $.626 \mathrm{E}-01$ & 54133 & $.129 \mathrm{E}-02$ \\
\hline 55133 & $.697 E-01$ & 54134 & $.917 E-01$ & 55134 & $.609 E-02$ \\
\hline 56134 & $.316 E-03$ & 54135 & $.948 E-11$ & 55135 & $.453 \mathrm{E}-02$ \\
\hline 56135 & $.127 E-05$ & 54136 & .155 & 55136 & $.546 \mathrm{E}-04$ \\
\hline 56136 & $.424 \mathrm{E}-03$ & 55137 & $.763 \mathrm{E}-01$ & 56137 & $.421 \mathrm{E}-03$ \\
\hline 56138 & $.761 E-01$ & 57139 & $.719 E-01$ & 56140 & $.537 E-02$ \\
\hline 57140 & $.813 E-03$ & 58140 & $.712 \mathrm{E}-01$ & 58141 & $.168 \mathrm{E}-01$ \\
\hline 59141 & $.495 \mathrm{E}-01$ & 58142 & $.678 \mathrm{E}-01$ & 59142 & $.209 \mathrm{E}-08$ \\
\hline 60142 & $.601 E-03$ & 58143 & $.544 \mathrm{E}-05$ & 59143 & $.535 E-02$ \\
\hline 60143 & $.451 E-01$ & 58144 & $.483 E-01$ & 60144 & $.263 \mathrm{E}-01$ \\
\hline 60145 & $.405 E-01$ & 60146 & $.398 E-01$ & 60147 & $.159 \mathrm{E}-02$ \\
\hline 61147 & $.151 \mathrm{E}-01$ & 62147 & $.758 \mathrm{E}-03$ & 60148 & $.228 \mathrm{E}-01$ \\
\hline 61148 & $.590 \mathrm{E}-04$ & 61601 & $.839 E-04$ & 62148 & $.244 \mathrm{E}-02$ \\
\hline 61149 & $.280 E-04$ & 62149 & $.765 E-03$ & 60150 & $.108 E-01$ \\
\hline 62150 & $.206 \mathrm{E}-01$ & 61151 & $.312 \mathrm{E}-06$ & 62151 & $.973 E-03$ \\
\hline 63151 & $.348 E-06$ & 62152 & $.912 \mathrm{E}-02$ & 63152 & $354 \mathrm{E}-06$ \\
\hline 64152 & $.407 \mathrm{E}-06$ & 62153 & $.994 \mathrm{E}-05$ & 63153 & $.702 \mathrm{E}-02$ \\
\hline 62154 & $.263 \mathrm{E}-02$ & 63154 & $.107 \mathrm{E}-02$ & 64154 & $.129 \mathrm{E}-04$ \\
\hline 63155 & $.314 E-03$ & 64155 & $.160 \mathrm{E}-05$ & 63156 & $.615 \mathrm{E}-03$ \\
\hline 64156 & $.282 \mathrm{E}-02$ & 63157 & $.278 E-09$ & 64157 & $.257 \mathrm{E}-04$ \\
\hline 64158 & $.153 \mathrm{E}-02$ & 65159 & $.159 \mathrm{E}-03$ & 64160 & $.674 \mathrm{E}-04$ \\
\hline 65160 & $.845 E-05$ & 66160 & $.386 \mathrm{E}-05$ & 66161 & $.232 \mathrm{E}-04$ \\
\hline 66162 & $.172 E-04$ & 66163 & $.117 E-04$ & 66164 & $.240 \mathrm{E}-05$ \\
\hline 67165 & $.398 E-05$ & 68166 & $.727 \mathrm{E}-06$ & 68167 & $.123 \mathrm{E}-07$ \\
\hline
\end{tabular}




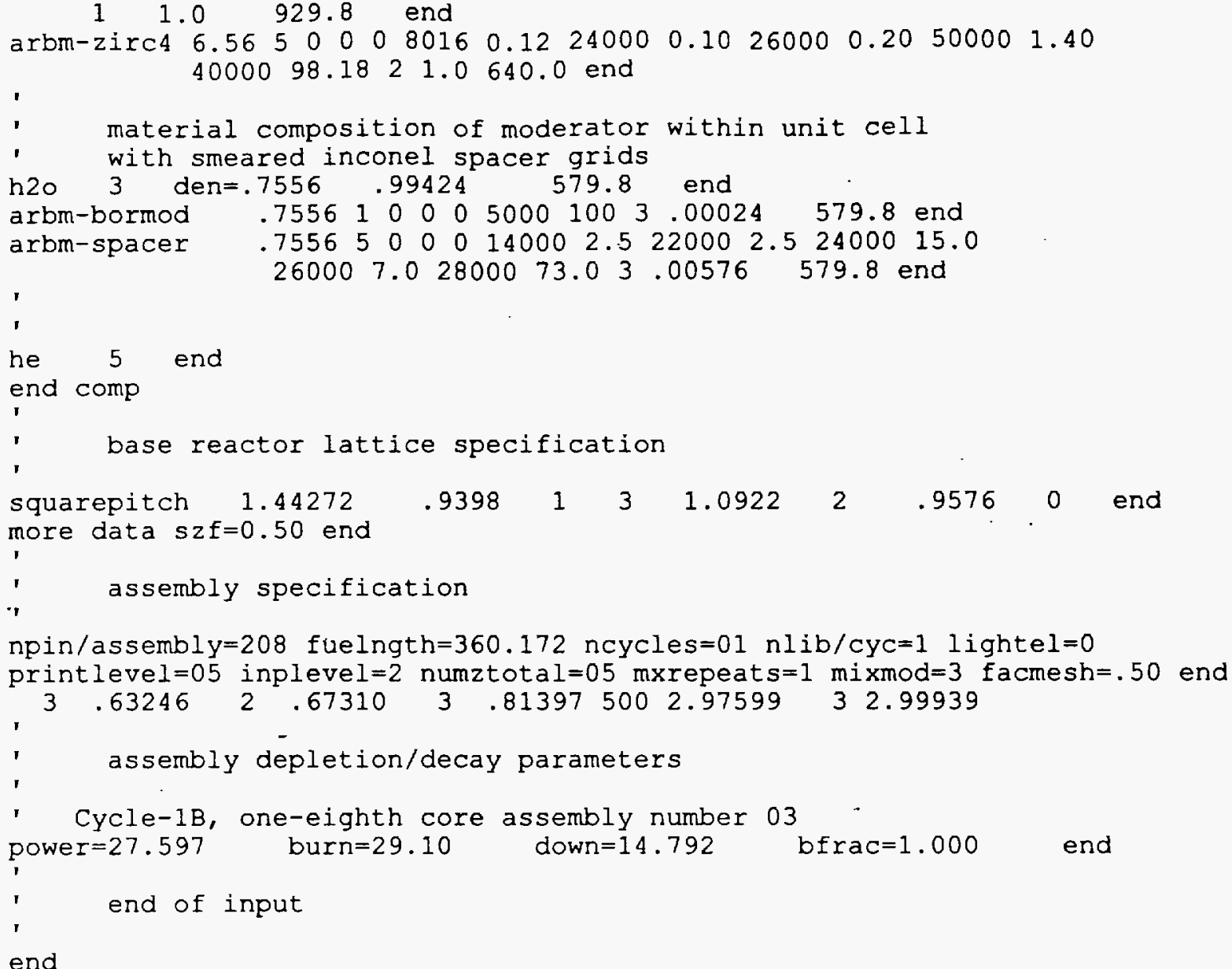

Figure 4.9.1-3 Calculation 3 of the Isotopic Exclusion Test Depletion Calculation

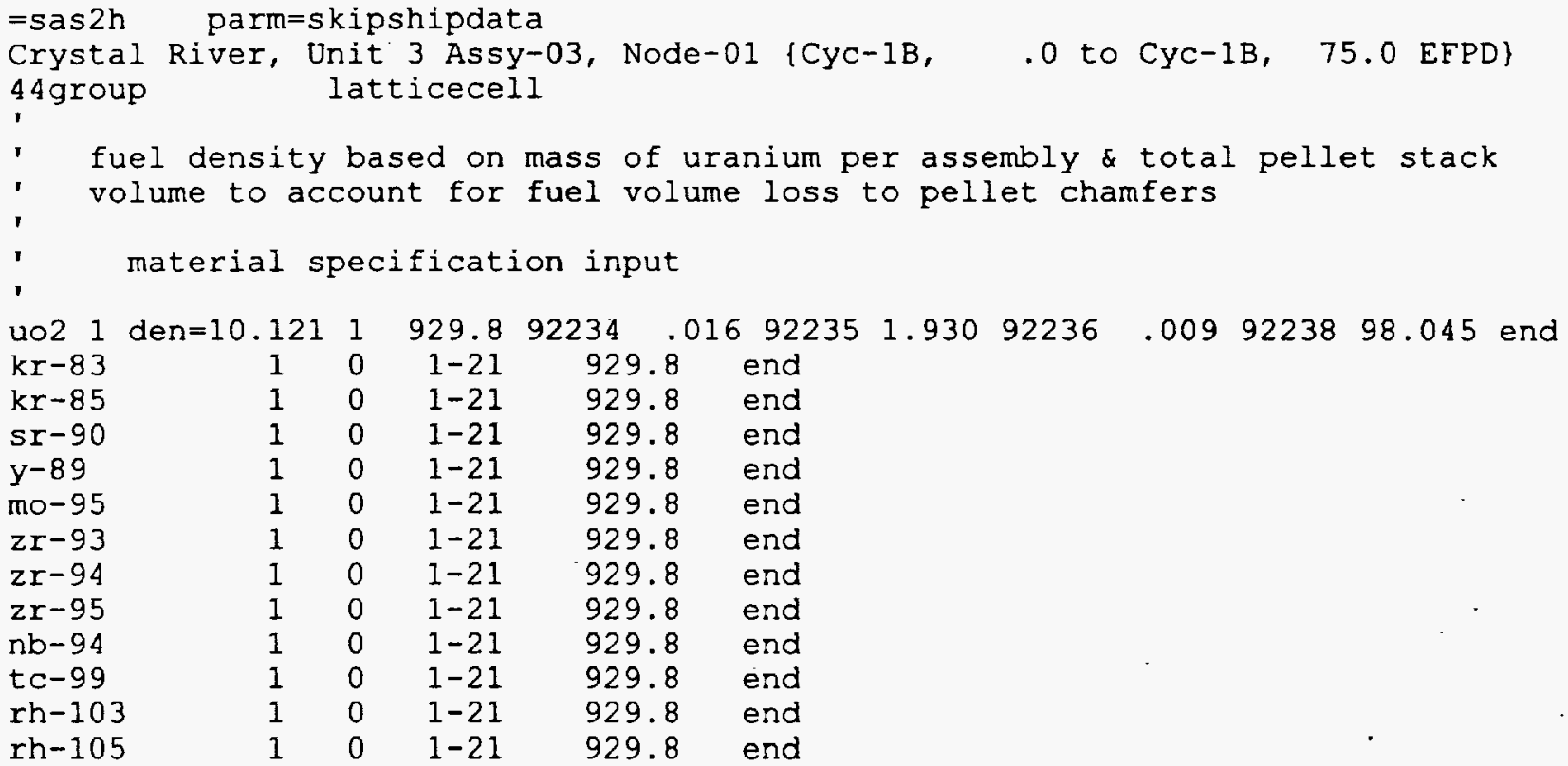




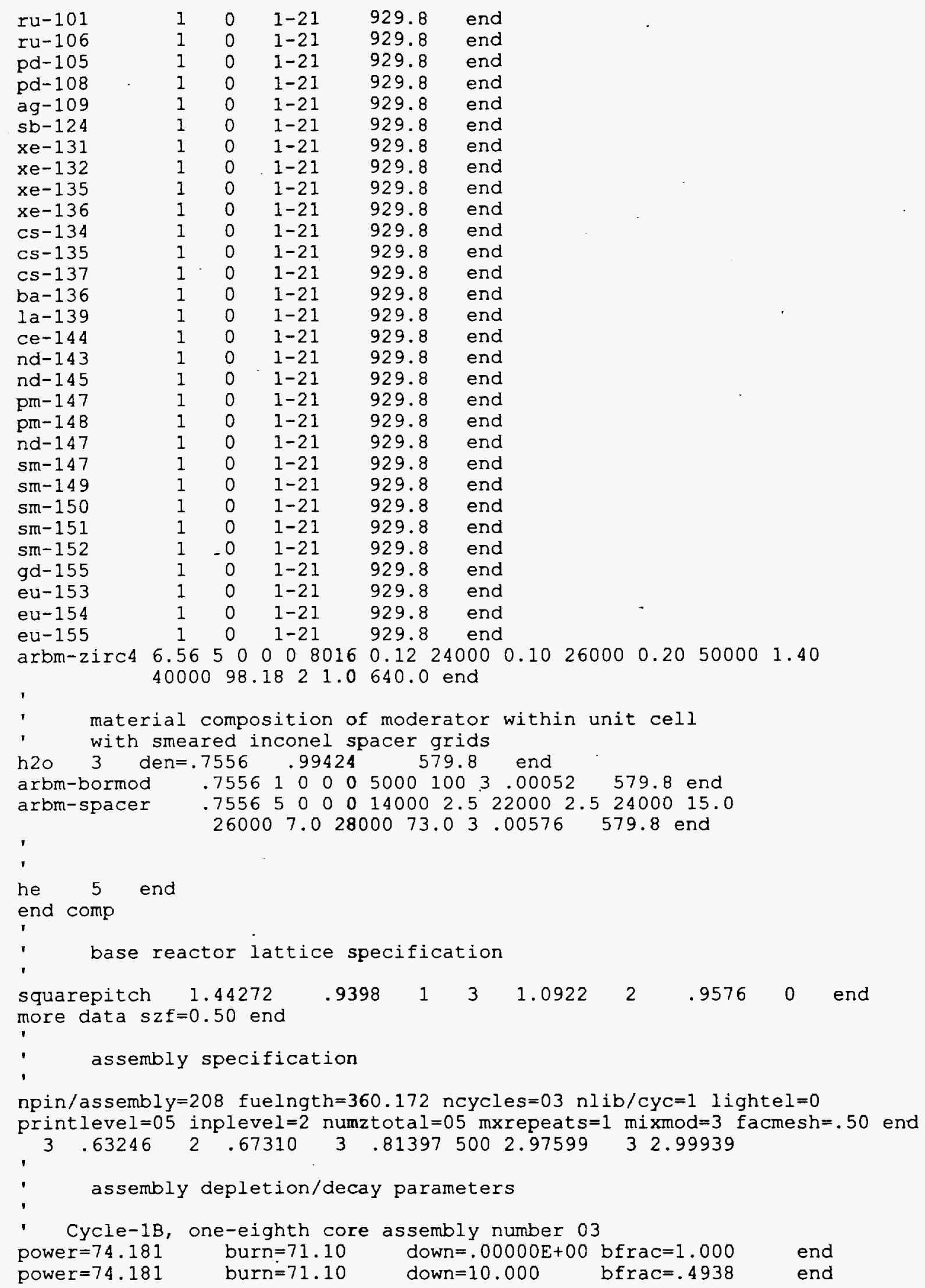


Title: CRC Depletion Calculations for the Rodded Assemblies in Batches 1, 2, 3, and $1 \mathrm{X}$ of Crystal River Unit 3 Document Identifier: BBA000000-01717-0200-00040 REV 00 Date Printed: 7/31/97 Attachment I, Page 39 of 84

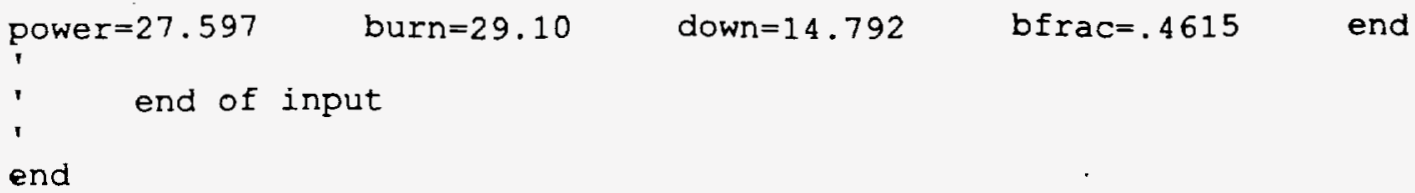

The burnable poison initial charge composition for continuing a fuel assembly depletion calculation is developed using the depleted abundances of B-10 and B-11 in the burnable poison material. These depleted abundances of B-10 and B-11 are obtained from the appropriate previous SAS2H depletion and decay calculation's output. The depletion of other isotopes in the burnable poison composition are not tracked in the CRAFT calculation. The isotopes in the burnable poison material other than B-10 and B-11 are respecified in the burnable poison composition of the continuing depletion calculation with their initial abundances. The total mass of all isotopes in the burnable poison composition is calculated to assist in determining the weight percentages of each isotope in the composition and the density of the composition. The burnable poison composition is then defined as an arbitrary material specification in the SAS2H input deck with the nodal moderator temperature applied.

\subsubsection{Calculations Performed within the CONTINUATION_WRITER Subroutine}

- The density of the fuel composition in the CRAFT generated continuing depletion SAS2H input deck must be calculated by the CONTINUATION_WRITER subroutine. This calculation is performed by simply dividing the total mass of the charge fuel composition (including oxygen) in the node by the total fuel volume in the node. The charge fuel composition (excluding oxygen) is obtained from the appropriate previous SAS2H calculation's output. The oxygen contribution and fuel volume of the node are calculated in the same manner as previously described in the STANDARD_WRITER subroutine description.

- The weight percentages of each isotope in the depleted initial charge compositions for the fuel and burnable poison are calculated by using the following equation.

$$
\begin{gathered}
\text { Weight Percent } \\
\text { of Constituent in Material }
\end{gathered}=\frac{\text { Mass of Constituent }}{\text { Total Material Mass }} * 100
$$

- The default burnable poison material is $\mathrm{Al}_{2} \mathrm{O}_{3}-\mathrm{B}_{4} \mathrm{C}$. If this burnable poison material is specified for use in the BPRA of a continuing depletion calculation, the initial mass abundances of the aluminum, oxygen, and carbon in the fresh material must be calculated for use is defining the depleted burnable material composition for the continuation case. The first step in calculating the mass abundances of these elements is to use the following equation to calculate the mass of $\mathrm{B}_{4} \mathrm{C}$ in the $\mathrm{Al}_{2} \mathrm{O}_{3}-\mathrm{B}_{4} \mathrm{C}$ material in the node. 


$$
\begin{gathered}
B_{4} C \text { Mass } \\
\text { in Node }
\end{gathered}=\left(\frac{B_{4} C \text { wt\% in } \mathrm{Al}_{2} \mathrm{O}_{3}-\mathrm{B}_{4} C}{100}\right) *\left(\begin{array}{c}
\text { Density of } \\
\mathrm{Al}_{2} \mathrm{O}_{3}-B_{4} C
\end{array}\right) *\left(\begin{array}{c}
\text { Burnable Poison } \\
\text { Volume in Node }
\end{array}\right)
$$

The carbon mass in the $\mathrm{Al}_{2} \mathrm{O}_{3}-\mathrm{B}_{4} \mathrm{C}$ material of the node may then be calculated using the following equation.

$$
\text { Carbon Mass in } \mathrm{Al}_{2} \mathrm{O}_{3}-\mathrm{B}_{4}=\left(\mathrm{B}_{4} \mathrm{C} \text { Mass in Node }\right) *(0.217374)
$$

The aluminum mass in the $\mathrm{Al}_{2} \mathrm{O}_{3}-\mathrm{B}_{4} \mathrm{C}$ of the node is calculated using the following equation.

$\underset{\text { in } \mathrm{Al}_{2} \mathrm{O}_{3}-B_{4} C}{\text { Aluminum Mass }}=\left(\frac{100-B_{4} C \text { wt\% }}{100}\right) *\left(\begin{array}{l}\text { Density of } \\ \mathrm{Al}_{2} \mathrm{O}_{3}-B_{4} C\end{array}\right) *\left(\begin{array}{l}\text { Burnable Poison } \\ \text { Volume in Node }\end{array}\right) *\left(\frac{2 * 26.981539}{101.961278}\right)$

The oxygen mass in the $\mathrm{Al}_{2} \mathrm{O}_{3}-\mathrm{B}_{4} \mathrm{C}$ of the node is calculated using the following equation.

$\begin{aligned} & \text { Oxygen Mass } \\ & \text { in } \mathrm{Al}_{2} \mathrm{O}_{3}-B_{4} \mathrm{C}\end{aligned}=\left[\left(\frac{100-B_{4} C \text { wt\% }}{100}\right) *\left(\begin{array}{l}\text { Density of } \\ \mathrm{Al}_{2} \mathrm{O}_{3}-B_{4} C\end{array}\right) *\left(\begin{array}{l}\text { Burnable Poison } \\ \text { Volume in Node }\end{array}\right)\right]-\left(\begin{array}{c}\text { Aluminum Mass } \\ \text { in } \mathrm{Al}_{2} \mathrm{O}_{3}-B_{4} C\end{array}\right)$

The total mass of the $\mathrm{Al}_{2} \mathrm{O}_{3}-\mathrm{B}_{4} \mathrm{C}$ material in the node for the continuation case is the sum of the aluminum, oxygen, and carbon masses calculated from the fresh burnable poison description, plus the depleted B-10 and B-11 masses in the burnable poison of the node obtained from the appropriate previous SAS2H depletion and decay calculation's output. The volume of the burnable poison material in the node must be calculated using the following equation for use in calculating the density of the depleted burnable poison material.

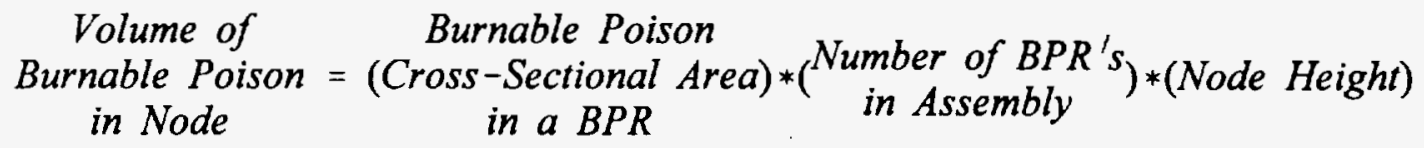

where the burnable poison cross-sectional area is defined in the CRAFT input deck.

The density of the depleted burnable poison for the continuing depletion SAS2H case is calculated by dividing the total mass of the depleted burnable poison material in the node by the burnable poison volume in the node. The weight percentages of the constituents of the $\mathrm{Al}_{2} \mathrm{O}_{3}-\mathrm{B}_{4} \mathrm{C}$ material are calculated and used by CRAFT in generating the $\mathrm{SAS} 2 \mathrm{H}$ input deck. 
- The CRAFT code has the ability to model burnable poison materials other than $\mathrm{Al}_{2} \mathrm{O}_{3}$ $\mathrm{B}_{4} \mathrm{C}$. If a burnable poison material other than $\mathrm{Al}_{2} \mathrm{O}_{3}-\mathrm{B}_{4} \mathrm{C}$ is specified, the CONTINUATION_WRITER subroutine must calculate the appropriate depleted composition for the continuing depletion SAS2H case. The first step in determining the depleted burnable poison material composition is to calculate the total mass of the depleted burnable poison in the node using the following equation.

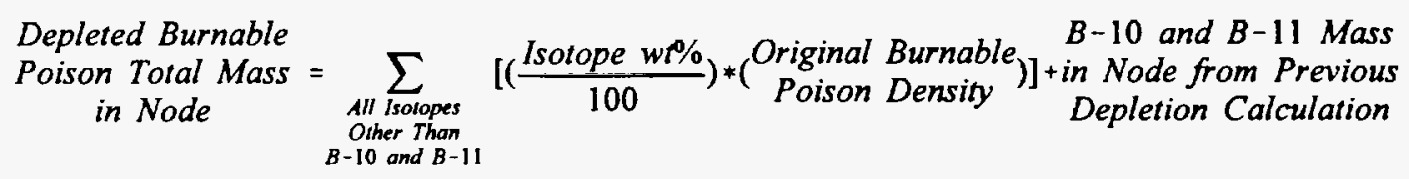

The density of the depleted burnable poison composition is then calculated by dividing the total depleted burnable poison mass in the node by the total burnable poison material volume in the node.

The weight percents of the constituents of the burnable poison composition other than B10 and B-11 are calculated using the following equation.

$\begin{gathered}\text { Weight Percent of } \\ \begin{array}{c}\text { Constituent in } \\ \text { Burnable Poison other } \\ \text { than B-10 and B-11 }\end{array}\end{gathered}=\frac{\left.\begin{array}{c}\text { Original wt\% } \\ \text { of Constituent }\end{array}\right) *\left(\begin{array}{c}\text { Original Burnable } \\ \text { Poison Density }\end{array}\right) *\left(\begin{array}{l}\text { Burnable Poison } \\ \text { Volume in Node }\end{array}\right)}{\text { Total Mass of Depleted Burnable Poison in Node }} * 100$

The weight percentages of the constituents of the burnable poison material are calculated and used by CRAFT in generating the SAS2H input deck.

\subsection{CUTTER Subroutine}

The cutter subroutine creates a consolidated output file for each CRAFT generated SAS2H calculation. This output file contains the time/date stamp from the SAS2H calculation output file, the echo of the SAS2H input deck from the SAS2H output file, and the portion of the final ORIGEN calculation's output produced as part of the SAS2H calculation which contains the light element, actinide, and fission product material compositions relevant to CRC evaluations. The output files generated by the CUTTER subroutine contain the statepoint calculation's base filename followed by the "*.cut" suffix. Section 8 contains a detailed description of the CRAFT generated filenames.

\subsection{RETRIEVER Subroutine}

The RETRIEVER subroutine reads through the appropriate "*.cut" file to obtain the fuel and burnable poison initial charge compositions for the next SAS2H calculation. Additionally, the RETRIEVER subroutine writes a file which contains a listing of all isotopes and their 
concentrations which were present in the ORIGEN output of the SAS2H calculation, but not utilized in the initial charge composition of the next SAS2H calculation. This file is identified by the initial filename identifier corresponding to the SAS2H case which is being generated followed by a "*.notes" suffix. The RETRIEVER subroutine calculates the total mass of the depleted fuel composition in the node which will be used as the initial charge for the next SAS2H calculation. The total oxygen mass in the node, which is calculated in the CONTINUATION_WRITER subroutine, is included in the total fuel mass calculated by RETRIEVER. The weight percentages of each isotope in the fuel composition are then calculated by RETRIEVER to be transferred through an array designation to the CONTINUATION_WRITER subroutine where they will be implemented into the appropriate SAS2H input deck.

\subsection{ZEROS Subroutine}

The ZEROS subroutine is a utility for converting integer values less than 100 to a two character string representation with leading zeros if necessary. 


\section{CRAFT Input Summary}

The following table summarizes the input card formats and parameters required to perform a CRAFT calculation. The CRAFT input deck filename must be "datain".

\begin{tabular}{|c|c|c|c|}
\hline $\begin{array}{c}\text { Card } \\
\text { Number } \\
\end{array}$ & $\begin{array}{c}\text { Special } \\
\text { Notes } \\
\end{array}$ & $\begin{array}{l}\text { Card } \\
\text { Format } \\
\end{array}$ & Card Description \\
\hline 1 & & 1 Character & $\begin{array}{l}{[\mathrm{Y}] \text { to signal that this case should pick up }} \\
\text { from a previous statepoint, any other } \\
\text { character equals alternative }\end{array}$ \\
\hline $1 \mathrm{~A}$ & $\star$ & Integer & $\begin{array}{l}\text { Relative cycle number at which to begin the } \\
\text { calculation \{If Input Card (IC) } 1=\text { "Y"\} }\end{array}$ \\
\hline 1B & $\star$ & Integer & $\begin{array}{l}\text { Relative statepoint number within the startup } \\
\text { cycle at which to begin the calculation } \\
\text { \{If IC } 1=\text { "Y"\} }\end{array}$ \\
\hline 2 & & 21 Characters & Problem identifier (i.e., Crystal River, Unit 3) \\
\hline 3 & & 3 Characters & $\begin{array}{l}\text { Problem prefix to be used as an identifier in } \\
\text { all filenames }\end{array}$ \\
\hline 4 & & 15 Characters & $\begin{array}{l}\text { SCALE cross-section library to be utilized by } \\
\text { SAS2H }\end{array}$ \\
\hline 5 & & Real & $w t \%$ U-235 enrichment in $\mathrm{UO}_{2}$ \\
\hline 6 & & Real & Mass of $U$ per assembly $(\mathrm{g})$ \\
\hline 7 & & Real & Number of fuel rods in assembly \\
\hline 8 & & Real & Rod pitch in assembly $(\mathrm{cm})$ \\
\hline 9 & & Real & Fuel pellet diameter $(\mathrm{cm})$ \\
\hline 10 & & Real & Fuel rod cladding inner diameter $(\mathrm{cm})$ \\
\hline 11 & & Real & Fuel rod cladding outer diameter $(\mathrm{cm})$ \\
\hline 12 & & Real & Active fuel length $(\mathrm{cm})$ \\
\hline 13 & & 1 Character & $\begin{array}{l}{[\mathrm{Y}] \text { to indicate that the assembly contains }} \\
\text { axial blanket fuel, any other character equals } \\
\text { alternative }\end{array}$ \\
\hline $13 \mathrm{~A}$ & $\star$ & Real & $\begin{array}{l}\text { wt } \% \text { U-235 enrichment in } \mathrm{UO}_{2} \text { for axial } \\
\text { blanket fuel \{If IC } 13=\text { "Y"\} }\end{array}$ \\
\hline
\end{tabular}


Title: CRC Depletion Calculations for the Rodded Assemblies in Batches 1, 2, 3, and $1 \mathrm{X}$ of Crystal River Unit 3 Document Identifier: BBA000000-01717-0200-00040 REV 00 Date Printed: 7/31/97 Attachment I, Page 44 of 84

\begin{tabular}{|c|c|c|c|}
\hline $\begin{array}{c}\text { Card } \\
\text { Number }\end{array}$ & $\begin{array}{c}\text { Special } \\
\text { Notes } \\
\end{array}$ & $\begin{array}{c}\text { Card } \\
\text { Format } \\
\end{array}$ & Card Description \\
\hline $13 \mathrm{~B}$ & $\star$ & Integer & $\begin{array}{c}\text { Number of CRC axial nodes containing axial } \\
\text { blanket fuel }\{\text { If IC } 13=\text { "Y" }\}\end{array}$ \\
\hline $13 \mathrm{C}$ & $\star, \Re$ & Integer & $\begin{array}{l}\text { CRC axial node number(s) (1=top node) } \\
\text { containing axial blanket fuel \{If IC } 13=" Y "\}\end{array}$ \\
\hline 14 & & 7 Characters & $\begin{array}{l}\text { Spacer grid material identification (ZIRC-4, } \\
\text { INCONEL, SS304, SS304S, SS316, SS316S) }\end{array}$ \\
\hline $14 \mathrm{~A}$ & & Real & $\begin{array}{l}\text { Volume fraction of spacer grids in the } \\
\text { moderator of the fuel assembly }\end{array}$ \\
\hline 15 & & 10 Characters & $\begin{array}{c}\text { Fuel cladding material identification (ZIRC-4 } \\
\text { or ZIRCALLOY4, SS304, SS304S, SS316, } \\
\text { SS316S) }\end{array}$ \\
\hline $15 \mathrm{~A}$ & & Real & Average fuel cladding temperature $(\mathrm{K})$ \\
\hline 16 & - & 1 Character & $\begin{array}{c}{[\mathrm{Y}] \text { to indicate if a cladding specification }} \\
\text { other than Zirc-4 is required by any CR, BPR, } \\
\text { or APSR }\end{array}$ \\
\hline $16 \mathrm{~A}$ & $\star$ & Integer & $\begin{array}{l}\text { Total number of special cladding material } \\
\text { compositions to be specified other than Zirc-4 } \\
\text { \{If IC } 16=\text { "Y"\} }\end{array}$ \\
\hline $16 \mathrm{~B}$ & $\star, \Re$ & Integer & $\begin{array}{l}\text { Material mixture number to be used in } \\
\text { SAS2H calculations for special cladding } \\
\text { composition \{If IC } 16=\text { "Y"\} }\end{array}$ \\
\hline $16 \mathrm{C}$ & $\star, \Re$ & 6 Characters & $\begin{array}{l}\text { Special cladding material identification } \\
\text { (INCONEL, SS304, SS304S, SS316, } \\
\text { SS316S) \{If IC } 16=\text { "Y"' }\end{array}$ \\
\hline 17 & & Real & System pressure (psia) \\
\hline 18 & & 1 Character & $\begin{array}{l}{[\mathrm{Y}] \text { to indicate if the assembly ever contains a }} \\
\text { BPRA, any other character equals alternative }\end{array}$ \\
\hline $18 \mathrm{~A}$ & $\star$ & Integer & $\begin{array}{l}\text { Number of reactor cycles in which the } \\
\text { assembly contains a BPRA \{If IC } 18=\text { "Y"\} }\end{array}$ \\
\hline $18 \mathrm{~B}$ & $\star$ & Integer, Integer & $\begin{array}{l}\text { Number of different BPRA designs inserted } \\
\text { in assembly throughout its irradiation history, } \\
\text { Number of BP material used other than } \\
\mathrm{Al}_{2} \mathrm{O}_{3}-\mathrm{B}_{4} \mathrm{C} \text { \{If IC } 18=\text { "Y" }\end{array}$ \\
\hline
\end{tabular}


Title: CRC Depletion Calculations for the Rodded Assemblies in Batches 1, 2, 3, and 1 X of Crystal River Unit 3 Document Identifier: BBA000000-01717-0200-00040 REV 00 Date Printed: 7/31/97 Attachment I, Page 45 of 84

\begin{tabular}{|c|c|c|c|}
\hline $\begin{array}{c}\text { Card } \\
\text { Number }\end{array}$ & $\begin{array}{c}\text { Special } \\
\text { Notes }\end{array}$ & $\begin{array}{c}\text { Card } \\
\text { Format }\end{array}$ & Card Description \\
\hline $18 \mathrm{C}$ & $\star, \Re$ & $\begin{array}{l}\text { Real, Real, } \\
\text { Real, Integer, } \\
\text { Integer, Integer }\end{array}$ & $\begin{array}{c}\text { Density of burnable poison }(\mathrm{g} / \mathrm{cc}), \mathrm{B}_{4} \mathrm{C} \text { wt\% } \\
\text { in burnable poison, Cross-sectional area of } \\
\text { burnable poison in BPR }\left(\mathrm{cm}^{2}\right), \text { Number of } \\
\text { BPR's in BPRA, SAS2H material mixture } \\
\text { number for BPR cladding, SAS2H material } \\
\text { mixture number for BP material } \\
\text { \{If IC } 18=\text { "Y"\} }\end{array}$ \\
\hline $18 \mathrm{D}$ & $\star, \Re$ & Integer & $\begin{array}{c}\text { Number of radial zones in BPRA Path B } \\
\text { model \{If IC } 18=\text { "Y"\} }\end{array}$ \\
\hline $18 \mathrm{E}$ & $\star, \Re$ & Integer, Real & $\begin{array}{c}\text { Material mixture number for zone of BPRA } \\
\text { Path B model, Outer radii (cm) for zone of } \\
\text { BPRA Path B model (This combination must } \\
\text { be specified from inner zone to outer zone.) } \\
\text { \{If IC } 18=\text { "Y"\} }\end{array}$ \\
\hline $18 \mathrm{~F}$ & 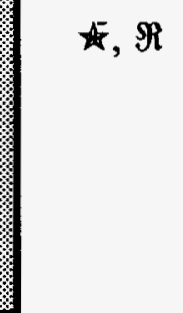 & Integer, Real & $\begin{array}{l}\text { Material mixture number for zone of Path B } \\
\text { model with removed BPRA, Outer radii (cm) } \\
\text { for zone of Path B model with removed } \\
\text { BPRA (This combination must be specified } \\
\text { from inner zone to outer zone.) } \\
\text { \{If IC } 18=\text { "Y"\} }\end{array}$ \\
\hline $18 \mathrm{G}$ & $\star, \Re$ & Integer, Real & $\begin{array}{l}\text { Material mixture number for zone of Path B } \\
\text { model for BPRA region above the BP } \\
\text { absorber region, Outer radii (cm) for zone of } \\
\text { Path B model for BPRA region above the BP } \\
\text { absorber region (This combination must be } \\
\text { specified from inner zone to outer zone.) } \\
\text { \{If IC } 18=\text { "Y"\} }\end{array}$ \\
\hline $18 \mathrm{H}$ & $\star, \Re$ & $\begin{array}{l}5 \text { Characters, } \\
\text { Integer }\end{array}$ & $\begin{array}{c}\text { Material in BPR above the BP absorber } \\
\text { material (i.e., "AL2O3"), Corresponding } \\
\text { SAS2H material mixture number } \\
\text { \{If IC } 18=\text { "Y" }\}\end{array}$ \\
\hline $18 \mathrm{I}$ & $\star, \Re$ & Integer & $\begin{array}{c}\text { Number of isotopes in material composition } \\
\text { above the BP absorber material in the BPR } \\
\{\text { If IC } 18=\text { "Y" }\} \text { \& } \\
\{\text { Value \#1 of IC } 18 \mathrm{H} \neq \text { "AL2O3" }\}\end{array}$ \\
\hline
\end{tabular}




\begin{tabular}{|c|c|c|c|}
\hline $\begin{array}{c}\text { Card } \\
\text { Number }\end{array}$ & $\begin{array}{c}\text { Special } \\
\text { Notes }\end{array}$ & $\begin{array}{l}\text { Card } \\
\text { Format }\end{array}$ & Card Description \\
\hline $18 \mathrm{~J}$ & $\star, \Re$ & Integer, Real & $\begin{array}{l}\text { SCALE nuclide identifier in material } \\
\text { composition above the BP absorber material } \\
\text { in the BPR, Corresponding wt } \% \text { of nuclide in } \\
\text { material composition }\{\text { If IC } 18=\text { "Y" } \& \\
\text { \{Value \#1 of IC } 18 \mathrm{H} \neq \text { "AL2O3" }\end{array}$ \\
\hline $18 \mathrm{~K}$ & $\star$ & Integer & $\begin{array}{l}\text { SAS2H material mixture number to be used } \\
\text { for the BP material specified in } 18 \mathrm{~L} \text { and } 18 \mathrm{M} \\
\text { \{If IC } 18=\text { "Y" } \& \text { \{Value \#2 of IC } 18 \mathrm{~B}>0\}\end{array}$ \\
\hline $18 \mathrm{~L}$ & $\star$ & Integer & $\begin{array}{c}\text { Number of isotopes in the BP absorber } \\
\text { material mixture \{If IC } 18=\text { "Y" } \& \& \\
\{\text { Value } \# 2 \text { of IC } 18 \mathrm{~B}>0\}\end{array}$ \\
\hline $18 \mathrm{M}$ & $\star, \Re$ & Integer, Real & $\begin{array}{l}\text { SCALE nuclide identifier in BP absorber } \\
\text { material mixture, wt } \% \text { for nuclide in mixture } \\
\{\text { If IC } 18=" Y "\} \&\{\text { Value } \# 2 \text { of IC } 18 B>0\}\end{array}$ \\
\hline $18 \mathrm{~N}$ & $\star, \Re$ & $\begin{array}{l}\text { Integer, Integer, } \\
\text { Integer, Integer }\end{array}$ & $\begin{array}{l}\text { Relative cycle number containing BPRA, } \\
\text { Relative BPRA design number, Top axial } \\
\text { node containing BPRA, Bottom axial node } \\
\text { containing BPRA \{If IC } 18=\text { "Y" }\end{array}$ \\
\hline 19 & & Integer & $\begin{array}{l}\text { Number of radial zones in standard Path B } \\
\text { model }\end{array}$ \\
\hline 20 & $\Re$ & Integer, Real & $\begin{array}{l}\text { Material mixture number for zone of standard } \\
\text { Path B model, Outer radii }(\mathrm{cm}) \text { for zone of } \\
\text { standard Path B model (This combination } \\
\text { must be specified from inner zone to outer } \\
\text { zone.) }\end{array}$ \\
\hline 21 & & Integer & $\begin{array}{l}\text { Number of cross-section libraries to be } \\
\text { created per irradiation step }\end{array}$ \\
\hline 22 & & Integer & SAS2H print level \\
\hline 23 & & Real & Zone mesh factor for use by XSDRNPM \\
\hline 24 & & 7 Character & $\begin{array}{c}\text { [SPECIAL] to indicate the input of } 7 \\
\text { XSDRNPM calculational control parameters } \\
\text { to follow, any other character string indicates } \\
\text { no XSDRNPM calculational control } \\
\text { parameter input }\end{array}$ \\
\hline
\end{tabular}




\begin{tabular}{|c|c|c|c|}
\hline $\begin{array}{c}\text { Card } \\
\text { Number }\end{array}$ & $\begin{array}{l}\text { Special } \\
\text { Notes }\end{array}$ & $\begin{array}{c}\text { Card } \\
\text { Format } \\
\end{array}$ & Card Description \\
\hline $24 \mathrm{~A}$ & $\star$ & Real & $\begin{array}{l}\text { XSDRNPM calculational control parameter: } \\
\text { Spatial Mesh Factor (SZF < } 1 \text { for finer, SZF > } \\
\text { 1 for coarser), Default }=1 \\
\text { \{If IC } 24=\text { "SPECIAL" }\end{array}$ \\
\hline $24 B$ & $\star$ & Integer & $\begin{array}{l}\text { XSDRNPM calculational control parameter: } \\
\text { Order of Angular Quadrature, Default }=8 \\
\text { \{If IC } 24=\text { "SPECIAL"\} }\end{array}$ \\
\hline $24 \mathrm{C}$ & $\star$ & Integer & $\begin{array}{l}\text { XSDRNPM calculational control parameter: } \\
\text { Maximum Number of Inner Iterations, } \\
\text { Default = } 20 \text { If IC } 24 \text { = "SPECIAL" }\end{array}$ \\
\hline 24D & $\star$ & Integer & $\begin{array}{l}\text { XSDRNPM calculational control parameter: } \\
\text { Maximum Number of Outer Iterations, } \\
\text { Default = } 25 \text { \{If IC } 24=\text { "SPECIAL"\} }\end{array}$ \\
\hline $24 \mathrm{E}$ & $\star$ & Real & $\begin{array}{l}\text { XSDRNPM calculational control parameter: } \\
\text { Overall Convergence Criteria, Default = } \\
0.0001 \text { \{If IC } 24=\text { "SPECIAL"\} }\end{array}$ \\
\hline $24 \mathrm{~F}$ & $\star$ & Real & $\begin{array}{l}\text { XSDRNPM calculational control parameter: } \\
\text { Scalar Flux Point Convergence, Default = } \\
0.0001 \text { \{If IC } 24=\text { "SPECIAL"\} }\end{array}$ \\
\hline $24 \mathrm{G}$ & $\star$ & Integer & $\begin{array}{l}\text { XSDRNPM calculational control parameter: } \\
\text { IUS }=1 \text { for upscatter scaling to speed } \\
\text { convergence, IUS = } 0 \text { for no scaling, Default } \\
=0 \text { \{If IC } 24=\text { "SPECIAL" }\}\end{array}$ \\
\hline 25 & & Integer & $\begin{array}{l}\text { Number of reactor cycles in which the } \\
\text { assembly is inserted }\end{array}$ \\
\hline 26 & $\Re$ & 2 Characters & $\begin{array}{l}\text { Reactor cycle identifier in which assembly is } \\
\text { inserted }\end{array}$ \\
\hline 27 & $\Re$ & Integer & $\begin{array}{l}\text { Number of CRC statepoints in reactor cycle } \\
\text { in which the assembly is inserted (BOC is } \\
\text { always considered statepoint } 1 \text { in a cycle) }\end{array}$ \\
\hline 28 & $\Re$ & Real & Statepoint EFPD \\
\hline 29 & $\Re$ & Real & Length to statepoint in calendar days \\
\hline 30 & $\Re$ & Real & Downtime at statepoint \\
\hline
\end{tabular}


Title: CRC Depletion Calculations for the Rodded Assemblies in Batches 1, 2, 3, and IX of Crystal River Unit 3 Document Identifier: BBA000000-01717-0200-00040 REV 00 Date Printed: 7/31/97 Attachment l, Page 48 of 84

\begin{tabular}{|c|c|c|c|}
\hline $\begin{array}{c}\text { Card } \\
\text { Number }\end{array}$ & $\begin{array}{l}\text { Special } \\
\text { Notes }\end{array}$ & $\begin{array}{c}\text { Card } \\
\text { Format }\end{array}$ & Card Description \\
\hline 31 & $\Re$ & Real & Days of downtime at EOC \\
\hline 32 & $\Re$ & Real & Total cycle length in EFPD \\
\hline 33 & $\Re$ & Real & Total cycle length in calendar days \\
\hline 34 & $\Re$ & Integer & Integer position of assembly in cycle \\
\hline 35 & & 1 Character & $\begin{array}{c}\text { Flag to signal if constant or variable } \\
\text { irradiation step histories will be specified } \\
{[\mathrm{Y}=\text { variable, } N=\text { constant }]}\end{array}$ \\
\hline 36 & $\star, \Re$ & Integer & $\begin{array}{l}\text { Relative cycle number to which the following } \\
\text { boron letdown data applies } \\
\text { \{If IC } 35=\text { "N"\} }\end{array}$ \\
\hline 37 & $\star, \Re$ & Integer & $\begin{array}{l}\text { Relative statepoint number in the relative } \\
\text { cycle to which the following boron letdown } \\
\text { data applies (BOC statepoint equals 1) } \\
\text { \{If IC } 35=\text { "N"\} }\end{array}$ \\
\hline 38 & $\star, \Re$ & Real & $\begin{array}{l}\text { Irradiation step length in EFPD } \\
\text { \{If IC } 35=\text { "N" }\end{array}$ \\
\hline 39 & $\star, \Re$ & Real & $\begin{array}{l}\text { Number of irradiation steps to next statepoint } \\
\text { \{If IC } 35=\text { "N"\} }\end{array}$ \\
\hline 40 & $\star, \Re$ & Real & $\begin{array}{l}\text { Mid-point ppmb concentration for irradiation } \\
\text { step \{If IC } 35=" \mathrm{~N} " \text { \} }\end{array}$ \\
\hline 41 & $\star, \Re$ & Integer & $\begin{array}{l}\text { Relative cycle number to which the following } \\
\text { boron letdown data applies } \\
\text { \{If IC } 35=\text { "Y"\} }\end{array}$ \\
\hline 42 & $\star, \Re$ & Integer & $\begin{array}{l}\text { Relative statepoint number in the relative } \\
\text { cycle to which the following boron letdown } \\
\text { data applies (BOC statepoint equals 1) } \\
\text { (If IC } 35=\text { "Y" }\end{array}$ \\
\hline 43 & $\star, \Re$ & Real & $\begin{array}{l}\text { Number of irradiation steps to next statepoint } \\
\text { \{If IC } 35=\text { "Y" }\end{array}$ \\
\hline 44 & $\star, \Re$ & Real, Real & $\begin{array}{l}\text { Irradiation step length in EFPD, Mid-point } \\
\text { ppmb concentration for irradiation step } \\
\text { \{If IC } 35=\text { "Y" }\end{array}$ \\
\hline 45 & & Integer & Number of axial nodes for CRC calculation \\
\hline
\end{tabular}


Title: CRC Depletion Calculations for the Rodded Assemblies in Batches 1, 2, 3, and $1 \mathrm{X}$ of Crystal River Unit 3 Document Identifler: BBA000000-01717-0200-00040 REV 00 Date Printed: 7/31/97 Attachment l, Page 49 of 84

\begin{tabular}{|c|c|c|c|}
\hline $\begin{array}{c}\text { Card } \\
\text { Number }\end{array}$ & $\begin{array}{c}\text { Special } \\
\text { Notes }\end{array}$ & $\begin{array}{l}\text { Card } \\
\text { Format }\end{array}$ & Card Description \\
\hline 46 & $\Re$ & Real, Real & Node number, Node height $(\mathrm{cm})$ \\
\hline 47 & & 6 Characters & $\begin{array}{c}\text { 'RODDED' if any control rod assembly data is } \\
\text { to be provided, any other character string } \\
\text { equals alternative }\end{array}$ \\
\hline $47 \mathrm{~A}$ & $\star$ & Integer & $\begin{array}{l}\text { Number of previously defined irradiation } \\
\text { steps in which the assembly contains a CRA } \\
\text { \{If IC } 47=\text { "RODDED"\} }\end{array}$ \\
\hline 47A.1 & $\star, \Re$ & Integer & $\begin{array}{l}\text { Number of delineated axial assembly sections } \\
\text { containing the CRA during the irradiation } \\
\text { step of interest \{If IC } 47=\text { "RODDED" }\end{array}$ \\
\hline 47B & $\star, \Re$ & $\begin{array}{l}\text { Integer, Integer, } \\
\text { Integer, Integer, } \\
\text { Integer, Integer, } \\
\quad \text { Integer }\end{array}$ & $\begin{array}{l}\text { Relative cycle number containing the CRA, } \\
\text { Relative statepoint in cycle (BOC=stpt } 1 \text { ), } \\
\text { Relative irradiation step number, } \\
\text { Top axial node number containing CRA, } \\
\text { Bottom axial node number containing CRA, } \\
\text { CRA absorber material mixture, } \\
\text { CRA design description number } \\
\text { \{If IC } 47=\text { "RODDED"\} }\end{array}$ \\
\hline $47 \mathrm{C}$ & $\star$ & Integer & $\begin{array}{l}\text { Number of different CRA absorber material } \\
\text { mixtures that will be specified for use in this } \\
\text { fuel assembly \{If IC } 47=\text { "RODDED" }\end{array}$ \\
\hline 47D & $\star, \Re$ & Integer & $\begin{array}{l}\text { SAS2H material mixture identifier for CRA } \\
\text { absorber material mixture } \\
\text { \{If IC } 47 \text { = "RODDED" }\end{array}$ \\
\hline $47 \mathrm{E}$ & $\star, \Re$ & Integer & $\begin{array}{l}\text { Number of isotopes in CRA absorber material } \\
\text { mixture \{If IC } 47=\text { "RODDED" }\end{array}$ \\
\hline $47 \mathrm{~F}$ & $\star, \Re$ & Integer, Real & $\begin{array}{l}\text { SCALE nuclide identifier in CRA absorber } \\
\text { material mixture, wt } \% \text { for nuclide in mixture } \\
\text { [If IC } 47=\text { "RODDED") }\end{array}$ \\
\hline $47 \mathrm{G}$ & $\star$ & Integer & $\begin{array}{l}\text { Number of different CRA designs that will be } \\
\text { specified for use with this fuel assembly } \\
\text { \{If IC } 47=\text { "RODDED" }\end{array}$ \\
\hline
\end{tabular}




\begin{tabular}{|c|c|c|c|}
\hline $\begin{array}{c}\text { Card } \\
\text { Number }\end{array}$ & $\begin{array}{c}\text { Special } \\
\text { Notes }\end{array}$ & $\begin{array}{c}\text { Card } \\
\text { Format }\end{array}$ & Card Description \\
\hline $47 \mathrm{H}$ & $\star, \Re$ & Real, Integer & $\begin{array}{l}\text { CRA absorber material density for design, } \\
\text { SAS2H material mixture number for CR } \\
\text { cladding in CRA design } \\
\text { \{If IC } 47=\text { "RODDED" }\end{array}$ \\
\hline $47 \mathrm{I}$ & $\star, \Re$ & Integer & $\begin{array}{l}\text { Number of radial zones in the Path B unit cell } \\
\text { model for the assembly containing CRA } \\
\text { design \{If IC } 47=\text { "RODDED" }\end{array}$ \\
\hline $47 \mathrm{~J}$ & $\star, \Re$ & Integer, Real & $\begin{array}{l}\text { Zone mixture identifier for use in CRA design } \\
\text { Path B unit cell model, Corresponding zone } \\
\text { outer radii (cm) \{If IC } 47=\text { "RODDED"\} }\end{array}$ \\
\hline $47 \mathrm{~K}$ & $\star, \Re$ & Integer, Real & $\begin{array}{l}\text { Zone mixture identifier for use in Path B unit } \\
\text { cell model after the CRA is removed, } \\
\text { Corresponding zone outer radii (cm) } \\
\text { \{If IC } 47=\text { "RODDED" }\end{array}$ \\
\hline 48 & & 6 Characters & $\begin{array}{l}\text { 'RODDED' if any axial power shaping rod } \\
\text { assembly data is to be provided, any other } \\
\text { character string equals alternative }\end{array}$ \\
\hline $48 \mathrm{~A}$ & $\star$ & Integer & $\begin{array}{l}\text { Number of previously defined irradiation } \\
\text { steps in which the assembly contains an } \\
\text { APSR assembly \{If IC } 48=\text { "RODDED" }\end{array}$ \\
\hline $48 \mathrm{~B}$ & $\star, \Re$ & $\begin{array}{l}\text { Integer, Integer, } \\
\text { Integer, Integer, } \\
\text { Integer, Integer, } \\
\text { Integer, Integer }\end{array}$ & $\begin{array}{l}\text { Relative cycle number containing the APSR, } \\
\text { Relative statepoint in cycle (BOC=stpt } 1 \text { ), } \\
\text { Relative irradiation step number in cycle, } \\
\text { Top axial node number containing APSR, } \\
\text { Bottom axial node number containing APSR, } \\
\text { APSR absorber material mixture number, } \\
\text { APSR assembly design description number, } \\
\text { APSR follow rod material mixture number } \\
\text { \{If IC } 48 \text { = "RODDED"\} }\end{array}$ \\
\hline $48 \mathrm{C}$ & $\star$ & Integer & $\begin{array}{c}\text { Number of different APSR assembly absorber } \\
\text { material mixtures that will be specified for } \\
\text { use with this fuel assembly } \\
\text { \{If IC } 48=\text { "RODDED" }\end{array}$ \\
\hline $48 \mathrm{D}$ & $\star, \Re$ & Integer & $\begin{array}{l}\text { SAS2H material mixture identifier for APSR } \\
\text { assembly absorber material mixture } \\
\text { \{If IC } 48=\text { "RODDED" }\end{array}$ \\
\hline
\end{tabular}




\begin{tabular}{|c|c|c|c|}
\hline $\begin{array}{c}\text { Card } \\
\text { Number }\end{array}$ & $\begin{array}{c}\text { Special } \\
\text { Notes }\end{array}$ & $\begin{array}{l}\text { Card } \\
\text { Format }\end{array}$ & Card Description \\
\hline $48 \mathrm{E}$ & $\star, \Re$ & Integer & $\begin{array}{l}\text { Number of isotopes in APSR assembly } \\
\text { absorber material mixture } \\
\text { \{If IC } 48=\text { "RODDED" }\end{array}$ \\
\hline $48 \mathrm{~F}$ & $\star, \Re$ & Integer, Real & $\begin{array}{l}\text { SCALE nuclide identifier in APSR absorber } \\
\text { material mixture, wt\% for nuclide in mixture } \\
\text { \{If IC } 48=\text { "RODDED" }\}\end{array}$ \\
\hline $48 G$ & $\star$ & Integer & $\begin{array}{c}\text { Number of different APSR assembly designs } \\
\text { that will be specified for use with this fuel } \\
\text { assembly \{If IC } 48=\text { "RODDED"\} }\end{array}$ \\
\hline $48 \mathrm{H}$ & $\star, \Re$ & Real, Integer & $\begin{array}{l}\text { APSR absorber material density for APSR } \\
\text { assembly design, SAS2H material mixture } \\
\text { number for APSR cladding in APSRA design } \\
\text { \{If IC } 48=\text { "RODDED" }\end{array}$ \\
\hline $48 I$ & $\star, \Re$ & Integer & $\begin{array}{l}\text { Number of radial zones in the Path B unit cell } \\
\text { model for the assembly containing APSR } \\
\text { assembly design- }\{\text { If IC } 48=\text { "RODDED" }\end{array}$ \\
\hline $48 \mathrm{~J}$ & $\star, \Re$ & Integer, Real & $\begin{array}{c}\text { Zone mixture identifier for use in APSR } \\
\text { assembly design Path B unit cell model, } \\
\text { Corresponding zone outer radii (cm) } \\
\text { \{If IC } 48=\text { "RODDED"\} }\end{array}$ \\
\hline $48 \mathrm{~K}$ & $\star, \Re$ & Integer, Real & $\begin{array}{l}\text { Zone mixture identifier for use in Path B unit } \\
\text { cell model after the APSR assembly is } \\
\text { removed, Corresponding zone outer radii } \\
\text { (cm) \{If IC } 48=\text { "RODDED" }\end{array}$ \\
\hline $48 \mathrm{~L}$ & $\star, \Re$ & Integer, Real & $\begin{array}{c}\text { Zone mixture identifier for use in Path B unit } \\
\text { cell model for the follow rod section of the } \\
\text { APSR assembly, Corresponding zone outer } \\
\text { radii }(\mathrm{cm}) \text { \{If IC } 48=\text { "RODDED" }\end{array}$ \\
\hline 49 & $\Re$ & Integer & $\begin{array}{l}\text { Number of axial nodes for fuel temperature } \\
\text { input }\end{array}$ \\
\hline 50 & $\Re$ & Real, Real & $\begin{array}{l}\text { Axial node number for fuel temperature input, } \\
\text { Corresponding axial node height }(\mathrm{cm})\end{array}$ \\
\hline 51 & $\Re$ & Real & Axial node fuel temperature input data $(F)$ \\
\hline 52 & $\Re$ & Integer & $\begin{array}{c}\text { Number of axial nodes for moderator specific } \\
\text { volume input }\end{array}$ \\
\hline
\end{tabular}


Title: CRC Depletion Calculations for the Rodded Assemblies in Batches 1, 2, 3, and IX of Crystal River Unit 3 Document Identifier: BBA000000-01717-0200-00040 REV 00 Date Printed: 7/31/97 Attachment I, Page 52 of 84

\begin{tabular}{|c|c|c|c||}
\hline $\begin{array}{c}\text { Card } \\
\text { Number }\end{array}$ & $\begin{array}{c}\text { Special } \\
\text { Notes }\end{array}$ & $\begin{array}{c}\text { Card } \\
\text { Format }\end{array}$ & Card Description \\
\hline 53 & $\Re$ & Real, Real & $\begin{array}{c}\text { Axial node number for moderator specific } \\
\text { volume input, Corresponding axial node } \\
\text { height (cm) }\end{array}$ \\
\hline 54 & $\Re$ & Real & $\begin{array}{c}\text { Axial node moderator specific volume input } \\
\text { data }(\mathrm{f} \wedge \text { 3/lb) }\end{array}$ \\
\hline 55 & $\Re$ & Integer & Number of axial nodes for burnup input data \\
\hline 56 & $\Re$ & Real, Real & $\begin{array}{c}\text { Axial node number for burnup input, } \\
\text { Corresponding axial node height (cm) }\end{array}$ \\
\hline 57 & $\Re$ & Real & Axial node burnup input data (GWd/MTU) \\
\hline
\end{tabular}

$\star$ : The existence of these input cards is dependent on certain previous input card values. The detailed descriptions for these input cards in Section 7 explain the various dependencies.

$\Re: \quad$ These are recursive input cards that must be entered multiple times in a specific grouping format. The detailed descriptions for the recursive input cards in Section 7 explain the specific grouping formats and number of required input iterations.

The continuous shaded boxes in the special notes column indicate groupings of recursive input cards. The format and content of these recursive groupings are explained in the detailed input descriptions in Section 7.

\section{CRAFT Code Limits and Execution Instructions}

The following listing describes the CRAFT code limitations.

1) The maximum number of irradiation steps allowed in a given CRAFT generated SAS2H input deck is 23 .

2) The maximum number of isotopes allowed in a CR or APSR absorber material specification is 10 .

3) The maximum number of concentric zones allowed in a SAS2H Path B model is 15.

4) The maximum number of axial nodes allowed in any axial format is 50 .

5) The maximum number of reactor cycles in which an assembly may be inserted is 10 .

6) The maximum number of CRC statepoints allowed in a single reactor cycle (BOC counts as one statepoint) is 20 .

7) The maximum number of BPRA design description specifications allowed is 10 .

8) The maximum number of different $C R$ absorber material mixtures allowed is 25. 
9) The maximum number of CRA design description specifications allowed is 10 .

10) The maximum number of APSR absorber material mixtures allowed is 25.

11) The maximum number of APSRA design description specifications allowed is 10 .

The procedure for performing a fuel assembly depletion calculation with CRAFT Version 1.0 consists of the following four steps:

1) Create a CRAFT input deck for the assembly depletion calculation.

2) Assure that the CRAFT executable file, the CRAFT input deck entitled "datain", the "batch43" executable file, and the "sedexecute" executable file are in the same directory. The "batch43" executable file is a script file which is used by CRAFT to execute the SCALE code system. An ASCII listing of the "batch43" script is shown in Table 6-1. The "sedexecute" executable file is a script file which is used in conjunction with the CRAFT code to create the consolidated output files described in Section 8 of this document. An ASCII listing of the "sedexecute" script is shown in Table 6-2.

3) Assure that the "sed" line editor is loaded onto the computer system and is in the command path (i.e., executable from the command line through the issuance of the "sed" command).

4) Execüte CRAFT.

Table 6-1 Listing of the "batch43" Script Required for the Execution of CRAFT

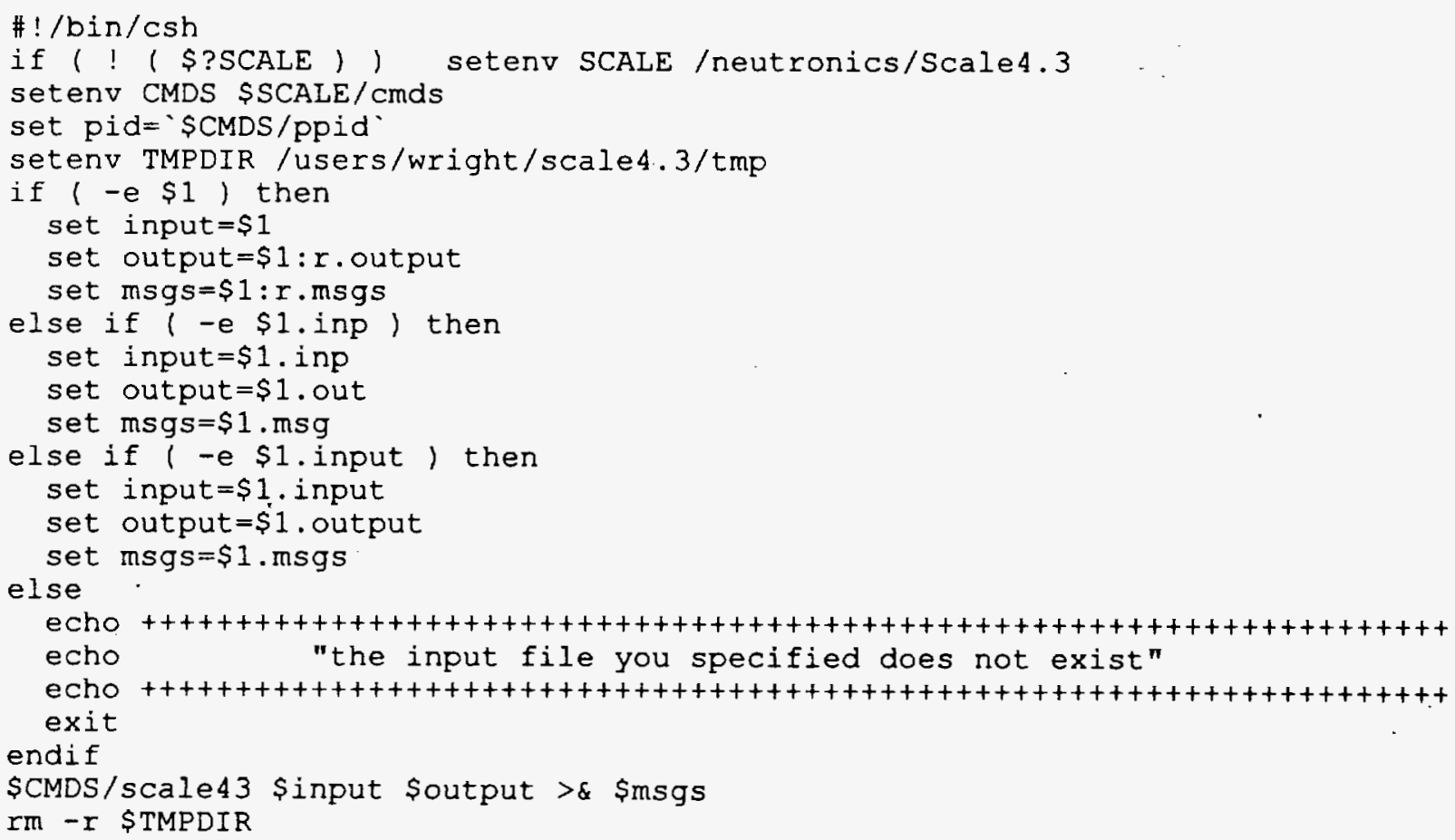

The stucture of the "batch43" script shown in Table 6-1 is only understandable if examined in the context in which it is used in the CRAFT code. 


\section{Table 6-2 Listing of the "sedexecute" Script Required for the Execution of CRAFT}

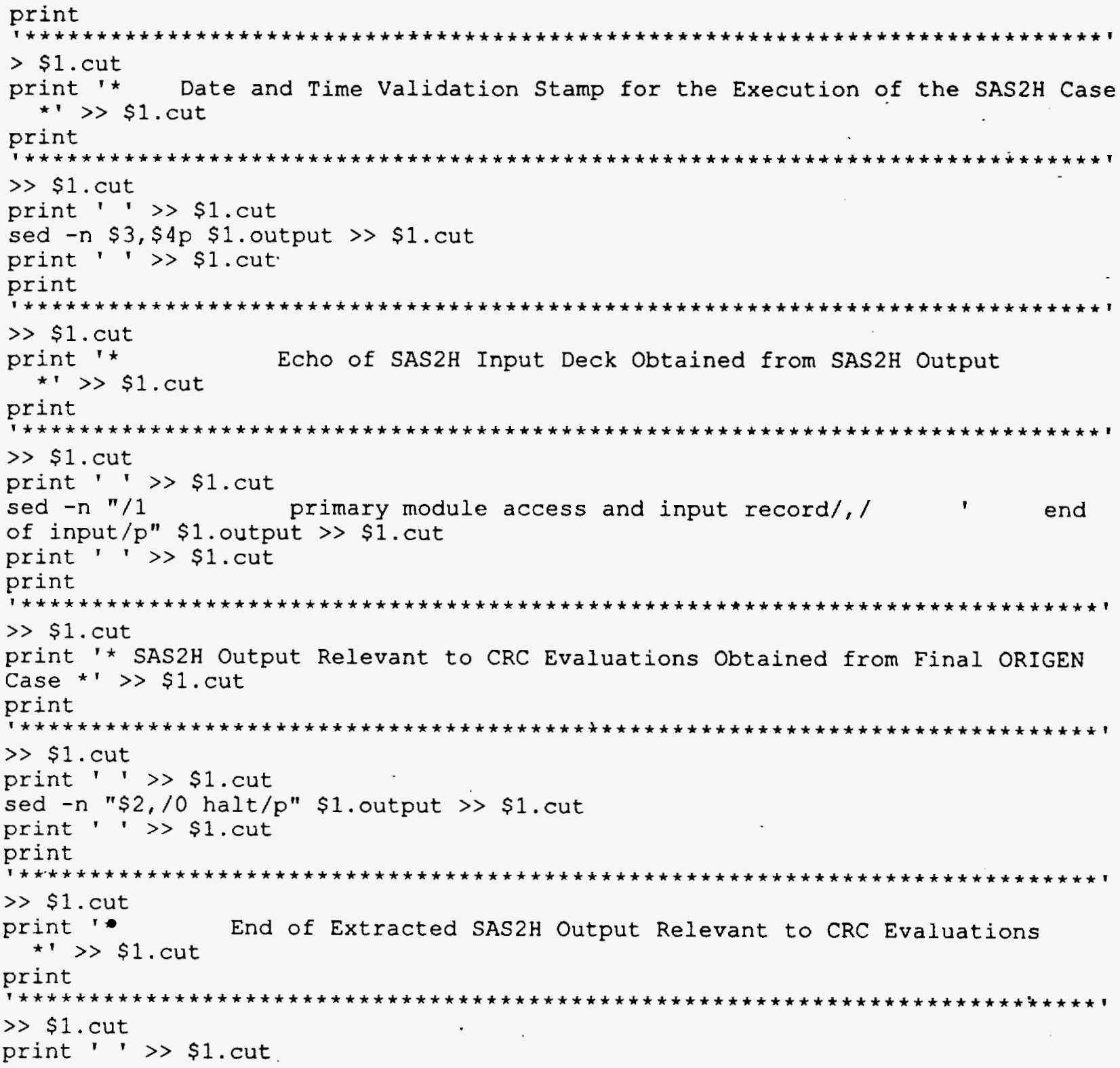

The stucture of the "sedexecute" script shown in Table 6-1 is only understandable if examined in the context in which it is used in the "CUTTER" subroutine of the CRAFT code. The "sed" command issued in the "sedexecute" script initiates the execution of the sed line editor. 


\section{Detailed Descriptions of CRAFT Input Cards}

\section{Input Card \\ Number}

The CRAFT code is capable of continuing an assembly depletion/decay calculation from a statepoint other than the $\mathrm{BOC}$ statepoint of relative cycle number one. The requirements for continuing a CRAFT calculation from an arbitrary statepoint include the following--

1) all CRAFT input for the statepoints prior to the continuation statepoint must be specified in the CRAFT input deck for the continuation calculation;

2) all "*.cut" files from the last statepoint calculation prior to the continuation statepoint, for each node, must be present in the CRAFT execution directory.

If the CRAFT calculation is a continuation calculation, an uppercase letter "Y" should be placed in column 1 of this card. Otherwise, any character other than " $Y$ " will signal that the CRAFT calculation is to begin from BOC of relative cycle number one as defined in the CRAFT input deck.

1A : $\quad$ This card should only be specified if the value of card number 1 is "Y". This card should contain an integer value representing the relative cycle number as specified in the CRAFT input deck from which the calculation should commence. The relative cycle number refers to the sequential cycle number in which the assembly is inserted. The relative cycle number is not the cycle identifier. For example, if a CRAFT calculation is to be performed for an assembly inserted in the actual reactor cycles 1 and 4 , input data for the assembly would be provided to CRAFT for cycles 1 and 4, in that order. Cycle 1 would be considered relative cycle number 1 , and cycle 4 would be considered relative cycle number 2 .

1B : This card should only be specified if the value of card number 1 is "Y". This card should contain an integer value representing the relative statepoint, within the continuation relative cycle. number provided on card $1 \mathrm{~A}$, from which the calculation should commence. 


\section{Input Card \\ Number}

\section{Detailed Description}

This card should contain a 21 character problem identifier which will be placed on all SAS2H input decks and echoed throughout the SAS2H output. The problem identifier must be placed in columns 1 through 21 of this card. An example of a problem identifier would be "Crystal River, Unit 3".

This card should contain a 3 character prefix which will be used as the initial 3 characters of each file generated in the CRAFT calculation. The prefix must be placed in columns 1 through 3 of this card. An example of a prefix meaningful for use with the problem identifier example previously provided would be "CR3".

This card should contain the identifier for the SCALE crosssection library which is to be used in all of the SAS2H calculations generated by the CRAFT calculation. Available SCALE cross-section libraries include the following--

1) 44GROUPNDF5 or 44group;

2) 27BURNUPLIB;

3) 27GROUPNDF4;

4) 238GROUPNDF5;

5) HANSEN-ROACH.

The 44group cross-section library is recommended for use in all CRAFT calculations relevant to Commercial Reactor Critical evaluations.

This card should contain the weight percent of U-235 in the $\mathrm{UO}_{2}$ fuel of the assembly. This value should not be adjusted to compensate for axial blanket fuel. Axial blanket fuel descriptions provided later in the CRAFT input deck will override the enrichment specified on this card as appropriate.

This card should contain the total mass of uranium metal in the fuel assembly in units of grams per assembly.

This card should contain the number of fuel rods in the assembly.

This card should contain the rod pitch in the assembly in units of cm. 


\section{Input Card \\ Number}

9

10

11

12

13

$13 \mathrm{~A}$

13B

$13 \mathrm{C}$

14

\section{Detailed Description}

This card should contain the nominal fuel pellet diameter in the assembly in units of $\mathrm{cm}$.

This card should contain the nominal fuel rod cladding inner diameter in the assembly in units of $\mathrm{cm}$.

This card should contain the nominal fuel rod cladding outer diameter in the assembly in units of $\mathrm{cm}$.

This card should contain the nominal active fuel length in the assembly in units of $\mathrm{cm}$.

The CRAFT code is capable of modeling fuel assemblies which utilize axial blanket fuel designs. If the assembly utilizes an axial blanket fuel design, an uppercase letter "Y" should be placed in column 1 of this card. If the assembly does not utilize an axial blanket fuel design, any character other than "Y" should be specified.

This card should only be specified if the value of card number 13 is "Y". This card should contain the weight percent of U-235 in the $\mathrm{UO}_{2}$ fuel of the axial blanket region of the assembly.

This card should only be specified if the value of card number 13 is "Y". This card should contain an integer number representing the number of $\mathrm{CRC}$ axial nodes that will contain the axial blanket fuel.

This card should only be specified if the value of card number 13 is "Y". This card should contain a single integer value which identifies a CRC axial node containing axial blanket fuel. This input card must be repeated a number of times equal to the value specified on input card 13B.

This card should contain a 7 character name, beginning in column 1, which specifies the spacer grid material. The currently available spacer grid material specifications include--
1) ZIRC-4
2) INCONEL
2) $\mathrm{SS} 304$
3) SS304S
4) $\operatorname{SS} 316$
5) SS316S. 


\section{Input Card Number}

$14 \mathrm{~A}$

15

$15 \mathrm{~A}$

16

$16 \mathrm{~A}$

\section{Detailed Description}

This card should contain a value representing the volume fraction of the moderated region of the fuel assembly which is displaced by spacer grid material. The sum of the moderator volume fraction and the spacer grid volume fraction should equal one. The moderator and spacer grid volumes present in the assembly-to-assembly spacing region may also be included in calculation of the spacer grid volume fraction to be input on this card.

This card should contain the identification of the fuel cladding material. The identification must be specified in columns 1 through 10 . The currently available cladding material specifications include--

$\begin{array}{ll}\text { 1) } & \text { Zirc-4 or ZIRCALLOY4 } \\ \text { 2) } & \text { INCONEL } \\ \text { 3) } & \text { SS304 } \\ \text { 4) } & \text { SS304S } \\ \text { 5) } & \text { SS316 } \\ 6) & \text { SS316S. }\end{array}$

This card should contain an average fuel rod cladding temperature value in units of degrees Kelvin that will be used consistently throughout the CRAFT generated SAS2H calculations.

The CRAFT code is capable of modeling CRA's, APSRA's, and BPRA's with cladding material compositions other than the default Zirc-4. If any cladding material must be specified other than the default Zirc-4, an uppercase letter "Y" should be placed in column 1 of this card. If Zirc-4 is the only cladding material utilized in the CRAFT calculation, any character other than "Y" should be specified.

This card should only be specified if the value of card number 16 is "Y". This card should contain an integer value specifying the number of additional cladding materials to be specified other than the default cladding material Zirc-4. 


\section{Input Card}

Number

\section{Detailed Description}

Input cards $16 \mathrm{~B}$ and $16 \mathrm{C}$ represent an input grouping that must be specified recursively for each cladding material as denoted on input card 16A. This means that input cards $16 \mathrm{~B}$ and $16 \mathrm{C}$ would be input for the first cladding material, and then input again for the second cladding material, etc., until all of the cladding materials other than Zirc- 4 which are utilized in the CRAFT calculation, as specified on input card 16A, have been described.

16B : This card should only be specified if the value of card number 16 is "Y". This card should contain an integer value representing the material mixture number which corresponds to a cladding material specification that may be specified in the SAS2H input decks generated by the CRAFT calculation.

16C : This card should only be specified if the value of card number 16 is "Y". This card should contain either a 5 or 6 character identifier corresponding to the cladding material. The cladding material identifiers currently available in CRAFT include the following--
1) SS304
2) SS304S
3) $\mathrm{SS} 316$
4) SS316S.
5) INCONEL

17 : This card should contain the system pressure in units of psi.

18 : The CRAFT code is capable of modeling an assembly that contains a BPRA. Usually, fuel assemblies may contain a BPRA in one cycle but not in subsequent cycles. If the fuel assembly for which the CRAFT calculation is to be performed contains a BPRA in any of its specified reactor cycles, an uppercase letter "Y" should be placed in column 1 of this card. Any other character signifies that the assembly never contains a BPRA.

18A : $\quad$ This card should only be specified if the value of card number 18 is "Y". This card should contain an integer value representing the number of reactor cycles in which the fuel assembly contains a BPRA. 


\section{Input Card Number}

$18 \mathrm{~B}$

\section{Detailed Description}

This card should only be specified if the value of card number 18 is "Y". This card should contain two integer values delineated by spaces. The first value represents the number of different BPRA designs inserted in the fuel assembly during its irradiation history. The second value represents the number of $\mathrm{BP}$ absorber materials other than the default, $\mathrm{Al}_{2} \mathrm{O}_{3}-\mathrm{B}_{4} \mathrm{C}$, which are utilized during the irradiation of the assembly as specified in the CRAFT calculation.

Input cards $18 \mathrm{C}$ through $18 \mathrm{~J}$ represent an input grouping that must be specified recursively for each BPRA design as denoted on input card 18B. This means that input cards $18 \mathrm{C}$ through $18 \mathrm{~J}$ would be input for BPRA design 1, and then input again for BPRA design 2, etc., until all of the number of BPRA designs specified on input card 18B have been described.

18C : This card should only be specified if the value of card number 18 is "Y". This card should contain 6 values delineated by spaces. The first value should be the density of the $\mathrm{Al}_{2} \mathrm{O}_{3}-\mathrm{B}_{4} \mathrm{C}$ burnable absorber material. The second value should be the weight percent of the $\mathrm{B}_{4} \mathrm{C}$ in the $\mathrm{Al}_{2} \mathrm{O}_{3}-\mathrm{B}_{4} \mathrm{C}$ absorber material. The third value should be the cross-sectional area of the burnable poison material in a single BPR. The fourth value should be the number of BPR's in the BPRA. The fifth value should be the BPR cladding material mixture number to be utilized in the CRAFT generated SAS2H calculations. The sixth value should be the BP absorber material mixture number to be utilized in the CRAFT generated SAS2H calculations.

18D : $\quad$ This card should only be specified if the value of card number 18 is "Y". This card should contain the integer number of radial zones that will be used to describe the SAS2H Path B model for the assembly node containing the BPRA.

18E : This card should only be specified if the value of card number 18 is "Y". This card contains the description of a single radial zone in the SAS2H Path B model for the assembly containing the BPRA. This card should contain two values delineated by spaces. The first of which should be an integer value representing the SAS2H material mixture number for the Path B model radial zone which this card represents. The second value should be the outer radius $(\mathrm{cm})$ of the Path B model radial zone which this card represents. This input card must be repeated a number of times equal to that specified on input card 18D. 


\section{Input Card Number}

$18 \mathrm{~F}$

$18 \mathrm{G}$

\section{Detailed Description}

This card should only be specified if the value of card number 18 is "Y". If an assembly contains a BPRA in one cycle but not in another, an alternative SAS2H Path B model must be provided that describes the assembly after removal of the BPRA. This alternative Path $B$ model must contain the same number of radial zones as the Path B model with the BPRA inserted. This card contains the description of a single radial zone in the SAS2H Path B model for the assembly node with the BPRA removed. This card should contain two values delineated by spaces. The first of which should be an integer value representing the SAS2H material mixture number for the Path B model radial zone which this card represents. The second value should be the outer radius $(\mathrm{cm})$ of the Path B model radial zone which this card represents. This input card must be repeated a number of times equal to that specified on input card 18D.

This card should only be specified if the value of card number 18 is "Y". Some BPR designs incorporate a non-absorbing region above the poison region in the BPR. To accomodate this type of BPR design an alternative SAS2H Path B model must be provided that describes the BPR assembly above the poison region of the BPR. This alternative Path B model must contain the same number of radial zones as the Path $B$ model with the BPRA inserted. This card contains the description of a single radial zone in the SAS2H Path B model for the assembly node containing the BPRA region above the poison region of the BPR. This card should contain two values delineated by spaces. The first of which should be an integer value representing the SAS2H material mixture number for the Path $B$ model radial zone which this card represents. The second value should be the outer radius $(\mathrm{cm})$ of the Path B model radial zone which this card represents. This input card must be repeated a number of times equal to that specified on input card 18D. 


\section{Input Card Number}

$18 \mathrm{H}$

$18 \mathrm{I}$

$18 \mathrm{~J}$

$18 \mathrm{~K}$

$18 \mathrm{~L}$

\section{Detailed Description}

This card should only be specified if the value of card number 18 is "Y". This card contains a five character entry followed by an integer entry. This card should contain the 5 character string "AL2O3" if the material in the non-absorbing region of the BPR (above the poison region of the BPR) is composed of $\mathrm{Al} 2 \mathrm{O} 3$. Any other character string indicates that a material other than $\mathrm{Al} 2 \mathrm{O} 3$ is present in the $\mathrm{BPR}$ above the poison region. The integer entry of this card should be the SAS2H material mixture number for the material within the BPR above the BP absorbing region.

This card should only be specified if the value of card number 18 is "Y", and the character string specified on input card $18 \mathrm{H}$ is not "AL2O3". This card should contain an integer value indicating the number of isotopes in the composition of the material contained within the BPR above the poison region.

This card should only be specified if the value of card number 18 is "Y", and the character string specified on input card $18 \mathrm{H}$ is not "AL2O3". This card should contain an integer value and a floating-point value. The first value specified on this card should be an integer representing the SCALE nuclide identifier for a constituent of the material composition within the BPR above the poison region. The second value should be a floatingpoint value representing the corresponding wt $\%$ of this nuclide in the material composition. This input card should be repeated a number of times equal to that specified on input card $18 \mathrm{I}$.

This card should only be specified if the last value of card number $18 \mathrm{~B}$ is greater than zero. This card should contain an integer value representing the SAS2H material mixture number for the BP absorber material being specified on cards $18 \mathrm{~L}$ and $18 \mathrm{M}$.

This card should only be specified if the last value of card number $18 \mathrm{~B}$ is greater than zero. This card should contain an integer value specifying the number of isotopes in the BP absorber material mixture specified on input card $18 \mathrm{~K}$. 


\section{Input Card \\ Number}

$18 \mathrm{M}$

$18 \mathrm{~N}$

19

\section{Detailed Description}

This card should only be specified if the last value of card number $18 \mathrm{~B}$ is greater than zero. This card should contain two values delineated by spaces. The first value should be the SCALE nuclide identifier corresponding to a constituent of the $\mathrm{BP}$ absorber material mixture specified on input card $18 \mathrm{~K}$. The second value should be the weight percent of the nuclide, identified by the first value, in the BP absorber material mixture specified on input card $18 \mathrm{~K}$. If the $\mathrm{BP}$ absorber material contains boron, the SCALE nuclide identifiers for B-10 and B11 must be specified explicitly. This input card must be repeated a number of times equal to that specified on input card $18 \mathrm{~L}$ such that data for all nuclides in the BP absorber material mixture are provided, and the sum of the weight percents of the nuclides in the mixture equals 100 .

This card should only be specified if the value of card number 18 is "Y". This input card contains four integer values delineated by spaces. The first value is the relative cycle number containing a BPRA. The second value is the relative BPRA design number corresponding to the order in which information was provided in the groupings of input cards $18 \mathrm{C}$ through $18 \mathrm{~F}$. The third value is the upper CRC axial node number containing the BPRA (the topmost CRC node number is always considered 1). The fourth value is the lower $C R C$ axial node number containing the BPRA. This input card must be repeated a number of times equal to the value specified on input card $18 \mathrm{~A}$.

This card should contain an integer value representing the number of radial zones in the SAS2H Path B model for the fuel assembly as it would be if the assembly never contained a BPRA, a CRA, or an APSR assembly during its irradiation history. This is called the standard Path B model.

This card contains the description of a single radial zone in the standard Path B model for the fuel assembly. This card should contain two values delineated by spaces. The first of which should be an integer value representing the SAS2H material mixture number for the Path B model radial zone which this card represents. The second value should be the outer radius $(\mathrm{cm})$ of the Path B model radial zone which this card represents. This input card must be repeated a number of times equal to that specified on input card 19. 


\section{Input Card Number}

21

22

23

24

$24 \mathrm{~A}$

24B

\section{Detailed Description}

This card should contain an integer value representing the number of cross-section libraries that are to be produced for each irradiation step in the SAS2H calculations generated by CRAFT. The number of cross-section libraries per irradiation step for CRC evaluations should be set to 1 .

This card should contain an integer value representing the SAS2H print level desired for the output of SAS2H calculations generated by CRAFT. The minimum print level allowed for $\mathrm{CRC}$ evaluations is 5 . A complete listing and description of the available print levels is provided on page S2.5.18 of reference 1 .

This card should contain the zone mesh factor that should be utilized by XSDRNPM in the SAS2H calculations generated by CRAFT. A description of the zone mesh factor is provided on page S2.5.5 of reference 1 .

The CRAFT calculation allows the specification of special XSDRNPM control parameters that will be utilized in SAS2H calculations generated by CRAFT. If any of the special control parameters described in cards $24 \mathrm{~A}$ through $24 \mathrm{G}$ are to be specified, the character string "SPECIAL" must be provided in columns 1 through 7 of this card. Any other character string specification indicates that the default XSDRNPM control parameters are to be utilized.

This card should only be specified if the value of card number 24 is "SPECIAL". This card contains the XSDRNPM calculational control parameter SZF. The size of the largest spatial mesh interval can be adjusted by entering a value for SZF. SZF less than 1 indicates a finer mesh spacing. SZF greater than one indicates a coarser mesh spacing. SZF equal to 1 is the default.

This card should only be specified if the value of card number 24 is "SPECIAL". This card contains the XSDRNPM calculational control parameter ISN. The ISN value specifies the order of angular quadrature for XSDRNPM. Quadrature sets are geometry-dependent quantities that are defaulted to a value of 8 . 


\section{Input Card \\ Number}

$24 \mathrm{C}$

24D

$24 \mathrm{E}$

$24 \mathrm{~F}$

$24 \mathrm{G}$

\section{Detailed Description}

This card should only be specified if the value of card number 24 is "SPECIAL". This card contains the XSDRNPM calculational control parameter IIM. The IIM value specifies the maximum number of inner iterations to be used by XSDRNPM. The default value is 20 .

This card should only be specified if the value of card number 24 is "SPECIAL". This card contains the XSDRNPM calculational control parameter ICM. The ICM value specifies the maximum number of outer iterations to be used by XSDRNPM. The default value is 25 .

This card should only be specified if the value of card number 24 is "SPECIAL". This card contains the XSDRNPM calculational control parameter EPS. The EPS value specifies the overall convergence criteria. This value is used by XSDRNPM after each outer iteration to determine if the problem has converged. The default value of EPS is 0.0001 . A smaller value tightens the convergence criteria, and a larger value loosens the convergence criteria.

This card should only be specified if the value of card number 24 is "SPECIAL". This card contains the XSDRNPM calculational control parameter PTC. The PTC value specifies the point flux convergence criteria used by XSDRNPM to determine if convergence has been achieved after an inner iteration. The default value of PTC is 0.0001 . A smaller value tightens the convergence criteria, and a larger value loosens the convergence criteria.

This card should only be specified if the value of card number 24 is "SPECIAL". This card contains the XSDRNPM calculational control parameter IUS. The IUS value is a flag to direct XSDRNPM to use an upscatter scaling technique to accelerate the solution or force convergence. The default value is 0 , which indicates that upscatter scaling is not used. An IUS value of 1 directs XSDRNPM to use the upscatter scaling technique. The default value is 0 .

This card should specify an integer number of reactor cycles in which the fuel assembly is inserted in the CRAFT calculation. 


\section{Input Card}

Number

\section{Detailed Description}

Input cards 26 through 34 represent an input grouping that must be specified recursively for each reactor cycle in which the fuel assembly is inserted in the CRAFT calculation as denoted on input card 25. This means that input cards 26 through 34 would be input for the first reactor cycle, and then input again for the second reactor cycle, etc., until all of the number of reactor cycles specified on input card 25 have been described.

This card should contain a 2 character reactor cycle identifier that will be used to identify the cycle on appropriate SAS2H input decks generated by the CRAFT calculation. For example, if the first reactor cycle were identified as "Cycle-1A", the value of this input card should be " $1 \mathrm{~A}$ ". If a reactor cycle were identified as "Cycle-1", the value of this input card should be "01", etc...

This card should contain an integer value specifying the number of CRC statepoints in the reactor cycle specified on input card number 25. The BOC is always considered statepoint 1 in a CRC evaluation. For example, if the reactor cycle specified on card 25 contained one mid-cycle CRC statepoint, the value specified on this card would be 2 .

Input cards 28 through 30 represent an input grouping that must be specified recursively for each CRC statepoint in the reactor cycle as denoted on input card 27. This means that input cards 28 through 30 would be input for the first statepoint (BOC), and then input again for the second statepoint, etc., until all of the number of CRC statepoints in the reactor cycle as specified on input card 27 have been described.

28

29

30

31
This card should contain a value specifying the EFPD for the statepoint. If the first statepoint in a reactor cycle (BOC) is being described, the value of this card should be 0 .

This card should contain a value specifying the length in . calendar days from the BOC to the CRC statepoint. If the first statepoint in a reactor cycle (BOC) is being described, the value of this card should be 0 .

This card should contain a value specifying the downtime in calendar days for the reactor shutdown at the CRC statepoint. If the first statepoint in a reactor cycle (BOC) is being described, the value of this card should be 0 .

This card should contain a value specifying the downtime in calendar days at the EOC reactor shutdown. 


\section{Input Card Number}

32

33

34

35

\section{Detailed Description}

This card should contain a value specifying the total EFPD for the reactor cycle from the BOC startup to the EOC shutdown.

This card should contain a value specifying the total cycle length in calendar days from the BOC startup to the EOC shutdown.

This card should contain an integer value less than 100 that specifies the position of the fuel assembly in the symmetrical representation of the reactor core. Typically, a CRC evaluation is performed using core symmetry to reduce the overall calculation time required to perform the evaluation. When core symmetry is used, the input parameters utilized in the CRAFT calculation for each node of an assembly are the average of the parameters from each symmetric core location corresponding to the assembly node. Usually, one-eighth core symmetry is utilized in performing CRC evaluations.

This card should contain a single character to signal to CRAFT whether variable or constant irradiation step description data will be provided. The variable irradiation step description input allows the specification of unique irradiation step durations for each irradiation step in a statepoint calculation. This option may be useful when modeling rodded cycles. The constant irradiation step duration applies the same irradiation step length to a specified number of irradiation steps in a given statepoint calculation. The character "Y" placed in column one of the input card specifies variable irradiation step duration input. The character "N" placed in column one of the input card specifies constant irradiation step duration input.

Input cards 36 through 40 should be specified only if the value of input card 35 is "N". Input cards 36 through 40 represent an input grouping that must be specified recursively for each reactor cycle in which the fuel assembly is inserted in the CRAFT calculation as denoted on input card 25 . This means that input cards 36 through $\mathbf{4 0}$ would be input for the first reactor cycle, and then input again for the second reactor cycle, etc., until all of the number of reactor cycles specified on input card 25 have been described. 


\section{Input Card Number}

36

\section{Detailed Description}

This card should only be specified if the value of card number 35 is "N". This card should contain an integer value specifying the relative cycle number to which the input data provided in the current grouping of input cards 36 through 40 apply. For example, if the CRAFT calculation involved two reactor cycles labeled Cycle- 1 and Cycle-5, the relative cycle number corresponding to Cycle-5 would be specified as 2 .

Input cards 37 through 40 represent an input grouping that must be specified recursively. for the SAS2H calculations commencing from each statepoint in the relative reactor cycle specified on input card 36. This means that input cards 37 through 40 would be input for the first statepoint calculation (BOC to statepoint 2) in the reactor cycle, and then input again for the second statepoint calculation (perhaps statepoint 2 to statepoint 3) in the reactor cycle, etc., until all of the statepoint calculations in the reactor cycle, as specified on input card 27 corresponding to the appropriate reactor cycle, have been described. The last iteration of input cards 37 through $\mathbf{4 0}$ for a given reactor cycle should correspond to the last mid-cycle statepoint to EOC SAS2H calculation.

This card should only be specified if the value of card number 35 is "N". This card should contain an integer value corresponding to the relative statepoint calculation number in the reactor cycle for which input data is being provided. The BOC to mid-cycle statepoint 2 calculation is always considered relative statepoint calculation 1 . The last mid-cycle statepoint to EOC calculation is always considered the last relative statepoint calculation in a given reactor cycle.

$38 \quad: \quad$ This card should only be specified if the value of card number 35 is "N". This card should contain a value specifying the irradiation step length in EFPD for the SAS2H statepoint calculation for which input data is being provided. If the value on input card 35 is "N", the CRAFT code only allows the use of a fixed irradiation step length in each generated SAS2H calculation. However, different irradiation step lengths may be specified for different CRAFT generated SAS2H calculations.

This card should only be specified if the value of card number 35 is "N". This card should contain an integer value specifying the number of irradiation steps to be utilized in the CRAFT generated SAS2H calculation corresponding to the statepoint calculation for which input data is being provided. 


\section{Input Card \\ Number}

40

\section{Detailed Description}

This card should only be specified if the value of card number 35 is "N". This card should contain the soluble boron concentration in units of ppmb at the mid-point of a given irradiation step in the current statepoint calculation for which input data is being provided. This input card must be repeated a number of times equal to that specified on input card 39. The order of repetition of this input card should be such that the initial ppmb concentration corresponds to the first irradiation step, and the final ppmb concentration corresponds to the last irradiation step in the statepoint calculation of interest.

Input cards 41 through 44 should be specified only if the value of input card 35 is "Y". Input cards 41 through 44 represent an input grouping that must be specified recursively for each reactor cycle in which the fuel assembly is inserted in the CRAFT calculation as denoted on input card 25. This means that input cards 41 through 44 would be input for the first reactor cycle, and then input again for the second reactor cycle, etc., until all of the number of reactor cycles specified on input card 25 have been described.

This card should only be specified if the value of card number 35 is "Y". This card should contain an integer value specifying the relative cycle number to which the input data provided in the current grouping of input cards 41 through 44 apply. For example, if the CRAFT calculation involved two reactor cycles labeled Cycle- 1 and Cycle-5, the relative cycle number corresponding to Cycle- 5 would be specified as 2 .

Input cards 42 through 44 represent an input grouping that must be specified recursively for the SAS2H calculations commencing from each statepoint in the relative reactor cycle specified on input card 41 . This means that input cards 42 through 44 would be input for the first statepoint calculation (BOC to statepoint 2) in the reactor cycle, and then input again for the second statepoint calculation (perhaps statepoint 2 to statepoint 3 ) in the reactor cycle, etc., until all of the statepoint calculations in the reactor cycle, as specified on input card 27 corresponding to the appropriate reactor cycle, have been described. The last iteration of input cards 42 through 44 for a given reactor cycle should correspond to the last mid-cycle statepoint to EOC SAS2H calculation. 


\section{Input Card Number}

42

43

44

45

46

\section{Detailed Description}

This card should only be specified if the value of card number 35 is "Y". This card should contain an integer value corresponding to the relative statepoint calculation number in the reactor cycle for which input data is being provided. The BOC to mid-cycle statepoint 2 calculation is always considered relative statepoint calculation 1 . The last mid-cycle statepoint to EOC calculation is always considered the last relative statepoint calculation in a given reactor cycle.

This card should only be specified if the value of card number 35 is "Y". This card should contain an integer value specifying the number of irradiation steps to be utilized in the CRAFT generated SAS2H calculation corresponding to the statepoint calculation for which input data is being provided.

: $\quad$ This card should only be specified if the value of card number 35 is "Y". This card should contain two real values delineated by spaces. The first value on this card should specify the irradiation step length in EFPD for the SAS2H statepoint calculation for which input data is being provided. The second value on this card should specify the soluble boron concentration in units of ppmb at the mid-point of a given irradiation step in the current statepoint calculation for which input data is being provided. This input card must be repeated a number of times equal to that specified on input card 43. The order of repetition of this input card should be such that the initial ppmb concentration corresponds to the first irradiation step, and the final ppmb concentration corresponds to the last irradiation step in the statepoint calculation of interest.

This card should contain an integer value corresponding to the number of axial nodes utilized in the CRC evaluation.

This card contains two integer values delineated by spaces. The first value specifies an axial node number in the CRC axial format. The second value specifies the corresponding node height in units of $\mathrm{cm}$. This card must be repeated a number of times equal to that specified on input card 45 . The repetition of this card should be performed such that the CRC axial node data is provided in sequential order (i.e., node 1 through node $\mathrm{N}$, where $\mathrm{N}$ is the final node). Node 1should always be specified as the top node of the fuel assembly. 


\section{Input Card \\ Number}

47

$47 \mathrm{~A}$

47A. 1

\section{Detailed Description}

The CRAFT code is capable of modeling an assembly that contains a CRA. If the fuel assembly for which the CRAFT calculation is to be performed contains a CRA in any of its specified reactor cycles, the character string "RODDED" should be placed in columns 1 through 6 of this card. Any other character string signifies that the assembly never contains a CRA.

This card should only be specified if the value of card number 47 is "RODDED". This card should contain an integer value specifying the number of previously defined irradiation steps in the CRAFT calculation in which the fuel assembly contains a CRA.

This card should only be specified if the value of card number 47 is "RODDED". This card should contain an integer value specifying the number of axial section of the fuel assembly which contain a CRA during the irradiation step for which data is being provided. This card should be repeated the number of times specified in card number 47A. 


\section{Input Card Number}

$47 \mathrm{~B}$

$47 \mathrm{C}$

\section{Detailed Description}

This card should only be specified if the value of card number 47 is "RODDED". This card must be repeated a number of times equal to that specified on input card 47A.1. This card should contain 8 integer values delineated by spaces. The first integer value specifies the relative cycle number in the CRAFT calculation in which a CRA is inserted. The second integer value specifies the relative statepoint calculation number in which a CRA is inserted in the cycle identified by the first value of this card. The BOC to statepoint 1 is always considered statepoint calculation 1 . The third value specifies the relative irradiation step number in the statepoint calculation identified by the second value of this card in which the CRA is inserted. The fourth value specifies the upper CRC axial node of the axial assembly section containing the CRA in the relative irradiation step specified by the third value of this card. The top node in the $\mathrm{CRC}$ axial format is always node 1 . The fifth value specifies the lower CRC axial node of the axial assembly section containing the CRA in the relative irradiation step specified by the third value of this card. The CRAFT code is capable of modeling numerous CRA absorber material mixtures and CRA designs for insertion in an assembly throughout its irradiation history. The sixth value specifies the CRA absorber material mixture number for SAS2H corresponding to the CRA described on this card. The CRA absorber material specifications and mixture numbers are specified on input cards $47 \mathrm{C}$ through $47 \mathrm{~F}$. The seventh value specifies the CRA design description number corresponding to the CRA described on this card. The CRA design inputs are specified on input cards $47 \mathrm{G}$ through $47 \mathrm{~K}$. The CRA design description number corresponds to the relative position in which the relevant CRA design description input is provided in the CRAFT input deck.

This card should only be specified if the value of card number 47 is "RODDED". This card should contain an integer value specifying the number of different CRA absorber material mixtures which must be specified for use in the various CRA designs which are inserted in the fuel assembly during its irradiation history relevant to the CRAFT calculation. 


\section{Input Card}

Number

\section{Detailed Description}

Input cards 47D through 47F represent an input grouping that must be specified recursively for each CRA absorber material mixture used in the CRAFT calculation as denoted on input card 47C. This means that input cards $47 \mathrm{D}$ through $47 \mathrm{~F}$ would be input for the first CRA absorber material mixture, and then input again for the second CRA absorber material mixture, etc., until all of the CRA absorber material mixtures specified on input card $47 \mathrm{C}$ have been described.

47D : $\quad$ This card should only be specified if the value of card number 47 is "RODDED". This card should contain an integer value denoting the material mixture number that should be utilized in the CRAFT generated SAS2H calculations to identify the CRA absorber material mixture for which input is being provided.

47E : This card should only be specified if the value of card number 47 is "RODDED". This card should contain an integer value specifying the number of isotopes in the CRA absorber material mixture specified on input card 47D.

47F $\quad: \quad$ This card should only be specified if the value of card number 47 is "RODDED". This card should contain two values delineated by spaces. The first value should be the SCALE nuclide identifier corresponding to a constituent of the CRA absorber material mixture specified on input card 47D. The second value should be the weight percent of the nuclide, identified by the first value, in the CRA absorber material mixture specified on input card 47D. This input card must be repeated a number of times equal to that specified on input card $47 \mathrm{E}$ such that data for all nuclides in the CRA absorber material mixture are provided, and the sum of the weight percents of the nuclides in the mixture equals 100 .

47G : This card should only be specified if the value of card number 47 is "RODDED". This card should contain an integer value specifying the number of different CRA design descriptions that will be specified for use in the CRAFT calculation.

Input cards $47 \mathrm{H}$ through $47 \mathrm{~K}$ represent an input grouping that must be specified recursively for each CRA design used in the CRAFT calculation as denoted on input card $47 \mathrm{G}$. This means that input cards $47 \mathrm{H}$ through $47 \mathrm{~K}$ would be input for the first CRA design description, and then input again for the second CRA design description, etc., until all of the CRA design descriptions specified on input card 47G have been described. The order in which the CRA design descriptions are provided determines the relative CRA design number which corresponds to the description. 


\section{Input Card \\ Number}

$47 \mathrm{H}$

471

$47 \mathrm{~J}$

$47 \mathrm{~K}$

\section{Detailed Description}

This card should only be specified if the value of card number 47 is "RODDED". This card contains two values delineated by spaces. The first value should specify the absorber material density in units of $\mathrm{g} / \mathrm{cc}$ for the CRA design for which input is being provided. The second value should be an integer specifying the SAS2H material mixture number for the CR cladding in the CRA design for which input is being provided.

This card should only be specified if the value of card number 47 is "RODDED". This card should contain an integer value specifying the number of radial zones utilized in the SAS2H Path $\mathrm{B}$ model for the fuel assembly containing the CRA design for which input is being provided.

This card should only be specified if the value of card number 47 is "RODDED". This card contains the description of a single radial zone in the SAS2H Path B model for the fuel assembly containing the CRA design for which input is being provided. This card should contain two values delineated by spaces. The first of which should be an integer value representing the SAS2H material mixture number for the Path B model radial zone which this card represents. The second value should be the outer radius of the Path B model radial zone which this card represents. This input card must be repeated a number of times equal to that specified on input card $47 \mathrm{I}$.

This card should only be specified if the value of card number 47 is "RODDED". If an assembly contains a CRA in one cycle but not in another, an alternative SAS2H Path B model must be provided that describes the assembly after removal of the CRA. This alternative Path $B$ model must contain the same number of radial zones as the Path B model with the CRA inserted. This card contains the description of a single radial zone in the SAS2H Path B model for the assembly after the removal of the CRA design for which input is being provided. This card should contain two values delineated by spaces. The first of which should be an integer value representing the SAS2H material mixture number for the Path $\mathrm{B}$ model radial zone which this card represents. The second value should be the outer radius of the Path B model radial zone which this card represents. This input card must be repeated a number of times equal to that specified on input card 47I. 


\section{Input Card \\ Number}

48

$48 \mathrm{~A}$

\section{Detailed Description}

The CRAFT code is capable of modeling a fuel assembly that contains a APSRA. If the fuel assembly for which the CRAFT calculation is to be performed contains an APSRA in any of its specified reactor cycles, the character string "RODDED" should be placed in columns 1 through 6 of this card. Any other character string signifies that the assembly never contains an APSRA.

This card should only be specified if the value of card number 48 is "RODDED". This card should contain an integer value specifying the number of previously defined irradiation steps in the CRAFT calculation in which the fuel assembly contains an APSRA. 


\section{Input Card Number}

$48 \mathrm{~B}$

\section{Detailed Description}

This card should only be specified if the value of card number 48 is "RODDED". This card must be repeated a number of times equal to that specified on input card 48A. This card should contain 7 integer values delineated by spaces. The first integer value specifies the relative cycle number in the CRAFT calculation in which a APSRA is inserted. The second integer value specifies the relative statepoint calculation number in which a APSRA is inserted in the cycle identified by the first value of this card. The $B O C$ to statepoint 1 is always considered statepoint calculation 1 . The third value specifies the relative irradiation step number in the statepoint calculation identified by the second value of this card in which the APSRA is inserted. The fourth value specifies the upper CRC axial node of the axial assembly section containing the APSRA in the relative irradiation step specified by the third value of this card. The top node in the CRC axial format is always node 1 . The fifth value specifies the lower CRC axial node of the axial assembly section containing the APSRA in the relative irradiation step specified by the third value of this card. The CRAFT code is capable of modeling numerous APSRA absorber material mixtures and APSRA designs for insertion in an assembly throughout its irradiation history. The sixth value specifies the APSRA absorber material mixture number for SAS2H corresponding to the APSRA described on this card. The APSRA absorber material specifications and mixture numbers are specified on input cards $48 \mathrm{C}$ through $48 \mathrm{~F}$. The seventh value specifies the APSRA design description number corresponding to the APSRA described on this card. The APSRA design inputs are specified on input cards $48 \mathrm{G}$ through $48 \mathrm{~K}$. The APSRA design description number corresponds to the relative position in which the relevant APSRA design description input is provided in the CRAFT input deck. The eighth value is the SAS2H material mixture number corresponding to the APSR follow rod material.

This card should only be specified if the value of card number 48 is "RODDED". This card should contain an integer value specifying the number of different APSRA absorber material mixtures which must be specified for use in the various APSRA designs which are inserted in the fuel assembly during its irradiation history relevant to the CRAFT calculation. 
Input Card

Number

\section{Detailed Description}

Input cards $48 D$ through $48 F$ represent an input grouping that must be specified recursively for each APSRA absorber material mixture used in the CRAFT calculation as denoted on input card $48 \mathrm{C}$. This means that input cards $48 \mathrm{D}$ through $48 \mathrm{~F}$ would be input for the first APSRA absorber material mixture, and then input again for the second APSRA absorber material mixture, etc., until all of the APSRA absorber material mixtures specified on input card $48 \mathrm{C}$ have been described.

$48 \mathrm{D}$

This card should only be specified if the value of card number 48 is "RODDED". This card should contain an integer value denoting the material mixture number that should be utilized in the CRAFT generated SAS2H calculations to identify the APSRA absorber material mixture for which input is being provided.

48E - : $\quad$ This card should only be specified if the value of card number 48 is "RODDED". This card should contain an integer value specifying the number of isotopes in the APSRA absorber material mixture specified on input card 48D.

48F : $\quad$ This card should only be specified if the value of card number 48 is "RODDED". This card should contain two values delineated by spaces. The first value should be the SCALE nuclide identifier corresponding to a constituent of the APSRA absorber material mixture specified on input card 48D. The second value should be the weight percent of the nuclide, identified by the first value, in the APSRA absorber material mixture specified on input card 48D. This input card must be repeated a number of times equal to that specified on input card $48 \mathrm{E}$ such that data for all nuclides in the APSRA absorber material mixture are provided, and the sum of the weight percents of the nuclides in the mixture equals 100 .

48G : $\quad$ This card should only be specified if the value of card number 48 is "RODDED". This card should contain an integer value specifying the number of different APSRA design descriptions that will be specified for use in the CRAFT calculation. 


\section{Input Card}

Number

\section{Detailed Description}

Input cards $48 \mathrm{H}$ through $48 \mathrm{~L}$ represent an input grouping that must be specified recursively for each APSRA design used in the CRAFT calculation as denoted on input card 48G. This means that input cards $48 \mathrm{H}$ through $48 \mathrm{~L}$ would be input for the first APSRA design description, and then input again for the second APSRA design description, etc., until all of the APSRA design descriptions specified on input card 48G have been described. The order in which the APSRA design descriptions are provided determines the relative APSRA design number which corresponds to the description.

$48 \mathrm{H} \quad$ : This card should only be specified if the value of card number 48 is "RODDED". This card should contain two values delineated by spaces. The first value should specify the absorber material density in units of $\mathrm{g} / \mathrm{cc}$ for the APSRA design for which input is being provided. The second value should be an integer specifying the SAS2H material mixture for the APSR cladding in the APSRA for which input is being provided.

48I - : $\quad$ This card should only be specified if the value of card number 48 is "RODDED". This card should contain an integer value specifying the number of radial zones utilized in the SAS2H Path B model for the fuel assembly containing the APSRA design for which input is being provided.

48J : This card should only be specified if the value of card number 48 is "RODDED". This card contains the description of a single radial zone in the SAS2H Path B model for the fuel assembly containing the APSRA design for which input is being provided. This card should contain two values delineated by spaces. The first of which should be an integer value representing the SAS2H material mixture number for the Path $B$ model radial zone which this card represents. The second value should be the outer radius of the Path B model radial zone which this card represents. This input card must be repeated a number of times equal to that specified on input card $48 \mathrm{I}$. 


\section{Input Card \\ Number}

$48 \mathrm{~K}$

$48 \mathrm{~L}$

\section{Detailed Description}

This card should only be specified if the value of card number 48 is "RODDED". If an assembly contains a APSRA in one cycle but not in another, an alternative SAS2H Path B model must be provided that describes the assembly after removal of the APSRA. This alternative Path B model must contain the same number of radial zones as the Path $B$ model with the APSRA inserted. This card contains the description of a single radial zone in the SAS2H Path B model for the assembly after the removal of the APSRA design for which input is being provided. This card should contain two values delineated by spaces. The first of which should be an integer value representing the SAS2H material mixture number for the Path B model radial zone which this card represents. The second value should be the outer radius of the Path B model radial zone which this card represents. This input card must be repeated a number of times equal to that specified on input card $48 I$.

This card should only be specified if the value of card number 48 is "RODDED". APSRA designs typically utilize follow rods which are not of the same material composition as the APSR cladding. To facilitate modeling of the APSR follow rod region, an alternative SAS2H Path B model must be provided that describes the follow rod region of the APR's above the poison region in the APSRA. This alternative Path $B$ model must contain the same number of radial zones as the Path B model with the APSRA inserted. This card contains the description of a single radial zone in the SAS2H Path B model for the follow rod region of the APSRA design for which input is being provided. This card should contain two values delineated by spaces. The first of which should be an integer value representing the SAS2H material mixture number for the Path $\mathrm{B}$ model radial zone which this card represents. The second value should be the outer radius of the Path B model radial zone which this card represents. This input card must be repeated a number of times equal to that specified on input card $48 \mathrm{I}$. 
Input Card

Number

\section{Detailed Description}

Input cards 49 through 51 represent an input grouping that must be specified recursively for each statepoint calculation to be generated by the CRAFT calculation. This means that input cards 49 through 51 would be input for the first statepoint calculation (BOC to statepoint 2 of relative cycle number 1 ), and then input again for the second statepoint calculation, etc., until all of the statepoint calculations to be generated by CRAFT have been addressed (the final statepoint calculation would be that ending at the final statepoint in the last relative cycle).

: This card should contain two values delineated by spaces. The first value should be the appropriate node number in the fuel temperature axial format for the statepoint calculation for which input is being provided. The second value should be the node height corresponding to the axial node number identified by the first value. This input card specification should be repeated the number of times identified on input card 49 . The nodal format input specified with this card should be ordered sequentially such that node 1 represents the top node of the fuel assembly.

$51 \quad$ : This card should contain an exposure weighted average fuel temperature value in units of degrees Fahrenheit for the appropriate node in the fuel temperature input axial format corresponding to the statepoint calculation for which input data is being provided. This input card specification should be repeated the number of times identified on input card 49 . The data provided in the sequential repetition of this input card should be ordered to correspond to the nodal input format described by the previous repetition of input card 50 .

Input cards 52 through 54 represent an input grouping that must be specified recursively for each statepoint calculation to be generated by the CRAFT calculation. This means that input cards 52 through 54 would be input for the first statepoint calculation (BOC to statepoint 2 of relative cycle number 1 ), and then input again for the second statepoint calculation, etc., until all of the statepoint calculations to be generated by CRAFT have been addressed (the final statepoint calculation would be that ending at the final statepoint in the last relative cycle). 


\section{Input Card \\ Number}

53

54

\section{Detailed Description}

This card should contain two values delineated by spaces. The first value should be the appropriate node number in the moderator specific volume axial format for the statepoint calculation for which input is being provided. The second value should be the node height corresponding to the axial node number identified by the first value. This input card specification should be repeated the number of times identified on input card 52. The nodal format input specified with this card should be ordered sequentially such that node 1 represents the top node of the fuel assembly.

This card should contain an exposure weighted average moderator specific volume value in units of $\mathrm{ft}^{3} / \mathrm{b}$ for the appropriate node in the moderator specific volume input axial format corresponding to the statepoint calculation for which input data is being provided. This input card specification should be repeated the number of times identified on input card 52. The data provided in the sequential repetition of this input card should be ordered to correspond to the nodal input format described by the previous repetition of input card 53 .

Input cards 55 through 57 represent an input grouping that must be specified recursively for each statepoint calculation to be generated by the CRAFT calculation. This means that input cards 55 through 57 would be input for the first statepoint calculation (BOC to statepoint 2 of relative cycle number 1), and then input again for the second statepoint calculation, etc., until all of the statepoint calculations to be generated by CRAFT have been addressed (the final statepoint calculation would be that ending at the final statepoint in the last relative cycle).

55

56
This card should contain an integer value specifying the number of axial nodes in the axial format in which the current burnup input data is being provided.

This card should contain two values delineated by spaces. The first value should be the appropriate node number in the burnup axial format for the statepoint calculation for which input is being provided. The second value should be the node height corresponding to the axial node number identified by the first value. This input card specification should be repeated the number of times identified on input card 55 . The nodal format input specified with this card should be ordered sequentially such that node 1 represents the top node of the fuel assembly. 


\section{Input Card Number}

57

\section{Detailed Description}

This card should contain an exposure weighted average burnup value in units of GWd/MTU corresponding to the total burnup of the node at the beginning of the statepoint calculation for which input data is being provided. This input card specification should be repeated the number of times identified on input card 55. The data provided in the sequential repetition of this input card should be ordered to correspond to the nodal input format described by the previous repetition of input card 56 .

\section{CRAFT Output Description}

The CRAFT code generates five types of files identified as either "*.input", "*.output", "*.cut", "*.msgs", or "*.notes", where the "*" is the base file set identifier for the statepoint calculation of interest. The "*.cut" and "*.notes" files are the only files that must be retained for CRC evaluation and documentation purposes. All files are generated in the working directory in which the CRAFT calculation is performed.

All CRAFT generated filenames utilize the following format: "\{Base File Set Identifier\}.\{suffix $\} "$. Where the suffix corresponds to one of the five file types previously mentioned, and the base file set identifier is a 25 character name containing essential information necessary to delineate one CRAFT generated SAS2H calculation from another.

The base file set identifier for a statepoint calculation contains the following information:

1) reactor identifier (three character);

2) one-eighth core symmetry assembly number in current reactor cycle (two digit);

3) axial node number (node 1 is always the top node) (two digit);

4) reactor cycle number in which the SAS2H calculation starts (two character);

5) EFPD statepoint at which the SAS2H calculation starts (truncated to three digits);

6) reactor cycle number in which the SAS2H calculation ends (two character);

7) EFPD statepoint at which the SAS2H calculation ends (truncated to three digits).

The format of the base file set identifier is as follows where the numbers identified as \#\{number\} correspond to one of the seven items previously listed-- \#1 A \#2 N \#3 DC \#4 T \#5 AC \#6 T \#7. The base file set identifier does not contain any spaces.

The "*.input" files contain a CRAFT generated SAS2H input deck. The "* output" files contain a complete SAS2H calculation output file. The "*.cut" files contain the corresponding SAS2H input deck followed by an output extraction, from the final ORIGEN pass of the SAS2H calculation, which contains data relevant to CRC evaluations. The "*.msgs" files contain the standard run-time messages associated with the SAS2H calculation. The "*.notes" files contain a 
listing of the isotopes and their concentration which were left behind in generating the initial charge fuel composition for a continuation SAS2H calculation. The "*.notes" files are only generated for CRAFT generated SAS2H calculations which are continuing depletion and decay calculations. The "*.cut" and "*.notes" files contain all of the information which is required to perform CRC evaluations or repeat calculations as necessary for quality assurance purposes. The remainder of the CRAFT generated files may be discarded once the "*.cut" and "*.notes" files have been produced correctly.

\section{Modifications Made Between CRAFT Version 1.0 and Version 2.0}

Two modifications were made to the CRAFT Version 2.0 source code to create CRAFT Version 3.0. The CRAFT Version 2.0 code is documented in Attachment I of reference 4. The Version 3.0 code modifications are documented in this section. The modifications do not affect the validity of any of the previous results obtained using either the CRAFT Version 1.0 or CRAFT Version 2.0 codes.

Modification 1:

In Version 3.0, the following lines were added to the code as presented on page 10 of the source code listing in Appendix A.

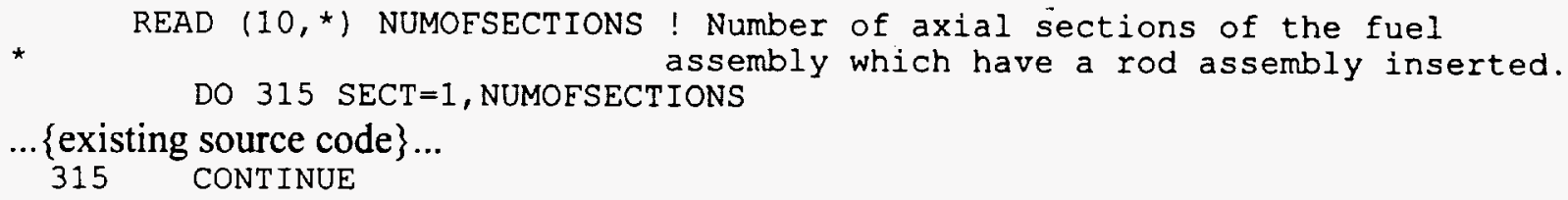

The purpose of this modification is to allow the CRAFT code to model a non-continuous number of axial assembly sections containing a CRA during an individual irradiation time step in the depletion calculations.

Modification 2:

In Version 3.0, the following modification was made to a line of the code as presented on page 57 of the source code listing in Appendix A.

Format of the modified line of source code in Version 2.0:

IF (BPMIX(BPRA_DESCRIPTION_ID).EQ.0) THEN

Format of the modified line of source code in Version 3.0:

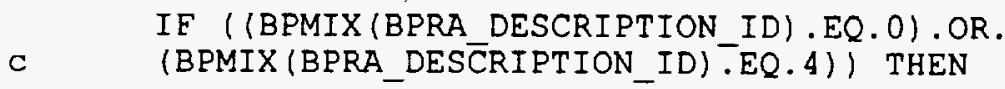

The purpose of this modification is to enable CRAFT to label the burnable poison material as 
$\mathrm{Al}_{2} \mathrm{O}_{3}-\mathrm{B}_{4} \mathrm{C}$ in the $\mathrm{SAS} 2 \mathrm{H}$ input decks if a SAS2H material mixture number for the burnable poison is entered in the CRAFT input deck as either " 0 " or " 4 ". This modification is purely for commentary purposes and does not affect the calculational flow as dictated by the CRAFT code.

\section{References}

1) SCALE 4.3: Modular Code System for Performing Standardized Computer Analyses forLicensing Evaluation User Manuals, Volumes 0 through 3, Oak Ridge National Laboratory, Document Number: CCC-545.

2) S. M. Bowman, O. W. Hermann, and M. C. Brady. Scale-4 Analysis of Pressurized Water Reactor Critical Configurations: Volume 2-Sequoyah Unit 2 Cycle 3, Oak Ridge National Laboratory, Document Number: ORNL/TM-12294/V2.

3) F. W. Walker, J. R. Parrington, and F. Feiner. Nuclides and Isotopes, Fourteenth Edition, General Electric Company, 1989.

4) K. D. Wright. CRC Depletion Calculations for the Non-Rodded Assemblies in Batches 4 and 5 of Crystal River Unit 3, Office of Civilian Radioactive Waste Management, U. S. Department of Energy, Document Identifier: BBA000000-01717-0200-00033 REV 00. 


\section{- Attachment II}

\section{CRAFT Version 3.0 Fortran Source Code Listing}

This is Appendix A: CRAFT Version 3.0

Fortran Source Code Listing of the CRAFT

User Information Documented in Attachment I 


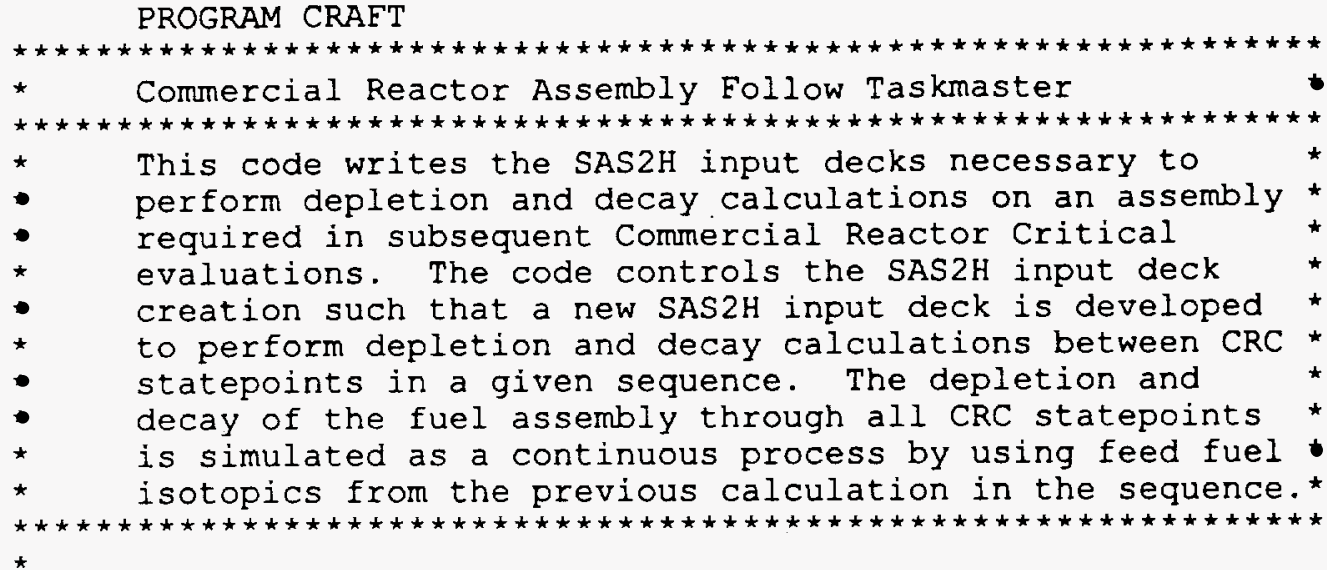

INTEGER ^4 BPZONE (10), $\operatorname{BPMA}(15,10), \operatorname{LMA}(15,10), \operatorname{LUZONE,~}$ C LMB (15), NLIB, PLEVEL, ISN, IIM, ICM, IUS, NBR, AXNUM, C $\operatorname{FTNUM}(20), \operatorname{MONUM}(20), \operatorname{BUNUM}(20), \mathrm{CT} 1, \mathrm{CT} 2$,

C APSRINS $(10,20,23,50)$, APSRSTEPNUM,

C APSRMIXNUM, APSRMIXID(25),

C CRINS $(10,20,23,50)$, CRSTEPNUM,

C CRMIXNUM, CRMIXID(25), CRNUMISOS(25),

C CRISOID $(25,10)$, AXBLANK(50), AXBLANKNODNUM,

C STPTS (10), CYCPOS (10), APSRNUMISOS (25), APSRISOID $(25,10)$,

c STPTSUM, BPRADESNUM, CRDESNUM, CRZONE(10), CRMA $(15,10)$,

c $\operatorname{LMC}(15,10), \operatorname{APSRDESNUM}, \operatorname{APSRZONE}(10), \operatorname{APSRMA}(15,10)$,

C $\operatorname{LMD}(15,10), \operatorname{BPCYCID,} \operatorname{BPTN}(10), \operatorname{BPBN}(10), \mathrm{DES}, \mathrm{BPCYCNUM}$,

C $\operatorname{BPDESID}(10), \operatorname{CRDES}(10,20,23,50), \operatorname{APSRDES}(10,20,23,50)$,

C RELATIVE STPT NUM, RELATIVE APSR MIX ID,

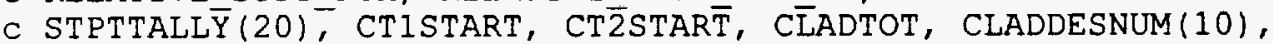

C BPRCLAD (10), CRCLAD (10), APSRCLAD (10), BPMIXNUM, BPMIX (10),

C BPMIXID $(10)$, BPNUMISOS $(20), \operatorname{BPISOID}(10,20), \operatorname{VARSTEPNEM}(10,20)$,

C $\operatorname{BPRFM}(15,10), \operatorname{BPFMNUMISOS}(25), \operatorname{BPFISOID}(25,10), \operatorname{ABOVEBPNUM}(10)$,

C $\operatorname{APSRFM}(15,10)$, APSREOLLOWMIX $(10,20,23,50)$

REAL CLTEMP, PRESS, $\operatorname{BPDEN}(10), \operatorname{BPRA}(15,10), \operatorname{CRISOWTPCT}(25,10)$, C $\operatorname{LRA}(15,10)$, LRB (15), MESH, SZF, EPS, PTC, APSRISOWTPCT $(25,10)$, C $\operatorname{NODES}(50,2), \operatorname{BLETDOWN}(10,20,25), \operatorname{AXBLANKRICH,} \operatorname{STPTDAT}(10,20,3)$, c $\operatorname{FtNdES}(50,2,20), \operatorname{FTdAT}(50,20), \operatorname{MONDES}(50,2,20), \operatorname{MOdAT}(50,20)$, C $\operatorname{BUNDES}(50,2,20), \operatorname{BUDAT}(50,20), \operatorname{RICH}, \operatorname{FMASS}, \operatorname{RODS}, \operatorname{CYCLEN}(10,2)$, C PITCH, FOD, CID, COD, LENGTH, CYCDOWN (10), CRDEN(10),

C CRRA $(15,10), \operatorname{LRC}(15,10), \operatorname{APSRDEN}(10), \operatorname{APSRRA}(15,10), \operatorname{LRD}(15,10)$,

C BPWTPCT (10), HTOT, $\operatorname{FDHT}(20), \operatorname{MDHT}(20), \operatorname{BDHT}(20), \operatorname{FTIN}(50,20)$,

C $\operatorname{MOIN}(50,20), \operatorname{BUIN}(50,20), \operatorname{GRAMS}(50), \operatorname{POWER}(50,20)$,

C FTFINAL $(50,20)$, MODDENEINAL $(50,20)$, MODTEMPEINAL $(50,20)$,

C DENDAT $(29,10), \operatorname{BPISOWTPCT}(10,20)$, $\operatorname{BPXSECT}(10)$, UCSPACERFRAC,

C VARBLETDOWN $(10,20,25,25)$, $\operatorname{VARPOWER}(10,20,25,50), \operatorname{BPRFR}(15,10)$,

C BPFISOWTPCT $(25,10)$, APSRER $(15,10)$

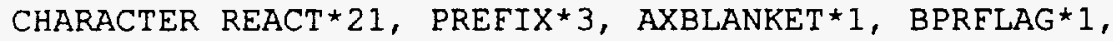
C FUELCLAD ${ }^{2} 10, \operatorname{FLAG} 2 \star 7, \operatorname{CYCLEID}(10) \star 2, \operatorname{CRSTAT} \star 6$,

C APSRSTAT $\star 6, \operatorname{LIB} * 15, \mathrm{NM} \star 31, \operatorname{CLADDESNAME}(10) \star 7$,

C SPACERMAT*7, STEPCONTROL $* 1, \operatorname{ABOVEBP}(10) \star 5$

$\star$

Data input for table of subcooled water density ( $/ \mathrm{ccc}$ ) at various temperatures (F) and pressures (psia).

(REFERENCE: Radiation Shielding Information Center Number CCC-545," "SCALE 4.2, Modular Code System for Performing Standardized Computer Analyses for Licensing Evaluation, 
* Volume 1, Page S2.5.14, Table S2.5.2.)

DATA $((\operatorname{DENDAT}(E, Q), Q=1,10), E=1,29) / 0.0,3000.0,2500.0$,

C $2000.0,1500.0,1000.0$,

c $800.0,600.0,400.0,200.0,50.0,1.0084,1.0069,1.0055,1.0040$,

c $1.0025,1.0019$,

c $1.0013,1.0007,1.000,100,1.0018,1.0004,0.9989,0.9975,0.9960$,

c $0.9954,0.9948,0.9942,0.9936,150.0,0.9893,0.9878,0.9864,0.9849$,

c $0.9834,0.9828,0.9822,0.9815,0.9809,200,0.9725,0.9709,0.9694$,

c $0.9679,0.9663,0.9656,0.9650,0.9644,0.9637,250.0,0.9522,0.9505$,

c $0.9489,0.9472,0.9455,0.9449,0.9442,0.9435,0.9428,300,0.9289$,

c $0.9271,0.9252,0.9234,0.9215,0.9208,0.9200,0.9192,0.9185,350.0$,

c $0.9026,0.9006,0.8985,0.8964,0.8943,0.8934,0.8925,0.8916,0$,

c $400.0,0.8733,0.8709,0.8685,0.8660,0.8634,0.8624,0.8613,0.8603,0$,

c $450.0,0.8405,0.8375,0.8345,0.8314,0.8281,0.8268,0.8255,0,0$,

c $500.0,0.8029,0.7992,0.7952,0.7911,0.7869,0.7851,0,0,0$,

c $510.0,0.7947,0.7907,0.7866,0.7822,0.7776,0,0,0,0$,

c $520.0,0.7862,0.7820,0.7776,0.7729,0.7680,0,0,0,0$,

c $530.0,0.7775,0.7729,0.7682,0.7632,0.7579,0,0,0,0$,

c $540.0,0.7683,0.7635,0.7584,0.7530,0.7472,0,0,0,0$,

c $550.0,0.7589,0.7537,0.7482,0.7423,0,0,0,0,0$,

c $560.0,0.7490,0.7434,0.7374,0.7310,0,0,0,0,0$,

c $570.0,0.7386,0.7326,0.7261,0.7190,0,0,0,0,0$,

c $580.0,0.7278,0.7212,0.7141,0.7062,0,0,0,0,0$,

c $590.0,0.7164,0.7092,0.7012,0.6923,0,0,0,0,0$,

c $600.0,0.7043,0.6963,0.6874,0,0,0,0,0,0$,

c $610.0,0.6915,0.6825,0.6724,0,0,0,0,0,0$,

c $620.0,0.6777,0.6676,0.6558,0,0,0,0,0,0$,

c $630.0,0.6629,0.6512,0.6370,0,0,0,0,0,0$,

c $640.0,0.6467,0.6329,0,0,0,0,0,0,0$,

c $650.0,0.6288,0.6119,0,0,0,0,0,0,0$,

c $660.0,0.6086,0.5866,0,0,0,0,0,0,0$,

c $670.0,0.5850,0,0,0,0,0,0,0,0$,

c $680.0,0.5559,0,0,0,0,0,0,0,01$

write $(*, *)$ 'calling data aquisition'

CALL DATA AQUISITION (BPZŌNE, BPMA,

C LMB, NLIB, PLEVEL, ISN, IIM, ICM, IUS, NBR, AXNUM,

C FTNUM, MONUM, BUNUM, APSRINS,

C APSRSTEPNUM, APSRMIXNUM, APSRMIXID, CRINS,

C CRSTEPNUM, CRMIXNUM, CRMIXID, CRNUMISOS,

C CRISOID, AXBLANK, AXBLANKNODNUM, STPTS,

C CYCPOS, APSRNUMISOS, APSRISOID, STPTSUM,

C BPRADESNUM, CRDESNUM, CRZONE, CRMA, LMC,

C APSRDESNUM, APSRZONE, APSRMA, LMD,

C BPCYCID, BPTN, BPBN, DES, BPCYCNUM, BPDESID,

C CRDES, APSRDES, LMA, LUZONE,

C CLTEMP, PRESS, BPDEN, BPRA, CRISOWTPCT,

C LRA, LRB, MESH, SZF, EPS, PTC, APSRISOWTPCT,

C NODES, BLETDOWN, AXBLANKRICH, STPTDAT,

C FTNDES, FTDAT, MONDES, MODAT,

C BUNDES, BUDAT, RICH, FMASS, RODS, CYCLEN,

C PITCH, FOD, CID, COD, LENGTH, CYCDOWN, CRDEN,

C CRRA, LRC, APSRDEN, APSRRA, LRD,

c BPWTPCT, REACT, PREFIX, AXBLANKET, BPRFLAG,

C FUELCLAD, FLAG2, CYCLEID, CRSTAT,

c APSRSTAT, LIB, BPXSECT, BPRODS, CTISTART,

C CT2START, CLADTOT, CLADDESNUM, CLADDESNAME,

C BPRCLAD, CRCLAD, APSRCLAD, BPMIXNUM, BPMIX, BPMIXID,

C BPNUMISOS, BPISOID, BPISOWTPCT, UCSPACERFRAC, 
C SPACERMAT, STEPCONTROL, VARBLETDOWN, VARSTEPNUM,

C BPRFM, BPFMNUMISOS, BPFISOID, ABOVEBPNUM, APSRFM,

C BPRFR, BPFISOWTPCT, APSRER, ABOVEBP, APSRFOLLOWMIX)

*

write $(*, *)$ 'calling std height'

CALL STD HEIGHT (AXNUM, FTNUM,

C MONUM, BUNUM, HTOT, NODES, STPTSUM,

C EDHT, FTNDES, MDHT, MONDES,

c BDHT, BUNDES)

*

write $\left({ }^{\star},{ }^{*}\right)$ 'calling fueltemp format'

CALI FUELTEMP FORMAT (STPTSUM, AXNUM, FTNUM,

c NODES, FTNDES, FTDAT, FTIN)

*

write $\left(*,{ }^{*}\right)$ 'calling modspecvol format'

CALI MODSPECVOL_FORMAT (STPTSUM, AXNUM, MONUM,

C NODES, MONDES, MODAT, MOIN)

*

write $\left({ }^{*},{ }^{*}\right)$ 'calling burnup_format'

CALL BURNUP FORMAT (STPTSUM, AXNUM, BUNUM,

C NODES, BUNDEES, BUDAT, BUIN)

write $\left({ }^{*},{ }^{*}\right)$ 'calling power calcs'

CALL POWER CALCS (NBR, AXNUMM, STPTSUM, STPTTALLY,

C STPTS, GRĀMS, FMASS, NODES, HTOT, BUIN,

C STPTDAT, POWER, CYCLEN, STEPCONTROL, VARBLETDOWN,

C VARSTEPNUM, VARPOWER)

write $(*, *)$ 'calling units_conversion'

CALL UNITS CONVERSION (STPTSUM, AXNUM, FTEINAL,

C ETIN, MODDENFINAL, MOIN, PRESS, MODTEMPEINAL,

C DENDAT)

write $(*, *)$ 'calling execution control'

CALL EXECUTION_CONTROL (NBR, RELATIVE_STPT_NUM,

C CT1, CT2, CT3, AXNUM, CYCPOS, AXBLANK,

c BPDESID, CRINS, CRDES,

C CRMIXNUM, CRMIXID, CRNUMISOS, CRISOID,

C APSRINS, APSRMIXNUM, APSRMIXID,

C RELATIVE APSR MIX ID, APSRNUMISOS,

C APSRISOI $\bar{D}$, IS $\bar{N}$, I $\bar{I} M$, ICM, IUS, PLEVEL,

C BPZONE, BPMA, CRZONE, CRMA,

C LMC, APSRZONE, APSRMA, LMD,

C BPTN, BPBN, STPTS, APSRDES,

C STPTDAT, AXBLANKRICH, GRAMS,

C NODES, RODS, RICH, FTFINAL,

C MODDENFINAL, MODTEMPFINAL,

C BLETDOWN, BPWTPCT, BPDEN, CRDEN,

C CRISOWTPCT, APSRDEN, APSRISOWTPCT,

C PITCH, FOD, COD, CID, SZF, EPS, PTC, MESH,

C BPRA, CRRA, LRC, APSRRA,

C LRD, POWER, CYCDOWN, PREFIX,

C NM, CYCLEID, REACT, LIB, AXBLANKET,

c FUELCLAD, BPRFLAG, CRSTAT, APSRSTAT, FLAG2,

C LUZONE, LMB, LRB, BPXSECT, BPRODS,

C CTISTART, CT2START, STPTTALLY, CLADTOT,

C CLADDESNUM, CLADDESNAME, BPRCLAD, CRCLAD,

C APSRCLAD, CLTEMP, BPMIXNUM, BPMIX, BPMIXID,

C BPNUMISOS, BPISOID, BPISOWTPCT, UCSPACERFRAC,

C SPACERMAT, STEPCONTROL, VARBLETDOWN, VARSTEPNUM, 
C VARPOWER, BPRFM, BPFMNUMISOS, BPFISOID,

C ABOVEBPNUM, APSREM, BPRFR, BPFISOWTPCT,

$\star$

C APSRER, ABOVEBP, APSREOLLOWMIX)

END

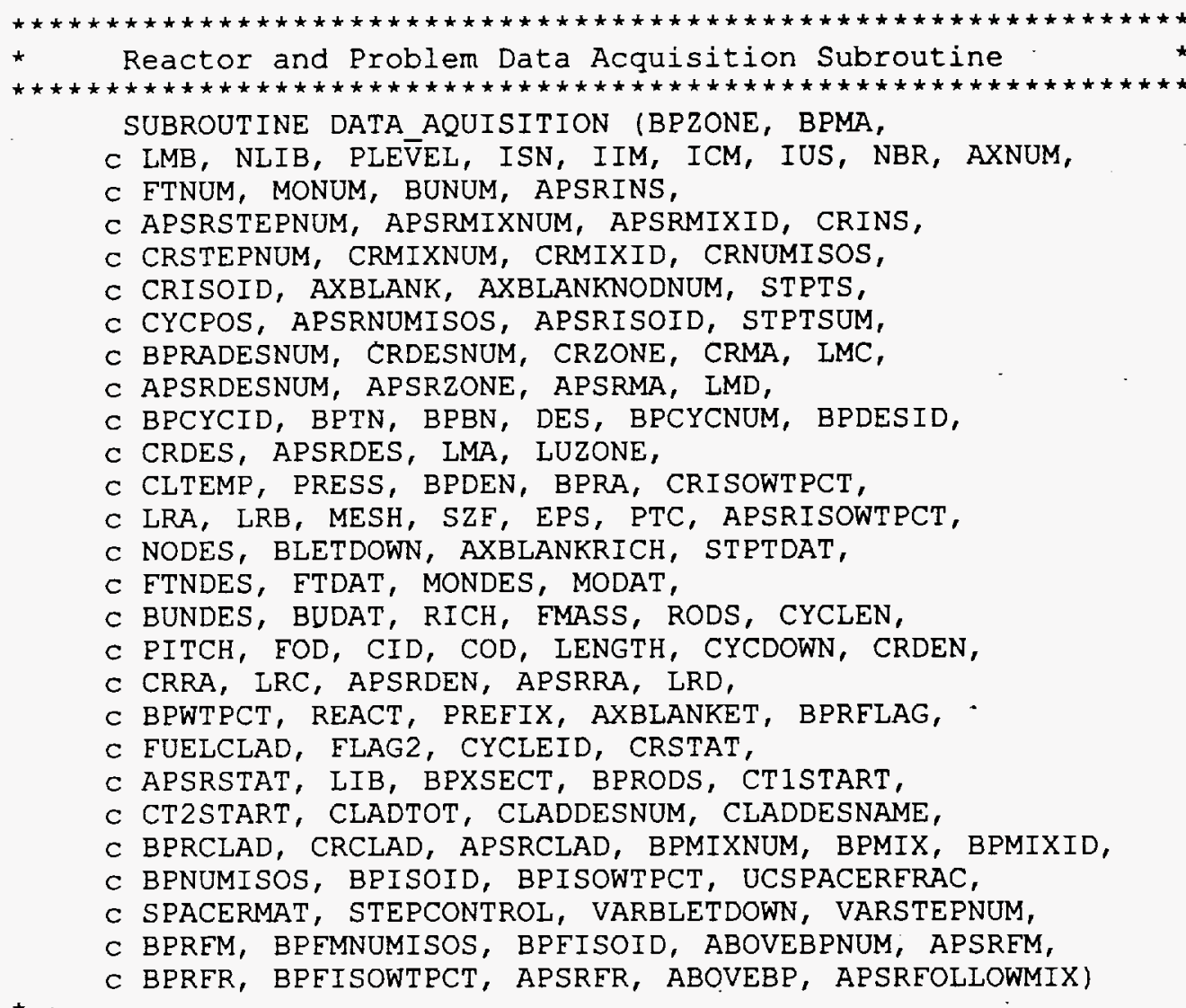

INTEGER * 4 BPZONE (10), $\operatorname{BPMA}(15,10), \operatorname{LMA}(15,10), \operatorname{LUZONE,}$ C LMB (15), NLIB, PLEVEL, ISN, IIM, ICM, IUS, NBR, AXNUM, C ETNUM(20), MONUM (20), BUNUM (20), CT1, CT2, CT3, C APSRINS $(10,20,23,50)$, APSRSTEPNUM, APSRCYC, APSRSTEP,

C TOPN, BOTN, APSRMIX, APSRMIXNUM, APSRMIXID(25),

C CRINS $(10,20,23,50)$, CRSTEPNUM, CRCYC, CRSTEP, CYCHOLDER, c CRMIX, STPTHOLDER, CRMIXNUM, CRMIXID(25), CRNUMISOS (25), c CRISOID $(25,10)$, AXBLANK $(50)$, AXBLANKNODNUM, AXBLANKTEMP, C STPTS (10), CYCPOS (10), APSRNUMISOS (25), APSRISOID $(25,10)$, C STPTSUM, BPRADESNUM, CRDESNUM, $\operatorname{CRZONE}(10), \operatorname{CRMA}(15,10)$,

C $\operatorname{LMC}(15,10), \operatorname{APSRDESNUM}, \operatorname{APSRZONE}(10), \operatorname{APSRMA}(15,10)$,

c $\operatorname{LMD}(15,10)$, BPCYCID, $\operatorname{BPTN}(10), \operatorname{BPBN}(10), \mathrm{DES}, \mathrm{BPCYCNUM,}$

C $\operatorname{BPDESID}(10), \operatorname{CRDES}(10,20,23,50), \operatorname{APSRDES}(10,20,23,50)$,

C BPRODS(10), CTISTART, CT2START, APSRSTPT, CRSTPT,

C CLADTOT, CLADDESNUM(10), BPRCLAD(10), CRCLAD (10),

C APSRCLAD (10), BPMIXNUM, BPMIX (10), BPMIXID(10),

C BPNUMISOS $(20)$, $\operatorname{BPISOID}(10,20)$, $\operatorname{VARSTEPNUM}(10,20)$,

C $\operatorname{BPRFM}(15,10)$, $\operatorname{BPFMNUMISOS}(25), \operatorname{BPFISOID}(25,10)$,

c ABOVEBPNUM(10), APSREM $(15,10), \operatorname{FMIX}, \operatorname{APSRFOLLOWMIX}(10,20,23,50)$,

C NUMOFSECTIONS, SECT 
C $\operatorname{LRA}(15,10), \operatorname{LRB}(15), \mathrm{MESH}, \mathrm{SZF}, \operatorname{EPS}, \operatorname{PTC}, \operatorname{APSRISOWTPCT}(25,10)$,

C NODES $(50,2), \operatorname{BLETDOWN}(10,20,25), \operatorname{AXBLANKRICH,} \operatorname{STPTDAT}(10,20,3)$,

c ETNDES $(50,2,20), \operatorname{ETDAT}(50,20), \operatorname{MONDES}(50,2,20), \operatorname{MODAT}(50,20)$,

C $\operatorname{BUNDES}(50,2,20), \operatorname{BUdAT}(50,20), \operatorname{RICH}, \operatorname{FMASS}, \operatorname{RODS}, \operatorname{CYCLEN}(10,2)$,

C PITCH, FOD, CID, COD, LENGTH, CYCDOWN(10), CRDEN(10),

c $\operatorname{CRRA}(15,10), \operatorname{LRC}(15,10), \operatorname{APSRDEN}(10), \operatorname{APSRRA}(15,10), \operatorname{LRD}(15,10)$,

C BPWTPCT (10), BPXSECT $(10), \operatorname{BPISOWTPCT}(10,20)$, UCSPACERFRAC,

c VARBLETDOWN $(10,20,25,25), \operatorname{BPRER}(15,10), \operatorname{BPFISOWTPCT}(25,10)$,

C APSRER $(15,10)$

*

$\star \quad$ Hardwired ASSYFOLLOW limitations:

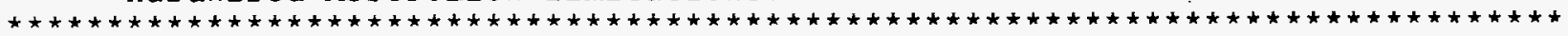

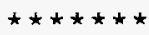

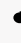

$\star$

$\star$

2 FORMAT (AI)

IF (PICKUPFLAG.EQ.' 'Y') THEN $\operatorname{READ}(10, *)$ CTISTART ELSE

OPEN (UNIT=10, FILE='datain', STATUS='OLD')

REWIND (UNIT $=10$ )

$\operatorname{READ}(10,2)$ PICKUPFLAG ! PICKUPFLAG is a signal to begin the assembly depletion and decay calculation at a point other than the beginning of the assembly's irradiation history as specified in the input deck

\section{ENDIF}

CTISTART $=1$

CT2START $=1$

$\operatorname{READ}(10,10)$ REACT ! REACT is the problem identification 
*

*

10 FORMAT (A21)

20 FORMAT (A3)

30 FORMAT (A2)

40 FORMAT (A15) (up to 21 characters).

! PREFIX is a 3 character prefix to be placed at the beginning of all SAS2H input decks produced.

! LIB is a 15 character identification of the cross-section library requested for use in the SCALE code system.

Fuel Batch Data Acquisition

$\operatorname{READ}\left(10,{ }^{*}\right) \operatorname{RICH} ! \operatorname{RICH}$ is the fuel assy wto U-235 in UO2 enrichment.

$\operatorname{READ}\left(10,{ }^{*}\right)$ FMASS ! FMASS is the fuel assy loading of uranium in g/assy.

READ $(10, *)$ RODS ! RODS is the number of fuel rods in the assy.

READ $(10, *)$ PITCH ! PITCH is the fuel rod pitch in the assy.

$\operatorname{READ}(10, *)$ FOD! FOD is the fuel rod outer diameter in $\mathrm{cm}$.

$\operatorname{READ}(10, \star)$ CID ! CID is the clad inner diameter in Cm.

$\operatorname{READ}(10, *) \operatorname{COD} ! \operatorname{COD}$ is the clad outer diameter in $\mathrm{cm}$.

$\operatorname{READ}(10, *)$ LENGTH ! LENGTH is the active fuel length in $\mathrm{cm}$.

READ $(10,70)$ AXBLANKET ! Flag for axial blanket modelling.

70 FORMAT (A1).

IF (AXBLANKET.EQ. 'Y') THEN

READ $(10, *)$ AXBLANKRICH ! Axial blanket fuel U-235 enrichment.

*

Initialize AXBLANK array

DO $80 \mathrm{CT} 1=1,50$

AXBLANK $(\mathrm{CT} 1)=0$

80 CONTINUE

* Gather data for AXBLANK array

$\operatorname{READ}\left(10,{ }^{*}\right)$ AXBLANKNODNUM ! Number of nodes with axial

$\star$

DO $90 \mathrm{CT} 1=1$, AXBLANKNODNUM

blanket fuel.

$\operatorname{READ}(10, *)$ AXBLANKTEMP ! Node containing axial

blanket fuel.

AXBLANK(AXBLANKTEMP) $=1$ ! Identify axial blanket fuel

$\star$

90 CONTINUE node location in AXBLANK.

ENDIF

* Spacer data acquisition

READ $(10,92)$ SPACERMAT

92 FORMAT (A7)

$\operatorname{READ}(10, *)$ UCSPACERFRAC

* Cladding data acquisition

READ $(10,100)$ FUELCLAD

100 FORMAT (A10)

$\operatorname{READ}\left(10,{ }^{\star}\right)$ CLTEMP

READ $(10,101)$ CLADFLAG

101 FORMAT (A1)

IF (CLADFLAG.EQ.' $Y$ ') THEN

$\operatorname{READ}(10, *)$ CLADTOT

DO $108 \mathrm{CT} 1=1$, CLADTOT

$\operatorname{READ}(10, *)$ CLADDESNUM(CT1)

105

108

$\operatorname{READ}(10,105)$ CLADDESNAME (CT1)

CONTINUE

ENDIF 
- System Pressure

READ $(10, *)$ PRESS

READ $(10,110)$ BPRFLAG

110 FORMAT (AI)

IE (BPRELAG.EQ.' $Y$ ') THEN

$\operatorname{READ}\left(10,{ }^{\star}\right)$ BPCYCNUM ! Number of cycles with BPRA

$\operatorname{READ}(10, \star)$ BPRADESNUM, BPMIXNUM

DO 145 CT $2=1$, BPRADESNUM

$\star$

Get BP density, B4C wto in Al203-B4C,

* $\quad B P x$-sectional area, \#BP rods, and BPR clad mix num

C BPRODS (CT2), BPRCLAD(CT2), BPMIX(CT2)

* Larger BPRA unit cell data acquisition

$\operatorname{READ}(10, \star)$ BPZONE (CT2)

DO 112 CT $1=1, \mathrm{BPZONE}(\mathrm{CT} 2)$ $\operatorname{READ}\left(10,{ }^{\star}\right) \operatorname{BPMA}(\mathrm{CT} 1, \mathrm{CT} 2), \mathrm{BPRA}(\mathrm{CT} 1, \mathrm{CT} 2)$

112 CONTINUE

* Larger standard unit cell for use with BPRAs

DO 114 CT $1=1$, BPZONE (CT2)

$\operatorname{READ}(10, *) \operatorname{LMA}(\mathrm{CT} 1, \mathrm{CT} 2), \operatorname{LRA}(\mathrm{CT} 1, \mathrm{CT} 2)$

114 CONTINUE

DO $116 \mathrm{CT} 1=1, \mathrm{BPZONE}$ (CT2)

$\operatorname{READ}(10, \star) \operatorname{BPREM}(\mathrm{CT} 1, \mathrm{CT} 2), \operatorname{BPRFR}(\mathrm{CT} 1, \mathrm{CT} 2)$

116 CONTINUE

READ (10,118) ABOVEBP(CT2), ABOVEBPNUM(CT2)

118 FORMA T (A5, 1X, I3)

IF (ÄBOVEBP (CT2). NE. 'AL2O3') THEN

READ $(10, *)$ BPEMNUMISOS (CT2)

DO $120 \mathrm{CT} 1=1, \mathrm{BPFMNUMISOS}(\mathrm{CT} 2)$

$\operatorname{READ}\left(10,{ }^{*}\right)$ BPFISOID (CT2,CT1),

120

BPEISOWTPCT (CT 2, CT1)

ENDIF

145 CONTINUE

DO 147 CT $1=1,10$

DO 146 CT $2=1,20$

$\mathrm{BPISOID}(\mathrm{CT} 1, \mathrm{CT} 2)=0$

146

147 CONTINUE

CONTINUE

IF (BPMIXNUM. NE. O) THEN

DO $150 \mathrm{CT} l=1$, BPMIXNUM

READ $(10, \star)$ BPMIXID(CT1) ! SAS2H Mixture ID for CR. READ $(10, *)$ BPNUMISOS (CT 1 )

DO 149 CT2 $=1$, BPNUMISOS (CT1)

$\operatorname{READ}\left(10,{ }^{*}\right)$ BPISOID (CT1, CT2), BPISOWTPCT (CT1, CT2)

149

150 CONT INUE CONTINUE

ENDIF

DO 156 CT $1=1,10$

156 CONTINUE

DO 157 CT $1=1$, BPCYCNUM

$\operatorname{READ}(10, *)$ BPCYCID, BPDESID (BPCYCID), BPTN (BPCYCID),

157

C BPBN (BPCYCID)

ENDIF

* Larger standard unit cell

$\operatorname{READ}(10, \star)$ LUZONE

DO 170 CT $1=1$, LUZONE 
170 CONTINUE

$\operatorname{READ}(10, *) \operatorname{LMB}(\mathrm{CT} 1), \operatorname{LRB}(\mathrm{CT} 1)$

* Contol parameter data acquisition

$\operatorname{READ}(10, *)$ NLIB

$\operatorname{READ}(10, *)$ PLEVEL

$\operatorname{READ}(10, *)$ MESH

READ $(10,180)$ FLAG2

180 FORMAT (A7)

IF (FLAG2.EQ.'SPECIAL') THEN

$\operatorname{READ}(10, *)$ SZF

$\operatorname{READ}(10, *)$ ISN

$\operatorname{READ}(10, *)$ IIM

$\operatorname{READ}(10, *)$ ICM

$\operatorname{READ}(10, *)$ EPS

$\operatorname{READ}(10, *)$ PTC

ENDIF

$\operatorname{READ}(10, *)$ IUS

* Reactor history data acquisition

$\operatorname{READ}(10, *)$ NBR

DO 210 CTI 1 , NBR

READ $(10,190)$ CYCLEID (CT1)

190

FORMAT (A2)

$\operatorname{READ}(10, *)$ STPTS (CT1)

DO 200 CT $2=1$, STPTS (CT1)

$\operatorname{READ}\left(10,{ }^{*}\right)$ STPTDAT (CT1, CT2, 1)

READ_ $(10, *)$ STPTDAT (CT1, CT2, 2)

$\operatorname{READ}(10, *)$ STPTDAT (CT1, CT2,3)

200 CONTINUE

$\operatorname{READ}(10, *)$ CYCDOWN (CT1)

$\operatorname{READ}(10, *)$ CYCLEN (CT 1, 1)

$\operatorname{READ}(10, *)$ CYCLEN $($ CT 1,2$)$

$\operatorname{READ}(10, *)$ CYCPOS (CT1)

210 CONTINUE

STEPCONTROL $=$ ' $N$ '

READ $(10,212)$ STEPCONTROL

212 FORMAT (AI)

IF (STEPCONTROL.EQ. 'N') THEN

DO 220 CT $1=1, N B R$

$\operatorname{READ}\left(10,{ }^{*}\right)$ CYCHOLDER

DO 217 CT $2=1$, STPTS (CYCHOLDER)

$\operatorname{READ}(10, *)$ STPTHOLDER

$\operatorname{READ}(10, *)$ BLETDOWN (CYCHOLDER, STPTHOLDER, 1)

READ $(10, *)$ BLETDOWN (CYCHOLDER, STPTHOLDER, 2 )

DO 213 CT $3=3$, (INT (BLETDOWN (CYCHOLDER, STPTHOLDER, 2)) +2)

READ $(10, *)$ BLETDOWN (CYCHOLDER, STPTHOLDER, CT 3 )

213

217 CONTINUE

220 CONTINUE

ELSEIF (STEPCONTROL.EQ. 'Y') THEN

DO 240 CT $1=1$, NBR

READ $(10, *)$ CYCHOLDER

DO 235 CT $2=1$, STPTS (CYCHOLDER)

READ $(10, *)$ STPTHOLDER

READ $(10, *)$ VARSTEPNUM (CYCHOLDER, STPTHOLDER)

DO 230 CT $3=1$, VARSTEPNUM (CYCHOLDER, STPTHOLDER)

READ $(10, *)$ VARBLETDOWN (CYCHOLDER, STPTHOLDER, CT 3, 1),

C

230

235

240 CONTINUE

CONTINUE

CONTINUE 


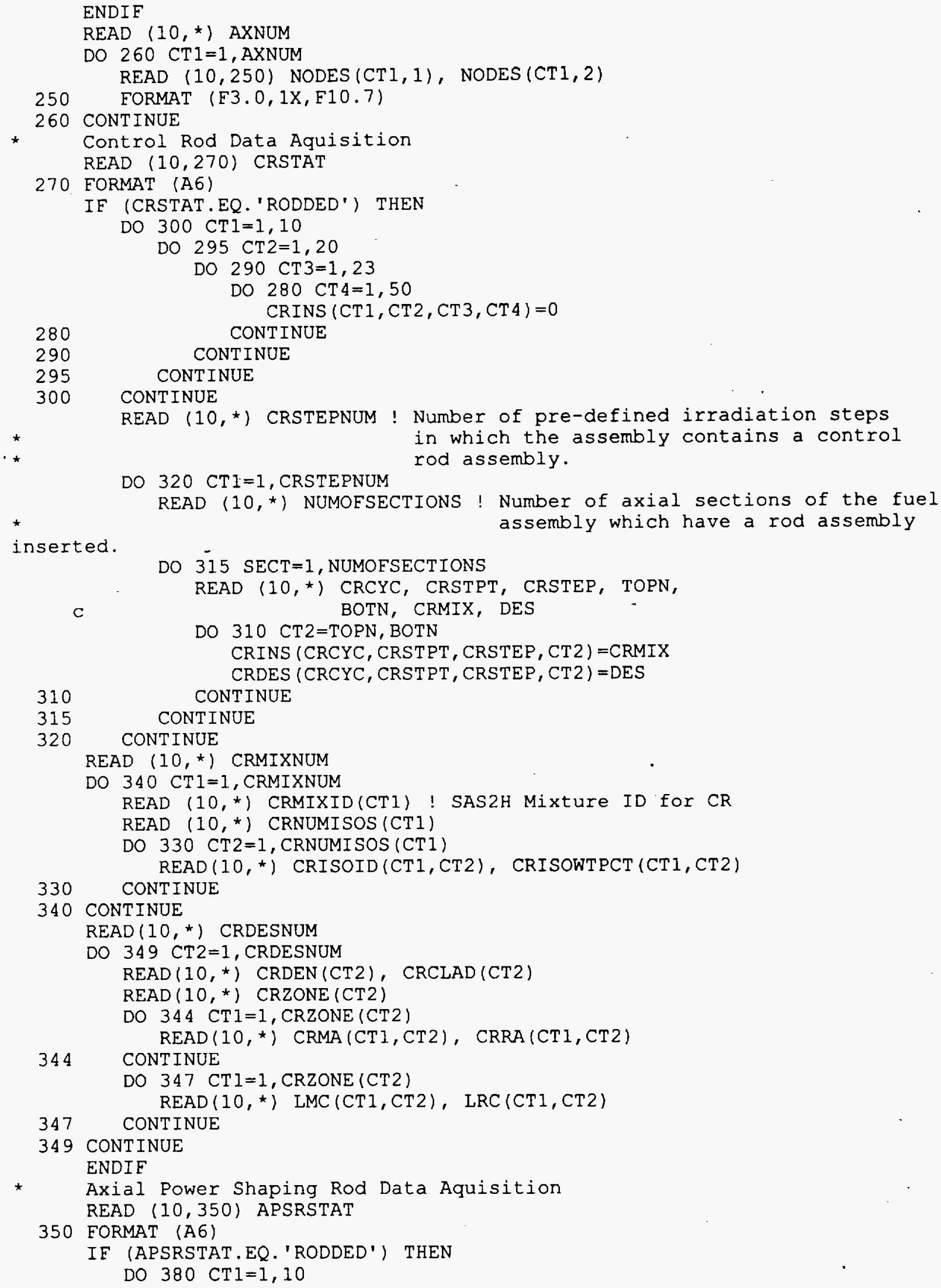




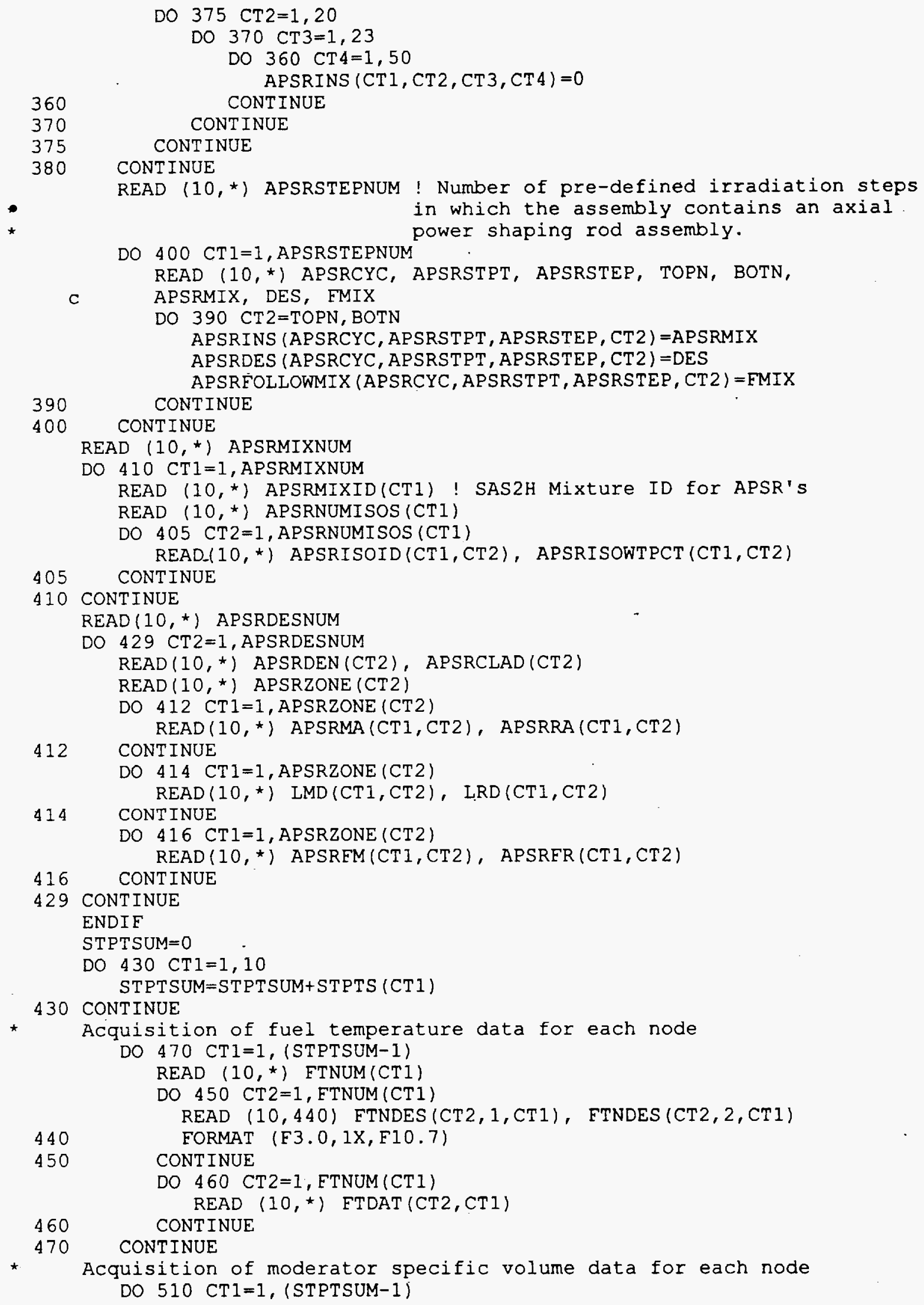




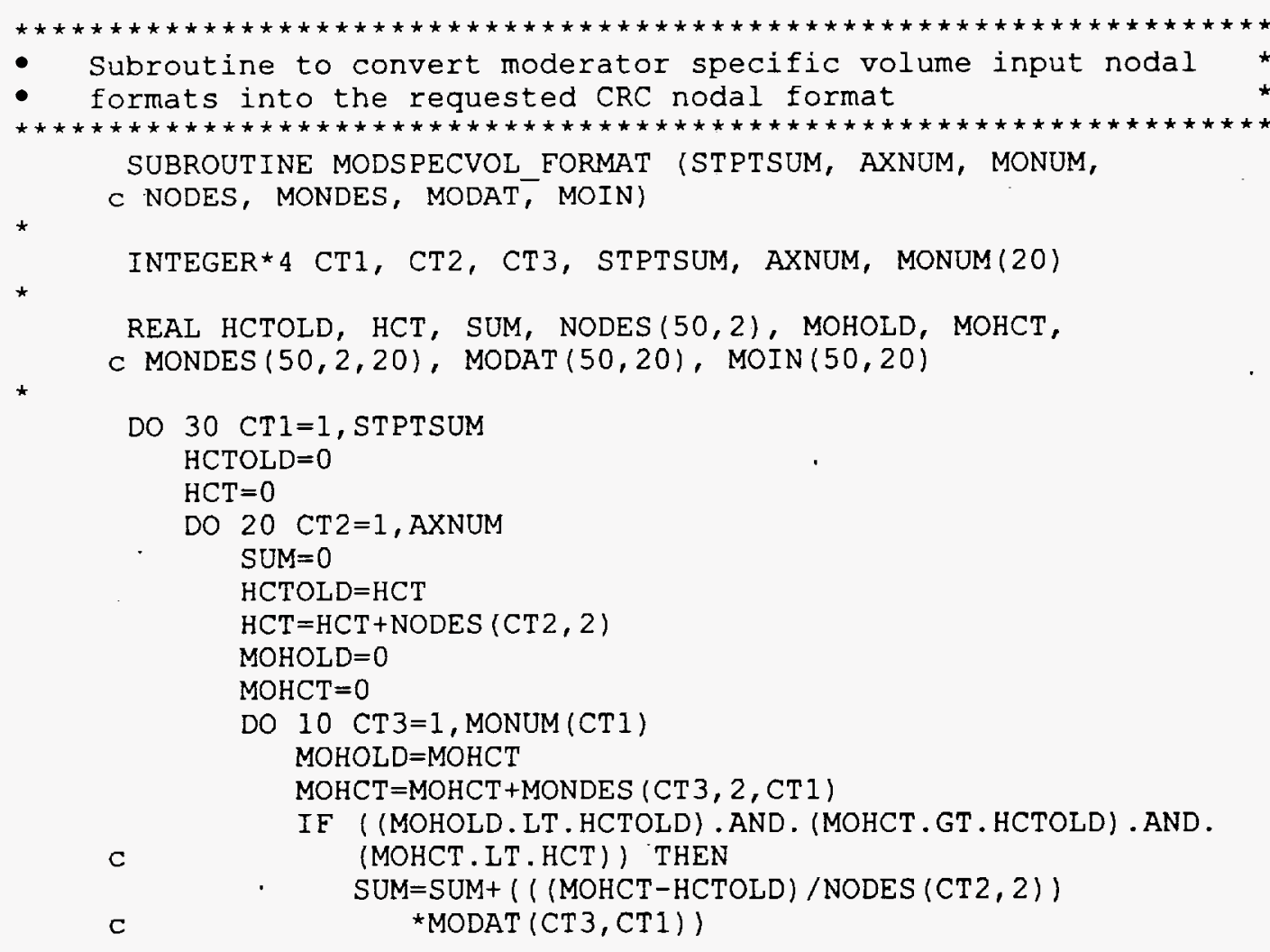




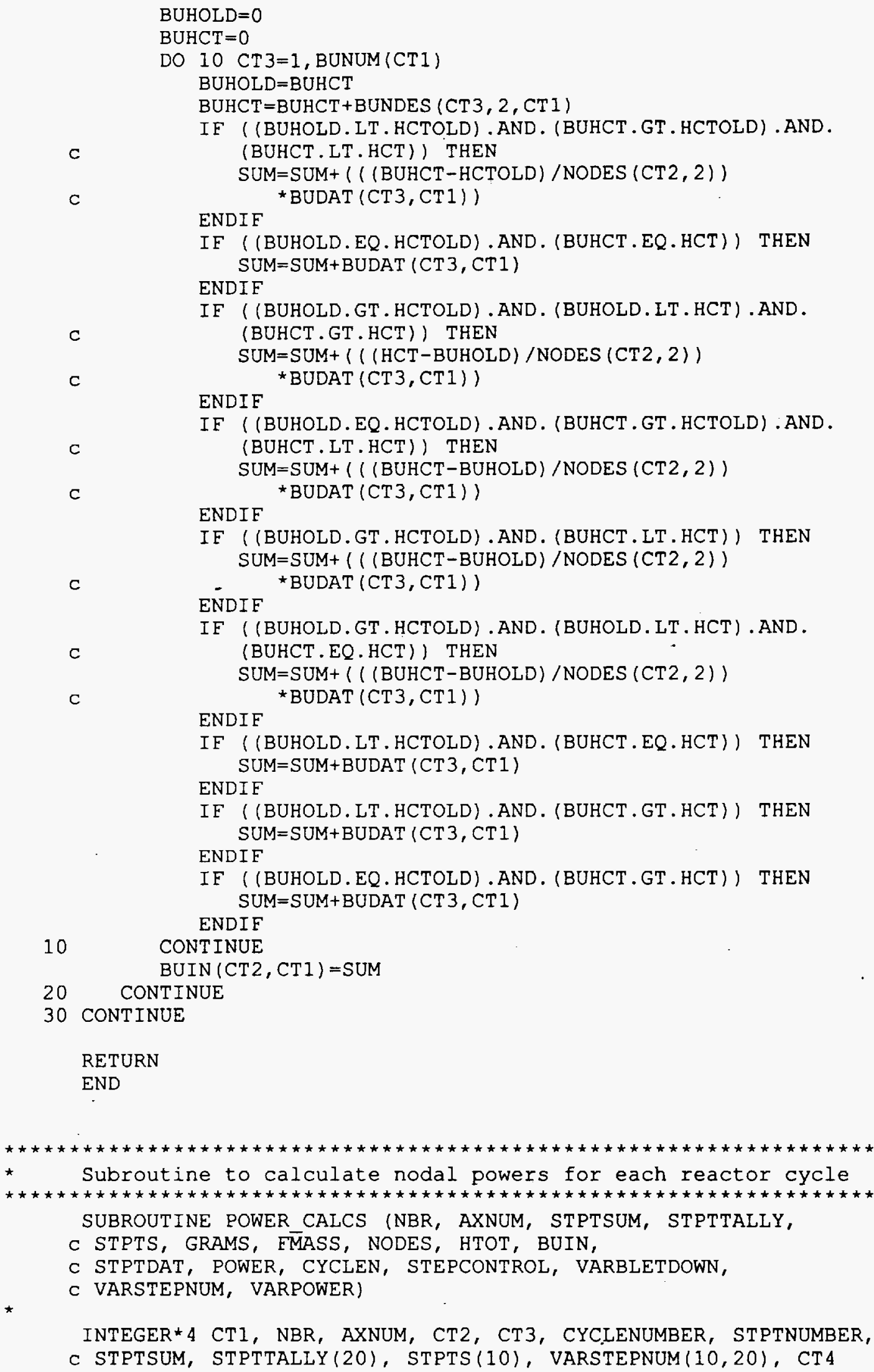




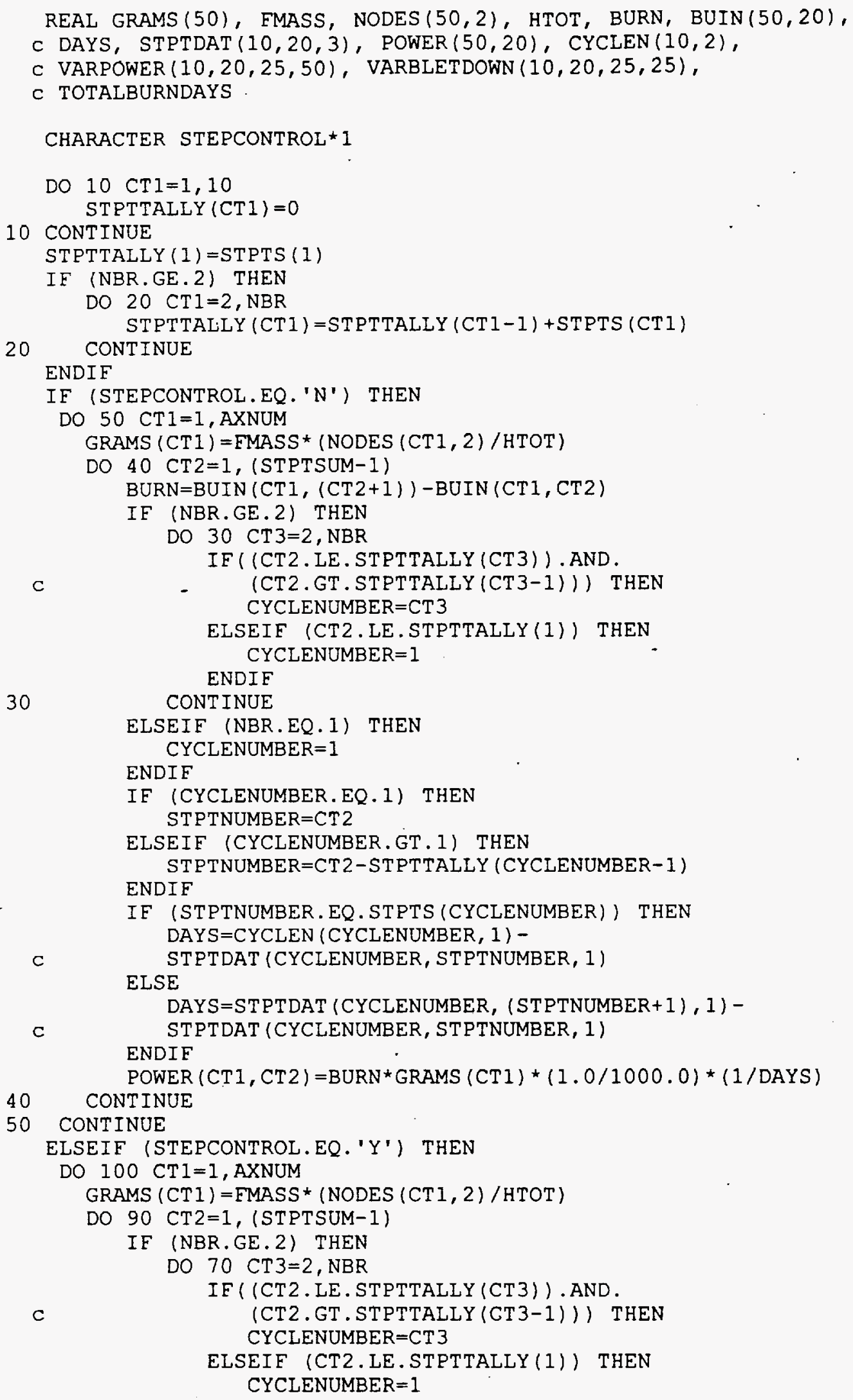




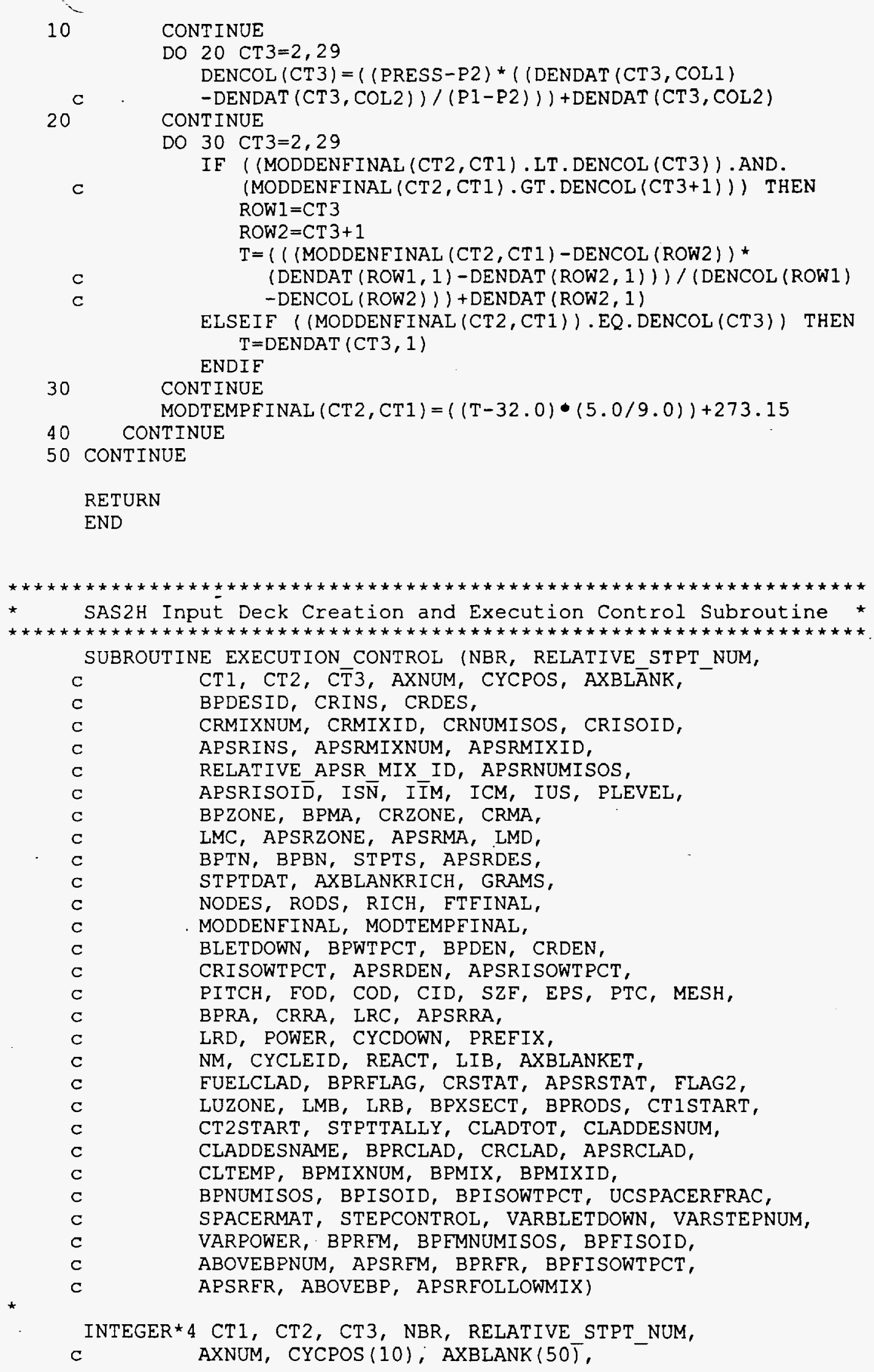


* CT2 is the statepoint incrementer within cycle CT1

DO 10 CT2=CT2GOVALUE, CT2ENDVALUE

RELATIVE STPT_NUM=RELATIVE_STPT_NUM+1

IF ((CT1.EQ.1).AND. (CT2.EQ.1)) THEN

CALL STANDARD WRITER (RELATIVE STPT NUM, CTI,

C

c

$\mathrm{C}$

$\mathrm{C}$

C

C CT2, CT3, AXNUMM, CYCPOS, AXBLANKK, BPDESID, CRINS, CRDES, CRMIXNUM, CRMIXID, CRNUMISOS, CRISOID, APSRINS, APSRMIXNUM, APSRMIXID, RELATIVE APSR MIX_ID, APSRNUMISOS, APSRISOID, IS $\overline{\mathrm{N}}$, I $\bar{I} M$, ICM, IUS, PLEVEL, BPZONE, BPMA, CRZONE, CRMA, LMC, APSRZONE, APSRMA, LMD, BPTN, BPBN, STPTS, APSRDES, STPTDAT, AXBLANKRICH, GRAMS, NODES, RODS, RICH, FTFINAL, MODDENFINAL, MODTEMPEINAL, BLETDOWN, BPWTPCT, BPDEN, CRDEN, CRISOWTPCT, APSRDEN, APSRISOWTPCT, PITCH, FOD, COD, CID, SZF, EPS, PTC, MESH, BPRA, CRRA, LRC, APSRRA, LRD, POWER, CYCDOWN, PREFIX, NM, CYCLEID, REACT, LIB, AXBLANKET, FUELCLAD， BPRFLAG， CRSTAT， APSRSTAT， FLAG2, LUZONE, LMB, LRB, PREVIOUSNAME, FINALDOWNTIME, BPRA_INSERTED, CLADTOT, CLADDESNUM, CLADDESNAME, BPRCLAD, CRCLAD, APSRCLAD, CLTEMP, BPMIXNUM, BPMIX, BPMIXID, BPNUMISOS, BPISOID, BPISOWTPCT, UCSPACERERAC, SPACERMAT, STEPCONTROL, VARBLETDOWN, VARSTEPNUM, VARPOWER, BPRFM, BPFMNUMISOS, BPFISOID, ABOVEBPNUM, APSRFM, BPRFR, BPFISOWTPCT, APSRFR, ABOVEBP, APSRFOLLOWMIX, CTISTART, CT2GOVALUE, APSRINSOLD) SASEXECOMMAND $(1: 8)=$ 'batch 43 ' SASEXECOMMAND $(9: 33)=\mathrm{NM}(1: 25)$ SASEXERESULT $=$ SYSTEM (SASEXECOMMAND) IF (SASEXERESULT.LT.O) THEN WRITE $(*, *)$ 'AN ERROR OCCURRED DURING SAS2H', 'EXECUTION OF', NM $(1: 25)$ ENDIF ELSE CALL CUTTER (NM)

CALL CONTINUATION WRITER (RELATIVE STPT NUM,

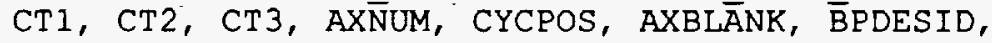
CRINS, CRDES, CRMIXNUM, CRMIXID, CRNUMISOS, CRISOID, APSRINS, APSRMIXNUM, APSRMIXID, RELATIVE APSR MIX ID, APSRNUMISOS, APSRISOID, ISN, II $\bar{M}, I C \bar{M}$, I USS, PLEVEL, BPZONE, BPMA, CRZONE, CRMA, LMC, APSRZONE, APSRMA, LMD, BPTN, BPBN, STPTS, APSRDES, STPTDAT, AXBLANKRICH, GRAMS, NODES, RODS, RICH, FTEINAL, MODDENFINAL, MODTEMPEINAL, BLETDOWN, BPWTPCT, BPDEN, CRDEN, CRISOWTPCT, APSRDEN, APSRISOWTPCT, PITCH, FOD, COD, CID, SZF, EPS, PTC, MESH, BPRA, CRRA, LRC, APSRRA, LRD, POWER, CYCDOWN, PREFIX; NM, CYCLEID, REACT, LIB， AXBLANKET, EUELCLAD, 
Title: CRC Depletion Calculations for the Rodded Assemblies in Batches 1, 2, 3, and $1 \mathrm{X}$ of Crystal River Unit 3 Document Identifier: BBA000000-01717-0200-00040 REV 00 Date Printed: 4/3/97 Attachment II, Page 23 of 82

c APSRINSOLD)

$\star$

INTEGER ${ }^{4}$ RELATIVE STPT NUM, CT1, CT2, CT3, AXNUM, c NUMSTPT1, NUMSTPT $\overline{2}$, NUMSTPT3, CYCPOS(10), AXBLANK(50),

c BPDESID(10), BPRA DESCRIPTION ID, CT4, CT5, CRINS $(10,20,23,50)$,

C CR MIXTURE ID, CR DESCRIPTION, CRDES $(10,20,23,50)$, CRMIXNUM,

c CRM̄IXID (25), RELATTIVE_CR MIX_ID, CRNUMISOS (25),

C CRISOID $(25,10)$, APSRIN̄S $(\overline{1} 0,2 \overline{0}, 23,50)$, APSR_MIXTURE_ID,

C APSR DESCRIPTION, APSRMIXNUM, APSRMIXID (25),

c RELATTIVE APSR MIX ID, APSRNUMISOS (25), APSRISOID $(25,10)$,

C ISN, IIM, ICM, IUS, $\operatorname{PLEVEL}, \operatorname{BPZONE}(10), \operatorname{BPMA}(15,10)$,

C $\operatorname{CRZONE}(10), \operatorname{CRMA}(15,10), \operatorname{LMC}(15,10), \operatorname{APSRZONE}(10)$,

C APSRMA $(15,10), \operatorname{LMD}(15,10), \operatorname{BPTN}(10), \operatorname{BPBN}(10), \operatorname{STPTS}(10)$,

c APSRDES $(10,20,23,50)$, LUZONE, LMB (15), NUMSTPT4, NUMSTPT5,

C NUMSTPT 6, CLADTOT, CLADDESNUM(10), BPRCLAD(10), CRCLAD(10),

c APSRCLAD (10), APSRCLNUM, CRCLNUM, BPRCLNUM, BPMIXNUM,

C BPMIX(10), BPMIXID(10), BPNUMISOS(20), BPISOID $(10,20)$,

C $\operatorname{VARSTEPNUM}(10,20), \operatorname{BPRFM}(15,10), \operatorname{BPFMNUMISOS}(25)$,

c $\operatorname{BPFISOID}(25,10), \operatorname{ABOVEBPNUM}(10), \operatorname{APSRFM}(15,10)$,

C APSRFOLLOWMIX $(10,20,23,50)$, FOLNODKEEP,

C FOLSTEPKEEP, APSRFOLNUM, APSRFOLLOWDATA $(10,20,23,50)$,

C CTISTART, CT2GOVALUE, APSRINSOLD $(10,20,23,50)$

REAL STPTDAT $(10,20,3)$, ENR, AXBLANKRICH, OXYGMS, GRAMS (50), C UO2GMS, FVOL, PI, NODES $(50,2)$, RODS, FDEN, WT234,

c WT235, WT236, WT238, RICH, FTFINAL $(50,20)$,

c MODDENEINAL $(50,20)$, MODTEMPFinAL $(50,20), \operatorname{BLETdOWN}(10,20,25)$,

c BPWTPCT (10), BPDEN(10), ALERAC, OFRAC, CRDEN(10),

c CRISOWTPCT $(25,10), \operatorname{APSRDEN}(10), \operatorname{APSRISOWTPCT}(25,10)$,

C PITCH, FOD, COD, CID, SZF, EPS, PTC, MESH, BPRA $(15,10)$,

C $\operatorname{CRRA}(15,10), \operatorname{LRC}(15,10), \operatorname{APSRRA}(15,10), \operatorname{LRD}(15,10)$,

C DOWNTIME, BORON FRACTION, POWER $(50,20)$, CYCDOWN(10), LRB (15),

C FINALDOWNTIME, CLTEMP, BPISOWTPCT $(10,20)$, UCSPACERERAC,

C BORATEDMODVF, BORONVE, UCMODREGIONDEN,

C VARBLETDOWN $(10,20,25,25)$, $\operatorname{VARPOWER}(10,20,25,50)$,

c $\operatorname{BPRFR}(15,10)$, $\operatorname{BPFISOWTPCT}(25,10), \operatorname{APSRFR}(15,10)$

CHARACTER CHNODE*2, CHID*2, PREFIX*3, CHSTPT1*1, CHSTPT2*1,

C CHSTPT $3 \star 1, \mathrm{NM} * 31, \operatorname{CYCLEID}(10) \star 2, \operatorname{REACT} \star 21, \mathrm{LIB} * 15$,

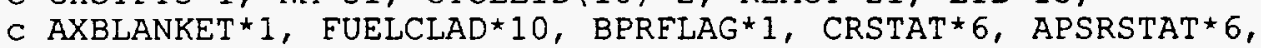

c FLAG $2 \star 7$, IRRAD STEPS $\star 2$, PLEVELCH ${ }^{\star} 2$, BPZONECH ${ }^{\star} 2$, CRZONECH ${ }^{\star} 2$,

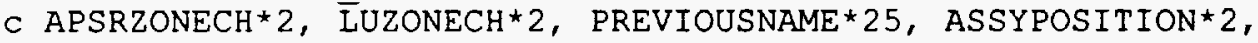

c CHSTPT $4 \star 1, \operatorname{CHSTPT} 5 \star 1, \operatorname{CHSTPT} 6 \star 1, \operatorname{CLADDESNAME}(10) \star 7$,

c SPACERMAT $\star 7$, STEPCONTROL $\star 1, \operatorname{ABOVEBP}(10) \star 5$

*

LOGICAL BPRA_INSERTED, CR_INSERTED, CRCOMPFLAG, APSR_INSERTED, C APSRCOMPFLA $\bar{G}$, BPRA_FOLLOW, APSRBOTFLAG, FOLLOWIN

$\star$

PI=3.14159265359

* Determination of the input deck filename

CALL ZEROS (CT3, CHNODE)

CALL ZEROS (CYCPOS (CT1), CHID)

NUMSTPT $1=$ INT (STPTDAT (CT I, CT 2,1$) / 100.0)$

CHSTPT $1=$ CHAR $($ NUMSTPT $1+48)$

NUMSTPT $2=$ INT $((\operatorname{STPTDAT}(\mathrm{CT} 1, \mathrm{CT} 2,1)-($ NUMSTPTI 1 100) $) / 10.0)$

CHSTPT $2=$ CHAR (NUMSTPT $2+48)$

NUMSTPT $3=$ INT $(($ STPTDAT $(C T 1$, CT 2,1$)-($ NUMSTPT $1 * 100)-$

C (NUMSTPT2*10))

CHSTPT $3=$ CHAR $($ NUMSTPT $3+48)$

IF (CT2.LT.STPTS (CT1)) THEN 


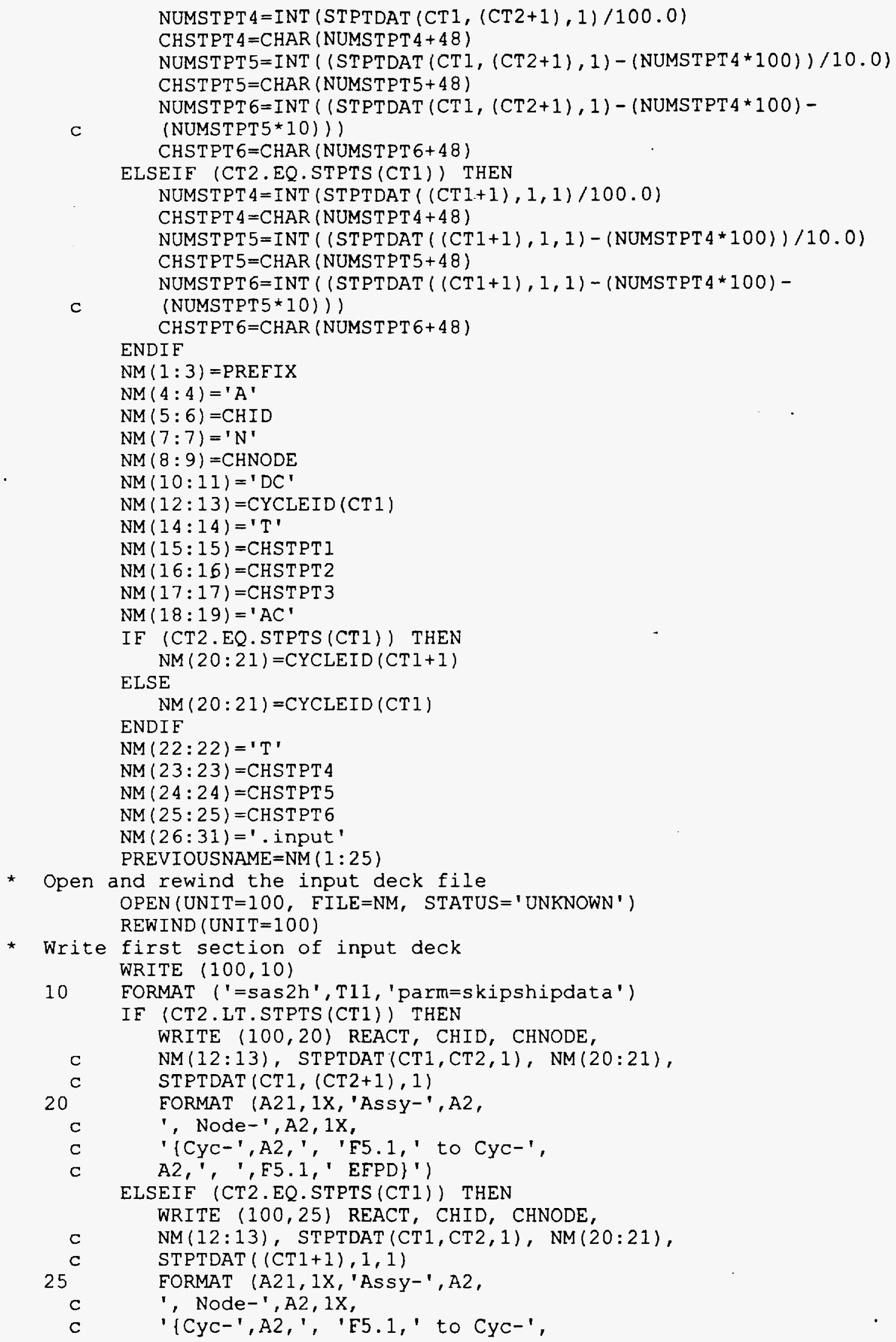




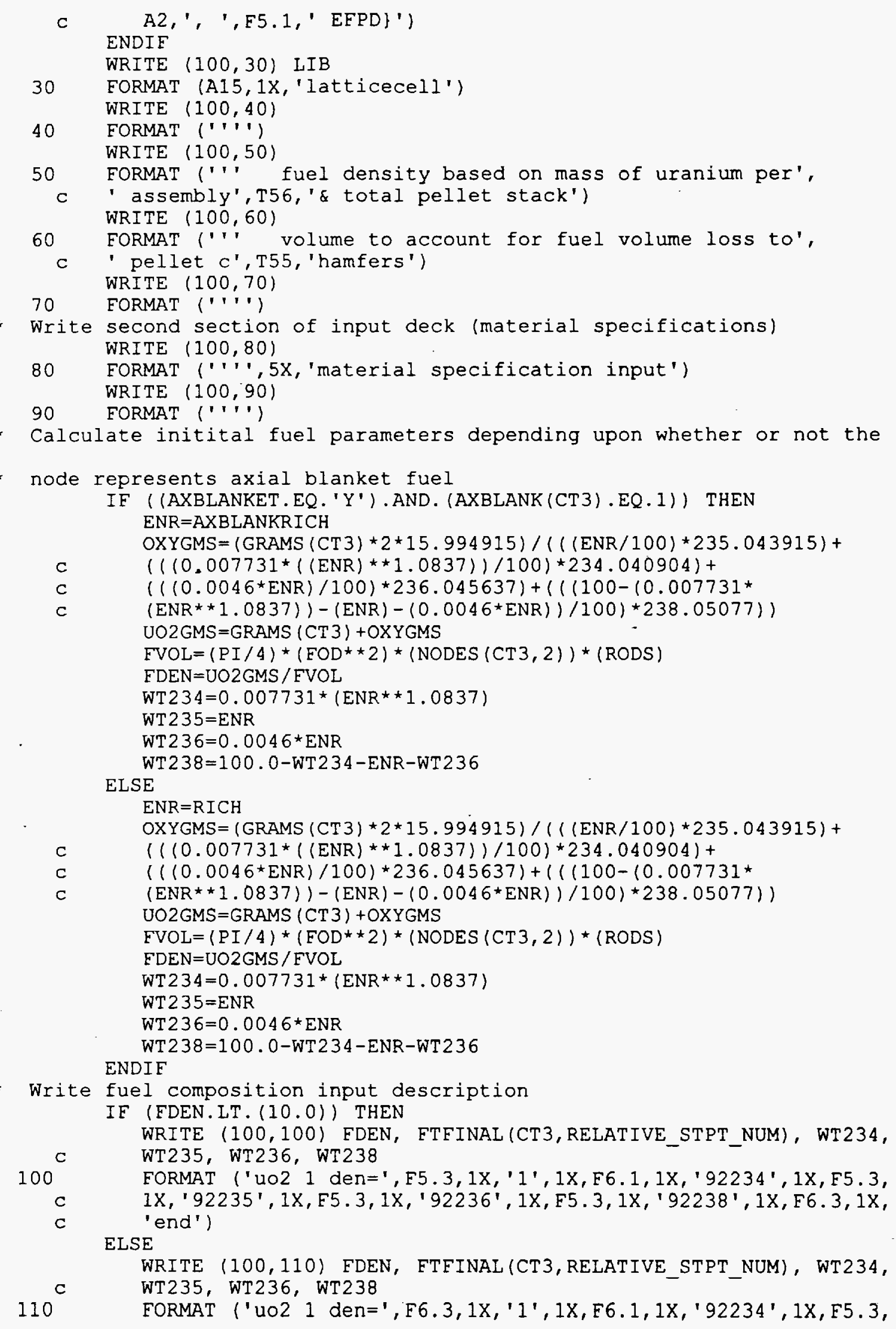




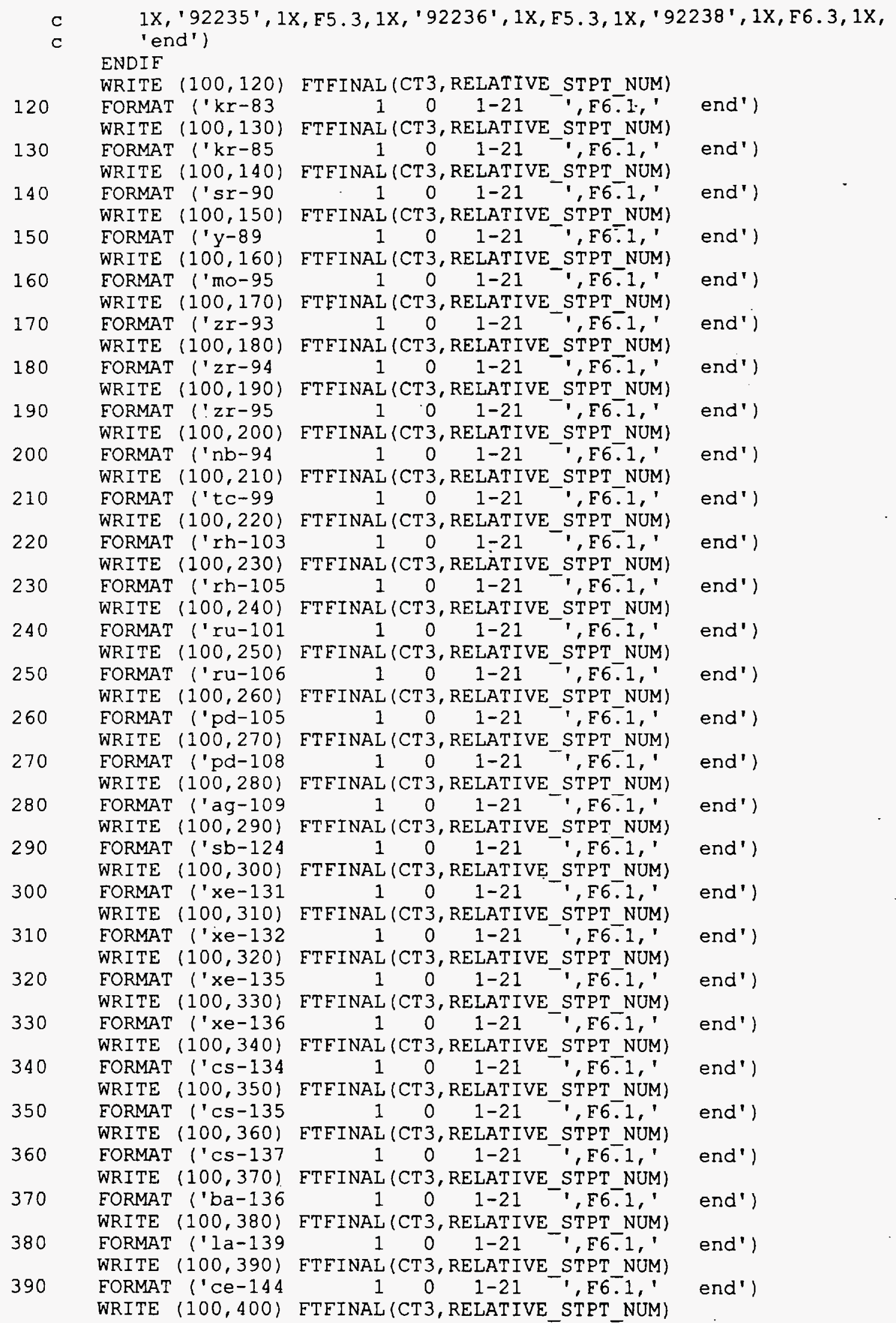


Title: CRC Depletion Calculations for the Rodded Assemblies in Batches 1, 2, 3, and $1 \mathrm{X}$ of Crystal River Unit 3 Document Identifier: BBA000000-01717-0200-00040 REV 00 Date Printed: 4/3/97 Attachment II, Page 28 of 82

557

559

* Write

560 WRITE $(100,560)$

560 FORMAT (''') ENDIF

' 1.02400017 .0250552 .01$)$

WRITE $(100,557)$

moderator material specifications

BORATEDMODVF $=1.0$-UCSPACERERAC

IF (STEPCONTROL.EQ. 'N') THEN

BORONVF=BLETDOWN $(\mathrm{CT} 1, \mathrm{CT} 2,3)$ * $(1 \mathrm{E}-6)$ * BORATEDMODVE

ELSEIF (STEPCONTROL.EQ. ' $Y$ ') THEN

BORONVF =VARBLETDOWN $(\mathrm{CT} 1, \mathrm{CT} 2,1,2) \star(1 E-6) \star$ BORATEDMODVE

C

IF ( (SPACERMAT.EQ.'ZIRC-4') . AND.

561

(UCSPACERFRAC.GT.(0.0))) THEN

WRITE $(100,561)$

FORMAT ('' material composition of moderator',

c ' within unit cell')

WRITE $(100,562)$

562

C

FORMAT ('' with smeared zirc-4 spacer grids') UCMODREGIONDEN $=($ MODDENFINAL (CT3, RELATIVE_STPT_NUM).

BORATEDMODVF $)+\left(6.56^{\star}\right.$ UCSPACERFRAC $)$

IF (MODDENEINAL (CT3, RELATIVE STPT NUM) .LT. (1.0)) THEN WRITE $(100,563)$ UCMODREGIONDEN, BORATEDMODVF,

C MODTEMPEINAL (CT3, RELATIVE STPT NUM)

563

564 ELSE FORMAT ('h20 3 den=', $\bar{E} 5.4, \overline{3}$, F6.5, 3X, F7.1,3X, 'end')

WRITE $(100,564)$ UCMODREGIONDEN, BORATEDMODVE, MODTEMPEINAL (CT3, RELATIVE STPT NUM)

ENDIF

FORMAT ('h2o 3 den $=$ ', $\overline{\mathrm{F}} 6.4, \overline{3} \mathrm{x}, \mathrm{F} 6.5,3 \mathrm{X}, \mathrm{F} 7.1,3 \mathrm{x}$, 'end')

WRITE $(100,565)$ UCMODREGIONDEN, BORONVF,

565

c

566

c

c

567

c

c

MODTEMPFINAL (CT 3, RELATIVE STPT NUM)

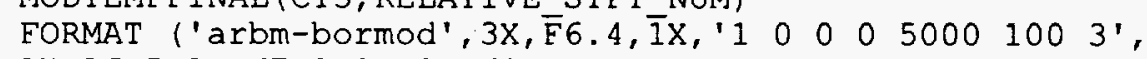

$1 \mathrm{X}, \mathrm{F} 6.5,1 \mathrm{X}, \mathrm{F} 7.1,1 \mathrm{X}$, 'end')

WRITE $(100,566)$ UCMODREGIONDEN

FORMAT ('arbm-spacer',3X,F6.4,1X,'50 0080160.12 ',

' $\left.240000.10260000 .25^{\prime}\right)$

WRITE $(100,567)$ UCSPACERERAC,

MODTEMPEINAL (CT 3, RELATIVE_STPT_NUM)

FORMAT (T17'50000 $1.4040 \overline{0} 009 \overline{8} .18$ 3', 1X, F6.5, 1X, F7.1, 1X, 'end')

ELSEIE ( (SPACERMAT.EQ. 'INCONEL') . AND.

(UCSPACERFRAC.GT. (0.0))) THEN

WRITE $(100,568)$

568

$\mathrm{C}$

FORMAT (' '

' within unit cell')

WRITE $(100,569)$

FORMAT ('" with smeared inconel spacer grids')

C BORATEDMODVF $)+(8.3 \star$ UCSPACERFRAC $)$

IF (MODDENEINAL (CT3, RELATIVE STPT NUM) .LT. (1.0)) THEN WRITE $(100,570)$ UCMODREGIONDEN, BORATEDMODVF,

C MODTEMPEINAL (CT3, RELATIVE STPT NUM)

570 ELSE FORMAT ('h20 3 den $=$ ', $\bar{F} 5.4, \overline{3} X, F 6: 5,3 \mathrm{X}, \mathrm{F} 7.1,3 \mathrm{X}$, 'end')

- WRITE $(100,571)$ UCMODREGIONDEN, BORATEDMODVF,

C MODTEMPEINAL (CT 3, RELATIVE STPT NUM) 
571

572

c ENDIF

FORMAT ('h20 3 den=', F6.4,3X,F6.5, 3X, F7.1, 3X, 'end') WRITE $(100,572)$ UCMODREGIONDEN, BORONVF, MODTEMPFINAL (CT 3, RELATIVE_STPT_NUM) FORMAT ('arbm-bormod', 3X, F $6.4, \overline{1 X}, ' 100005000100 \quad 3$ ', IX, F6.5, 1X, F7.1, IX, 'end' ' WRITE $(100,573)$ UCMODREGIONDEN
FORMAT ('arbm-spacer', 3X,F6.4,1X,'5 $0000014000 \quad 2.5$ ', - $\left.220002.52400015 .0^{\prime}\right)$ WRITE $(100,574)$ UCSPACERFRAC, 


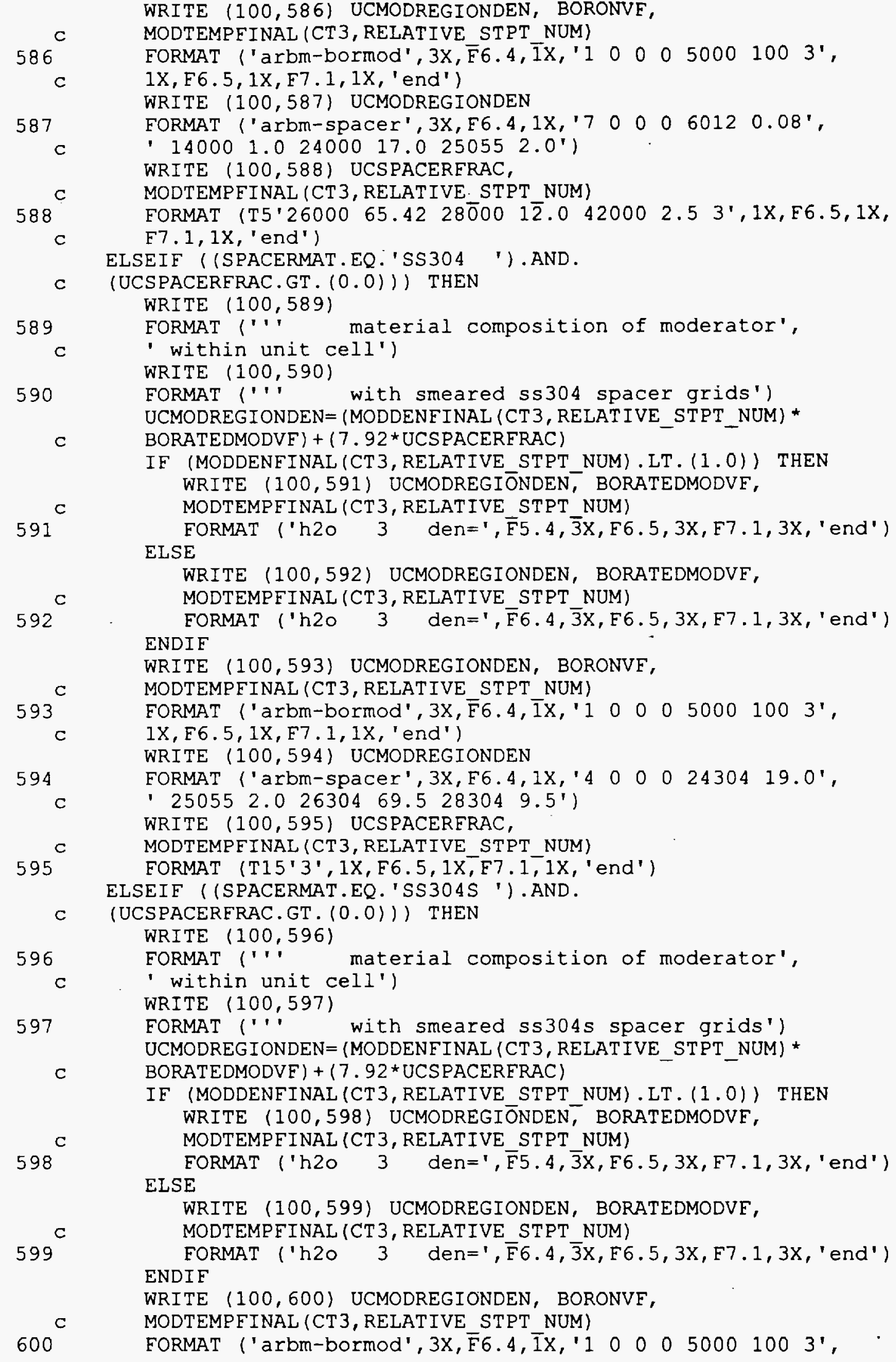




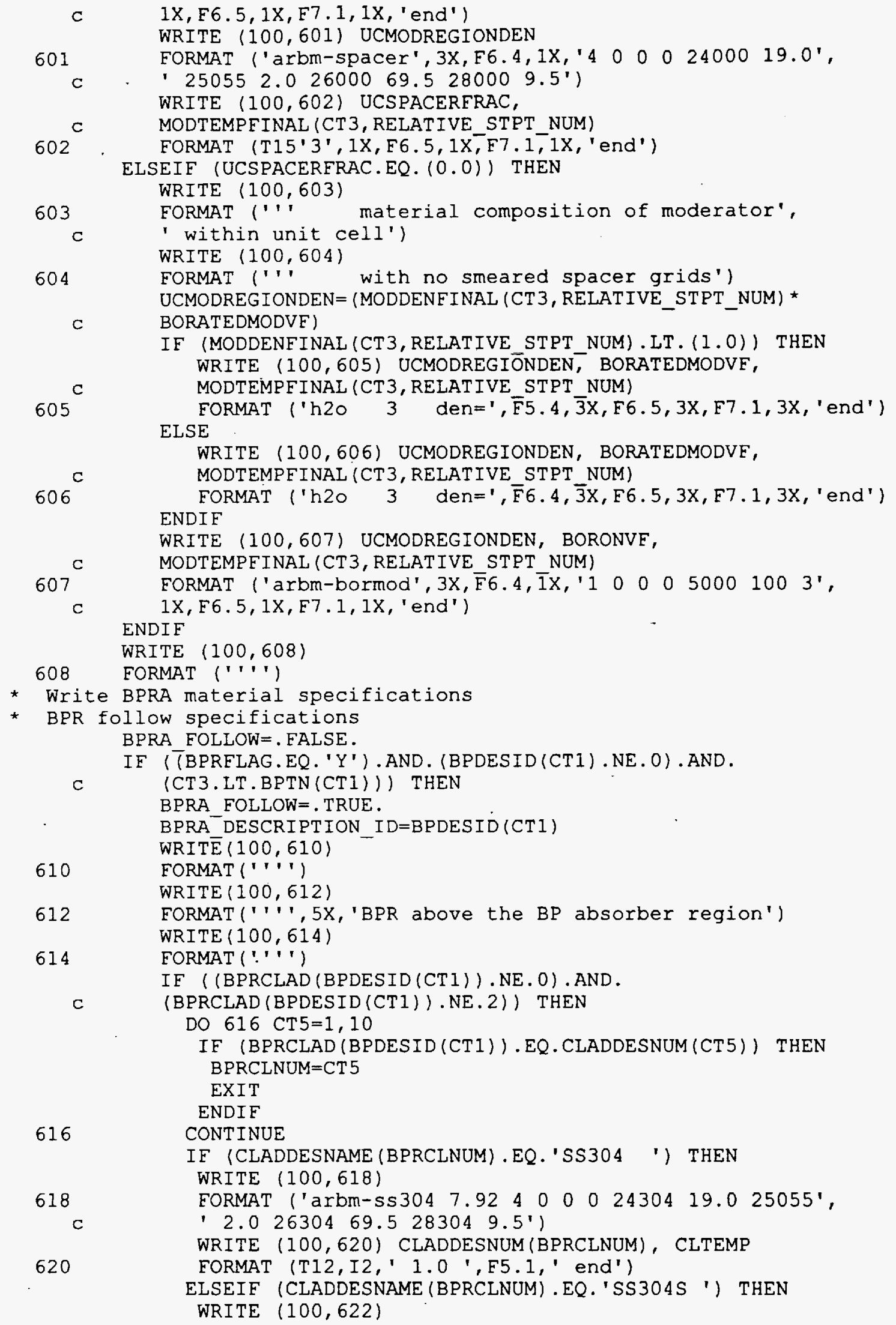




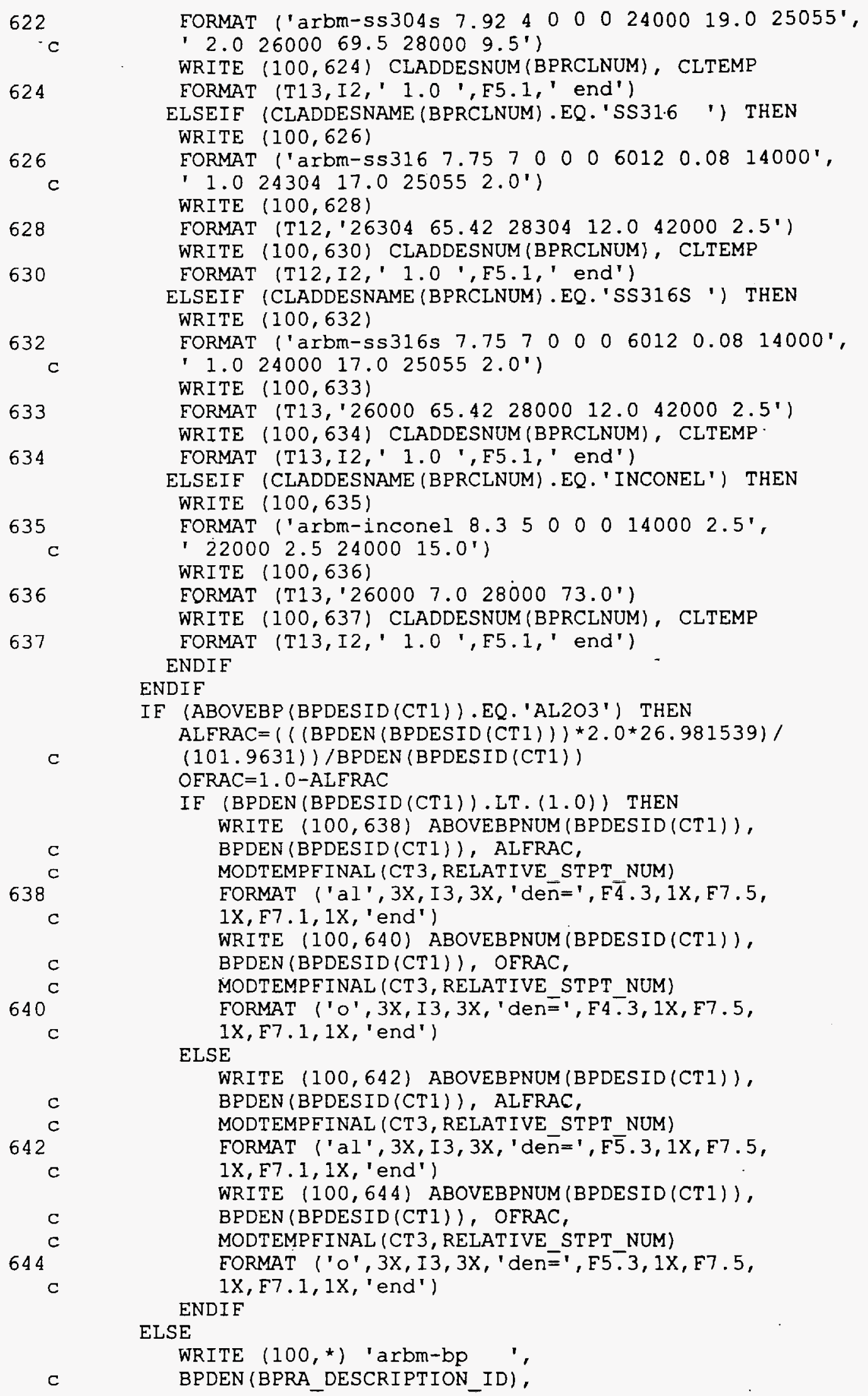

622

- $\mathrm{C}$

624

626

c

628

630

632

c

633

634

635

C

636

637

c

638

c

$\mathrm{C}$

640

c

C

642

c

c

$644^{c}$

c

C

FORMAT ('arbm-ss304s $7.9240002400019 .025055^{\prime}$, ' $\left.2.02600069 .5280009 .5^{\prime}\right)$

WRITE $(100,624)$ CLADDESNUM(BPRCLNUM), CLTEMP

FORMAT (T13,I2,' 1.0 ',F5.1,' end')

ELSEIF (CLADDESNAME (BPRCLNUM).EQ.'SS31.6 ') THEN WRITE $(100,626)$

FORMAT ('arbm-ss316 7.757000060120 .08 14000', ' $1.024304 \quad 17.0250552 .0^{\prime}$ ')

WRITE $(100,628)$

FORMAT (T12,'26304 65.42 $\left.28304 \quad 12.0 \quad 42000 \quad 2.5^{\prime}\right)$

WRITE $(100,630)$ CLADDESNUM(BPRCLNUM), CLTEMP

EORMAT (T12,I2,' 1.0 ',F5.1,' end')

ELSEIF (CLADDESNAME (BPRCLNUM) .EQ.'SS316S') THEN

WRITE $(100,632)$

FORMAT ('arbm-ss316s 7.75700000120 .0814000 ',

' $\left.1.02400017 .0250552 .0^{\prime}\right)$

WRITE $(100,633)$

FORMAT (T13,'26000 65.42 $\left.28000 \quad 12.0 \quad 42000 \quad 2.5^{\prime}\right)$

WRITE $(100,634)$ CLADDESNUM(BPRCLNUM), CLTEMP

FORMAT (T13,I2,' 1.0', F5.1,' end')

ELSEIF (CLADDESNAME (BPRCLNUM). EQ. 'INCONEL') THEN

WRITE $(100,635)$

FORMAT ('arbm-inconel 8.350000140002 .5 ',

' $\left.220002.52400015 .0^{\prime}\right)$

WRITE $(100,636)$

EORMAT (T13,'26000 7.0 28000 73.0')

WRITE $(100,637)$ CLADDESNUM(BPRCLNUM), CLTEMP

FORMAT (T13, I2,' 1.0 ',F5.1,' end') ENDIF

ENDIF

IF (ABOVEBP (BPDESID(CT1)).EQ. 'AL2O3') THEN

$A L E R A C=(((B P D E N(B P D E S I D(C T 1))) * 2.0 * 26.981539) /$

(101.9631))/BPDEN (BPDESID (CT1))

OFRAC $=1.0-A L F R A C$

IF (BPDEN(BPDESID(CT1)).LT. (1.0)) THEN

WRITE $(100,638)$ ABOVEBPNUM(BPDESID(CT1)),

BPDEN (BPDESID(CT1)), ALFRAC,

MODTEMPFINAL (CT 3, RELATIVE_STPT_NUM)

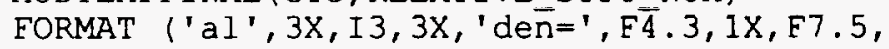

1X, F7.1, 1X, 'end')

WRITE $(100,640)$ ABOVEBPNUM(BPDESID(CT1)),

BPDEN (BPDESID (CT1)), OFRAC,

MODTEMPEINAL (CT 3, RELATIVE STPT NUM)

FORMAT ('O', 3X, I3, 3X, 'den $=$ ', F4.3, 1X, F7.5,

ELSE

IX, E7.1, IX, 'end')

WRITE $(100,642)$ ABOVEBPNUM(BPDESID(CT1)),

BPDEN (BPDESID (CT1)), ALFRAC,

MODTEMPEINAL (CT3, RELATIVE STPT NUM)

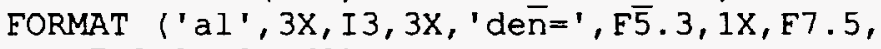

1X, F7.1, 1X, 'end' '

WRITE $(100,644)$ ABOVEBPNUM(BPDESID(CT1)),

BPDEN (BPDESID (CT1)), OFRAC,

MODTEMPEINAL (CT 3, RELATIVE_STPT_NUM) ENDIF

FORMAT ('o', $3 \mathrm{X}, \mathrm{I3}, 3 \mathrm{X}, \mathrm{\prime}^{\mathrm{d}} \mathrm{den}=\mathrm{\prime}, \mathrm{F5} .3,1 \mathrm{X}, \mathrm{F} 7.5$, $1 \mathrm{X}, \mathrm{F} 7.1,1 \mathrm{X}$, 'end')

ELSE

WRITE $\left(100,{ }^{\star}\right)$ 'arbm-bp ',

BPDEN (BPRA_DESCRIPTION_ID), 


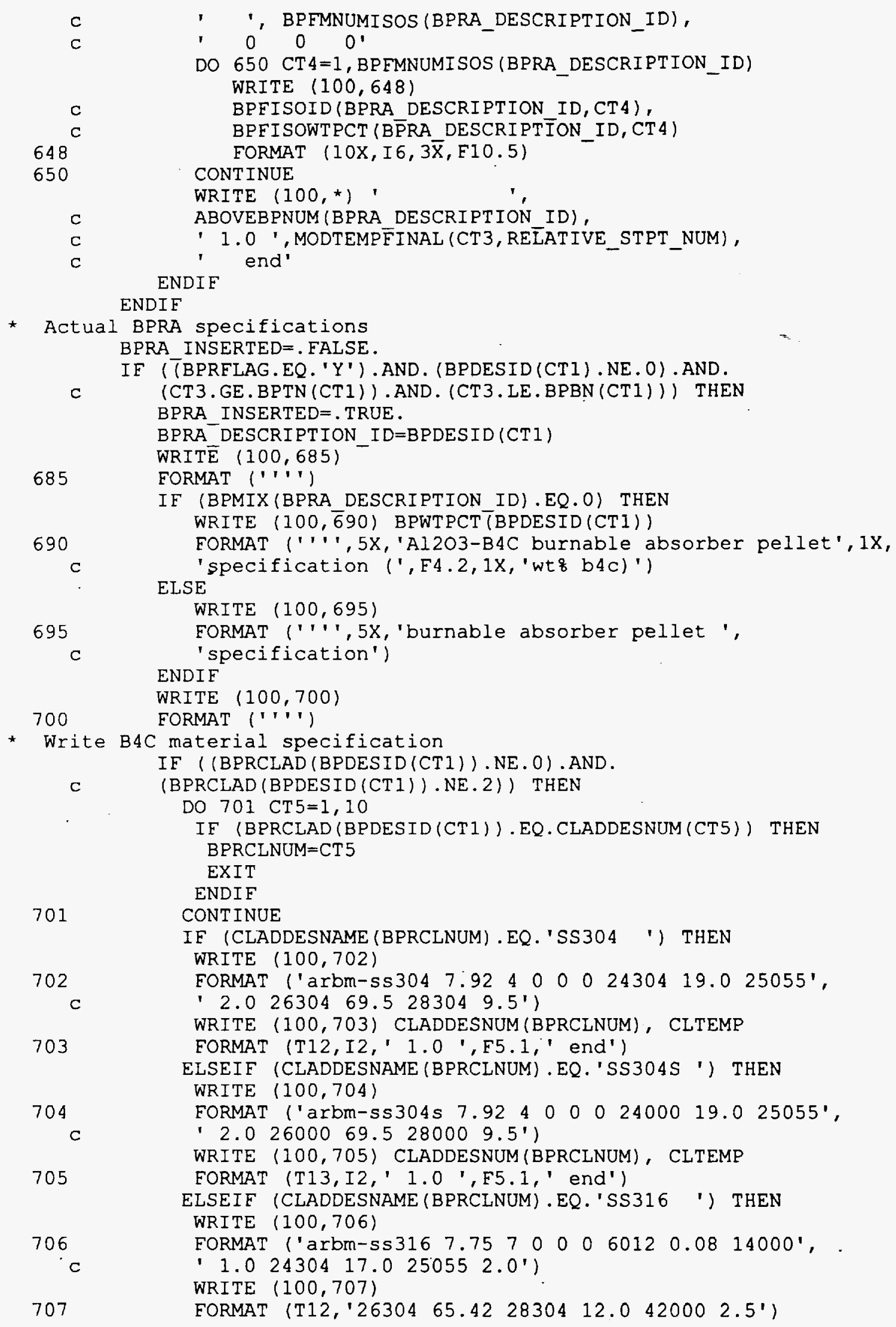




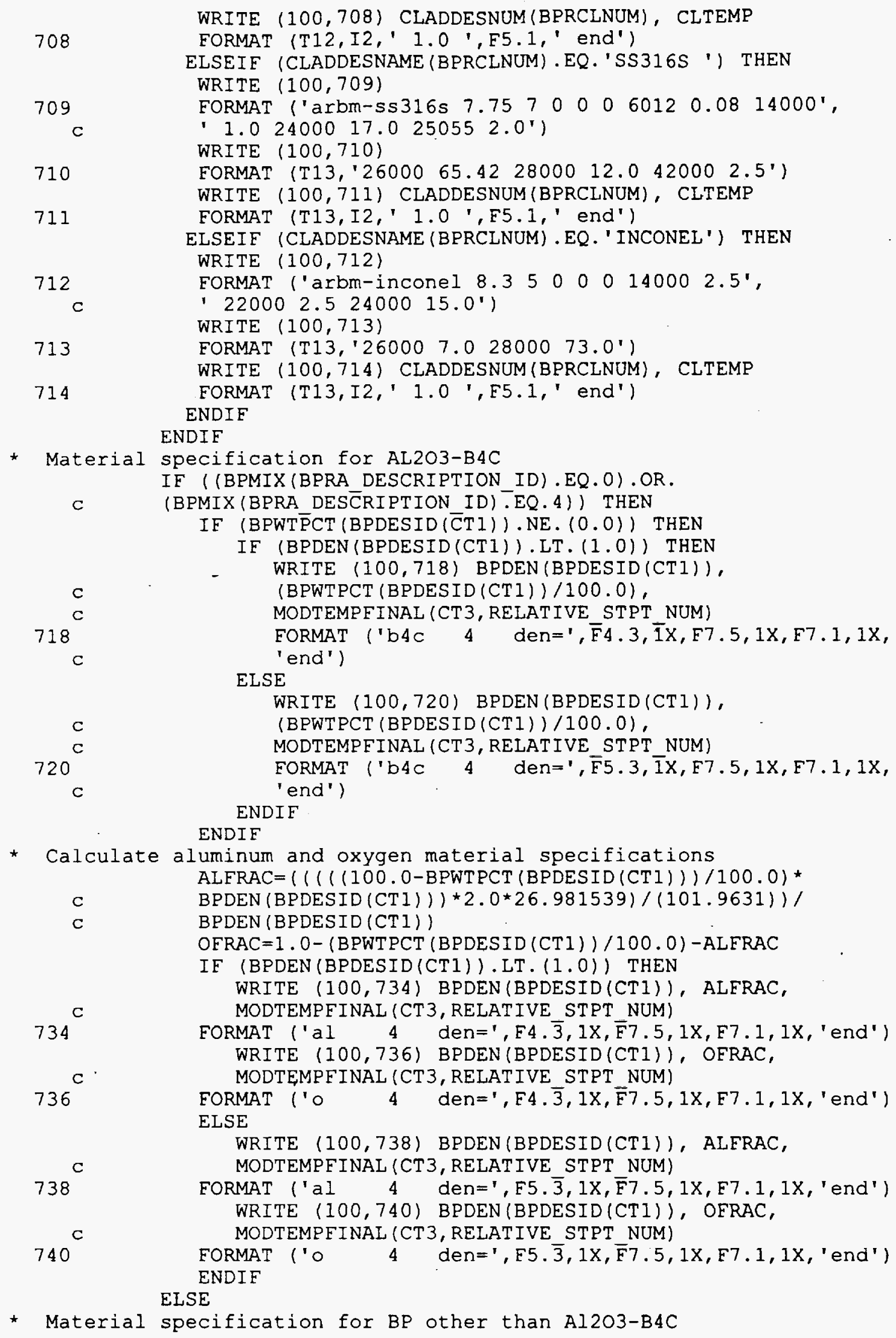

* Material specification for BP other than Al203-B4C 
742

c

C

C

745

750

C

ENDIF ENDIF

DO 742 CT $4=1$, BPMIXNUM

IF (BPMIXID(CT 4) .EQ.BPMIX(BPRA_DESCRIPTION_ID)) THEN ENDIF RELATIVE_BP_MIX_ID=CT 4

CONT INUE

WRITE $\left(100,{ }^{*}\right)$ 'arbm-bp ', BPDEN (BPRA_DESCRIPTION_ID),

' ', BPNUMISOS (RELATIVE_BP_MIX_ID),

$\begin{array}{lll}0 & 0 & 0 \\ 0\end{array}$

DO 750 CT $4=1$, BPNUMISOS (RELATIVE BP MIX_ID)

WRITE $(100,745)$ BPISOID(RELATIVE_BP_MIX_ID,CT4),

BPISOWTPCT (RELATIVE_BP_MIX_ID, CT $\overline{4}$ )

EORMAT (1OX, I $6,3 \mathrm{X}, \mathrm{F} \overline{1} 0 . \overline{5})$

CONTINUE

WRITE $\left(100,{ }^{\star}\right)$ ' ', BPMIX (BPRA_DESCRIPTION_ID), ' 1.0 ', MODTEMPFINAL (CT 3, RELATIVE_STPT_NUM), ' eñd'

control rod material specification

CR INSERTED $=$. FALSE.

$I^{-}$(CRSTAT.EQ.'RODDED') THEN

CRCOMPELAG $=$. FALSE.

DO 760 CT $4=1,23$

IF (CRINS(CT1, CT2, CT 4,CT3).NE.0) THEN

CRCOMPFLAG $=$. TRUE.

CR INSERTED $=$. TRUE.

- CR_MIXTURE_ID=CRINS (CT1, CT2, CT 4, CT 3)

CR DESCRIPT ION=CRDES (CT $1, \mathrm{CT} 2, \mathrm{CT} 4, \mathrm{CT} 3)$ EXIT

ENDIF

$760 \quad$ CONTINUE

IF (CRCOMPFLAG. EQ. . TRUE.) THEN

DO 770 CT $4=1$, CRMIXNUM

IF (CRMIXID (CT 4).EQ.CR MIXTURE_ID) THEN ENDIF

RELATIVE CR MIX ID $=\bar{C}$ T 4

CONTINUE

WRITE $(100,780)$

780

790

800

FORMAT (',')

WRITE $(100,790)$

FORMAT (''', T5,' control rod material specification') WRITE $(100,800)$

FORMAT (''')

IF (CRCLAD (CR DESCRIPTION). NE. O) THEN

DO $801 \mathrm{CT} 5=\overline{1}, 10$

IF (CRCLAD(CR DESCRIPTION) . EQ.CLADDESNUM(CT5)) THEN

CRCLNUM $=$ CT 5

EXIT

ENDIF

801 CONTINUE

IF (CLADDESNAME (CRCLNUM) .EQ.'SS304 ') THEN WRITE $(100,802)$

802

803

804

c

FORMAT ('arbm-ss304 $7.9244000024304 \quad 19.025055$ ',

'2.0 $\left.26304 \quad 69.5 \quad 28304 \quad 9.5^{\prime}\right)$

WRITE $(100,803)$ CLADDESNUM(CRCLNUM), CLTEMP

FORMAT (T12, I2,' 1.0 ', F5.1,' end')

ELSEIF (CLADDESNAME (CRCLNUM).EQ.'SS304S ') THEN

WRITE $(100,804)$

FORMAT ('arbm-ss304s 7.924400002400019 .025055 ',

'2.0 $\left.2600069.528000 \quad 9.5^{\prime}\right)$

WRITE $(100,805)$ CLADDESNUUM(CRCLNUM), CLTEMP 
805

806

C

807

808

809

C

810

811

812

c

813

814

C

$815^{\mathrm{C}}$

820

C ENDIF ENDIE ENDIF

FORMAT (T13, I2,' 1.0 ',F5.1,' end')

ELSEIF (CLADDESNAME (CRCLNUM).EQ.'SS316 ') THEN

WRITE $(100,806)$

FORMAT ('arbm-ss316 $7.75 \quad 7 \quad 0 \quad 0 \quad 060120.0814000$ ',

' $1.024304 \quad 17.0250552 .0$ ')

WRITE $(100,807)$

FORMAT (T12,'26304 $65.42 \quad 28304 \quad 12.0 \quad 42000 \quad 2.5$ ')

WRITE $(100,808)$ CLADDESNUM(CRCLNUM), CLTEMP

FORMAT (T12, I2,' 1.0 ', F5.1,' end')

ELSEIF (CLADDESNAME (CRCLNUM).EQ.'SS316S ') THEN

WRITE $(100,809)$

FORMAT ('arbm-ss316s $7.75 \quad 7 \quad 0 \quad 0 \quad 0 \quad 60120.08 \quad 14000$ ',

'1.0 $\left.2400017.0250552 .0^{\prime \prime}\right)$

WRITE $(100,810)$

FORMAT (T13,'26000 $\left.65.42 \quad 28000 \quad 12.0 \quad 42000 \quad 2.5^{\prime}\right)$

WRITE $(100,811)$ CLADDESNUM(CRCLNUM), CLTEMP

FORMAT (T13, I2,' 1.0 ', F5.1,' end')

ELSEIF (CLADDESNAME (CRCLNUM) .EQ. 'INCONEL') THEN

WRITE $(100,812)$

FORMAT ('arbm-inconel $8.350000140002 .5^{\prime}$,

' 220002.524000 15.0')

WRITE $(100,813)$

FORMAT (T13,'26000 7.02800073 .0 ')

WRITE $(100,814)$ CLADDESNUM(CRCLNUM), CLTEMP

FORMAT (T13,I2,' 1.0 ', F5.1,' end')

ENDIF

ENDIF

WRITE $(100, *)$ 'arbm-cr ', CRDEN(CR DESCRIPTION),

'DO 820 CT $4=1$, CRNUMISOS (RELATIVE_CR_MIX_ID)

WRITE $(100,815)$ CRISOID (RELATIVE $\bar{E}$ CR_MIX_ID, CT4), CRISOWTPCT (RELATIVE_CR_MIX_ID，CT4)

FORMAT $(10 \mathrm{X}, \mathrm{I} 5,3 \mathrm{X}, \mathrm{F} \overline{1} \mathrm{O} . \overline{5})$

CONTINUE

WRITE $\left(100,{ }^{*}\right) '$ ' CR_MIXTURE_ID, ' 1.0 ',
MODTEMPFINAL (CT3, RELATIVE_STPT_NUM), '- end'

* Write APSR material specification

IF ((CTI.EQ.CTISTART). AND. (CT2 EQ.CT2GOVALUE) . AND.

c

(CT 3.EQ.1)) THEN

DO 824 CT $4=1,10$

DO 823 CT $5=1,20$

DO 822 СТ $6=1,23$

DO 821 CT $7=1,50$

APSRINSOLD $(\mathrm{CT} 4, \mathrm{CT} 5, \mathrm{CT} 6, \mathrm{CT} 7)=$

C

821

822

823

824

C
APSRINS (CT $4, \mathrm{CT} 5, \mathrm{CT} 6, \mathrm{CT} 7)$ CONTINUE

CONTINUE

CONTINUE

CONTINUE

ENDIF

APSR INSERTED $=$, FALSE.

IF ( $\bar{A} P S R S T A T . E Q$. 'RODDED') THEN

DO 830 CT $4=1,23$

APSRBOT $F L A G=$. FALSE.

DO 825 CT $5=50,1,-1$

IF ((APSRINSOLD (CT1, CT2, CT 4, CT5). NE. 0). AND.

(APSRBOTELAG.EQ...FALSE.)) THEN

APSR_DESCRIPTION=APSRDES (CT $1, \mathrm{CT} 2, \mathrm{CT} 4, \mathrm{CT} 5)$ 


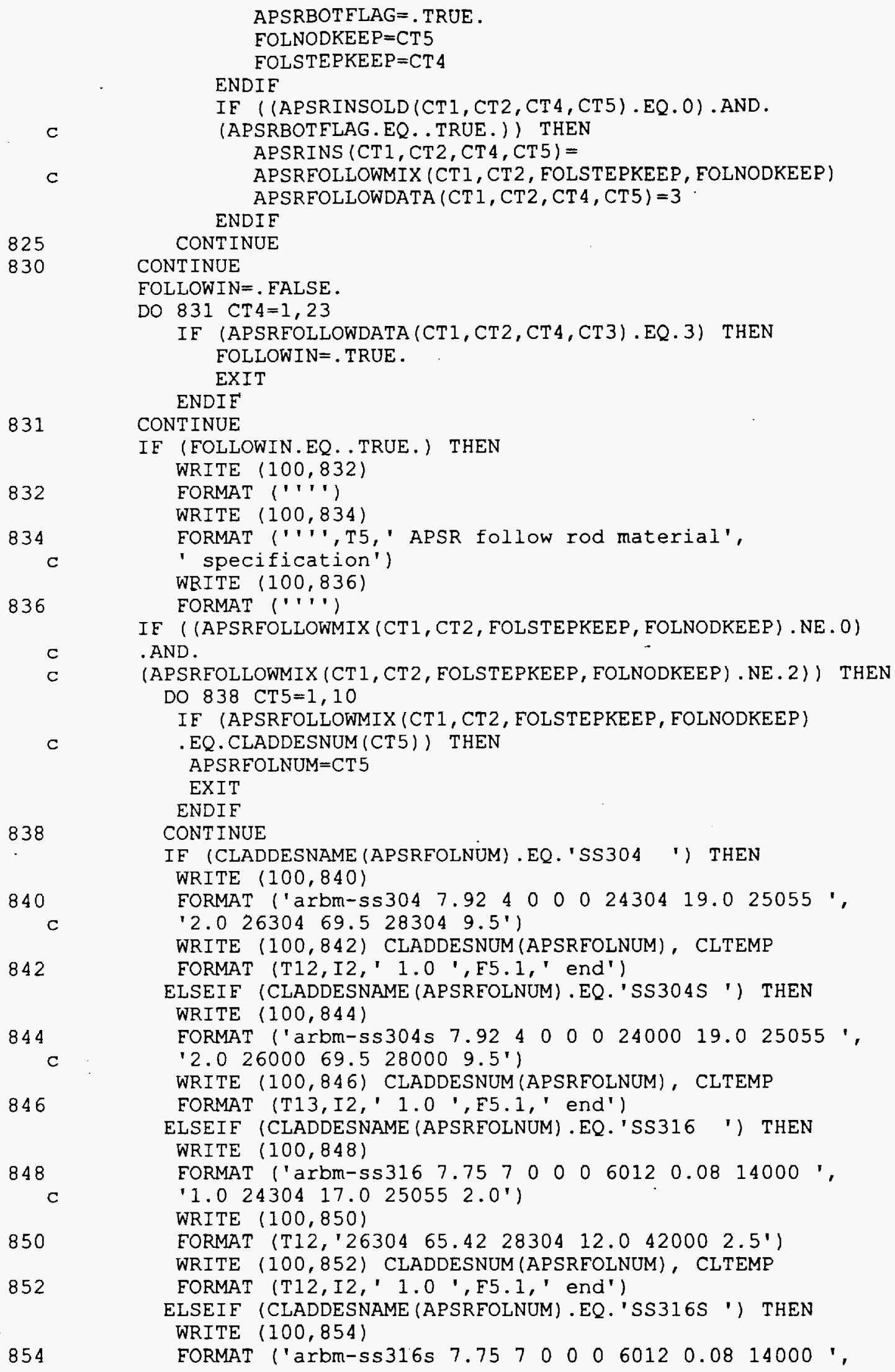


856

858

860

$\mathrm{C}$

862

864

c

c

865

866

868

870

C

880

881

882

$\mathrm{C}$

883

884 C
1.02400017 .0250552 .0 ')

WRITE $(100,856)$

FORMAT (T13,'26000 $65.42 \quad 28000 \quad 12.0 \quad 42000 \quad 2.5$ ')

WRITE $(100,858)$ CLADDESNUM(APSRFOLNUM), CLTEMP

FORMAT (T13,I2,' 1.0 ', F5.1,' end')

ELSEIF (CLADDESNAME (APSRFOLNUM).EQ. 'INCONEL') THEN

WRITE $(100,860)$

FORMAT ('arbm-inconel 8.3 50000140002.5 ',

' 220002.52400015 .0 ')

WRITE $(100,862)$

FORMAT (T13,'26000 7.0 2800073.01$)^{\prime}$

WRITE $(100,864)$ CLADDESNUM(APSRFOLNUM), CLTEMP

FORMAT (T13,I2,' 1.0 ', F5.1,' end')

ENDIF

ENDIF

ENDIF

APSRCOMPFLAG = . FALSE. .

DO 865 CT $4=1,23$

IF. ( (APSRINS (CT1, CT2, CT 4, CT 3) . NE. 0) . AND.

(APSRINS (CT1, CT2, CT 4, CT 3 ). NE.

APSRFOLLOWMIX (CT 1, CT2, FOLSTEPKEEP, FOLNODKEEP))) THEN

APSRCOMPFLAG $=$. TRUE.

APSR INSERTED $=$. TRUE.

APSR MIXTURE ID=APSRINS (CT $1, \mathrm{CT} 2, \mathrm{CT} 4, \mathrm{CT} 3$ )

APSR_DESCRIPTION=APSRDES (CT 1, CT 2, CT 4, CT 3)

EXIT

EXIT

CONTINUE

IF (APSRCOMPELAG.EQ. TRUE.) THEN

DO 866 CT $4=1$, APSRMIXNUM

IF (APSRMIXID (CT 4).EQ.APSR_MIXTURE_ID) THEN ENDIF

CONTINUE

WRITE $(100,868)$

FORMAT (' ' ' ')

WRITE $(100,870)$

FORMAT (''', T5,' axial power shaping rod material',

' specification')

WRITE $(100,880)$

FORMAT (' '')

IF (APSRCLAD (APSR_DESCRIPTION).NE.0) THEN

DO 881 CT $5=1,10^{-}$

IF (APSRCLAD (APSR_DESCRIPTION) .EQ.CLADDESNUM(CT5)) THEN

APSRCLNUM $=$ CT 5

EXIT

ENDIF

CONTINUE

IF (CLADDESNAME (APSRCLNUM) .EQ.'SS304 ') THEN

WRITE $(100,882)$

FORMAT ('arbm-ss304 7.92400002430419 .025055 ',

'2.0 $\left.26304 \quad 69.528304 \quad 9.5^{\prime}\right)$

WRITE $(100,883)$ CLADDESNUM(APSRCLNUM), CLTEMP

FORMAT (T12, I2,' 1.0 ', F5.1,' end')

ELSEIF (CLADDESNAME (APSRCLNUM).EQ.'SS304S ') THEN

WRITE $(100,884)$

FORMAT ('arbm-ss304s 7.92400002400019 .025055 ', '2.0 2600069.5280009 .5 ')

WRITE $(100,885)$ CLADDESNUM(APSRCLNUM), CLTEMP

885

FORMAT (T13,I2,' 1.0 ',F5.1,' end') 
886

c

887

888

889

c

890

891

892

C

893

894

c

c

895

900

c ENDIF ENDIF

ELSEIF (CLADDESNAME (APSRCLNUM).EQ.'SS316 ') THEN

WRITE $(100,886)$

FORMAT ('arbm-ss316 $7.75 \quad 7 \quad 0 \quad 0 \quad 06012 \quad 0.08 \quad 14000$ ',

' $1.024304 \quad 17.0250552 .0^{\prime \prime}$ )

WRITE $(100,887)$

FORMAT (T12,'26304 $\left.65.42 \quad 28304 \quad 12.042000 \quad 2.5^{\prime}\right)$

WRITE $(100,888)$ CLADDESNUM(APSRCLNUM), CLTEMP

FORMAT (T12, I2,' 1.0 ',F5.1,' end')

ELSEIF (CLADDESNAME (APSRCLNUM). EQ.'SS316S') THEN

WRITE $(100,889)$

FORMAT ('arbm-ss316s 7.757000060120 .0814000 ',

' $\left.1.02400017 .0250552 .0^{\prime}\right)$

WRITE $(100,890)$

FORMAT $\left(T 13, ' 26000 \quad 65.42 \quad 28000 \quad 12.0 \quad 42000 \quad 2.5^{\prime}\right)$

WRITE $(100,891)$ CLADDESNUM(APSRCLNUM), CLTEMP

FORMAT (T13,I2,' 1.0 ', F5.1,' end')

ELSEIF (CLADDESNAME (APSRCLNUM) . EQ. 'INCONEL') THEN

WRITE $(100,892)$

FORMAT ('arbm-inconel 8.350000140002 .5 ';

' $\left.220002.52400015 .0^{\prime}\right)$

WRITE $(100,893)$

FORMAT (T13,'26000 7.0 $\left.2800073.0^{\prime}\right)$

WRITE $(100,894)$ CLADDESNUM (APSRCLNUM), CLTEMP

FORMAT (T13, I2,' 1.0 ', E5.1,' end') ENDIE

ENDIE

WRITE $(100, *)$ 'arbm-apsr ', APSRDEN (APSR_DESCRIPTION);

DO 900 CT $4=1$, APSRNUMISOS (RELATIVE APSR_MIX_ID)

WRITE $(100,895)$ APSRISOID (RELATIVE_APSR_MIX_ID, CT4),

APSRISOWTPCT (RELATIVE_APSR_MIX_ID, CT 4 )

FORMAT (10X, I5, 3X, F10.5)

CONT INUE

WRITE $(100,)^{*}$ ' ', APSR MIXTURE_ID, ' 1.0 ', MODTEMPFINAL (CT3, RELATIVE_STPT_NŪM), ' - end'

* Write fuel rod fill gas material specification

WRITE $(100,910)$

910 FORMAT (''')

WRITE $(100,920)$

920 FORMAT ('he 5 end')

WRITE $(100,930)$

930 FORMAT ('end comp')

* Write base reactor lattice specifications

WRITE $(100,940)$

940 FORMAT ( ' ' ' )

WRITE $(100,950)$

950 FORMAT (''' base reactor lattice specification')

WRITE $(100,960)$

960 FORMAT ( $\cdots)$

WRITE $(100,970)$ PITCH, FOD, COD, CID

970 FORMAT ('squarepitch', 3X, F7.5, 3X,F6.4, 3X, '1 3', 3X, F6.4, c $3 \mathrm{X},{ }^{\prime} 2 ', 3 \mathrm{X}, \mathrm{F} 6.4,3 \mathrm{x}, \mathrm{\prime}^{\prime} \mathrm{O}$ end')

* The following writing routine for 'SPECIAL' input data

* has not been formatted to compensate for FORTRAN's ingenious

* incapability to print leading zeros in numeric fields.

* Errors will occur in the FIDO input if null space exists

* between an equal sign and the appropriate value. Therefore,

* the IIM and ICM factors must always be at least 10 . 


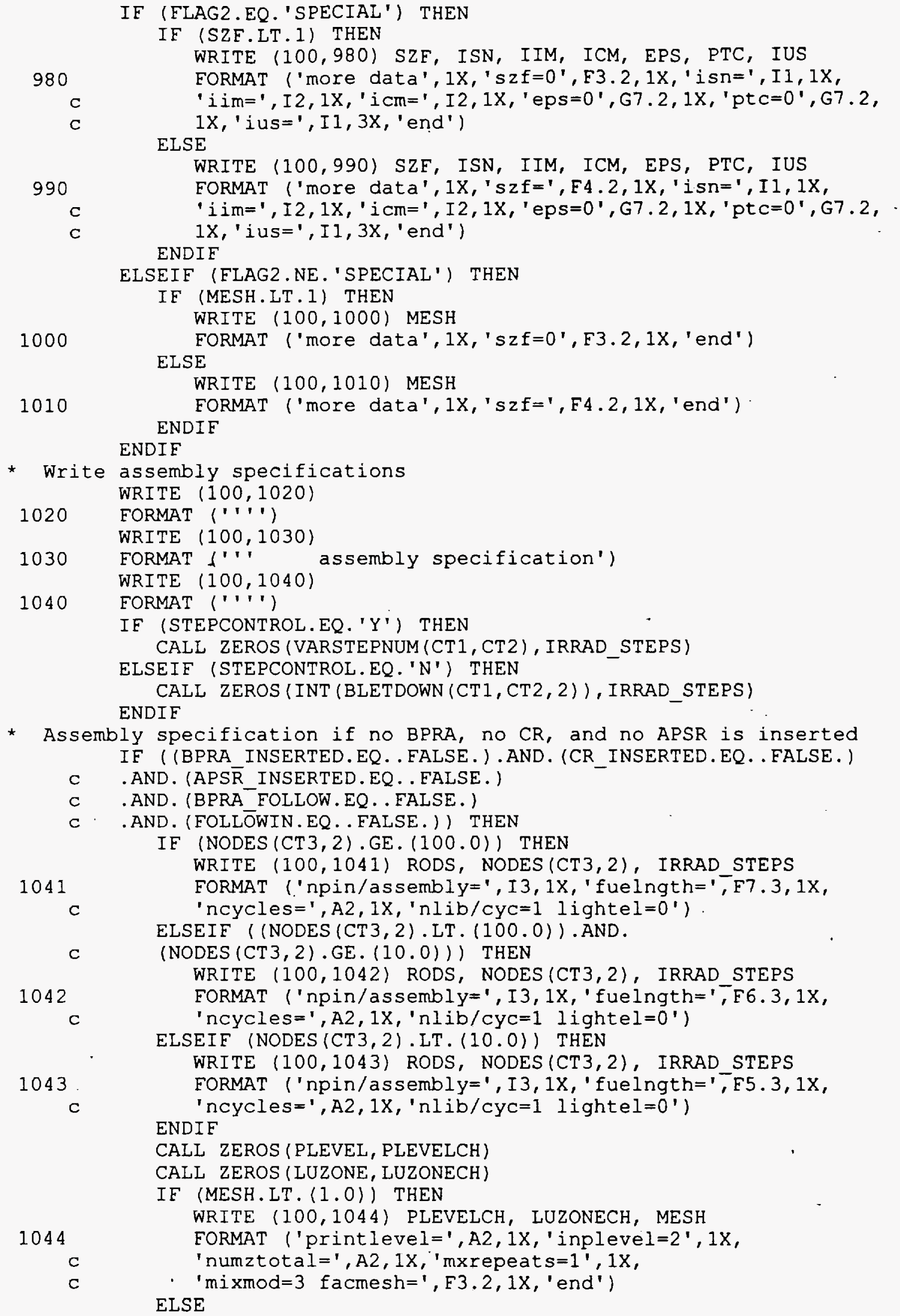




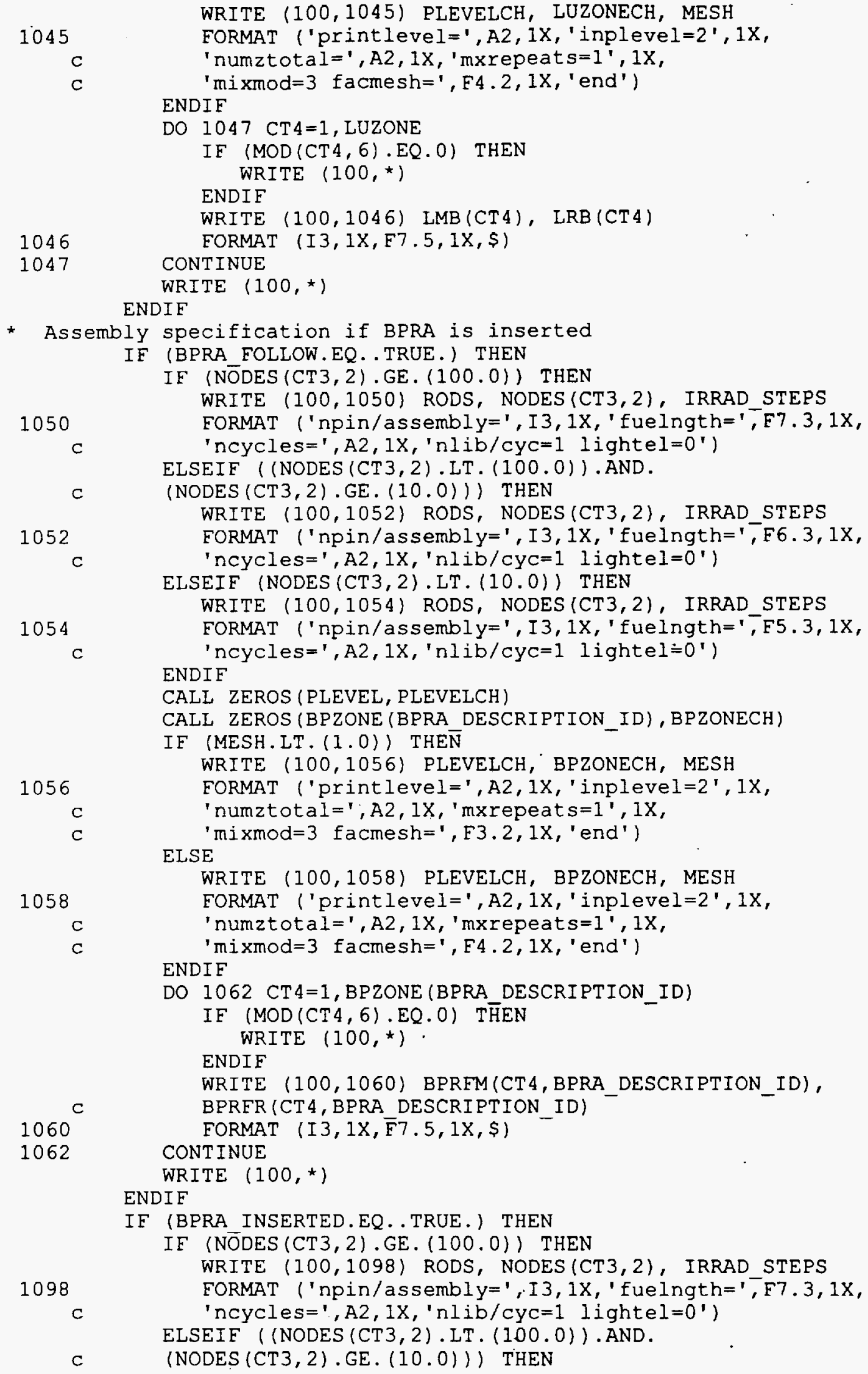




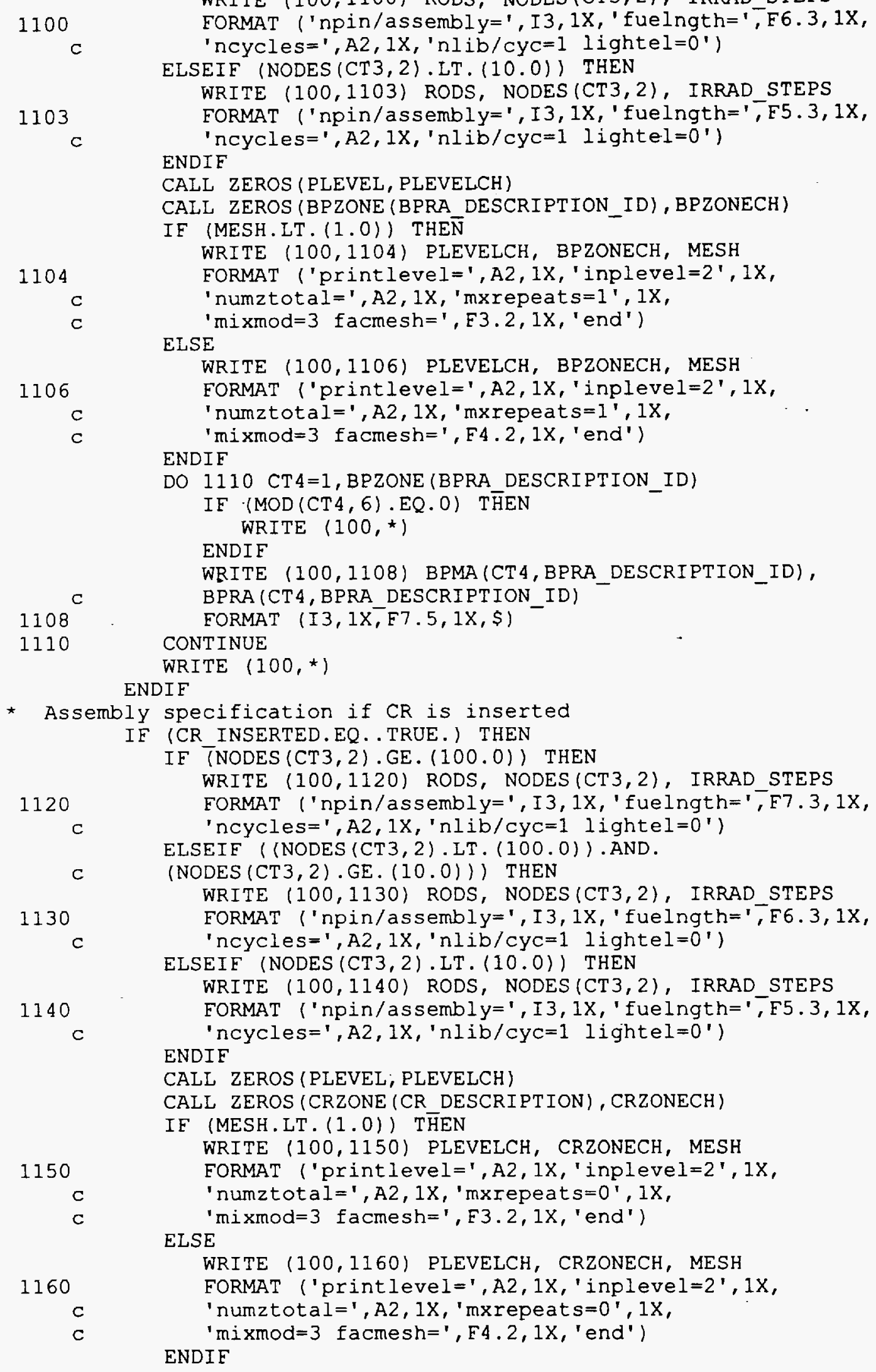


IF (STEPCONTROL.EQ.' 'N') THEN

DO 1169 CT 4=1, INT (BLETDOWN (CT1, CT2, 2))

IF (CRINS (CT1, CT2, CT 4, CT3). NE. 0) THEN

DO 1164 CT5 $=1$, CRZONE (CR_DESCRIPTION)

IE (MOD $(C T 5,6), E Q .0)$ THEN

1169 CONTINUE

ELSEIF (STEPCONTROL.EQ. 'Y') THEN

DO $1210 \mathrm{CT} 4=1$, VARSTEPNUM (CT 1, CT2)

IF (CRINS (CT1, CT2, CT 4, CT3). NE. 0) THEN

DO 1180 CT5=1, CRZONE (CR_DESCRIPTION)

IE (MOD $(\mathrm{CT} 5,6) . \mathrm{EQ} .0)^{-T H E N}$

ENDIF

WRITE $(100, *)$

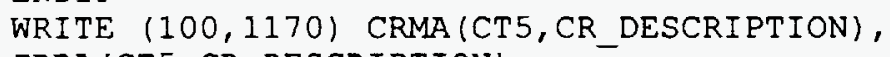

* Assembly specification if APSR is inserted

IF ((APSR INSERTED.EQ..TRUE.).OR. (FOLLOWIN.EQ. . TRUE.)) THEN

IF (NODES $(C T 3,2) . G E .(100.0))$ THEN

WRITE $(100,1220)$ RODS, NODES $(C T 3,2)$, IRRAD_STEPS

1220 FORMAT ('npin/assembly=', I3, 1X, 'fuelngth=', F7.3, 1X, 'ncycles=', A2, 1X, 'nlib/cyc=1 lightel=0') ELSEIE ((NODES (CT 3,2) . LT . (100.0)). AND.

C (NODES $(\mathrm{CT} 3,2) . \mathrm{GE} .(10.0))$ THEN WRITE $(100,1230)$ RODS, NODES $(C T 3,2)$, IRRAD_STEPS

1230 FORMAT ('npin/assembly=', I3,1X,' fuelngth=', F6.3, 1X, 


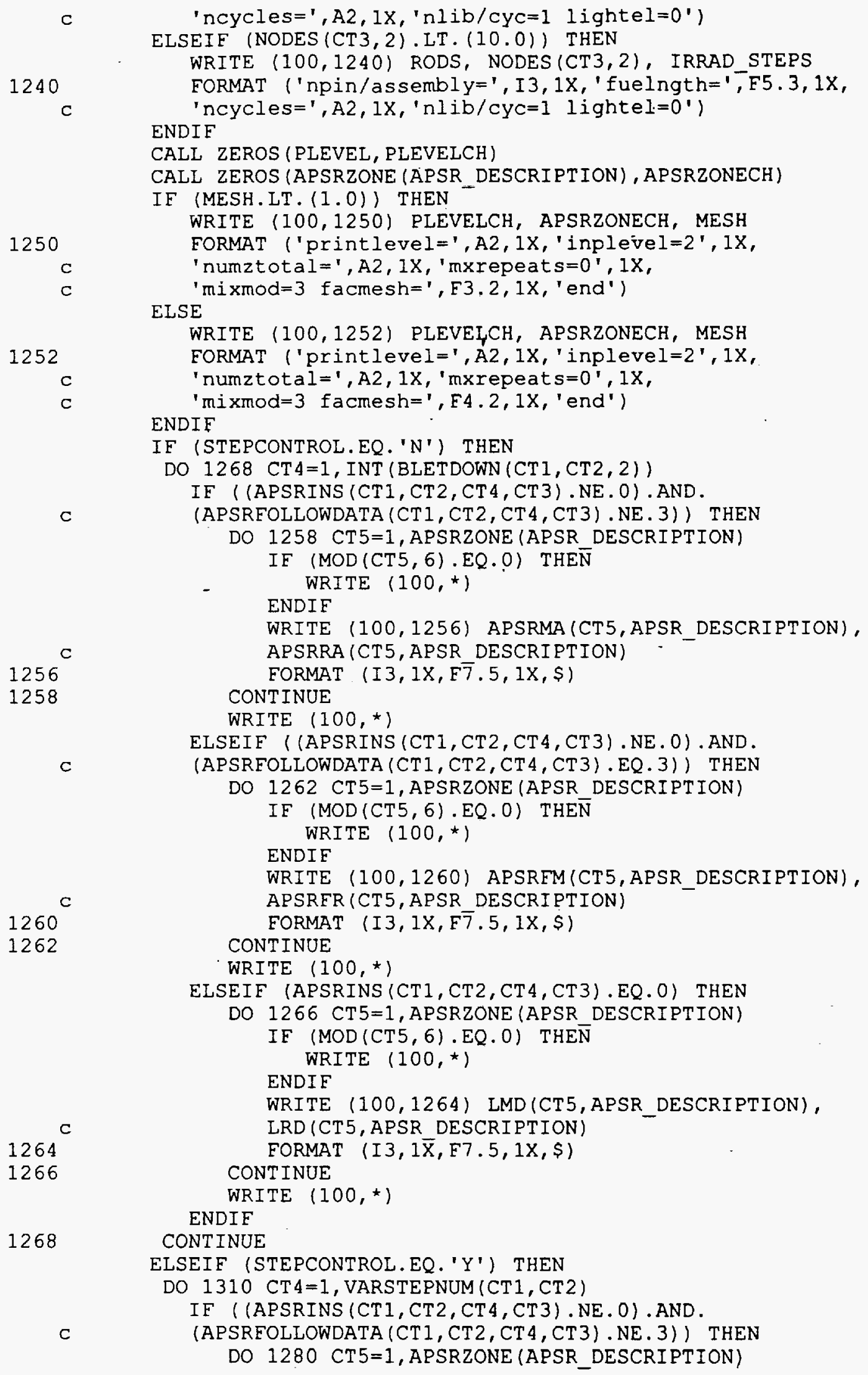




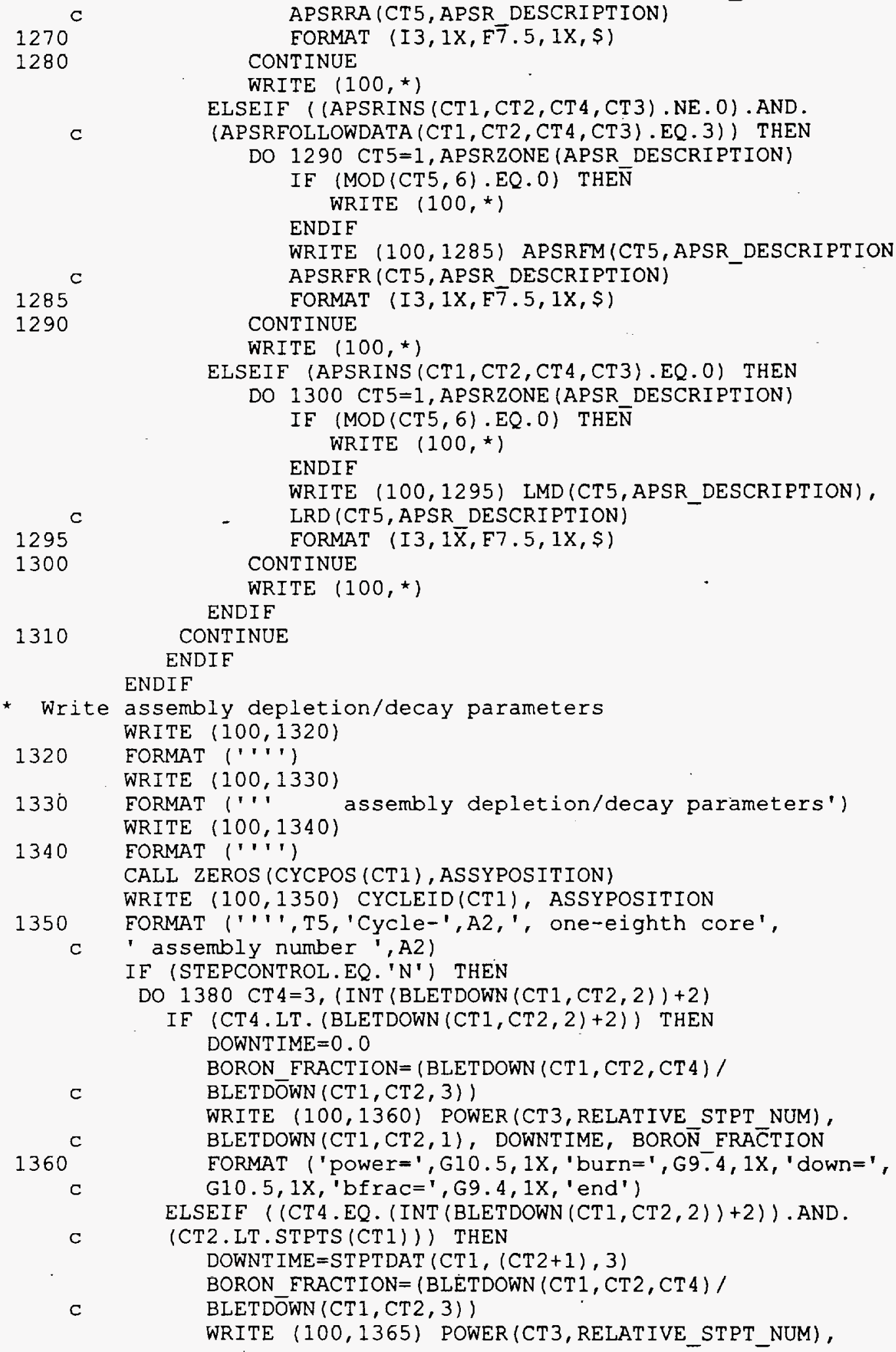




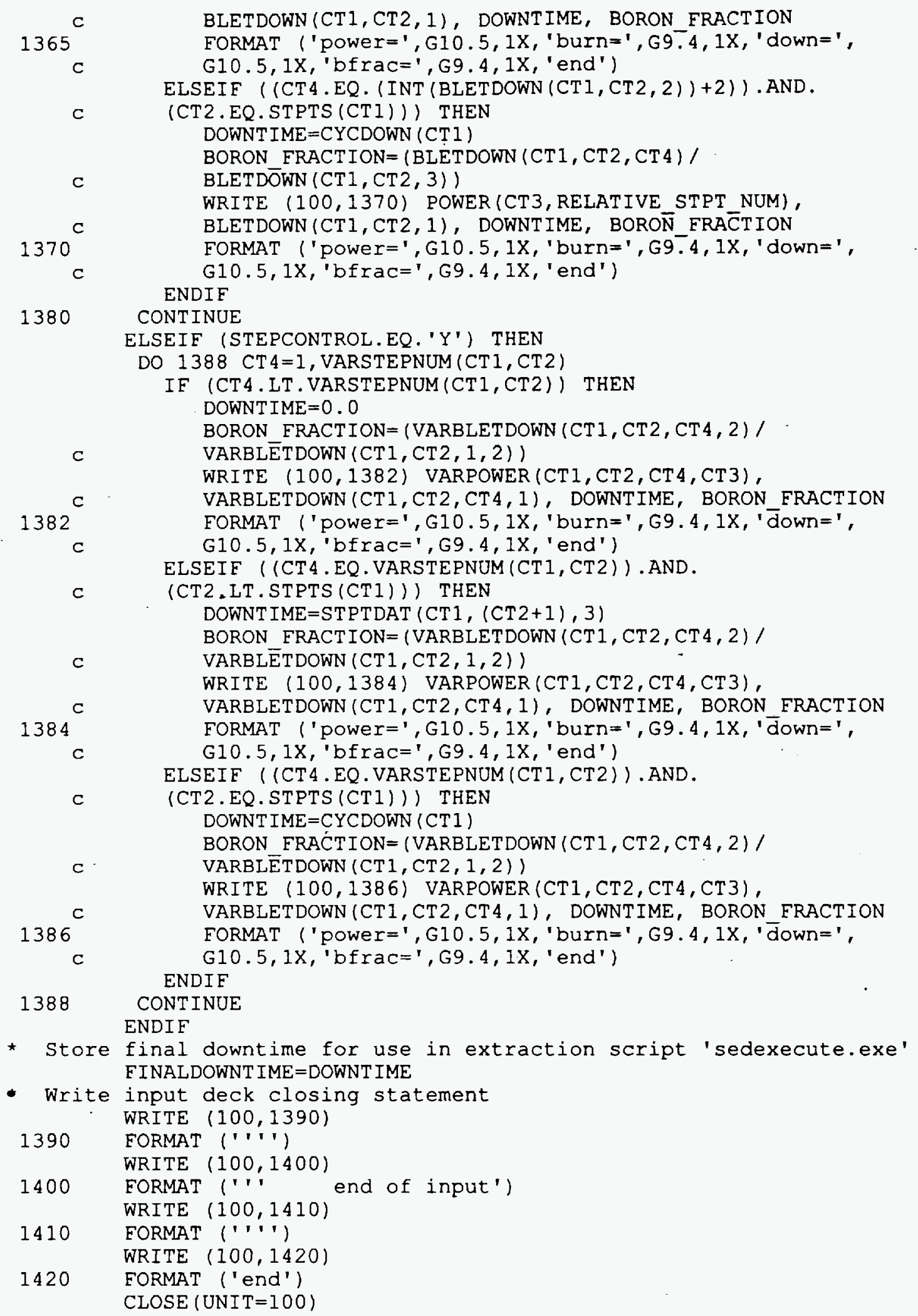




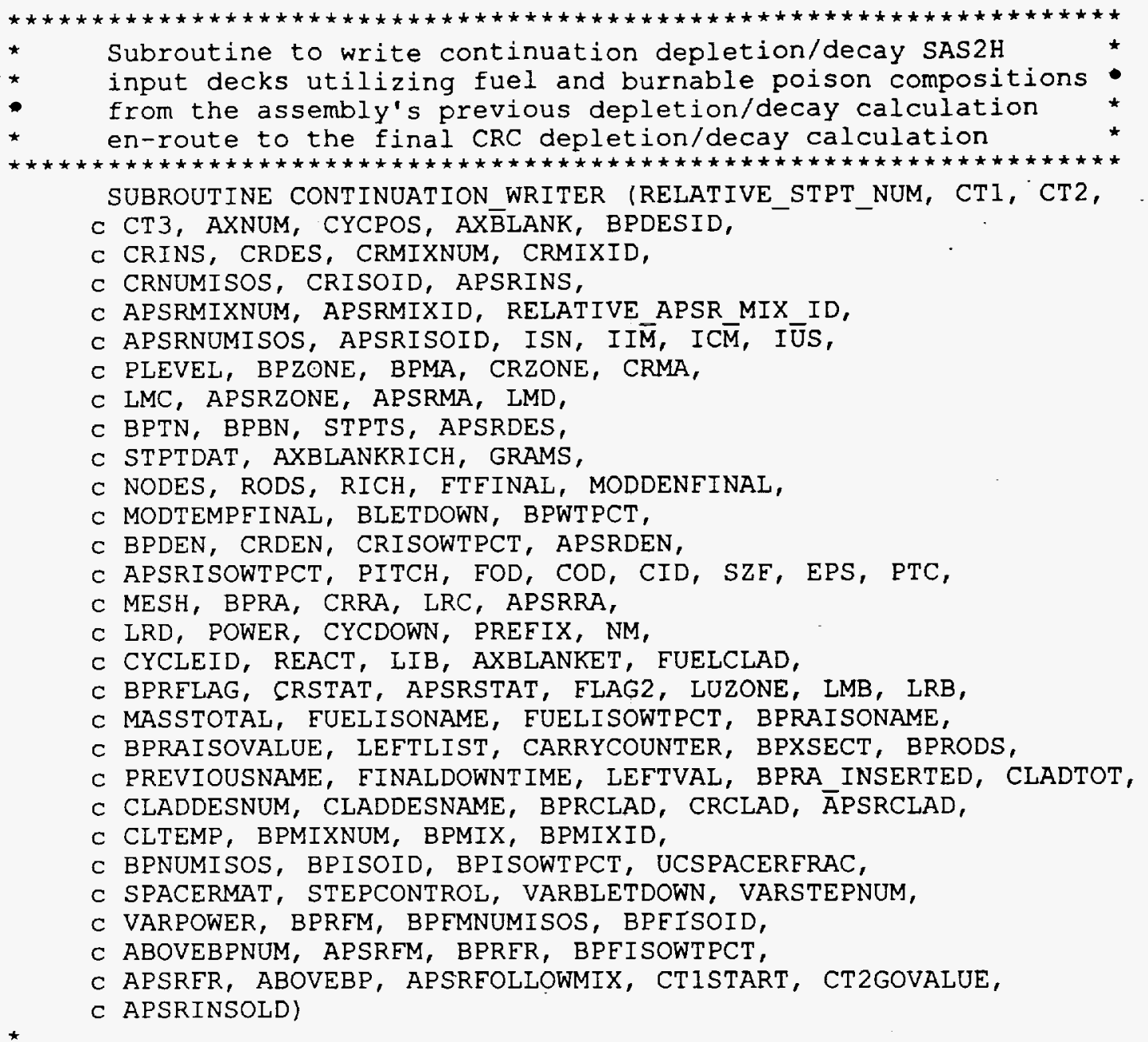

INTEGER ${ }^{4}$ RELATIVE STPT NUM, CT1, CT2, CT3, AXNUM, C NUMSTPT 1, NUMSTPT $\overline{2}$, NUMSTPT3, CYCPOS $(10), \operatorname{AXBLANK}(50)$, C BPDESID(10), BPRA_DESCRIPTION_ID, CT4, CT5, CRINS $(10,20,23,50)$, C CR MIXTURE ID, CR DESCRIPTION, CRDES $(10,20,23,50)$, CRMIXNUM, C CRM̄IXID (25), RELATTIVE CR MIX ID, CRNUMISOS (25), 
REAL STPTDAT $(10,20,3)$, ENR, AXBLANKRICH, OXYGMS, GRAMS $(50)$,

C FVOL, PI, NODES $(50,2)$, RODS, FDEN,

C RICH, FTFINAL $(50,20)$,

c MODDENFINAL $(50,20)$, MODTEMPFinaL $(50,20), \operatorname{BLETdOWN}(10,20,25)$,

c BPWTPCT (10), BPDEN(10), ALFRAC, OFRAC, CRDEN(10),

c CRISOWTPCT $(25,10), \operatorname{APSRDEN}(10), \operatorname{APSRISOWTPCT}(25,10)$,

C PITCH, FOD, COD, CID, SZF, EPS, PTC, MESH, BPRA $(15,10)$,

c $\operatorname{CRRA}(15,10), \operatorname{LRC}(15,10), \operatorname{APSRRA}(15,10), \operatorname{LRD}(15,10)$,

c DOWNTIME, BORON_FRACTION, POWER $(50,20)$, CYCDOWN $(10), \operatorname{LRB}(15)$,

C MASSTOTAL, FUELISOWTPCT (1000), BPRAISOVALUE(2), BPXSECT (10),

C BPVOL, FINALDOWNTIME, LEFTVAL (1000), CLTEMP,

C BPISOWTPCT $(10,20)$, UCSPACERFRAC, BORATEDMODVF,

C BORONVF, UCMODREGIONDEN, B4CMASS, ALMASS, OMASS, CMASS,

C NEWBPMASSTOTAL, NEWBPDEN, ALWTPCT, OWTPCT, CWTPCT, BIOWTPCT,

C B1IWTPCT, VARBLETDOWN $(10,20,25,25)$, $\operatorname{VARPOWER}(10,20,25,50)$,

C $\operatorname{BPRFR}(15,10), \operatorname{BPEISOWTPCT}(25,10), \operatorname{APSRFR}(15,10)$

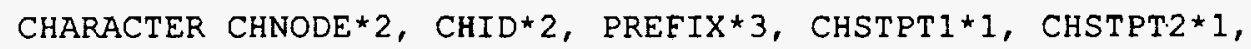

C CHSTPT $3 * 1, N M * 31, \operatorname{CYCLEID}(10) * 2, \operatorname{REACT} * 21, \operatorname{LIB} * 15$,

C AXBLANKET ${ }^{\star} 1$, FUELCLAD $\star 10$, BPRFLAG* 1 , CRSTAT $\star 6$, APSRSTAT $* 6$,

c FLAG2 ${ }^{\star} 7$, IRRAD STEPS 2 2, PLEVELCH $\star 2, \mathrm{BPZONECH} \star 2, \mathrm{CRZONECH} \star 2$,

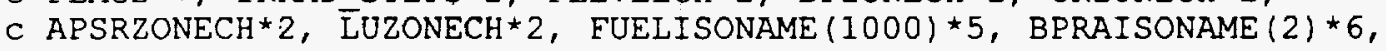

C LEFTLIST $(1000) \star 6$, PREVIOUSNAME $\star 25$, PCHSTPT $1 \star 1$, PCHSTPT $2 \star 1$,

c PCHSTPT $3 \star 1$, ASSYPOSITION $* 2$, CHSTPT $4 \star 1$, CHSTPT $5 \star 1$, CHSTPT $6 \star 1$,

c PCHSTPT $4 * I$, PCHSTPT $5 * 1$, PCHSTPT $6 * 1$, PCHID*2, CLADDESNAME $(10) * 7$,

C SPACERMAT*7, STEPCONTROL*1, ABOVEBP $(10) \star 5$

LOGICAL BPRA_INSERTED, CR_INSERTED, CRCOMPFLAG, APSR_INSERTED,

C APSRCOMPFLA $\bar{G}$, BPRA_FOLLOW, APSRBOTFLAG, FOLLOWIN

$\mathrm{PI}=3.14159265359$

* Determination of the input deck filename

CALL ZEROS (CT 3 , CHNODE)

CALL ZEROS (CYCPOS (CTI), CHID)

IF ( (CT2-1).EQ.0) THEN

PNMCT $1=\mathrm{CT} 1-1$

PNMCT $2=S T$ PTS $($ PNMCT 1 )

ELSE

PNMCT $1=\mathrm{CT} 1$

PNMCT $2=\mathrm{CT} 2-1$

ENDIF

CALL ZEROS (CYCPOS (PNMCT1), PCHID)

* Determine new filename

NUMSTPT $1=$ INT $($ STPTDAT $(C T 1, C T 2,1) / 100.0)$

CHSTPT $1=$ CHAR $($ NUMSTPT $1+48)$

NUMSTPT $2=\operatorname{INT}((\operatorname{STPTDAT}(\mathrm{CT} 1, \mathrm{CT} 2,1)-(\operatorname{NUMSTPT} 1 \star 100)) / 10.0)$

CHST PT $2=$ CHAR (NUMSTPT $2+48$ )

NUMSTPT $3=$ INT $(($ STPTDAT $(C T 1, C T 2,1)-($ NUMSTPT $1 * 100)-$

C (NUMSTPT $2 * 10))$ )

CHSTPT $3=$ CHAR (NUMST PT $3+48$ )

IF (CT2.LT.STPTS (CT1)) THEN

NUMSTPT $4=\operatorname{INT}($ STPTDAT $(C T),(C T 2+1), 1) / 100.0)$

CHST PT $4=$ CHAR (NUTSTPT $4+48)$

NUMSTPT $5=\operatorname{INT}((\operatorname{STPTDAT}(\mathrm{CT} 1,(\mathrm{CT} 2+1), 1)-($ NUMSTPT $4 * 100)) / 10.0)$

CHST PT $5=$ CHAR $($ NUMST PT $5+48)$

NUMSTPT $6=$ INT $(($ STPTDAT $($ CT $1,($ CT $2+1), 1)-($ NUMSTPT 4 * 100$)-$

C (NUMSTPT $5 * 10)$ ))

CHST PT $6=$ CHAR (NUMST PT $6+48$ )

ELSEIE (CT2.EQ.STPTS (CT1)) THEN 


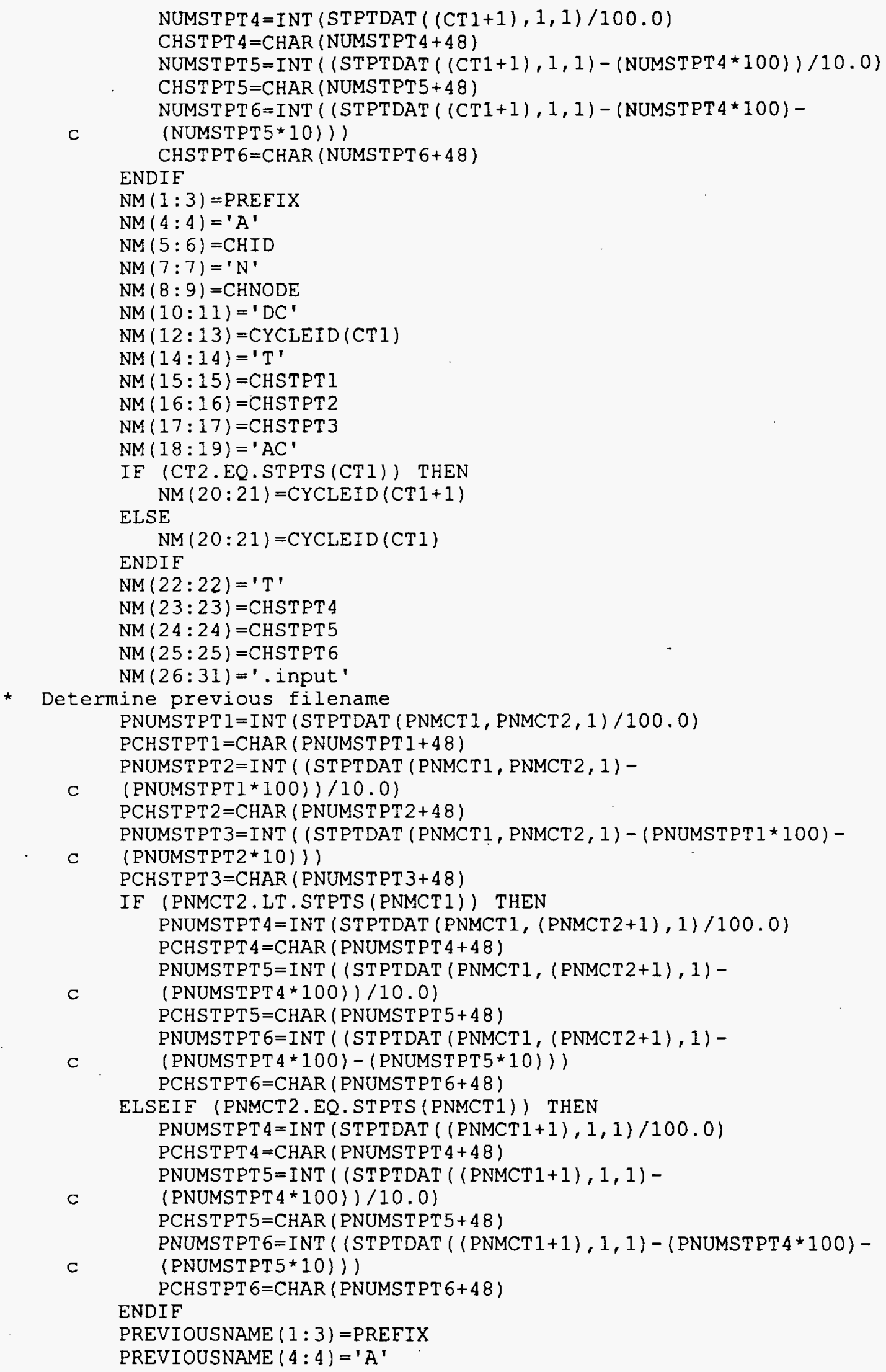




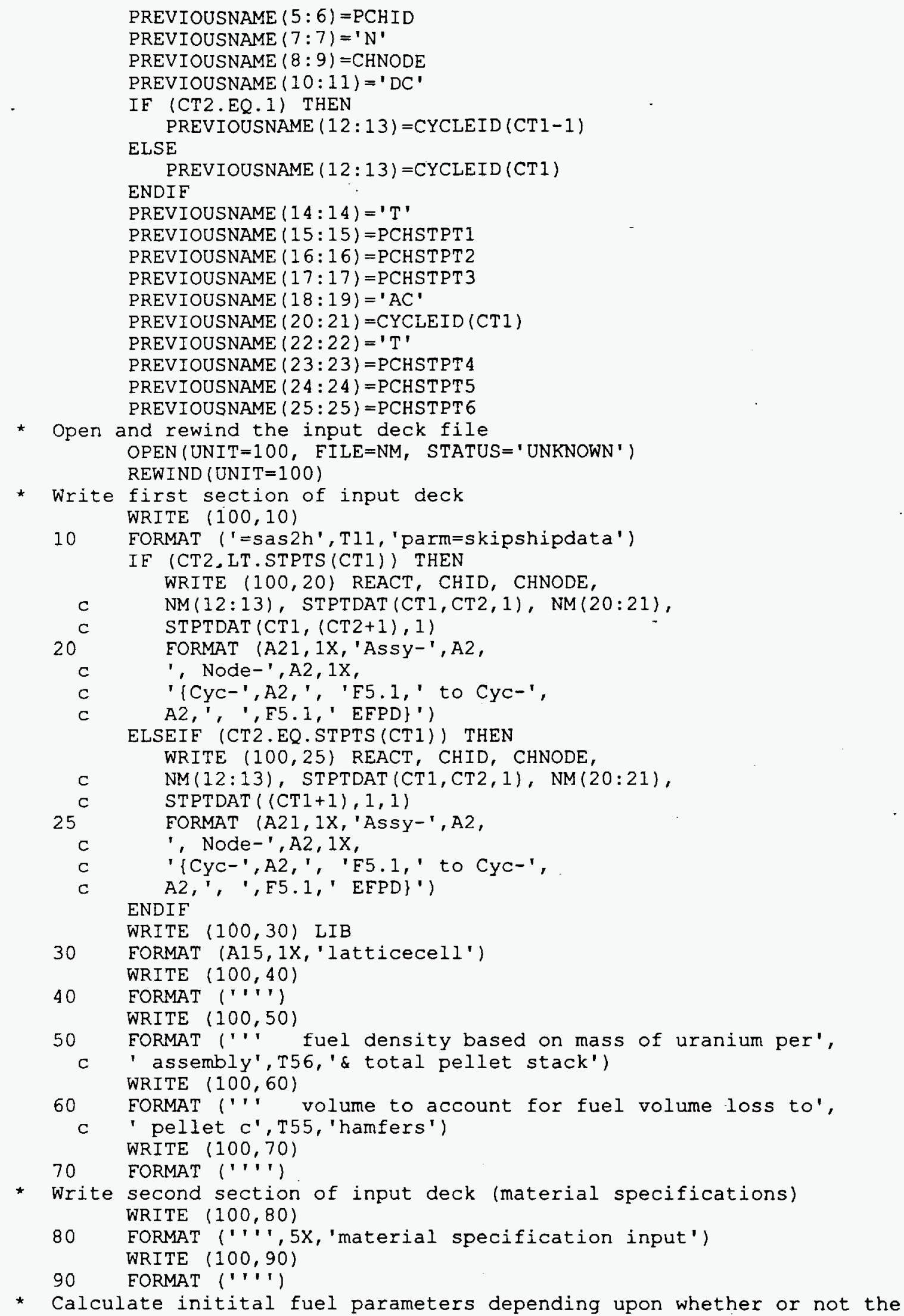


* node represents axial blanket fuel

IE ((AXBLANKET.EQ. ' $Y$ ') . AND. (AXBLANK (CT3).EQ.1)) THEN $E N R=A X B L A N K R I C H$

ELSE

ENDIF $E N R=R I C H$

OXYGMS $=($ GRAMS $($ CT3 $) \star 2 \star 15.994915) /((($ ENR $/ 100) \star 235.043915)+$

C $\quad(((0.007731 *((E N R) \star \star 1.0837)) / 100) \star 234.040904)+$

C $\quad(((0.0046 \star E N R) / 100) \star 236.045637)+(((100-(0.007731 \star$

c $(E N R \star \star 1.0837))-(E N R)-(0.0046 \star E N R)) / 100) \star 238.05077))$

* Determine if the burnable poison charge isotopics should be retrieved BPRA INSERTED $=$. FALSE.

IF ('(BPRFLAG.EQ.' 'Y') . AND. (BPDESID (CT1) . NE. 0) . AND.

C (CT3.GE.BPTN (CT1)).AND. (CT3.LE.BPBN(CT1))) THEN ENDIF BPRA INSERTED $=$. TRUE.

* Call subroutine to retrieve charge for fuel and bp isotopics CALL RETRIEVER (OXYGMS, MASSTOTAL,

C FUELISONAME, FUELISOWTPCT, BPRAISONAME,

C BPRAISOVALUE, LEETLIST, CARRYCOUNTER,

C PREVIOUSNAME, LEFTVAL, NM, BPRA_INSERTED)

- Calculate the nodal fuel volume, fuel-density, and oxygen wto FVOL $=(P I / 4) \star(F O D \star \star 2) \star(\operatorname{NODES}(\mathrm{CT} 3,2)) \bullet(\operatorname{RODS})$

FDEN=MASSTOTAL $/$ FVOL

OXYWTPCT $=($ OXYGMS $/$ MASSTOTAL $) \star 100.0$

FUELISOTOPENUMBER=CARRYCOUNTER+ 1

- Write fuel composition input description

IF (FDEN.LT.(10.0)) THEN

WRITE $(100,100)$ FDEN, FUELISOTOPENUMBER, OXYWTPCT

100 FORMAT ('arbm-fuel', IX, GI0.3, IX, I3, 1X, '0 0 0', 1X,

$\mathrm{C}$

ELSE '8016', 1X, G10.3)

(100,110) FDEN, FUELISOTOPENUMBER, OXY FORMAT ('arbm-fuel', $1 \mathrm{X}, \mathrm{G} 10.3,1 \mathrm{X}, \mathrm{I} 3,1 \mathrm{X}$, ' 0 O 0 ', $1 \mathrm{X}$, c

ENDIF '8016',1X, G10.3)

DO 130 CT $4=1$, CARRYCOUNTER

IF (MOD (CT 4,3).EQ.0) THEN

ENDIF

WRITE $(100, *)$

WRITE $(100,120)$ FUELISONAME (CT 4), FUELISOWTPCT (CT4)

120 FORMAT $(5 \mathrm{X}, \mathrm{A} 5,1 \mathrm{X}, \mathrm{GI0} .3,1 \mathrm{X}, \$)$

130 CONTINUE

WRITE $(100$, *)

WRITE $(100,140)$ FTFINAL (CT3,RELATIVE_STPT_NUM)

140 FORMAT (5X,' '1',3X,'1.0',3X,F6.1,' énd')

* Write cladding material specifications

* Additional cladding material specifications may be added to the

* following IF statement as required IF ((FUELCLAD.EQ. 'ZIRC-4 ').OR.

C (FUELCLAD.EQ.'ZIRCALLOY4')) THEN WRITE $(100,532)$

532 FORMAT ('arbm-zirc4 $6.56500080160 .1224000^{\prime}$,

C $\left.\quad 0.10260000 .20500001 .40^{\prime}\right)$ WRITE $(100,535)$ CLTEMP

535 FORMAT (T12,'40000 98.1821 .0 ',F5.1,' end')

$$
\text { ELSEIF (FUELCLAD.EQ.'SS304 ') THEN }
$$

WRITE $(100,537)$

537 FORMAT ('arbm-ss3047.924000 24304 19.0 25055', 


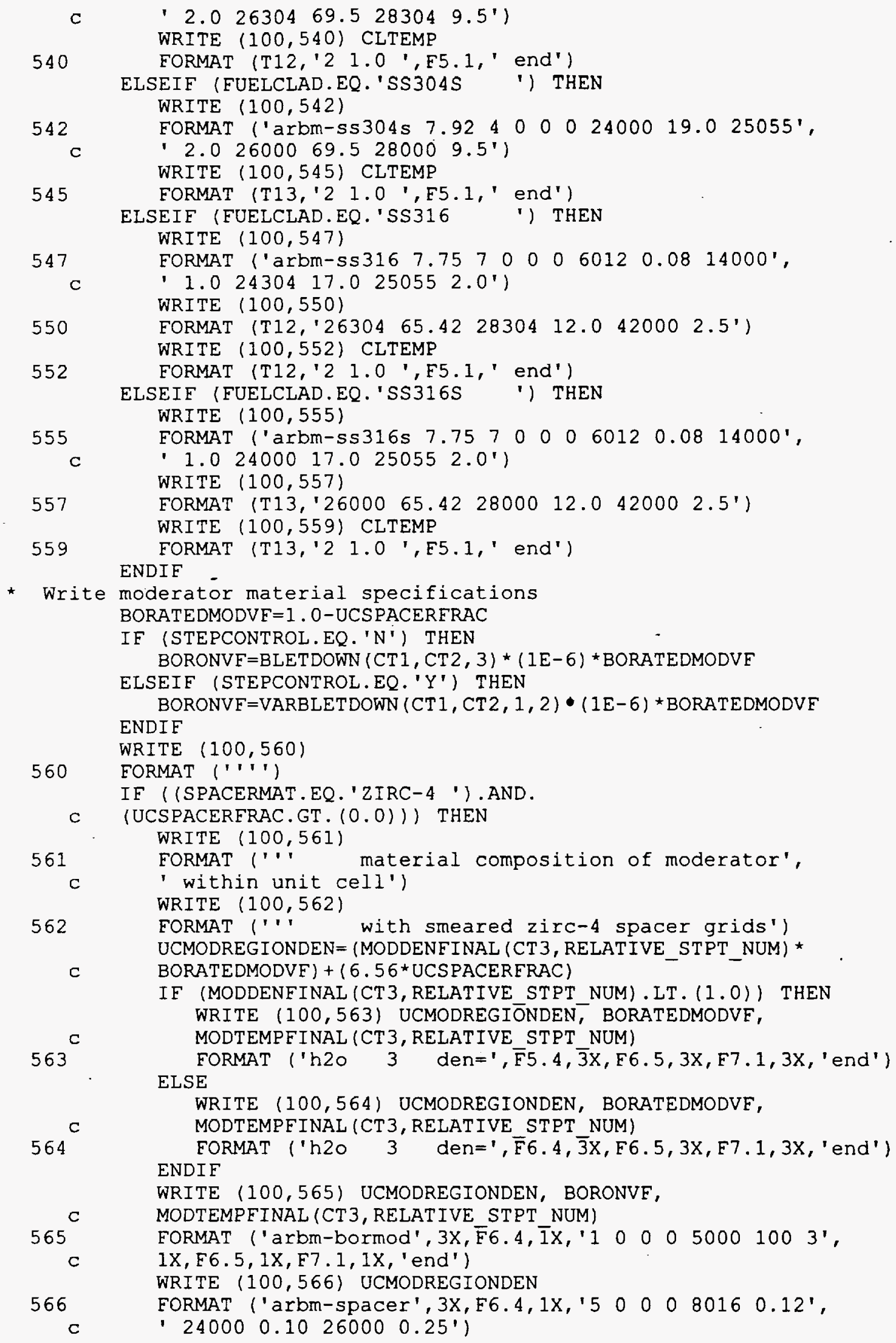




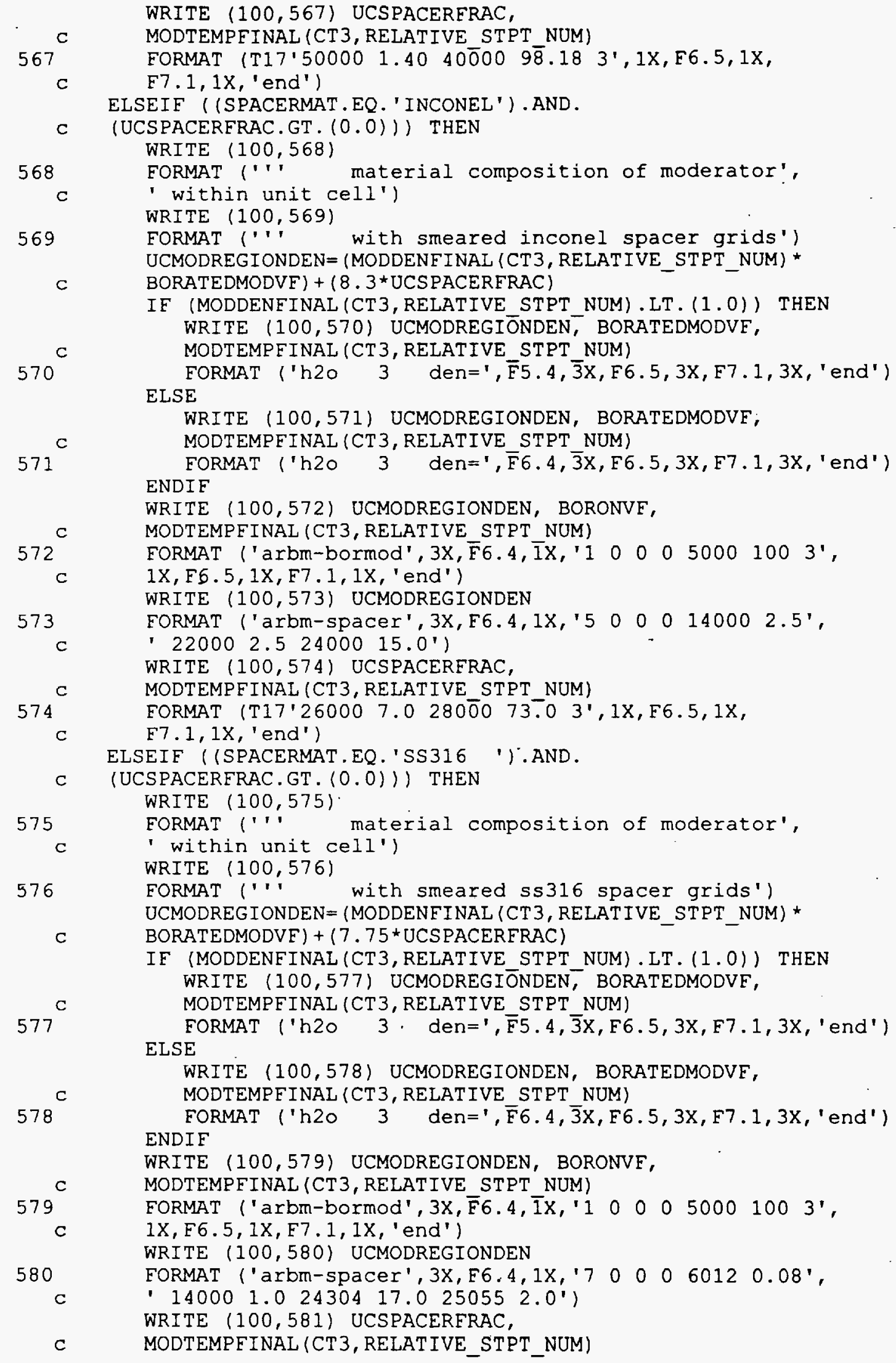

C UCMODREGIONDEN=(MODDENFINAL (CT3,RELATIVE_STPT_NUM) * BORATEDMODVF $)+(8.3 *$ UCSPACERFRAC $)$

IF (MODDENEINAL (CT3, RELATIVE STPT NUM) . LT. (1.0)) THEN WRITE $(100,570)$ UCMODREGIONDEN, BORATEDMODVE, MODTEMPEINAL (CT 3, RELATIVE STPT NUM) 
581

C

C

582

C

583

584

585

$$
586^{\circ}
$$$$
\mathrm{c}
$$

C

$588^{c}$

$$
\mathrm{C}
$$

c

589

c

590

c

C

591

592

C

$$
593^{c}
$$

$c$

$595^{c}$

FORMAT (T5'26304 65.422830412 .0420002 .5 3', 1X, F6.5, 1X, F7.1, 1X, 'end')

ELSEIF ((SPACERMAT.EQ.'SS316S '). AND.

(UCSPACERFRAC.GT. (0.0))) THEN

WRITE $(100,582)$

FORMAT ("' material composition of moderator',

' within unit celI')

WRITE $(100,583)$

FORMAT ("' with smeared ss316s spacer grids') UCMODREGIONDEN= (MODDENFINAL (CT3, RELATIVE_STPT_NUM) * BORATEDMODVF $)+(7.75$ *UCSPACERFRAC $)$

IF (MODDENEINAL (CT3, RELATIVE STPT NUM) .LT. (1.0)) THEN WRITE $(100,584)$ UCMODREGIONDEN, BORATEDMODVF, MODTEMPEINAL (CT 3, RELATIVE_STPT_NUM) ELSE FORMAT ('h20 3 den $=$ ', $\overline{\mathrm{F}} 5.4, \overline{3} \mathrm{X}, \mathrm{F} 6.5,3 \mathrm{X}, \mathrm{F} 7.1,3 \mathrm{X}$, 'end')

WRITE $(100,585)$ UCMODREGIONDEN, BORATEDMODVF, MODTEMPFINAL (CT 3, RELATIVE STPT NUM) ENDIF FORMAT ('h20 3 den=', $\overline{\mathrm{F}} 6.4, \overline{3} \mathrm{X}, \mathrm{F} 6.5,3 \mathrm{X}, \mathrm{F} 7.1,3 \mathrm{X}$, 'end')

WRITE $(100,586)$ UCMODREGIONDEN, BORONVF, MODTEMPFINAL (CT 3, RELATIVE STPT NUM)

FORMAT ('arbm-bormod', 3X, $\overline{\mathrm{F}} 6.4, \overline{\mathrm{X} X}$, ' 1000000100 3', IX, F6. 5, 1X, F7.1, 1X, 'end')

WRITE $(100,587)$ UCMODREGIONDEN

FORMAT ('arbm-spacer', 3X, F6.4, 1X,'70000612 0.08',

' $140001.02400017 .0250552 .0^{\prime}$ '

WRITE $(100,588)$ UCSPACERERAC,

MODTEMPFINAL (CT3, RELATIVE_STPT_NUM)

FORMAT (T5'26000 $65.4228 \overline{0} 001 \overline{2} .0420002 .53$ ', IX, F6.5, 1X, F7.1, 1X, 'end')

ELSEIF ((SPACERMAT.EQ.'SS304 '). AND.

(UCSPACERFRAC.GT . (0.0))) THEN

WRITE $(100,589)$

FORMAT ('"' material composition of moderator',

' within unit cell')

WRITE $(100,590)$

FORMAT ("' with smeared ss304 spacer grids')

UCMODREGIONDEN $=($ MODDENFINAL (CT 3, RELATIVE_STPT_NUM) *

BORATEDMODVF $)+(7.92 *$ UCSPACERFRAC $)$

IF (MODDENFINAL (CT 3, RELATIVE STPT NUM) . LT. (1.0)) THEN WRITE $(100,591)$ UCMODREGIONDEN, BORATEDMODVE, MODTEMPFINAL (CT 3, RELATIVE STPT NUM)

ELSE FORMAT ('h2o 3 den=', $\overline{5} 5.4, \overline{3} X, F 6.5,3 \mathrm{X}, \mathrm{F} 7.1,3 \mathrm{X}$, 'end')

WRITE $(100,592)$ UCMODREGIONDEN, BORATEDMODVF, MODTEMPFINAL (CT3, RELATIVE STPT NUM)

ENDIE

FORMAT ('h20 3 den $=$ ', $\overline{\mathrm{F}} 6.4, \overline{3} \mathrm{X}, \mathrm{F} 6.5,3 \mathrm{X}, \mathrm{F} 7.1,3 \mathrm{X}$, 'end')

WRITE $(100,593)$ UCMODREGIONDEN, BORONVE,

MODTEMPFINAL (CT3, RELATIVE STPT NUM)

FORMAT ('arbm-bormod', $3 \mathrm{X}, \overline{\mathrm{F}} 6.4, \overline{1} \mathrm{X}, ' 10005000100$ 3',

1X, F6.5, IX, F7.1, 1X, 'end')

WRITE $(100,594)$ UCMODREGIONDEN

FORMAT ('arbm-spacer', 3X,F6.4,1X,'4 000024304 19.0',

' $250552.02630469 .528304 \quad 9.5^{\prime}$ )

WRITE $(100,595)$ UCSPACERERAC,

MODTEMPEINAL (CT 3, RELATIVE_STPT_NUM)

FORMAT (T15'3',1X, F6.5,1X,F7.1, 1X, 'end')

ELSEIF ((SPACERMAT.EQ.'SS304S ').AND. 


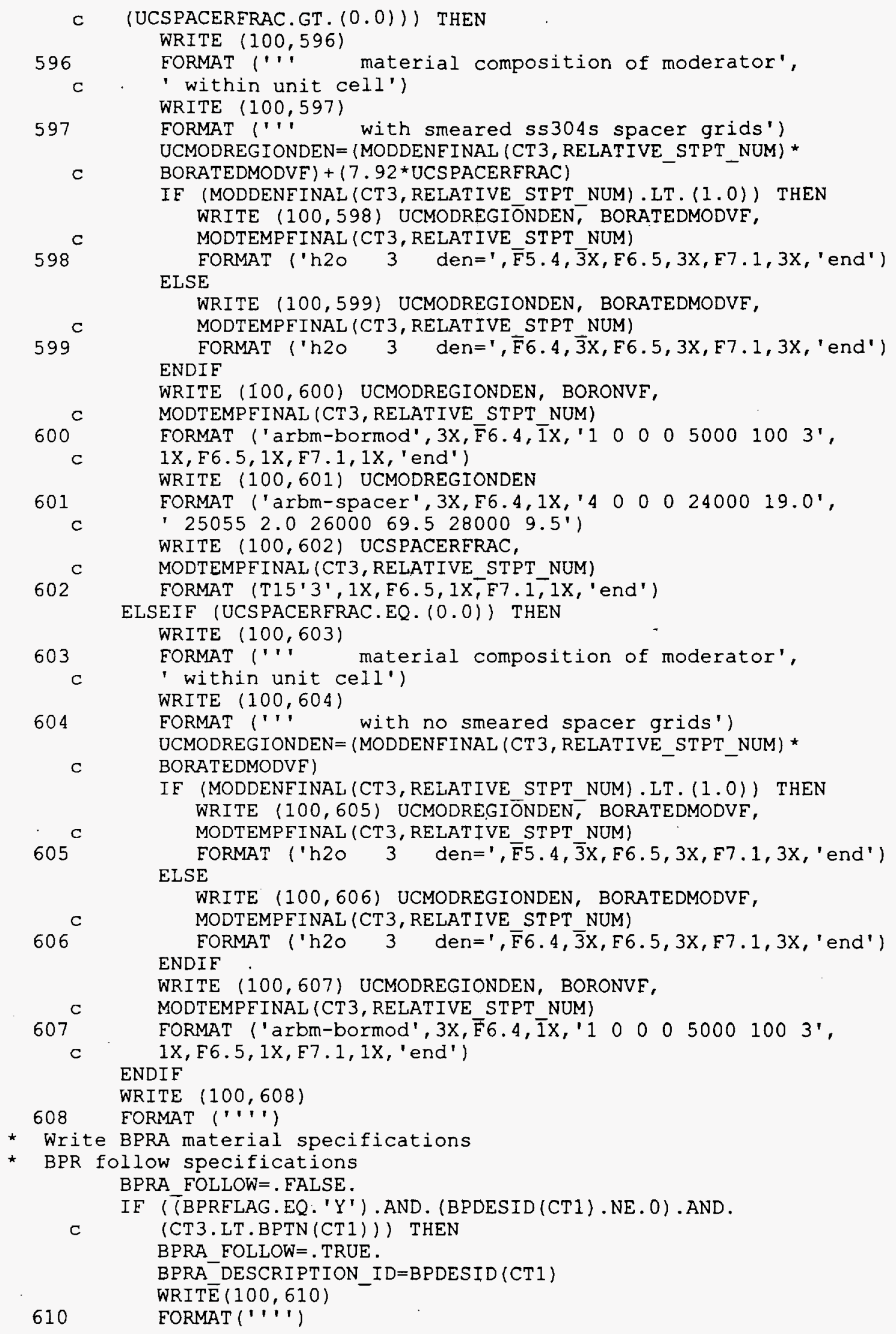




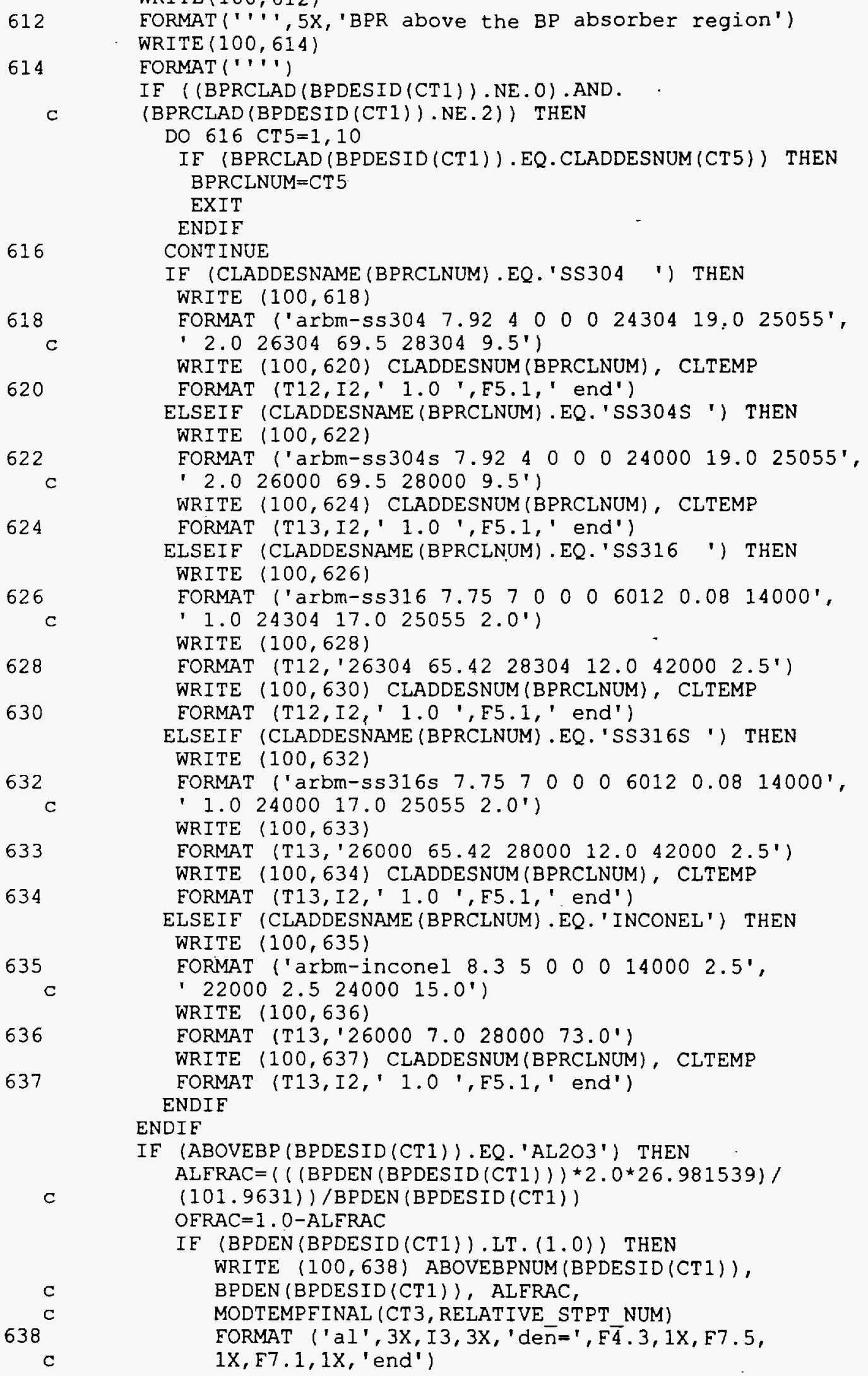




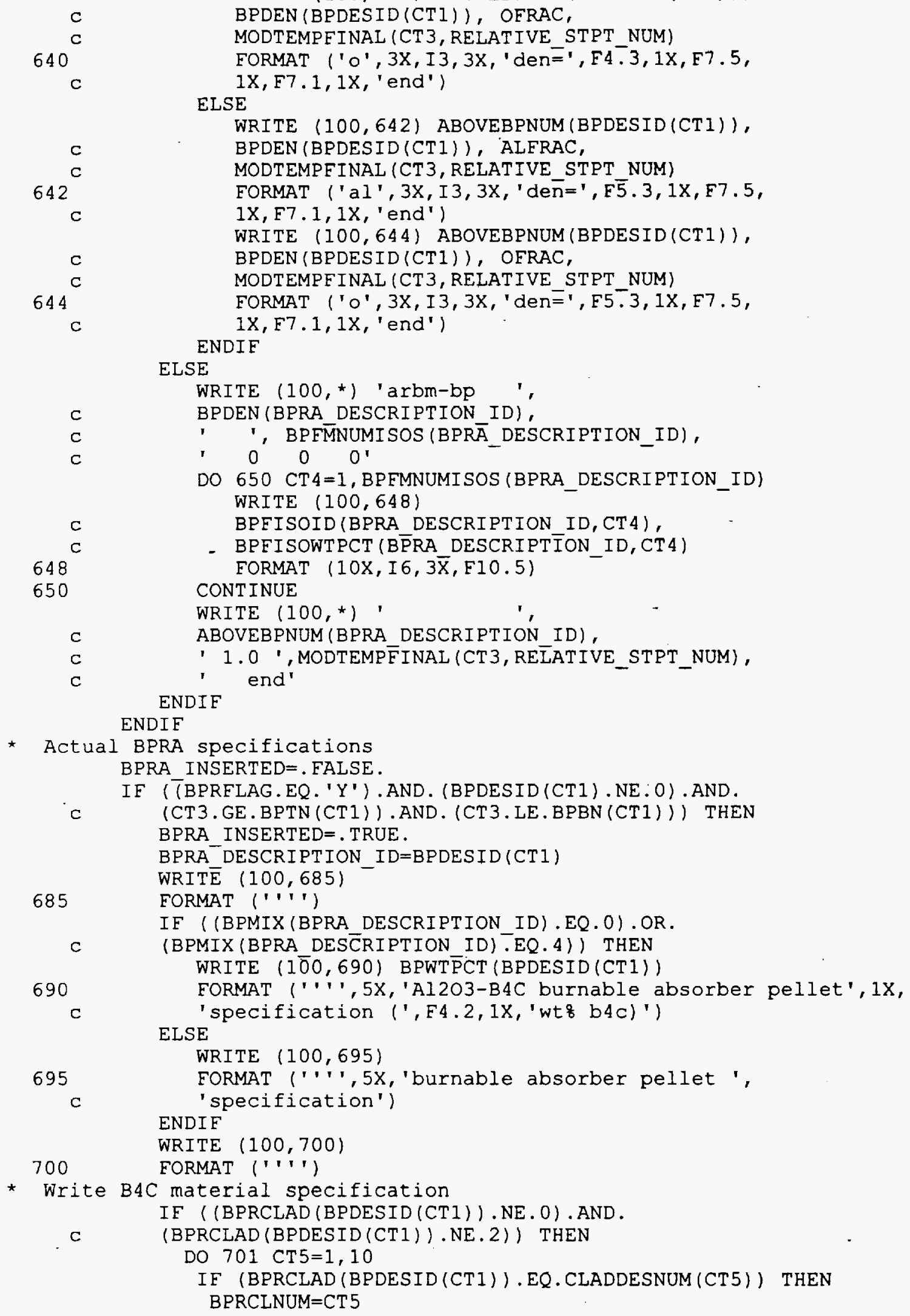




\section{1}

702

c

703

704

c

705

706

c

707

708

709

$\mathrm{c}$

710

711

712 c

713

714

\section{ENDIF}

* Material specification if it is a BOC to statepoint 1 calculation IF (CT2.EQ.1) THEN

* Material specification for AL203-B4C

C IF ((BPMIX (BPRA DESCRIPTION_ID).EQ.0).OR. (BPMIX (BPRA DESCRIPTION ID).$E Q .4)$ ) THEN

IF (BPWTPCT (BPDESID $(\bar{C} \bar{T} 1)$ ).NE. (0.0)) THEN

IF (BPDEN (BPDESID (CT1)).LT. (1.0)) THEN WRITE $(100,715)$ BPDEN(BPDESID(CT1)),

C

C

715

C

C

$716^{\mathrm{C}}$

c

EXIT

IE (CLADDESNAME (BPRCLNUM) .EQ.'SS304 ') THEN WRITE $(100,702)$

' $\left.2.026304 \quad 69.528304 \quad 9.5^{\prime}\right)$

WRITE $(100,703)$ CLADDESNUM(BPRCLNUM), CLTEMP

WRITE $(100,704)$

' $2.02600069 .5280009 .5^{\prime}$ )

WITE $(100,705)$ CIADDESNUM (BPRCLNUM) , CLTEMP

WRITE $(100,706)$

' $\left.1.02430417 .0250552 .0^{\prime}\right)$

RITE $(100,707)$

WRITE (100,708) CLADDESNUM(BPRCLNUM), CLTEMP

FORMAT (T12, I2,' 1.0 ', F5.1,' end')

WRITE $(100,709)$

EORMAT ('arbm-ss316s $7.7570006012 \quad 0.08$ 14000',

WRITE $(100,710)$

FORMAT (T13, $\left.2600065.422800012 .0 \quad 420002.5^{\prime}\right)$

(100,711) CLADDESNUM (BPRCLNUM) , CLTEMP

WRITE $(100,712)$

' $\left.220002.52400015 .0^{\prime}\right)$

RITE $(100,713)$

(T13,'26000 7.0 $\left.2800073.0^{\prime}\right)$

WRITE $(100,714)$ CLADDESNUM(BPRCLNUM), CLTEMP

FORMAT (T13, I2,' 1.0 ', F5.1,' end')

ENDIF

(BPWTPCT (BPDESID (CT1))/100.0), MODTEMPEINAL (CT 3, RELATIVE_STPT_NUM)

FORMAT ('b4c 4 den=', $\overline{\mathrm{F}} 4.3, \overline{\mathrm{X}}, \mathrm{F} 7.5,1 \mathrm{X}, \mathrm{F} 7.1,1 \mathrm{X}$, 'end')

ELSE

WRITE (100,716) BPDEN(BPDESID(CT1)),

(BPWTPCT (BPDESID (CT1))/100.0),

MODTEMPFINAL (CT3, RELATIVE STPT NUM)

FORMAT ('b4c 4 den=', $\overline{\mathrm{F}} 5.3, \overline{\mathrm{X}} \mathrm{X}, \mathrm{F} 7.5,1 \mathrm{X}, \mathrm{F} 7.1,1 \mathrm{X}$, 'end' ' 
ENDIF

* Calculate aluminum and oxygen material specifications

ALFRAC $=(((() 100.0-B P W T P C T(B P D E S I D(C T 1))) / 100)$ *

C $\operatorname{BPDEN}(\operatorname{BPDESID}(\mathrm{CT} 1))) \star 2 \star 26.981539) /(101.9631)) /$

C BPDEN (BPDESID (CT1))

OERAC $=1-($ BPWTPCT $($ BPDESID $(C T 1)) / 100.0)-A L F R A C$

WRITE (100,718) BPDEN(BPDESID(CT1)), ALFRAC,

$718^{\mathrm{C}}$ MODTEMPEINAL (CT 3, RELATIVE_STPT NUM)

$\begin{array}{cl}718 & \text { FORMAT ('al } 4 \text { den=', F5.3, 1X, F7.5, 1X, F7.1, 1X, 'end') } \\ \text { WRITE (100,720) BPDEN(BPDESID(CT1)), OFRAC, } \\ 720\end{array}$

* Material specification for BP other than A1203-B4C

DO 722 CT $4=1$, BPMIXNUM

IF (BPMIXID (CT4) .EQ.BPMIX (BPRA_DESCRIPTION_ID)) THEN ENDIF

722 CONTINUE

RELATIVE_BP_MIX_ID=CT 4

WRITE $\left(100,{ }^{\star}\right)$ 'arbm-bp ', BPDEN (BPRA_DESCRIPTION_ID),

C $\quad, \quad$ BPNUMISOS (RELATIVE_BP_MIX_ID),

DO 726 CT $4=1$, BPNUMISOS (RELATIVE_BP_MIX_ID)

WRITE $(100,724)$ BPISOID(RELATTIVE_BP_MIX_ID, CT4),

724

726 BPISOWTPCT (RELATIVE_BP MIX_ID, CT $\overline{4}$ )

- FORMAT (IOX, I $6,3 \mathrm{X}, \mathrm{F} \overline{1} 0 . \overline{5})$

CONTINUE

WRITE $(100, *)$ ', BPMIX (BPRA_DESCRIPTION_ID),

C ' 1.0 ',MODTEMPEINAL (CT3, RELATIVE_STPT_NUM), ' eñd' ENDIF

* Material specification if it is not a BOC to statepoint 1 calculation ELSEIF (CT2.NE.1) THEN

BPVOL=BPXSECT $($ BPRA DESCRIPTION ID) *

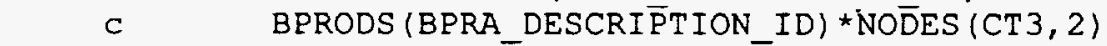

* Material specification for $\mathrm{AL} 2 \mathrm{O} 3-\overline{\mathrm{B}} 4 \mathrm{C}$

IF ((BPMIX (BPRA_DESCRIPTION_ID).EQ.0).OR.

C (BPMIX (BPRA DESCRIPTION ID) .EQ.4) THEN

B4CMASS $=(\overline{B P W T P C T}($ BPDE $\bar{S} I D(C T 1)) / 100.0)$ *

C $\quad$ BPDEN (BPDESID (CT1))*BPVOL

ALMASS $=((((100-B P W T P C T(B P D E S I D(C T 1))) / 100.0) *$

C BPDEN (BPDESID (CT1)))*BPVOL $)^{\star}((2 \star 26.981539) / 101.961278)$

OMASS $=((((100-B P W T P C T(B P D E S I D(C T 1))) / 100.0)$ *

C BPDEN (BPDESID(CT1)))*BPVOL) - ALMASS

$\mathrm{CMASS}=\mathrm{B} 4 \mathrm{CMASS} \star 0.217374$

NEWBPMASSTOTAL=ALMASS +OMASS +CMASS+BPRAISOVALUE $(1)+$

C BPRAISOVALUE (2)

NEWBPDEN=NEWBPMASSTOTAL/BPVOL

ALWTPCT $=($ ALMASS $/$ NEWBPMASSTOTAL $) \star 100.0$

OWTPCT $=($ OMASS $/$ NEWBPMASSTOTAL $) \star 100.0$

CWTPCT $=($ CMASS $/$ NEWBPMASSTOTAL $) \star 100.0$

B1 OWTPCT $=($ BPRAISOVALUE $(1) /$ NEWBPMASSTOTAL $) \star 100.0$

B1 IWTPCT $=($ BPRAISOVALUE $(2) /$ NEWBPMASSTOTAL $) \star 100.0$

IF (BPWTPCT (BPDESID (CT1)).NE. (0.0)) THEN

728 FORMAT ('arbm-bp', IX, F7.3,1X,'5 0000 ') WRITE $(100,728)$ NEWBPDEN

IF (BPRAISOVALUE (1). NE.0) THEN WRITE $(100,730)$ BPRAISONAME (1),

C B1OWTPCT

$730 \quad$ FORMAT (5X, A6, 1X, G10.3) ENDIF 


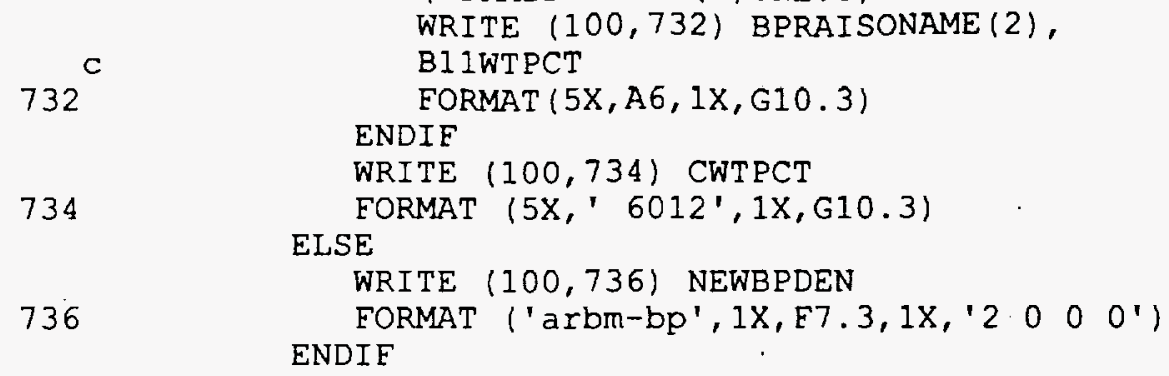




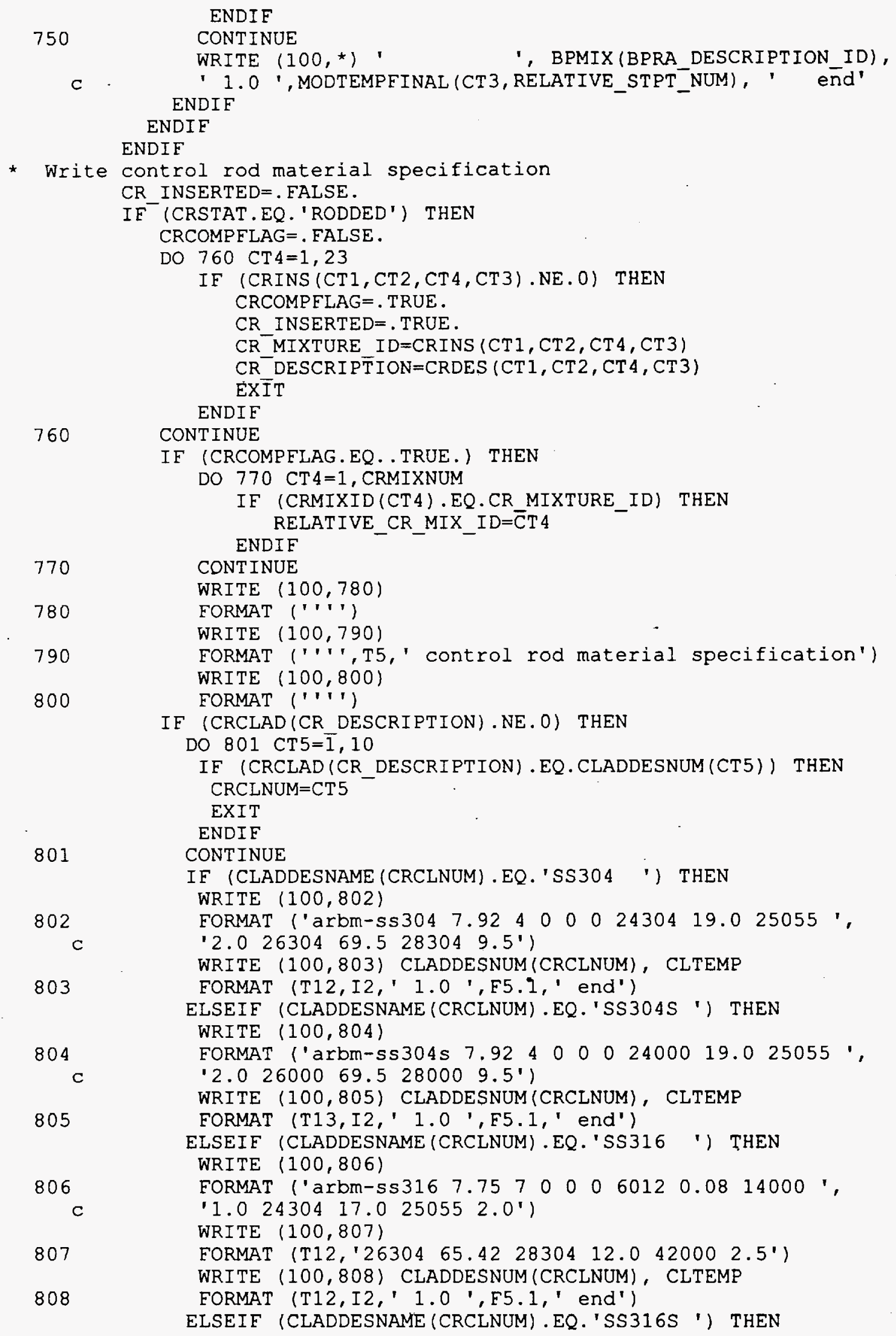


809

c

810

811

812

$\mathrm{c}$

813

814

$c$

815
820

C ENDIE ENDIF

WRITE $(100,809)$

FORMAT ('arbm-ss316s 7.7577000060120 .0814000 ',

' 1.02400017 .0250552 .0 ')

WRITE $(100,810)$

FORMAT (T13,'26000 65.42 $\left.28000 \quad 12.0 .42000 \quad 2.5^{\prime}\right)$

WRITE $(100,811)$ CLADDESNUM(CRCLNUM), CLTEMP

FORMAT (T13,I2,' 1.0 ',F5.1,' end')

ELSEIF (CLADDESNAME (CRCLNUM).EQ. 'INCONEL') THEN

WRITE $(100,812)$

FORMAT ('arbm-inconel 8.350000140002 .5 ',

' $\left.220002.52400015 .0^{\prime}\right)$

WRITE $(100,813)$

FORMAT (T13,'26000 7.0 $2800073.0^{\prime}$ )

WRITE $(100,814)$ CLADDESNUM(CRCLNUM), CLTEMP

FORMAT (T13,I2,' 1.0 ', F5.1,' end') ENDIF

ENDIF

WRITE (100,*) 'arbm-cr ', CRDEN(CR_DESCRIPTION),

' ', CRNUMISOS (RELATIVE_CR_MIX_ID) ,' 0 o 0 '

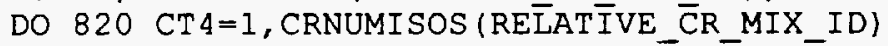

WRITE $(100,815)$ CRISOID (RELATIVE CR MIX_ID, CT4), CRISOWTPCT (RELATIVE CR MIX_ID, CTT4)

FORMAT $(10 X, I 5,3 X, \bar{E} \overline{1} 0 . \overline{5})$

CONTINUE

WRITE $\left(100,{ }^{\star}\right)$ ' ', CR_MIXTURE_ID, '1.0',

MODTEMPEINAL (CT3, RELATIVE_STPT_NUM), '- end'

* Write APSR material specification

C (CT3.EQ.1)) THEN

DO 824 CT $4=1,10$

DO 823 CT $5=1,20$

DO 822 CT $6=1,23$

DO $821 \mathrm{CT} 7=1,50$

APSRINSOLD $(\mathrm{CT} 4, \mathrm{CT} 5, \mathrm{CT} 6, \mathrm{CT} 7)=$

C

821

822

823

824

C

CONT INUT
CONTINUE

APSRINS (CT 4, CT 5, CT 6, CT7)

CONTINUE

CONTINUE

ENDIE

APSR INSERTED = FALSE.

IF (APPSRSTAT.EQ. 'RODDED') THEN

DO 830 CT $4=1,23$

APSRBOT FLAG $=$. FALSE.

DO 825 CT5 $=50,1,-1$

IE ((APSRINSOLD (CT1, CT 2, CT 4, CT 5) . NE. 0) . AND.

(APSRBOTELAG.EQ.. FALSE.)) THEN

APSR DESCRIPTION=APSRDES (CT1, CT2, CT 4, CT5)

APSR $\bar{B} O T F L A G=$. TRUE .

FOLNODKEEP $=$ CT 5

FOLSTEPKEEP $=$ CT 4

ENDIE

IE ( (APSRINSOLD (CT1, CT2, CT 4, CT5) . EQ. 0) . AND.

c

(APSRBOTFLAG.EQ..TRUE.)) THEN

APSRINS $(\mathrm{CT} 1, \mathrm{CT} 2, \mathrm{CT} 4, \mathrm{CT} 5)=$

APSRFOLLOWMIX (CT 1, CT 2, FOLSTEPKEEP, FOLNODKEEP)

APSRFOLLOWDATA $(\mathrm{CT} 1, \mathrm{CT} 2, \mathrm{CT} 4, \mathrm{CT} 5)=3$

ENDIF 


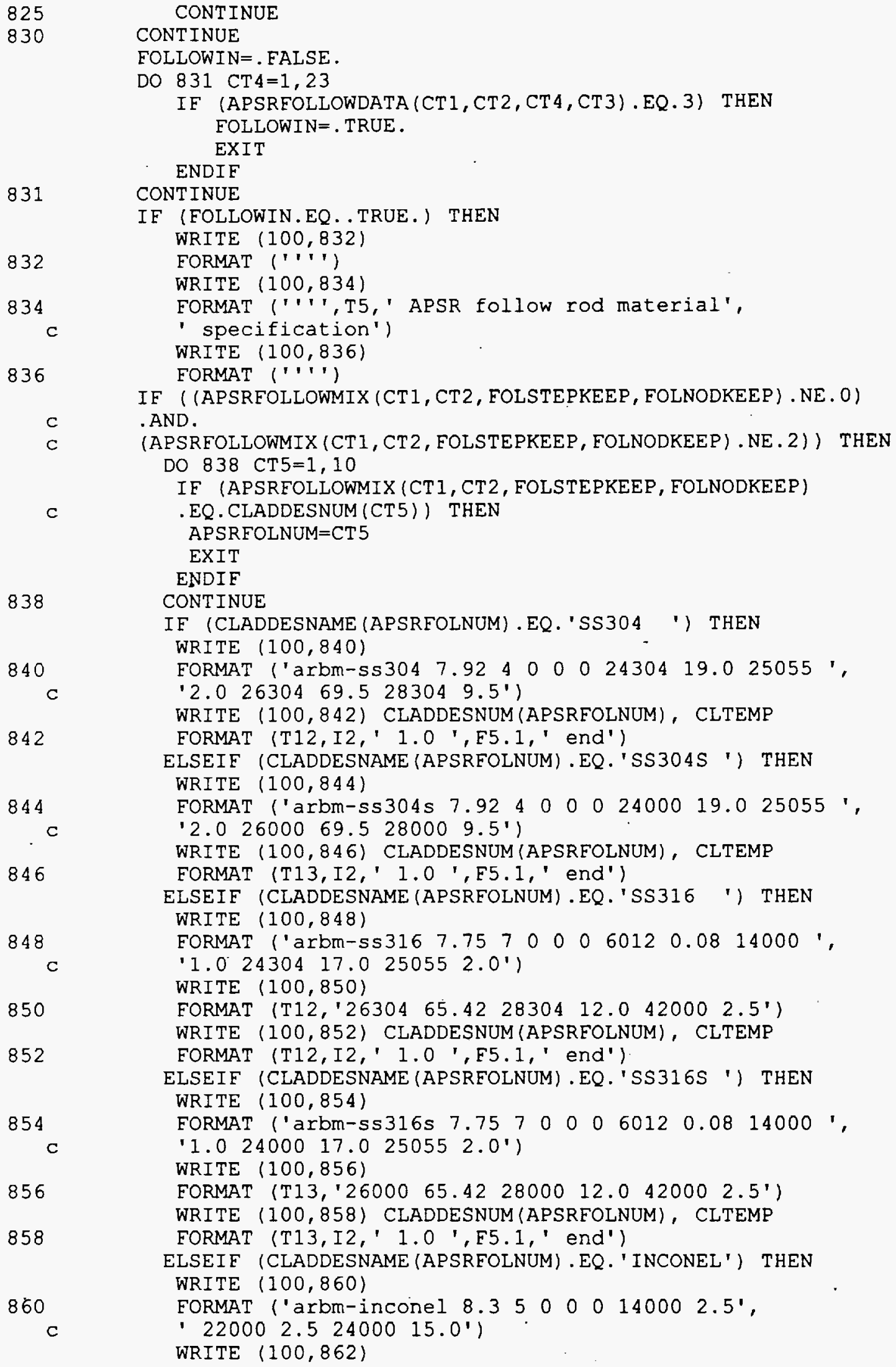




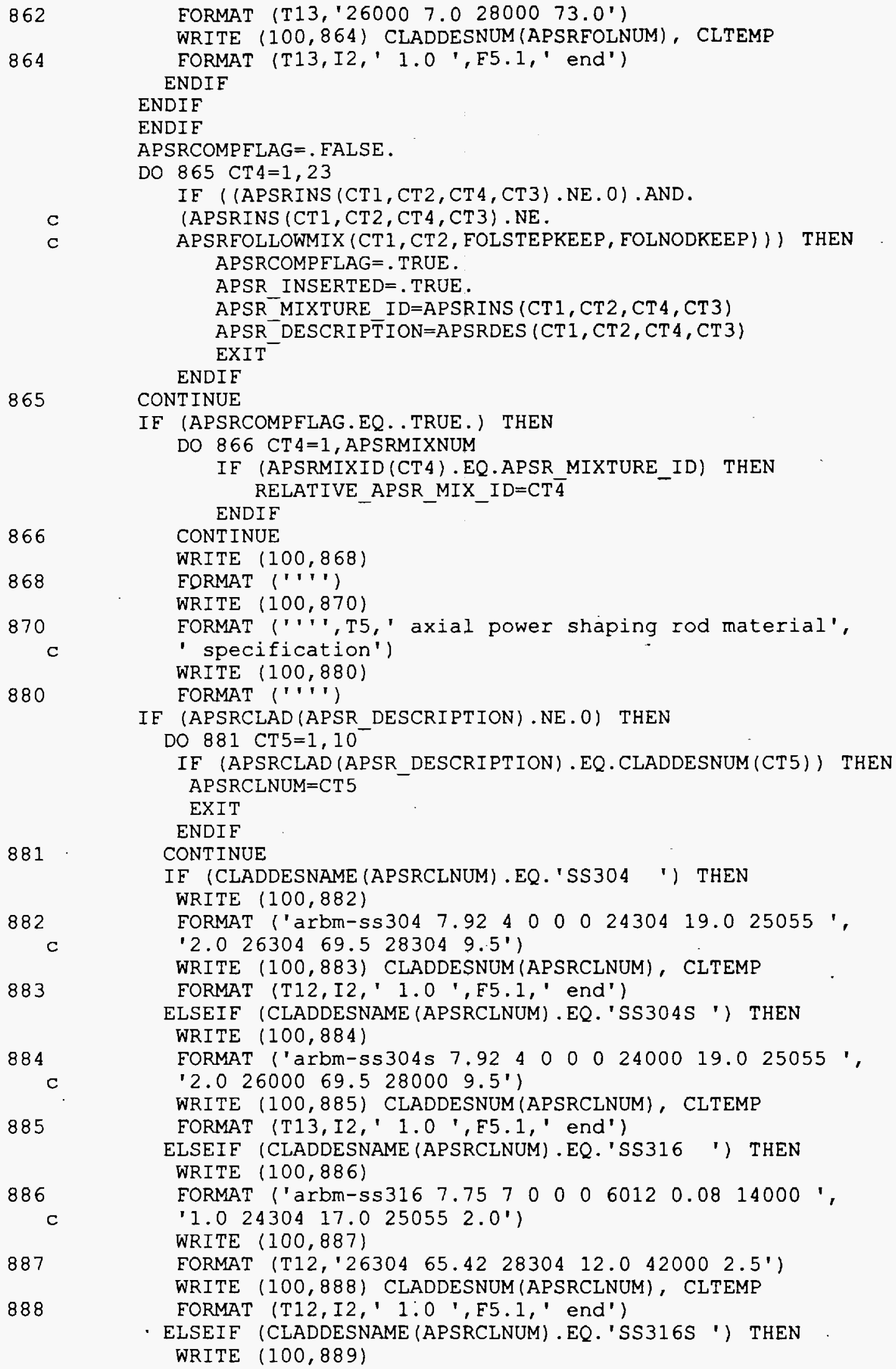




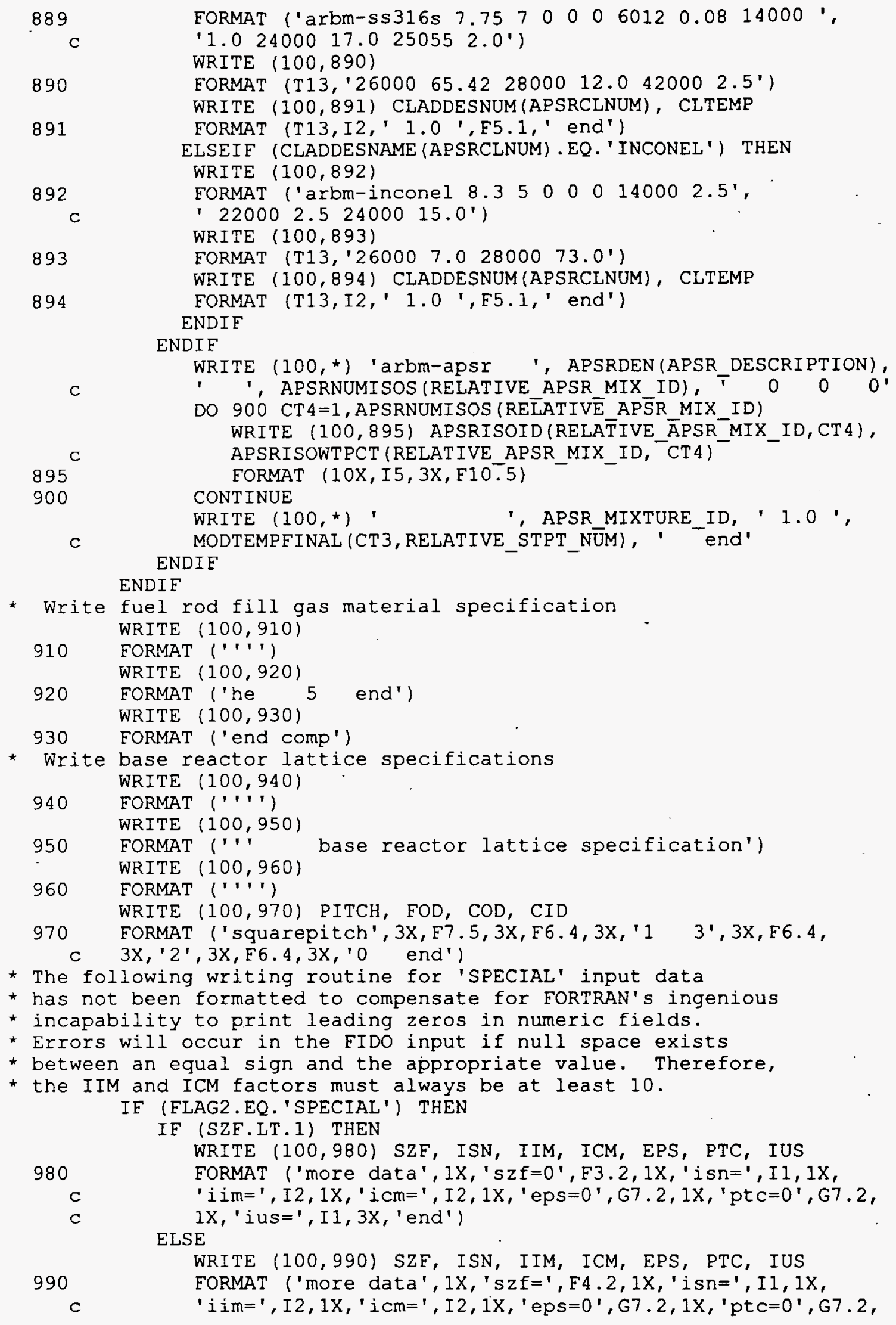




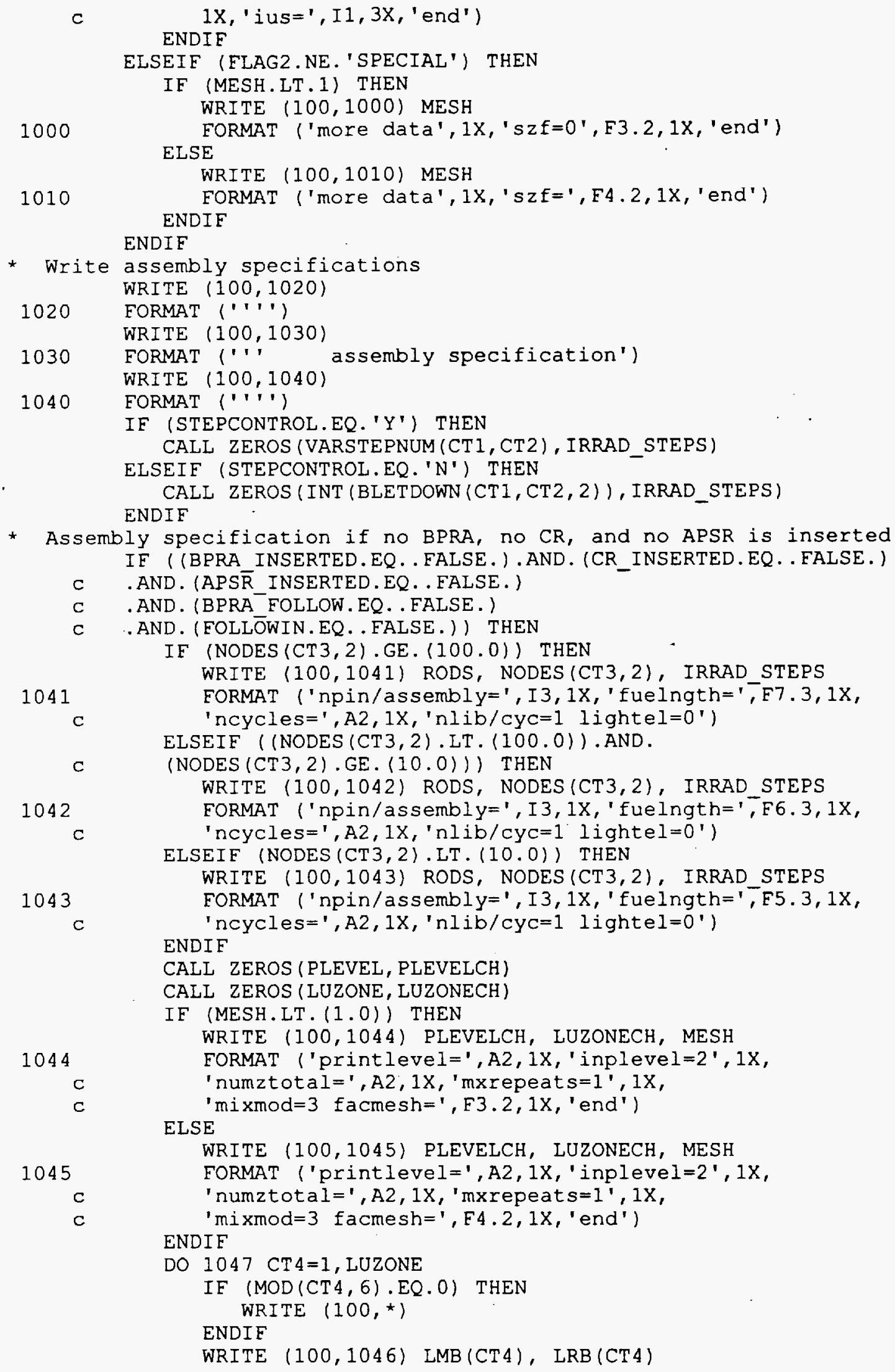


1046

1047

ENDIE

FORMAT (I $3,1 \mathrm{X}, \mathrm{F} 7.5,1 \mathrm{X}, \$)$

CONTINUE

WRITE $(100, \star)$

* Assembly specification if BPRA is inserted

IF (BPRA FOLLOW.EQ.. TRUE.) THEN

IE (NODDES $(C T 3,2)$.GE. (100.0)) THEN

WRITE $(100,1050)$ RODS, NODES $(C T 3,2)$, IRRAD STEPS

1050

C FORMAT ('npin/assembly=', I3, 1X, ' fuelngth=', F7.3, 1X, 'ncycles=', A2, IX, 'nlib/cyc=1 lightel=0')

ELSEIF ((NODES $(C T 3,2)$.LT. $(100.0))$.AND.

C

(NODES $(C T 3,2)$. GE. $(10.0)))$ THEN

WRITE $(100,1052)$ RODS, NODES $(C T 3,2)$, IRRAD STEPS

1052 FORMAT ('npin/assembly=', I3, IX, ' fuelngth=', F6.3, IX,

C

'ncycles=', A2, $1 \mathrm{x}$, 'nlib/cyc=1 lightel=0')

ELSEIF (NODES $(\mathrm{CT} 3,2)$. LT. $(10.0)$ ) THEN

WRITE $(100,1054)$ RODS, NODES $($ CT 3,2$)$, IRRAD STEPS

1054 FORMAT ('npin/assembly=', I3, 1X, ' fuelngth=', $55.3,1 \mathrm{X}$, ENDIF 'ncycles=', A2, 1X, 'nlib/cyc=1 lightel=0')

CALL ZEROS (PLEVEL, PLEVELCH)

CALI ZEROS (BPZONE (BPRA_DESCRIPTION_ID), BPZONECH)

IF (MESH.LT. (1.0)) THE $\bar{N}$ WRITE $(100,1056)$ PLEVELCH, BPZONECH, MESH FORMAT ('printlevel=', A2, 1X, 'inplevel=2', 1X, ' numztotal=' , A2, 1X, 'mxrepeats=1', 1X,

WRITE $(100,1058)$ PLEVELCH, BPZONECH; MESH

' numztotal=' , A2, 1X, 'mxrepeats $=1$ ', $1 \mathrm{X}$, ENDIF

'mixmod=3 facmesh=', F4.2, 1X, 'end')

DO 1062 CT $4=1$, BPZONE (BPRA_DESCRIPTION_ID)

IF (MOD $(C T 4,6) . E Q .0)$ THEN

ENDIF

WRITE $(100, *)$

WRITE $(100,1060)$ BPRFM (CT4,BPRA_DESCRIPTION_ID), FORMAT ('npin/assembly=', I3, 1X, 'fuelngth=',F7.3, 1X, 'ncycles=', A2, 1X, 'nlib/cyc=1 lightel=0')

ELSEIF ((NODES (CT3,2) . LT . (100.0)) . AND.

1102 FORMAT ('npin/assembly=', I3, 1X, 'fuelngth=1, F5.3,1X, ENDIF

'ncycles=', A2, 1X, 'nlib/cyc=1 lightel=0')

CALL ZEROS (PLEVEL, PLEVELCH)

CALL ZEROS (BPZONE (BPRA_DESCRIPTION_ID), BPZONECH) 
1104

c

C

1106

C

\section{ENDIF}

IF (MESH.LT. (1.0)) THEN

WRITE $(100,1104)$ PLEVELCH, BPZONECH, MESH

FORMAT ('printlevel=', A2, 1X, 'inplevel=2', 1X,

'numztotal=' , A2, 1X, 'mxrepeats=1', IX,

ELSE

'mixmod=3 facmesh=',F3.2, 1X, 'end').

WRITE $(100,1106)$ PLEVELCH, BPZONECH, MESH

FORMAT ('printlevel=', A2, 1X, 'inplevel=2', 1X,

' numztotal='., A2, IX, 'mxrepeats=1', IX,

'mixmod=3 facmesh=', F4.2,1X, 'end')

DO 1110 CT 4=1, BPZONE (BPRA DESCRIPTION ID)

IF (MOD (CT 4,6).EQ.0) THEN

ENDIF

WRITE $(100, \star)$

WRITE $(100,1108)$ BPMA (CT 4,BPRA_DESCRIPTION_ID),

C BPRA (CT 4 , BPRA DESCRIPTION ID)

CONTINUE

1108

ENDIF

WRITE $(100, *)$

* Assembly specification if $C R$ is inserted

IF (CR INSERTED.EQ. . TRUE.) THEN

IF (NODES $($ CT 3,2).GE. (100.0)) THEN

WRITE $(100,1120)$ RODS, NODES $(C T 3,2)$, IRRAD STEPS

1120

c

c

1130

c

1140

C

1150

C

C

1160

C

C

c

1161 EORMAT ('npin/assembly=', I 3, 1X, ' fuelngth=', $57.3,1 \mathrm{X}$, 'ncycles=', A2, $1 \mathrm{x}$, 'nlib/cyc=1 lightel=0')

ELSEIF ((NODES $(C T 3,2)$. LT . $(100.0))$. AND .

(NODES $(C T 3,2)$. GE. $(10.0))$ ) THEN

WRITE $(100,1130)$ RODS, NODES $(C T 3,2)$, IRRAD STEPS FORMAT ('npin/assembly=', I3,1X, ' fuelngth=', F6.3,1X, 'ncycles=', A2, 1X, 'nlib/cyc=1 lightel=0')

ELSEIF (NODES (CT 3,2).LT. (10.0)) THEN WRITE $(100,1140)$ RODS, NODES $(C T 3,2)$, IRRAD STEPS FORMAT ('npin/assembly=', I3, 1X, 'fuelngth=',F5.3, 1X, ENDIF

'ncycles=', A2, 1X, 'nlib/cyc=1 lightel=0')

CALL ZEROS (PLEVEL, PLEVELCH)

CALL ZEROS (CRZONE (CR_DESCRIPTION), CRZONECH)

IF (MESH.LT. (1.0)) THEN

WRITE $(100,1150)$ PLEVELCH, CRZONECH, MESH

FORMAT ('printlevel=', A2, 1X, 'inplevel=2', 1X,

'numztotal=', A2, 1X, 'mxrepeat $s=0$ ', 1X, ELSE

'mixmod=3 facmesh=', E3.2, 1X, 'end')

WRITE $(100,1160)$ PLEVELCH, CRZONECH, MESH

FORMAT ('printlevel=' , A2, $1 \mathrm{X}$, ' inplevel=2', $1 \mathrm{X}$,

' numztotal=', A2, $1 \mathrm{X}$, 'mxrepeats $=0$ ', $1 \mathrm{x}$, ENDIF

' mixmod=3 facmesh=',F4.2,1X,'end')

IF (STEPCONTROL.EQ. ' $N$ ') THEN

DO 1168 CT 4=1, INT (BLETDOWN (CT1, CT2, 2))

IF (CRINS (CT1, CT2, CT 4, CT 3). NE.0) THEN

DO 1162 CT5=1, CRZONE (CR_DESCRIPTION)

IF (MOD $(C T 5,6)$. EQ.0) THEN

ENDIF

WRITE $(100, \star)$

WRITE $(100,1161)$ CRMA (CT5,CR_DESCRIPTION), CRRA (CT5, CR_DESCRIPTION)

FORMAT $(I 3, \overline{1 X}, \mathrm{~F} 7.5,1 \mathrm{X}$, \$) 
CONTINUE

WRITE $(100, *)$

ELSEIF (CRINS (CT1,CT2, CT 4, CT 3) .EQ.0) THEN

DO 1166 CT $5=1$, CRZONE (CR DESCRIPTION)

IF (MOD $(C T 5,6)$. EQ.0) THEN

ENDIF

WRITE $\left(100,{ }^{\star}\right)$

WRITE $(100,1164)$ LMC(CT5,CR DESCRIPTION),

1164 LRC (CT 5, CR DESCRIPTION)

1166 FORMAT (I $3,1 \mathrm{X}, \mathrm{F} 7.5,1 \mathrm{X}, \$)$

ENDIF

1168 CONTINUE CONTINUE WRITE $(100, *)$

\section{ELSEIF (STEPCONTROL.EQ.' Y') THEN}

DO 1210 CT $4=1$, VARSTEPNUM (CT 1, CT 2)

IF (CRINS (CT1, CT2, CT 4, CT 3). NE.O) THEN

DO 1180 CT5=1, CRZONE (CR DESCRIPTION)

IF (MOD $(C T 5,6)$.EQ.0) THEN

WRITE $\left(100,{ }^{*}\right)$

ENDIF

WRITE $(100,1170)$ CRMA (CT5,CR_DESCRIPTION),

CRRA (CT5, CR DESCRIPTION)

1180

CONTINUE

FORMAT (I $3, \overline{1} X, F 7.5,1 X, \$)$

WRITE $(100, *)$

ËLSEIF (CRINS (CT1, CT2, CT4, CT3).EQ.0) THEN

DO 1200 CT $5=1$, CRZONE (CR_DESCRIPTION)

IF (MOD $(C T 5,6)$.EQ .0) THEN

ENDIF

WRITE $(100, *)$

WRITE $(100,1190)$ LMC (CT5,CR_DESCRIPTION),

1200 LRC (CT5, CR DESCRIPTION)

CONTINUE FORMAT (I3, 1X, E7 . 5, 1X, \$)

ENDIF

WRITE $(100, *)$

$1210 \quad$ CONTINUE

ENDIF

ENDIF

* Assembly specification if APSR is inserted

IF ((APSR INSERTED.EQ.. TRUE.). OR. (FOLLOWIN.EQ. TRUE.)) THEN

IF (NODESS(CT 3,2).GE. (100.0)) THEN

1220 WRITE $(100,1220)$ RODS, NODES $($ CT 3,2), IRRAD_STEPS FORMAT ('npin/assembly=', I 3, 1X,' fuelngth=', F7.3,1X, 'ncycles=', A2, 1X, 'nlib/cyc=1 lightel=0')

ELSEIF ((NODES (CT 3,2). LT . (100.0)). AND.

(NODES $(C T 3,2)$. GE. $(10.0)))$ THEN

WRITE $(100,1230)$ RODS, NODES $(C T 3,2)$, IRRAD STEPS

1230 FORMAT ('npin/assembly=', I3, 1X, ' fuelngth=', F6.3,1X, 'ncycles =', A2, 1X, 'nlib/cyc=1 lightel=0')

ELSEIF (NODES (CT 3,2).LT.(10.0)) THEN WRITE $(100,1240)$ RODS, NODES $(C T 3,2)$, IRRAD STEPS

1240 FORMAT ('npin/assembly=', I 3, 1X, 'fuelngth=',F5.3,1X,

c 'ncycles=', A2, 1X, 'nlib/cyc=1 lightel=0') ENDIF

CALL ZEROS (PLEVEL, PLEVELCH)

CALL ZEROS (APSRZONE (APSR DESCRIPTION), APSRZONECH)

IF (MESH. LT. (1.0)) THEN

WRITE $(100,1250)$ PLEVELCH, APSRZONECH, MESH 
1250

C

C

1252

C

c

1256

1258

C

1260

1262

1264

1266

1268

c.

C

1270

1280

FORMAT ('printlevel=', A2, 1X, 'inplevel=2',1X,

'numztotal=', A2, 1X, 'mxrepeats=0', 1X, ELSE

'mixmod=3 facmesh=',F3.2,1X, 'end')

WRITE $(100,1252)$ PLEVELCH, APSRZONECH, MESH

FORMAT ('printlevel=', A2, 1X, 'inplevel=2', 1X,

' numztotal=', A2, 1X, 'mxrepeats $=0$ ', $1 \mathrm{X}$,

ENDIF

'mixmod=3 facmesh=', $F 4.2,1 \mathrm{X}$ ' 'end')

IF (STEPCONTROL.EQ. 'N') THEN

DO 1268 CT $4=1$, INT (BLETDOWN (CT1, CT2, 2))

IF ((APSRINS (CT1, CT2, CT 4, CT 3) . NE.0) . AND.

(APSRFOLLOWDATA (CT 1, CT2, CT 4, CT 3 ). NE.3)) THEN

DO 1258 CT $5=1$, APSRZONE (APSR DESCRIPTION)

IF (MOD $(C T 5,6)$. EQ.0) THE $\bar{N}$

ENDIF

WRITE $(100, *)$

WRITE $(100,1256)$ APSRMA (CT5,APSR_DESCRIPTION),

APSRRA (CT5, APSR_DESCRIPTION)

CONTINUE

WRITE $\left(100,{ }^{*}\right)$

ELSEIF ( (APSRINS (CT 1, CT 2, CT 4, CT 3) .NE. 0) . AND.

(APSRFOLLOWDATA (CT 1, CT 2, CT 4, CT 3).EQ.3)) THEN

DO 1262 CT5=1, APSRZONE (APSR DESCRIPTION)

IF (MOD $(C T 5,6) . E Q .0)$ THE $\bar{N}$

ENDIF

WRITE $(100, *)$

WRITE $(100,1260)$ APSRFM(CT5,APSR_DESCRIPTION),

APSRFR (CT 5, APSR DESCRIPTION)

FORMAT $(I 3,1 X, F \overline{7} .5,1 X, \$)$

CONTINUE

WRITE $(100, *)$

ELSEIF (APSRINS (CT1,CT2,CT4,CT3).EQ. 0) THEN

DO 1266 CT5=1, APSRZONE (APSR DESCRIPTION)

IF (MOD $(C T 5,6) . E Q .0)$ THEN

ENDIF WRITE $(100$, *)

WRITE $(100,1264)$ LMD (CT5,APSR_DESCRIPTION),

C LRD (CT5, APSR DESCRIPTION)

FORMAT $(I 3,1 \bar{X}, F 7.5,1 X, \$)$

CONTINUE

ENDIF

WRITE $(100, *)$

CONTINUE

ELSEIF (STEPCONTROL.EQ.' $Y$ ') THEN

DO 1310 CT $4=1$, VARSTEPNUM (CT 1, CT2)

IF ((APSRINS (CT1,CT2, CT 4, CT3) . NE.0) . AND.

(APSRFOLLOWDATA (CT 1, CT2, CT 4, CT 3).NE. 3)) THEN

DO $1280 \mathrm{CT} 5=1$, APSRZONE (APSR DESCRIPTION)

IF (MOD $(C T 5,6)$.EQ.0) THEN

ENDIF

WRITE $(100, *)$

WRITE $(100,1270)$ APSRMA(CT5, APSR DESCRIPTION), APSRRA (CT5, APSR DESCRIPTION)

CONTINUE

WRITE $(100, *)$ 


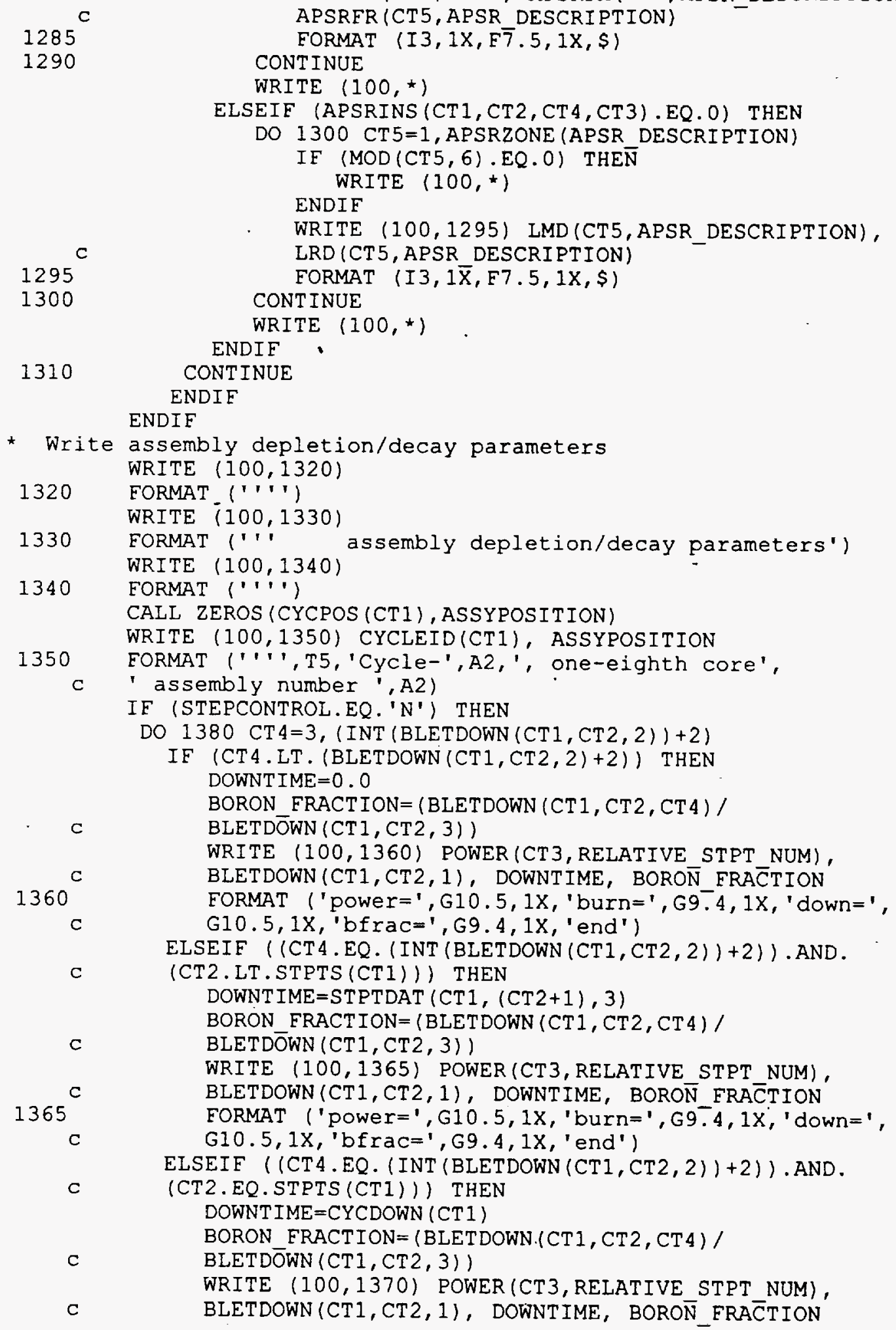


1370

1380 ENDIF

FORMAT ('power=' , G10.5, 1X, 'burn=', G9.4,1X, 'down=', CONTINUE

ELSEIE (STEPCONTROL.EQ.' 'Y') THEN

DO 1388 CT $4=1$, VARSTEPNUM (CT 1, CT 2$)$

IF (CT 4.LT.VARSTEPNUM(CT 1, CT2)) THEN

DOWNT IME $=0.0$

BORON FRACTION $=($ VARBLETDOWN $(\mathrm{CT} 1, \mathrm{CT} 2, \mathrm{CT} 4,2) /$

C

VARBLETDOWN (CT1, CT2, 1,2))

WRITE $(100,1382)$ VARPOWER (CT1, CT2, CT 4, CT3),

$1382^{\mathrm{C}}$

c

c

VARBLETDOWN (CT 1, CT2, CT 4, 1), DOWNTIME, BORON_ERACTION

FORMAT ('power=', G10.5, IX, 'burn=' , G9.4, 1X, 'down=',

G10.5, 1X, 'bfrac=', G9.4, $1 \mathrm{X}$, 'end' ')

ELSEIF ((CT4 .EQ.VARSTEPNUM (CT1, CT2)). AND.

(CT2.LT.STPTS (CT1))) THEN

DOWNT IME $=$ ST PTDAT $(C T 1,(C T 2+1), 3)$

BORON_FRACTION $=($ VARBLETDOWN $($ CT 1, CT 2, CT 4,2$) /$

VARBLE TDOWN (CT 1, CT $2,1,2)$ )

WRITE $(100,1384)$ VARPOWER (CT1, CT2, CT4, CT3),

$1384^{c}$

VARBLETDOWN (CT 1, CT 2, CT 4, 1), DOWNTIME, BORON

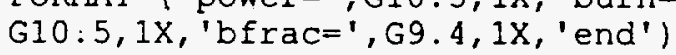

ELSEIF ((CT4.EQ.VARSTEPNUM (CT1, CT2)). AND.

c

(CT2.EQ.STPTS (CT1))) THEN

DOWNT I ME $=$ CYCDOWN (CT 1$)$

BORON FRACTION $=($ VARBLETDOWN $(\mathrm{CT} 1, \mathrm{CT} 2, \mathrm{CT} 4,2) /$

C

VARBLE $\bar{E} T$ DOWN (CT 1, CT $2,1,2)$ )

WRITE $(100,1386)$ VARPOWER (CT1, CT2, CT4, CT3),

1386

VARBLETDOWN (CT1, CT2, CT 4, 1), DOWNTIME, BORON_FRACTION

C

1388

ENDIF

FORMAT ('power=', G10.5, 1X, 'burn=' , G9. $4,1 \mathrm{X}$, ' $\mathrm{d}$ own=' ,

ENDIF

* Store final downtime for use in extraction script 'sedexecute.exe' FINALDOWNTIME=DOWNTIME

* Write input deck closing statement

WRITE $(100,1390)$

1390 FORMAT (''')

1400 FRITE $(100,1400)$ end of input')

1410 FORMAT $(\cdots, \cdots)$

WRITE $(100,1420)$

1420 FORMAT ('end')

CLOSE $($ UNIT $=100)$

RETURN

END

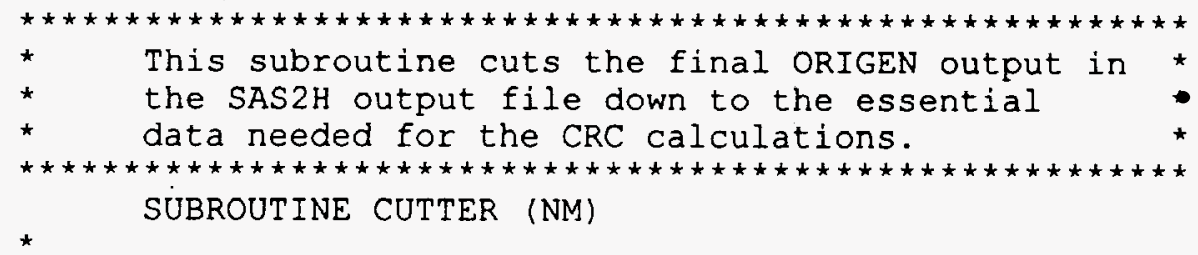

INTEGER * 4 LINECOUNTER, CUTLINE， NUM1， NUM2, C NUM3, NUM4, NUM5, NUM6, NUM7, SEDEXERESULT, 
C VERIFCOUNTER, VERIFCUTLINE, VERIEENDCUTLINE,

C OUTPUTREMOVALRESULT

*

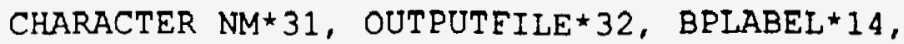

C LINVAL $\star 7$, SEDEXECOMMAND $* 60$, FORMATLABEL 29 ,

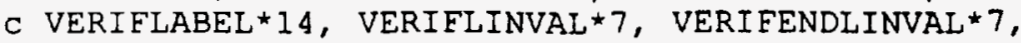

C OUTPUTREMOVAL $\$ 35$

*

LOGICAL BPEIND, NUMZEROFLAG, VERIFFIND

OUTPUTFILE $(1: 25)=\mathrm{NM}(1: 25)$

OUTPUTFILE $(26: 32)=$ ' . output'

OPEN (UNIT=700, FILE=OUTPUTEILE, STATUS='OLD')

REWIND (700)

LINECOUNTER $=0$

$B P F I N D=$. FALSE.

DO 14 WHILE (BPFIND.EQ..FALSE.)

LINECOUNTER $=$ LINECOUNTER +1

$\operatorname{READ}(700,12)$ BPLABEL

FORMAT (T98, A14)

IF (BPLABEL.EQ.' light elements') THEN

$\operatorname{READ}(700$, *)

READ $(700 ; 13)$ FORMATLABEL

13 FORMAT (T 46;A29)

IF (FORMATLABEL.EQ. 'nuclide concentrations, grams') THEN ELSE BPFIND $=$. TRUE.

BACKSPACE (700)

ENDIF

ENDIE

14 CONTINUE

NUMZEROFLAG $=$. FALSE.

CUTLINE=LINECOUNTER-2

NUMI $=$ INT $($ CUTLINE $/ 1000000.0)$

IF ((NUM1.EQ.0). AND. (NUMZEROELAG.EQ..FALSE.)) THEN $\operatorname{LINVAL}(1: 1)=$ ' '

ELSE

$\operatorname{LINVAL}(1: 1)=\mathrm{CHAR}(\operatorname{NUM} 1+48)$

NUMZEROFLAG $=$. TRUE .

ENDIF

NUM2 $=$ INT $(($ CUTLINE $-($ NUM1 $\star 1000000)) / 100000.0)$

IF ((NUM2 . EQ.0). AND. (NUMZEROFLAG.EQ..FALSE.)) THEN $\operatorname{INNVAL}(2: 2)=$ ' '

ELSE

$\operatorname{LINVAL}(2: 2)=\mathrm{CHAR}(\mathrm{NUM} 2+48)$

NUMZEROFLAG $=$. TRUE.

ENDIF

C (NUMI*1000000))/10000.0)

IF ((NUM3.EQ.0). AND. (NUMZEROFLAG.EQ. FALSE.)) THEN $\operatorname{LINVAL}(3: 3)=;$

ELSE

$\operatorname{LINVAL}(3: 3)=\mathrm{CHAR}(\operatorname{NUM} 3+48)$

ENDIF

NUMZEROFLAG $=$. TRUE.

NUM4 $=$ INT $(($ CUTLINE $-(N U M 3 * 10000)-$

C

$(N U M 2 * 100000)-(N U M 1 * 1000000)) / 1000.0)$

IF ((NUM4.EQ.0).AND. (NUMZEROFLAG.EQ..FALSE.)) THEN

$\operatorname{LINVAL}(4: 4)=$ ' ' 


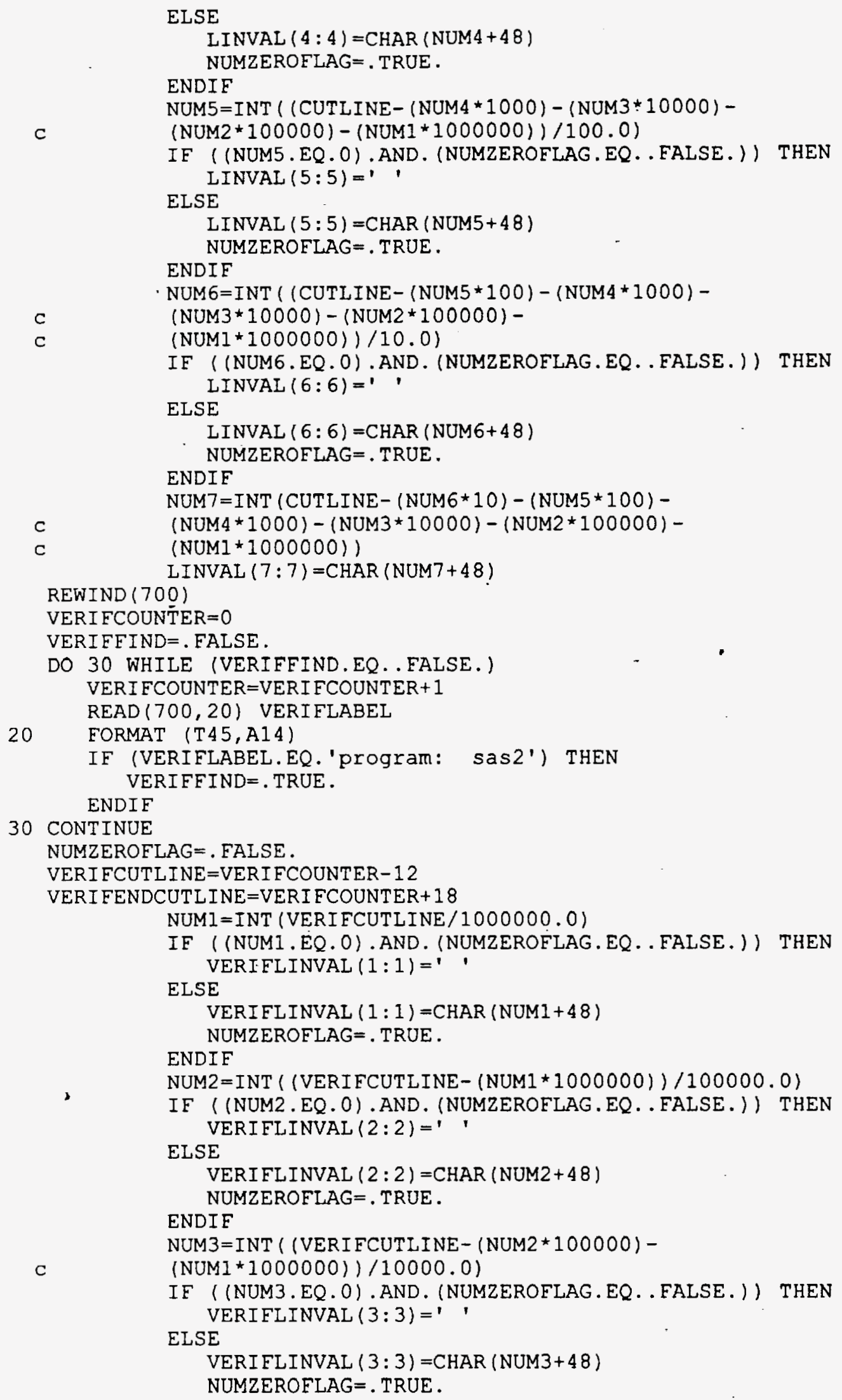




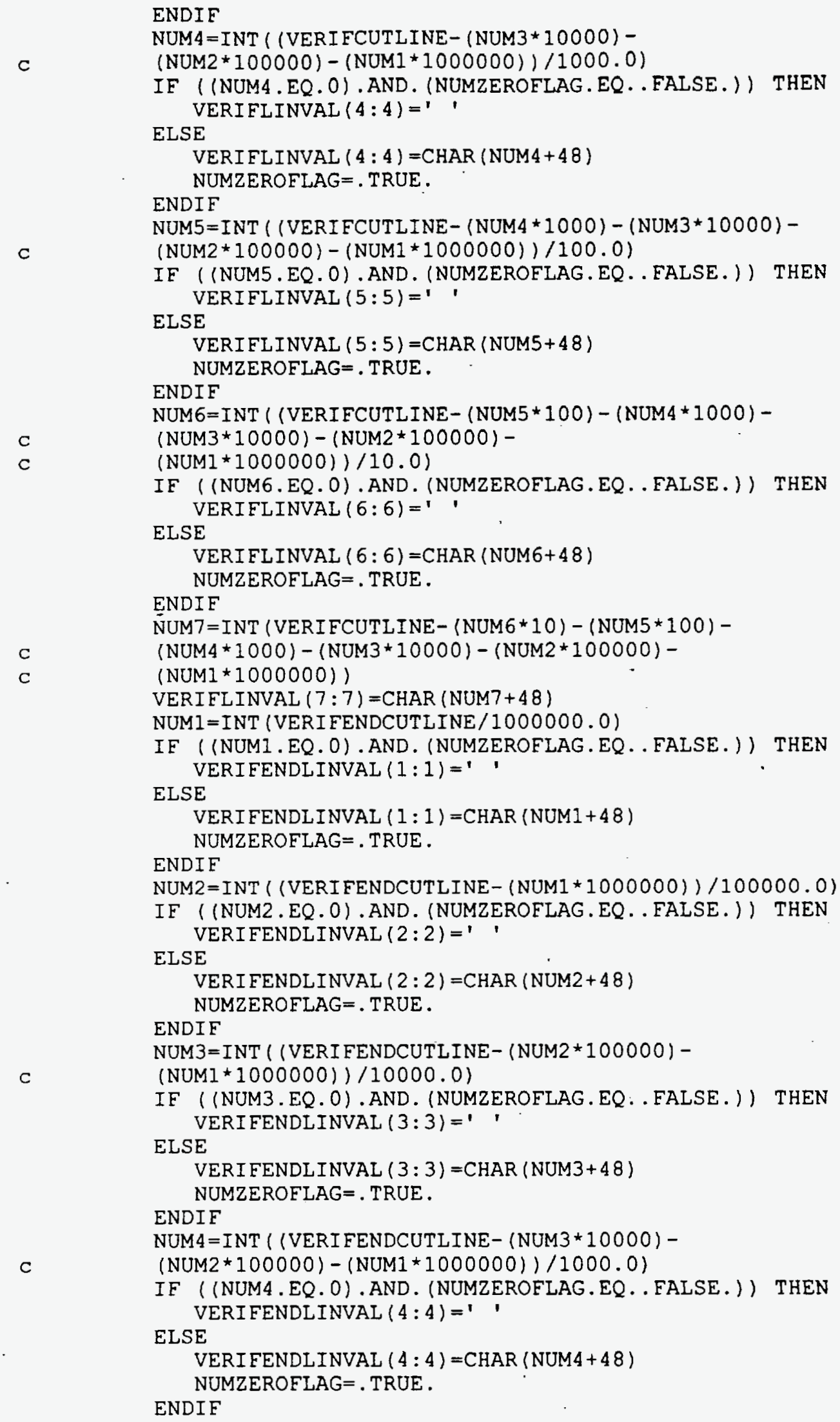




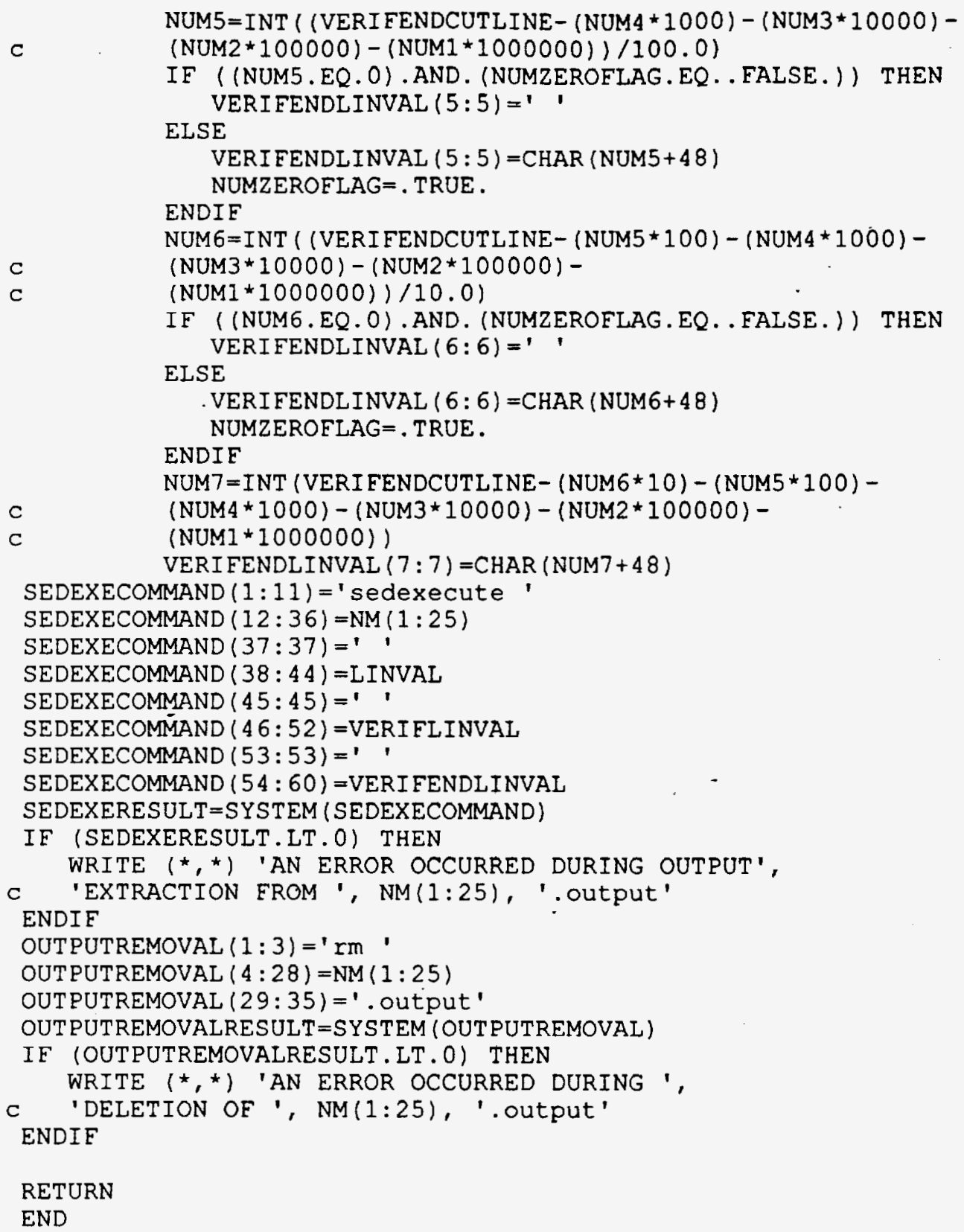

NUM5 $=$ INT $(($ VERIFENDCUTLINE $-($ NUM4 $\star 1000)-(N U M 3 * 10000)-$

IF ((NUM5.EQ.0).AND. (NUMZEROFLAG.EQ..FALSE.)) THEN LSE

VERIFENDLINVAL $(5: 5)=$ CHAR (NUM5 +48$)$

ENDIE

$\left(\right.$ NUM3 $\left.{ }^{\star} 10000\right)-($ NUM2 2100000$)-$

IF ((NUM6.EQ.0). AND. (NUMZEROFLAG.EQ. FALSE.)) THEN

VERIFENDLINVAL $(6: 6)='$ '

VERI FENDLINVAL $(6: 6)=$ CHAR $($ NUM $6+48)$

ENDIE

NUM7 = INT $($ VERIFENDCUTLINE $-($ NUM6* 10$)-($ NUM5 * 100) -

(NUM1*1000000))

VERIFENDLINVAI $(7: 7)=$ CHAR $($ NUM $7+48)$

SEDEXECOMMAND $(1: 11)=$ 'sedexecute '

COMMAN $(12: 36)=$ NM $(1: 25)$

SEDEXECOMMAND $(38: 44)=$ LINVAL

SEDEXECOMMAND $(45: 45)='$ '

SEDEXECOMMAND $(53: 53)='$ '

SEDEXECOMMAND $(54: 60)=$ VERI FENDLINVAL

SEDEXERESULT $=$ SYSTEM (SEDEXECOMMAND)

IE (SEDEXERESULT.LT.0) THEN

ENDIE

OUT PUTREMOVALRESULT =SYSTEM (OUT PUTREMOVAL)

(OUTPUTREMOVALRESULT.LT. O) THEN
WRITE $\left({ }^{\star},{ }^{*}\right)$ 'AN ERROR OCCURRED DURING ',

ENDIE

END

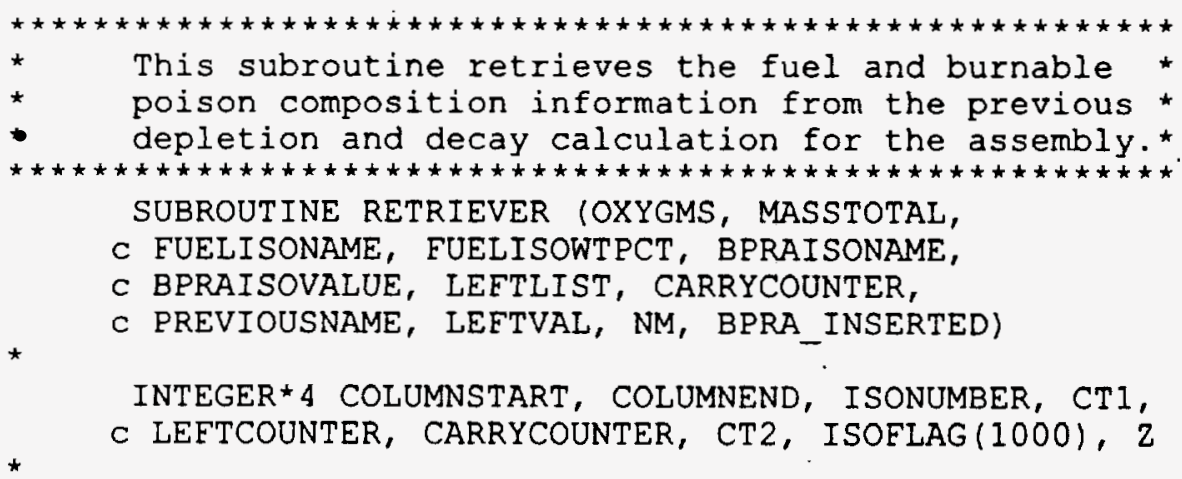


REAL ISOVALUE (1000), BPRAISOVALUE (2), MASSTOTAL,

C FUELISOVALUE (1000), FUELISOWTPCT (1000), OXYGMS,

C LEETVAL (1000)

*

CHARACTER ROWFLAG $\star 7, \operatorname{COL} 1 \star 8, \operatorname{COL} 2 \star 8, \operatorname{COL} 3 \star 8, \operatorname{COL} 4 \star 8$, C COL $5 \star 8, \operatorname{COL} 6 \star 8, \operatorname{COL} 7 \star 8, \operatorname{COL} 8 \star 8, \operatorname{ACTINIDELABEL} \star 9$,

C FORMATLABEL $\star 29$, ISOLABEL $\star 6$, I SONAME $(1000) \star 6$,

C FISSPRODLABEL * 16, BPRAISONAME (2) *6, ORIGNAME $(297) \star 6$,

C LIBRARYID $(297) \star 5$, $\operatorname{EUELISONAME}(1000) * 5, \operatorname{LEFTLIST}(1000) * 6$,

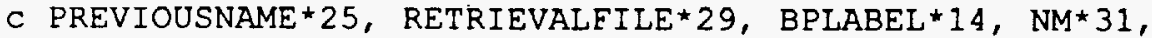

C NOTESFILE`31

LOGICAL ROWFLAGLOG, ACTINIDEFIND, FISSPRODFIND, BPFIND, C BPRA INSERTED

DATA (LIBRARYID(Z), $Z=1,297$ ) /' 1001',

C '1002',' $1003^{\prime}, ' 2003^{\prime}, ' 2004^{\prime}, ' 3006^{\prime}$ '

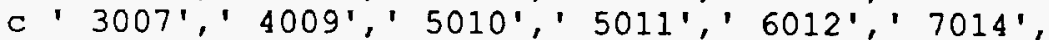

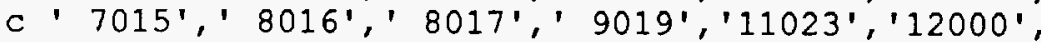

c '13027','14000','15031','16000','16032','17000',

c '19000','20000','22000','23000','24000', '25055',

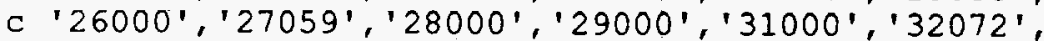

c '32073','32074', '32076', '33075', '34074', '34076',

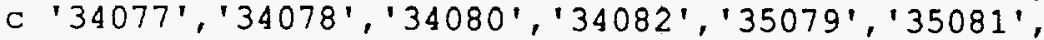

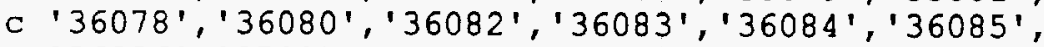

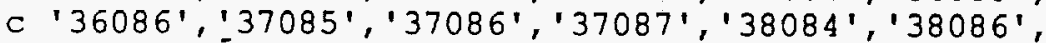

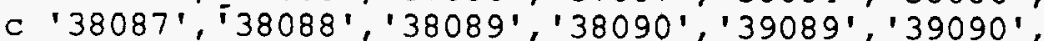

c '39091', '40000', '40090', '40091', '40092', '40093',

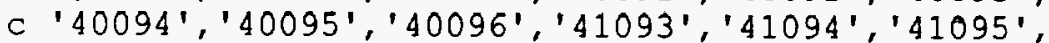

c '42000','42092','42094', '42095', '42096', '42097',

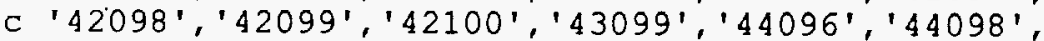

c '44099','44100', '44101', '44102', '44103', '44104',

c '44105','44106', '45103', '45105', '46102', '46104',

c '46105', '46106', '46107', '46108', '46110', '47107',

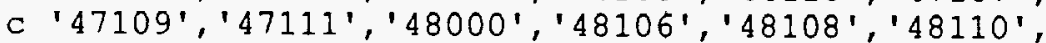

c '48111', '48112', '48113', '48114', '48116', '48601',

c '49113','49115','50112','50114','50115','50116',

c '50117', '50118', '50119', '50120', '50122', '50123',

c '50124', '50125', '50126', '51121', '51123', '51124',

c '51125', '51126', '52120', '52122', '52123', '52124',

c '52125', '52126', '52128', '52130', '52132', '52601',

c '52611', '53127', '53129', '53130', '53131', '53135',

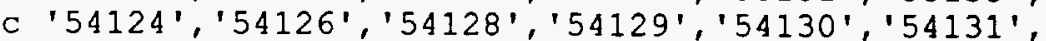

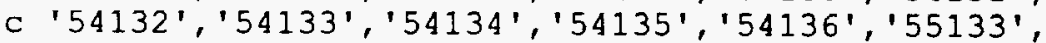

c '55134','55135', '55136', '55137', 56134', $56135^{\prime}$ ',

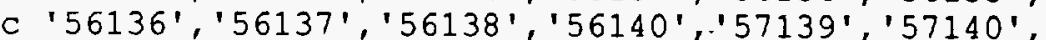

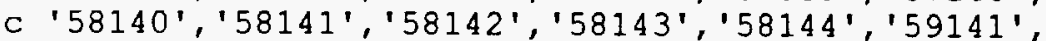

c '59142', '59143', '60142', '60143', '60144', '60145',

c '60146', '60147', '60148', '60150', '61147', '61148',

c '61149', '61151', '61601', '62144','62147','62148',

c '62149', '62150', '62151', '62152', '62153', '62154',

c '63000', '63151', '63152', '63153', '63154', '63155',

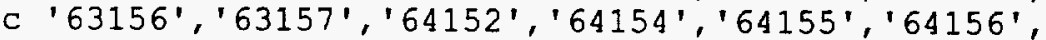

c '64157','64158','64160','65159', '65160', '66160',

c '66161', '66162', '66163', '66164', '67165', '68166',

c '68167','71175', $71176^{\prime}, ' 72000^{\prime}, ' 72174^{\prime}, ' 72176^{\prime}$ ',

c '72177', $72178^{\prime}, ' 72179^{\prime}, \cdot 72180^{\prime}, ' 73181^{\prime}, ' 73182^{\prime}$,

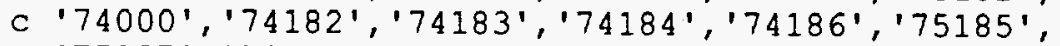

c '75187', '79197', '82000', '83209', '90230', '90232',

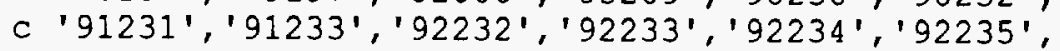




$$
\begin{aligned}
& \text { C'u238','np237 ', 'np238 ', 'pu236 ', 'pu237' ', } \\
& \text { c 'pu238','pu239' ', 'pu240 ','pu241','pu242 ', } \\
& \text { c 'pu243 ', 'pu244 ', 'am241 ', 'am242 ',' 'am243 ', } \\
& \text { c 'am242m', 'cm241 ', ' } \mathrm{cm} 242 \text { ', ' } \mathrm{cm} 243 \text { ' }^{\prime},{ }^{\prime} \mathrm{cm} 244 \text { ', }
\end{aligned}
$$

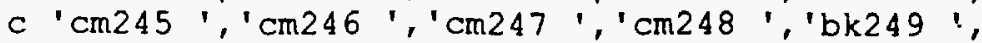

$$
\begin{aligned}
& \text { c ' cf249', 'cf250', ' cf251 ', 'cf252', 'cf253', } \\
& \text { c 'es253'/ }
\end{aligned}
$$

RETRIEVALFILE $(1: 25)=$ PREVIOUSNAME

RETRIEVALFILE $(26: 29)=$ ' . cut'

NOTESFILE $(1: 25)=\mathrm{NM}(1: 25)$

NOTESFILE $(26: 31)=$ '. notes'

OPEN (UNIT $=300$, FILE=RETRIEVALFILE, STATUS='OLD')

OPEN (UNIT $=500$, FILE=NOTESFILE, STATUS= 'UNKNOWN')

REWIND (300)

REWIND (500)

DO 5 CT $1=1,1000$

ISOVALUE $($ CT 1$)=0.0$

FUELISOVALUE (CT 1$)=0.0$

EUELISOWTPCT $(\mathrm{CT} 1)=0.0$

LEFTVAL $=0.0$

I SONAME $=$ '

FUELISONAME =

LEFTLIST $=$ '

ISOFLAG $=0$

5 CONTINUE

ROWFLAGLOG $=$. FALSE .

DO 11 WHILE (ROWFLAGLOG.EQ..FALSE.)

READ $(300,10)$ ROWFLAG, COL1, COL2, COL3,

C COL $4, \operatorname{COL} 5, \operatorname{COL} 6, \operatorname{COL} 7, \operatorname{COL} 8$

10 FORMAT (T15, A7, T24, A8, T34, A8, T44, A8, T54, A8,

C $\quad \mathrm{T} 64, \mathrm{~A} 8, \mathrm{~T} 74, \mathrm{~A} 8, \mathrm{~T} 84, \mathrm{~A} 8, \mathrm{~T} 94, \mathrm{~A} 8$ )

IF (ROWFLAG.EQ. 'initial') THEN ROWFLAGLOG $=$. TRUE.

ENDIE

11 CONTINUE

IF (COL1.NE.' COLUMNSTART $=23$

COLUMNEND $=32$

ENDIF

IF (COL2.NE.' COLUMNSTART $=33$

ENDIF COLUMNEND $=42$

IF (COL3.NE.' COLUMNSTART $=43$ COLUMNEND $=52$

ENDIF

IF (COL4.NE.' COLUMNSTART $=53$ COLUMNEND $=62$

ENDIE
IE (COL5.NE.' COLUMNSTART $=63$ COLUMNEND $=72$

ENDIF

IF (COL6.NE.' COLUMNSTART $=73$

') THEN COLUMNEND $=82$

ENDIF

') THEN

') THEN

') THEN

1) THEN

-) THEN 


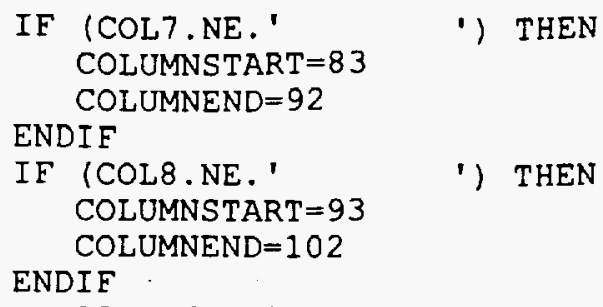

\section{* Get $B-10$ and $B-11$ composition data for BPRA}

IF (BPRA_INSERTED.EQ.. TRUE.) THEN

BPRAI SOVALUE (1) $=0.0$

BPRAISOVALUE $(2)=0.0$

BPRAISONAME $(1)=$ '

BPRAISONAME $(2)=$ '

REWIND (300)

$B P F I N D=$. FALSE.

DO 14 WHILE (BPFIND.EQ..FALSE.)

12

READ $(300,12)$ BPLABEL

2 FORMAT (T98,A14)

IF (BPLABEL.EQ.' 'light elements') THEN

$$
\operatorname{READ}(300, \star)
$$

13 FORMAT (T46, A29)

IF (FORMATLABEL.EQ. 'nuclide concentrations, grams') THEN

$$
\text { ENDİF }
$$
ENDIF

14 CONTINUE

DO 24 CT $1=1,25$

22

READ $(300,22)$ BPRAISONAME (1)

FORMAT (T6, A6)

IF (BPRAISONAME (1).EQ.' b 10 ') THEN

BACKSPACE (300)

EXIT

24 CONTINUE

CONTINUE
READ 1300

26 FORMAT (T $6, A 6, T<$ COLUMNSTART $>$, G10.2)

READ $(300,29)$ BPRAISONAME (2), BPRAISOVALUE (2)

29 FORMAT ( T 6, A6, T<COLUMNSTART>, G10.2)

IF (BPRAISONAME (1).EQ.' b 10 ') THEN

ENDIF BPRAISONAME $(1)={ }^{\prime} 5010^{\prime}$

IF (BPRAISONAME (2).EQ.' b 11 ') THEN ENDIF BPRAISONAME (2) $=$ ' 5011'

ENDIF

* Get fuel composition data

REWIND (300)

ACTINIDEFIND $=$. FALSE.

DO 50 WHILE (ACTINIDEFIND.EQ..FALSE.)

READ (300,30) ACTINIDELABEL

30 FORMAT (T103, A9)

IF (ACTINIDELABEL.EQ.' actinides') THEN $\operatorname{READ}(300, *)$

40 FORMAT (T46, A29)

IF (FORMATLABEL.EQ. 'nuclide concentrations, grams') THEN ENDIF 
Title: CRC Depletion Calculations for the Rodded Assemblies in Batches 1, 2, 3, and IX of Crystal River Unit 3

Document Identifier: BBA000000-01717-0200-00040 REV 00 Date Printed: 4/3/97 Attachment II, Page 81 of 82

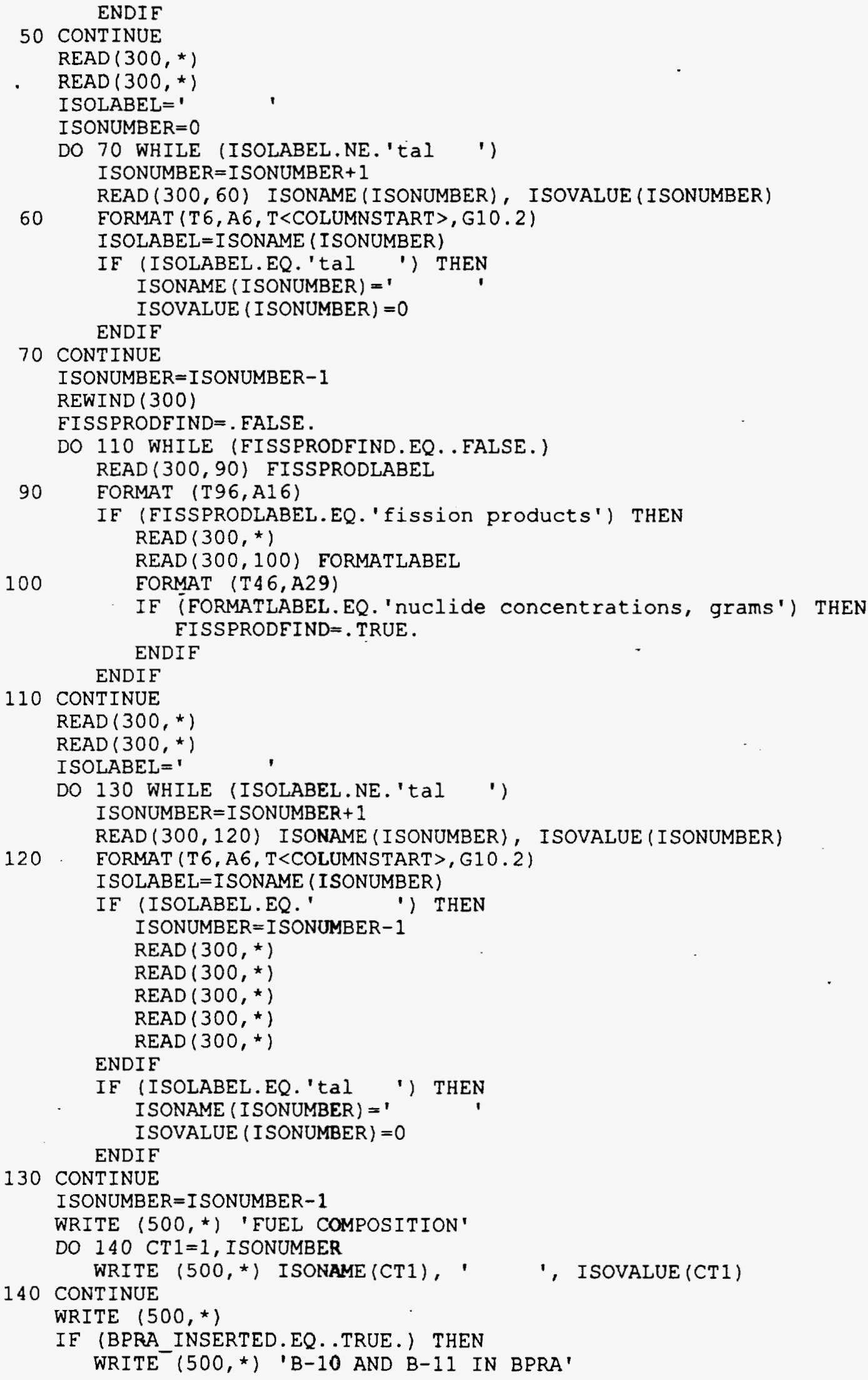




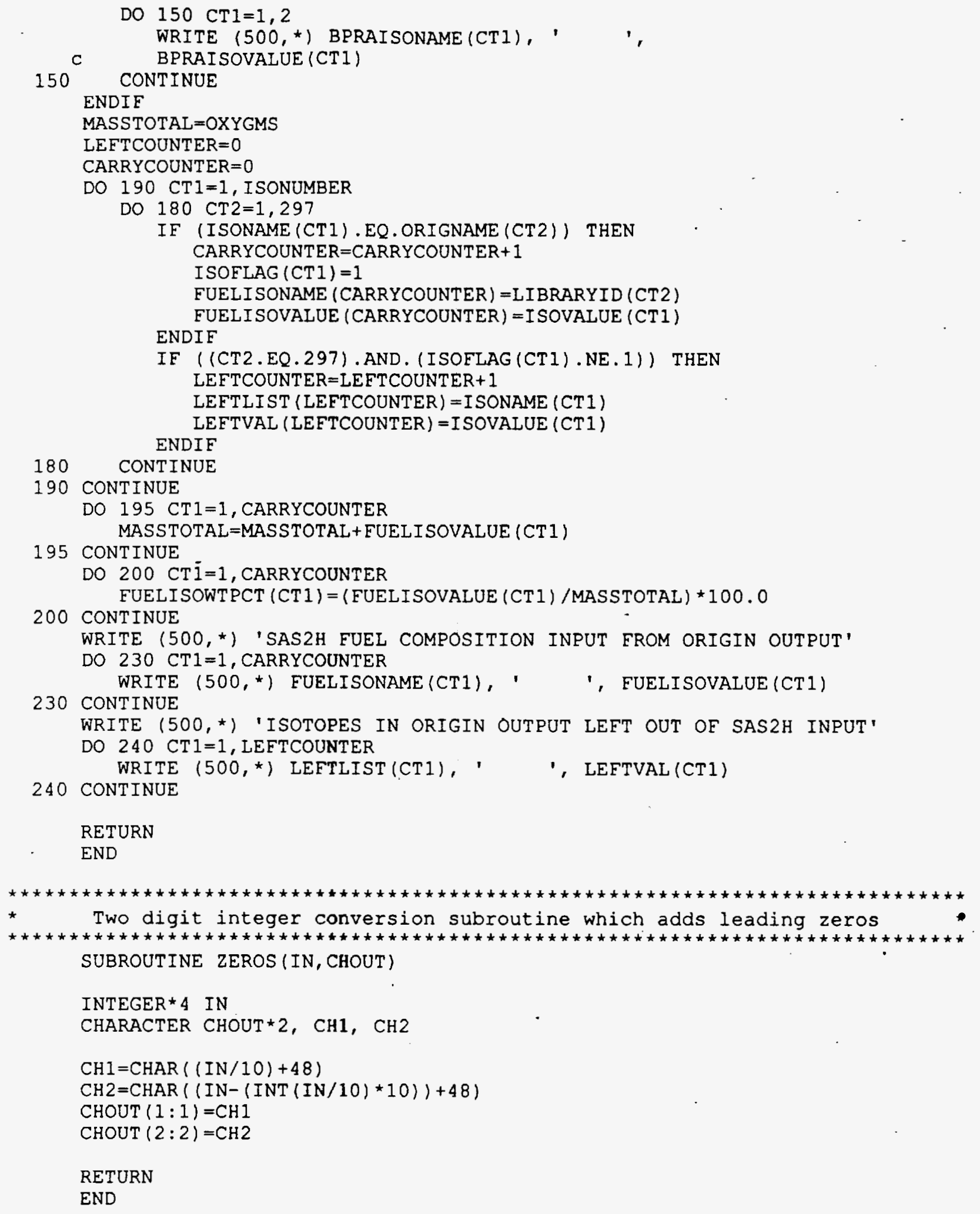




\section{Attachment III}

\section{RLAYOUT Version 1.0 Program Description}




\section{RLAYOUT Version 1.0 \\ Rodded Assembly Irradiation History Layout Developer}

Developed by Kenneth D. Wright

Framatome Cogema Fuels

High-Level Waste Division

under contract with the

Management and Operating Contractor for the

Yucca Mountain High-Level Radioactive Waste Repository Project 


\section{Table of Contents}

Item

Page

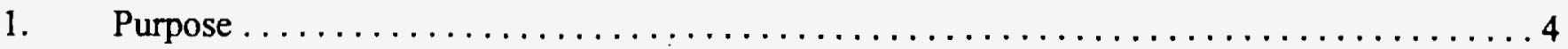

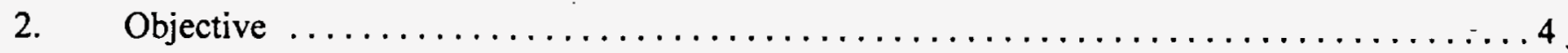

3. Methodology $\ldots \ldots \ldots \ldots \ldots \ldots \ldots \ldots \ldots \ldots \ldots \ldots \ldots \ldots \ldots \ldots \ldots \ldots \ldots$

4. Required File Structures for the Input to RLAYOUT $\ldots \ldots \ldots \ldots \ldots \ldots \ldots \ldots \ldots$

5. Calculations Performed by the RLAYOUT Program $\ldots \ldots \ldots \ldots \ldots \ldots \ldots \ldots \ldots \ldots$

6. Example of an Irradiation History Layout for a Rodded Assembly Developed by RLAYOUT . 8

7. RLAYOUT Version 1.0 Fortran Source Code Listing $\ldots \ldots \ldots \ldots \ldots \ldots \ldots \ldots \ldots$ 


\section{Purpose}

The RLAYOUT program was written to support Commercial Reactor Critical (CRC) evaluations. The CRC evaluations are performed as part of the disposal criticality methodology development for the proposed Yucca Mountain High-Level Radioactive Waste Repository.

\section{Objective}

The objective of the RLAYOUT program is to develop the irradiation time step layouts for CRC depletion calculations performed by SAS2H such that the assembly axial node irradiation histories are simulated according to relevant data. The data that is relevant to determining an appropriate irradiation time step layout for a CRC SAS2H depletion calculation includes the following:

1) the control rod assembly (CRA) or axial power shaping rod assembly (APSRA) insertion time in a given axial node of an assembly during each CRC statepoint depletion calculation;

2) the boron letdown data measured at nominal full-power conditions;

3) the minimum desired cross-section update frequency to be implemented in the CRC SAS2H depletion calculations.

The RLAYOUT program is intended to be used without supporting documentation through the implementation of run-time user defined input prompts.

\section{Methodology}

The methodology employed by the RLAYOUT program is to prompt the user for a minimum set of required information, and develop appropriate irradiation layouts for the CRC statepoint calculations in which the assembly of interest contains either a CRA or an APSRA. The developed irradiation layouts will provide the following information:

1) the number of SAS2H irradiation time steps required for each statepoint depletion calculation;

2) the duration of each SAS2H irradiation time step required for each statepoint depletion calculation;

3) the soluble boron concentration at the mid-point of each SAS2H irradiation time step required for each statepoint depletion calculation;

4) the identification of which axial assembly nodes in which SAS2H irradiation time steps should have either a CRA or an APSRA inserted.

The RLAYOUT program prompts the user for a minimum set of input information required to develop the irradiation history layouts. This minimum set of input information includes the following:

1) the assembly archive identifier (i.e., the label designated to the assembly such that it may be uniquely identified in the CRC depletion calculation archives); 
2) the number of axial nodes to be utilized in the CRAFT calculations;

3) the number of rodded CRC statepoint calculations required for the assembly depletion;

4) the number of reactor cycles in which the assembly is inserted and has a rodded statepoint calculation (this number is used to identify the number of files corresponding to the boron letdown data for the relevant reactor cycles);

5) the filenames corresponding to the boron letdown data for each reactor cycle relevant to the assembly CRC depletion calculations;

6) the cycle identifier (2 characters), and each rodded statepoint depletion calculation's starting and ending EFPD values (floating point);

7) the filename containing the CRA and/or APSRA nodal insertion history data.

In SAS2H, the duration of an irradiation interval may be separated into a number of time steps of variable length. Typically, an irradiation interval is a CRC statepoint depletion calculation interval, or the continuous irradiation time required to go from one CRC statepoint to another. To follow the CRA or APSRA insertion histories, detailed intra-cycle variable irradiation time steps are required. This is due to the fact that the CRs and APSRs are only present in a given axial node of an assembly for a given period of exposure during a statepoint depletion calculation. A user specified number of cross-section library updates are performed during each time step of an irradiation interval. The boron letdown curve of the reactor cycle may also be followed by specifying, at each irradiation step, a fraction of the soluble boron concentration defined in the base moderator material specification. This boron concentration is applied uniformly over the irradiation time step. The boron concentration fraction at the mid-point of each irradiation time step is specified in the SAS2H depletion calculations of this analysis to appropriately follow boron letdown curves. Considering the cross-section update frequency, the boron letdown data, and the absorber rod assembly insertion histories, the following three primary requirements apply to determining an appropriate reactor cycle irradiation layout for a rodded assembly.

1) The duration of each time step should be specified such that a maximum of 80 days of irradiation is not exceeded between cross-section updates. The SAS2H calculations in this analysis utilize one cross-section update per irradiation step. Therefore, the maximum duration of any time step in any reactor cycle irradiation layout of this analysis should not exceed 80 days. The 80 day limit is an arbitrary limit based on engineering judgement. The 80 day irradiation time step limit should assure that the isotopic concentrations of the system (primarily fuel and borated moderator) will not alter the neutron spectrum radically enough to cause a time step of the depletion calculation to be performed without the availability of cross-sections which have been properly weighted with an appropriate neutron spectrum and spatial flux.

2) Any radical perturbations in the boron letdown curve should be followed by defining irradiation time step durations such that the average boron concentration over each time step is representative of the actual boron letdown. Usually, the 80 day time step limit imposed for cross-section update frequency is adequate to properly follow a reactor cycle's boron letdown curve.

3) The duration of each time step should be specified such that the insertion of a CRA or APSRA in a given assembly axial node may be modeled for the correct exposure time in 
terms of EFPD. A more detailed description of the meaning this statement is warranted. In SAS2H, there is an option to vary the Path B unit cell model between irradiation steps as long as the number of radial zones in the Path B models of a given SAS2H calculation (i.e., statepoint depletion calculation) remain the same. Therefore, an assembly axial node represented in a given SAS2H statepoint depletion calculation that has a CRA or APSRA insertion history for a specified period of exposure (that is a fraction of the exposure covered by the statepoint depletion calculation) may be modeled appropriately by changing the Path $B$ model from one representing the insertion of a CRA or APSRA to one representing the removal of a CRA or APSRA at the appropriate time step (corresponding to the CRA or APSRA removal time).

All three of the requirements previously described must be correctly addressed in the SAS2H input decks developed for each axial node of an assembly in each statepoint depletion calculation. Assuring that the cross-section update frequency and the boron letdown curves are properly modeled is usually a by-product of developing the irradiation layouts for the statepoint depletion calculations containing either CRA or APSRA insertion history. The irradiation time step layout for a given statepoint depletion calculation must be developed such that breakpoints exist between irradiation time steps, that allow for the appropriate removal or insertion of a CRA or APSRA, to obtain the correct integrated neutron spectrum exposure for each axial node of the assembly. It becomes obvious then that the complexity of the irradiation time step layout for a given statepoint calculation is proportional to the number of CRC axial nodes being modeled and the frequency of CRA or APSRA movement during the assembly depletion. The time steps developed to model CRA or APSRA insertion histories are also designed to encompass the cross-section update and boron letdown requirements. RLAYOUT automates this development of appropriate irradiation time step layouts for the statepoint depletion calculations of an assembly containing either a CRA or APSRA insertion history.

\section{Required File Structures for the Input to RLAYOUT}

The RLAYOUT program utilizes two types of input data files. The first type of input data file contains the boron letdown data for a given reactor cycle that is needed by RLAYOUT to develop the SAS2H irradiation layouts. The second type of input data file contains the nodal CRA and/or APSRA insertion times for each CRC statepoint depletion calculation. Each of these files must follow a specific layout to be read properly by the RLAYOUT program.

The files containing boron letdown data should only contain data for a single reactor cycle. The format of these boron letdown data files should consist of the following:

1) the lines of input data should begin at the top of the file;

2) there should be no blank lines present in the input data;

3) each line should contain two values delineated by spaces;

4) the first value on a line of input should be the EFPD value corresponding to the boron data that is subsequently provided on the same input line;

5) the second value on a line of input should be the measured soluble boron concentration (ppm) in the moderator at the corresponding EFPD value;

6) a minimum of one blank line should appear at the bottom of the file. 
The files containing rod insertion time data should provide the rod insertion time data for each statepoint depletion calculation to be performed for the assembly in which a CRA and/or APSRA is inserted. The rod insertion time data for each statepoint depletion calculation should be provided in the order in which the statepoint depletion calculations are performed. Each set of rod insertion time data for each statepoint depletion calculation must contain a line of input corresponding to the specified number of axial nodes as input by the user when executing the RLAYOUT program. The rod insertion time input data file for an assembly should correspond to a specific format as follows:

1) the node number corresponding to a line of input containing a rod insertion time should begin in column one of the input file;

2) the node number "1" (always the top node) should be used to delineate a set of nodal rod insertion times for a given CRC statepoint depletion calculation;

3) the rod insertion time on a given line of input should be specified in terms of EFPD of rod insertion during the statepoint calculation (this value should be based on an average power that is calculated from the same nodal burnup data that will be utilized in the corresponding CRAFT calculation);

4) the rod insertion time on a given line of input should be located in columns 60 through 74 ;

5) each set of nodal rod insertion time input data provided for each CRC statepoint depletion calculation must contain data for the full number of axial nodes as specified by the user in the input to RLAYOUT;

6) the nodal input data for a given CRC statepoint depletion calculation must always be specified in sequential order according to node number;

7) any comments or other data may appear in the input files as long as it does not interfere with the format described in numbers one through six above.

\section{Calculations Performed by the RLAYOUT Program}

The RLAYOUT program performs only one calculation. This is a simple linear interpolation calculation within the user provided boron letdown data to determined the soluble boron concentration at the midpoint of each irradiation time step that is defined by RLAYOUT. The RLAYOUT code utilizes the following standard linear interpolation scheme: 


$$
\frac{\text { Target Value }-x_{1}}{\text { Reference Value }-y_{1}}=\frac{x_{2}-x_{1}}{y_{2}-y_{1}}
$$

where,

Target Value $=$ the value for which the interpolation is being performed to obtain; Reference Value = the known value which has a one-to-one correspondence to the Target Value;

$x_{1}=$ the target parameter value displayed in the table which corresponds to $y_{1}$;

$x_{2}=$ the target parameter value displayed in the table which corresponds to $y_{2}$;

$y_{1}=$ the reference parameter value displayed in the table which is the largest value less than the Reference Value;

$y_{2}=$ the reference parameter value displayed in the table which is the smallest value greater than the Reference Value.

\section{Example of an Irradiation History Layout for a Rodded Assembly Developed by RLAYOUT}

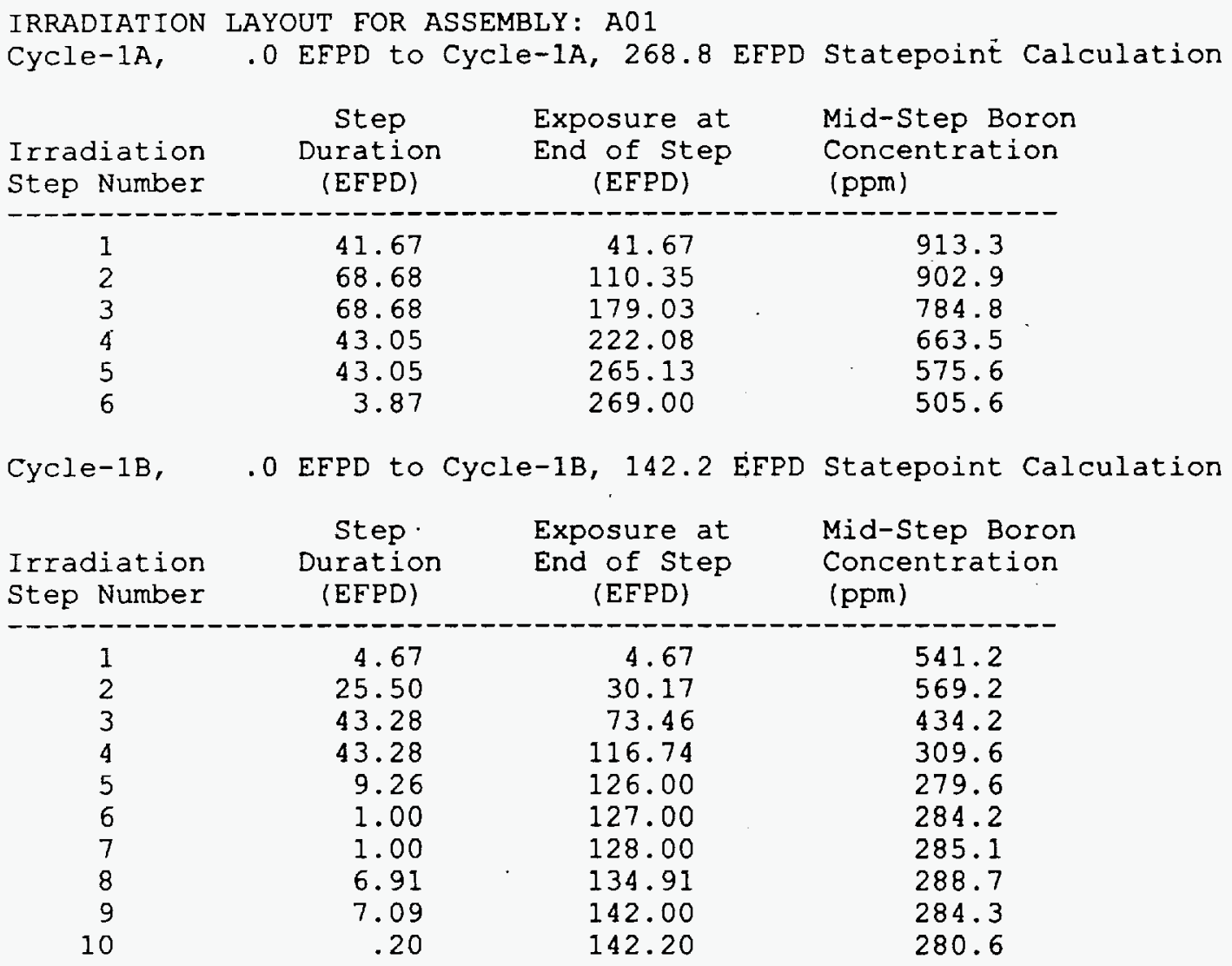

Cycle-1B, 142.2 EFPD to Cycle-1B, 171.3 EFPD Statepoint Calculation 
Title: CRC Depletion Calculations for the Rodded Assemblies in Batches 1, 2, 3, and $1 \mathrm{X}$ of Crystal River Unit 3 Doc. Identifier: BBA000000-01717-0200-00040 REV 00 Date Printed: 04/04/97

Attachment III, Page 9 of 24

\begin{tabular}{cccc}
$\begin{array}{c}\text { Irradiation } \\
\text { Step Number }\end{array}$ & $\begin{array}{c}\text { Step } \\
\text { Duration } \\
\text { (EFPD) }\end{array}$ & $\begin{array}{c}\text { Exposure at } \\
\text { End of Step } \\
\text { (EFPD) }\end{array}$ & $\begin{array}{c}\text { Mid-Step Boron } \\
\text { Concentration } \\
\text { (Ppm) }\end{array}$ \\
\hline 1 & 6.57 & 6.57 & 277.1 \\
2 & 5.45 & 12.02 & 266.5 \\
3 & 16.98 & 29.00 & 204.7 \\
4 & .10 & 29.10 & 157.6
\end{tabular}

NODAL ROD ASSEMBLY INSERTION LAYOUT FOR FUEL ASSEMBLY: A01

COLUMN A: Cycle-1A, .0 EFPD to Cycle-1A, 268.8 EFPD Statepoint Calculation COLUMN B: Cycle-1B, $\quad .0$ EFPD to Cycle-1B, 142.2 EFPD Statepoint Calculation COLUMN C: Cycle-1B, 142.2 EFPD to Cycle-1B, 171.3 EFPD Statepoint Calculation

$X=$ Rod assembly inserted in corresponding node during the irradiation step

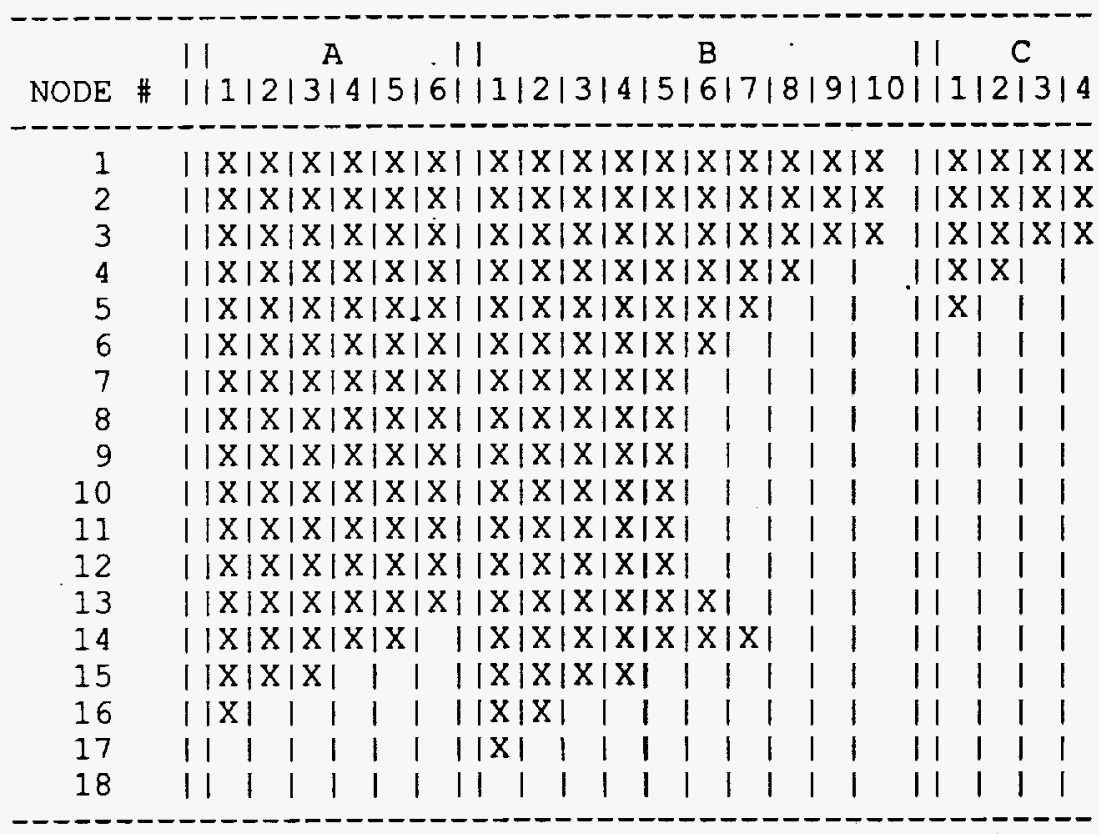

\section{RLAYOUT Version 1.0 Fortran Source Code Listing}

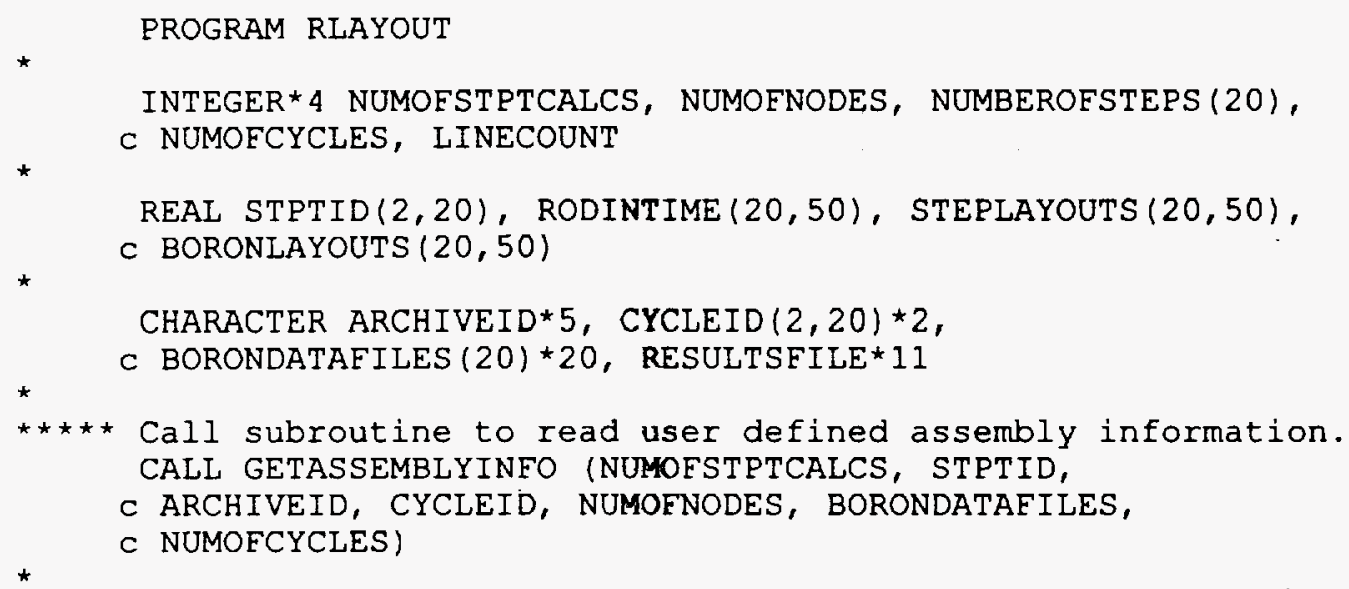




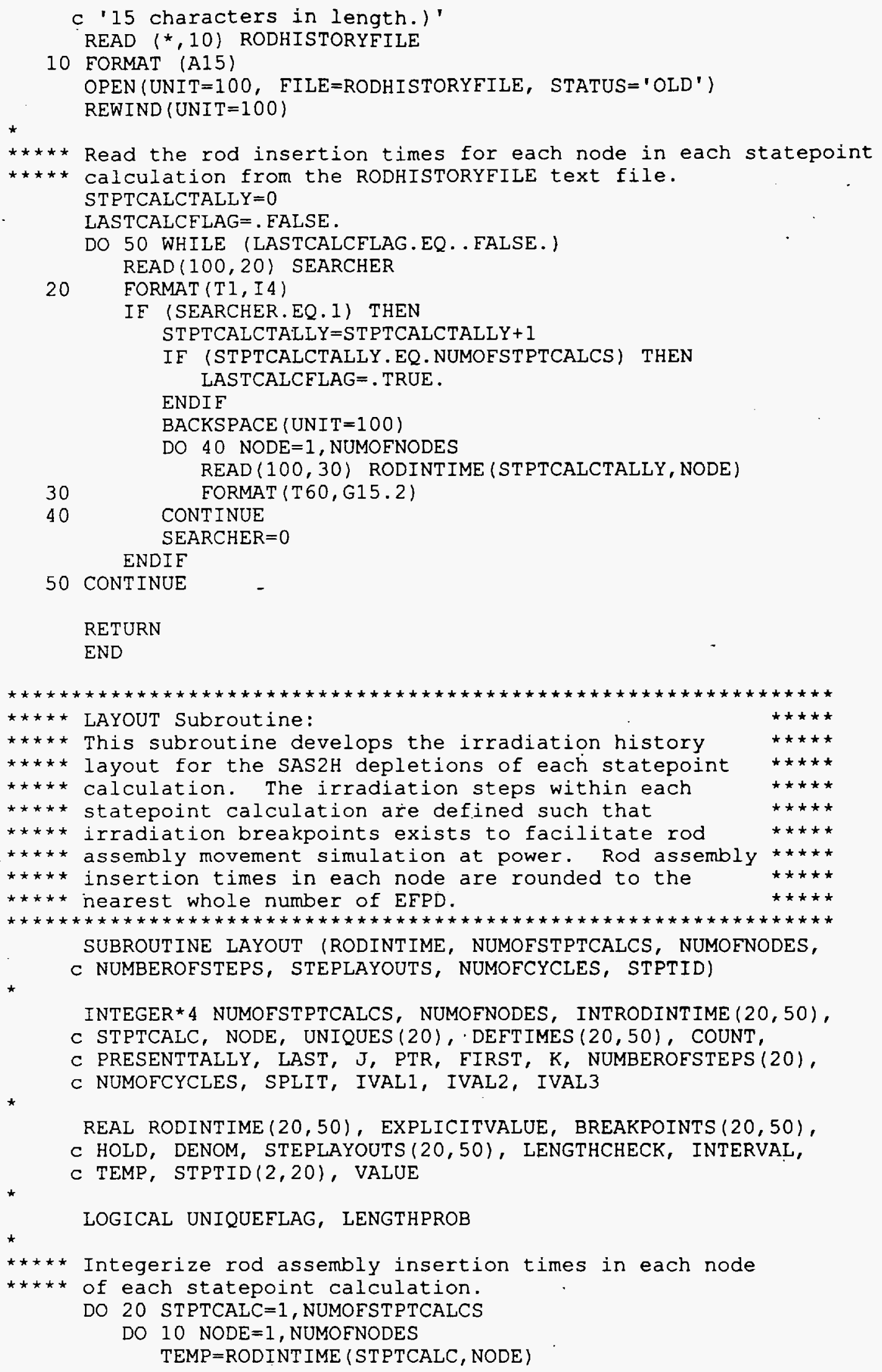

INTEGER * 4 NUMOFSTPTCALCS, NUMOFNODES, INTRODINTIME $(20,50)$, C STPTCALC, NODE, UNIQUES (20), DEFTIMES $(20,50)$, COUNT, C PRESENTTALLY, LAST, J, PTR, FIRST, $K$, NUMBEROFSTEPS (20), c NUMOFCYCLES, SPLIT, IVAL1, IVAL2, IVAL3

REAL RODINTIME $(20,50)$, EXPLICITVALUE, $\operatorname{BREAKPOINTS}(20,50)$, C HOLD, DENOM, STEPLAYOUTS $(20,50)$, LENGTHCHECK, INTERVAL, c TEMP, $\operatorname{STPTID}(2,20)$, VALUE

LOGICAL UNIQUEFLAG, LENGTHPROB

***** Integerize rod assembly insertion times in each node

$\star \star \star \star *$ of each statepoint calculation.

DO 20 STPTCALC $=1$, NUMOFSTPTCALCS

DO 10 NODE $=1$, NUMOFNODES

TEMP=RODINT IME (ST PTCALC, NODE) 
10

$\star$

$\star \star \star \star \star$ Determine the number of unique rod assembly insertion times

$\star \star \star \star *$ for each statepoint calculation, and record these unique rod

$\star \star \star * \star$ assembly insertion times in an array entitled DEFTIMES.

DO 50 STPTCALC $=1$, NUMOFSTPTCALCS

UNIQUES (STPTCALC) $=0$

DO 40 NODE $=1$, NUMOFNODES

IE (NODE.EQ.1) THEN

UNIQUES (ST PTCALC) =UNIQUES (STPTCALC $)+1$

C DEFTIMES (STPTCALC, UNIQUES (STPTCALC) ) = INTRODINT IME (ST PTCALC, NODE)

ELSEIF (NODE.GT.1) THEN

UNIQUEFLAG $=$. TRUE.

DO 30 COUNT $=1$, UNIQUES (STPTCALC)

IF (INTRODINT IME (STPTCALC, NODE) . EQ.

C

30

C ENDIF

ELSEIF (NODE.EQ.NUMOFNODES) THEN

UNIQUEFLAG $=$. TRUE.

DO 35 COUNT=1, UNIQUES (STPTCALC)

IF (STPTID (2, STPTCALC).EQ.

c

35

C

$$
\text { ENDIF }
$$

DEFTIMES (STPTCALC, COUNT)) THEN

UNIQUEFLAG = . FALSE.

ENDIF

EXIT

CONTINUE

IF (UNIQUEFLAG.EQ. . TRUE.) THEN

UNIQUES (STPTCALC) =UNIQUES (STPTCALC) +1 DEFTIMES (STPTCALC, UNIQUES (STPTCALC) ) = STPTID (2, STPTCALC) -STPTID (1, STPTCALC) ENDIF

40 CONTINUE

50 CONTINUE

$\star \star \star \star \star$ Determine which rod assembly insertion times occur only once in

$\star \star \star \star \star$ a statepoint calculation, and define these insertion times

$\star \star \star \star \star$ using the explicit floating point value as originally read from

$\star \star \star \star \star$ the rod insertion history data text file (UNIT $=100$ ). The

$\star \star \star \star *$ BREAKPOINTS array contains the irradiation step breakpoint values

$\star \star \star \star \star$ necessary to define the irradition history layout.

DO 80 STPTCALC $=1$, NUMOFSTPTCALCS

DO 70 COUNT $=1$, UNIQUES (STPTCALC)

PRESENTTALLY $=0$ 


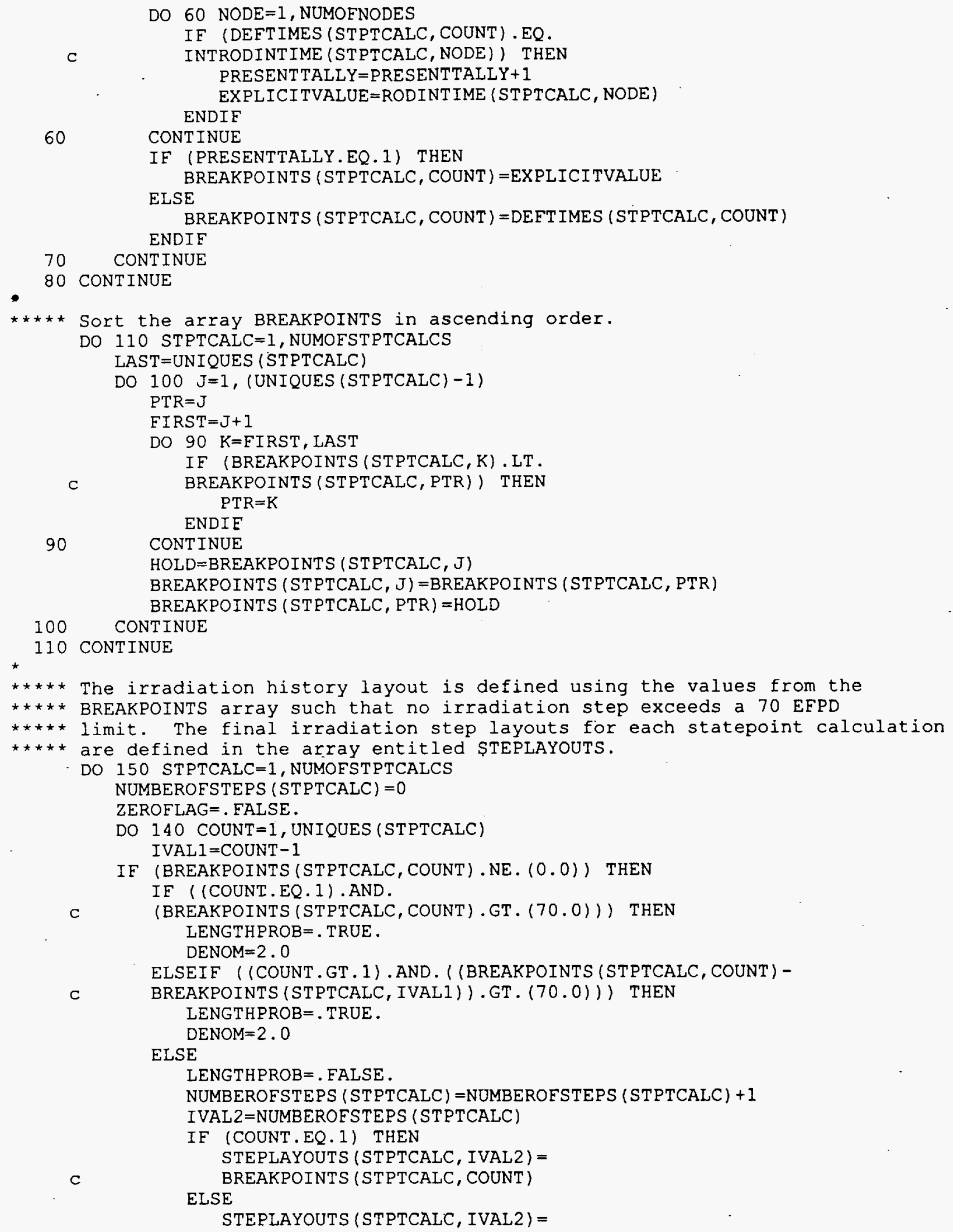




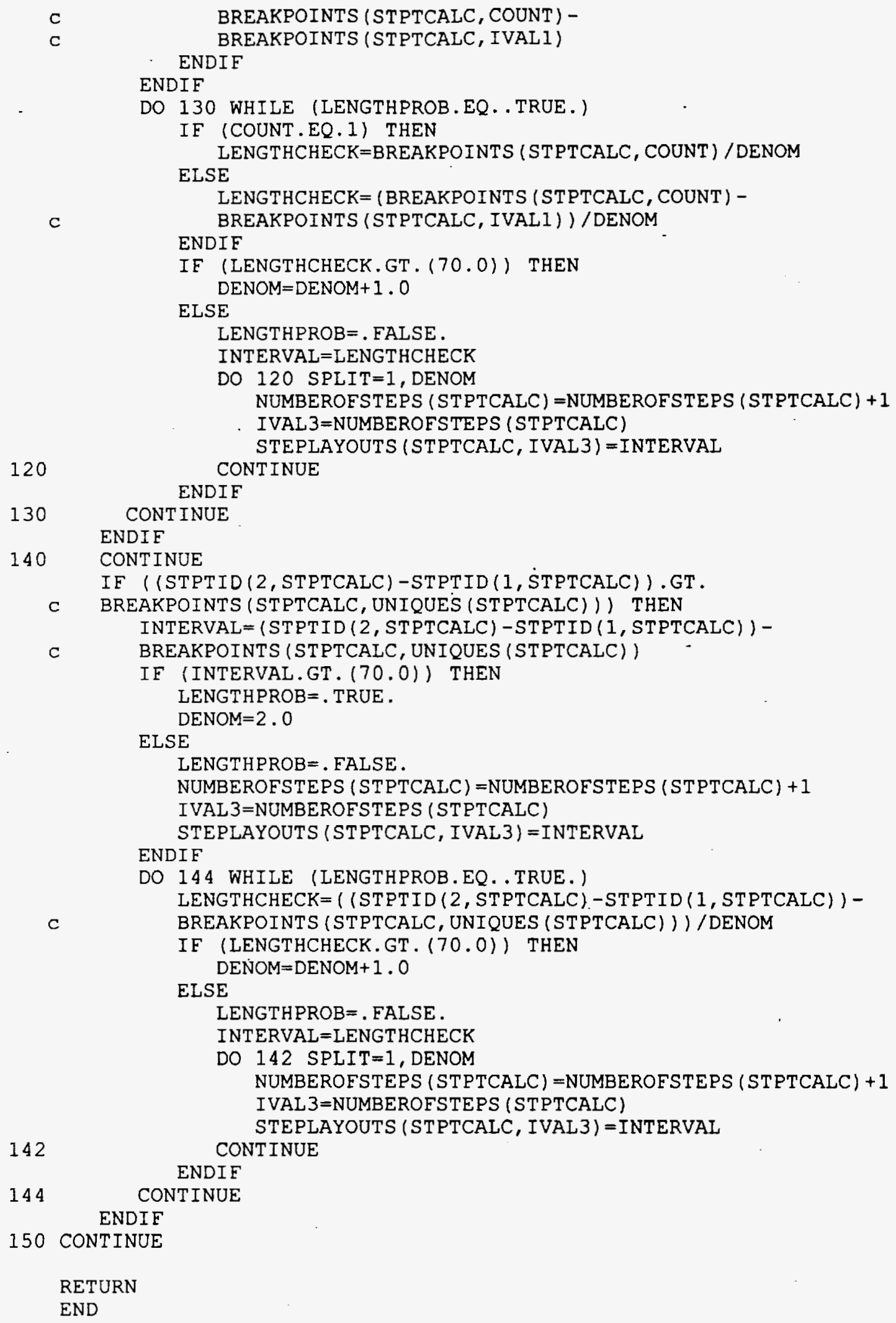

c

$\mathrm{c}$

C

120

130

140

c

C

142

144

150 CONTINUE

BREAKPOINTS (STPTCALC, COUNT) ENDIF

BREAKPOINTS (STPTCALC, IVALI)

ENDIF

DO 130 WHILE (LENGTHPROB.EQ. TRUE.)

IF (COUNT.EQ.1) THEN ELSE

LENGTHCHECK=BREAKPOINTS (STPTCALC, COUNT) /DENOM

LENGTHCHECK= (BREAKPOINTS (STPTCALC, COUNT) ENDIF BREAKPOINTS (STPTCALC, IVALI)) /DENOM

IE (IENGTHCHECK.GT. $(70.0)$ ) THEN

$D E N O M=D E N O M+1.0$

ELSE

LENGTHPROB=. FALSE.

INTERVAL=LENGTHCHECK

DO 120 SPLIT $=1$, DENOM

NUMBEROFSTEPS (STPTCALC) =NUMBEROFSTEPS (STPTCALC) +1

IVAL $3=$ NUMBEROFSTEPS (STPTCALC)

STEPLAYOUTS (STPTCALC, IVAL3) = INTERVAL

ENDIF

CONTINUE

CONT INUE

ENDIE

CONTINUE

IF ((STPTID $(2, \operatorname{STPTCALC})-\operatorname{STPTID}(1, \dot{\operatorname{STPTCALC}})) . \mathrm{GT}$.

C BREAKPOINTS (STPTCALC, UNIQUES (STPTCALC)) ) THEN

INTERVAL = (STPTID (2, STPTCALC) -STPTID (1, STPTCALC) ) -

BREAKPOINTS (STPTCALC, UNIQUES (STPTCALC))

IF (INTERVAL.GT. $(70.0)$ ) THEN

LENGTHPROB $=$. TRUE .

ELSE

DENOM $=2.0$

LENGTHPROB $=$. FALSE.

NUMBEROFSTEPS (STPTCALC) $=$ NUMBEROFSTEPS (STPTCALC) +1

IVAL $3=$ NUMBEROFSTEPS (STPTCALC)

STEPLAYOUTS (STPTCALC, IVAL3) = INTERVAL

ENDIF

DO 144 WHILE (LENGTHPROB.EQ. . TRUE.)

LENGTHCHECK $=((\operatorname{STPTID}(2, \operatorname{STPTCALC})-\operatorname{STPTID}(1$, STPTCALC $))-$ BREAKPOINTS (STPTCALC, UNIQUES (STPTCALC)) ) /DENOM

IF (LENGTHCHECK.GT. (70.0)) THEN $D E N O M=D E N O M+1.0$

ELSE

LENGTHPROB $=$. FALSE.

INTERVAL $=$ LENGTHCHECK

DO 142 SPLIT $=1$, DENOM

NUMBEROFSTEPS (STPTCALC) =NUMBEROFSTEPS $($ STPTCALC $)+1$

IVAL $3=$ NUMBEROFSTEPS (STPTCALC)

STEPLAYOUTS (STPTCALC, IVAL3) = INTERVAL

ENDIF

CONTINUE

CONTINUE

ENDIF

RETURN

END 


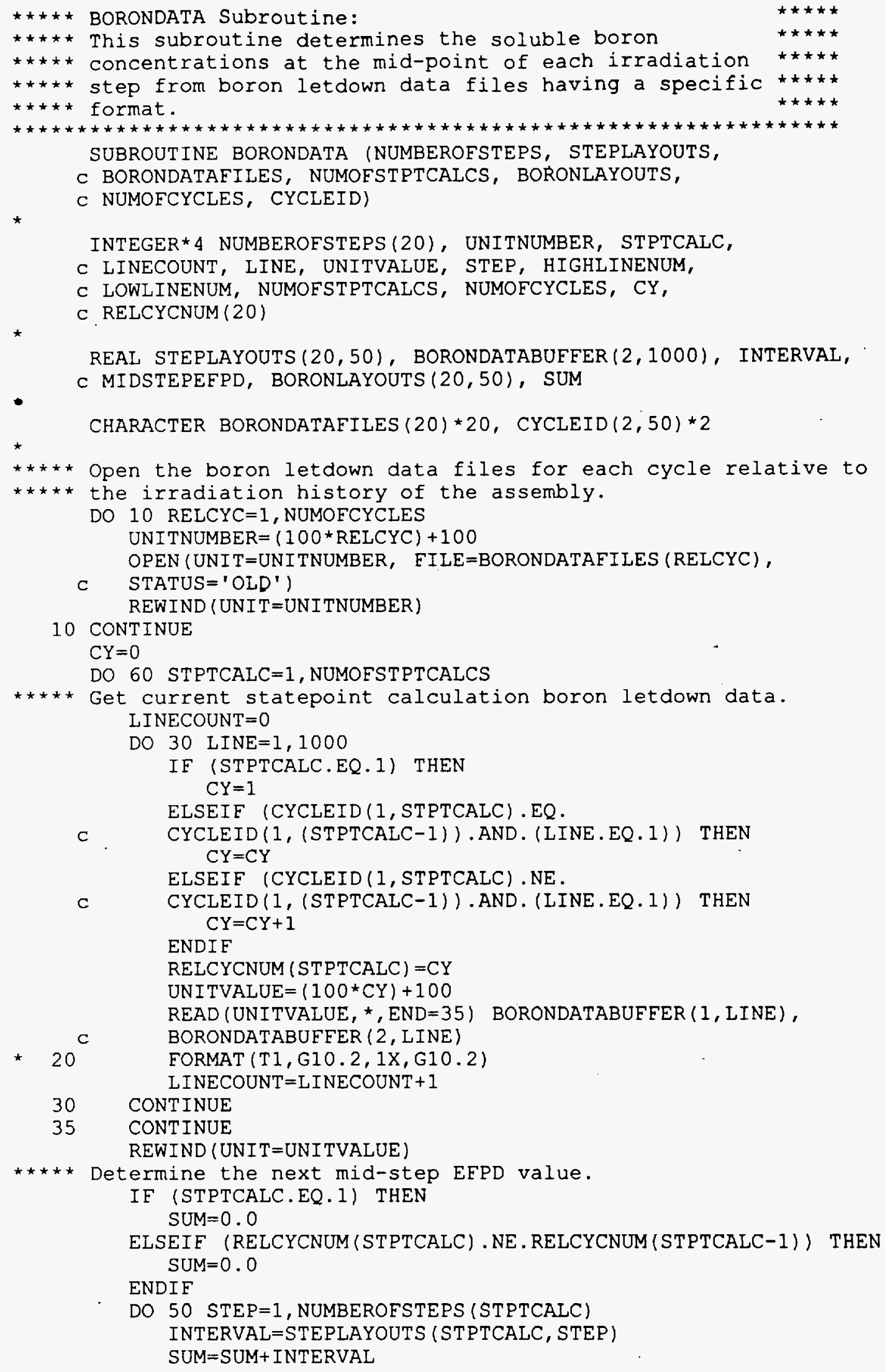


C

C

40

50

60

RETURN

END

MIDSTEPEFPD $=S U M-($ INTERVAL $/ 2.0)$

DO $40 \mathrm{~L}=2$, LINECOUNT

IF ((BORONDATABUEFER (I,L).GT.MIDSTEPEFPD) . AND.

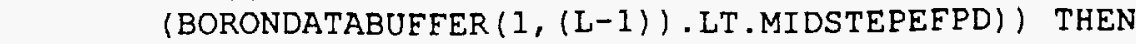

HIGHLINENUM $=\mathrm{L}$

LOWLINENUM $=\mathrm{L}-1$

BORONLAYOUTS (STPTCALC, STEP) $=$

( ( (BORONDATABUEFER ( 2 , HIGHIINENUM) -

BORONDATABUFEER (2, LOWLINENUM)) /

(BORONDATABUFFER ( 1 , HIGHLINENUM) BORONDATABUFFER ( 1 , LOWLINENUM) ) ) *

(MIDSTEPEFPD-BORONDATABUFEER (1, LOWLINENUM)) ) + BORONDATABUFEER (2, LOWLINENUM)

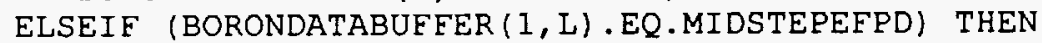

BORONLAYOUTS (STPTCALC, STEP) =BORONDATABUFFER $(2, \mathrm{~L}$ )

ELSEIF (MIDSTEPEFPD.GT.
BORONDATABUFFER ( 1 , LINECOUNT)) THEN

HIGHLINENUM $=$ LINECOUNT

LOWLINENUM=LINECOUNT -1

BORONLAYOUTS $($ STPTCALC, STEP $)=$

( ( (BORONDATABUFFER (2, HIGHLINENUM) -

BORONDATABUFFER (2, LOWLINENUM)) /

(BORONDATABUFFER ( 1 , HIGHLINENUM) BORONDATABUFFER (I, LOWLINENUM)) ) *

(MIDSTEPEFPD-BORONDATABUFFER (1, LOWLINENUM)) ) + BORONDATABUFFER (2, LOWLINENUM)

ELSEIF (MIDSTEPEEPD. LT.

BORONDATABUFFER $(1,1)$ ) THEN

HIGHLINENUM $=2$

LOWLINENUM $=1$

BORONLAYOUTS (STPTCALC, STEP) $=$

( ( (BORONDATABUFFER (2, HIGHLINENUM) -

BORONDATABUFEER (2, LOWLINENUM)) /

(BORONDATABUFEER (1, HIGHLINENUM) -

BORONDATABUFFER ( 1 , LOWLINENUM) ) )

(MIDSTEPEFPD-BORONDATABUFFER $(1$, LOWLINENUM) ) ) +

ENDIF

BORONDATABUFFER (2, LOWLINENUM)

CONT INUE

CONT INUE

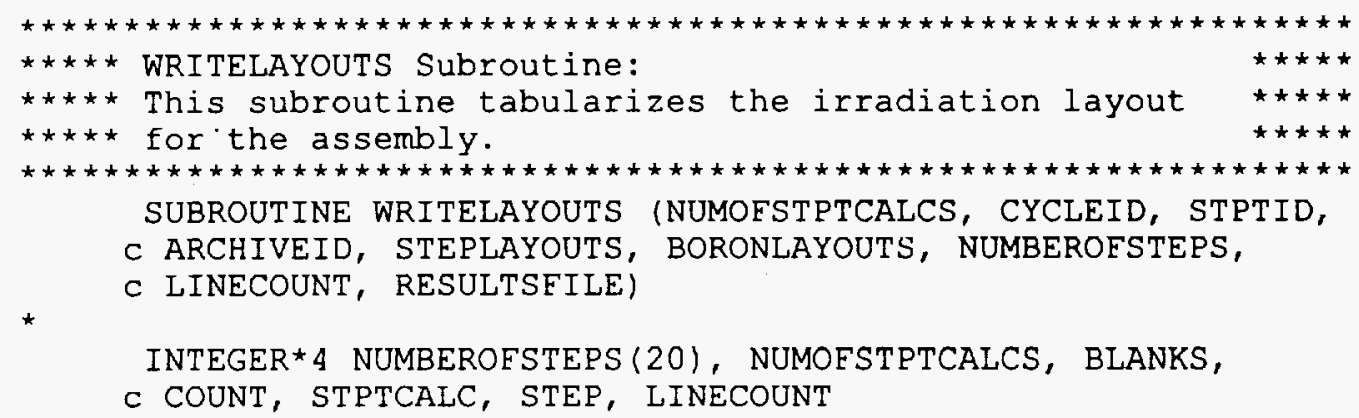

REAL STEPLAYOUTS $(20,50), \operatorname{BORONLAYOUTS}(20,50), \operatorname{STPTID}(2,20)$, C TOTAL 
CHARACTER CYCLEID $(2,20) \star 2, \operatorname{ARCHIVEID\star 5,~RESULTSFILE\star ~} 11$

$\star \star \star \star \star$ Open the file which will contain the irradiation history

$\star \star \star \star \star$ tabularization. LINECOUNT $=0$

BLANKS $=0$

DO 10 COUNT $=1,5$

IE (ARCHIVEID (COUNT: COUNT) .EQ.' ') THEN BLANKS $=$ BLANKS +1

ENDIF

10 CONTINUE

RESULTSFILE $(1:(11-$ BLANKS -6$))=$

C ARCHIVEID ( $1:(5-B L A N K S))$

RESULTSEILE ( (11-BLANKS-5):(11-BLANKS-1) ) =' . hist '

RESULTSFILE $((11$-BLANKS $): 11)='$

OPEN (UNIT $=50$, FILE=RESULTSFILE, STATUS = ' UNKNOWN ' )

REWIND (UNIT $=50$ )

$\star \star \star \star \star$ Write irradiation history layout information for

**** each statepoint calculation.

WRITE $(50,20)$ ARCHIVEID

20 FORMAT (T1,' IRRADIATION LAYOUT FOR ASSEMBLY: ',A5)

LINECOUNT $=$ L INECOUNT +1

DO 100 STPTCALC $=1$, NUMOFSTPTCALCS

TOTAL $=0.0$

WRITE $(50,30)$ CYCLEID (1, STPTCALC), STPTID(1, STPTCALC),

C CYCLEID (2, STPTCALC), STPTID $(2, \operatorname{STPTCALC})$

30 FORMAT (T1, 'Cycle-', A2, ', ', F5.1,' EFPD to Cycle-', A2,

C ', ',F5.1,' EFPD Statepoint Calculation')

LINECOUNT $=$ L INECOUNT +1

WRITE $(50, *)$

LINECOUNT $=$ LINECOUNT +1

WRITE $(50,40)$

40 FORMAT (T19,'Step', $7 \mathrm{X}$, 'Exposure at', 5X, 'Mid-step Boron') LINECOUNT $=$ LINECOUNT +1

WRITE $(50,50)$

50 FORMAT (T1,' 'Irradiation', 5X, 'Duration', 5X,

c 'End of Step',5X, 'Concentration')

LINECOUNT $=$ LINECOUNT +1

WRITE $(50,60)$

60 FORMAT (T1, 'Step Number', 6X, '(EFPD)', 9X, '(EFPD) ', 7X,

C '(Ppm)')

LINECOUNT $=$ LINECOUNT +1

WRITE $(50,70)$

70 FORMAT (TI, '-

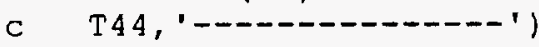

LINECOUNT $=$ LINECOUNT +1

DO 90 STEP=1, NUMBEROESTEPS (STPTCALC)

TOTAL=TOTAL+STEPLAYOUTS (STPTCALC, STEP)

WRITE $(50,80)$ STEP, STEPLAYOUTS (STPTCALC, STEP),

C TOTAL, BORONLAYOUTS (STPTCALC, STEP)

80 FORMAT (T1, 4X, I2, IIX, F6.2, 9X, F6.2, 11X, F6.1)

LINECOUNT $=$ LINECOUNT +1

90

CONTINUE

WRITE $(50$, *)

LINECOUNT $=$ LINECOUNT +1

100 CONTINUE

CLOSE (UNIT $=50$ )

RETURN 
END

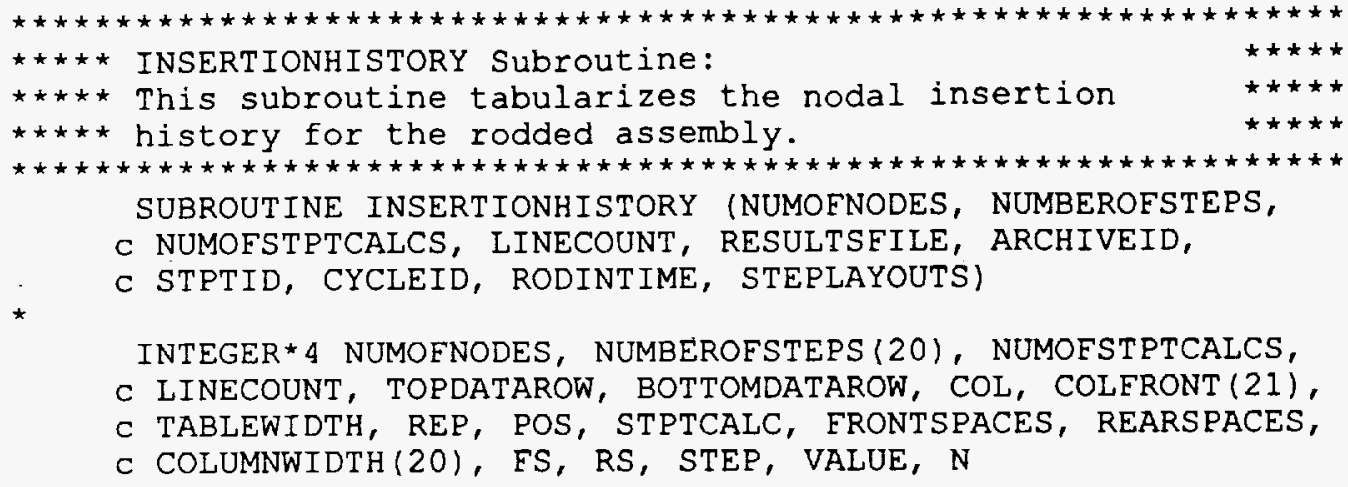

INTEGER * 4 NUMOFNODES, NUMBEROFSTEPS(20), NUMOFSTPTCALCS, C LINECOUNT, TOPDATAROW, BOTTOMDATAROW, COL, COLFRONT (21), C TABLEWIDTH, REP, POS, STPTCALC, FRONTSPACES, REARSPACES, C COLUMNWIDTH(20), FS, RS, STEP, VALUE, $N$

REAL $\operatorname{STPTID}(2,20), \operatorname{RODINTIME}(20,50), \operatorname{STEPLAYOUTS}(20,50)$, C TEMP, SUM

CHARACTER RESULTSFILE* $11, \operatorname{ARCHIVEID} \star 5, \operatorname{CYCLEID}(2,20) \star 2$

$\star$ LOGICAL MULTIPLE, SPECIALFLAG

$\star$

$\star \star \star \star \star$ Open the file which will contain the nodal insertion history

$\star \star \star \star \star$ tabularization for the rodded assembly. OPEN (UNIT $=50$, EILE=RESULTSFILE, STATUS= ' OLD' )

REWIND (UNI T $=50$ )

DO 2 POS $=1$, LINECOUNT $\operatorname{READ}(50$, *)

2 CONTINUE

$\star \star \star \star \star$ Determine the column and row delimiters for use in producing

$\star \star \star \star \star$ the table of nodal rod insertion history. TOPDATAROW $=15+$ NUMOFSTPTCALCS+LINECOUNT BOTTOMDATAROW=TOPDATAROW $+(2 *($ NUMOFNODES -1$))$ TABLEWIDTH $=0$

DO 5 COUNT $=1$, NUMOFSTPTCALCS COLUMNWIDTH (COUNT) =NUMBEROFSTEPS (COUNT)

TABLEWIDTH=TABLEWIDTH+NUMBEROFSTEPS (COUNT)

IF (NUMBEROFSTEPS (COUNT) . GT .9) THEN TABLEWIDTH=TABLEWIDTH+NUMBEROFSTEPS (COUNT) - 9 COLUMNWIDTH (COUNT) =COLUMNWIDTH (COUNT) +

c NUMBEROESTEPS (COUNT) -9

ENDIE

TABLEWIDTH=TABLEWIDTH+NUMBEROFSTEPS (COUNT) -1

COLUMNWIDTH (COUNT) $=$ COLUMNWIDTH $($ COUNT $)+$

C NUMBEROFSTEPS (COUNT) - 1

5 CONTINUE

TABLEWIDTH=TABLEWIDTH $+(2$ * (NUMOESTPTCALCS -1$))+10$

DO 10 COL $=1$, (NUMOFSTPTCALCS +1 )

IF (COL.EQ.1) THEN COLFRONT $($ COL $)=1$

ELSEIF (COL.EQ.2) THEN COLFRONT $(\mathrm{COL})=11$ ELSE

C

COLFRONT $($ COL $)=$ COLFRONT $($ COL -1$)+$

10 CONTINUE COLUMNWIDTH $(\mathrm{COL}-1)+3$

$\operatorname{WRITE}(50, *)$ 


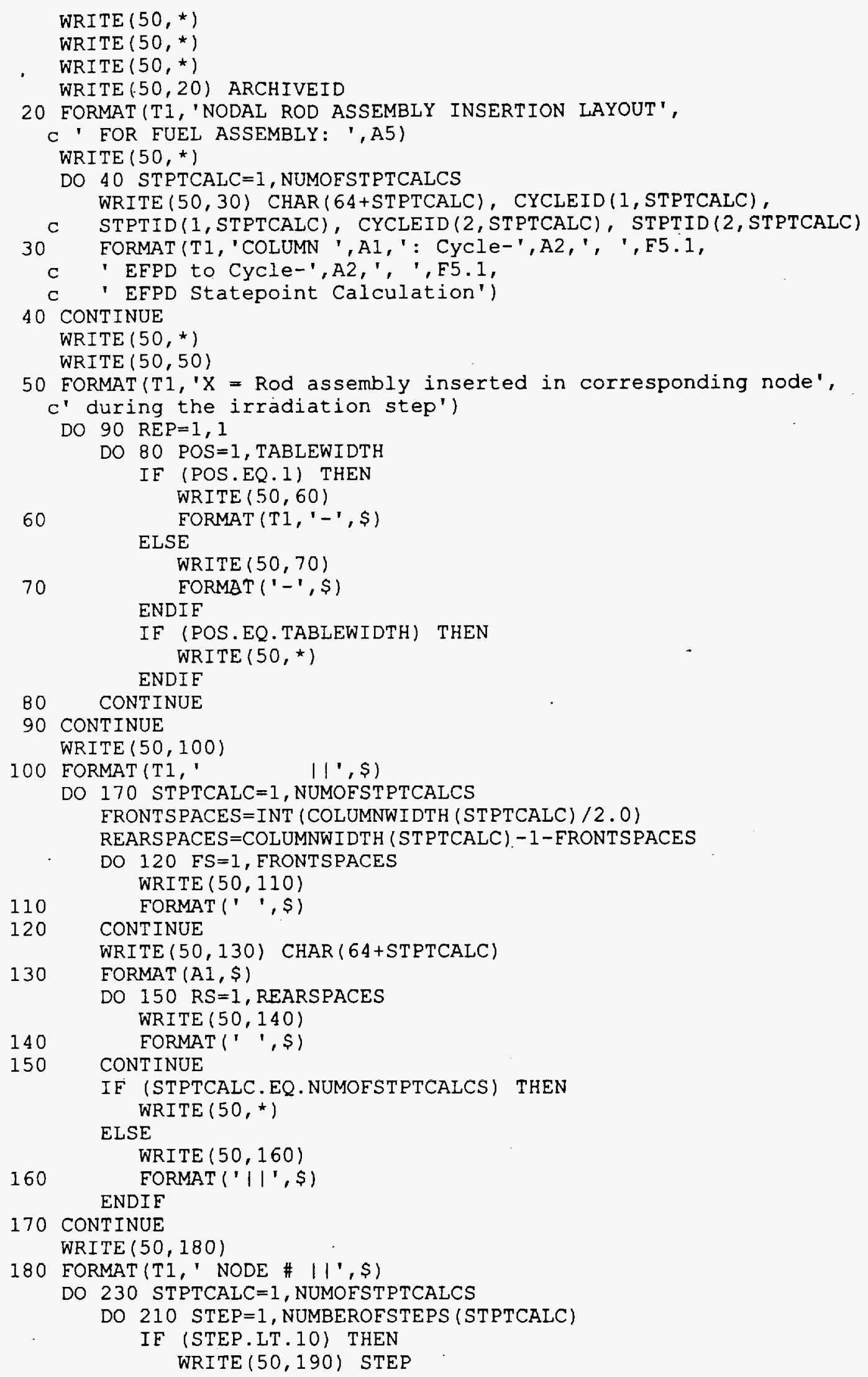


190

200

\section{5}

210

$$
220
$$

230

CONTINUE

DO 270 REP $=1,1$

DO 260 POS $=1$, TABLEWIDTH

IF (POS.EQ.1) THEN .

WRITE $(50,240)$

240

250

$$
\text { ELSE }
$$$$
\text { FORMAT (T1, '-', \$) }
$$

WRITE $(50,250)$

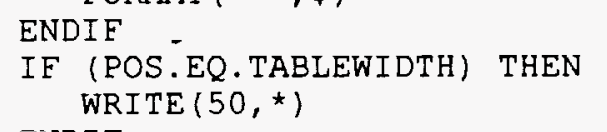

260

270 CONTINUE

$$
\text { CONTINUE }
$$

DO 530 NODE $=1$, NUMOFNODES

$$
\text { WRITE }(50,280) \text { NODE }
$$

280

(T $1,3 X, 12,3 X, 111, \$)$

DO 520 STPTCALC $=1$, NUMOFST PTCALCS

DO 510 STEP $=1$, NUMBEROFSTEPS (STPTCALC)

IF (STEP.EQ.1) THEN

SUM=STEPLAYOUTS (STPTCALC, STEP)

ELSE

SUM $=$ SUM+STEPLAYOUTS (STPTCALC, STEP)

ENDIF

IF ((RODINTIME (STPTCALC, NODE) -

C

INT (RODINT IME (STPTCALC, NODE))) . LT . (0.5)) THEN TEMP = INT (RODINT IME (STPTCALC, NODE))

ELSEIF ((RODINT IME (STPTCALC, NODE) -

c

INT (RODINT IME (STPTCALC, NODE) )) . GE. (0.5)) THEN TEMP=INT (RODINT IME (STPTCALC, NODE) +1.0)

\section{ENDIF}

VALUE $=0$

MULT I PLE $=$. FALSE .

DO $282 \mathrm{~N}=1$, NUMOFNODES

IF ((RODINTIME (STPTCALC, N) -

C

INT (RODINTIME (STPTCALC,N))) . LT . (0.5)) THEN VALUE =INT (RODINTIME (STPTCALC, N))

$c$ ELSEIF ((RODINTIME (STPTCALC, N) INT (RODINTIME (STPTCALC, N) )) .GE. (0.5)) THEN ENDIF VALUE =INT $($ RODINT IME $($ STPTCALC, N $)+1.0)$

IF ((N.NE. NODE) . AND. (TEMP.EQ.VALUE)) THEN MULTIPLE $=$. TRUE. 
282

C

C

285

290

300

C

305

310

320
ENDIF

CONTINUE

IF (MULTIPLE.EQ.. FALSE.) THEN

TEMP=RODINT IME (STPTCALC, NODE)

ENDIE

IE ((STEP.EQ. NUMBEROFSTEPS (STPTCALC)) . AND.

(STEPLAYOUTS (STPTCALC, STEP) . LT. (0.5))) THEN

TEMP=RODINT IME (ST PTCALC, NODE)

SPECIALELAG = . TRUE.

ELSE

ENDIE

SPECIALFLAG $=$. FALSE.

IF (SPECIALFLAG.EQ..FALSE.) THEN

IF ((SUM.LE.TEMP) .OR. ((SUM-TEMP) .LT. (0.5))) THEN

IF (STEP.GT.9) THEN

IF ((STEP.EQ. NUMBEROFSTEPS (STPTCALC)) . AND.

(STPTCALC.EQ.NUMOFSTPTCAICS)) THEN

WRITE $(50,285)$

FORMAT ('X', \$)

ELSEIF (STEP.NE.NUMBEROFSTEPS (STPTCALC)) THEN

WRITE $(50,290)$

FORMAT ('X |', \$)

ELSE

WRITE $(50,300)$

ENDIF

ELSEIF (STEP.LE.9) THEN

IF ((STEP.EQ. NUMBEROFSTEPS (STPTCALC)) . AND.

(STPTCALC.EQ. NUMOFSTPTCALCS)) THEN

WRITE (50, 305)

EORMAT (' $X$ ', $\$$ )

ELSEIE (STEP.NE.NUMBEROFSTEPS (STPTCALC)) THEN WRITE $(50,310)$

ELSE

FORMAT ('X)', \$)

WRITE $(50,320)$

ENDIF

FORMAT (' $\mathrm{X}||$ ', \$)

ENDIF

ELSE

IF (STEP.GT.9) THEN

IF ((STEP.EQ. NUMBEROFSTEPS (STPTCALC)) . AND.

(STPTCALC.EQ. NUMOFSTPTCALCS)) THEN

WRITE $(50,330)$

FORMAT (' ', \$)

ELSEIF (STEP.NE. NUMBEROFSTEPS (STPTCALC)) THEN WRITE $(50,340)$

FORMAT (' 1 ', \$)

ELSE

WRITE $(50,350)$

ENDIF

ELSEIF (STEP.LE. 9) THEN

IF ((STEP. EQ. NUMBEROFSTEPS (STPTCALC)) . AND.

(STPTCALC.EQ.NUMOFSTPTCALCS)) THEN

WRITE $(50,360)$

FORMAT (' ', \$)

ELSEIE (STEP.NE. NUMBEROFSTEPS (STPTCALC)) THEN WRITE $(50,370)$

FORMAT (' 1 ', \$) 
380

ELSE

$\operatorname{WRITE}(50,380)$

FORMAT (' ||$\left.^{\prime}, \$\right)$

$$
\text { ENDIF }
$$

ENDIF

\section{ENDIF}

ELSEIF (SPECIALFLAG.EQ..TRUE.) THEN

IF ((SUM. LE. TEMP) .OR. ((SUM-TEMP) . LE. $(0.0001)$ )) THEN

IF (STEP.GT.9) THEN

IF ((STEP.EQ. NUMBEROFSTEPS (STPTCALC)). AND.

390 FORMAT ('X ', $\$$ )

ELSEIF (STEP.NE. NUMBEROFSTEPS (STPTCALC)) THEN WRITE $(50,400)$

400 FORMAT (' $\mathrm{X} \mid$ ', \$)

$$
\text { ELSE }
$$

WRITE $(50,410)$

410

FORMAT (' $\mathrm{X}||$ ', $\$$ )

$$
\text { ENDIF }
$$

ELSEIF (STEP.LE.9) THEN

IF ((STEP. EQ. NUMBEROFSTEPS (STPTCALC)) . AND.

420

(STPTCALC.EQ.NUMOFSTPTCALCS)) THEN

WRITE $(50,420)$

FORMAT ('X', \$)

ELSEIF (STEP.NE. NUMBEROFSTEPS (STPTCALC)) THEN WRITE $(50,430)$

430 FORMAT ('XI', \$)

ELSE

440

WRITE $(50,440)$

ENDIF

FORMAT (' $X||$ ', \$)

\section{ENDIF}

ELSE

IF (STEP.GT.9) THEN

IF ((STEP.EQ. NUMBEROESTEPS (STPTCALC)) . AND.

(STPTCALC.EQ.NUMOFSTPTCALCS)) THEN

WRITE $(50,450)$

450

EORMAT(' ', \$)

ELSEIF (STEP.NE. NUMBEROFSTEPS (STPTCALC)) THEN WRITE $(50,460)$

460 FORMAT (' 1 ', \$)

$$
\text { ELSE }
$$

WRITE $(50,470)$

470

FORMAT(' $\mid 1$, , \$)

ENDIF

ELSEIF (STEP.LE. 9) THEN

IF ((STEP. EQ. NUMBEROFSTEPS (STPTCALC)). AND.

(STPTCALC.EQ.NUMOFSTPTCALCS)) THEN

WRITE $(50,480)$

480

490

FORMAT(' ', \$)

ELSEIF (STEP.NE.NUMBEROFSTEPS (STPTCALC)) THEN

WRITE $(50,490)$

ELSE

FORMAT (' $\mid ', \$)$

500

WRITE $(50,500)$

ENDIF

EORMAT (' $\mid 1, \$)$

- ENDIF

ENDIF 


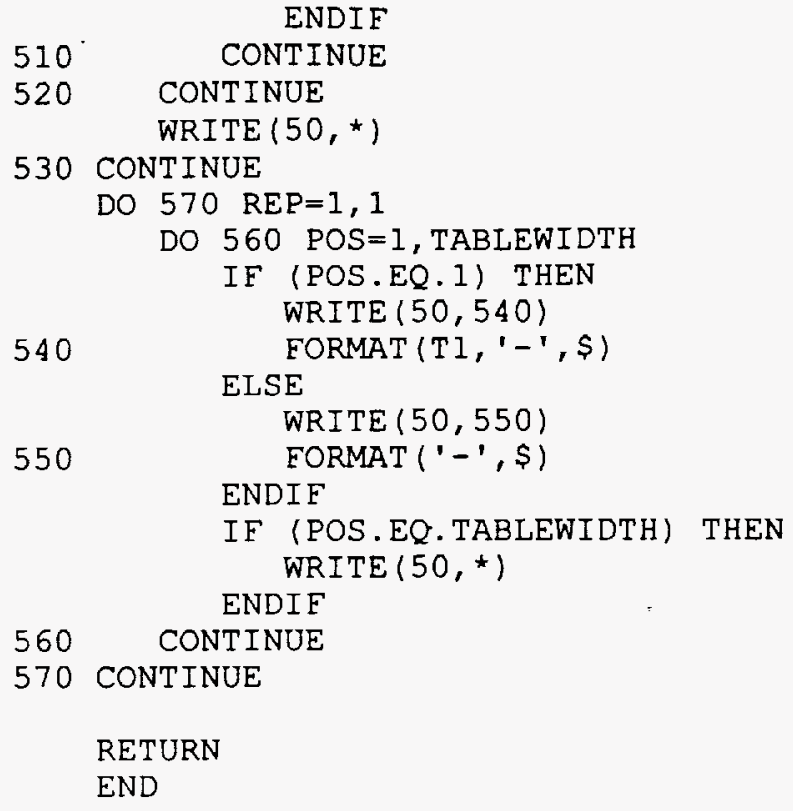




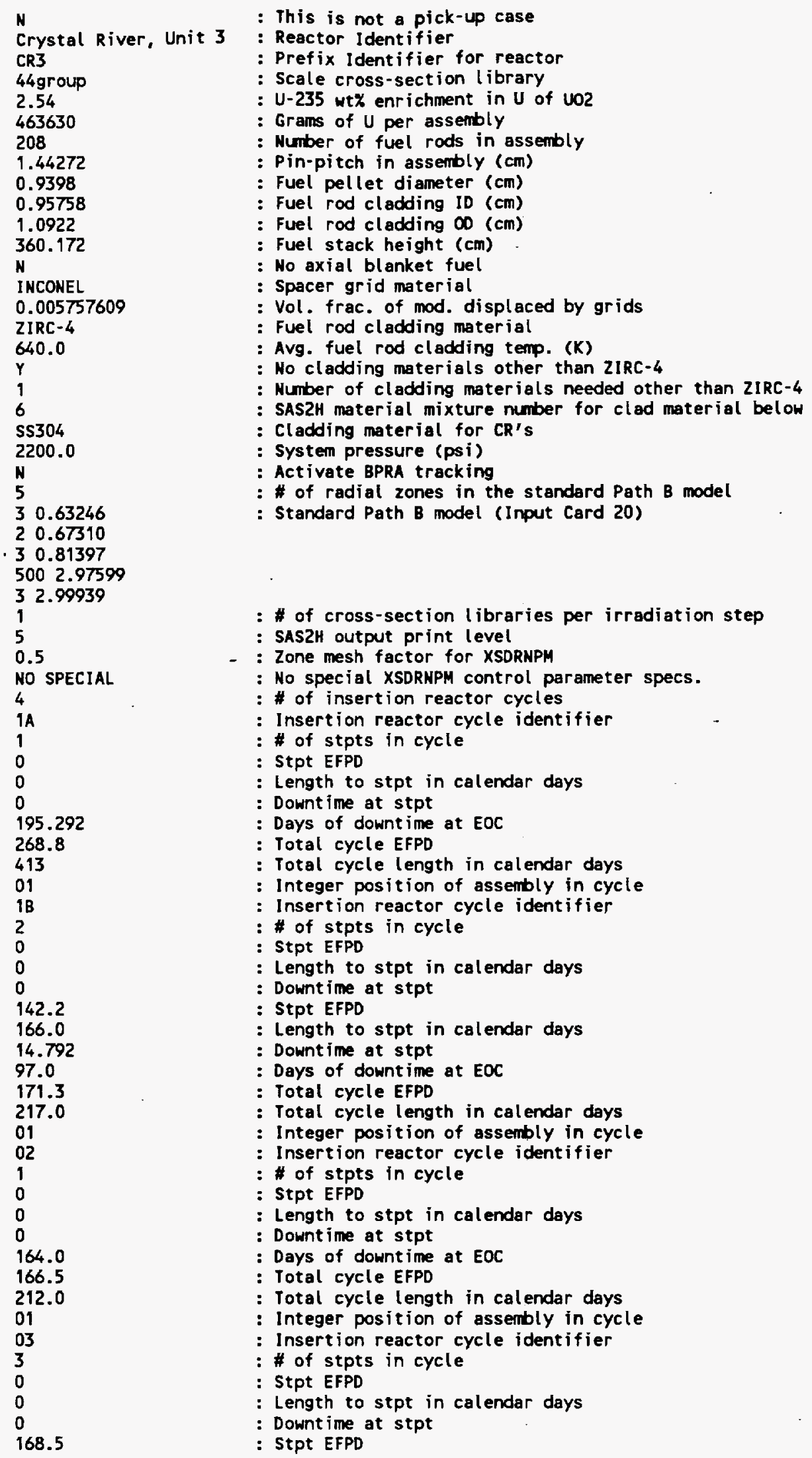




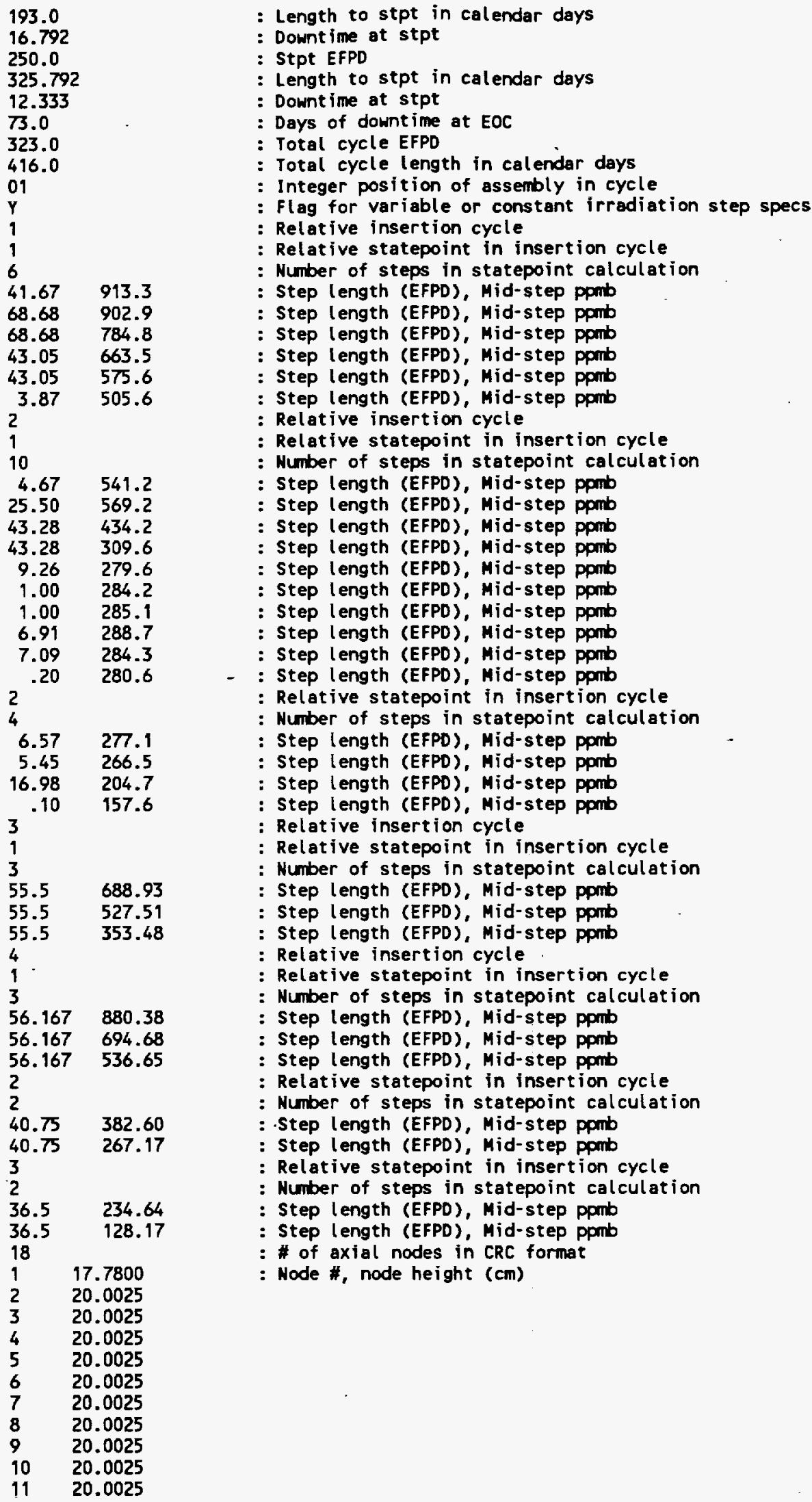


: Number of irradiation steps with CRA inserted

: Number of axial section with CRA inserted in step 1

: Input card $47 \mathrm{~B}$

: Number of axial section with CRA inserted in step 2

: Input card 47B

: Number of axial section with CRA inserted in step 3

: Input card 47B

: Number of axial section with CRA inserted in step 4

: Input card 47B

: Number of axial section with CRA inserted in step 5

: Input card 47B

: Number of exial section with CRA inserted in step 6

: Input card $47 \mathrm{~B}$

: Number of axial section with CRA inserted in step 7

: Input card 47B

: Number of axial section with CRA inserted in step 8

: Input card 47B

: Number of axial section with CRA inserted in step 9

: Input card 478

: Number of axial section with CRA inserted in step 10

- Input card 47B

: Number of axial section with CRA inserted in step 11

: Input card 47B

- : Number of axial section with CRA inserted in step 12

- Input card 47B

- Input card 47B

: Number of axial section with CRA inserted in-step 13

: Input card 47B

: Input card 47B

: Number of axial section with CRA inserted in step 14

: Input card 47B

: Number of axial section with CRA inserted in step 15

: Input card 47B

: Number of axial section with CRA inserted in step 16

: Input card 478

: Number of axial section with CRA inserted in step 17

: Input card 47B

: Number of axial section with CRA inserted in step 18

: Input card 47B

: Number of axial section with CRA inserted in step 19

: Input card 47B

: Number of axial section with CRA inserted in step 20

: Input card $47 \mathrm{~B}$

: Number of different CRA absorber material mixtures

: SAS2H material mixture number for CRA absorber

: Number of isotopes or elements in the CRA absorber

: SCALE isotope 1D, Isotope wtX

: SCALE isotope ID, I sotope wtX

: SCALE isotope ID, Isotope wtX

: SCALE isotope ID, Isotope wtX

: Number of CRA designs

: CR absorber density, CR clad SASZH mat. mix. number

: Number of radial zones in Path $B$ model with CRA inserted

: Path B model CRA inserted (Input Card 47J)

: Path B model CRA removed (Input Card 47K) 
Apr 03 14:28 1997 File Name: A01i.dat BBA000000-01717-0200-00040 RÉV 00 ATTACHMENT IV - Page 4

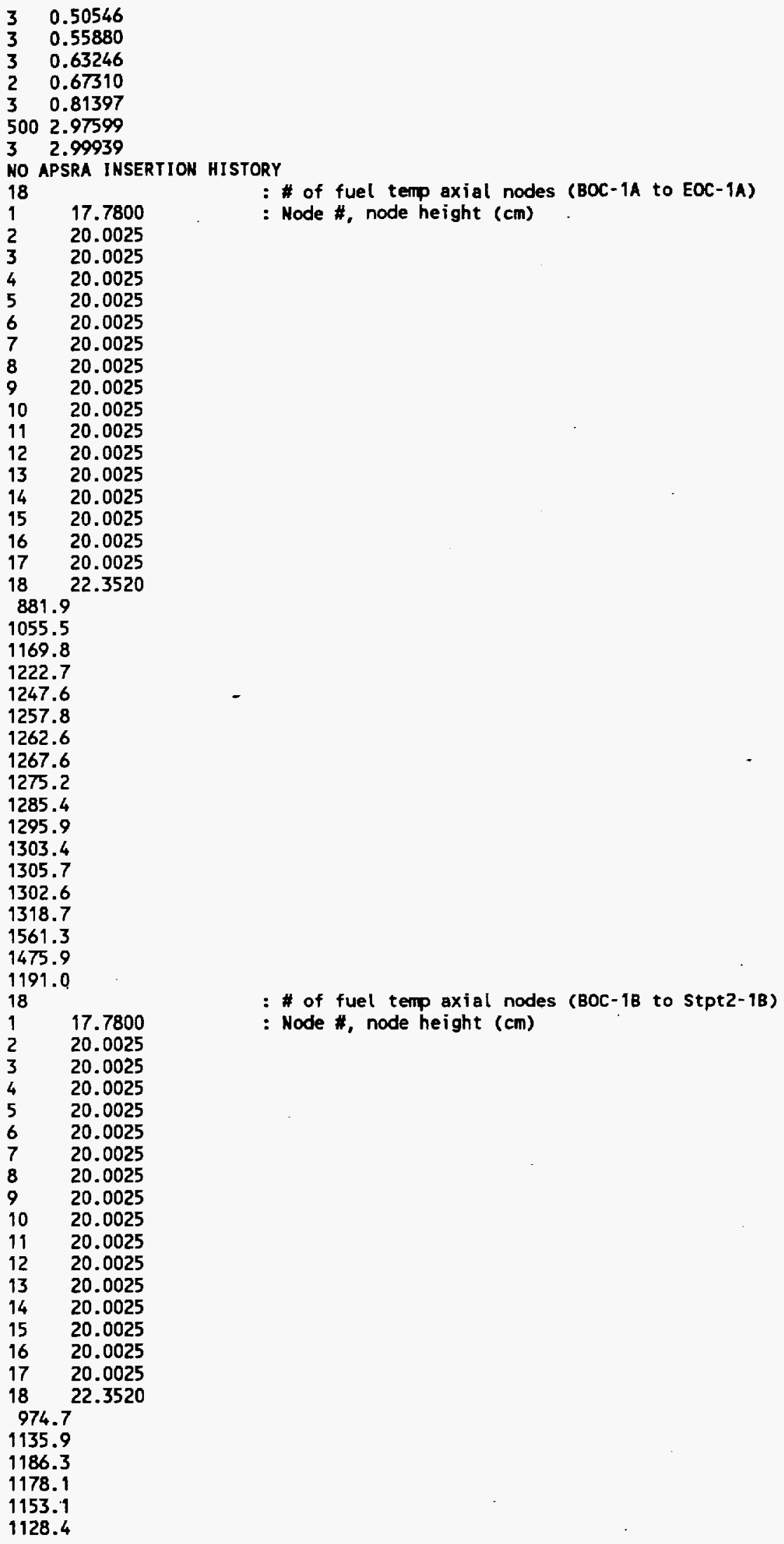




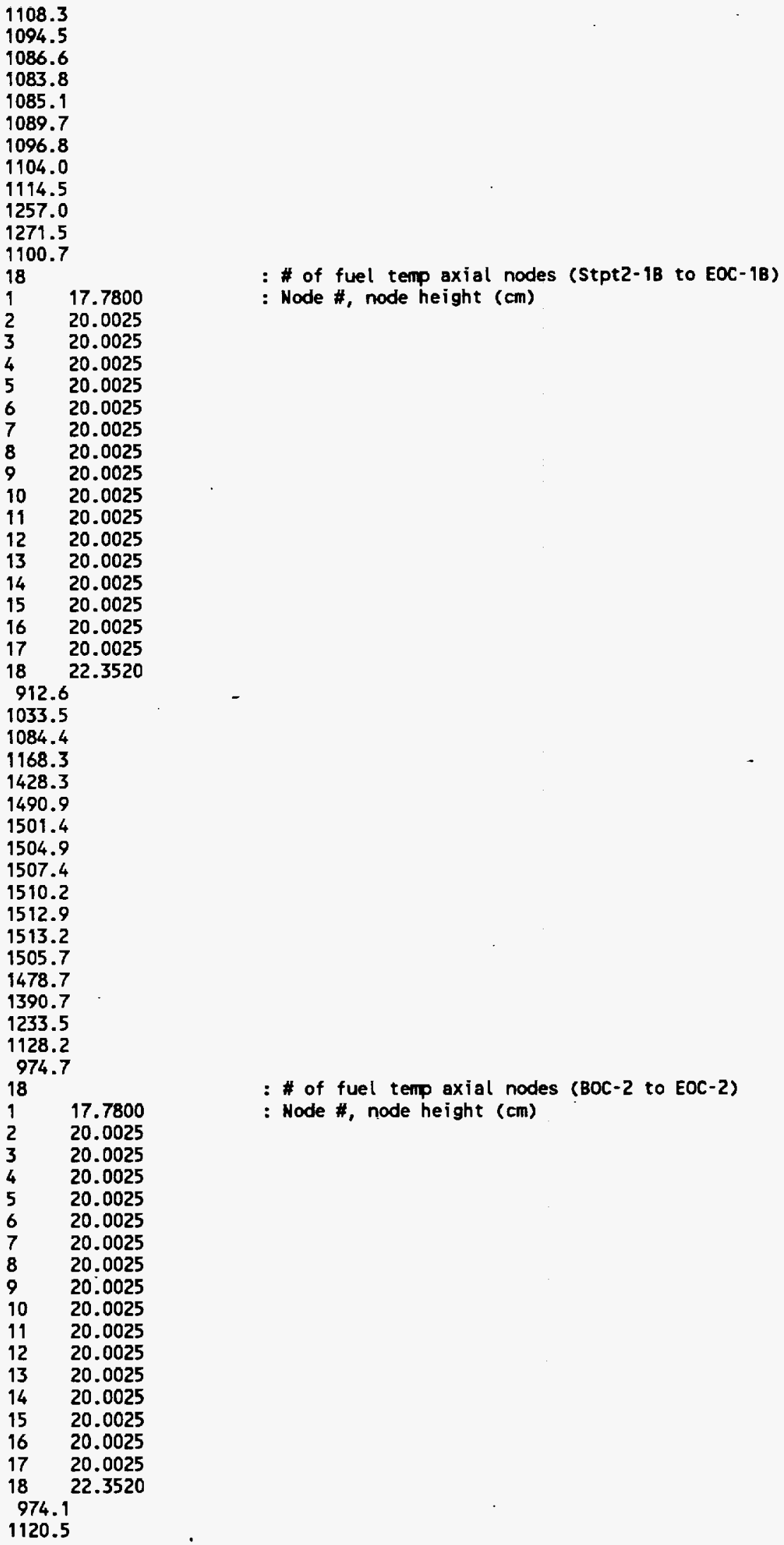


Apr 03 14:28 1997 File Name: A01i.dat BBA000000-01717-0200-00040 REV 00 ATtACHMENT IV - Page 6

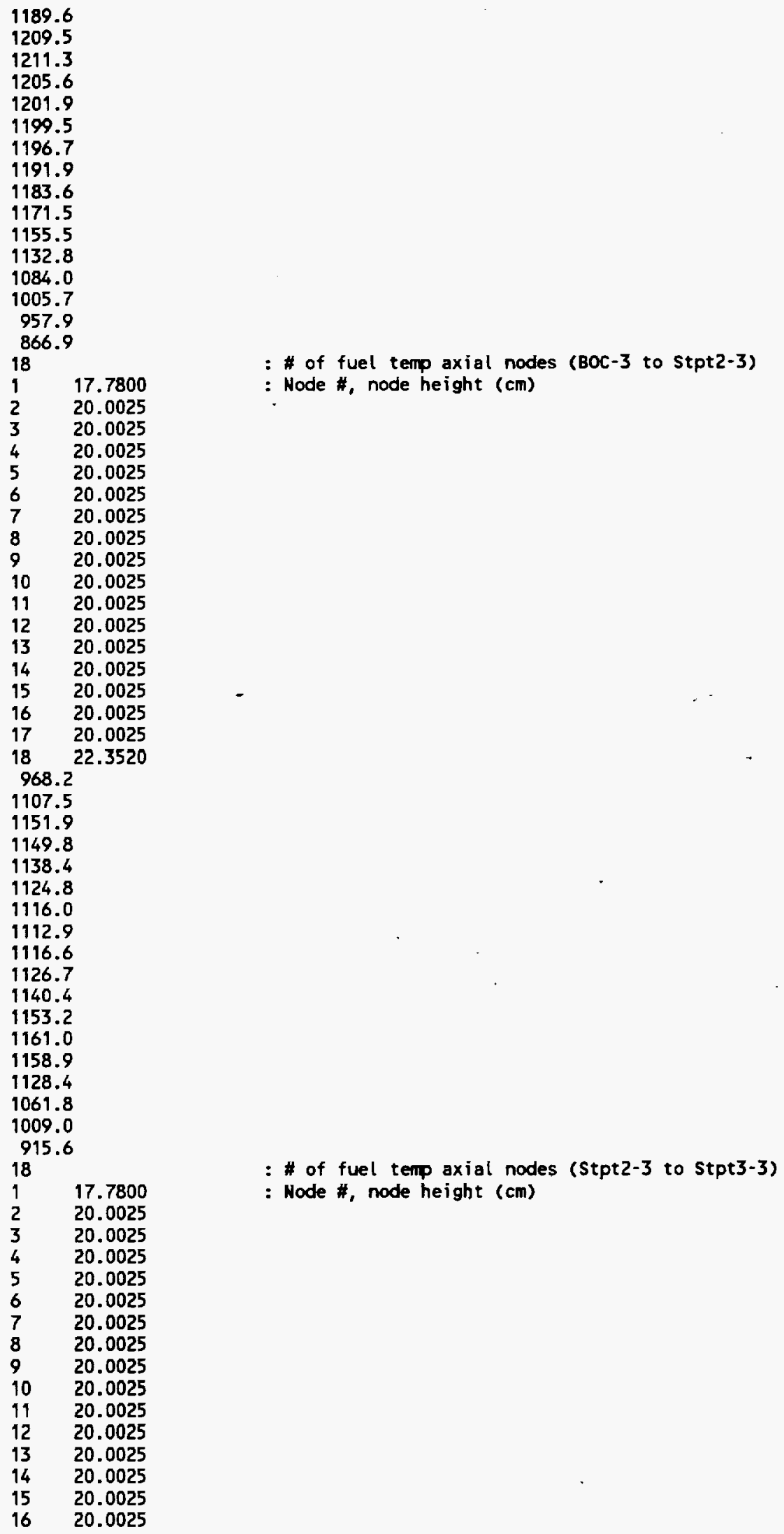




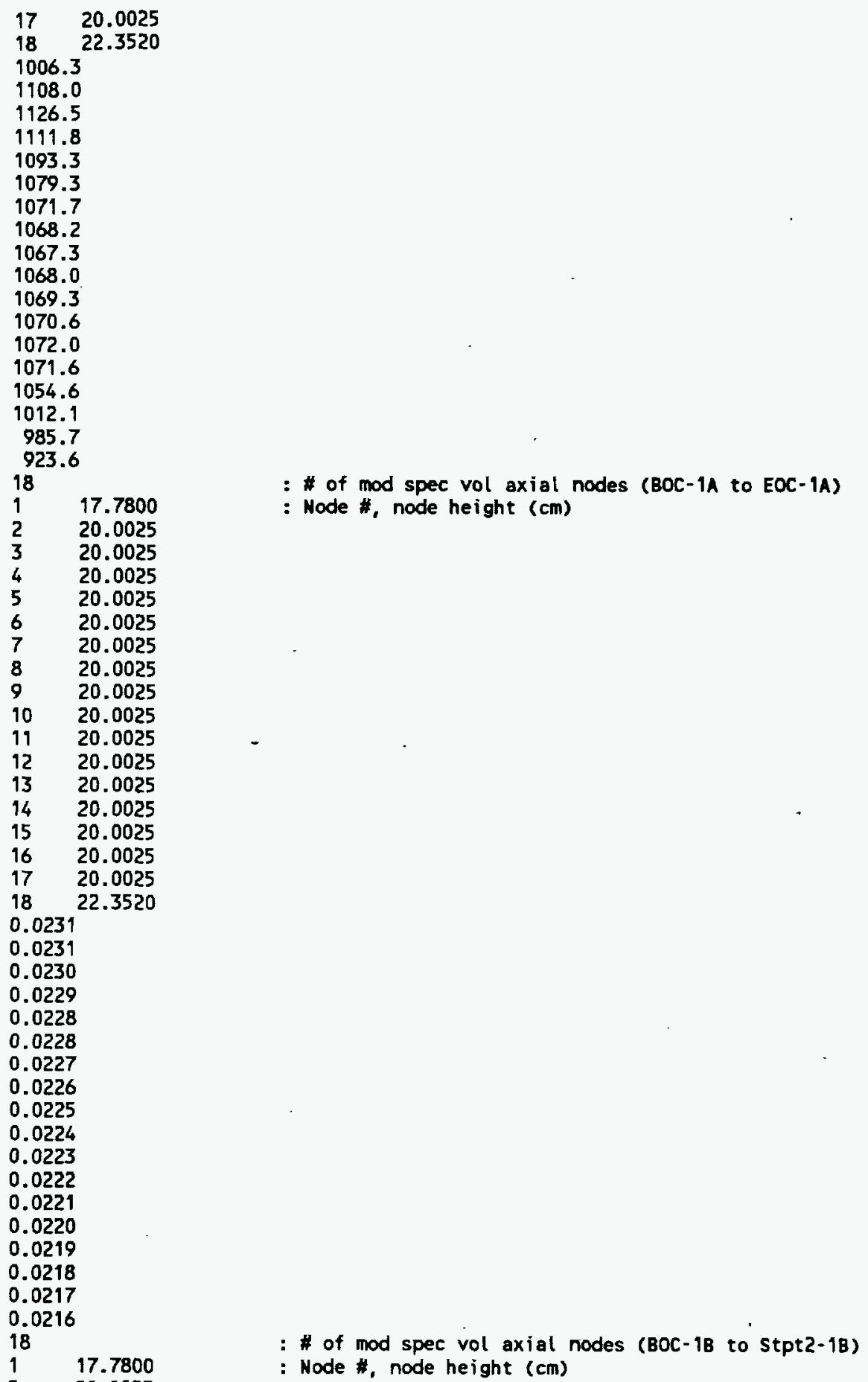


Apr 03 14:28 1997 File Name: A01i.dat BBA000000-01717-0200-00040 REV 00 ATTACHMENT IV - Page 8

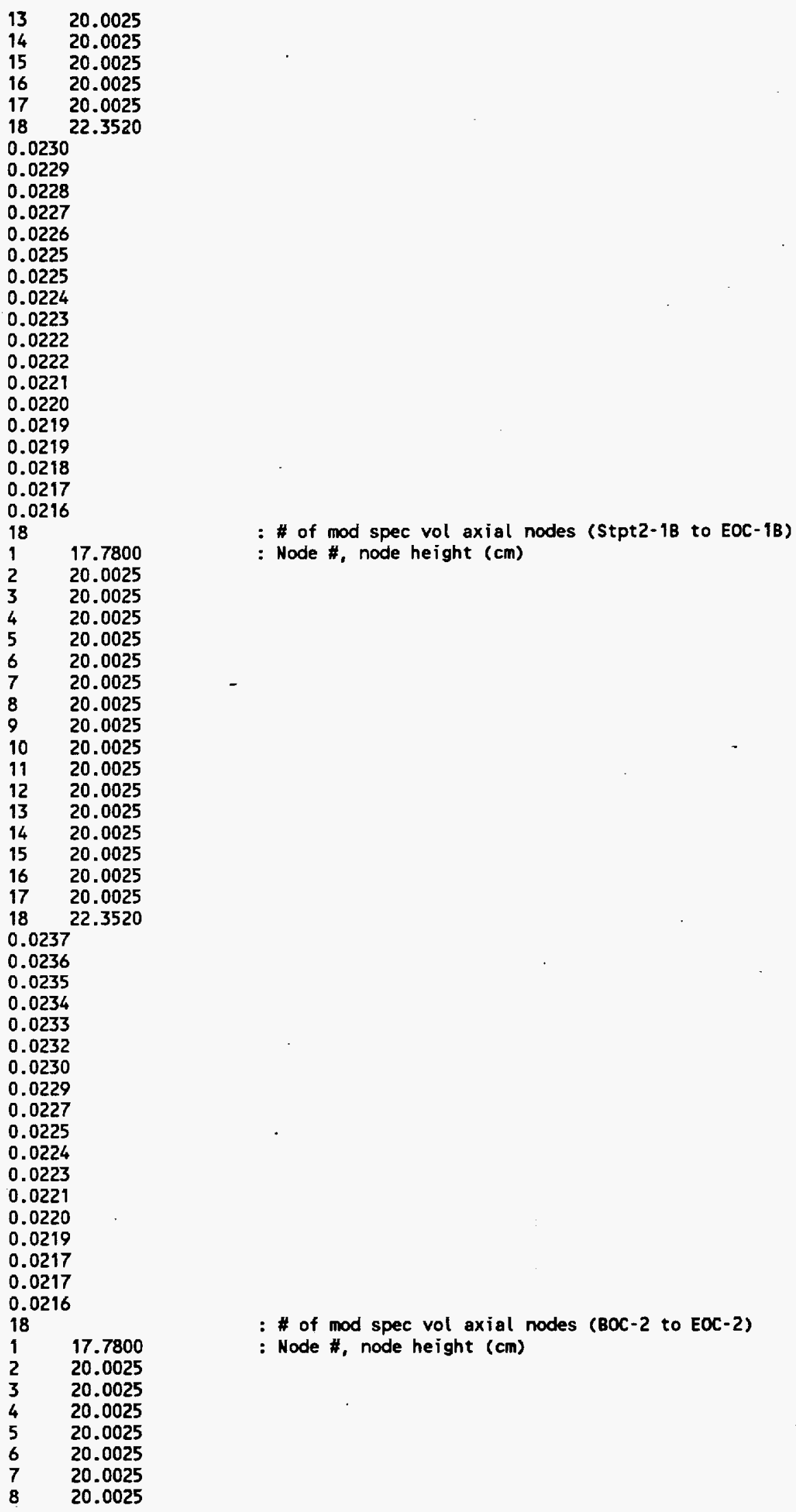




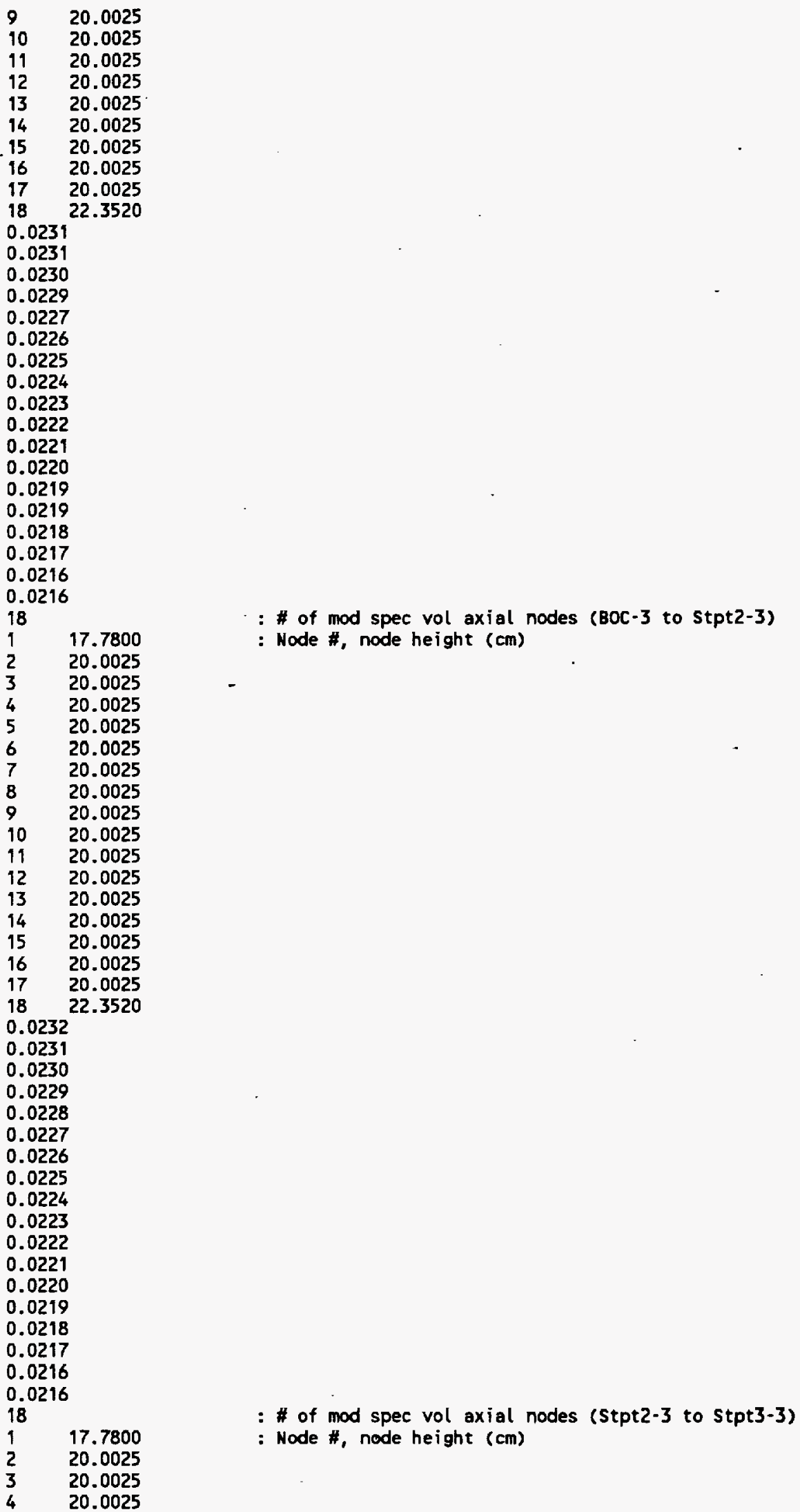


Apr 03 14:28 1997 File Name: A01i.dat BBA000000-01717-0200-00040 RÉV 0O ATTACHMENT IV - Page 10

20.0025

20.0025

20.0025

20.0025

20.0025

20.0025

20.0025

20.0025

20.0025

20.0025

20.0025

20.0025

20.0025

22.3520

0.0232

0.0231

0.0230

0.0229

0.0228

0.0227

0.0226

0.0225

0.0224

0.0223

0.0222

0.0221

0.0220

0.0219

0.0218

0.0217

0.0217

0.0216

17.7800

: \# of burnup axial nodes (BOC-1A)

20.0025

: Node \#, node height $(\mathrm{cm})$

20.0025

20.0025

20.0025

20.0025

20.0025

20.0025

20.0025

20.0025

20.0025

20.0025

20.0025

20.0025

20.0025

20.0025

20.0025

22.3520

0.0

0.0

0.0

0.0

0.0

0.0

0.0

0.0

0.0

0.0

0.0

0.0

0.0

0.0

0.0

0.0

0.0

0.0

18

: \# of burnup axial nodes (BOC-18) 
Apr 03 14:28 1997 File Name: A01i.dat BBA000000-01717-0200-00040 REV 00 ATtACHMENT IV - Page 11

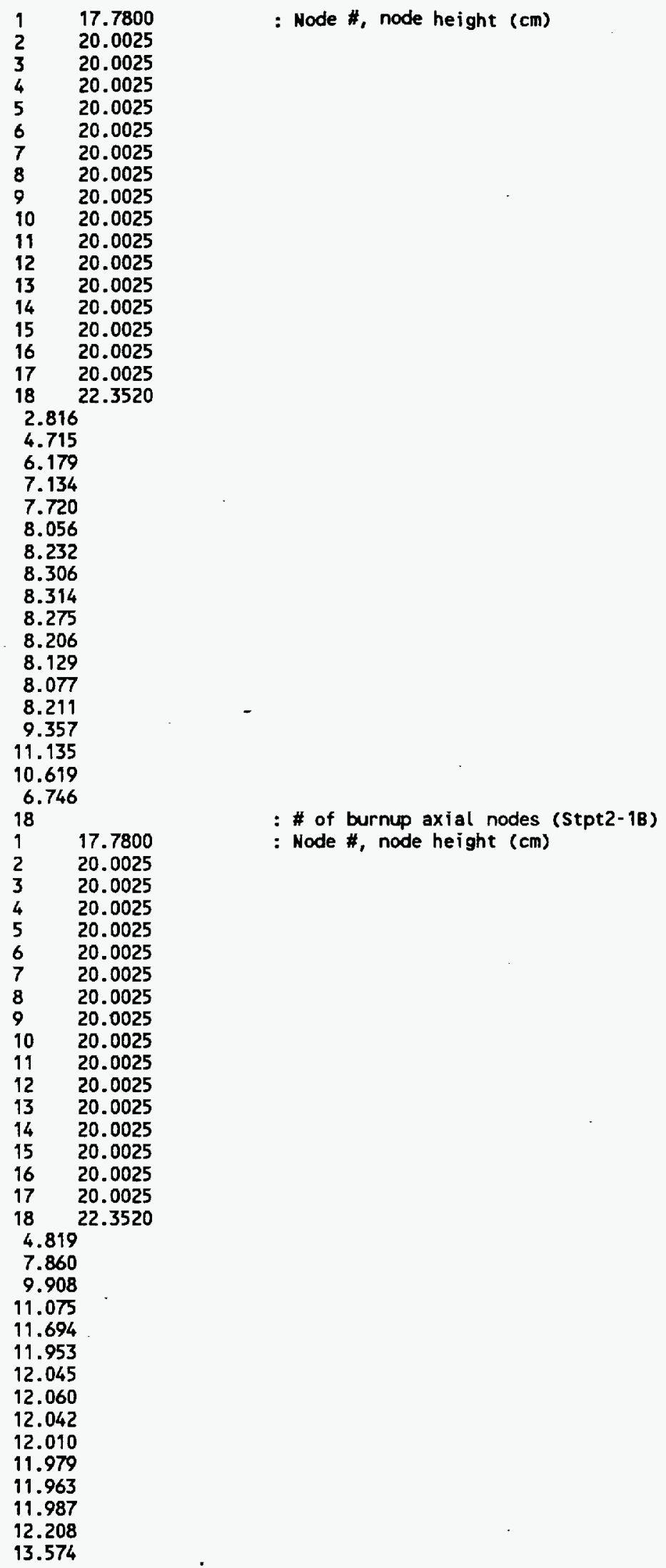


Apr 03 14:28 1997 File Name: A01i.dat BBA000000-01717-0200-00040 REV 00 ATtACHmENT IV - Page 12

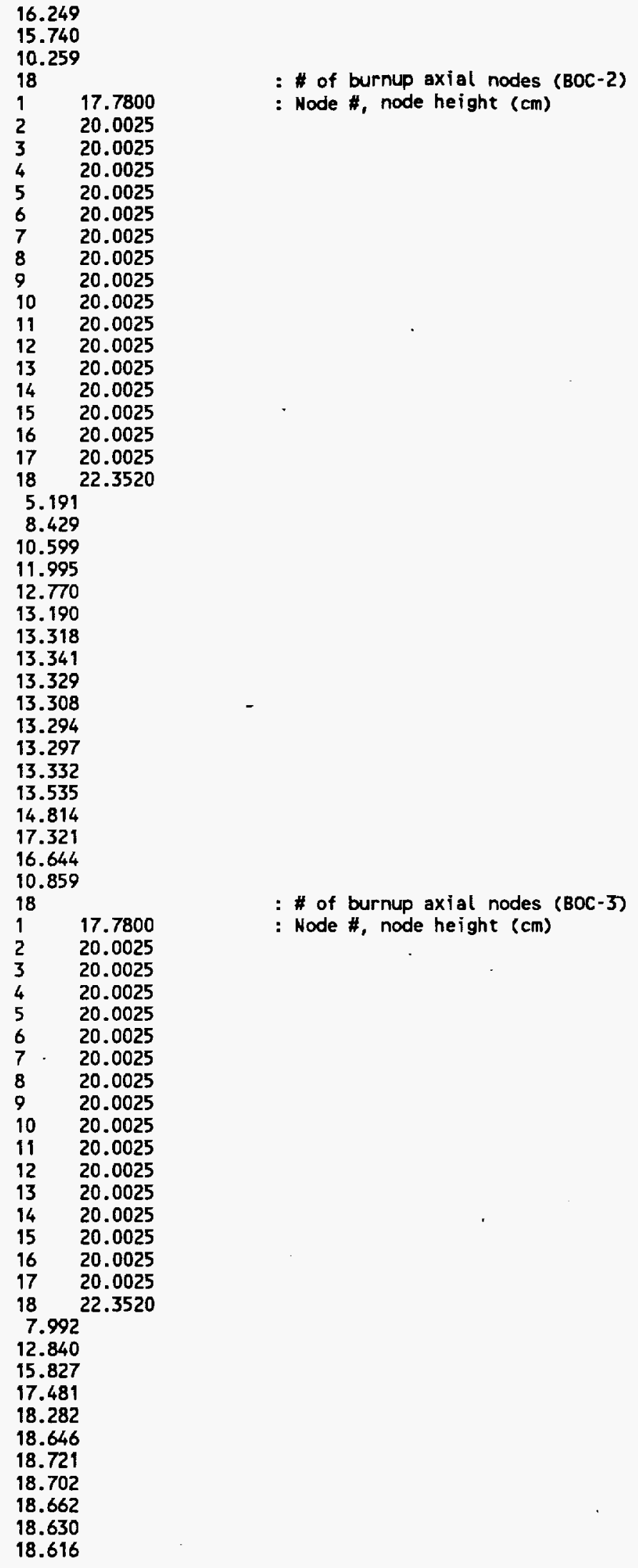


Apr 03 14:28 1997 File Name: A01i.dat BBA000000-01717-0200-00040 REV 00 ATTACKMENT IV - Page 13

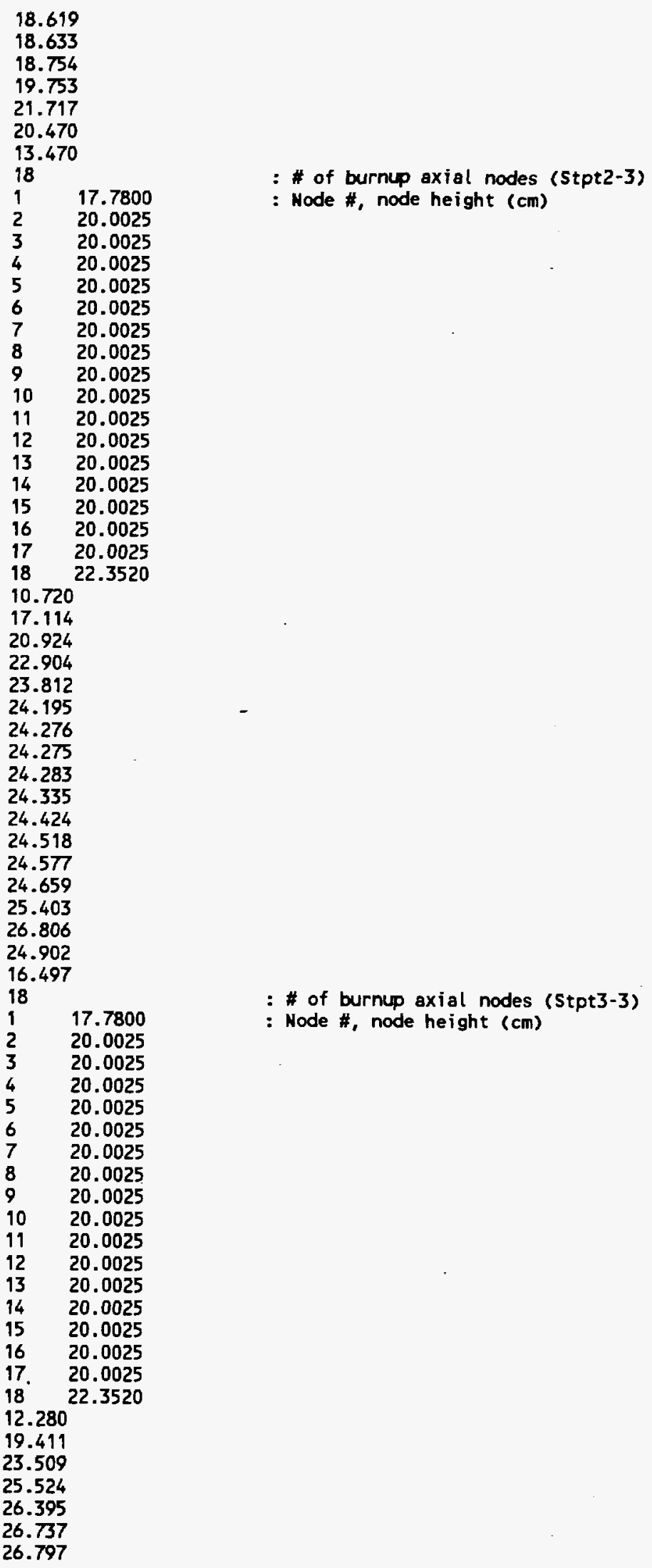


Apr 03 14:28 1997 file Name: A01i.dat BBA000000-01717-0200-00040 RÉV ÓO ATTACHMENT IV - Page 14

26.793

26.815

26.893

27.016

27.148

27.247

27.358

28.056

29.285

27.150

18.111 


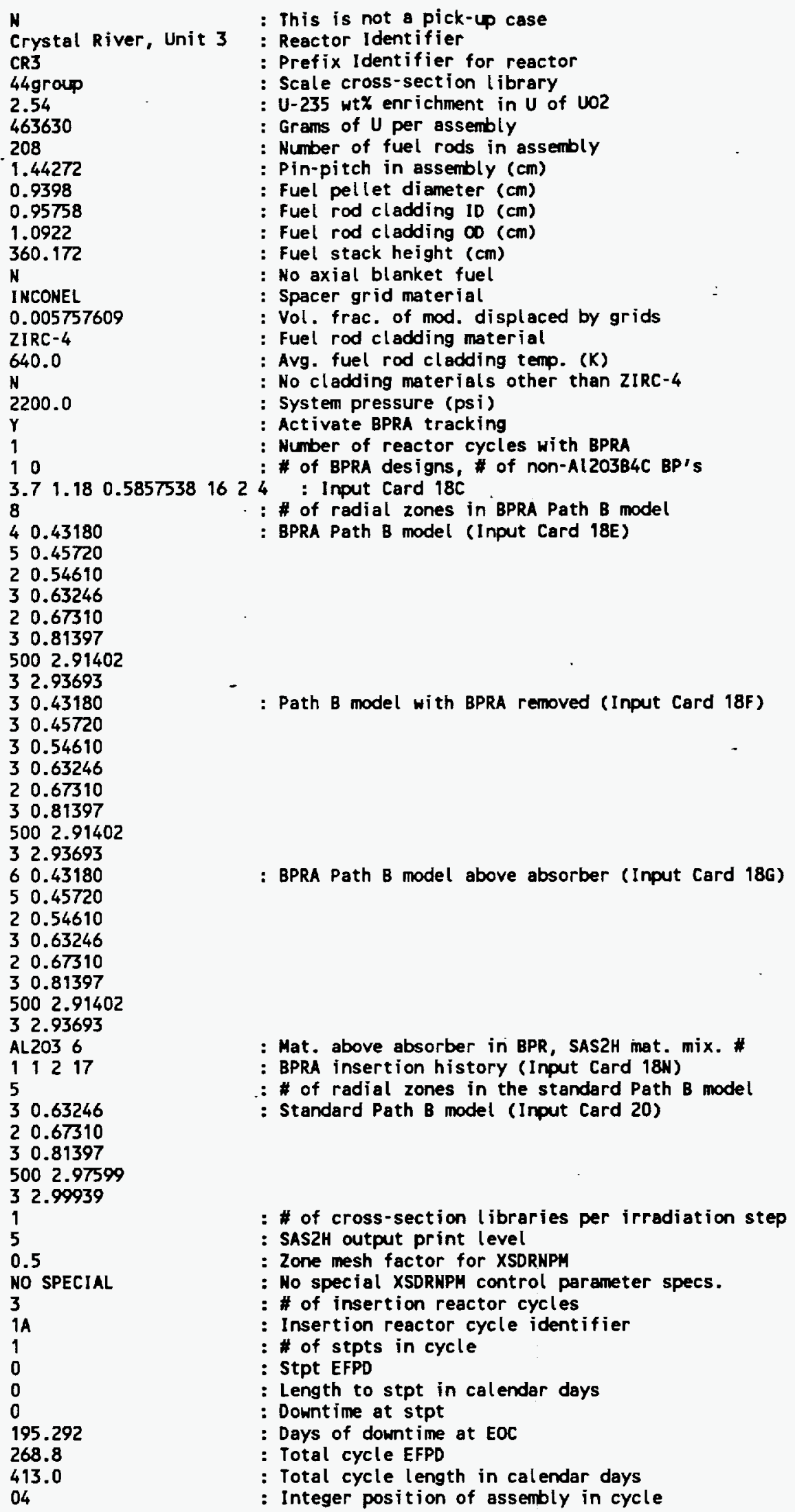




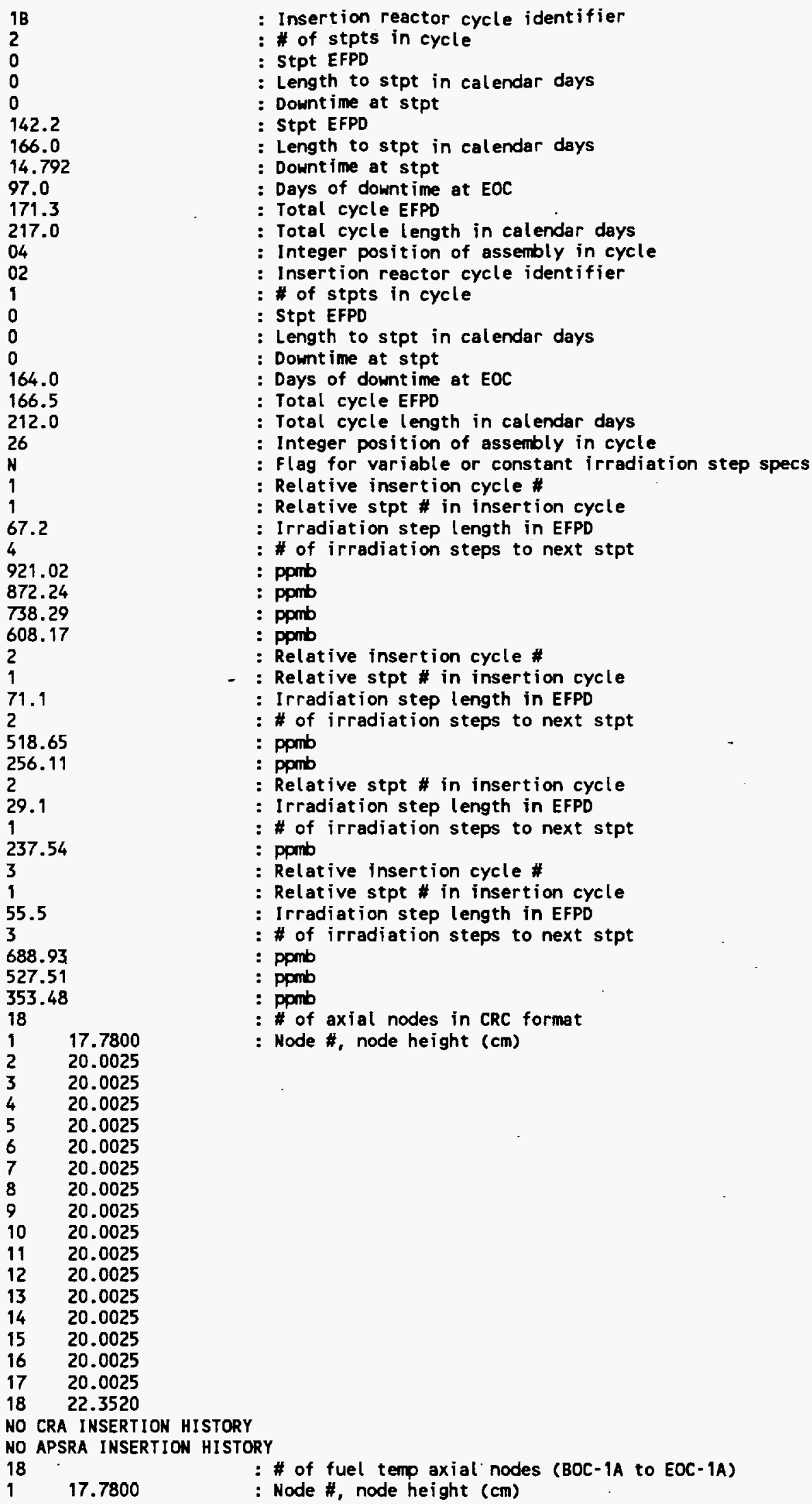


Apr 03 15:00 1997 File Name: A04i.dat BBA000000-01717-0200-00040 REV 00 ATtACHMENT V - Page 3

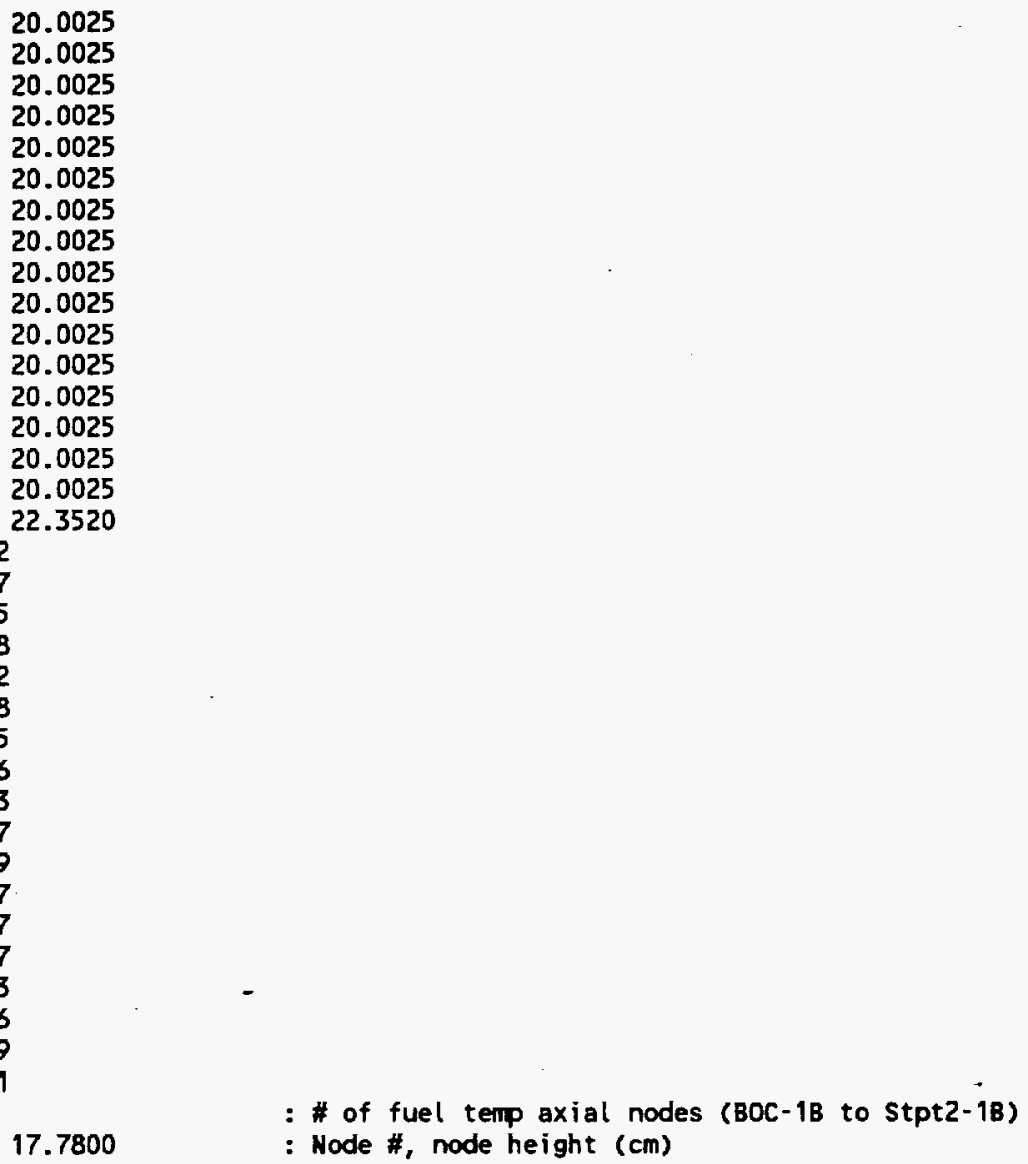

: \# of fuel temp axial nodes (BOC-1B to stpt2-18)

: Node \#, node height $(\mathrm{cm})$

1298.2

1279.0

1268.6

1264.8

1266.9

1275.4

1289.5

1308.3

1330.3

1344.4 
Apr 03 15:00 1997 File Name: A04i.dat BBA000000-01717-0200-00040 REV 00 ATTACHMENT V - Page 4

1308.8

1109.5

18

$1 \quad 17.7800$

20.0025

20.0025

20.0025

20.0025

20.0025

20.0025

20.0025

20.0025

20.0025

20.0025

20.0025

20.0025

20.0025

20.0025

20.0025

20.0025

22.3520

1111.1

1267.6

1288.1

1255.8

1226.7

1209.2

1199.5

1193.5

1188.7

1185.4

1186.3

1191.8

1197.4

1199.5

1196.3

1183.5

1141.3

983.4

18

17.7800

20.0025

20.0025

20.0025

20.0025

20.0025

20.0025

20.0025

20.0025

20.0025

20.0025

20.0025

20.0025

20.0025

20.0025

20.0025

$17 \quad 20.0025$

$18 \quad 22.3520$

0.0239

0.0238

0.0237

0.0235

0.0234

0.0232

0.0231

0.0229

0.0228

0.0226

0.0225

0.0223

: \# of fuel tenp axial nodes (Stpt2-1B to EOC-1B)

: Node \#, node height $(\mathrm{cm})$

\# of mod spec vol axial nodes (BOC-1A to EOC-1A)

: Node \#, node height $(\mathrm{cm})$ 


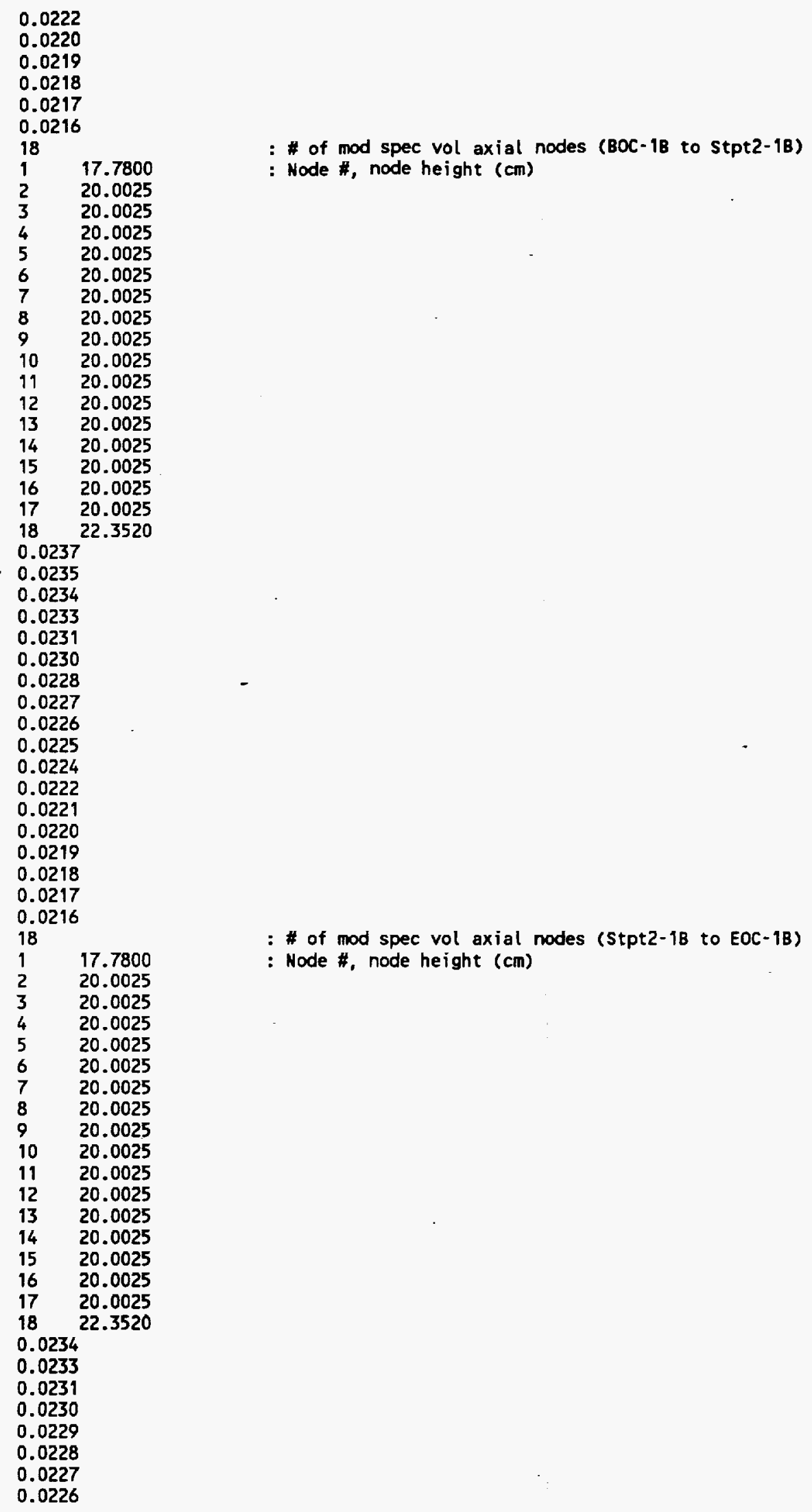




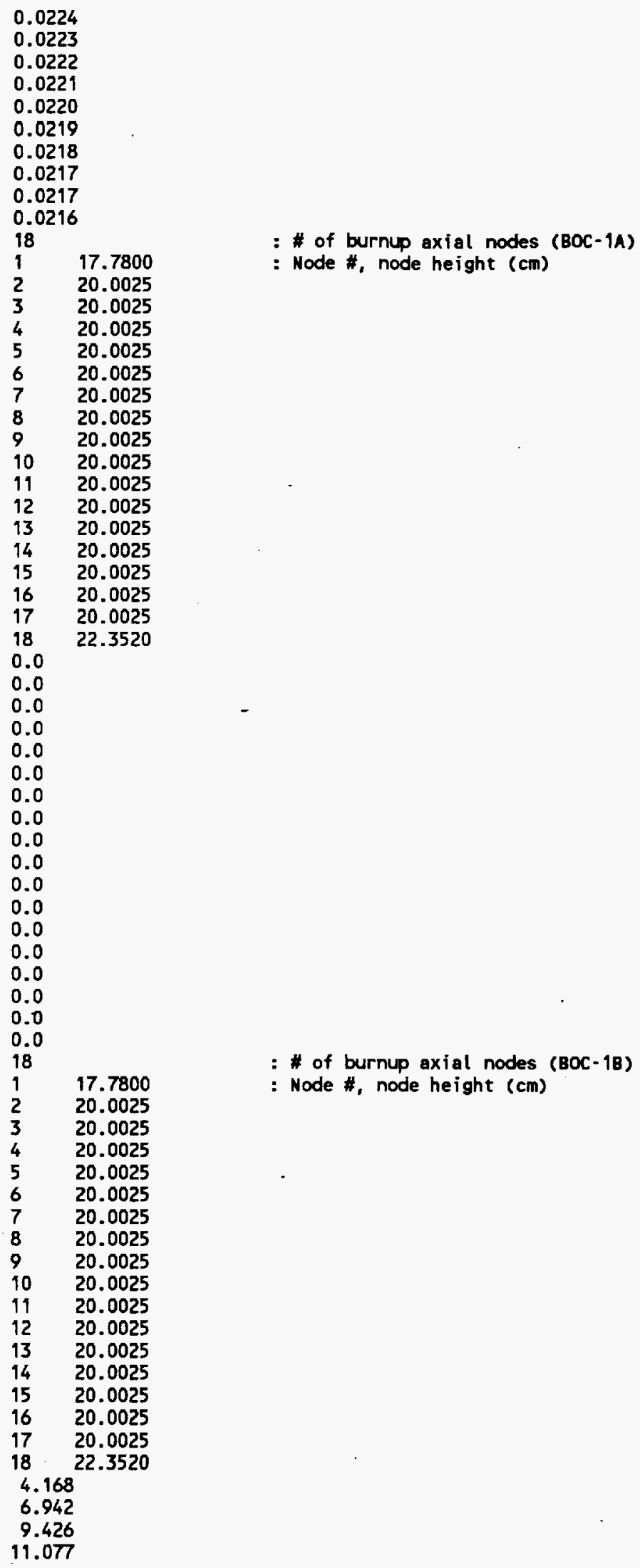


Apr 03 15:00 1997 File Name: A04i.dat BBA000000-01717-0200-00040 REV 00 ATtAChMENT V - Page 7

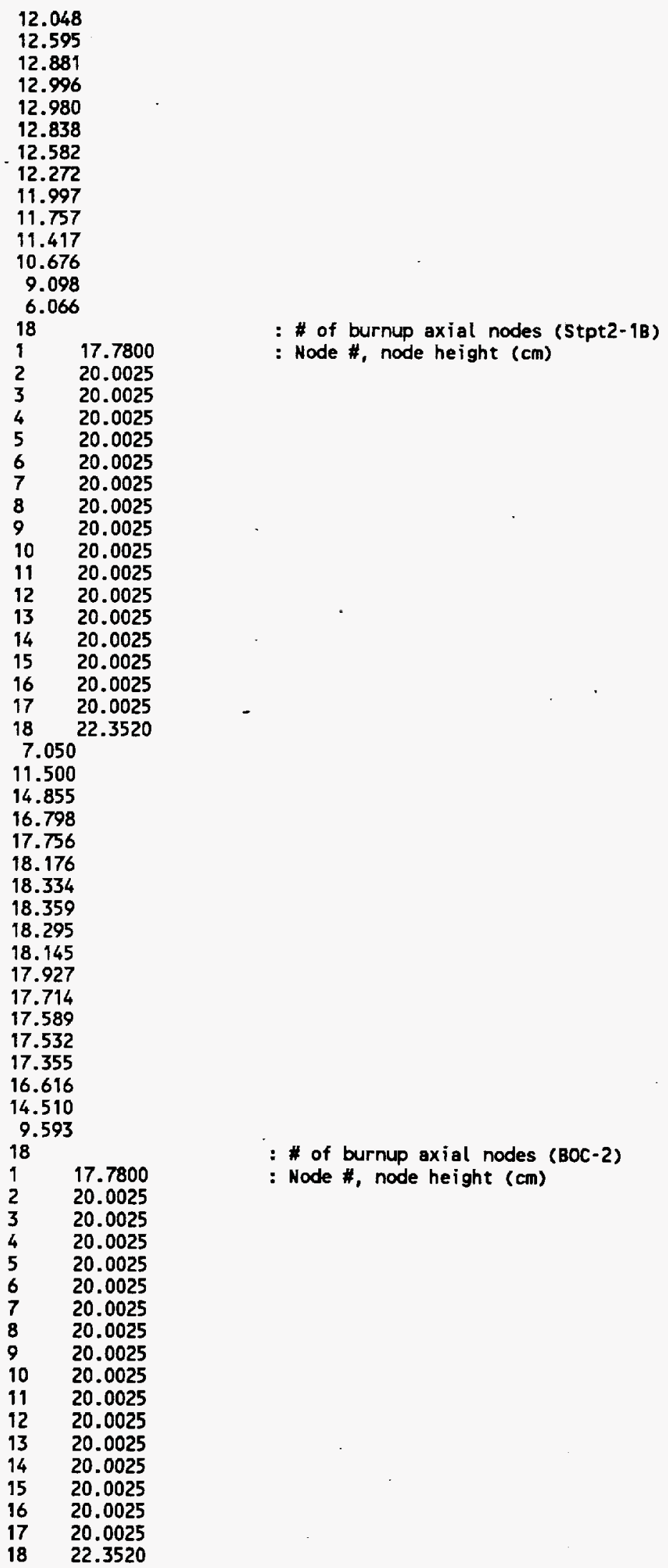


Apr 03 15:00 1997 file Name: A04i.dat BBA000000-01717-0200-00040 REV 00 ATTACHMENT $V$ - Page 8
7.708
12.474
15.921
17.847
18.769
19.161
19.298
19.306
19.230
19.077
18.874
18.692
18.604
18.575
18.406
17.637
15.425
10.199 


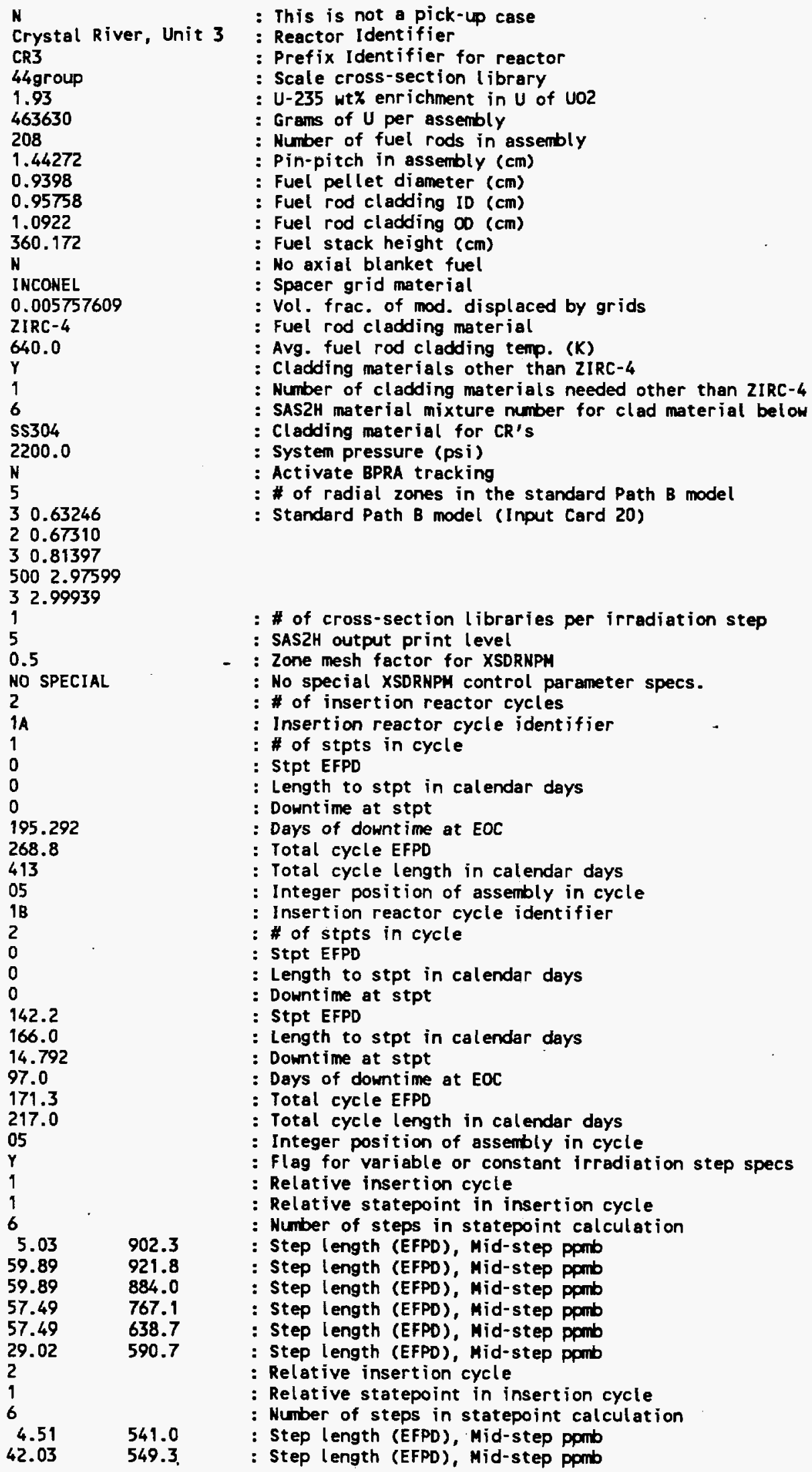




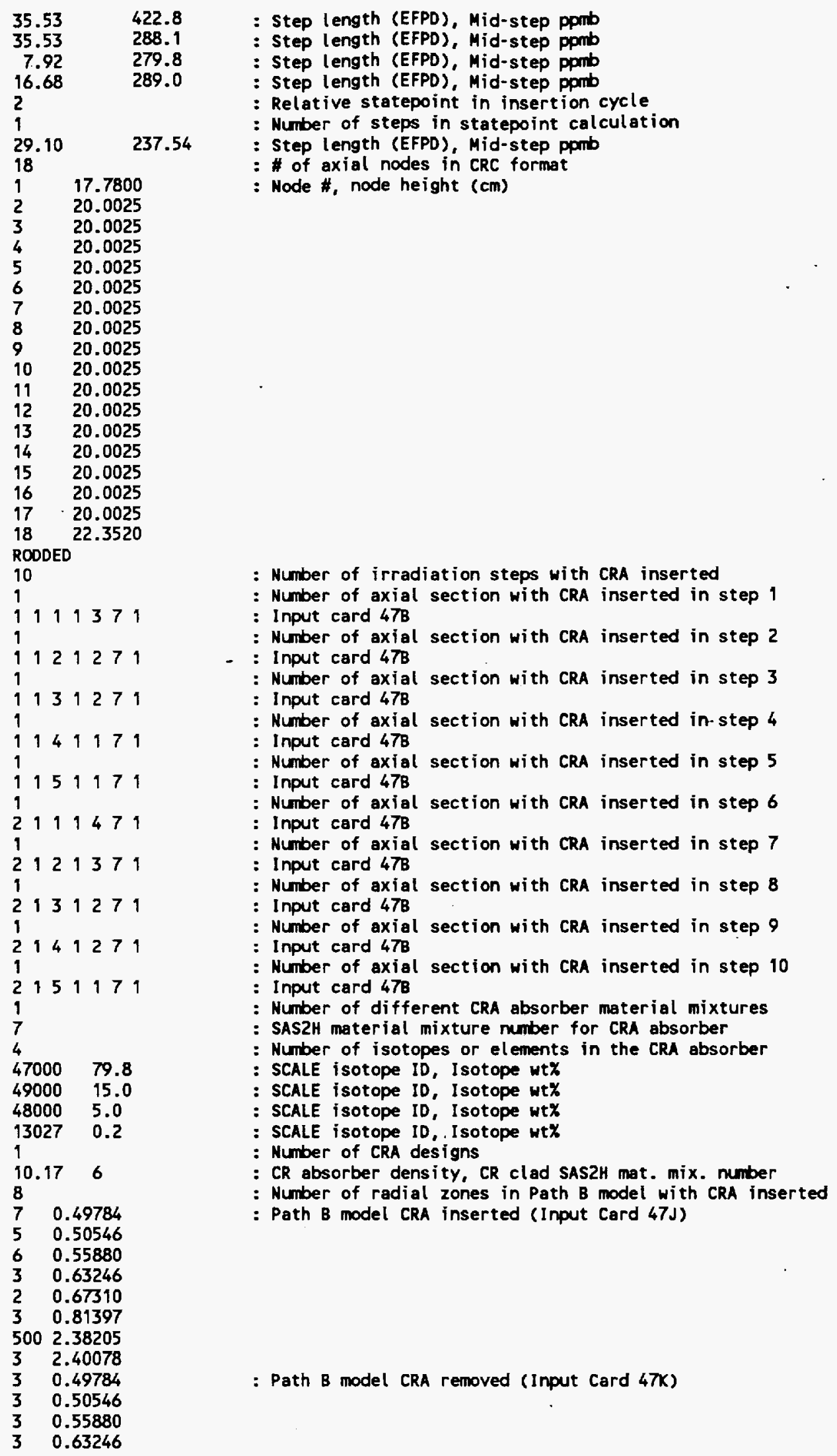




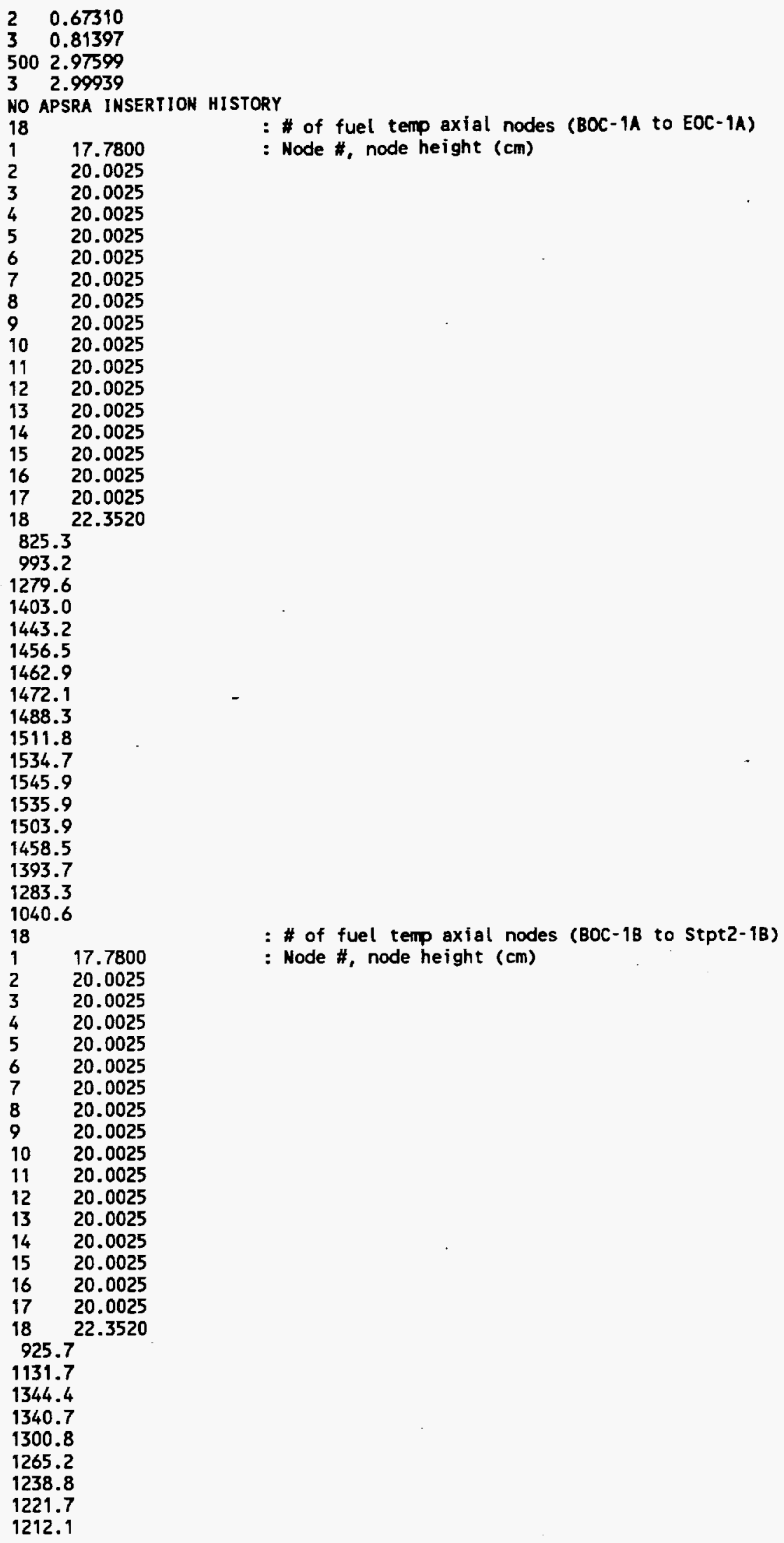


Apr 03 15:00 1997 File Name: A05i.dat BBA000000-01717-0200-00040 REV 00 ATTACHMENT VI - Page 4

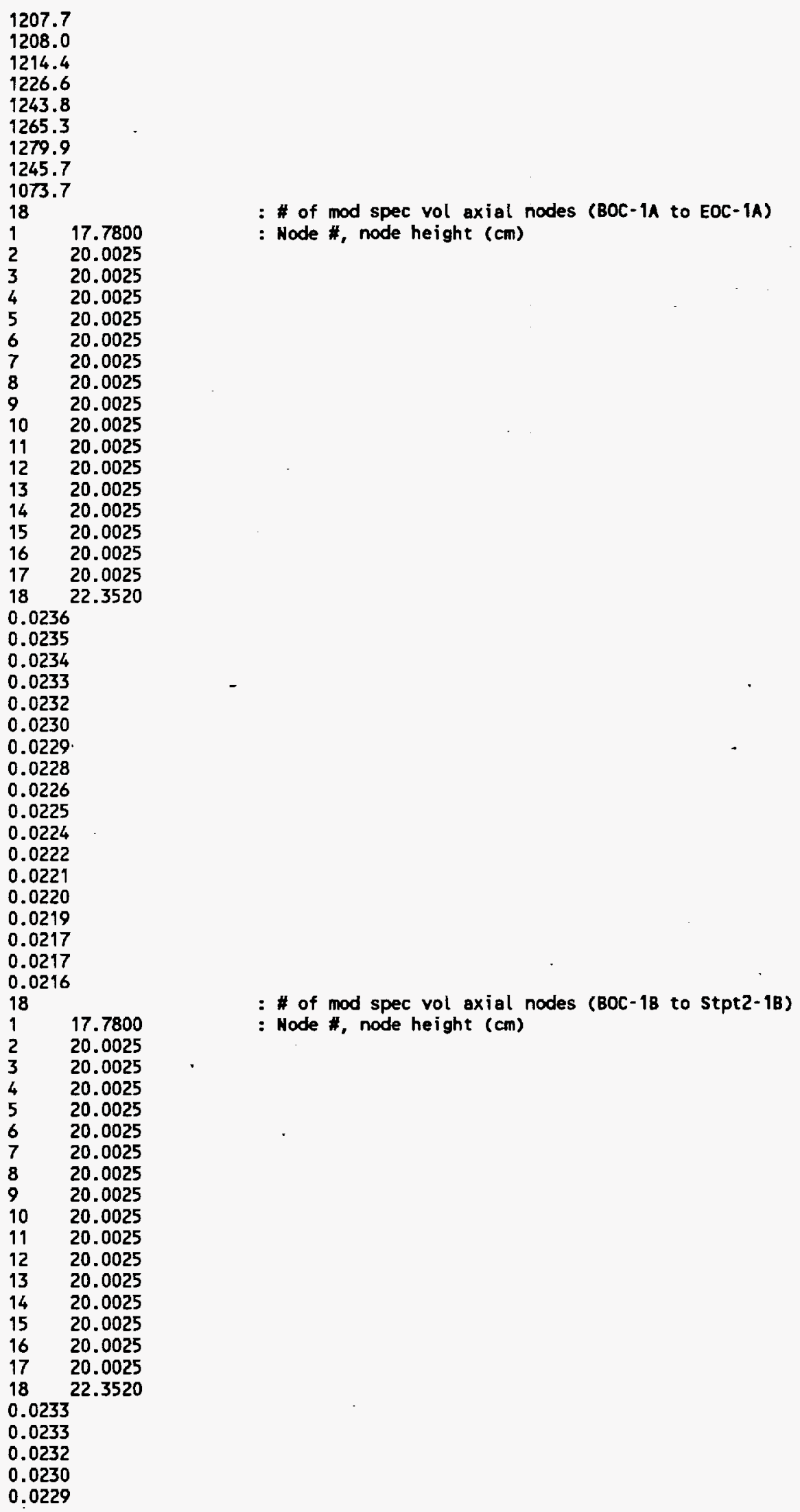




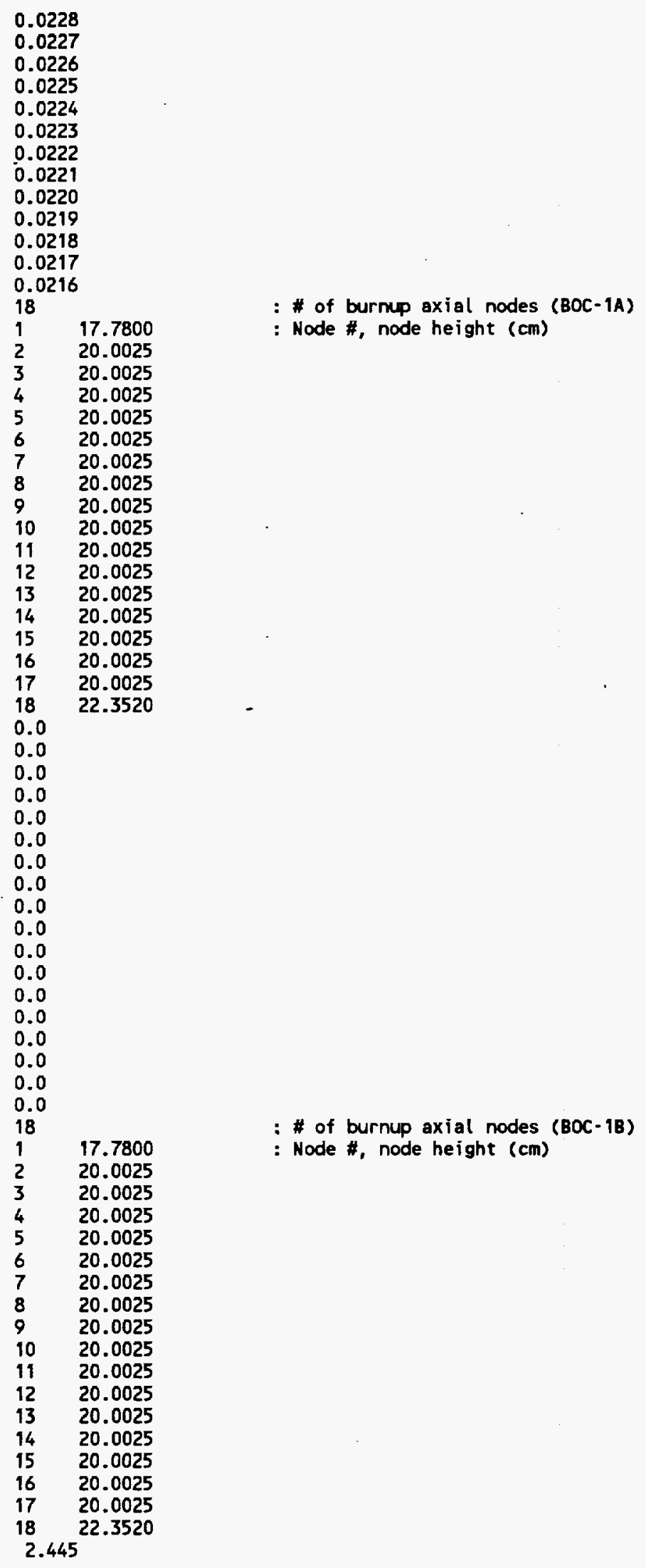


Apr 03 15:00 1997 File Name: A05i.dat BBA000000-01717-0200-00040 REV 00 ATTACHMENT VI - Page 6

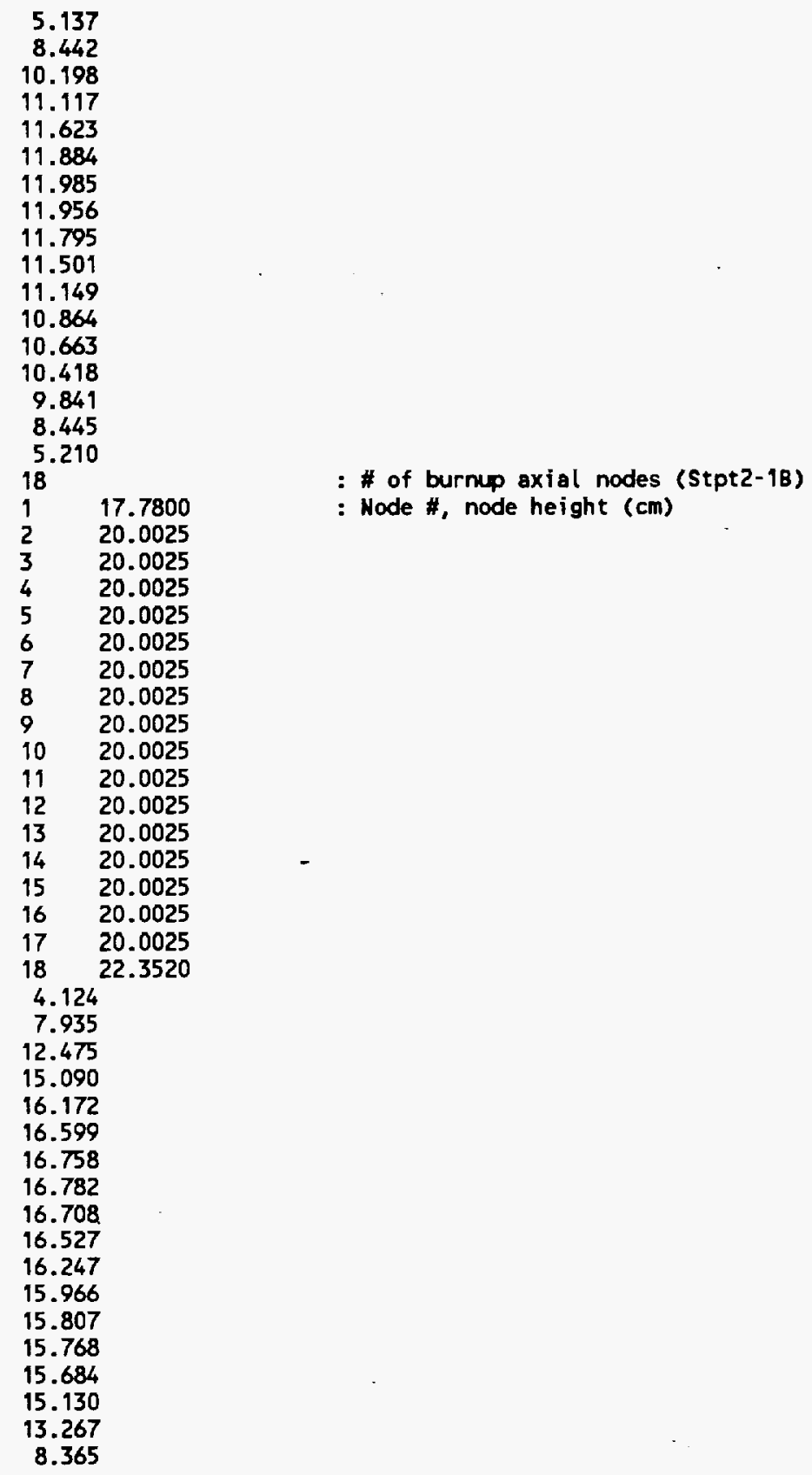




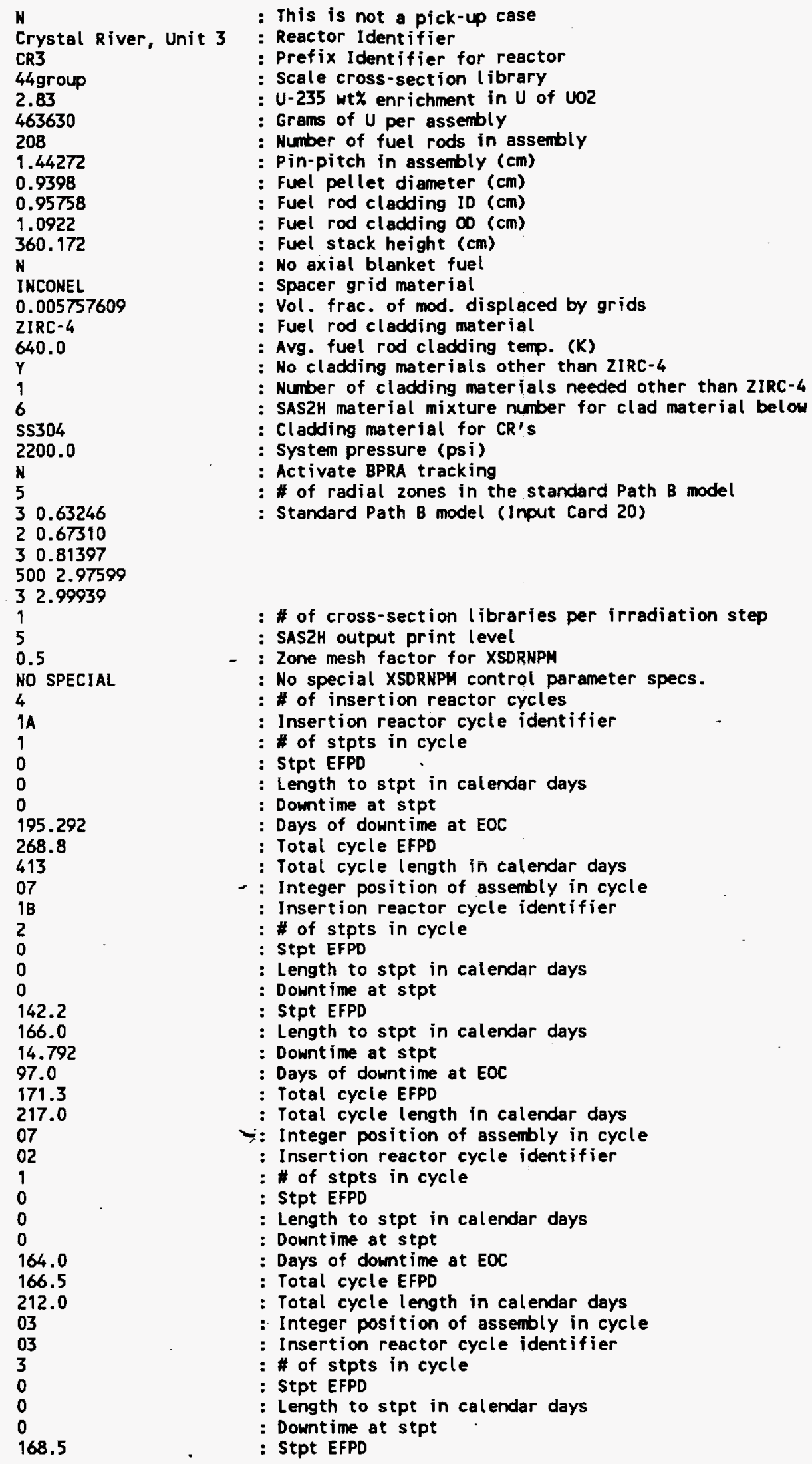




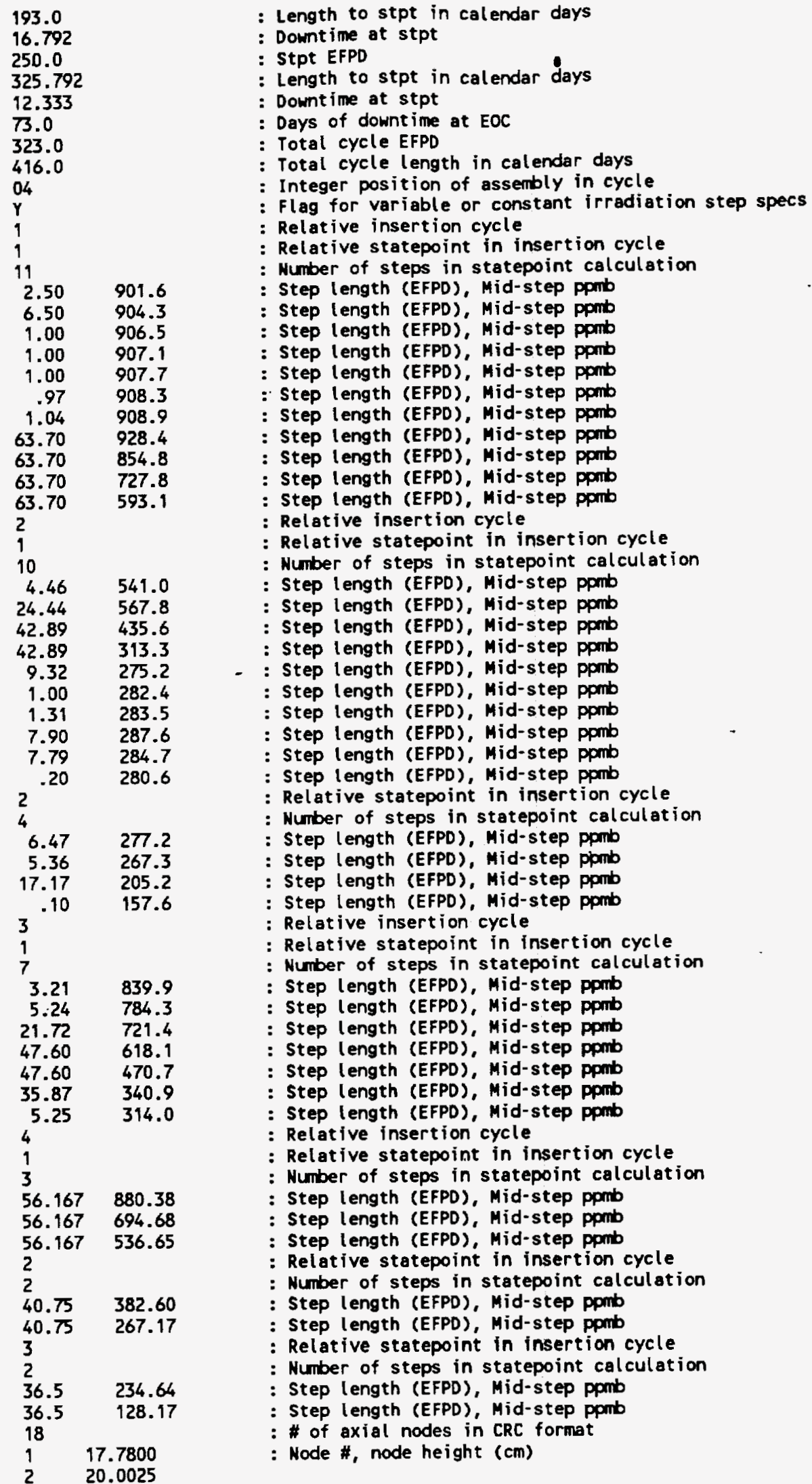


20.0025

20.0025

20.0025

20.0025

20.0025

20.0025

20.0025

20.0025

20.0025

20.0025

20.0025

20.0025

20.0025

20.0025

20.0025

22.3520

OODED

27

11111571

11211471

2

1131771

113111471

1141571

114131471

1151371

$\begin{array}{lllllll}1 & 1 & 5 & 14 & 14 & 71\end{array}$

1161271

171171

1111771

1211671

21311571

21411571

21511471

2161771

$2 \begin{array}{llllll}1 & 6 & 14 & 14 & 7 & 1\end{array}$

171571

181471

191371

1101371

2211571

2221471

2231371

2241371

3111571

3121471

3131371
: Number of irradiation steps with CRA inserted

: Number of axial section with CRA inserted in step 1

: Input card 478

: Number of axial section with CRA inserted in step 2

: Input card 478

: Number of axial section with CRA inserted in step 3

: Input card 478

: Input card 478

: Number of axial section with CRA inserted in step 4

: Input card 47B

: Input card $47 \mathrm{~B}$

: Number of axial section with CRA inserted in step 5

: Input card $47 \mathrm{~B}$

- Input card 47B

- Number of axial section with CRA inserted in step 6

: Input card 478

: Number of axial section with CRA inserted in step 7

: Input card 47B

: Number of axial section with CRA inserted in step 8

: Input card 478

: Number of axial section with CRA inserted in step 9

: Input card $47 \mathrm{~B}$

: Number of axial section with CRA inserted in step 10

: Input card 47B

: Number of axial section with CRA inserted in step 11

: Input card 47B

: Number of axial section with CRA inserted in step 12

: Input card 47B

: Number of axial section with CRA inserted in step 13

: Input card 47B

: Input card 47B

: Number of axial section with CRA inserted in step 14

: Input card 478

: Number of axial section with CRA inserted in step 15

: Input card 47B

: Number of axial section with CRA inserted in step 16

: Input card 478

: Number of axial section with CRA inserted in step 17

: Input card 47B

: Number of axial, section with CRA inserted in step 18

: Input card 47B

: Number of axial section with CRA inserted in step 19

: Input card 47B

: Number of axial section with CRA inserted in step 20

: Input card 478

: Number of axial section with CRA inserted in step 21

: Input card 478

: Number of axial section with CRA inserted in step 22

: Input card 47B

: Number of axial section with CRA inserted in step 23

: Input card 478

: Number of axial section with CRA inserted in step 24

: Input card 47B 


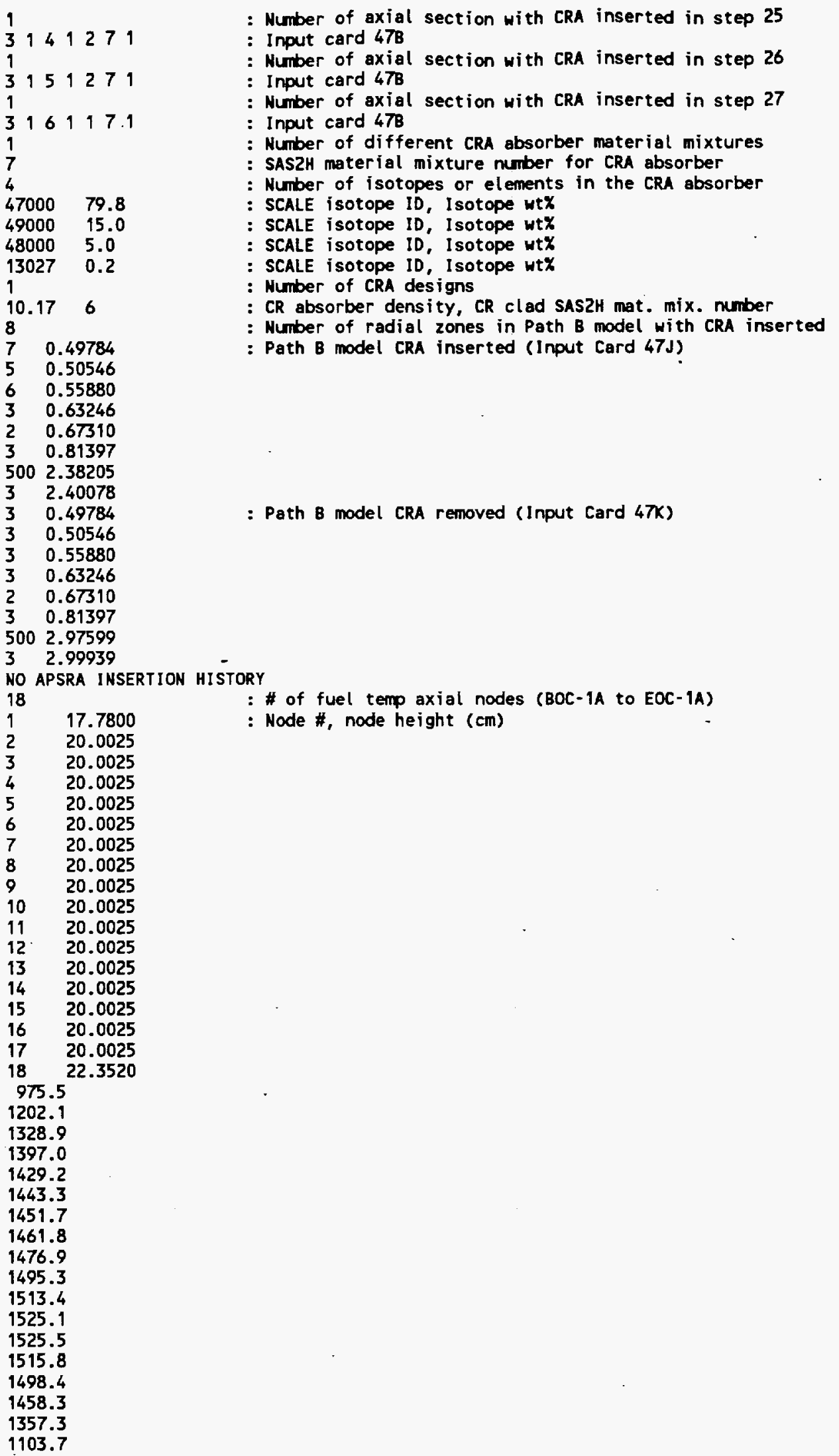


Apr 03 15:00 1997 File Name: A07i.dat BBA000000-01717-0200-00040 REV 00 ATtACHMENT VII - Page 5

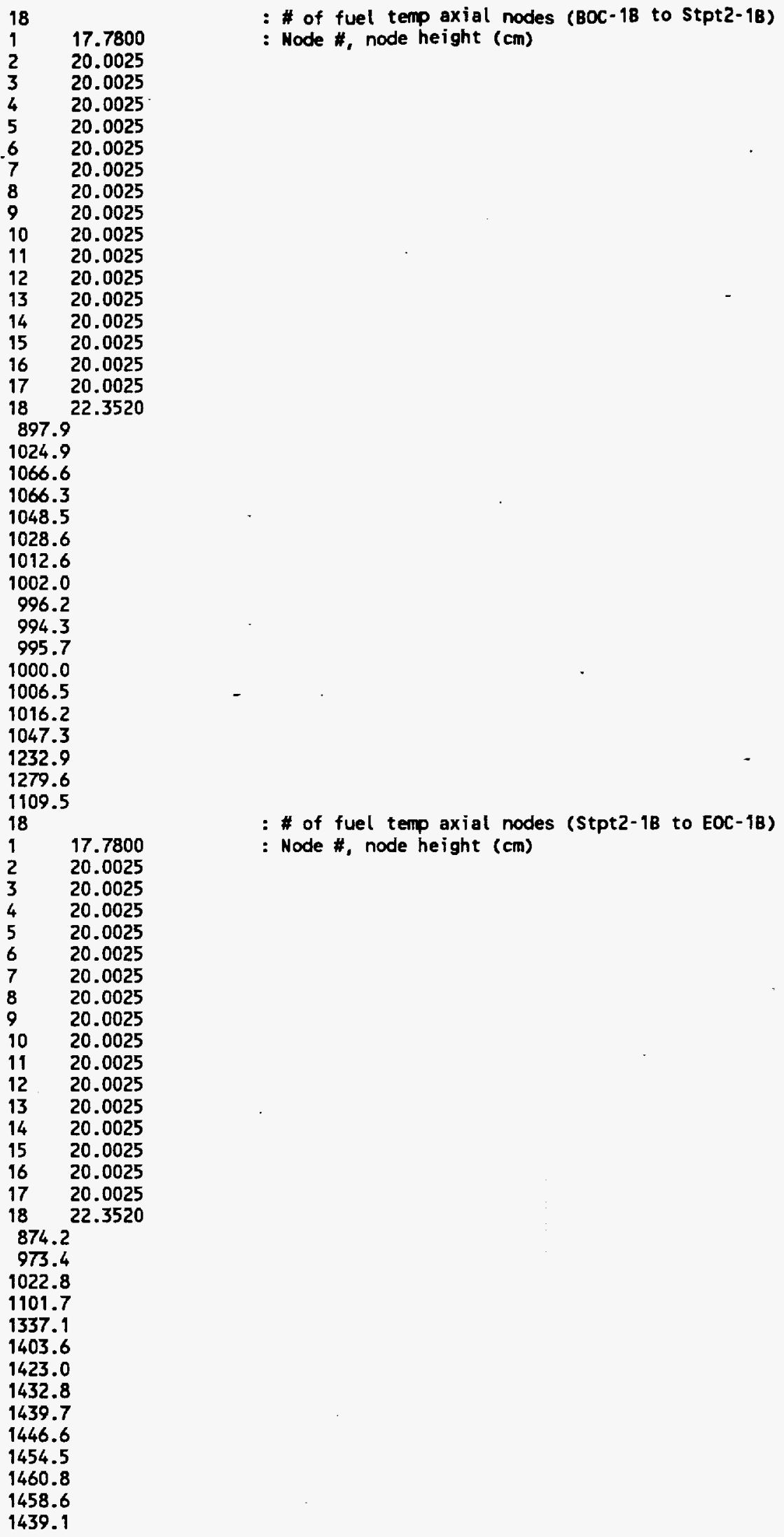


Apr 03 15:00 1997 File Name: A07i.dat BBA000000-01717-0200-00040 REV 00 ATTACHMENT VII - Pege 6

1385.4

1288.2

1195.1

1021.7

18

17.7800

: of fuel temp axial nodes (BOC-2 to EOC-2)

20.0025

: Node \#, node height $(\mathrm{cm})$

20.0025

20.0025

20.0025

20.0025

20.0025

20.0025

20.0025

20.0025

20.0025

20.0025

20.0025

20.0025

20.0025

20.0025

20.0025

22.3520

871.5

977.1

1121.4

1241.2

1250.0

1239.1

1232.8

1229.6

1226.0

1218.8

1206.9

$\$ 190.9$

1172.9

1154.5

1132.7

1101.9

1073.3

963.8

18

$1 \quad 17.7800$

20.0025

20.0025

20.0025

20.0025

20.0025

20.0025

20.0025

20.0025

20.0025

20.0025

20.0025

20.0025

20.0025

20.0025

20.0025

20.0025

$18 \quad 22.3520$

1020.4

1176.9

1200.7

1188.3

1167.2

1145.2

1126.3

1111.6

1106.1

1113.7

: \# of fuel temp axial nodes (BOC-3 to stpt2-3)

: Node \#, node height (cm) 
Apr 03 15:00 1997 File Name: A07i.dat BBA000000-01717-0200-00040 REV 00 ATtACHMENT VII - Page 7

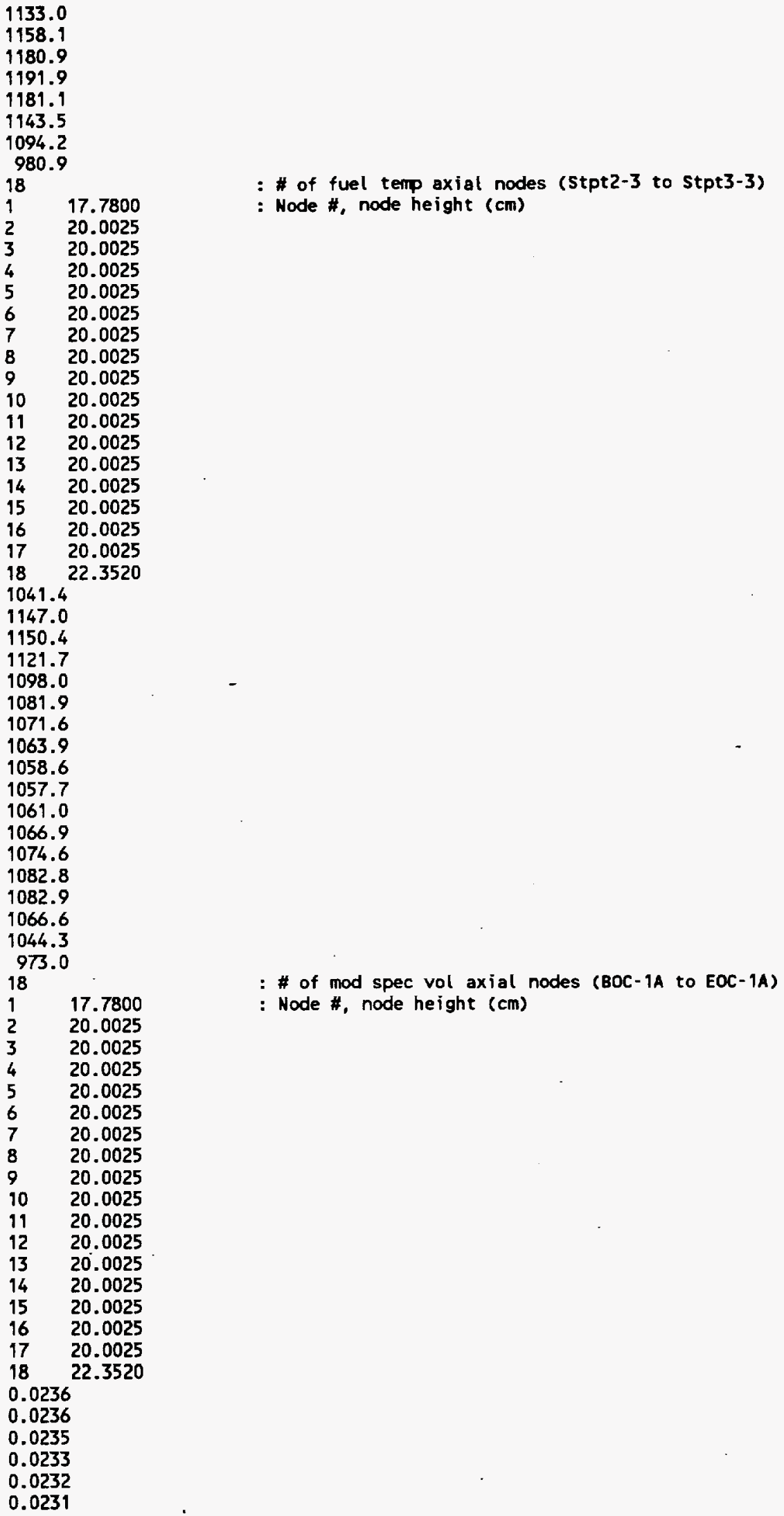




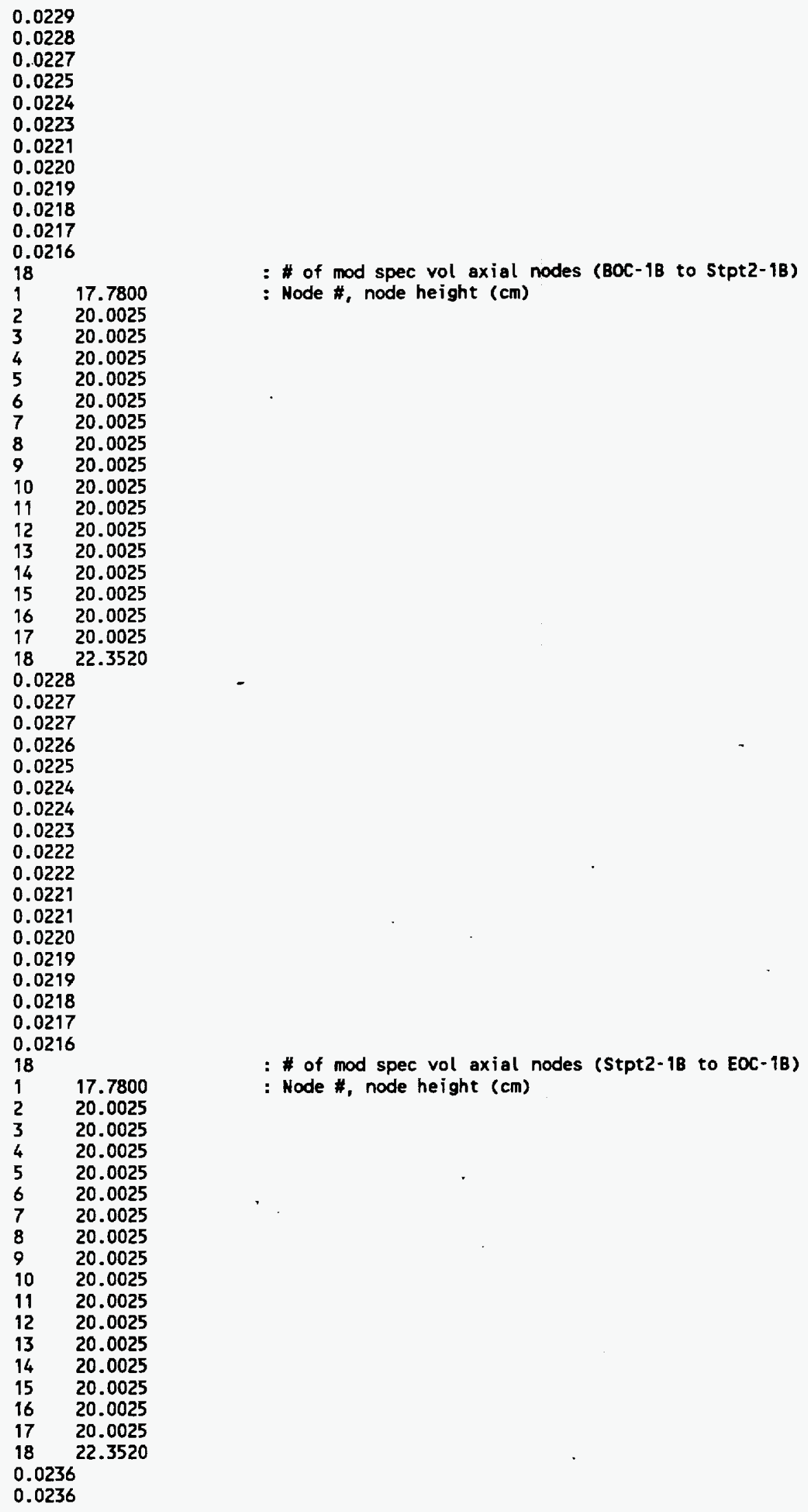


Apr 03 15:00 1997 File Name: A07i.dat BBA000000-01717-0200-00040 REV 00 ATTACHMENT VII - Page 9

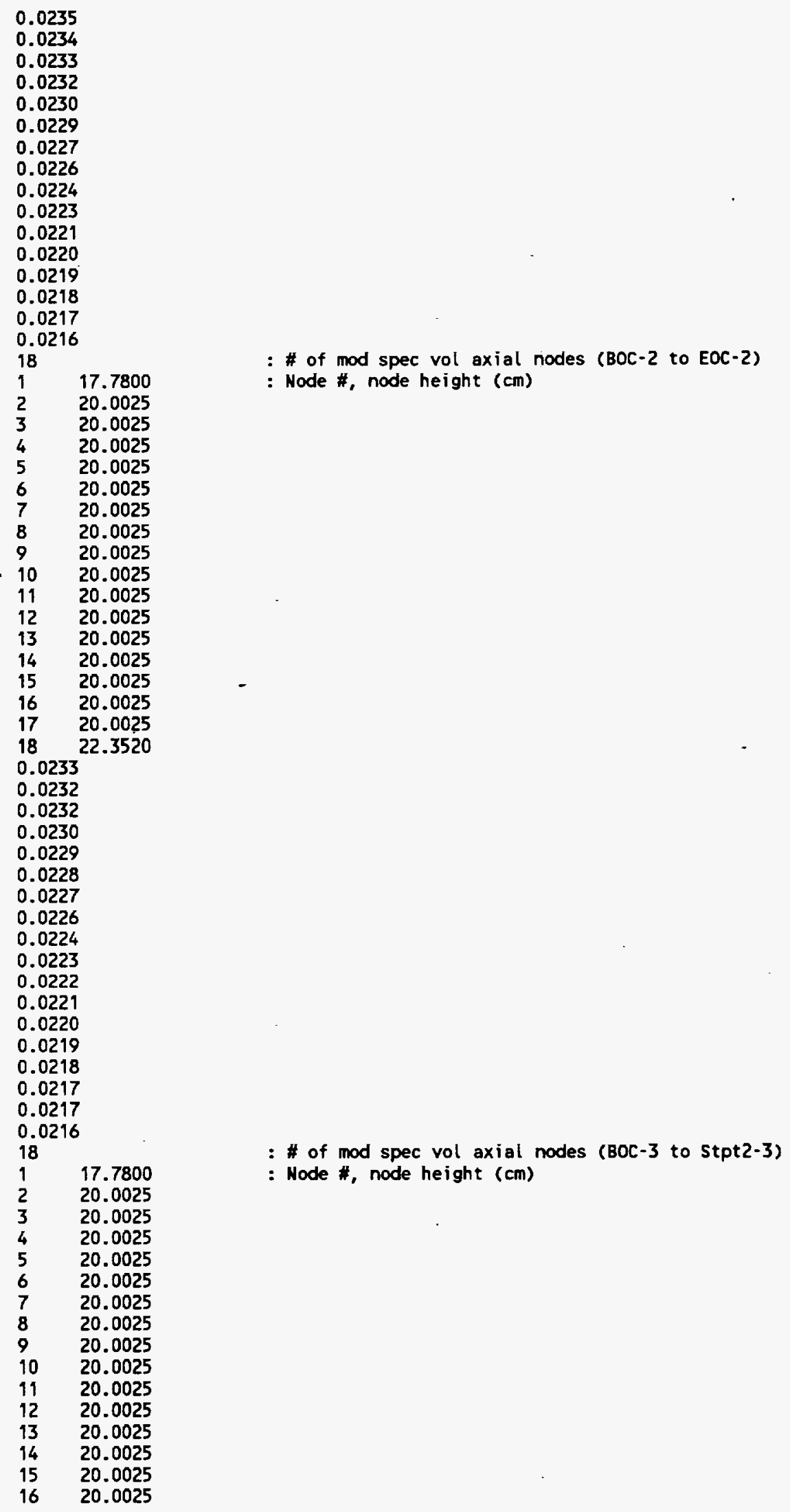


Apr 03 15:00 1997 File Name: A07i.dat BBA000000-01717-0200-00040 REV 00 ATtACHMENT VII - Page 10

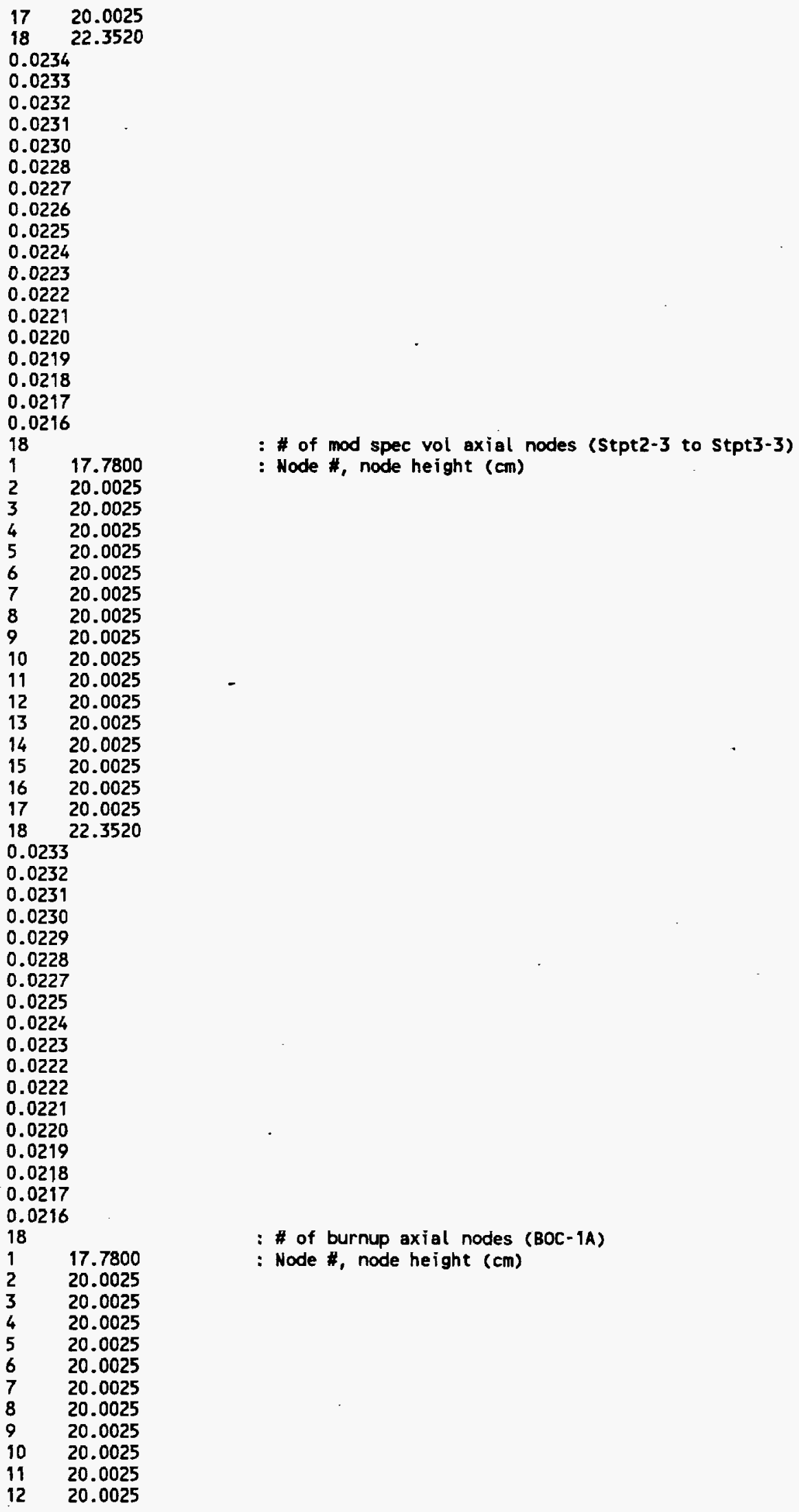


Apr 03 15:00 1997 File Name: A07i.dat BBa000000-01717-0200-00040 REV 00 ATtACHMENT VII - Page 11

20.0025

20.0025

20.0025

20.0025

20.0025

22.3520

0.0

0.0

0.0

0.0

0.0

0.0

0.0

0.0

0.0

0.0

0.0

0.0

0.0

0.0

0.0

0.0

0.0

0.0

17.7800

20.0025

20.0025

20.0025

20.0025

20.0025

20.0025

20.0025

20.0025

20.0025

20.0025

20.0025

20.0025

20.0025

20.0025

20.0025

20.0025

22.3520

3.645

6.341

8.389

9.750

10.606

11.121

11.414

11.561

11.597

11.542

11.417

11.273

11.175

11.168

11.291

10.892

9.417

5.813

18

17.7800

: \# of burnup axial nodes (BOC-1B)

: Node \#, node height $(\mathrm{cm})$

20.0025

20.0025

20.0025

20.0025

20.0025

20.0025

20.0025

\footnotetext{
: \# of burnup axial nodes (Stpt2-1B)
}

: Node \#, node height $(\mathrm{cm})$ 


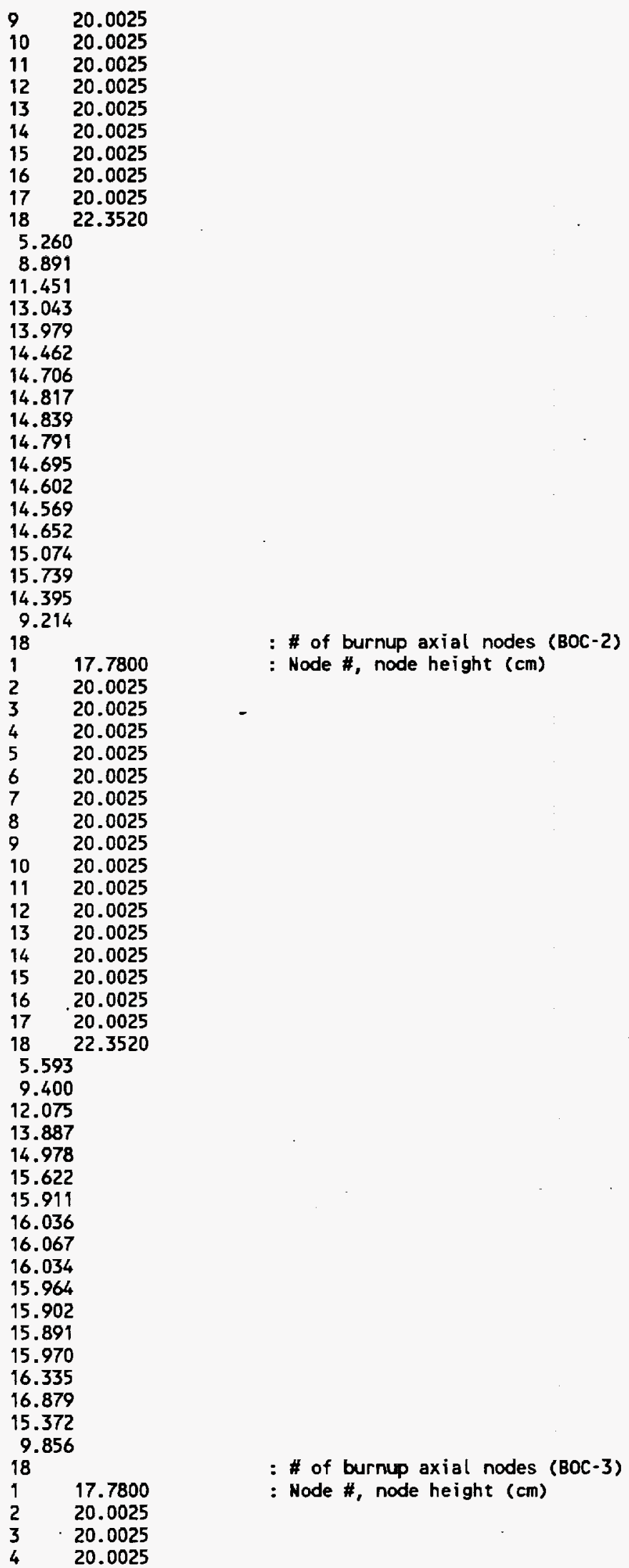




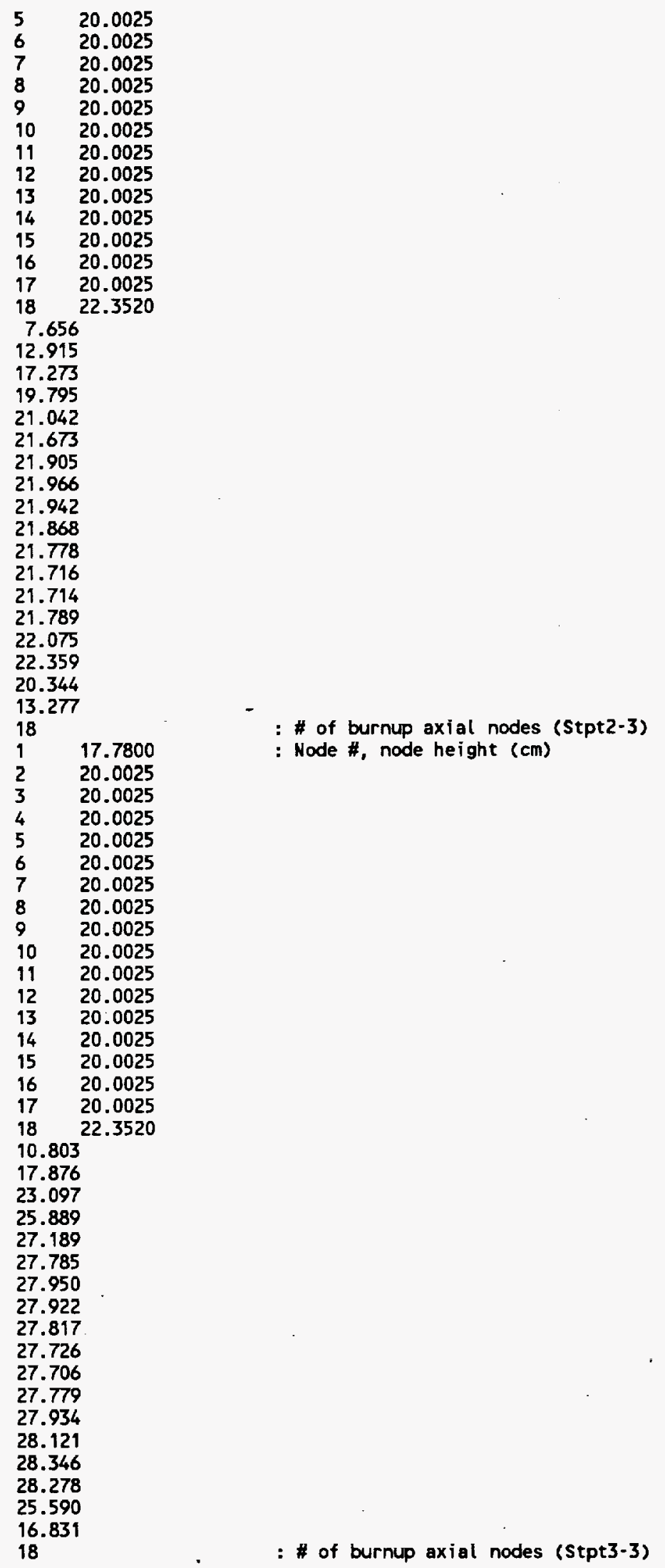


Apr 03 15:00 1997 file Name: A07i.dat BBA000000-01717-0200-00040 REV 00 ATTACHMENT VII - Page 14

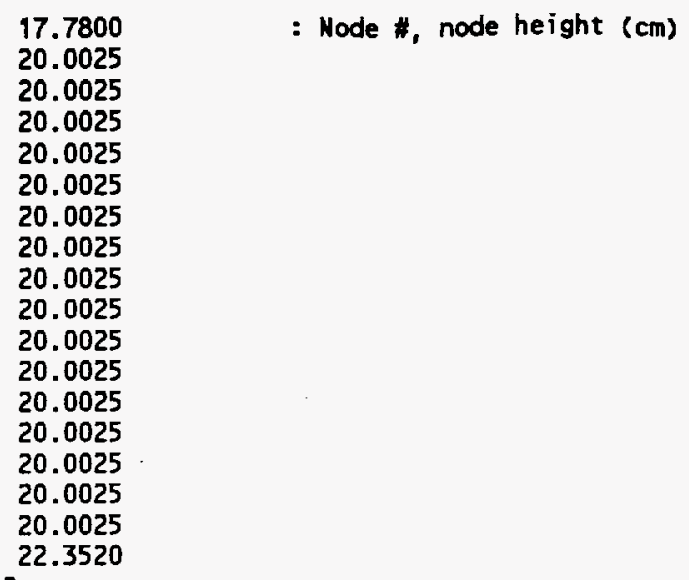

: Node \#, node height $(\mathrm{cm})$

31.101

28.192

18.697 


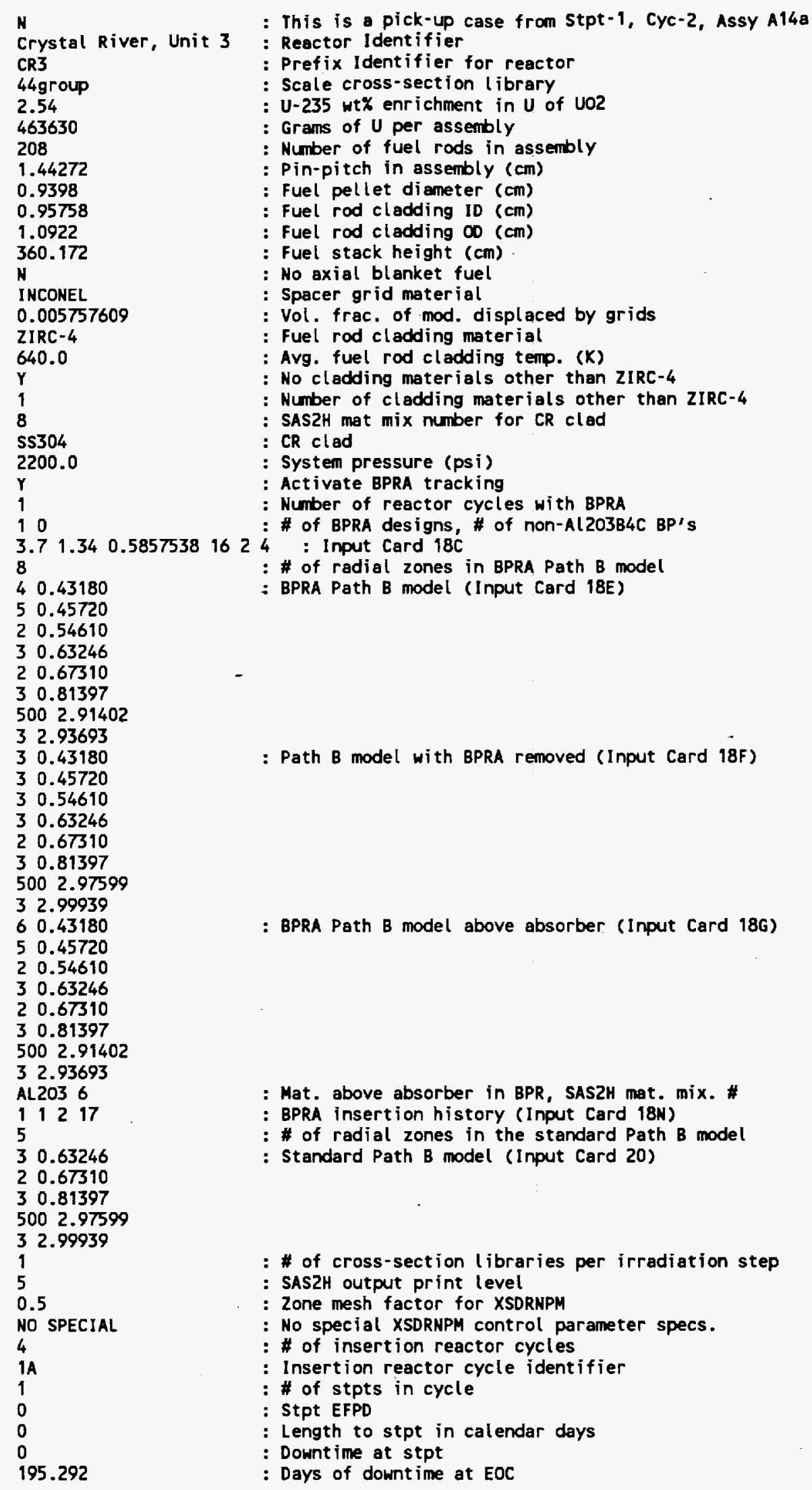




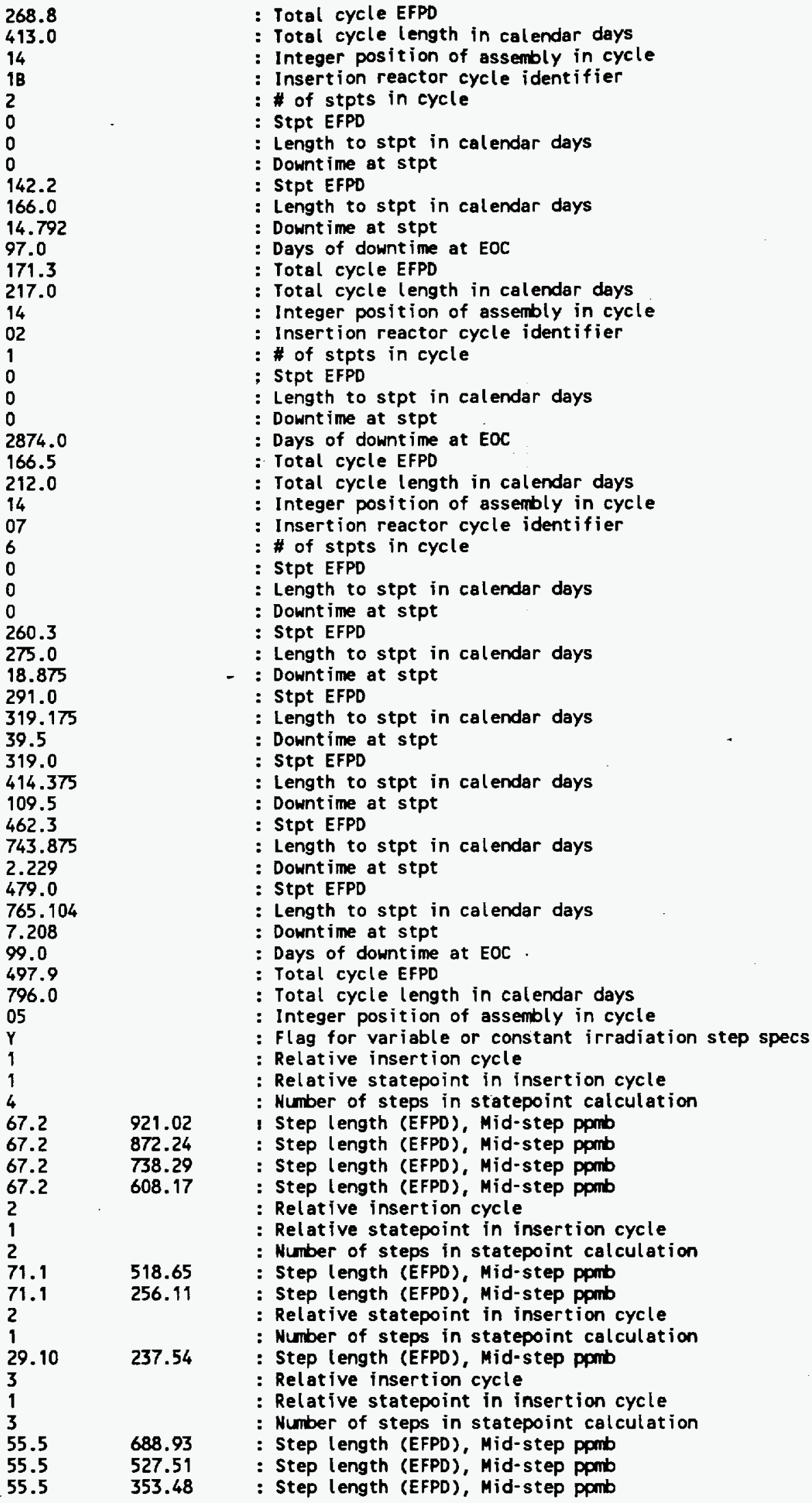




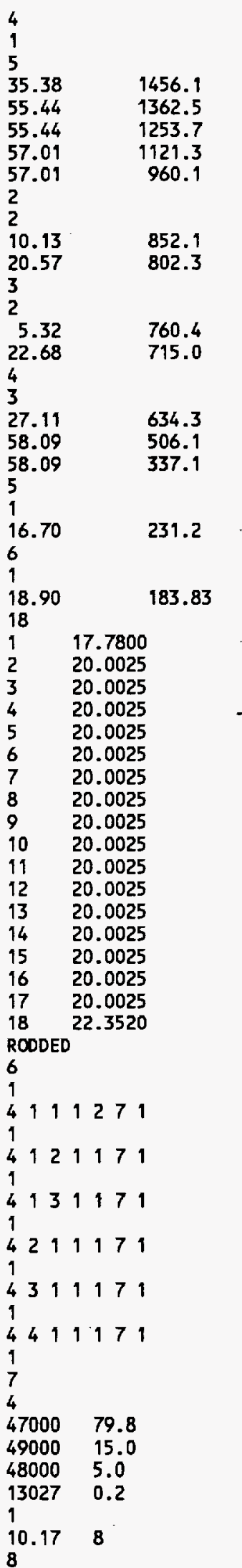

: Relative insertion cycle

: Relative statepoint in insertion cycle

: Number of steps in statepoint calculation

: Step length (EFPD), Mid-step ppmb

: Step length (EFPD), Mid-step ppmb

: Step length (EFPD), Mid-step pomb

: Step length (EFPD), Mid-step ppmb

: Step length (EFPD), Mid-step ppmb

: Relative statepoint in insertion cycle

: Number of steps in statepoint calculation

: Step length (EFPD), Mid-step ppmb

: Step length (EFPD), Mid-step ppib

Relative statepoint in insertion cycle

Number of steps in statepoint calculation

: Step length (EFPD), Mid-step ppmb

: Step length (EFPD), Mid-step ppmb

: Relative statepoint in insertion cycle

- Number of steps in statepoint calculation

: Step length (EFPD), Mid-step ppmb

: Step length (EFPD), Mid-step ppmb

Step length (EFPD), Mid-step ppmb

Relative statepoint in insertion cycle

Number of steps in statepoint calculation

: Step length (EFPD), Mid-step ppmb

: Relative statepoint in insertion cycle

: Number of steps in statepoint calculation

: Step length (EFPD), Mid-step ppmb

: \# of axial nodes in CRC format

: Node \#, node height (cm)

: Number of irradiation steps with CRA inserted

: Number of axial section with CRA inserted in step 1

Input card $47 \mathrm{~B}$

: Number of axial section with CRA inserted in step 2

- Input card 47B

: Number of axial section with CRA inserted in step 3

: Input card 47B

: Number of axial section with CRA inserted in step 4

: Input card 47B

: Number of axial section with CRA inserted in step 5

: Input card 47B

Number of axial section with CRA inserted in step 6

: Input card 47B

- Number of different CRA absorber material mixtures

: SAS2H material mixture number for CRA absorber

Number of isotopes or elements in the CRA absorber

SCALE isotope ID, Isotope wTX

SCALE isotope ID, Isotope wtX

: SCALE isotope ID, Isotope wtX

SCALE isotope ID, I sotope wtX

Number of CRA designs

: CR absorber density, CR clad SAS2H mat. mix. number

: Number of radial zones in Path B model with CRA inserted 
Apr 03 15:00 1997 File Name: A14i.dat BBA000000-01717-0200-00040 RÉV 00 ATTACHMENT VIII - Page 4

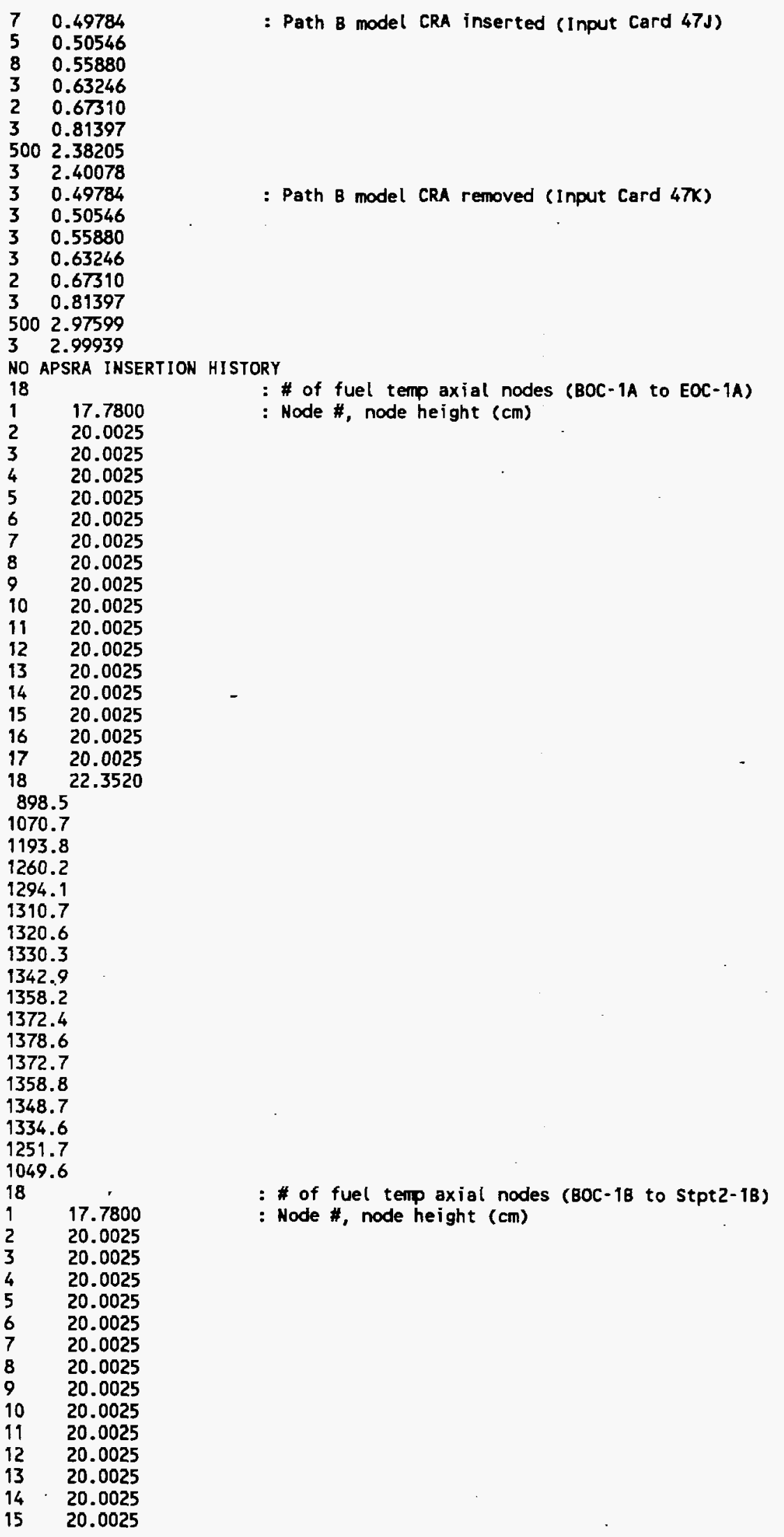




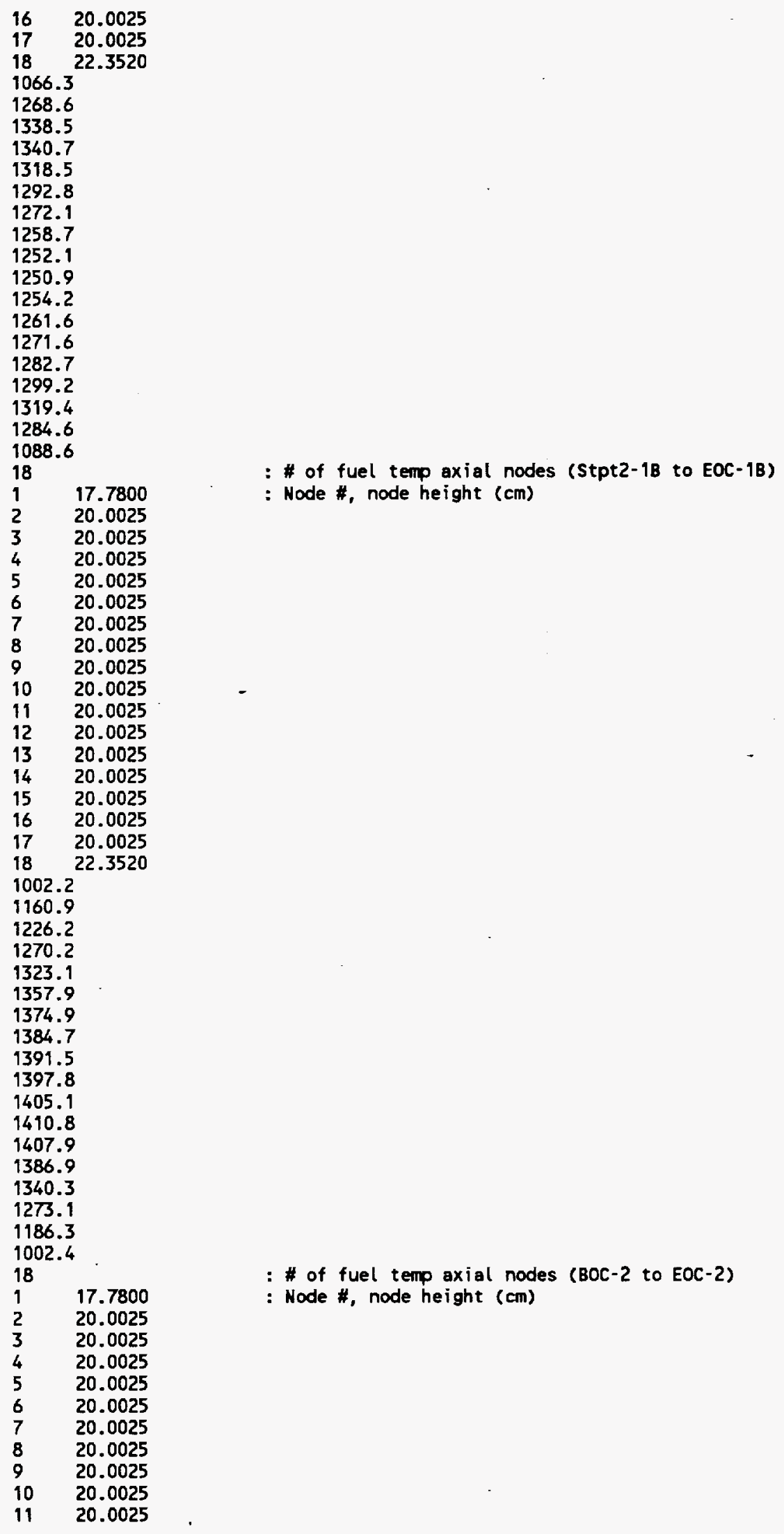


Apr 03 15:00 1997 File Name: A14i.dat BBA000000-01717-0200-00040 REV 00 ATTACHMENT VIII - Page 6

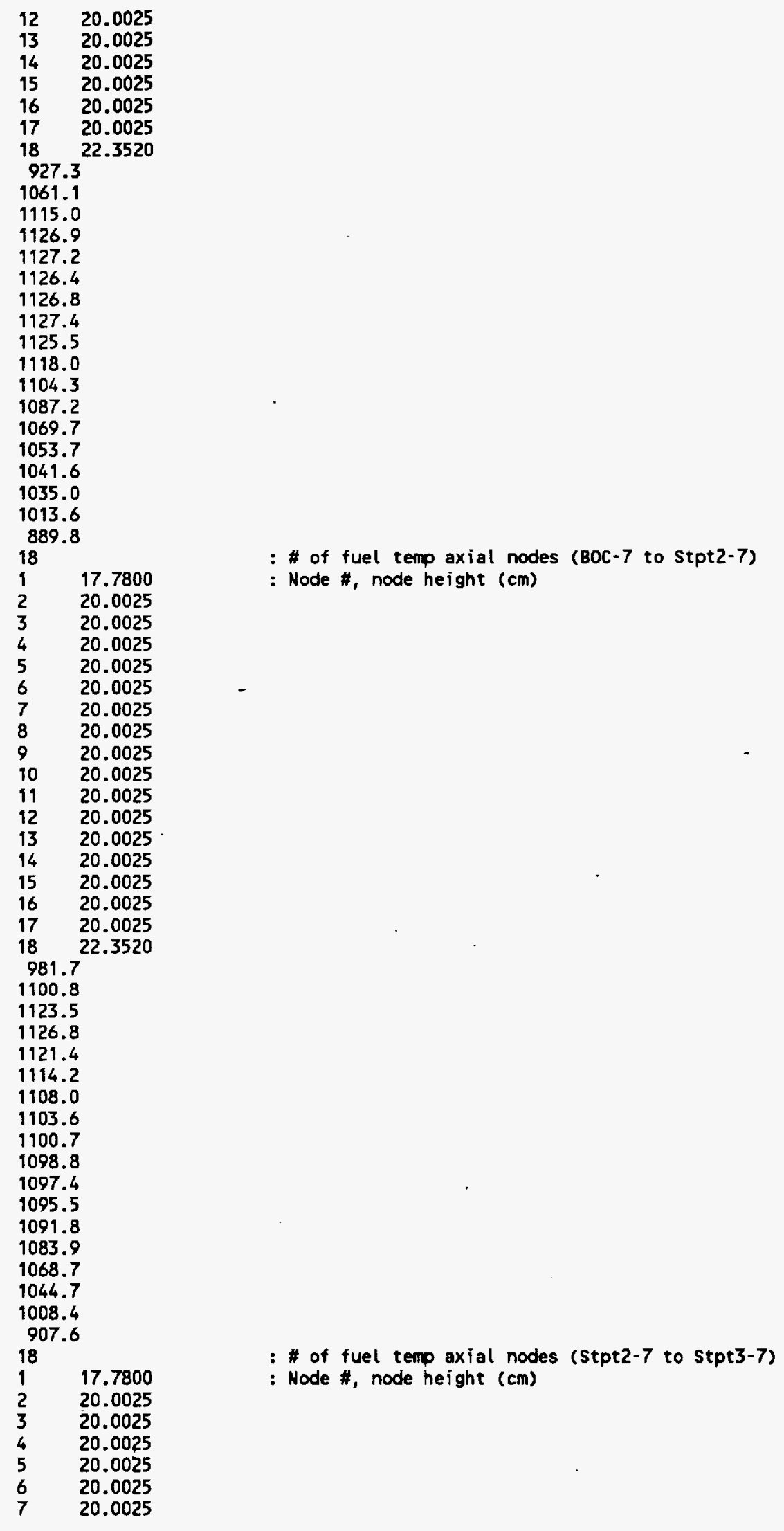




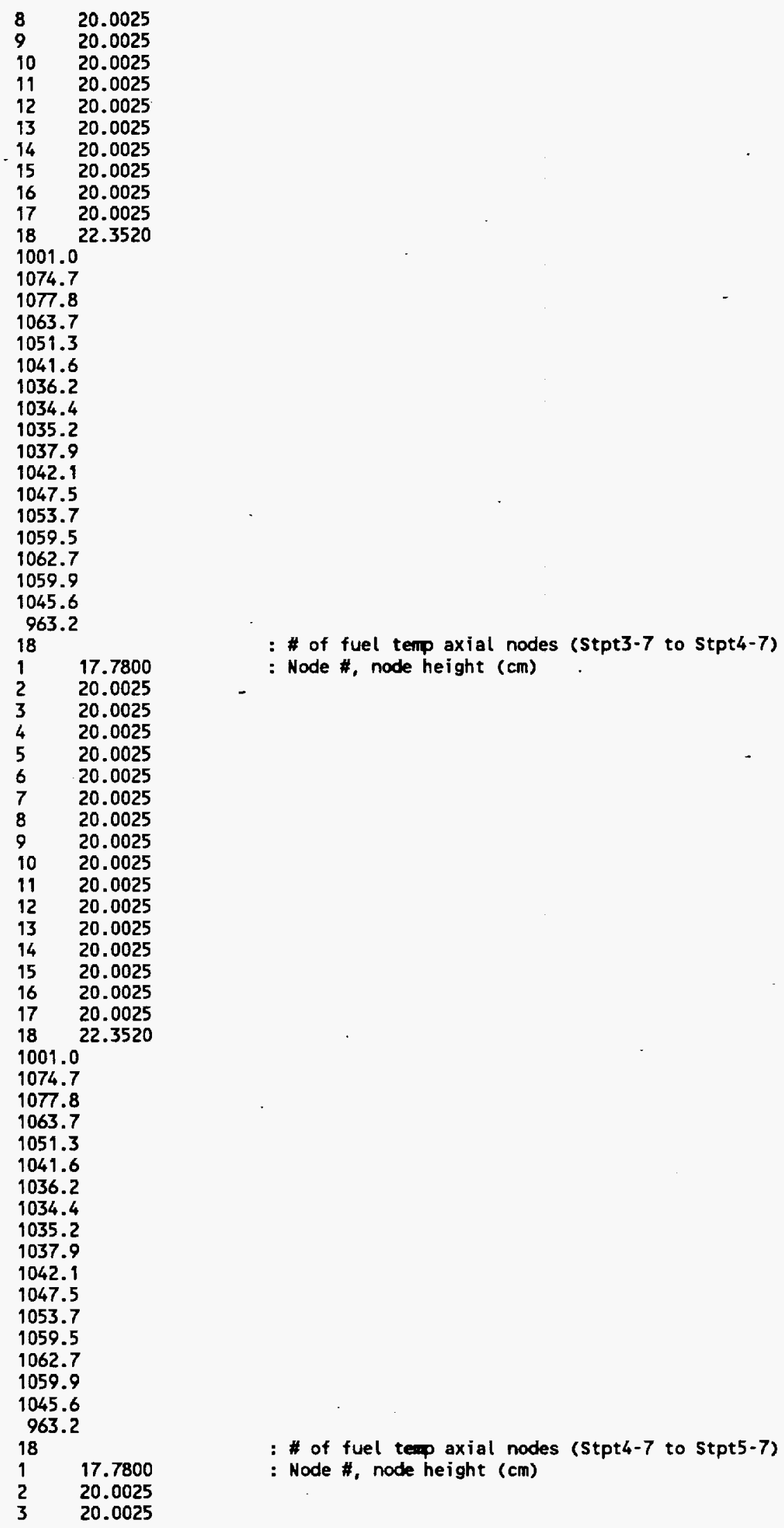




$\begin{array}{ll}4 & 20.0025 \\ 5 & 20.0025 \\ 6 & 20.0025 \\ 7 & 20.0025 \\ 8 & 20.0025 \\ 9 & 20.0025 \\ 10 & 20.0025 \\ 11 & 20.0025 \\ 12 & 20.0025 \\ 13 & 20.0025 \\ 14 & 20.0025 \\ 15 & 20.0025 \\ 16 & 20.0025 \\ 17 & 20.0025 \\ 18 & 22.3520\end{array}$

1014.2

1079.0

1075.1

1062.0

1049.6

1039.4

1033.0

1029.6

1028.4

1028.8

1030.4

1033.0

1036.7

1040.9

1044.2

1042.5

1028.2

957.4

$18 \quad 17.7800$

$2 \quad 20.0025$

$3 \quad 20.0025$

$4 \quad 20.0025$

$5 \quad 20.0025$

$6 \quad 20.0025$

$7 \quad 20.0025$

$8 \quad 20.0025$

$9 \quad 20.0025$

$10 \quad 20.0025$

$11 \quad 20.0025$

$12 \quad 20.0025$

$13 \quad 20.0025$

$14 \quad 20.0025$

$15 \quad 20.0025$

$16 \quad 20.0025$

$17 \quad 20.0025$

$18 \quad 22.3520$

1014.2

1079.0

1075.1

1062.0

1049.6

1039.4

1033.0

1029.6

1028.4

1028.8

1030.4

1033.0

1036.7

1040.9

1044.2

1042.5

1028:2

957.4

: \# of fuel temp axial nodes (Stpt5-7 to Stpt6-7)

: Node \#, node height $(\mathrm{cm})$ 
Apr 03 15:00 1997 File Name: A14i.dat BBA000000-01717-0200-00040 REV 00 ATTACHHENT VIII - Page 9

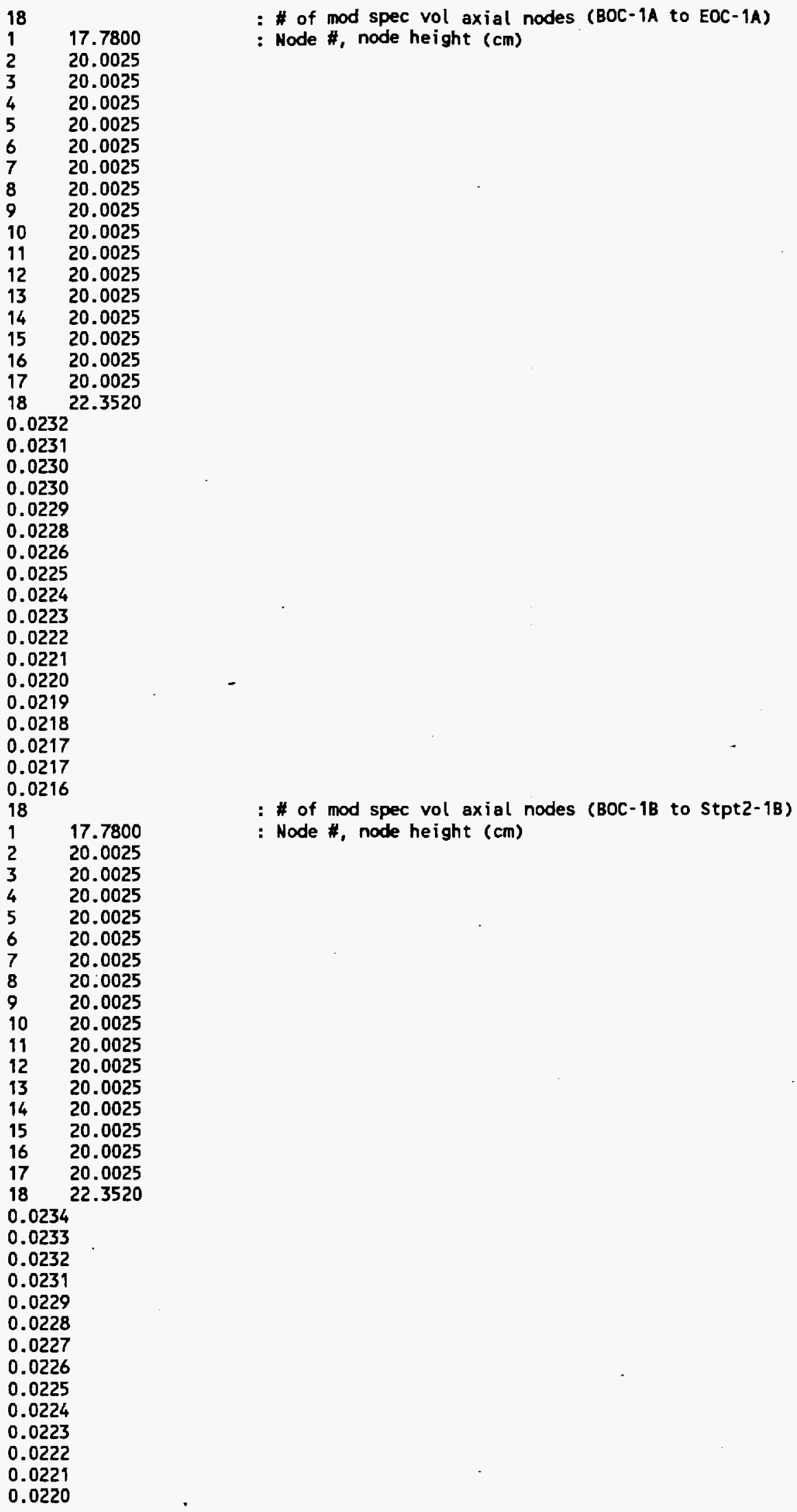




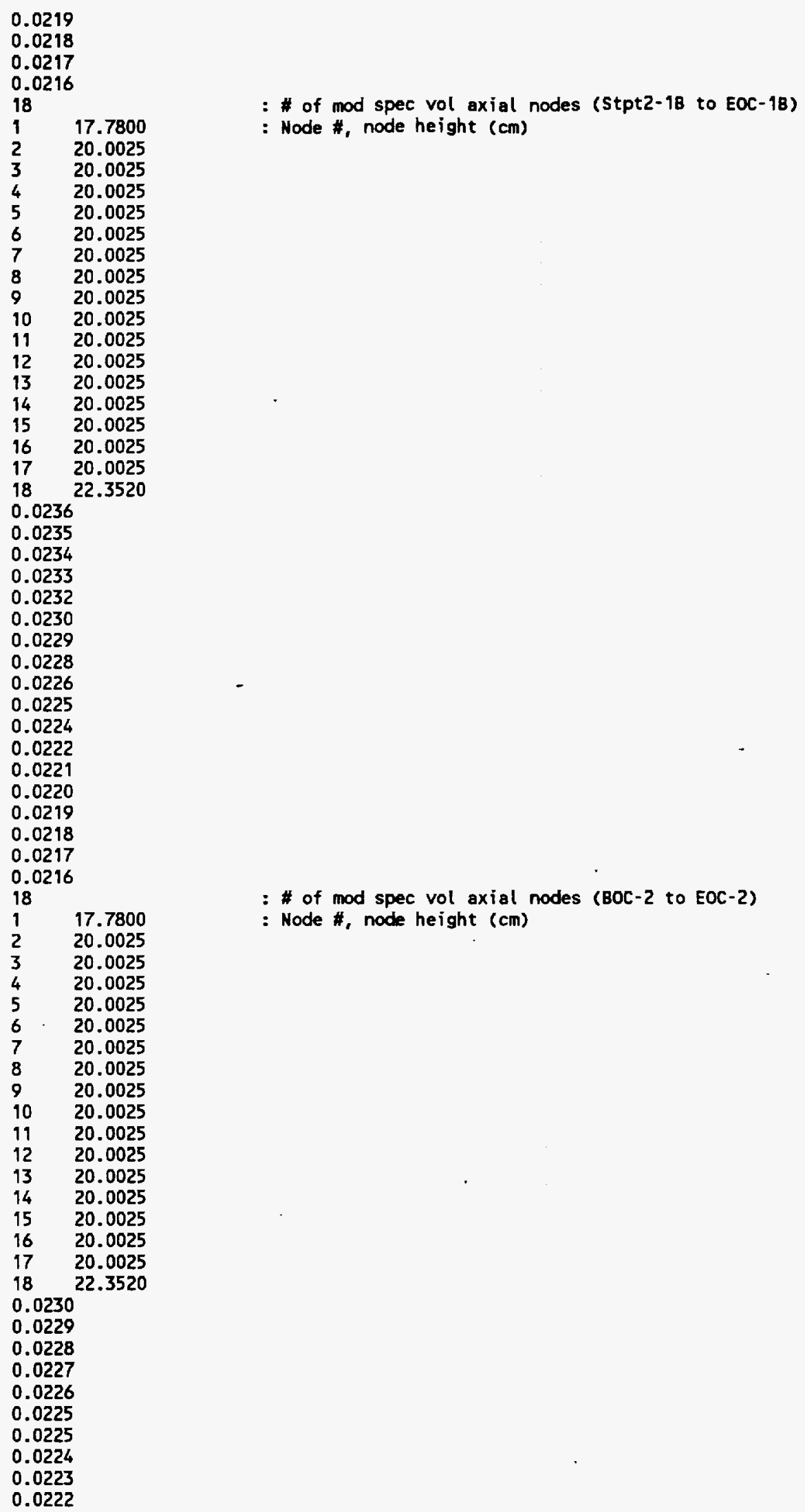




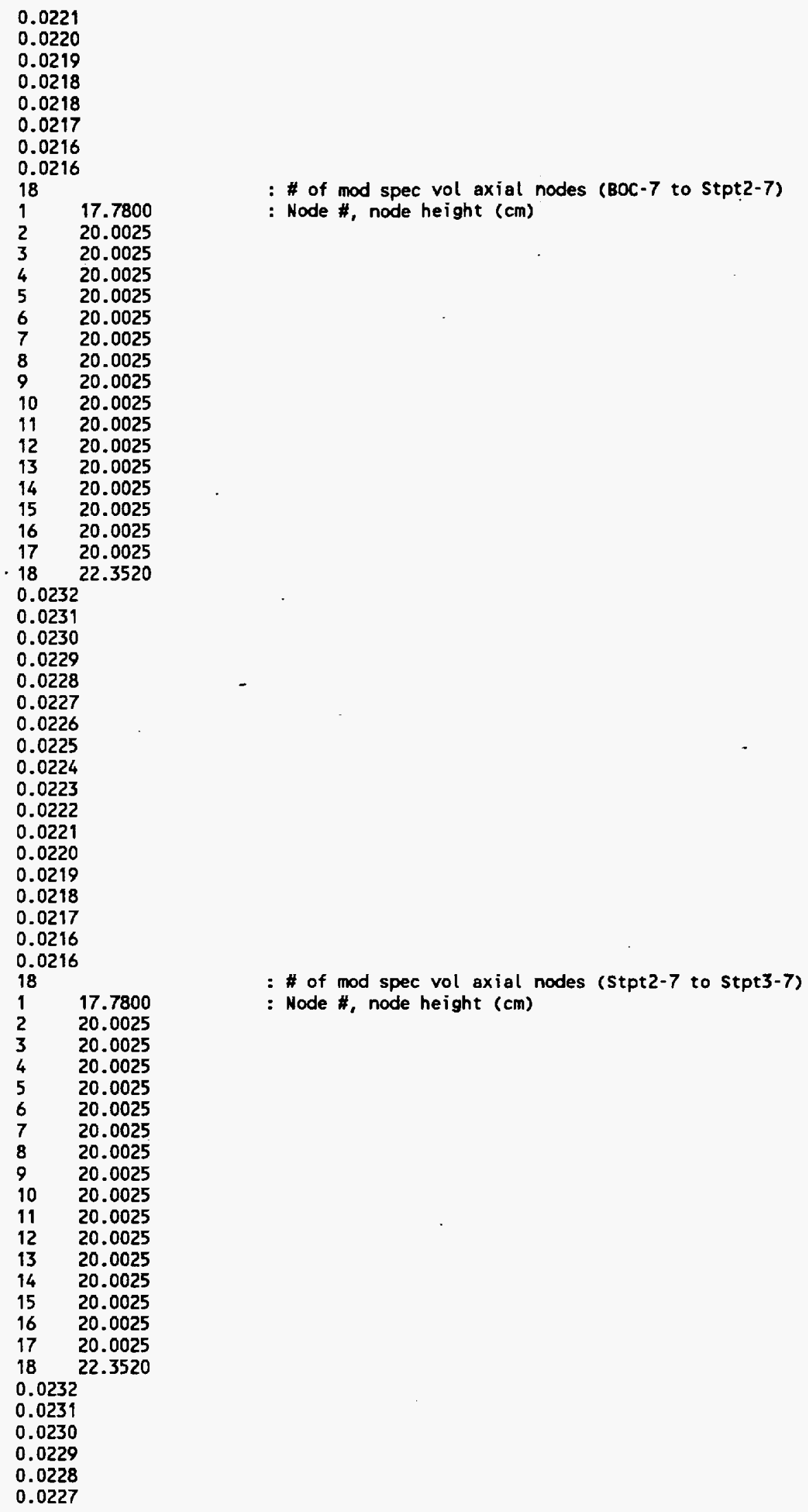




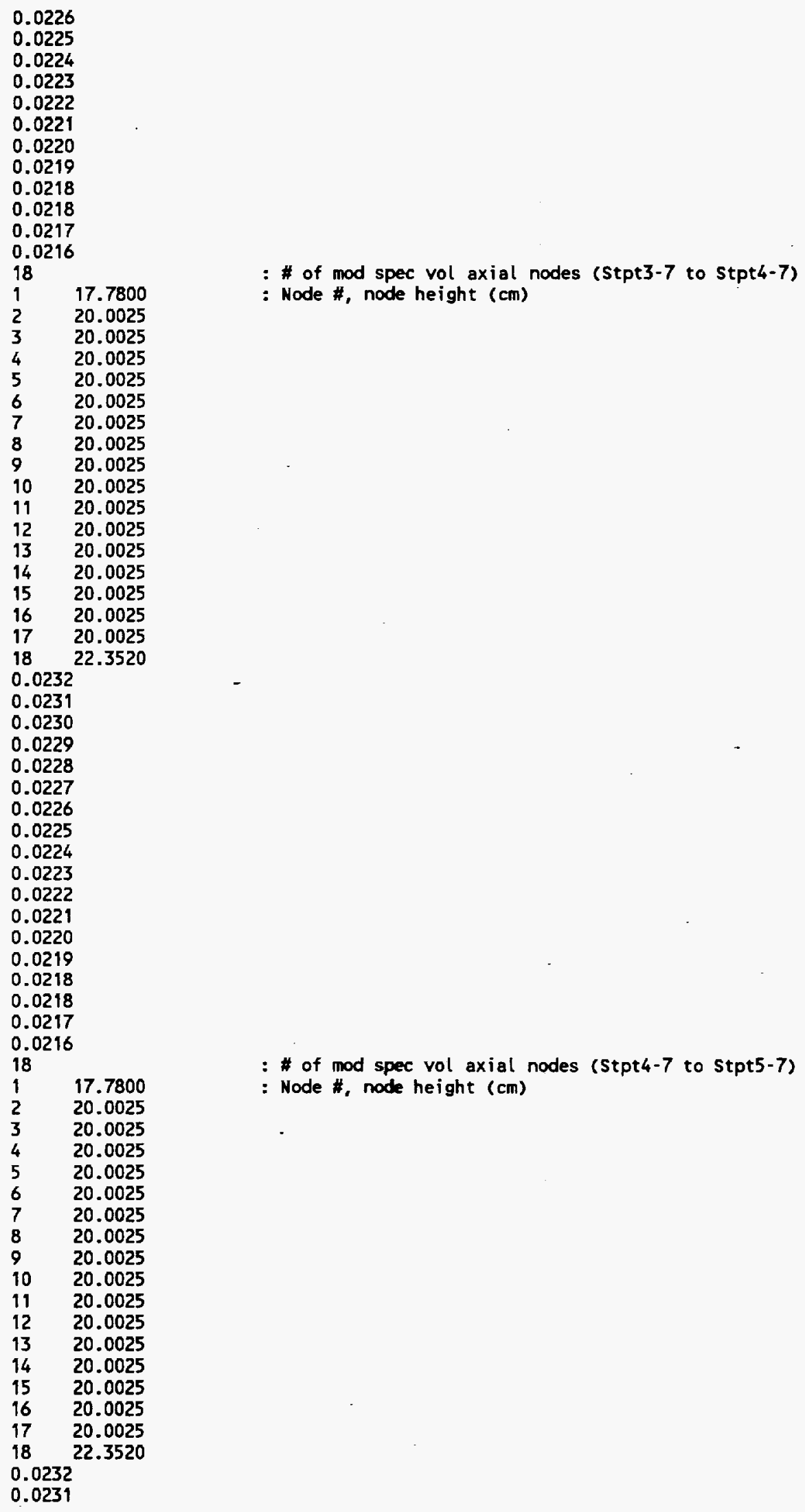




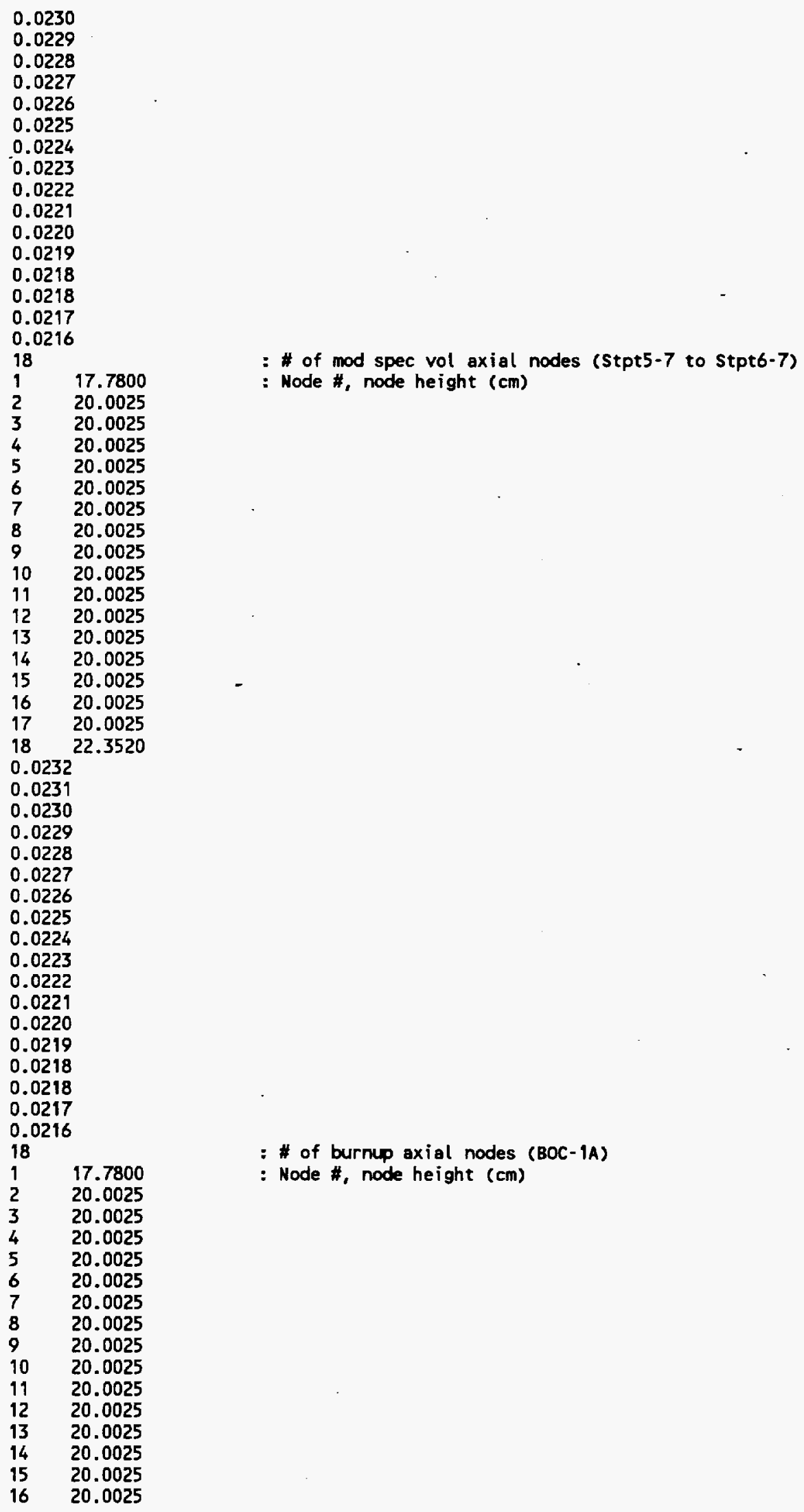


20.0025

22.3520

0.0

0.0

0.0

0.0

0.0

0.0

0.0

0.0

0.0

0.0

0.0

0.0

0.0

1

2

3

40.0025

$5 \quad 20.0025$

$6 \quad 20.0025$

$7 \quad 20.0025$

$8 \quad 20.0025$

9 20.0025

$10 \quad 20.0025$

$11 \quad 20.0025$

$12 \quad 20.0025$

$13 \quad 20.0025$

$14 \quad 20.0025$

$15 \quad 20.0025$

$16 \quad 20.0025$

$17 \quad 20.0025$

$18 \quad 22.3520$

3.042

4.939

6.561

7.668

8.361

8.770

8.995

9.097

9.105

9.030

8.888

8.731

8.635

8.663

8.790

8.660

7.628

5.173

18

17.7800

: \# of burnup axial nodes (BOC-1B)

: Node \#, node height (cm)

20.0025

20.0025

20.0025

20.0025

20.0025

20.0025

20.0025

20.0025

20.0025

20.0025

20.0025

: \# of burnup axial nodes (Stpt2-1B)

: Node \#, node height $(\mathrm{cm})$ 
Apr 03 15:00 1997 File Name: A14i.dat BBA000000-01717-0200-00040 REV 00 ATtACHMENT VIII - Page 15

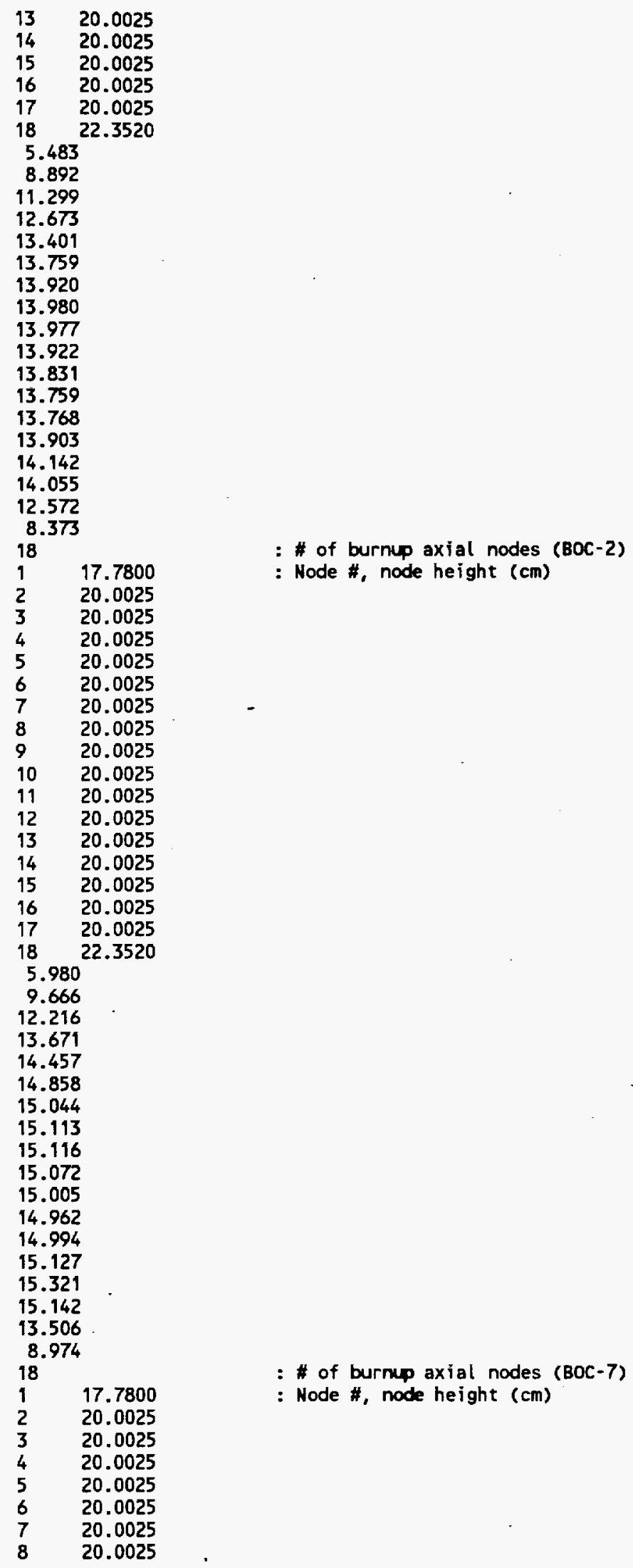


Apr 03 15:00 1997 File Name: A14i.dat BBA000000-01717-0200-00040 REV 00 ATTACHMENT VIII - Page 16

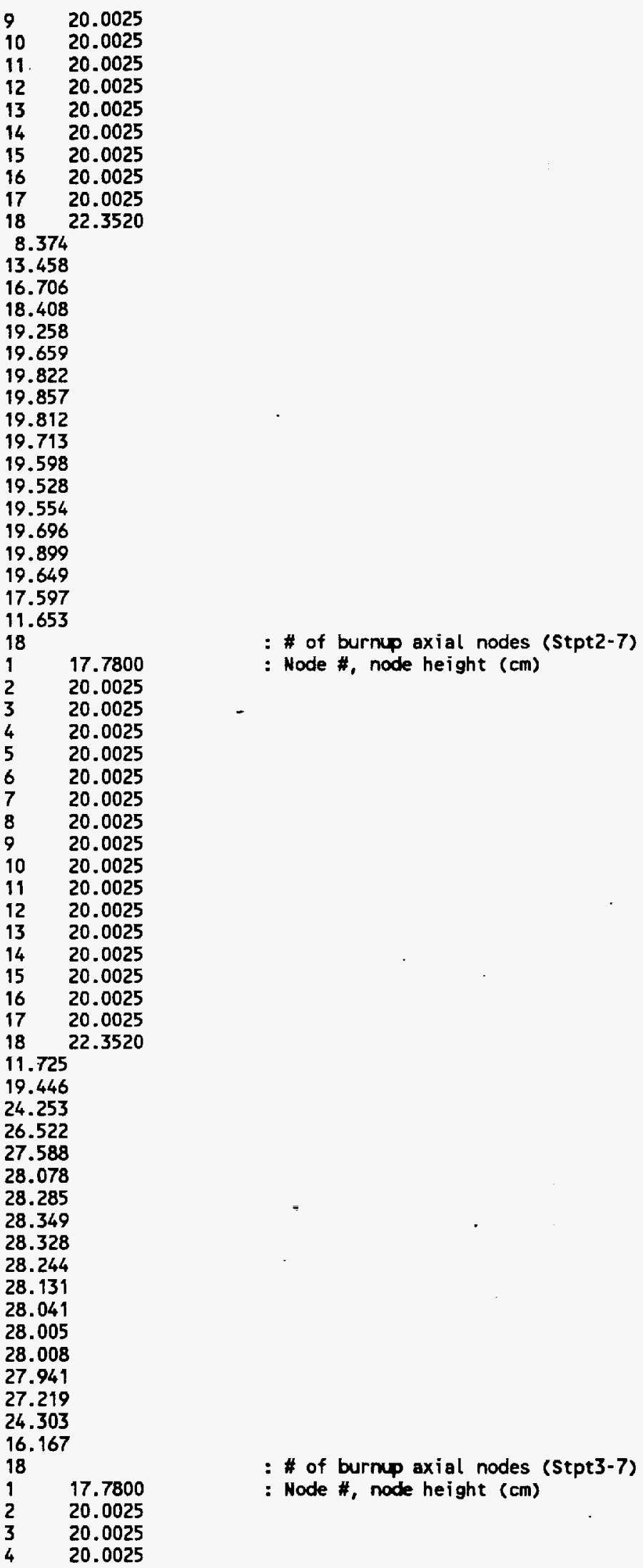


Apr 03 15:00 1997 File Name: A14i.dat BBA000000-01717-0200-00040 REV 00 ATTACHMENt VIII - Page 17

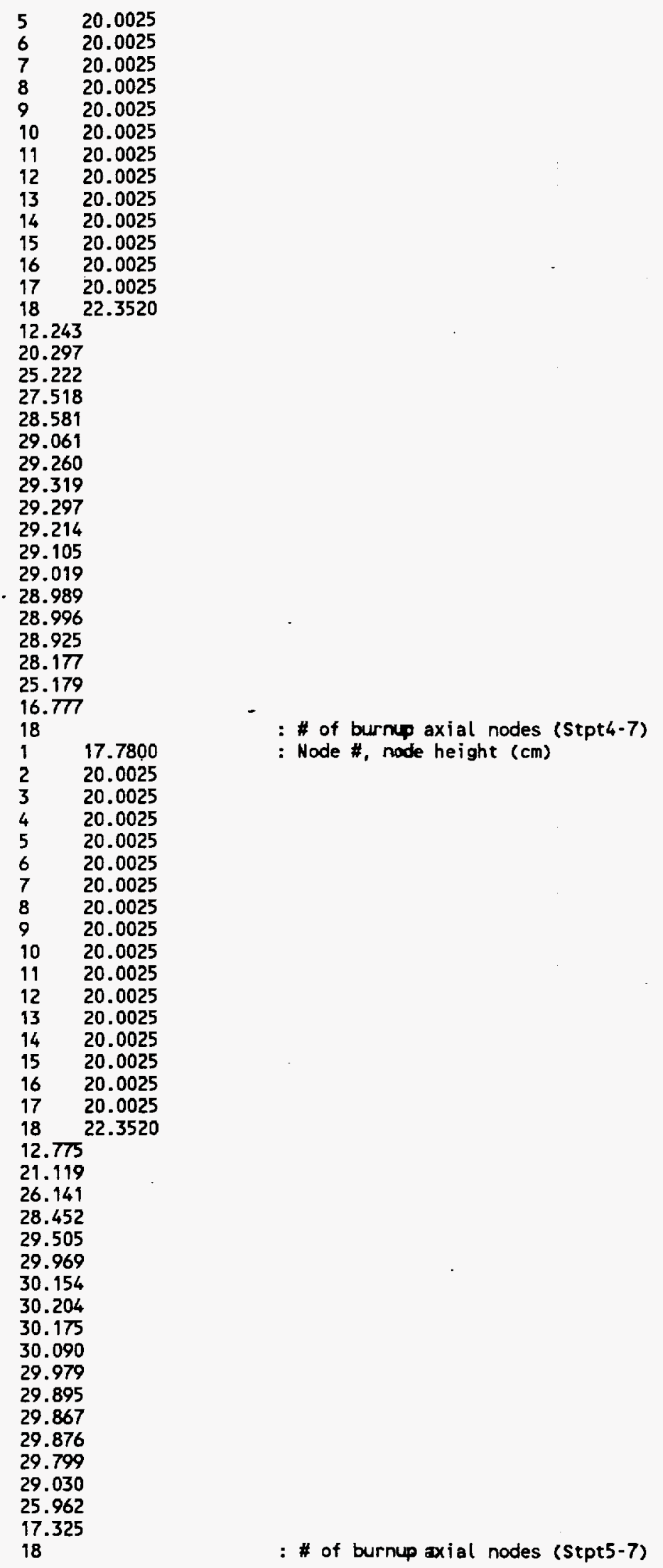




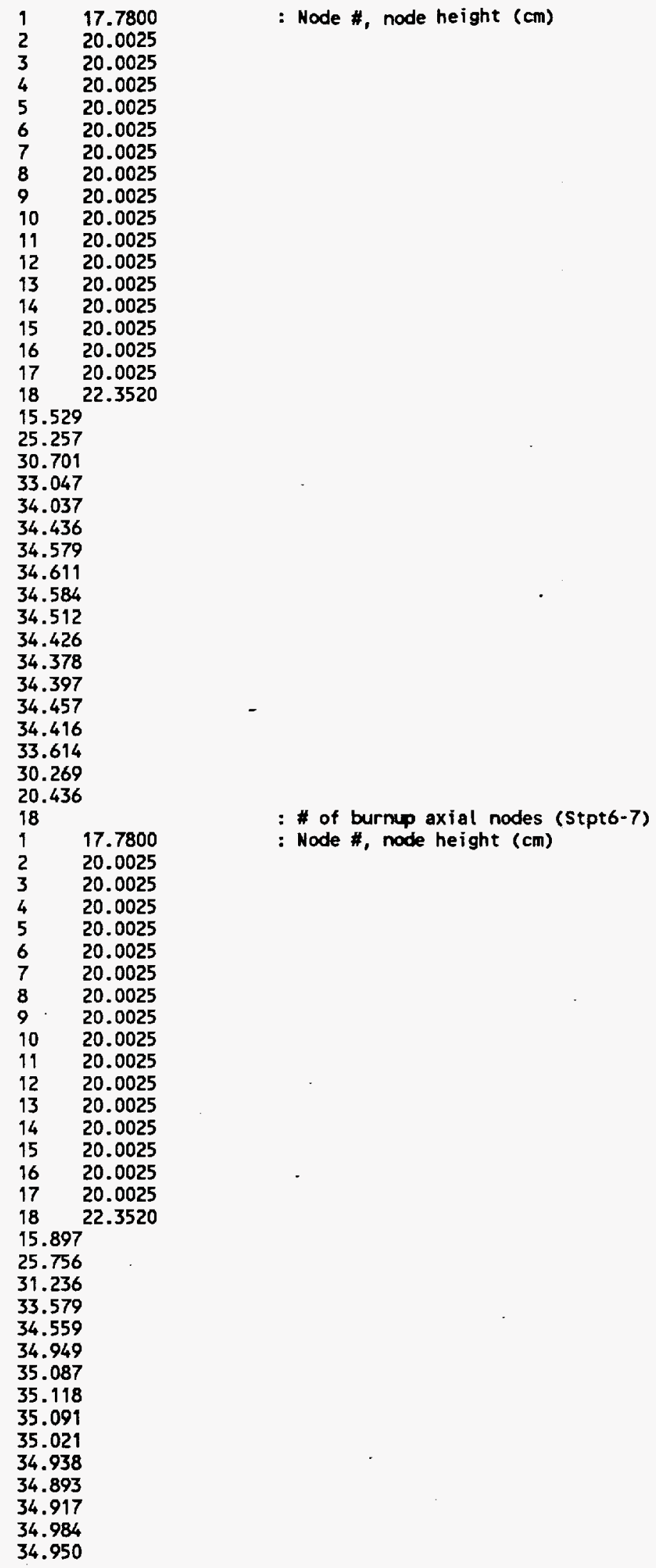


34.150

30.781

20.815 


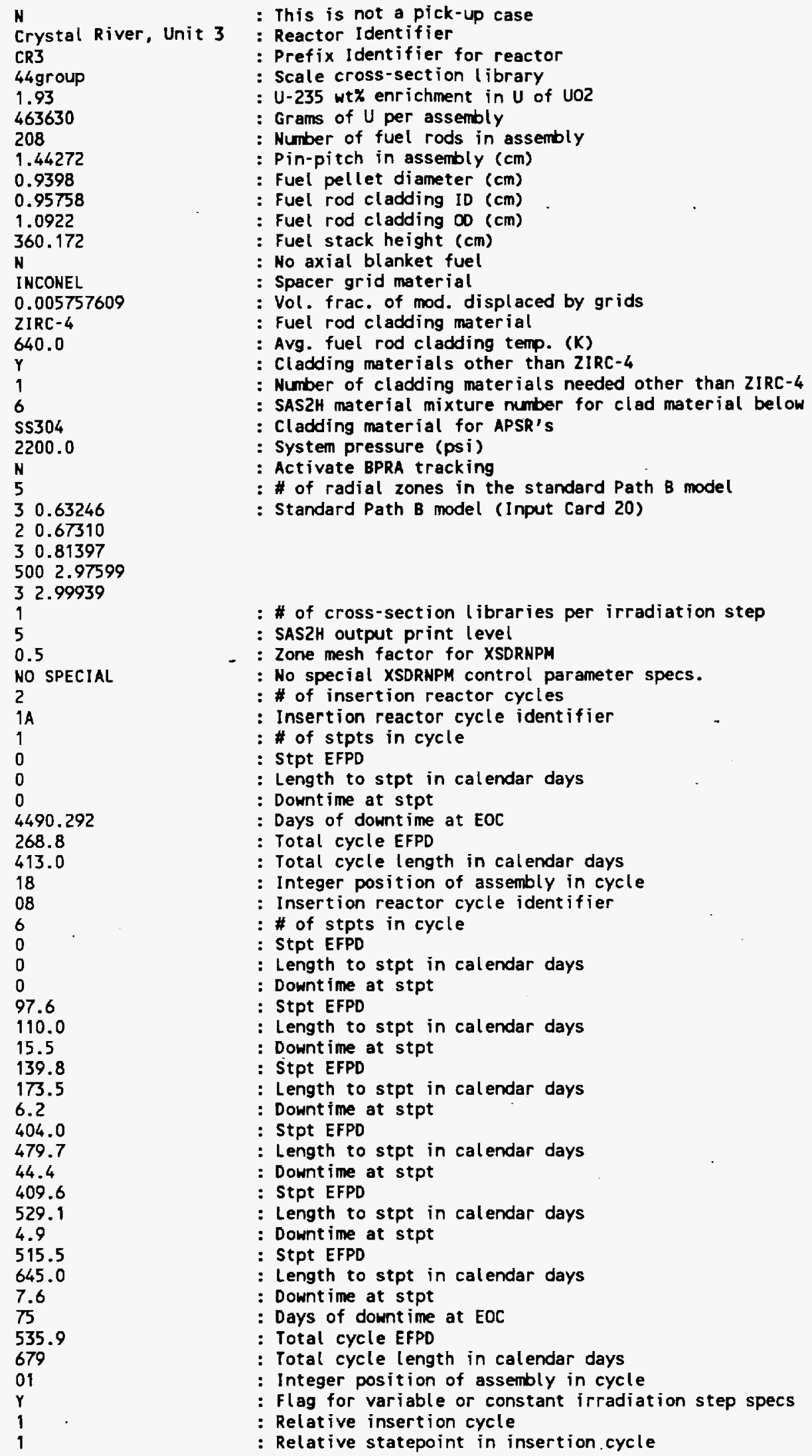




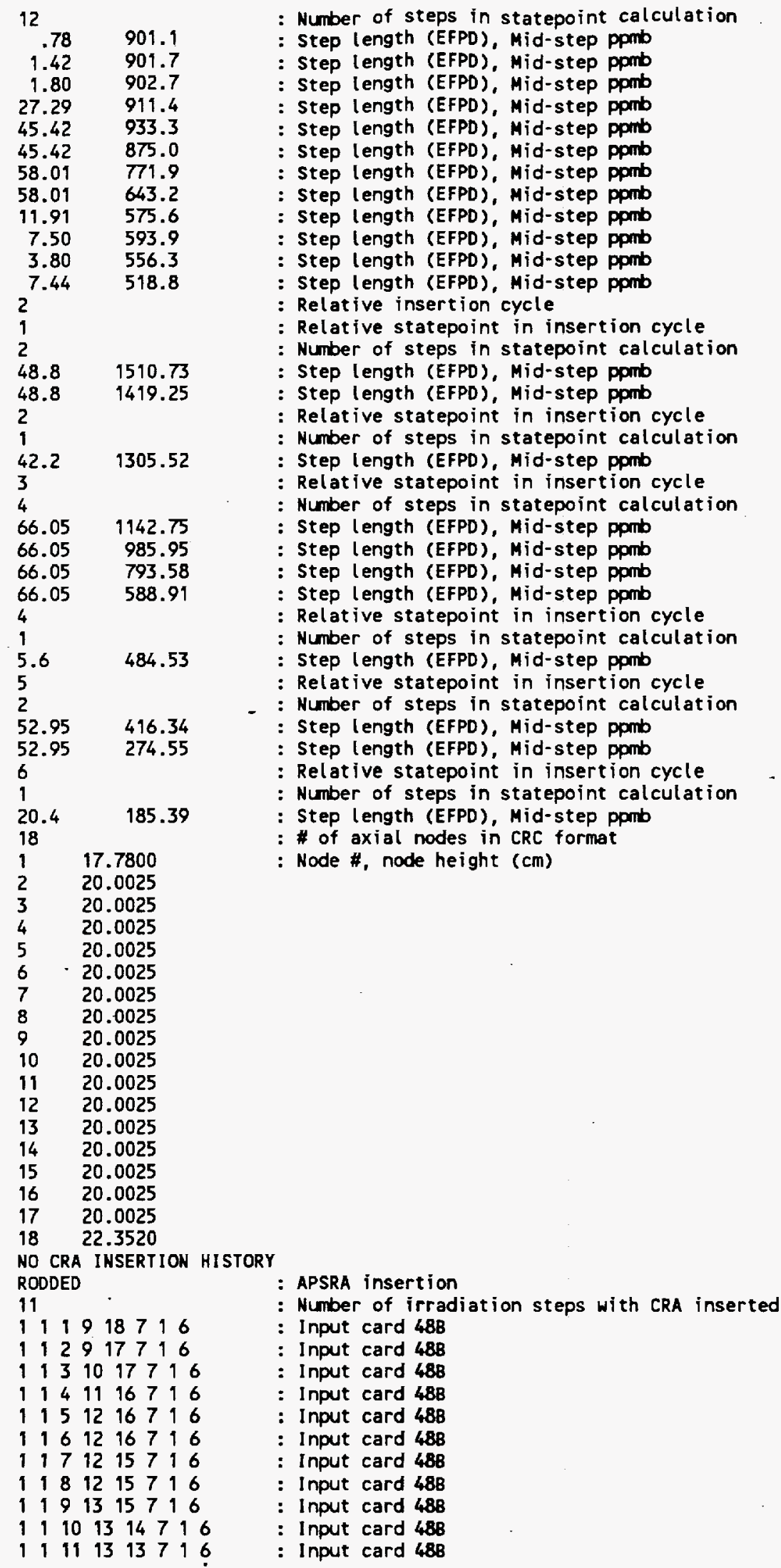




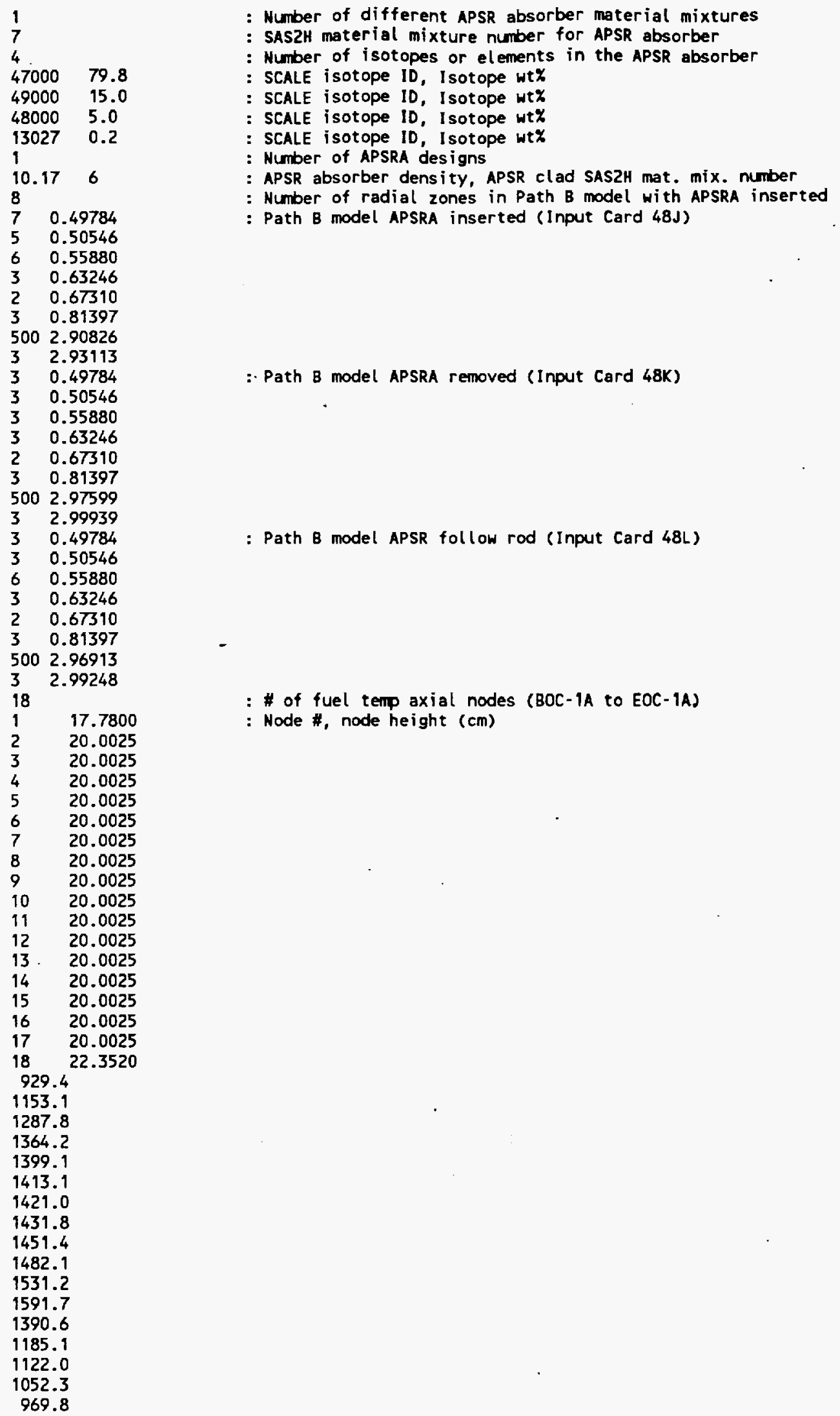




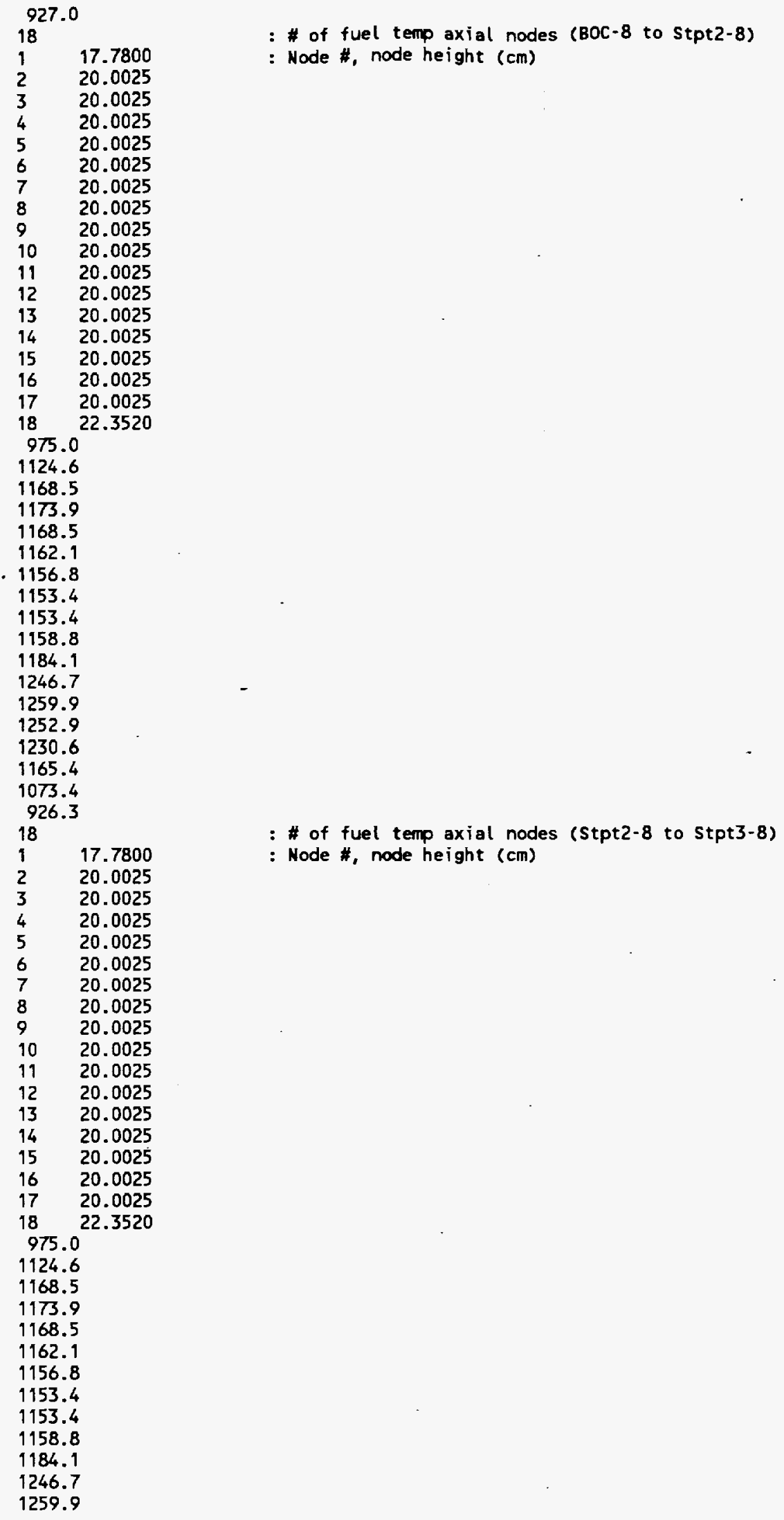


Jul 30 11:33 1997 File Name: A18zi.dat BBA000000-01717-0200-00040 REV 00 ATTACHMENT IX - Page 5

1252.9

1230.6

1165.4

1073.4

926.3

18

17.7800

: \# of fuel temp axial nodes (Stpt3-8 to Stpt4-8)

20.0025

: Node \#, node height $(\mathrm{cm})$

20.0025

20.0025

20.0025

20.0025

20.0025

20.0025

20.0025

20.0025

20.0025

20.0025

20.0025

20.0025

20.0025

20.0025

20.0025

22.3520

977.2

1101.7

1134.2

1128.2

1116.0

1105.3

1097.4

1092.3

1090.9

1094.6

1115.0

1166.1

1178.9

1178.4

1169.2

1129.3

1063.3

931.6

18

17.7800

20.0025

20.0025

20.0025

20.0025

20.0025

20.0025

20.0025

20.0025

20.0025

20.0025

20.0025

20.0025

20.0025

20.0025

20.0025

20.0025

$18 \quad 22.3520$

977.2

1101.7

1134.2

1128.2

1116.0

1105.3

1097.4

1092.3

1090.9

: \# of fuel temp axial nodes (Stpt4-8 to Stpt5-8)

: Node \#, node height $(\mathrm{cm})$ 
Jul 30 11:33 1997 File Name: A18zi.dat BBA000000-01717-0200-00040 REV 00 ATTACHMENT IX - Page 6

1094.6

1115.0

1166.1

1178.9

1178.4

1169.2

1129.3

1063.3

931.6

18

$1 \quad 17.7800$

: \# fuel temp axial nodes (Stpt5-8 to Stpt6-8)

20.0025

: Node \#, node height (cm)

\subsection{5}

20.0025

20.0025

20.0025

20.0025

20.0025

20.0025

20.0025

20.0025

20.0025

20.0025

20.0025

20.0025

20.0025

20.0025

$18 \quad 22.3520$

983.9

1074.1

1076.1

1058.8

1041.2

1027.9

1018.7

1012.7

1009.9

1010.8

1022.9

1055.0

1064.7

1068.9

1071.7

1058.6

1030.9

944.7

18

$1 \quad 17.7800$

20.0025

20.0025

20.0025

20.0025

20.0025

20.0025

20.0025

20.0025

20.0025

20.0025

20.0025

20.0025

20.0025

20.0025

20.0025

20.0025

$18 \quad 22.3520$

0.0232

0.0232

0.0231

0.0230

0.0229

: \# of mod spec vol axial nodes (BOC-1A to EOC-1A)

: Node \#, node height $(\mathrm{cm})$ 


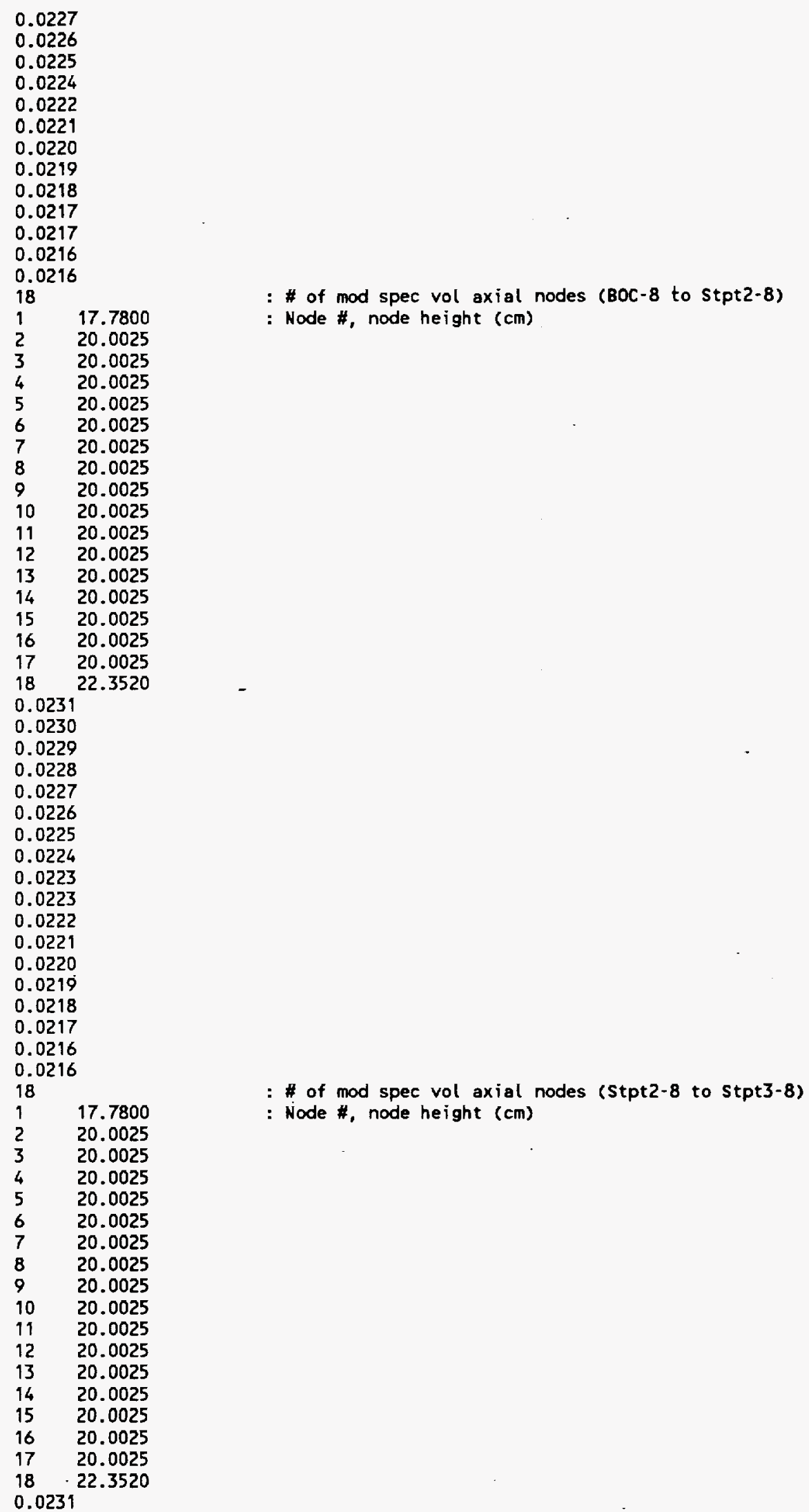


Jul 30 11:33 1997 File Name: A18zi.dat BBA000000-01717-0200-00040 REV 00 ATTACHMENT IX - Page 8

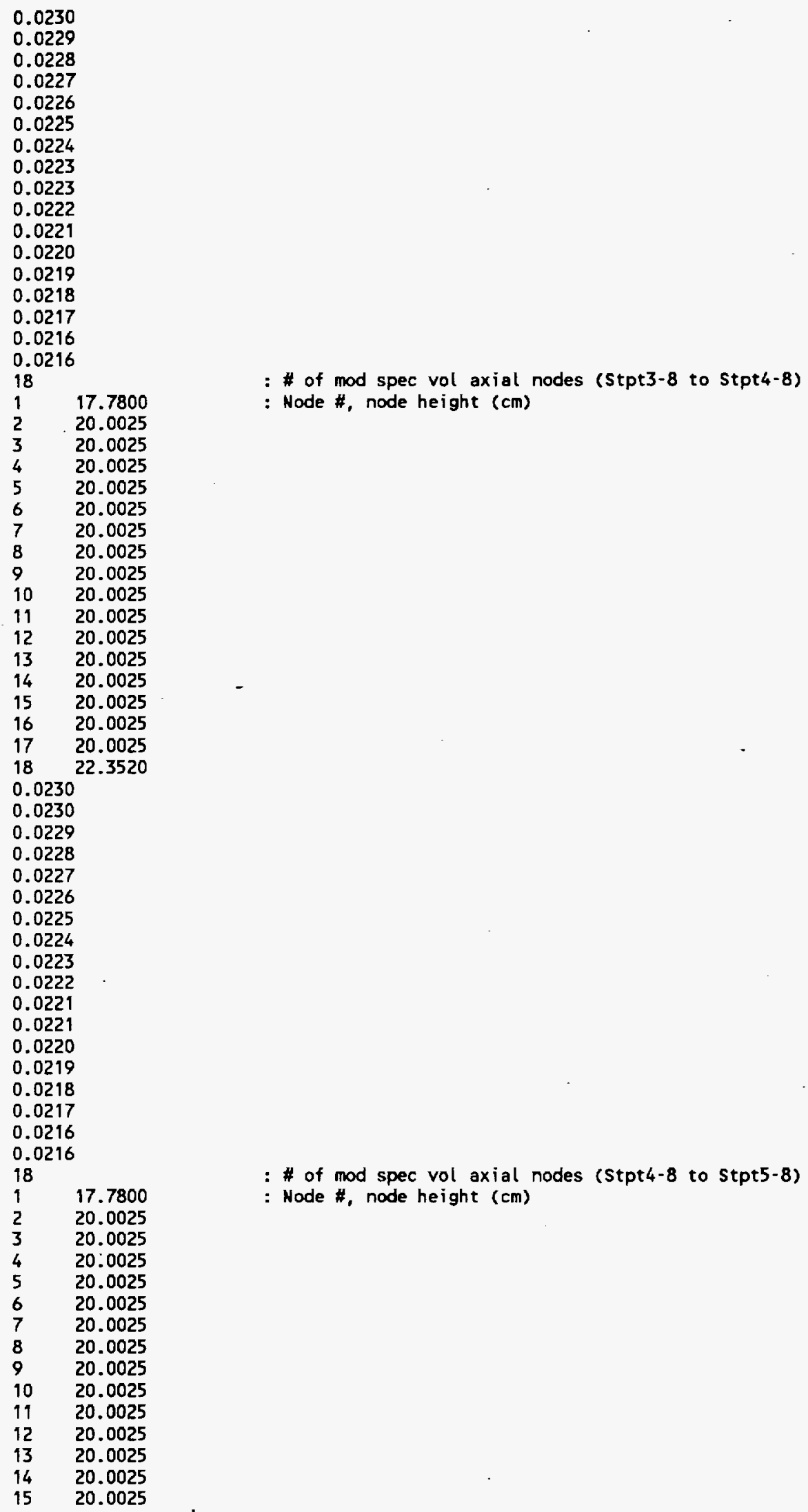




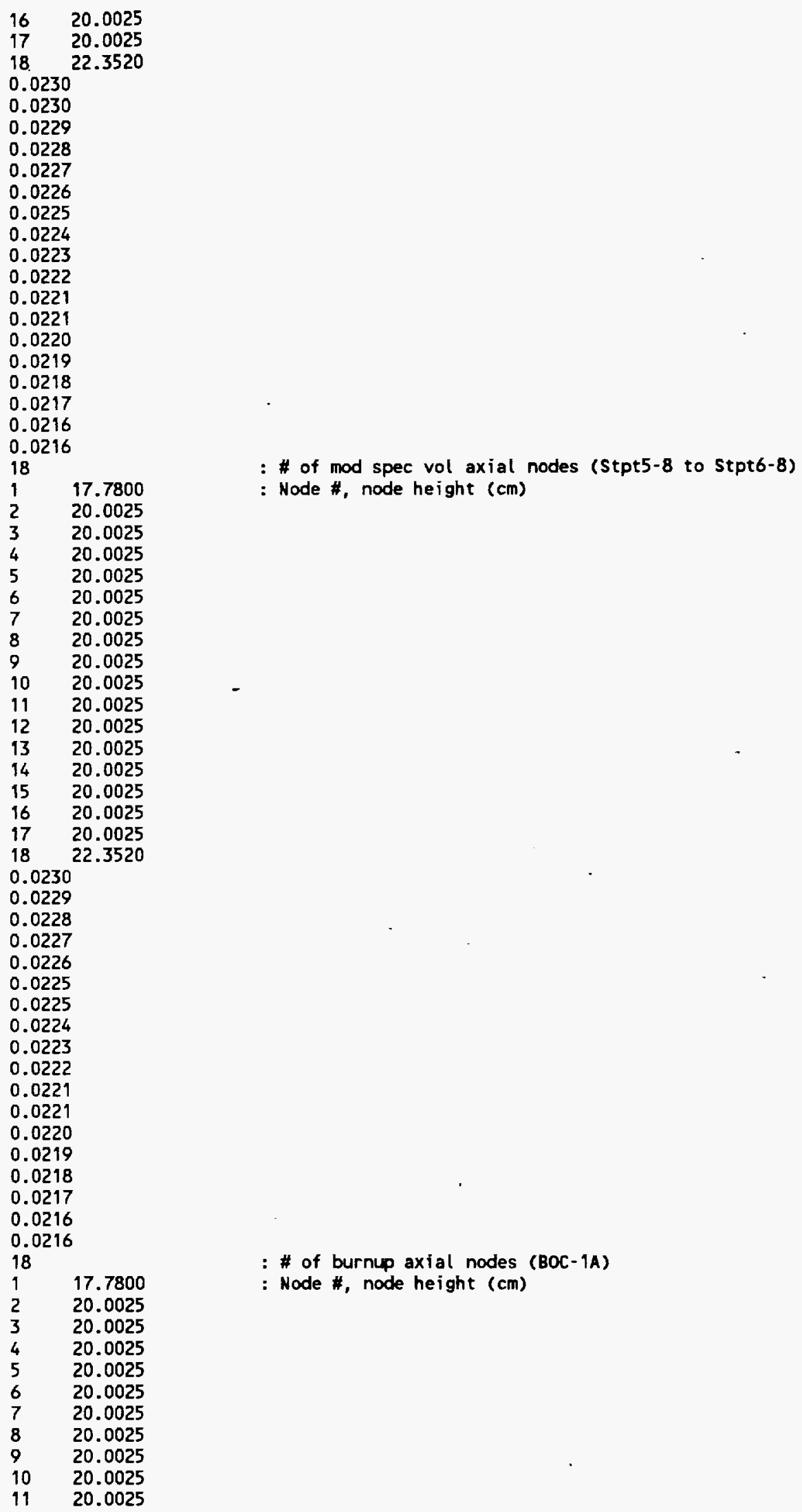


20.0025

20.0025

20.0025

20.0025

20.0025

20.0025

22.3520

0.0

0.0

0.0

0.0

0.0

0.0

0.0

0.0

0.0

0.0

0.0

0.0

0.0

0.0

0.0

0.0

0.0

0.0

18

.1

2

4

5

7

7

9

10

11

13

$14 \quad 20.0025$

$15 \quad 20.0025$

$16 \quad 20.0025$

$17 \quad 20.0025$

$18 \quad 22.3520$

3.416

6.039

8.119

9.507

10.341

10.809

11.047

11.116

10.998

10.622

9.391

6.666

5.922

5.786

5.910

6.969

7.477

4.839

18

17.7800

20.0025

20.0025

20.0025

20.0025

20.0025

20.0025
: \# of burnup axial nodes (BOC-8)

: Node \#, node height $(\mathrm{cm})$
: \# of burnup axial nodes (Stpt2-8)

: Node \#, node height $(\mathrm{cm})$ 
Jul 30 11:33 1997 File Name: A18zi.dat BBA000000-01717-0200-00040 REV 00 ATTACHMENT IX - Page 11

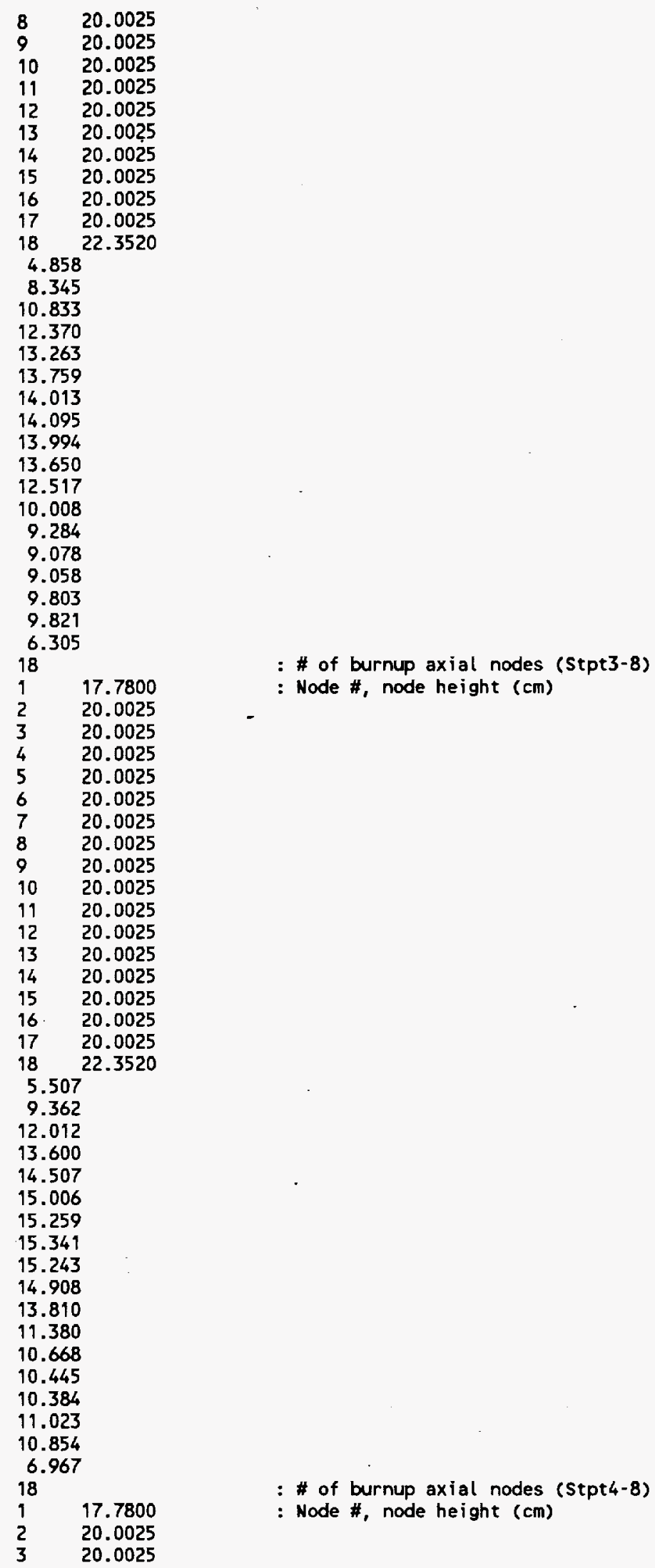




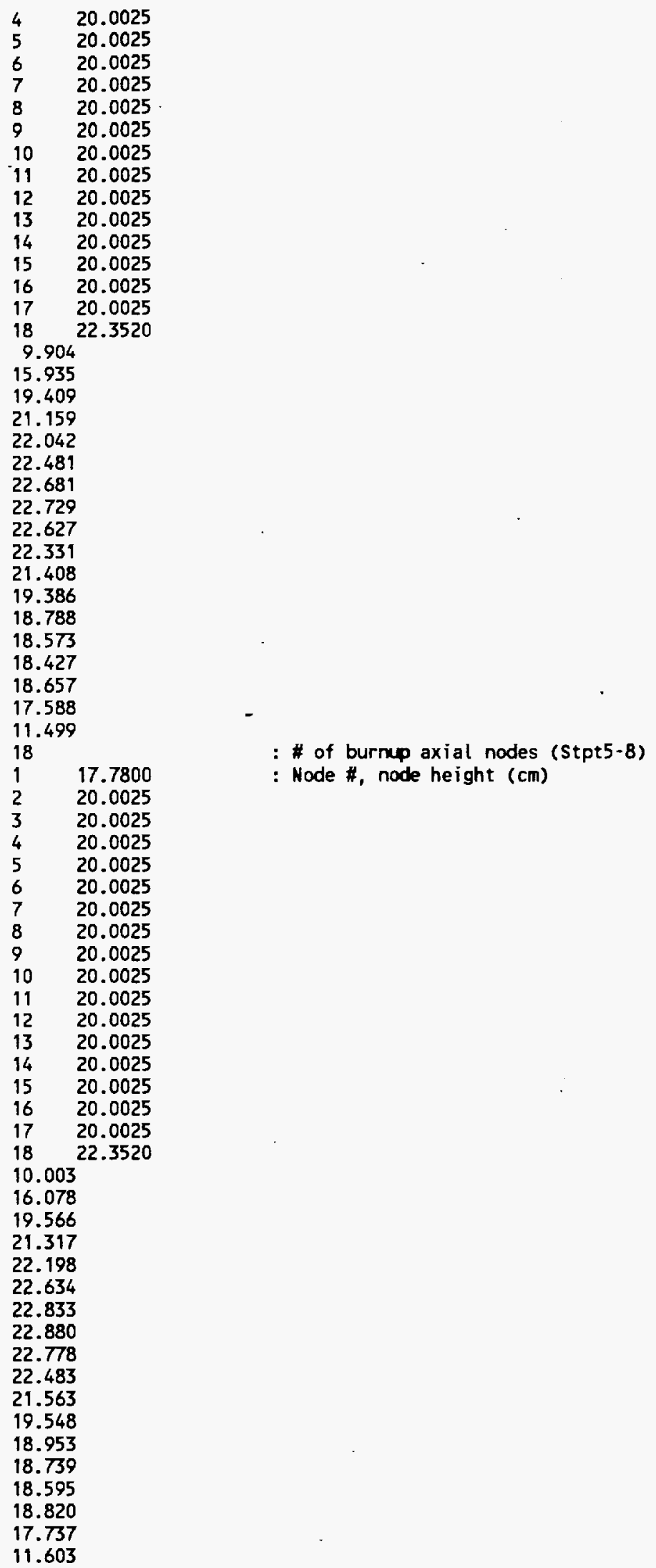


Jul 30 11:33 1997 File Name: A18zi.dat BBA000000-01717-0200-00040 REV 00 ATTACHMENT IX - Page 13

$\begin{array}{ll}18 & \\ 1 & 17.7800 \\ 2 & 20.0025 \\ 3 & 20.0025 \\ 4 & 20.0025 \\ 5 & 20.0025 \\ 6 & 20.0025 \\ 7 & 20.0025 \\ 8 & 20.0025 \\ 9 & 20.0025 \\ 10 & 20.0025 \\ 11 & 20.0025 \\ 12 & 20.0025 \\ 13 & 20.0025 \\ 14 & 20.0025 \\ 15 & 20.0025 \\ 16 & 20.0025 \\ 17 & 20.0025 \\ 18 & 22.3520 \\ 11.964 & \\ 18.845 & \\ 22.566 & \\ 24.308 & \\ 25.131 & \\ 25.516 & \\ 25.678 & \\ 25.702 & \\ 25.591 & \\ 25.302 & \\ 24.433 & \\ 22.549 & \\ 22.002 & \\ 21.820 & \\ 21.698 & \\ 21.858 & \\ 20.539 & \\ 13.607 & \\ & \end{array}$

: \# of burnup axial nodes (Stpt6-8)

: Node \#, node height $(\mathrm{cm})$ 


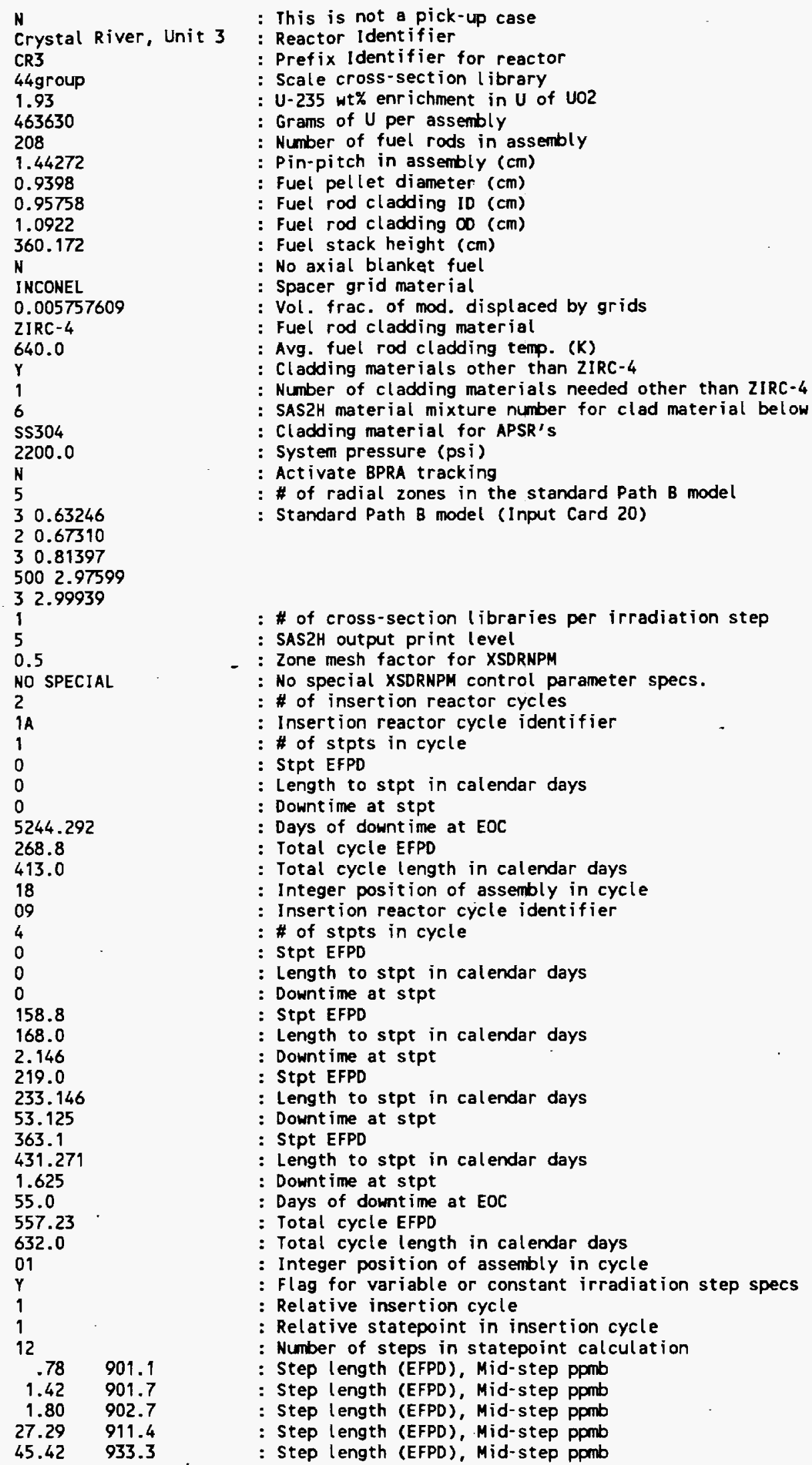




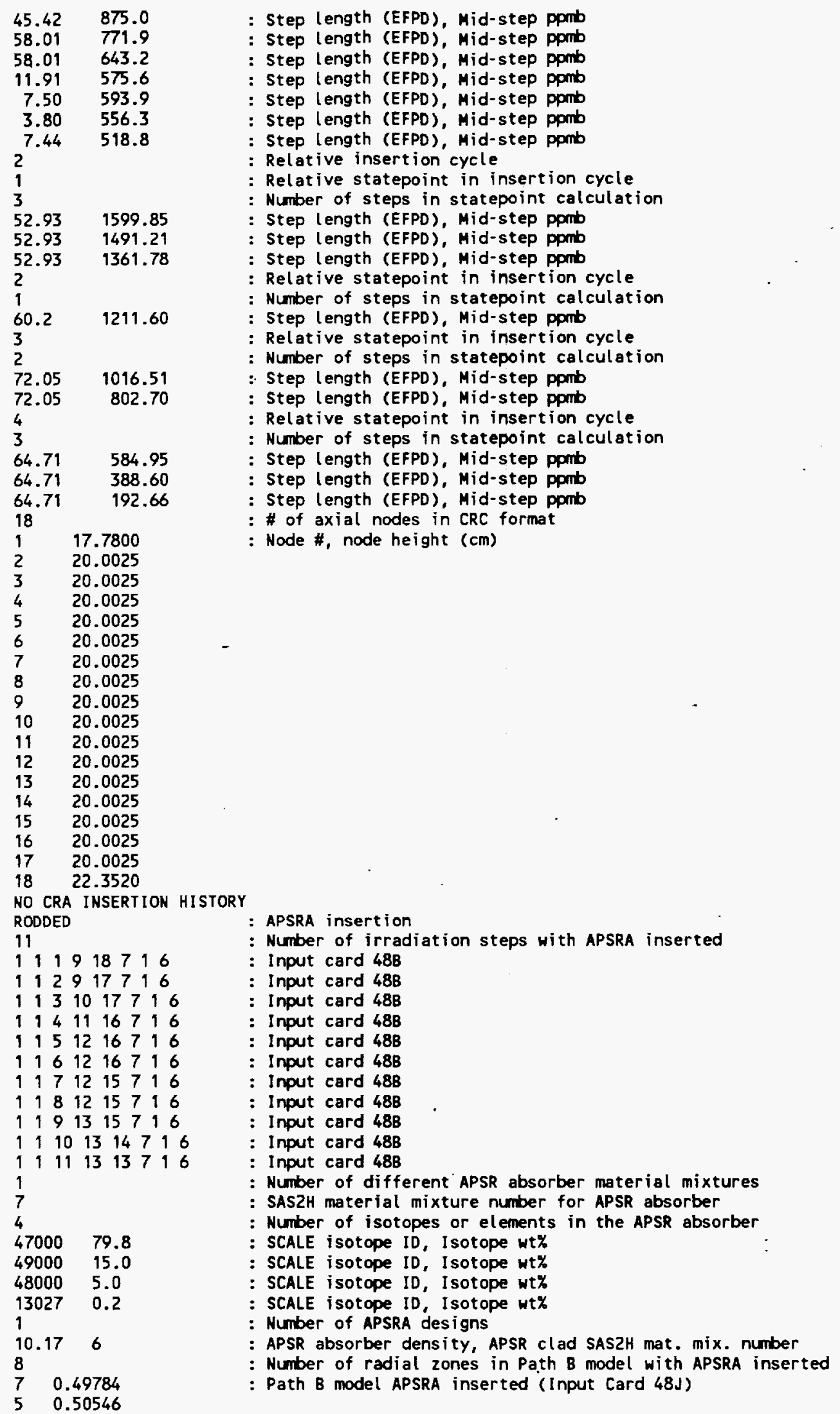


Jul 30 11:35 1997 File Name: A18azi.dat BBA000000-01717-0200-00040 REV 00 ATtACHMENT X - Page 3

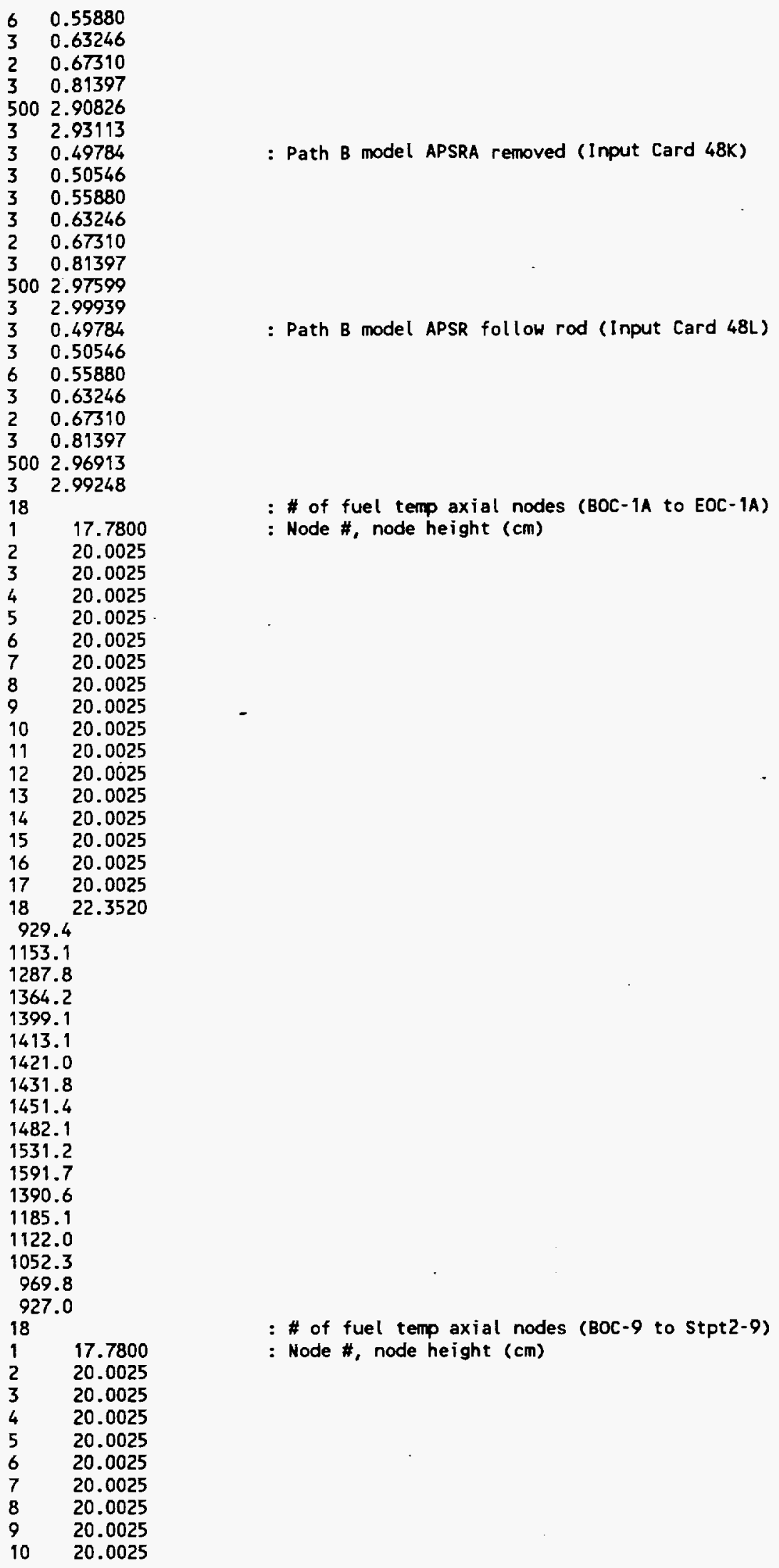




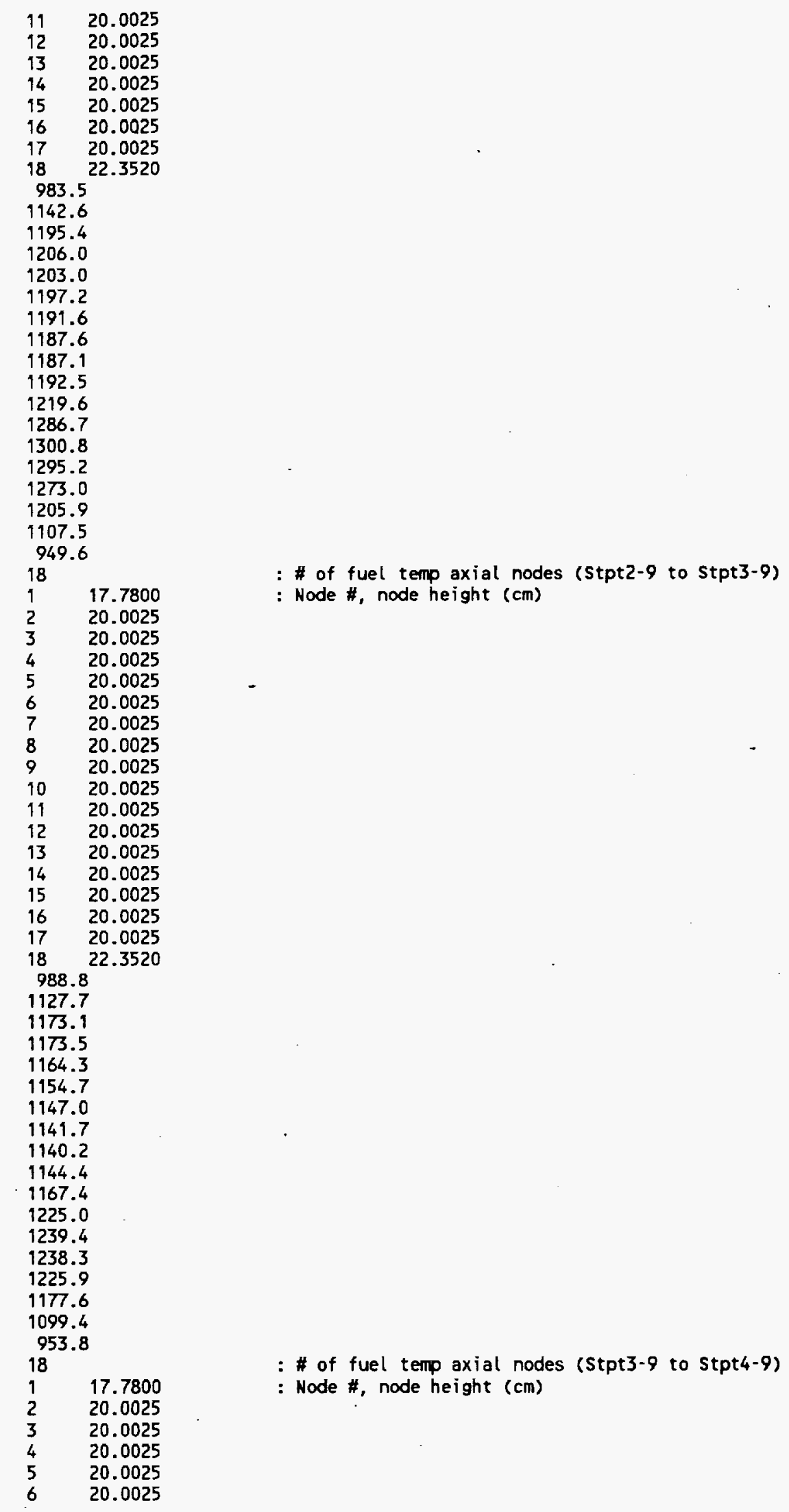


Jul 30 11:35 1997 File Name: A18azi.dat BBA000000-01717-0200-00040 REV 00 ATTACHMENT X - Page 5

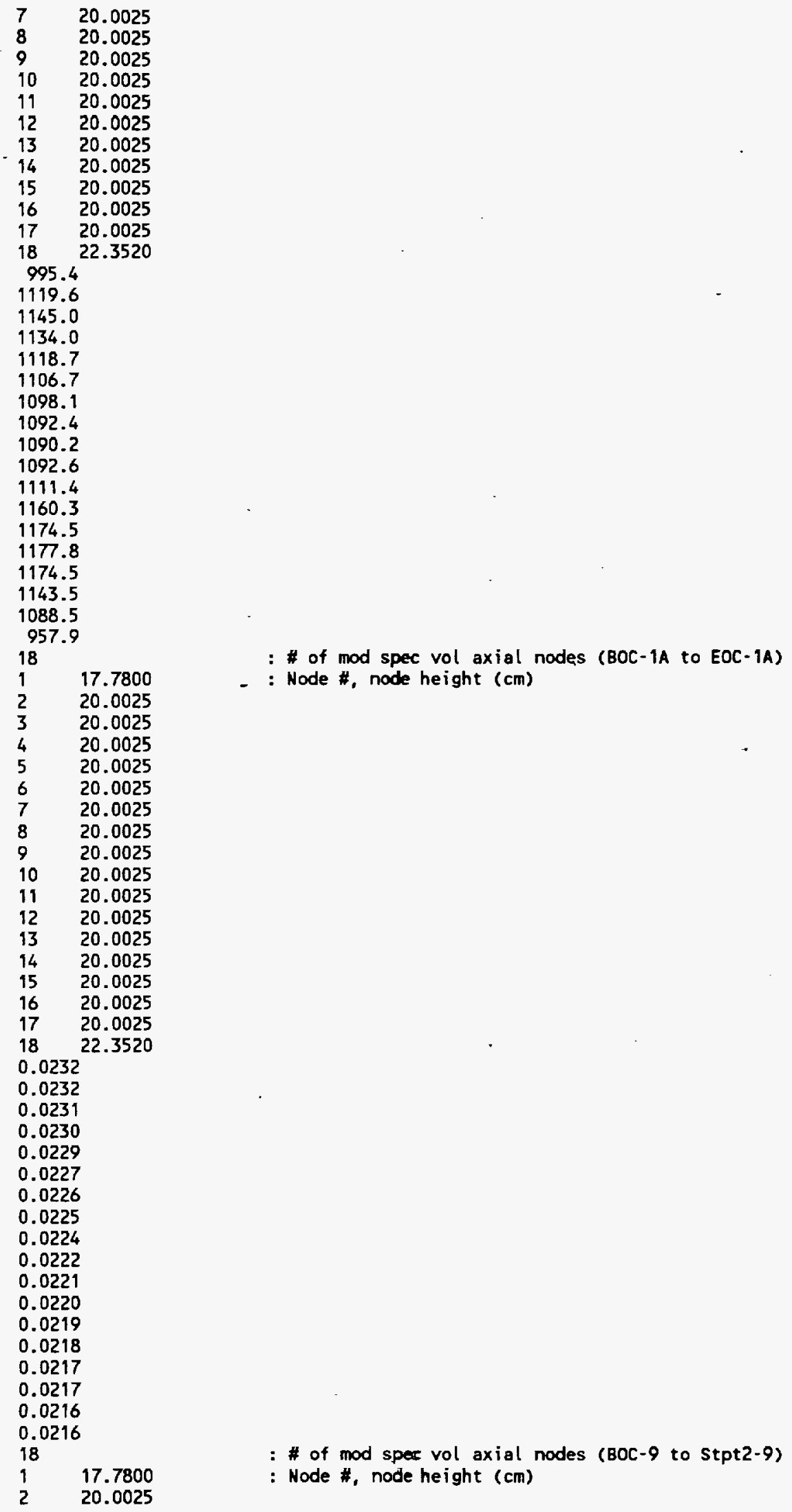




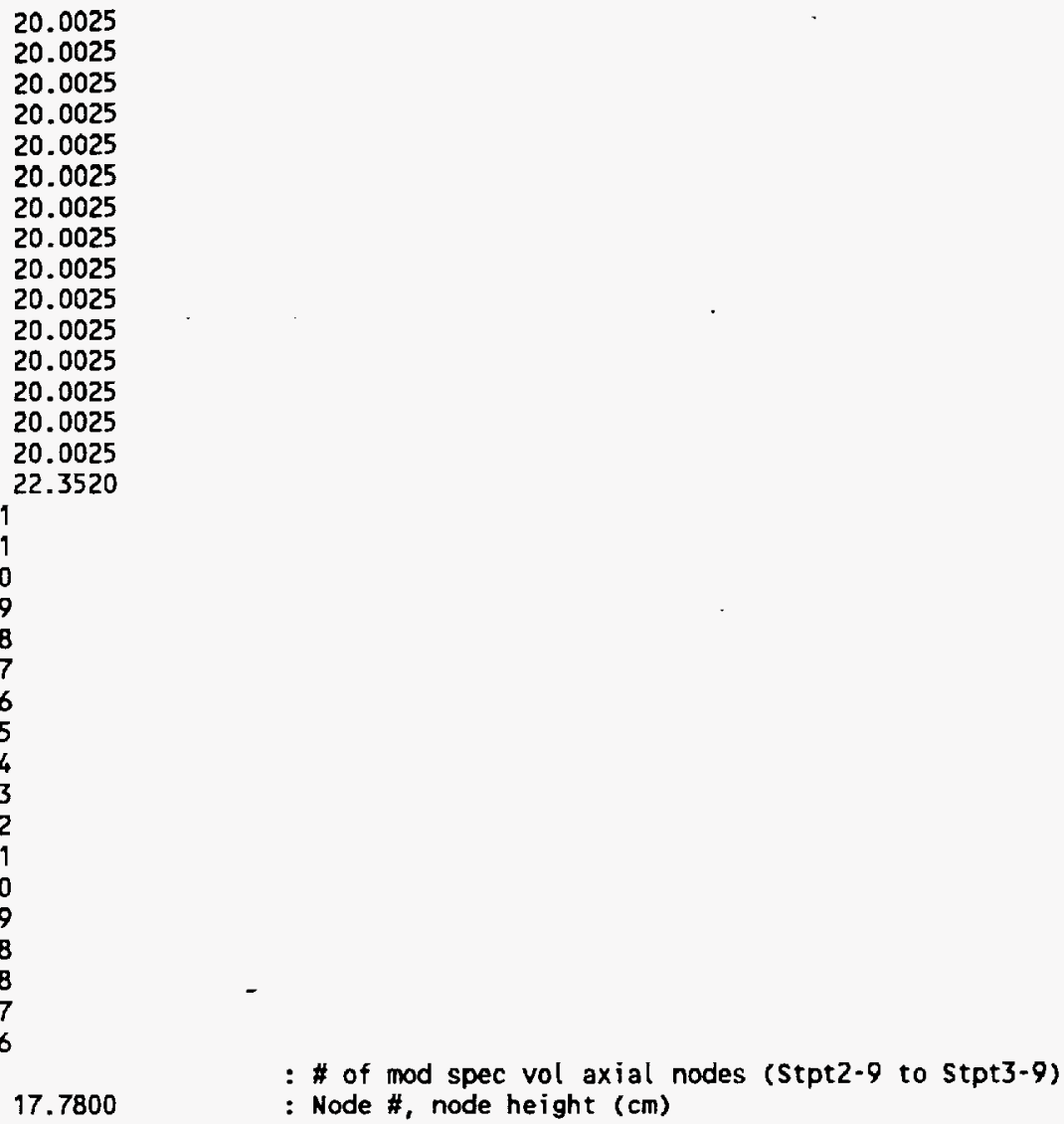


Jul 30 11:35 1997 File Name: A18azi.dat BBA000000-01717-0200-00040 REV DO ATTACHMENT X - Page 7

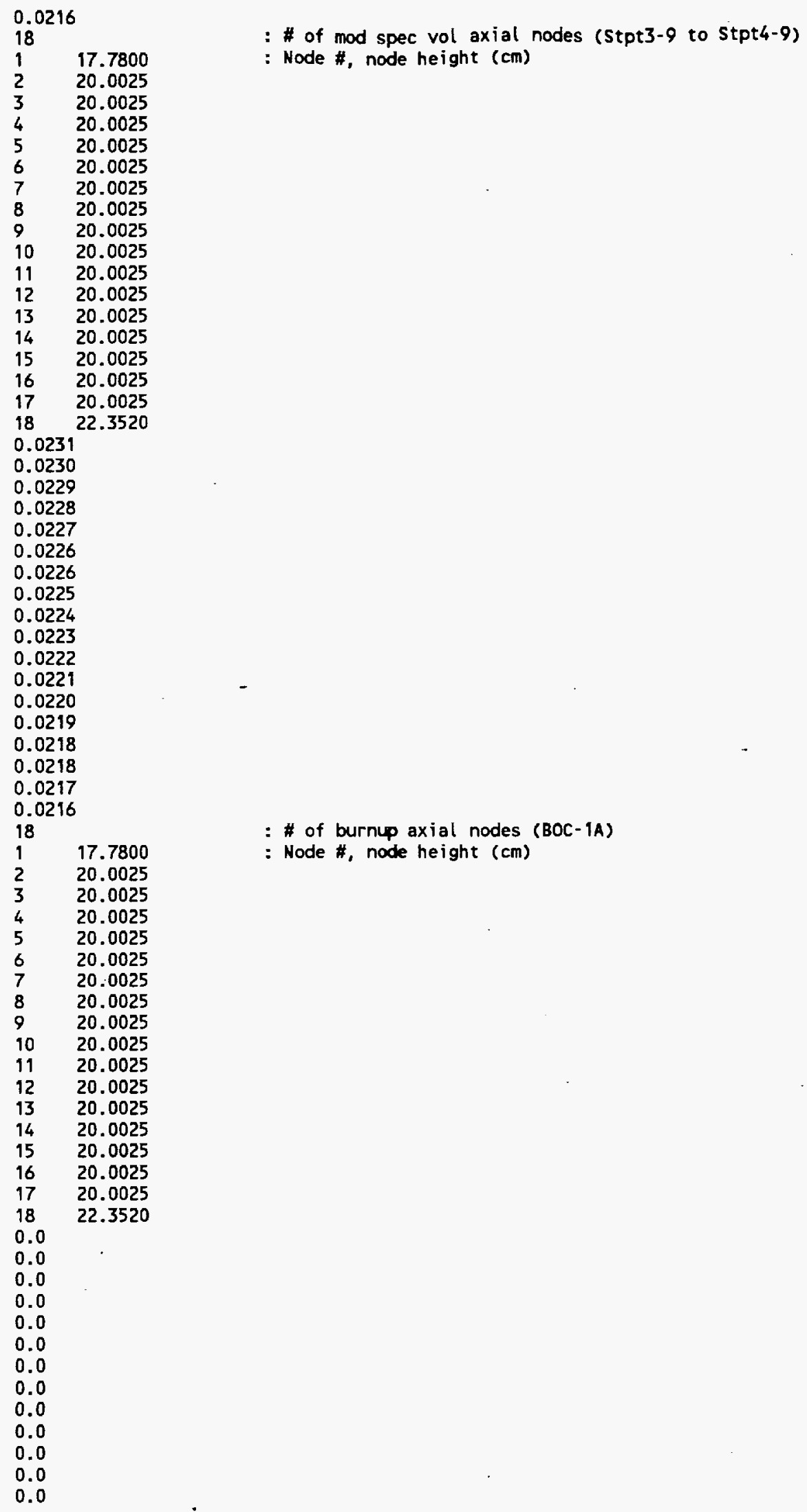




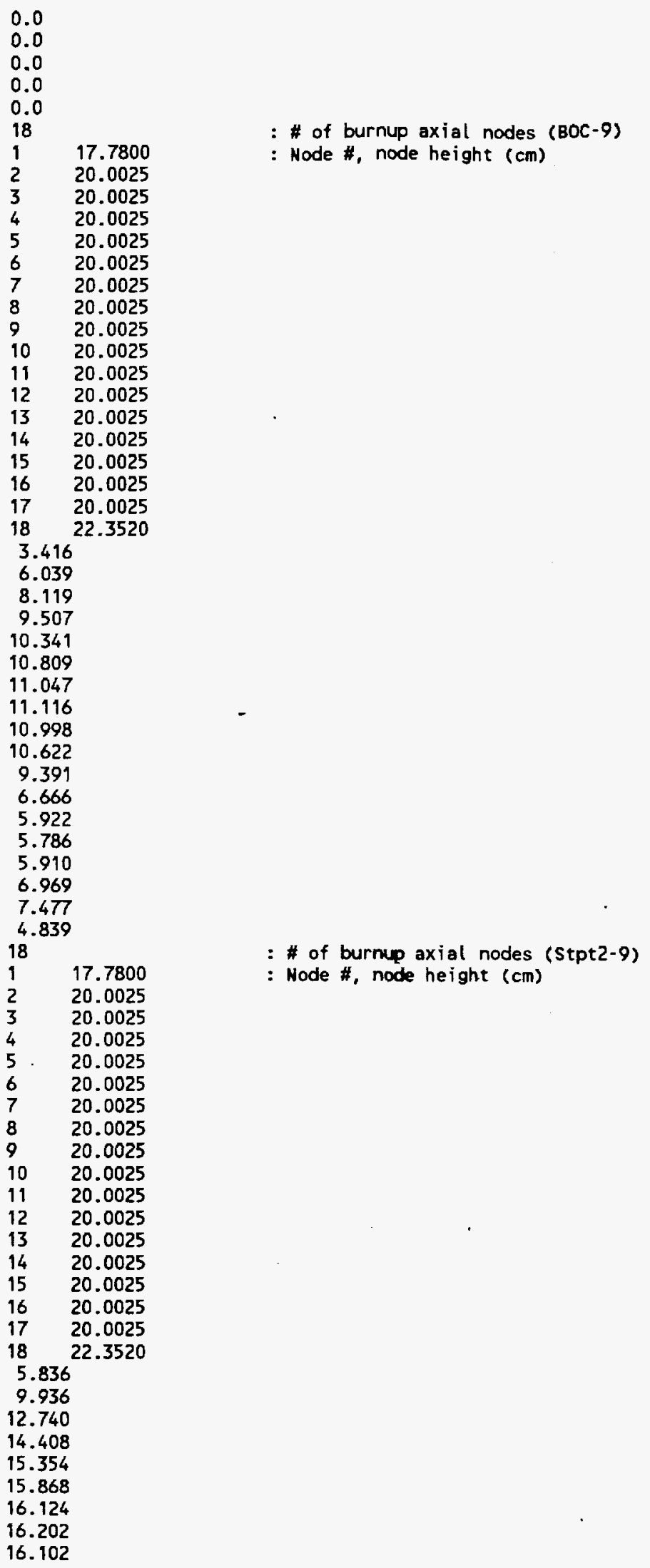


Jul 30 11:35 1997 File Name: A18azi.dat BBA000000-01717-0200-00040 REV 00 ATTACHMENT $X$ - Page 9

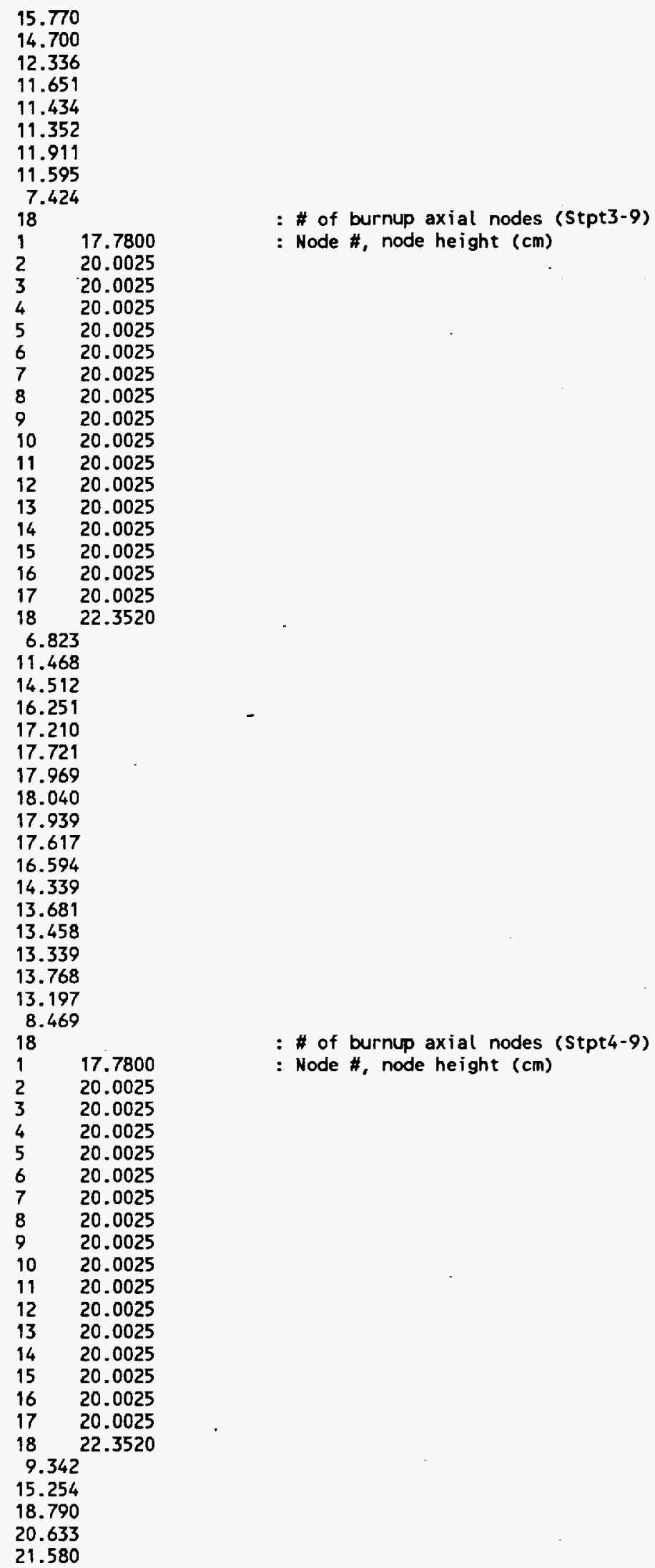


Jul 30 11:35 1997 File Name: A18azi.dat BBA000000-01717-0200-00040 REV 00 ATTACHMENT X - Page 10

22.052

22.265

22.313

22.207

21.908

20.985

18.963

18.383

18.182

18.034

18.247

17.168

11.149 


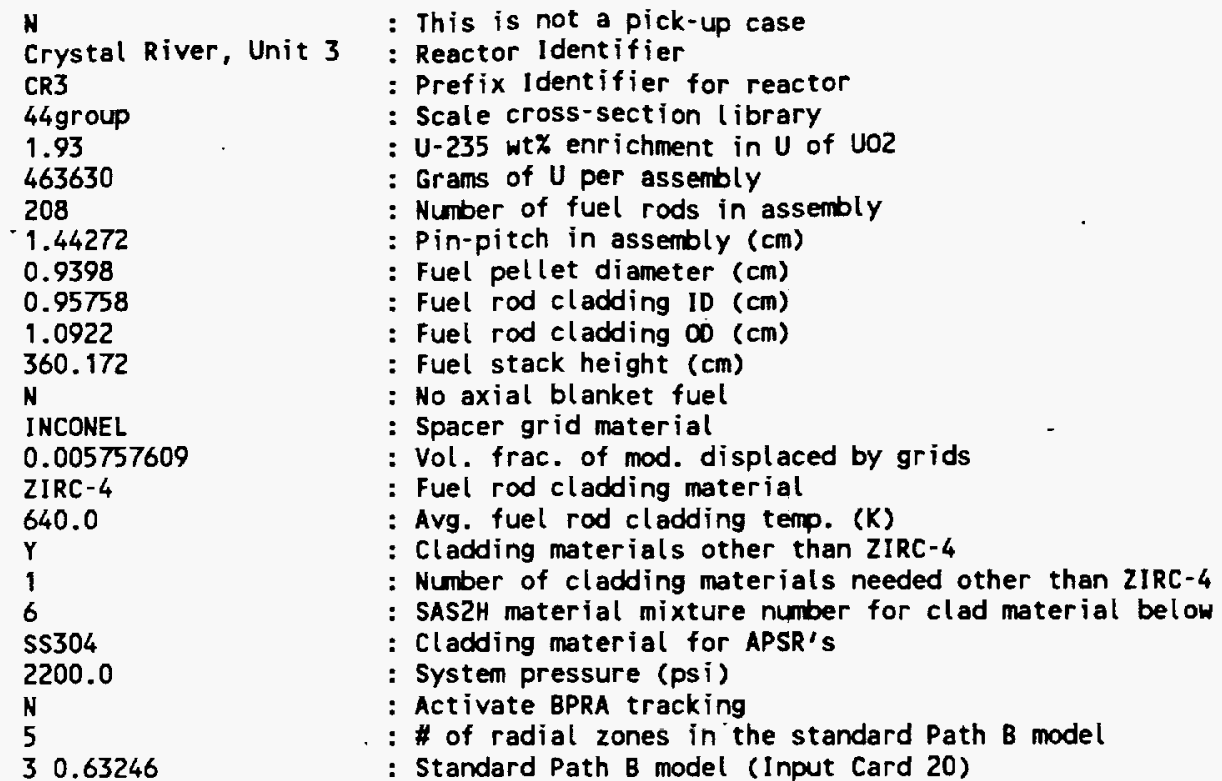

20.67310

30.81397

5002.97599

32.99939

1

5

0.5

NO SPECIAL

$1 \mathrm{~A}$

1

0

0

195.292

268.8

413

18

$1 \mathrm{~B}$

2

\# of cross-section libraries per irradiation step

: SAS2H output print level

: Zone mesh factor for XSDRNPM

: No special XSORNPM control parameter specs.

: \# of insertion reactor cycles

: Insertion reactor cycle identifier

\# of stpts in cycle

: Stpt EFPD

: Length to stpt in calendar days

: Downt ime at stpt

: Days of downtime at EOC

: Total cycle EFPD

: Total cycle length in calendar days

: Integer position of assembly in cycle

: Insertion reactor cycle identifier

: \# of stpts in cycle

: Stpt EFPD

: Length to stpt in calendar days

: Downtime at stpt

: Stpt EFPD

: Length to stpt in calendar days

: Downt ime at stpt

: Days of downt ime at EOC

: Total cycle EFPD

: Total cycle length in calendar days

: Integer position of assembly in cycle

: Flag for variable or constant irradiation step specs

: Relative insertion cycle

: Relative statepoint in insertion cycle

: Number of steps in statepoint calculation

: Step length (EFPD), Mid-step ppmb

: Step length (EFPD), Mid-step pomb

: Step length (EFPD), Mid-step ppmb

: Step length (EFPD), Mid-step pomb

: Step length (EFPD), Mid-step ppmb

: Step length (EFPD), Mid-step ppmb

: Step length (EFPD), Mid-step ppmb

: Step length (EFPD), Mid-step ppmb

: Step length (EFPD), Mid-step pomb

: Step length (EFPD), Mid-step ppmb

: Step length (EFPD), Mid-step ppmb 


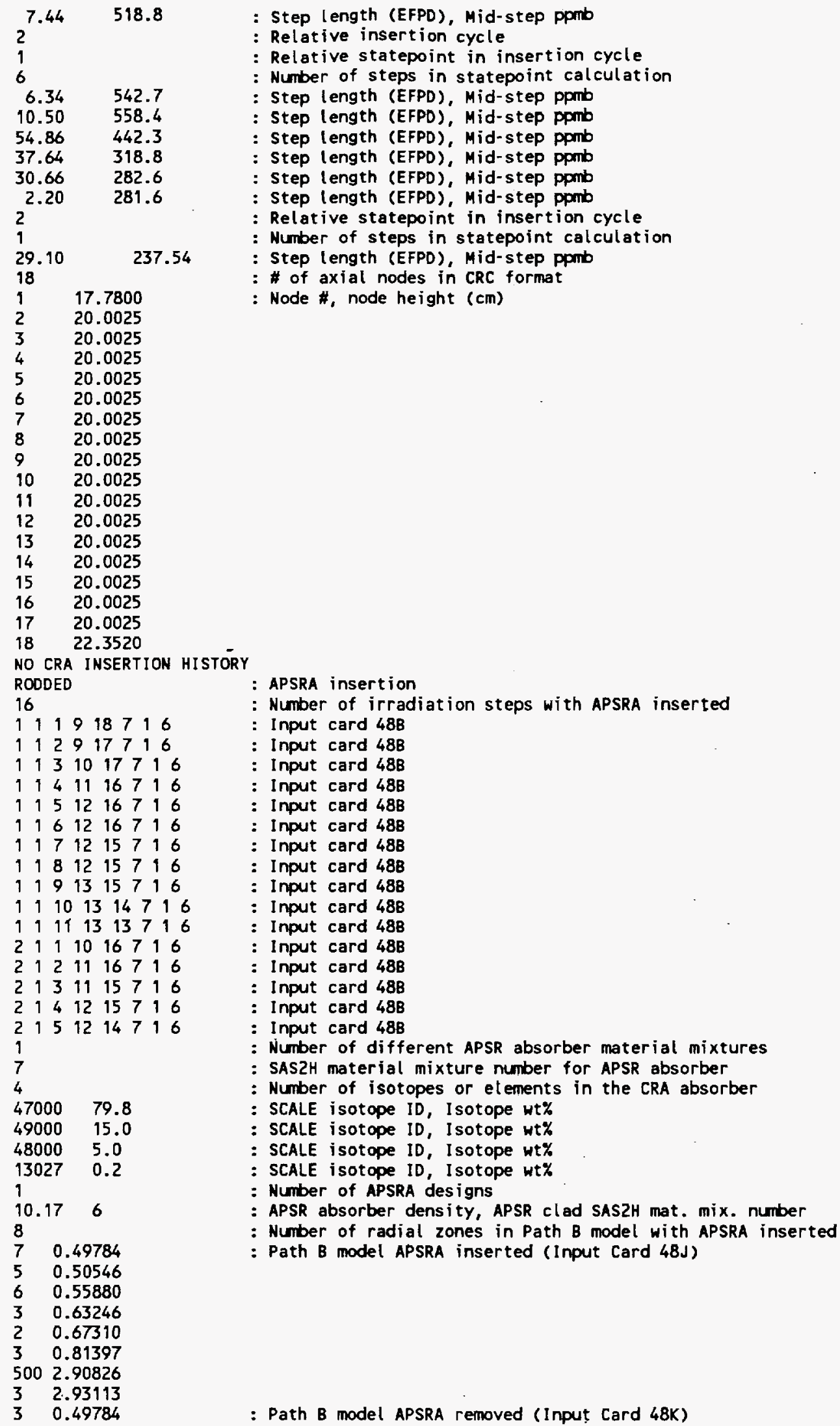


Jul 30 11:35 1997 File Name: A18bzi.dat BBA000000-01717-0200-00040 REV d0 ATTACHMENT XI - Page 3

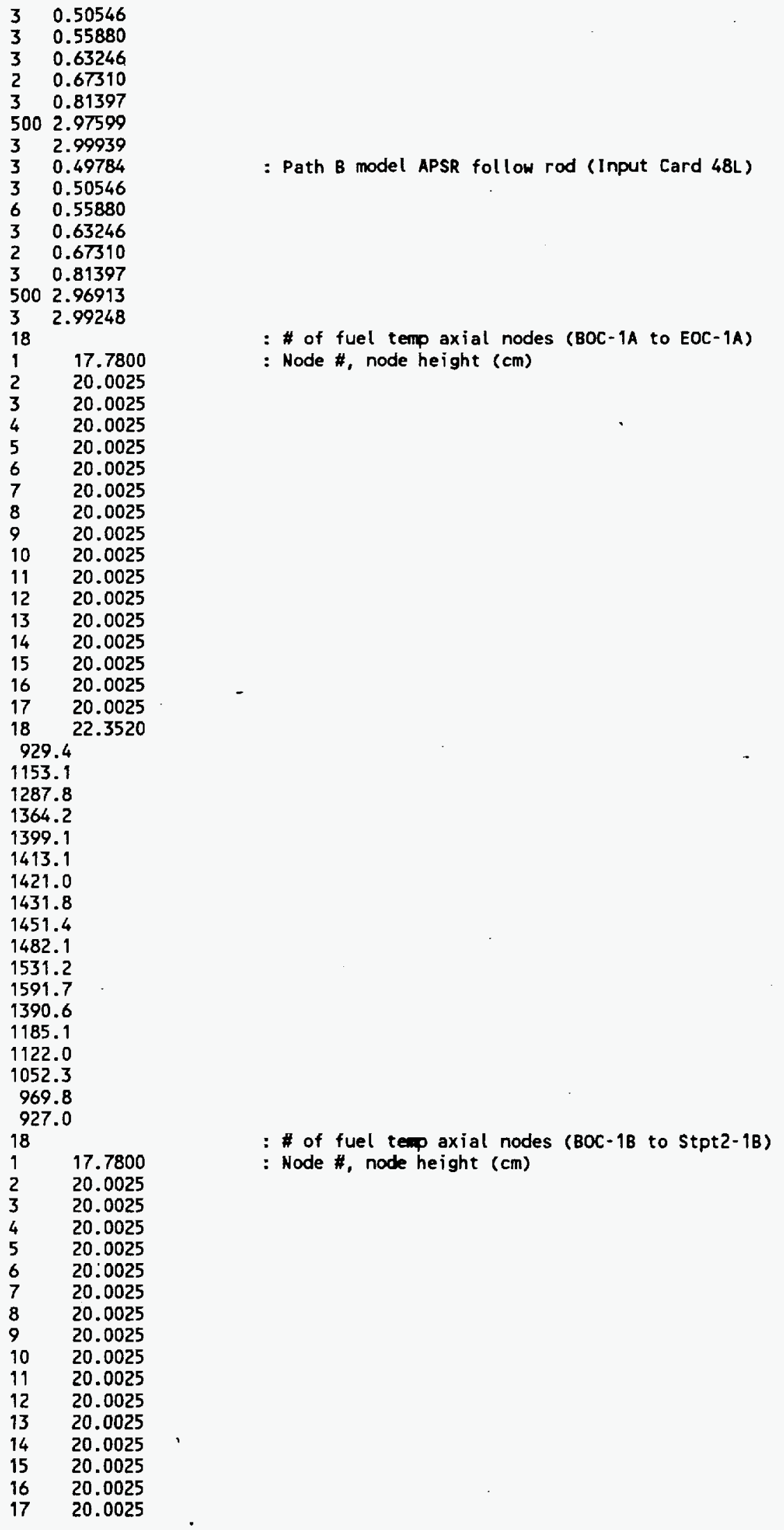


Jul 30 11:35 1997 File Name: A18bzi.dat BBA000000-01717-0200-00040 REV 00 ATTACHMENT XI - Page 4

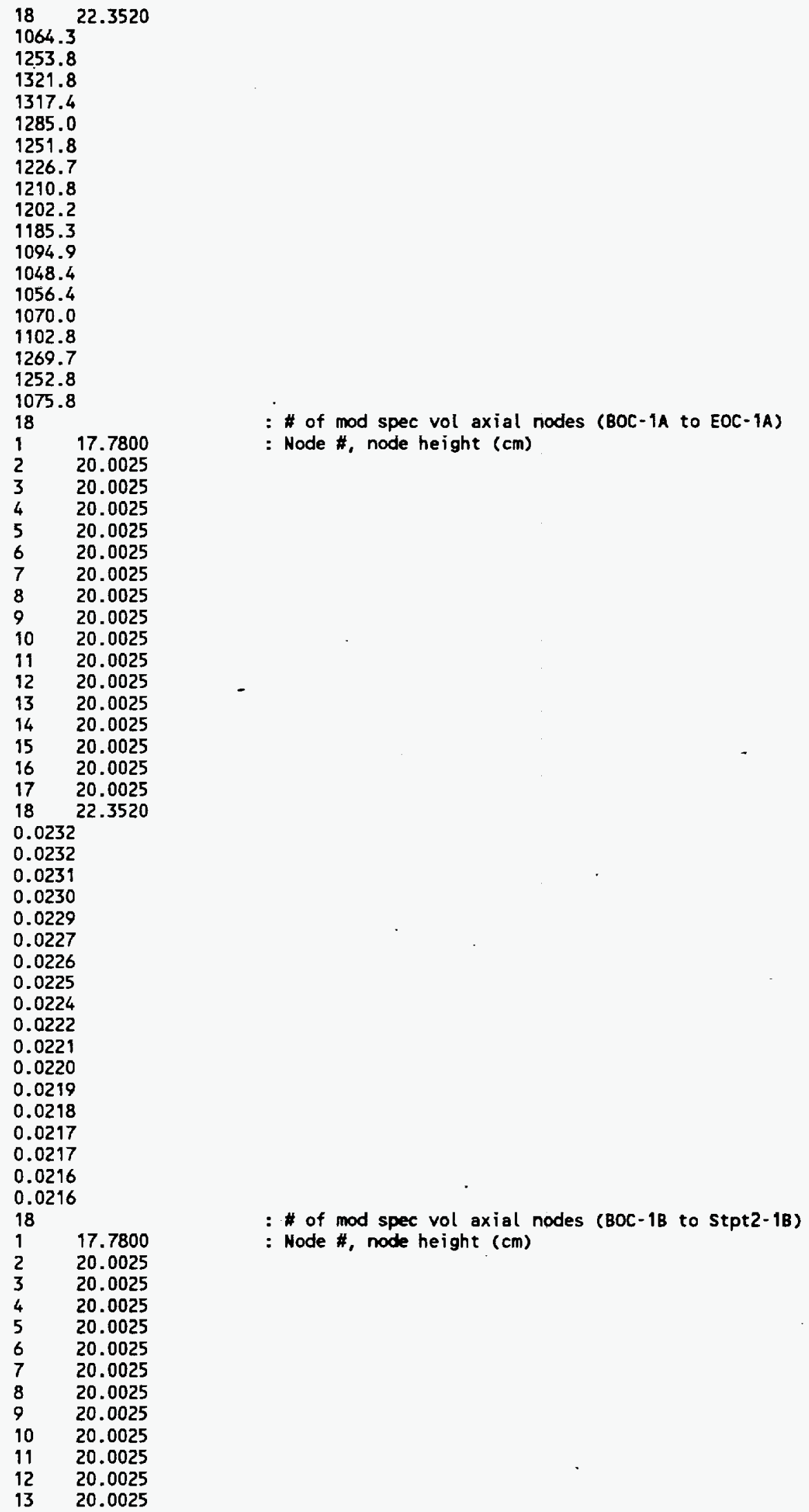


Jul 30 11:35 1997 file Name: A18bzi.dat BBA000000-01717-0200-00040 REV 00 ATTACHMENT XI - Page 5

20.0025

20.0025

20.0025

20.0025

22.3520

0.0231

0.0231

0.0229

0.0228

0.0227

0.0226

0.0225

0.0224

0.0223

0.0222

0.0221

0.0220

0.0220

0.0219

0.0218

0.0218

0.0217

0.0216

17.7800

20.0025

20.0025

20.0025

20.0025

20.0025

20.0025

20.0025

20.0025

20.0025

20.0025

20.0025

20.0025

20.0025

20.0025

20.0025

20.0025

22.3520

0.0

0.0

0.0

0.0

0.0

0.0

0.0

0.0

0.0

0.0

0.0

0.0

0.0

0.0

0.0

0.0

0.0

17.7800

20.0025

20.0025

20.0025

20.0025

20.0025

20.0025

20.0025

20.0025
: \# of burnup axial nodes (BOC-1A)

: Node \#, node height ( $\mathrm{cm}$ )

\footnotetext{
: \# of burnup axial nodes (BOC-1B)

: Node \#, node height $(\mathrm{cm})$
} 
Jul 30 11:35 1997 File Name: A18bzi.dat BBA000000-01717-0200-00040 REV 00 ATTACHMENT XI - Page 6

20.0025

20.0025

20.0025

20.0025

20.0025

20.0025

20.0025

20.0025

822.3520

3.416

6.039

8.119

9.507

10.341

10.809

11.047

11.116

10.998

10.622

9.391

6.666

5.922

5.786

5.910

6.969

7.477

4.839

18

20.0025

20.0025

20.0025

20.0025

20.0025

20.0025

20.0025

20.0025

20.0025

20.0025

20.0025

20.0025

20.0025

20.0025

20.0025

20.0025

18. 22.3520

5.861

9.956

12.792

14.424

15.255

15.627

15.760

15.750

15.564

14.951

12.957

9.795

9.090

9.113

9.779

11.959

12.257

7.957

: \# of burnup axial nodes (Stpt2-1B)

: Node \#, node height (cm) 


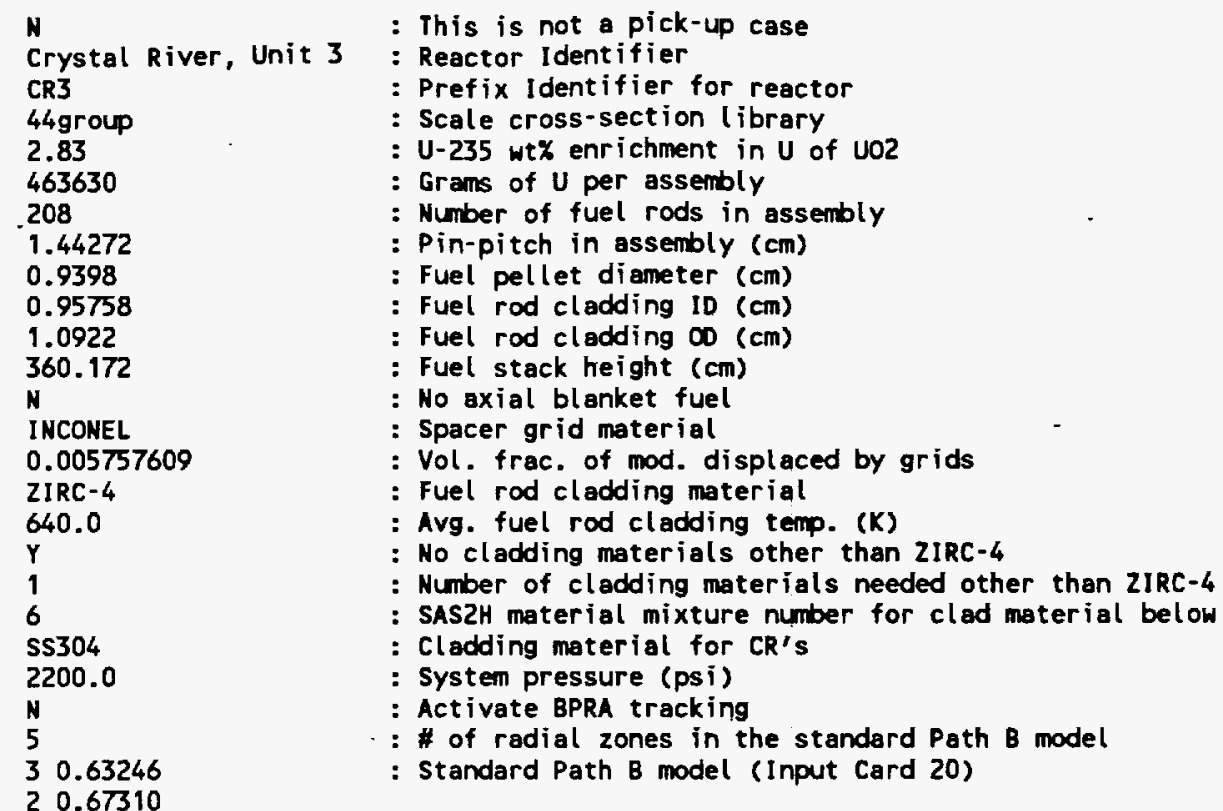

: \# of cross-section libraries per irradiation step : SASZH output print level

- : Zone mesh factor for XSDRNPM

: No special XSDRNPM control parameter specs.

: \# of insertion reactor cycles

: Insertion reactor cycle identifier

: \# of stpts in cycle

: Stpt EFPD

: Length to stpt in calendar days

: Downtime at stpt

: Days of downtime at EOC

: Total cycle EFPD

: Total cycle length in calendar days

: Integer position of assembly in cycle

: Insertion reactor cycle identifier

: \# of stpts in cycle

: Stpt EFPD

: Length to stpt in calendar days

: Downtime at stpt

: Stpt EFPD

: Length to stpt in calendar days

: Downtime at stpt

: Days of downt ime at EOC

: Total cycle EFPD

: Total cycle length in calendar days

: Integer position of assembly in cycle

: Insertion reactor cycle identifier

: \# of stpts in cycle

: Stpt EFPD

: Length to stpt in calendar days

: Downtime at stpt

: Days of downt ime at EOC

: Total cycle EFPD

: Total cycle length in calendar days

: Integer position of assembly in cycle

: Insertion reactor cycle identifier

: \# of stpts in cycle

: Stpt EFPD

: Length to stpt in calendar days

: Downtime at stpt

: Stpt EFPD 


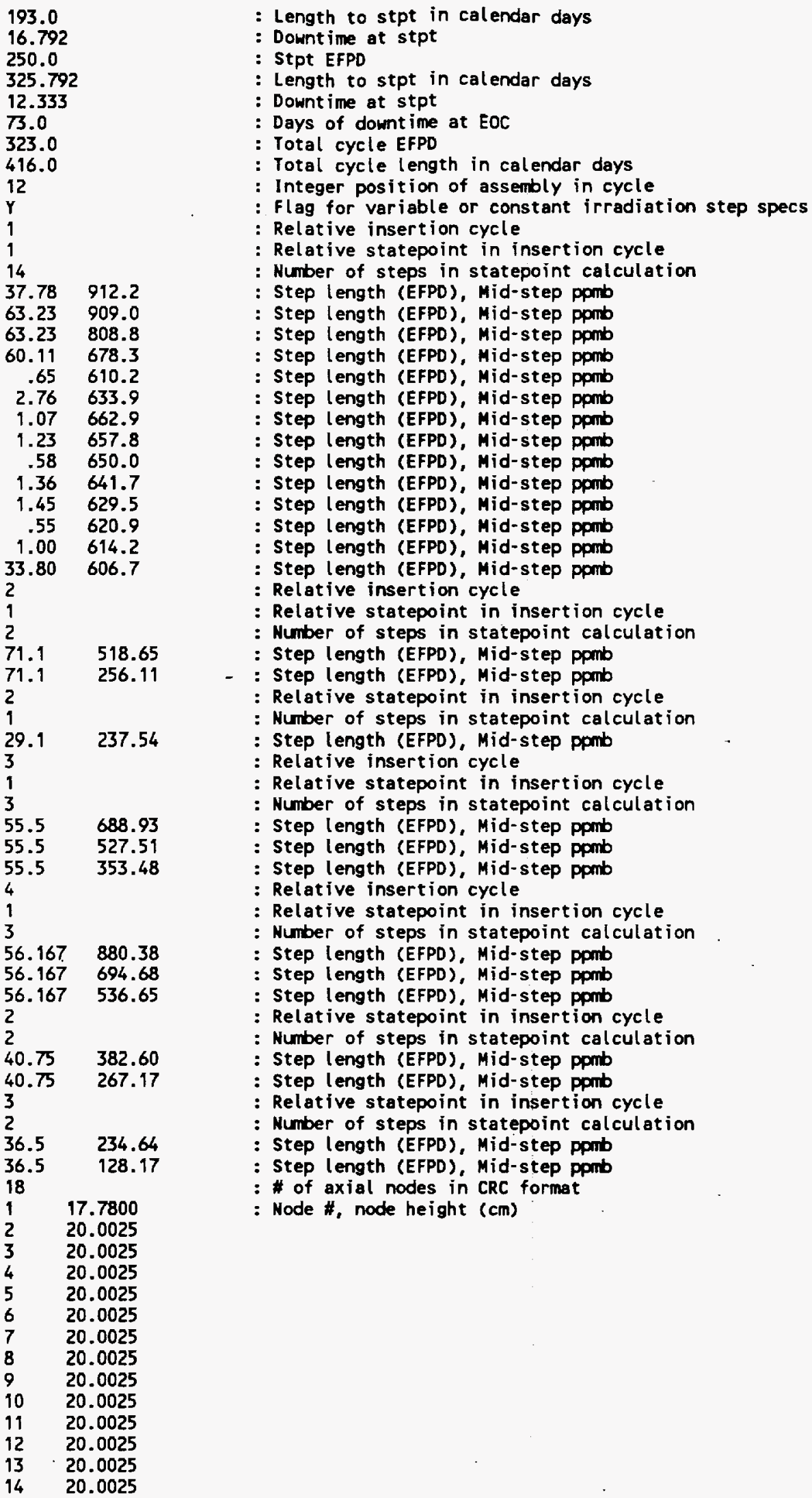




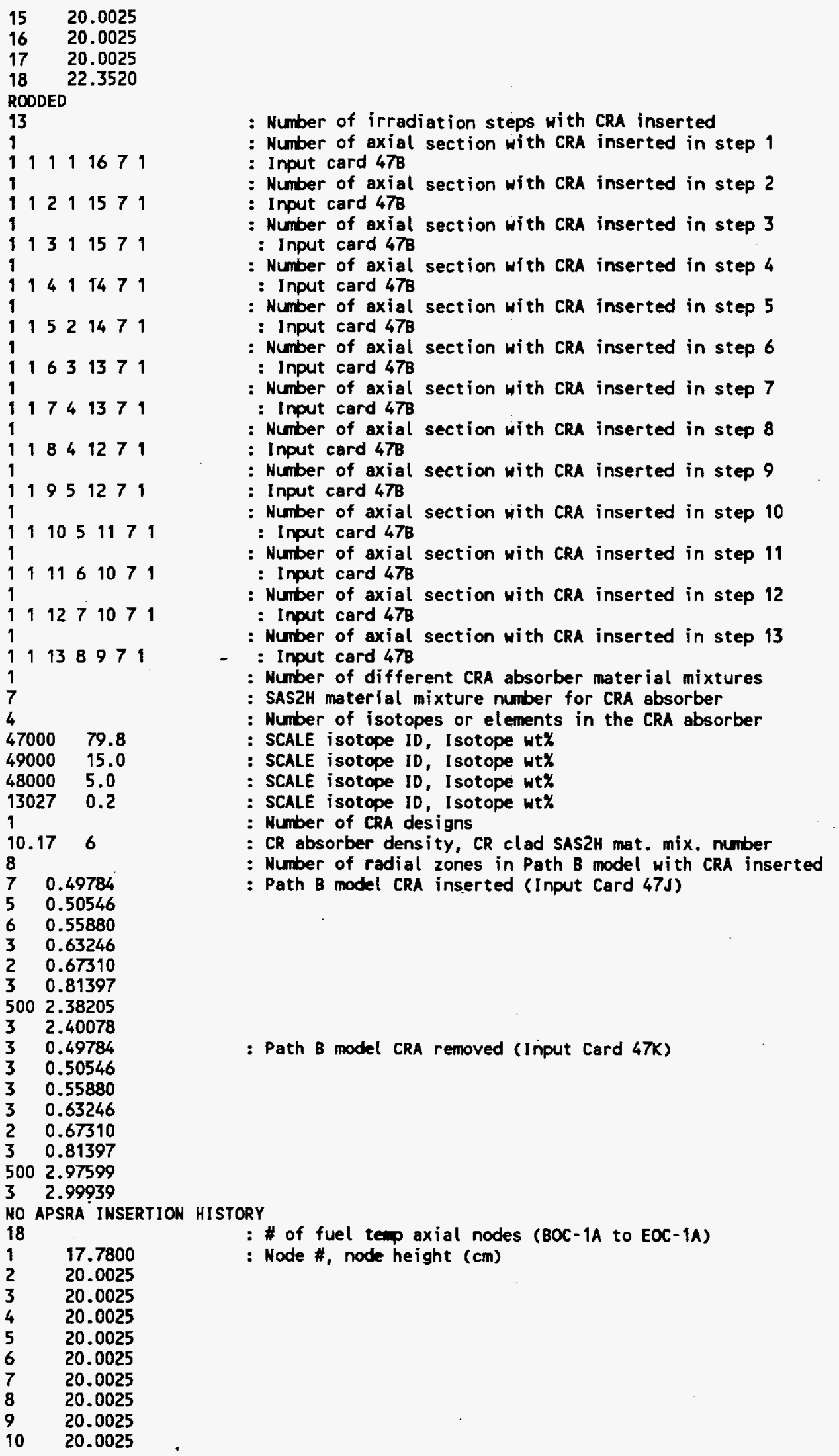


Apr 03 14:26 1997 File Name: A20i.dat BBA000000-01717-0200-00040 REV 00 ATTACHMENT XII - Page 4

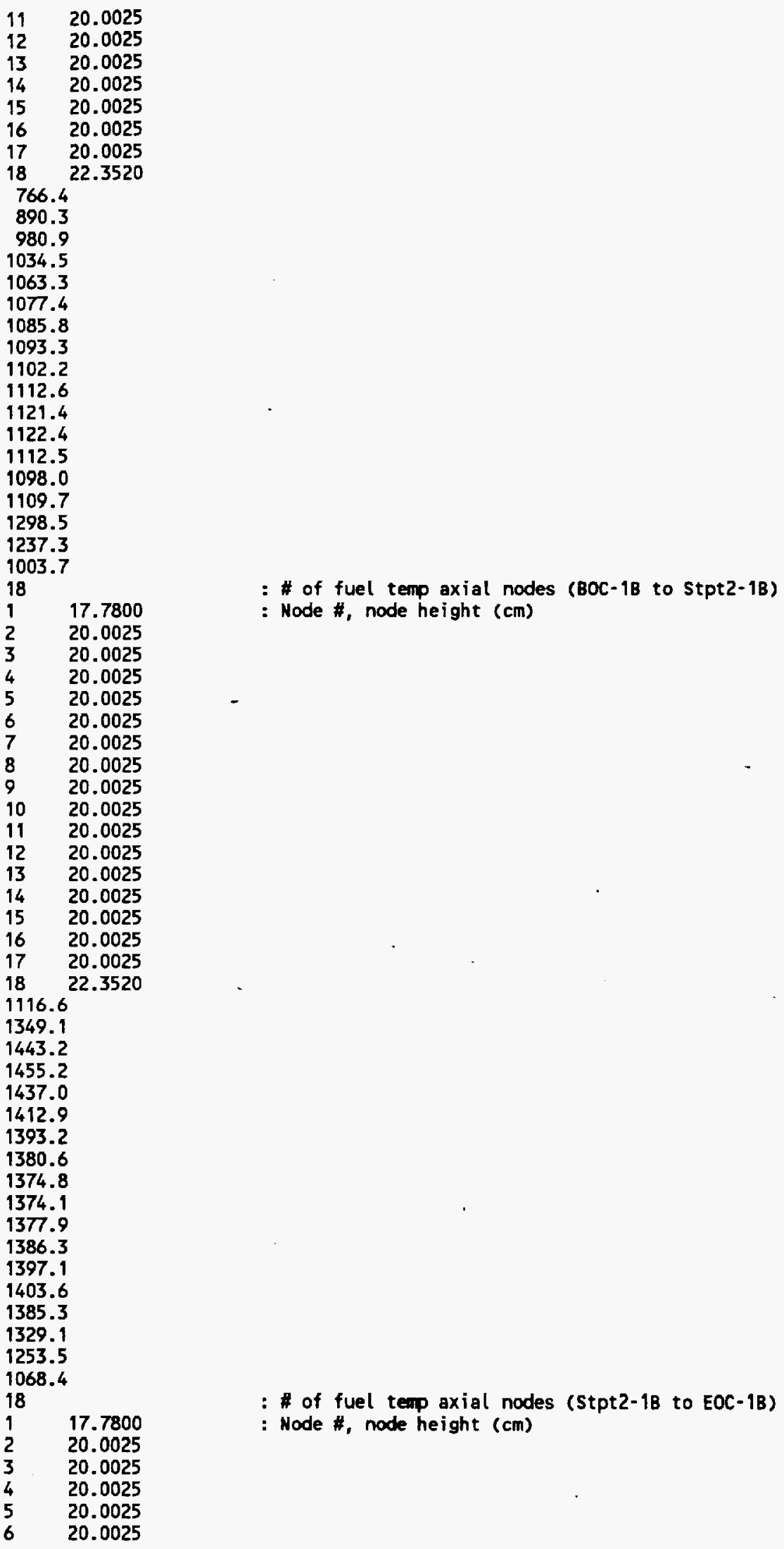


Apr 03 14:26 1997 File Name: A20i.dat BBA000000-01717-0200-00040 REV 00 ATTACHMENT XII - Page 5

20.0025

20.0025

20.0025

20.0025

20.0025

20.0025

20.0025

20.0025

20.0025

20.0025

20.0025

22.3520

1038.6

1220.9

1294.5

1327.4

1351.3

1370.9

1385.1

1394.4

1400.1

1404.1

1409.1

1414.7

1412.9

1393.5

- 1337.7

1244.4

1150.2

985.1

18

17.7800

: \# of fuel temp axial nodes (BOC-2 to EOC-2)

20.0025

- : Node \#, node height $(\mathrm{cm})$

20.0025

20.0025

20.0025

20.0025

20.0025

20.0025

20.0025

20.0025

20.0025

20.0025

20.0025

20.0025

20.0025

20.0025

20.0025

22.3520

1050.3

1217.7

1311.6

1360.1

1380.6

1389.8

1397.4

1404.3

1404.1

1379.8

1318.8

1260.1

1220.0

1193.8

1179.2

1168.9

1141.1

1005.6

18

$1 \quad 17.7800$

$2 \quad 20.0025$ 
Apr 03 14:26 1997 File Name: A20i.dat BBA000000-01717-0200-00040 REV 00 ATtACHMENT XII - Page 6

$$
\begin{aligned}
& 20.0025 \\
& 20.0025 \\
& 20.0025 \\
& 20.0025 \\
& 20.0025 \\
& 20.0025 \\
& 20.0025 \\
& 20.0025 \\
& 20.0025 \\
& 20.0025 \\
& 20.0025 \\
& 20.0025 \\
& 20.0025 \\
& 20.0025 \\
& 20.0025 \\
& 22.3520
\end{aligned}
$$

1167.1

1207.6

1211.1

1201.2

1184.3

1159.4

1126.3

1103.4

1107.0

1130.3

1169.9

1221.0

1248.4

1234.1

1185.8

1126.2

999.8

1044.7

1137.9

1153.8

1140.6

1125.2

1113.4

1103.9

1090.3

1071.2

1065.2

1070.7

1081.5

1099.8

1121.4

1120.3

1098.5

1075.1

: \# of fuel temp axial nodes (Stpt2-3 to Stpt3-3)

: Node \#, node height $(\mathrm{cm})$ 
Apr 03 14:26 1997 File Name: A20i.dat BBA000000-01717-0200-00040 REV 00 ATTACHMENT XII - Page 7

995.2

18

$1 \quad 17.7800$

$2 \quad 20.0025$

$3 \quad 20.0025$

$4 \quad 20.0025$

$5 \quad 20.0025$

$6 \quad 20.0025$

$7 \quad 20.0025$

$8 \quad 20.0025$

$9 \quad 20.0025$

$10 \quad 20.0025$

$11 \quad 20.0025$

$12 \quad 20.0025$

$13 \quad 20.0025$

$14 \quad 20.0025$

$15 \quad 20.0025$

$16 \quad 20.0025$

$17 \quad 20.0025$

$18 \quad 22.3520$

0.0226

0.0226

0.0225

0.0225

0.0224

0.0224

0.0223

0.0222

0.0222

0.0221

0.0220

0.0220

0.0219

0.0219

0.0218

0.0217

0.0216

0.0216

18

1

17.7800

$2 \quad 20.0025$

$3 \quad 20.0025$

$4 \quad 20.0025$

$5 \quad 20.0025$

$6 \quad 20.0025$

$7 \quad 20.0025$

$8 \quad 20.0025$

$9 \quad 20.0025$

$10 \quad 20.0025$

$11 \quad 20.0025$

$12 \quad 20.0025$

$13 \quad 20.0025$

$14 \quad 20.0025$

$15 \quad 20.0025$

$16 \quad 20.0025$

$17 \quad 20.0025$

$18 \quad 22.3520$

0.0236

0.0235

0.0234

0.0232

0.0231

0.0229

0.0228

0.0227

0.0226

0.0224

0.0223

0.0222

0.0221
: \# of mod spec vol axial nodes (BOC-1A to EOC-1A)

: Node \#, node height (cm)
: \# of mod spec vol axial nodes (BOC-1B to Stpt2-1B)

: Node \#, node height (cm) 


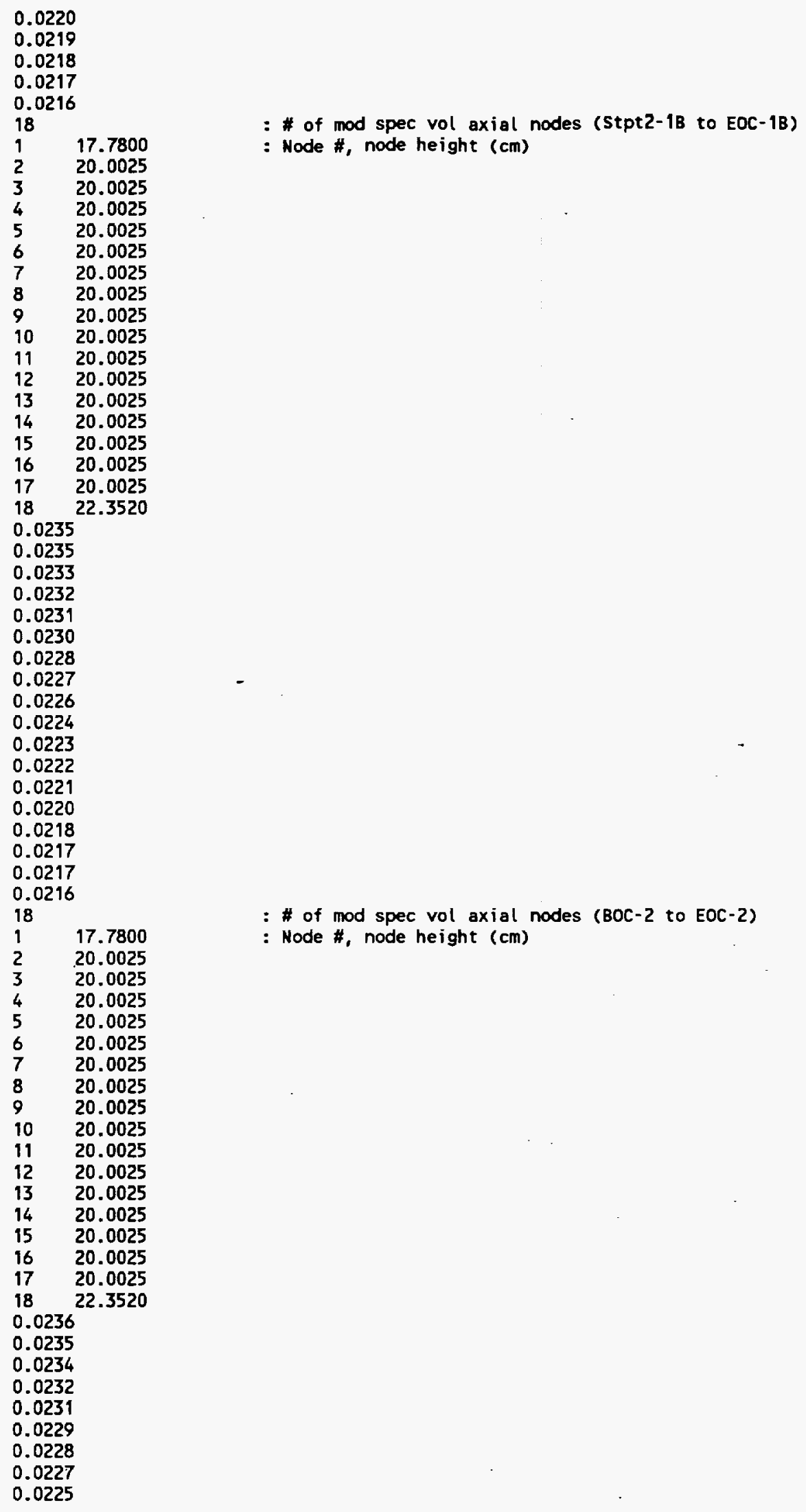


Apr 03 14:26 1997 File Name: A20i.dat BBA000000-01717-0200-00040 REV 00 ATtACHMENT XII - Page 9

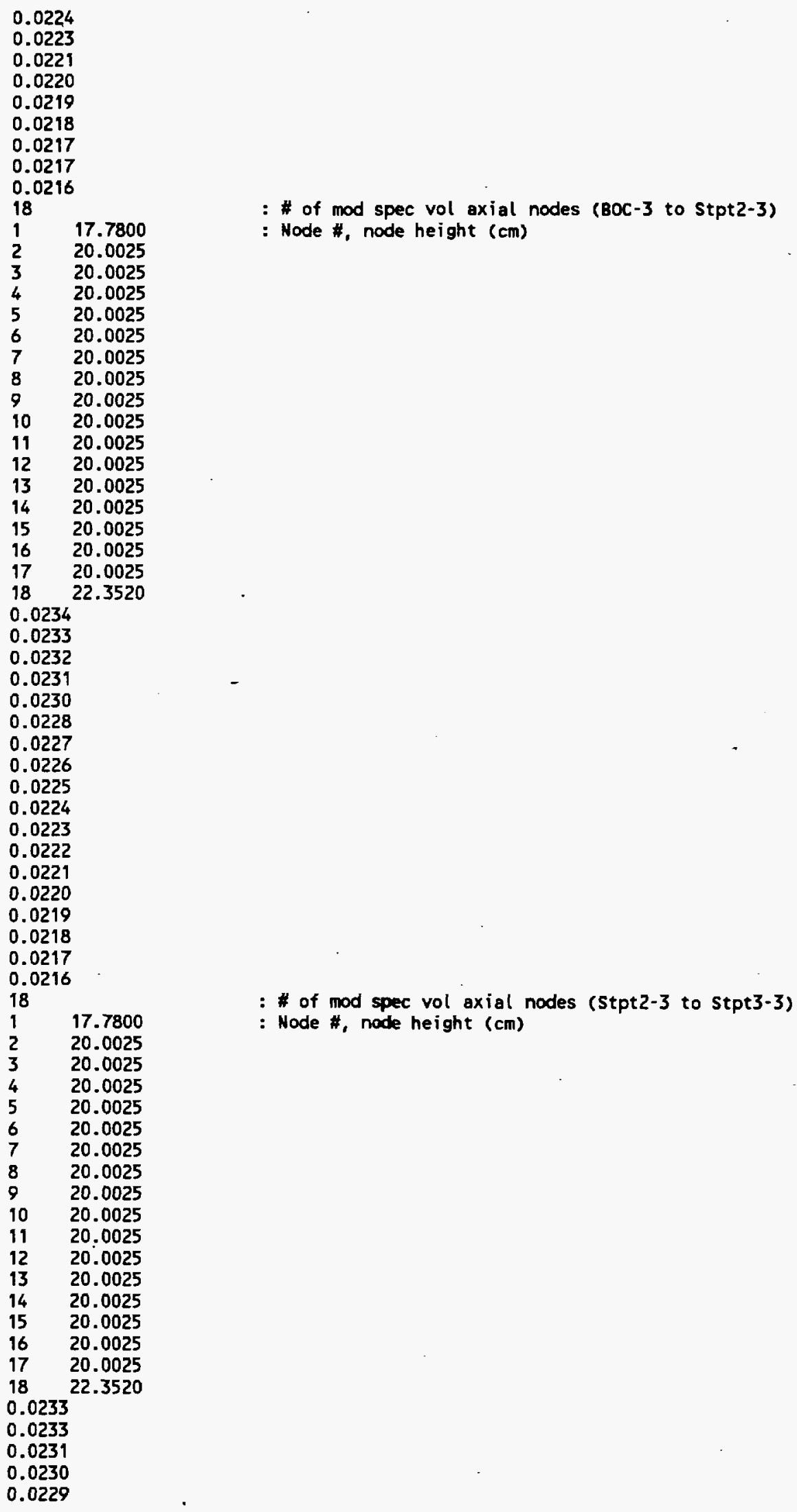




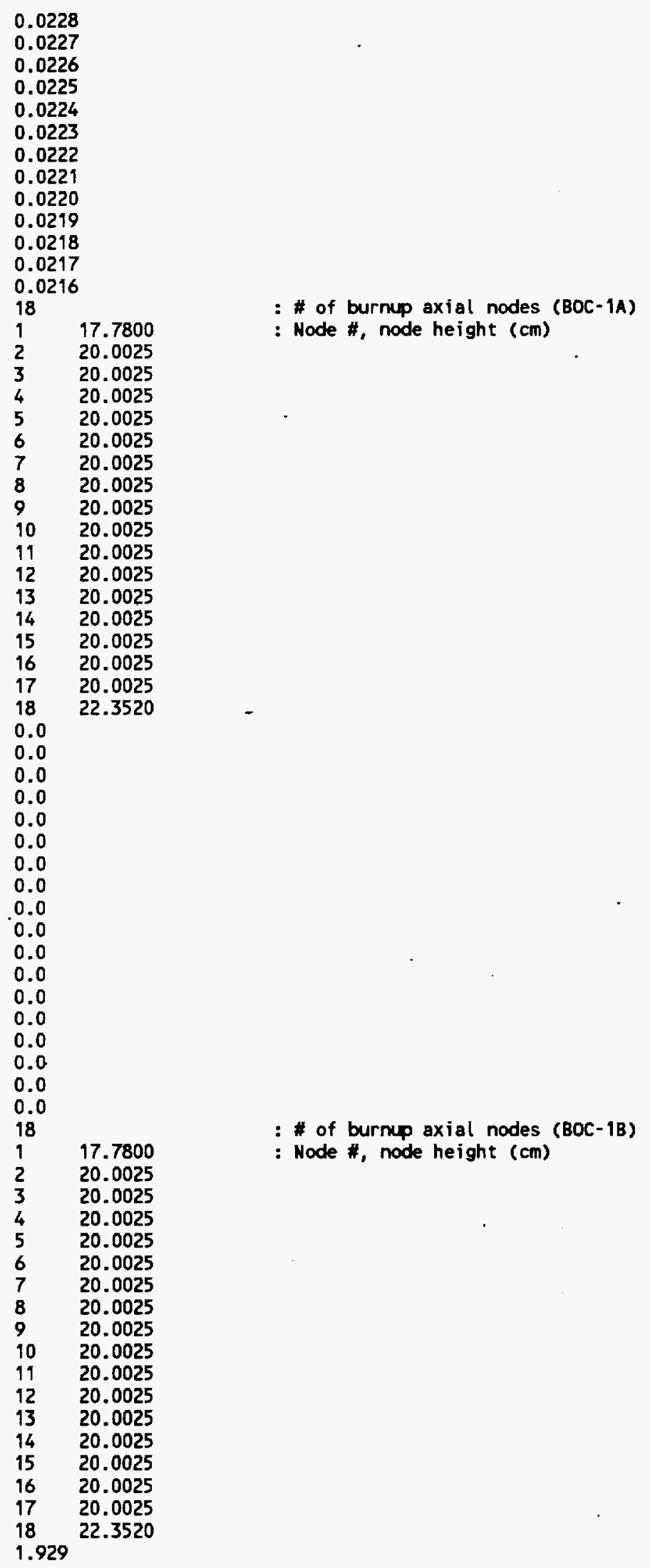


Apr 03 14:26 1997 file Name: A20i.dat BBA000000-01717-0200-00040 REV 00 ATtACHMENT XII - Page 11

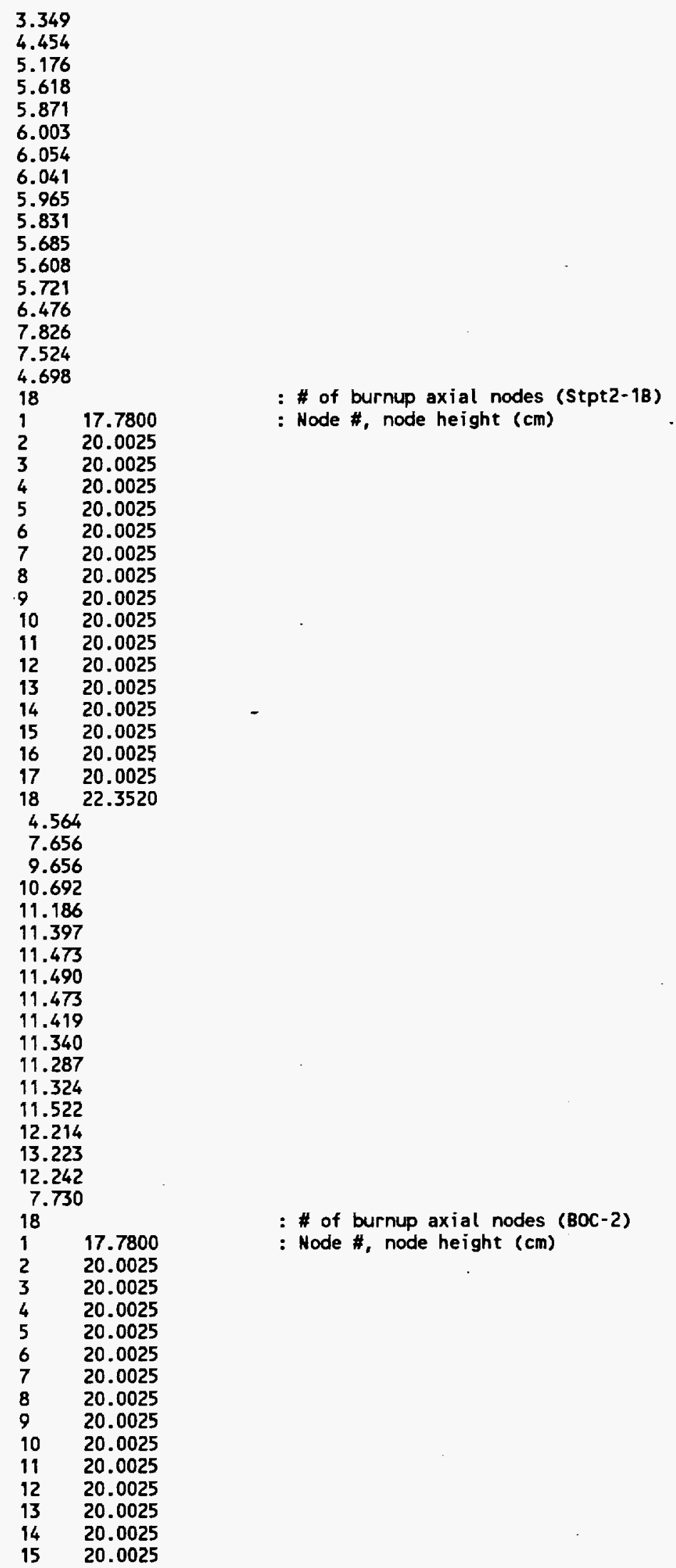


Apr 03 14:26 1997 File Name: A20i.dat BBA000000-01717-0200-00040 REV 00 ATtACHMENT XI1 - Page 12

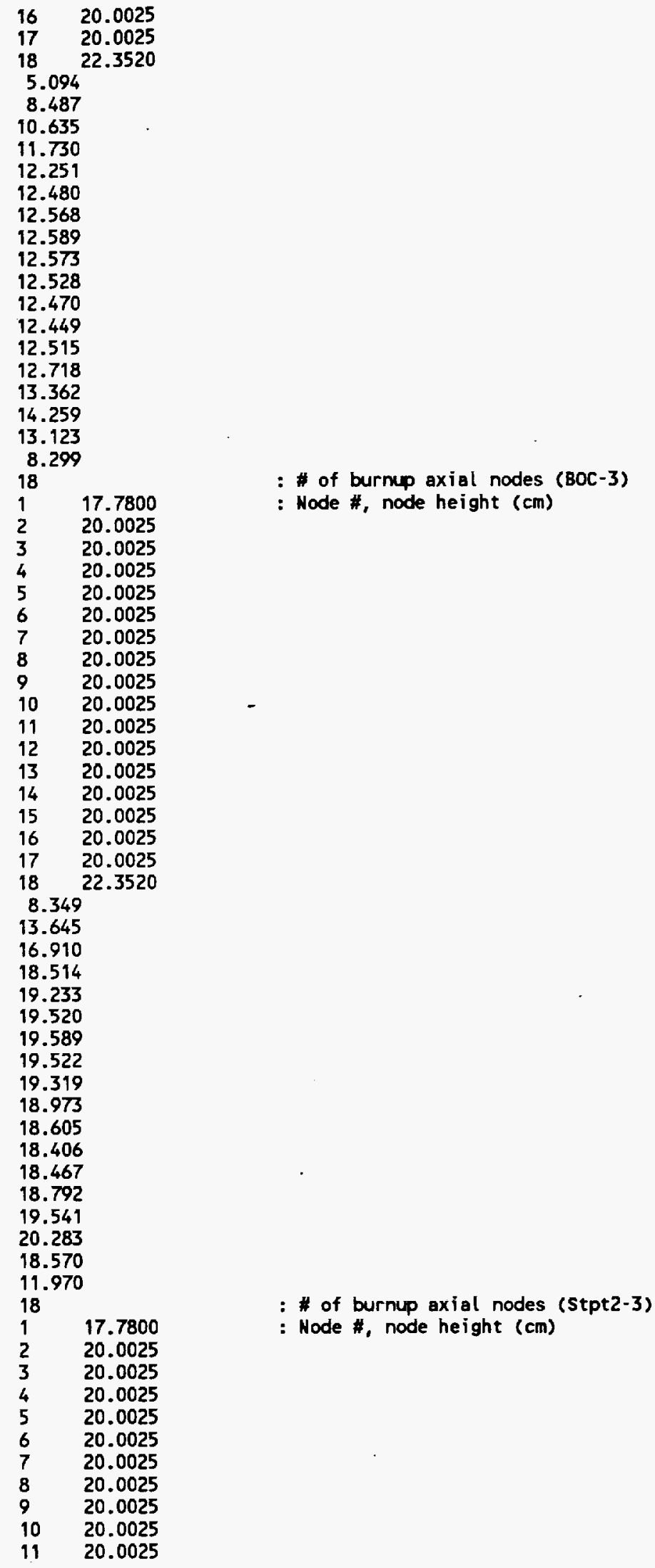


Apr 03 14:26 1997 File Name: A20i.dat BBA000000-01717-0200-00040 REV 00 ATTACHMENT XII - Page 13

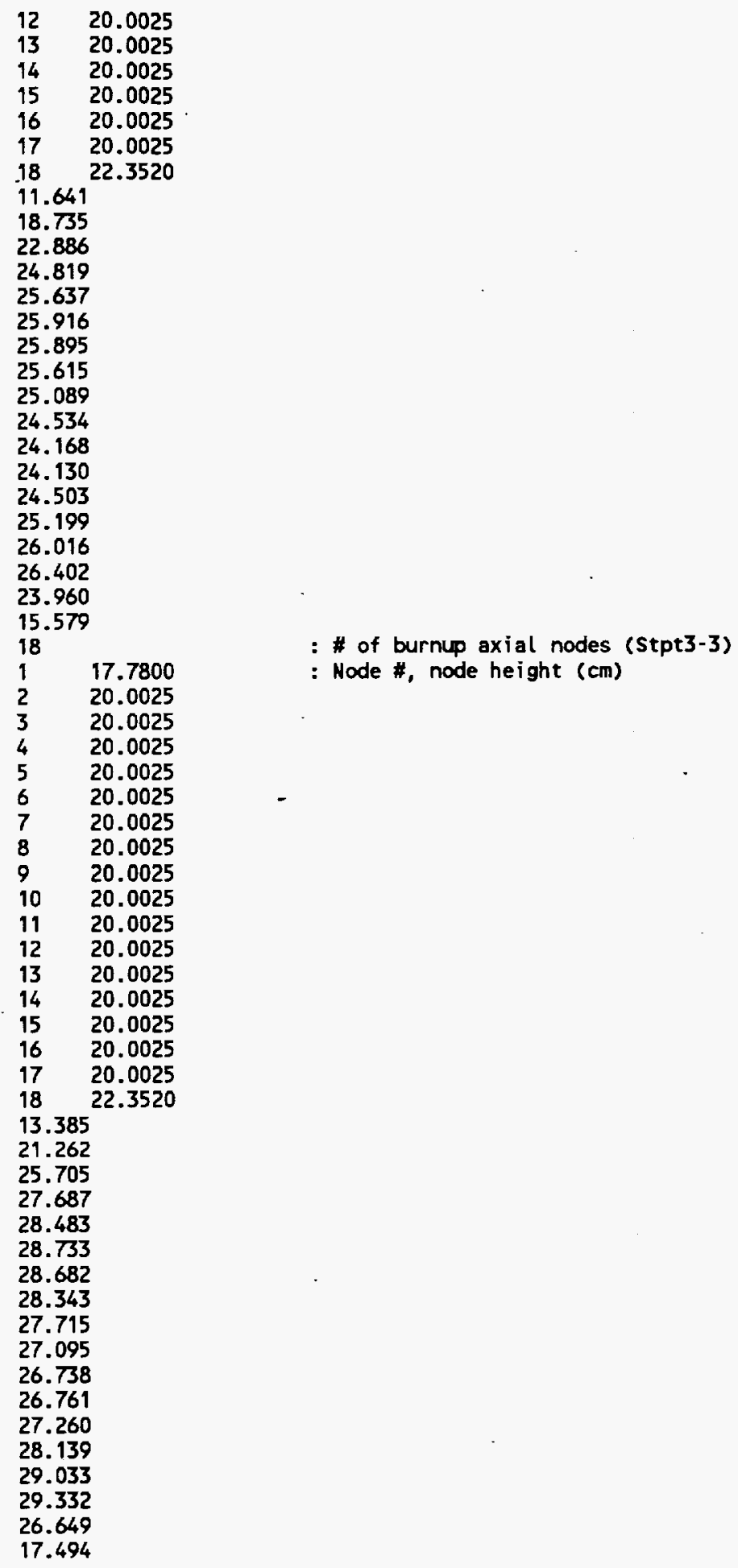




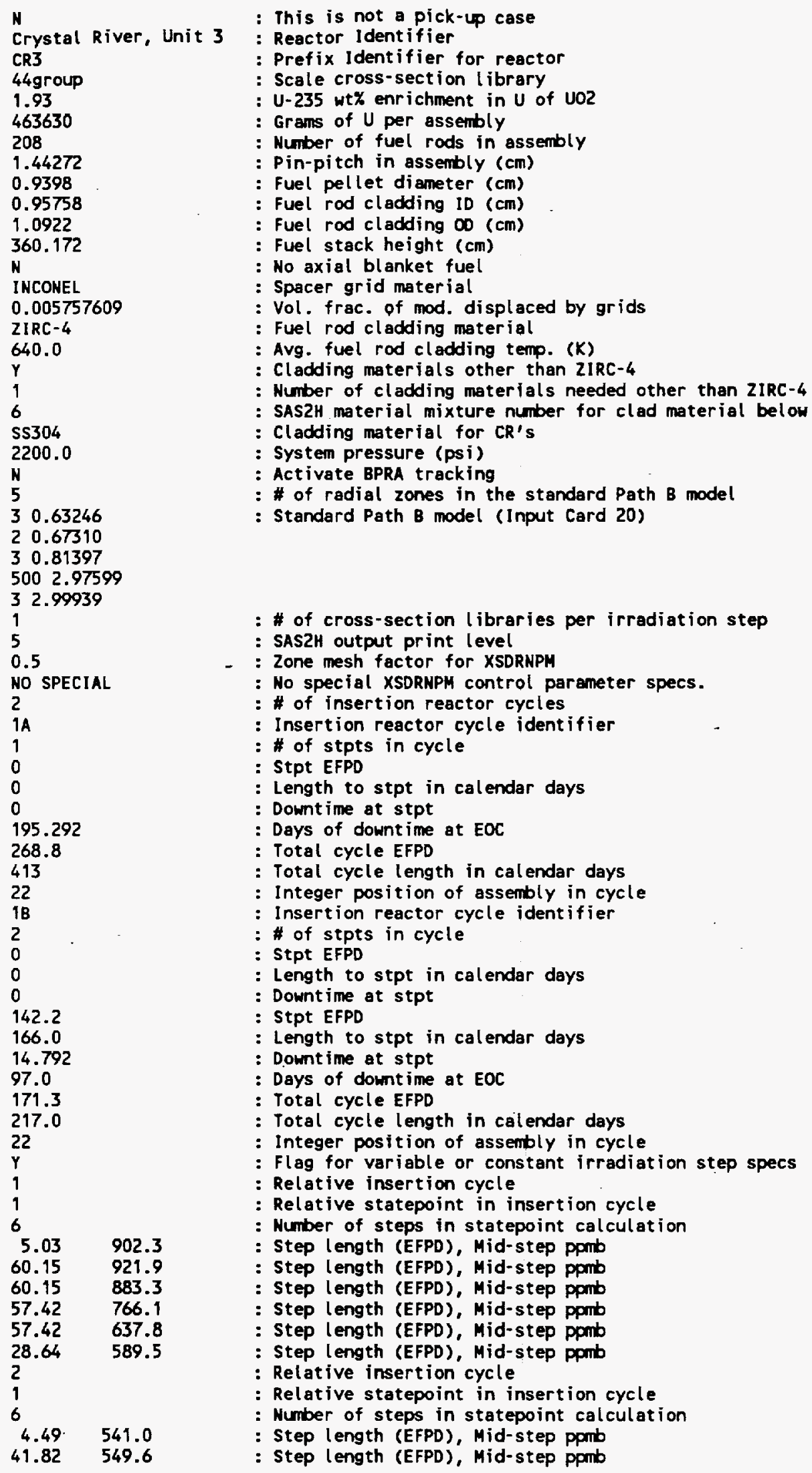

Crystal River, Unit 3 : Reactor Identifier

Prefix Identifier for reactor

: Scale cross-section library

: U-235 wt\% enrichment in U of UO2

: Grams of U per assembly

: Number of fuel rods in assembly

: Pin-pitch in assembly $(\mathrm{cm})$

: Fuel pellet diameter $(\mathrm{cm})$

: Fuel rod cladding 10 ( $\mathrm{cm}$ )

: Fuel rod cladding $\infty 0(\mathrm{~cm})$

: Fuel stack height $(\mathrm{cm})$

: No axial blanket fuel

: Spacer grid material

: Vol. frac. of mod. displaced by grids

: Fuel rod cladding material

: Avg. fuel rod cladding temp. $(K)$

: Cladding materials other than 2IRC-4

: Number of cladding materials needed other than 2IRC-4

: SAS2H material mixture number for clad material below

: Cladding material for CR's

: System pressure (psi)

: Activate BPRA tracking

: \# of radial zones in the standard Path $B$ model

: Standard Path B model (Input Card 20)

: \# of cross-section libraries per irradiation step

: SAS2H output print level

: Zone mesh factor for XSDRNPH

: No special XSDRNPM control parameter specs.

: \# of insertion reactor cycles

: Insertion reactor cycle identifier

: \# of stpts in cycle

: Stpt EFPD

: Length to stpt in calendar days

: Downt ime at stpt

: Days of downt ime at EOC

: Total cycle EFPD

: Total cycle length in calendar days

: Integer position of assembly in cycle

: Insertion reactor cycle identifier

: \# of stpts in cycle

: Stpt EFPD

: Length to stpt in calendar days

: Downtime at stpt

: StPt EFPD

: Length to stpt in calendar days

: Downtime at stpt

: Days of downtime at EOC

: Total cycle EFPD

: Total cycle length in calendar days

: Integer position of assembly in cycle

: Flag for variable or constant irradiation step specs

: Relative insertion cycle

: Relative statepoint in insertion cycle

: Number of steps in statepoint calculation

: Step length (EFPD), Mid-step pprib

: Step length (EFPD), Mid-step ppmb

: Step length (EFPD), Mid-step ppmb

: Step length (EFPD), Mid-step ponb

: Step length (EFPD), Mid-step ppmb

: Step length (EFPD), Mid-step pomb

: Relative insertion cycle

: Relative statepoint in insertion cycle

: Number of steps in statepoint calculation

: Step length (EFPD), Mid-step ppmb

: step length (EFPD), Mid-step ppmb 


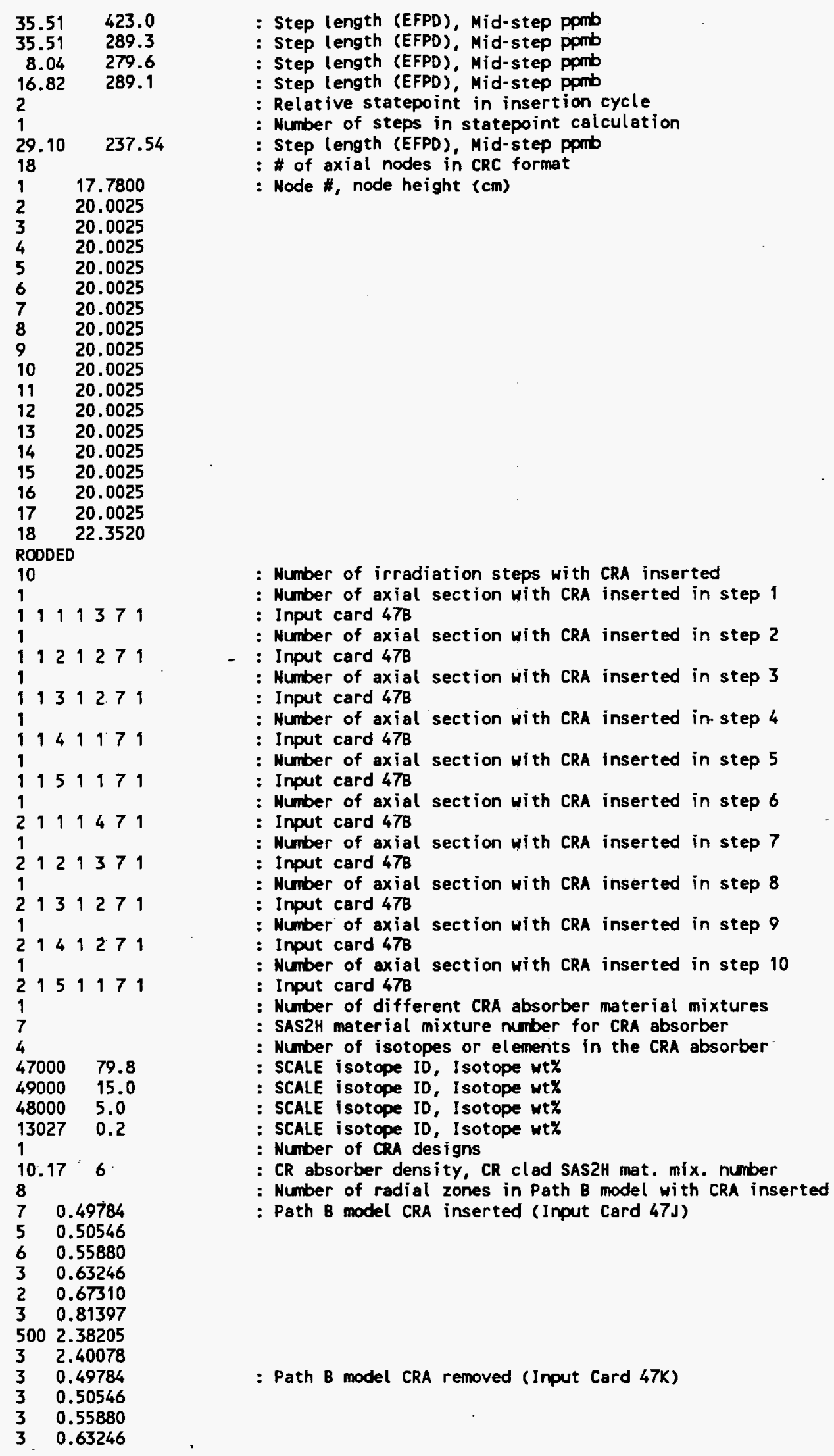




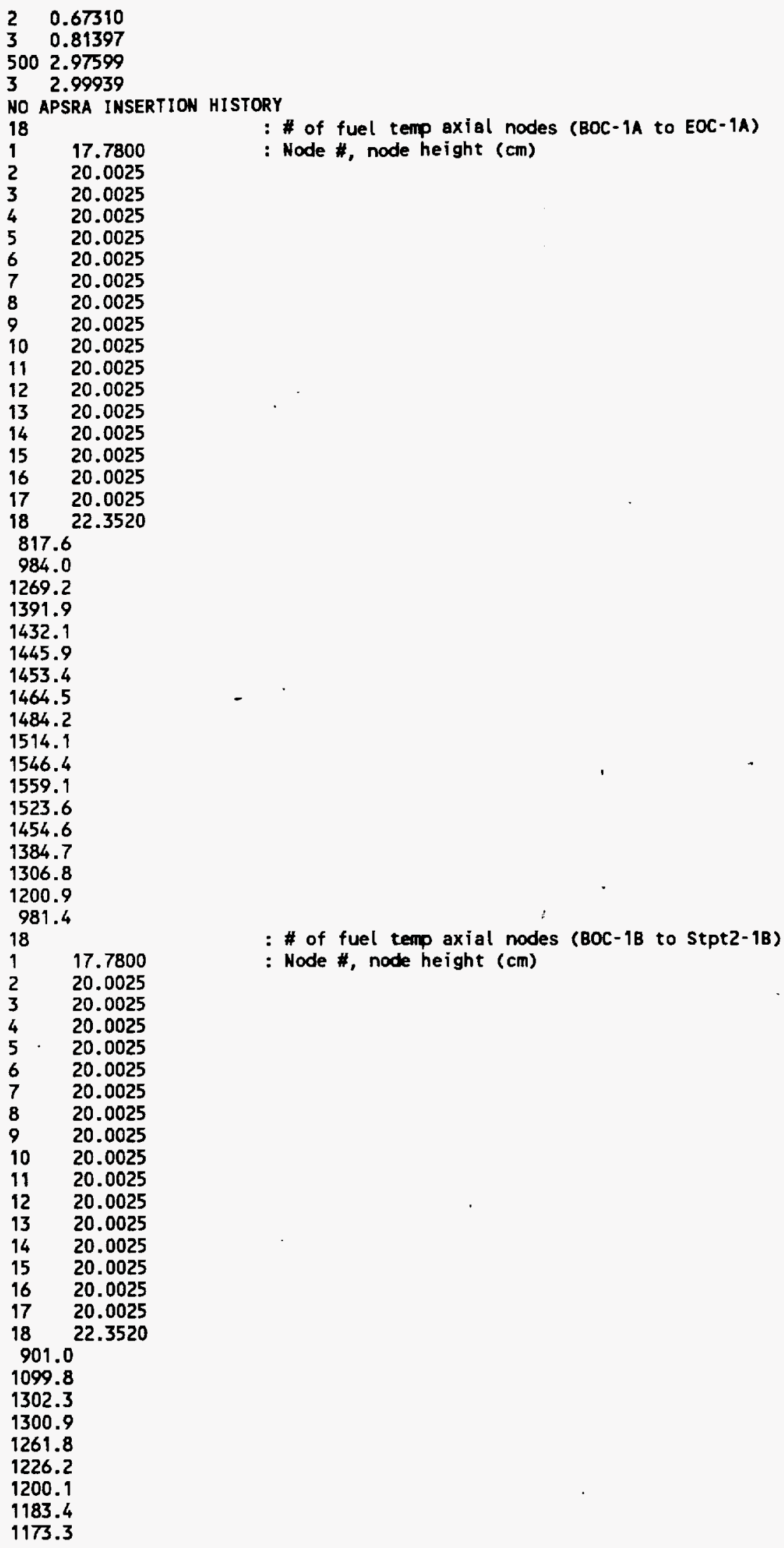




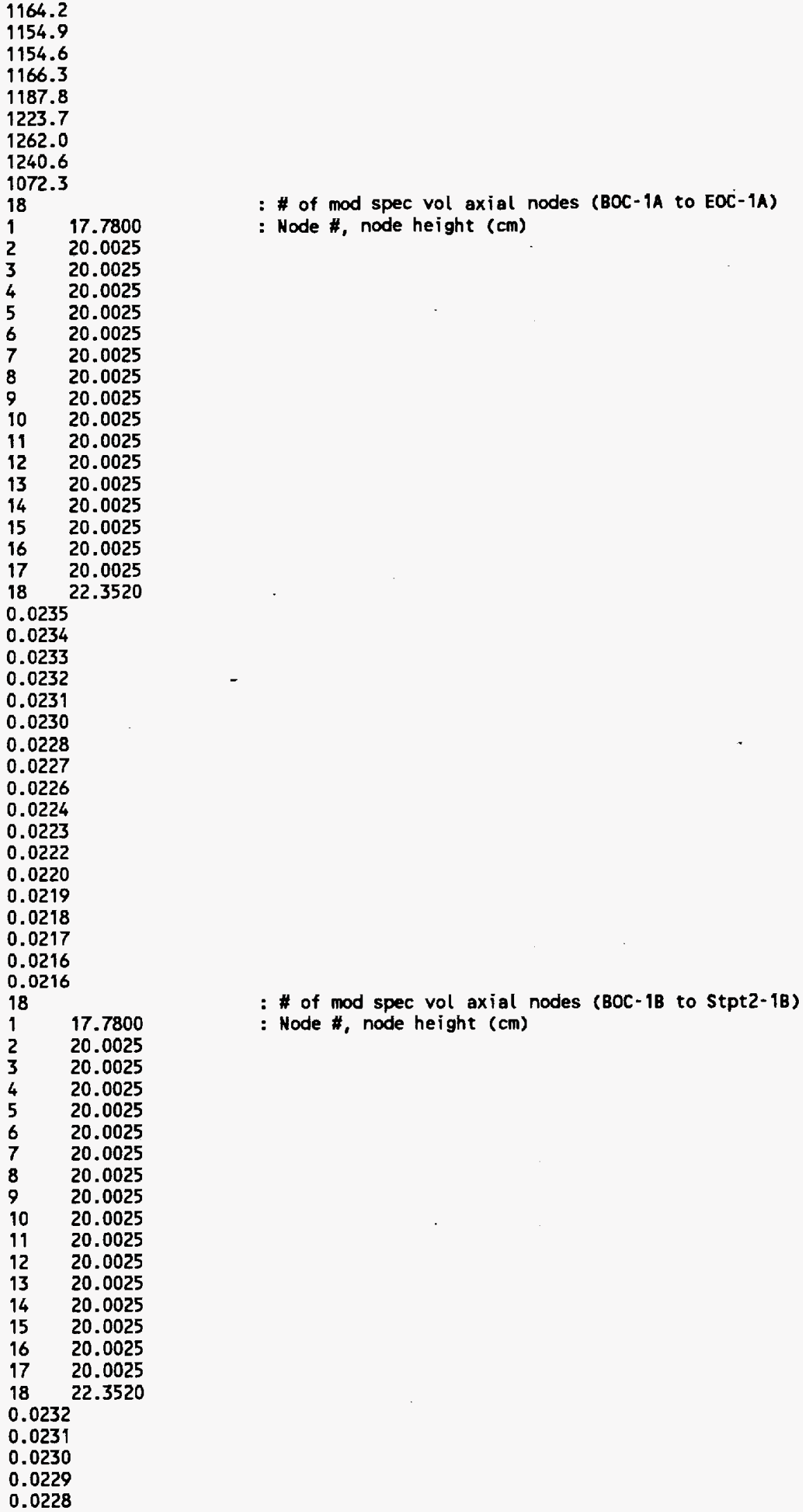


Apr 03 15:01 1997 File Name: A22i.dat BBA000000-01717-0200-00040 REV 00 ATTACHMENT XIII - Page 5

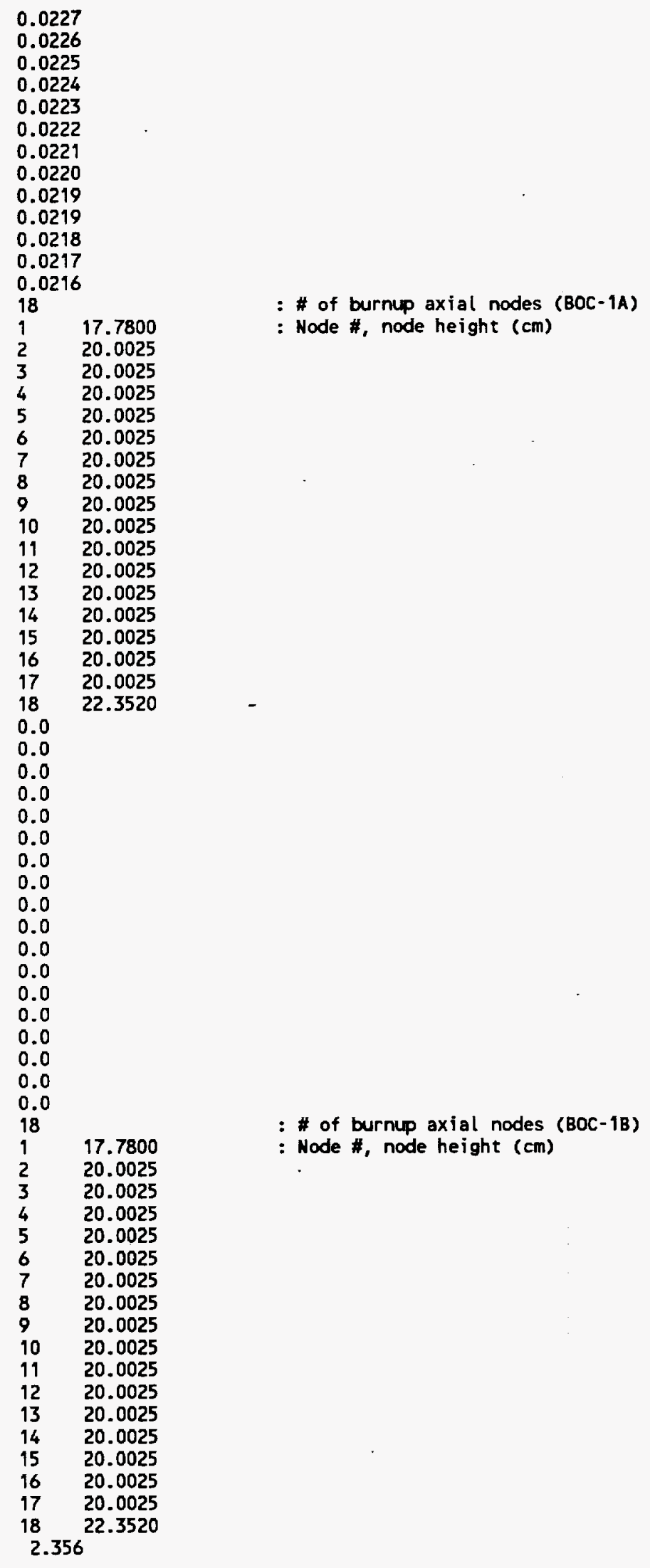


Apr 03 15:01 1997 File Name: A22i.dat BBA000000-01717-0200-00040 REV 00 ATTACHMENT XIII - Page 6

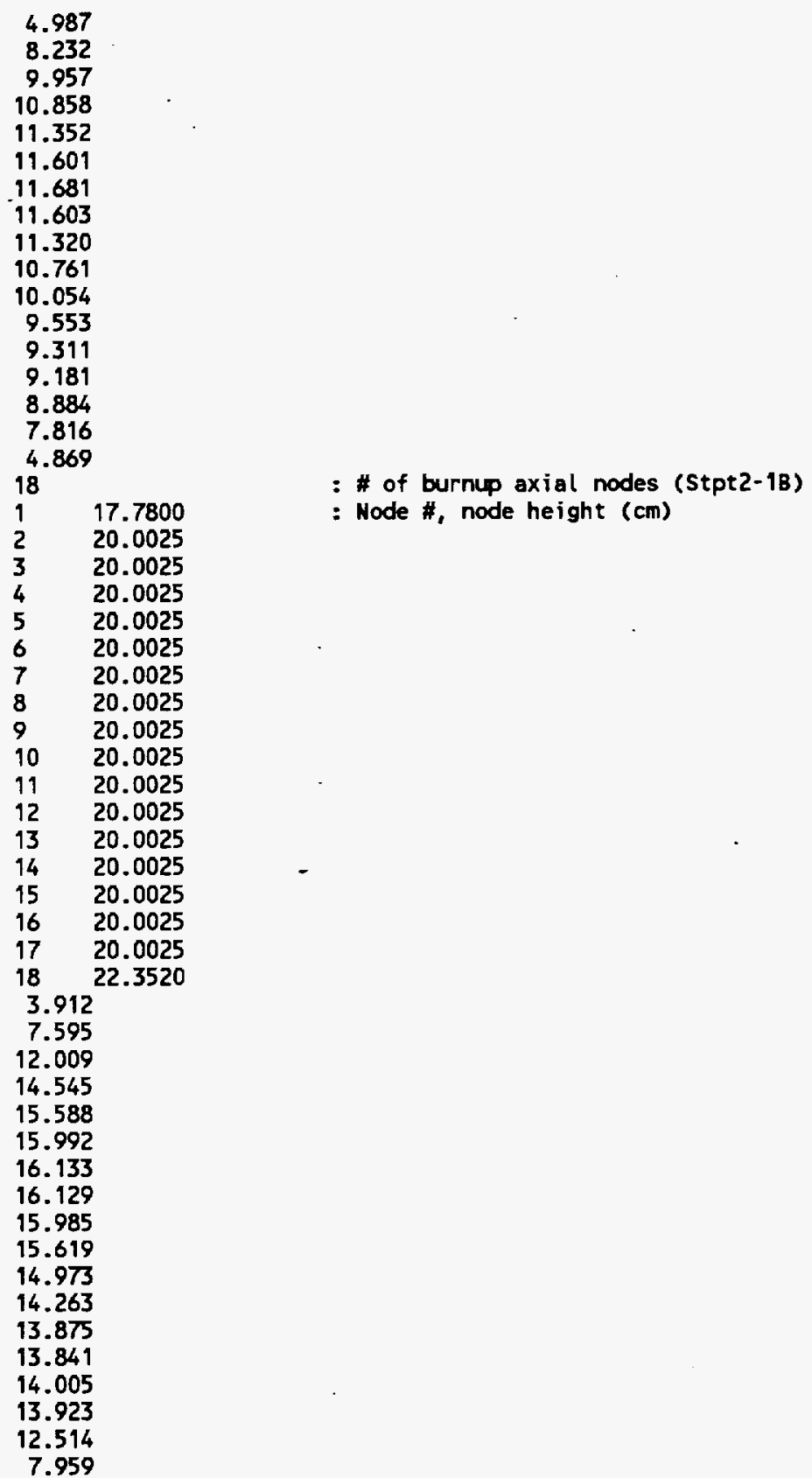




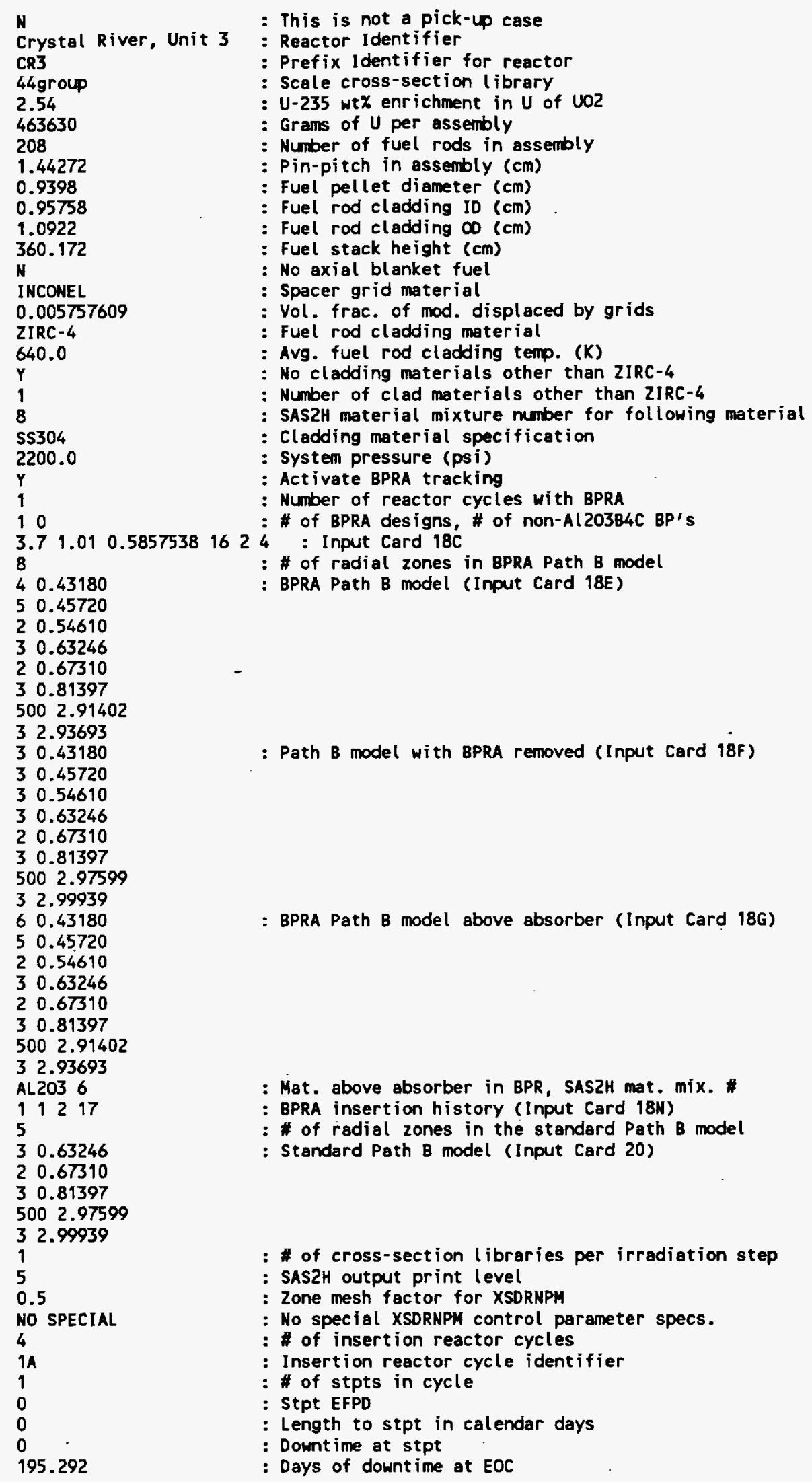




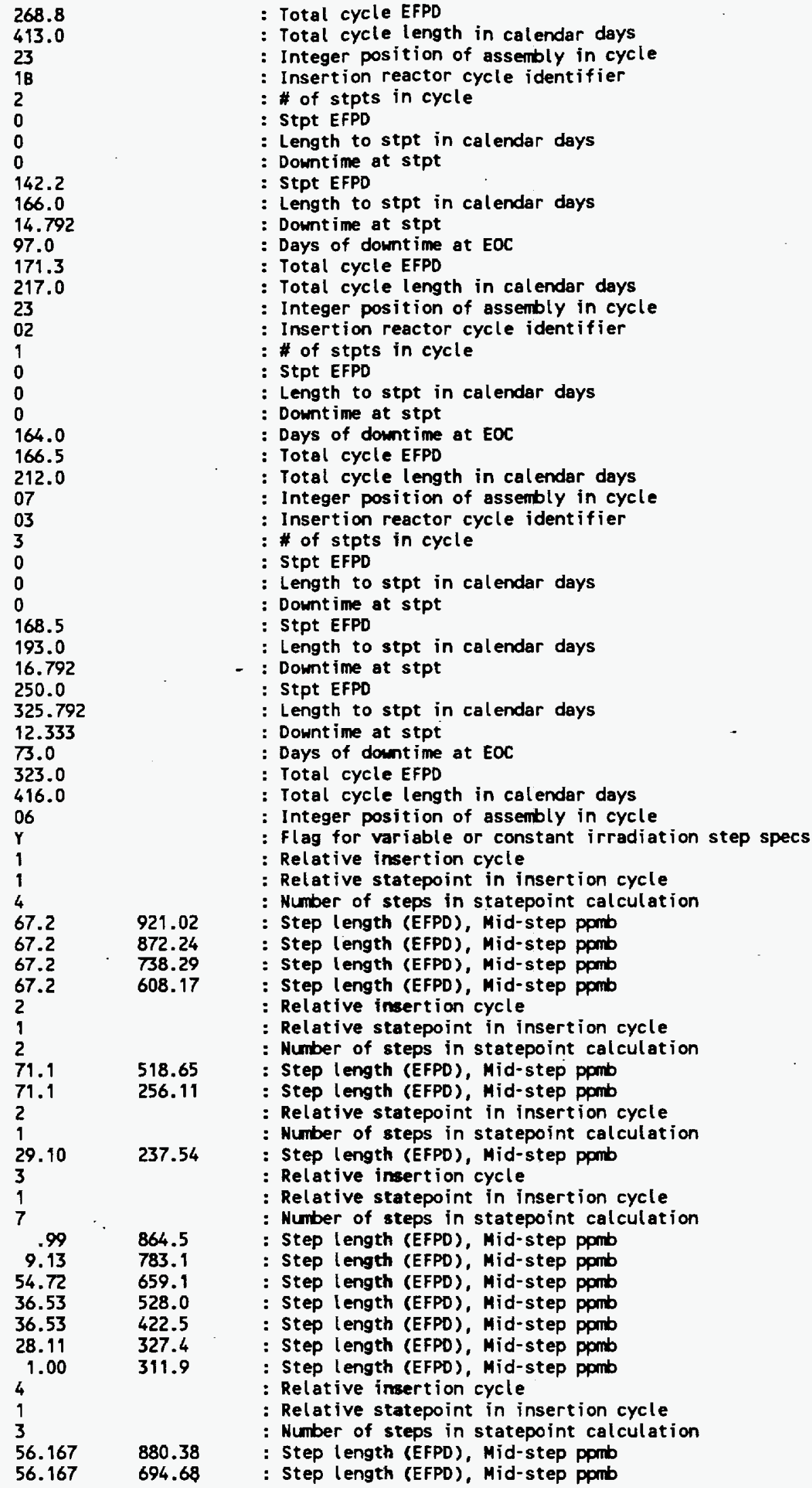




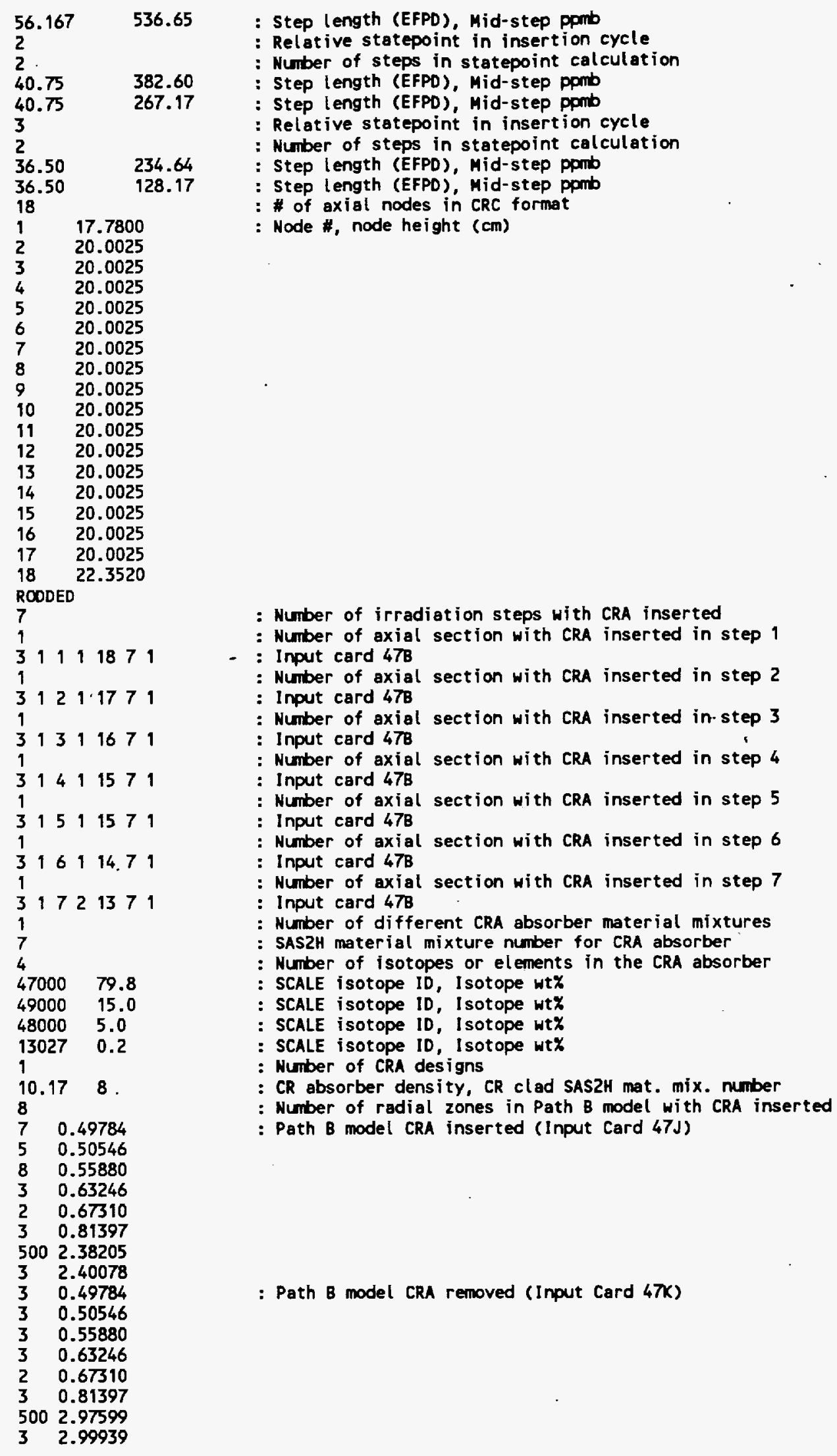


Apr 03 15:01 1997 File Name: A23i.dat BBA000000-01717-0200-00040 REV 00 ATTACHMENT XIV - Page 4

NO APSRA INSERTION HISTORY

18

$1 \quad 17.7800$

20.0025

20.0025

20.0025

20.0025

20.0025

20.0025

20.0025

20.0025

20.0025

20.0025

20.0025

20.0025

20.0025

20.0025

20.0025

20.0025

22.3520

938.7

1149.6

1304.2

1395.0

1436.6

1454.3

1464.9

1478.0

1498.6

1526.7

1557.5

1567.9

1520.3

1441.6

1372.0

1295.7

1192.2

1005.8

18

17.7800

20.0025

20.0025

20.0025

20.0025

20.0025

20.0025

20.0025

20.0025

20.0025

20.0025

20.0025

20.0025

20.0025

20.0025

20.0025

20.0025

22.3520

1052.6

1250.7

1331.4

1329.1

1292.3

1255.8

1229.3

1213.1

1204.0

1195.3

1183.6

1182.9

1195.7
: \# of fuel temp axial nodes (BOC-1A to EOC-1A)

: Node \#, node height $(\mathrm{cm})$

: \# of fuel temp axial nodes (BOC-1B to Stpt2-1B)

: Node \#, node height $(\mathrm{cm})$ 
Apr 03 15:01 1997 File Name: A23i.dat BBA000000-01717-0200-00040 REV 00 ATtACHMENT XIV - Page 5

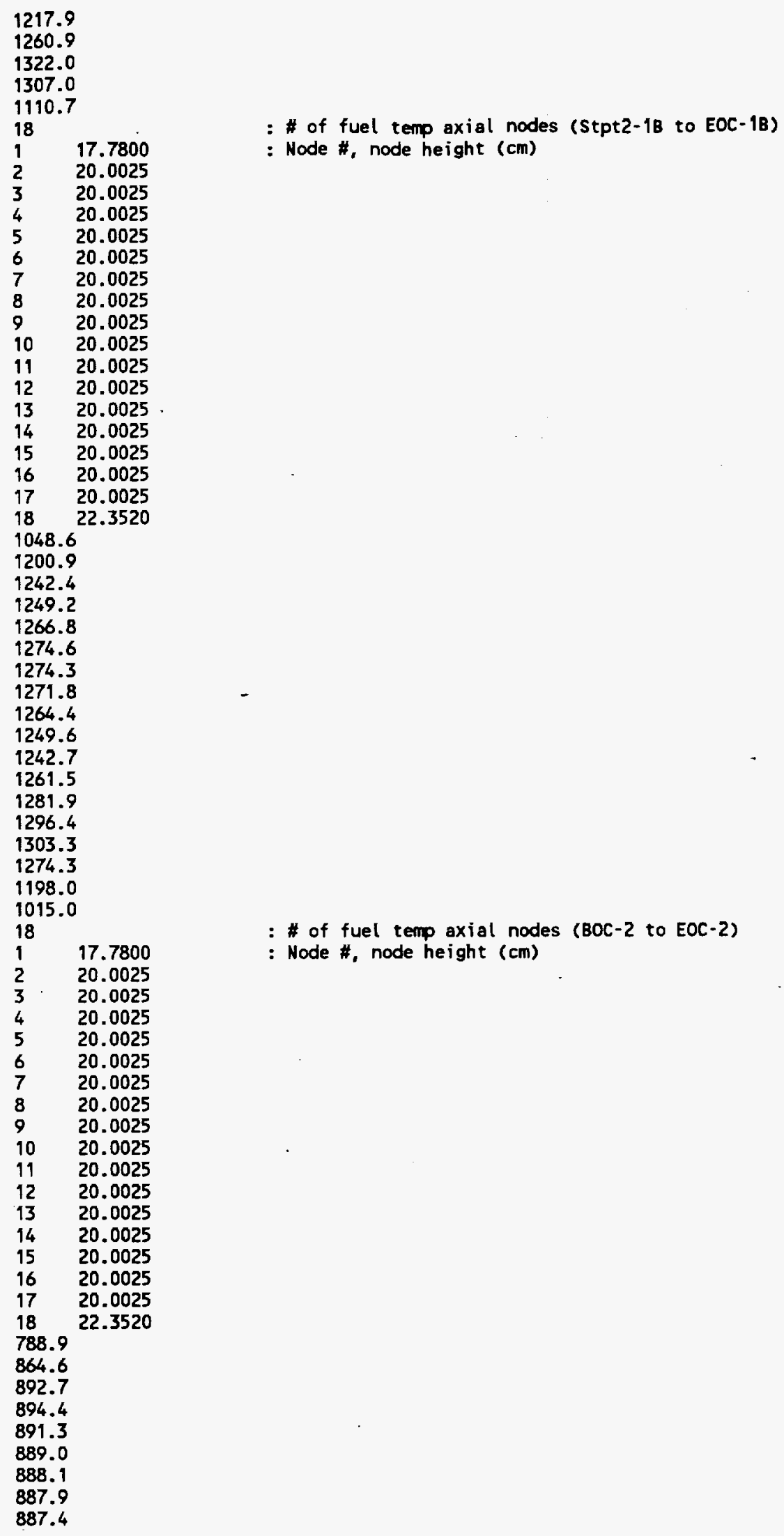




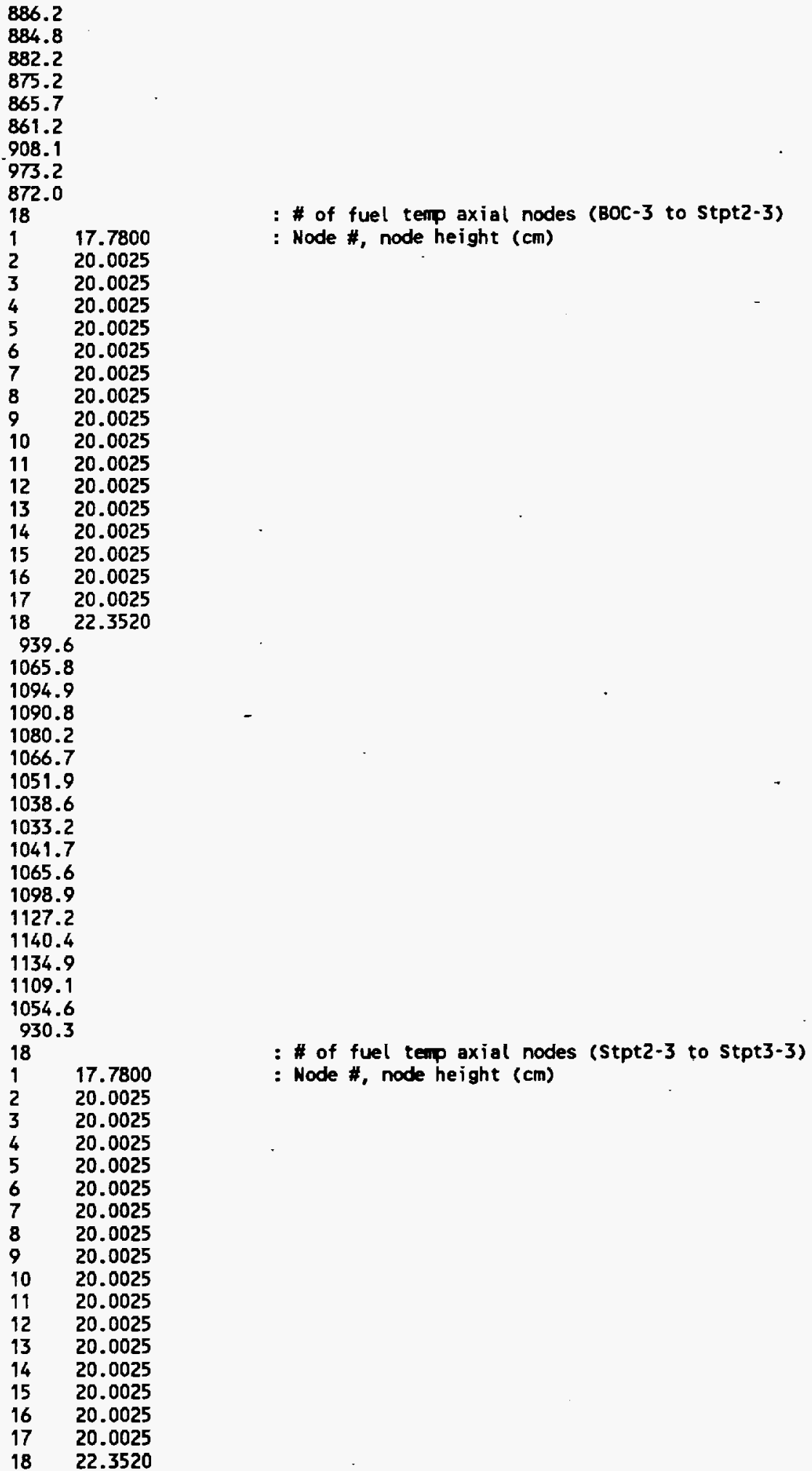


1032.4

1024.9

1019.4

1016.6

1020.0

1031.2

1046.7

1060.4

1074.7

1086.1

1072.9

1041.8

953.9

$1 \quad 17.7800$

: \# of mod spec vol axial nodes (BOC-1A to EOC-1A)

20.0025

20.0025

20.0025

20.0025

20.0025

20.0025

20.0025

20.0025

20.0025

20.0025

20.0025

20.0025

20.0025

20.0025

20.0025

20.0025

22.3520

0.0235

0.0234

0.0233

0.0232

0.0231

0.0230

0.0228

0.0227

0.0226

0.0224

0.0223

0.0222

0.0220

0.0219

0.0218

0.0217

0.0216

0.0216

18

17.7800

: Node \#, node height (cm)

20.0025

20.0025

20.0025

20.0025

20.0025

20.0025

20.0025

20.0025

20.0025

20.0025

20.0025

20.0025

20.0025

$8 \cdot 22.3520$

: \# of mod spec vol axial nodes (BOC-1B to stpt2-1B)

: Node \#, node height ( $\mathrm{cm}$ )

0.0233 
Apr 03 15:01 1997 File Name: A23i.dat BBA000000-01717-0200-00040 REV 00 ATTACHMENT XIV - Page 8

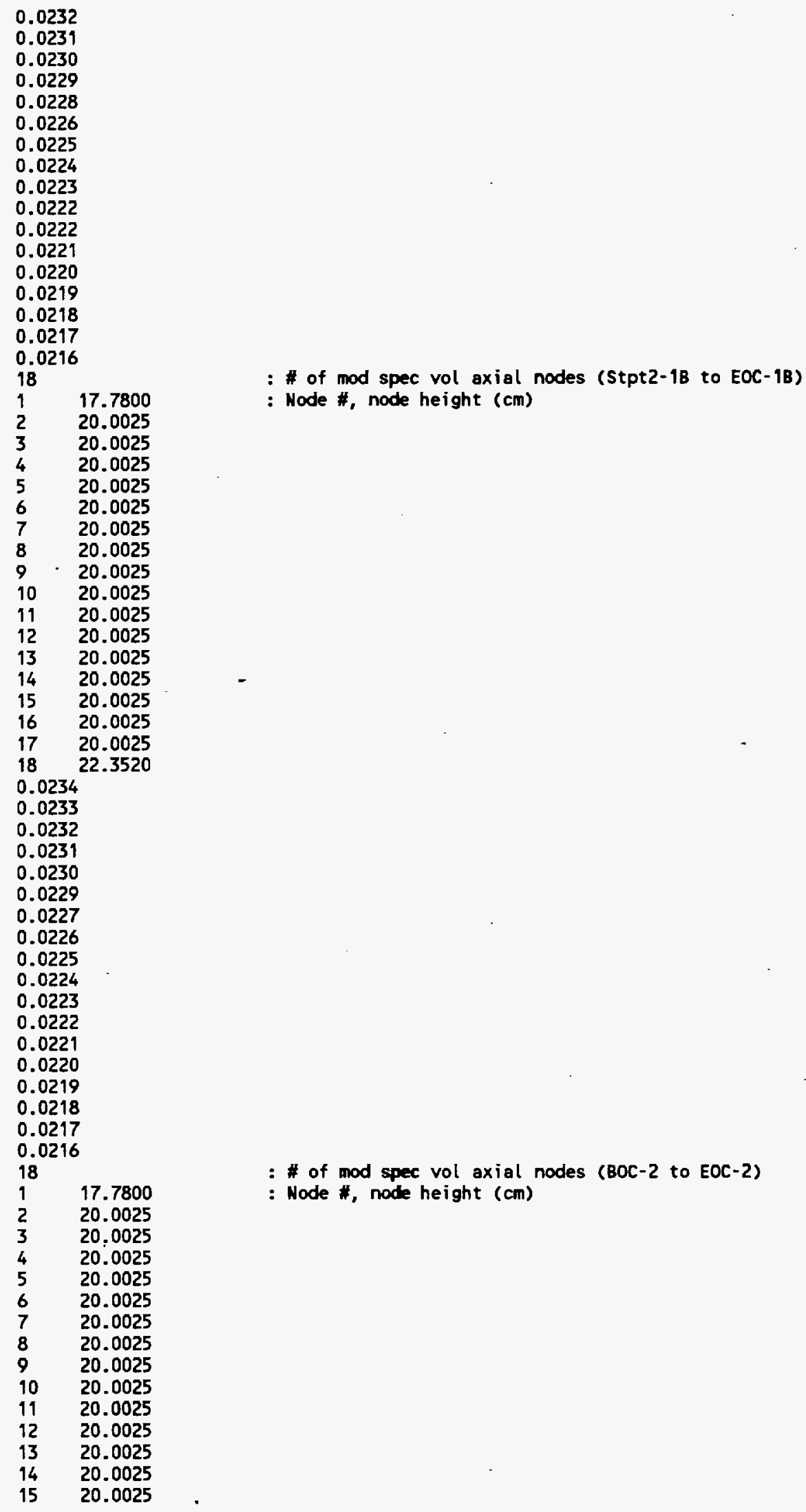


Apr 03 15:01 1997 File Name: A23i.dat BBA000000-01717-0200-00040 REV 00 ATTACHMENT XIV - Page 9

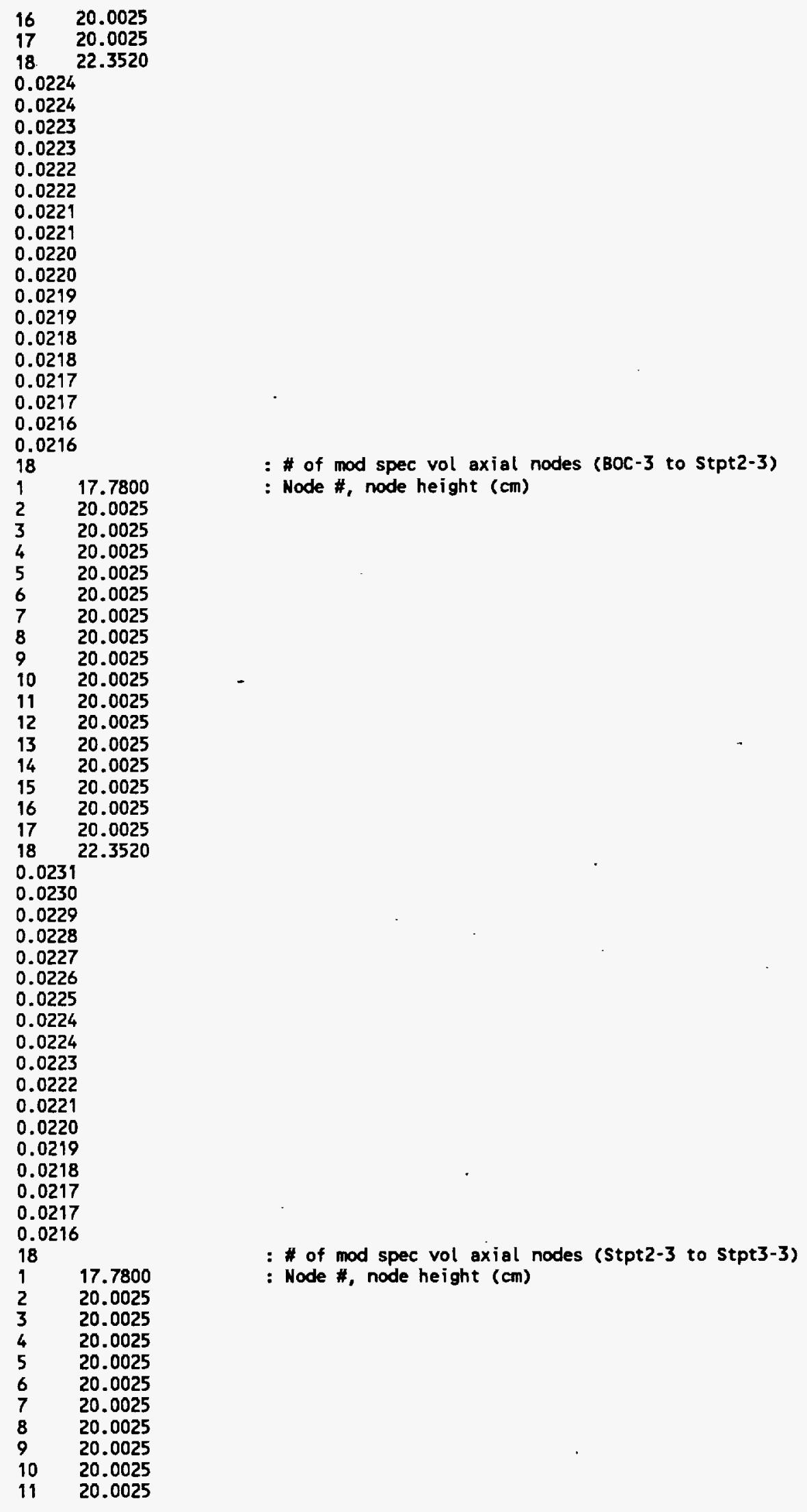


Apr 03 15:01 1997 File Name: A23i.dat BBA000000-01717-0200-00040 REV 00 ATTACHMENT XIV - Page 10

20.0025

20.0025

20.0025

20.0025

20.0025

20.0025

22.3520

0.0231

0.0230

0.0229

0.0228

0.0227

0.0226

0.0225

0.0224

0.0224

0.0223

0.0222

0.0221

0.0220

0.0219

0.0218

0.0217

0.0217

0.0216

18

17.7800

20.0025

20.0025

20.0025

20.0025

20.0025

20.0025

20.0025

20.0025

20.0025

20.0025

20.0025

20.0025

20.0025

20.0025

20.0025

20.0025

22.3520

: Node \#, node height $(\mathrm{cm})$

0.0

0.0

0.0

0.0

0.0

0.0

0.0

0.0

0.0

0.0

0.0

0.0

0.0

0.0

0.0

0.0

0.0

18

17.7800

: \# of burnup axial nodes (BOC-1B)

20.0025

: Node \#, node height $(\mathrm{cm})$ 


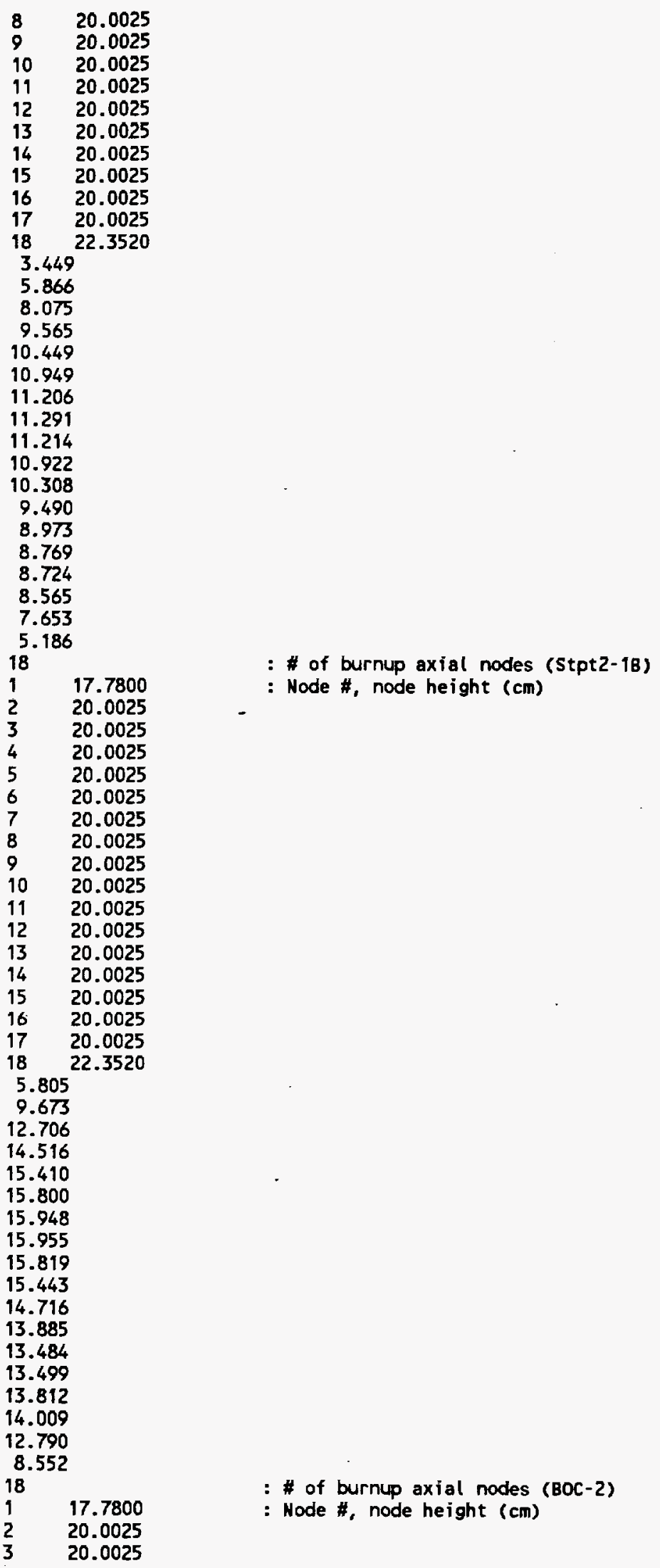


Apr 03 15:01 1997 File Name: A23i.dat BBA000000-01717-0200-00040 REV 00 ATtACHMENT XIV - Page 12

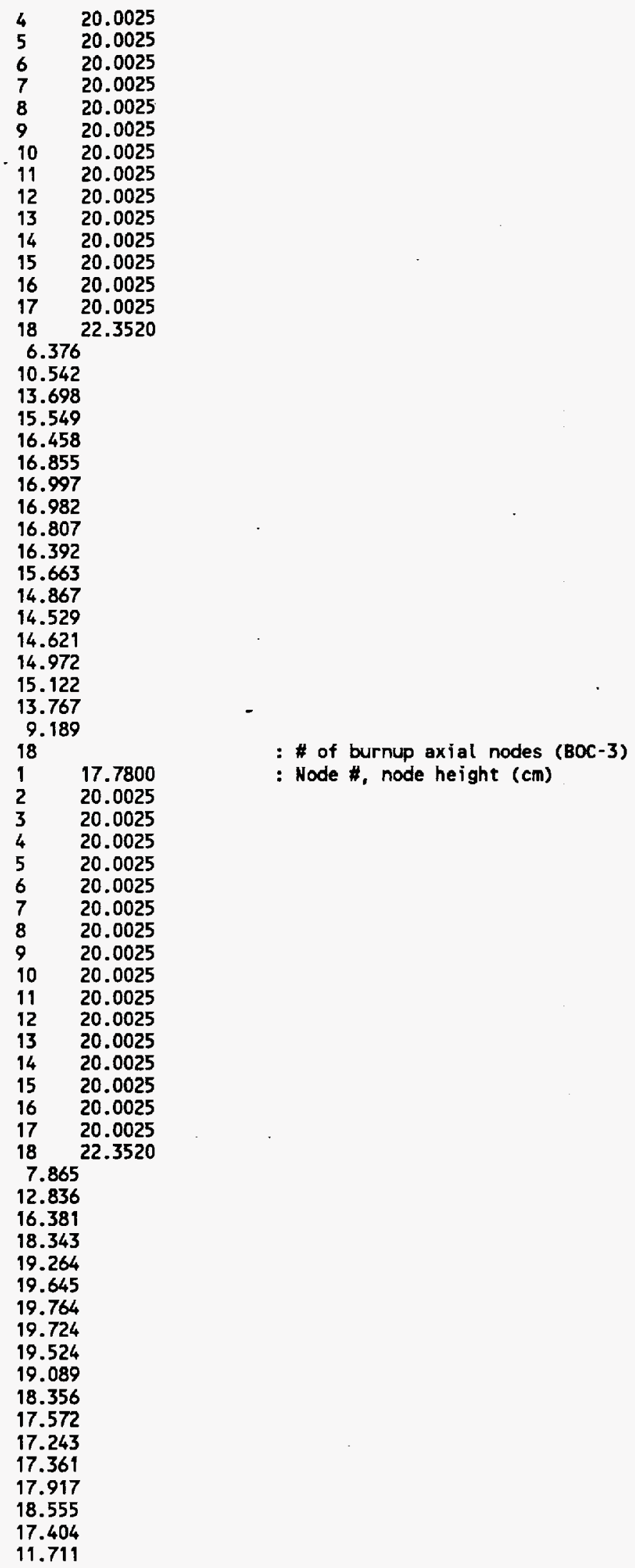


Apr 03 15:01 1997 File Name: A23i.dat BBA000000-01717-0200-00040 REV 00 ATtACHMENT XIV - Page 13

18

17.7800

20.0025

20.0025

20.0025

20.0025

20.0025

20.0025

20.0025

20.0025

20.0025

20.0025

20.0025

20.0025

20.0025

20.0025

20.0025

20.0025

22.3520

10.524

16.971

21.207

23.400

24.380

24.747

24.805

24.668

24.366

23.889

23.219

22.586

22.437

22.707

23.302

23.728

21.941

14.702

18

$1 \quad 17.7800$

20.0025

20.0025

20.0025

20.0025

20.0025

20.0025

20.0025

20.0025

20.0025

20.0025

20.0025

20.0025

20.0025

20.0025

20.0025

20.0025

$18 \quad 22.3520$

11.962

19.074

23.549

25.776

26.735

27.077

27.114

26.957

26.639

26.167

25.535

24.973

24.914

25.305
: \# of burnup axial nodes (Stpt2-3)

: Hode \#, node height (cm)

: \# of burnup axial nodes (Stpt3-3)

: Node \#, node height $(\mathrm{cm})$ 
Apr 03 15:01 1997 File Name: A23i.dat BBA000000-01717-0200-00040 REV 00 ATTACHMENT XIV - Pege 14

26.011

26.384

24.346

16.375 


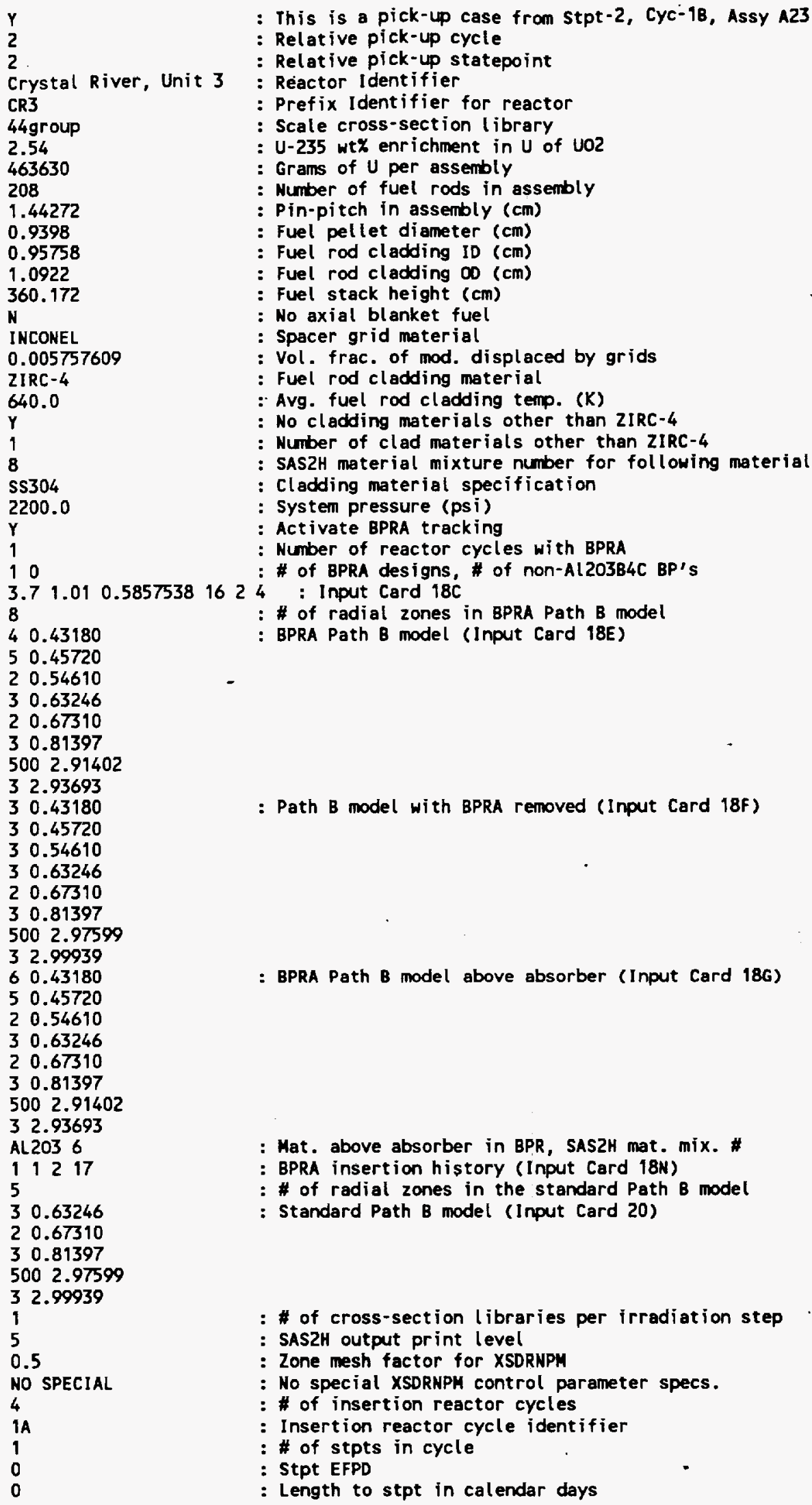


0

195.292

268.8

413.0

23

18

2

0

142.2

166.0

14.792

97.0

171.3

217.0

23

02

1

0

164.0

166.5

212.0

16

.03

3

168.5

193.0

16.792

250.0

325.792

12.333

73.0

323.0

416.0

22

Y

1

4

67.2

67.2

67.2

67.2

2

1

$71.1 \quad 518.65$

$71.1 \quad 256.11$

2

29.10

3

7

1.06

8.96

51.85

37.40

37.40

29.34

1.00

4

1
: Downt ime at stpt

: Days of downtime at EOC

: Total cycle EFPD

: Total cycle length in calendar days

: Integer position of assembly in cycle

: Insertion reactor cycle identifier

: \# of stpts in cycle

: Stpt EFPD

: Length to stpt in calendar days

: Downt ime at stpt

: Stpt EFPD

: Length to stpt in calendar days

: Downtime at stpt

: Days of downtime at EOC

: Total cycle EFPO

: Total cycle length in calendar days

: Integer position of assembly in cycle

: Insertion reactor cycle identifier

: \# of stpts in cycle

: stpt EFPD

: Length to stpt in calendar days

: Downtime at stpt

: Days of downtime at EOC

: Total cycle EFPD

- Total cycle length in calendar days

: Integer position of assembly in cycle

- Insertion reactor cycle identifier

\# of stpts in cycle

: Stpt EFPD

: Length to stpt in calendar days

Downtime at stpt

Stpt EFPD

: Length to stpt in calendar days

Downt ime at stpt

: Stpt EFPD

: Length to stpt in calendar days

: Downtime at stpt

: Days of downtime at EOC

: Total cycle EFPD

: Total cycle length in calendar days

: Integer position of assembly in cycle

: Flag for variable or constant irradiation step specs

: Relative insertion cycle

: Relative statepoint in insertion cycle

: Number of steps in statepoint calculation

: step length (EFPD), Mid-step ppmb

: Step length (EFPD), Mid-step ppmb

: Step length (EFPD), Mid-step ppmb

: Step length (EFPD), Mid-step ppmb

: Relative insertion cycle

: Relative statepoint in insertion cycle

- Number of steps in statepoint calculation

: Step length (EFPD), Mid-step ppmb

: Step length (EFPD), Mid-step ppmb

: Relative statepoint in insertion cycle

: Number of steps in statepoint calculation

: Step length (EFPD), Mid-step ppmb

: Relative insertion cycle

: Relative statepoint in insertion cycle

: Number of steps in statepoint calculation

: Step length (EFPD), Mid-step ppmb

: Step length (EFPD), Mid-step ppmb

: Step length (EFPD), Mid-step ppmb

: Step length (EFPD), Mid-step ppmb

: Step length (EFPD), Mid-step ppmb

: Step (ength (EFPD), Mid-step ppmb

: Step (ength (EFPD), Mid-step ppmb

: Relative insertion cycle

: Relative statepoint in insertion cycle

: Number of steps in statepoint calculation 


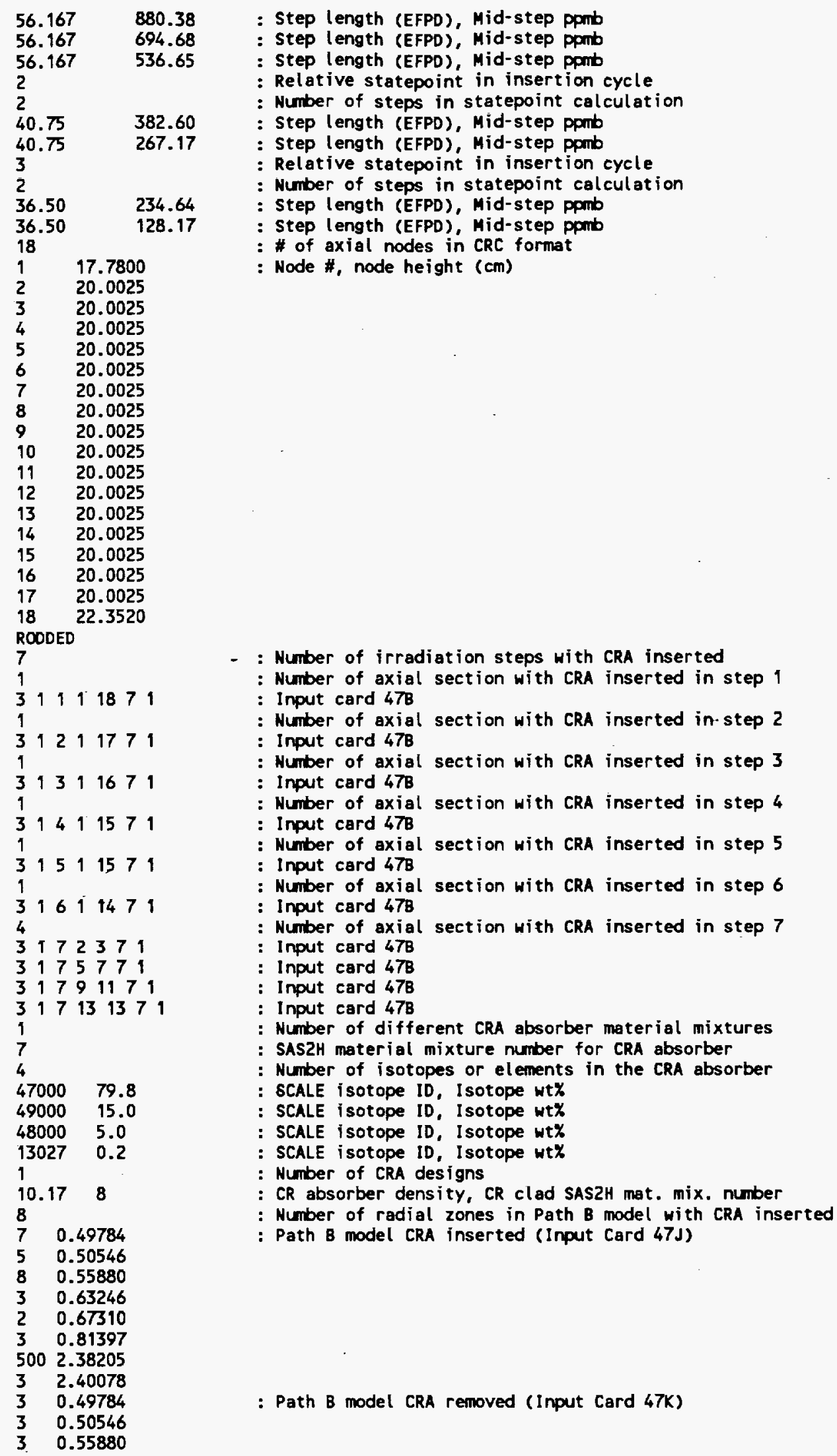




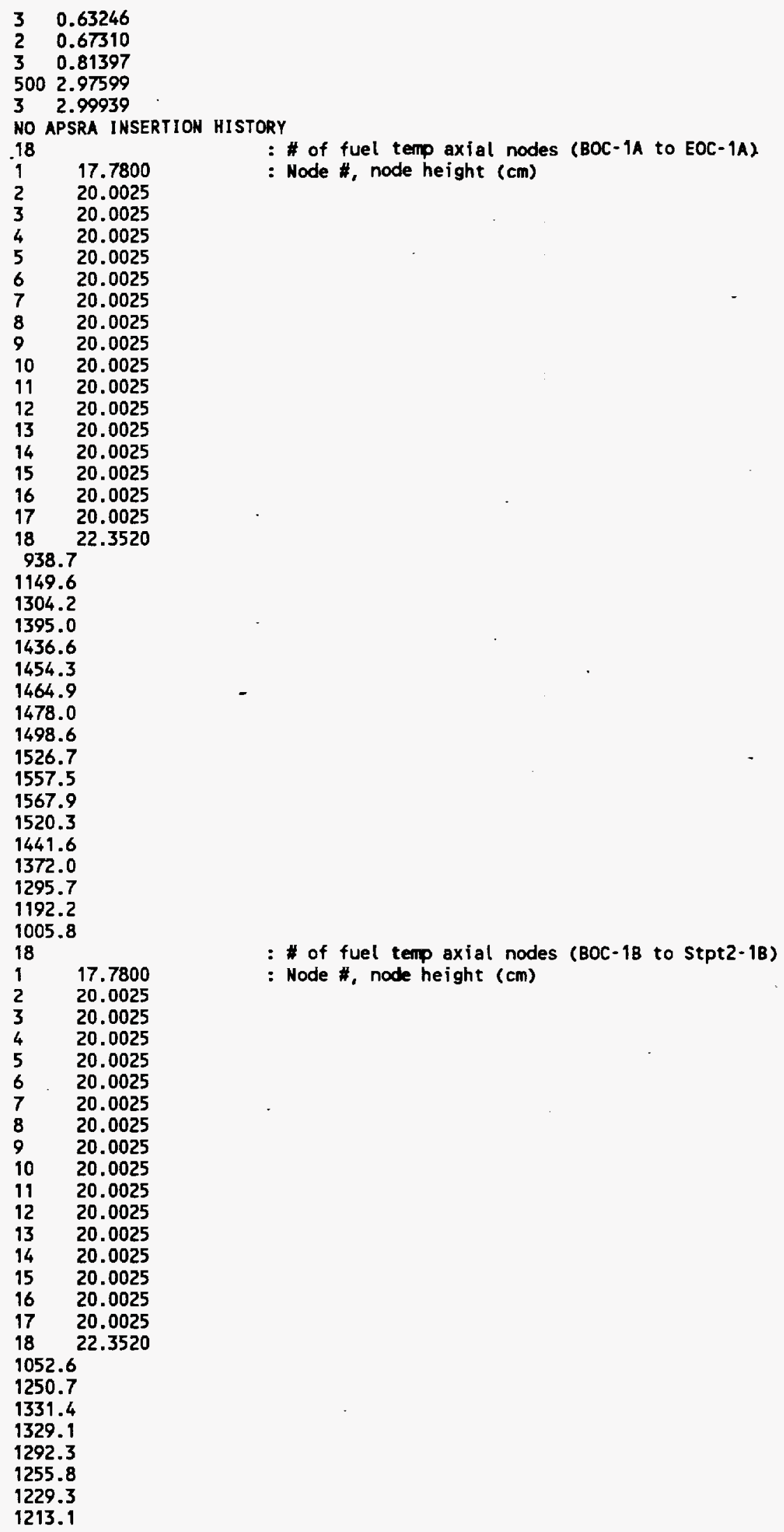




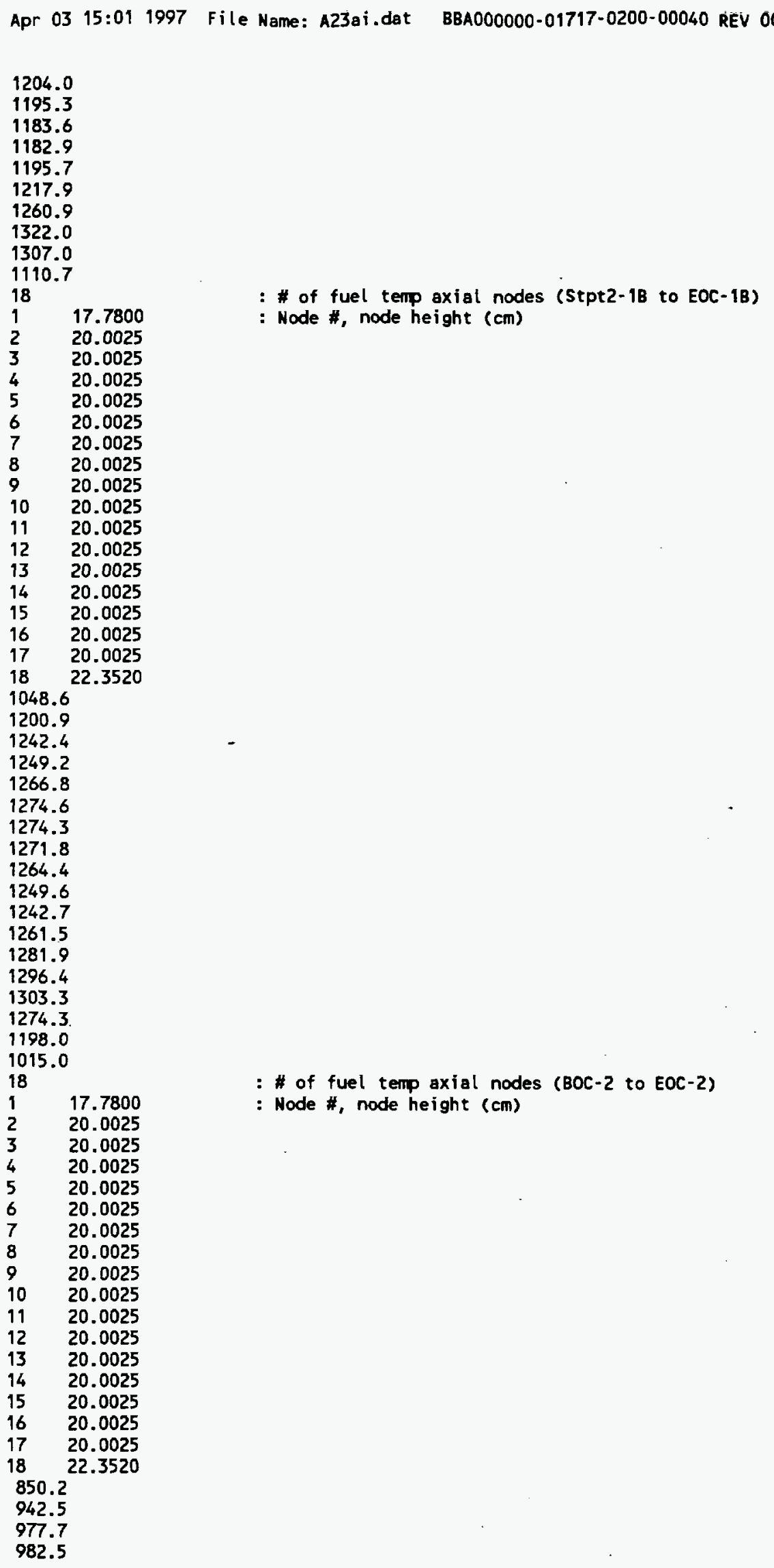


Apr 03 15:01 1997 File Name: A23ai.dat BBA000000-01717-0200-00040 REV 00 ATTACHMENT XV - Page 6

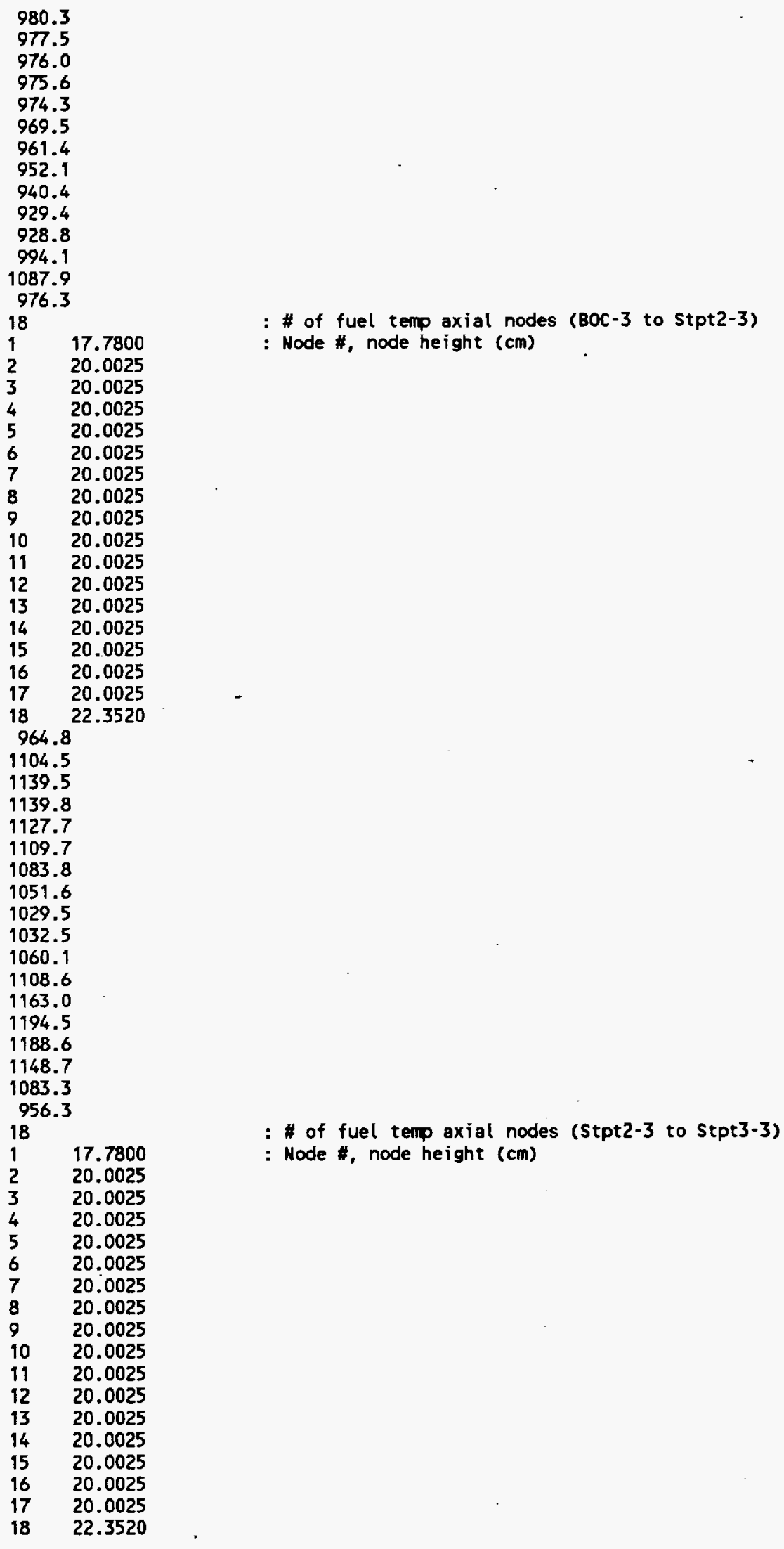




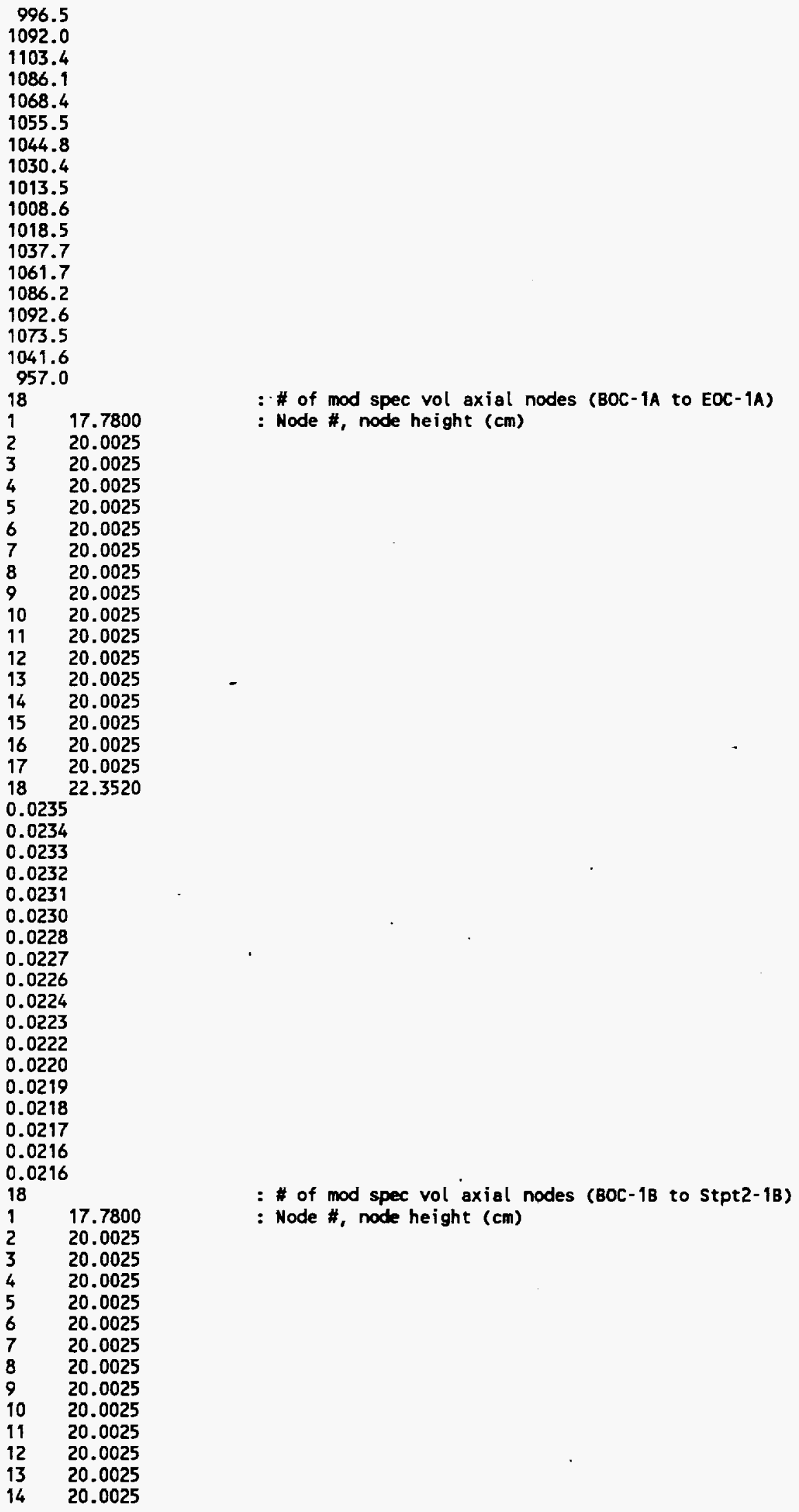




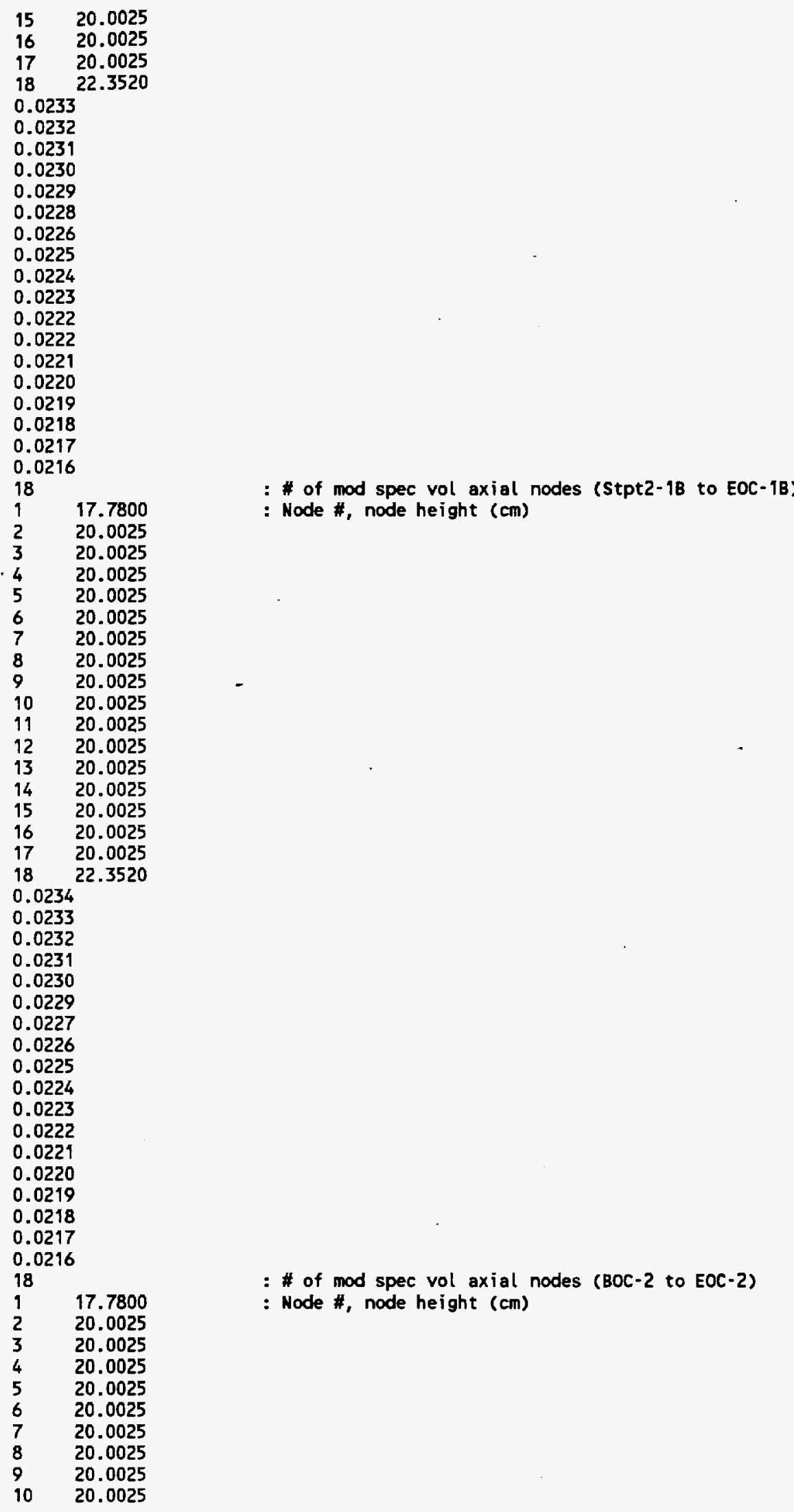




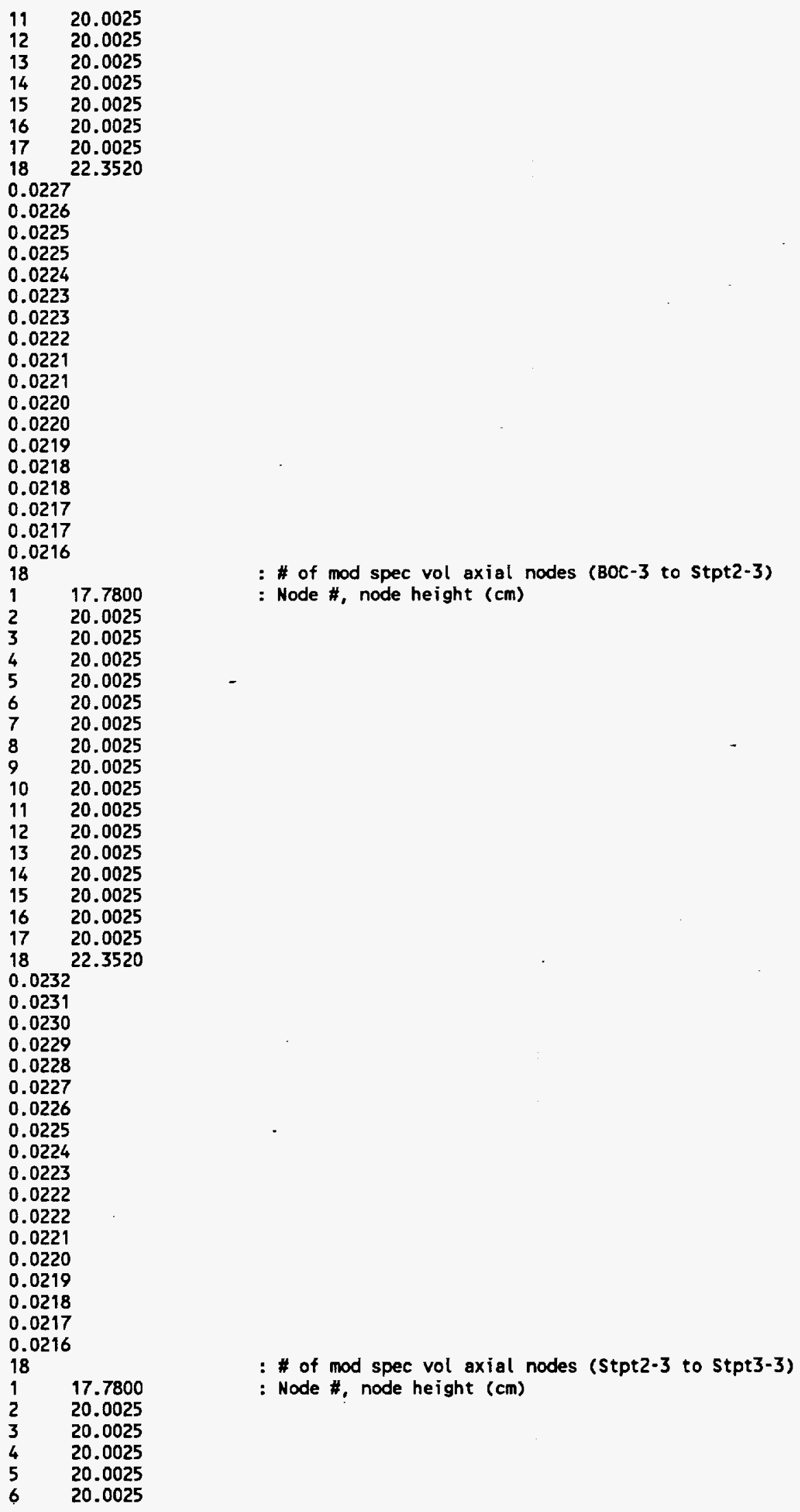




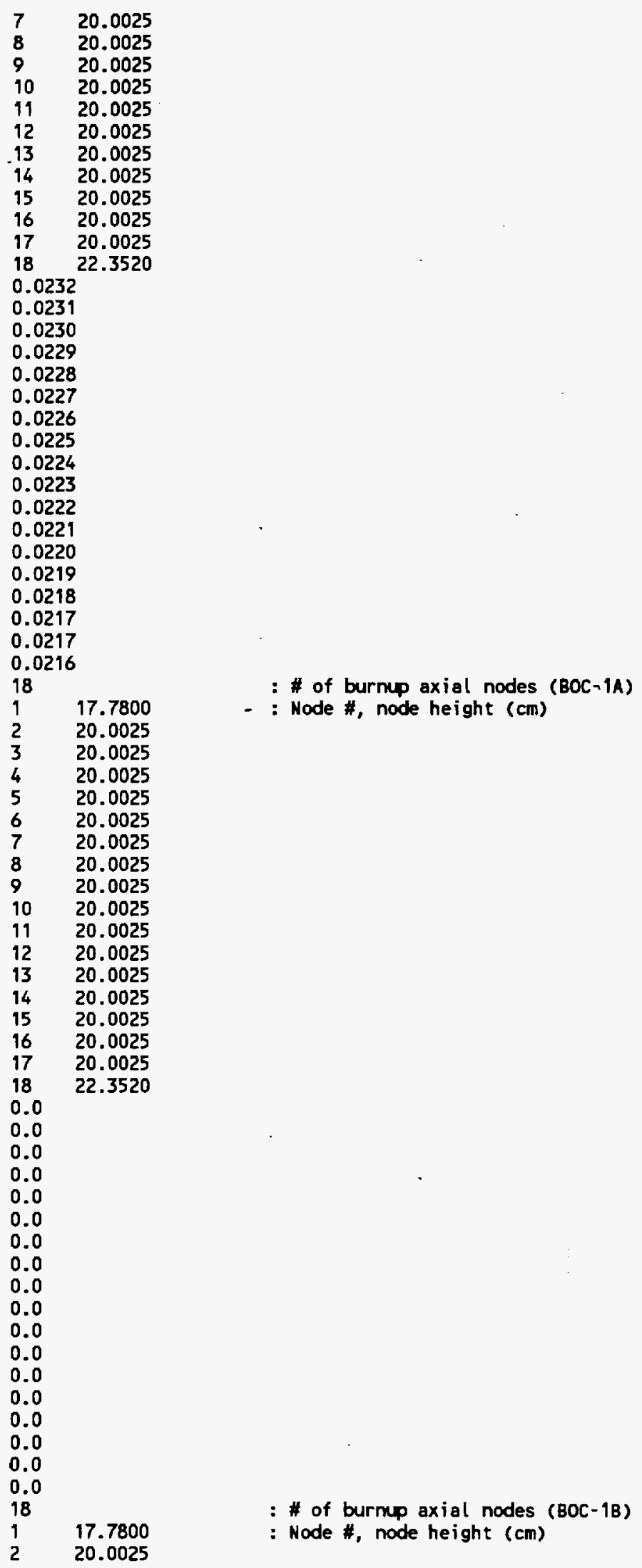


Apr 03 15:01 1997 file Name: A23ai.dat BBA000000-01717-0200-00040 REV 00 ATTACHMENT XV - Page 11

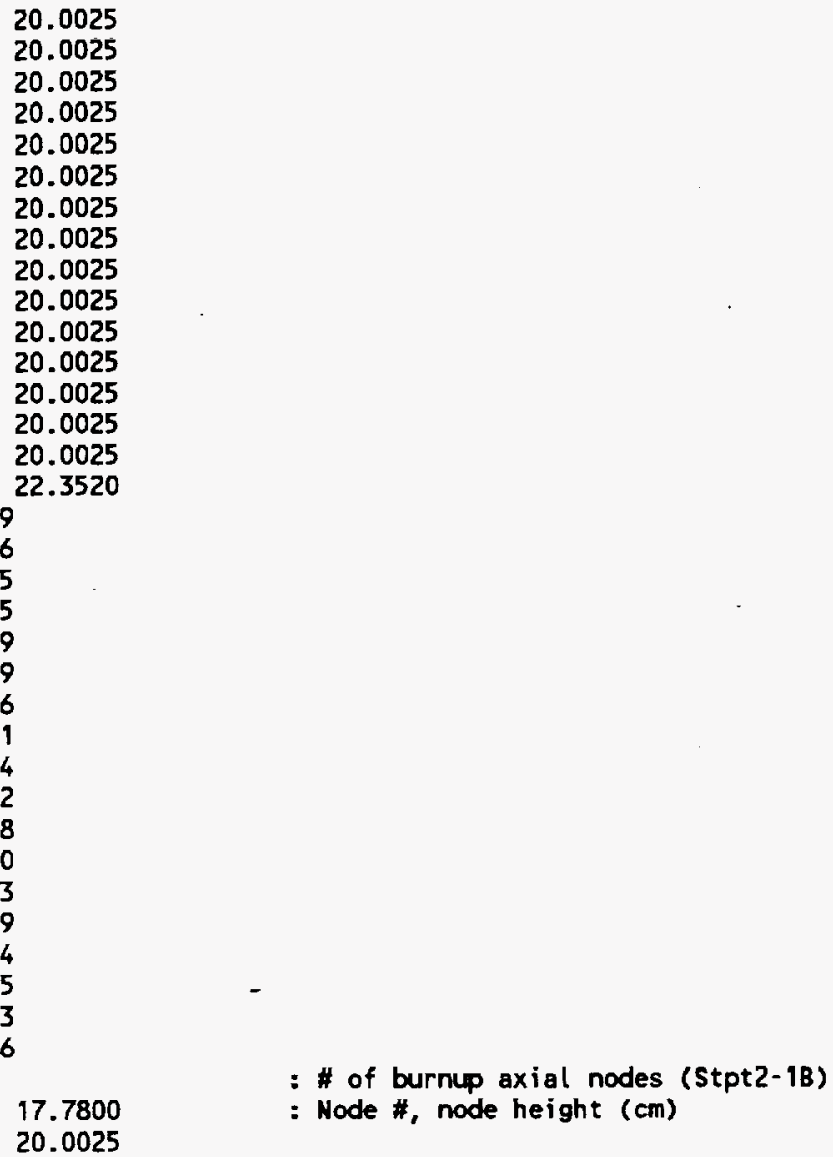

15.410

15.800

15.948

15.955

15.819

15.443

14.716

13.885

13.484

13.499

13.812

14.009

12.790 


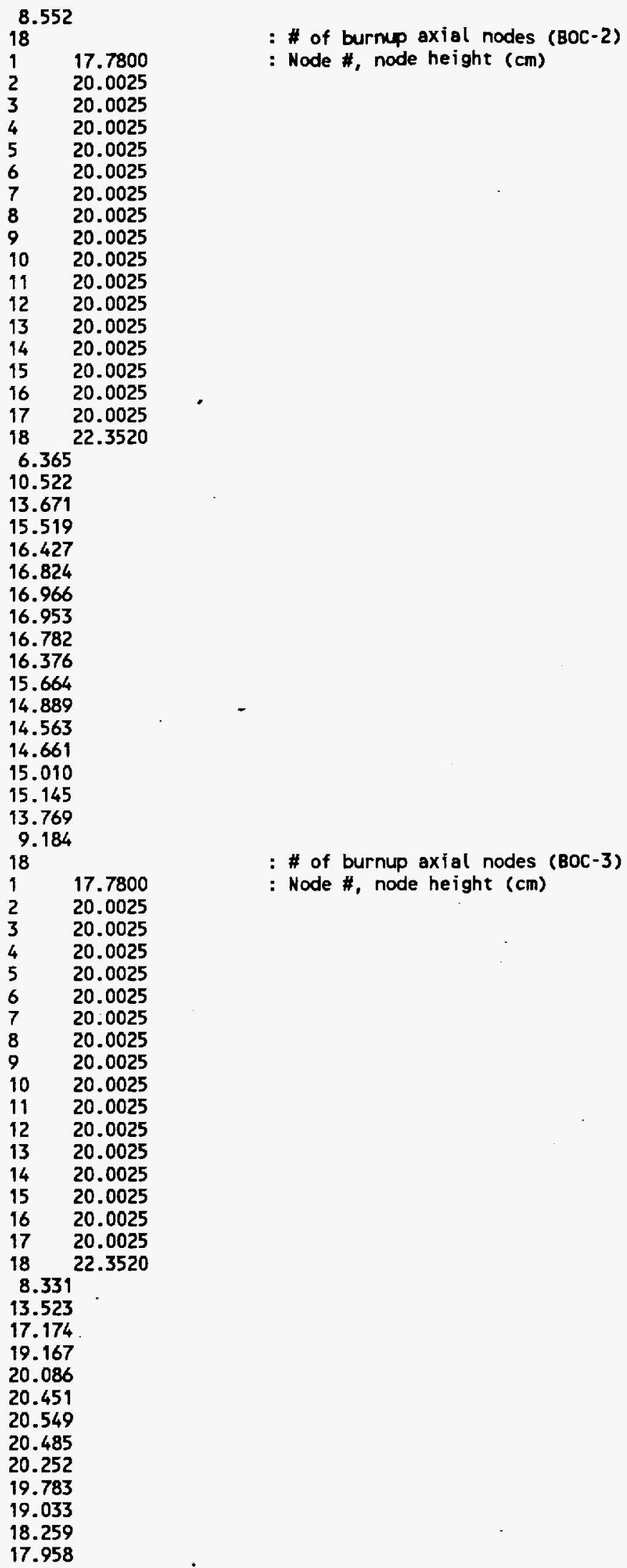


Apr 03 15:01 1997 File Name: A23ai.dat BBA000000-01717-0200-00040 REV 00 ATTACHMENT XV - Page 13

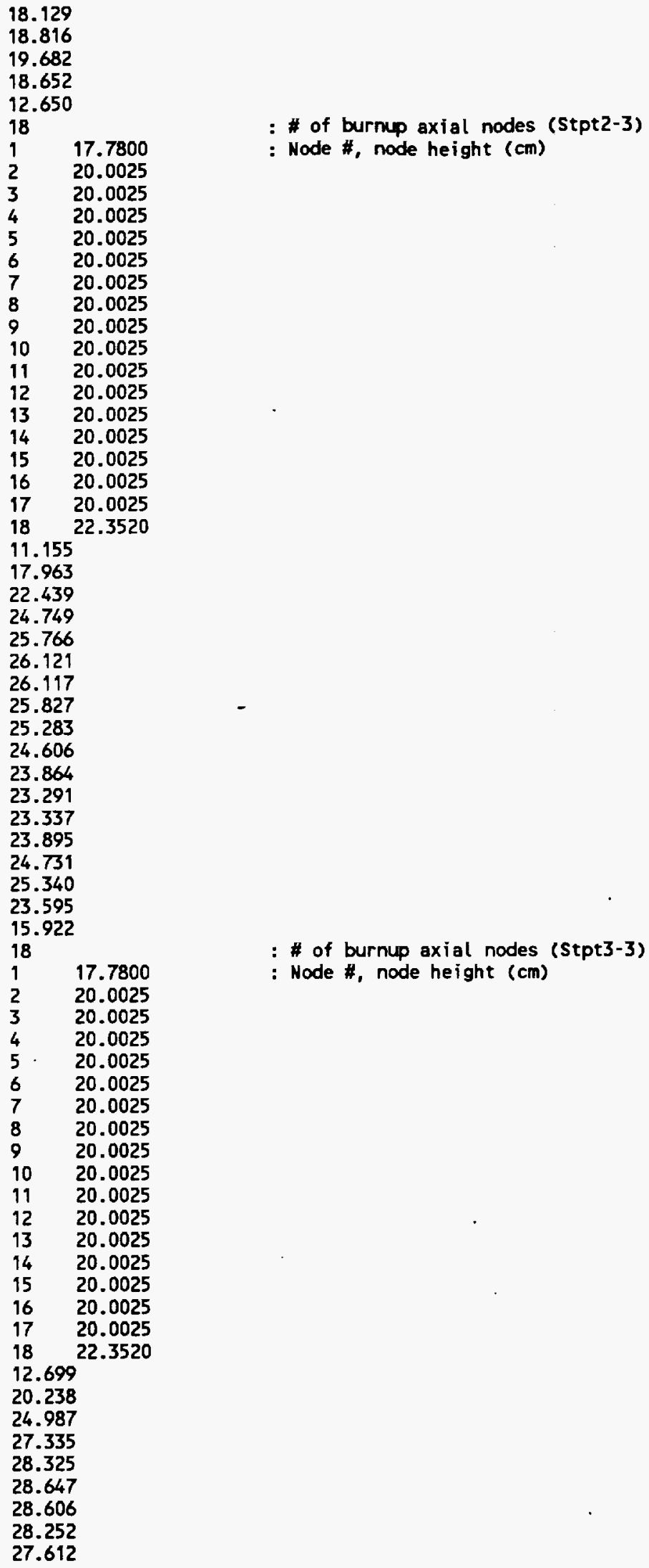


Apr 03 15:01 1997 File Name: A23ai.dat BBA000000-01717-0200-00040 REV 00 ATTACHMENT XV - Page 14

26.872

26.143

25.648

25.835

26.583

27.535

28.085

26.085

17.667 


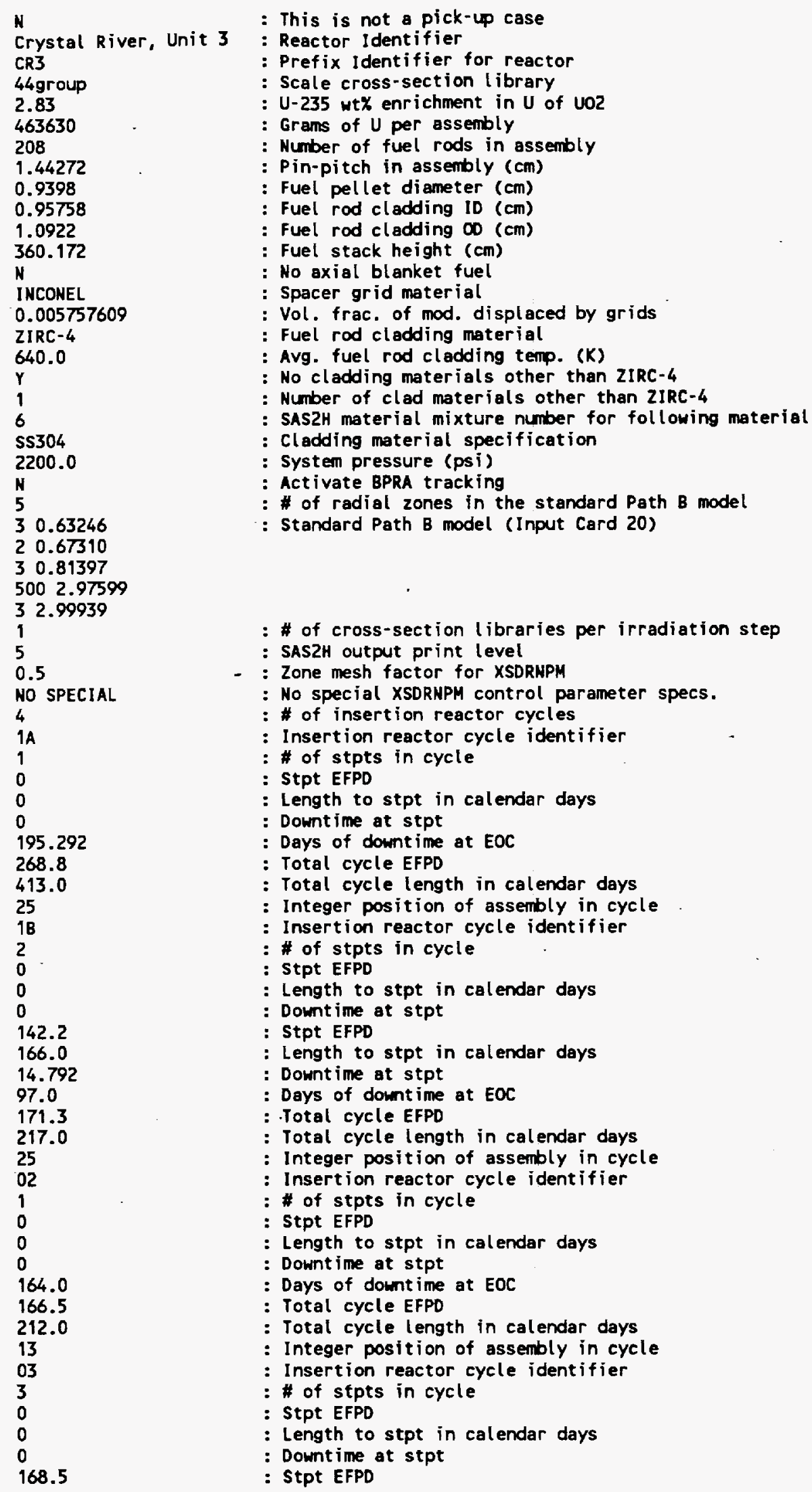




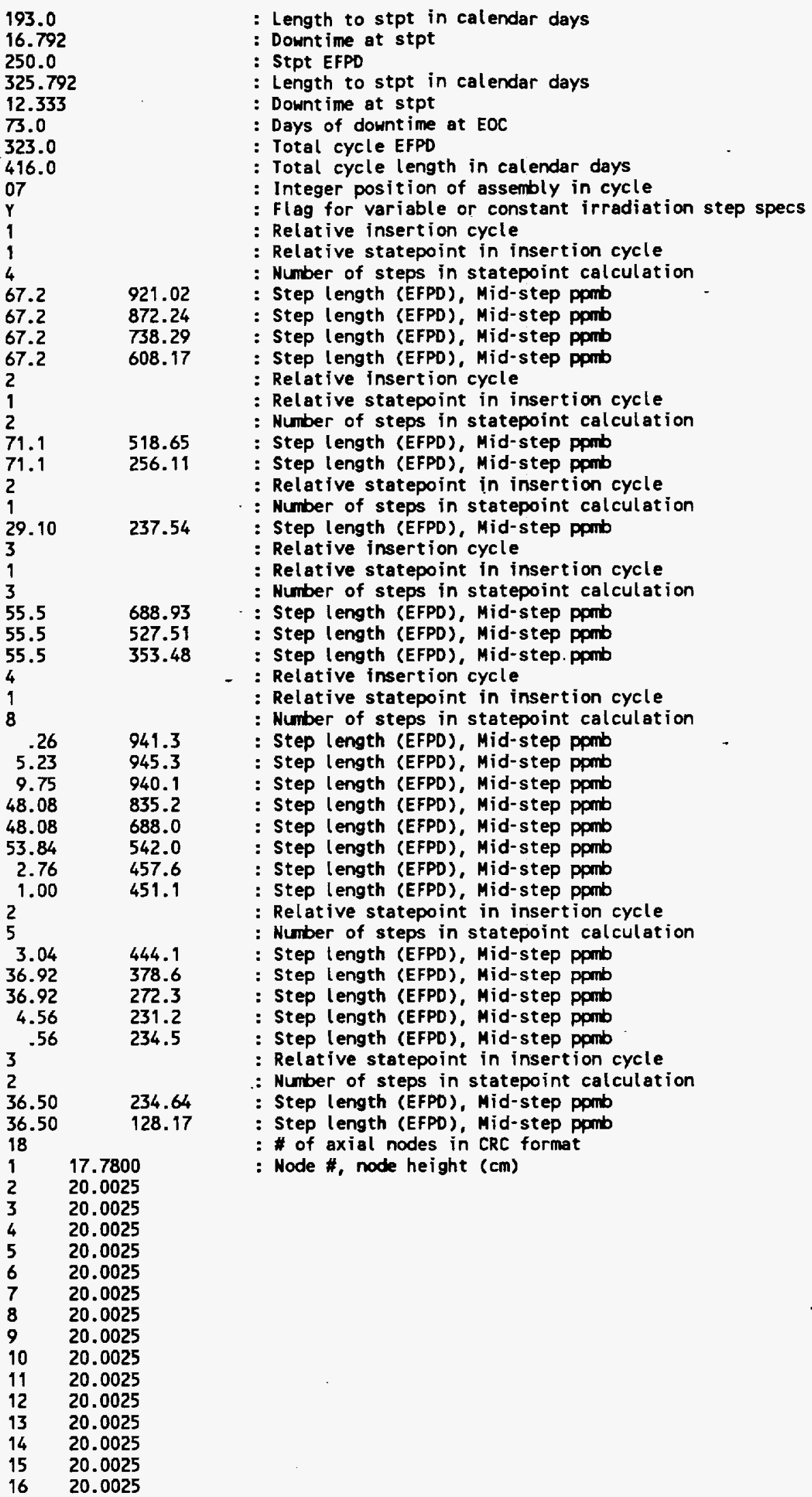




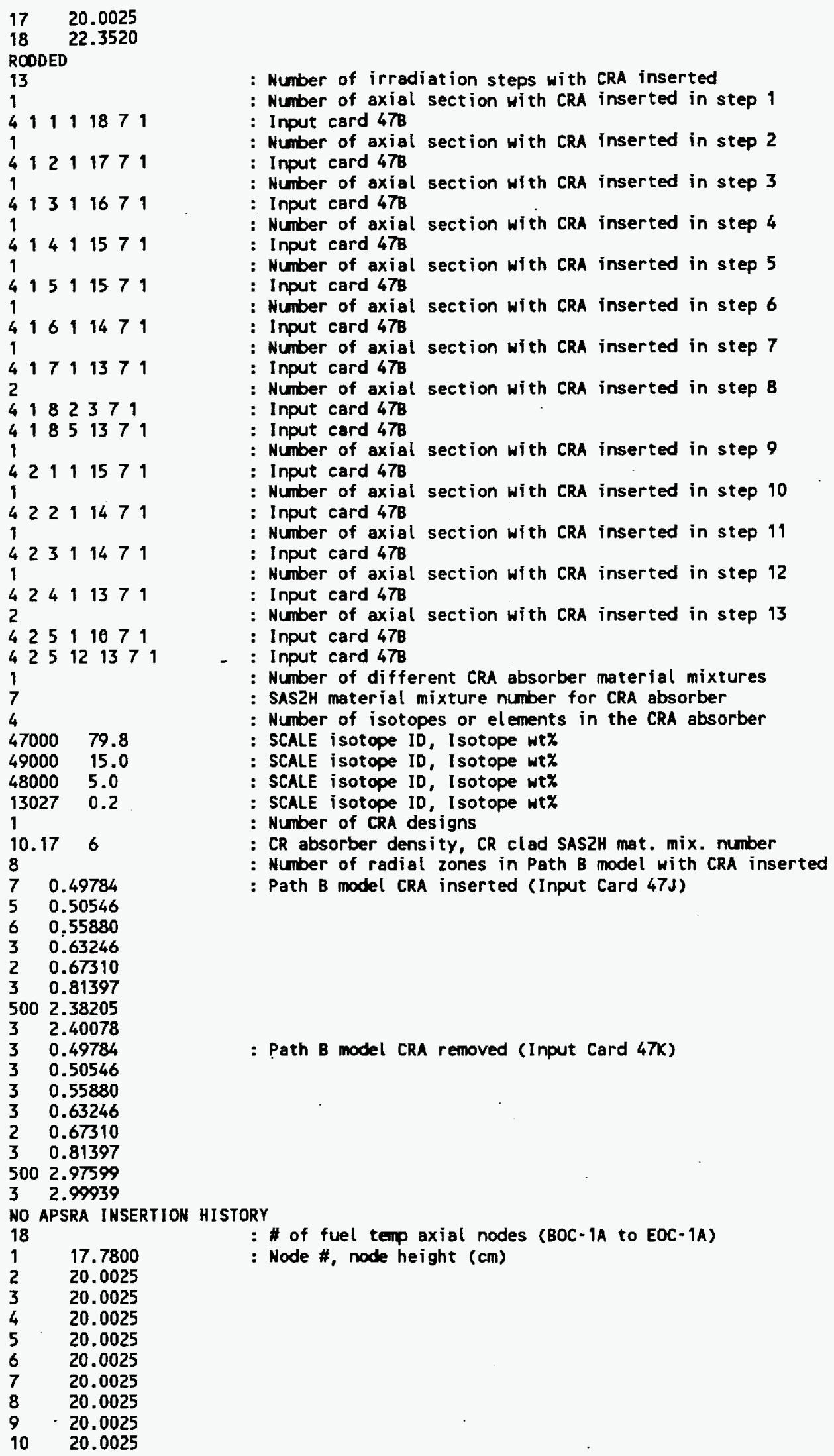


Apr 03 15:01 1997 File Name: A25i.dat BBA000000-01717-0200-00040 REV 00 ATTACHMENT XVI -... Page 4

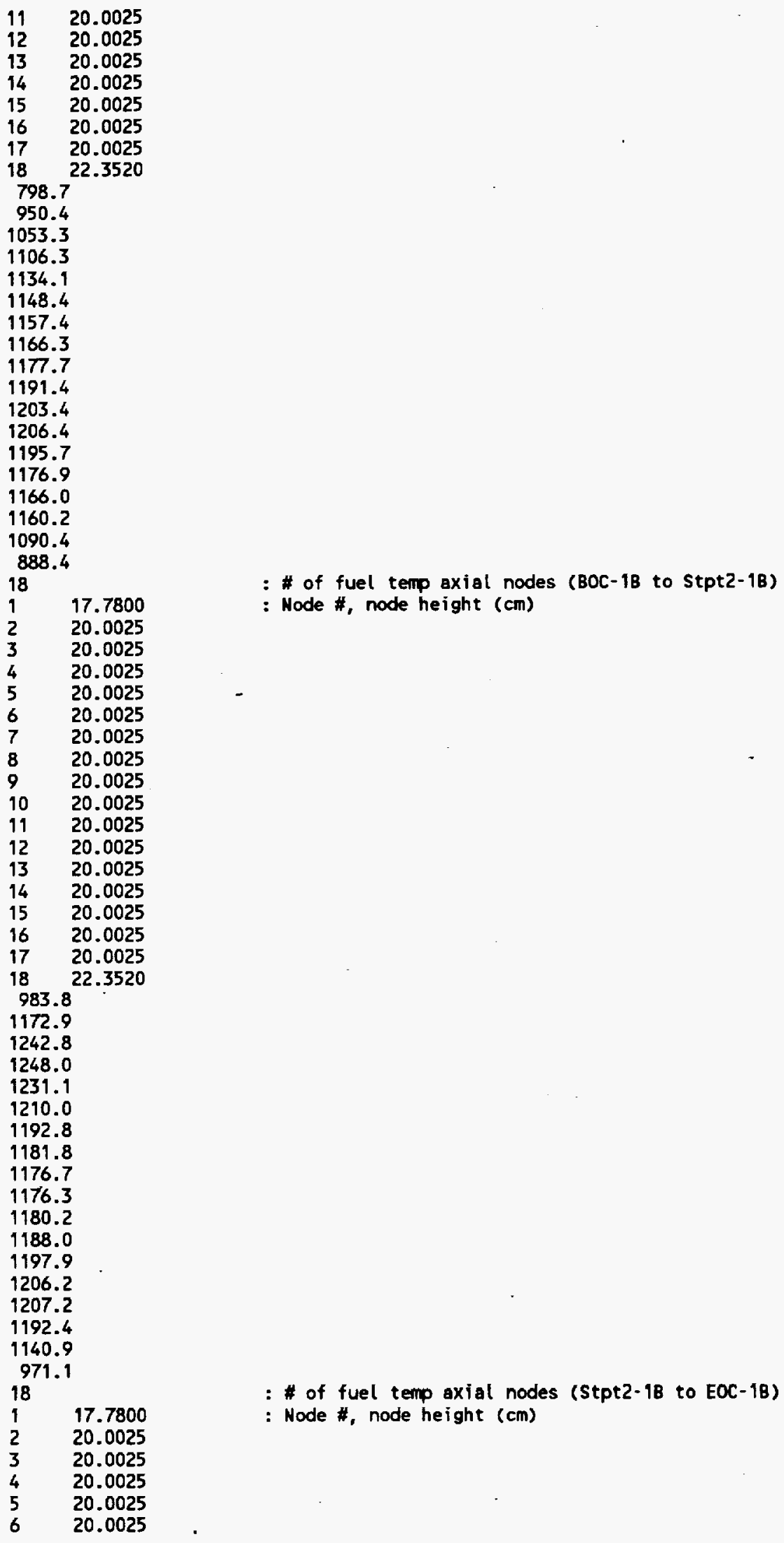


Apr 03 15:01 1997 File Name: A25i.dat BBA000000-01717-0200-00040 REV 00 ATTACHMENT XVI - Page 5
20.0025
20.0025
20.0025
20.0025
20.0025
20.0025
20.0025
20.0025
20.0025
20.0025
20.0025

22.3520

928.5

1079.4

1137.8

1160.7

1174.0

1184.1

1191.3

1196.0

1199.1

1202.1

1207.3

1213.6

1215.0

1204.0

1175.3

1128.6

1059.8

910.1

18

$1 \quad 17.7800$

20.0025

: \# fuel temp axial nodes (BOC-2 to EOC-2)

20.0025

- : Node \#, node height $(\mathrm{cm})$

20.0025

20.0025

20.0025

20.0025

20.0025

20.0025

20.0025

20.0025

20.0025

20.0025

20.0025

20.0025

20.0025

20.0025

$18 \quad 22.3520$

1077.0

1241.9

1306.2

1319.7

1320.1

1320.1

1322.6

1326.0

1324.7

1309.0

1275.9

1240.4

1211.8

1191.5

1184.6

1187.8

1163.2

1020.3

18

$1 \quad 17.7800$

: \# of fuel temp axial nodes (BOC-3 to stpt2-3)

20.0025

: Node \#, node height $(\mathrm{cm})$ 
Apr 03 15:01 1997 File Name: A25i.dat BBA000000-01717-0200-00040 REV 00 ATTACHMENT XVI - Page 6

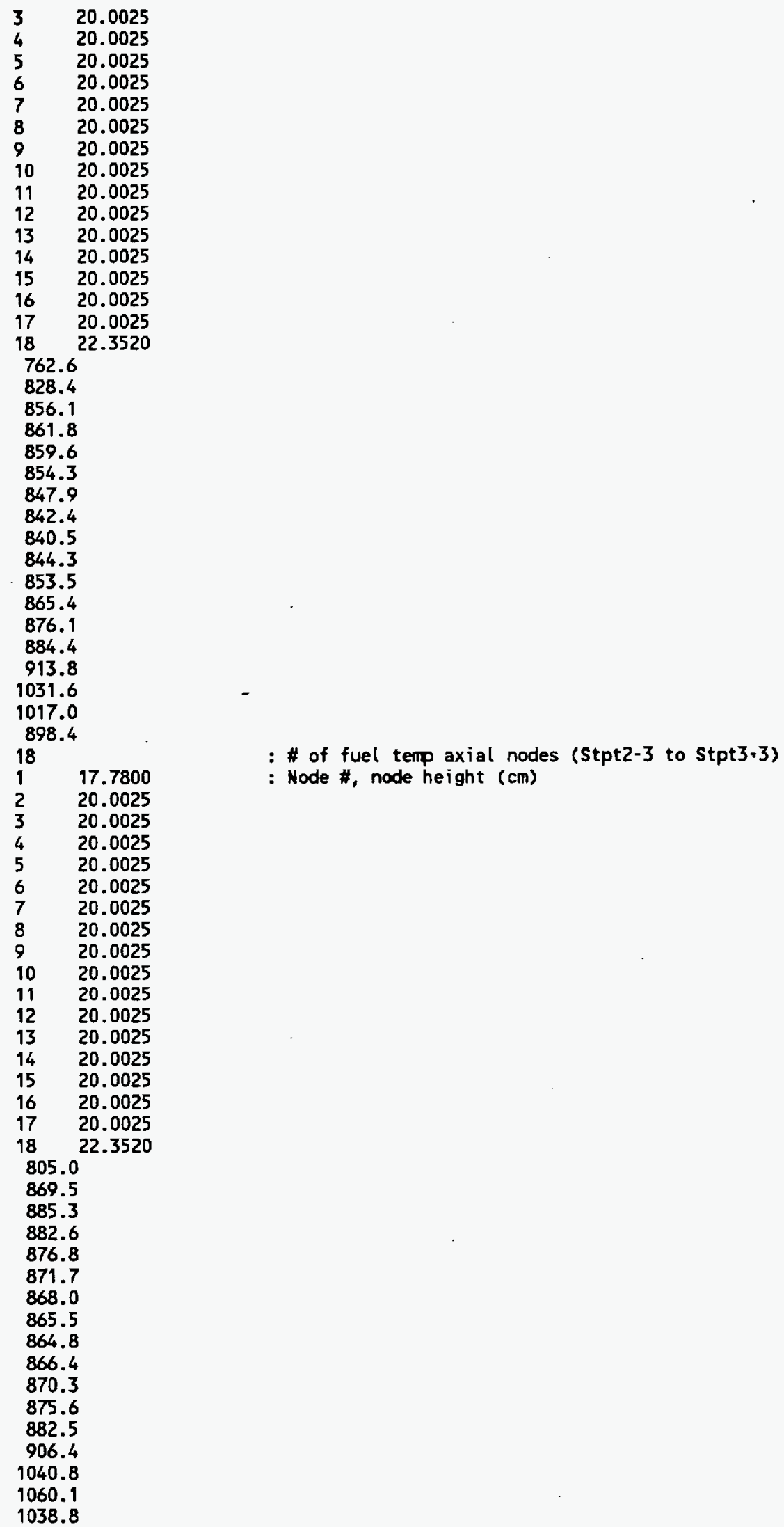


950.7

18

17.7800

20.0025

20.0025

20.0025

20.0025

20.0025

20.0025

20.0025

20.0025

20.0025

20.0025

20.0025

20.0025

20.0025

20.0025

20.0025

$17 \quad 20.0025$

$18 \quad 22.3520$

0.0227

0.0227

0.0226

0.0226

0.0225

0.0224

0.0223

0.0223

0.0222

0.0221

0.0220

0.0220

0.0219

0.0218

0.0217

0.0217

0.0216

0.0216

18

17.7800

20.0025

20.0025

20.0025

20.0025

20.0025

20.0025

20.0025

20.0025

20.0025

20.0025

20.0025

20.0025

20.0025

20.0025

20.0025

20.0025

$18 \quad 22.3520$

0.0230

0.0230

0.0229

0.0228

0.0227

0.0226

0.0225

0.0224

0.0223

0.0222

0.0221

0.0221

0.0220
: \# of mod spec vol axial nodes (BOC-1A to EOC-1A)

: Wode \#, node height $(\mathrm{cm})$
: of mod spec vol axial nodes (BOC-1B to Stpt2-1B)
: Node \#, node height (cm) 
Apr 03 15:01 1997 File Name: A25i.dat BBA000000-01717-0200-00040 REV 00 ATTACHMENT XVI - Page 8

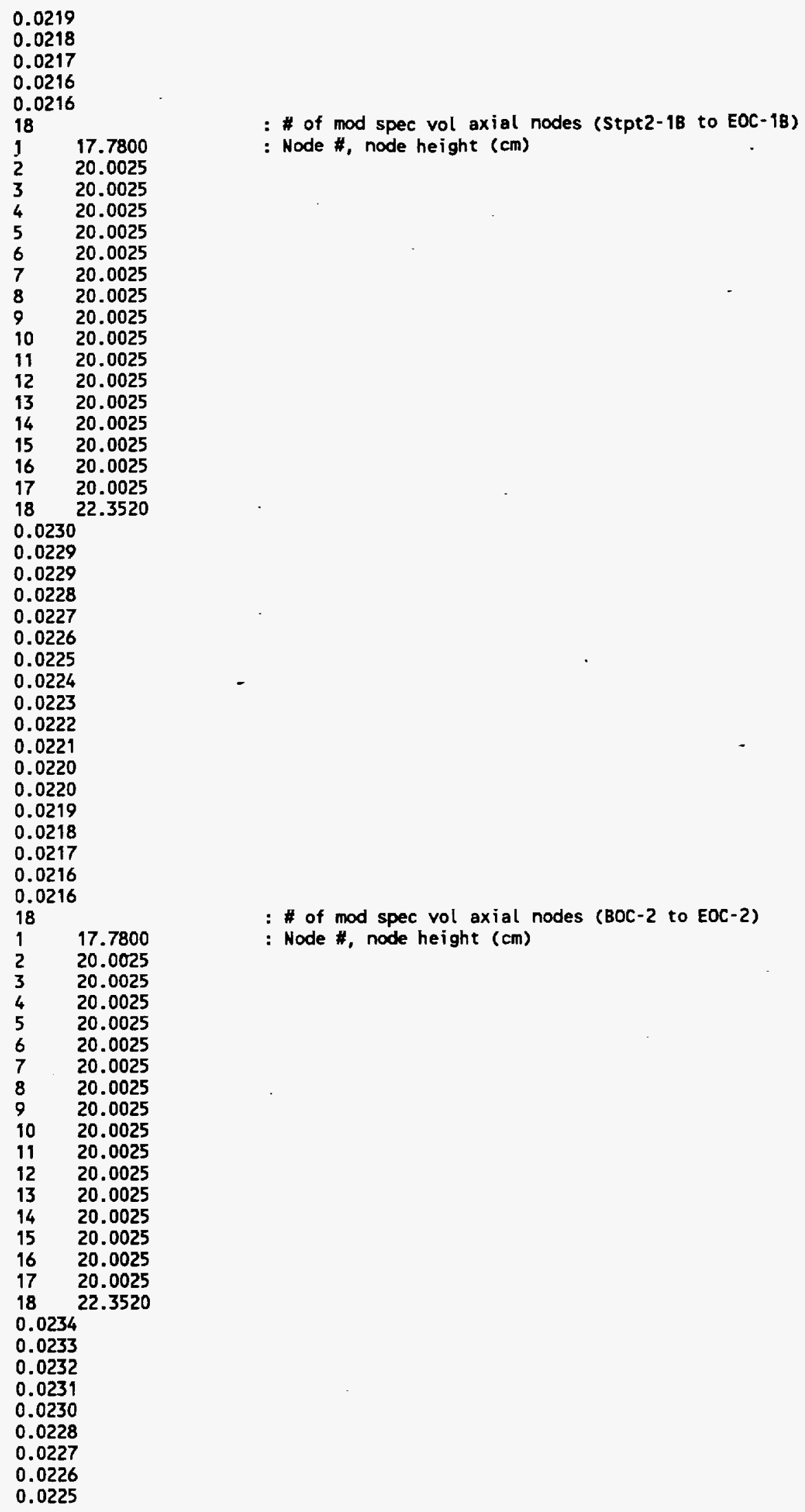


Apr 03 15:01 1997 File Name: A25i.dat BBA000000-01717-0200-00040 REV 00 ATtACHMENT XVI - Page 9

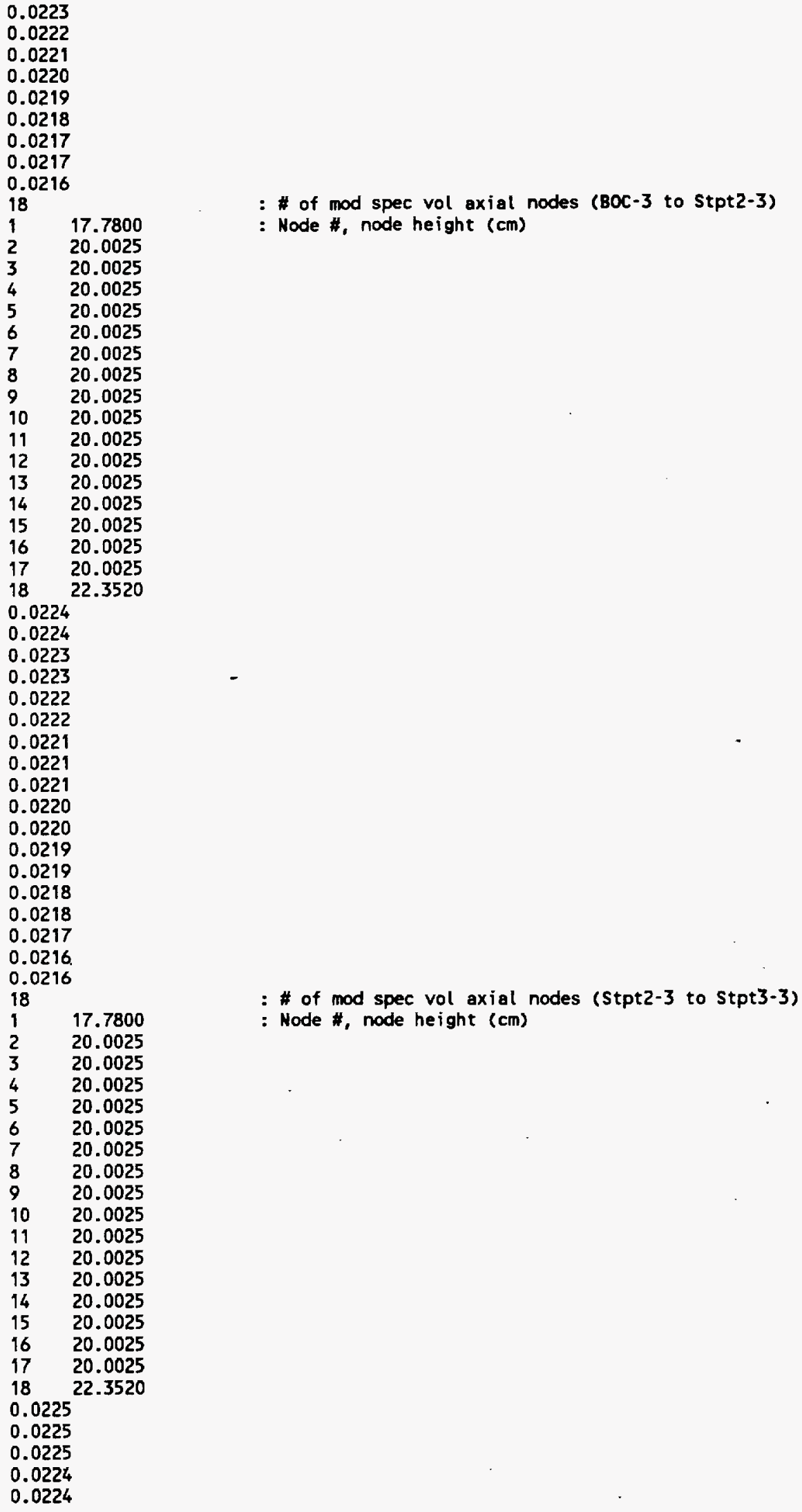


Apr 03 15:01 1997 File Name: A25i.dat BBA000000-01717-0200-00040 REV 00 ATtACHMENT XVI - Page 10

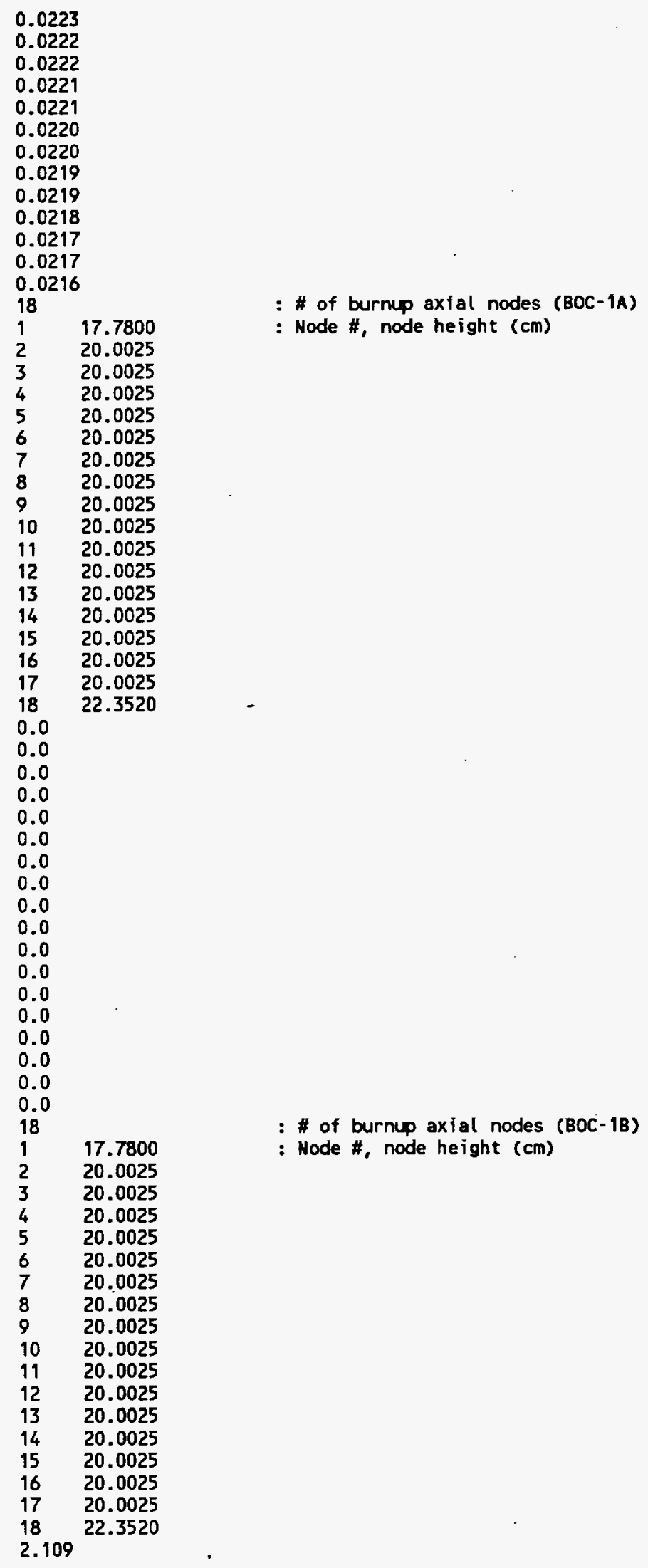


Apr 03 15:01 1997 File Name: A25i.dat B8A000000-01717-0200-00040 REV 00 ATtACHMENT XVI - Page 11

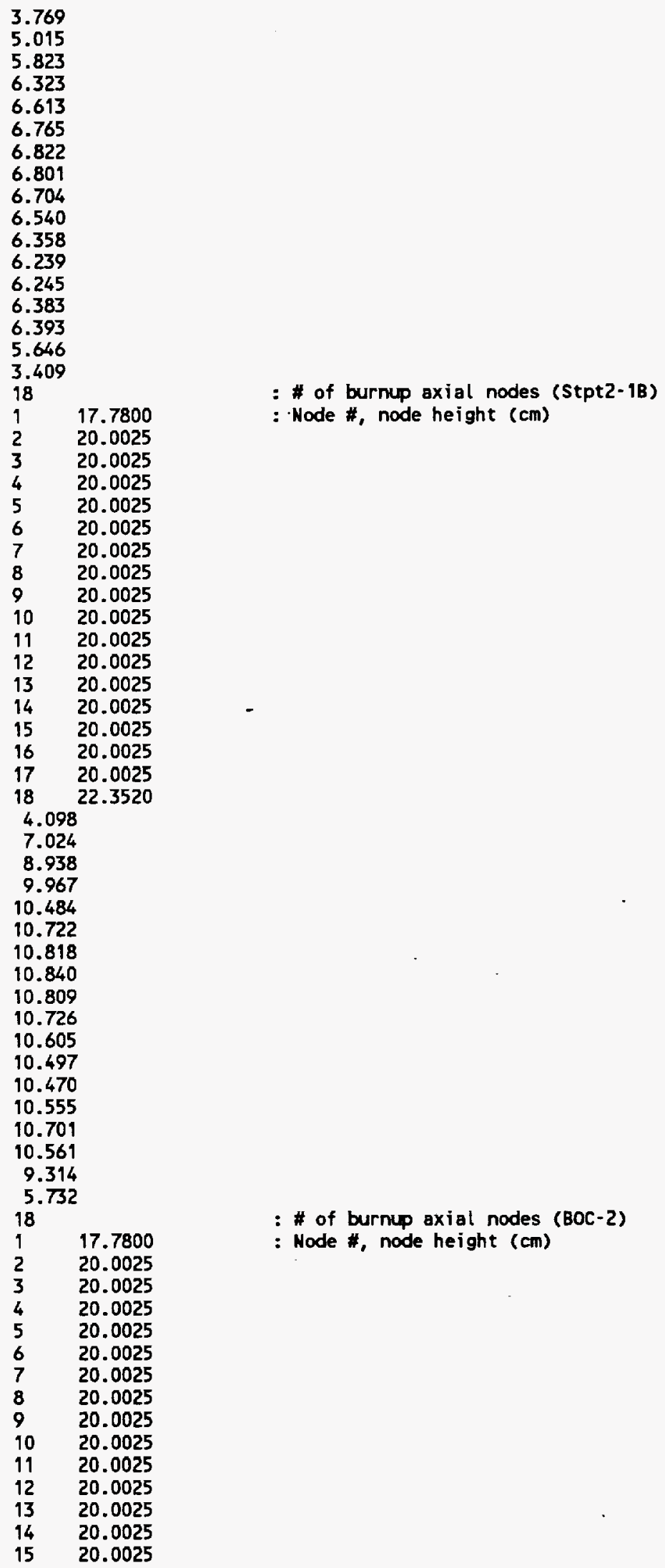


Apr 03 15:01 1997 File Name: A25i.dat BBA000000-01717-0200-00040 REV 00 ATtACHMENT XVI - Page 12

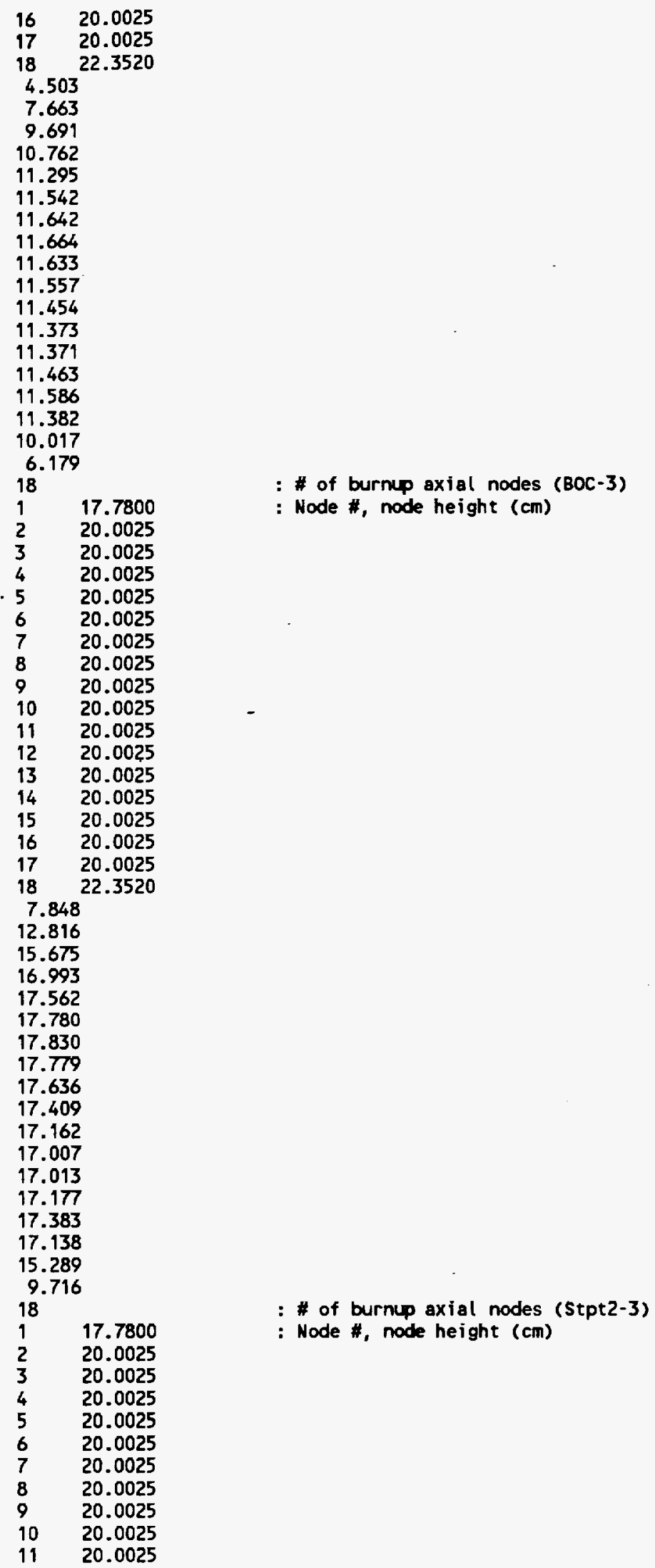




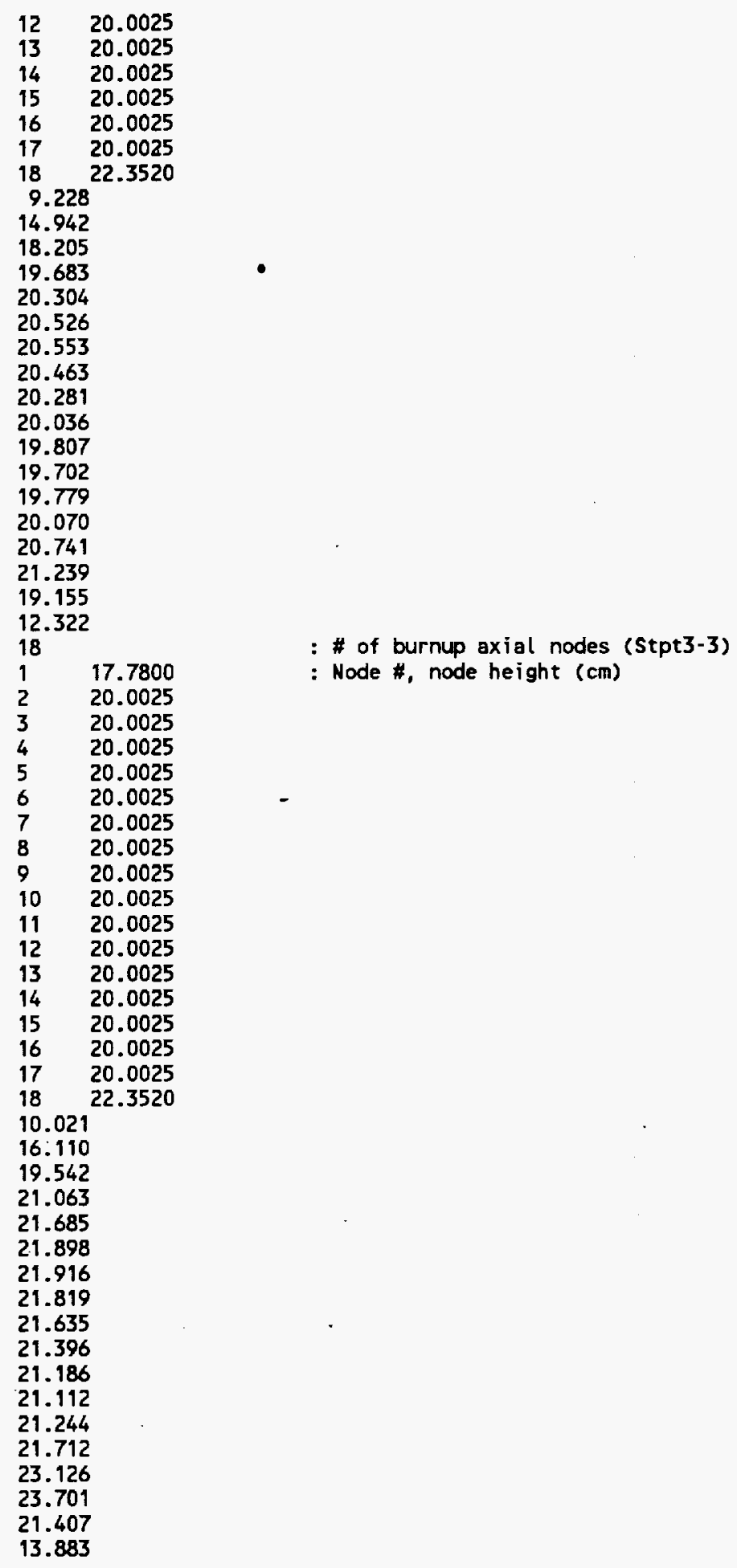




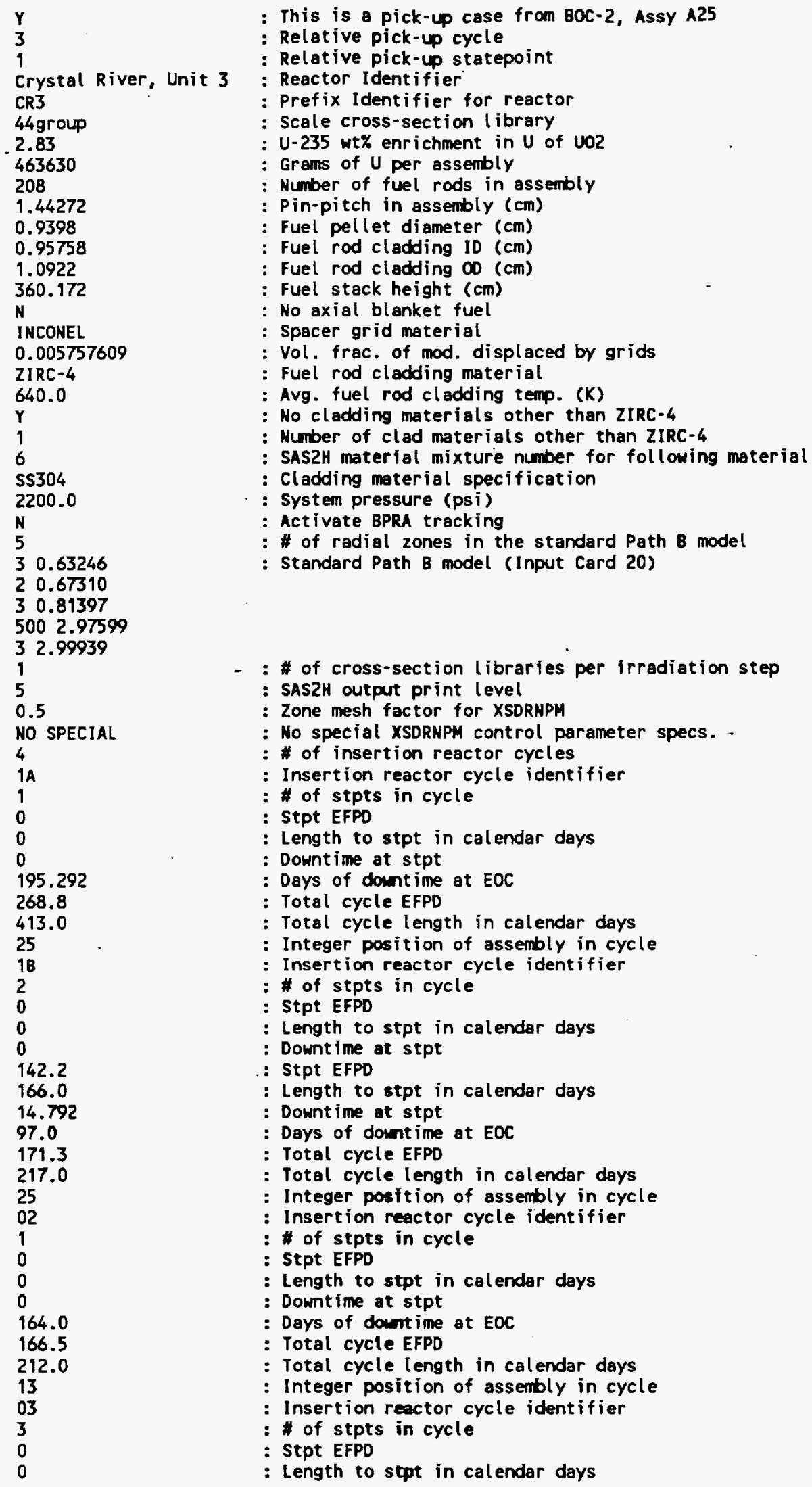


0

168.5

193.0

16.792

250.0

325.792

12.333

73.0

323.0

416.0

26

$Y$

1

67.2

67.2

67.2

67.2

2

1

71.1

71.1

2

29.10

3

$\frac{1}{3}$

55.5

55.5

55.5

4

1

6

6.84

3.33

66.47

39.84

39.84

12.18

2

8.99

62.99

9.52

3

2

36.50

36.50

18

1

2

3

4

5

6

7

8

9

10

11

12

13

14

15

16

17
18
921.02

872.24

738.29

608.17

518.65

256.11

237.54

688.93

527.51

353.48

946.1

945.6

830.5

672.2

546.9

471.5

435.5

333.5

231.6

234.64

128.17

17.7800

20.0025

20.0025

20.0025

20.0025

20.0025

20.0025

20.0025

20.0025

20.0025

20.0025

20.0025

20.0025

20.0025

20.0025

20.0025

20.0025

22.3520
: Downtime at stpt

: Stpt EFPD

: Length to stpt in calendar days

: Downtime at stpt

: Stpt EFPD

: Length to stpt in calendar days

: Downtime at stpt

: Days of downtime at EOC

- Total cycle EFPD

: Total cycle length in calendar days

- Integer position of assembly in cycle

: Flag for variable or constant irradiation step specs

: Relative insertion cycle

: Relative statepoint in insertion cycle

: Number of steps in statepoint calculation

: Step length (EFPD), Mid-step ppmb

: Step length (EFPD), Mid-step ppmb

: Step length (EFPD), Mid-step ppmb

: Step length (EFPD), Mid-step ppmb

: Relative insertion cycle

: Relative statepoint in insertion cycle

: Number of steps in statepoint colculation

: Step length (EFPD), Mid-step ppmb

: Step length (EFPD), Mid-step ppmb

: Relative statepoint in insertion cycle

: Number of steps in statepoint calculation

: Step length (EFPD), Mid-step ppmb

: Relative insertion cycle

: Relative statepoint in insertion cycle

: Number of steps in statepoint calculation

: Step length (EFPD), Mid-step ppmb

: Step length (EFPD), Mid-step ppmb

: Step length (EFPO), Mid-step ppmb

: Relative insertion cycle

: Relative statepoint in insertion cycle

: Number of steps in statepoint calculation

: Step length (EFPD), Mid-step ppmb

Step length (EFPD), Mid-step ppmb

: Step length (EFPD), Mid-step Ppmb

: Step length (EFPD), Mid-step ppmb

: Step length (EFPD), Mid-step ppmb

: Step length (EFPD), Mid-step ppmb

: Relative statepoint in insertion cycle

: Number of steps in statepoint calculation

: Step length (EFPD), Mid-step ppmb

: Step length (EFPD), Mid-step ppmb

: Step length (EFPD), Mid-step ppmb

: Relative statepoint in insertion cycle

: Number of steps in statepoint calculation

: Step length (EFPD), Mid-step ppmb

: Step length (EFPD), Mid-step ppmb

: \# of axial nodes in CRC format

: Node \#, node height (cm) 


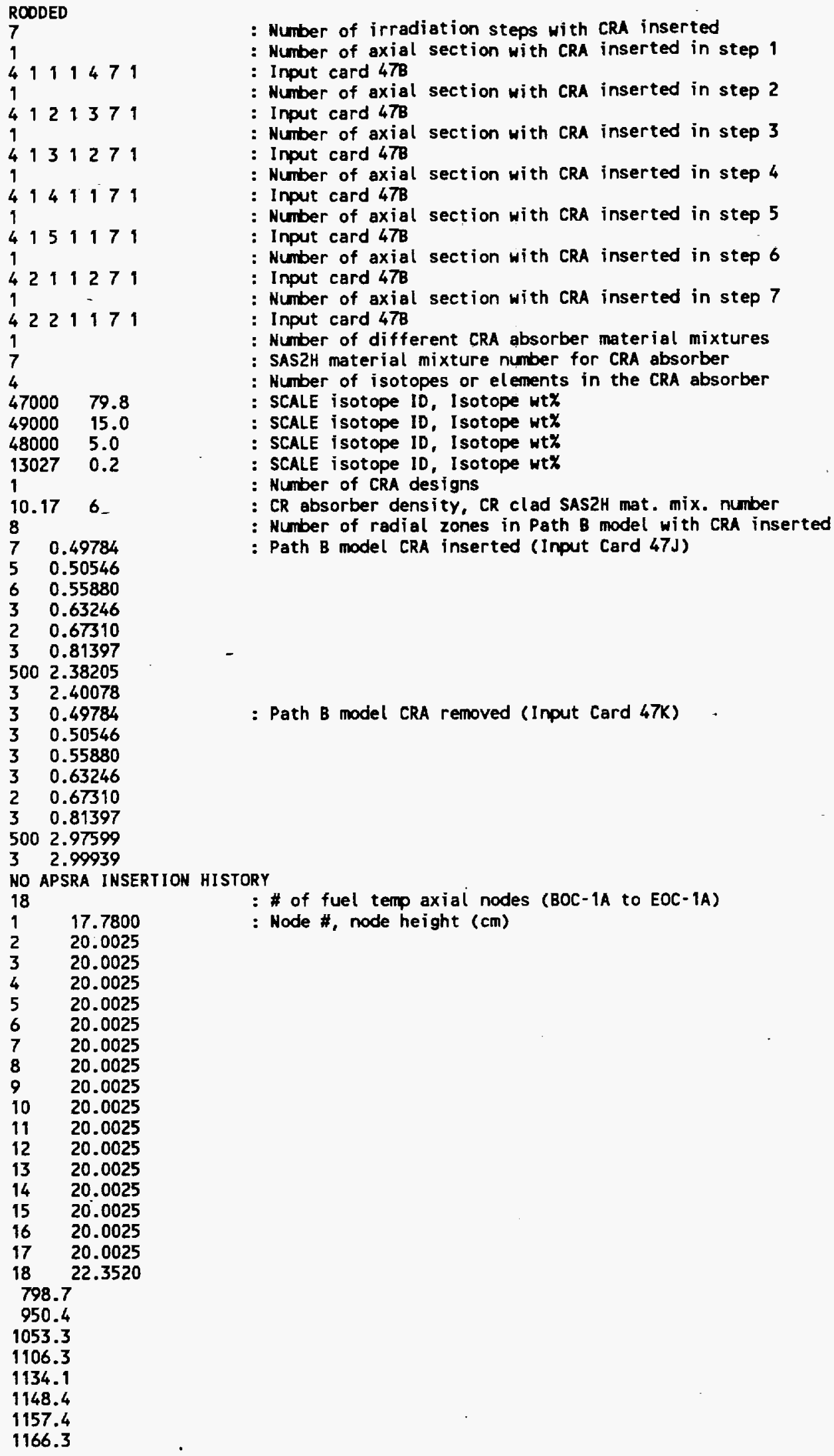


Apr 03 15:01 1997 File Name: A25ai.dat BBA000000-01717-0200-00040 REV 00 ATTACHMENT XVII - Page 4

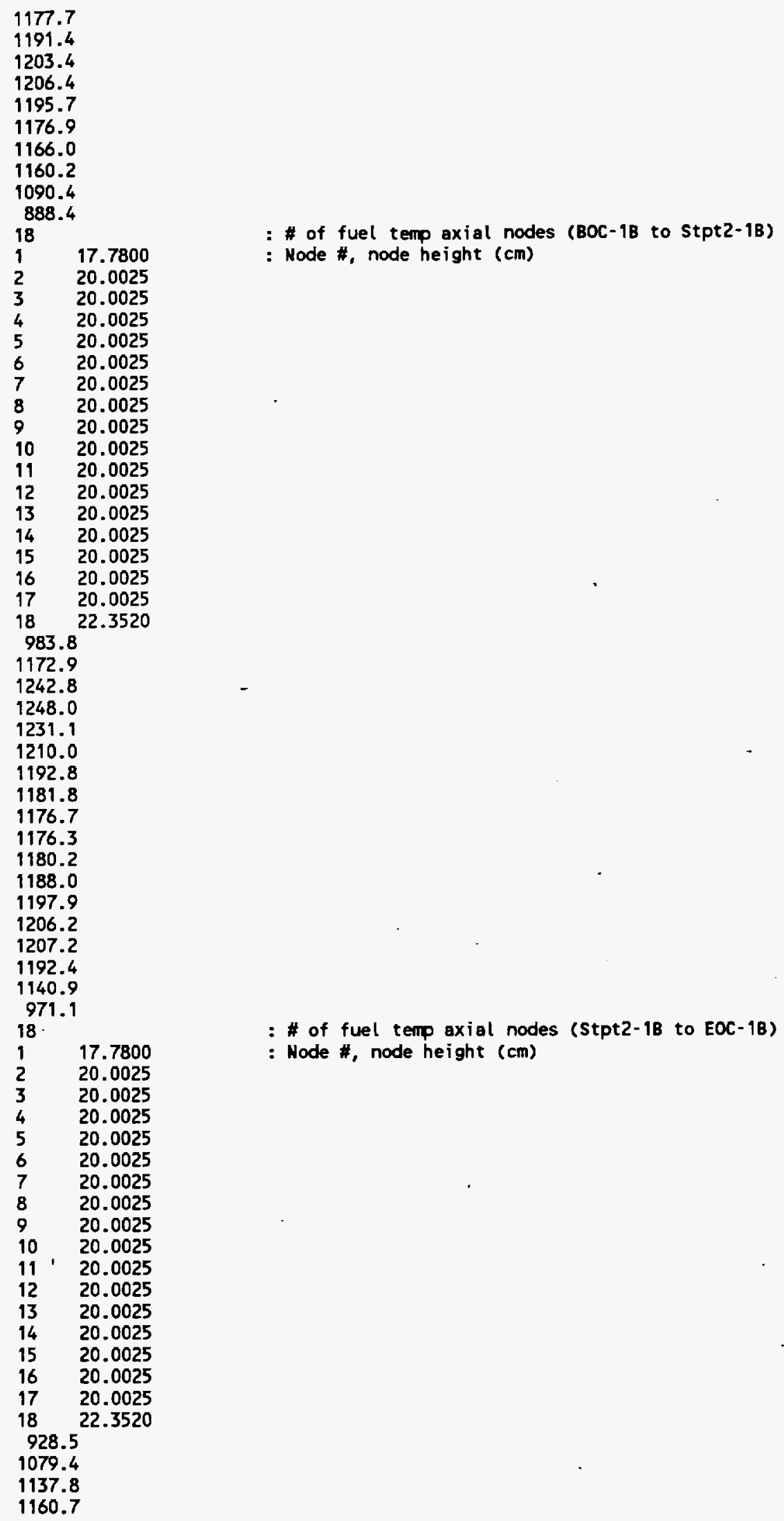




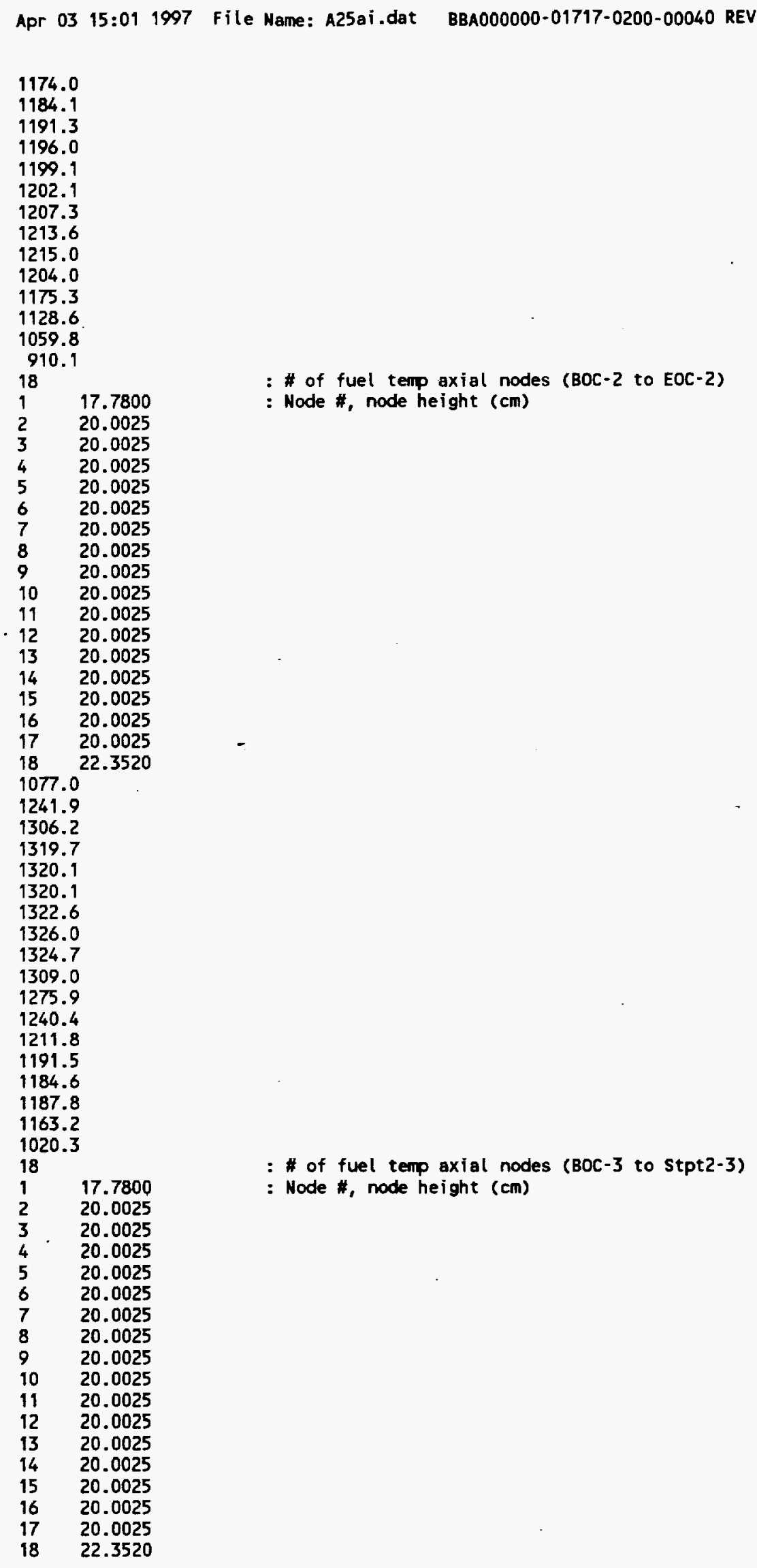




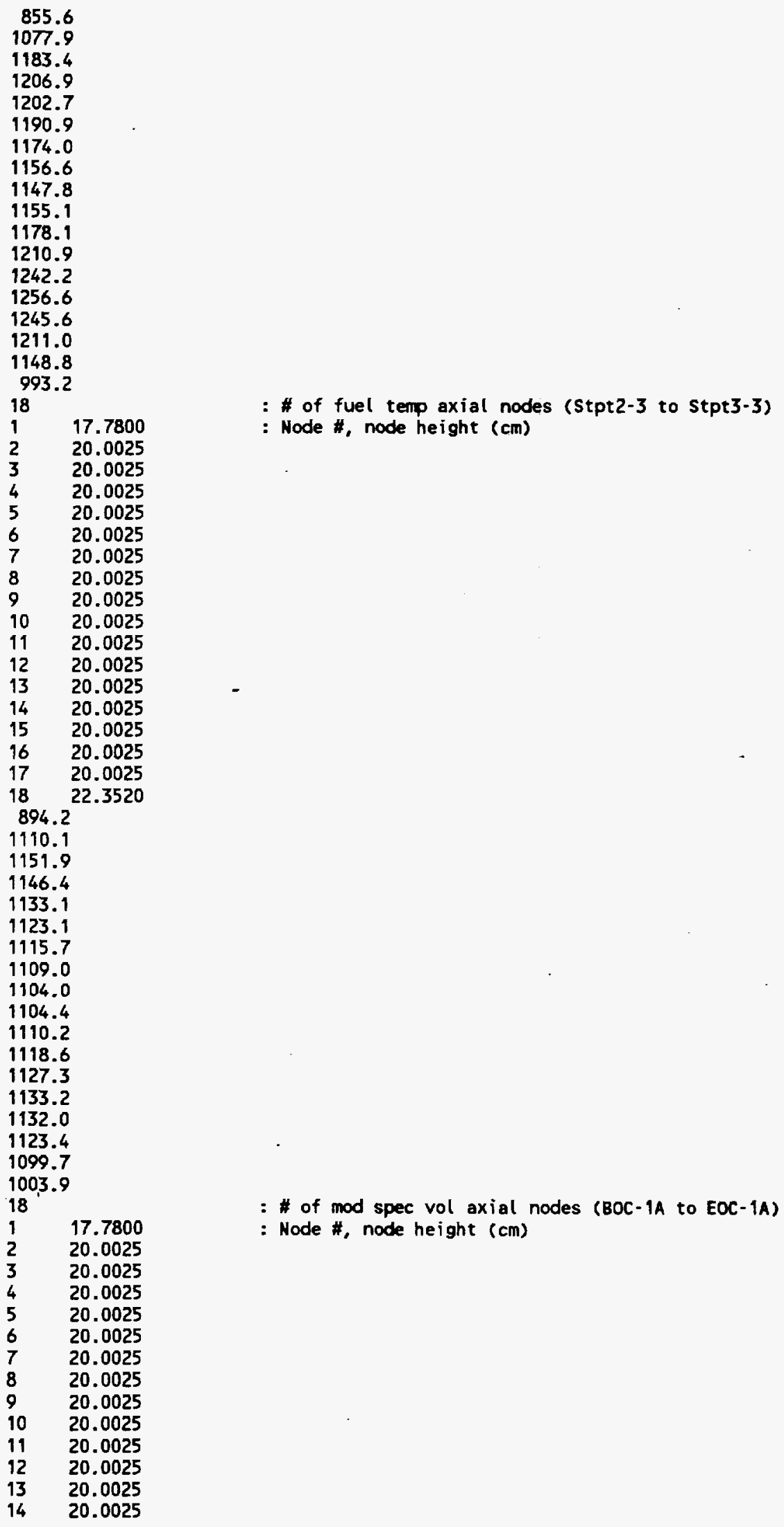


Apr 03 15:01 1997 File Name: A25ai.dat BBA000000-01717-0200-00040 REV 00 ATTACHMENT XVII - Page 7

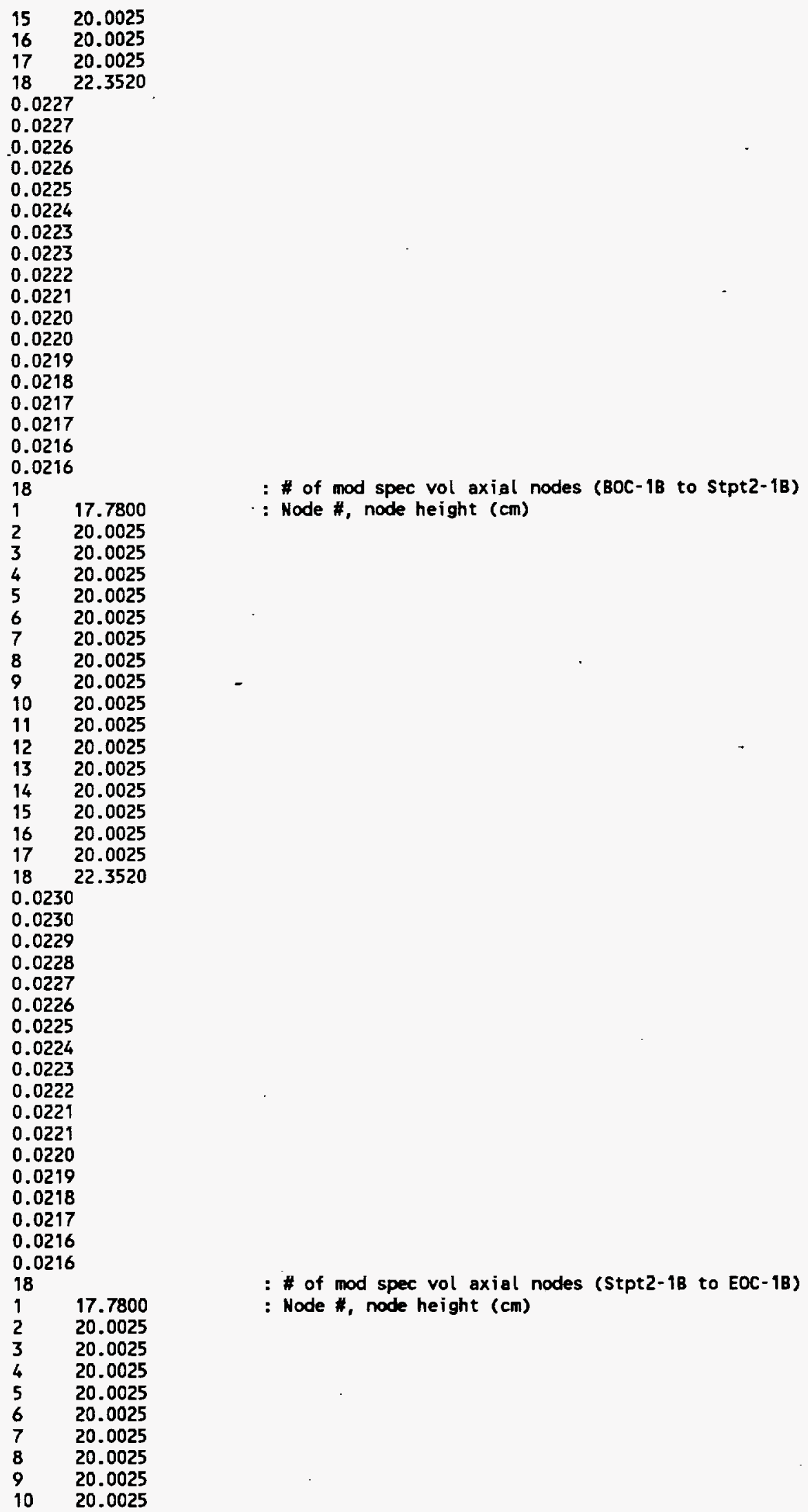




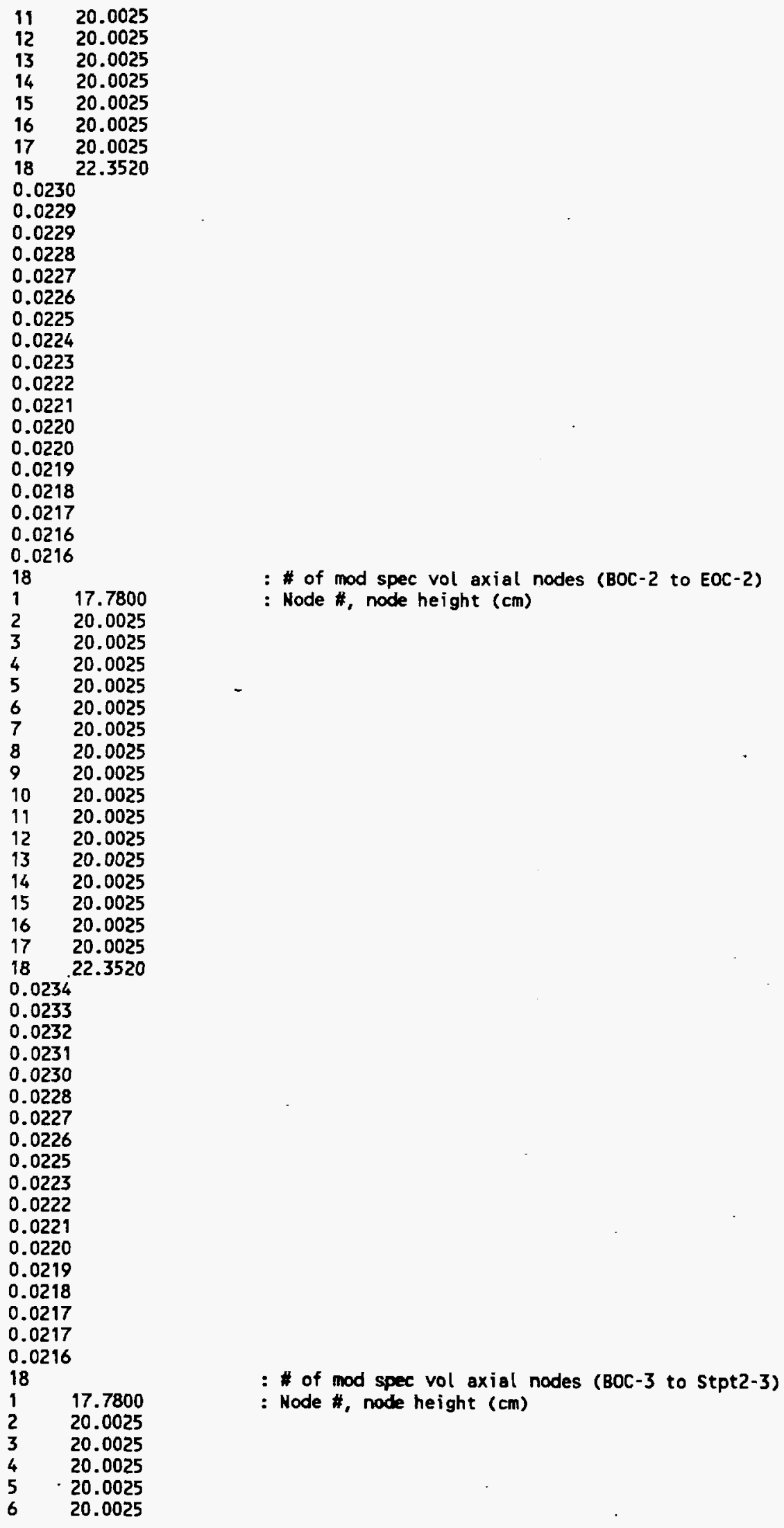


Apr 03 15:01 1997 File Name: A25ai.dat BBA000000-01717-0200-00040 REV 00 ATTACHMENT XVII - Page 9

20.0025

20.0025

20.0025

20.0025

20.0025

20.0025

20.0025

20.0025

20.0025

20.0025

20.0025

$18 \quad 22.3520$

0.0234

0.0233

0.0232

0.0231

0.0230

0.0229

0.0227

0.0226

0.0225

0.0224

0.0223

0.0222

0.0221

0.0220

0.0219

0.0218

0.0217

0.0216

$1 \quad 17.7800$

20.0025

20.0025

20.0025

20.0025

20.0025

20.0025

20.0025

20.0025

20.0025

20.0025

20.0025

20.0025

20.0025

20.0025

20.0025

20.0025

$18 \quad 22.3520$

0.0233

0.0233

0.0232

0.0230

0.0229

0.0228

0.0227

0.0226

0.0225

0.0224

0.0223

0.0222

0.0221

0.0220

0.0219

0.0218

0.0217

0.0216

$\begin{array}{ll}18 & \\ 1 & 17.7800 \\ 2 & 20.0025\end{array}$

: \# of mod spec vol axial nodes (Stpt2-3 to stpt3-3)

- : Node \#, node height $(\mathrm{cm})$

: \# of burnup axial nodes (BOC-1A)

: Node \#, node height $(\mathrm{cm})$ 
Apr 03 15:01 1997 File Name: A25ai.dat BBA000000-01717-0200-00040 REV 00 ATtACHMENT XVII - Page 10

20.0025

20.0025

20.0025

20.0025

20.0025

20.0025

20.0025

20.0025

20.0025

20.0025

20.0025

20.0025

20.0025

20.0025

20.0025

22.3520

0.0

0.0

0.0

0.0

0.0

0.0

0.0

0.0

0.0

0.0

0.0

0.0

0.0

0.0

0.0

0.0

18

$1 \quad 17.7800$

20.0025

20.0025

20.0025

20.0025

20.0025

20.0025

20.0025

20.0025

20.0025

20.0025

20.0025

20.0025

20.0025

20.0025

20.0025

20.0025

$18 \quad 22.3520$

2.109

3.769

5.015

5.823

6.323

6.613

6.765

6.822

6.801

6.704

6.540

6.358

6.239

6.245

6.383

6.393

5.646

: \# of burnup axial nodes (BOC-1B)

: Node \#, node height $(\mathrm{cm})$ 
Apr 03 15:01 1997 File Name: A25ai.dat BBA000000-01717-0200-00040 REV 00 AtTACHMENT XVII - Page 11

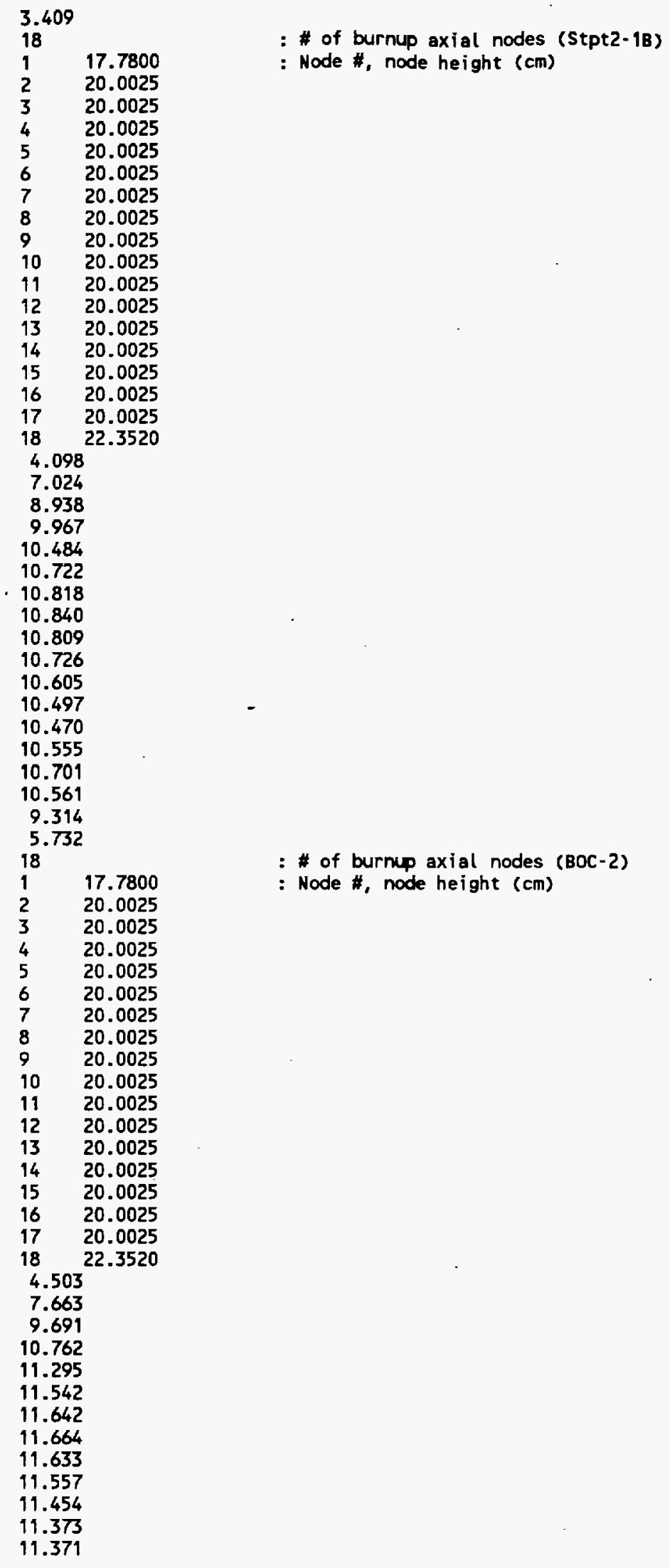


Apr 03 15:01 1997 File Name: A25ai.dat BBA000000-01717-0200-00040 REV 00'ATTACHMENT XVII - Page 12

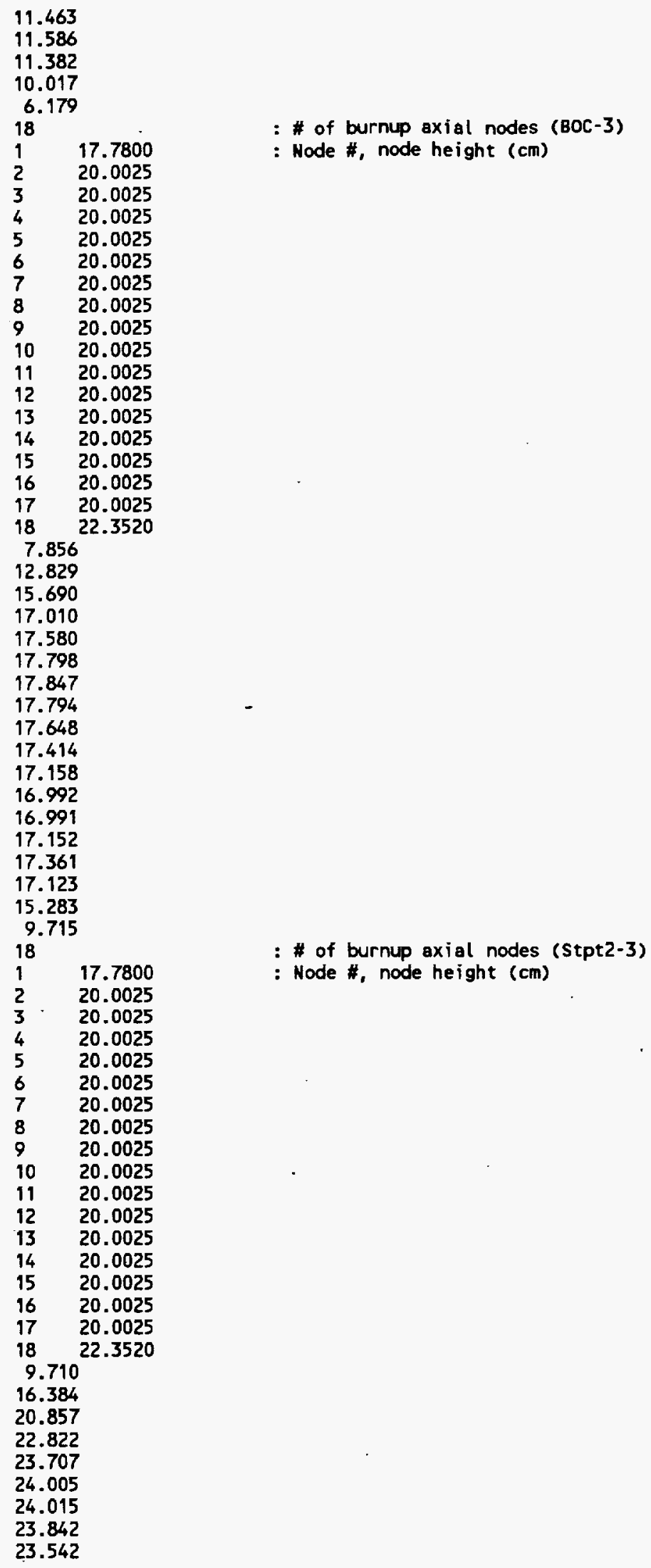


Apr 03 15:01 1997 File Name: A25ai.dat BBA000000-01717-0200-00040 REV 00 ATtACHMENT XVII - Page 13

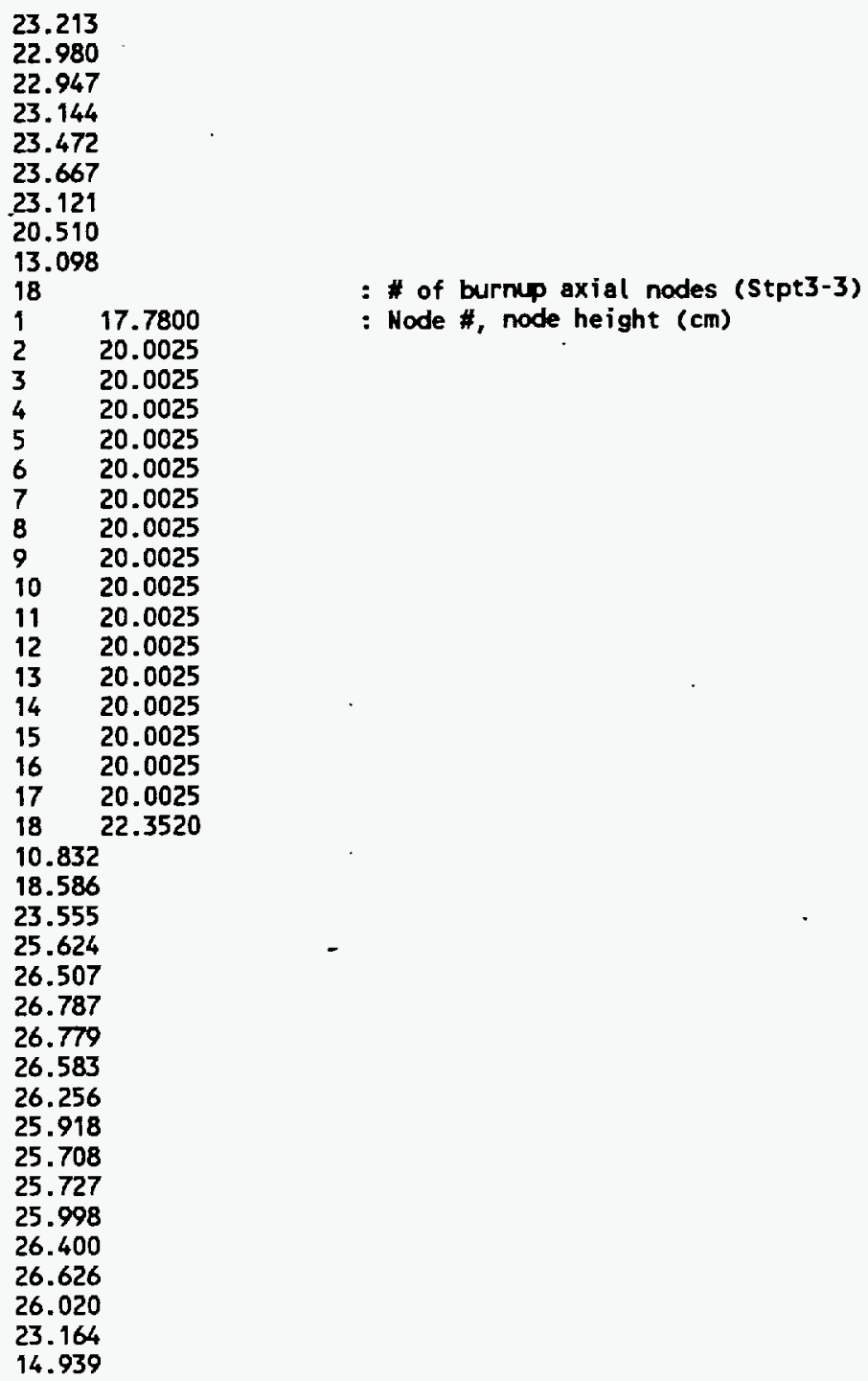




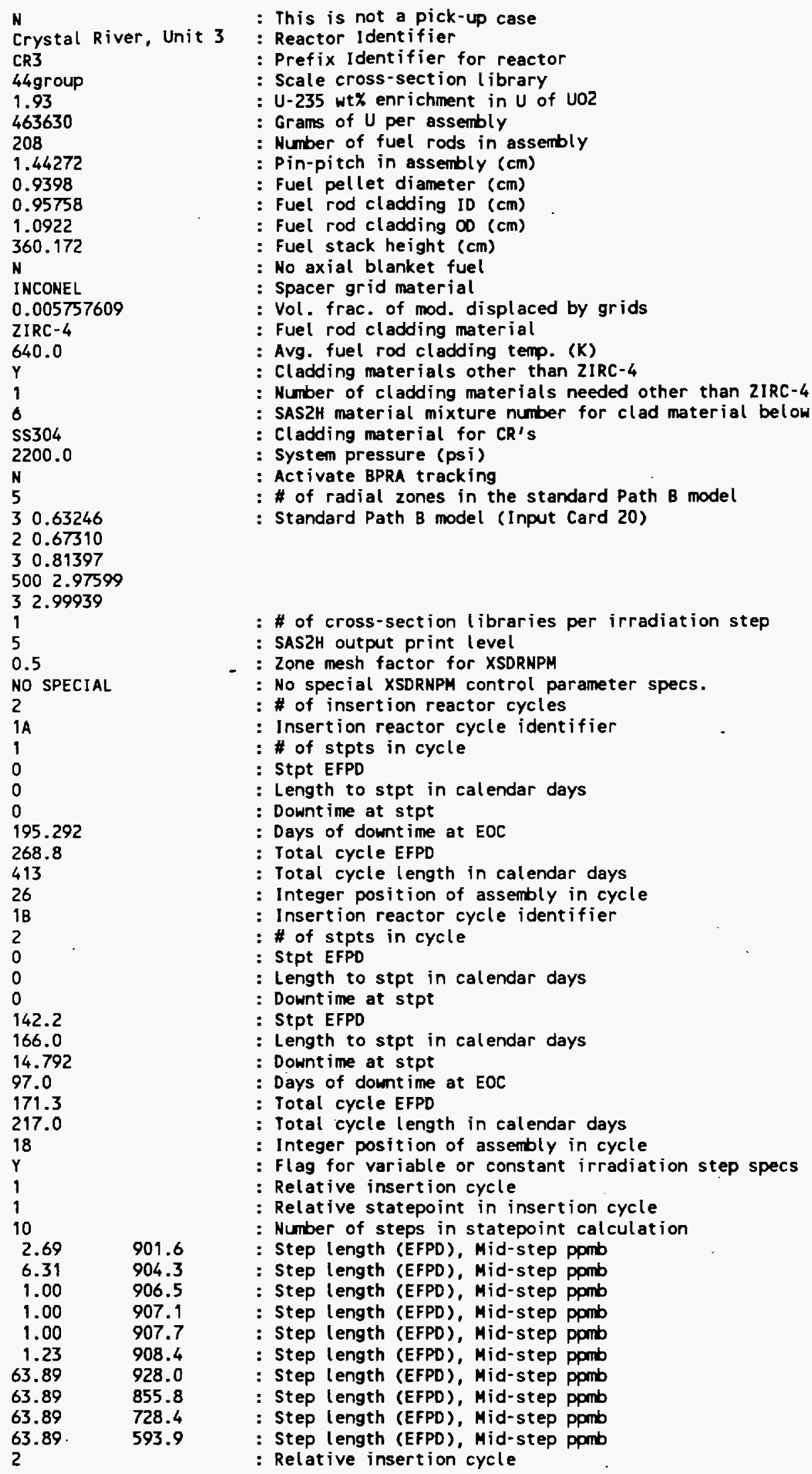




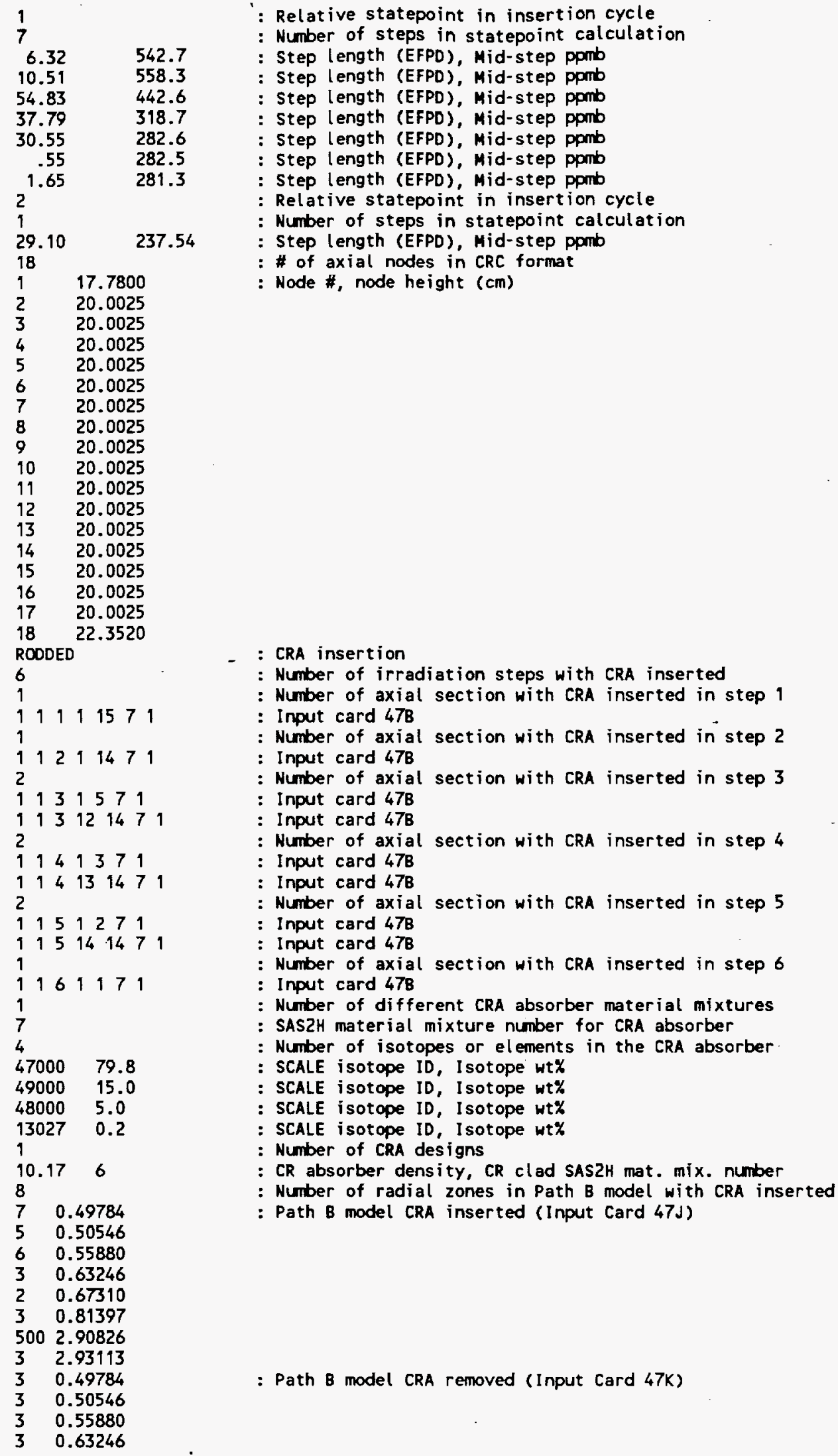




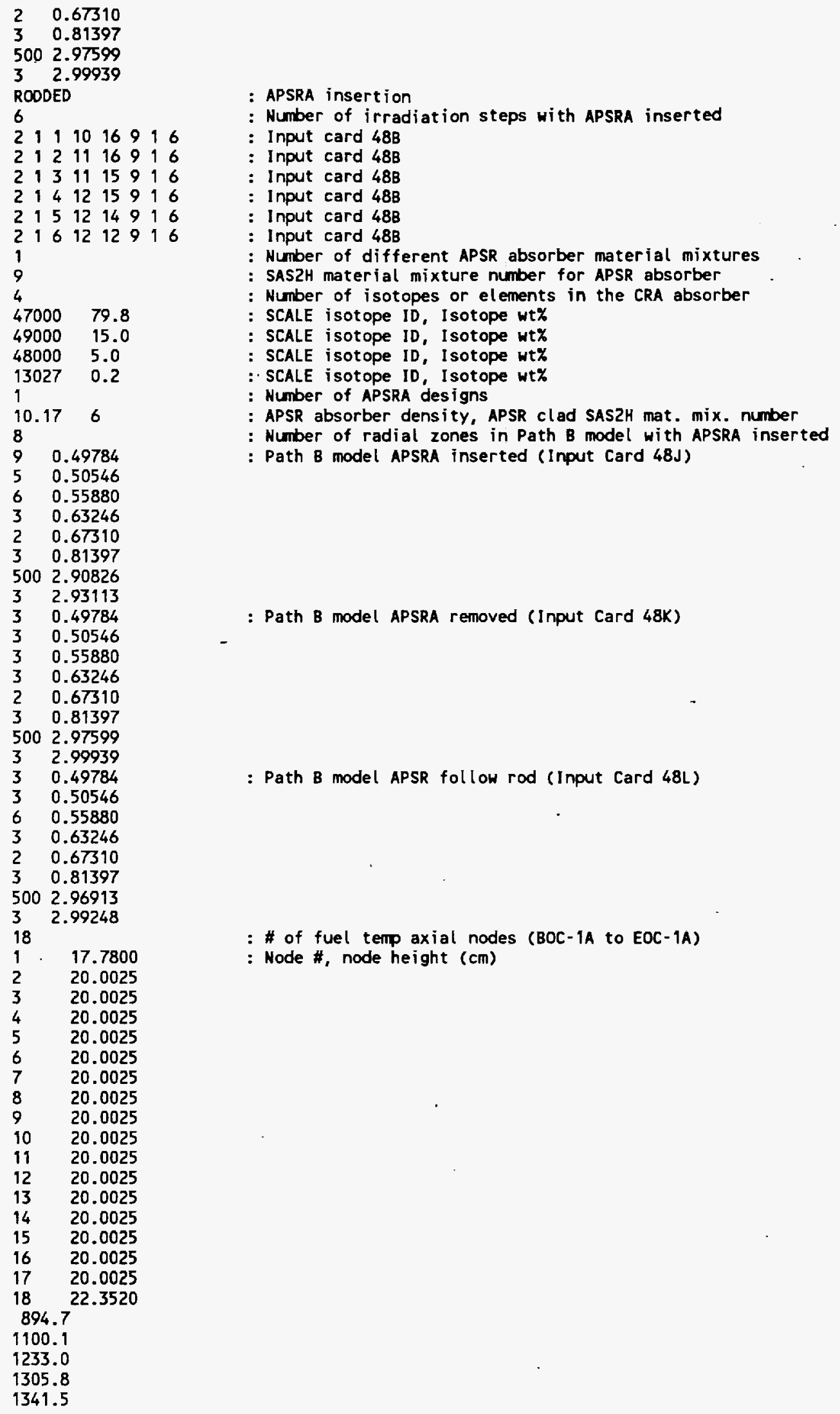


1357.9

1368.1

1380.0

1397.3

1420.2

1442.6

1449.8

1429.5

1388.0

1336.0

1269.4

1168.3

944.6

18

$1 \quad 17.7800$

$2 \quad 20.0025$

320.0025

$4 \quad 20.0025$

$5 \quad 20.0025$

$6 \quad 20.0025$

$7 \quad 20.0025$

$8 \quad 20.0025$

$9 \quad 20.0025$

$10 \quad 20.0025$

$11 \quad 20.0025$

$12 \quad 20.0025$

$13 \quad 20.0025$

$14 \quad 20.0025$

$15 \quad 20.0025$

$16 \quad 20.0025$

$17 \quad 20.0025$

$18 \quad 22.3520$

1080.1

1285.2

1358.8

1357.7

1325.9

1292.0

1266.0

1248.9

1237.5

1213.7

1097.8

999.0

993.9

1006.5

1041.6

1238.9

1270.0

1093.8

$18 \quad 17.7800$

: \# of fuel temp axial nodes (BOC-1B to Stpt2-1B)

: Node \#, node height $(\mathrm{cm})$

$2 \quad 20.0025$

$3 \quad 20.0025$

$4 \quad 20.0025$

$5 \quad 20.0025$

$6 \quad 20.0025$

$7 \quad 20.0025$

$8 \quad 20.0025$

$9 \quad 20.0025$

$10 \quad 20.0025$

1120.0025

$12 \quad 20.0025$

$13 \quad 20.0025$

$14 \quad 20.0025$

$15 \quad 20.0025$

$16 \quad 20.0025$

$17 \quad 20.0025$

$18 \quad 22.3520$

0.0232

: \# of mod spec vol axial nodes (BOC-1A to EOC-1A)

: Node \#, node height $(\mathrm{cm})$ 


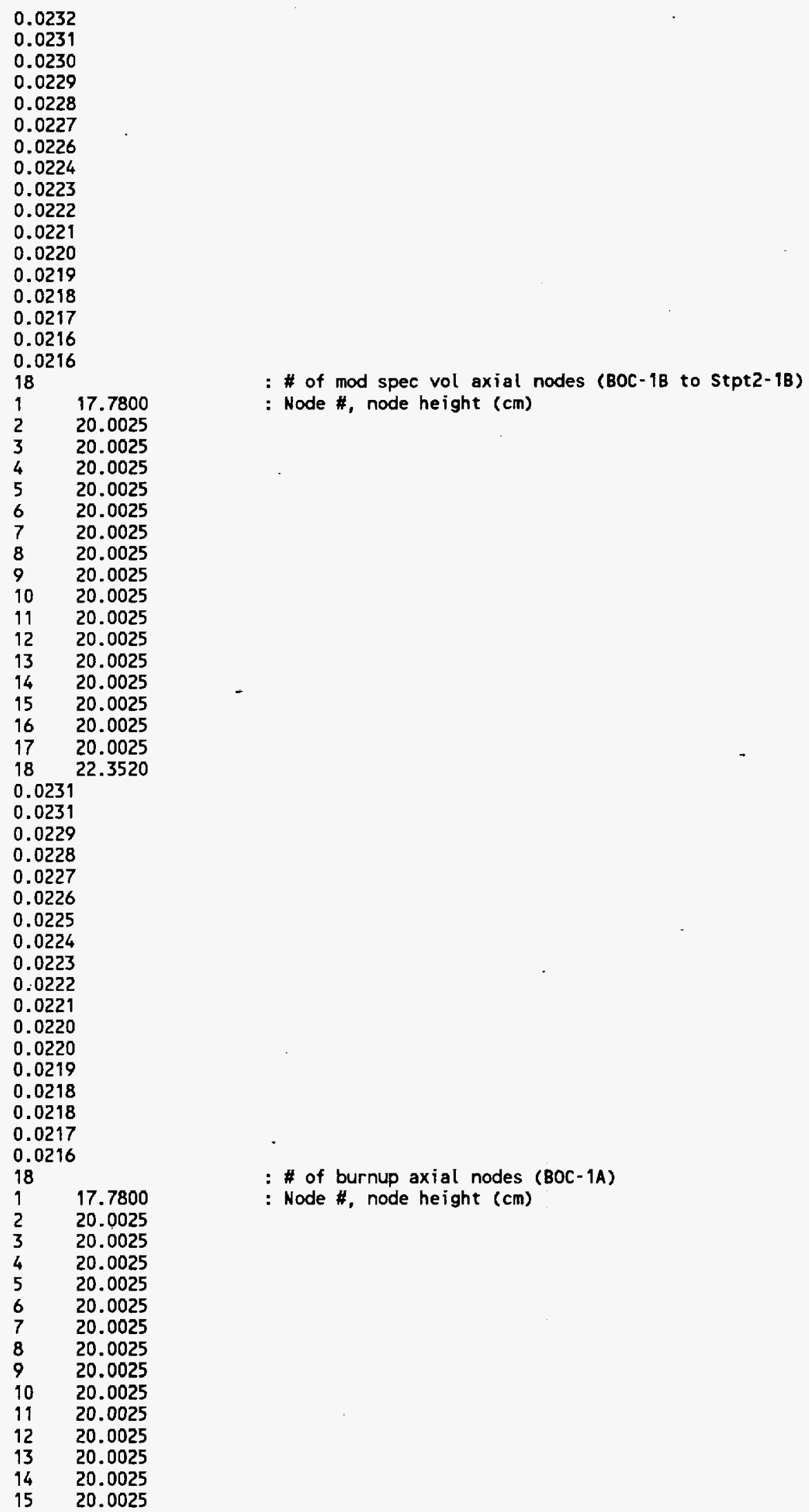


20.0025

20.0025

22.3520

0.0

0.0

0.0

0.0

0.0

0.0

0.0

0.0

0.0

0.0

0.0

0.0

0.0

0.0

0.0

0.0

18

2

6

7

$9 \quad 20.0025$

$10 \quad 20.0025$

$11 \quad 20.0025$

. $12 \quad 20.0025$

$13 \quad 20.0025$

$14 \quad 20.0025$

$15 \quad 20.0025$

$16 \quad 20.0025$

$17 \quad 20.0025$

$18 \quad 22.3520$

2.845

5.061

6.838

8.044

8.786

9.214

9.439

9.518

9.470

9.282

8.954

8.566

8.260

8.097

8.098

7.767

6.697

4.116

18

17.7800

20.0025

20.0025

20.0025

20.0025

20.0025

20.0025

20.0025

20.0025

$10 \quad 20.0025$

$11 \quad 20.0025$
: \# of burnup axial nodes (BOC-1B)

: Node \#, node height $(\mathrm{cm})$
: \# of burnup axial nodes (Stpt2-1B)

: Node \#, node height $(\mathrm{cm})$ 
Jul 30 11:36 1997 File Name: A26zi.dat BBA000000-01717-0200-00040 REV 00 ATTACHMENT XVIII - Page 7
20.0025
20.0025
20.0025
20.0025
20.0025
20.0025

$18 \quad 22.3520$

5.336

9.077

11.651

13.123

13.869

14.202

14.320

14.314

14.182

13.718

12.512

11.504

11.196

11.180

11.693

12.576

11.509

7.278 


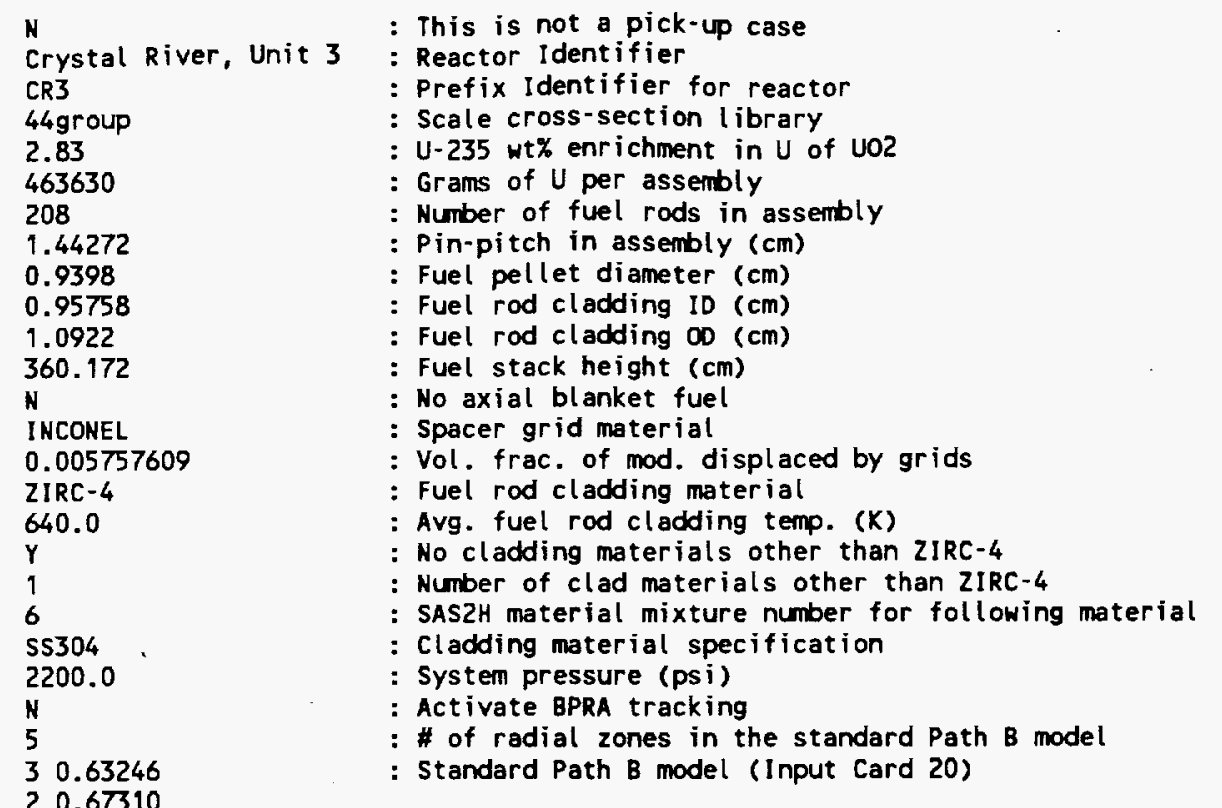

: \# of cross-section libraries per irradiation step : SASZH output print level

: Zone mesh factor for XSDRNPM

: No special XSDRMPM control parameter specs.

: \# of insertion reactor cycles

: Insertion reactor cycle identifier

: \# of stpts in cycle

: Stpt EFPD

: Length to stpt in calendar days

: Downt ime at stpt

: Days of downtime at EOC

: Total cycle EFPD

: Total cycle length in calendar days

: Integer position of assembly in cycle

: Insertion reactor cycle identifier

: \# of stpts in cycle

: Stpt EFPD

: Length to stpt in calendar days

: Downt ime at stpt

: Stpt EFPD

: Length to stpt in calendar days

: Downtime at stpt

: Days of dount ime at EOC

: Total cycle EFPD

: Total cycle length in calendar days

: Integer position of assembly in cycle

: Insertion reactor cycle identifier

: \# of stpts in cycle

: Stpt EFPD

: Length to stpt in calendar days

: Dountime at stpt

: Days of downt ime at EOC

: Total cycle EFPD

: Total cycle length in calendar days

: Integer position of assembly in cycle

: Insertion reactor cycle identifier

: \# of stpts in cycle

: Stpt EFPD

: Length to stpt in calendar days

: Downt ime at stpt

: Stpt EFPD 


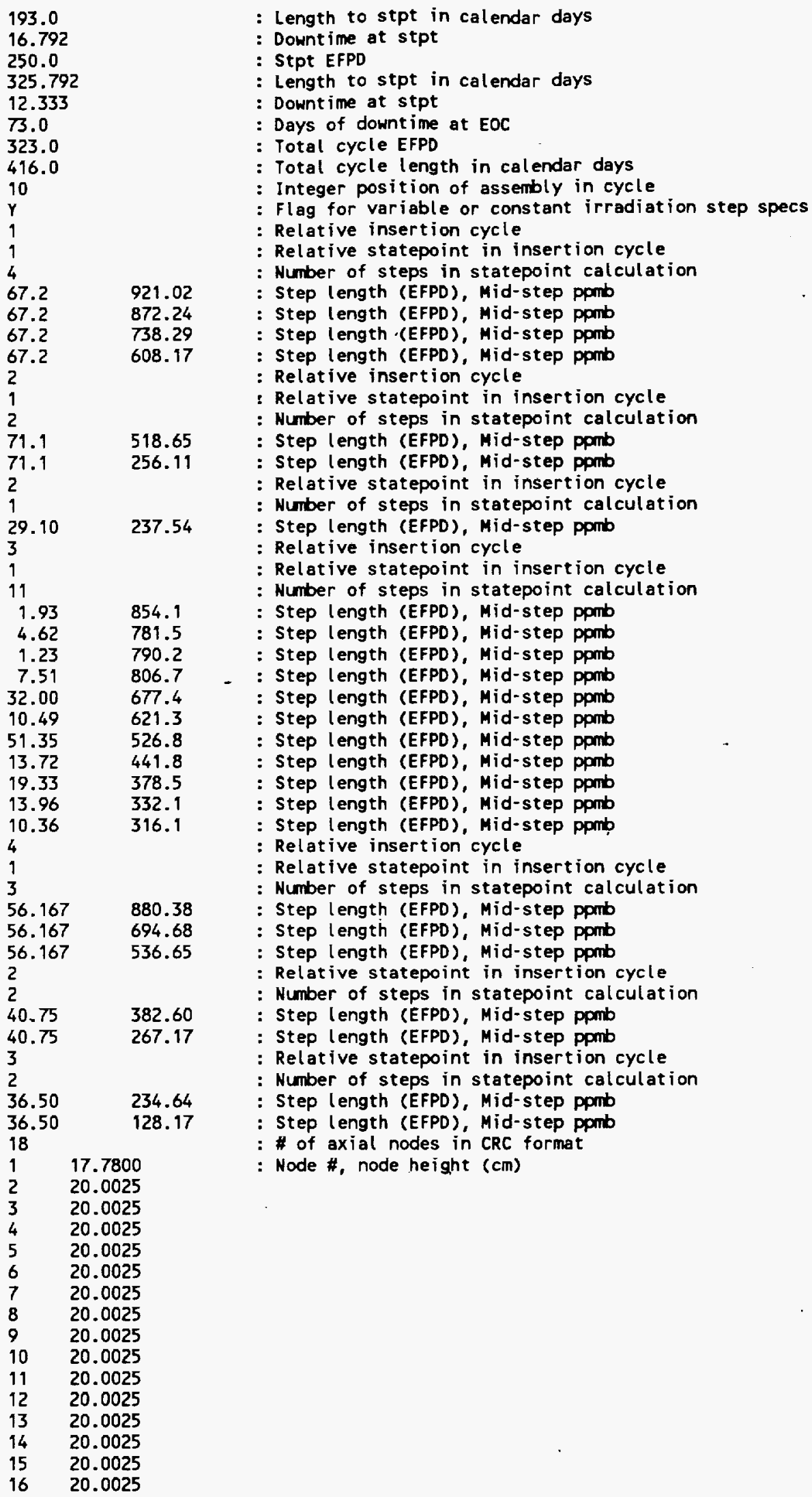




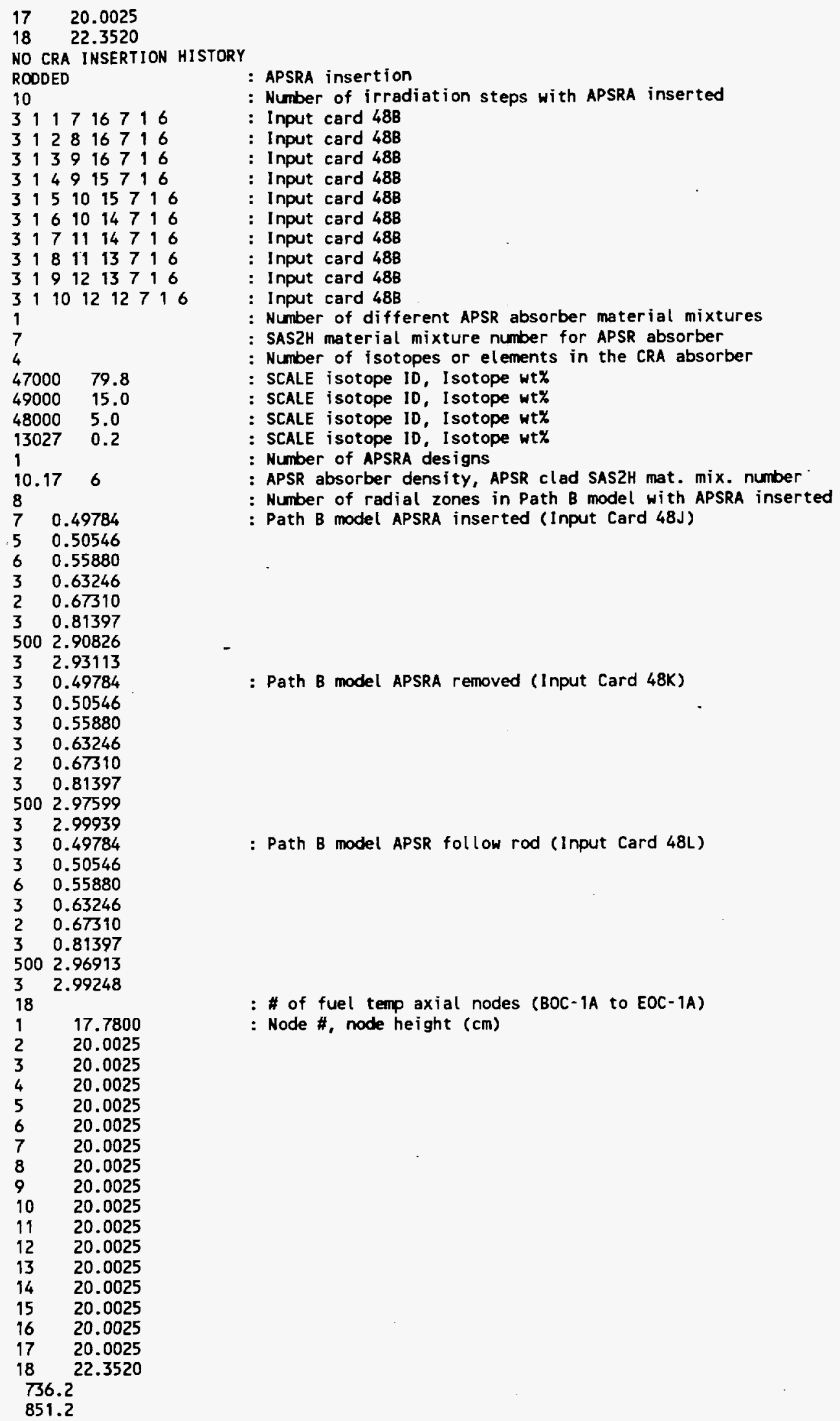

851.2

: APSRA insertion

: Number of irradiation steps with APSRA inserted

: Input card 488

: Input card 48B

: Input card 488

: Input card 488

: Input card 488

- Input card 488

: Input card 488

: Input card 488

: Input card 488

: Input card 488

: Number of different APSR absorber material mixtures

: SAS2H material mixture number for APSR absorber

: Number of isotopes or elements in the CRA absorber

: SCALE isotope ID, Isotope wt\%

: SCALE isotope 1D, I sotope wt\%

: SCALE isotope ID, I sotope WT\%

: SCALE isotope 10, I sotope wt\%

: Number of APSRA designs

: APSR absorber density, APSR clad SASZH mat. mix. number

: Number of radial zones in Path $B$ model with APSRA inserted

: Path B model APSRA inserted (Input Card 48J)

: Path B model APSRA removed (Input Card 48K)

: Path B model APSR follow rod (Input Card 48L)

\author{
: \# of fuel temp axial nodes (BOC-1A to EOC-1A)
}

: Node \#, node height $(\mathrm{cm})$ 
Jul 30 11:37 1997 File Name: A28zi.dat BBA000000-01717-0200-00040 REV 00 ATtACHMENT XIX - Page 4

931.2

978.1

1002.3

1015.2

1023.4

1030.9

1039.7

1049.5

1057.3

1058.6

1050.4

1034.4

1013.1

979.7

912.5

770.4

18

17.7800

: \# of fuel temp axial nodes (BOC-1B to Stpt2-1B)

20.0025

: Node \#, node height $(\mathrm{cm})$

20.0025

20.0025

20.0025

20.0025

20.0025

20.0025

20.0025

20.0025

20.0025

20.0025

20.0025

20.0025

20.0025

20.0025

20.0025

22.3520

833.2

976.4

1035.4

1043.6

1031.3

1014.9

1001.4

992.7

988.6

988.4

991.5

997.7

1006.3

1016.4

1026.4

1026.7

988.9

845.1

18

17.7800

\# of fuel temp axial nodes (Stpt2-1B to EOC-1B)

20.0025

: Node \#, node height $(\mathrm{cm})$ 
Jul 30 11:37 1997 file Name: A28zi.dat BBA000000-01717-0200-00040 REV do ATTACHMENT XIX - Page 5

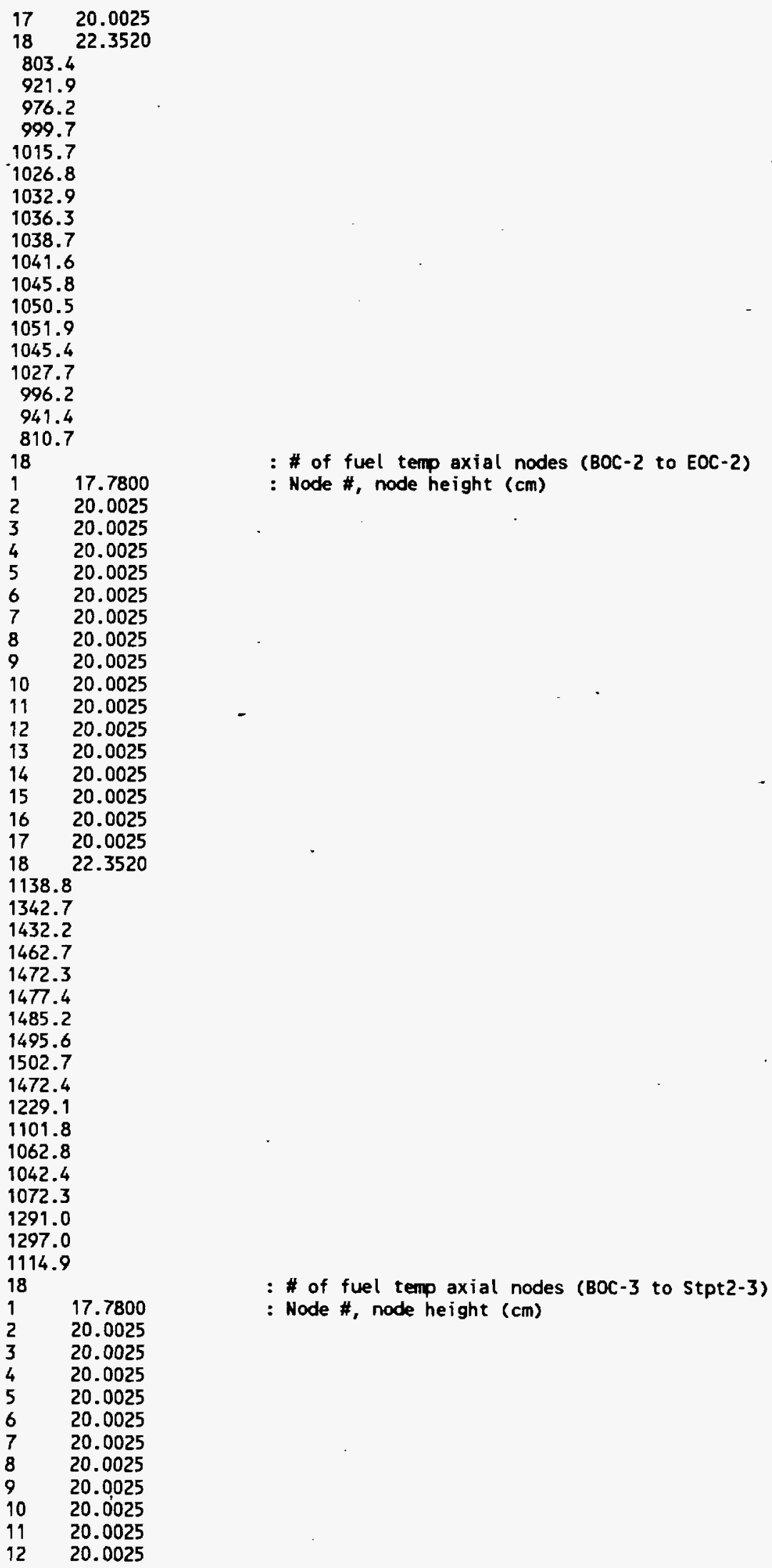


$18 \quad 22.3520$

982.8

1136.1

1199.8

1209.1

1199.9

1186.1

1174.0

1168.1

1175.9

1208.6

1256.3

1295.9

1316.0

1316.2

1299.1

1282.4

1236.5

1066.8

17.7800

20.0025

20.0025

20.0025

20.0025

20.0025

20.0025

20.0025

20.0025

20.0025

20.0025

20.0025

20.0025

20.0025

20.0025

20.0025

20.0025

$18 \quad 22.3520$

1010.3

1130.6

1156.0

1146.9

1132.6

1121.3

1114.5

1111.8

1115.2

1131.3

1152.6

1166.6

1173.1

1180.6

1187.8

1178.8

1153.8

1049.9

18

1

17.7800

20.0025

20.0025

20.0025

20.0025

20.0025

.20 .0025

20.0025
: \# of fuel temp axial nodes (Stpt2-3 to Stpt3-3)

: Node \#, node height $(\mathrm{cm})$
: \# of mod spec vol axial nodes (BOC-1A to EOC-1A)
: Node \#, node height $(\mathrm{cm})$ 


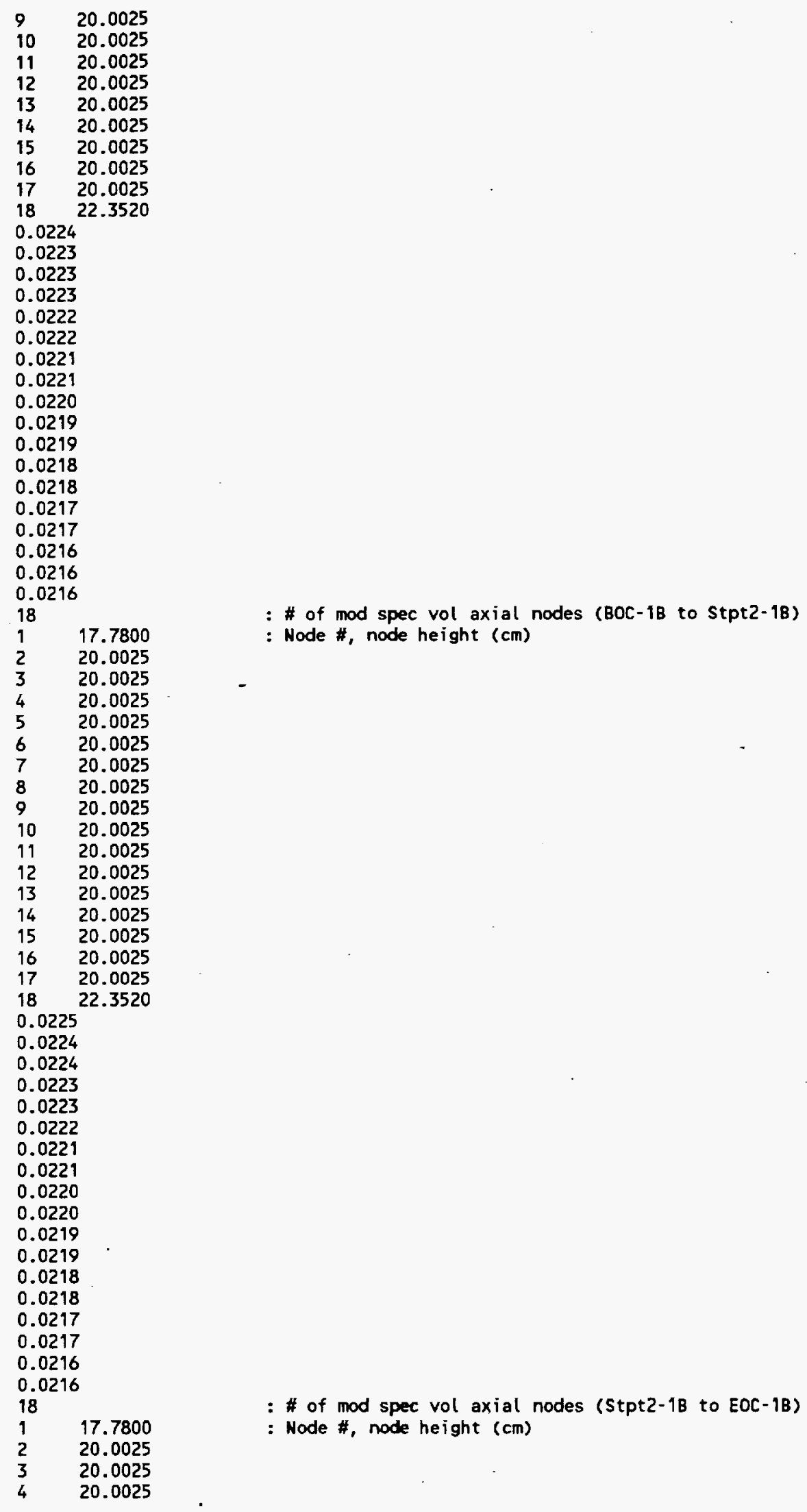




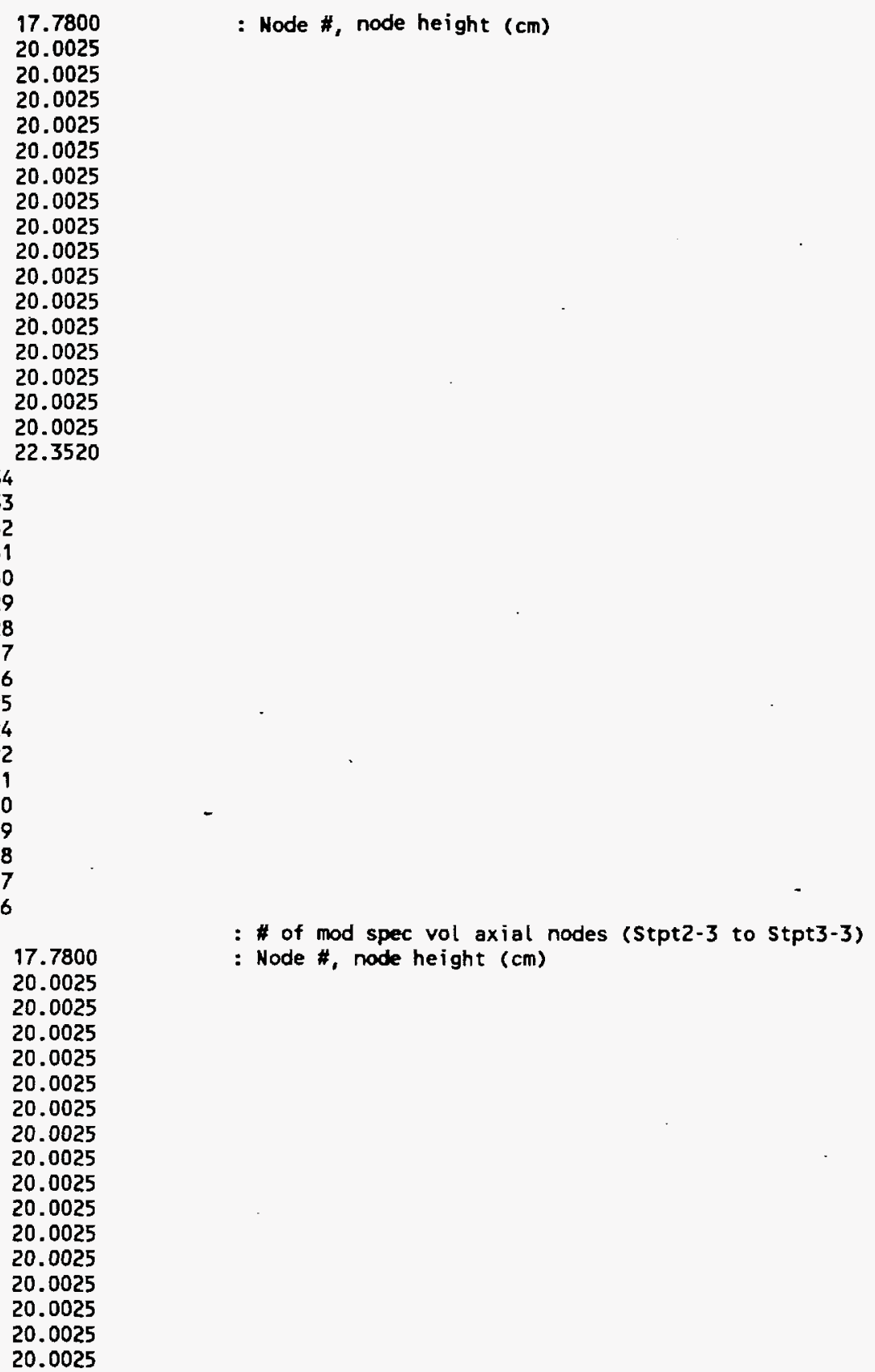




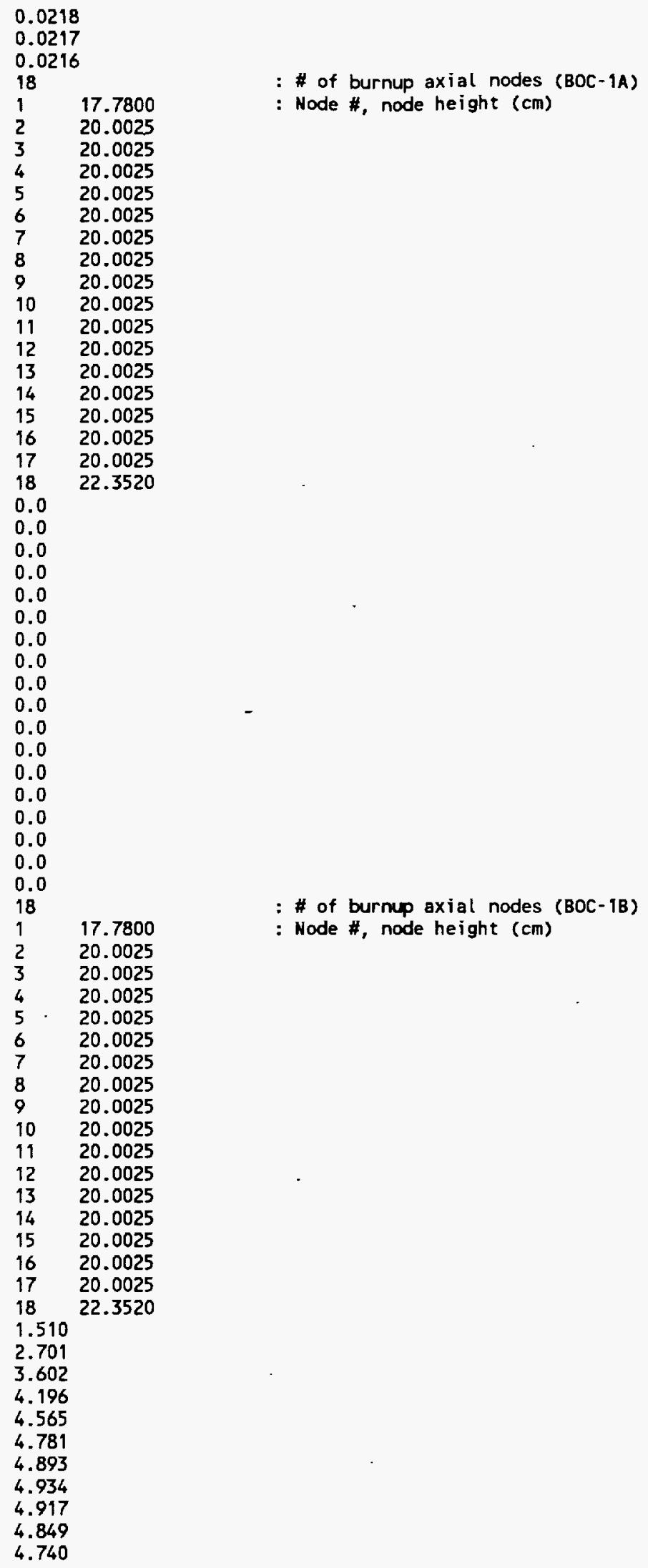




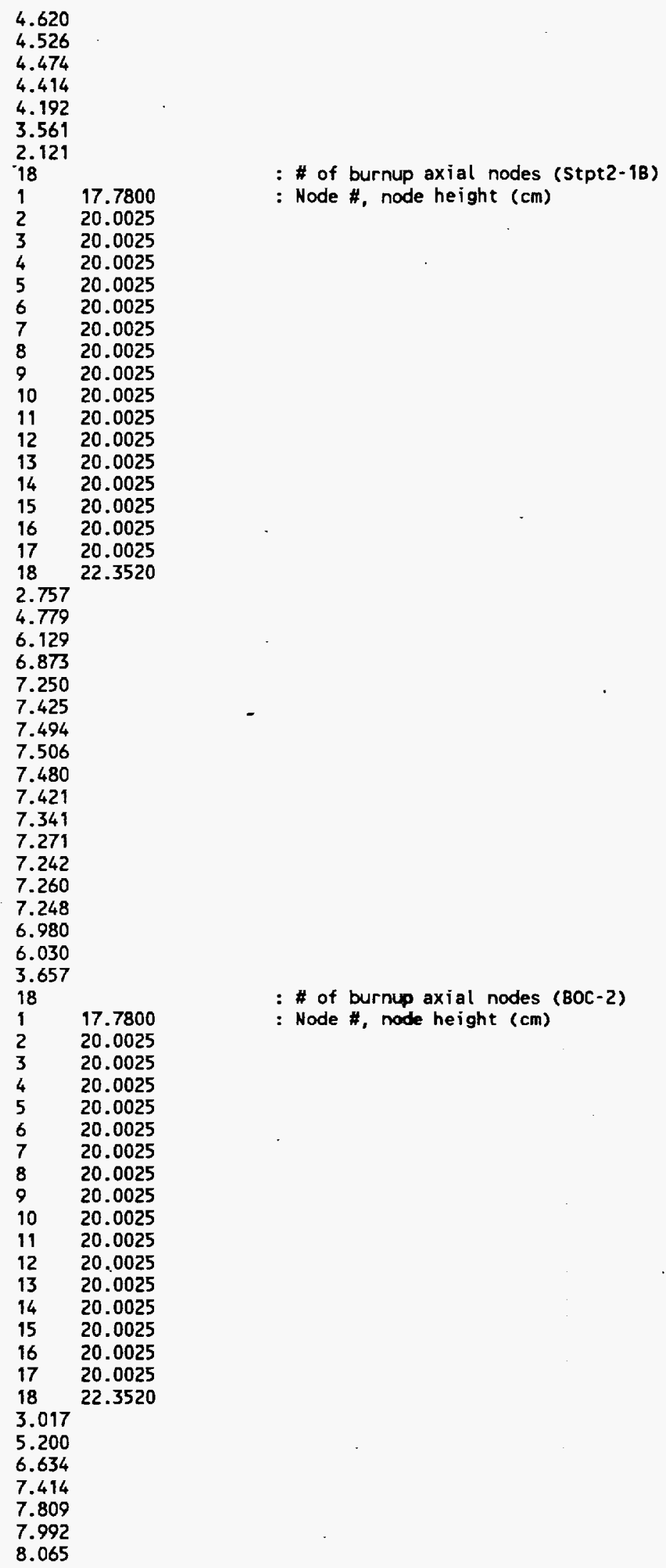


Jul 30 11:37 1997 File Name: A28zi.dat BBA000000-01717-0200-00040 REV 00 ATTACHMENT XIX - Page 12

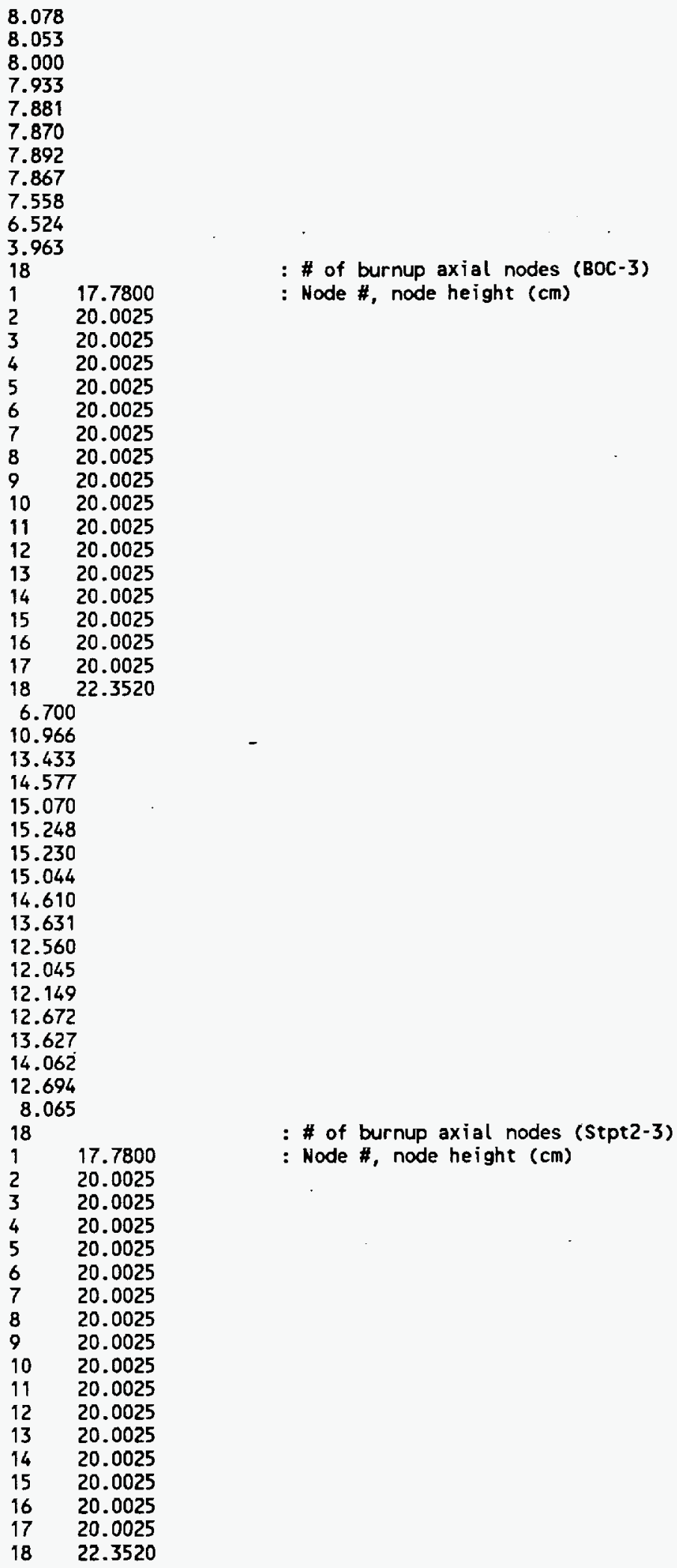


Jul 30 11:37 1997 File Name: A28zi.dat BBA000000-01717-0200-00040 REV 00 ATTACHMEnT XIX - Page 13

20.263

20.898

21.104

21.072

20.869

20.464

19.642

18.816

18.517

18.757

19.343

20.264

20.525

18.523

11.954

18

$1 \quad 17.7800$

20.0025

20.0025

20.0025

20.0025

20.0025

20.0025

20.0025

20.0025

20.0025

20.0025

20.0025

20.0025

20.0025

20.0025

20.0025

20.0025

$18 \quad 22.3520$

10.947

17.603

21.346

22.952

23.563

23.738

23.686

23.477

23.089

22.330

21.595

21.379

21.699

22.398

23.432

23.658

21.413

13.987

\author{
: \# of burnup axial nodes (Stpt3-3) \\ : Node \#, node height (cm)
}




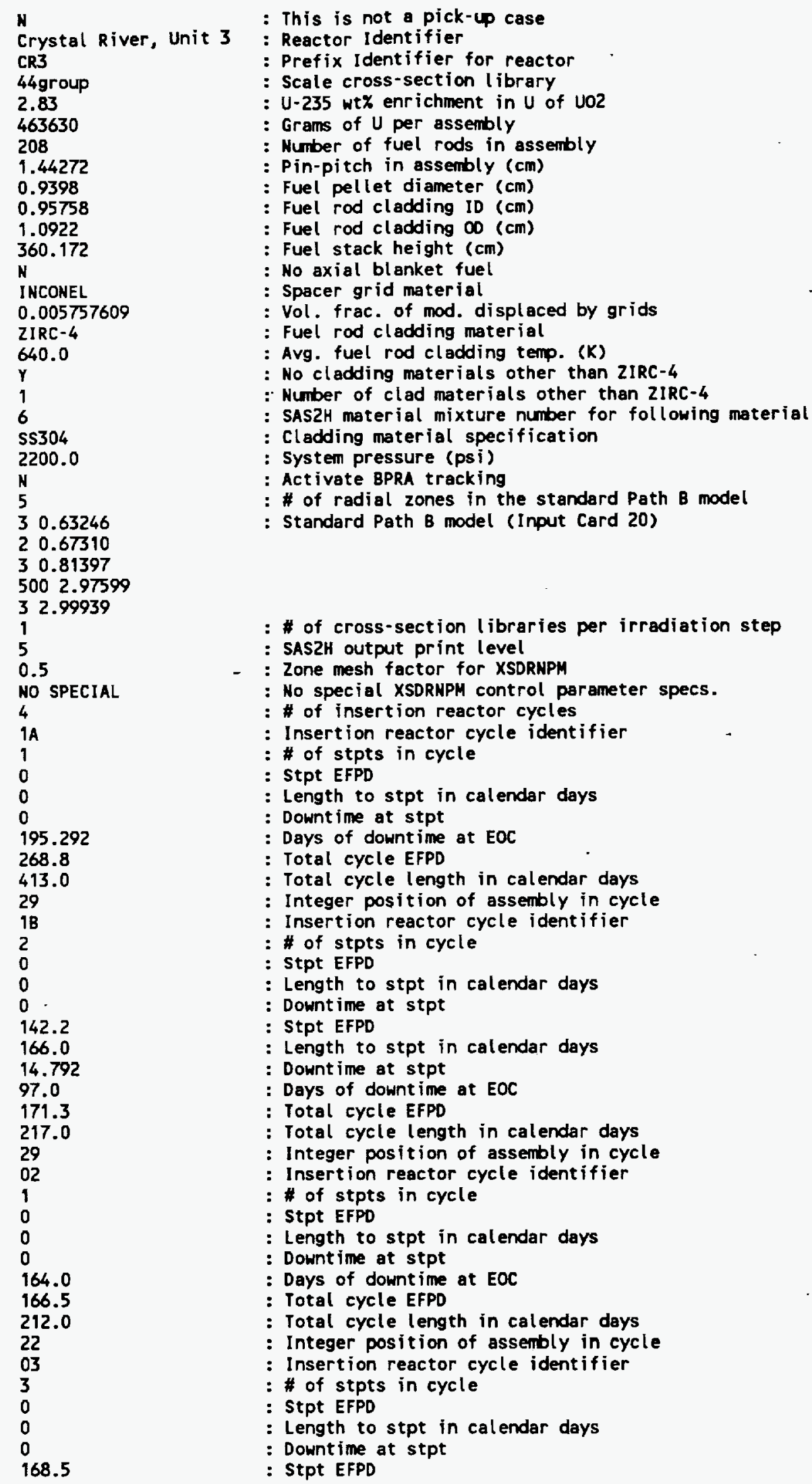




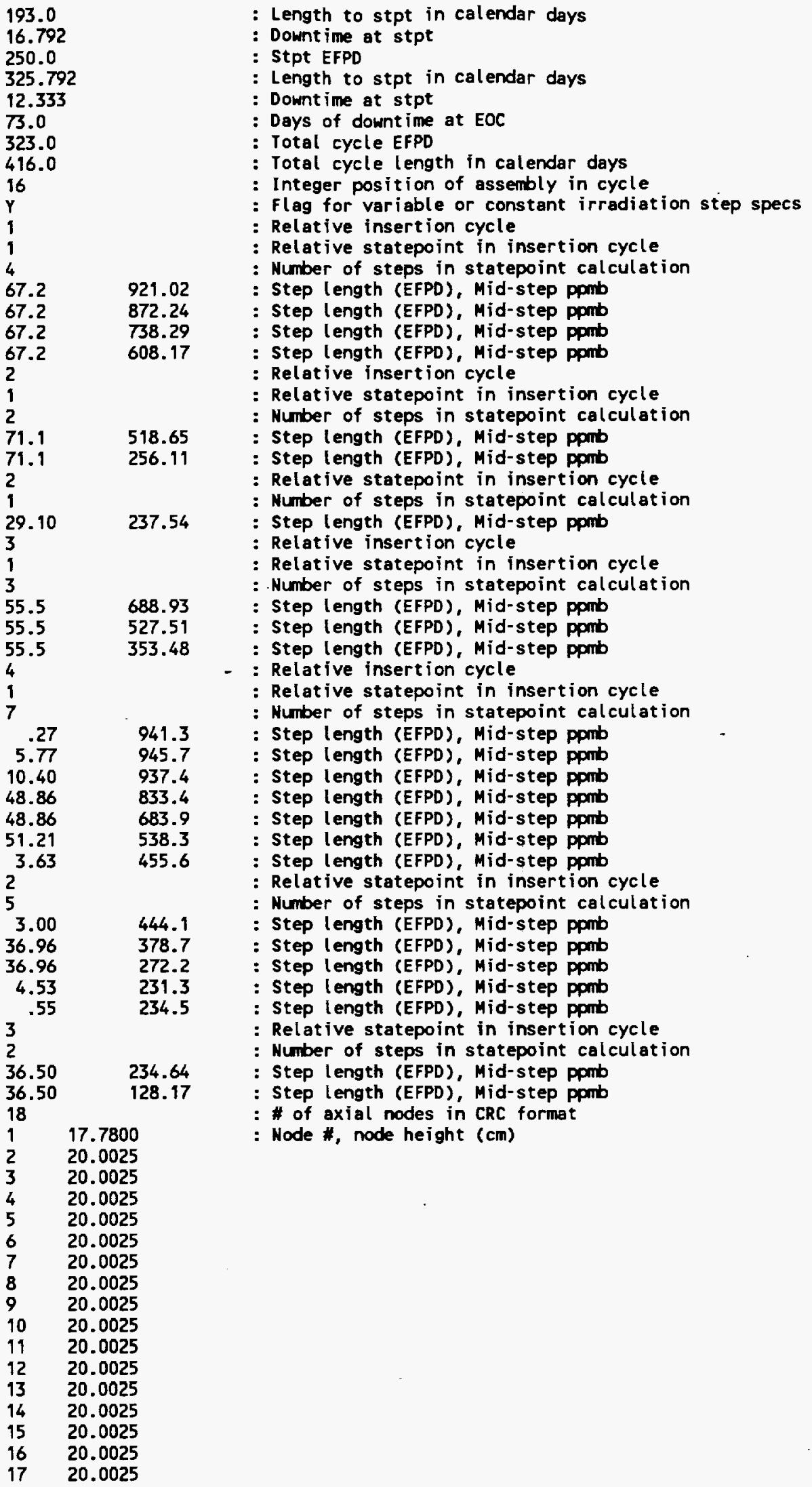




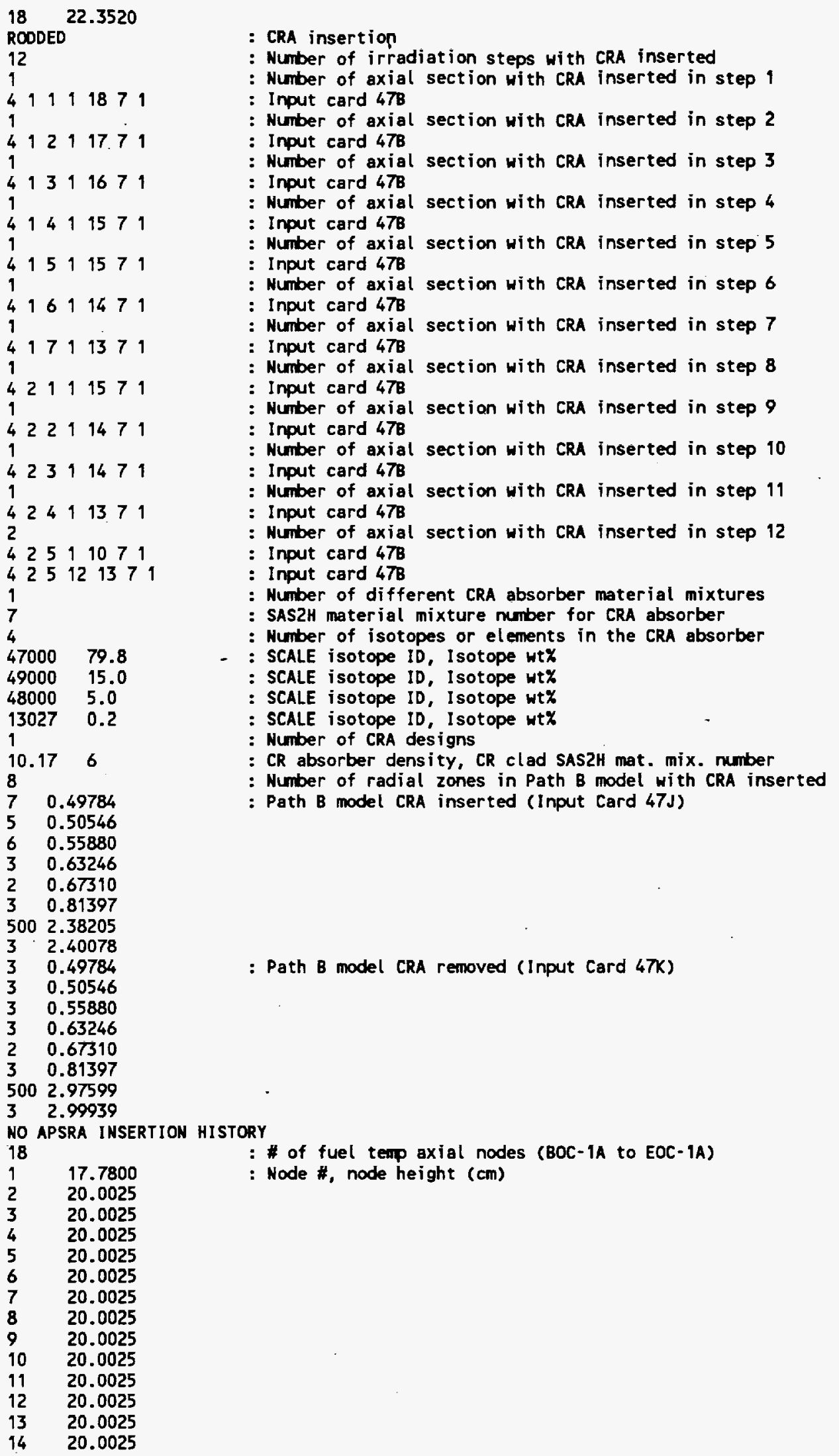


Apr 03 15:02 1997 File Name: A29i.dat BBA000000-01717-0200-00040 REV 00 ATtACHMENT XX - Page 4

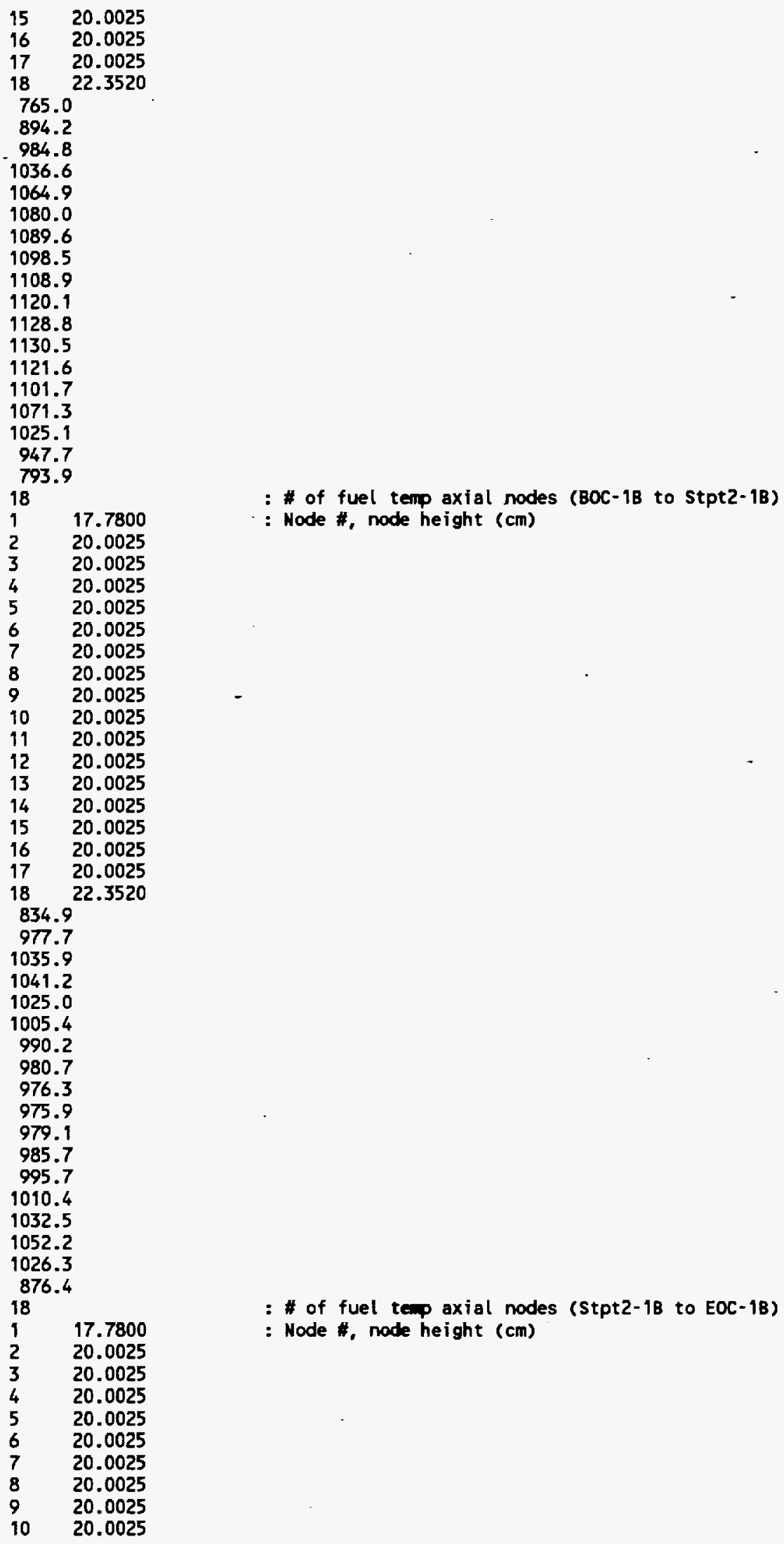


Apr 03 15:02 1997 File Name: A29i.dat BBA000000-01717-0200-00040 REV 00 ATtACHMENT XX - Page 5

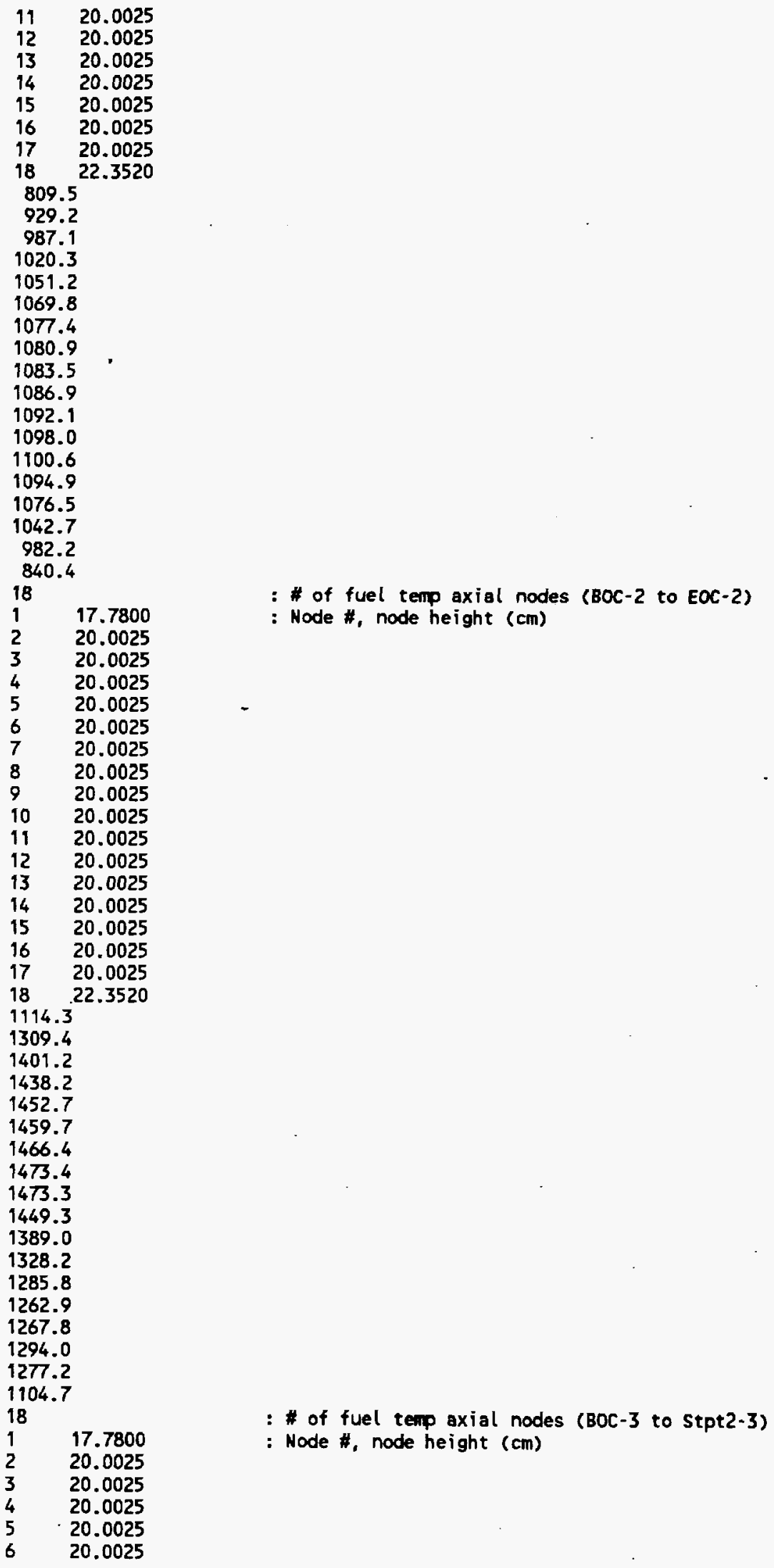


Apr 03 15:02 1997 Fille Name: A29i.dat BBA000000-01717-0200-00040 REv 00 ATTACHMENT XX - Page 6

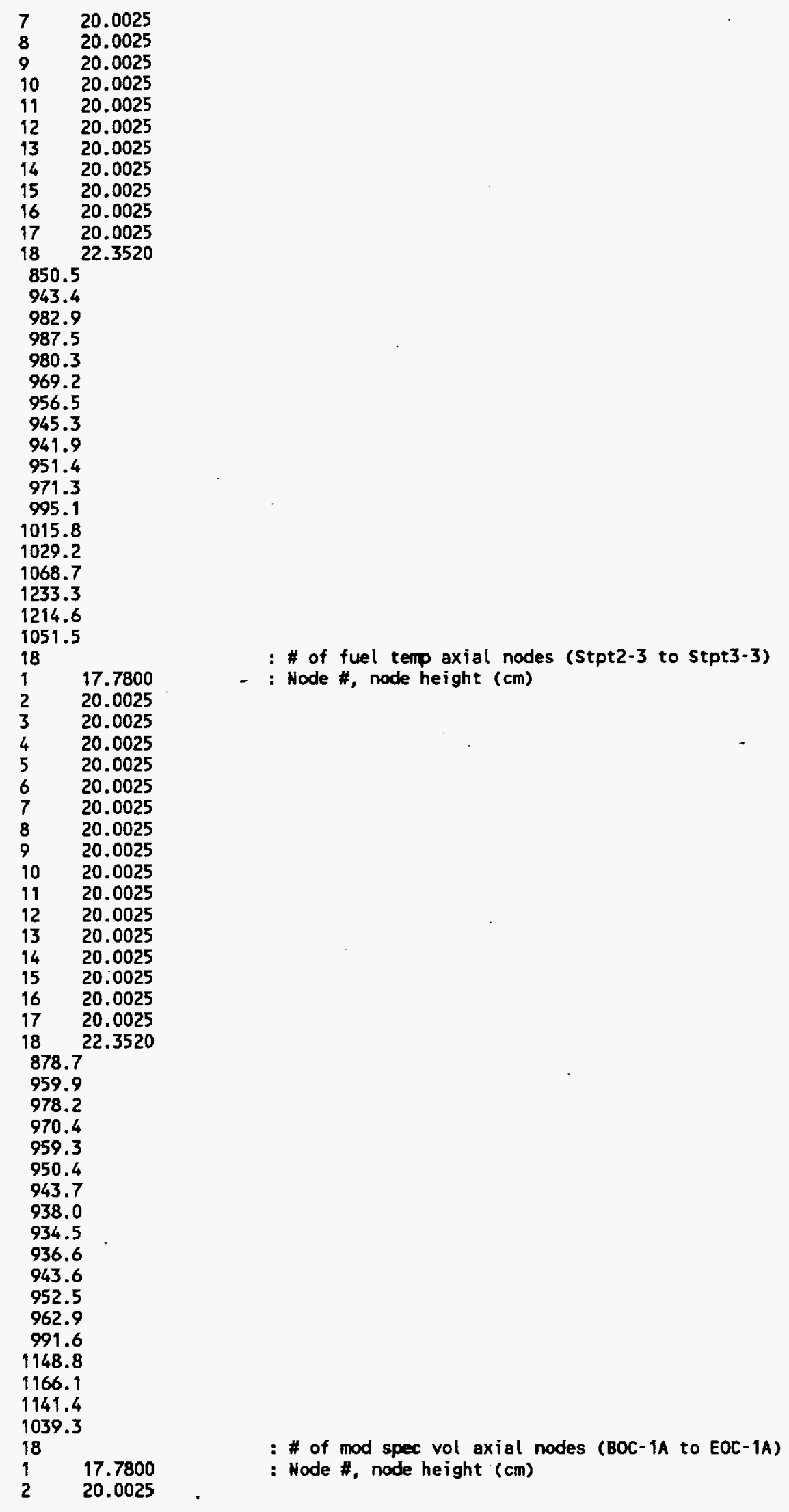




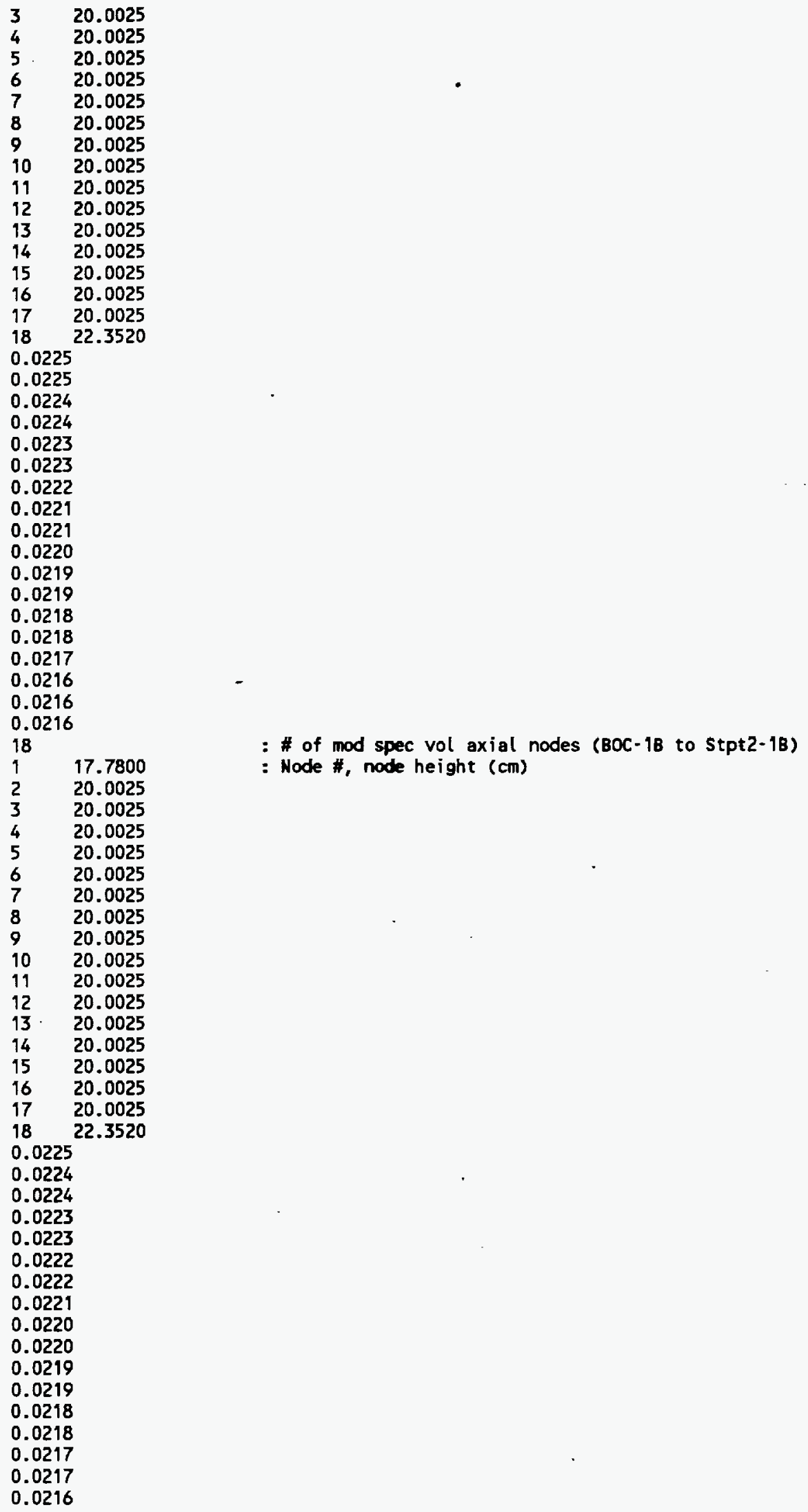


apr 03 15:02 1997 File Name: A29i.dat B8A000000-01717-0200-00040 REV 00 ATtACHMENT XX - Page 8

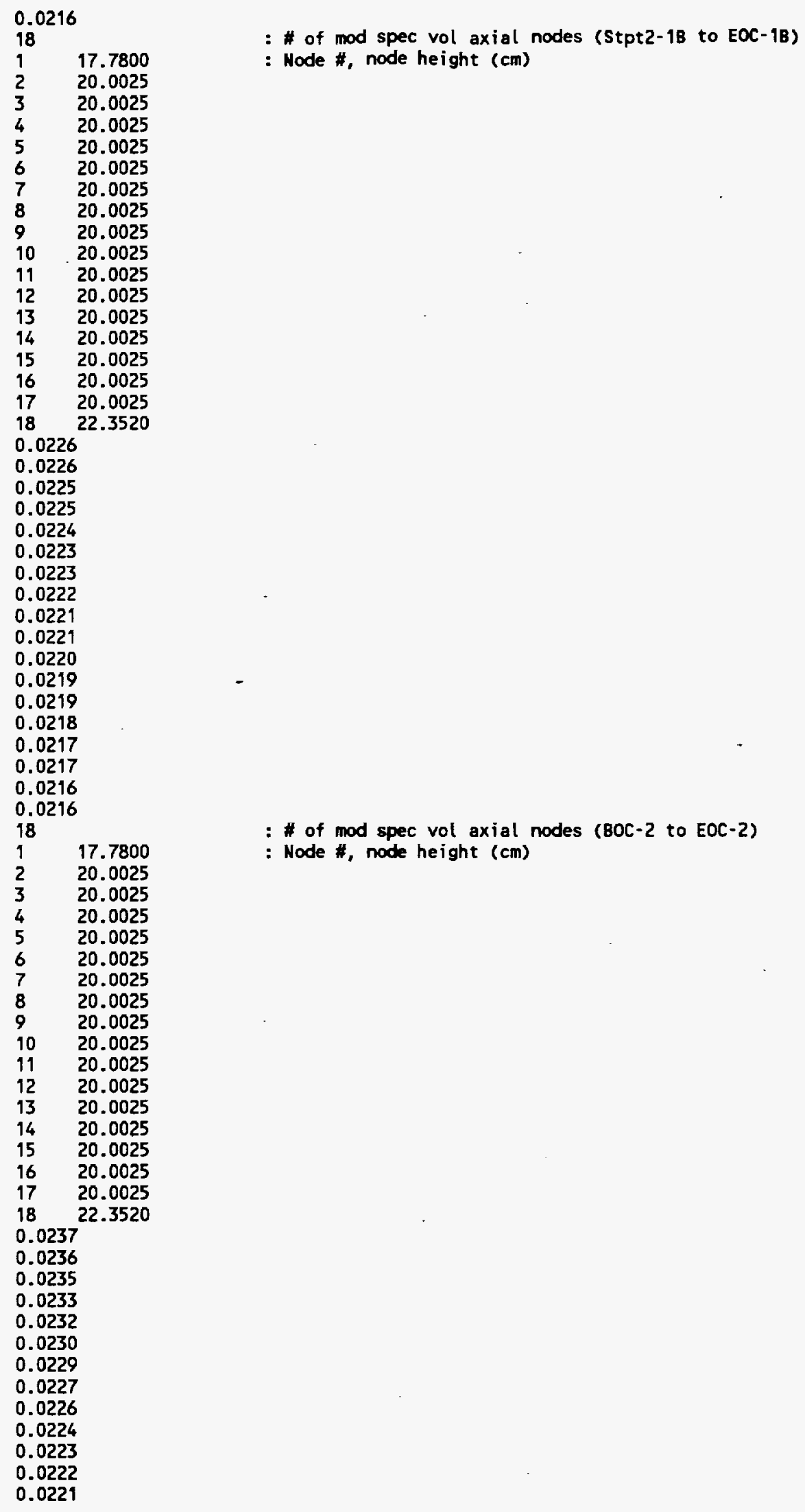


Apr 03 15:02 1997 File Name: A29i.dat BBA000000-01717-0200-00040 REV 00 ATTACHMENT XX - Page 9

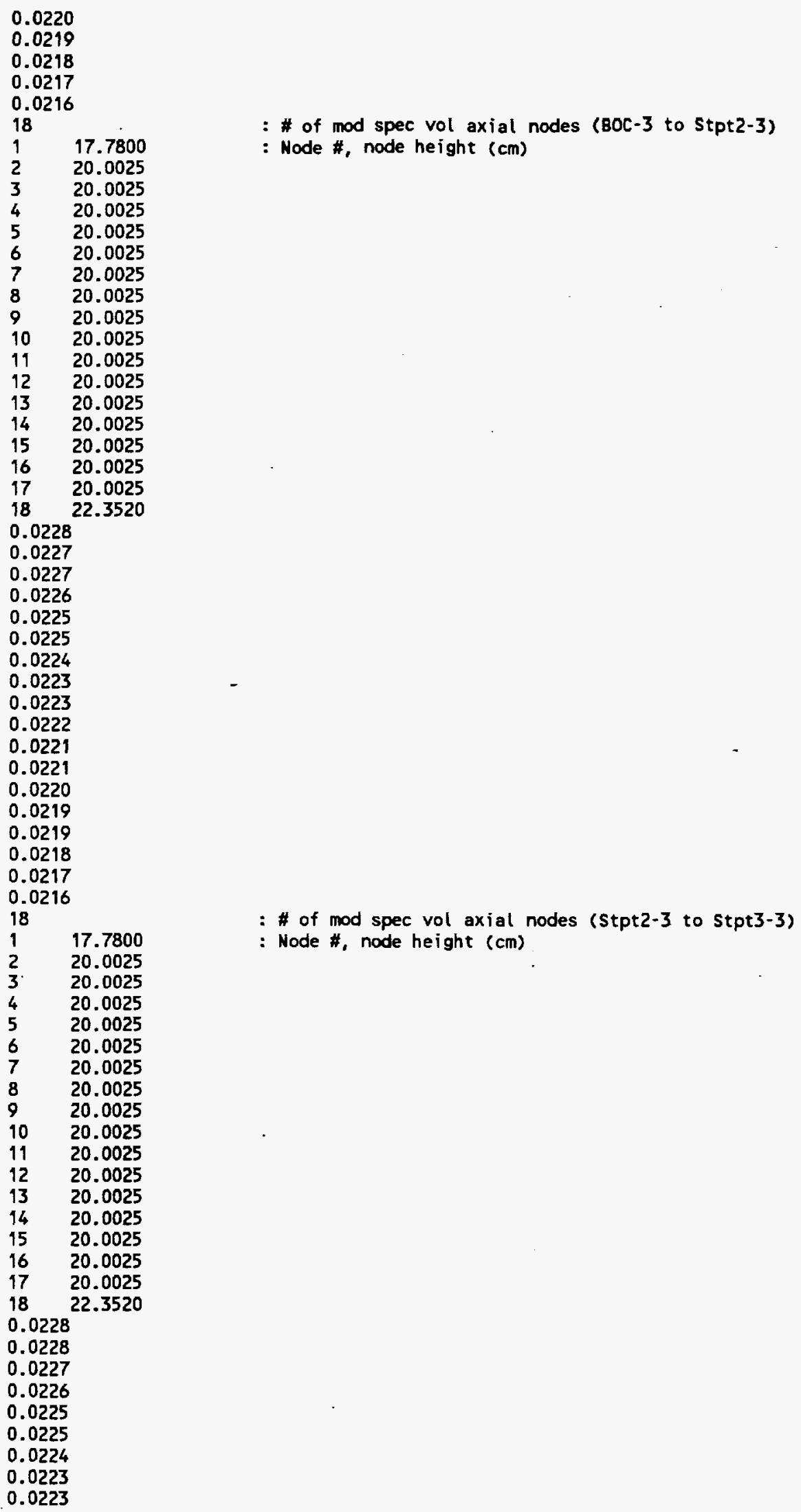




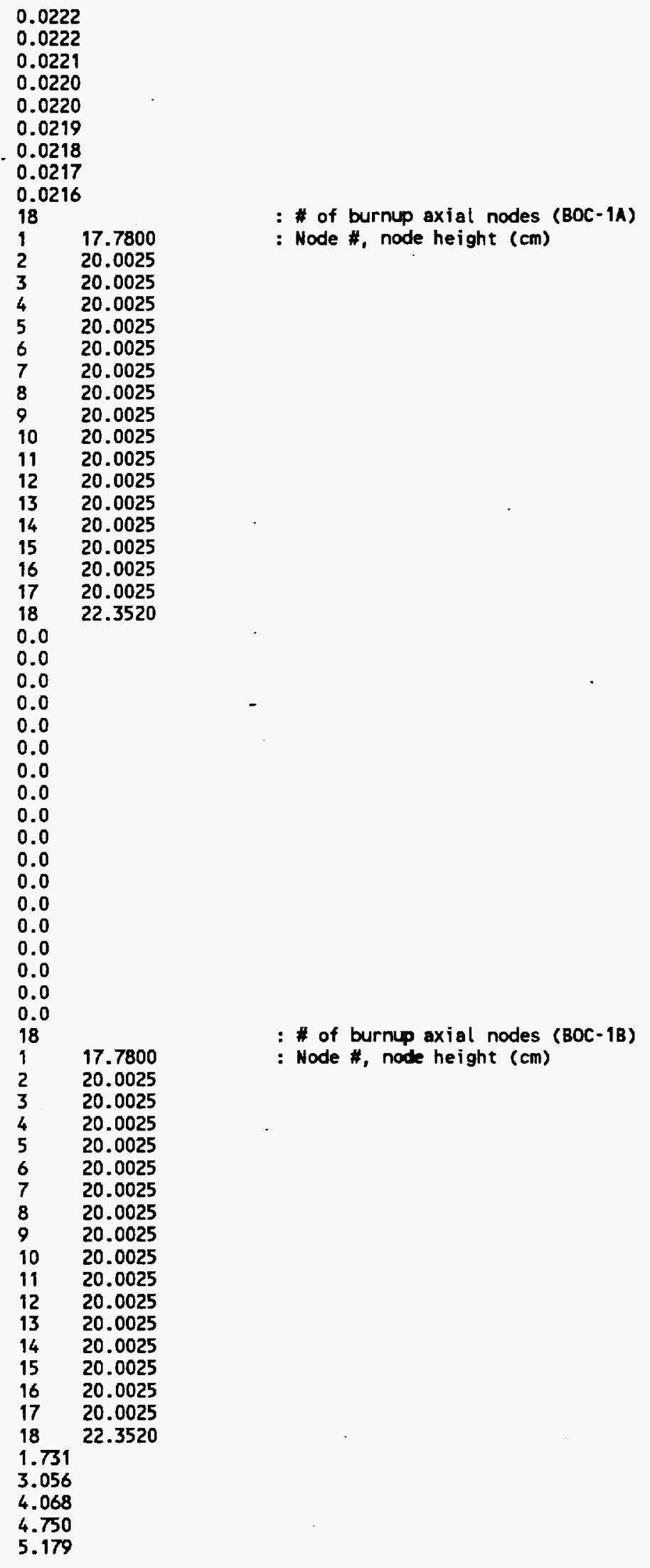


Apr 03 15:02 1997 File Name: A29i.dat B8A000000-01717-0200-00040 REV 00 ATtACHAENT XX - Page 11

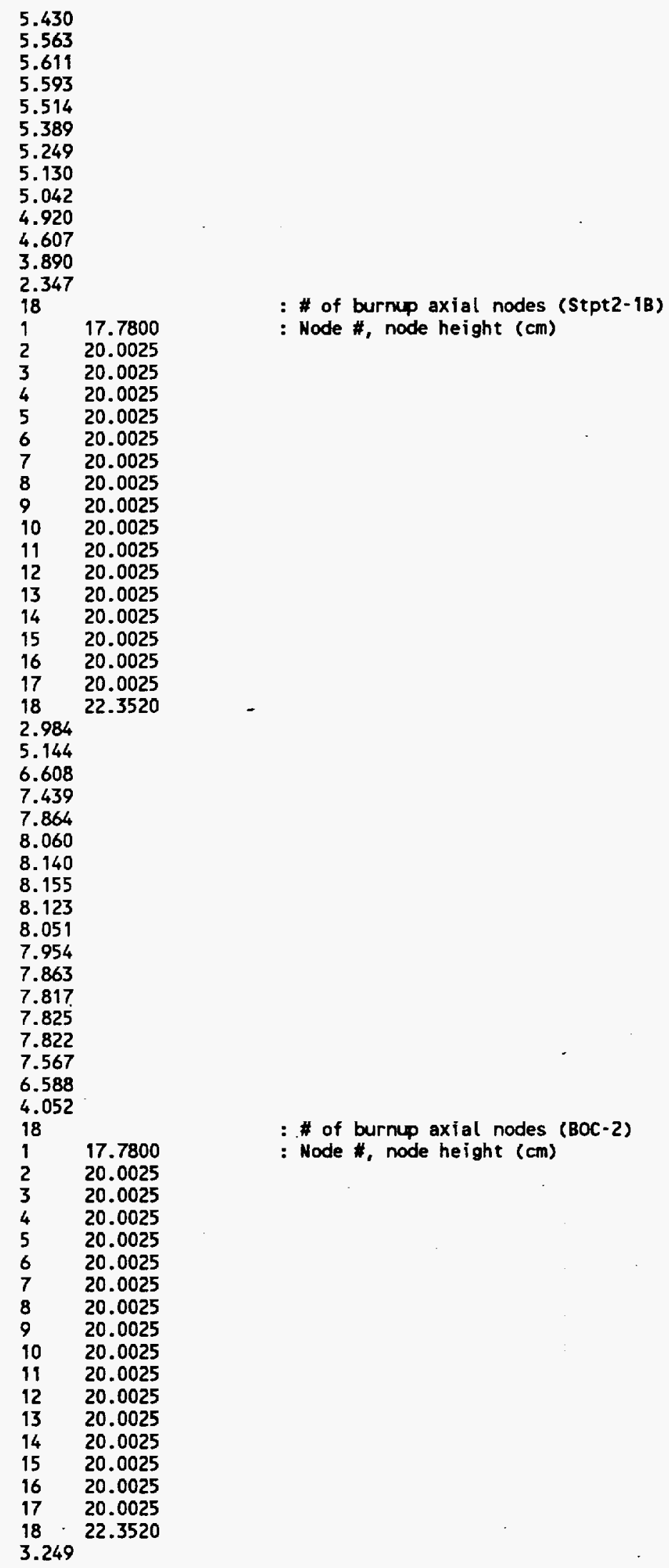


Apr 03 15:02 1997 File Name: A29i.dat BBA000000-01717-0200-00040 REV 00 ATtACHment XX - Pege 12

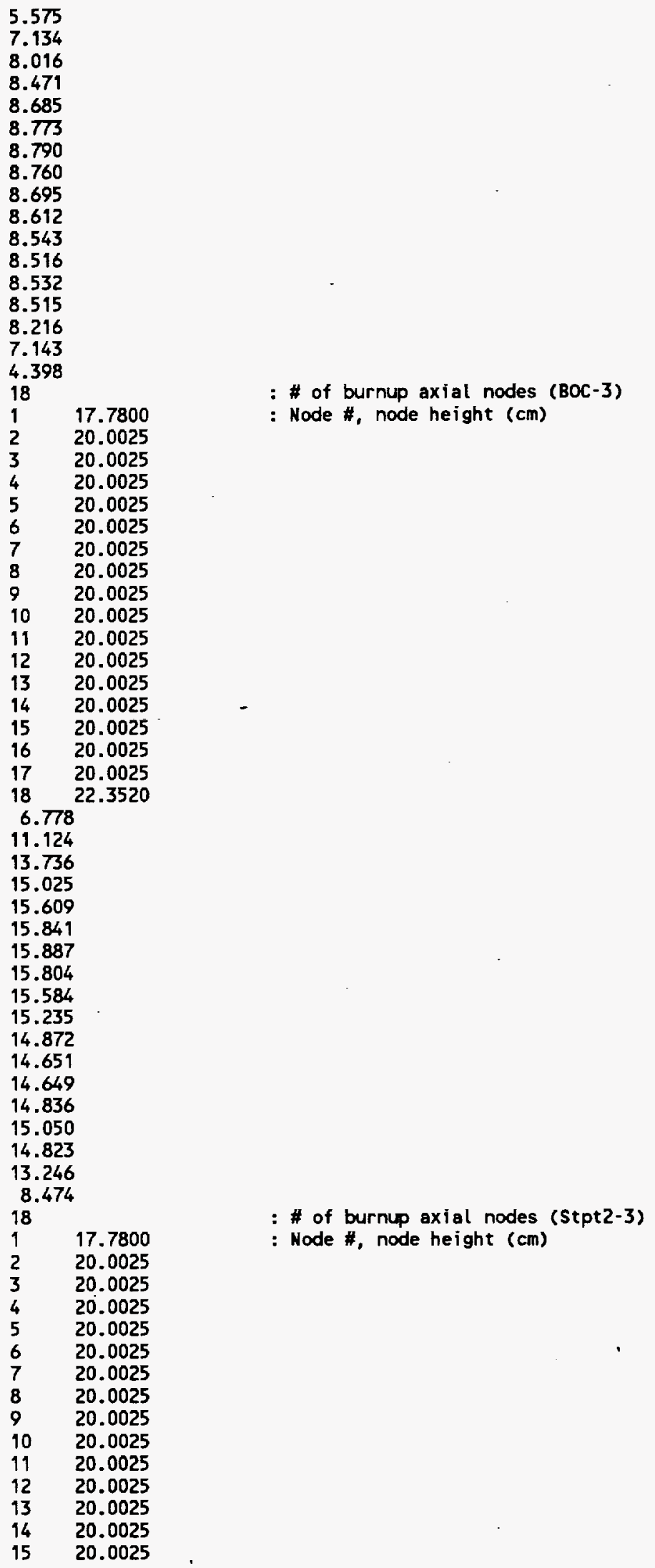


Apr 03 15:02 1997 File Name: A29i.dat BBA000000-01717-0200-00040 REV 00 ATTACHMENT XX - Pege 13

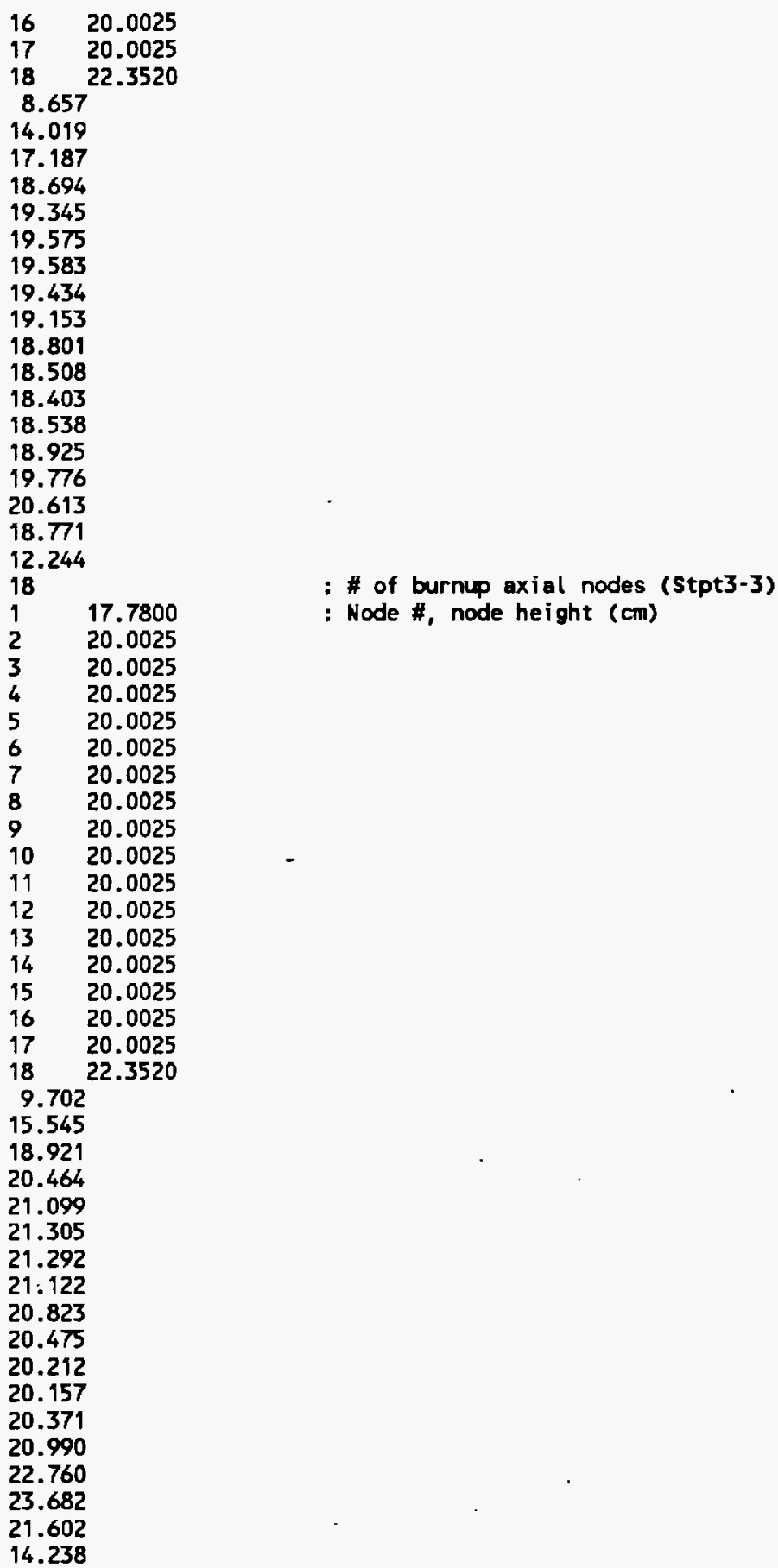




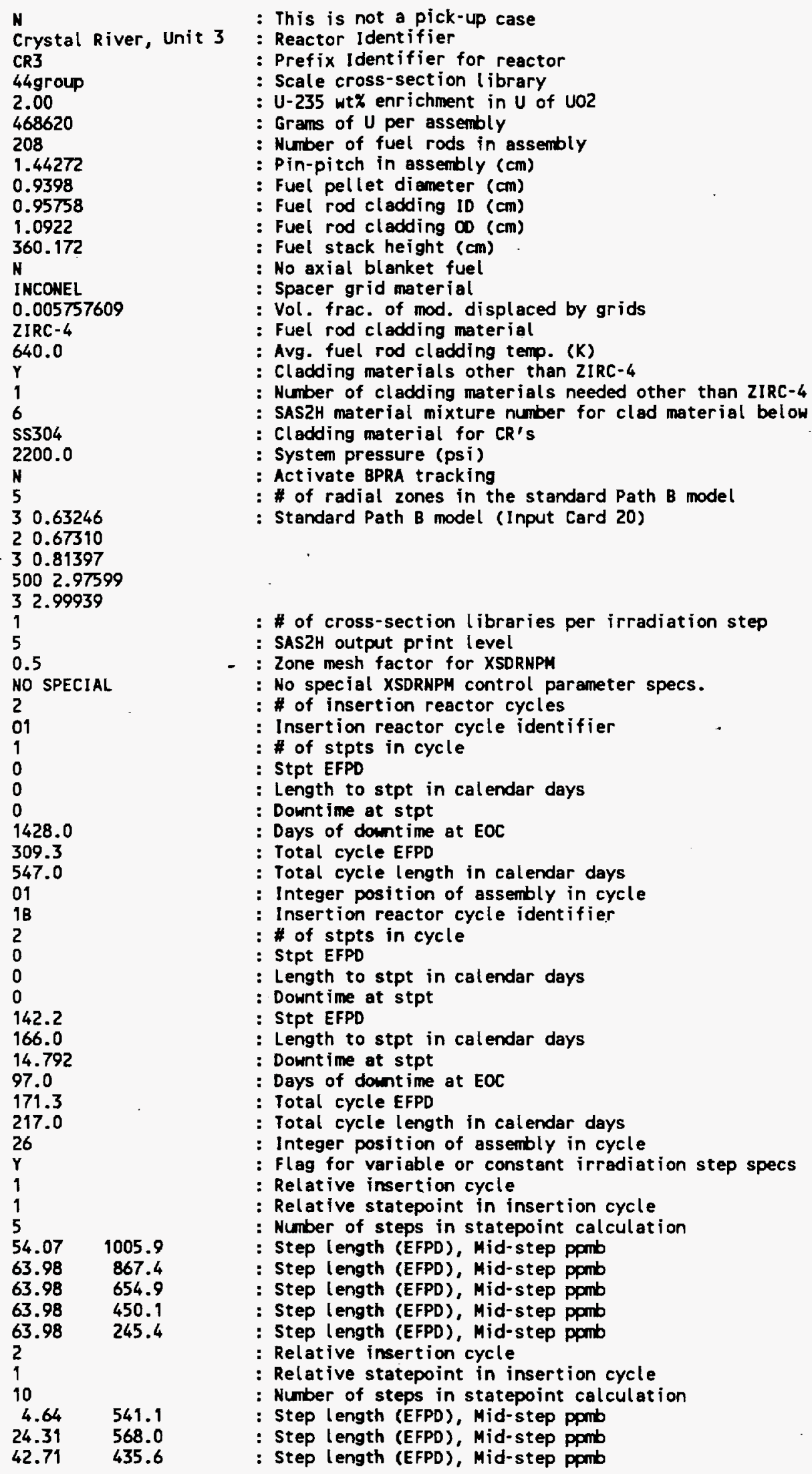




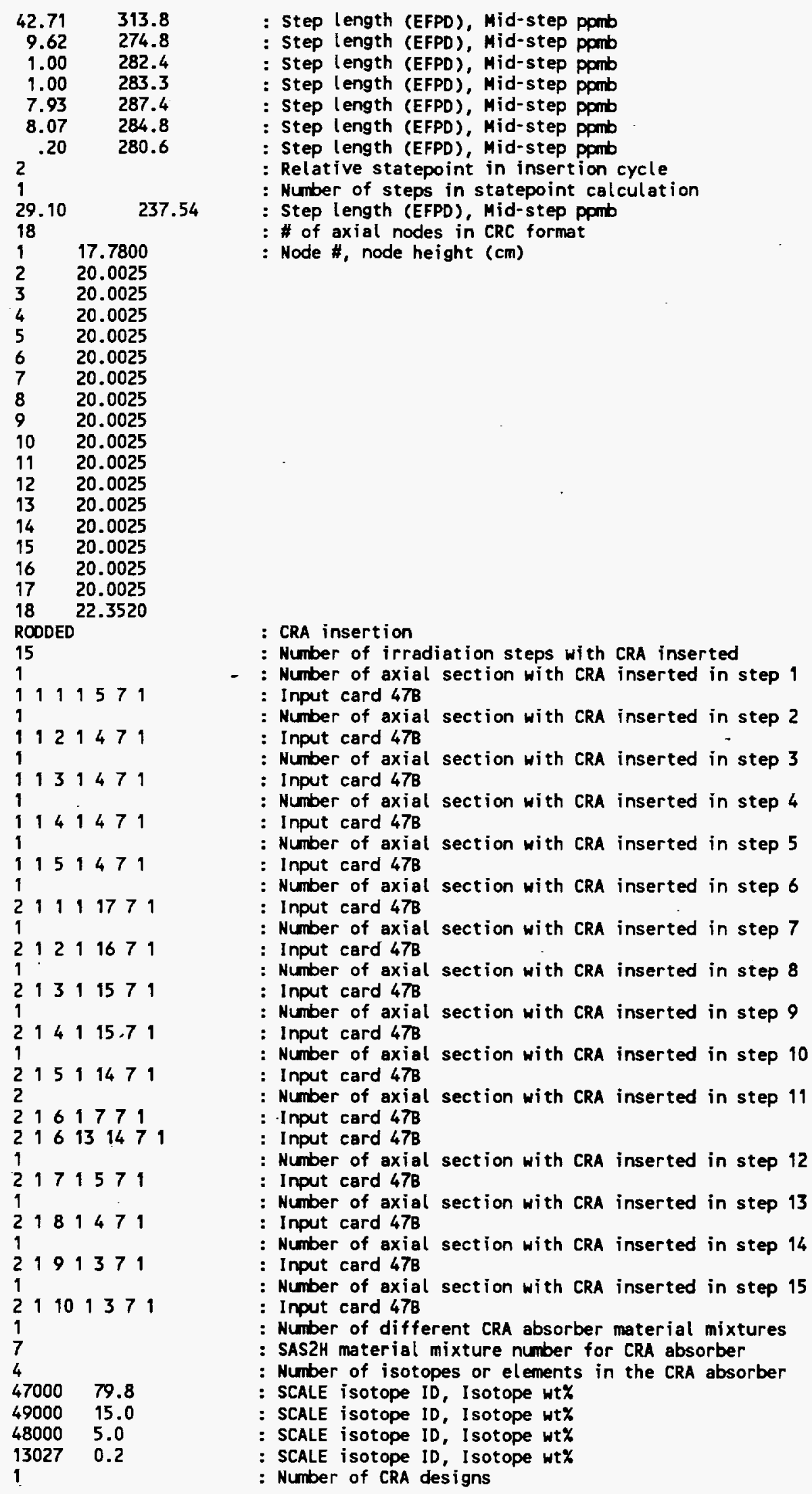




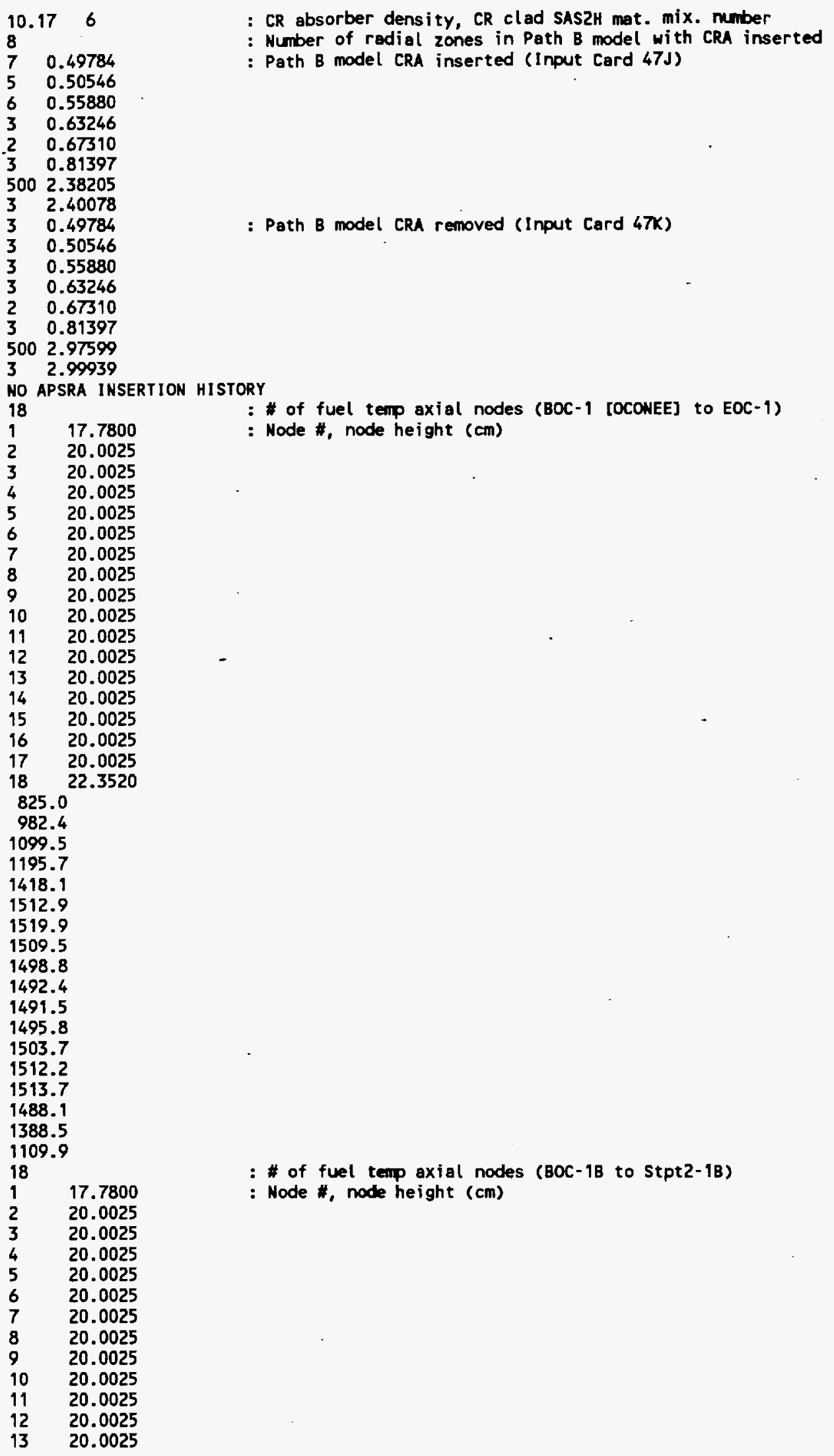


Apr 03 15:02 1997 File Name: 01i.dat BBA000000-01717-0200-00040 REV 00 ATTACHMENT XXI - Page 4

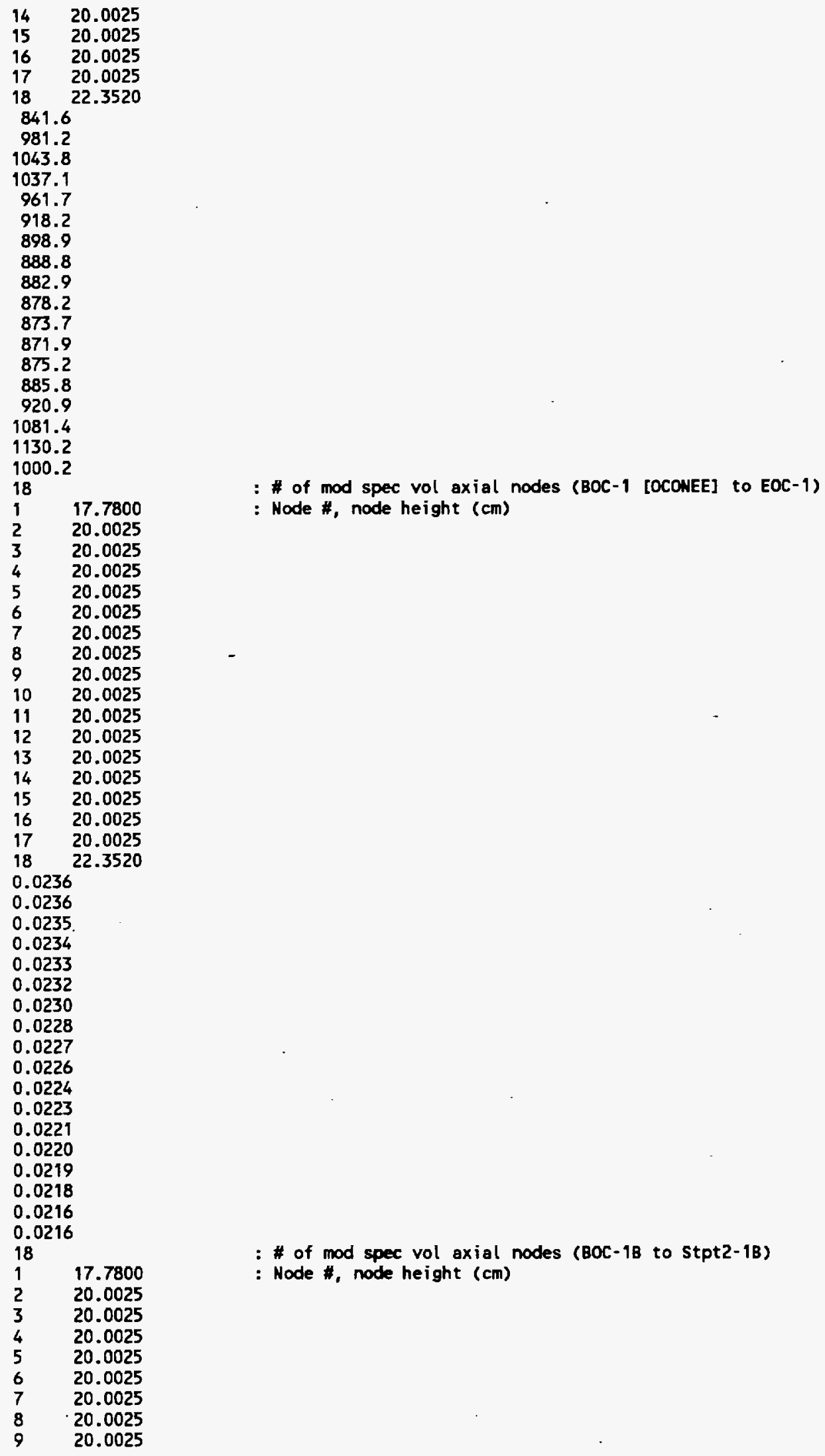


Apr 03 15:02 1997 file Name: 01i.dat BBA000000-01717-0200-00040 REV 00 ATTACHMENT XXI - Pege 5

20.0025

20.0025

20.0025

20.0025

20.0025

20.0025

20.0025

20.0025

22.3520

0.0225

0.0225

0.0224

0.0223

0.0223

0.0222

0.0222

0.0221

0.0221

0.0220

0.0220

0.0219

0.0219

0.0218

0.0218

0.0217

0.0217

0.0216

18

17.7800

20.0025

20.0025

20.0025

20.0025

20.0025

20.0025

20.0025

20.0025

20.0025

20.0025

20.0025

20.0025

20.0025

20.0025

20.0025

20.0025

22.3520

0.0

0.0

0.0

0.0

0.0

0.0

0.0

0.0

0.0

0.0

0.0

0.0

0.0

0.0

0.0

0.0

0.0

0.0

18

$1 \quad 17.7800$

20.0025

20.0025

20.0025

20.0025
: \# of burnup axial nodes (BOC-1 [OCONEE])

: Node \#, mode height (cm)
: of burnup axial nodes (BOC-1B)
: Node \#, node height $(\mathrm{cm})$ 
Apr 03 15:02 1997 File Name: 01i.dat BBA000000-01717-0200-00040 REV 00 ATtACHMENT XXI - Page 6

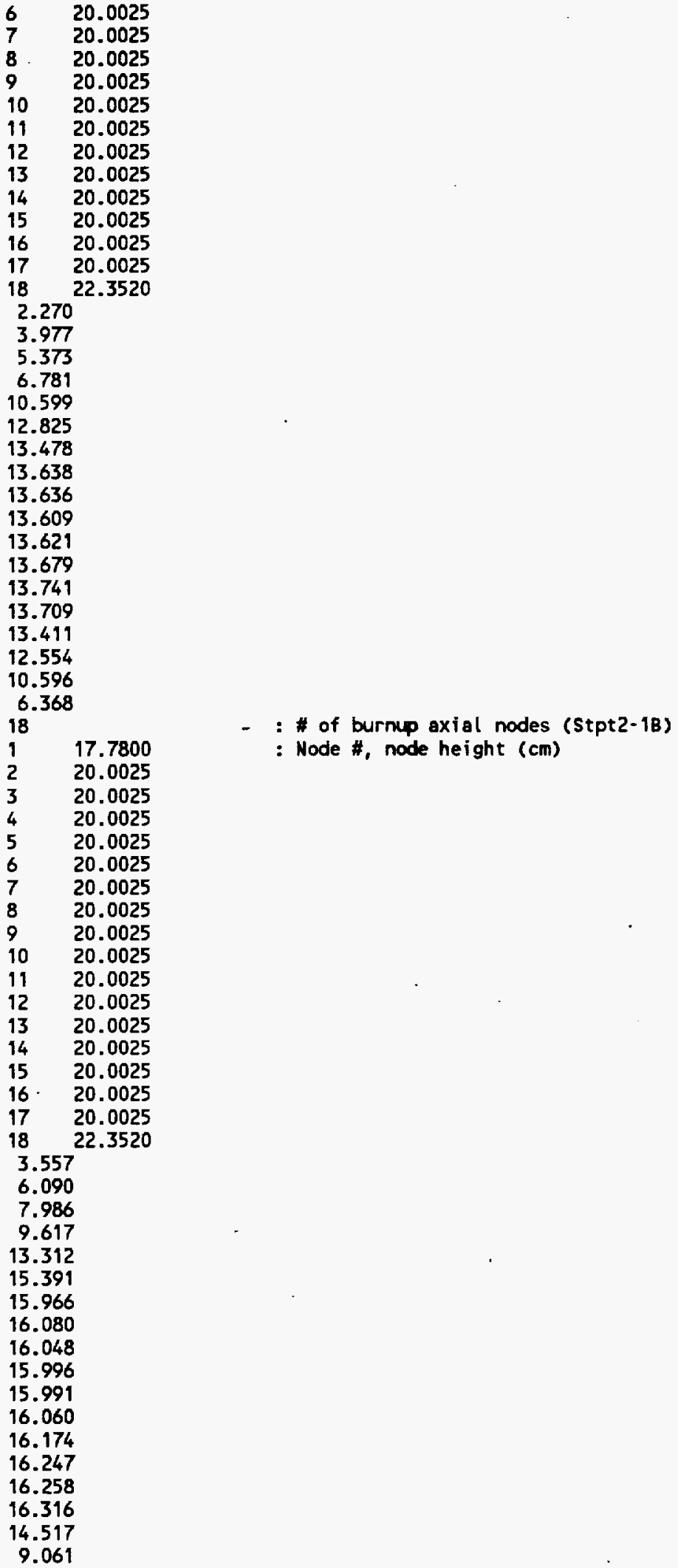




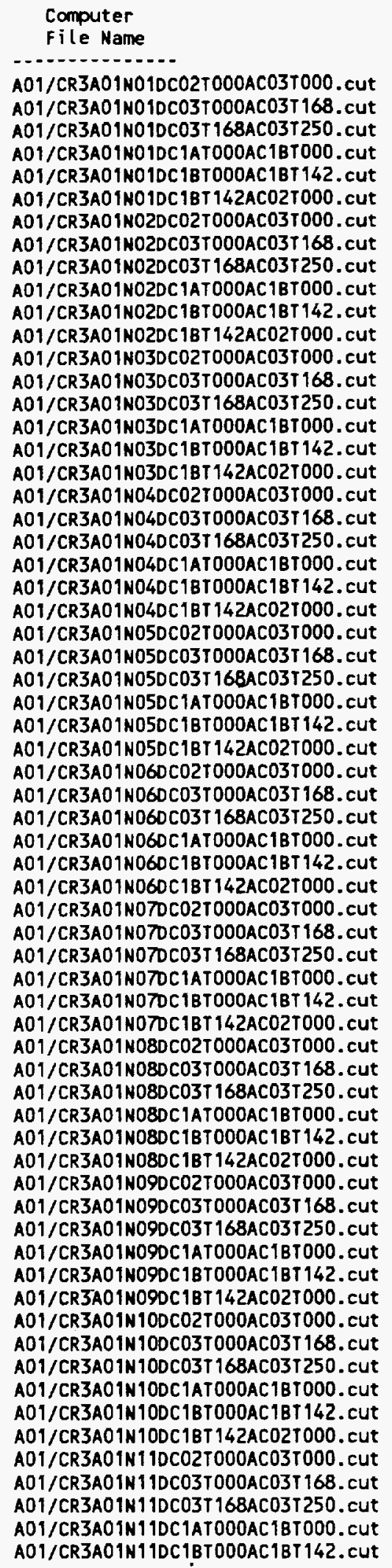

\begin{tabular}{|c|c|c|c|c|c|}
\hline $\begin{array}{l}\text { Tape Backup } \\
\text { File Name }\end{array}$ & $\begin{array}{l}\text { Number of } \\
\text { Print Pages }\end{array}$ & & $\begin{array}{l}\text { e Date } \\
\text { utput) }\end{array}$ & $\begin{array}{c}\text { File size } \\
\text { (Bytes) }\end{array}$ & $\begin{array}{r}\text { File Type } \\
\text { (Format) }\end{array}$ \\
\hline - & $\cdots$ & -- & $\cdots$ & - & $\cdots$ \\
\hline aX.Lf1 & 29 & Apr & 31997 & 136725 & Ascll \\
\hline$a x . L f 2$ & 33 & Apr & 31997 & 151964 & ASCII \\
\hline ax.Lf3 & 32 & Apr & 31997 & 151120 & ASCII \\
\hline ax.Lf4 & 33 & $\mathrm{Apr}$ & 31997 & 147474 & ASCII \\
\hline ax.Lf5 & 41 & Apr & 31997 & 180414 & ASCII \\
\hline ax.Lfo & 32 & Apr & 31997 & 145880 & ASCII \\
\hline ax.Lf7 & 30 & Apr & 31997 & 138804 & ASCII \\
\hline aX.Lf8 & 33 & Apr & 31997 & 154905 & ASCII \\
\hline aX.Lf9 & 33 & Apr & 31997 & 153531 & ASCII \\
\hline aXL.f10 & 33 & Apr & 31997 & $149798^{\circ}$ & ASCII \\
\hline aXL.f11 & 41 & Apr & 31997 & 182248 & ASCII \\
\hline aXL. $f 12$ & 32 & Apr & 31997 & 148042 & ASCII \\
\hline$a \times L . f 13$ & 30 & Apr & 31997 & 139638 & ASCII \\
\hline aXL.f14 & 34 & Apr & 31997 & 157170 & ASCII \\
\hline aXL. $f 15$ & 33 & Apr & 31997 & 155182 & ASCII \\
\hline aXL. $f 16$ & 33 & Apr & 31997 & 150960 & ASCII \\
\hline$a \times L . f 17$ & 41 & Apr & 31997 & 183580 & ASCII \\
\hline aXL.f18 & 32 & Apr & 31997 & 148710 & ASCII \\
\hline aXL.f19 & 30 & Apr & 31997 & 140306 & ASCII \\
\hline aXL. $f 20$ & 34 & Apr & 31997 & 157502 & ASCII \\
\hline aXL.f21 & 33 & Apr & 31997 & 155680 & ASCII \\
\hline aXL.f22 & 33 & Apr & 31997 & 151458 & ASCII \\
\hline$a \times L . f 23$ & 41 & Apr & 31997 & 183514 & ASCII \\
\hline aXL. $f 24$ & 32 & Apr & 31997 & 149225 & ASCII \\
\hline aXL. $f 25$ & 30 & Apr & 31997 & 140306 & ASCII \\
\hline aXL. $\$ 26$ & 34 & Apr & 31997 & 157585 & Ascil \\
\hline aXL. f27 & 33 & Apr & 31997 & 156012 & ASCII \\
\hline aXL.f28 & 33 & Apr & 31997 & 151790 & ASCII \\
\hline aXL.f29 & 41 & $\mathrm{Apr}$ & 31997 & 183763 & ASCII \\
\hline aXL. $f 30$ & 32 & Apr & 31997 & 149225 & ASCII \\
\hline aXL. +31 & 30 & Apr & 31997 & 140389 & ASCII \\
\hline aXL. $\lceil 32$ & 34 & Apr & 31997 & 157585 & ASCII \\
\hline aXL. $f 33$ & 33 & Apr & 31997 & 156095 & ASCII \\
\hline aXL. $\$ 34$ & 34 & Apr & 31997 & 152039 & ASCII \\
\hline aXL. $\uparrow 35$ & 41 & Apr & 31997 & 183763 & ASCII \\
\hline aXL.f36 & 31 & Apr & 31997 & 144637 & ASCII \\
\hline aXL. $\{37$ & 30 & Apr & 31997 & 140389 & ASCII \\
\hline$a \times L . f 38$ & 34 & Apr & 31997 & 157585 & ASCII \\
\hline aXL.f39 & 33 & Apr & 31997 & 156095 & ASCII \\
\hline aXL. $f 40$ & 34 & Apr & 31997 & 152122 & ASCII \\
\hline aXL. $\$ 41$ & 41 & Apr & 31997 & 183763 & ASCII \\
\hline aXL. $f 42$ & 31 & Apr & 31997 & 144637 & ASCII \\
\hline aXL. $f 43$ & 30 & Apr & 31997 & 140389 & ASCII \\
\hline aXL. $\$ 44$ & 34 & Apr & 31997 & 157585 & ASCII \\
\hline aXL. $f 45$ & 33 & $\mathrm{Apr}$ & 31997 & 156095 & ASCII \\
\hline$a X L . \$ 46$ & 34 & Apr & 31997 & 152122 & ASCII \\
\hline aXL. $f 47$ & 41 & Apr & 31997 & 183763 & ASCII \\
\hline aXL. $f 48$ & 31 & Apr & 31997 & 144637 & ASCII \\
\hline aXL. $f 49$ & 30 & $\mathrm{Apr}$ & 31997 & 140389 & ASCII \\
\hline aXL. $f 50$ & 34 & Apr & 31997 & 157385 & ASCII \\
\hline aXL. $f 51$ & 33 & Apr & 31997 & 156095 & ASCII \\
\hline aXL. $f 52$ & 34 & Apr & 31997 & 152122 & ASCII \\
\hline aXL. $f 53$ & 41 & Apr & 31997 & 183763 & ASCII \\
\hline aXL. f54 & 31 & Apr & 31997 & 144637 & ASCII \\
\hline aXL. $\$ 55$ & 30 & Apr & 31997 & 140389 & ASCII \\
\hline aXL. $f 56$ & 34 & Apr & 31997 & 157585 & ASCII \\
\hline aXL. $f 57$ & 33 & Apr & 31997 & 156095 & ASCII \\
\hline aXL. $f 58$ & 34 & Apr & 31997 & 152122 & ASCII \\
\hline aXL.f59 & 41 & $\mathrm{Apr}$ & 31997 & 183763 & ASCII \\
\hline aXL. $f 60$ & 31 & $\mathrm{Apr}$ & 31997 & 144637 & ASCII \\
\hline aXL.f61 & 30 & Apr & 31997 & 140389 & ASCII \\
\hline$a X L . f 62$ & 34 & Apr & 31997 & 157585 & ASCII \\
\hline$a X L . f 63$ & 33 & Apr & 31997 & 156012 & ASCII \\
\hline aXL: f 64 & 34 & Apr & 31997 & 152039 & ASCII \\
\hline aXL.f65 & 4 & Apr & 31997 & 183763 & ASCII \\
\hline
\end{tabular}


A01/CR3A01H11DC1BT 142AC02T000.cut A01/CR3A01N12DC02TO0OACO3TO0O.cut A01/CR3A01N12DCO3TOOOACO3T168. cut A01/CR3A01N12DC03T 168AC03T250. cut A01/CR3A01N12DC1ATO00AC1BT000.cut A01/CR3A01N12DC1BT000AC1BT142.cut A01/CR3A01N12DC1BT 142AC02T000.cut A01/CR3A01N13DCO2TO00AC03T000.cut A01/CR3A01N13DC03T000AC03T168.cut A01/CR3A01N13DC03T168ACO3T250. cut A01/CR3A01N13DC1ATO00AC1BT000.cut A01/CR3A01N13DC1BT000AC1BT142. cut A01/CR3A01N13DC1BT142AC02T000.cut A01/CR3A01N14DC02T000AC03T000.cut A01/CR3A01N14DC03T000AC03T168. cut A01/CR3A01N14DC03T 168AC03T250. cut A01/CR3A01N14DC1AT000AC18T000. cut A01/CR3A01N14DC1BT000AC1BT142. cut A01/CR3A01N14DC1BT 142ACO2T000. cut A01/CR3A01N15DC02TO00ACO3T000. cut A01/CR3A01N15DC03T000AC03T168. cut A01/CR3A01N15DC03T168AC03T250. cut A01/CR3A01N15DC1AT000AC1BT000.cut A01/CR3A01N15DC1BT000AC1BT 142. cut A01/CR3A01N15DC1BT 142AC02T000.cut A01/CR3A01N16DC02TO00AC03T000. cut A01/CR3A01N160C03T000AC03T168. cut A01/CR3A01N160C03T 168AC03T250. cut A01/CR3A01N160C1AT000ACIBT000. Cut A01/CR3A01N160C1BT000AC1BT142. cut A01/CR3A01N16DC1BT 142AC02T000. cut A01/CR3A01N17DC02TO00ACO3T000.cut A01/CR3A01N17DC03TO00AC03T168. cut A01/CR3A01N170C03T168AC03T250. cut A01/CR3A01N170C1ATODOAC1BTO00. Cut A01/CR3A01N170C1BT000AC1BT142. cut A01/CR3A01N17DC1BT142ACO2T000. CUT A01/CR3A01N180C02TO00AC03T000. Cut A01/CR3A01N180C03TO00AC03T168.cut A01/CR3A01N18DC03T 168AC03T250. cut A01/CR3A01N18DC1ATO00AC1BT000. cut A01/CR3A01N180C1BTO0OACIBT142. CUT A01/CR3A01N180C1BT142AC02T000. cut

\footnotetext{
Computer

File Name

A04/CR3A04N010C1ATOOOAC1BT000. CUt A04/CR3A04N01DC1BTO0OAC1BT142. cut A04/CR3A04NO1DCIBT 142ACO2T000. CUT A04/CR3A04N02DC1ATOOOAC1BTO00. CUT A04/CR3A04N02DC1BTO0OAC18T142. cut A04/CR3A04NO2DC1BT142AC02TO00. Cut A04/CR3A04N03DC1ATOOOAC1BT000. CuT A04/CR3A04N03DC1BTO00AC1BT142. Cut A04/CR3A04N03DC1BT142AC02T000. Cut A04/CR3A04N04DC1ATOOOAC1BT000. cut A04/CR3A04N04DC1BT000AC1BT142. CUT A04/CR3A04N04DC1BT142ACO2TO0O. CUT A04/CR3A04N05DC1AT000AC1BT000. Cut A04/CR3A04N05DC1BT000AC1BT142. CUT A04/CR3A04N05DC1BT142AC02T000. CUt A04/CR3A04N06DC1AT000AC1BT000. CUt A04/CR3A04N06DC1BTD00AC1BT142.cUt A04/CR3A04N060C1BT142ACO2T000. Cut A04/CR3A04N07DC1ATOOOAC1BTO00. CUT A04/CR3A04N07DC1BT000AC1BT142. CUt A04/CR3A04N0TDC1BT 142ACO2TODO. CUT A04/CR3A04N08DC1ATOOOAC1BTO0O.CUt
}

\begin{tabular}{|c|}
\hline 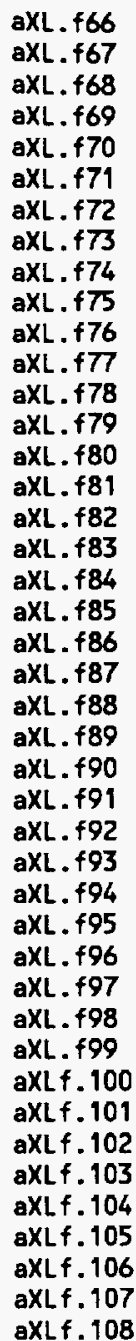 \\
\hline
\end{tabular}

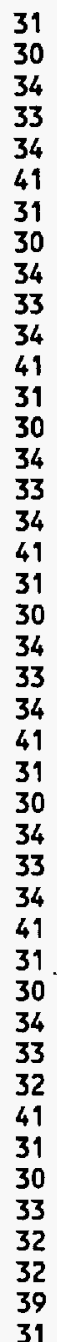

Apr 31997

Apr 31997

Apr 31997

Apr 31997

Apr 31997

Apr 31997

Apr 31997

Apr 31997

Apr 31997

Apr 31997

Apr 31997

Apr 31997

Apr 31997

Apr 31997

Apr 31997

Apr 31997

Apr 31997

Apr 31997

Apr 31997

Apr 31997

Apr 31997

Apr 31997

Apr 31997

Apr 31997

Apr 31997

Apr 31997

Apr 31997

Apr 31997

Apr 31997

Apr 31997

Apr 31997

Apr 31997

Apr 31997

Apr 31997

- Apr 31997

Apr 31997

Apr 31997

Apr 31997

Apr 31997

Apr 31997

Apr 31997

Apr 31997

Apr 31997

144637

140389

157585

156012

152039

183763

144637

140389

157585

156012

151956

183763

144637

140389

157585

156012

151973

183763

144637

140472

157668

156261

152139

184095

144803

140675

158004

156344

152117

184200

145069

140343

157834

156012

145851

183324

144903

138310

154735

153328

144706

174966

143492

ASCII

ASCII

ASCII

ASCII

ASCII

ASCII

ASCII

ASCII

ASCII

ASCII

ASCII

ASCII

ASCII

ASCII

ASCII

ASCII

ASC11

ASCII

ASCII

ASCII

ASCII

ASCII

ASCII

ASCII

ASCII

ASCII

ASCII

ASCII

ASCII

ASCII

ASCII

ASCII

ASCII

ASCII

ASCII

ASCII

ASCII

ASCII

ASCII

ASCII

ASCII

ASCII

ASCII

\begin{tabular}{llccc}
$\begin{array}{c}\text { Tape Backup } \\
\text { File Name }\end{array}$ & $\begin{array}{c}\text { Number of } \\
\text { Print Pages }\end{array}$ & $\begin{array}{c}\text { File Dote } \\
\text { (Output) }\end{array}$ & $\begin{array}{c}\text { File Size } \\
\text { (Bytes) }\end{array}$ & $\begin{array}{c}\text { File Type } \\
\text { (Format) }\end{array}$ \\
\hline aXLf.109 & 30 & Apr 31997 & 137145 & ASCII \\
aXLf.110 & 31 & Apr 31997 & 148274 & ASCII \\
aXLf.111 & 28 & Apr 31997 & 132982 & ASCII \\
aXLf.112 & 31 & Apr 31997 & 140469 & ASCII \\
aXLf.113 & 32 & Apr 31997 & 150606 & ASCII \\
aXLf.114 & 28 & Apr 31997 & 134812 & ASCII \\
aXLf.115 & 31 & Apr 31997 & 141465 & ASCII \\
aXLf.116 & 32 & Apr 31997 & 152100 & ASCII \\
aXLf.117 & 29 & Apr 31997 & 135310 & ASCII \\
aXLf.118 & 31 & Apr 31997 & 141946 & ASCII \\
aXLf.119 & 32 & Apr 31997 & 153305 & ASCII \\
aXLf.120 & 29 & Apr 31997 & 135642 & ASCII \\
aXLf.121 & 31 & Apr 31997 & 142278 & ASCII \\
aXLf.122 & 33 & Apr 31997 & 153724 & ASCII \\
aXLf.123 & 29 & Apr 31997 & 135895 & ASCII \\
aXLf.124 & 31 & Apr 31997 & 142693 & ASCII \\
aXLf.125 & 33 & Apr 31997 & 153724 & ASCII \\
aXLf.126 & 29 & Apr 31997 & 135895 & ASCII \\
aXLf.127 & 31 & Apr 31997 & 142859 & ASCII \\
aXLf.128 & 33 & Apr 31997 & 153834 & ASCII \\
aXLf.129 & 29 & Apr 31997 & 135895 & ASCII \\
aXLf.130 & 31 & Apr 31997 & 142859 & ASCII
\end{tabular}


A04/CR3A04N08DC1BT000AC1BT142.cut A04/CR3A04N08DCIBT 142ACO2T000. cut A04/CR3A04N09DC1ATO0OAC1BT000. cut A04/CR3A04N09DCIBTO0OAC1BT 142. cut A04/CR3A04N090C1BT 142AC02T000. cut A04/CR3A04N10DC1ATO0OAC1BT000. cut A04/CR3A04N10DC1BTO00AC1BT142. cut A04/CR3A04N10DC1BT 142ACO2T000.cut A04/CR3A04N11DC1ATO00AC1BT000.cut A04/CR3A04N11DC1BTO0OAC1BT142.cut A04/CR3A04N11DCIBT 142AC02T000. cut A04/CR3A04N120C1ATO0OAC1BTO00. cut A04/CR3A04N120C1BTO00AC1BT 142. cut A04/CR3A04N12DC1BT142AC02T000. cut A04/CR3A04N13DC1ATO00AC1BT000. cut A04/CR3A04N13DC1BTDOOAC1BT 142. cut A04/CR3A04N13DC1BT142AC02T000. cut A04/CR3A04N14DC1ATOOOAC1BT000. cut A04/CR3A04N14DC1BTO0OAC1BT 142. cut A04/CR3A04N14DC1BT142AC02T000. cut A04/CR3A04N15DC1ATO00AC1BT000. cut A04/CR3A04N15DC1BTOOOAC1BT 142. cut A04/CR3A04N15DC1BT 142AC02T000.cut A04/CR3A04N160C1ATOOOAC1BTO00. cut A04/CR3A04N16DC1BTODOAC1BT 142. cut A04/CR3A04N 160C1BT 142AC02T000. cut A04/CR3A04N170C1ATOOOAC1BTO00. Cut A04/CR3A04N17DC1BT 000AC1BT 142. CUT A04/CR3A04N17DC1BT142AC02T000. cut A04/CR3A04N18DC1ATO00AC1BTO00.cut A04/CR3A04N18DC1BTO00AC1BT 142. cut A04/CR3A04N18DC1BT142AC02T000. cut

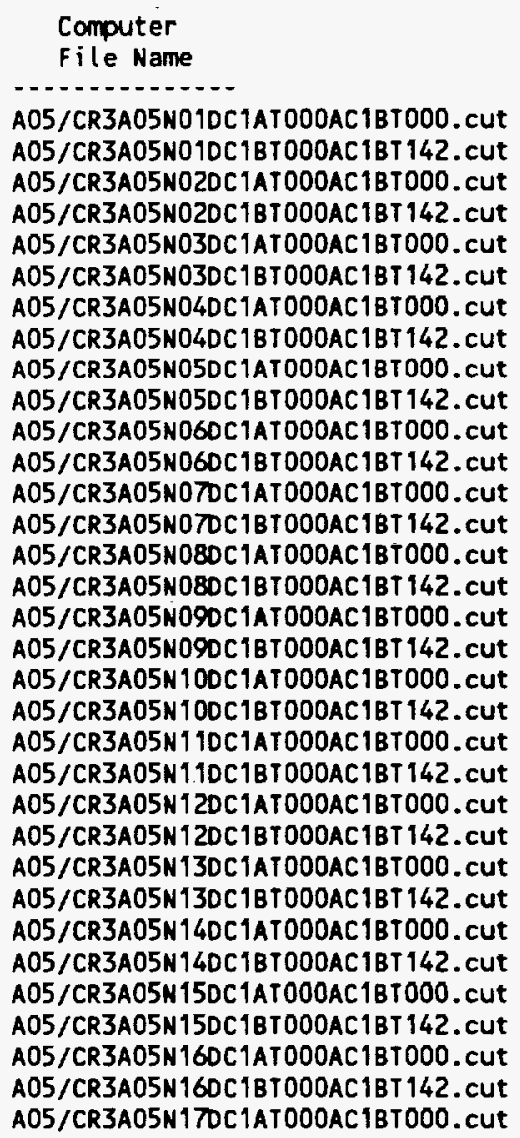

$\begin{array}{ll}\text { aXLf.131 } & 33 \\ \text { aXLf.132 } & 29 \\ \text { aXLf.133 } & 31 \\ \text { aXLf.134 } & 33 \\ \text { aXLf.135 } & 29 \\ \text { aXLf.136 } & 31 \\ \text { aXLf.137 } & 33 \\ \text { aXLf.138 } & 29 \\ \text { aXLf.139 } & 31 \\ \text { aXLf.140 } & 33 \\ \text { aXLf.141 } & 29 \\ \text { aXLf.142 } & 31 \\ \text { aXLf.143 } & 33 \\ \text { aXLf.144 } & 29 \\ \text { aXLf.145 } & 31 \\ \text { aXLf.146 } & 33 \\ \text { aXLf.147 } & 29 \\ \text { aXLf.148 } & 31 \\ \text { aXLf.149 } & 33 \\ \text { aXLf.150 } & 29 \\ \text { aXLf.151 } & 31 \\ \text { aXLf.152 } & 33 \\ \text { aXLf.153 } & 29 \\ \text { aXLf.154 } & 31 \\ \text { aXLf.155 } & 32 \\ \text { aXLf.156 } & 29 \\ \text { aXLf.157 } & 31 \\ \text { aXLf.158 } & 32 \\ \text { aXLf.159 } & 28 \\ \text { aXLf.160 } & 30 \\ \text { aXLf.161 } & 32 \\ \text { aXLf.162 } & 28 \\ & \end{array}$

33
29
31
33
29
31
33
29
31
33
33
29
31
33
29
31
33
33
29
31
33
29
31
31
33
29
31
32
32
29
31

Apr 31997

Apr 31997

Apr 31997

Apr 31997

Apr 31997

Apr 31997

Apr 31997

- Apr 31997

Apr 31997

Apr 31997

Apr 31997

Apr 31997

Apr 31997

Apr 31997

Apr 31997

Apr 31997

Apr 31997

Apr 31997

Apr 31997

Apr 31997

Apr 31997

Apr 31997

Apr 31997

Apr 31997

Apr 31997

Apr 31997

Apr 31997

Apr 31997

Apr 31997

Apr 31997

Apr 31997

Apr 31997
153751

135895

142859

153751

135812

142693

153751

135729

142693

153668

135646

142278

153668

135646

142029

153471

135729

141946

153554

135812

141946

153637

135729

141697

152493

135476

141299

151851

135144

137951

149274

133729

Filesize

(Bytes)

........

146637

165454

150313

168723

150289

169865

146283

170492

146847

166510

146930

166510

146930

166427

147013

166427

146930

166427

146930

166261

146930

166261

146764

166178

146432

166178

146266

166269

146283

166344

145868

165573

145453
ASCII

ASCII

ASCII

ASCII

ASCII

ASCII

ASCII

ASCII

ASCII

ASCII

ASCII

ASCII

ASCII

ASCII

ASCII

ASCl1

ASCII

ASCII

ASC II

ASCII

ASCI]

ASCII

ASCII

ASCII

ASCII

ASCII

ASCII

ASCII

ASCII

ASCII

ASCII

ASCII

File Type

(Format)

ASCII

ASCII

ASCII

ASCII

ASCII

ASCII

ASCII

ASCII

ASCII

ASCII

ASCII

ASCII

ASCII

ASCII

ASCII

ASCII

ASCII

ASCII

ASCI I

ASCII

ASCII

ASCII

ASCII

ASCII

ASCI I

ASCI I

ASCI I

ASCI I

ASCII

ASCI I

ASCII

ASCII

ASCII 
A05/CR3A05N170C18TO00AC1BT 142. cut A05/CR3A05N18DC1ATO00AC1BT000.cut A05/CR3A05N18DC1BTO0OAC1BT142.cut

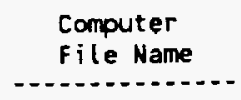

A07/CR3A03N01DC02TO0OAC03T000. cut A07/CR3A03N02DCO2TOOOACO3T000. cut A07/CR3A03N03DCO2TOOOACO3TO00. cut A07/CR3A03N040CO2TOOOAC03T000. cut A07/CR3A03N05DC02TO00AC03T000. cut A07/CR3A03N06DC02TOOOACO3T000. cut A07/CR3A03NOTDC02TO0OACO3T000. cut A07/CR3A03N08DC02T000ACO3T000. cut A07/CR3A03NO9DCO2TO0OACO3TO0O. cut A07/CR3A03N10DC02T000AC03T000. cut A07/CR3A03N110C02TOOOAC03TO0O. cut A07/CR3A03N12DC02TO00AC03T000. cut A07/CR3A03N13DC02T000AC03T000. cut A07/CR3A03N14DC02T000AC03T000. cut A07/CR3A03N15DC02TO00ACO3TO00. cut A07/CR3A03N16DC02T000AC03T000. cut A07/CR3A03N170C02TO00ACO3T000. cut A07/CR3A03N18DC02TO00AC03T000. cut A07/CR3A04N01DC03TOOOAC03T168. cut A07/CR3A04N01DC03T 168ACO3T250. cut A07/CR3A04N02DC03TO0OAC03T168. cut A07/CR3A04N02DC03T168AC03T250. cut A07/CR3A04N03DC03TO0OAC03T168. cut A07/CR3A04N03DC03T 168AC03T250. cut A07/CR3A04N04DC03TO0OAC03T168. cut A07/CR3A04N04DC03T168AC03T250. cut A07/CR3A04N05DCO3TODOAC03T168.cut A07/CR3A04N050C03T168AC03T250. cut A07/CR3A04N060C03TOODACO3T 168. cut A07/CR3A04N060C03T168AC03T250. cut A07/CR3A04N0TDCO3TOOOACO3T168. cut A07/CR3A04N0TDCO3T168ACO3T250.cut A07/CR3A04N08DC03TO0OACO3T168. Cut AO7/CR3A04N080C03T168AC03T250.cut A07/CR3A04N090C03TODOAC03T168.cut AO7/CR3A04NOSOC03T168AC03T250. cut A07/CR3A04N100C03TD00AC03T168.cut A07/CR3A04N10DC03T 168AC03T250.cut A07/CR3A04N110C03T000AC03T168.cut A07/CR3A04N11DCO3T 168AC03T250.cut A07/CR3A04N12DCO3TOOOACO3T168.cut A07/CR3A04N12DC03T168AC03T250.cut A07/CR3A04N 13DCO3TOOOAC03T 168.cut A07/CR3A04N 13DC03T 168AC03T250. cut A07/CR3A04N14DCO3TOOOACO3T168. cut A07/CR3AO4N 14DC03T 168AC03T250. cut A07/CR3A04N15DCO3TO00AC03T168. Cut A07/CR3A04N 15DCO3T 168AC03T250. cut A07/CR3A04N16DCO3T000ACO3T 168.cut A07/CR3A04N160C03T 168AC03T250. Cut A07/CR3A04N170C03TO00AC03T168.cut A07/CR3A04N17DCO3T168AC03T250. cut A07/CR3A04N18DCO3T000AC03T 168. cut A07/CR3A04N18DCO3T 168AC03T250. cut A07/CR3A07N01DC1ATO00AC1BT000. CUT A07/CR3A07N01DC1BT000AC1BT142. cut A07/CR3A07NO1DC1BT 142AC02T000. CUT A07/CR3A07N02DC1ATOOOAC1BT000. Cut A07/CR3A07N02DC1BTO00AC1BT142.cut A07/CR3A07N02DC1BT 142AC02T000. Cut A07/CR3A07N03DC1ATO00AC1BTD00. Cut A07/CR3A07N03DC1BT000AC1BT 142. cut

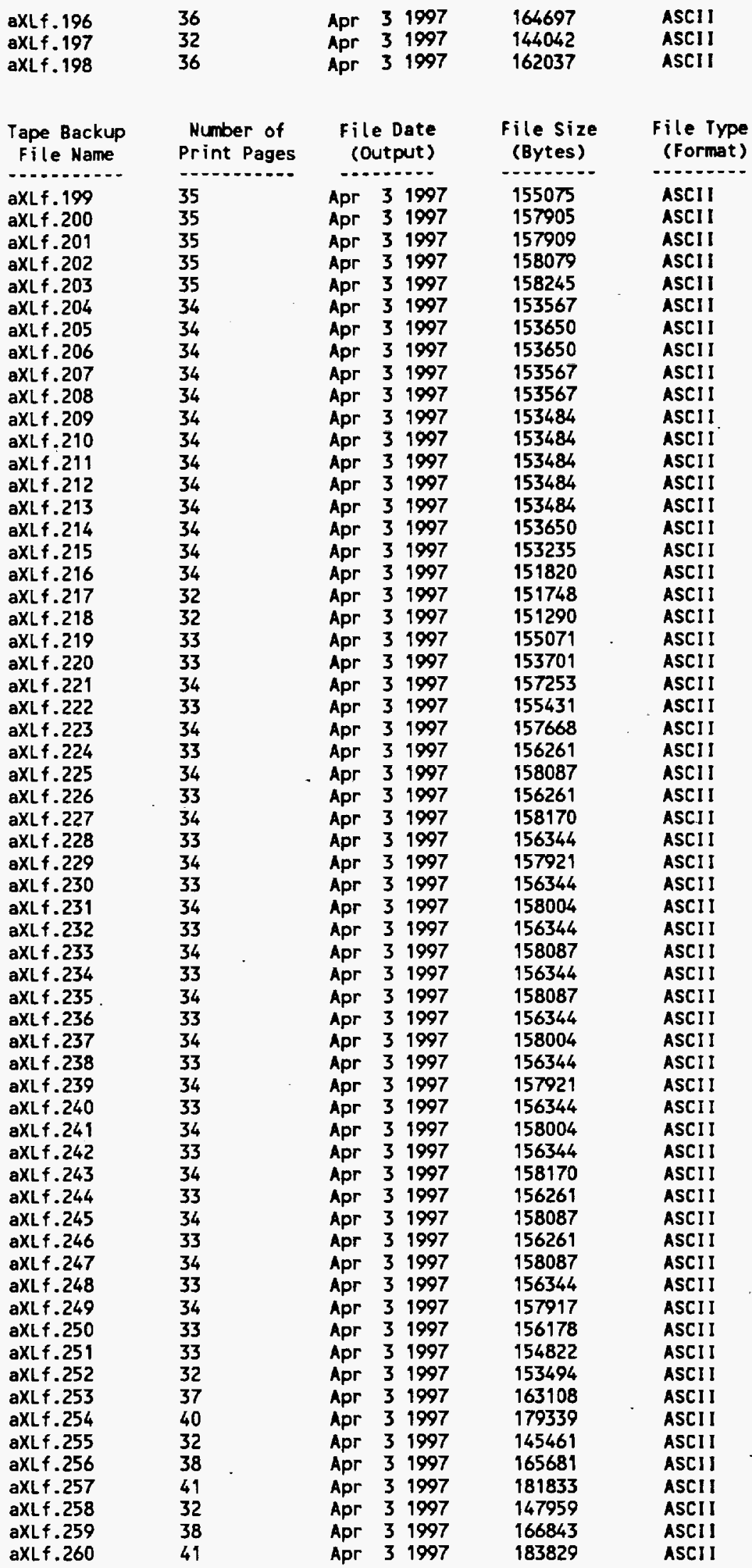


A07/CR3A07N03DC1BT 142AC02T000. cut A07/CR3A07N04DC1ATOOOAC1BT000. Cut A07/CR3A07N04DC1BTO00AC1BT142.CUt A07/CR3A07N04DC1BT 142ACO2T000. cut A07/CR3A07N05DC1ATOOOACIBTO00. cut A07/CR3A07N05DC1BT000AC18T142. Cut A07/CR3A07N05DC1BT 142ACO2T000. Cut A07/CR3A07N060C1ATO0OAC1BT000. CUt A07/CR3A07N06DC1BTO00AC18T142. cut A07/CR3A07N06DC1BT 142AC02T000. cut A07/CR3A07N0TDC1ATO00AC1BT000. cut A07/CR3A07N0TOC1BTO0OAC1BT142. cut A07/CR3A07N07DC1BT 142AC02T000. CUt A07/CR3A07N08DC1ATOOOAC1BTO00. Cut A07/CR3A07N08DC1BT000AC1BT142. Cut A07/CR3A07N08DC1BT 142AC02T000. cut A07/CR3A07N09DC1ATO00AC1BT000. cut A07/CR3A07N09DC1BT000AC1BT142. Cut A07/CR3A07N090C1BT 142AC02T000. cut A07/CR3A07N10DC1AT000AC1BT000. Cut A07/CR3A07N10DC1BT000AC1BT142. cut A07/CR3A07N10DC1BT142AC02T000. cut A07/CR3A07N11DC1AT000AC1BT000. CUT A07/CR3A07N11DC1BTDOOAC1BT142. Cut A07/CR3A07N11DC1BT142AC02T000. Cut A07/CR3A07N12DC1ATDOOAC1BT000. cut A07/CR3A07N12DC1BT000AC1BT142. cut A07/CR3AOTN12DC1BT142ACO2T000. CUT A07/CR3A07N13DC1ATO00AC1BT000. CUT A07/CR3A07N13DC1BTD00AC18T142. CUt A07/CR3A07N130C1BT 142ACO2TO00. CUT A07/CR3A07N140C1ATO00AC1BT000. CUT A07/CR3A07N140C1BT D0OAC1BT 142. cUt A07/CR3A07N14DC1BT142ACOZTO00. CUt A07/CR3A07N15DC1ATDO0AC1BT000.CUT A07/CR3A07N15DC1BT 000AC1BT142. CUT A07/CR3A07N15DC1BT142ACO2T000. cUt A07/CR3A07N160C1ATDOOAC1BT000.CUt A07/CR3A07N160C1BT000AC1BT142. CUT A07/CR3A07N160C1BT 142AC02T000. CUt A07/CR3A07N17DC1ATO0OAC1BT000.cut A07/CR3A07N170C1BTO0OAC1BT 142.cUt A07/CR3A07N1TOC1BT 142AC02T000.cut A07/CR3A07N18DC1ATOOOAC1BTO00.CUT A07/CR3A07N18DCIBTO0OAC1BT 142.cut A07/CR3A07N 18DC1BT 142AC02T000.cut

\footnotetext{
Computer

File Name

A14/CR3A05N01DCOTTOOOAC07T260. cut A14/CR3A05N01DC07T260AC07T291. Cut A14/CR3A05N01DC07T291AC07T319.cUt A14/CR3A05N01DC07T319AC071462.cut A14/CR3A05N01DC07T462AC07T479. Cut A14/CR3A05N02DC07TT000AC07T260. cut A14/CR3A05N02DC07T260AC07T291. Cut A14/CR3A05N02DC07T291AC07T319.cut A14/CR3A05N02DC07T319AC07T462. cut A14/CR3A05N02DC07T462AC07T479.cut A14/CR3A05N03DC07TO00AC07T260. cut A14/CR3A05N03DC07T260AC07T291. cut A 14/CR3A05N03DC07T291AC07T319.cut A14/CR3A05N03DC071319AC071462.cut A14/CR3A05N03DC07T462AC07T479. cut A14/CR3A05N04DC07T000AC07T260.cut A14/CR3A05N04DC07T260AC07T291. cut A14/CR3A05N04DC07T291AC07T319.cut A14/CR3A05N04DC07T319AC07T462.cut
}

\begin{tabular}{|c|}
\hline 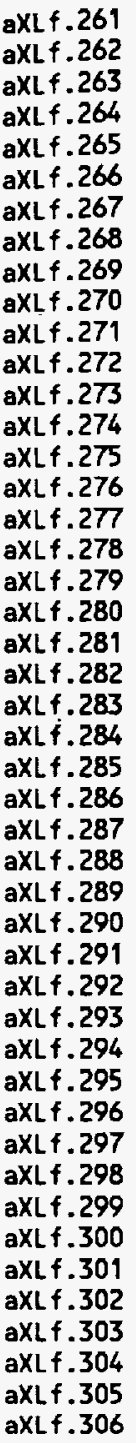 \\
\hline
\end{tabular}

32
38
41
32
38
41
32
38
41
31
38
41
31
38
41
31
38
41
31
38
41
31
38
41
31
38
41
31
38
41
31
38
41
31
38
41
31
37
41
31
37
41
31
37
39
31

$\begin{array}{lll}\text { Apr } & 3 & 1997 \\ \text { Apr } & 3 & 1997 \\ \text { Apr } & 3 & 1997 \\ \text { Apr } & 3 & 1997 \\ \text { Apr } & 3 & 1997 \\ \text { Apr } & 3 & 1997 \\ \text { Apr } & 3 & 1997 \\ \text { Apr } & 3 & 1997 \\ \text { Apr } & 3 & 1997 \\ \text { Apr } & 3 & 1997 \\ \text { Apr } & 3 & 1997 \\ \text { Apr } & 3 & 1997 \\ \text { Apr } & 3 & 1997 \\ \text { Apr } & 3 & 1997 \\ \text { Apr } & 3 & 1997 \\ \text { Apr } & 3 & 1997 \\ \text { Apr } & 3 & 1997 \\ \text { Apr } & 3 & 1997 \\ \text { Apr } & 3 & 1997 \\ \text { Apr } & 3 & 1997 \\ \text { Apr } & 3 & 1997 \\ \text { Apr } & 3 & 1997 \\ \text { Apr } & 3 & 1997 \\ \text { Apr } & 3 & 1997 \\ \text { Apr } & 3 & 1997 \\ \text { Apr } & 3 & 1997 \\ \text { Apr } & 3 & 1997 \\ \text { Apr } & 3 & 1997 \\ \text { Apr } & 3 & 1997 \\ \text { Apr } & 3 & 1997 \\ \text { Apr } & 3 & 1997 \\ \text { Apr } & 3 & 1997 \\ \text { Apr } & 3 & 1997 \\ \text { Apr } & 3 & 1997 \\ \text { Apr } & 3 & 1997 \\ \text { Apr } & 3 & 1997 \\ \text { Apr } & 3 & 1997 \\ \text { Apr } & 3 & 1997 \\ \text { Apr } & 3 & 1997 \\ \text { Apr } & 3 & 1997 \\ \text { Apr } & 3 & 1997 \\ \text { Apr } & 3 & 1997 \\ \text { Apr } & 3 & 1997 \\ \text { Apr } & 3 & 1997 \\ \text { Apr } & 3 & 1997 \\ \text { Apr } & 3 & 1997 \\ & & \end{array}$

148950

167507

183763

149308

167490

183846

149474

167573

183846

144803

167739

184141

144803

167739

184307

144969

167739

184307

144886

167739

184307

144803

167573

184141

144886

167573

184058

144886

167573

183929

144886

167656

184095

144886

167241

184178

144886

161618

183407

144803

161386

182328

144488

159643

174302

142990

ASCII

ASCII

ASCII

ASCII

ASCII

ASCII

ASCII

ASCII

ASCII

ASCII

ASCII

ASCII

ASCII

ASCII

ASCII

ASCII

ASCII

ASCII

ASCII

ASCII

ASCII

ASCII

ASCII

ASCII

ASCII

ASCII

ASCII

ASCII

ASCII

ASCI I

ASCII

ASCII

ASCII

ASCII

ASCII

ASCII

ASCII

ASCII

ASCII

ASCII

ASCII

ASCII

ASCII

ASCII

ASCII

ASCII

\begin{tabular}{|c|c|}
\hline $\begin{array}{c}\text { Tape Backup } \\
\text { File Name }\end{array}$ & $\begin{array}{l}\text { Number of } \\
\text { Print Pages }\end{array}$ \\
\hline מ & \\
\hline aXLf. 307 & 36 \\
\hline axLf. .308 & 31 \\
\hline aXLf.309 & 30 \\
\hline aXL $f .310$ & 37 \\
\hline axLf. 311 & 32 \\
\hline axLf.312 & 36 \\
\hline axLf. .313 & 31 \\
\hline aXLf. $\mathbf{3 1 4}$ & 30 \\
\hline axLf. .315 & 37 \\
\hline aXLf. 316 & 32 \\
\hline aXLf. .317 & 36 \\
\hline axLf. .318 & 31 \\
\hline axLf.319 & 30 \\
\hline aXLf. $\mathbf{3 2 0}$ & 37 \\
\hline axL $f .321$ & 33 \\
\hline axLf. 322 & 36 \\
\hline axLf. .323 & 31 \\
\hline axLf. .324 & 30 \\
\hline aXLf. .325 & 37 \\
\hline
\end{tabular}

\begin{tabular}{cc}
$\begin{array}{c}\text { File Size } \\
\text { (Bytes) }\end{array}$ & $\begin{array}{c}\text { File Iype } \\
\text { (Format) }\end{array}$ \\
\hdashline 163855 & ASCII \\
147563 & ASCII \\
141608 & ASCII \\
174968 & ASCII \\
152245 & ASCII \\
167456 & ASCII \\
144921 & ASCII \\
139136 & ASCII \\
172737 & ASCI I \\
154680 & ASCII \\
164395 & ASCII \\
145423 & ASCII \\
140132 & ASCII \\
173658 & ASCII \\
155390 & ASCII \\
164814 & ASCII \\
145755 & ASCII \\
140219 & ASCII \\
174488 & ASCII
\end{tabular}


A14/CR3A05N04DC07T462AC07T479. Cut A14/CR3A05N05DC07TO00AC07T260. cut A14/CR3A05N05DC07T260AC07T291. cut A14/CR3A05N05DC07T291AC071319. cut A14/CR3A05N05DC07T319AC07T462. CUt A14/CR3A05N05DC07T462AC07T479. Cut A14/CR3A05N060C071000AC07T260. cut A14/CR3A05N06DC07T260AC07T291. cut A14/CR3A05N06DC07T291AC07T319. cut A14/CR3A05N060C07T319AC07T462. cut A14/CR3A05N060C07T1462AC07T479. cut A14/CR3A05N0TOCO7TOOOAC07T260.cut A14/CR3A05N070C07T260AC07T291. cut A14/CR3A05N07DC07T291AC07T319.cut A14/CR3A05N07DC07T319AC07T462. cut A14/CR3A05N07DC07T462AC07T479. cut A14/CR3A05N08DC07TO00AC07T260. cut A14/CR3A05N080C07T260AC07T291. cut A14/CR3A05N08DC07T291AC07T319.cut A 14/CR3A05N08DC07T319ACO7T 462. cut A14/CR3A05N08DC07T462AC07T479.cut A14/CR3A05N090C07TO00AC07T260. cut A14/CR3A05N09DC07T260AC07T291. cut A 14/CR3A05N09DC07T291AC07T319.cut A14/CR3A05N09DC07T319AC07T462. cut A14/CR3A05N09DC07T462AC07T479. cut A14/CR3A05N10DC07TO00AC07T260. cut A 14/CR3A05N10DC07T260AC07T291. cut A14/CR3A05N10DC07T291AC07T319.cut A14/CR3A05N10DC07T319AC07T462.cut A $14 / C R 3 A 05 N 10 D C 07 T 462 A C 07 T 479$. cut A 14/CR3A05N1 10C07TO00ACO7T 260. cut A14/CR3A05N110C07T260AC07T291. cut A14/CR3A05N110C07T291AC07T319.cut A14/CR3A05N11DC07T319AC07T462. cut A14/CR3A05N11DC07T462AC07T479. cut A14/CR3A05N12DC07TO00AC07T260. cut A14/CR3A05N12DC07T 260AC07T291. Cut A14/CR3A05N12DC07T291AC07T319. cut A14/CR3A05N12DC07T319AC07T462. cut A14/CR3A05N120C07T462AC071479. Cut A14/CR3A05N13DC07TO00AC07T260. cut A14/CR3A05N13DCO7T260AC07T291. cut A14/CR3A05N13DC07T291AC07T319. cut A14/CR3A05N13DC07T319AC07T462. Cut A14/CR3A05N13DC07T462AC07T479. cut A14/CR3A05N140C07TT000AC07T260.cut A14/CR3A05N14DC07T 260AC07T291. cut A 14/CR3A05N140CO7T291AC07T319.cut A14/CR3A05N140C07T319AC07T462. Cut A14/CR3A05N14DC07T462AC07T479.cut A14/CR3A05N15DC07TO00AC07T260.cut A14/CR3A05N15DCO7T260ACOTT291.cut A14/CR3A05N15DC07T291AC07T319.cut A14/CR3A05N150C07T319AC07T 462. cut A14/CR3A05N15DC07T462AC07T479.cut A14/CR3A05N160C07TTO00AC07T260.cut A14/CR3A05N160C07T260AC07T291.cut A14/CR3A05N160C07T291AC07T319.cut A14/CR3A05N160C07T319AC07T462.cut A14/CR3A05N160C07T 462ACOTT479.cut A14/CR3A05N170C07TO00AC07T260.cut A14/CR3A05N170C07T260AC07T291.cut A14/CR3A05N170C07T291AC07T319.cut A14/CR3A05N170C07T319AC077462.cut A14/CR3A05N170C07T462AC07T479. Cut A 14/CR3A05N18DC07T1000AC07T260.cut A14/CR3A05N18DC07T260AC07T291. cut A14/CR3A05N180C07T291AC07T319.cut A14/CR3A05N18DC07T319AC07T462. cut

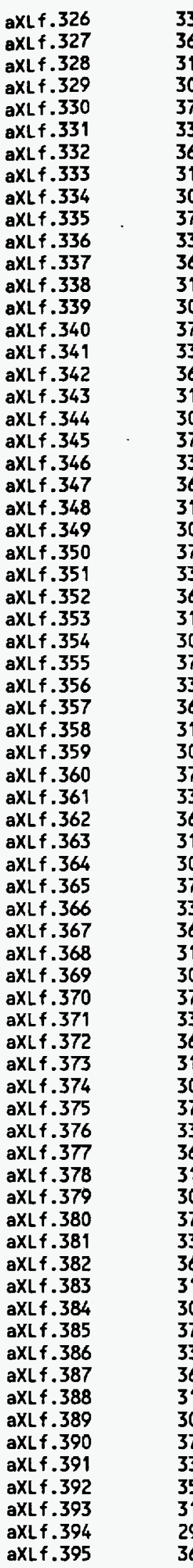

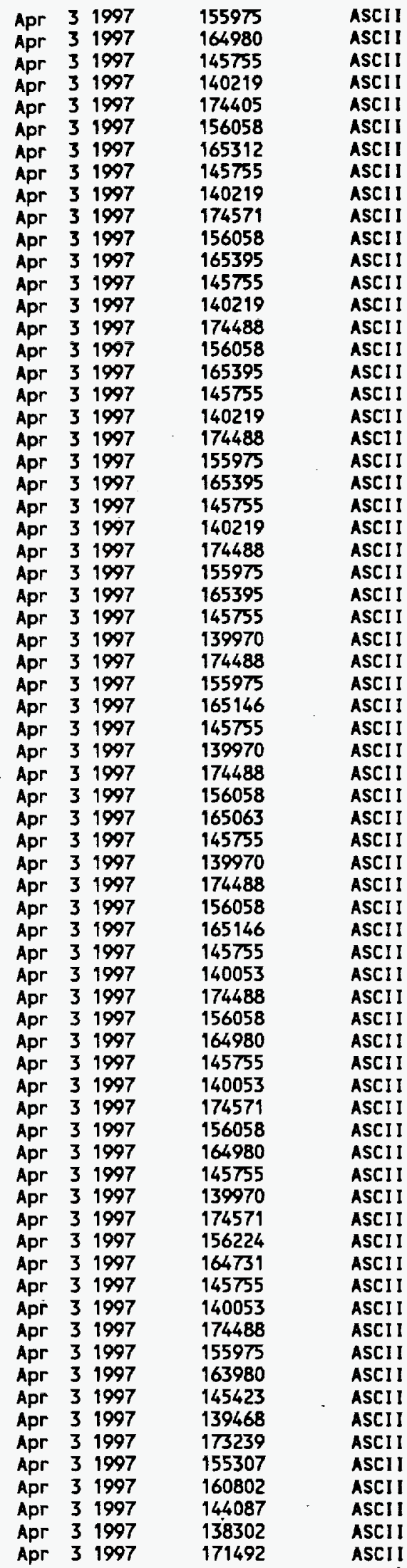


A14/CR3A05N18DC07T462AC07T479. cut A14/CR3A14N01DC02TO00AC07TODO. cut A14/CR3A14N01DC1ATO00AC1BT000. CUt A14/CR3A14N01DC1BTO00AC1BT142. cut A14/CR3A14N01DC1BT142AC02T000.cut A14/CR3A14N020C02TOOOAC07TO00. cut A14/CR3A14N02DC1ATOOOAC1BT000. cut A14/CR3A14N02DC1BTO00AC1BT 142. CUt A14/CR3A14N02DC1BT 142AC02T000.cut A14/CR3A14N03DC02TO00AC07TO00. cut A14/CR3A14N03DC1ATO00AC1BT000.cut A14/CR3A14N03DC1BTO0OAC1BT142. Cut A14/CR3A14NO3DC1BT 142AC02T000. cut A 14/CR3A14N04DC02TO00AC07T000. cut A14/CR3A14N04DC1AT000AC1BT000. Cut A14/CR3A14N04DC1BT000AC1BT 142. Cut A14/CR3A14N04DC1BT142AC02T000. cut A14/CR3A14NO5DC02TO00AC07T000. cut A14/CR3A14N05DC1ATO00AC1BT000. Cut A14/CR3A14N05DC1BT000AC1BT142. cut A14/CR3A14N05DC1BT 142AC02T000. cut A 14/CR3A14N06DC02TO00AC07T000. cut A14/CR3A14N060C1ATO00AC1BT000. CUt A14/CR3A14N060C1BT000AC1BT142. cut A14/CR3A14N060C1BT 142AC02T000.cut A14/CR3A14N070C02T000AC07T000.cut A14/CR3A14N0TDC1ATOOOAC1BT000.cut A14/CR3A14N0TDC1BTOOOAC1BT 142. cut A 14/CR3A14N0TDC1BT 142AC02T000. Cut A14/CR3A14N080CO2TO00AC07T000.cut A14/CR3A14N08DC1ATO00AC1BT000.cut A14/CR3A14N08DC1BTO0OAC1BT142.cut A14/CR3A14N08DC1BT142AC02T000.cut A14/CR3A14N09DC02TO00AC07T000. cut A14/CR3A14NO9DC1ATO0OAC1BT000.cut A14/CR3A14NO90C1BT000AC1BT142.cut A14/CR3A14N09DC1BT 142AC02T000.cut A14/CR3A14N10DC02T000AC07T000. cut A14/CR3A14N10DC1ATO00AC1BT000. cut A14/CR3A14N10DC1BTO00AC1BT142.cut A14/CR3A14N10DC1BT 142AC02T000. cut A14/CR3A14N11DC02T000AC07T000.cut A14/CR3A14N11DC1AT000AC1BT000.cut A14/CR3A14N11DC1BTO00AC1BT142.cut A14/CR3A14N11DC1BT142AC02T000. Cut A14/CR3A14N12DC02TO00AC07T000. cut A 14/CR3A14N12DC1ATO00AC1BT000.cut A14/CR3A14N120C1BTO0OAC1BT142. cut A14/CR3A14N12DC1BT142AC02T000.cut A14/CR3A14N13DC02T000AC07T000.cut A14/CR3A14N13DC1AT000AC1BT000. cut A14/CR3A14N13DC1BTOOOAC1BT142. cut A14/CR3A14N13DC1BT 142AC02T000. cut A14/CR3A14N14DC02TO00AC07T000. cut A14/CR3A14N14DC1ATO00AC1BT000. cut A14/CR3A14N14DC1BTO00AC1BT142. cut A14/CR3A14N14DC1BT142AC02T000. Cut A14/CR3A14N15DC02TO00AC07T000.cut A14/CR3A14N15DC1ATO00AC1BT000.cut A14/CR3A14N15DC1BT000AC1BT142. cut A14/CR3A14N15DC1BT142AC02T000.cut A14/CR3A14N160C02T000AC07T000. Cut A14/CR3A14N160C1ATO00AC1BT000.cut A14/CR3A14N160C1BTODOAC1BT142.cut A14/CR3A14N160C1BT142AC02T000. cut A14/CR3A14N170C02TO00AC07T000.cut A14/CR3A14N17DC1ATO0OAC1BT000.cut A 14/CR3A14N17DC1BTDOOAC1BT 142. Cut A14/CR3A14N170C1BT142AC02T000.cut A14/CR3A14N180C02T000AC07T000.cut

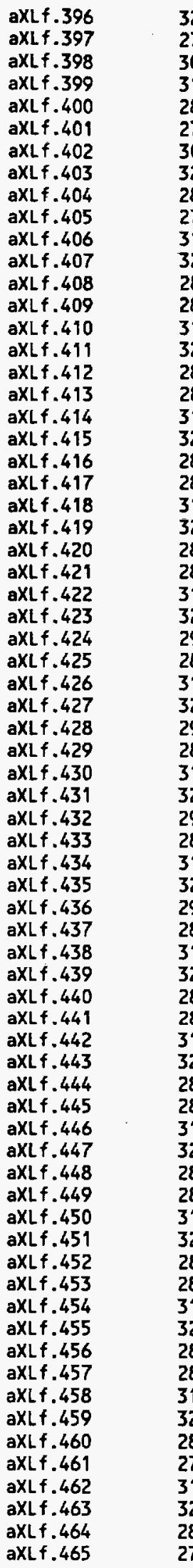

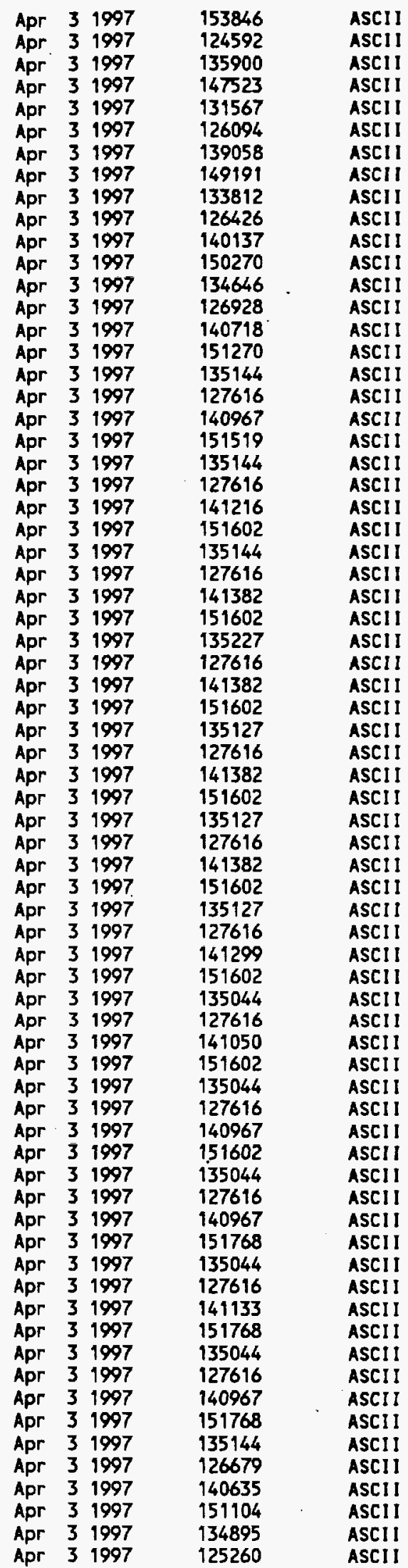

ASCII SCII

SCII

ASCII

SCII

ASCII

SCII

SCII

ASCII

SCII

SCII

SCII

ASCII

SCII

ASCII

SCII

SCII

ASCII

ASCII

ASCl 1

SCII

ASCI I

SCII

SCII

SCII

SCII

ASCII

ASCII

ASCII

SCII

ASCII 
Jul $3015: 371997$ File Name: maglist BBA000000-01717-0200-00040 REV 00 ATTACHMENT XL - Page 8

A14/CR3A14N18DC1AT000AC1BT000.cut A14/CR3A14N18DC1BTOOOAC1BT142.cut A14/CR3A14N18DC1BT 142AC02T000.cut
axLf. .466
aXLf. .467

aXLf.468 28 $\begin{array}{lll}\text { Apr } & 3 & 1997 \\ \text { Apr } & 3 & 1997 \\ \text { Apr } & 3 & 1997\end{array}$

137204

133397
ASCII

ASCII

ASCII

THE FOLLOWING FILES FOR ASSEMBLY A18 WERE SUPERCEEDED BY FILES UNDER ASSEMBLY DESIGNATION AI8Z DURING CHECKING.

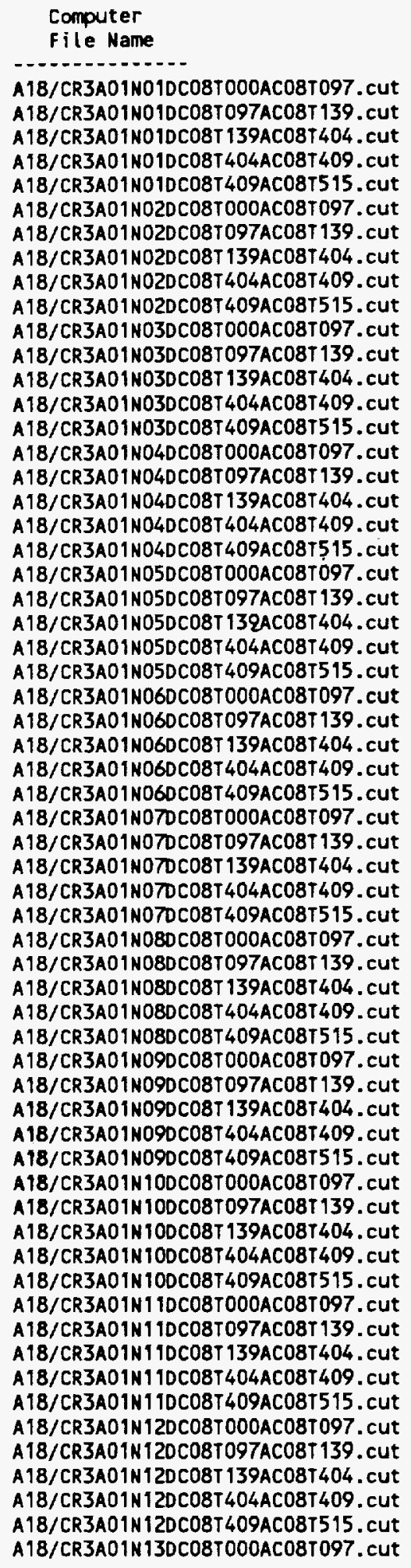

\begin{tabular}{|c|c|}
\hline $\begin{array}{l}\text { Tape Backup } \\
\text { File Name }\end{array}$ & $\begin{array}{l}\text { Number of } \\
\text { Print Pages }\end{array}$ \\
\hline - & \\
\hline $\begin{array}{l}\text { aXL }+.469 \\
\text { aXL } f .470\end{array}$ & $\begin{array}{l}31 \\
31\end{array}$ \\
\hline $\begin{array}{l}\text { aXLT. } \\
\text { aXL } f .471\end{array}$ & 32 \\
\hline aXL +.472 & 32 \\
\hline axL $f .473$ & 33 \\
\hline aXLf .474 & 32 \\
\hline aXLf. 475 & 32 \\
\hline aXLf. 476 & 33 \\
\hline aXLf. 477 & 33 \\
\hline aXLf. 478 & 33 \\
\hline aXLf .479 & 32 \\
\hline aXLf +.480 & 32 \\
\hline axLf. .481 & $3 \overline{3}$ \\
\hline axLf .482 & 33 \\
\hline $\mathrm{aXL}+.483$ & 34 \\
\hline aXL $f .484$ & 32 \\
\hline aXLf.485 & 32 \\
\hline aXLf.486 & 33 \\
\hline aXLf. .487 & 33 \\
\hline aXLf.488 & 34 \\
\hline axL $f .489$ & 32 \\
\hline aXLf.490 & 32 \\
\hline axLf.491 & 33 \\
\hline aXLf. 492 & 33 \\
\hline aXL $f .493$ & .34 \\
\hline aXLf.494 & 32 \\
\hline aXLf .495 & 32 \\
\hline aXLf. .496 & 33 \\
\hline aXLf.497 & 33 \\
\hline aXLf.498 & 34 \\
\hline aXLf.499 & 32 \\
\hline aXLf. .500 & 32 \\
\hline aXLf.501 & 33 \\
\hline axLf.502 & 33 \\
\hline aXLf.503 & 34 \\
\hline aXLf.504 & 32 \\
\hline aXLf.505 & 32 \\
\hline aXLf.506 & 33 \\
\hline axLf.507 & 33 \\
\hline aXLf..508 & 34 \\
\hline axLf.509 & 32 \\
\hline aXLf. .510 & 32 \\
\hline aXLf.511 & 33 \\
\hline aXLf.512 & 33 \\
\hline aXLf.513 & 34 \\
\hline axLf. 514 & 32 \\
\hline aXLf.515 & 32 \\
\hline aXLf.516 & 33 \\
\hline $\operatorname{axLf.517}$ & 33 \\
\hline aXLf.518 & 34 \\
\hline axLf.519 & 32 \\
\hline axLf. 520 & 32 \\
\hline aXLf.521 & 33 \\
\hline aXLf.522 & 33 \\
\hline aXLf. 523 & 34 \\
\hline axLf.524 & 32 \\
\hline aXLf.525 & 32 \\
\hline aXLf. .526 & 33 \\
\hline aXLf.527 & 33 \\
\hline aXLf.528 & 34 \\
\hline axLf .529 & 32 \\
\hline
\end{tabular}

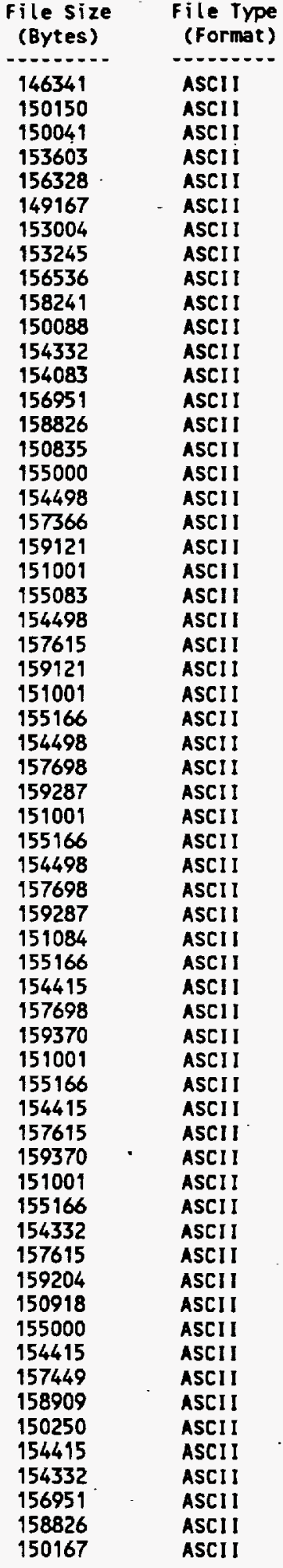


A18/CR3A01N13DC08T097AC08T 139. cut A18/CR3A01N13DC08T139AC08T404. cut A18/CR3A01N13DC08T404AC08T409.cut A18/CR3A01N13DC08T409AC08T515. cut A 18/CR3A01N14DC08T000AC08T097. Cut A18/CR3A01N14DC08T097AC08T139.cut A18/CR3A01N14DC08T139AC08T404.cut A 18/CR3A01N14DC08T404AC08T409. Cut A18/CR3A01N14DC08T409AC08T515.cut A18/CR3A01N15DC08TO00AC08T097.cut A 18/CR3A01N15DC08T097AC08T139.cut A 18/CR3A01N150C08T139AC08T404. Cut A18/CR3A01N15DC08T404AC08T409. cut A 18/CR3A0IN150C08T409AC08T515. cut A 18/CR3A01N160C08T000AC08T097. cut A 18/CR3A01N160C08T097AC08T 139.cut A18/CR3A01N160C08T 139AC08T404. cut A18/CR3A01N160C08T404AC08T409. Cut A18/CR3A01N16DC08T409AC08T515. Cut A18/CR3A01N17DC08T000AC08T097. Cut A18/CR3A01N17DC08T097ACOBT139. cut A18/CR3A01N17DC08T139AC08T404.Cut A18/CR3A01N170C08T404AC08T409. Cut A18/CR3A01N170C08T409ACO8T515. cut A18/CR3A01N18DC08TO00AC08T097. Cut A18/CR3A01N180C08T097AC08T139.cut A18/CR3A01N180C08T139ACOBT404.cut A18/CR3A01N180C08T404ACOBT409. cut A18/CR3A01N180C08T409AC08T515. CUT A18/CR3A18N010C1ATO00ACO8T000.cut A18/CR3A18N020C1ATODOACO8T000.cut A 18/CR3A18N030C1ATOOOACOBT000.CUt A 18/CR3A18N040C1ATO00AC08T000. Cut A18/CR3A18N05DC1ATDOOAC08T000.cut A18/CR3A18EN060C1ATDOOAC08T000. Cut A18/CR3A18NOTOC1ATODOAC08T000. Cut A18/CR3A18NOBOC1ATOOOACO8T000. Cut A18/CR3A18N090C1ATOOOAC08T000.cut A18/CR3A18N100C1ATO00AC08T000.cut A18/CR3A18N110C1ATOOOAC08T000. CUT A18/CR3A18N12DC1ATO00AC08T000.cut A18/CR3A18N130C1ATO00AC08T000.cut A18/CR3A18N14DC1AT000AC08T000.cut A18/CR3A18N15DC1ATOOOAC08T000.CUT A18/CR3A18N 16DC1AT000AC08T000.cut A18/CR3A18N1TDC1ATO00AC08T000. Cut A18/CR3A18N 18DC1ATO00AC08T000. Cut

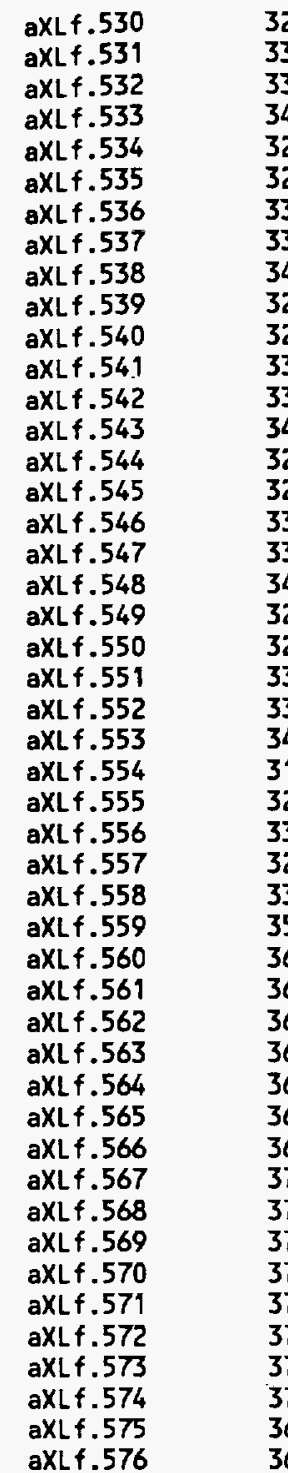

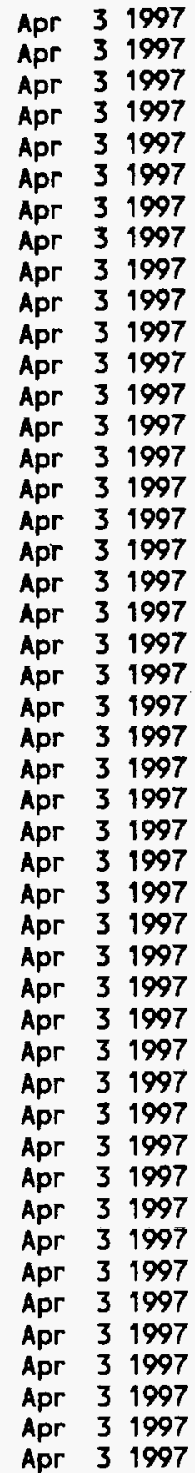

154249

156868

158660

149918

153917

153996

157034

158992

149835

153751

153996

157034

158992

150001

153917

153917

157117

158992

149337

153668

153581

156702

158573

147590

151316

151917

154682

157324

151789

153615

154362

154445

154611

154853

155019

155019

158196

158113

158286

157954

157436

157436

157436

157602

156274

154697
ASCII

ASCII

ASCII

ASCII

ASCII

ASCII

ASCII

ASCII

ASCII

ASCII

ASCII

ASCII

ASCII

ASCII

ASCII

ASCII

ASCII

ASCII

ASCII

ASCII

ASCII

ASCII

ASCII

ASCII

ASCII

ASCII

ASCII

ASCII

ASCII

ASCII

ASCII

ASCII

ASCII

ASCII

ASCII

ASCII

ASCII

ASCII

ASCII

ASCII

ASCII

ASCII

ASCII

ASCII

ASCII

ASCII

ASCII

THE FOLLOWING FILES FOR ASSEMBLY A18a WERE SUPERCEEDED BY FILES UNDER ASSEMBLY DESIGNATION A18aZ DURING CHECKING.

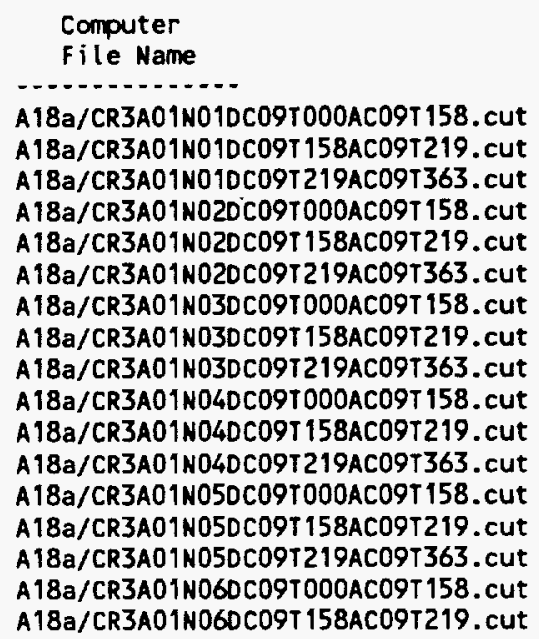

\begin{tabular}{ll}
$\begin{array}{c}\text { Tape Backup } \\
\text { File Name }\end{array}$ & $\begin{array}{c}\text { Number of } \\
\text { Print Pages }\end{array}$ \\
\hline aXLf.577 & 35 \\
aXLf.578 & 29 \\
aXLf.579 & 36 \\
aXLf.580 & 36 \\
aXLf.581 & 29 \\
aXLf.582 & 36 \\
aXLf.583 & 36 \\
aXLf.584 & 29 \\
aXLf.585 & 36 \\
aXLf.586 & 37 \\
aXLf.587 & 30 \\
aXLf.588 & 37 \\
aXLf.589 & 37 \\
aXLf.590 & 30 \\
aXLf.591 & 37 \\
aXLf.592 & 37 \\
aXLf.593 & 30
\end{tabular}

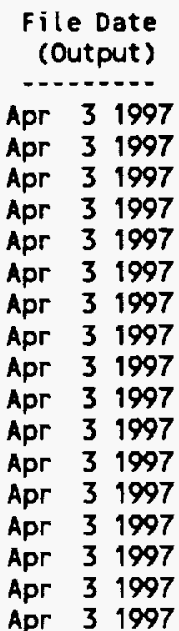

File Type (Format) ASCII

ASCII

ASCII

ASCII

ASCII

ASCII

ASCII

ASCII

ASCII

ASCII

ASCII

ASCII

ASCII

ASCI I

ASCII

ASCII

ASCI I 
A18a/CR3A01N06DC09T219ACO9T363. cut A 18a/CR3A01N070C09T000AC09T158. cut A18a/CR3A01N07DC09T158ACO9T219. cut A18a/CR3A01N07DC09T219ACO9T363. cut A18a/CR3A01N08DC09T000ACO9T158. cut A18a/CR3A01N08DC091158ACO9T219.cut A18a/CR3A01N0BDC09T219ACO9T363. cut A18a/CR3A01N09DC09T000ACO9T158. cut A 18a/CR3A01N090C09T158AC09T219. cut A 18a/CR3A01N090C09T219AC09T363. cut A18a/CR3A01N10DC09T000AC09T158. cut A18a/CR3A01N10DC09T158AC09T219. cut A18a/CR3A01N10DC09T219AC09T363.cut A18a/CR3A01N110C09T000AC09T158.cut A18a/CR3A01N110CO9T158AC09T219.cut A18a/CR3A01N110C09T219AC09T363. cut A18a/CR3A01N12DCO9TO0OACO9T158. cut A 18a/CR3A01N12DC09T158AC09T219. Cut A18a/CR3A01N12DC09T219AC09T363. cut A18a/CR3A01N13DC09T000AC09T158.cut A18a/CR3A01N13DCO9T158AC09T219.cut A18a/CR3A01N13DC09T219ACO9T363. cut A18a/CR3A01N14DC09T000ACO9T158.cut A 18a/CR3A01N14DC09T158AC09T219.cut A 18a/CR3A01N14DC09T219AC09T363. cut A 18a/CR3A01N15DCO9TOOOACO9T158. cut A 18a/CR3A01N15DCO9T158AC09T219.cut A18a/CR3A01N150C09T219AC09T363. cut A18a/CR3A01N160C09T000AC09T158. cut A18a/CR3A01N160CO9T158AC09T219.cut A18a/CR3A01N160C09T219AC09T363. cut A18a/CR3A01N170CO9T000ACO9T158. cut A 18a/CR3A01N170CO9T158AC09T219. cut A 18a/CR3A01N170CO9T219AC09T363. cut A18a/CR3A01N180CO9TO00ACO9T158. cut A18a/CR3A01N18DCO9T158AC09T219. cut A18a/CR3A01N180C09T219AC09T363. cut A18a/CR3A18N01DC1AT000AC09T000. cut A18a/CR3A18N020C1ATO00AC09T000. Cut A18a/CR3A18N03DC1ATO00AC09T000. cut A18a/CR3A18N04DC1ATD00AC09T000. cut A 18a/CR3A18N05DC1AT000ACO9T000. Cut A18a/CR3A18N06DC1ATO0OACO9T000. cut A 18a/CR3A18NOTDC1AT000AC09T000.cut A 18a/CR3A18N080C1ATO00ACO9T000. Cut A18a/CR3A18N090C1ATODOACO9T000. Cut A 18a/CR3A18N10DC1ATO00AC09T000.cut A18a/CR3A18N110C1ATO0OAC09T000.cut A18a/CR3A18N120C1ATOOOACO9T000.cut A 18a/CR3A18N13DC1ATDOOACO9T000.cut A18a/CR3A18N140C1ATOODACO9T000. Cut A18a/CR3A18N15DC1ATO00AC09T000. cut A18a/CR3A18N160C1ATO0OACO9T000.cut A18a/CR3A18N17DC1ATO00ACO9T000. cut A18a/CR3A18N180C1ATOOOACO9TO00.cut

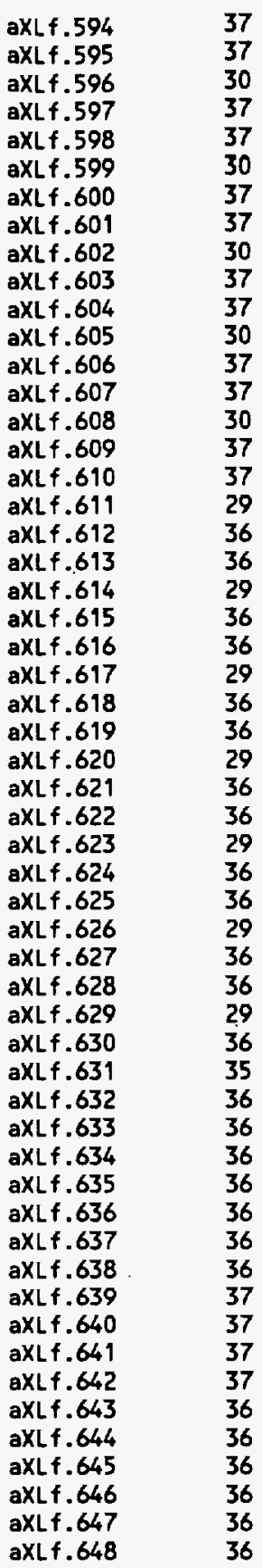

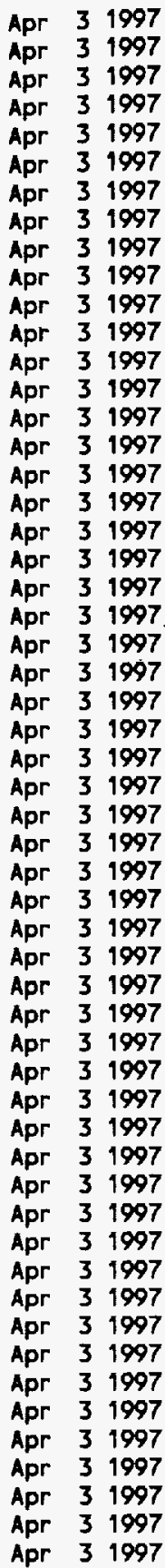

173720

172547

140721

173720

172547

140721

173720

172547

140638

173720

172464

140638

173720

172215

140306

173637

171879

139974

173139

171298

139642

172886

171215

139642

172886

171215

139559

172886

171298

139725

172969

170717

139642

172720

167974

136895

170641

150498

152324

153237

153532

153698

153774

154272

154272

157366

156868

157373

156829

156311

156228

156228

156477

155066

153240
ASCII

ASCII

ASCII

ASCII

ASCII

ASCII

ASCII

ASCII

ASCII

ASCII

ASCII

ASCII

ASCII

ASCII

ASCII

ASCII

ASCII

ASCII

ASCII

ASCII

ASCII

ASCII

ASCII

ASCII

ASCII

ASCII

ASCII

ASCII

ASCII

ASCII

ASCII

ASCII

ASCII

ASCII

ASCII

ASCII

ASCII

ASCII

ASCII

ASCII

ASCII

ASCII

ASCII

ASCII

ASCII

ASCII

ASCII

ASCI I

ASCII

ASCII

ASCII

ASCII

ASCII

ASCII

ASCII

THE FOLLONING FILES FOR ASSEMBLY A18b MERE SUPERCEEDED BY FILES UNDER ASSEMBLY DESIGNATION A18bZ DURING CHECKING.

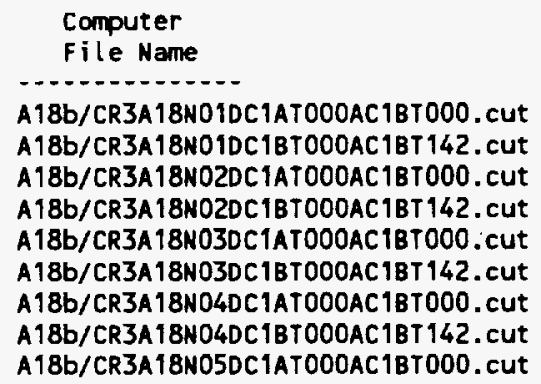

A18b/CR3A18NO1DC1ATOOOAC1BT000.cut A18b/CR3A18NO1DC1BTOOOAC1BT142. CUT A18b/CR3A18N030C1ATDOOAC1BT000.cut 18b/CR3A18N03DC1BTOOOAC1BT142. Cut A18b/CR3A18NO5DC1ATOOOAC1BT000. cut

$\begin{array}{ll}\text { Tape Backup } & \begin{array}{c}\text { Number of } \\ \text { File Name }\end{array} \\ \text { Print Pages }\end{array}$

\begin{tabular}{cc}
$\begin{array}{c}\text { File Size } \\
\text { (Bytes) }\end{array}$ & $\begin{array}{c}\text { File Type } \\
\text { (Format) }\end{array}$ \\
\hline 164042 & - \\
162716 & ASCII \\
166615 & ASCII \\
164878 & ASCII \\
167528 & ASCII \\
166625 & ASCII \\
168026 & ASCII \\
167252 & ASCII \\
168441 & ASCII \\
& ASCII
\end{tabular}


A18b/CR3A18N05DC1BT000AC1BT142. cut A 18b/CR3A18N06DC1AT000AC1BT000. Cut A 18b/CR3A18N060C1BTODOAC1BT 142. CUt A18b/CR3A18N070C1ATOOOAC1BT000. CUT A18b/CR3A18N0TDC18TO0OAC1BT142. Cut A 18b/CR3A18N080C1ATO0OAC1BT000. Cut A 18b/CR3A18N0BDC1BT000AC1BT142. Cut A18b/CR3A18N090C1AT000AC1BT000. CUT A 18b/CR3A18N090C1B TOOOAC1BT 142. Cut A18b/CR3A18N10DC1ATO0OAC1BT000. Cut A18b/CR3A18N100C1BTO0OAC1BT 142.cut A 18b/CR3A18N11DC1ATOOOACIBTO0O. cut A18b/CR3A18N11DC1BT000AC1BT142. cut A18b/CR3A18N120C1ATO0OAC1BT000. Cut A18b/CR3A18N12DC1BTDOOAC1BT142.cut A18b/CR3A18N130CIATO00AC1BT000.cut A18b/CR3A18N13DC1BTO0OAC1BT142.cut A 18b/CR3A18N14DC1AT000AC18T000.cut A18b/CR3A18N14DC1BTO0OAC1BT 142. cut A18b/CR3A18N15DC1AT000AC1BT000. cut A 18b/CR3A18N15DC1BTO0OAC1BT 142.cut A18b/CR3A18N160C1ATO0OAC1BT000. cut A 18b/CR3A18N160C1BTO00AC1BT142.cut A $18 \mathrm{~b} /$ CR3A18N17DC1AT000AC1BT000. cut A18b/CR3A18N17DC1BTO0OAC1BT 142.cut A 18b/CR3A18N18DC1ATOOOAC18T000. cUt A 18b/CR3A18N18DC1BTOOOAC1BT 142. Cut

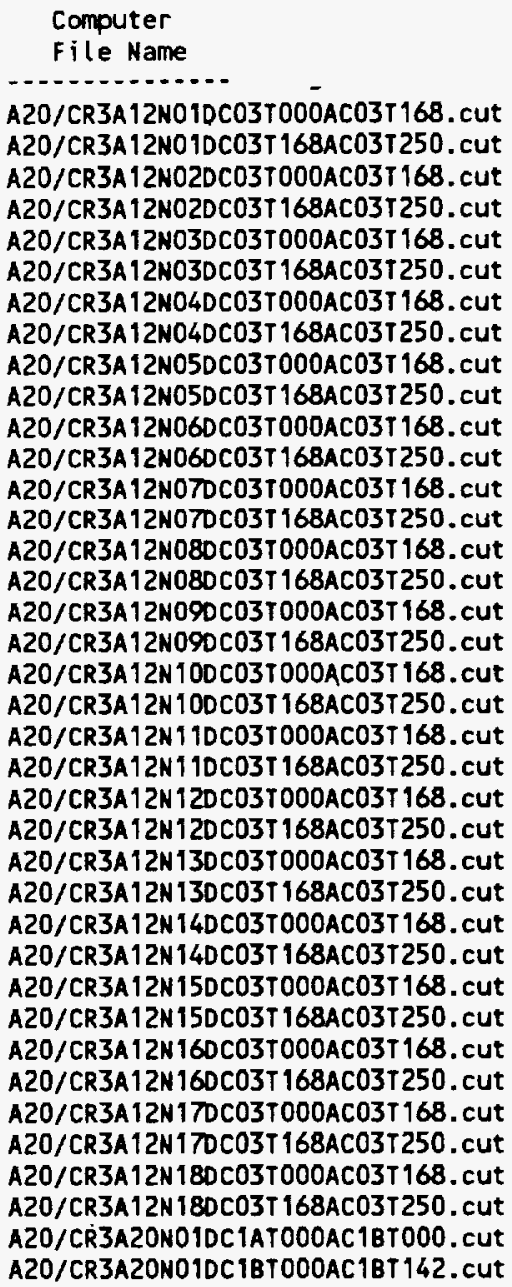

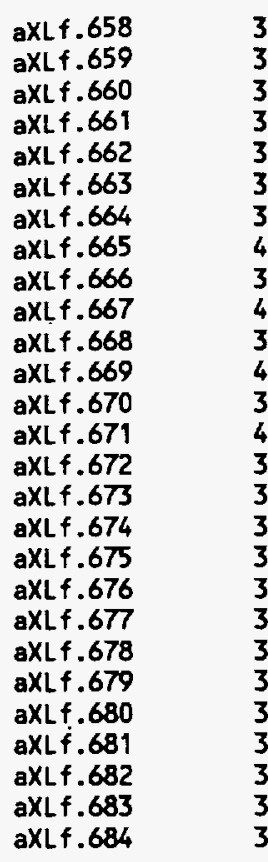

37
39
37
39
37
39
37
40
37
40
37
40
37
40
37
39
37
39
37
39
37
39
37
39
36
39
36

Apr 31997

Apr 31997

Apr 31997

Apr 31997

Apr 31997

Apr 31997

Apr 31997

Apr 31997

Apr 31997

Apr 31997

Apr 31997

Apr 31997

Apr 31997

Apr 31997

Apr 31997

Apr 31997

Apr 31997

Apr 31997

Apr 31997

Apr 31997

Apr 31997

Apr 31997

Apr 31997

Apr 31997

Apr 31997

Apr 31997

Apr 31997

Tape Backup

file Name

aXLf. 685

aXLf.686

aXLf.687

aXLf.688

aXLf. 689

aXLf.690

aXL $f: 691$

aXLf.692

aXLf.693

aXLf.694

aXLf.695

aXLf. 696

aXLf. 697

aXLf. 698

aXL $f .699$

aXL $f .700$

aXL $f .701$

aXLf.702

aXLf. 703

aXLf. 704

aXLf.705

aXLf.706

aXLf. 707

aXLf. 708

aXLf.709

aXL $f .710$

aXLf.711

aXLf. 712

aXLf. 713

aXLf. 714

aXL $f .715$

aXLf.716

aXLf.717

aXL $f .718$

aXLf.719

aXL $f .720$

aXLf. 721

aXLf. 722

Number of
Print Pages
33
32
33
33
34
33
34
33
34
33
34
33
34
33
34
33
34
33
34
33
34
33
34
33
34
33
34
33
34
33
34
33
34
33
33
32
40
31

File Date (Output)

Apr 31997

Apr 31997

Apr 31997

Apr 31997

Apr 31997

Apr 31997

Apr 31997

Apr 31997

Apr 31997

Apr 31997

Apr 31997

Apr 31997

Apr 31997

Apr 31997

Apr 31997

Apr 31997

Apr 31997

Apr 31997

Apr 31997

Apr 31997

Apr 31997

Apr 31997

Apr 31997

Apr 31997

Apr 31997

Apr 31997

Apr 31997

Apr 31997

Apr 31997

Apr 31997

Apr 31997

Apr 31997

Apr 31997

Apr 31997

Apr 31997

Apr 31997

Apr 31997
Apr 31997
167584

168590

167584

168839

167584

168922

167418

172099

167418

171850

170678

172389

170300

171642

169972

170875

169802

170875

169885

170958

169968

171124

169865

169772

164199

168195

161871

File size file Type

ASCII

ASCII

ASCII

ASCII

ASCII

ASCII

ASCII

ASCII

ASCII

ASCII

ASCII

ASCII

ASCII

ASCII

ASCII

ASCII

ASCII

ASCII

ASCII

ASCII

ASCII

ASCII

ASCII

ASCII

ASCII

ASCII

ASCII

(Bytes) (Format)

153075 ASCII

151539 ASCII

155569 ASCII

153618 ASCII

157004 ASCII

155348 ASCII

157385 ASCII

155929 ASCII

157385 ASCII

155929 ASCII

157385 ASCII

156012 ASCII

157668 ASCII

$156012^{\circ}$ ASCII

157668 ASCII

156012 ASCII

157751 ASCII

155680 - ASCII

157751 ASCII

155597 ASCI I

157668 ASCII

155431 ASCII

157385 ASCII

155431 ASCII

157502 ASCII

155763 ASCII

157585 ASCI I

155929 ASCII

157668 ASCII

155929 ASCII

157751 ASCII

156261 ASCII

157668 ASCII

155763 ASCII

154739 ASCII

152867 - ASCII

172227 ASCII

146523 ASCII 
A20/CR3A20N01DC1BT142ACO2T000.cut A2O/CR3A2ON02DC1ATOOOAC1BT000.cut A20/CR3AZONO2DC1BTOOOAC1BT142.cut A20/CR3A2ON02DC1BT 142AC02T000.cut A20/CR3A2ON03DC1ATOOOAC1BTO00.cut A20/CR3A2ON03DC1BTOOOAC1BT142. cut A20/CR3A2ON03DC1BT 142ACO2T000.CUt A20/CR3A2ONO4DC1ATO0OAC1BT000.cut A20/CR3A2ON04DC1BTO0OAC1BT 142. cut A20/CR3A2ONO4DC1BT 142AC02T000.CUT A20/CR3A2ON05DC1ATO0OAC1BT000.cUT A20/CR3A2ONO5DC1BTO0OAC1BT142. Cut A20/CR3A2ON05DC1BT 142AC02T000.cut A20/CR3A20N06DC1ATOOOAC1BT000. cut A20/CR3A20N06DC1BTO00AC1BT142. cut A20/CR3A20N06DC1BT142AC02T000. Cut A20/CR3A20NOTDC1ATOOOAC1BTO00. CUT A20/CR3A20N0TDC1BTO00AC1BT 142. cut A20/CR3A2ONOTDC1BT 142ACO2T000.cut A20/CR3A2ON080C1ATOOOAC1BT000. Cut A20/CR3A20N08DC1BTO0OAC1BT142. CUT A20/CR3A20N08DC1BT142AC02T000. CUT A20/CR3A20N09DC1ATO00AC1BT000. CUT A20/CR3A2ONO9DC1BTO0OAC1BT 142. CUT A20/CR3A2ON09DC1BT 142AC02T000. Cut A20/CR3A20N10DC1ATO00AC1BT000. CUT A20/CR3A20N10DC1BT000AC1BT142. CUT A20/CR3A20N10DC1BT 142AC02T000. cut A20/CR3A2ON11DC1ATO00AC1BTO00. Cut A20/CR3A20N110C1BT 000AC1BT 142. cut A20/CR3A2ON110C1BT142AC02T000. cut A20/CR3A20N12DC1ATODOAC1BT000. CUT A20/CR3A20N12DC1BTDOOAC1BT 142. cut A20/CR3A2ON12DC1BT 142ACO2T000. cut A20/CR3A20N130C1ATO00AC1BT000. cut A20/CR3A20N13DCIBTO0OACIBT 142. cut A20/CR3A2ON13DC1BT1 142AC02T000.cut A20/CR3A20N140C1ATOOOAC1BTO00.cut A20/CR3A20N14DC1BTO00AC1BT142. Cut A20/CR3A2ON140C1BT 142AC02T000. Cut A20/CR3A20N15DC1ATOOOAC1BTO00. CUT A20/CR3A2ON15DC1BTO00AC1BT142. cut A20/CR3A20N 150C1BT142AC02T000. cut A20/CR3A20N16DCIATOOOAC1BT000. CUT A20/CR3A2ON16DC1BTO00AC1BT142. cut A20/CR3A2ON160C1BT142AC02T000. Cut A20/CR3A20N1TDC1ATO0OAC1BTD00. Cut A20/CR3A2ON17DC1BTO0OAC1BT142. CUt A20/CR3A2ON170C1BT142AC02T000. cUt A20/CR3A20N180C1AT000AC1BT000. CUt A20/CR3A2ON180C1BTDODAC1BT 142. cut A20/CR3A20N180C1BT142ACO2T000. Cut A20/CR3A23N010C02TD00AC03T000.CUT A2O/CR3A23N02DCO2TOOOACO3TOOO.cut A20/CR3A23N03DC02TO0OACO3TO0O. cut A20/CR3A23N040CO2T000AC03T000.cut A20/CR3A23N05DC02TO0OAC03T000.CUT A20/CR3A23N060C02TO00ACO3T000.cut A20/CR3A23NOTOCO2TOOOACO3TOOO. Cut A2O/CR3A23N08DCO2TODOACO3TOOO.cut A20/CR3A23NO9OCO2TOOOACO3TOOO.cut A20/CR3A23N100C02TO00AC03T000.cut A20/CR3A23N11DC02T000AC03T000. cut A20/CR3A23N12DCO2TO0OACO3T000.cut A20/CR3A23N130C02TO0OAC03TO0O. Cut A20/CR3A23N140C02TO0OAC03T000. cut A2O/CR3A23N15DCO2TO0OACO3TO0O.cut A20/CR3A23N160C02T000AC03T000. cut A20/CR3A23N17DCO2TOOOACO3T000. cut A20/CR3A23N18DC02TO0OAC03T000. cut

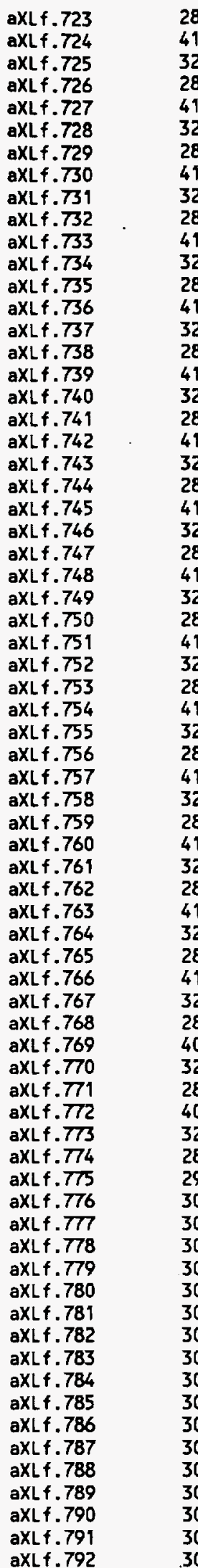

$28 \quad$ Apr 31997

32

28

41

32

28

41

28

41

32
28

41

32

41

32

28

41

28

41

32

28

41
32

28

41

32

28

32

28

41
32

32
28

41

32

28

41
32

28

41

32

40

40
32

28

40

32

28

29
30

30

30

30

30

30

30

30
30

30
30

30

30

30

30

aXL $f .792$

30 $\begin{array}{lll}\text { Apr } & 3 & 1997 \\ \text { Apr } & 3 & 1997 \\ \text { Apr } & 3 & 1997 \\ \text { Apr } & 3 & 1997 \\ \text { Apr } & 3 & 1997 \\ \text { Apr } & 3 & 1997 \\ \text { Apr } & 3 & 1997 \\ \text { Apr } & 3 & 1997 \\ \text { Apr } & 3 & 1997 \\ \text { Apr } & 3 & 1997 \\ \text { Apr } & 3 & 1997 \\ \text { Apr } & 3 & 1997 \\ \text { Apr } & 3 & 1997 \\ \text { Apr } & 3 & 1997 \\ \text { Apr } & 3 & 1997 \\ \text { Apr } & 3 & 1997 \\ \text { Apr } & 3 & 1997 \\ \text { Apr } & 3 & 1997 \\ \text { Apr } & 3 & 1997 \\ \text { Apr } & 3 & 1997 \\ \text { Apr } & 3 & 1997 \\ \text { Apr } & 3 & 1997 \\ \text { Apr } & 3 & 1997 \\ \text { Apr } & 3 & 1997 \\ \text { Apr } & 3 & 1997 \\ \text { Apr } & 3 & 1997 \\ \text { Apr } & 3 & 1997 \\ \text { Apr } & 3 & 1997 \\ \text { Apr } & 3 & 1997 \\ \text { Apr } & 3 & 1997 \\ \text { A } & 3 & 1997\end{array}$

$\begin{array}{lll}\text { Apr } & 3 & 1997 \\ \text { Apr } & 3 & 1997\end{array}$

Apr 31997

Apr 31997

Apr 31997

Apr 31997

Apr 31997

Apr 31997

Apr 31997

Apr 31997

Apr 31997

Apr 31997

Apr 31997

Apr 31997

Apr 31997

Apr 31997

Apr 31997

Apr 31997

Apr 31997

Apr 31997

Apr 31997

Apr 31997

Apr 31997

Apr 31997

Apr 31997

Apr 31997

Apr 31997

Apr 31997

Apr 31997

Apr 31997

Apr 31997

Apr 31997

Apr 31997

Apr 31997

Apr 31997

Apr 31997

Apr 31997

Apr 31997

Apr 31997

Apr 31997
Apr 31997
130986

174302

148772

133148

175903

149353

134148

176733

149938

134314

177148

150082

134480

177314

150414

134397

177480

150187

134563

177480

150187

134563

177480

150187

134563

177397

150187

134563

177231

150187

134646

177148

150082

134463

177148

150165

134546

177148

150165

134629

177314

150829

134795

176958

150938

134978

170066

150270

134480

168572

148523

132982

136725

139057

139721

140036

140202

140202

140202

140202

140036

140136

140223

140140

140140

140223

140306

140555

139721

138555

ASCII

ASCII

ASCII

ASCII

ASCII

ASCII

ASCII

ASCII

ASCII

ASCII

ASCII

ASCII

ASCII

ASCII

ASCII

ASCII

ASCII

ASCII

ASCII

ASCII

ASCII

ASCII

ASCII

ASCII

ASCII

ASCII

ASCII

ASCII

ASCII

ASCII

ASCII

ASCII

ASCII

ASCII

ASCII

ASCII

ASCII

ASCII

ASCII

ASCII

ASCII

ASCII

ASCII

ASCII

ASCII

ASCII

ASCII 


\begin{abstract}
Computer
File Name

A.2/CR3Az2N010

A22/CR3A22N01DC1ATO0OAC1BTO0O.cUt A22/CR3A22N01DC1BTOOOAC1BT142. cut A22/CR3A22N02DC1ATDOOAC1BTO0O. CUT A22/CR3A22N02DC1BTOOOAC1BT142. cut A22/CR3A22NO3DC1ATOOOAC1BTOOD. CUT A22/CR3A22NO3DC1BTOOOAC1BT 142. cut A22/CR3A22N04DC1ATOOOAC1BTODO. Cut A22/CR3A22N040C1BTOOOAC1BT 142. CUT A22/CR3A22N05OC1ATDOOAC1BTOOD. CUT A22/CR3A22N05DC1BTDOOAC1BT 142. cut A22/CR3A22NO60C1ATOOOAC1BT000.cut A22/CR3A22N060C1BTOOOAC1BT 142. cut A22/CR3A22NOTDC1ATOOOAC1BT000.cut A22/CR3A22NOTOC1BTOOOAC1BT 142. cut A22/CR3A22NOBDC1ATOOOAC1BT000.cut A22/CR3A22NOBOC1BTOOOAC1BT 142. cut A22/CR3A22N09DC1ATOOOAC1BT000.cut A22/CR3A22N090C1BTOOOAC1BT 142.cut A22/CR3A2ZN10DC1ATOOOAC1BTOOO.cut A22/CR3A22N10DC1BTODOAC1BT 142.cut A22/CR3A22N11DC1ATOOOAC1BTO0O.cut A22/CR3A22N110C1BTO0OAC1BT 142.cut A22/CR3A22N12DC1ATO00AC1BT000.cut A22/CR3A22N12DC1BTO0OAC1BT142.cut A22/CR3A22N130C1ATO0OAC1BT000.cut A22/CR3A22N 13DC1BTO0OAC1BT142.cut A22/CR3A22N 14DC1ATOOQAC1BT000.cut A22/CR3A22N 14DC1BTOOOAC1BT142.cut A22/CR3A22N 15DC1ATO00AC1BT000.cut A22/CR3A22N15DC1BTOOOAC1BT142. CUt A22/CR3A22N16DC1ATOOOAC1BT000.cut A22/CR3A22N16DC1BTO0OAC1BT142.cut A22/CR3A22N17DC1ATOOOAC1BT000.cut A22/CR3A22N17DC1BTOOOAC 1BT 142. cut A22/CR3A22N18DC1ATOOOAC1BT000. Cut A22/CR3A22N 18DC1BTOOOAC1BT 142.cut
\end{abstract}

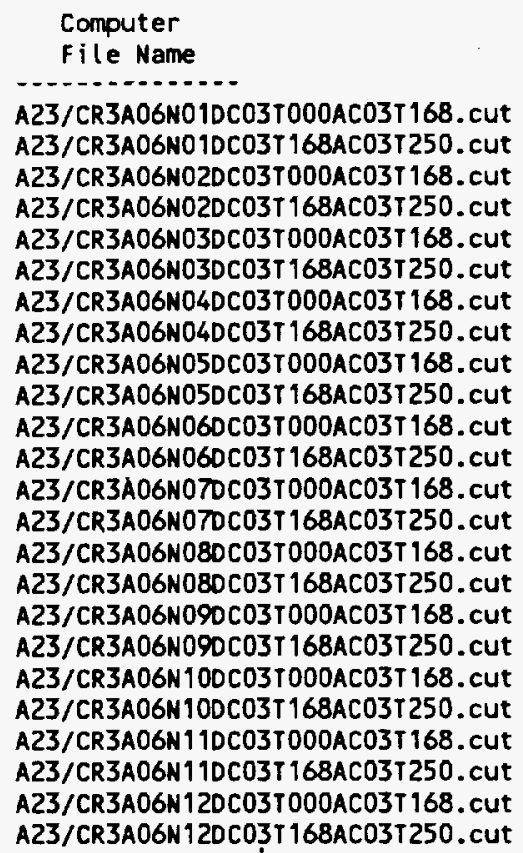

\begin{tabular}{|c|c|}
\hline $\begin{array}{c}\text { Tape Backup } \\
\text { File Name }\end{array}$ & $\begin{array}{l}\text { Number of } \\
\text { Print Pages }\end{array}$ \\
\hline - n & \\
\hline axLf. 793 & 33 \\
\hline aXLf. 794 & 36 \\
\hline aXLf.795 & 33 \\
\hline axLf. .796 & 37 \\
\hline axLf. .797 & 33 \\
\hline axLf.798 & 37 \\
\hline aXL $f .799$ & 32 \\
\hline aXL $f .800$ & 37 \\
\hline aXL $f .801$ & 33 \\
\hline axL f.802 & 36 \\
\hline axL $f .803$ & 33 \\
\hline aXLf. 804 & 36 \\
\hline aXLf .805 & 33 \\
\hline aXLf. 806 & 36 \\
\hline $\mathrm{aXL} f .807$ & 33 \\
\hline$a x L f .808$ & 36 \\
\hline aXLf. 809 & 33 \\
\hline $\mathrm{aXL}+.810$ & 36 \\
\hline axLf $f .811$ & 33 \\
\hline$a X L f .812$ & 36 \\
\hline aXLf. 813 & 32 \\
\hline axLf. 814 & 36 \\
\hline aXLf.815 & 32 \\
\hline axLff.816 & 36 \\
\hline axLf $f .817$ & 32 \\
\hline$a X L f .818$ & 36 \\
\hline axLf.819 & 32 \\
\hline aXLf. 820 & 36 \\
\hline aXLf $f .821$ & 32 \\
\hline$a \times L f .822$ & 36 \\
\hline axLff.823 & 32 \\
\hline aXLf.824 & 36 \\
\hline aXLf .825 & 32 \\
\hline$a \times L f .826$ & 36 \\
\hline aXLf .827 & 32 \\
\hline aXLf. .828 & 36 \\
\hline
\end{tabular}

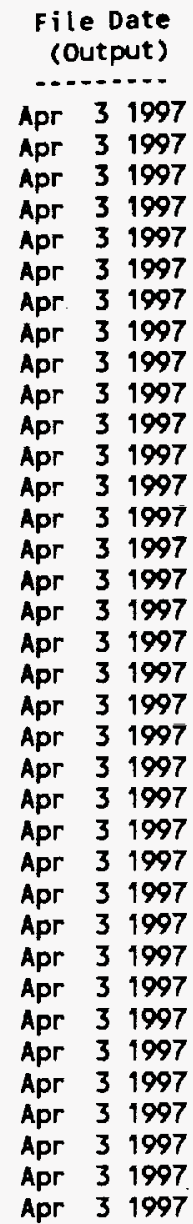

\begin{tabular}{cc}
$\begin{array}{c}\text { File Size } \\
\text { (Bytes) }\end{array}$ & $\begin{array}{c}\text { File Type } \\
\text { (Format) }\end{array}$ \\
\hdashline-1 & ASCII \\
146554 & ASCII \\
164264 & ASCII \\
150147 & ASCII \\
168474 & ASCII \\
150206 & ASCII \\
169616 & ASCII \\
146200 & ASCII \\
170077 & ASCII \\
146681 & ASCII \\
165490 & ASCII \\
146847 & ASCII \\
166178 & ASCII \\
146930 & ASCII \\
166178 & ASCII \\
146930 & ASCII \\
165407 & ASCII \\
146930 & ASCII \\
165241 & ASCII \\
146847 & ASCII \\
165075 & ASCII \\
146432 & ASCII \\
164992 & ASCII \\
146117 & ASCII \\
164992 & ASCII \\
145785 & ASCII \\
164909 & ASCII \\
145702 & ASCII \\
164697 & ASCII \\
145702 & ASCII \\
164863 & ASCII \\
145619 & ASCII \\
164946 & ASCII \\
145287 & ASCII \\
164365 & ASCII \\
143710 & ASCII \\
161954 & ASCII
\end{tabular}

\begin{tabular}{ll}
$\begin{array}{l}\text { Tape Backup } \\
\text { File Name }\end{array}$ & $\begin{array}{c}\text { Number of } \\
\text { Print Pages }\end{array}$ \\
\hline aXLf.829 & 33 \\
aXLf.830 & 32 \\
aXLf.831 & 33 \\
aXLf.832 & 32 \\
aXLf.833 & 34 \\
aXLf.834 & 33 \\
aXLf.835 & 34 \\
aXLf.836 & 33 \\
aXLf.837 & 34 \\
aXLf.838 & 33 \\
aXLf.839 & 34 \\
aXLf.840 & 33 \\
aXLf.841 & 34 \\
aXLf.842 & 33 \\
aXLf.843 & 34 \\
aXLf.844 & 33 \\
aXLf.845 & 34 \\
aXLf.846 & 33 \\
aXLf.847 & 34 \\
aXLf.848 & 33 \\
aXLf.849 & 34 \\
aXLf.850 & 33 \\
aXLf.851 & 34 \\
aXLf.852 & 33
\end{tabular}

\begin{tabular}{cc}
$\begin{array}{c}\text { File Size } \\
\text { (Bytes) }\end{array}$ & $\begin{array}{c}\text { File Type } \\
\text { (Format) }\end{array}$ \\
\hdashline 151877 & - \\
151037 & ASCII \\
154818 & ASCII \\
153448 & ASCII \\
157502 & ASCII \\
154826 & ASCII \\
157917 & ASCII \\
156012 & ASCII \\
157917 & ASCII \\
156012 & ASCII \\
157917 & ASCII \\
156012 & ASCII \\
157917 & ASCII \\
156012 & ASCII \\
157917 & ASCII \\
156012 & ASCII \\
157834 & ASCII \\
155929 & ASCII \\
157751 & ASCII \\
156012 & ASCII \\
157751 & ASCII \\
155846 & ASCII \\
157834 & ASCII \\
155075 & ASCII \\
& ASCII
\end{tabular}


A23/CR3A06N13DC03T000AC03T168. cut A23/CR3A06N13DC03T168AC03T250. cut A23/CR3A06N14DCO3TOOOACO3T168. cut A23/CR3A06N14DC03T168ACO3T250. cut A23/CR3A06N15DC03TO00ACO3T168. cut A23/CR3A06N150C03T168ACO3T250. cut A23/CR3A06N160C03TO00AC03T168. cut A23/CR3A06N160C03T168AC03T250. cut A23/CR3A06N170C03TO0OAC03T168. Cut A23/CR3A06N17DCO3T16BACO3T250. cut A23/CR3A06N180C03T000AC03T168. Cut A23/CR3A06N180C03T16BACO3T250. Cut A23/CR3A07N01DC02TO0OAC03T000. Cut A23/CR3A07N02DC02TO0OAC03T000. cut A23/CR3A07N03DC02TO0OAC03T000.cut A23/CR3A07N04DCO2TOOOACO3TOOO. cut A23/CR3A07N05DC02TO00ACO3TO00.cut A23/CR3A07N06DCO2TO00ACO3TO0O. Cut A23/CR3A07N070C02 TO0OACO3TOOO. Cut A23/CR3A07NO8DC02TOOOACO3TOOO. CUT A23/CR3A07N090C02TO0OACO3TO00.cut A23/CR3A07N100C02TDOOACO3TO0O. Cut A23/CR3A07N110C02T000AC03T000.cut A23/CR3A07N120CO2TO00ACO3TO0O. CUT A23/CR3A07N130C02TO00AC03T000. Cut A23/CR3A07N140C02TOOOACO3TO00.cut A23/CR3A07N15DC02TO0OACO3T000. Cut A23/CR3A07N160C02TO0OACO3TO00. cut A23/CR3A07N1TOCO2TO0OAC03TO0O. CUt A23/CR3A07N180C02T000AC03T000.cut A23/CR3A23N010C1AT00OAC1BT000. CUT A23/CR3A23N010C1BTOOOAC1BT142. CUT A23/CR3A23H010C1BT 142AC02T00O. cut A23/CR3A23N02DC1ATDOOAC1BT000. CUT A23/CR3A23N02DC1BTOOOAC1BT142. CUT A23/CR3A23NO2DC1BT142ACO2T00O. CUt A23/CR3A23NO3DC1ATODOAC1BTOOO. cut A23/CR3A23NO3DC1BTDOOAC1BT 142. CUt A23/CR3A23N030C1BT142ACO2T000.CUT A23/CR3A23N040C1ATOOOAC1BT000. CUt A23/CR3A23N04DC1BTO0OAC1BT142. cUt A23/CR3A23NO4DC1BT142ACO2T00O. Cut A23/CR3A23N05DC1ATDOOAC1BT000. CUT A23/CR3A23N05DC1BTDOOAC1BT 142.cut A23/CR3A23NO5DC1BT 142AC02T000.cut A23/CR3A23NO60C1ATOODAC1BT000.cut A23/CR3A23NO60C18TOOOAC1BT 142.cut A23/CR3A23N060C1BT 142AC02T000.cut A23/CR3A23NOTDC1ATOOOAC1BTOOO.CUT A23/CR3A23N07DC1BTOOOAC1BT142.cut A23/CR3A23NOTOC1BT 142ACO2T000. cut A23/CR3A23NOBDC1ATOOOAC1BTO00. Cut A23/CR3A23NOBDC1BTOOOAC1BT 142.CUT A23/CR3A23NO8DC1BT 142AC02T000. Cut A23/CR3A23NO9DC1ATO0OAC1BT000.cut A23/CR3A23NO9DC1BTO0OAC1BT 142. Cut A23/CR3A23N09DC1BT 142AC02T000.cut A23/CR3A23N10DC1ATOOOAC $1 B$ T000. CUT A23/CR3A23N10DC1BTO0OAC1BT142.cut A23/CR3A23N10DC1BT 142AC02T000. cut A23/CR3A23N11DC1ATO0OAC1BT000. Cut A23/CR3A23N11DC1BTO0OAC1BT142. CUt A23/CR3A23N11DC1BT 142AC02T000. Cut A23/CR3A23N12DC1ATO0OAC1BT000.cut A23/CR3A23N120C1BTO0OAC1BT142. Cut A23/CR3A23N12DC1BT 142ACO2T000.cut A23/CR3A23N13DC1ATOOOAC1BT000.cut A23/CR3A23N13DC1BT000AC1BT142.cut A23/CR3A23N13DC1BT 142ACO2T000. Cut A23/CR3A23N14DC1ATOOOAC1BT000.CUT

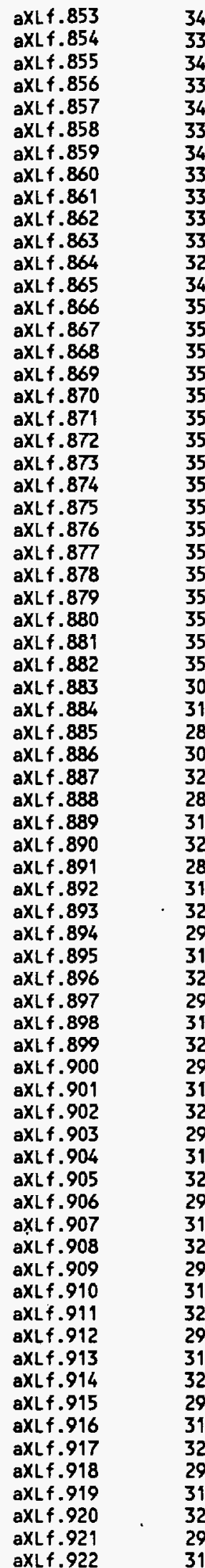

Apr 31997

Apr 31997

Apr 31997

Apr 31997

Apr 31997

Apr 31997

Apr 31997

Apr 31997

Apr 31997

Apr 31997

Apr 31997

Apr 31997

Apr 31997

Apr 31997

Apr 31997

Apr 31997

Apr 31997

Apr 31997

Apr 31997

Apr 31997

Apr 31997

Apr 31997

Apr 31997

Apr 31997

Apr 31997

Apr 31997

Apr 31997

Apr 31997

Apr 31997

Apr 31997

Apr 31997

Apr 31997

Apr 31997

Apr 31997

Apr 31997

Apr 31997

Apr 31997

Apr 31997

Apr 31997

Apr 31997

Apr $3 \quad 1997$

Apr 31997

Apr 31997

Apr 31997

Apr 31997

Apr 31997

Apr 31997

Apr 31997

Apr 31997

Apr 31997

Apr 31997

Apr 31997

Apr 31997

Apr 31997

Apr 31997

Apr $3 \quad 1997$

Apr 31997

Apr 31997

Apr 31997

Apr 31997

Apr 31997

Apr 31997

Apr 31997

Apr 31997

Apr 31997

Apr 31997

Apr 31997

Apr 31997

Apr 31997

Apr 31997

155763

157668

155929

157668

156012

157731

156095

156648

154909

154569

152867

154664

157584

158667

159082

159165

159248

159248

159248

159248

159165

158999

159082

159082

158494

158494

158199

157498

155087

136564

147693

131982

139805

149274

134061

140718

151270

135061

141465

151768

135310

141780

152017

135476

141780

152229

135559

141946

152229

135559

141946

152312

135559

141946

152312

135559

141780

152312

135476

141714

151934

135393

141465

151436

135227

141216

151519

135310

141050
ASCII

ASCII

ASCII

ASCII

ASCII

ASCII

ASCII

ASCII

ASCII

ASCII

ASCII

ASCII

ASCII

ASCII

ASCII

ASCII

ASCII

ASCII

ASCII

ASCII

ASCII

ASCI I

ASCII

ASCII

ASCII

ASCII

ASCII

ASCII

ASCII

ASCII

ASCII

ASCII

ASCII

ASCII

ASCII

ASCII

ASCII

ASCII

ASCII

ASCI I

ASCII

ASCII

ASCII

ASCII

ASCII

ASCII

ASCII

ASCII

ASCII

ASCII

ASCI

ASCII

ASCI

ASCII

ASCII

ASCII

ASCII

ASCII

ASCII

ASCII

ASCII

ASCII

ASCII

ASCII

ASCII

ASCII

ASCII

ASCII

ASCI I

ASCII 
A23/CR3A23N140C1BTO0OAC1BT142. cut A23/CR3A23N14DC1BT142AC02T000.cut A23/CR3A23N15DC1ATOOOAC1BTO00. cut A23/CR3A23N15DC1BTOOOAC1BT 142.cut A23/CR3A23N15DC1BT 142ACO2T000.cut A23/CR3A23N16DC1ATO00AC1BT000.cut A23/CR3A23N160C1BTOOOAC1BT142.cut A23/CR3A23N160C1BT142AC02T000. cut A23/CR3A23N17DCIATOOOAC18T000.cut A23/CR3A23NITDCIBTOOOAC1BT 142. CUT A23/CR3A23N17DC1BT 142AC02T000.cut A23/CR3A23N180C1ATOOOAC18TO00. cut A23/CR3A23N180C1BT000AC1BT142. cut A23/CR3A23N18DC1BT142AC02T000.cut

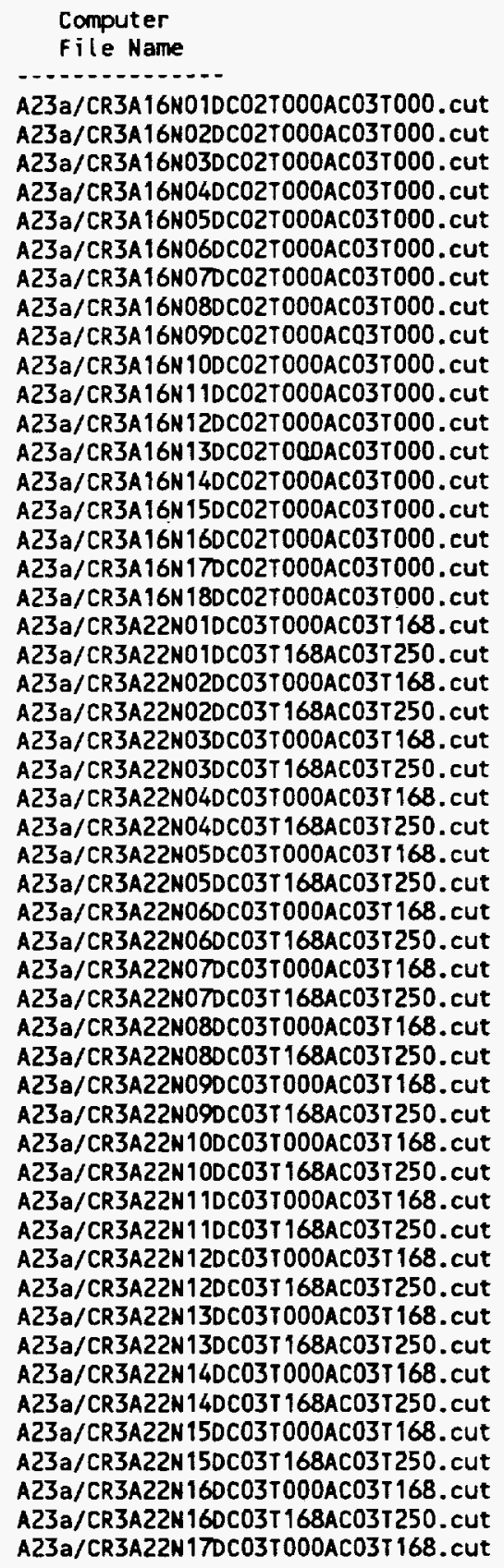

$\begin{array}{ll}\text { aXLf. } .923 & 32 \\ \text { aXLf. } 924 & 28 \\ \text { aXLf.925 } & 31 \\ \text { aXLf.926 } & 32 \\ \text { aXLf.927 } & 28 \\ \text { aXLf.928 } & 31 \\ \text { aXLf.929 } & 32 \\ \text { aXLf. } 930 & 28 \\ \text { aXLf.931 } & 31 \\ \text { aXLf.932 } & 32 \\ \text { aXLf.933 } & 28 \\ \text { aXLf. } 934 & 30 \\ \text { aXLf.935 } & 32 \\ \text { aXLf.936 } & 28\end{array}$

$\begin{array}{llll}32 & \text { Apr } 3 & 1997 \\ 28 & \text { Apr } & 3 & 1997 \\ 31 & \text { Apr } 3 & 1997 \\ 32 & \text { Apr } 3 & 1997 \\ 28 & \text { Apr } 3 & 1997 \\ 31 & \text { Apr } 3 & 1997 \\ 32 & \text { Apr } 31997 \\ 28 & \text { Apr } 31997 \\ 31 & \text { Apr } 31997 \\ 32 & \text { Apr } 31997 \\ 28 & \text { Apr } 31997 \\ 30 & \text { Apr } 31997 \\ 32 & \text { Apr } 31997 \\ 28 & \text { Apr } 31997\end{array}$

Tape Backup

file Name

axt $f .037$

aXLf.938

aXL $f .939$

aXLf. 940

aXLf.941

axLf. 942

aXLf. 943

aXLf. 944

aXL $f .945$

axLf.946

aXL $f .947$

aXL $f .948$

axLf. 949

aXL $f .950$

aXLf.951

aXL $f .952$

aXLf. 953

aXLf. 954

aXLf. 955

aXL $f .956$

aXLf.957

aXLf.958

aXLf.959

aXL $f .960$

aXLf.961

aXLf. 962

aXL $f .963$

aXLf.964

aXLf.965

aXLf.966

aXL $f .967$

aXLf.968

aXLf.969

aXL $f .970$

axLf.971

aXLf.972

aXL $f .973$

aXL $f .974$

aXL $f .975$

axLf.976

aXL $f .977$

aXL $f .978$

aXL $f .979$

aXLf. 980

aXLf. 981

aXLf. 982

aXLf. 983

aXLf.984

aXLf. 985

aXLf. 986

aXLf.987

Number of
Print Pages
35
35
35
35
35
35
35
35
35
35
35
35
35
35
35
35
35
35
33
32
33
33
34
33
34
33
34
33
34
33
34
33
34
33
34
33
34
33
34
33
34
33
34
33
34
33
34
33
34
33
34

$\begin{array}{ll}151436 & \text { ASCII } \\ 135144 & \text { ASCII } \\ 140884 & \text { ASCII } \\ 151602 & \text { ASCII } \\ 135044 & \text { ASCII } \\ 140884 & \text { ASCII } \\ 151685 & \text { ASCII } \\ 135144 & \text { ASCII } \\ 140635 & \text { ASCII } \\ 151187 & \text { ASCII } \\ 134978 & \text { ASCII } \\ 137204 & \text { ASCII } \\ 149108 & \text { ASCII } \\ 133480 & \text { ASCII }\end{array}$

file size file Type

(Bytes)

155245

158414

159202

159075

159534

159534

159534

158946

159534

159451

159368

158614

159119

158531

159136

158697

157830

155834

152379

151369

155320

153618

157585

155846

157751

156178

157917

156344

157917

156344

158000

156344

158083

156178

158000

156095

157751

155680

157668

156012

157834

156012

157751

156012

157668

156012

157668

156178

157834

156261

157834

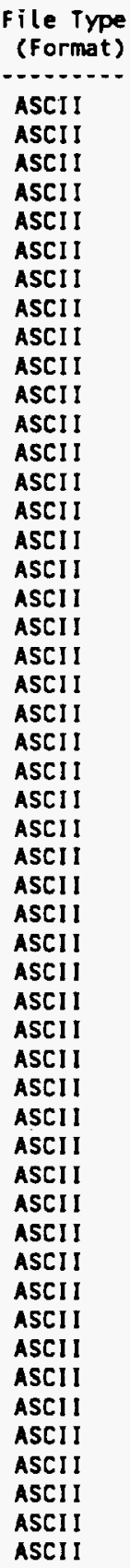


A23a/CR3A22N170C03T168AC03T250. cut A23a/CR3A22N180C03TO0OACO3T168. cut A23a/CR3A22N180C03T168AC03T250. cut A23a/CR3A23N010C1BTO0OAC1BT 142. CUT A23a/CR3A23N01DC1BT142AC02T000.cut A23a/CR3A23NO2DC1BTOOOAC1BT142. cut A23a/CR3A23N02DC1BT142ACO2T00O. cut A23a/CR3A23N030C1BTDOOAC1BT142. CUt A23a/CR3A23N03DC1BT142AC02T000. CUt A23a/CR3A23N04DC1BTOOOAC1BT 142. cut A23a/CR3A23N040C1BT 142ACO2T000.cut A23a/CR3A23N05DC1BTDOOAC1BT142. Cut A23a/CR3A23N050C1BT142AC02T000. CUt A23a/CR3A23N060C1BTO0OAC1BT 142. CUt A23a/CR3A23N060C1BT142ACO2T000. cut A23a/CR3A23NOTDC1BTDOOAC1BT142. cut A23a/CR3A23NOTDC1BT 142ACO2TO0O. CUT A23a/CR3A23N0BOC1BTOOOAC1BT 142. CUT A23a/CR3A23N08DC1BT 142AC02T000.cut A23a/CR3A23NO9OC1BTOODAC1BT 142. cut A23a/CR3A23N090C1BT142AC02T000.cut A23a/CR3A23N10DC1BTODOAC1BT 142.cut A23a/CR3A23N10DC1BT 142AC02T000.cut A23a/CR3A23N110C1BTDOOAC1BT 142.cut A23a/CR3A23N11DC1BT 142AC02T000.cut A23a/CR3A23N12DC1BTOOOAC1BT142. cut A23a/CR3A23N120C1BT142AC02T000. cut A23a/CR3A23N13DC1BTOOOAC1BT 142. cut A23a/CR3A23N130C1BT142ACO2T000.cut A23a/CR3A23N140C1BTOOOAC1BT 142. cut A23a/CR3A23N14DC1BT142AC02T000. CUt A23a/CR3A23N150C1BTOOOAC1BT 142. cut A23a/CR3A23N15DC1BT142AC02T000.cut A23a/CR3A23N16DC1BTO0OAC1BT 142.cut A23a/CR3A23N160C1BT142AC02T000.cut A23a/CR3A23N17DC1BTO0OAC1BT 142.cut A23a/CR3A23N1TDC1BT142AC02T000.cut A23a/CR3A23N180C1BTOOOAC1BT 142.cut A23a/CR3A23N180C1BT142AC02T000.cut

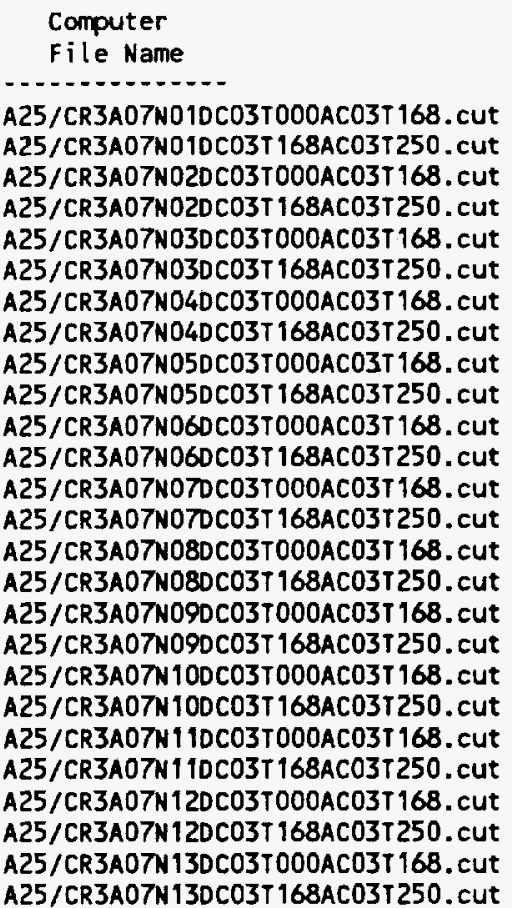

aXL $f .988$

aXLf.989

aXL $f .990$

aXL $f .991$

aXLf.992

aXLf.993

axL $f .994$

aXL $f .995$

axLf.996

aXLf.997

aXL $f .998$

aXL $f .999$

axL +1.000

$\operatorname{axL}+1.001$

aXL $f 1.002$

aXL +1.003

aXL +1.004

aXL +1.005

aXL +1.006

aXL +1.007

aXL +1.008

aXL +1.009

axL +1.010

axL 1.011

aXL +1.012

aXL $f 1.013$

aXLf1.014

aXLf1.015

aXL 1.016

aXL 1.017

aXL 1.018

aXL 1.019

aXL 1.020

aXL 1.021

aXL 1.022

aXL $f 1.023$

aXLf 1.024

aXL 1.025

aXL 1.026

Tape Backup
file Nane
-

aXL +1.027

aXL +1.028

aXL +1.029

aXL +1.030

aXL $f 1.031$

aXL $f 1.032$

aXL +1.033

aXL +1.034

aXL $f 1.035$

aXL 19.036

aXL 1.037

aXL +1.038

aXL 11.039

aXL $f 1.040$

aXL $f 1.041$

aXL $f 1.042$

aXL $f 1.043$

aXL 11.044

aXL 1.045

aXL 1 1.046

aXL +1.047

aXL 1 1.048

aXLf1.049

aXL +1.050

aXL 1.051

aXL $f 1.052$

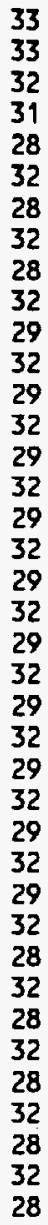

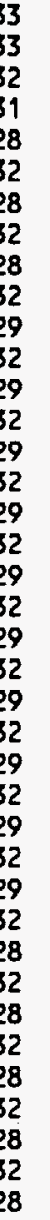

Number of
Print Pages
38
36
39
37
39
37
39
37
39
37
39
37
39
37
39
37
39
37
39
37
39
37
39
37
39
37

Apr 31997

Apr 31997

Apr 31997

Apr 31997

Apr 31997

Apr 31997

Apr 31997

Apr 31997

Apr 31997

Apr 31997

Apr 31997

Apr 31997

Apr 31997

Apr 31997

Apr 31997

Apr 31997

Apr 31997

Apr 31997

Apr 31997

Apr 31997

Apr 31997

Apr 31997

Apr 31997

Apr 31997

Apr 31997

Apr 31997

Apr 31997

Apr 31997

Apr 31997

Apr 31997

Apr 31997

Apr 31997

Apr 31997

Apr 31997

Арг 31997

Apr 31997

Apr 31997

Apr 31997

Apr 31997

156012

154822

153245

147693

131982

149274

133978

151270

134978

151768

135310

152017

135559

152229

135559

152229

135559

152312

135559

152312

135476

152312

135476

151934

135393

151436

135310

151519

135227

151436

135044

151602

135044

151685

135044

151187

134978

149108

133480

File Date (Output)

Apr 31997

Apr 31997

Apr 31997

Apr 31997

Apr 31997

Apr 31997

Apr 31997

Apr 31997

Apr 31997

Арг 31997

Apr 31997

Apr 31997

Apr 31997

Apr 31997

Apr 31997

Арг 31997

Apr 31997

Apr 31997

Apr 31997

Apr 31997

Apr 31997

Apr 31997

Apr 31997

Apr 31997

Apr 31997

Apr 31997
File size

(Bytes)

172583

165437

177606

168951

178270

169997

178204

170246

178519

170163

178519

170163

178519

170163

178436

170163

178436

170163

178436

170246

178436

169492

178436

170329

178436

170329

ASCII

ASCII

ASCII

ASCII

ASCII

ASCII

ASCII

ASCII

ASCII

ASCII

ASCII

ASCII

ASCII

ASCII

ASCII

ASCII

ASCII

ASCII

ASCII

ASCII

ASCII

ASCII

ASCII

ASCII

ASCII

ASCII

ASCII

ASCII

ASCII

ASCII

ASCII

ASCII

ASCI I

ASCII

ASCII

ASCII

ASCII

ASCII

ASCII

File Type 
A25/CR3A07N14DC03TO00AC03T168. Cut A25/CR3AOTN14DC03T168AC03T250. cut A25/CR3AOTN15DCO3TO00AC03T 168. cut A25/CR3A07N 15DC03T 168AC03T250. cut A25/CR3AO7N16DCO3TO0OAC03T168.cut A25/CR3A07N 160C03T168AC03T250.cut A25/CR3A07N17DCO3TO00AC03T168.cut A25/CR3A07N1TDC03T 168AC03T250.cut A25/CR3AOTN 18DC03TO00AC03T168.cut A25/CR3A07N18DCO3T 168AC03T250. cut A25/CR3A13N01DC02TO00AC03T000. CUt A25/CR3A13N02DC02TO00AC03TO00.Cut A25/CR3A13N03DCO2TOOOACO3TO0O. cut A25/CR3A13N04DC02TO00AC03T000. cut A25/CR3A13N05DC02TOOOAC03T000. cut A25/CR3A13N06DC02TO0OAC03T000.cut A25/CR3A13NOTOC02TO0OAC03T000. cut A25/CR3A13N08DC02TO00AC03T000.cut A25/CR3A13NO9DC02TO00AC03T000. cut A25/CR3A13N10DC02TOOOAC03T000. CUt A25/CR3A13N110C02TO00AC03T000. cut A25/CR3A13N 12DCO2TO00AC03T000. cut A25/CR3A13N 13DC02TO0OACO3T000. cut A25/CR3A13N14DCO2TO00AC03T000. cut A25/CR3A13N 15DC02TO00AC03T000.cut A25/CR3A 13N160C02TO00AC03T000. cut A25/CR3A13N17DCO2TO00AC03T000. cut A25/CR3A13N18DCO2TOOOACO3T000. Cut A25/CR3A25N01DCIATO00AC1BT000. CUT A25/CR3A25N01DC1BTO00AC1BT142. cut A25/CR3A25N01DC1BT 142AC02T000. Cut A25/CR3A25N02DC1ATOOOAC1BT000.cut A25/CR3A25N02DC1BTO0OAC1BT142. CUt A25/CR3A25N02DC1BT 142AC02T000.cut A25/CR3A25NO3DC1ATO00AC1BT000.CUT A25/CR3A25N03DC1BT000AC1BT142. Cut A25/CR3A25NO3DC1BT 142ACO2T000. cut A25/CR3A25N04DC1AT000AC1BT000.cut A25/CR3A25N04DC1BTO0OAC1BT142.cut A25/CR3A25N04DC1BT 142AC02T000. CUt A25/CR3A25N05DC1ATO00AC18T000.cut A25/CR3A25N05DC1BTO00AC1BT142. Cut A25/CR3A25N05DC1BT142AC02T000.cut A25/CR3A25NO60C1ATOOOAC1BT000. cut A25/CR3A25NO6DC1BT000AC1BT142. CUt A25/CR3A25N060C1BT142AC02T000. Cut A25/CR3A25N0TDC1ATO0OAC1BT000. CUT A25/CR3A25NOTDC1BTO0OAC1BT 142. CUT A25/CR3A25N07DC1BT 142AC02T000. Cut A25/CR3A25N08DC1ATO0OAC1BTO0D.cut A25/CR3A25N08DC1BT000AC1BT 142. cut A25/CR3A25N08DC1BT142AC02T000.cut A25/CR3A25N090C1ATO00AC1BTO00.cut A25/CR3A25N09DC1BT000AC1BT 142. cut A25/CR3A25N090C1BT 142ACO2TO00. cut A25/CR3A25N100C1AT000AC1BT000. cut A25/CR3A25N100C1BTD00AC1BT 142. cut A25/CR3A25N100C1BT142AC02T000. cut A25/CR3A25N11DC1AT000ACIBTO00. cut A25/CR3A25N11DC1BT000ACIBT 142. cut A25/CR3A25N11DC1BT142AC02T000.cut A25/CR3A25N12DC1ATO00AC1BT000. cut A25/CR3A25N12DC1BT000ACIBT142. cut A25/CR3A25N12DC1BT142AC02T000.cut A25/CR3A25N13DC1ATOOOACIBTO00. cut A25/CR3A25N13DC1BT000AC1BT 142. Cut A25/CR3A25N13DC1BT142AC02T000. cut A25/CR3A25N14DC1ATO00ACIBT000.cut A25/CR3A25N14DC1BT000AC1BT142. cut A25/CR3A25N140C1BT142AC02T000. CUt

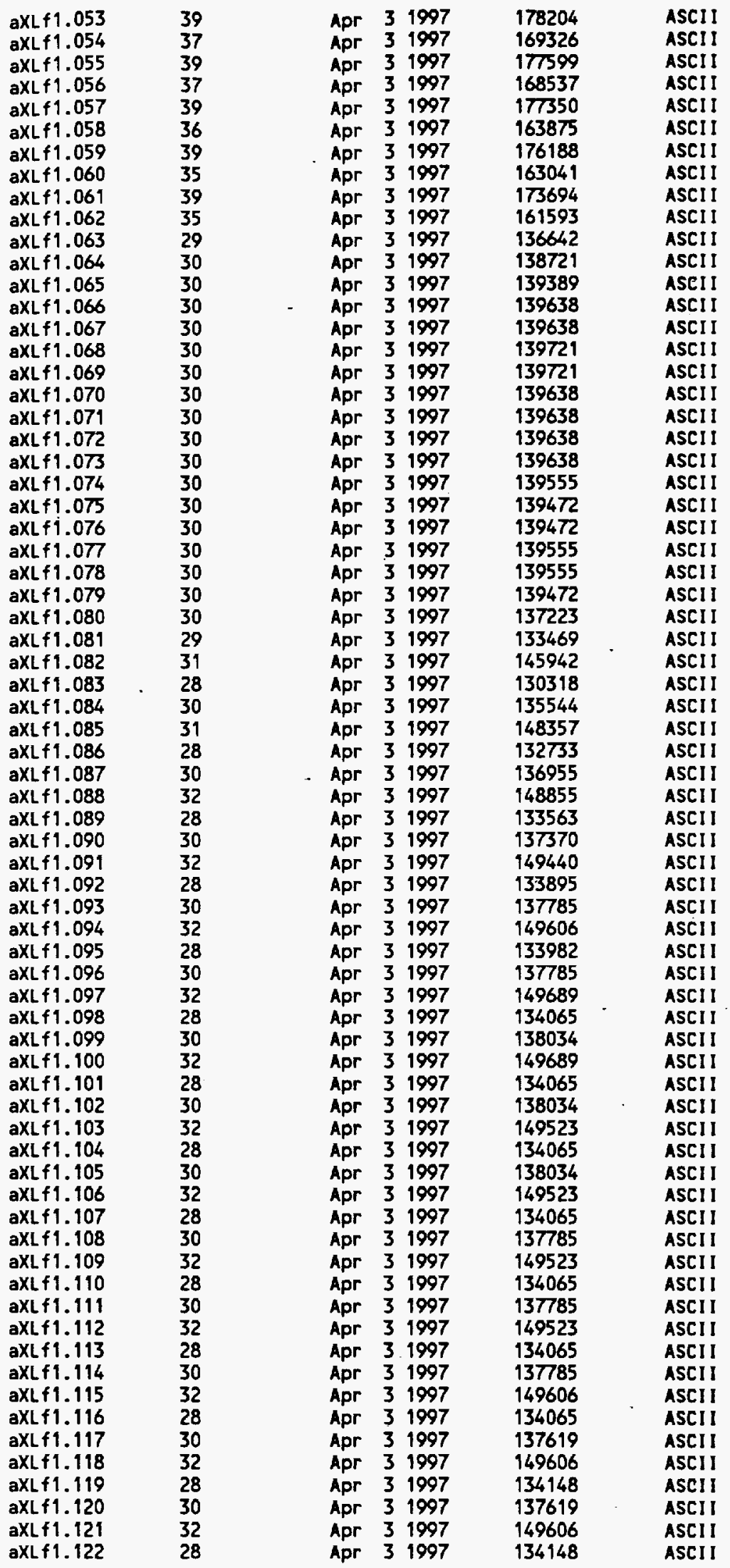


A25/CR3A25N15DC1ATDOOAC1BTO00. Cut A25/CR3A25N15OC1BTDOOAC1BT142.cut A25/CR3A25N15DC1BT142ACO2TO00. Cut A25/CR3A25N160C1ATOOOAC1BT000. CUT A25/CR3A25N16DC1BTDOOAC1BT142. CUT A25/CR3A25N160C1BT142AC02T000. cut A25/CR3A25N17DC1ATO0OAC1BT000. CUT A25/CR3A25N170C1BTOOOAC1BT 142. cut A25/CR3A25N17DC1BT 142ACO2T000. Cut A25/CR3A25N18DC1ATOOOAC1BTD00. Cut A25/CR3A25N18DC1BTO00AC1BT142. cut A25/CR3A25N18DC1BT 142ACO2TO00. CUt

Computer
File Name
A25a/CR3A13N010C02T000AC03T000. cut A25a/CR3A13NO2DC02T000AC03T000. cut A25a/CR3A13N03DC02TOOOAC03T000. CUt A25a/CR3A13N04DC02TOOOAC03T000. cut A25a/CR3A13N05DC02TO0OAC03T000. cut A25a/CR3A13N060C02TO00AC03T000. cut A25a/CR3A13N0TDCO2TOOOACO3TO00. cut A25a/CR3A13N08DC02T000AC03T000. cut A25a/CR3A 13N09DC02T000AC03T000. cut A25a/CR3A13N100C02T000AC03T000. cut A25a/CR3A13N110C02T000AC03T000.cut A25a/CR3A13N12DC02TO0OAC03T000. cut A25a/CR3A13N13DC02TOOOAC03T000. cut A25a/CR3A13N14DC02TO00AC03T000. cut A25a/CR3A13N15DC02TOQOAC03T000. Cut A25a/CR3A13N 160C02TO00AC03T000. cut A25a/CR3A13N170C02TO00AC03T000. cut A25a/CR3A13N18DC02T000ACO3T000.cut A25a/CR3A25N01DC1BT 142AC02T000.cut A25a/CR3A25N02DC1BT 142ACO2T000. cut A25a/CR3A25N03DC1BT 142AC02T000.cut A25a/CR3A25N04DC1BT 142AC02T000. cut A25a/CR3A25N05DC1BT 142AC02T000. cut A25a/CR3A25N06DC1BT 142AC02T000. cut A25a/CR3A25NOTDCIBT 142AC02T000. cut A25a/CR3A25N080C1BT 142AC02T000. cut A25a/CR3A25N090C1BT 142AC02T000. CUT A25a/CR3A25N10DC1BT 142AC02T000. cut A25a/CR3A25N110C1BT142AC02T000. cut A25a/CR3A25N12DC1BT 142AC02T000. cut A25a/CR3A25N130CIBT 142AC02T000. cut A25a/CR3A25N14DC1BT142AC02T000. cut A25a/CR3A25N15DC1BT 142AC02T000. CUt A25a/CR3A25N160C1BT142AC02T000. cut A25a/CR3A25N17DC1BT 142AC02T000. cut A25a/CR3A25N180C1BT142AC02TO00. Cut A25a/CR3A26N010CO3TO0OAC03T168.cut A25a/CR3A26N010C03T168ACO3T250. cut A25a/CR3A26N02DC03TO0OAC03T168. cut A25a/CR3A26N02DC03T168AC03T250. cut A25a/CR3A26N03DCO3TOOOAC03T168. cut A25a/CR3A26N03DC03T168AC03T250. cut A25a/CR3A26N04DC03TO0OAC03T168.cut A25a/CR3A26N04DC03T168AC03T250. cut A25a/CR3A26N05DC03TO00ACO3T168.cut A25a/CR3A26N05DC03T 168AC03T250.cut A25a/CR3A26N060C03TO00AC03T168.cut A25a/CR3A26N060C03T168AC03T250. cut A25a/CR3A26N07DCO3TO00ACO3T168. cut A25a/CR3A26N07DC03T168AC03T250. cut A25a/CR3A26N08DC03T 000AC03T 168. cut A25a/CR3A26N08DC03T 168AC03T250. cut A25a/CR3A26N09DC03TO0OAC03T168. cut

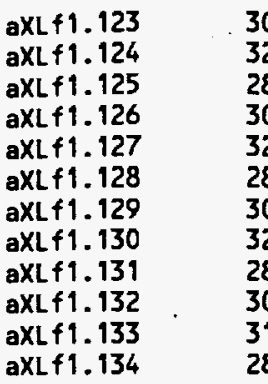

$\begin{array}{lll}\text { Apr } & 3 & 1997 \\ \text { Apr } & 3 & 1997 \\ \text { Apr } & 3 & 1997 \\ \text { Apr } & 3 & 1997 \\ \text { Apr } & 3 & 1997 \\ \text { Apr } & 3 & 1997 \\ \text { Apr } & 3 & 1997 \\ \text { Apr } & 3 & 1997 \\ \text { Apr } & 3 & 1997 \\ \text { Apr } & 3 & 1997 \\ \text { Apr } & 3 & 1997 \\ \text { Apr } & 3 & 1997\end{array}$

137785

149606

134148

137785

149606

133982

137370

148942

133480

135295

147776

131733

ASCII

ASCII

ASCI I

ASCII

ASCII

ASCII

ASCII

ASCII

ASCII

ASCII

ASCII

ASCII

\begin{tabular}{|c|c|c|c|c|c|}
\hline $\begin{array}{c}\text { Tape Backup } \\
\text { File Name }\end{array}$ & $\begin{array}{l}\text { Number of } \\
\text { Print Pages }\end{array}$ & $\begin{array}{l}\text { Fil } \\
\text { (C }\end{array}$ & $\begin{array}{l}\text { e Date } \\
\text { utputs) }\end{array}$ & $\begin{array}{c}\text { File size } \\
\text { (Bytes) }\end{array}$ & $\begin{array}{l}\text { File Type } \\
\text { (Format) }\end{array}$ \\
\hline axLf1.135 & 29 & $\mathrm{Apr}$ & 31997 & 136642 & AsCII \\
\hline aXLf1.136 & 30 & $\mathrm{Apr}$ & 31997 & 138721 & ASCII \\
\hline aXL 1.137 & 30 & Apr & 31997 & 139389 & ASCI I \\
\hline aXLf1.138 & 30 & Apr & 31997 & 139638 & ASCII \\
\hline aXLf1.139 & 30 & Apr & 31997 & 139721 & ASCII \\
\hline aXLf1.140 & 30 & Apr & 31997 & 139721 & ASCII \\
\hline aXLf1.141 & 30 & Apr & 31997 & 139721 & ASCII \\
\hline aXLf1.142 & 30 & Apr & 31997 & 139638 & ASCII I \\
\hline aXLf1.143 & 30 & Арг & 31997 & 139638 & ASCII \\
\hline aXLf1.144 & 30 & Apr & 31997 & 139638 & ASCII \\
\hline aXLf1.145 & 30 & Apr & 31997 & 139638 & ASCII \\
\hline aXLf1.146 & 30 & Apr & 31997 & 139555 & ASCII \\
\hline aXLf1.147 & 30 & Apr & 31997 & 139472 & ASCI I \\
\hline aXLf1.148 & 30 & Apr & 31997 & 139472 & ASCII \\
\hline aXLf1.149 & 30 & Apr & 31997 & 139555 & ASCII \\
\hline aXLf1.150 & 30 & Apr & 31997 & 139555 & ASCII \\
\hline aXLf1.151 & 30 & Apr & 31997 & 139472 & ASCII \\
\hline aXLf1.152 & 30 & Apr & 31997 & 137223 & ASCII \\
\hline aXL $f 1.153$ & 28 & Apr & 31997 & 130318 & ASCII \\
\hline aXLf1.154 & 28 & Apr & 31997 & 132733 & ASCII \\
\hline aXLf1.155 & 28 & Apr & 31997 & 133563 & ASCII \\
\hline aXLf1.156 & 28 & Apr & 31997 & 133895 & ASCII \\
\hline aXLf1.157 & 28 & Apr & 31997 & 133982 & ASCII \\
\hline aXLf1.158 & 28 & Apr & 31997 & 134065 & ASCI I \\
\hline aXLf1.159 & 28 & Apr & 31997 & 134065 & ASCII \\
\hline aXLf1.160 & 28 & Apr & 31997 & 134065 & ASCII \\
\hline aXLf1.161 & 28 & Apr & 31997 & 134065 & ASCII \\
\hline aXLf1.162 & 28 & Apr & 31997 & 134065 & ASCII \\
\hline aXLf1.163 & 28 & Apr & 31997 & 134065 & ASCII \\
\hline aXLf1.164 & 28 & Apr & 31997 & 134065 & ASCII \\
\hline aXLf1.165 & 28 & Apr & 31997 & 134148 & ASCII \\
\hline aXLf1.166 & 28 & Apr & 31997 & 134148 & ASCII \\
\hline aXLf1.167 & 28 & Apr & 31997 & 134148 & ASCII \\
\hline aXLf1.168 & 28 & Apr & 31997 & 133982 & ASCII \\
\hline aXLf1.169 & 28 & Apr & 31997 & 133480 & ASCII \\
\hline $\operatorname{aXL} \$ 1.170$ & 28 & Apr & 31997 & 131733 & ASCII \\
\hline aXLf1.171 & 36 & Apr & 31997 & 166310 & ASCII I \\
\hline aXLf1.172 & 34 & Apr & 31997 & 158812 & ASCII \\
\hline aXLf1.173 & 37 & Apr & 31997 & 169832 & ASCII \\
\hline aXL $f 1.174$ & 34 & Apr & 31997 & 161098 & ASCII \\
\hline aXLf1.175 & 37 & Apr & 31997 & 170662 & ASCII \\
\hline aXLf1.176 & 34 & Apr & 31997 & 157535 & ASCI I \\
\hline aXLf1.177 & 37 & Apr & 31997 & 171433 & ASCII \\
\hline aXLf1.178 & 34 & Apr & 31997 & 158518 & ASCII \\
\hline aXLf1.179 & 36 & $\mathrm{Apr}$ & 31997 & 166680 & ASCII \\
\hline aXLf1.180 & 34 & Apr & 31997 & 158684 & ASCII \\
\hline aXLf1.181 & 36 & Apr & 31997 & 166763 & ASCII \\
\hline aXLf1.182 & 34 & Apr & 31997 & 158850 & ASCII \\
\hline aXLf1.183 & 37 & Apr & 31997 & 166929 & ASCII \\
\hline aXLf1.184 & 34 & Apr & 31997 & 158850 & ASCII \\
\hline aXLf 1.185 & 36 & Apr & 31997 & 166763 & ASCII \\
\hline aXLf1.186 & 34 & Apr & 31997 & 158684 & ASCI I \\
\hline aXLf1.187 & 36 & Apr & 31997 & 580 & ASCII \\
\hline
\end{tabular}


A25a/CR3A26N090C03T168AC03T250.cut A25a/CR3A26N10DCO3TOOOACO3T168. cut A25a/CR3A26N100CO3T168AC03T250.cut A25a/CR3A26N11DC03TOOOAC03T168.cut A25a/CR3A26N110C03T168ACO3T250. cut A25a/CR3A26N12DCO3TOOOACO3T168. cut A25a/CR3A26N120C03T 168AC03T250. cut A25a/CR3A26N13DCO3TOOOAC03T168.cut A25a/CR3A26N130C03T168AC03T250. cut A25a/CR3A26N14DC03TO00AC03T168.cut A25a/CR3A26N140CO3T168AC03T250. cut A25a/CR3A26N15DCO3TOOOAC03T168. cut A25a/CR3A26N15DC03T168AC03T250. cut A25a/CR3A26N16DC03TO00AC03T168. cut A25a/CR3A26N 160C03T168AC03T250. cut A25a/CR3A26N1TDC03T000AC03T168. Cut A25a/CR3A26N1TDC03T168AC03T250. cut A25a/CR3A26N18DC03T000AC03T168.cut A25a/CR3A26N18DC03T168AC031250. CUt

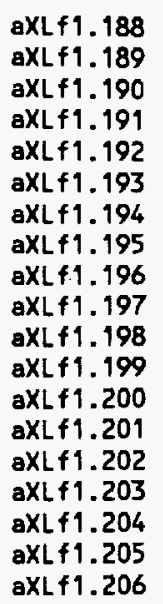

34
36
34
36
34
36
34
36
34
36
34
36
34
36
34
36
34
36
33

$\begin{array}{ll}158684 & \text { ASCII } \\ 166680 & \text { ASCII } \\ 158518 & \text { ASCII } \\ 166597 & \text { ASCII } \\ 158435 & \text { ASCII } \\ 166514 & \text { ASCII } \\ 158518 & \text { ASCII } \\ 166514 & \text { ASCII } \\ 158518 & \text { ASCII } \\ 166597 & \text { ASCII } \\ 158601 & \text { ASCII } \\ 166680 & \text { ASCII } \\ 158601 & \text { ASCII } \\ 166514 & \text { ASCII } \\ 158601 & \text { ASCII } \\ 165660 & \text { ASCII } \\ 157286 & \text { ASCII } \\ 163415 & \text { ASCII } \\ 155373 & \text { ASCII }\end{array}$

THE FOLLOWING FILES FOR ASSEMBLY A26 WERE SUPERCEEDED BY FILES UNDER ASSEMBLY DESIGNATION A26Z DURING CHECKING.

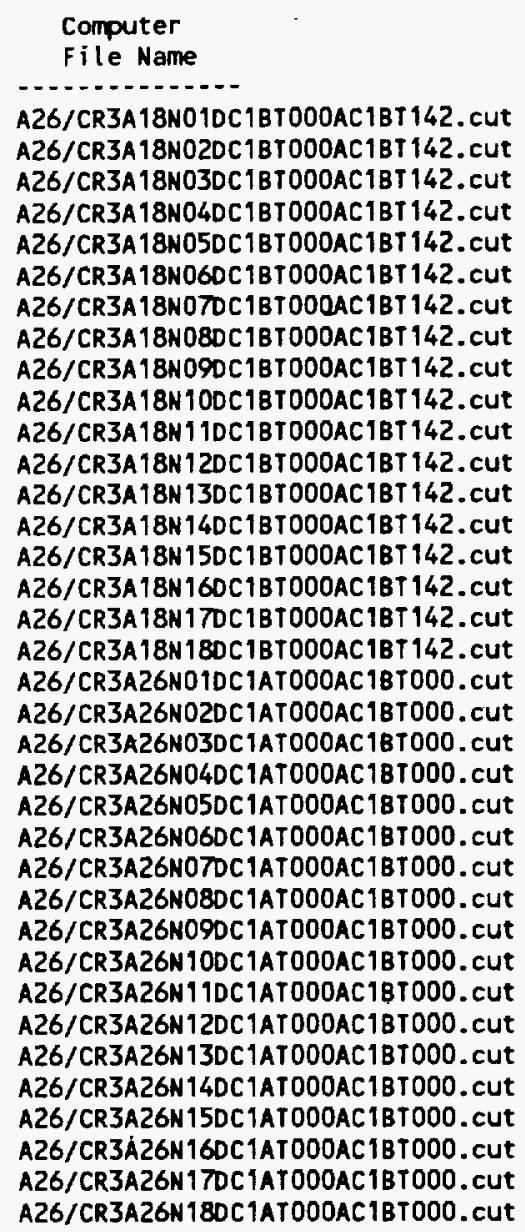

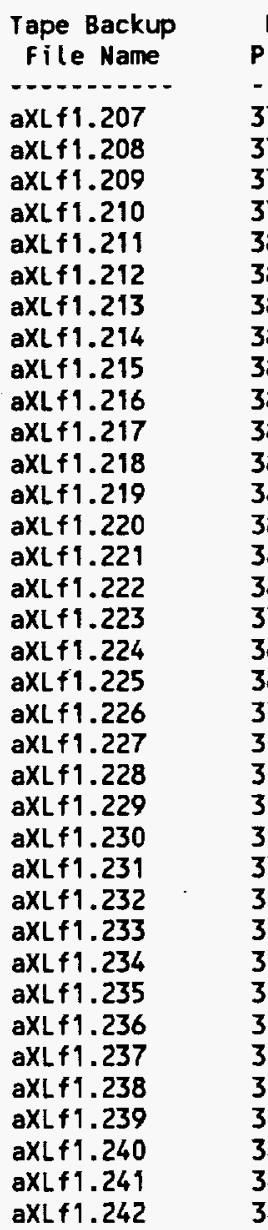

Number of
Print Pages
37
37
37
37
38
38
38
38
38
38
38
38
38
38
38
38
37
36
36
37
37
37
37
37
37
37
37
37
37
37
37
37
37
36
36
36

37
37
37
37
37
37
37

$\begin{array}{ccc}\text { Fi le } & \text { Date } \\ \text { (Output) } \\ -1 \text { Apr } & 3 & 1997 \\ \text { Apr } & 3 & 1997 \\ \text { Apr } & 3 & 1997 \\ \text { Apr } & 3 & 1997 \\ \text { Apr } & 3 & 1997 \\ \text { Apr } & 3 & 1997 \\ \text { Apr } & 3 & 1997 \\ \text { Apr } & 3 & 1997 \\ \text { Apr } & 3 & 1997 \\ \text { Apr } & 3 & 1997 \\ \text { Apr } & 3 & 1997 \\ \text { Apr } & 3 & 1997 \\ \text { Apr } & 3 & 1997 \\ \text { Apr } & 3 & 1997 \\ \text { Apr } & 3 & 1997 \\ \text { Apr } & 3 & 1997 \\ \text { Apr } & 3 & 1997 \\ \text { Apr } & 3 & 1997 \\ \text { Apr } & 3 & 1997 \\ \text { Apr } & 3 & 1997 \\ \text { Apr } & 3 & 1997 \\ \text { Apr } & 3 & 1997 \\ \text { Apr } & 3 & 1997 \\ \text { Apr } & 3 & 1997 \\ \text { Apr } & 3 & 1997 \\ \text { Apr } & 3 & 1997 \\ \text { Apr } & 3 & 1997 \\ \text { Apr } & 3 & 1997 \\ \text { Apr } & 3 & 1997 \\ \text { Apr } & 3 & 1997 \\ \text { Apr } & 3 & 1997 \\ \text { Apr } & 3 & 1997 \\ \text { Apr } & 3 & 1997 \\ \text { Apr } & 3 & 1997 \\ \text { Apr } & 3 & 1997 \\ \text { Apr } & 3 & 1997\end{array}$

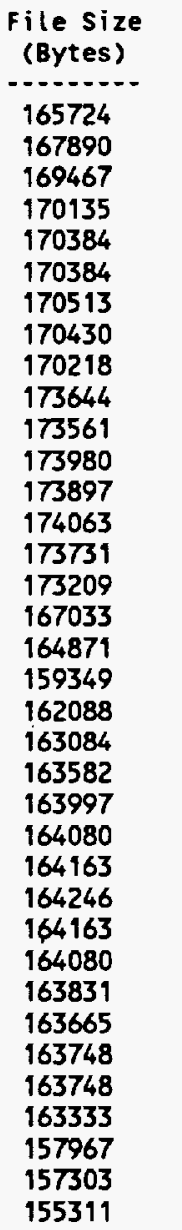

File Trpe

(Format)

ASCII

ASCII

ASCII

ASCII

ASCII

ASCII

ASCII

ASCII

ASCII

ASCII

ASCII

ASCII

ASCII

ASCII

ASCII

ASCII

ASCII

ASCII

ASCII

ASCII

ASCII

ASCII

ASCII

ASCII

ASCII

ASCII

ASCII

ASCII

ASCII

ASCII

ASCII

ASCII

ASCII

ASCII

ASCII

ASCII

THE FOLLONING FILES FOR ASSEMBLY A28 IERE SUPERCEEDED BY FILES UNDER ASSEMBLY DESIGNATION A28Z DURING CHECKING.

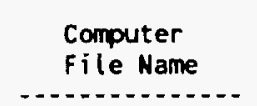

A28/CR3A10N01DC03T000AC03T168.cut A28/CR3A10N010C03T168AC03T250. CUT A28/CR3A10N0ZDCO3̦TOOOACO3T168.cut

\begin{tabular}{|c|c|c|}
\hline $\begin{array}{c}\text { Tape Backup } \\
\text { File Name }\end{array}$ & $\begin{array}{l}\text { Number of } \\
\text { Print Pages }\end{array}$ & $\begin{array}{c}\text { File Date } \\
\text { (Output) }\end{array}$ \\
\hline $\begin{array}{l}\text { aXL } f 1.243 \\
\text { aXL } f 1.244 \\
a X L f 1.245\end{array}$ & $\begin{array}{l}32 \\
32 \\
33\end{array}$ & $\begin{array}{lll}\text { Apr } & 3 & 1997 \\
\text { Apr } & 3 & 1997 \\
\text { Apr } & 3 & 1997\end{array}$ \\
\hline
\end{tabular}

$\begin{gathered}\text { File size } \\ \text { (Bytes) }\end{gathered}$
-150341
150622
154407

File Type
(Format)
ASCII
ASCII
ASCII 
A28/CR3A1ONO2DC03T 168AC03T250. Cut A28/CR3A10N03DC03TO0OACO3T168.cut A28/CR3A10N03DC03T168AC03T250. Cut A28/CR3A10N04DC03TO00AC03T168.cut A28/CR3A 10N04DC03T168ACO3T250.cut A28/CR3A1ON05DC03TO0OAC03T168. cut A28/CR3A 1ON05DC03T168AC03T250.cut A28/CR3A 10N06DC03TO00ACO3T168. cut A28/CR3A 10N06DC03T 168ACO3T250. cut A28/CR3A 1ONOTDC03TO0OAC03T 168. cut A28/CR3A 1ONOTDC03T168AC03T250. cut A28/CR3A 10N08DC03T O00AC03T 168. cut A28/CR3A 10N080C03T168AC03T250.cut A28/CR3A 10N09DC03TO00AC03T16B. cut A28/CR3A10N09DC03T168AC03T250. cut A28/CR3A 10N 10DC03T000AC03T168. cut A28/CR3A10N 10DCO3T168ACO3T250. cut A28/CR3A10N11DC03T000AC03T168. cut A28/CR3A1ON11DC03T 168AC03T250. cut A28/CR3A10N 12DC03T000AC03T168. cut A28/CR3A10N12DC03T168AC03T250.cut A28/CR3A10N13DC03T 000AC03T168. cut A28/CR3A10N13DC03T 168AC03T250. Cut A28/CR3A10N14DC03T000AC03T168. cut A28/CR3A10N14DC03T168AC03T250. cut A28/CR3A10N15DC03T000AC03T168.cut A28/CR3A10N15DC03T168AC03T250. cut A28/CR3A10N16DC03T000AC03T168.CUT A28/CR3A10N160C03T168AC03T250. cut A28/CR3A10N17DC03T000AC03T168.cut A28/CR3A10N170C03I168AC03T250. cut A28/CR3A10N180C03TO0OAC03T168. Cut A28/CR3A10N18DC03T 168AC03T250. cut A28/CR3A18N01DC02TO0OACO3TO0O. cut A28/CR3A18N02DC02TOOOACO3TO00. CUT A28/CR3A18N03DC02TOOOACO3TO00. cut A28/CR3A18N04DC02TO0OACO3TO00. cut A28/CR3A18N05DC02TO0OACO3TO00.cut A28/CR3A 18N06DC02TO00AC03T000.cut A28/CR3A18N07DC02TO0OAC03T000, cut A28/CR3A 18N08DC02TOOOAC03T000. cut A28/CR3A18N09DCO2TO0OACO3TO00.cut A28/CR3A18N10DC02 TO00AC03T000.cut A28/CR3A18N110C02T000AC03T000. cut A28/CR3A18N120C02T000AC03T000. cut A28/CR3A18N13DC02TO0OAC03T000. Cut A28/CR3A18N14DC02TO0OAC03T000. Cut A28/CR3A18N15DC02TOOOAC03TO0O. cut A28/CR3A18N160C02TOOOAC03T000. CUT A28/CR3A18N170C02TO00AC03T000. Cut A28/CR3A18N180C02T000AC03T000. cut A28/CR3A28NO1DC1ATOOOAC1BTO0O. CUT A28/CR3A28N010C18TOOOAC1BT142. CUT A28/CR3A28N01DC1BT142AC02T000. CUT A28/CR3A28NO2DC1ATOOOAC1BTO00. cut A28/CR3A28NO2DC1BTODOAC1BT142. cut A28/CR3A28N02DC18T142AC02T000.CUT A28/CR3A28N030C1ATOOOAC1BT000. CUT A28/CR3A28NO3DC1BTOOOAC1BT142. cut A28/CR3A28NO3DC1BT 142ACO2TO0O. CUt A28/CR3A28N04DC1ATOOOAC1BTDOD.CUT A28/CR3A28N04DC1BTDOOAC1BT142. CUT A28/CR3A28NO4DC1BT142AC02T000. CUt A28/CR3A28N05DC1ATOOOAC1BT000. Cut A28/CR3A28N05DC1BTO0OAC1BT 142. CUT A28/CR3A28N05DC18T142AC02T000.cut A28/CR3A28NO6DC1ATO0OAC1BTODO. CUt A28/CR3A28N06DC1BTDOOAC1BT 142. CUt A28/CR3A28N060C1BT142ACOZT000.cut A28/CR3A28NOTDC1ATOOOAC1BT000.cut

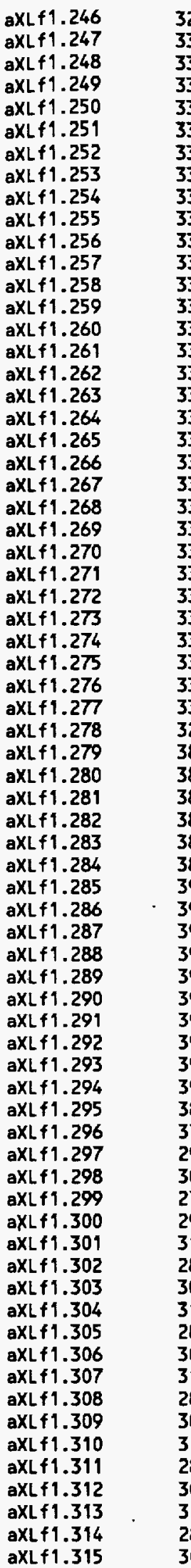

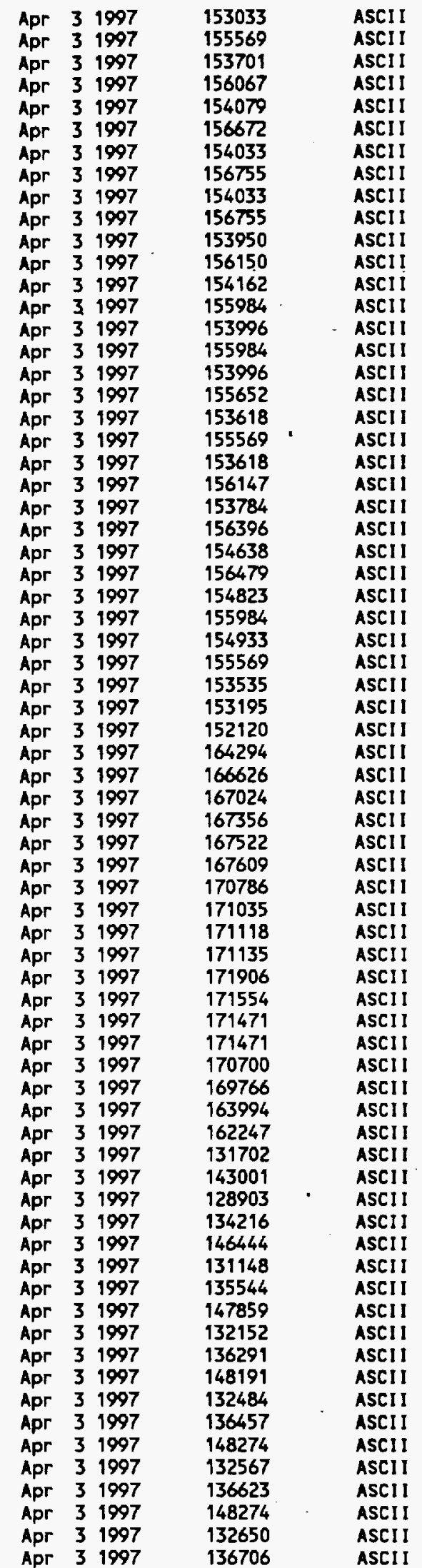


A28/CR3A28NOTOC1BTOOOAC1BT142.cut A28/CR3A28NOTDC1BT 142ACO2T000.cut A28/CR3A28NOBOC1ATOOOAC1BTOOO. CUT A28/CR3A28N080C1BTDOOAC1BT142.cut A28/CR3A2BNOBOC1BT 142AC02TOOO.CUT A28/CR3A28NO9OC1ATOOOAC1BT000.cUT A28/CR3A28NOSOC1BTOOOAC1BT 142.cut A28/CR3A28NO90C1BT 142AC02T000.cut A28/CR3A28N100C1ATOOOAC1BT000.cut A28/CR3A28N10DC1BTDOOAC1BT142. CUt A28/CR3A28N100C1BT 142AC02T000.cut A28/CR3A28N110C1ATOOOAC1BTO00.cut A28/CR3A28N11DC1BTOOOAC1BT142.cut A28/CR3A28N110C1BT 142AC02T000.cut A28/CR3A28N120C1ATOOOAC1BT000.CUT A28/CR3A28N120C1BTOOOAC1BT 142.cut A28/CR3A28N120C1BT 142AC02T000.cut A28/CR3A28N13DC1ATOOOAC1BT000.cut A28/CR3A28N130C1BT 000AC1BT 142.cUt A28/CR3A28N13DC1BT142AC02T000. cut A28/CR3A28N14DC1ATODOAC1BT000.CUT A28/CR3A28N14DC1BTOOOAC1BT 142.cut A28/CR3A28N140C1BT 142AC02T000.cut A28/CR3A28N15DC1ATOOOAC1BT000.cut A28/CR3A28N15DC1BTODOAC1BT142.cut A28/CR3A28N15DC1BT 142AC02T000.cut A28/CR3A28N160C1AT000AC1BT000.cut A28/CR3A28N160C1BTOOOAC1BT 142. cut A28/CR3A28N160C1BT 142AC02T000.cut A28/CR3A28N1TDC1ATOOOAC1BTO0O.cut A28/CR3A28N170C1BT O0OAC1BT142.cut A28/CR3A28N17DC1BT1 142AC02T000. Cut A28/CR3A28N180C1ATODOAC1BT000.cut A28/CR3A2BN18DC1BTOOOAC1BT 142.cut A28/CR3A28N180C1BT142ACO2T000. cut

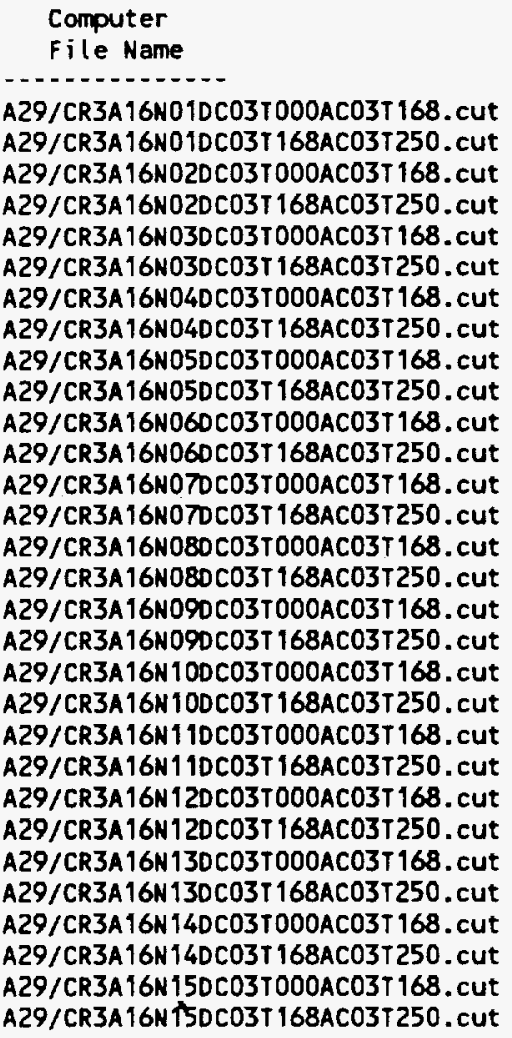

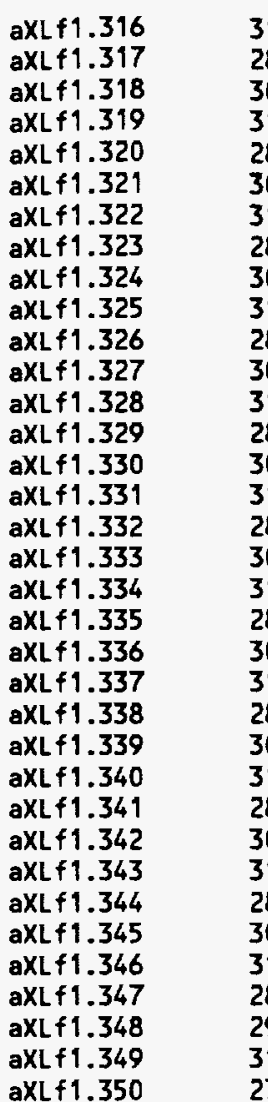

31
28
30
31
28
30
31
28
30
31
28
30
31
28
30
31
28
30
31
28
30
31
28
30
31
28
30
31
28
30
31
28
29
31
27

148274

132733

136872

148274

132733

136789

148274

132733

136623

148274

132733

136623

148274

132733

136457

148274

132816

136374

148274

132816

136374

148357

132816

136291

148357

132816

136291

148274

132567

135378

147776

132069

133801

145776

129903

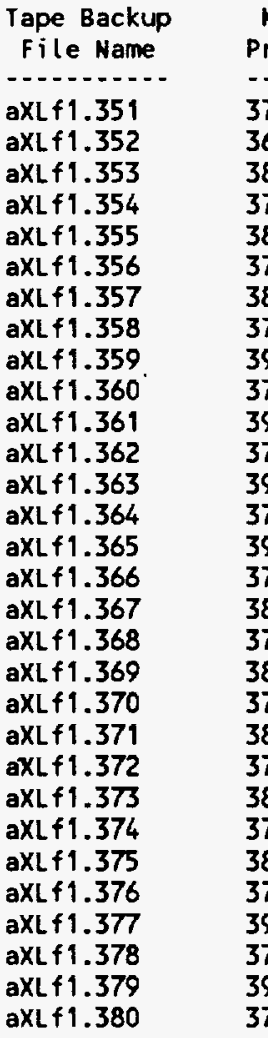

\begin{tabular}{|c|c|c|c|c|}
\hline $\begin{array}{l}\text { Number of } \\
\text { Print Pages }\end{array}$ & & $\begin{array}{l}\text { Date } \\
\text { utput) }\end{array}$ & $\begin{array}{c}\text { File size } \\
\text { (Bytes) }\end{array}$ & $\begin{array}{r}\text { File Type } \\
\text { (Format) }\end{array}$ \\
\hline 37 & Apr & $\begin{array}{ll}3 & 1997\end{array}$ & 169910 & ASCII \\
\hline 36 & Apr & 31997 & 166586 & ASCII \\
\hline 38 & Apr & $\begin{array}{ll}31997\end{array}$ & 174428 & ASCII \\
\hline 37 & Apr & $\begin{array}{ll}31997\end{array}$ & 169121 & ASCII \\
\hline 38 & Apr & 31997 & 174677 & ASCII \\
\hline 37 & Ap & $\begin{array}{ll}31997 \\
\end{array}$ & 169997 & ASCII \\
\hline 38 & Apr & $\begin{array}{ll}31997 \\
\end{array}$ & 175092 & ASCII \\
\hline 37 & $\mathrm{Apr}$ & 31997 & 170163 & ASCII \\
\hline 39 & Apr & 31997 & 175424 & ASCII I \\
\hline 37 & Apr & 31997 & 170416 & ASCII \\
\hline 39 & Арг & 31997 & 175424 & ASCII \\
\hline 37 & Apr & 31997 & 170416 & ASCII \\
\hline 39 & Арг & $\begin{array}{ll}31997 \\
\end{array}$ & 175424 & ASCII I \\
\hline 37 & Apr & $\begin{array}{ll}3 & 1997\end{array}$ & 170416 & ASCII \\
\hline 39 & Apr & $\begin{array}{ll}31997\end{array}$ & 175424 & ASCII \\
\hline 37 & Apr & $\begin{array}{ll}31997\end{array}$ & 170333 & Ascil \\
\hline 38 & Арг & 31997 & 175258 & ASCII \\
\hline 37 & Apr & $\begin{array}{ll}31997 \\
\end{array}$ & 170333 & ASCII \\
\hline 38 & Apr & $\begin{array}{ll}31997 \\
\end{array}$ & 175092 & ASCII \\
\hline 37 & Apr & 31997 & 170246 & ASCII \\
\hline 38 & Apr & 31997 & 175009 & ASCII \\
\hline 37 & Apr & 31997 & 169492 & ASCII \\
\hline 38 & Apr & 31997 & 175009 & ASCII \\
\hline 37 & Арг & 31997 & 170163 & ASCII \\
\hline 38 & Apr & 31997 & 175175 & ASCII \\
\hline 37 & Apr & $\begin{array}{ll}31997 \\
\end{array}$ & 170416 & ASCII \\
\hline 39 & $\mathrm{Apr}$ & $\begin{array}{ll}31997 \\
\end{array}$ & 175192 & ASCII \\
\hline 37 & Apr & 31997 & 169201 & ASCII \\
\hline 39 & Apr & 3. 1997 & 173026 & ASCII I \\
\hline 37 & Apr & 31997 & 168976 & ASCII \\
\hline
\end{tabular}

ASCII ASCII ASCII ASCII ASCII ASCII

ASCII

ASCII

ASCII

ASCII

ASCII

ASCII

ASCII

ASCII

ASCII

ASCII

ASCII

ASCII

ASCII

ASCII

ASCII

ASCII

ASCII

ASCII

ASCII

ASCII

ASCII

ASCII

ASCII

ASCII

ASCII

ASCII

ASCII

ASCII

ASCII

(e Type

SCII

ASCII

ASCII

ASCII

SCII

ASCII

SCII

SCII

ASCII 
A29/CR3A16N16DC03T000AC03T168. cut A29/CR3A16N16DC03T 168AC03T250. cut A29/CR3A 16N17DCO3TOOOACO3T168. cut A29/CR3A16N17DCO3T168AC03T250. cut A29/CR3A16N18DC03TOOOAC03T168. cut A29/CR3A16N.18DC03T168AC03T250. cut A29/CR3A22N01DC02TO0OAC03T000. cut A29/CR3A22N02DC02TOOOACO3T000.cut A29/CR3A22N03DC02TOOOACO3TO0O. cut A29/CR3A22N04DC02TOOOAC03T000.cut A29/CR3A22N05DC02TOOOACO3T000. cut A29/CR3A22N06DC02TOOOACO3T000. cut A29/CR3A22NOTDC02TO0OAC03T000. cut A29/CR3A22N08DC02TO00AC03T000. cut A29/CR3A22N090C02TOOOACO3TO00. cut A29/CR3A22N 10DC02TOOOACO3T000. cut A29/CR3A22N1 1DC02TOOOACO3TOOO. cut A29/CR3A22N12DC02TOOOAC03T000. cut A29/CR3A22N 13DCO2TOOOACO3T000.cut A29/CR3A22N14DC02TO0OACO3T000.cut A29/CR3A22N15DCO2TO0OACO3TO00. cut A29/CR3A22N16DC02TO00AC03TO00. Cut A29/CR3A22N170C02TO00AC03T000. cut A29/CR3A22N18DCO2TOOOAC03T000.cut A29/CR3A29N01DC1ATO00AC1BT000. cut A29/CR3A29N01DC1BTOOOAC18T142. Cut A29/CR3A29N01DC1BT142ACO2T000. cut A29/CR3A29N02DC1ATOOOAC1BT000. Cut A29/CR3A29N02DC1BT000AC1BT142. cut A29/CR3A29N02DC1BT142ACO2T000.cut A29/CR3A29N03DC1ATOOOAC1BT000. CUT A29/CR3A29N03DC1BTOOOAC1BT142. CUT A29/CR3A29N03DC1BT142AC02T000.cut A29/CR3A29N04DC1ATO00AC1BT000. cut A29/CR3A29N040C1BTO00AC1BT142. Cut A29/CR3A29N04DC1BT 142AC02T000. Cut A29/CR3A29N05DC1ATO00AC1BT000. Cut A29/CR3A29N05DC1BTOOOAC1BT142. cut A29/CR3A29N05DC1BT142AC02TO00. cut A29/CR3A29N060C1ATO0OAC1BT000. Cut A29/CR3A29N060C1BTO00AC1BT 142. cut A29/CR3A29N06DC1BT 142AC02TO00. cut A29/CR3A29NOTDC1ATO00AC1BTD00. CUT A29/CR3A29NOTDC1BTOODAC1BT142. CUT A29/CR3A29NOTDC1BT142ACO2T000. cut A29/CR3A29N08DC1ATODOAC1BTODO.cut A29/CR3A29N08DC1BTDOOAC1BT142.cut A29/CR3A29N08DC1BT 142AC02T000.cut A29/CR3A29N090C1ATODOAC1BT000. cut A29/CR3A29N09DC1BT O0OAC1BT142.cut A29/CR3A29N090C18T142ACO2T000.cut A29/CR3A29N100C1ATODOAC1BT000.cut A29/CR3A29N100C1BT DOOAC1BT142. cut A29/CR3A29N10DC1BT 142AC02T000. cut A29/CR3A29N110C1ATDOOAC1BT000.cut A29/CR3A29N110C1BTOOOAC1BT142.cut A29/CR3A29N11DC1BT 142ACO2T000.cut A29/CR3A29N120C1AT DOOAC1BT000.cut A29/CR3A29N12DC1BT D0OAC1BT142.cut A29/CR3A29N120C1BT 142AC02T000.cut A29/CR3A29N130C1ATOOOAC1BT000.cut A29/CR3A29N130C1BTDOOAC1BT 142.cut A29/CR3A29N13DC1BT 142AC02T000. Cut A29/CR3A29N14DC1ATOOOAC1BT000.cut A29/CR3A29N14DC1BTO0OAC1BT142.cut A29/CR3A29N140C1BT142AC02T000.cut A29/CR3A29N150C1ATOOOAC1BTO00.cut A29/CR3A29N150C1BTO00AC1BT142.cut A29/CR3A29N15DC1BT 142AC02T000.cut A29/CR3A29N160C1ATO0OAC1BT000. CUT

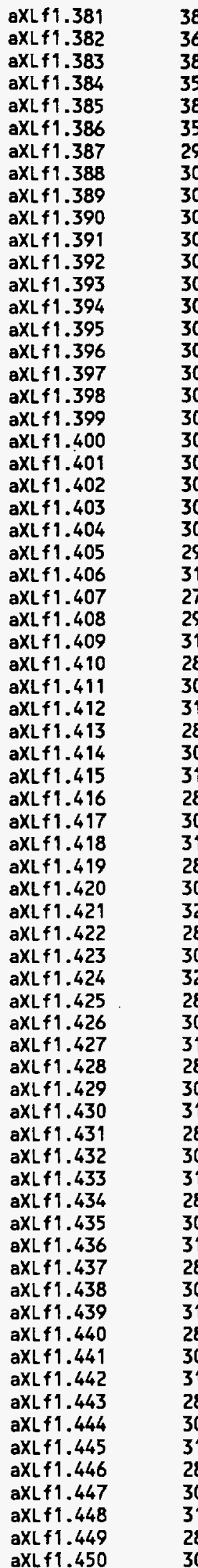

Apr 31997

Apr 3199

Apr 31997

Apr 31997

Apr 31997

Apr 31997

Apr 31997

Apr 31997

Apr 31997

Apr 31997

Apr 31997

Apr 31997

Apr 31997

Apr 31997

Apr 31997

Apr 31997

Apr 31997

Apr 31997

Apr 31997

Apr 31997

Apr 31997

Apr 31997

Apr 31997

Apr 31997

Apr 31997

Apr 31997

Apr 31997

Apr 31997

Apr 31997

Apr 31997

Apr 31997

Apr 31997

Apr 31997

Apr 31997

Apr 31997

Apr 31997

Apr 31997

Apr 31997

Apr 31997

Apr 31997

Apг 31997

Apr 31997

Apr 31997

Apr 31997

Apr 31997

Apr 31997

Apr 31997

Apr 31997

Apr 31997

Apr 31997

Apr 31997

Apr 31997

Apr 31997

Apr 31997

Apr 31997

Apr 31997

Apr 31997

Apr 31997

Apr 31997

Apr 31997

Apr 31997

Apr 31997

Apr 31997

Apr 31997

Apr 31997

Apr 31997

Apr 31997

Apr 31997

Apr 31997

Apr 31997
174089

164231

173259

163128

170470

161547

136053

138468

138700

139285

139285

139285

139285

139285

139202

139119

139136

139053

139053

139053

139036

138953

138634

137057

132805

143503

129484

134714

146776

131148

136208

148108

132318

136623

148274

132816

137121

148440

132899

137287

148527

133065

137370

148527

133148

137370

148444

133148

137370

148444

133148

137287

148444

133065

137204

148357

133065

137121

148357

133065

137038

148357

133065

136955

148440

133065

136789

148440

133065

136457
ASCII

ASCII

ASCII

ASCII

ASCII

ASCII

ASCII

ASCII

ASCII

ASCII

ASCII

ASCII

ASCII

ASCII

ASCII

ASCII

ASCII

ASCII

ASCII

ASCI I

ASCII

ASCII

ASCII

ASCII

ASCII

ASCII

ASCII

ASCII

ASCII

ASCII

ASCII

ASClI

ASCII

ASCII

ASCII

ASCII

ASCII

ASCII

ASCII

ASCII

ASCII

ASCII

ASCII

ASCII

ASCII

ASCII

ASCII

ASCII

ASCII

ASCII

ASCII

ASCII

ASCII

ASCII

ASCII

ASCII

ASCII

ASCII

ASCII

ASCII

ASCII

ASCII

ASCII

ASCII

ASCII

ASCII

ASCII

ASCII

ASCII

ASCII 
A29/CR3A29N160C1BTD00AC1BT 142. cUt A29/CR3A29N160C1BT142ACO2T000.cut A29/CR3A29N170C1ATODOAC1BTO00. CUT A29/CR3A29N17DC1BTDOOAC1BT 142. CUT A29/CR3A29N170C1BT142ACO2T000. cut A29/CR3A29N180C1ATO0OAC1BTO0O. CUT A29/CR3A29N180C1BTO0OAC1BT 142. CUt A29/CR3A29N180C1BT142ACO2T000.CUt

Computer
File Name

01/CR3A01N010C01TO0OAC1BT000. CUt 01/CR3A01N02DCO1TO0OAC1BT000. CUT 01/CR3A01N03DCO1TOOOAC1BTD00.cut 01/CR3A01N04DCO1 TOOOAC1BTDOO. cut 01/CR3A01N05DCO1TO0OAC1BT000. cut 01/CR3A01N06DCO1TO0OAC1BT000. CUT 01/CR3A01N0TDCO1 TOOOAC1BT000. CUT 01/CR3A01N080CO1TODOAC1BTO0O. Cut O1/CR3A01N090C01TO0OAC1BTDOD.CUT 01/CR3A01N10DC01TO00AC1BT000. Cut O1/CR3A01N110CO1T000AC1BT000. CUT 01/CR3A01N12DC01T000AC1BT000. cut 01/CR3A01N13DCO1TO00AC1BT000.cut 01/CR3A01N14DCO1TO00AC1BT000. cut 01/CR3A01N15DCO1T000AC1BT000. CUT 01/CR3A01N16DC01T000AC1BTO00. CUT OI/CR3A01N17DCO1T000AC1BT000. Cut 01/CR3A01N18DC01TO0OAC1BT000. CUT 01/CR3A26N01DC1BT 000AC1BT142. cut 01/CR3A26N02DC1BTO00AC1BT142. CUT 01/CR3A26N03DC1BTO00AC1BT 142. CUt O1/CR3A26N04DC1BTO00AC1BT142. CUT 01/CR3A26N05DC1BTOOOAC1BT 142. Cut 01/CR3A26N06DC1BTO00AC1BT142. cut 01/CR3A26NOTDC1BTO0OAC1BT142. cut 01/CR3A26N08DC1BTOOOAC1BT 142. cut 01/CR3A26N09DC1BTOOOAC1BT 142. CUT 01/CR3A26N100C18TO00AC1BT142. CUT 01/CR3A26N11DC1BTOOOAC1BT 142. CUt 01/CR3A26N12DC1BT 000AC1BT142. Cut 01/CR3A26N13DC1BTO00AC1BT142. CUT 01/CR3A26N14DC1BT000AC1BT142. cut 01/CR3A26N15DC1BTOOOAC1BT 142. cut 01/CR3A26N16DC1BTO00AC1BT142. CUT 01/CR3A26N17DC1BTO00AC1BT142. CUt 01/CR3A26N18DC1BTO0OAC1BT142. CUt

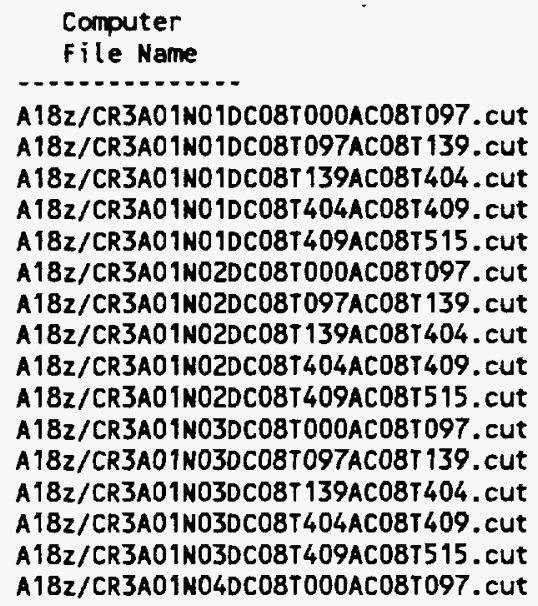

$\begin{array}{ll}\text { axL } f 1.451 & 3 \\ \text { axL } f 1.452 & 28 \\ \text { aXL } f 1.453 & 3 \\ \text { aXLf1.454 } & 3 \\ \text { aXLf1.455 } & 28 \\ \text { aXLf1.456 } & 29 \\ \text { aXLf1.457 } & 3 \\ \text { axL } f 1.458 & 28\end{array}$

$\begin{array}{llll}31 & \text { Apr } & 3 & 1997 \\ 28 & \text { Apr } & 3 & 1997 \\ 30 & \text { Apr } & 3 & 1997 \\ 31 & \text { Apr } & 3 & 1997 \\ 28 & \text { Apr } & 3 & 1997 \\ 29 & \text { Apr } & 3 & 1997 \\ 31 & \text { Apr } & 31997 \\ 28 & \text { Apr } & 31997\end{array}$

148357

132982

135544

148025

132401

133967

145946

130567

ASCII

ASCII

ASCII

ASCII

ASCII

ASCII

ASCII

ASCII

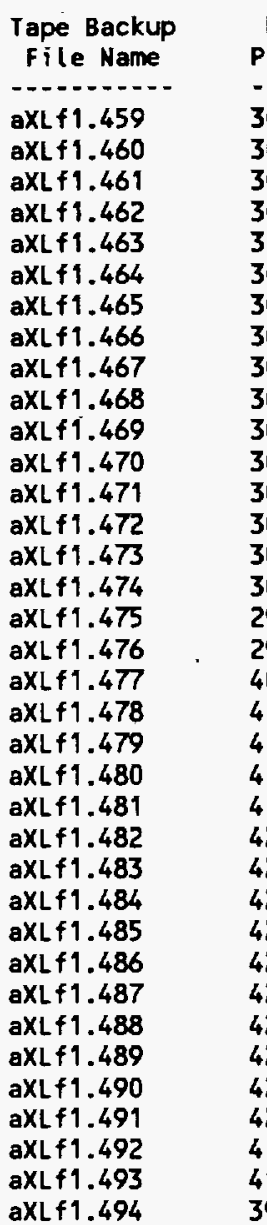

Number of
Print Pages
30
30
30
30
30
31
30
30
30
30
30
30
30
30
30
30
30
29
29
40
41
41
41
41
42
42
42
42
42
42
42
42
42
42
41
41
39

File Date
(Output)

Apr 31997

Apr 31997

Apr 31997

Apr 31997

Apr 31997

Apr 31997

Apr 31997

Apr 31997

Apr 31997

Apr 31997

Apr 31997

Apr 31997

Apr 31997

Apr 31997

Apr 31997

Apr 31997

Apr 31997

Apr 31997

Apr 31997

Apr 31997

Apr 31997

Apr 31997

Apr 31997

Apr 31997

Apr 31997

Apr 31997

Apr 31997

Apr 31997

Apr 31997

Apr 31997

Apr 31997

Apr 31997

Apr 31997

Apr 31997

Apr 31997

Apr 31997

File size

(Bytes)

134260

136857

137438

137770

138534

134358

134690

134856

134856

134773

134607

134773

134856

134607

134524

134109

133125

132129

178485

181315

182311

182664

184453

185038

185291

185291

185291

185208

185125

185208

185291

185291

185374

184018

182225

174701

File Type

(Format)

AsCII

ASCII

ASCII

ASCII

Ascl I

AsCII

ASCII

ASCII

AsCII

ASCII

ASCII

ASCII

ASCII

ASCII

ASCII

ASCII

ASCI I

ASCII

ASCI I

ASCII

ASCII

ASCII

ASCII

ASCII

ASCII

ASCII

ASCII

ASCII

ASCII

ASCII

ASCII

ASCII

ASCII

ASCI I

ASCI I

ASCII

Tape Backup
File Name

\begin{tabular}{cc}
$\begin{array}{c}\text { File Size } \\
\text { (Bytes) }\end{array}$ & $\begin{array}{c}\text { File Type } \\
\text { (Format) }\end{array}$ \\
\hdashline $146 . \ldots$ & ASCII \\
146424 & ASCII \\
150233 & ASCI \\
150041 & ASCII \\
153769 & ASCII \\
156328 & ASCII \\
149167 & ASCII \\
153004 & ASCII \\
153245 & ASCII \\
156536 & ASCII \\
158241 & ASCII \\
150088 & ASCII \\
154332 & ASCII \\
154083 & ASCII \\
156951 & ASCII \\
158826 & ASCII \\
150835 & ASCII
\end{tabular}


A182/CR3A01N04DC08T097AC08T139.cut A182/CR3A01N04DCO8T 139AC08T404. Cut A182/CR3A01N04DC08T404AC08T409.cut A182/CR3A01NO4DCO8T 409AC08T515.cut A18Z/CR3A01N05DCOBTO0OAC08T097.cut A1B2/CR3A01N05DCO8T097AC08T139.cut A18z/CR3A01N05DC08T139AC08T404. cut A18z/CR3A01N05DC08T404AC08T409.cut A18z/CR3A01N05DC08T409AC08T515.cut A182/CR3A01N060C08T000AC08T097.cut A182/CR3A01N060C08T097AC08T 139. Cut A182/CR3A01N060C08T 139AC08T404.cut A18z/CR3A01N060C08T404AC08T409.cut A18z/CR3A01N060CO8T409AC08T515.cut A18z/CR3A01NOTDCOBTO00AC08T097.cut A 18z/CR3A01N07DCOBTO97ACOBT 139.cut A18Z/CR3A01NOTOCOBT 139AC08T404.CUT A18z/CR3A01N070C08T 404AC08T409.cut A18z/CR3AO1NOTDCOBT 409AC08T515.cut A18z/CR3A01NOBOCOBTOOOACOBT097.cut A 18z/CR3A01N08DCOBTO97AC08T 139.cut A 182/CR3A01NOBOCOBT 139AC08T 404. Cut A18z/CR3AOINOBDCOBT 404AC08T409.cut A18z/CR3AO1NOBDCO8T 409AC08T515.cut A18z/CR3A01N090COBTO0OAC08T097.cut A18z/CR3AO1NO9DCOBTO97AC08T 139. cut A182/CR3A01NO9OCOBT 139ACO8T404.cut A 18z/CR3A01NOSDCOBT 404AC08T409. cut A182/CR3A01N090C08T409AC08T515.cut A18Z/CR3A01N10DCOBT000AC08T097.cut A 18z/CR3A01N100C08T097AC08T139.cut A18z/CR3A01N100C081139AC08T404.cut A18z/CR3A01N100COBT404ACO8T409.cut A182/CR3A01N100COBT409AC08T515. cut A18z/CR3A01N110C08T000AC08T097.cut A18z/CR3A01N110C08T097AC08T139.cut A 18z/CR3A01N110C08T139AC08T404.cut A 18Z/CR3A01N1 10C0BT 404AC08T409.cut A 18Z/CR3A01N110COBT409ACO8T515.cut A18z/CR3A01N120COBTODOAC08T097. cut A182/CR3A01N120COBT097ACOBT 139. CUt A 182/CR3A01N120C08T139ACOBT404. CUt A18z/CR3A01N12DC08T404AC08T409. cut A18Z/CR3A01N120COBT409ACO8T515. cut A18z/CR3A01N130C08TODOACOBT097. CUT A 18z/CR3A01N13DCOBT097ACOBT 139. cut A18Z/CR3A01N130C08T139AC08T404.cut A 18z/CR3A01N130C08T404AC08T409.cut A182/CR3A01N13DC08T409AC08T515.cut A18Z/CR3A01N14DCOBTO0OAC08T097.cut A18z/CR3A01N140CO8T097AC08T 139.cut A18z/CR3A01N140C08T139AC08T404.cut A 18z/CR3A01N14DC08T404AC08T409.cut A 182/CR3A01N14DC08T409AC08T515 : cut A18Z/CR3A01N150C08TD0OAC08T097.cut A 182/CR3A01N15DCOBT 097ACO8T 139.cut A18z/CR3A01N150C08T139AC08T404.cut A18Z/CR3A01N15DC081404AC08T409. cut A 18z/CR3A01N150C08T409AC08T515. cut A 18z/CR3A01N160COBTO0OAC08T097.cut A 18z/CR3A01N160C08T097AC08T139.cut A18z/CR3A01N160C08T139AC08T404 . cut A18Z/CR3A01N160C08T404AC08T409.cut A18z/CR3AO1N160COBT409ACO8T515.cut A 18Z/CR3A01N170C08T000AC08T097. cut A 18Z/CR3A01N170C08T097AC08T 139.cut A182/CR3AO1N17DCOBT 139ACOBT404.CUt A18z/CR3A01N170C08T404AC08T409.cut A18z/CR3A01N170C081409AC08T515.cut A 18Z/CR3A01N18DC08T000AC08T097. cut

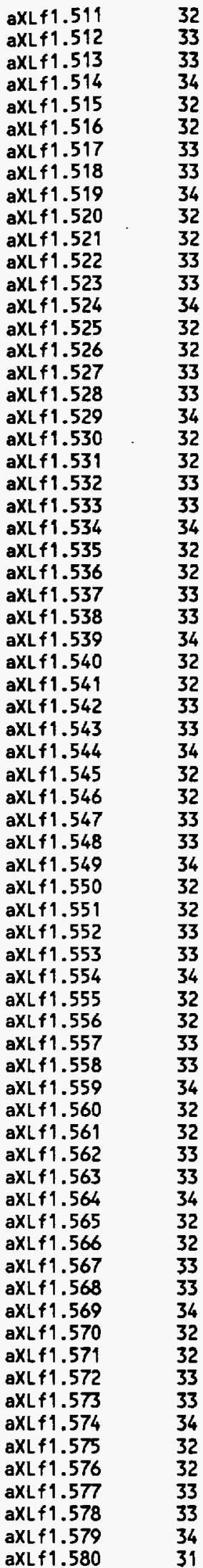

Jul 171997 Jul 171997 Jul 171997 Jul 171997 Jul 171997 Jul 171997 Jul 171997 Jul 171997 Jul 171997 Jul 171997 Jul 171997 Jul 171997 Jul 171997 Jul 171997 Jul 171997 Jul 171997 Jul 171997 Jul 171997 Jul 171997 Jul 171997 Jul 171997 Jul 171997 JuL 171997 Jul 171997 Jul 171997 Jul 171997 Jul 171997 Jul 171997 Jul 171997 Jul 171997 Jul 171997 Jul 171997 Jul 171997 Jul 171997 Jul 171997 Jul 171997 Jul 181997 Jul 181997 Jul $18 \quad 1997$ Jul $18 \quad 1997$ Jul $18 \quad 1997$ Jul 181997 Jul 181997 Jul 181997 Jul 181997 Jul $18 \quad 1997$ Jul $18 \quad 1997$ Jul $18 \quad 1997$ Jul $18 \quad 1997$ Jul 181997 Jul 181997 Jul $18 \quad 1997$ Jul $18 \quad 1997$ Jul 181997 Jul 181997 Jul 181997 Jul 181997 Jul 181997 Jul 181997 Jul 181997 Jul $18 \quad 1997$ Jul 181997 Jul $18 \quad 1997$ Jul 181997 Jul 181997 Jul 181997 Jul $18 \quad 1997$ Jul 181997 Jul 181997 Jul 181997
155000

154498

157366

159121

151001

155083

154498

157615

159121

151001

155166

154498

157698

159287

151001

155166

154415

157698

159370

151167

155166

154415

157698

159370

151001

155166

154415

157698

159287

151084

155166

154332

157615

159204

150918

155000

154415

157449

158909

150333

154502

154332

156951

158826

150167

154170

154249

157117

158992

149665

154000

154079

157034

158992

149752

153834

153996

157117

158992

149835

153917

154000

157117

158992

149337

153668

153581

156702

158573

147590
ASCII

ASCII

ASCII

ASCII

ASCII

ASCII

ASCII

ASCII

ASCII

ASCII

ASCII

ASCII

ASCII

ASCII

ASCII

ASCII

ASCII

ASCII

ASCII

ASCII

ASCII

ASCII

ASCII

ASCII

ASCII

ASCII

ASCII

ASCII

ASCII

ASCII

ASCII

ASCII

ASCII

ASCII

ASCII

ASCII

ASCII

ASCII

ASCII

ASCII

ASCII

ASCII

ASCII

ASCII

ASCII

ASCII

ASCII

ASCII

ASCII

ASCII

ASCII

ASCII

ASCII

ASCII

ASCII

ASCI I

ASCII

ASCII

ASCI I

ASCII

ASCII

ASCII

ASCII

ASCII

ASCI I

ASCII

ASCII

ASCII

ASCII

ASCII 
A18z/CR3A01N18DC08T097AC08T139.cut A18z/CR3A01N18DC08T139AC08T404. Cut A18z/CR3A01N180C08T404AC08T409. Cut A18z/CR3A01N180C08T409AC08T515. cut A18Z/CR3A18N01DC1ATO0OACO8TO00. CUT A18Z/CR3A18N02DC1AT000AC08T000. CUT A 18z/CR3A18N03DC1AT000AC08T000. cut A18Z/CR3A 18N040C1AT000AC08T000. CUT A18z/CR3A18N05DC1AT000AC08T000. Cut A18Z/CR3A18N06DC1ATO0OAC08TO0O. CUT A18Z/CR3A18NOTOC1AT000AC08T000. Cut A18Z/CR3A18N08DC1AT000AC08T000. Cut A 18Z/CR3A18N090C1AT000AC08T000. CUT A18Z/CR3A18N10DC1AT000AC08T000. cut A18Z/CR3A18N11DC1AT000AC08T000. cut A18z/CR3A18N12DC1AT000AC08T000. CUt A182/CR3A18N13DC1AT000AC08T000. CUT A18z/CR3A18N14DC1AT000AC08T000. Cut A18Z/CR3A 18N15DC1AT000AC08T000. cut A18z/CR3A18N16DC1AT000AC08T000.cut A182/CR3A18N17DC1AT000AC08T000. CUT A18Z/CR3A 18N180C1AT000AC08T000. cut

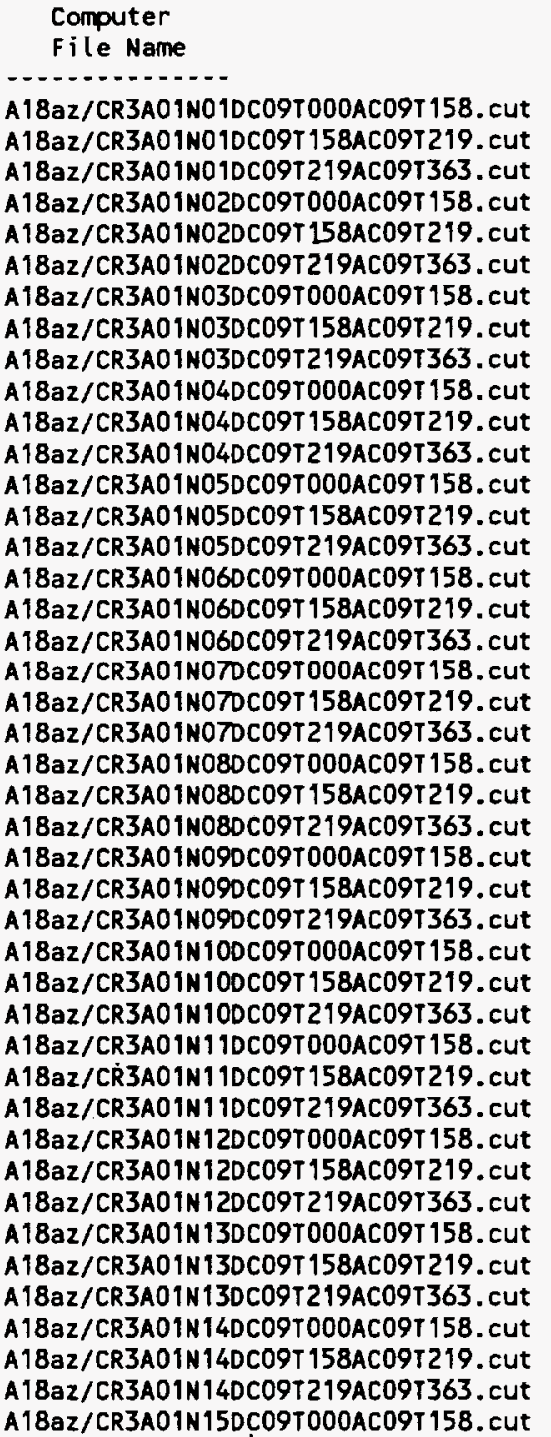

$\begin{array}{ll}\text { aXLf1.581 } & 32 \\ \text { aXLf1.582 } & 33 \\ \text { aXLf1.583 } & 32 \\ \text { aXLf1.584 } & 33 \\ \text { aXLf1.585 } & 36 \\ \text { aXLf1.586 } & 36 \\ \text { aXLf1.587 } & 36 \\ \text { aXLf1.588 } & 36 \\ \text { aXLf1.589 } & 36 \\ \text { aXLf1.590 } & 36 \\ \text { aXLf1.591 } & 36 \\ \text { aXLf1.592 } & 36 \\ \text { aXLf1.593 } & 37 \\ \text { aXLf1.594 } & 37 \\ \text { aXLf1.595 } & 37 \\ \text { aXLf1.596 } & 37 \\ \text { aXLf1.597 } & 37 \\ \text { aXLf1.598 } & 37 \\ \text { aXLf1.599 } & 37 \\ \text { aXLf1.600 } & 37 \\ \text { aXLf1.601 } & 36 \\ \text { aXLf1.602 } & 36\end{array}$

32
33
32
33
36
36
36
36
36
36
36
36
37
37
37
37
37
37
37
37
36
36

Tape Backup File Name

aXL $f 1.603$

aXLf1.604

axLf1.605

aXLf1.606

aXLf1.607

aXL $f 1.608$

aXLf1.609

aXL 11.610

$\operatorname{aXL} 1.611$

$\operatorname{aXL} f 1.612$

aXLf1.613

aXL 11.614

aXLf1.615

$\operatorname{aXL} 1.616$

aXLf1.617

aXL 1.618

aXLf1.619

aXL 1.620

aXLf1.621

axL 1.622

aXL $f 1.623$

aXLf1.624

aXL 1.625

aXLf1.626

aXL 11.627

aXL 1.628

axL 1.629

aXLf 1.630

aXL 1.631

aXL 1.632

aXL $f 1.633$

aXL 1.634

aXL 19.635

aXL 19.636

aXL 1.637

aXL 1.638

aXL 1.639

aXLf1.640

aXLf1.641

aXL 11.642

aXL $f 1.643$

aXL 1.644

aXL 1.645

35

29

36

36

36

36

29

36

37

30

37

37

37

37

30

37

37

30

37
37

30

37

37

30

37

37

30

37

37

30

37

36

29

36

36

29

36

36

29

36 $\begin{array}{lll}\text { Jul } & 18 & 1997 \\ \text { Jul } 18 & 1997 \\ \text { jul } 18 & 1997 \\ \text { Jul } 18 & 1997 \\ \text { Jul } 17 & 1997 \\ \text { Jul } 17 & 1997 \\ \text { Jul } 17 & 1997 \\ \text { Jul } 17 & 1997 \\ \text { Jul } 17 & 1997 \\ \text { Jul } 17 & 1997 \\ \text { Jul } 17 & 1997 \\ \text { Jul } 17 & 1997 \\ \text { Jul } 17 & 1997 \\ \text { Jul } 17 & 1997 \\ \text { Jul } & 17 & 1997 \\ \text { Jul } & 18 & 1997 \\ \text { Jul } 18 & 1997 \\ \text { Jul } & 18 & 1997 \\ \text { Jul } & 18 & 1997 \\ \text { Jul } & 18 & 1997 \\ \text { Jul } 18 & 1997 \\ \text { Jul } & 18 & 1997\end{array}$

Number of

Print Pages
File Date (Output)

Jul 171997

Jul 171997

Jul 171997

Jul 171997

Jul 171997

Jul 171997

Jul 171997

Jul 171997

Jul 171997

Jul 171997

Jul 171997

Jul 171997.

Jul 171997

Jul 171997

Jul 171997

Jul 171997

Jul 171997

Jul 171997

Jul 171997

Jul 171997

Jul 171997

Jul 171997

Jul 171997

Jul 171997

Jul 171997

Jul 171997

Jul 171997

Jul 171997

Jul 171997

Jul 171997

Jul 171997

Jul 171997

Jul 171997

Jul 171997

Jul 171997

Jul 179997

Jul 171997

Jul 171997

Jul 171997

Jul 171997

Jul 171997
Jul 171997

Jul 171997
151316

151917

154682

157324

151872

153615

154362

154528

154694

154853

155351

155351

158445

157864

158203

157954

157353

157270

157353

157436

156108

154697

\section{File size File Type \\ (Bytes) \\ 166393 \\ 135895 \\ (Format) \\ ASCII \\ ASCII}

168811

169800

139223

171969

171551

139808

173301

171966

140306

173720

172464

140555

173720

172547

140721

173720

172547

140721

173720

172630

140721

173720

172547

140721

173720

172464

140638

173720

172215

140306

173637

171713

139891

173222

171298

139642

172886

171381

139642

172886

171381

ASCII

ASCII

Ascil

ASCII

ASClI

ASCII

ASCII

ASClI

ASCII

AsCII

ASCII

ASCII.

ASCI I

ASCII

ASCII

ASCII

ASCII

ASCII

ASCII

ASCII

ASCII

ASCII

ASCII

ASCII

ASCII

ASCII

ASCII

ASCII

ASCII

ASCII

ASCII

ASCII

ASCII

ASCII

ASCII

ASCII

ASCII

ASCII

ASCII

ASCII

ASCII

ASCII

ASCII

ASCII

ASCII

ASCII

ASCII

ASCII

ASCII

ASCII

ASCII

ASCII

ASCII

ASCII

ASCII

ASCII 
A18az/CR3A01N15DC09T158AC09T219.cut A18az/CR3A01H15DCO9T219AC09T363.cut A18az/CR3A01N16DCO9T000ACO9T 158.cut A18az/CR3A01N160C09T158AC09T219. Cut A18az/CR3A01N160CO9T219AC09T363. Cut A18az/CR3A01N170C09T000AC09T 158. cut A18az/CR3A01N17DCO9T158ACO9T219.cut A18az/CR3A01N170CO9T219ACO9T363.cut A18az/CR3A01N180CO9T000ACO9T 158. cut A18az/CR3A01N18DC09T158ACO9T219. Cut A18az/CR3A01N18DC09T219ACO9T363.cut A18az/CR3A18NO1DC1ATOOOACO9TOOO. CUT A18aZ/CR3A18N02DC1ATODOACO9T000. CUT A18aZ/CR3A18N030C1ATOOOACO9TO00. CUT A18aZ/CR3A18N040C1AT000AC09T000. CUT A18az/CR3A18N050C1ATO00ACO9T000. Cut A18az/CR3A18N060C1ATODOACO9T000. cut A18az/CR3A18N0T0C1ATOOOACO9T000. cut A18az/CR3A18N080C1ATO00ACO9T000. Cut A18az/CR3A18N09DC1ATO0OACO9T000. Cut A18az/CR3A18N10DC1ATO00AC09T000. cut A18az/CR3A18N11DC1ATO00ACO9T000. cut A18az/CR3A18N120C1ATO00ACO9T000. Cut A18az/CR3A18N13DC1AT000AC09T000. cut A18az/CR3A18N14DC1AT000AC09T000. CUT A18az/CR3A18N15DC1AT000ACO9T000. Cut A18az/CR3A18N160C1AT000AC09T000. cut A18az/CR3A18N17DC1ATO00ACO9T000. cut A18az/CR3A18N18DC1AT000AC09T000. Cut

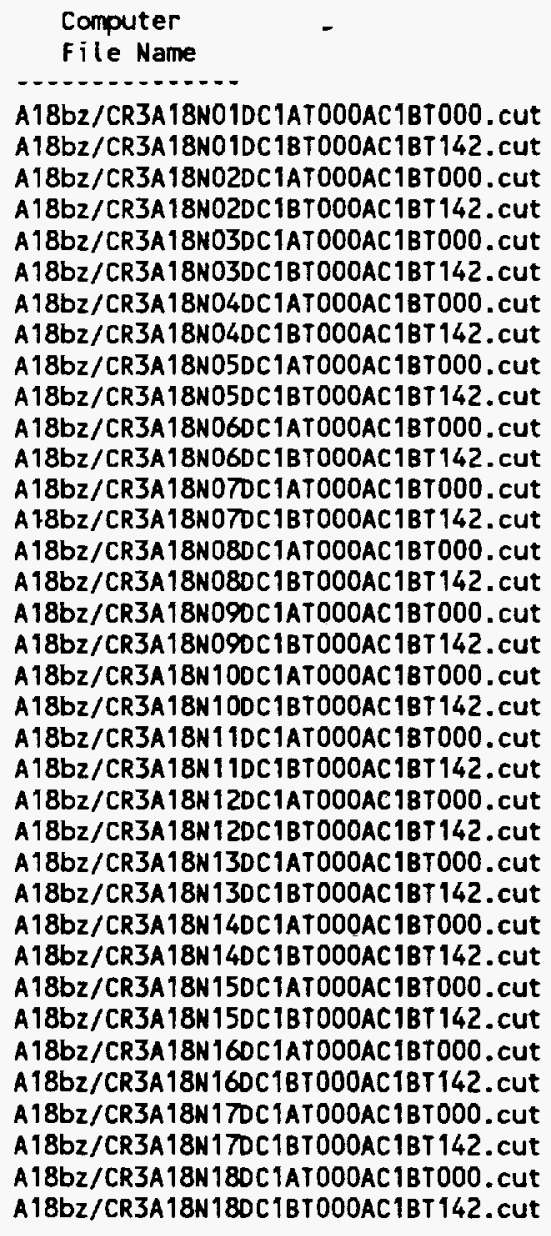

\begin{tabular}{|c|}
\hline 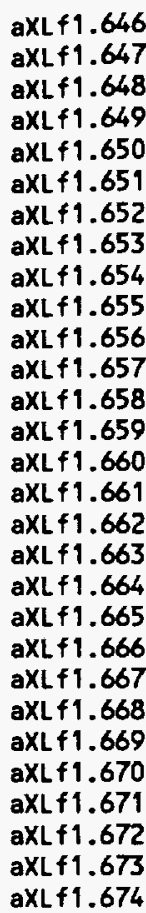 \\
\hline
\end{tabular}

\begin{tabular}{ll} 
Tape Backup & $\begin{array}{c}\text { Number of } \\
\text { Fi I le Name }\end{array}$ \\
Print Pages \\
\hline aXLf1.675 & 38 \\
aXLf1.676 & 36 \\
aXLf1.677 & 38 \\
aXLf1.678 & 36 \\
aXLf1.679 & 39 \\
aXLf1.680 & 36 \\
aXLf1.681 & 39 \\
aXLf1.682 & 37 \\
aXLf1.683 & 39 \\
aXLf1.684 & 37 \\
aXLf1.685 & 39 \\
aXLf1.686 & 37 \\
aXLf1.687 & 39 \\
aXLf1.688 & 37 \\
aXLf1.689 & 39 \\
aXLf1.690 & 37 \\
aXLf1.691 & 40 \\
aXLf1.692 & 37 \\
aXLf1.693 & 40 \\
aXLf1.694 & 37 \\
aXLf1.695 & 40 \\
aXLf1.696 & 37 \\
aXLf1.697 & 39 \\
aXLf1.698 & 37 \\
aXLf1.699 & 39 \\
aXLf1.700 & 37 \\
aXLf1.701 & 39 \\
aXLf1.702 & 37 \\
aXLf1.703 & 39 \\
aXLf1.704 & 37 \\
aXLf1.705 & 39 \\
aXLf1.706 & 37 \\
aXLf1.707 & 39 \\
aXLf1.708 & 36 \\
aXLf1.709 & 39 \\
aXLf1.710 & 36 \\
&
\end{tabular}

$\begin{array}{lll}\text { Jul } & 17 & 1997 \\ \text { Jul } & 17 & 1997 \\ \text { Jul } 17 & 1997 \\ \text { Jul } 17 & 1997 \\ \text { Jul } & 17 & 1997 \\ \text { Jul } & 17 & 1997 \\ \text { Jul } & 17 & 1997 \\ \text { Jul } & 17 & 1997 \\ \text { Jul } & 17 & 1997 \\ \text { Jul } & 17 & 1997 \\ \text { Jul } & 17 & 1997 \\ \text { Jul } 17 & 1997 \\ \text { Jul } & 17 & 1997 \\ \text { Jul } & 17 & 1997 \\ \text { Jul } & 17 & 1997 \\ \text { Jul } & 17 & 1997 \\ \text { Jul } & 17 & 1997 \\ \text { Jul } & 17 & 1997 \\ \text { Jul } & 17 & 1997 \\ \text { Jul } & 17 & 1997 \\ \text { Jul } & 17 & 1997 \\ \text { Jul } & 17 & 1997 \\ \text { Jul } & 17 & 1997 \\ \text { Jul } & 17 & 1997 \\ \text { Jul } & 17 & 1997 \\ \text { Jul } & 17 & 1997 \\ \text { Jul } & 17 & 1997 \\ \text { Jul } & 17 & 1997 \\ \text { Jul } & 17 & 1997\end{array}$

13955

172886

171298

$\$ 39725$

172969

170717

139642

172720

167974

136895

170641

150581

152158

153237

153615

153698

154106

154355

154355

157449

156951

157041

156829

156145

156145

156145

156145

154983

153157

File Date

(Output)

July 171997

July 171997

July 171997

July 171997

July 171997

July 171997

July 171997

July 171997

July 171997

July 171997

July 171997

July 171997

July 171997

July 171997

July 171997

July 171997

July 171997

July 171997

July 171997

July 171997

July 171997

July 171997

July 171997

July 171997

July 171997

July 171997

July 171997

July 171997

July 171997

July 171997

July 171997

July 181997

July 181997

July 181997

July 181997

July 181997

\begin{tabular}{cc}
$\begin{array}{c}\text { File Size } \\
\text { (Bytes) }\end{array}$ & $\begin{array}{c}\text { File Type } \\
\text { (Format) }\end{array}$ \\
\hline 164208 & - \\
162716 & ASCII \\
166615 & ASCII \\
164795 & ASCII \\
167528 & ASCII \\
166542 & ASCII \\
168026 & ASCII \\
167252 & ASCII \\
168441 & ASCII \\
167584 & ASCII \\
168839 & ASCII \\
167667 & ASCII \\
168922 & ASCII \\
167667 & ASCII \\
168922 & ASCII \\
167501 & ASCII \\
172182 & ASCII \\
167335 & ASCII \\
171684 & ASCII \\
170512 & ASCII \\
172306 & ASCII \\
170134 & ASCII \\
171476 & ASCII \\
169118 & ASCII \\
170792 & ASCII \\
168948 & ASCII \\
170792 & ASCII \\
168948 & ASCII \\
170875 & ASCII \\
168948 & ASCII \\
171041 & ASCII \\
169450 & ASCII \\
169689 & ASCII \\
164199 & ASCII \\
168195 & ASCII \\
161871 & ASCII \\
& ASCII
\end{tabular}

ASCII

ASCII

ASCII

ASCII

ASCII

ASCII

ASCII

ASCII

ASCII

ASCII

ASCII

ASCII

ASCII

ASCII

ASCII

ASCII

ASCII

ASCII

ASCII

ASCII

ASCII

ASCII

ASCII

ASCII

ASCII

ASCII

ASCI I

ASCII

ASCII

ASCII

ASCII

SCI I

ASCII

ASCII

ASCII

ASCII

SCII

ASCII

SCII

ASCI I

ASCII

SCII

SCII

SCII

ASCII 


\begin{abstract}
Computer
File Name

A

A262/CR3A18N01DC1BTOOOAC1BT142. Cut A262/CR3A18N02DC1BTODOAC1BT142. CUt A262/CR3A18N03DC1BTO0OAC1BT142. Cut A262/CR3A18N04DC1BTO00AC1BT142.cut A262/CR3A18NO5DC1BTO0OAC1BT142. cut A26Z/CR3A18N060C1BTO0OAC1BT142. cut A262/CR3A18N07DC1BTO0OAC1BT142. CUT A26Z/CR3A18N08DC1BTOOOAC1BT142.CUT A262/CR3A18N09DC1BT000AC1BT142. CUT A262/CR3A18N10DC1BTDOOAC1BT142. CUt A262/CR3A18N11DC1BTOOOAC1BT142.CUT A262/CR3A18N12DC1BTODOAC1BT142. CUT A262/CR3A18N130C1BTO00AC1BT142. CUt A262/CR3A18N140C1BTDOOAC1BT142.CUT A262/CR3A18N15DC1BTDOOAC1BT142.cUt A262/CR3A18N160C1BTDOOAC1BT142. CUT A262/CR3A18N170C1BTDOOAC1BT142.CUt A262/CR3A18N180C1BTODOAC1BT142. CUt A26Z/CR3A26N010C1ATOOOAC1BTO0O. Cut A262/CR3A26N020C1ATOOOAC1BT000. CUt A262/CR3A26N03DC1ATOOOAC1BTO00. CUT A262/CR3A26N04DC1ATOOOAC1BT000.CUT A262/CR3A26N05DC1ATOOOAC1BT000. CUt A26Z/CR3A26NO60C1ATOOOAC1BTO00.cut A262/CR3A26NOTDC1ATOOOAC1BT000.CUT A262/CR3A26N080C1ATOOOAC1BTO00. CUT A262/CR3A26NO9OC1ATOOOAC1BT000. CUt A262/CR3A26N100C1ATO0OAC1BTO00.cut A262/CR3A26N110C1ATO00AC1BT000.CUT A262/CR3A26N12DC1ATOOOAC1BTO00.CUT A262/CR3A26N130C1ATOOOAC1BTO00.cUT A262/CR3A26N14DC1ATOOOAC1BTO00.cut A262/CR3A26N15DC1ATO00AC1BTO00.cut A262/CR3A26N160C1ATO00AC1BT000.cut A262/CR3A26N1TDC1ATO0OAC1BTO00.cUT A26Z/CR3A26N180C1ATOOOAC1BTO00.CUt
\end{abstract}

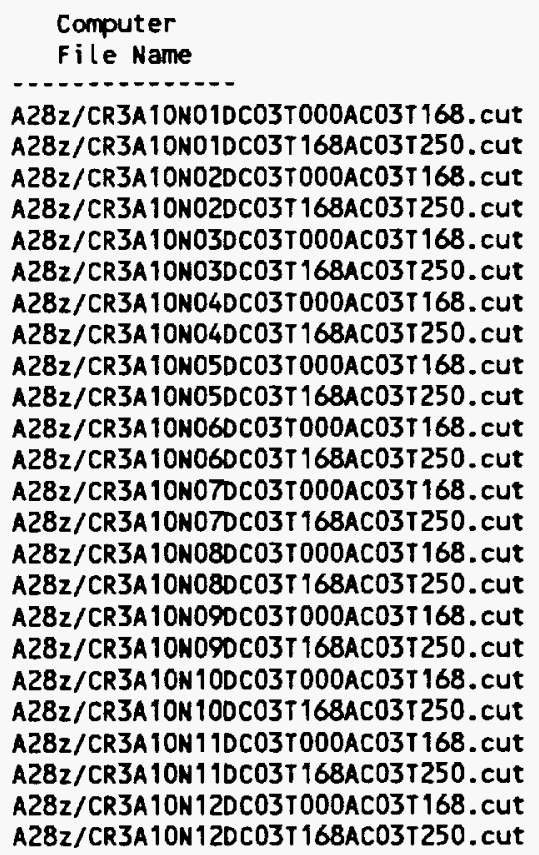

Tape Backup
File Name

$\begin{gathered}\text { Number of } \\ \text { Print Pages }\end{gathered}$
37
37
37
37
37
38
38
38
38
38
38
38
38
38
38
38
38
37
36
36
37
37
37
37
37
37
37
37
37
37
37
37
37
37
36
36
36

File Date
(Output)

July 171997

July 171997

July 171997

July 171997

July 171997

July 171997

July 171997

July 171997

July 171997

July 171997

July 171997

July 171997

July 171997

July 171997

July 171997

July 181997

July 181997

July 181997

July 171997

July 171997

July 171997

July 171997

July 171997

July 171997

July 171997

July 171997

July 171997

July 171997

July 171997

- July 171997

July 171997

July 171997

July 171997

July 171997

July 181997

July 181997

\begin{tabular}{cc}
$\begin{array}{c}\text { File Size } \\
\text { (Bytes) }\end{array}$ & $\begin{array}{c}\text { File Type } \\
\text { (Format) }\end{array}$ \\
\hdashline 165641 & - \\
167807 & ASCII \\
169550 & ASCII \\
170052 & ASCII \\
170301 & ASCII \\
170513 & ASCII \\
170513 & ASCII \\
170430 & ASCII \\
170430 & ASCII \\
173478 & ASCII \\
173478 & ASCII \\
173209 & ASCII \\
173126 & ASCII \\
173043 & ASCII \\
172794 & ASCII \\
172877 & ASCII \\
167033 & ASCII \\
164871 & ASCII \\
159183 & ASCII \\
162005 & ASCII \\
163001 & ASCII \\
163582 & ASCII \\
163831 & ASCII \\
163997 & ASCII \\
163997 & ASCII \\
163997 & ASCII \\
163997 & ASCII \\
163997 & ASCII \\
163831 & ASCII \\
163582 & ASCII \\
163582 & ASCII \\
163582 & ASCII \\
163333 & ASCII \\
157967 & ASCII \\
157303 & ASCII \\
155311 & ASCII \\
& ASCII
\end{tabular}

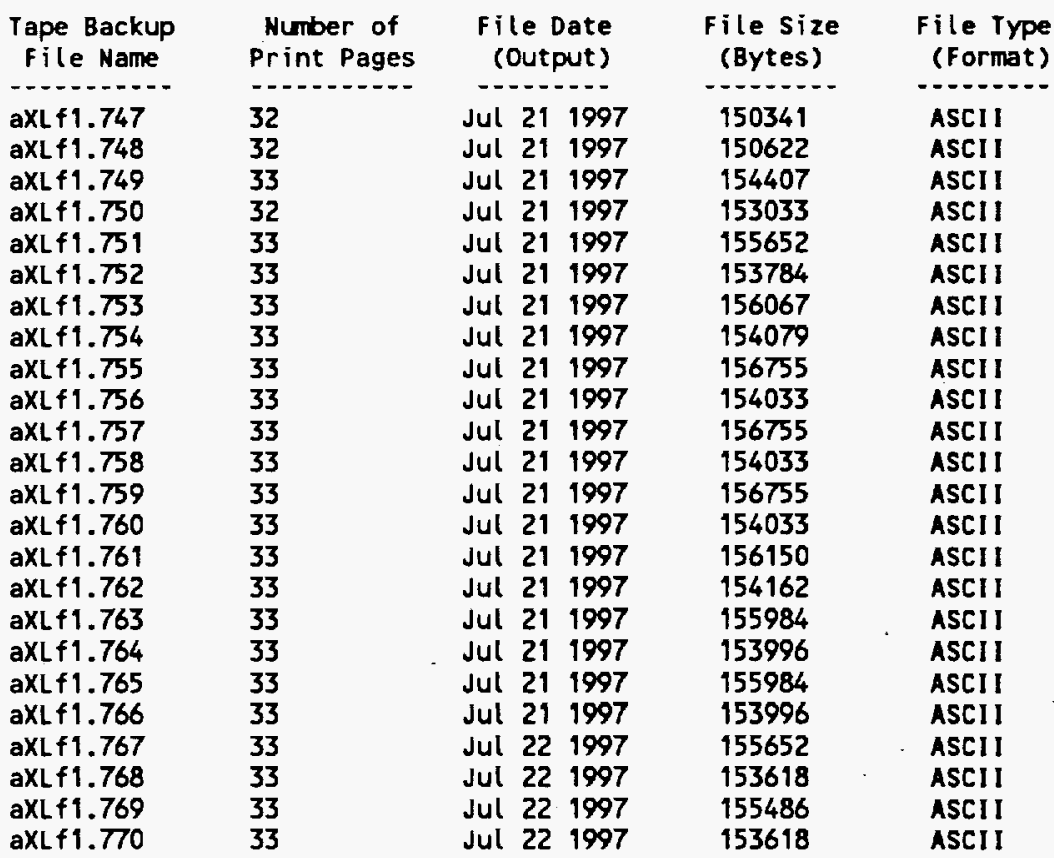


A282/CR3A10N13DC03T000AC03T168.cut A28Z/CR3A10N13DCO3T168ACO3T250.cut A28z/CR3A10N14DC03TO00AC03T168. cut A28z/CR3A10N14DC03T168AC03T250.cut A28z/CR3A10N150C03T000ACO3T 168. cut A28Z/CR3A10N15DCO3T168ACO3T250. CUt A28z/CR3A10N160C03T000AC03T168.cut A28z/CR3A10N160C03T168AC03T250. cut A282/CR3A10N17DC03TO00AC03T168. Cut A28Z/CR3A10N170C03T168AC03T250. Cut A28z/CR3A10N180C03T000AC03T168.cut A28z/CR3A10N18DC03T168AC031250. cut A28Z/CR3A18N01DC02T000AC03T000. cut A28z/CR3A18N020C02TO0OACO3TO00.cut A28Z/CR3A18N03DC02TOOOACO3TOOO.cut A28z/CR3A18N04DC02T000ACO3T000.cut A28Z/CR3A18N05DC02TODOACO3TO00.CUT A28Z/CR3A18N060C02T000AC03T000. cut A28z/CR3A 18N070C02TO00AC03T000. cut A28z/CR3A18N080C02TO0OAC03T000. cut A282/CR3A18N090C02TO00ACO3TOOD. CUt A28Z/CR3A18N100C02TO0OACO3TO00. CUT A28Z/CR3A18N11DC02T000AC03T000.cut A28Z/CR3A18N12DC02TOOOACO3TO00. cut A282/CR3A18N 13DC02TO0OAC03TO00. cut A28Z/CR3A18N14DC02TO00AC03TO00. CUt A282/CR3A18N15DC02T000ACO3TO00.cut A28z/CR3A18N 160C02T000AC03T000.cut A28z/CR3A18N170C02T000AC03TO00. cut A28Z/CR3A18N 180C02TO00AC03T000. cut A28Z/CR3A28N01DC1ATO00AC1BTO00. CUT A28Z/CR3A28N01DC1BTOQOAC1BT142.cut A28Z/CR3A28N01DC1BT142AC02T000. cut A28Z/CR3A28N02DC1ATOOOAC1BTO00. Cut A28z/CR3A28N02DC1BT000AC1BT 142. CUt A28Z/CR3A28N02DC1BT142AC02TO00. Cut A282/CR3A28N03DC1ATO0OAC1BT000.cut A282/CR3A28N03DC1BTDOOAC1BT142. CUT A28Z/CR3A28N03DC1BT142ACO2T000.cut A28Z/CR3A28N04DC1ATO00AC1BT000.cut A28Z/CR3A28N04DC1BTO00AC1BT142. Cut A282/CR3A28N04DC1BT 142AC02TO00. CUt A28Z/CR3A28N05DC1AT000AC1BTO00. cut A28z/CR3A28N05DC1BTO00AC1BT 142.cut A28z/CR3A28N05DC1BT 142ACO2T000. cut A282/CR3A28N06DC1ATO0OACIBT000. Cut A28Z/CR3A28N06DC1BTO0OAC1BT 142. cut A28z/CR3A28N06DC1BT 142AC02T000. CUt A28Z/CR3A28NOTDC1ATOOOACIBTO00.cut A28Z/CR3A28N0TDC1BTOOOAC1BT142. cut A28Z/CR3A28NOTDC1BT 142ACO2TO00.cut A28Z/CR3A28N080C1ATOOOAC1BTOOO. CUT A28Z/CR3A28N08DC1BT 000AC1BT 142. CUT A282/CR3A28N08DC1BT142AC02TOOO. Cut A28z/CR3A28N09DC1ATOOOAC1BTO00.cut A28z/CR3A28N09DC1BT 00OAC1BT142. CUt A28z/CR3A28N09DC18T142AC02T000.cut A28Z/CR3A28N10DC1ATO0OAC1BT000. cut A28Z/CR3A28N10DC1BTDOOAC1BT 142. cut A28Z/CR3A28N10DC1BT 142AC02T000.cut A28z/CR3A28N11DC1ATOOOAC1BT000.cut A28Z/CR3A28N11DC1BTOOOAC1BT142. cut A28z/CR3A28N11DC1BT142AC02T000. cut A28z/CR3A28N120C1ATOOOACIBTO00. cut A28Z/CR3A28N120C1BTO0OAC1BT142.cut A28Z/CR3A28N120C1BT 142AC02TO00.cut A282/CR3A28N13DC1ATOOOAC1BTO00. Cut A28Z/CR3A28N13DC1BT000AC1BT142. CUt A28Z/CR3A28N130C1BT142ACO2T000. Cut A28Z/CR3A28N14DC1ATOOOAC1BT000.cut

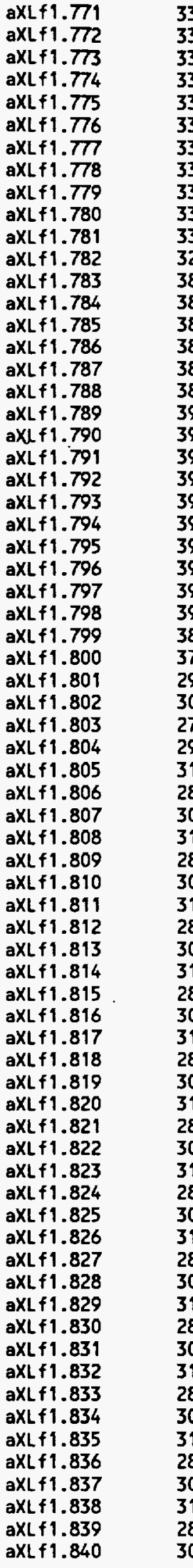

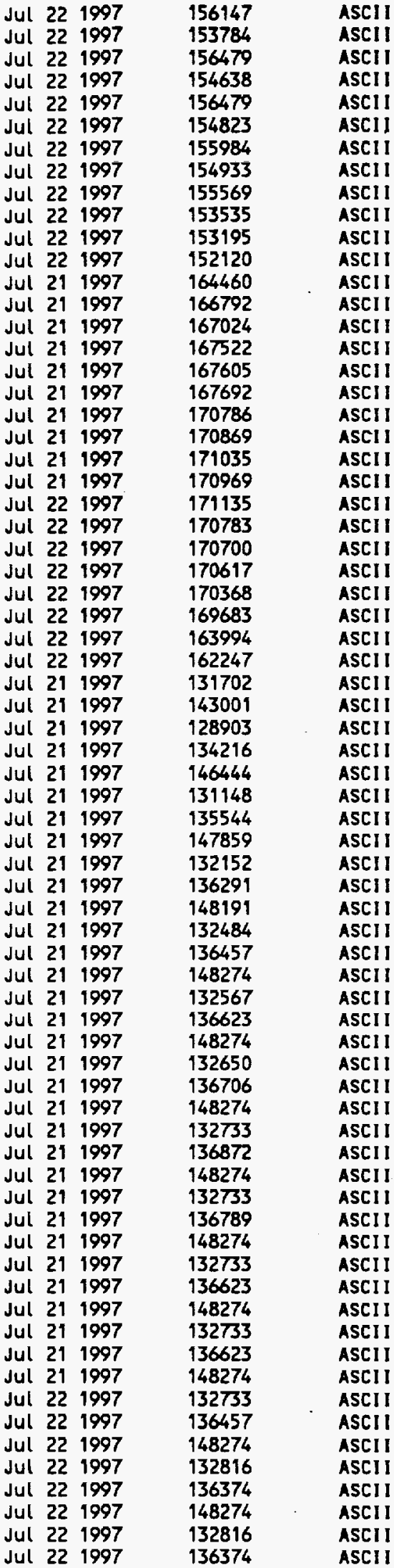

ASCII ASCII

II

ASCII

ASCII

ASCII

ASCII

SCII

ASCII

ASCII

ASCII

SCII

ASCII

ASCII

SCII

ASCII

SCII

ASCII

ASCII

ASCII

ASCII

ASCII

ASCII

CII

SCII

CII

SCII

SCII

SCII

ASCII

SCII

SCII

ASCII

ASCII

ASCII

ASCII

ASCII

ASCII

ASCII 


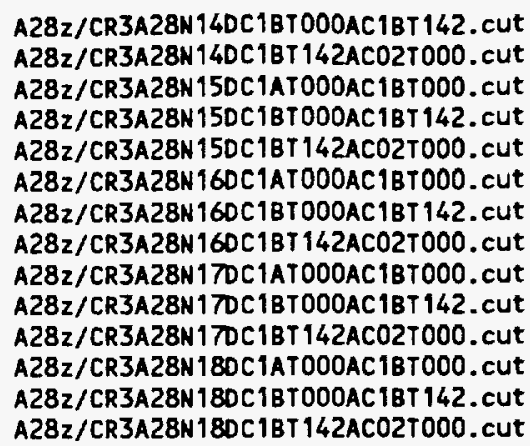

A282/CR3A28N14DC1BTOOOAC1BT142.cUt A28z/CR3A28N140C1BT142ACO2T000. CUT A28: /CR3A28N15DC1BTOOOAC1BT142. CUE A28Z/CR3A2BN150C1BT142ACO2TOOO. CUT A282/CR3A28N16DC1ATOOOAC1BT000.CUT A282/CR3A28N160C1BT142ACO2T000. CUT A282/CR3A28N1TOC1ATODOAC1BTO00.CUT A28Z/CR3A28N180C1ATOOOAC1BTO0O.CUT A282/CR3A28N180C1BT142ACO2T000. CUT

$\begin{array}{ll}\text { aXL } f 1.841 & 31 \\ \text { aXL } f 1.842 & 28 \\ \text { aXL } f 1.843 & 30 \\ \text { aXL } f 1.844 & 31 \\ \text { aXL } f 1.845 & 28 \\ \text { aXL } f 1.846 & 30 \\ \text { aXL } f 1.847 & 31 \\ \text { aXL } f 1.848 & 28 \\ \text { aXLf1.849 } & 30 \\ \text { aXLf1.850 } & 31 \\ \text { aXLf1.851 } & 28 \\ \text { aXLf1.852 } & 29 \\ \text { aXLf1.853 } & 31 \\ \text { aXLf1.854 } & 27\end{array}$

Jul 221997

Jul 221997

Jul 221997

Jul 221997

Jul 221997

Jul 221997

Jul 221997

Jul 221997

Jul 221997

Jul 221997

Jul $22 \quad 1997$

Jul 221997

Jul 221997

Jul 221997

148357

132816

136291

148357

132816

136291

148274

132567

135378

147776

132069

133801

145776

129903
ASCII

ASCII

ASCII

ASCII

ASCII

ASCII

ASCI I

ASCII

ASCII

ASCII

ASCII

ASCII

ASCII

ASCII 


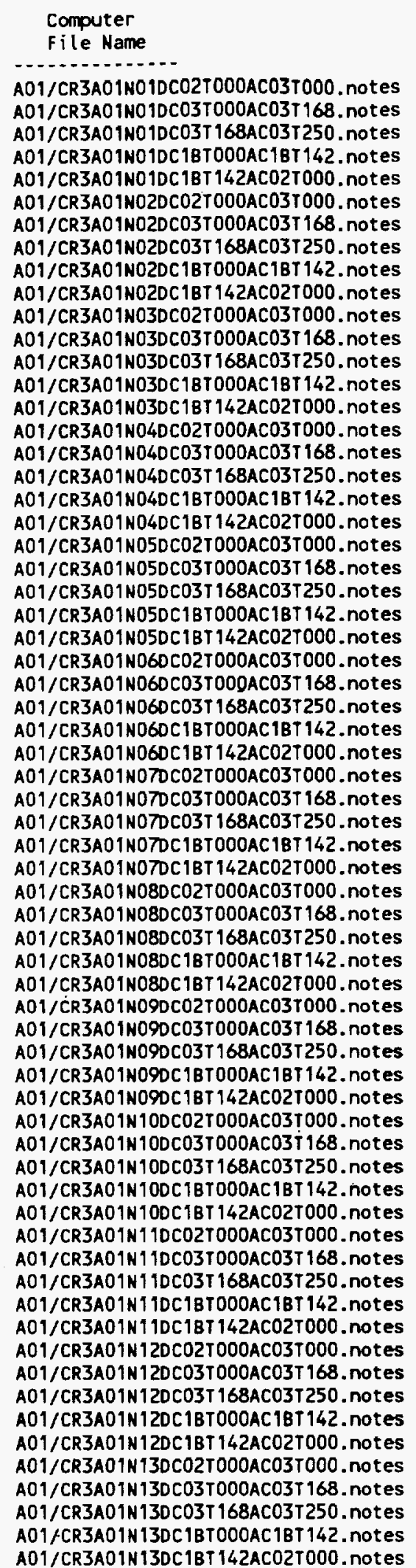

\begin{tabular}{|c|c|}
\hline $\begin{array}{c}\text { Tape Backup } \\
\text { File Name }\end{array}$ & $\begin{array}{l}\text { Number of } \\
\text { Print Pages }\end{array}$ \\
\hline - & \\
\hline aXL.If1 & 7 \\
\hline axL. If & 7 \\
\hline aXL. I f 3 & 8 \\
\hline aXL. If 4 & 7 \\
\hline$a \times L . I f 5$ & 8 \\
\hline aXL. If 6 & 7 \\
\hline aXL. If7 & 7 \\
\hline aXL. If8 & 8 \\
\hline aXL. If9 & 7 \\
\hline aXLI.f10 & 8 \\
\hline aXLI. $f 11$ & 7 \\
\hline aXL1. $f 12$ & 7 \\
\hline axLI.f13 & 8 \\
\hline aXL1. 194 & 7 \\
\hline axLi.f15 & 8 \\
\hline axLI. $\$ 16$ & 7 \\
\hline aXLI. .17 & 8 \\
\hline aXLI. $f 18$ & 9 \\
\hline aXLI .f19 & 7 \\
\hline aXLI. $f 20$ & 8 \\
\hline aXLI. $f 21$ & 7. \\
\hline axL I. $\$ 22$ & 8 \\
\hline aXLI . $\$ 23$ & 9 \\
\hline aXLI. +24 & 7 \\
\hline aXL1. +25 & 8 \\
\hline aXLI. $\$ 26$ & 7 \\
\hline axLI.f27 & 8 \\
\hline aXLI. +28 & 9 \\
\hline aXLI.f29 & 7 \\
\hline aXLI. $\$ 30$ & 8 \\
\hline axLI . $f 31$ & 7 \\
\hline aXLI. $\$ 32$ & 8 \\
\hline axLI.f33 & 9 \\
\hline axLI. $\$ 34$ & 7 \\
\hline aXL $1 . \$ 35$ & 8 \\
\hline aXLI. $\$ 36$ & 7 \\
\hline aXL1. $\$ 37$ & 8 \\
\hline aXLI.f38 & 9 \\
\hline aXLI . $\$ 39$ & 7 \\
\hline aXLI. $\$ 40$ & 8 \\
\hline aXLI. .441 & 7 \\
\hline$a X L 1 . f 42$ & 8 \\
\hline axL 1. $f 43$ & 9 \\
\hline aXLI. $f 44$ & 7 \\
\hline aXLI. $\$ 45$ & 8 \\
\hline aXLI. +46 & 7 \\
\hline aXL1. 147 & 8 \\
\hline aXL I. 148 & 9 \\
\hline axLI.$\$ 49$ & 7 \\
\hline $\mathrm{aXL} I . f 50$ & 8 \\
\hline aXL1. 151 & 7 \\
\hline aXL I. 152 & 8 \\
\hline aXL1. 153 & 9 \\
\hline axL1.f54 & 7 \\
\hline aXL I. $\$ 55$ & 8 \\
\hline aXL1. 156 & 7 \\
\hline aXL1.f57 & 8 \\
\hline aXL I. $\$ 58$ & 9 \\
\hline aXLI.f59 & 7 \\
\hline aXL1.f60 & 8 \\
\hline aXLI.f61 & 7 \\
\hline aXLI. 662 & 8 \\
\hline aXL I. 663 & 9 \\
\hline aXL1. 164 & 7 \\
\hline aXLI. 655 & 8 \\
\hline
\end{tabular}

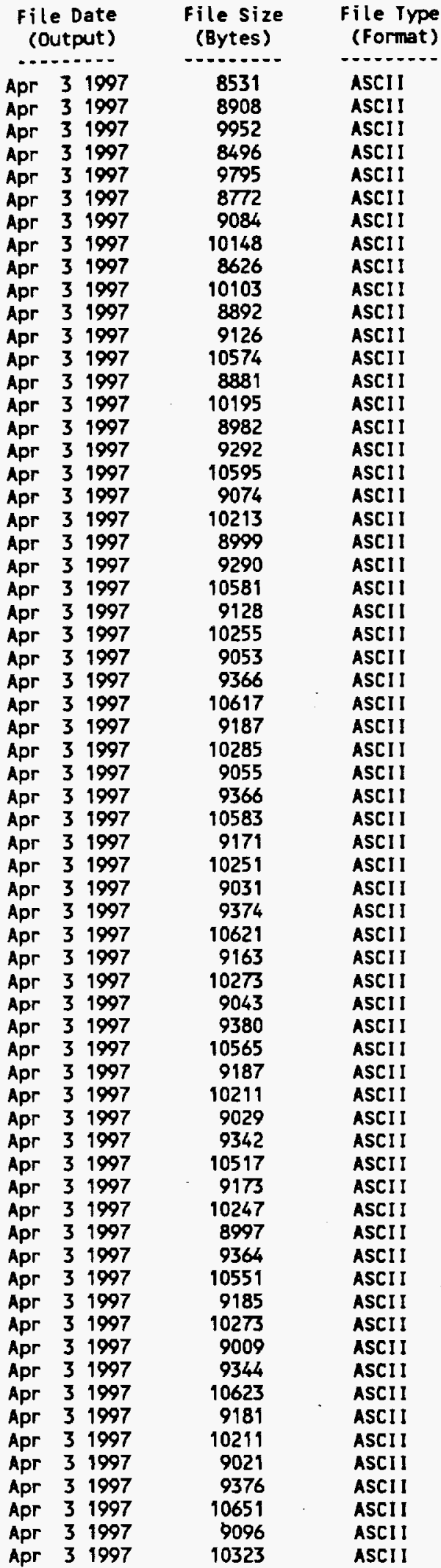


A01/CR3A01N14DCO2T000AC03T000. notes A01/CR3A01N14DCO3TO0OACO3T168. notes A01/CR3A01N14DCO3T168AC03T250. notes A01/CR3A01N14DC1BT000AC1BT142. notes A01/CR3A01N14DC1BT142AC02T000. notes A01/CR3A01N 15DC02T000AC03T000. notes A01/CR3A01N15DC03TO00AC03T168. notes A01/CR3A01N 15DC03T 168ACO3T250. notes A01/CR3A01N15DC1BT000AC1BT142.notes A01/CR3A01N 15DC1BT 142AC02T000. notes A01/CR3A01N16DC02T000ACO3T000. notes A01/CR3A01N 160C03T000AC03T168. notes A01/CR3A01N16DC03T168AC03T250. notes A01/CR3A01N16DC1BT000AC1BT142. notes A01/CR3A01N16DC1BT 142AC02T000. notes A01/CR3A01N17DC02TO00ACO3T000.notes A01/CR3A01N17DC03T000AC03T168. notes A01/CR3A01N17DC03T168ACO3T250. notes A01/CR3A01N17DC1BT000AC1BT142. notes A01/CR3A01N17DC1BT142AC021000. notes A01/CR3A01N180C02TO00ACO3TO00. notes A01/CR3A01N18DC03T000ACO3T168. notes A01/CR3A01N18DC03T168AC03T250. notes A01/CR3A01N18DC1BT000AC1BT 142. notes A01/CR3A01N18DC1BT142AC02T000. notes

\section{Computer \\ File Name}

AO4/CR3AOLNOIDC

A04/CR3A04N01DC1BT000AC1BT 142. notes A04/CR3A04N01DC1BT142AC02T000. notes A04/CR3A04N02DC1BTO00AC1BT 142. notes A04/CR3A04N02DC1BT 142AC02T000. notes A04/CR3A04N03DC1BT000AC1BT142. notes A04/CR3A04N03DC1BT 142AC02T000. notes A04/CR3A04N04DC1BTO00AC1BT142. notes A04/CR3A04N04DC1BT142AC02T000. notes A04/CR3A04N05DC1BT000AC1BT142. notes A04/CR3A04N05DC1BT142AC02T000. notes A04/CR3A04N06DC1BTO00AC1BT142. notes A04/CR3A04N06DC1BT142AC02TD00. notes A04/CR3A04N07DC1BTO00AC1BT 142. notes A04/CR3A04N07DC1BT 142ACO2T000. notes A04/CR3A04N08DC1BT000AC18T142. notes A04/CR3A04N08DC1BT 142AC02T000. notes A04/CR3A04N090C1BT000AC1BT 142. notes A04/CR3A04N090C1BT 142ACO2TO00. notes A04/CR3A04N10DC1BT000AC1BT142. notes A04/CR3A04N10DC1BT142ACO2T000. notes A04/CR3A04N11DC1BTO00AC1BT142.notes A04/CR3A04N11DC1BT 142AC02T000.notes A04/CR3A04N12DC1BT000AC1BT142. notes A04/CR3A04N12DC1BT142AC02T000. notes A04/CR3A04N13DC1BTO00AC1BT 142. notes A04/CR3A04N13DC1BT 142AC02T000. notes A04/CR3A04N14DC1BT000AC1BT 142. notes A04/CR3A04N14DC1BT142AC02TO00.notes A04/CR3A04N15DC18TO00AC1BT142.notes A04/CR3A04N15DC1BT142AC02T000. notes A04/CR3A04N160C1BTO00AC1BT142. notes A04/CR3A04N16DC1BT142AC02T000.notes A04/CR3A04N17DC1BTO00AC1BT142. notes A04/CR3A04N17DC1BT142ACO2T000.notes A04/CR3A04N180C1BTO00AC1BT 142. notes A04/CR3A04N180C1BT142AC02T000. notes

Computer

Fi le Name

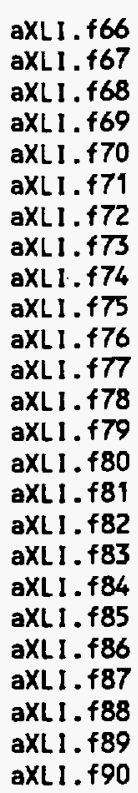

Tape Backup

fi le Name

aXLI. 991

aXLI. 992

aXLI. 993

aXL I. 994

aXL1. 995

aXLI. 996

aXL I. 997

aXL I. 998

aXL1.f99

aXL If . 100

aXLIf. 101

aXLIf. 102

aXLIf. 103

axLif. 104

aXLIf. 105

aXLIf. 106

aXLIf. 107

aXLIf. 108

aXLIf. 109

aXLIf. 110

axLIf. 111

aXLIf. 112

aXLIf. 113

aXLIf. 114

axLIf. 115

aXLIf. 116

aXLIf. 117

axLIf. 118

aXLIf. 119

aXLIf. 120

aXLIf. 121

aXLIf. 122

aXLIf. 123

aXLIf .124

aXLIf. 125

aXLIf. 126

Tape Backup

file Name

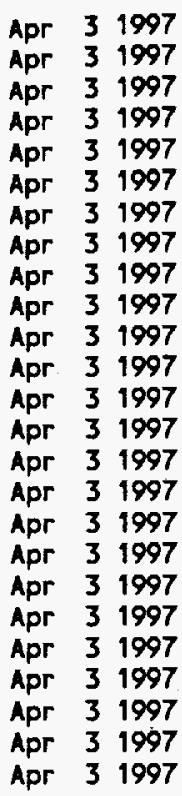

9031

9358

10613

9975

10271

9043

9378

10521

9145

10323

9003

9447

10585

9145

10459

8966

9227

10577

9195

10323

8837

9053

10214

8871

10148

ASCII

ASCII

ASCII

ASCII

ASCII

ASCII

ASCII

ASCII

ASCII

ASCII

ASCII

ASCII

ASCI!

ASCII

ASCII

ASCII

ASCII

ASCII

ASCII

ASCII

ASCII

ASCII

ASCII

ASCII

ASCII

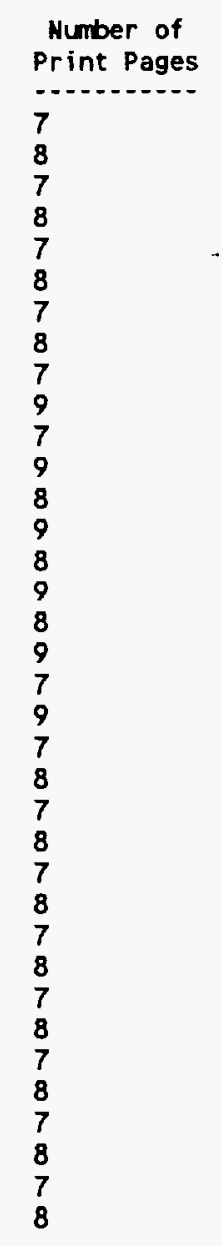

\section{File Date \\ (Output)}

Apr 31997

Apr 31997

Apr 31997

Apr 31997

Apr 31997

Apr 31997

Apr 31997

Apr 31997

Apr 31997

Apr 31997

Apr 31997

Apr 31997

Apr 31997

Apr 31997

Apr 31997

Apr 31997

Apr 31997

Apr 31997

Apr 31997

Apr 31997

Apr - 31997

Apr 31997

Apr 31997

Apr 31997

Apr 31997

Apr 31997

Apr 31997

Apr 31997

Apr 31997

Apr 31997

Apr 31997

Apr 31997

Apr 31997

Apr 31997

Apr 31997

Apr 31997

File size

(Bytes)

8508

9951

8953

10326

9177

10277

9139

10452

9148

10637

9256

10647

9290

10645

9266

10603

9274

10647

9198

10637

9242

10563

9160

10565

9123

10603

9207

10645

9213

10563

9171

10413

9193

10343

8919

10044

File Type

(Format)

ASCII

ASCII

ASCII

ASCII

ASCII

ASCII

ASCII

ASCII

ASCII

ASCII

ASCII

ASCII

ASCII

ASCI!

ASCII

ASCII

ASCI I

ASCII

ASCII

ASCII

ASCII

ASCII

ASCII

ASCII

ASCII

ASCII

ASCII

ASCII

ASCII

ASCII

ASCII

ASCII

ASCII

ASCII

ASCII

ASCII

Number of
Print Pages
(Output)

File Size

(Bytes)

File Type

(Format) 
A05/CR3A05N01DC1BTO00AC1BT142, notes A05/CR3A05N02DC1BTO00AC1BT142.notes A05/CR3A05N03DC1BTO0OAC1BT142. notes A05/CR3A05N04DC1BTO00AC1BT 142. notes A05/CR3A05N05DC1BTO0OAC18T142. notes A05/CR3A05N06DC1BT000AC1BT 142. notes A05/CR3A05NOTDC1BTO00AC1BT142.notes A05/CR3A05N08DC1BTO00AC1BT142. notes A05/CR3A05N09DC1BTODOAC1BT142. notes A05/CR3A05N10DC1BT000AC1BT142. notes A05/CR3A05N11DC1BT000AC1BT142. notes A05/CR3A05N12DC1BT000AC1BT142. notes A05/CR3A05N 13DC1BT000AC1BT 142. notes A05/CR3A05N 14DC1BT000AC1BT 142. notes A05/CR3A05N 15DC1BT000AC1BT142. notes A05/CR3A05N 160C1BT000AC1BT142. notes A05/CR3A05N17DC1BTO00AC1BT142. notes A05/CR3A05N18DC1BT000AC1BT142. notes

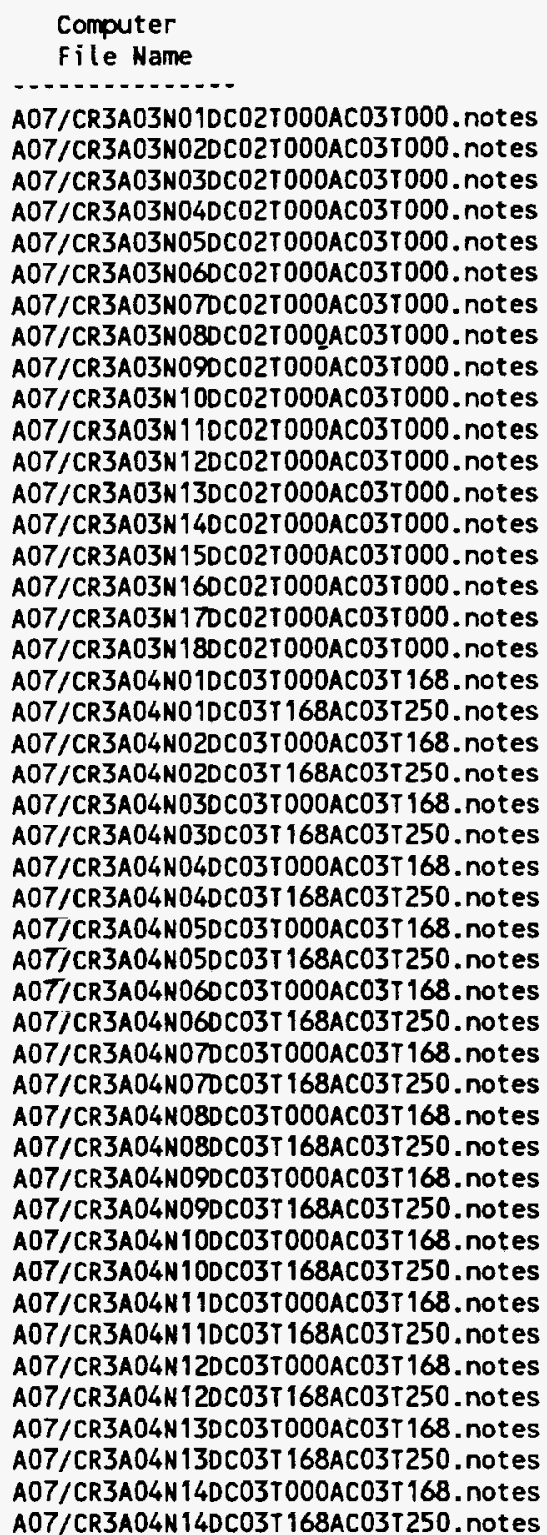

axi $1+.127$

aXLIf. 128

aXLIf. 129

aXLIf. 130

aXLIf. 131

aXLIf. 132

aXL.If.133

axL1f. 134

aXLIf. 135

aXLIf. 136

aXL If. 137

aXL If. 138

aXLIf. 139

aXLIf. 140

aXLIf. 141

aXLIf. 142

axLIf. 143

aXLIf. 144

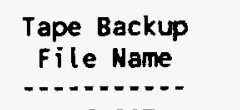

aXLIf. 145

aXLIf. 146

aXLIf. 147

aXLIf. 148

aXLIf. 149

aXLIf. 150

aXLIf. 151

aXLIf. 152

aXLIf. 153

aXLIf. 154

aXLIf. 155

aXLIf. 156

aXLIf. 157

aXLI $f .158$

axLIf. 159

aXLIf. 160

aXLIf. 161

aXLIf. 162

aXLIf. 163

aXLIf. 164

axLIf. 165

aXLIf. 166

aXLI f.167

aXLIf. 168

aXLIf. 169

aXLIf .170

aXLIf. 171

aXLIf. 172

axLIf.173

aXLIf. 174

axL1f. 175

aXLIf. 176

aXLIf. 177

aXLIf. 178

aXLIf. 179

aXLIf. 180

aXLIf. 181

aXLIf .182

aXLIf. 183

aXLIf. 184

aXLIf. 185

aXLIf .186

aXLIf. 187

aXLIf. 188

aXLIf. 189

aXLIf. 190
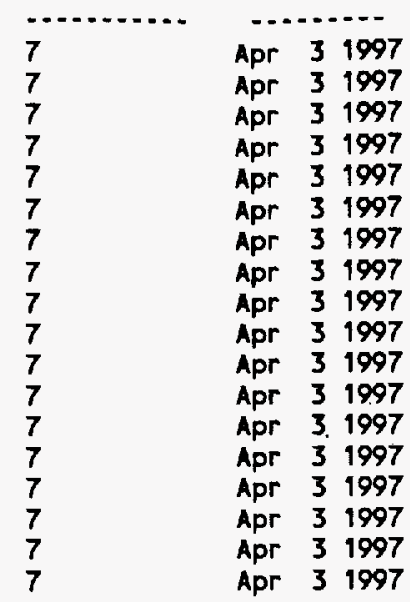

$\begin{array}{ll}8388 & \text { ASCII } \\ 8734 & \text { ASCII } \\ 9028 & \text { ASCII } \\ 9139 & \text { ASCII } \\ 9143 & \text { ASCII } \\ 9177 & \text { ASCII } \\ 9115 & \text { ASCII } \\ 9231 & \text { ASCII } \\ 9159 & \text { ASCII } \\ 9105 & \text { ASCII } \\ 9101 & \text { ASCII } \\ 9149 & \text { ASCII } \\ 9125 & \text { ASCII } \\ 9169 & \text { ASCII } \\ 9167 & \text { ASCII } \\ 9143 & \text { ASCII } \\ 9024 & \text { ASCII } \\ 8825 & \text { ASCII }\end{array}$

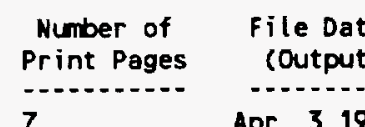

Apr 31997

$\begin{array}{lll}\text { Apr } & 3 & 1997 \\ \text { Apr } & 3 & 1997\end{array}$

Apr 31997

Apr 31997

Apr 31997

Apr 31997

Apr 31997

Apr 31997

Apr 31997

Apr 31997

Apr 31997

Apr 31997

Apr 31997

Apr 31997

Apr 31997

Apr 31997

Apr 31997

Apr 31997

Apr 31997

Apr 31997

Apr 31997

Apr 31997

Apr 31997

Apr 31997

Apr 31997

Apr 31997

Apr 31997

Apr 31997

Apr 31997

Apr 31997

Apr 31997

Apr 31997

Apr 31997

Apr $3 \quad 1997$

Apr 31997

Apr 31997

Apr 31997

Apr 31997

Apr 31997

Apr 31997

Apr 31997

Apr 31997

Apr 31997

Apr 31997

Apr 31997

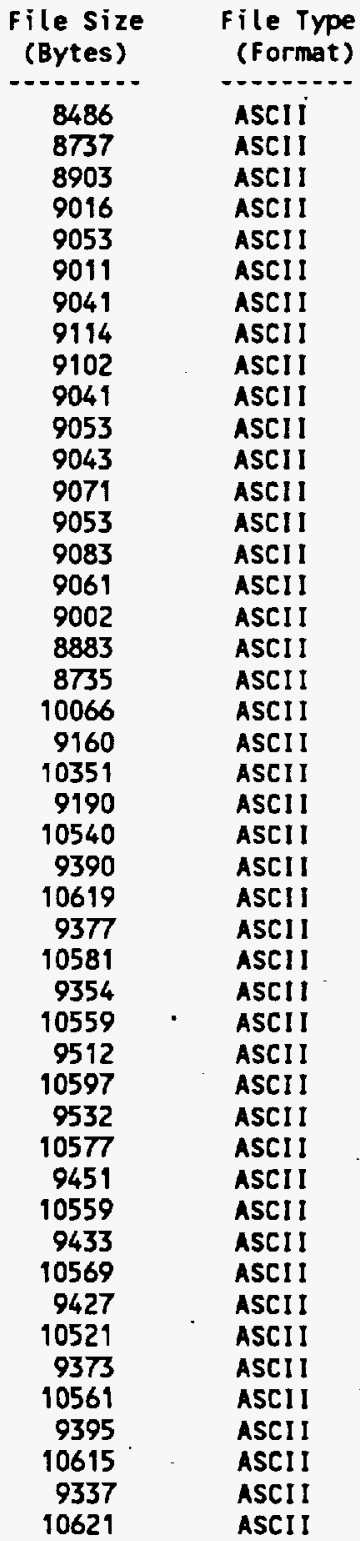


A07/CR3A04N15DC03TO00AC03T168. notes A07/CR3A04N15DCO3T 168ACO3T250. notes A07/CR3A04N16DCO3TOOOACO3T168. notes A07/CR3A04N160C03T 168ACO3T250. notes A07/CR3A04N17DCO3TOOOAC03T168.notes A07/CR3A04N170CO3T 168ACO3T250.notes A07/CR3A04N180C03TO0OAC03T 168. notes A07/CR3A04N 18DC03T 168AC03T250. notes A07/CR3A07NO1DC1BTO0OAC1BT142.notes A07/CR3A07NO1DC 1BT 142AC02T000.notes A07/CR3A07N02DC1BT000AC1BT142.notes A07/CR3A07N02DC1BT 142AC02T000.notes A07/CR3A07N03DC1BTO00AC1BT 142. notes A07/CR3A07N03DC1BT 142AC02T000. notes A07/CR3A07N04DC1BTOOOAC1BT142.notes A07/CR3A07N04DC1BT 142AC02T000. notes A07/CR3A07N05DC1BTOOOAC1BT142. notes A07/CR3A07N05DC1BT 142AC02T000. notes A07/CR3A07N060C1BTO0OAC1BT142. notes A07/CR3A07N06DC1BT 142AC02T000. notes A07/CR3A07NOTDC1BTO0OAC1BT142. notes A07/CR3A07NOTDC1BT 142AC02T000. notes A07/CR3A07NOBDC1BTOOOAC1BT142. notes AOT/CR3A07NOBDC1BT 142AC02TOO0.nOtes A07/CR3A07N090C1BTO0OAC1BT142.notes A07/CR3A07NOSOC1BT 142AC02T000.notes A07/CR3A07N10DC1BTD00AC1BT142.notes A07/CR3A07N100C1BT 142AC02T000.notes A07/CR3A07N110C1BTO00AC1BT142.notes A07/CR3A07N11DC1BT 142ACO2T000.notes A07/CR3A07N120C1BTO00AC1BT 142. notes A07/CR3A07N12DC1BT 142AC02T000. notes AO7/CR3A07N13DC1BTOOOAC1BT142.notes A07/CR3A07N13DC1BT 142AC02T000.notes AO7/CR3A07N140C1BTOOOAC1BT142.notes A07/CR3A07N140C1BT142AC02T000.notes AO7/CR3A07N15DC1BTOOOAC1BT142.notes A07/CR3A07N15DC1BT142AC02T000. notes A07/CR3A07N160C1BTO00AC1BT 142. notes AO7/CR3A07N160C1BT142AC02T000.notes AO7/CR3A07N170C1BTO0OAC1BT 142.notes AO7/CR3A07N170C1BT 142AC02T000.notes A07/CR3A07N180C1BTO0OAC1BT142.notes AO7/CR3A07N1BDC1BT 142AC02T000. notes

\section{Computer
File Name}

A14/CR3A05N010C07TO00AC07T260. notes A14/CR3A05N010C07T260AC07T291.notes A14/CR3A05N01DC07T291AC07T319.notes A14/CR3A05N01DC07T319AC07T462.notes A14/CR3A05N010C07T 462AC07T479. notes A14/CR3A05N020CO7TOOOAC07T260. notes A14/CR3A05N02DCO7T260AC07T291. notes A14/CR3A05N02DC07T291AC07T319. notes A14/CR3A05N020C07T319AC07T462.notes A14/CR3A05N02DC07T 462AC07T479. notes A 14/CR3A05N03DCO7TOOOACOTT260. notes A14/CR3A05N03DC07T260AC07T291. notes A14/CR3A05N030C07T291AC07T319. notes A14/CR3A05N030C07T319AC07T462.notes A14/CR3A05N030C07T462AC07T479. notes A 14/CR3A05N040C07T000AC07T260. notes A14/CR3A05N040C07T260AC07T291. notes A14/CR3A05N040C07T291AC07T319. notes A14/CR3A05N04DC07T319AC07T462.notes A14/CR3A05N04DC07T462AC07T479. notes A14/CR3A05N05DC07TO0OAC07T260.notes

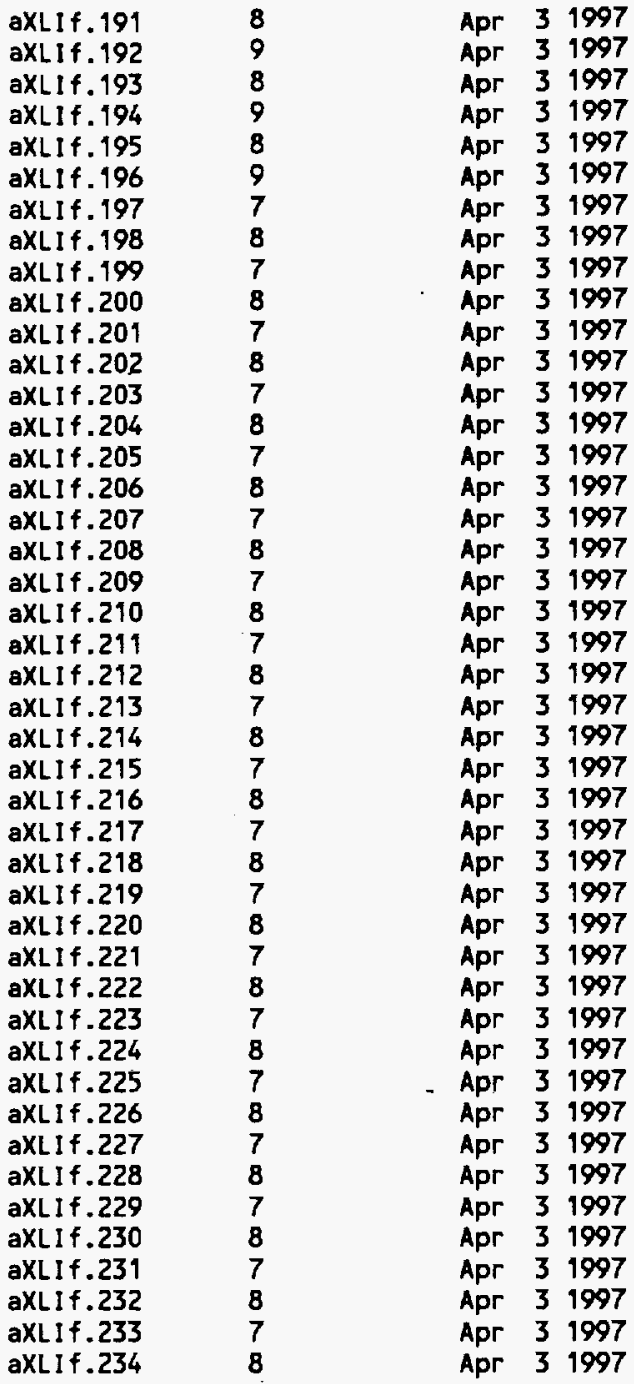

\begin{tabular}{ll}
$\begin{array}{c}\text { Tape Backup } \\
\text { File Name }\end{array}$ & $\begin{array}{c}\text { Number of } \\
\text { Print Pages }\end{array}$ \\
\hline aXLIf.235 & - \\
aXLIf.236 & 8 \\
aXLIf.237 & 7 \\
aXLIf.238 & 7 \\
aXLIf.239 & 9 \\
aXLIf .240 & 6 \\
aXLIf.241 & 8 \\
aXLIf.242 & 8 \\
aXLIf.243 & 8 \\
aXLIf .244 & 10 \\
aXLIf.245 & 6 \\
aXLIf.246 & 9 \\
aXLIf.247 & 8 \\
aXLIf .248 & 8 \\
aXLIf.249 & 10 \\
aXLIf.250 & 7 \\
aXLIf.251 & 9 \\
aXLIf.252 & 8 \\
aXLIf.253 & 8 \\
aXLIf.254 & 10 \\
aXLIf.255 & 7
\end{tabular}

$\begin{array}{rr}9403 & \text { ASCII } \\ 10549 & \text { ASCII } \\ 9419 & \text { ASCII } \\ 10597 & \text { ASCII } \\ 9253 & \text { ASCII } \\ 10565 & \text { ASCII } \\ 9096 & \text { ASCII } \\ 10272 & \text { ASCII } \\ 8489 & \text { ASCII } \\ 9647 & \text { ASCII } \\ 8843 & \text { ASCII } \\ 10042 & \text { ASCII } \\ 8991 & \text { ASCII } \\ 10214 & \text { ASCII } \\ 9235 & \text { ASCII } \\ 10251 & \text { ASCII } \\ 9201 & \text { ASCII } \\ 10173 & \text { ASCII } \\ 9169 & \text { ASCII } \\ 10259 & \text { ASCII } \\ 9169 & \text { ASCII } \\ 10316 & \text { ASCII } \\ 9141 & \text { ASCII } \\ 10302 & \text { ASCII } \\ 9205 & \text { ASCII } \\ 10284 & \text { ASCII } \\ 9147 & \text { ASCII } \\ 10290 & \text { ASCII } \\ 9211 & \text { ASCII } \\ 10298 & \text { ASCII } \\ 9209 & \text { ASCII } \\ 10260 & \text { ASCII } \\ 9159 & \text { ASCII } \\ 10338 & \text { ASCII } \\ 9193 & \text { ASCII } \\ 10342 & \text { ASCII } \\ 9119 & \text { ASCII } \\ 10288 & \text { ASCII } \\ 9173 & \text { ASCII } \\ 10366 & \text { ASCII } \\ 9061 & \text { ASCII } \\ 10251 & \text { ASCII } \\ 8845 & \text { ASCII } \\ 10042 & \text { ASCII } \\ \end{array}$

\begin{tabular}{cc}
$\begin{array}{c}\text { File Size } \\
\text { (Bytes) }\end{array}$ & $\begin{array}{r}\text { File Iype } \\
\text { (Format) }\end{array}$ \\
\hline 7540 & ASCII \\
10025 & ASCII \\
9113 & ASCII \\
8640 & ASCII \\
11785 & ASCII \\
7697 & ASCII \\
10374 & ASCII \\
9649 & ASCII \\
9170 & ASCII \\
12246 & ASCII \\
7645 & ASCII \\
10791 & ASCII \\
9718 & ASCII \\
9435 & ASCII \\
12330 & ASCII \\
7840 & ASCII \\
10783 & ASCII \\
9772 & ASCII \\
9355 & ASCII \\
12317 & ASCII \\
7932 & ASCII
\end{tabular}

ASCII

ASCII

SCII

ASCII

ASCII

SCII

ASCII

ASCII

SCII

ASCII

ASCI

ASCI

SCII

SCI

SCII

ASCI I

ASCII

ASCII

SCII

ASCII

SCII

ASCI I

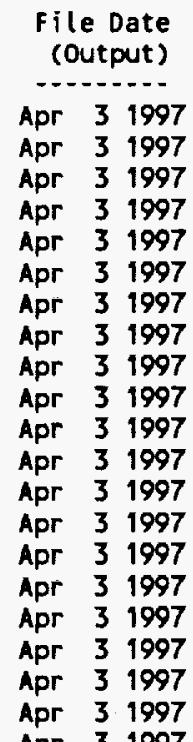


A14/CR3A05N05DC07T260AC07T291. notes A14/CR3A05N05DC07T291AC07T319. notes A14/CR3A05N05DC07T319AC07T462. notes A14/CR3A05N05DC07T462AC07T479. notes A14/CR3A05N06DC07TO00OAC07T260. notes A 14/CR3A05N06DC07T260ACOTT291. notes A14/CR3A05N06DC07T291AC07T319. notes A 14/CR3A05N06DC07T319AC07T462. notes A14/CR3A05N06DC07T462AC07T479. notes A14/CR3A05N07DC07TO00AC07T260.notes A 14/CR3A05N07DC07T260AC07T291. notes A14/CR3A05N07DC07T291AC07T319.notes A14/CR3A05N070C07T319AC07T462. notes A 14/CR3A05N07DC07T462AC07T479. notes A 14/CR3A05N08DC07TO0OAC07T260. notes A 14/CR3A05N08DC07T260AC07T291. notes A 14/CR3A05N08DC07T291AC07T319.notes A 14/CR3A05N08DC07T319AC07T462. notes A 14/CR3A05N08DC07T462AC07T479. notes A 14/CR3A05N09DC07TO00AC07T260. notes A 14/CR3A05N09DC07T260AC07T291. notes A14/CR3A05N090C07T291AC07T319.notes A14/CR3A05N09DC07T319AC07T462. notes A14/CR3A05N09DC07T462AC077479. notes A14/CR3A05N100C07T000AC07T260. notes A 14/CR3A05N10DC07T260AC07T291. notes A14/CR3A05N100C07T291AC07T319. notes A 14/CR3A05N10DC07T319AC07T462.notes A14/CR3A05N100C07T462AC07T479. notes A 14/CR3A05N110C07T000AC07T260. notes A14/CR3A05N110C07T260AC07T291. notes A 14/CR3A05N11DC07T29 LAC07T319. notes A 14/CR3A05N110C07T319AC07T462.notes A 14/CR3A05N110C07T462AC07T479. notes A 14/CR3A05N120C07T000AC07T260.notes A14/CR3A05N12DC07T260AC07T291. notes A 14/CR3A05N12DC07T291AC07T319. notes A 14/CR3A05N12DC07T319AC07T462. notes A14/CR3A05N12DC07T462AC07T479. notes A 14/CR3A05N13DC07T000AC07T260.notes A14/CR3A05N13DCO7T260AC07T291. notes A14/CR3A05N13DC07T291AC07T319.notes A14/CR3A05N13DC07T319AC07T462. notes A14/CR3A05N13DC07T462AC07T479. notes A 14/CR3A05N14DC07T000AC07T260. notes A14/CR3A05N140C07T260AC07T291. notes A14/CR3A05N14DC07T291AC07T319. notes A14/CR3A05N14DC07T319ACO7T462. notes A14/CR3A05N140C07T462AC071479. notes A14/CR3A05N15DC07T000AC07T260. notes A14/CR3A05N15DC07T260AC07T291. notes A14/CR3A05N15DC07T291AC07T319. notes A14/CR3A05N15DC07T319AC07T462. notes A14/CR3A05N15DC07T462AC07T479. notes A14/CR3A05N160C07T000AC07T260. notes A14/CR3A05N160C07T260AC07T291. notes A14/CR3A05N160C07T291AC07T319. notes A14/CR3A05N16DC07T319AC07T462. notes A14/CR3A05N160C07T462AC07T479. notes A14/CR3A05N17DC07TO00AC07T260.notes A 14/CR3A05N17DC07T260AC07T291. notes A14/CR3A05N17DC07T291AC07T319. notes A14/CR3A05N17DC077319AC07T462. notes A14/CR3A05N17DC07T462AC071479. notes A14/CR3A05N180C07T000AC07T260. notes A14/CR3A05N180C07T260AC07T291. notes A14/CR3A05N18DC07T291AC07T319. notes A14/CR3A05N18DC07T319AC07T462. notes A14/CR3A05N180C07T462AC07T479. notes A14/CR3A14N01DC02T000AC07T000. notes

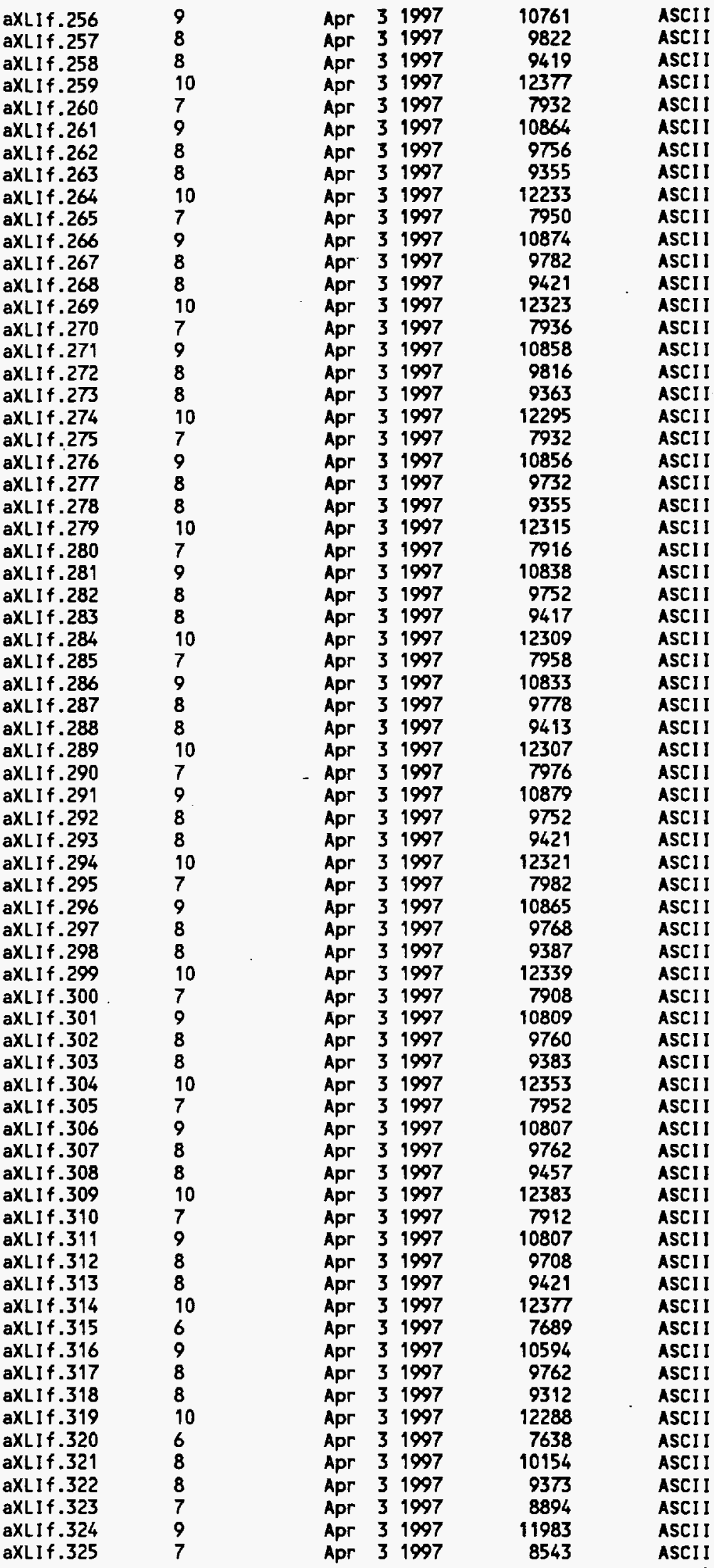


A14/CR3A14NO1DC1BTOOOAC1BT 142.notes A14/CR3A14NO10C1BT 142ACO2T000.notes A14/CR3A14N02DCO2TO0OACO7T000. notes A14/CR3A14NO2DC1BT000AC1BT142.notes A14/CR3A14NO2DC1BT 142ACO2TO00.notes A14/CR3A14N03DC02TO00AC07T000.notes A14/CR3A14NO3DC1BTO0OAC1BT142.notes A14/CR3A14N03DC1BT 142ACO2T000.notes A14/CR3A14N04DC02TO0OACO7TO00.notes A14/CR3A14NO4DC1BTOOOAC1BT142.notes A14/CR3A14N04DC1BT 142AC02T000.notes A14/CR3A14N05DC02TO0OAC07T000.notes A14/CR3A14N05DC1BTOOOAC1BT142, notes A14/CR3A14NO5DC1BT 142AC02T000.notes A14/CR3A14N06DC02TO0OACO7T000.notes A14/CR3A14N06DC1BTOOOAC1BT142.notes A14/CR3A14N060C1BT 142AC02T000.notes A14/CR3A14NOTDC02TOOOAC07TO00.notes A14/CR3A14NOTDC1BTOOOAC1BT142.notes A14/CR3A14NOTDC1BT142ACO2T000.notes A14/CR3A14N08DC02TO0OAC07TO00.notes A14/CR3A14NOBDC1BTO0OAC1BT142. notes A14/CR3A14NOBDC1BT 142AC02T000.notes A14/CR3A14NO90C02T000ACO7T000.notes A14/CR3A14NO9DC1BTO00AC1BT142.notes A14/CR3A14N10DC02T000AC07T000.notes A14/CR3A14N100C1BT 142AC02T000.notes A14/CR3A14N110C02TO00AC07T000. notes A14/CR3A14N110C1BT000AC1BT142.notes A14/CR3A14N110C1BT 142AC02T000. notes A14/CR3A14N120C02TOOOACOTTO00. notes A14/CR3A14N12DC1BTO00AC1BT142.notes A14/CR3A14N12DC1BT142AC02T000.notes A14/CR3A14N13DC02TO00AC07T000. notes A14/CR3A14N13DC1BTOOOAC1BT142.notes A 14/CR3A14N13DC1BT 142AC02T000.notes A14/CR3A14N14DC02TO00AC07T000. notes A14/CR3A14N14DC1BTO0OAC1BT142.notes A14/CR3A14N14DC1BT142ACO2T000.notes A14/CR3A14N15DC02T000AC07T000.notes A14/CR3A14N15DC1BTO00AC1BT 142.notes A14/CR3A14N15DC1BT142AC02T000.notes A14/CR3A14N160CO2TO00AC07T000.notes A14/CR3A14N160C1BTO0OAC1BT 142.notes A14/CR3A14N160C1BT 142AC02T000. notes A14/CR3A14N170CO2TO0OACOTTO00.notes A14/CR3A14N170C1BT000AC1BT142.notes A14/CR3A14N1TOC1BT142AC02T000.notes A14/CR3A14N180C02TO0OACOTTO00.notes A14/CR3A14N18DC1BTO0OAC1BT142.notes A14/CR3A14N18DC1BT 142ACO2T000. notes A14/CR3A14NDODC1BT 142ACO2T000.notes A14/CR3A14N10DC1BTOOOAC1BT142.notes

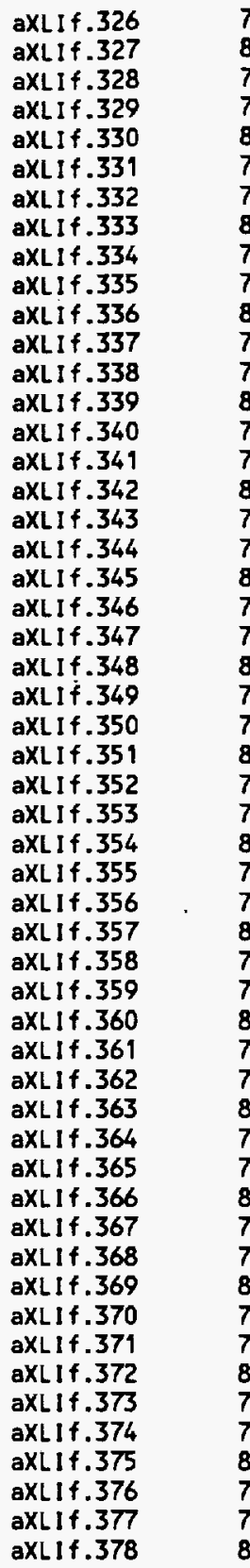

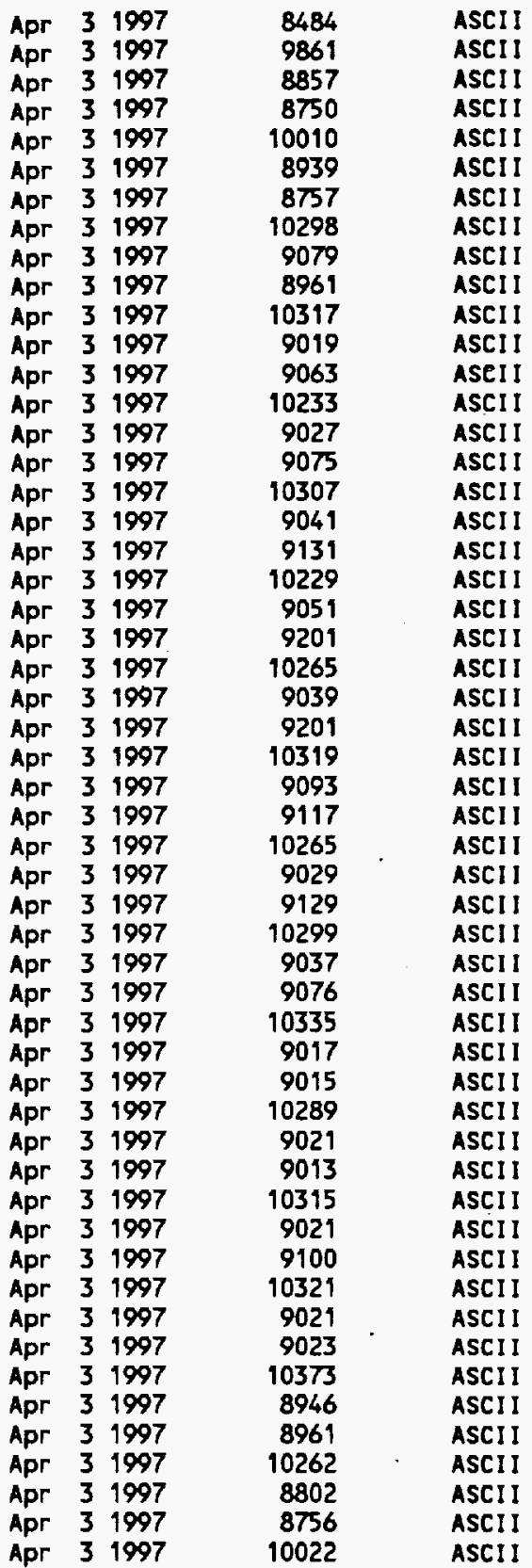

THE FOLLONING FILES FOR ASSEMBLY A18 UERE SUPERCEEDED BY FILES UNDER ASSEMBLY DESIGHATION A18Z DURING CHECKING.

\begin{tabular}{ll}
$\begin{array}{c}\text { Tape Backup } \\
\text { File Name }\end{array}$ & $\begin{array}{c}\text { Number of } \\
\text { Print Pages }\end{array}$ \\
\hline aXLIf.379 & 6 \\
aXLIf.380 & 8 \\
aXLIf.381 & 8 \\
aXLIf.382 & 8 \\
aXLIf.383 & 8 \\
aXLIf.384 & 6 \\
aXLIf.385 & 8 \\
aXLIf.386 & 8 \\
aXLIf.387 & 8 \\
aXLIf.388 & 8 \\
aXLIf.389 & 6
\end{tabular}

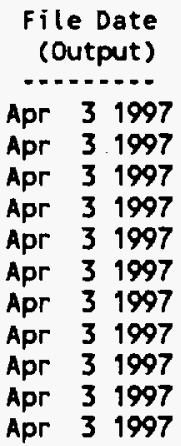

File Type

(Format)

ASCII

ASCII

ASCII

ASCII

ASCII

ASCII

ASCII

ASCII

ASCII

ASCII

ASCII 
18/CR3A01N03DC08T097AC08T139, notes A18/CR3A01N03DC08T 139AC08T404. notes A18/CR3A01N03DC08T404AC08T409. notes A18/CR3AO1N03DCOBT409AC08T515. notes A18/CR3A01N04DCO8TOOOAC08T097.notes A18/CR3A01N04DCO8T097AC08T139. notes A 18/CR3A01N04DCO8T 139AC08T404.notes A18/CR3A01N04DC08T404AC08T409.notes A18/CR3A01N04DC08T409AC08T515.notes A 18/CR3A01N05DC08T000AC08T097.notes A18/CR3A01N05DC08T097AC08T139. notes A18/CR3A01N05DC08T 139AC08T404. notes A18/CR3A01N05DC08T404AC08T409. notes A18/CR3A01N05DC08T409AC08T515.notes A18/CR3A01N06DC08T000AC08T097.notes A18/CR3A01N06DC08T097AC08T139. notes A18/CR3A01N060C08T139AC08T404 notes A18/CR3A01N06DC08T404AC08T409.notes A18/CR3A01N06DC08T409AC0BT515.notes A18/CR3A01N07DC08TO00AC08T097. notes A18/CR3A01N07DC08T097AC081139. notes A18/CR3A01N07DC08T139AC08T404 notes A18/CR3A01N0TDC08T404AC08T409. notes A18/CR3A01N07DC08T409AC081515 notes A18/CR3A01N080C08TO00AC08T097. notes A18/CR3A01N08DC08T097AC081 139. notes A18/CR3A01N08DC08T 139AC08T404. notes A18/CR3A01N08DC08T404AC08T409. notes A18/CR3A01N08DC08T409AC08T515. notes A18/CR3A01N09DC08T000AC08T097. notes A18/CR3A01N09DC08T097AC08T139. notes A18/CR3A01N09DC08T139AC08T404.notes A18/CR3A01N090C08T404AC08T409. notes A18/CR3A01N09DC08T409AC08T515. notes A 18/CR3A01N100C08T000AC08T097. notes A18/CR3A01N 10DC08T097AC08T139. notes A18/CR3A01N1ODC08T139AC08T404. notes A 18/CR3A01N10DC08T404AC08T409. notes A 18/CR3A01N 10DC08T409AC08T515.notes A18/CR3A01N11DC08T000AC08T097. notes A18/CR3A01N11DC08T097AC08T139. notes A18/CR3A01N11DC08T139AC081404. notes A 18/CR3A01N11DC08T404AC08T409.notes A 18/CR3A01N11DC08T409AC08T515. notes A18/CR3A01N12DC08T000AC08T097. notes A18/CR3A01N12DC08T097AC081 139. notes A18/CR3A01N12DC08T139AC08T404. notes A 18/CR3A01N12DC08T404AC08T409. notes A 18/CR3A01N12DC08T409AC08T515.notes A 18/CR3A01N13DC08T000AC08T097. notes A 18/CR3A01N13DC08T097AC08T139.notes A 18/CR3A01N13DC08T 139AC08T404. notes A18/CR3A01N13DC08T404AC08T409. notes A 18/CR3A01N13DC08T409AC08T515. notes A 18/CR3A01N14DC08TO00AC08T097. notes A 18/CR3A01N14DC08T097AC08T139. notes A 18/CR3A01N14DC08T139AC08T404. notes A 18/CR3A01N14DC08T404AC08T409.notes A 18/CR3A01N 14DC08T409AC08T515. notes A18/CR3A01N15DC08T000AC08T097. notes A 18/CR3A01N15DC08T097AC08T139. notes A18/CR3A01N15DC08T139AC08T404.notes A 18/CR3A01N15DC08T404AC08T409.notes A 18/CR3A01N15DC081409AC08T515.notes A 18/CR3A01N 160C08T 000AC08T097. notes A18/CR3A01N160C08T097AC08T139.notes A 18/CR3A01N16DC08T 139AC08T404. notes A 18/CR3A01N16DC08T404AC08T409.notes A18/CR3A01N160C08T409AC08T515. notes A 18/CR3A01N17DC08T000AC08T097. notes
aXLIf .390

aXLIf.391

aXLIf.392

aXLIf .393

aXLIf .394

aXLIf .395

aXLIf. 396

aXLIf. 397

axLIf.398

aXLIf. 399

aXLIf. 400

aXLIf .401

aXLIf .402

aXL If .403

aXLIf. 404

axLIf .405

aXLIf. 406

aXLIf .407

aXLIf. 408

aXLIf. 409

aXLIf .410

aXLIf. 411

aXLIf. 412

aXLI $\$ .413$

aXLIf. 414

aXL If .415

aXL If. 416

aXLIf. .417

aXL I .418

aXLIf .419

aXLIf. 420

aXLIf .421

aXL If .422

axLIf. 423

aXLIf. 424

aXLIf .425

aXLIf. 426

aXLIf .427

aXLIf . 428

aXLI $f .429$

aXLIf .430

aXLIf. 431

aXLIf . 432

aXLI +.433

aXLIf. 434

aXLIf .435

aXLIf .436

aXLIf. 437

aXLIf. 438

aXLIf. 439

aXLIf .440

aXLIf. 441

aXLIf .442

aXLI $f .443$

aXLIf. 444

aXLIf $\mathbf{4} .445$

aXLIf. 446

aXLIf .447

aXLIf. 448

aXL If . 449

aXLIf .450

aXLIf. 451

aXLIf. 452

aXLIf. 453

aXLIf .454

aXLIf. 455

aXLIf. 456

aXLIf .457

aXLIf. 458

aXLIf. 459

\begin{tabular}{|c|c|c|c|}
\hline Apr & 31997 & $10146^{\circ}$ & ASCII \\
\hline Apr & 31997 & 10905 & ASCII \\
\hline Apr & 31997 & 10262 & ASCII \\
\hline Apr & 31997 & 10649 & ASCII \\
\hline Apr & 31997 & 7796 & ASCII \\
\hline $\mathrm{Apr}$ & 31997 & 10282 & ASCII \\
\hline Apr & 31997 & 11082 & ASCII \\
\hline Apr & 31997 & 10278 & ASCII \\
\hline Apr & 31997 & 10708 & ASCII \\
\hline Apr & 31997 & 7802 & ASCII \\
\hline Apr & 31997 & 10380 & ASCII \\
\hline Apr & 31997 & 11048 & ASCII \\
\hline Apr & 31997 & 10300 & ASCII \\
\hline Apr & 31997 & 10650 & ASCII \\
\hline Apr & 31997 & 7740 & ASCII \\
\hline Apr & 31997 & 10324 & ASCII \\
\hline Apr & 31997 & 11105 & ASCII \\
\hline Apr & 31997 & 10284 & ASCII \\
\hline Apr & 31997 & 10712 & ASCII \\
\hline Apr & 31997 & 7756 & ASCII \\
\hline Apr & 31997 & 10374 & ASCII \\
\hline Apr & 31997 & 11033 & ASCII \\
\hline Apr & 31997 & 10274 & ASCII \\
\hline Apr & 31997 & 10662 & ASCII \\
\hline Apr & 31997 & 7736 & ASCII \\
\hline Apr & 31997 & 10404 & ASCII \\
\hline Apr & 31997 & 11095 & ASCII \\
\hline Apr & 31997 & 10258 & ASCII \\
\hline Apr & 31997 & 10674 & ASCII \\
\hline Apr & 31997 & 7764 & ASCII \\
\hline Apr & 31997 & 10348 & ASCII \\
\hline Apr & 31997 & 11085 & ASCII \\
\hline Apr & 31997 & 10286 & ASCII \\
\hline Apr & 31997 & 10698 & ASCII \\
\hline Apr & 31997 & 7822 & ASCII \\
\hline Apr & 31997 & 10318 & ASCII \\
\hline Apr & 31997 & 11113 & ASCII \\
\hline Apr & 31997 & 10260 & ASCII \\
\hline Apr & 31997 & 10676 & ASCII \\
\hline Apr & 31997 & 7750 & ASCII \\
\hline Apr & 31997 & 10298 & ASCII \\
\hline Apr & 31997 & 11038 & ASCII \\
\hline Apr & 31997 & 10286 & ASCII \\
\hline Apr & 31997 & 10706 & ASCII \\
\hline Арг & 31997 & 7632 & ASCI I \\
\hline Apr & 31997 & 10284 & ASCII \\
\hline Арг & 31997 & 11020 & ASCII \\
\hline Apr & 31997 & 10246 & ASCII \\
\hline Apr & 31997 & 10647 & ASCII \\
\hline Apr & 31997 & 7606 & ASCII \\
\hline Apr & 31997 & 10252 & ASCII \\
\hline Apr & 31997 & 10979 & ASCII \\
\hline Apr & 31997 & 10282 & ASCII \\
\hline Apr & 31997 & 10615 & ASCII \\
\hline Apr & 31997 & 7626 & ASCII \\
\hline Apr & 31997 & 10154 & ASCII \\
\hline Apr & 31997 & 10960 & ASCII \\
\hline Ap & 31997 & 10232 & ASCII \\
\hline Apr & 31997 & 10714 & ASCII \\
\hline Арг & 31997 & 7628 & ASCII \\
\hline Apr & 31997 & 10130 & ASCII \\
\hline Ap & 31997 & 10904 & ASCII \\
\hline Apr & 31997 & 10284 & ASCII \\
\hline Apr & 31997 & 10668 & ASCII \\
\hline Apr & 31997 & 7658 & ASCII \\
\hline Apr & 31997 & 10164 & ASCII \\
\hline Apr & 31997 & 10937 & ASCI I \\
\hline Ap? & 31997 & 10218 & ASCII \\
\hline $\mathrm{Apr}$ & 31997 & 10756 & ASCII \\
\hline Apr & 31997 & 7623 & ASCII \\
\hline
\end{tabular}


A 18/CR3A01N17DC08T097AC08T139. notes A18/CR3A01N170C08T139ACOBT404. notes A 18/CR3A01N17DC08T404AC08T409. notes A18/CR3A01N170C08T409ACOBT515. notes A18/CR3A01N180C08TO00ACOBT097. notes A18/CR3A01N180C08T097ACOBT139. notes A 18/CR3A01N180C08T 139ACOBT404. notes A18/CR3A01N180C08T404ACOBT 409. notes A 18/CR3A01N180C08T409ACOBT515. notes

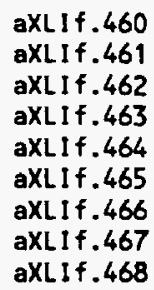

$\begin{array}{lll}\text { Apr } & 3 & 1997 \\ \text { Apr } & 3 & 1997 \\ \text { Apr } & 3 & 1997 \\ \text { Apr } & 3 & 1997 \\ \text { Apr } & 3 & 1997 \\ \text { Apr } & 3 & 1997 \\ \text { Apr } & 3 & 1997 \\ \text { Apr } & 3 & 1997 \\ \text { Apr } & 3 & 1997\end{array}$

ASCI I

ASCII

ASCII

ASCII

ASCII

ASCII

ASCII

ASCII

ASCII

THE FOLLOWING FILES FOR ASSEMBLY A18a WERE SUPERCEEDED BY FILES UNDER ASSEMBLY DESIGNATION A18az DURING CHECKING.

\section{Computer
File Name}

A18a/CR3A01N010CO9TO0OACO9T158. notes A18a/CR3A01N01DC09T158AC09T219. notes A18a/CR3A01N01DC09T219AC09T363. notes A 18a/CR3A01N02DC09TO0OAC09T 158. notes A18a/CR3A01N020C09T158AC09T219.notes A18a/CR3A01N020C091219AC09T363. notes A18a/CR3A01N03DCO9TOOOAC09T 158. notes A18a/CR3A01N03DCO9T 158ACO9T219.notes A18a/CR3A01N030CO9T219AC09T363.notes A18a/CR3A01N04DCO9TO00ACO9T158. notes A18a/CR3A01N04DCO9T 158ACO9T219. notes A18a/CR3A01N04DCO9T219AC09T363.notes A18a/CR3A01N050CO9T000ACO9T 158. notes A18a/CR3A01N05DC09T158AC09T219. notes A18a/CR3A01N05DC09T219AC09T363. notes A18a/CR3A01N06DC09T000AC09T 158. notes A18a/CR3A01N06DCO9T 158ACO9T219.notes A18a/CR3A01N060C09T219AC09T363. notes A18a/CR3A01N070C09T000AC09T158. notes A18a/CR3A01NOTDC09T 158AC09T219. notes A18a/CR3A01N070C09T219AC09T363. notes A18a/CR3A01N08DC09T000AC09T158.notes A18a/CR3A01NO8DCO9T158AC09T219. notes A18a/CR3A01NO8DCO9T219AC09T363. notes A18a/CR3A01N090C09T000AC09T158. notes A18a/CR3A01N090C09T 158AC09T219. notes A18a/CR3A01N09DC09T219AC09T363. notes A18a/CR3A01N10DCO9T000AC09T158. notes A18a/CR3A01N 10DC09T 158AC09T219. notes A18a/CR3A01N 10DC091219AC09T363. notes A18a/CR3A01N11DC09TO00AC09T158. notes A18a/CR3A01N11DC09T 158AC09I219. notes A18a/CR3A01N11DC09T219AC09T363. notes A18a/CR3A01N12DC09T000AC09T158. notes A18a/CR3A01N12DC09T158AC091219. notes A18a/CR3A01N12DC09T219AC09T363. notes A18a/CR3A01N13DC09T000AC09T158. notes A18a/CR3A01N13DCO9T158AC09T219. notes A18a/CR3A01N13DC091219AC09T363. notes A18a/CR3A01N14DC09T000AC09T158. notes A18a/CR3A01N14DC09T158AC09T219. notes A18a/CR3A01N14DC09T219AC09T363. notes A18a/CR3A01N15DC09T000AC09T158. notes A18a/CR3A01N15DC09T158AC09T219. notes A18a/CR3A01N150C09T219AC09T363. notes A18a/CR3A01N160CO9TO0OACO9T 158. notes A18a/CR3A01N160C09T158AC09T219.notes A18a/CR3A01N160C091219AC09T363. notes A18a/CR3A01N1TDC09TOOOACO9T 158.notes A 18a/CR3A01N170C091 158ACO9T219.notes A 18a/CR3A01N17DC09T219AC09T363. notes A18a/CR3A01N180C09T000AC09T158. notes A18a/CR3A01N180C09T158AC09T219. notes A18a/CR3A01N18DC091219AC09T363. notes
Tape Backup Number of File Name

aXL1 1.469

aXLIf. 470

aXLIf. 471

aXLIf. 472

aXLIf. 473

aXLIf. .474

aXLIf.475

aXLI +.476

aXL1 +.477

aXLIf. 478

aXL If . 479

aXLIf . 480

aXLIf. 481

aXLIf. .482

aXLIf. 483

aXLIf. 484

aXLIf. 485

aXLIf. 486

aXLIf. .487

aXLIf. 488

aXL1f. 489

aXL $1 f .490$

aXLIf. 491

aXLIf. 492

aXLIf. 493

aXL1f.494

aXLIf. 495

aXLIf. 496

aXLif. 497

aXLIf. 498

aXL If .499

aXLIf. 500

aXLIf. 509

aXLIf. 502

aXLI $f .503$

aXLIf.504

aXLIf .505

aXLI $f .506$

aXLI $f .507$

aXLIf. 508

aXLIf. 509

aXLIf. 510

aXLI If. 511

aXLIf. 512

aXLIf. 513

aXLIf. 514

aXLI $f .515$

aXLIf. 516

aXLIf. 517

aXLIf. 518

aXLI If.519

aXLIf. 520

aXLIf. 521

axLIf. 522

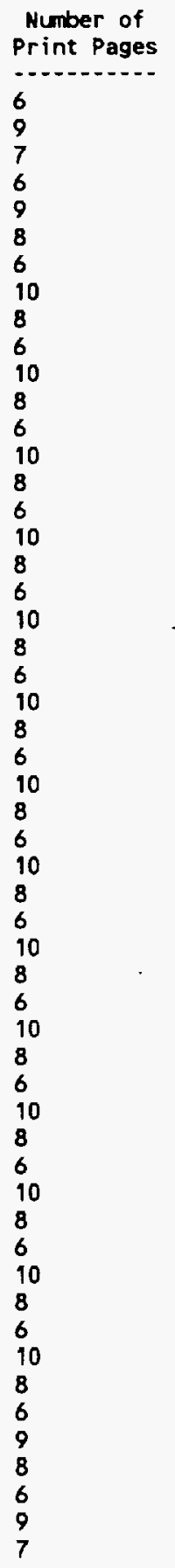

\begin{tabular}{|c|c|}
\hline $\begin{array}{c}\text { File size. } \\
\text { (Bytes) }\end{array}$ & $\begin{array}{l}\text { File Type } \\
\text { (Format) }\end{array}$ \\
\hline - & 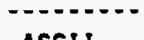 \\
\hline $\begin{array}{r}7436 \\
11403\end{array}$ & $\begin{array}{l}\text { ASCII } \\
\text { ASCII }\end{array}$ \\
\hline 9078 & ASCII \\
\hline 7528 & ASCII \\
\hline 11852 & ASCII \\
\hline 9403 & ASCII \\
\hline 7808 & ASCII \\
\hline 12176 & ASCII \\
\hline 9638 & ASCII \\
\hline 7780 & ASCII \\
\hline 12202 & ASCII \\
\hline 9801 & ASCII \\
\hline 7808 & ASCII \\
\hline 12349 & ASCII \\
\hline 9850 & ASCII \\
\hline 7782 & ASCII \\
\hline 12311 & ASCII \\
\hline 9812 & ASCII \\
\hline 7784 & ASCII \\
\hline 12317 & ASCII \\
\hline 9854 & ASCII \\
\hline 7772 & ASCII \\
\hline 12333 & ASCII \\
\hline 9802 & ASCII \\
\hline 7780 & ASCII \\
\hline 12347 & ASCII \\
\hline 9832 & ASCII \\
\hline 7830 & ASCII \\
\hline 12313 & ASCII \\
\hline 9794 & ASCII \\
\hline 7774 & ASCII \\
\hline 12203 & ASCII \\
\hline 9717 & ASCII \\
\hline 7636 & ASCII \\
\hline 12192 & ASCII \\
\hline 9729 & ASCII \\
\hline 7602 & ASCII \\
\hline 12066 & ASCII \\
\hline 9660 & ASCII \\
\hline 7644 & ASCII \\
\hline 12044 & ASCII \\
\hline 9626 & ASCII \\
\hline 7618 & ASCII \\
\hline 12152 & ASCII \\
\hline 9612 & ASCII \\
\hline 7707 & ASCII \\
\hline 12124 & ASCII \\
\hline 9616 & ASCII \\
\hline 7684 & ASCII \\
\hline 12096 & ASCII \\
\hline 9535 & ASCII \\
\hline 7490 & ASCII \\
\hline 11627 & ASCII \\
\hline 9183 & ASCII \\
\hline
\end{tabular}


THE FOLLOWING FILES FOR ASSEMBLY A18b WERE SUPERCEEDED BY FILES UNDER ASSEMBLY DESIGNATION AIBbZ DURING CHECKING.

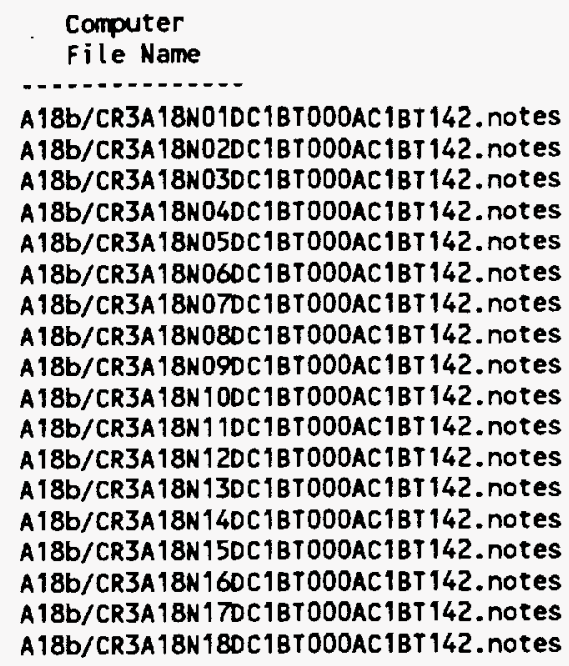

Computer
File Name

A20/CR3A12N01DC03T000AC03T168. notes A20/CR3A12N01DC03T 168AC03T250. notes A20/CR3A12N02DC03T000AC03T168. notes A20/CR3A12N02DC03T 168AC03T250. notes A20/CR3A12N03DC03T000ACO3T168. notes A20/CR3A12N03DC03T 168AC03T250. notes A20/CR3A12N04DC03T000AC03T168. notes A20/CR3A12N04DC03T168AC03T250. notes A20/CR3A12N05DC03T000AC03T 168. notes A20/CR3A12N05DC03T168AC03T250.notes A20/CR3A12N060C03T000AC03T 168. notes A20/CR3A12N06DC03T 168AC03T250. notes A20/CR3A12N070C03T000ACO3T168. notes A20/CR3A12N0TOC03T168AC03T250. notes A20/CR3A12N080C03TO00ACO3T168. notes A20/CR3A12N08DC03T 168AC03T250. notes A20/CR3A12N090C03T000AC03T168. notes A20/CR3A12N09DC03T 168AC03T250. notes A20/CR3A12N10DC03T000AC03T168. notes A20/CR3A12N100C03T 168AC03T250.notes A20/CR3A12N11DC03TOOOAC03T168. notes A20/CR3A12N110C03T 168AC03T250. notes A20/CR3A12N12DC03TO00AC03T 168. notes A20/CR3A12N12DC03T 168AC03T250.notes A20/CR3A12N13DC03T000AC03T168.notes A20/CR3A12N13DCO3T 168AC03T250. notes A20/CR3A12N14DC03TO00AC03T168. notes A20/CR3A12N14DC03T 168AC03T250.notes A20/CR3A12N15DC03TO00AC03T168. notes A20/CR3A12N15DC03T 168AC03T250.notes A20/CR3A12N160C03TO00AC03T168. notes A20/CR3A12N160C03T 168AC03T250.notes A20/CR3A12N17DC03TOOOAC03T168. notes A20/CR3A12N17DC03T 168AC03T250.notes A20/CR3A12N18DCO3TOOOACO3T168. notes A20/CR3A12N18DC03T 168AC03T250. notes A20/CR3A20N01DC1BTDOOAC $1 B T 142$. notes A20/CR3A20N01DC1BT 142AC02T000. notes A20/CR3A20N02DC1BTO0OAC1BT 142. notes A20/CR3A20N02DC1BT 142AC02T000. notes A20/CR3A20N03DC1BTO0OAC1BT 142. notes A20/CR3A2ONO3DC1BT 142AC02TOOO.notes

\begin{tabular}{|c|c|}
\hline $\begin{array}{c}\text { Tape Backup } \\
\text { File Name }\end{array}$ & $\begin{array}{l}\text { Number of } \\
\text { Print Pages }\end{array}$ \\
\hline - & $-\ldots$ \\
\hline aXl If .523 & 7 \\
\hline aXL If .524 & 7 \\
\hline aXLIf .525 & 7 \\
\hline aXL If.526 & 7 \\
\hline aXL If .527 & 7 \\
\hline axl If . 528 & 7 \\
\hline aXL I f . 529 & 7 \\
\hline aXLIf .530 & 7 \\
\hline aXLIf .531 & 7 \\
\hline aXLIf .532 & 7 \\
\hline aXLIf .533 & 7 \\
\hline aXLIf. .534 & 7 \\
\hline aXL If .535 & 7 \\
\hline aXL If .536 & 7 \\
\hline aXLIf .537 & 7 \\
\hline aXLIf .538 & 7 \\
\hline aXL I f .539 & 7 \\
\hline aXL If .540 & 7 \\
\hline
\end{tabular}

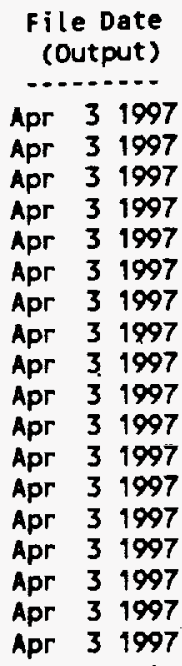

Tape Backup
Fil le Name

File Name

aXLI $f .541$

aXLIf .542

aXLIf 543

aXLIf.544

aXLIf $f .545$

aXL1 1 . 546

aXLIf.547

aXLI I .548

aXLI I . 549

aXLI $f .550$

aXLIf.551

aXLIf 552

aXLIf .553

aXLI I 554

aXLIf. 555

aXLIf.556

aXLIf $f .557$

aXLI I. 558

aXLIf. 559

aXLIf .560

aXLIf.561

aXL1f.562

aXLIf 563

aXLIf 5664

aXLI $f .565$

aXLIf.566

aXLI I . 567

ax́LIf 568

aXLI f.569

aXLI $f .570$

aXLIf 571

aXLI f. 572

aXLIf. 573

aXLIf. 574

aXLIf.575

aXLI I .576

aXLIf. 577

aXLIf .578

aXLIf. 579

aXLIf.580

aXLIf 581

aXLIf. 582

\begin{tabular}{l} 
Number of \\
Print Pages \\
\hline 7 \\
8 \\
7 \\
8 \\
8 \\
8 \\
8 \\
9 \\
8 \\
9 \\
8 \\
9 \\
8 \\
9 \\
8 \\
9 \\
8 \\
9 \\
8 \\
9 \\
8 \\
9 \\
8 \\
9 \\
8 \\
9 \\
8 \\
9 \\
8 \\
9 \\
8 \\
9 \\
7 \\
8 \\
7 \\
8 \\
7 \\
8 \\
7 \\
8 \\
7 \\
8 \\
\end{tabular}

\begin{tabular}{cc}
$\begin{array}{c}\text { File size } \\
\text { (Bytes) }\end{array}$ & $\begin{array}{c}\text { File Type } \\
\text { (Format) }\end{array}$ \\
\hline 8460 & - \\
\hline 8671 & ASCII \\
8930 & ASCII \\
9029 & ASCII \\
8995 & ASCII \\
9001 & ASCII \\
8979 & ASCII \\
8965 & ASCII \\
8989 & ASCII \\
8999 & ASCII \\
9025 & ASCII \\
8751 & ASCII \\
8659 & ASCII \\
8659 & ASCII \\
8663 & ASCII \\
8773 & ASCII \\
8833 & ASCII \\
8650 & ASCII
\end{tabular}

file size file Type

(Bytes) (Format)

8783

0918 ASCII

8904 ASCII

10159 ASCI

9025 ASCII

10350 ASCII

9104 ASCII

10377 ASCII

9206 ASCI

10383 ASCII

9206 ASCII

10363 ASCII

9210 ASCII

10365 ASCII

9212 ASCII

10377 ASCII

9106 ASCII

10383 ASCII

9116 ASCII

10387 ASCII

9118 ASCII

10397 ASCI

9069 ASCII

10397 ASCII

9067 ASCII

10375 - ASCII

9128 ASCII

10377 ASCII

9098 ASCII

10347 ASCII

$9152 \quad A S C I$

10331 ASCII

8976 ASCI

10342 ASCII

8998 ASCII

9982 ASCII

8222 ASCII

9704 ASCI

8397 ASCII

9920 ASCI

8416 ASCII 
A20/CR3A20N04DC1BTO0OAC1BT142.notes A20/CR3A20N04DC1BT 142ACO2T000.notes A20/CR3A20N05DC1BTOOOAC1BT 142.notes A20/CR3A20N05DC1BT 142AC02T000. notes A20/CR3A20N06DC1BTOOOAC1BT142.notes A20/CR3A2ON06DC1BT142ACO2T000.notes A20/CR3A2ONOTDC1BTOOOAC1BT 142. notes A20/CR3A20NOTDC1BT142ACO2T000.notes A20/CR3AZON080C1BTO00AC1BT142.notes A20/CR3A2ON080C1BT 142AC02T000.notes A20/CR3A20NO9DC1BTOOOAC1BT142.notes A20/CR3A20NO9DC1BT 142AC02T000. notes A20/CR3A20N 10DC1BTO00AC1BT142. notes A20/CR3A2ON 100C1BT 142AC02T000. notes A20/CR3A20N11DC1BTO00AC1BT142. notes A20/CR3A2ON11DC1BT 142AC02T000. notes A20/CR3A2ON12DC1BTO0OAC1BT142. notes A20/CR3A2ON12DC1BT 142AC02T000. notes A20/CR3A2ON13DC1BTOOOAC1BT142. notes A20/CR3A2ON13DC1BT142AC02T000. notes A20/CR3A20N14DC1BTO00AC1BT142. notes A20/CR3A20N14DC1BT 142AC02T000. notes A20/CR3A2ON15DC1BTO0OAC1BT142.notes A20/CR3A20N15DC1BT142AC02T000. notes A20/CR3A20N160C1BTO00AC1BT142. notes A20/CR3A2ON16DCiBT 142AC02T000. notes A20/CR3A20N17DC1BTO0OAC1BT142. notes A20/CR3A2ON1TDC1BT142AC02T000. notes A20/CR3A2ON180C1BTO00AC1BT142. notes A20/CR3A20N180C1BT142AC02TO00. notes A20/CR3A23N01DC02TO0OAC03T000. notes A20/CR3A23N02DC02TOOOACO3T000. notes A20/CR3A23N03DC02TO0OAC03T000. notes A20/CR3A23N04DC02TO0OAC03T000. notes A20/CR3A23N05DC02TODOAC03T000. notes A20/CR3A23N060C02TO00AC03T000. notes A2O/CR3A23NOTDC02TODOACO3TO0O. notes A20/CR3A23N08DC02TD00AC03T000. notes A20/CR3A23N090C02TOOOACO3T000. notes A20/CR3A23N10DC02TODOAC03T000. notes A20/CR3A23N110C02T000ACO3T000. notes A20/CR3A23N12DC02TO0OAC03T000. notes A20/CR3A23N13DC02TO00AC03T000. notes A20/CR3A23N14DC02TO00AC03T000. notes A20/CR3A23N15DCO2TO0OAC03T000. notes A20/CR3A23N16DC02TO00AC03T000. notes A20/CR3A23N170C02T000AC03T000.notes A20/CR3A23N180C02TO0OAC03T000. notes

A22/CR3A22N010C1BTO0OAC1BT142.notes A22/CR3A22N02DC1BTO0OAC1BT142.notes A22/CR3A22N030C1BTO0OAC1BT 142.notes A22/CR3A22N04DC1BTO0OAC1BT142.notes A22/CR3A22N05DC1BTOOOAC1B 1 142. notes A22/CR3A22N060C1BTOOOAC1BT142. notes A22/CR3A22NOTDC1BTO0OAC1BT142.notes A22/CR3A22N0BDC1BTOOOAC1BT142. notes A22/CR3A22NO9OC1BTO0OAC1BT142. notes A22/CR3A22N10DC1BTO0OAC1BT142.notes A22/CR3A22N11DC1BTOOOAC1BT142. notes A22/CR3A22N12DC1BTO0OAC1BT142.notes A22/CR3A22N130C1BTODOAC1BT 142.notes A22/CR3A22N14DC1BT000AC1BT142.notes A22/CR3A22N15DC1BTOOOAC1BT142. notes A22/CR3A22N160C1BTO0OAC1BT142. notes A22/CR3A22N1TOC1BTOOOAC1BT 142. notes
}

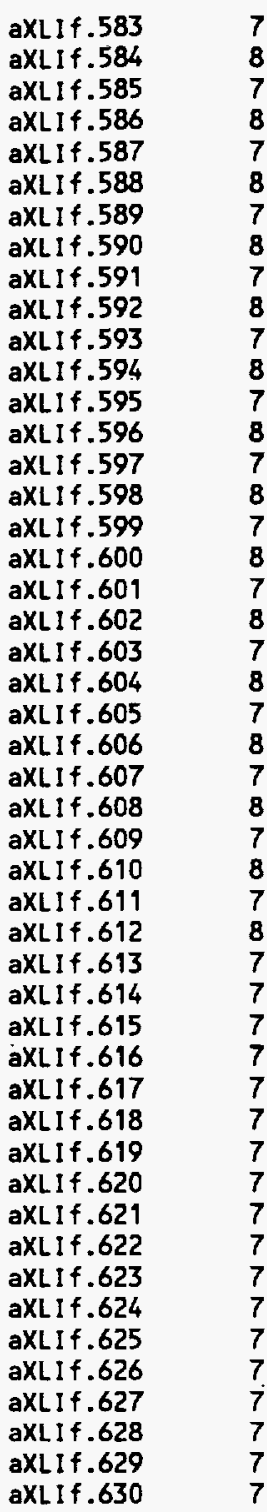

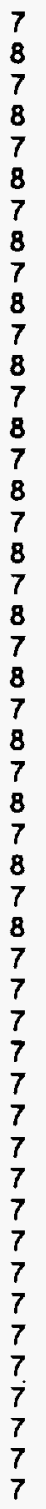

Apr 31997

\begin{tabular}{|c|c|}
\hline $\begin{array}{l}\text { Tape Backup } \\
\text { File Name }\end{array}$ & $\begin{array}{l}\text { Number of } \\
\text { Print Pages }\end{array}$ \\
\hline 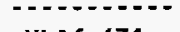 & \\
\hline aXLIf .631 & 7 \\
\hline aXLIf .632 & 7 \\
\hline aXLIf .633 & 7 \\
\hline aXLIf .634 & 7 \\
\hline aXLIf .635 & 7 \\
\hline aXLIf.636 & 7 \\
\hline aXLIf .637 & 7 \\
\hline aXLIf .638 & 7 \\
\hline aXLIf .639 & 7 \\
\hline aXLIf .640 & 7 \\
\hline aXLIf. 641 & 7 \\
\hline aXLIf. 642 & 7 \\
\hline aXLIf .643 & 7 \\
\hline aXLIf .644 & 7 \\
\hline aXLIf .645 & 7 \\
\hline aXLIf .646 & 7 \\
\hline aXLIf .647 & 7 \\
\hline
\end{tabular}

Apr 31997

Apr 31997

Apr 31997

Apr $3 \quad 1997$

Apr 31997

Apr 31997

Apr 31997

Apr 31997

Apr 31997

Apr 31997

Apr 31997

Apr 31997

Apr 31997

Apr 31997

Apr 31997

Apr 31997

Apr 31997

Apr 31997

Apr 31997

Apr 31997

Apr 31997

Apr 31997

Apr 31997

Apr 31997

Apr 31997

Apr 31997

Apr 31997

Apr 31997

Apr 31997

Apr 31997

Apr 31997

Apr 31997

Apr 31997

Apr 31997

Apr 31997

Apr 31997

Apr 31997

Apr 31997

Apr 31997

Apr 31997

Apr 31997

Apr 31997

Apr 31997

Apr 31997

Apr 31997

Apr 31997

Apr 31997

8659

10050

8729

10092

8733

10098

8776

10094

8732

10094

8740

10090

8717

10094

8741

10100

8739

10102

8735

10144

8725

10144

8653

10237

8821

10131

8780

10092

8591

9807

8380

8725

8742

8769

8735

8747

8783

8789

8789

8801

8842

8850

8836

8844

8895

8830

8797

8626

ASCI I

ASCII

ASCII

ASCII

ASCII

ASCII

ASCII

ASCII

ASCII

ASCII

ASCII

ASCII

ASCII

ASCII

ASCII

ASCII

ASCII

ASCII

ASCII

ASCII

ASCII

ASCII

ASCII

ASCII

ASCII

ASCII

ASCII

ASCII

ASCII

ASCII

ASCII

ASCII

ASCII

ASCII

ASCII

ASCII

ASCII

ASCII

ASCII

ASCII

ASCII

ASCII

ASCII

ASCII

ASCII

ASCII

ASCII

ASCII

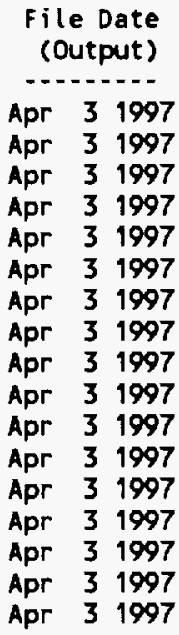

\begin{tabular}{cc}
$\begin{array}{c}\text { File Size } \\
\text { (Bytes) }\end{array}$ & $\begin{array}{c}\text { File Type } \\
\text { (Format) }\end{array}$ \\
\hline 8420 & ASCII \\
8748 & ASCII \\
9076 & ASCII \\
9113 & ASCII \\
9171 & ASCII \\
9137 & ASCII \\
9179 & ASCII \\
9137 & ASCII \\
9149 & ASCII \\
9183 & ASCII \\
9145 & ASCII \\
9189 & ASCII \\
9155 & ASCII \\
9101 & ASCII \\
9097 & ASCII \\
9149 & ASCII \\
9005 & ASCII
\end{tabular}


ASCII

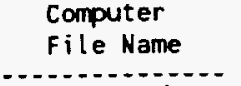

A23/CR3A06N01DC03TO0OACO3T 168 . notes A23/CR3A06N01DC03T168ACO3T250. notes A23/CR3A06N02DC03TOOOACO3T168. notes A23/CR3A06N02DC03T 168AC03T250.notes A23/CR3A06N03DCO3TO0OACO3T168. notes A23/CR3A06N03DC03T 168ACO3T250. notes A23/CR3A06N04DC03TO0OAC03T168. notes A23/CR3A06N04DC03T 168ACO3T250. notes A23/CR3A06N05DC03TO0OACO3T168. notes A23/CR3A06N05DC03T 168AC03T250. notes A23/CR3A06N06DC03TO0OACO3T168. notes A23/CR3A06N06DC03T 168AC03T250. notes A23/CR3A06N07DC03TO0OACO3T168. notes A23/CR3A06NOTDC03T 168ACO3T250. notes A23/CR3A06N08DC03TOOOACO3T168. notes A23/CR3A06N08DC03T168AC03T250. notes A23/CR3A06N09DC03TO00AC03T168. notes A23/CR3A06N09DC03T 168AC03T250. notes A23/CR3A06N10DC03TOOOAC03T168. notes A23/CR3A06N 10DC03T 168AC03T250. notes A23/CR3A06NIIDC03TO00AC03T168. notes A23/CR3A06N11DC03T168ACO3T250. notes A23/CR3A06N 120C03TO00AC03T168. notes A23/CR3A06N12DC03T168AC03T250. notes A23/CR3A06N130C03TO00AC03T168. notes A23/CR3A06N13DC03T168AC03T250. notes A23/CR3A06N140C03T000AC03T168. notes A23/CR3A06N14DC03T168AC03T250. notes A23/CR3A06N15DC03TO0OAC03T168. notes A23/CR3A06N15DC03T168AC03T250. notes A23/CR3A06N160C03TO00AC03T168. notes A23/CR3A06N 16DC03T168AC03T250. notes A23/CR3A06N17DCO3TO00AC03T168. notes A23/CR3A06N17DC03T168AC03T250. notes A23/CR3A06N18DC03TO00AC03T168. notes A23/CR3A06N 18DC03T168AC03T250. notes A23/CR3A07N01DCO2TOOOACO3TO00. notes A23/CR3A07N02DC02TO0OAC03T000. notes A23/CR3A07N03DC02TOOOACO3TO00. notes A23/CR3A07N04DC02TO00ACO3T000. notes A23/CR3AOTN05DC02TO0OACO3TO00. notes A23/CR3A07N06DC02TO0OACO3TO00. notes A23/CR3A07N07DC02TOOOACO3TO00. notes A23/CR3A07N080C02T TOOAACO3T000.notes A23/CR3AO7NO9DC02TOOOACO3TO00. notes A23/CR3A07N10DC02TO0OACO3T000. notes A23/CR3A07N110C02TODOACO3.T000.notes A23/CR3A07N120C02TO0OACO3T000.notes A23/CR3A07N13DC02TO0OACO3TO00. notes A23/CR3A07N140C02TO00AC03T000. notes A23/CR3A07N15DC02TDODACO3TO00. notes A23/CR3A07N160C02TO0OAC03T000.notes A23/CR3A07N170C02TO0OAC03TO00.notes A23/CR3A07N180C02TO0OAC03TO00.notes A23/CR3A23N010C1BTOOOAC1BT 142.notes A23/CR3A23NO1DC1BT142AC02T000.notes A23/CR3A23N02DC1BTO0OAC1BT 142. notes A23/CR3A23H02DC1BT 142ACO2TOO0. notes A23/CR3A23NO3DC1BTOOOAC1BT142.notes A23/CR3A23N03DC1BT142AC02T000.notes A23/CR3A23N040C1BTDOOAC1BT 142.notes A23/CR3A23N04DC1BT 142AC02T000.notes A23/CR3A23N05DC1BTO0OAC1BT 142. notes A23/CR3A23N050C1BT 142AC02T000. notes

\begin{tabular}{|c|c|c|c|c|c|}
\hline \multirow{2}{*}{$\begin{array}{l}\text { Tape Backup } \\
\text { File Name }\end{array}$} & $\begin{array}{l}\text { Number of } \\
\text { Print Pages }\end{array}$ & \multicolumn{2}{|c|}{$\begin{array}{c}\text { File Date } \\
\text { (Output) }\end{array}$} & \multirow{2}{*}{$\begin{array}{c}\text { File Size } \\
\text { (Bytes) }\end{array}$} & \multirow{2}{*}{$\begin{array}{r}\text { File Type } \\
\text { (Format) }\end{array}$} \\
\hline & & $=\ldots$ & - . - & & \\
\hline aXLIf. 649 & 7 & $\mathrm{Apr}$ & 31997 & 8573 & ASCII \\
\hline aXLIf. 650 & 8 & Apr & 31997 & 9738 & ASCII \\
\hline aXLIf .651 & 7 & $\mathrm{Apr}$ & 31997 & 8828 & ASCII \\
\hline axLIf .652 & 8 & Apr & 31997 & 9980 & ASCII \\
\hline axL If .653 & 7 & Apr & 31997 & 8950 & ASCII \\
\hline aXL. If .654 & 8 & Apr & 31997 & 10334 & ASCII \\
\hline aXLIf .655 & 7 & $\mathrm{Apr}$ & 31997 & 8983 & ASCII \\
\hline aXLIf. 656 & 9 & Apr & 31997 & 10395 & ASCII \\
\hline aXLIf .657 & 8 & Apr & 31997 & 9021 & ASCII \\
\hline aXLIf .658 & 9 & Apr & 31997 & 10375 & ASCII \\
\hline aXLIf.659 & 8 & Apr & 31997 & 9029 & ASCII \\
\hline aXLIf .660 & 9 & Apr & 31997 & 10369 & ASCII \\
\hline aXLif.661 & 8 & $\mathrm{Apr}$ & 31997 & 9023 & ASCII \\
\hline aXL If . .662 & 9 & Apr & 31997 & 10363 & ASCII \\
\hline aXLIf 1.663 & 8 & Apr & 31997 & 9023 & ASCII \\
\hline aXLIf .664 & 9 & Apr & 31997 & 10379 & ASCII \\
\hline aXLIf .665 & 8 & Apr & 31997 & 9023 & ASCII \\
\hline aXLIf .666 & 9 & $\mathrm{Apr}$ & 31997 & 10379 & ASCII \\
\hline aXLIf .667 & 8 & Apr & 31997 & 9021 & ASCII \\
\hline aXLIf .668 & 9 & $\mathrm{Apr}$ & 31997 & 10375 & ASCII \\
\hline aXLIf .669 & 7 & Арг & 31997 & 8944 & ASCII \\
\hline aXLIf .670 & 9 & $\mathrm{Apr}$ & 31997 & 10391 & ASCII \\
\hline aXLIf .671 & 7 & Apr & 31997 & 8944 & ASCII \\
\hline axLif .672 & 9 & Apr & 31997 & 10389 & ASCII \\
\hline axLIf. 673 & 7 & $\mathrm{Apr}$ & 31997 & 8936 & ASCII \\
\hline axLIf .674 & 9 & Apr & 31997 & 10395 & ASCII \\
\hline axLIf .673 & 7 & $\mathrm{Apr}$ & 31997 & 8936 & ASCII \\
\hline aXLIf .676 & 9 & Apr & 31997 & 10389 & ASCII \\
\hline aXLIf .677 & 7 & Apr & 31997 & 8932 & ASCII \\
\hline aXLIf .678 & 9 & Apr & 31997 & 10395 & ASCII \\
\hline aXLIf .679 & 7 & $\mathrm{Apr}$ & 31997 & 8936 & ASCII \\
\hline axLIf .680 & 9 & Apr & 31997 & 10387 & ASCII \\
\hline aXLIf.681 & 7 & Apr & 31997 & 8922 & ASCII \\
\hline aXLIf . .682 & 8 & Apr & 31997 & 10294 & ASCII \\
\hline aXLIf .683 & 7 & $\mathrm{Apr}$ & 31997 & 8862 & ASCII \\
\hline axLIf .684 & 8 & Apr & 31997 & 9957 & ASCII \\
\hline aXLIf .685 & 7 & $\mathrm{Apr}$ & 31997 & 8561 & ASCII \\
\hline aXLIf .686 & 7 & $\mathrm{Apr}$ & 31997 & 8722 & ASCII \\
\hline aXLIf .687 & 7 & Apr & 31997 & 8842 & ASCII \\
\hline aXLIf .688 & 7 & $\mathrm{Apr}$ & 31997 & 8835 & ASCII \\
\hline aXLIf .689 & 7 & $\mathrm{Apr}$ & 31997 & 8825 & ASCII \\
\hline aXLIf . 690 & 7 & Apr & 31997 & 8829 & ASCII \\
\hline axLIf .691 & 7 & Apr & 31997 & 8835 & ASCII \\
\hline aXLIf .692 & 7 & Apr & 31997 & 8835 & ASCI I \\
\hline axL If .693 & 7 & $\mathrm{Apr}$ & 31997 & 8827 & ASCII \\
\hline aXLIf .694 & 7 & Apr & 31997 & 8835 & ASCII \\
\hline aXLIf .695 & 7 & Apr & 31997 & 8843 & ASCII \\
\hline aXL If .696 & 7 & $\mathrm{Apr}$ & 31997 & 8859 & ASCII \\
\hline aXL $1 f .697$ & 7 & Apr & 31997 & 8869 & ASCII \\
\hline axL If . .698 & 7 & Apr & 31997 & 8873 & ASCII \\
\hline aXL If . .699 & 7 & Apr & 31997 & 8859 & ASCII \\
\hline axL If . 700 & 7 & Apr & 31997 & 8857 & ASCII \\
\hline aXLIf .701 & 7 & Apr & 31997 & 8844 & ASCII \\
\hline axLIf .702 & 7 & Apr & 31997 & 8701 & ASCII \\
\hline axL If . 703 & 7 & $\mathrm{Apr}$ & 31997 & 8411 & ASCII \\
\hline aXLIf .704 & 8 & Apr & 31997 & 9677 & ASCII \\
\hline aXL If .705 & 7 & Apr & 31997 & 8705 & ASCII \\
\hline aXLIf .706 & 8 & Apr & 31997 & 9907 & ASCII \\
\hline aXLif .707 & 7 & Apr & 31997 & 8839 & ASCi1 \\
\hline aXL if 708 & 8 & Apr & 31997 & 10101 & ASCII \\
\hline aXL If 709 & 7 & Apr & $\begin{array}{ll}3 & 1997\end{array}$ & 9031 & ASCII \\
\hline aXLIf 7.710 & 8 & Apr & 31997 & 10097 & ASCII \\
\hline aXLif.711 & 7 & Apr & 31997 & 9029 & ASCII \\
\hline & 8 & Apr & 31997 & 10071 & AOCII \\
\hline
\end{tabular}


A23/CR3A23N060C1BTO00AC1BT142.notes A23/CR3A23N06DC1BT 142AC02T000. notes A23/CR3A23NOTOC1BTOOOAC1BT142. notes A23/CR3A23NOTDC1BT 142AC02T000. notes A23/CR3A23NO8DC1BTOOOAC1BT 142. notes A23/CR3A23NO8DC1BT 142AC02T000.notes A23/CR3A23N090C1BTO0OAC1BT142.notes A23/CR3A23N09DC1BT 142AC02T000.notes A23/CR3A23N10DC1BT000AC1BT142.notes A23/CR3A23N10DC1BT 142AC02T000.notes A23/CR3A23N11DC1BTO00AC1BT142.notes A23/CR3A23N110C1BT142AC02T000.notes A23/CR3A23N120C1BT000AC1BT142.notes A23/CR3A23N12DC1BT 142AC02T000.notes A23/CR3A23N13DC1BT000AC1BT142.notes A23/CR3A23N13DC1BT 142AC02T000.notes A23/CR3A23N14DC1BTO00AC1BT142. notes A23/CR3A23N14DC1BT 142AC02T000. notes A23/CR3A23N15DC1BTO0OAC1BT142. notes A23/CR3A23H 15DC1BT 142AC02T000. notes A23/CR3A23N16DC1BTO00AC1BT142.notes A23/CR3A23N 160C1BT142AC02T000. notes A23/CR3A23N17DC1BT000AC1BT142. notes A23/CR3A23N17DC1BT142AC02T000. notes A23/CR3A23N180C1BT000AC1BT 142. notes A23/CR3A23N18DC1BT142AC02T000. notes

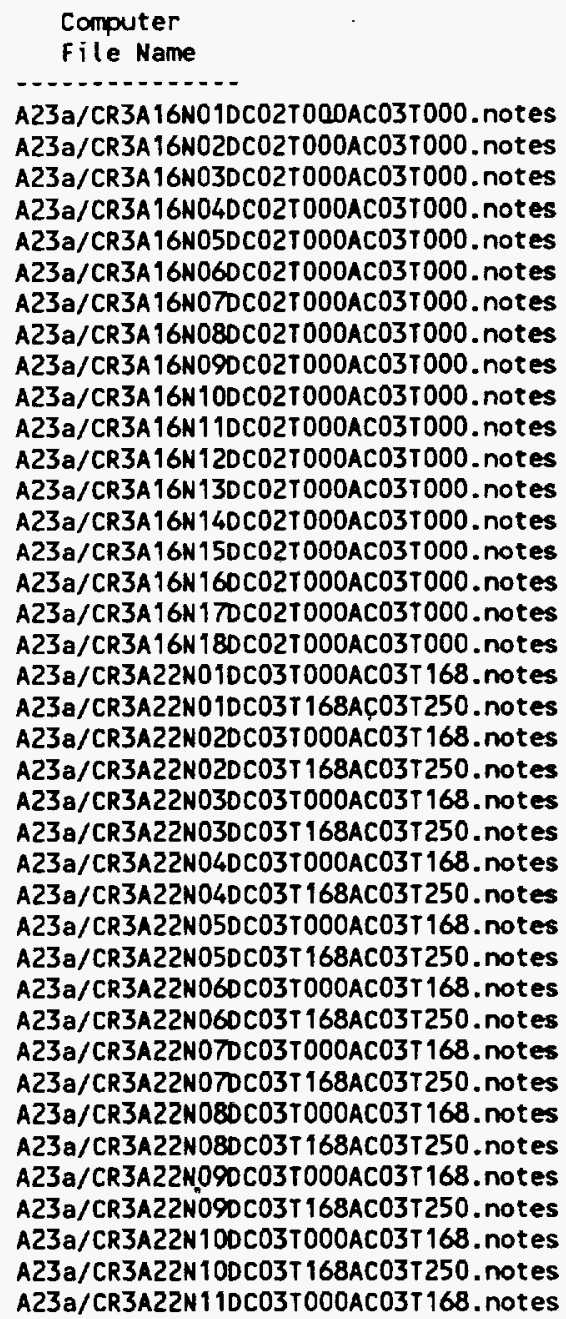

aXLIf.713

axLIf. .714

aXLIf. .715

aXLIf. .716

aXLIf.717

aXLIf. 718

aXLIf.719

aXLIf .720

aXLIf. 721

aXLIf. 722

aXLIf. 723

aXLIf. 724

aXLIf. .725

aXLIf. 726

aXLIf. 727

aXLIf. 728

aXLIf. 729

aXLIf. 730

axLIf. 731

aXLIf. 732

axLIf. 733

aXL1f. 734

aXL1f. 735

aXLIf. 736

axLIf. 737

aXL If. 738

\begin{tabular}{|c|c|}
\hline $\begin{array}{c}\text { Tape Backup } \\
\text { File Nane }\end{array}$ & $\begin{array}{l}\text { Number of } \\
\text { Print Pages }\end{array}$ \\
\hline - & \\
\hline aXLIf.739 & 7 \\
\hline aXLIf .740 & 7 \\
\hline aXL If .741 & 7 \\
\hline axLIf .742 & 7 \\
\hline aXL If .743 & 7 \\
\hline aXLIf. 744 & 7 \\
\hline aXLIf .745 & 7 \\
\hline aXLIf .746 & 7 \\
\hline$a \times L I f .747$ & 7 \\
\hline aXLIf. 748 & 7 \\
\hline aXLIf. 749 & 7 \\
\hline aXL If . 750 & 7 \\
\hline aXLIf. 751 & 7 \\
\hline aXLIf .752 & 7 \\
\hline aXLIf. 753 & 7 \\
\hline aXL1f. 754 & 7 \\
\hline aXLIf. 755 & 7 \\
\hline aXLIf. 756 & 7 \\
\hline aXL If. 757 & 7 \\
\hline aXLIf. 758 & 8 \\
\hline aXLIf. 759 & 7 \\
\hline aXLIf. 760 & 8 \\
\hline aXLIf.761 & 7 \\
\hline aXLIf. 762 & 8 \\
\hline aXLIf. 763 & 8 \\
\hline aXL If. 764 & 9 \\
\hline aXLIf. 765 & 8 \\
\hline aXLIf.766 & 9 \\
\hline axL If. 767 & 8 \\
\hline aXLIf.768 & 9 \\
\hline aXLIf. 769 & 8 \\
\hline aXL If . 770 & 9 \\
\hline aXLIf. 771 & 8 \\
\hline aXL If. 772 & 9 \\
\hline aXLIf. 773 & 8 \\
\hline axLIf. 774 & 9 \\
\hline axL If. 775 & 8 \\
\hline aXLIf. 776 & 9 \\
\hline axLIf. 777 & 8 \\
\hline
\end{tabular}

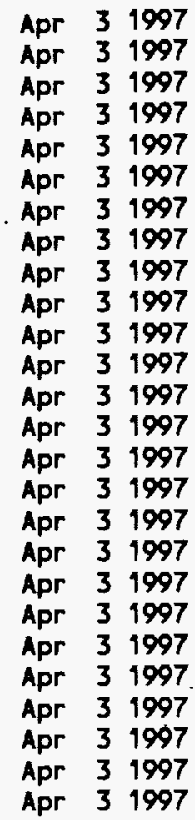

File Date (Output)

Apr 31997

Apr 31997

Apr 31997

Apr 31997

Apr 31997

Apr 31997

Apr 31997

Арг 31997

Apr 31997

Apr 31997

Apr 31997

Apr 31997

Apr 31997

Apr 31997

Apr 31997

Apr 31997

Apr 31997

Apr 31997

Apr 31997

Apr 31997

Apr 31997

Apr 31997

Apr 31997

Apr 31997

Apr 31997

Apr 31997

Apr 31997

Apr 31997

Apr 31997

Apr $3 \quad 1997$

Apr 31997

Apr 31997

Apr 31997

Apr 31997

Apr 31997

Apr 31997

Apr 31997

Apr 31997

Apr 31997
9019

10063

9013

10047

9025

10049

9009

10061

9019

10069

9033

10069

9039

10107

8991

10099

8932

10095

8867

10093

8877

10137

8823

10129

8630

9854

\begin{tabular}{cc}
$\begin{array}{c}\text { File size } \\
\text { (Bytes) }\end{array}$ & $\begin{array}{c}\text { File Type } \\
\text { (Format) }\end{array}$ \\
\hline 8559 & - \\
8667 & ASCII \\
8838 & ASCII \\
8831 & ASCII \\
8831 & ASCII \\
8823 & ASCII \\
8833 & ASCII
\end{tabular}

8835

8837

8843

8843

8855

8867

8867

8855

8857

8844

8701

8674

9811

8944

10077

8982

10346

9166

10365

9206

10347

9218

10337

9210

10337

9204

10349

9210

10351

9178

10363

9120
ASCII

ASCII

ASCII

ASCII

ASCII

ASCII

ASCII

ASCII

ASCII

ASCII

ASCII

ASCII

ASCII

ASCII

ASCII

ASCII

ASCII

ASCII

ASCII

ASCII

ASCII

ASCII

ASCII

ASCII

ASC II

ASCII

ASCII

ASCII

ASCII

ASCII

ASCII

ASCII

ASCII

ASCII

ASCII

ASCII

ASCII

ASCII

ASCII

ASCII

ASCII

ASCII

ASCII

ASCII

ASC I I

ASCII

ASCII

ASCII

ASCII

ASCII

ASCII

ASCII

ASCII

ASCII

ASCII

ASCII

ASCII

ASCII 
A23a/CR3A22N110C03T168AC03T250.notes A23a/CR3A22N12DC03TO0OACO3T168. notes A23a/CR3A22N12DC03T168AC03T250. notes A23a/CR3A22N 13DCO3TOOOACO3T168. notes A23a/CR3A22N13DC03T 168AC03T250. notes A23a/CR3A22N140CO3TO0OAC03T168. notes A23a/CR3A22N140C03T168ACO3T250. notes A23a/CR3A22N150C03TO00AC03T168. notes A23a/CR3A22N15DCO3T 168AC03T250. notes A23a/CR3A22N160CO3TO0OACO3T168. notes A23a/CR3A22N160C03T 168AC03T250.notes A23a/CR3A22N17DCO3TOOOACO3T 168. notes A23a/CR3A22N1TDC03T168AC03T250.notes A23a/CR3A22N180CO3TOOOACO3T 168. notes A23a/CR3A22N180CO3T 168ACO3T250. notes A23a/CR3A23N01DC1BT 142AC02T000. notes A23a/CR3A23NO2DC1BT 142AC02T000.notes A23a/CR3A23N03DC1BT 142AC02T000.notes A23a/CR3A23N04DC1BT 142AC02T000.notes A23a/CR3A23N05DC1BT 142AC02T000.notes A23a/CR3A23N060C1BT 142AC02T000. notes A23a/CR3A23NOTDC1BT142AC02T000. notes A23a/CR3A23N08DC1BT 142AC02T000. notes A23a/CR3A23NO9DC1BT 142AC02T000. notes A23a/CR3A23N 10DC1BT 142AC02T000. notes A23a/CR3A23N110C1BT 142AC02T000. notes A23a/CR3A23N120C1BT142AC02T000. notes A23a/CR3A23N 13DC1BT142AC02T000. notes A23a/CR3A23N 14DC1BT142AC02T000. notes A23a/CR3A23N 15DC1BT142AC02T000.notes A23a/CR3A23N 160C1BT 142AC02T000. notes A23a/CR3A23N17DC1BT142AC02T000.notes A23a/CR3A23N18DC1BT 142AC02T000. notes

\section{Computer$$
\text { File Name }
$$

A25/CR3A07N01DC03T000AC03T168. notes A25/CR3A07N01DC03T168AC03T250. notes A25/CR3A07N02DC03TO00AC03T168. notes A25/CR3A07N02DC03T168AC03T250.notes A25/CR3A07N03DC03TO0OACO3T168. notes A25/CR3A07N03DCO3T168AC03T250.notes A25/CR3A07N04DC03TOOOAC03T168.notes A25/CR3A07N04DCO3T168AC03T250.notes A25/CR3A07N05DC03TO00AC03T168. notes A25/CR3A07N05DCO3T 168AC03T250. notes A25/CR3A07N06DC03TOOOAC03T168. notes A25/CR3A07N06DC03T 168AC03T250. notes A25/CR3A07N070C03TO00AC03T168. notes A25/CR3A07N0TDCO3T168AC03T250. notes A25/CR3A07N08DC03TO0OAC03T168. notes A25/CR3A07N08DC03T 168AC03T250. notes A25/CR3A07N09DC03TO0OAC03T168. notes A25/CR3A07N09DC03T168AC03T250. notes A25/CR3A07N10DC03TO00AC03T168. notes A25/CR3A07N10DCO3T168AC03T250. notes A25/CR3A07N110CO3TO00ACO3T168. notes A25/CR3A07N1 1DC03T168AC03T250. notes A25/CR3A07N12DC03T000AC03T168. notes A25/CR3A07N12DC03T168AC03T250. notes A25/CR3A07N13DC03T000AC03T168. notes A25/CR3A07N13DC03T168ACO3T250. notes A25/CR3A07N14DC03T000AC03T168. notes A25/CR3A07N140C03T168AC03T250. notes A25/CR3A07N 15DC03TO00AC03T168. notes A25/CR3A07N15DC03T168ACO3T250. notes A25/CR3A07N16DC03TO0DAC03T168. notes A25/CR3A07N160C03T168AC03T250. notes

\begin{tabular}{|c|}
\hline 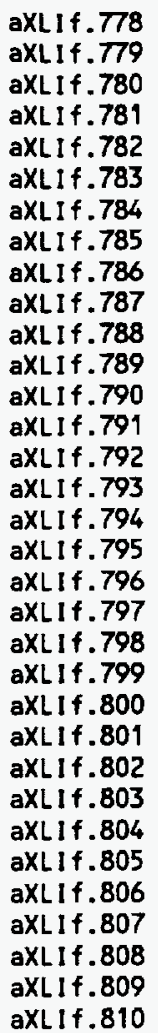 \\
\hline
\end{tabular}

Apr 31997

Apr 31997

Apr 31997

Apr 31997

Apr 31997

Apr 31997

Apr 31997

Apr 31997

Apr 31997

Apr 31997

Apr 31997

Apr 31997

Apr 31997

Apr 31997

Apr 31997

Apr 31997

Apr 31997

Apr 31997

Apr 31997

Apr 31997

Apr 31997

Apr 31997

Apr 31997

Apr 31997

Apr 31997

Apr 31997

Apr 31997

Apr 31997

Apr 31997

Apr 31997

Apr 31997

Apr 31997

Apr 31997

Tape Backup File Name

axLIf. 811

aXLIf. 812

aXLIf. 813

aXLIf. 814

aXLIf. 815

aXLIf. 816

aXLIf. 817

aXLIf. 818

aXL If 819

aXL1f.820

aXLIf.821

axLIf. 822

aXLIf. 823

aXLIf. 824

aXLIf. 825

aXLIf 826

aXLIf. 827

aXLIf .828

aXLIf. 829

aXLIf .830

aXLIf. 831

aXLIf 832

aXLIf .833

aXLI $f .834$

aXLIf .835

aXLIf. 836

aXLIf .837

aXLI f. 838

aXLIf. 839

aXL1f. 840

aXLIf 841

aXLIf. 842

\begin{tabular}{l} 
Number of \\
Print Pages \\
7 \\
8 \\
8 \\
7 \\
8 \\
7 \\
8 \\
7 \\
8 \\
7 \\
8 \\
8 \\
8 \\
8 \\
8 \\
7 \\
8 \\
7 \\
8 \\
7 \\
8 \\
7 \\
8 \\
7 \\
8 \\
7 \\
8 \\
7 \\
8 \\
7 \\
8 \\
7 \\
8 \\
\hline
\end{tabular}

File Date (Output)

Apr 31997

Apr 31997

Apr 31997

Apr 31997

Apr 31997

Apr 31997

Apr 31997

Apr 31997

Apr 31997

Apr 31997

Apr 31997

Apr 31997

Apr 31997

Apr 31997

Apr 31997

Apr 31997

Apr 31997

Apr 31997

Apr 31997

Apr 31997

Apr 31997

Apr 31997

Apr 31997

Apr 31997

Apr 31997

Apr 31997

Apr 31997

Apr 31997

Apr 31997

Apr 31997

Apr 31997
Apr 31997

$\begin{array}{rr}10381 & \text { ASCII } \\ 9090 & \text { ASCII } \\ 10397 & \text { ASCII } \\ 8998 & \text { ASCII } \\ 10393 & \text { ASCII } \\ 9037 & \text { ASCII } \\ 10379 & \text { ASCII } \\ 9069 & \text { ASCII } \\ 10375 & \text { ASCII } \\ 9136 & \text { ASCII } \\ 10371 & \text { ASCII } \\ 9071 & \text { ASCII } \\ 10373 & \text { ASCII } \\ 8912 & \text { ASCII } \\ 9988 & \text { ASCII } \\ 9677 & \text { ASCII } \\ 9907 & \text { ASCII } \\ 10101 & \text { ASCII } \\ 10097 & \text { ASCII } \\ 10071 & \text { ASCII } \\ 10063 & \text { ASCII } \\ 10047 & \text { ASCII } \\ 10049 & \text { ASCII } \\ 10061 & \text { ASCII } \\ 10069 & \text { ASCII } \\ 10069 & \text { ASCII } \\ 10107 & \text { ASCII } \\ 10099 & \text { ASCII } \\ 10095 & \text { ASCII } \\ 10093 & \text { ASCII } \\ 10137 & \text { ASCII } \\ 10129 & \text { ASCII } \\ 9854 & \text { ASCII }\end{array}$

\begin{tabular}{cc}
$\begin{array}{c}\text { File Size } \\
\text { (Bytes) }\end{array}$ & File Type \\
\hline & (Format) \\
\hline 8860 & ASCII \\
9716 & ASCII \\
9160 & ASCII \\
10099 & ASCII \\
9200 & ASCII \\
10126 & ASCII \\
9208 & ASCII \\
10256 & ASCII \\
9216 & ASCII \\
10210 & ASCII \\
9251 & ASCII \\
10222 & ASCII \\
9255 & ASCII \\
10234 & ASCII \\
9142 & ASCII \\
10220 & ASCII \\
9182 & ASCII \\
10260 & ASCII \\
9166 & ASCII \\
10224 & ASCII \\
9180 & ASCII \\
10270 & ASCII \\
9270 & ASCII \\
10264 & ASCII \\
9228 & ASCII \\
10272 & ASCII \\
9114 & ASCII \\
10278 & ASCII \\
9158 & ASCII \\
10275 & ASCII \\
9182 & ASCII \\
10381 & ASCII
\end{tabular}


A25/CR3A07N17DC03TODOAC03T168.notes A25/CR3A07N17DC03T168ACO3T250. notes A25/CR3A07N18DCO3TO0OACO3T168. notes A25/CR3A07N180C03T168AC03T250. notes A25/CR3A13N010C02TO0OAC03T000. notes A25/CR3A13H020C02TODOACO3TO00. notes A25/CR3A13N030C02TO0OACO3TOOO. notes A25/CR3A13N04DCO2TD00AC03TO00. notes A25/CR3A13N05DC02TO00AC03T000. notes A25/CR3A13N060C02TDOOACO3TO00. notes A25/CR3A13NOTOCO2TDOOACO3TO00. notes A25/CR3A13N080C02TDOOACO3TO00. notes A25/CR3A13N090CO2TO0OACO3T000. notes A25/CR3A13N100C02TD00ACO3T000. notes A25/CR3A13N11DC02T000AC03T000. notes A25/CR3A13N120C02TO0OACO3TO00. notes A25/CR3A13N130C02TO0OACO3T000. notes A25/CR3A13N140C02TODOACO3TODO. notes A25/CR3A13N15DC02TO0OACO3T000. notes A25/CR3A13N160C02TO0OACO3TO00. notes A25/CR3A13H1TDCO2TODOACO3TO00. notes A25/CR3A13N180C02TOOOACO3TO00. notes A25/CR3A25N01DC1BTOOOAC1BT 142.notes A25/CR3A25N010C1BT 142AC02T000. notes A25/CR3A25N02DC1BTODOAC1BT142.notes A25/CR3A25N02DC1BT142AC02T000.notes A25/CR3A25N03DC1BTOOOAC1BT142.notes A25/CR3A25N030C1BT142ACO2T000. notes A25/CR3A25N04DC1BTDOOAC1BT142. notes A25/CR3A25N04DC1BT 142ACO2T000. notes A25/CR3A25N05DC1BTO0OAC1BT 142. notes A25/CR3A25N050C1BT142AC02T000. notes A25/CR3A25N060C1BTODOAC1BT142. notes A25/CR3A25N06DC1BT142ACO2T000. notes A25/CR3A25NOTOC1BTOOOAC1BT142.notes A25/CR3A25NOTDC1BT142ACO2T000. notes A25/CR3A25NOBOC1BTOOOAC1BT 142. notes A25/CR3A25N080C1BT 142ACO2T000.notes A25/CR3A25N09DC1BTO00AC1BT 142.notes A25/CR3A25N090C1BT142AC02T000. notes A25/CR3A25N100C1BTO00AC1BT142. notes A25/CR3A25N10DC1BT142ACO2T000. notes A25/CR3A25N11DC1BTO00AC1BT142.notes A25/CR3A25N110C1BT142ACO2T000.notes A25/CR3A25N12DC1BTO00AC1BT142.notes A25/CR3A25N12DC1BT142ACO2T000.notes A25/CR3A25N13DC1BT000AC1BT142. notes A25/CR3A25N13DC1BT 142AC02T000. notes A25/CR3A25N14DC1BTO0OAC1BT142. notes A25/CR3A25N14DC1BT 142AC02T000. notes A25/CR3A25N 15DC1B TO00AC1BT 142. notes A25/CR3A25N15DC1BT 142AC02T000. notes A25/CR3A25N16DC1BTOOOAC1BT142. notes A25/CR3A25N 16DC1BT 142AC02T000. not es A25/CR3A25N17OC1BTO0OAC1BT142.notes A25/CR3A25N1TOC1BT 142AC02T000. notes A25/CR3A25N18DC1BT000AC1BT 142. notes A25/CR3A25N18DC1BT 142AC02T000. notes

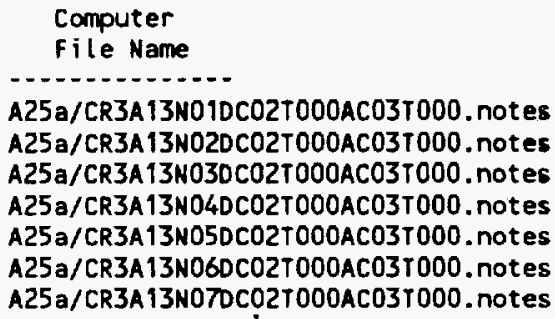

aXLIf. 843

aXLIf. 844

aXLIf. 845

aXLIf. 846

aXLIf.847

aXLIf .848

aXLIf. 849

aXLIf.850

aXL1f.851

aXLIf.852

aXLIf. 853

aXLIf.854

aXLIf. 855

aXLIf. 856

aXL1f.857

aXLIf. 858

aXLIf.859

aXLIf.860

aXLIf. 861

aXLIf .862

aXLIf. 863

aXLIf.864

aXL1f. 865

aXLIf.866

aXLIf. 867

aXLIf .868

aXLIf .869

aXLI $f .870$

aXLIf. 871

aXLIf.872

aXLIf.873

aXLIf .874

aXL I $f .875$

aXLIf.876

aXLIf.877

aXLIf .878

aXLIf. 879

aXLIf .880

aXLIf . 881

aXLIf. 882

aXL If .883

aXLIf. 884

aXLIf .885

aXL If .886

aXL If .887

aXLIf. 888

aXLIf .889

aXLIf .890

aXL1 $f .891$

aXLIf. 892

aXLIf.893

aXLIf. 894

aXLIf. 895

aXLIf. 896

aXLIf. 897

aXLIf. 898

aXL If .899

aXLIf. 900

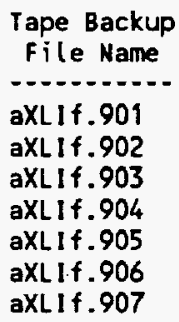

Apr 31997

Apr 31997

Apr 31997

Apr 31997

Apr 31997

Apr 31997

Apr 31997

Apr 31997

Apr 31997

Apr 31997

Apr 31997

Apr 31997

Apr 31997

Apr 31997

Apr 31997

Apr 31997

Apr 31997

Apr 31997

Apr 31997

Apr 31997

Apr 31997

Apr 31997

Apr 31997

Apr 31997

Apr 31997

Apr 31997

Apr 31997

Apr 31997

Apr 31997

Apr 31997

Apr 31997

Apr 31997

Apr 31997

Аpr 31997

Apr 31997

Apr 31997

Apr 31997

Apr 31997

Apr 31997

Apr 31997

Apr 31997

Apr 31997

Apr 31997

Apr 31997

Apr 31997

Apr 31997

Apr 31997

Apr 31997

Apr 31997

Apr 31997

Apr 31997

Apr 31997

Apr 31997

Apr 31997

Apr 31997

Apr 31997

Apr 31997

Apr 31997

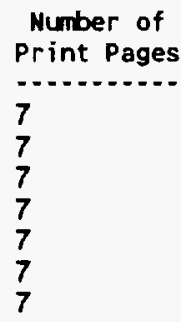

File Date (Output)

$\begin{array}{lll}\text { Apr } & 3 & 1997 \\ \text { Apr } & 3 & 1997\end{array}$

Apr 31997

Apr 31997

Apr 31997

Apr 31997

Apr 31997

9218

10224

8955

10119

8477

8767

8875

8845

8835

8853

8859

8843

8873

8905

8827

8954

8898

8928

8962

8889

8867

8614

8316

9765

8479

10003

8713

10056

8803

10046

8837

10124

8819

10166

8895

10136

8919

10158

8947

10144

8811

10210

8821

10126

8847

10124

8835

10076

8821

10142

8871

10108

8873

10174

8756

10044

8497
9930

ASCII

ASCII

ASCII

ASCII

ASCII

ASCII

ASCII

ASCII

ASCII

ASCII

ASCII

ASCI I

ASCII

ASCII

ASCII

ASCII

ASCII

ASCII

ASCII

ASCII

ASCII

ASCII

ASCII

ASCII

ASCII

ASCII

ASCII

ASCII

ASCII

ASCII

ASCII

ASCII

ASCII

ASCII

ASCII

ASCII

ASCII

ASCII

ASCI.I

ASCII

ASCII

ASCII

ASCII

ASCII

ASCII

ASCII

ASCII 
A25a/CR3A13N080C02T000AC03T000.notes A25a/CR3A13NO9OCO2TOOOACO3T000. notes A25a/CR3A13N10DC02TO00AC03T000. notes A25a/CR3A13N11DCO2TO00AC03T000.notes A25a/CR3A13N12DC02TO00ACO3T000. notes A25a/CR3A13N13DC02TO00AC03T000. notes A25a/CR3A13N14DC02TO0OACO3T000.notes A25a/CR3A13N150C02T000AC03T000. notes A25a/CR3A13N 160C02TO00AC03T000. notes A25a/CR3A13N170C02TO00AC03T000.notes A25a/CR3A13N180C02T000AC03T000. notes A25a/CR3A26N01DC03T000AC03T168.notes A25a/CR3A26N010C03T 168AC03T250. notes A25a/CR3A26N02DC03TO00AC03T168. notes A25a/CR3A26N020C03T168AC03T250. notes A25a/CR3A26N03DC03TO0OACO3T168. notes A25a/CR3A26N03DCO3T 168AC03T250.notes A25a/CR3A26N04DC03TO0OAC03T168. notes A25a/CR3A26N04DC03T 168AC03T250.notes A25a/CR3A26N05DC03TO00AC03T168. notes A25a/CR3A26N05DC03T 168ACO3T250. notes A25a/CR3A26N060C03TO00AC03T168. notes A25a/CR3A26N060C03T 168ACO3T250.notes A25a/CR3A26N070C03TO0OAC03T168.notes A25a/CR3A26N070CO3T168ACO3T250.notes A25a/CR3A26N080C03TO00AC03T168. notes A25a/CR3A26N080C03T168AC03T250. notes A25a/CR3A26N090C03T000AC03T168. notes A25a/CR3A26N090C03T 168AC03T250.notes A25a/CR3A26N100C03TO00ACO3T 168. notes A25a/CR3A26N100C03T168AC03T250. notes A25a/CR3A26N11DC03T000AC03T 168. notes A25a/CR3A26N110C03T168AC03T250. notes A25a/CR3A26N120C03TO00ACO3T168. notes A25a/CR3A26N12DC03T168ACO3T250. notes A25a/CR3A26N13DC03TO0OAC03T 168. notes A25a/CR3A26N130C03T 168AC03T250. notes A25a/CR3A26N14DC03T000AC03T 168.notes A25a/CR3A26N 140C03T168ACO3T250.notes A25a/CR3A26N150C03T000AC03T 168. notes A25a/CR3A26N15DC03T168AC03T250.notes A25a/CR3A26N160C03TO00AC03T168. notes A25a/CR3A26N 160C03T 168AC03T250.notes A25a/CR3A26N17DC03TO00AC03T 168.notes A25a/CR3A26N17DC03T168AC03T250.notes A25a/CR3A26N 18DC03TO00AC03T 168. notes A25a/CR3A26N18DC03T168ACO3T250. notes
aXLIf.908

aXLIf. 909

aXLIf. 910

aXLIf. 911

aXLIf. 912

aXLIf. 913

aXLIf. 914

axLIf. 915

aXLIf. 916

aXLIf. 917

aXLIf .918

aXLIf. 919

aXLIf. 920

aXLIf. 921

aXLIf. 922

aXLIf. 923

aXLIf. 924

aXLIf. 925

aXLI $f .926$

aXL1f. 927

aXLIf. 928

aXLIf. 929

aXLIf.930

axLIf. 931

aXLIf. 932

aXLIf .933

aXLIf.934

aXL1 1.935

aXLIf. 936

aXLIf. 937

aXLI $f .938$

aXL1 $f .939$

aXLIf .940

aXLIf .941

aXLIf. 942

aXLIf .943

aXLIf. 944

aXLIf. 945

aXLIf. 946

axLIf. 947

aXLIf. 948

aXLIf. 949

axLIf.950

axL1f.951

aXLIf. .952

aXLIf. 953

aXLIf. 954

7
7
7
7
7
7
7
7
7
7
7
7
8
7
8
7
8
7
8
8
9
8
9
8
9
7
9
7
8
7
8
8
7
8
8
7
9
9
7
8
8
8
8

Apr 31997

Apr 31997

Apr 31997

Apr 31997

Apr 31997

Apr 31997

Apr 31997

Apr 31997

Apr 31997

Apr 31997

Apr 31997

Apr 31997

Apr 31997

Apr 31997

Apr 31997

Apr 31997

Apr 31997

Apr 31997

Apr 31997

Apr 31997

Apr 31997

Apr 31997

Apr 31997

Apr 31997

Apr 31997

Apr 31997

Apr 31997

Apr 31997

Apr 31997

Apr 31997

Apr 31997

Apr 31997

Apr 31997

Apr 31997

Apr 31997

Apr 31997

Apr 31997

Apr 31997

Apr 31997

Apr 31997

Apr 31997

Apr 31997

Apr 31997

Apr 31997

Apr 31997

Apr 31997

Apr 31997

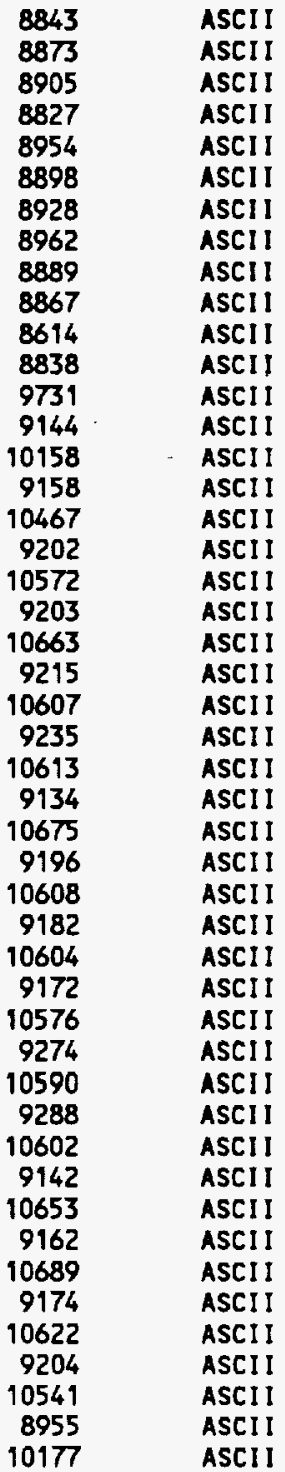

THE FOLLONING FILES FOR ASSEMBLY A26 UERE SUPERCEEDED BY FILES UNDER ASSEMBLY DESIGNATION A26Z DURING CHECKING.

\section{Computer \\ File Name}

A26/CR3A18N010C1BTO0OAC1BT142. notes A26/CR3A18N02DC1BTO0OAC1BT142.notes A26/CR3A18N03DC1BTO0OAC18T142.notes A26/CR3A18N04DC1BTOOOAC18T142.notes A26/CR3A18N05DC1BTO00AC18T142.notes A26/CR3A18N06DC1BTO00AC1BT142.notes A26/CR3A18NOTDC1BTOOOAC1BT142. notes A26/CR3A18N080C1BTO0OAC1BT142. notes A26/CR3A18NO9OC1BTOOOAC1BT142.notes A26/CR3A18N10DC1BTO00AC1BT142. notes A26/CR3A18N11DC1BTO00AC1BT142. notes A26/CR3A18N12DC1BTO00AC1BT142. notes A26/CR3A18N13DC1BT000AC1BT142. notes A26/CR3A18N14DC1BTOOOAC1BT142.notes A26/CR3A18N15DC1BTO00AC1BT142. notes A26/CR3A18N16DC1BTOOOAC1BT142. notes A26/CR3A18N170C1BT000AC1BT142. notes

\begin{tabular}{ll}
$\begin{array}{c}\text { Tape Backup } \\
\text { Fi I le Name }\end{array}$ & $\begin{array}{c}\text { Number of } \\
\text { Print Pages }\end{array}$ \\
\hline aXLIf.955 & 7 \\
aXLIf.956 & 7 \\
aXLIf.957 & 7 \\
aXLIf.958 & 7 \\
aXLIf.959 & 7 \\
aXLIf.960 & 7 \\
aXLIf.961 & 7 \\
aXLIf.962 & 7 \\
aXLIf.963 & 7 \\
aXLIf.964 & 7 \\
aXLIf.965 & 7 \\
aXLIf.966 & 7 \\
aXLIf 967 & 7 \\
aXLIf.968 & 7 \\
aXLIf.969 & 7 \\
aXLIf.970 & 7 \\
aXLIf.971 & 7
\end{tabular}

\begin{tabular}{|c|c|}
\hline $\begin{array}{c}\text { File Size } \\
\text { (Bytes) }\end{array}$ & $\begin{array}{l}\text { File Type } \\
\text { (Format) }\end{array}$ \\
\hline 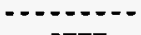 & . \\
\hline 8333 & ASCII \\
\hline 8650 & ASCII \\
\hline 8742 & ASCII \\
\hline 8922 & ASCII \\
\hline 9007 & ASCII \\
\hline 9029 & ASCII \\
\hline $\begin{array}{l}9013 \\
0070\end{array}$ & ASCII \\
\hline $\begin{array}{l}9029 \\
9015\end{array}$ & $\begin{array}{l}\text { ASCII } \\
\text { ASCIII }\end{array}$ \\
\hline 9023 & ASCII \\
\hline 8999 & ASCII \\
\hline 8916 & ASCII \\
\hline 8922 & ASCII \\
\hline 8936 & ASCII \\
\hline & ASCI I \\
\hline 8885 & ASCII \\
\hline & ASCII \\
\hline
\end{tabular}


A26/CR3A18N180C1BTO00AC1BT142.notes $\quad$ aXLIf.972 $\quad 7 \quad$ Apr $31997 \quad$ ASCII

THE FOLLONING FILES FOR ASSEMBLY A28 WERE SUPERCEEDED BY FILES UNDER ASSERBLY DESIGNATION A28Z DURING CHECKING.

Computer
Fi le Name

A28/CR3A10N01DC03TO00AC03T168. notes A28/CR3A10N01DC03T 168AC03T250.notes A28/CR3A1ON02DC03TO0OACO3T168. notes A28/CR3A1ON02DC03T 168AC03T250. notes A28/CR3A1ONO3DC03TOOOAC03T168. notes A28/CR3A10N03DC03T 168AC03T250. notes A2B/CR3A1ON04DCO3TOOOACO3T168. notes A28/CR3A10N04DC03T 168AC03T250. notes A28/CR3A10N05DC03TO0OAC03T168. notes A2B/CR3A10N05DC03T 168AC03T250. notes A2B/CR3A10N06DC03TO00AC03T168. notes A28/CR3A1ON06DC03T 168AC03T250.notes A28/CR3A1ON07DC03TO00AC03T168. notes A28/CR3A1ONOTDCO3T 168AC03T250. notes A28/CR3A10N08DC03TO00AC03T168. notes A28/CR3A10N08DC03T 168AC03T250.notes A28/CR3A10N09DCO3T000AC03T168. notes A28/CR3A10N09DC03T 168AC03T250. notes A28/CR3A1ON 10DC03TO0OAC03T168.notes A2B/CR3A10N 10DC03T168AC03T250.notes A28/CR3A1ON11DCO3TO0OAC03T168. notes A28/CR3A1ON11DC03T168AC03T250. notes A28/CR3A1ON12DC03TO00AC03T168. notes A2B/CR3A10N12DC03T168AC03T250.notes A2B/CR3A10N13DC03T000AC03T168. notes A28/CR3A1ON13DC03T168AC03T250. notes A28/CR3A10N14DCO3T000AC03T168. notes A28/CR3A10N 14DCO3T168AC03T250. notes A28/CR3A10N 15DCO3TO00AC03T 168. notes A28/CR3A10N 15DC03T168AC03T250. notes A28/CR3A10N 160C03T000AC03T168. notes A28/CR3A10N16DCO3T 168AC03T250. notes A28/CR3A1ON17DC03TO00AC03T168. notes A28/CR3A10N17DCO3T 168AC03T250. notes A28/CR3A1ON18DC03TO00AC03T168. notes A28/CR3A10N 18DC03T 168AC03T250. notes A28/CR3A18N01DC02TO00AC03T000.notes A28/CR3A18N02DC02TO00AC03T000. notes A28/CR3A18N03DC02T000AC03T000. notes A28/CR3A18N04DC02T000AC03T000. notes A28/CR3A 18N05DC02T000AC03T000. notes A28/CR3A 18N060C02TO00AC03T000. notes A28/CR3A18N0TDC02TO0OAC03TO00. notes A28/CR3A 18N08DC02TO00AC03TO00. notes A28/CR3A 18N09DC02TO00ACO3TO00. notes A28/CR3A 18N 10DC02TO00AC03T000. notes A28/CR3A18N11DC02T000AC03T000. notes A28/CR3A 18N12DC02TO00AC03T000. notes A28/CR3A18N13DC02T000AC03T000.notes A28/CR3A 18N14DC02T000AC03T000.notes A28/CR3A18N15DC02TO00ACO3TO00. notes A28/CR3A18N16DC02T000AC03T000. notes A28/CR3A18N17DC02TO00ACO3T000. notes A28/CR3A18N18DC02T000AC03T000. notes A28/CR3A28N01DC1BTOOOAC1BT142.notes A28/CR3A28N01DC1BT142AC02T000.notes A28/CR3A28N02DC1BTOOOAC1BT142.notes A28/CR3A28N02DC1BT 142ACO2T000.notes A28/CR3A28N03DC1BTO0OAC1BT142.notes A28/CR3A28N03DC1BT142AC02T000. notes A28/CR3A28N04DC1BTO00AC1BT142.notes A28/CR3A28N04DC1BT142AC02T000, notes A28/CR3A28N05DC1BTDOOAC1BT142.notes
File Name

axLIf. 973

aXLIf 974

aXL If .975

aXLIf. 976

aXLIf. 977

aXLIf .978

aXLIf. 979

aXLIf. 980

aXL If .981

aXLIf.982

aXLIf. 983

aXLIf .984

aXLIf .985

aXLIf. 986

aXLIf .987

aXLIf .988

aXLIf .989

aXLIf .990

aXLIf.991

aXLIf .992

aXLIf .993

aXLIf .994

aXLIf .995

aXLIf. 996

aXL I $f .997$

aXLIf .998

aXLIf .999

aXLI $\$ 1.000$

aXLIf1.001

aXLI 1.002

aXLI $f 1.003$

aXLI 11.004

aXLIf1.005

aXLIf1.006

aXLIf1.007

aXLIf 1.008

aXLI 1.009

aXLI $f 1.010$

aXLIf1.011

aXLI 1.012

aXLI $f 1.013$

aXLIf1.014

aXLI 1.015

aXLIf1.016

aXLI $\$ 1.017$

aXLIf 1.018

aXL $1+1.019$

aXLIf1.020

axLIf1.021

aXLIf1.022

aXLI +1.023

aXL1 1.024

aXLI 1 1.025

aXL $\$ 1.026$

aXLIf1.027

aXLIf1.028

aXL1 11.029

aXLIf1.030

aXLI $f 1.031$

aXLIf1.032

aXLI 1.033

aXLI 1.034

aXLI 1.035

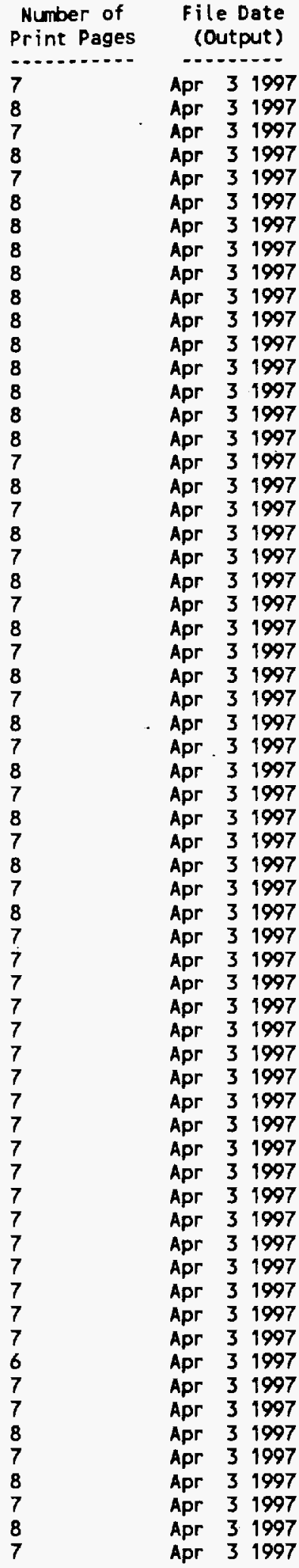

$\begin{array}{cc}\text { File Size } & \text { File Type } \\ \text { (Bytes) } & \text { (Format) }\end{array}$

-....... -.......

8841 ASCII

9841 ASCII

9166 ASCII

10282 ASCII

9157 ASCII

10343 ASCII

9307 ASCI

10411 ASCII

9242 ASCII

10587 ASCII

9252 ASCII

10589 ASCII

9244 ASCII

10519 ASCII

9264 ASCI

10447 ASCII

9118 ASCII

10487 ASCII

9166 ASCII

10529 ASCII

9156 ASCII

10471 ASCI

9144 ASCII

10541 ASCII

9188 ASCII

ASCI

ASCII

ASCII

ASCI

ASCI

ASCI

ASCI

ASCI

ASCII

ASCI

ASCI

ASCI

ASCI

ASCI

ASCII

ASCII

ASCII

ASCI

ASCII

ASCI

ASCI

ASCI

ASCI

ASCI

ASCII

ASCII

ASCI

ASCII

ASCII

ASCII

ASCII

ASCII

ASCII

8564 ASCII 
A28/CR3A28N05DC1BT 142AC02T000.notes A28/CR3A28N06DC1BTO0OAC1BT142. notes A28/CR3A28N06DC1BT 142AC02T000. notes A28/CR3A28NOTDC1BTO0OAC1BT142. notes A28/CR3A28N070C1BT142AC02T000. notes A28/CR3A28N08DC1BTOOOAC1BT142.notes A28/CR3A28N08DC1BT 142AC02T000.notes A28/CR3A28NO9DC1BTO0OAC1BT142. notes A28/CR3A28NO9DC1BT 142AC02T000.notes A28/CR3A28N10DC1BTDOOAC18T142. notes A28/CR3A28N100C1BT 142AC02T000.notes A28/CR3A28N110C1BTOOOAC1BT142. notes A28/CR3A28N11DCIBT 142ACO2T000.notes A28/CR3A28N12DC1BTO0OAC1BT 142.notes A28/CR3A2BN120C1BT 142AC02T000.notes A28/CR3A28N 13DC1BTO00AC1BT 142. notes A28/CR3A28N13DC1BT142AC02T000.notes A28/CR3A28N14DC1BTO0OAC1BT 142. notes A28/CR3A28N14DC1BT 142AC02T000.notes A28/CR3A28N 15DC1BTO00AC1BT 142.notes A28/CR3A28N15DC1BT142AC02T000. notes A28/CR3A28N160CIBTDOOAC1BT 142. notes A28/CR3A28N160C1BT 142AC02T000.notes A28/CR3A28N17DC1BTO00AC1BT142.notes A28/CR3A28N170C1BT 142AC02T000. notes A28/CR3A28N 180C1BTO00AC1BT 142. notes A28/CR3A28N 180C1BT 142AC02T000.notes

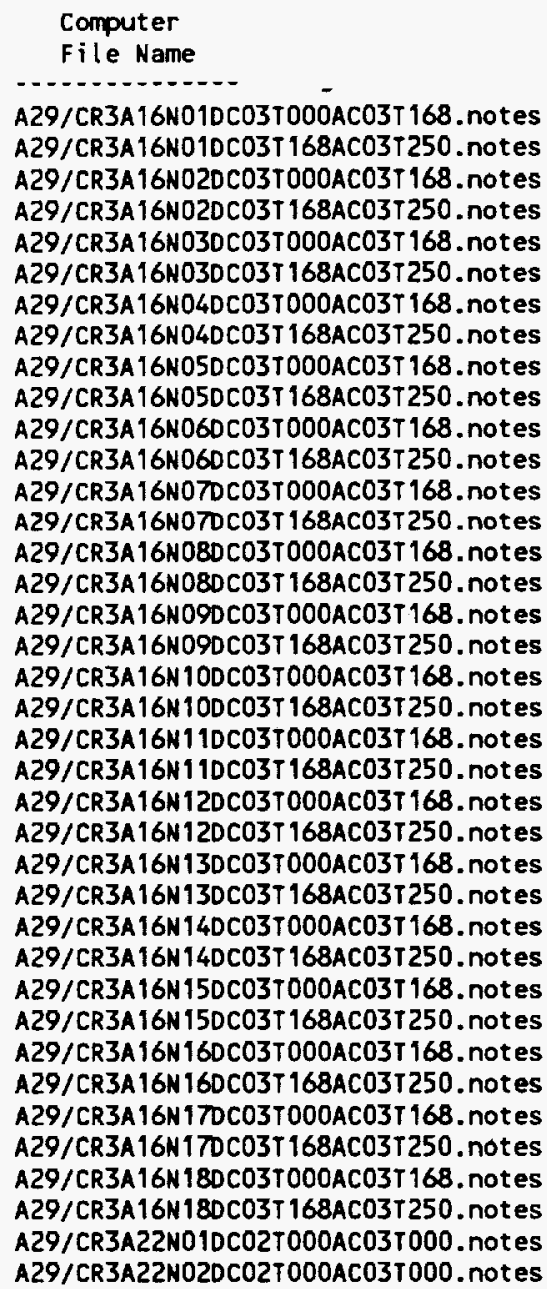

$\begin{array}{ll}\text { aXLIf } 1.036 & 8 \\ \text { aXLIf } 1.037 & 7 \\ \text { aXLIf } 1.038 & 8 \\ \text { aXLIf } 1.039 & 7 \\ \text { aXLIf1.040 } & 8 \\ \text { aXLIf } 1.041 & 7 \\ \text { aXLIf } 1.042 & 8 \\ \text { aXLIf } 1.043 & 7 \\ \text { aXLIf } 1.044 & 8 \\ \text { aXLIf } 1.045 & 7 \\ \text { aXLIf } 1.046 & 8 \\ \text { aXLIf } 9.047 & 7 \\ \text { aXLIf } 1.048 & 8 \\ \text { aXLIf } 1.049 & 7 \\ \text { aXLIf } 1.050 & 8 \\ \text { aXLIf } 1.051 & 7 \\ \text { aXLIf } 1.052 & 8 \\ \text { aXLIf } 1.053 & 7 \\ \text { aXLIf } 1.054 & 8 \\ \text { aXLIf } 1.055 & 7 \\ \text { aXLIf } 1.056 & 8 \\ \text { aXLIf } 1.057 & 7 \\ \text { aXLIf } 1.058 & 8 \\ \text { aXLIf } 1.059 & 7 \\ \text { aXLIf } 1.060 & 8 \\ \text { aXLIf } 1.061 & 7 \\ \text { aXLIf } 1.062 & 8\end{array}$

aXLIf1.062

\begin{tabular}{|c|c|}
\hline $\begin{array}{c}\text { Tape Backup } \\
\text { File Name }\end{array}$ & $\begin{array}{l}\text { Number of } \\
\text { Print Pages }\end{array}$ \\
\hline & \\
\hline aXLIf1.063 & 7 \\
\hline aXLIf 1.064 & 8 \\
\hline aXLIf 1.065 & 7 \\
\hline$a X L I f 1.066$ & 8 \\
\hline aXLI $\$ 1.067$ & 7 \\
\hline$a X L I f 1.068$ & 8 \\
\hline aXLI $f 1.069$ & 8 \\
\hline aXLI 1.070 & 8 \\
\hline aXLI f 1.071 & 8 \\
\hline aXLI f 1.072 & 8 \\
\hline aXLIf 9.073 & 8 \\
\hline aXLIf1.074 & 8 \\
\hline aXLI 11.075 & 8 \\
\hline aXLI I 1.076 & 8 \\
\hline aXLIf 1.077 & 8 \\
\hline aXLIf 1.078 & 8 \\
\hline aXLIf 1.079 & 7 \\
\hline aXLI I 1.080 & 8 \\
\hline aXLIf 1.081 & 7 \\
\hline aXLIf 1.082 & 8 \\
\hline aXLIf 9.083 & 7 \\
\hline aXLIf 1.084 & 8 \\
\hline aXLI 11.085 & 7 \\
\hline aXLIf1.086 & 8 \\
\hline aXLI 19.087 & 7 \\
\hline $\mathrm{aXLIf1.088}$ & 8 \\
\hline aXLIf 1.089 & 7 \\
\hline $\mathrm{aXLIf1.090}$ & 8 \\
\hline aXLIf1.091 & 7 \\
\hline aXLIf 1.092 & 8 \\
\hline aXLI 19.093 & 7 \\
\hline aXLIf 1.094 & 8 \\
\hline aXLI f 9.095 & 7 \\
\hline aXLIf 1.096 & 8 \\
\hline aXLIf 1.097 & 7 \\
\hline aXLIf 1.098 & 8 \\
\hline aXLIf 1.099 & 7 \\
\hline aXLIf 1.100 & 7 \\
\hline
\end{tabular}

$\begin{array}{rr}10037 & \text { ASCII } \\ 8578 & \text { ASCII } \\ 9971 & \text { ASCII } \\ 8604 & \text { ASCII } \\ 9991 & \text { ASCII } \\ 8623 & \text { ASCII } \\ 9959 & \text { ASCII } \\ 8625 & \text { ASCII } \\ 10027 & \text { ASCII } \\ 8594 & \text { ASCII } \\ 9973 & \text { ASCII } \\ 8648 & \text { ASCII } \\ 9935 & \text { ASCII } \\ 8590 & \text { ASCII } \\ 9987 & \text { ASCII } \\ 8594 & \text { ASCII } \\ 9983 & \text { ASCII } \\ 8588 & \text { ASCI I } \\ 10039 & \text { ASCII } \\ 8540 & \text { ASCII } \\ 10031 & \text { ASCII } \\ 8538 & \text { ASCII } \\ 10023 & \text { ASCII } \\ 8513 & \text { ASCII } \\ 9943 & \text { ASCII } \\ 8379 & \text { ASCII } \\ 9811 & \text { ASCII }\end{array}$

\begin{tabular}{|c|c|}
\hline $\begin{array}{c}\text { File size } \\
\text { (Bytes) }\end{array}$ & $\begin{array}{l}\text { File Type } \\
\text { (Format) }\end{array}$ \\
\hline - & - \\
\hline $\begin{array}{l}8747 \\
9559\end{array}$ & $\begin{array}{l}\text { ASCII } \\
\text { ASCII }\end{array}$ \\
\hline 9010 & ASCII \\
\hline 10032 & ASCII \\
\hline 8973 & ASCII \\
\hline 10040 & ASCII \\
\hline 9060 & ASCII \\
\hline 10042 & ASCII \\
\hline 9016 & ASCII \\
\hline 10153 & ASCII \\
\hline 9026 & ASCII \\
\hline 10155 & ASCII \\
\hline 9026 & ASCII \\
\hline 10157 & ASCII \\
\hline 9026 & ASCII \\
\hline 10175 & ASCII \\
\hline 8984 & ASCII \\
\hline 10077 & ASCII \\
\hline 9014 & ASCII \\
\hline 10018 & ASCII \\
\hline 8960 & ASCII \\
\hline 10036 & ASCII \\
\hline 8964 & ASCII \\
\hline 10038 & ASCII \\
\hline 8970 & ASCII \\
\hline 10113 & ASCII \\
\hline 8964 & ASCII \\
\hline 10131 & ASCII \\
\hline 8996 & ASCII \\
\hline 10165 & ASCII \\
\hline 8950 & ASCII \\
\hline 10237 & ASCII \\
\hline 8988 & ASCI I \\
\hline 10105 & ASCII \\
\hline 8801 & ASCII \\
\hline 9997 & ASCII \\
\hline 8187 & ASCII \\
\hline 8398 & ASCII \\
\hline
\end{tabular}


A29/CR3A22N030C02TOOOAC03TO0O.notes A29/CR3A22N 04DCO2TOOOACO3TO0O.notes A29/CR3A22N05DC02TOOOACO3TO0O. notes A29/CR3A22N060CO2TOOOACO3TOOO. notes A29/CR3A2ZNOTDCO2TOOOAC03TO0O. notes A29/CR3A22NNOBOCO2TOOOACO3TO00. notes A29/CR3A22N090CO2TOOOACO3TOOO. notes A29/CR3A22N100CO2TOOOACO3TO0O. notes A29/CR3A22N110C02TOOOACO3T000. notes A29/CR3A22N12DC02TOOOACO3TO00. notes A29/CR3A22N130C02TO0OACO3TO00. notes A29/CR3A22N140C02TOOOACO3TO00. notes A29/CR3A22N150C02TO0OACO3TO00. notes A29/CR3A22N160C02TO00AC03T000. notes A29/CR3A22N170C02TO0OACO3TO00. notes A29/CR3A22N180C02TO00AC03T000. notes A29/CR3A29N01DC1BTODOAC1BT142. notes A29/CR3A29N010C1BT 142ACO2TO00. notes A29/CR3A29N020C1BTODOAC1BT142. notes A29/CR3A29N02DC1BT142ACO2T000.notes A29/CR3A29N03DC1BT 000AC1BT142. notes A29/CR3A29N03DC1BT 142AC02T000. notes A29/CR3A29N04DC1BTO0OAC1BT142. notes A29/CR3A29N04DC1BT 142AC02TO00. notes A29/CR3A29N05DC1BTDO0AC1BT142. notes A29/CR3A29N05DC1BT 142AC02T000. notes A29/CR3A29N060C1BTO00AC1BT142. notes A29/CR3A29N060C1BT 142AC02T000. notes A29/CR3A29N07DC1BTO00AC1BT142. notes A29/CR3A29N07DC1BT 142AC02T000. notes A29/CR3A29N080C1BTO00AC1BT142. notes A29/CR3A29N08DC1BT142AC02T000. notes A29/CR3A29N090C1BTO00AC1BT142. notes A29/CR3A29N090C1BT142AC02T000. notes A29/CR3A29N10DC1BTO00AC 1BT 142. notes A29/CR3A29N10DC1BT142AC02T000. notes A29/CR3A29N11DC1BTO00AC1BT142.notes A29/CR3A29N11DC1BT142AC02T000. notes A29/CR3A29N12DC1BT000AC1BT142. notes A29/CR3A29N12DC1BT 142AC02T000. notes A29/CR3A29N13DC1BTO00AC1BT142. notes A29/CR3A29N13DC1BT142AC02T000. notes A29/CR3A29N14DC1BTO00AC1BT142. notes A29/CR3A29N14DC1BT 142AC02T000. notes A29/CR3A29N 15DC1BTO00AC1BT142. notes A29/CR3A29N 15DC1BT142AC02t000. notes A29/CR3A29N16DC18T000AC1BT142. notes A29/CR3A29N16DC1BT142AC02T000. notes A29/CR3A29N17DC1BTO00AC1BT142. notes A29/CR3A29N17DC1BT 142AC02TO00. notes A29/CR3A29N18DC1BTO00AC1BT142. notes A29/CR3A29N18DC1BT 142AC02T000. notes

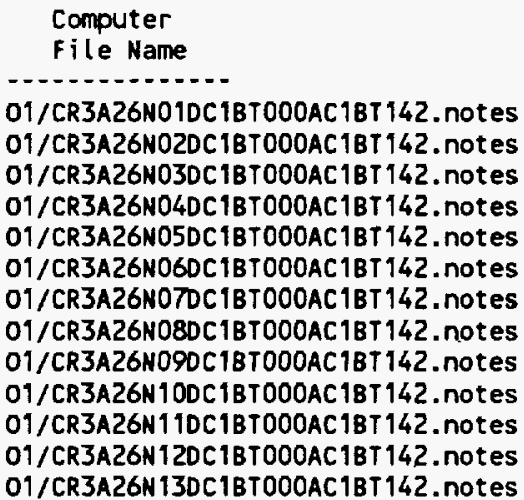

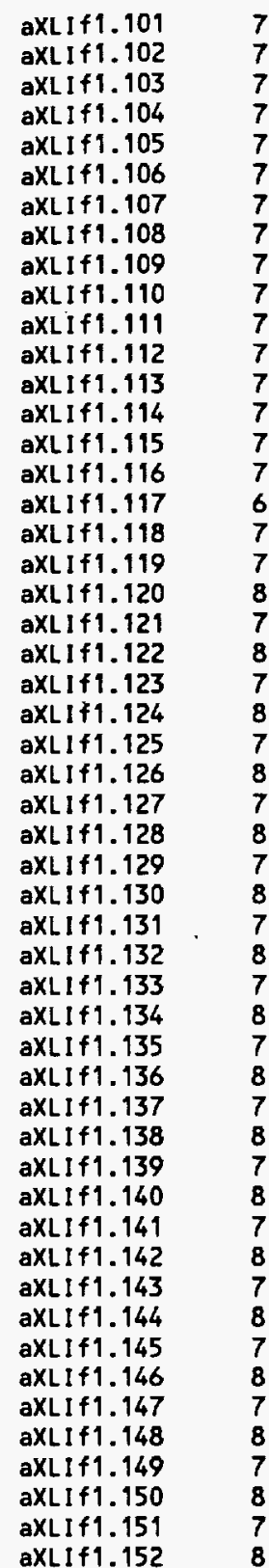

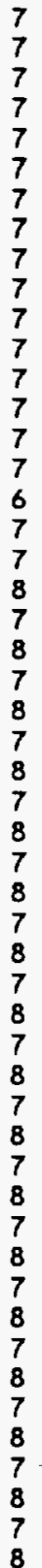

Apr 31997

Apr 31997

Apr 31997

Apr 31997

Apr 31997

Apr 31997

Apr 31997

Apr 31997

Apr 31997

Apr 31997

Apr 31997

Apr 31997

Apr 31997

Apr 31997

Apr 31997

Apr 31997

Apr 31997

Apr 31997

Apr 31997

Apr 31997

Apr 31997

Apr 31997

Apr 31997

Apr 31997

Apr 31997

Apr 31997

Apr 31997

Apr 31997

Apr 31997

Apr 31997

Apr 31997

Apr 31997

Apr 31997

Apr 31997

Apr 31997

Apr 31997

Apr 31997

Apr 31997

Apr 31997

Apr 31997

Apr 31997

Apr 31997

Apr 31997

Apr 31997

Apr 31997

Apr 31997

Apr 31997

Apr 31997

Apr 31997

Apr 31997

Apr 31997
Apr 31997

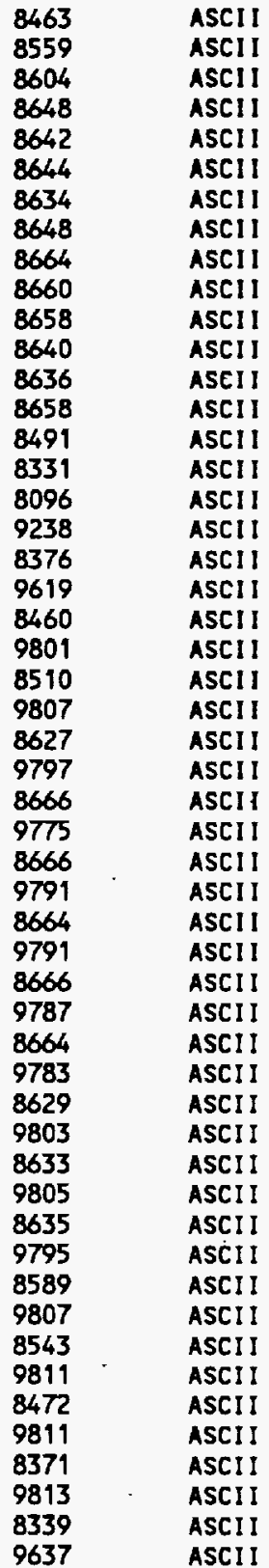

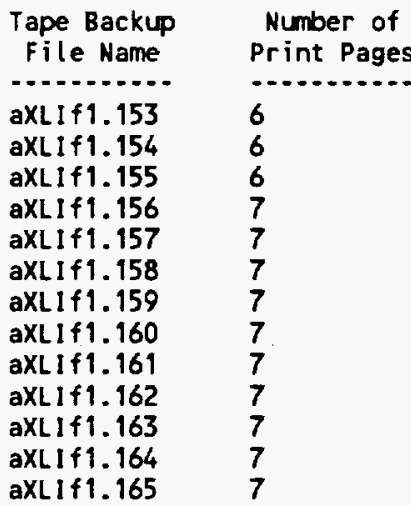

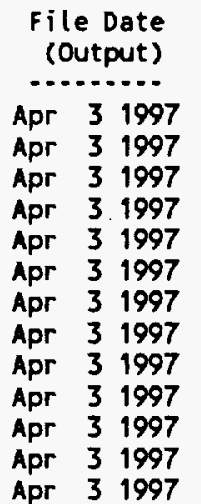

\begin{tabular}{cc}
$\begin{array}{c}\text { File size } \\
\text { (Bytes) }\end{array}$ & $\begin{array}{c}\text { File Type } \\
\text { (Format) }\end{array}$ \\
\hdashline$. \ldots . .$. & …..... \\
7435 & ASCII \\
7756 & ASCII \\
7757 & ASCII \\
7836 & ASCII \\
7904 & ASCII \\
8109 & ASCII \\
8140 & ASCII \\
8230 & ASCII \\
8230 & ASCII \\
8176 & ASCII \\
8180 & ASCII \\
8174 & ASCII \\
8230 & ASCII
\end{tabular}


01/CR3A26N14DC1BTO00AC1BT142. notes 01/CR3A26N15DC1BT000AC1BT142. notes 01/CR3A26N16DC1BTO00AC1BT142. notes 01/CR3A26N17DC1BTO0OAC1BT 142. notes 01/CR3A26N180C1BTO0OAC1BT142. notes

\begin{tabular}{l} 
Computer \\
File Name \\
\hline A
\end{tabular} A18z/CR3A01N01DC08T097AC08T139. notes A $18 z$ /CR3A01N01DC08T139AC08T404. notes A 182/CR3A01N01DC08T404AC08T409.notes A 18z/CR3A01N01DC08T409AC08T515. notes A18z/CR3A01N020C08TO00AC08T097. notes A18z/CR3A01N02DC08T097AC08T139. notes A 18z/CR3A01N02DC08T 139AC08T404. notes A $18 z$ /CR3AO1N02DC08T404AC08T409. notes A 182/CR3A01N020C08T409AC08T515. notes A 18z/CR3A01N03DC08TO0OAC08T097. notes A 182/CR3A01N03DC08T097AC08T139. notes A 18z/CR3A01N03DC08T139AC08T404. notes A18z/CR3A01N03DC08T404AC08T409. notes A18z/CR3A01N030C08T409AC08T515. notes A18z/CR3A01N04DC08TO0OAC08T097. notes A 18z/CR3A01N040C08T097AC08T139. notes A18z/CR3A01N04DC08T 139AC08T404 notes A 18z/CR3A01N04DC08T404AC08T409. notes A 18z/CR3A01N04DC08T409AC08T515. notes A 18z/CR3A01N05DC08T000AC08T097. notes A 18z/CR3A01N05DC08T027AC08T139. notes A 18z/CR3A01N05DC08T139AC08T404. notes A18z/CR3A01N05DC08T404AC08T409. notes A18z/CR3A01N05DC08T409AC08T515. notes A18z/CR3A01N060C08T000AC08T097. notes A18z/CR3A01N06DC08T097AC08T139. notes A 18z/CR3A01N060C08T139AC08T404. notes A18z/CR3A01N060C08T404AC08T409.notes A 18Z/CR3A01N060C08T409AC08T515.notes A18z/CR3A01N07DC08TO00AC08T097.notes A18z/CR3A01N07DC08T097AC08T139.notes A 18z/CR3A01N070C08T 139AC08T404.notes A 18z/CR3A01N0TDC08T404AC08T409.notes A 18z/CR3A01N070C08T409AC08T515. notes A 18z/CR3A01N08DC08TOOOAC08T097.notes A18Z/CR3A01N080C08T097AC08T139. notes A182/CR3A01N08DC08T139AC08T404.notes A 18Z/CR3A01N08DC08T404AC08T409. notes A18z/CR3A01N08DC08T409AC08T515. notes A18z/CR3A01N090C08T000AC08T097. notes A18z/CR3A01N090C08T097AC08T139. notes A18z/CR3A01N09DC08T 139AC08T404. notes A18z/CR3A01N090C08T404AC08T409. notes A18z/CR3A01N090C08T409AC08T515. notes A 18z/CR3A01N10DC08T000AC08T097. notes A18z/CR3A01N100C08T097AC08T139. notes A18z/CR3A01N10DC08T139AC08T404. notes A18z/CR3A01N10DC08T404AC08T409. notes A18z/CR3A01N100C08T409AC08T515. notes A18z/CR3A01N1 10C08T000AC08T097. notes A 18z/CR3A01N1 1DC08T097AC08T139. notes A18z/CR3A01N11DC08T139ACO8T404. notes A18z/CR3A01N110C08T404AC08T409. notes A18z/CR3A01N11DC08T409AC08T515. notes A 18z/CR3A01N120C08T000AC08T097. notes A 182/CR3A01N12DC08T097AC08T139. notes A 18z/CR3A01N12DC08T 139AC08T404.notes A 18z/CR3A01N120C08T404AC08T409. notes A18z/CR3A01N12DC08T409AC08T515. notes

aXLIf1.166
aXLIf1.167
aXLIf1.168
aXLIf1.169
aXLIf1.170

7
7
7
7
7

Apr 31997

Apr 31997

Apr 31997

Apr 31997

Apr 31997

8180
8140
8066
7918
7800

ASCII

ASCI

ASCII

ASCII

ASCII

\begin{tabular}{l} 
Tape Backup \\
File Name \\
\hline axtif1.171.
\end{tabular}

Number of
Print Pages
-6
8
8
8
8
6
8
8

\section{File Date \\ (Output)}

axLIf1.171

aXLIf1.172

aXL1f1.173

aXLIf9.174

aXL1f1.175

aXLI 11.176

aXLI I 1.177

aXLI I 1.178

aXLI 19.179

aXLI 191.180

aXLIf1.181

aXLIf1.182

aXLI $f 1.183$

aXLIf1.184

aXLI 1.185

aXLIf1.186

aXLI 1.187

aXLIf1.188

aXL1 1 1. 189

aXLIf1.190

aXLIf1.191

axL $1+1.192$

aXLIf1.193

aXLIf1.194

aXLI I 1.195

aXL If 1.196

aXL If 1.197

aXLIf1. 198

aXL If 1.199

aXLIf 1.200

aXLIf1.201

aXLIf 1.202

aXL If 1.203

aXLIf1.204

aXL If 1.205

aXLIf1.206

aXLIf1.207

aXL If 1.208

aXLIf1.209

aXLIf 1.210

aXLIf1.211

aXL If 1.212

aXLIf1.213

aXLIf1.214

aXLIf 1.215

aXLIf 1.216

aXL If 1.217

aXLIf1.218

aXL If1.219

aXLIf 1.220

aXL If 1.221

aXLIf1.222

aXLIf1.223

aXLIf1.224

aXLIf1.225

aXLI 1.226

aXLIf1.227

aXL I 1.228

aXL If1.229

aXL I +1.230

\section{Jul $17 \quad 1997$}

Jul 171997

Jul 171997

Jul 171997

Jul 171997

Jul 171997

Jul 171997

Jul 171997

Jul 171997

Jul 171997

Jul 179997

Jul 171997

Jul 171997

Jul 171997

Jul 171997

Jul 171997

Jul 171997

Jul 171997

Jul 171997

Jul 171997

Jul 171997

Jul 171997

Jul 171997

Jul 171997

Jul 171997

Jul 171997

Jul 171997

Jul 171997

Jul 171997

Jul 171997

Jul 171997

Jul 171997

Jul 171997

Jul 171997

Jul 171997

Jul 171997

Jul 171997

Jul 171997

Jul 171997

Jul 171997

Jul 171997

Jul 171997

Jul 171997

Jul 171997

Jul 171997

Jul 171997

Jul 171997

Jul 171997

Jul $17 \quad 1997$

Jul 171997

Jul $17 \quad 1997$

Jul 171997

Jul $18 \quad 1997$

Jul $18 \quad 1997$

Jul $18 \quad 1997$

Jul $18 \quad 1997$

Jul 181997

Jul $18 \quad 1997$

Jul 181997

Jul 181997

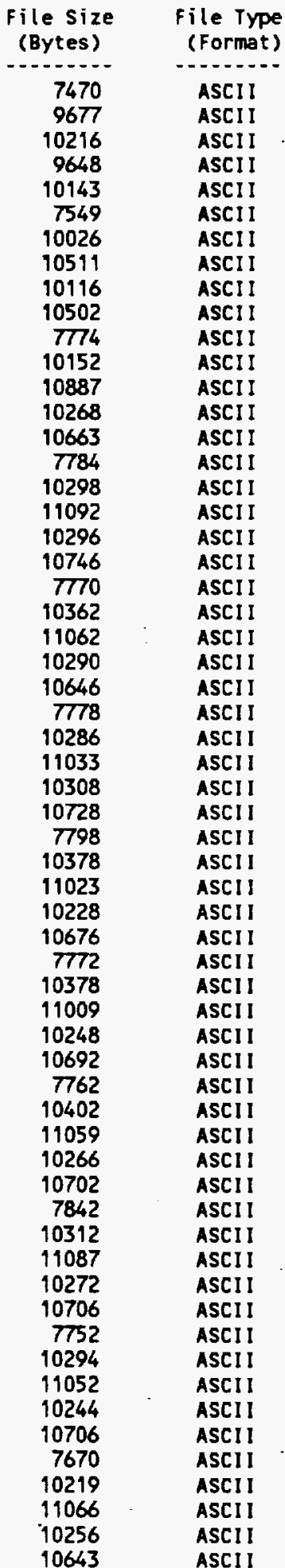


A18Z/CR3A01N13DC08T000AC08T097. notes A18Z/CR3A01N13DC08T097AC08T139. notes A182/CR3A01N13DC08T139AC08T404. notes A182/CR3A01N13DC08T404AC08T409. notes A182/CR3A01N13DC08T409AC08T515.notes A18z/CR3A01N14DC08T000AC08T097.notes A18z/CR3A01N14DC08T097AC08T 139.notes A 18z/CR3A01N14DCO8T 139AC08T404.notes A182/CR3A01N14DC08T404AC08T409.notes A18z/CR3A01N140CO8T409AC08T515.notes A18z/CR3A01N15DC08T000AC08T097. notes A182/CR3A01N15DC08T097AC08T 139.notes A182/CR3A01N15DC08T 139AC08T404.notes A182/CR3A01N15DC08T404AC08T409. notes A18Z/CR3A01N15DC08T409AC08T515. notes A18z/CR3A01N160C08T000AC08T097. notes A18Z/CR3A01N160CO8T097AC08T 139. notes A18z/CR3A01N160COBT 139ACOBT404 notes A18z/CR3A01N160COBT404AC08T409.notes A18z/CR3A01N160C08T409AC08T515. notes A18z/CR3AO1N1TDCOBTOODAC08T097. notes A18z/CR3A01N1TDCOBT097AC08T139. notes A18z/CR3A01N170COBT 139AC08T404.notes A18Z/CR3A01N170C08T404AC08T409. notes A18z/CR3A01N170COBT409AC08T515. notes A182/CR3A01N180C08TO00AC08T097. notes A18z/CR3A01N180COBT097AC08T139. notes A18z/CR3A01N180COBT 139AC08T404.notes A18z/CR3A01N180C08T404AC08T409.notes A 18z/CR3A01N180C081409AC08T515. notes

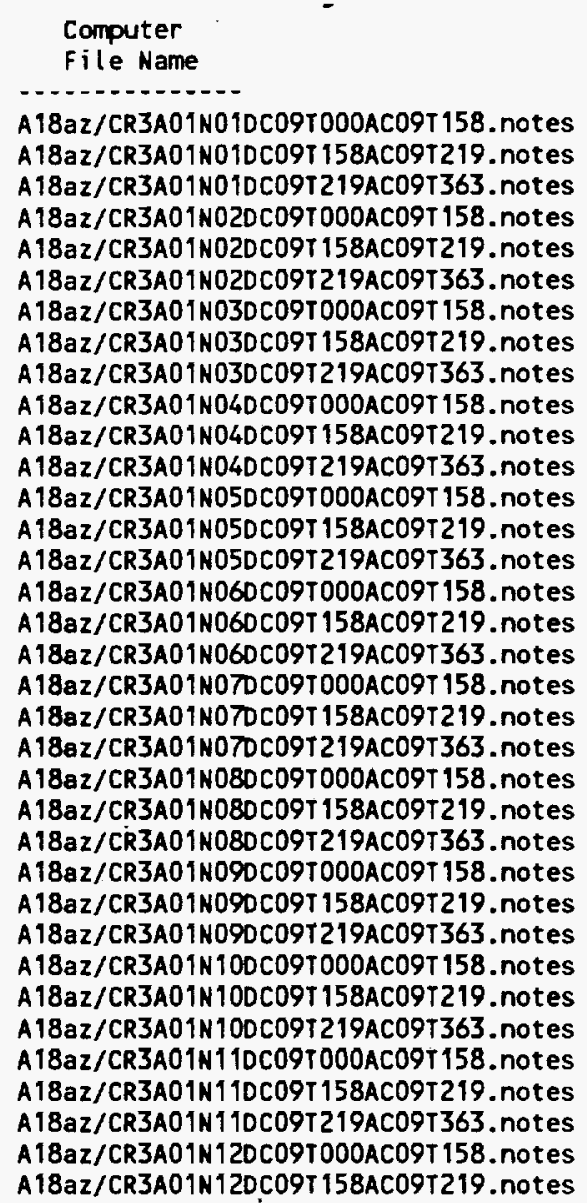

aXLI $f 1.231$

aXLI 1.232

aXLI +1.233

aXL $1+1.234$

aXL $1+1.235$

aXL $1+1.236$

aXLI 1.237

QXLI 11.238

aXL I 11.239

aXLI +1.240

aXL If 1.241

aXLIf1.242

aXL I 1.243

axLIf1.244

aXL I 1.245

aXL $1+1.246$

aXL $1+1.247$

aXL If 1.248

aXLIf1.249

aXLI $f 1.250$

aXLIf1.251

aXLI $f 1.252$

aXLI +1.253

aXL1f1.254

aXLI 1 1.255

aXLIf1.256

aXL I $f 1.257$

aXLIf1.258

aXL1 1 1.259

aXLI 1.260

Tape Backup File Name

aXLIf1.261

aXLIf1.262

aXLI 11.263

aXLI $f 1.264$

aXL I $f 1.265$

aXLIf 1.266

aXLI I 1.267

aXLI 1 1.268

aXLIf1.269

aXL I 1.270

aXLI 1.271

aXLI 1 1.272

aXLIf1.273

aXL I 1.274

aXLIf1.275

aXL $1+1.276$

aXL I 1.277

aXLI 1.278

aXLI 1.279

aXLI 11.280

aXL $1 f 1.281$

aXLI 1.282

aXL I 1 1.283

aXLI 1.284

aXL I 1.285

aXLIf 1.286

aXLIf1.287

aXLIf1.288

axL $1+1.289$

aXL $1+1.290$

aXLIf1.291

aXL $t f 1.292$

aXLIf1.293

aXLIf1.294

aXL I $f 1.295$

6
6
8
9
8
9
6
8
9
8
9
6
8
9
8
9
6
8
9
8
9
6
8
9
8
8
6
8
8
8
8

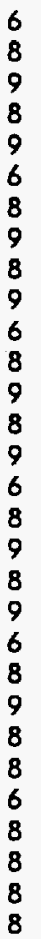

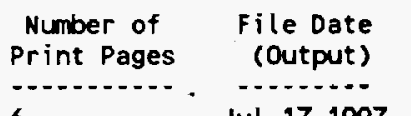

Jul 171997

Jul 171997

Jul 171997

Jul 171997

Jul 171997 .

Jul 171997

Jul 171997

Jul 171997

Jul 171997

Jul 171997

Jul 171997

Jul 171997

Jul 171997

Jul 171997

Jul 171997

Jul 171997

Jul 171997

Jul 171997

Jul 171997

Jul 171997

Jul 171997

Jul 171997

Jul 171997

Jul 171997

Jul 171997

Jul 171997

Jul 171997

Jul 171997

Jul 171997

Jul 171997

Jul 171997

Jul 171997

Jul 171997

Jul 171997

Jul 171997
7549

10215

10953

10252

10678

7500

10166

10928

10202

10708

7607

10138

10918

10218

10638

7653

10092

10905

10256

10740

7633

10099

10749

10143

10508

7476

9857

10322

9806

10315

File size

(Bytes)

(B)

7338

11219

8894

7400

11744

9245

7684

11990

9482

7690

11964

9563

7678

12133

9602

7656

.12141

9586

7644

12151

9586

7650

12145

9592

7646

12143

9594

7690

12133

9602

7696

12055

9563

7532

12002
ASCII

ASCII

ASCII

ASCII

ASCII

ASCII

ASCII

ASCII

ASCII

ASCII

ASCII

ASCII

ASCII

ASCII

ASCI!

ASCII

ASCII

ASCII

ASCII

ASCII

ASCII

ASCII

ASCII

ASCII

ASCII

ASCII

ASCII

ASCII

ASCII

ASCII

File Type

(Format)

ASCII

ASCII

ASCII

ASCII

ASCII

ASCII

ASCII

ASCII

ASCII

ASCII

ASCII

ASCII

ASCII

ASCII

ASCII

ASCII

ASCII

ASCII

ASCII

ASCII

ASCI I

ASCI I

ASCII

ASCII

ASCII

ASCII

ASCII

ASCII

ASCII

ASCII

ASCII

ASCII

ASCII

ASCII

ASCII 
A 18az/CR3A01N120C09T219AC09T363.notes A 18az/CR3A01N130CO9TOOOACO9T158. notes A18az/CR3A01N13DC09T158AC09T219.notes A 18az/CR3A01N13DC09T219ACO9T363. notes A18az/CR3A01N14DCO9TO00ACO9T158. notes A 18az/CR3A01N140CO9T158ACO9T219. notes A 18az/CR3A01N140CO9T219ACO9T363. notes A18az/CR3A01N150CO9TOOOACO9T158. notes A18az/CR3A01N150CO9T158ACO9T219. notes A18az/CR3A01N150C09T219AC09T363. notes A18az/CR3A01N160C09T000AC09T158. notes A18az/CR3A01N160C09T158AC09T219. notes A18az/CR3A01N16DC09T219AC09T363. notes A18az/CR3A01N170C09TO00AC09T158. notes A18az/CR3A01N170C09T158AC09T219. notes A18az/CR3A01N17DC09T219AC09T363. notes A18az/CR3A01N18DC09T000ACO9T158. notes A18az/CR3A01N180C09T158AC091219. notes A18az/CR3A01N18DC09T219AC09T363. notes

\section{Comouter \\ File Name}

A18bZ/CR3A18N01DC1BT000AC1BT142. notes A18bz/CR3A18N02DC1BTO0OAC1BT142. notes A18bz/CR3A18N03DC18T000AC1BT142. notes A18bz/CR3A18N04DC18TO00AC1BT142. notes A 18bz/CR3A18N05DC1BT000AC1BT142. notes A 18bz/CR3A18N06DC18T000AC1BT142. notes A18bz/CR3A18N07DC1BTO0OAC1BT 142. notes A18bz/CR3A18N08DC1BT000AC1BT142.notes A 18bz/CR3A18N090C1BT000AC1BT142. notes A 18bz/CR3A18N10DC1BT000AC1BT 142. notes A18bz/CR3A18N1 1DC1BTO0OAC1BT 142. notes A 18bz/CR3A18N12DC1BT000AC1BT142. notes A 18bz/CR3A18N130C1BTO0OAC1BT142. notes A 18bz/CR3A18N14DC1BTO00AC1BT142. notes A 18b2/CR3A18N15DC1BTO0OAC18T142.notes A18bz/CR3A18N160C1BTO00AC1BT 142.notes A18bz/CR3A18N17DC1BTO0OAC1BT142.notes A 18bz/CR3A18N180C1BTOOOAC1BT 142. notes
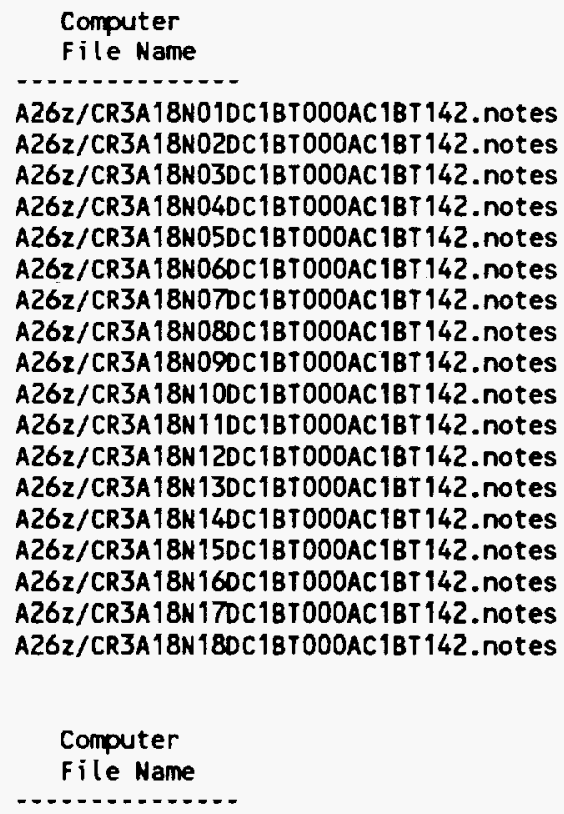

aXLI 11.296

aXLI 11.297

aXLI +1.298

aXLI 1.299

aXLI 19.300

QXLI 1 1.301

aXLI +1.302

aXLI 1.303

aXLIf1.304

aXLIf 1.305

aXLI 1.306

aXLI 19.307

aXLI 1 1.308

aXLI 1.309

aXLI 1.310

aXLI 1 1.311

aXL $1+1.312$

aXLIf 1.313

aXLI 1.314

Tape Backup file Name

aXLIf1.315

aXLI 1 1.316

aXL 1 f 1.317

aXLIf1.318

aXLI 1 1.319

aXLI 1 1.320

aXLI 19.321

aXL $1 \mathrm{f} 1.322$

aXL $1+1.323$

aXL1 11.324

aXLIf1.325

aXL $1+1.326$

aXLIf1.327

aXL $1+1.328$

aXLIf1.329

aXLIf1.330

aXL1f1.331

aXL $1+1.332$

Tape Backup File Name ..........

aXL $1+1.333$

aXLIf1.334

aXL If 1.335

aXLI 11.336

aXLI 191.337

aXLIf1.338

aXL I 9.339

aXLI I 1.340

aXLI 1.341

aXLI 1.342

aXLI 11.343

aXLI 1.344

aXLI 11.345

aXLIf1.346

axLIf1.347

aXLI I 1.348

aXLIf1.349

aXLI 1 1.350

Tape Backup.

File Name

8
6
10
8
6
10
8
6
10
8
6
10
8
6
9
8
6
9
7

8
6
10

6

10

10

8

10

8

8

6

7

Number of
Print Pages
-7
7
7
7
7
7
7
7
7
7
7
7
7
7
7
7
7
7

File Date
(Output)

July 171997

July 171997

July 171997

July 171997

July 171997

July 171997

July 171997

July 171997

July 171997

July 171997

July 171997

July 171997

July 171997

July 171997

July 171997

July 181997

July 181997

July 181997

\begin{tabular}{l} 
Number of \\
Print Pages \\
\hline 7 \\
7 \\
7 \\
7 \\
7 \\
7 \\
7 \\
7 \\
7 \\
7 \\
7 \\
7 \\
7 \\
7 \\
7 \\
7 \\
7 \\
7
\end{tabular}

File Date (Output)

July 171997 July 171997 July 171997 July 171997 July 171997 July 171997 July 171997 July 171997 July $17 \quad 1997$ July 171997 July 171997 July 171997 July 171997 July 171997

July 171997

July 181997

July 181997

July 181997

$\begin{array}{rr}9531 & \text { ASCII } \\ 7449 & \text { ASCII } \\ 11916 & \text { ASCII } \\ 9430 & \text { ASCII } \\ 7461 & \text { ASCII } \\ 11920 & \text { ASCII } \\ 9440 & \text { ASCII } \\ 7449 & \text { ASCII } \\ 11928 & \text { ASCII } \\ 9380 & \text { ASCII } \\ 7582 & \text { ASCII } \\ 11980 & \text { ASCII } \\ 9434 & \text { ASCII } \\ 7584 & \text { ASCII } \\ 11890 & \text { ASCII } \\ 9365 & \text { ASCII } \\ 7368 & \text { ASCII } \\ 11413 & \text { ASCII } \\ 9005 & \text { ASCII }\end{array}$

\begin{tabular}{cc}
$\begin{array}{c}\text { File Size } \\
\text { (Bytes) }\end{array}$ & $\begin{array}{r}\text { File Type } \\
\text { (Format) }\end{array}$ \\
\hline 8554 & ASCII \\
8813 & ASCII \\
9094 & ASCII \\
9183 & ASCII \\
9133 & ASCII \\
9177 & ASCII \\
9159 & ASCII \\
9129 & ASCII \\
9127 & ASCII \\
9205 & ASCII \\
9165 & ASCII \\
8883 & ASCII \\
8791 & ASCII \\
8801 & ASCII \\
8875 & ASCII \\
8943 & ASCII \\
8969 & ASCII \\
8804 & ASCII
\end{tabular}

\begin{tabular}{cc}
$\begin{array}{c}\text { File Size } \\
\text { (Bytes) }\end{array}$ & $\begin{array}{c}\text { File Type } \\
\text { (Format) }\end{array}$ \\
\hdashline 8427 & ASCII \\
8744 & ASCII \\
8860 & ASCII \\
9102 & ASCII \\
9127 & ASCII \\
9155 & ASCII \\
9185 & ASCII \\
9163 & ASCII \\
9181 & ASCII \\
9137 & ASCII \\
9187 & ASCII \\
9048 & ASCII \\
9092 & ASCII \\
9066 & ASCII \\
9110 & ASCII \\
9017 & ASCII \\
8832 & ASCII \\
8648 & ASCII \\
& ASCII
\end{tabular}

File size (Bytes)

File Type

Number of File Date

Print Pages (Output) 
A28Z/CR3A10N01DC03T000ACO3T168.notes A28Z/CR3A10N01DCO3T168AC03T250.notes A282/CR3A10N02DC03TOOOACO3T168. notes A28Z/CR3A10N02DCO3T 168ACO3T250. notes A28z/CR3A10N03DC03TOOOACO3T 168. notes A282/CR3A10N03DC03T168ACO3T250. notes A28Z/CR3A10N04DCO3TOOOACO3T 168. notes A28Z/CR3A10N04DC03T168ACO3T250. notes A28Z/CR3A10N05DC03T000AC03T168. notes A28z/CR3A10N05DC03T 168ACO3T250.notes A28z/CR3A10N060C03TO0OAC03T168. notes A28Z/CR3A10N06DC03T168AC03T250. notes A28z/CR3A 10N07DC03TO00AC03T168. notes A28Z/CR3A 1ON07DC03T168AC03T250. notes A28Z/CR3A 1ON08DC03TO0OAC03T 168. notes A282/CR3A10N08DC03T168AC03T250. notes A282/CR3A 10N090C03T000AC03T168. notes A28Z/CR3A10N09DC03T168AC03T250. notes A282/CR3A10N10DC03T000AC03T168. notes A282/CR3A10N10DC03T168AC03T250. notes A28Z/CR3A1ON11DC03TO00AC03T168. notes A282/CR3A10N11DC03T168AC03T250. notes A282/CR3A1ON12DCO3TO0OACO3T168. notes A28Z/CR3A1ON12DCO3T168ACO3T250.notes A28z/CR3A10N13DCO3TO00AC03T168.notes A28Z/CR3A10N13DCO3T168AC03T250. notes A282/CR3A10N14DCO3TO0OACO3T168. notes A28Z/CR3A10N14DCO3T 168ACO3T250.notes A28z/CR3A10N15DCO3TO00AC03T168.notes A28z/CR3A10N150C03T168AC03T250.notes A28Z/CR3A10N160C03TO0OACO3T168. notes A282/CR3A10N160C03T168AC03T250. notes A282/CR3A10N170C03TO00AC03T168. notes A28z/CR3A10N170C03T168AC03T250. notes A282/CR3A10N180C03TO00AC03T168. notes A28z/CR3A10N180C03T168AC03T250. notes A28z/CR3A18N01DC02TO0OAC03T000.notes A28z/CR3A 18N02DC02TO00AC03T000. notes A28z/CR3A18N03DC02T000AC03T000. notes A28z/CR3A18N04DC02TO00AC03T000. notes A28z/CR3A18N05DC02T000AC03T000.notes A2BZ/CR3A18N06DC02TO00AC03TO00. notes A28z/CR3A18NOTDC02T000ACO3T000. notes A282/CR3A18N08DC02TO00AC03T000. notes A28z/CR3A18N09DC02TO00AC03T000. notes A28z/CR3A18N 10DCO2TOOOACO3TO00. notes A28z/CR3A18N11DC02TO00AC03T000. notes A28z/CR3A18N12DC02TO0OAC03T000. notes A282/CR3A18N13DC02TO00AC03T000. notes A28Z/CR3A18N14DC02T000AC03T000. notes A28Z/CR3A18N15DC02TO00AC03T000. notes A28z/CR3A18N160C02TO00AC03T000. notes A282/CR3A18N17DC02TOOOAC03T000. notes A28z/CR3A18N180C02T000AC03T000. notes A28z/CR3A28N010C1BT000AC1BT142. notes A28z/CR3A28N01DC1BT 142AC02T000.notes A28Z/CR3A28N02DC1BTO0OAC1BT 142. notes A28Z/CR3A28N02DC1BT 142AC02T000. notes A28Z/CR3A28N03DC1BT000AC1BT 142.notes A28Z/CR3A28N03DC1BT142ACO2T000. notes A28Z/CR3A28N04DC1BT000AC1BT142.notes A28Z/CR3A28N04DC1BT 142AC02T000.notes A28Z/CR3A28N05DC1BT000AC1BT 142. notes A28Z/CR3A28N05DC1BT 142ACO2T000. notes A282/CR3A28N06DC1BT000AC18T142. notes A28Z/CR3A28N060C1BT142AC021000. notes A282/CR3A28NOTDC1BTO00AC1BT142. notes A2Bz/CR3A28NOTDC1BT142AC02T000.notes A282/CR3A28N08DC1BT000AC1BT142. notes A282/CR3A28N08DC1BT142AC02T000. notes
aXLIf 1.351

aXLIf1.352

aXLI 1.353

aXLIf1.354

aXLIf1.355

aXLIf1.356

aXLI 11.357

aXLIf1.358

aXLIf1.359

aXLIf1.360

aXLIf1.361

aXLIf1.362

aXLIf1.363

aXLIf1.364

aXLIf1.365

aXL $1+1.366$

aXL If1.367

aXLIf1.368

aXLIf1.369

aXLIf1.370

aXLIf1.371

axLI If 1.372

$\operatorname{axLIf1.373}$

aXLIf1.374

aXLI +1.375

aXLI 1.376

aXLI I 1.377

aXLI 1.378

aXLI 1 1.379

aXLI 1.380

aXLI I 1.381

aXLI 1.382

aXLI 1.383

aXLIf1.384

aXLI 11.385

aXLIf1.386

aXLIf1.387

aXLI $f 1.388$

aXLI 1 1.389

aXLI 1.390

aXLI 1.391

aXLI 1 1.392

aXLI 1.393

aXLIf1.394

aXLI 1.395

aXLI 1.396

aXLIf1.397

aXLIf1.398

aXLI $f 1.399$

aXLI 1.400

aXLI $f 1.401$

aXLIf1.402

aXLI $f 1.403$

aXLIf1.404

eXLIf 1.405

aXLIf1.406

aXLIf 1.407

aXLIf 1.408

aXL I 11.409

aXLI 11.410

बXLIf1.411

aXLI 1.412

aXLI 1.413

aXLI 11.414

aXLI 1.415

aXLIf1.416

aXLI $\$ 1.417$

aXLI $f 1.418$

aXLI $\$ 1.419$

aXLI $\$ 1.420$

7
8
7
8
7
8
8
8
8
8
8
8
8
8
8
8
7
8
7
8
7
8
7
8
7
8
7
8
7
8
7
8
7
8
7
8
7
7
7
7
7
7
7
8
7
7
7
7
7
7
7
7
7
7
7
7
7
7
7
7
7
7
7

Jul 211997 Jul 211997 Jul 211997 Jul 211997 Jul 211997 Jul 211997 Jul 211997 Jul 211997 Jul 211997 Jul 211997 Jul 211997 Jul 211997 Jul 219997 Jul 211997 Jul 211997 Jul 211997 Jul 211997 Jul 211997 Jul 211997 Jul $21 \quad 1997$ Jul 221997 Jul $22 \quad 1997$ Jul 221997 Jul 221997 Jul 221997 Jul 221997 Jul 221997 Jul 221997 Jul 221997 Jul 221997 Jul 221997 Jul 221997 Jul 221997 Jul 221997 Jul 221997 Jul 221997 Jul 211997 Jul 211997 Jul 211997 Jul 211997 Jul 211997 Jul 211997 Jul 211997 Jul 211997 Jul 211997 Jul 211997 Jul 221997 Jul 221997 Jul 221997 Jul 221997 Jul 221997 Jul 221997 Jul 221997 Jul 221997 Jul 211997 Jul 211997 Jul 211997 Jul 211997 Jul 211997 Jul 211997 Jul 211997 Jul 211997 Jul 211997 Jul 211997 Jul 211997 Jul 211997 Jul 211997 Jul 211997 Jul 211997 Jul 211997

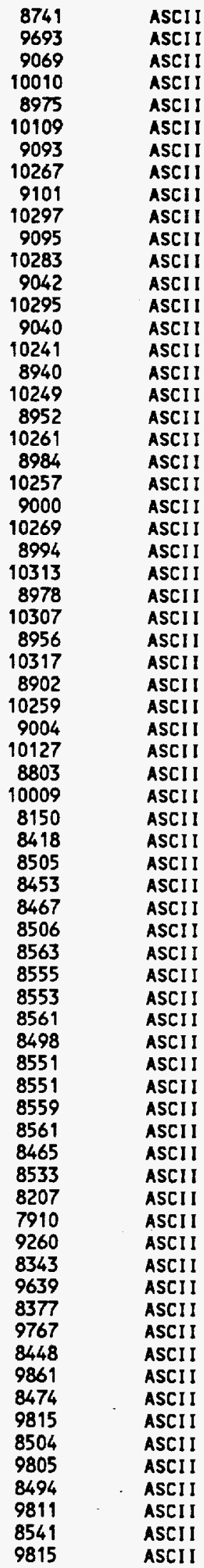


aXLI 1.421

axLIf 1.422

aXLIf 1.423

aXLIf 9.424

aXL1f1.425

aXLI 191.426

aXLI 1.427

aXLIf1.428

aXL1 1 1.429

aXLIf1.430

aXLIf 1.431

aXLIf1.432

aXLIf 1.433

aXLIf1.434

aXL1 1.435

aXLIf1.436

aXL 1 f 1.437

aXLIf 1.438

aXLIf1.439

aXLI $f 1.440$
Jul 211997

Jul 211997

Jul 211997

Jul 211997

Jul 211997

Jul 221997

Jul $22 \quad 1997$

Jul 221997

Jul 221997

Jul 221997

Jul 221997

Jul 221997

Jul 221997

Jul 221997

Jul 221997

Jul $22 \quad 1997$

Jul 221997

Jul 221997

Jul 221997

Jul 221997

$\begin{array}{ll}8545 & \text { ASCII } \\ 9823 & \text { ASCII } \\ 8496 & \text { ASCII } \\ 9801 & \text { ASCII } \\ 8534 & \text { ASCII } \\ 9821 & \text { ASCII } \\ 8478 & \text { ASCII } \\ 9811 & \text { ASCII } \\ 8470 & \text { ASCII } \\ 9817 & \text { ASCII } \\ 8470 & \text { ASCII } \\ 9815 & \text { ASCII } \\ 8432 & \text { ASCII } \\ 9845 & \text { ASCII } \\ 8458 & \text { ASCII } \\ 9859 & \text { ASCII } \\ 8385 & \text { ASCII } \\ 9779 & \text { ASCII } \\ 8253 & \text { ASCII } \\ 9683 & \text { ASCII }\end{array}$

